\title{
AปレI03 088683
}

NATL INST OF STANDARDS \& TECH R.I.C.

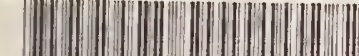

A11103088683

Symposium on Measure/Measurements for th QC100 .U57 NO.456, 1976 C.2 NBS-PUB-C 19

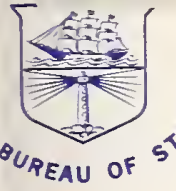

\section{NBS SPECIAL PUBLICATION 456}

\section{U.S. DEPARTMENT' OF COMMEREE / National Bureau of Standards}
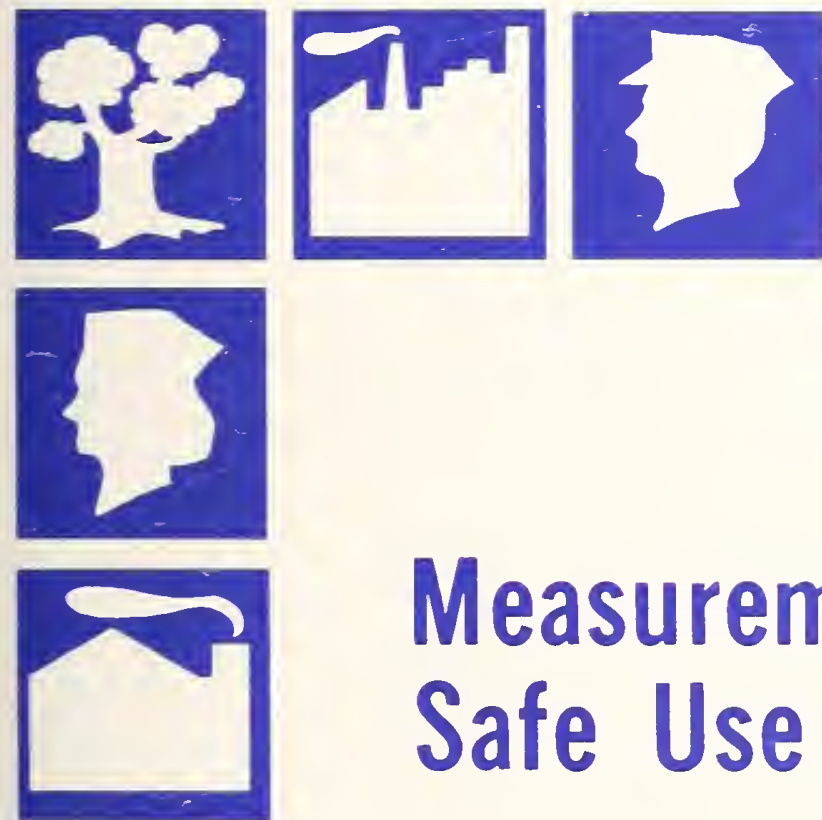

Measurements for the Safe Use

of

Radiation
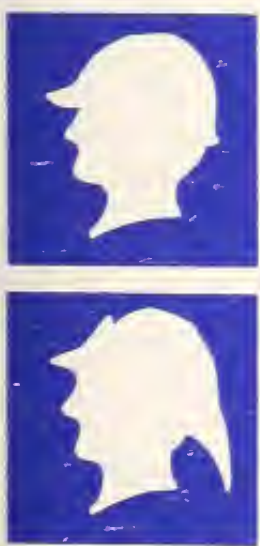


\section{NATIONAL BUREAU OF STANDARDS}

The Nitional Bureau of Standards ${ }^{1}$ was established by an act of Congress March 3, 1901. The Bureau's overall goal is to strengthen and advance the Nation's science and technology and facilitate their effective application for public benefit. To this end, the Bureau conducts research and provides: (1) a basis for the Nation's physical measurement system, (2) scientific and technological services for industry and government, (3) a technical basis for equity in trade, and (4) technical services to promote public safety. The Bureau consists of the Institute for Basic Standards, the Institute for Materials Research, the Institute for Applied Technology, the Institute for Computer Sciences and Technology, and the Office for Information Programs.

THE INSTITU'TE FOR BASIC STANDARDS provides the central basis within the United States of a complete and consistent system of physical measurement; coordinates that system with measurement systems of other nations; and furnishes essential services leading to accurate and uniform physical measurements throughout the Nation's scientific community, industry, and commerce. The Institute consists of the Office of Measurement Services, the Office of Radiation Measurement and the following Center and divisions:

Applied Mathematics - Electricity - Mechanics - Heat - Optical Physics - Center for Radiation Research: Nuclear Sciences; Applied Radiation - Laboratory Astrophysics ${ }^{2}$ - Cryogenics : - Electromagnetics "- Time and Frequency ".

THE INSTITUTE FOR MATERIALS RESEARCH conducts materials research leading to improved methods of measurement, standards, and data on the properties of well-characterized materials needed by industry, commerce, educational institutions, and Government; provides advisory and research services to other Government agencies; and develops, produces, and distributes standard reference materials. The Institute consists of the Office of Standard Reference Materials, the Office of Air and Water Measurement, and the following divisions:

Analytical Chemistry - Polymers - Metallurgy - Inorganic Materials - Reactor Radiation - Physical Chemistry.

THE INSTITUTE FOR APPLIED TECHNOLOGY provides technical services to promote the use of available technology and to facilitate technological innovation in industry and Government; cooperates with public and private organizations leading to the development of technological standards (including mandatory safety standards), codes and methods of test; and provides technical advice and services to Government agencies upon request. The Institute consists of the following divisions and Centers:

Standards Application and Analysis - Electronic Technology - Center for Consumer Product Technology: Product Systems Analysis; Product Engineering — Center for Building Technology: Structures, Materials, and Life Safety; Building Environment; Technical Evaluation and Application - Center for Fire Research: Fire Science; Fire Safety Engineering.

THE INSTITUTE FOR COMPUTER SCIENCES AND TECHNOLOGY conducts research and provides technical services designed to aid Government agencies in improving cost effectiveness in the conduct of their programs through the selection, acquisition, and effective utilization of automatic data processing equipment; and serves as the principal focus within the executive branch for the development of Federal standards for automatic data processing equipment, techniques, and computer languages. The Institute consists of the following divisions:

Computer Services - Systems and Software - Computer Systems Engineering — Information Technology.

THE OFFICE FOR INFORMATION PROGRAMS promotes optimum dissemination and accessibility of scientific information generated within NBS and other agencies of the Federal Government; promotes the development of the National Standard Reference Data System and a system of information analysis centers dealing with the broader aspects of the National Measurement System: provides appropriate services to ensure that the NBS staff has optimum accessibility to the scientific information of the world. The Office consists of the following organizational units:

Office of Standard Reference Ditti - Oifice of Information Activities - Office of Technical Publications - Library - Office of International Relations - Office of International Standards.

\footnotetext{
1 Hcadquarters and Lahoratories at Gailhershurg, Maryland, unless otherwise noted; mailing address Washington, D.C. 20234.

Locited at Boulder. Colorado 80302
} 


\section{- 197 Measurements for the Safe Use of Radiation}

Proceedings of an

NBS 75th ANNIVERSARY Symposium

held at the National Bureau of Standards

Gaithersburg, Maryland, March 1-4, 1976

\section{Edited by}

\section{Sherman P. Fivozinsky}

Institute for Basic Standards

National Bureau of Standards

Washington, D. C. 20234

\section{Sponsored by:}

National Bureau of Standards

American Association of Physicists in Medicine

Conference of Radiation Control Program Directors

Health Physics Society

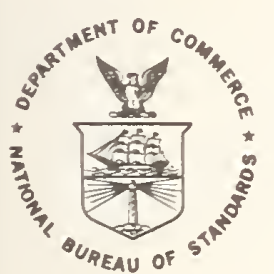

U.S. DEPARTMENT OF COMMERCE, Elliof L. Richardson, Secretary Edward O. Vetter, Under Secretary

Dr. Betsy Ancker-Johnson, Assistant Secretary for Science and Technology

S. NATIONAL BUREAU OF STANDARDS, Ernest Ambler, Acting Director

Issued November 1976 
Library of Congress Catalog Card Number: 76-600060

\section{National Bureau of Standards Special Publication 456}

Nat. Bur. Stand. (U.S.), Spec Publ. 456, 437 pages (Nov. 1976)

CODEN: XNBSAV 


\section{FOREWORD}

The explosive growth in the use of radiation in the major sciences and technologies has created an increasing need for Measurements for the Safe Use of Radiation. While medicine and industry place the greatest demands upon measurements at the present time, it is essential to recognize that radiation in a11 of its forms, non-ionizing as well as ionizing, is impacting our daily lives and our environment in more and more ways everyday. Our concern for safety in the use of such radiation is a matter of greatest importance.

It is most fortuitous that the Symposium on Measurements for the Safe Use of Radiation could be identified with the commemoration of the 75th Anniversary of the National Bureau of Standards. Thereby it places emphasis upon the new and urgent challenges faced by our institution in the years ahead because of the ever evolving increases in the social uses of advancing technology. NBS is gratified by the excellent results of the Symposium and pleased to have shared the sponsorship with the American Association of Physicists in Medicine, the Conference of Radiation Control Program Directors, and the Health Physics Society.

Arthur 0. McCoubrey, Director Institute for Basic Standards National Bureau of Standards 
These are the proceedings of the Symposium, "Measurements for the Safe Use of Radiation" held March 1-4, 1976, at the Nationa 7 Bureau of Standards, Gaithersburg, Maryland. The meeting was attended by about 250 scientists and engineers representing a wide range of private and government institutions with interests in radiation measurement.

The purpose of the Symposium was to draw attention to the rapidly increasing need for high quality measurements of ionizing and non-ionizing radiation, and to provide a forum for discussion of methodology of measurement, quality assurance, standards, and measurement assurance activities.

The program consisted of four invited paper sessions, three contributed paper sessions, three tutorials, and a panel discussion. The sessions divided the program into three broad areas: (1) Standardization and Measurement Assurance, (2) Therapeutic and Diagnostic Radiation Measurements, and

(3) Environmental and Personnel Protection Measurements. A total of 54 papers were presented. The tutorials were offered by NBS staff in parallel with the three contributed paper sessions. They were:

1. Radioactivity Standards and Measurements

2. Dosimetry Standards and Calibrations

3. Laser Measurements

A11 three were very well received, and I wish to thank Dr. W. B. Mann, Dr. R. Loevinger, and $\mathrm{Mr}$. A. Sanders for their cooperation in organizing and presenting, respectively, the three tutorials. In addition, tours of NBS' Center for Radiation Research, and FDA's
Bureau of Radiological Health took place the morning of March 3. I wish to thank Dr. J.E. Leiss and the staff of CRR, and $M r . R$. W. Schneider and the staff of BRH, for making available facilities and time for these activities.

The speech on U.S. Energy Policy delivered by Congressman Mike McCormack of Washington following the Symposium banquet was one of the high points of the meeting. An excerpted version of his talk has been published in the May 1976 issue of "Dimensions," an NBS magazine.

The opening remarks, papers; and panel discussion are printed in the order in which they were presented. To speed publication of these proceedings, all papers were submitted by the authors in camera ready form. No attempt was made to record comments or questions following each oral presentation, nor were the tutorials recorded.

When commercial equipment, instruments, and materials are mentioned or identified in these proceedings, it is intended only to adequately specify experimental procedures. In no case does such identification imply recommendation or endorsement by the National Bureau of Standards, nor does it imply that the material or equipment identified is necessarily the best available for the purpose.

The editor gratefully acknowledges the assistance of the National Bureau of Standards, Office of Technical Publications, and the extensive secretarial assistance of Mrs. Lynette Helfstein in the publication of these proceedings.

S. P. Fivozinsky

National Bureau of Standards 


\section{ABSTRACT}

These proceedings are the compilation of papers presented at the Symposium, "Measurements for the Safe Use of Radiation," held in Gaithersburg, Maryland, March 1-4, 1976. The symposium addressed the present status and future requirements for measurements of ionizing and non-ionizing radiation. Emphasis was placed on the needs for standardization and measurement assurance activities in medical, environmental, and personnel protection applications.

Key Words: calibrations, environment, measurements, medical, radiation, standards. 



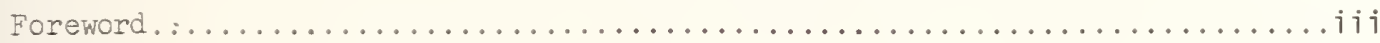

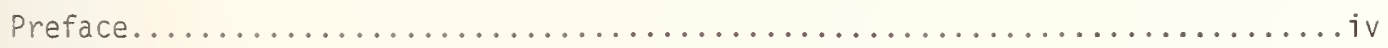

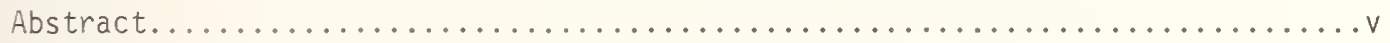

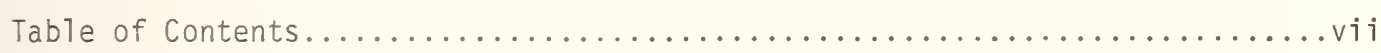

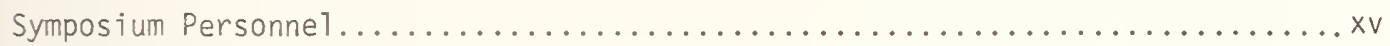

WELCOME

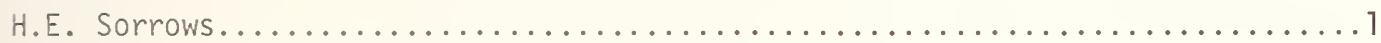

THE NATIONAL MEASUREMENT SYSTEM, THEN AND NOW

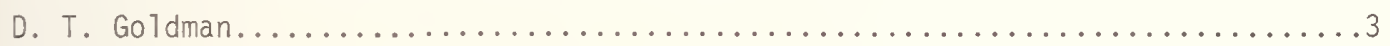

INVITED SESSION I

GENERAL

G. S. Parker, Chairman

IS1-1 Historical Development of the Need for Radiation Protection and Measurements in the United States

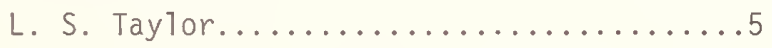

IS1-2 The Standards Laboratory and the Radiation Measurement System

R. S. Caswe11........................

IS1-3 The Federal Regulatory Agency and the Radiation Measurement System

R. H. Schneider...................25

IS1-4 State Responsibilities and the Radiation Measurement System

M. W. Parrott...................

CONTRIBUTED SESSION I

MEASUREMENT SYSTEM

R. Loevinger, Chairman

CS1-1 Ionization Chamber for Absorbed-Dose Calibration J. S. Pruitt and R. Loevinger...........37 
CS1-2 The Development and Application of Monoenergetic $X$-Ray Sources

J. H. Sparrow and C. E. Dick.

CS1-3 The Design of a Mobile Radiation Calibration Laboratory

R. C. Placious and J. C. Humphreys......47

CS1-4 Automated Calibration and Recordkeeping System for X-Ray Survey Instruments

T. R. Ohthaber, F. J. Prevo and

T. L. Miller......................53

CS1-5 Standardization of a Nationwide Gamma Radiation Calibration System

E. F. Williams, Jr., P. V. Barrans and

G. H. Brodie.......................59

CS1-6 A Reliable Calibration Program for Ionizing Radiation Measurement

A. Jacobson.

CS1-7 AIF-NBS Radioactivity Measurements Assurance Program for the Radiopharmaceutical Industry

R. Col1é..........................

CS7-8 ANSI-Quality Assurance Program in Radioactivity Measurements

C. W. Seidel and J. M. R. Hutchinson.....77

CS1-9 Filtered Beams at the NBS Reactor

R. B. Schwartz, I. G. Schroder and

E. D. McGarry.....................83

CS1-10 Neutron Source Calibrations at NBS for Calibration Checks of Neutron Radiation Instruments

V. Spiegei.......................87 
CS1-11 U1trasonic Measurements and Calibration

H. F. Stewart, M. E. Haran and

B. A. Herman.....................91

CS1-12 Methods for the Evaluation and Calibration of Microwave Survey Instruments

M. L. Swicord, H. I. Bassen

and $W$. A. Herman....................99

CS1-13 NBS U1traviolet Radiometric Standards

W. R. Ott........................107

INVITED SESSION II

THERAPEUTIC AND DIAGNOSTIC

RADIATION MEASUREMENT

J. R. Cameron, Chairman

IS2-1 Quality Assurance for Measurements in Therapy

R. J. Shalek, P. Kennedy, M. Stovall,

J. H. Cundiff, W. F. Gagnon,

W. Grant, III and W. F. Hanson..........111

IS2-2 Quality Assurance Measurements in Diagnostic Radiology

M. P. Siedband....................119

IS2-3 Quality Assurance for Measurements in Nuclear Medicine

B. A. Rhodes, W. B. Hladik and J.H. Gallagher.................131

IS2-4 State and Federal Regulatory Measurement Responsibilities Around Medical Facilities

L. H. Lanzl......................139

IS2-5 N.E.X.T. Program--and Radiation Measurements in the State

J.W. Shaver........................... 147 
CONTRIBUTED SESSION II

ENVIRONMENT AND PERSONNEL

PROTECTION

J. M. Matuszek, Chairman

CS2-1 Low Level Environmental Radiation Measurements with a Plastic Scintillation Detector System

A. P. Hull and G. S. Levine. .153

CS2-2 Precise Environmental Gamma-Ray Measurements Utilizing Standard Ge(Li) Detectors

R. M. Keyser, G. V. Wal ford and J. A. Cooper...................159

CS2-3 The Minimum-Detectable-Activity Concept

J. C. Lochamy......................169

CS2-4 use of NBS Mixed-Radionuclide Gamma-Ray Standards for Calibration of $\mathrm{Ge}(\mathrm{L} i)$ Detectors Used in the Assay of Environmental Radioactivity

B. M. Coursey....................173

CS2-5 The Safety and Reliability of Decentralized TLD

Carl-0 Wide11...................181

CS2-6 Error Analysis of Environmental Radiation Measurements Made with Integrating Detectors

G. De Planque Burke and T. F. Gesel1.... 187

CS2-7 Radio-Frequency Electromagnetic Radiation From Portable and Mobile Telecommunication Transmitters

J. F. Thiel.......................199

CS2-8 An Automated Measurement System for Determining Environmental Radiofrequency Field Intensities II

R. A. Tell, N. N. Hankin, J. C. Nelson,

T. W. Athey and D. E. Janes, Jr........203 
CS2-9 The Measurement of Optical Radiations:

Selected Practical Considerations

R. W. Peterson, J. M. Coakley, K. Mohan

and R. James.....................215

CS2-10 X-Ray Leakage from Conventional and High Voltage Electron Microscopes

D. F. Parsons, V. A. Phillips and

J. S. Lal7y.......................223

CS2-11 Intercomparison of Personnel Dosimeters

H. W. Dickson, W. F. Fox and

F. F. Haywood.....................225

INVITED SESSION II I

ENVIRONMENTAL AND PERSONNEL

PROTECTION MEASUREMENTS

W. A. Mills, Chairman

IS3-1 Environmental Radiation Measurements

J. E. McLaughtin..................233

IS3-2 Environmental Measurements and Regulatory Responsibilities

J. M. Matuszek...................249

IS3-3 Environmental Radioactivity Standards

A. N. Jarvis and D. G. Easterly........263

IS3-4 Personnel Monitoring Measurements

R. V. Griffith..................267

IS3-5 Personnel Monitoring Performance Standards

B. H. Weiss........................277 
CONTRIBUTED SESSION III

MEDICAL

N. Suntharalingam, Chairman

CS3-1 Optical Interferometric Measurements of UTtrasonic Radiation and Its Applications to Medicine

M. E. Haran and H. F. Stewart........285

CS3-2 Photon Mass Energy-Absorption Coefficient Air/Medium Ratios for Ionometric Dosimetry $0.1 \mathrm{keV}$ to $20 \mathrm{MeV}$

J. H. Hubbe11.....................299

CS3-3 Electron Dosimetry with Ionization Chambers M. J. Berger, S. M. Seltzer, S. R. Domen and $\mathrm{P}$. J. Lamperti........................

CS3-4 Dose Profiles in Tissue and in Air for Therapeutic Electron Beams

G. Osman. .313

CS3-5 Calorimetric Dose Measurements in Fast Neutron and Cobalt-60 Gamma-Ray Fields

J. C. McDona7d, J. S. Laughtin and

L. J. Goodman.

CS3-6 Scattered Radiation From a Neutron Collimator

F. H. Attix, L. S. August and

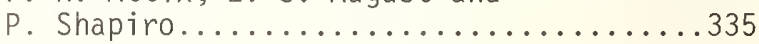

CS3-7 Dose Control in Radiation Therapy

K. R. Kase, B. E. Bjarngard, B. J. Maddox

and G. K. Svensson...................343

CS3-8 Dosimetry Procedures at the Northwest Medical Physics Center

D. Jones, D. Schumacher and

J. Washington. 
CS3-9 Intercomparison of Prescription Dose Delivery in the New England Region

J. S. Blinick, J. Spira, D. Cail and

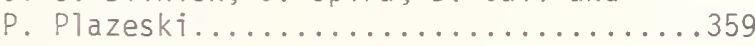

CS3-10 Electron-Therapy Dosimetry

M. Ehrlich and P. J. Lamperti.........365

CS3-11 Thyroid Monitoring and Minimizing I-125 Uptake

M. M. Gabel, K. W. Price and

G. R. Holeman.....................

CS3-12 Attenuation Phantoms for Patient Exposure Measurement During Radiographic Examinations Utilizing Phototiming Techniques

Pei-Jan Paut Lin....................381

CS3-13 Effects of Measuring Apparatus on X-Ray Attenuation Measurements

T. J. Morgan, L. B. Smith and

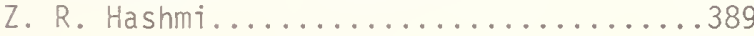

CS3-14 kVp Measurement for Dental X-Ray Units M. S. Moroz and J. A. Vukan............395

INVITED SESSION IV

STANDARDIZATION AND MEASUREMENT

ASSURANCE

R. S. Caswel 1, Cha irman

IS4-1 National Ionizing Radiation Standards

J. E. Leiss........................403

IS4-2 Non-Ionizing Electromagnetic Radiation Standardization

R. C. Baird and A. A. Sanders.........417 
PANEL DISCUSSION

E. D. Trout, Moderator

G. S. Parker

J. R. Cameron

W. A. Milis

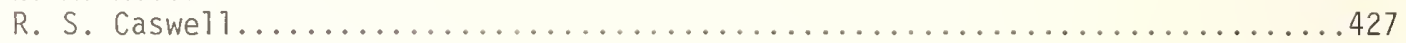

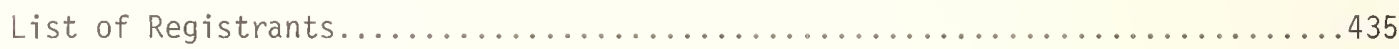

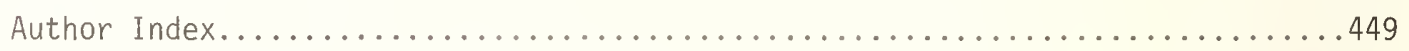


SYMPOSIUM PERSONNEL

SYMPOSIUM CHAIRMAN

James M. Wyckoff

National Bureau of Standards

PROGRAM COMMITTEE

Sherman P. Fivozinsky, Chairman

National Bureau of Standards

Richard Bluemle

South Carolina Department of Health

Paul Carson

University of Colorado Medical Center

Paul Eastvold

I11inois Department of Health

J.M. Robin Hutchinson

National Bureau of Standards

Thomas L. Johnson

Naval Research Laboratory

Robert Loevinger

National Bureau of Standards

Aaron Sanders

National Bureau of Standards

N. Suntharalingam

Thomas Jefferson University Hospital

Edward J. Vallario

U.S. Energy Research and Development Administration 


\section{.}




\title{
WELCOME
}

\author{
H. E. Sorrows \\ Associate Director for Programs \\ National Bureau of Standards
}

Ernie Ambler, the Director of the Bureau of Standards, sends his regards and regrets that he can't be with you. His response to the wishes of higher authority is my good fortune for it is certainly a privilege and an honor for me to welcome you.

This conference kicks off a series of special events designed to commemorate and to celebrate the 75th anniversary of the Bureau. Conferences like this one keep the Bureau as youthful and timely as ever. During the remainder of 1976 we are holding a number of symposia, lectures, and events which are appropriate for our birthday celebration and are designed to be beneficial to society in general and to the science and technology communities in particular.

In addition to being the keynote conference for our birthday celebration, this meeting presents an opportunity to provide a forum for the transfer of science and technology, one of the most important services of the Bureau of Standards. We are particularly pleased to join with the American Association of Physicists in Medicine, the Conference of Radiation Control Program Directors, and the Health Physics Society in spunsoring this meeting.

There is a third and even more self serving reason for our interest in this conference and that is our desire to seek better ways to provide our services to the nation. I only need observe that the theme of this conference "Measurements for the Safe Use of Radiation" is the objective of our radiation research and to remind you that the National Bureau of Standards would like to work with you in providing a system of measurement to the accuracy required, including the means for measurements to be made correctly and to be traceable to national standards, if necessary.

You have a tremendous responsibility and opportunity because the government and the informed public are looking to nuclear physics and to radiation technology for solutions to many of the nation's most pressing problems. Energy and health care are center stage; however, there are many other major problems and in all cases the solutions must be both safe and economic. A practical system of measurements will go a long way toward achieving optimum solutions.

I wish you a successful Symposium. You have all the ingredients. You have a timely topic, an excellent agenda, an inspiring banquet speaker and you even have beautiful weather. It's a pleasure to have you here. 



\section{T. Goldman}

Deputy Director, IBS

National Bureau of Standards

As an introductory talk to this conference, I would like to take the opportunity to introduce the concept of the National Measurement System which had been introduced by $\mathrm{Dr}$. Robert Huntoon about ten years ago. This is a concept by which we attempt to analyze the relationship of our activities at the National Bureau of Standards with needs of our user community on the outside. A reasonable definition of the National Measurement System is the network of measurement standards, techniques, procedures and standardizing laboratories in all of the measurement communities which generate the base on which day to day measurement operations are conducted at all levels of accuracy in the United States. This includes the international agreement on the basic SI units, maintained at the national standards laboratory, the National Bureau of Standards, and the various mechanisms for transferring the measurement capabilities maintained at the Bureau of Standards to the users who constitute the major portion of the measurement system.

Since this is the initial conference of the Bicentennial series at the National Bureau of Standards and is also the first of the symposia associated with the NBS 75th anniversary, I thought that perhaps a little historical review of standards might be in order. At the building of the pyramids the cubit, the biblical standard of length, was defined as the length between the tip of the middle finger and the elbow. Skipping over several millennia we find another lawful definition of unit of length, the rod, which was defined as the total length of the left feet of 16 men lined up as they left church on Sunday morning. The rationalists of the 18th century chose to define the meter as a new unit based on the decimal system. In fact, Jefferson tried to introduce this concept into the United States 200 years ago. However, as late as 1830 , the incipient national measurement system of the United States was somewhat limited in its extent and limited to weights and measures for commerce and taxation, especially for customs. The concept of utilizing the metric system of units was examined several times between the founding of the country and about 1830, and in each case for a variety of different reasons it was found not acceptable to the body politic at the time. Not only was the metric system not accepted but even the idea of compatible measurements although mentioned and featured in the Constitution appears not to have been used in practice. In fact, it's reported that in the city of Brooklyn during the 19th century there were several different standard feet and when you measured a lot you chose the unit of length that was most convenient for you.

In 1830 , there was no international agreement as yet, a national standards organization did not exist, and national standards didn't exist. However, to some extent there was a national agreement in terms of use of different instruments, especially weighing and length measuring instruments. Seventy years later as a result of the success demonstrated by our sister organization, the Physikalisch Technische Reichsanstalt, the German national standards laboratory which had been founded about 25 years earlier, 
a movement to establish the Bureau of Standards was undertaken. The Office of Weights and Measures was the building block upon which the National Bureau of Standards was established. Twentyfive years earlier the International Bureau of Weights and Measures had been established, and the idea of the metric system had been agreed upon formally by the United States (1875), so at least we were on our way to defining a national measurement system which could be used in practice. Need for it could be demonstrated as the electric industry grew up. It really made a difference if different voltages were measured by different things. No doubt, potential safety hazards in the uncertainty in the measurement of voltage was a factor, but commerce in the supply of electricity and electrical equipment was also important. However, there still was no accepted measurement system concept for many different units. Randy Caswell will be talking later this morring on that specific portion of the system which we would call the radiation measurement system. In 1901 radiation had just been discovered, but after the founding of the Nationai Bureau of Standards, a significant fraction of the initial publications of the Bureau of Standards which appeared in the form of handbooks was indeed devoted to the measurement of radiation quantities.

The National Bureau of Standards functions as the primary mechanism for linking the international standards generally as maintained at the International Bureau of Weights and Measures in Paris to the users in our country. We transfer that measurement capability by a variety of different methods: instruments, calibration services, measurement techniques or, in some cases, providing the numerical information which is needed for the transfer. The measurement system is composed of a variety of different users, including consumers, industrial organizations and the government. It is our responsibility to meet the needs of the system now and in the future.

To summarize, our job is to make sure that those who need measurement services, those who perform measurements, can do so with accuracy and known precision, reliably and compatibly and at reasonable cost. Measurements in one part of the country can be transferred to another part with assurance as to what the uncertainties are. In other words, our job at the Bureau of Standards is to service the National Measurements System and to make use of this expertise in the solution of specific national problems. With this introduction I am looking forward to hearing the discussion of one particular portion of the measurement system which will be the major topic of the remainder of the conference. Thank you. 
IIISTORICAL DEVELOPMENT OF THE NEED FOR RADIATION

PROTECTION AND MEASUREMENTS IN THE UNITED STATES

Lauriston S. Taylor

National Council on Radiation Protection and Measurements

Washington, D. C. 20014

Early means of radiation measurement included photographic film, chemical compounds, selenium and ionization of air, and all were uncertain and inaccurate until about 1920. By 1930, ionization measurements between different laboratories and countries agreed within 2 or $3 \%$. Also, quantitative limits for the exposure of radiation workers had been proposed and based on air ionization measurements - the roentgen. Accuracy requirements are $\pm 5 \%$ for radiation therapy but to maximize the benefits to patients greater attention must be given to measurement assurance or traceability. Accuracy for protection purposes will vary with the dose levels involved, for example, $\pm 10 \%$ at maximum permissible dose levels for radiation workers to perhaps $\pm 200 \%$ at the level of 10 mrems in a year for the population.

Since the purposes of this Conference center around problems of the measurement of ionizing radiation, I shall deal rather briefly with some of the areas of radiation measurement and the conditions which lead to their need. In this connection, one cannot avoid some speculation or even argument about the required accuracy for particular applications, but to put our present-day problems and our present-day thinking into some kind of mutual perspective, I believe it will be desirable to go back to the earliest steps taken in the radiation measurement field. (Incidentally, when I use the word radiation by itself, I refer to ionizing radiation.)

\section{The Purpose of Measurement}

Any kind of measurement, whether it be physical or biological, must have a purpose, and this may be very specific or fairly abstract but one just shouldn't run around measuring things for the sheer joy of it. Any measurement implies the definition of appropriate quantities which are to be measured and the appropriate units in which to express those measurements. Again if there were no specific purpose in a measurement, you would not need to worry about proper quantities and units. In research, in the clinical laboratory or in medicine, there may be any or all of a number of purposes in performing measurements. First, one has to be able to describe his own results so that others can determine what had been done, and how they could use it. Secondly, would be the opposite, where someone else had done some work and you wish to compare yours with it. Thirdly, would be the use of measurements to tie experiment to theory or the other way around. Physical research is at its best when an experiment suggests a theory that in turn suggests more experiments that test and improve the theory. While at times, some very crude experiments can be importantly indicative, the new offshoots of some of our theoretical developments require an accuracy that taxes our measurement capabilities to the extremes. In many instances, it is the ability to "measure the next decimal place" that distinguishes between two theoretical positions.

Measurements of any kind must preferably be made within some unified system of quantities and units. We have in the past used multiple systems and up to a point this was satisfactory since their interrelationships were known precisely. The main problem with different systems is likely to be one of convenience rather than fundamental significance. In the early history of radiation measurement, there were systems of measurement which defied the establishment of clear interrelationships, but these were supplanted by better systems as our scientific understanding of the problems developed along with the associated technology.

In the medical field, we have the current situation where quantities and units have been developed over the years and for the most part are technically sound but do not fall within the SI system of quantities and units. Therefore, steps are being taken to eventually phase them out. However, this is a matter centering mainly around uniformity of the system and certainly not the desire or convenience of the medical profession. There is an established relationship between the units used in 
radiology and those in the SI system and I suspect that nobody outside of the biomedical field would suffer anything but some small inconvenience in allowing continuance of the old system. But we must not disturb the purists.

The required accuracy of measurement of radiation depends to a considerable extent upon the purpose to which the measurements will be applied. Our technology places limitations upon accuracy, but it is usually found that by the time greater accuracy is required, our technology is on hand to accomplish it. It is usually the improvements in technology that create the new needs and so in most instances they go hand in hand.

It is all very well to be able to measure a quantity with high accuracy in, let us say, a central standards laboratory such as the NBS. However, it would serve no purpose if the NBS standards could not be intercompared with the standards in other laboratories and could not be applied through secondary instruments to applications in the field. Since there may be a number of steps between the measurement in a standards laboratory and a measurement in, let us say, a hospital, it is important that there be a high level of assurance that the end measurements carry the required accuracy. We thus have a direct necessity for what would be called "traceability" or "measurement assurance". Measurement assurance can be critical for a variety of reasons including commerce and manufacturing, safety or protection of people, legal compliance with regulations, medical applications, research - just to name a few.

The purposes of this conference cover all of these points and probably more, but to see where we are, it may be helpful to review some of the early background to see our progress: where we have been, where we are now, and what may be some of the problems that lie ahead.

This exercise will certainly help us preserve our sense of humor about the measurement problem and give us some feel for some of the things that are unrealistic. The program of this conference should constantly keep in mind what the real problems are and what are the problems that are of little or no significance and hence may be weeded out.

\section{Early Starts in the Measurement of Ionizing Radiation}

On the question of early radiation measurements, I shall deal primarily with problems in this country and more especially with those centering around the work of the National Bureau of Standards. That seems not inappropriate in view of the nature of this conference. Moreover, it might be pointed out that the Bureau of Standards has had direct involvement to one degree or another for over 60 years and, beginning nearly 50 years ago, has played a dominant role in the field of ionizing radiation protection and radiation measurement.

Measurement programs, relating primarily to the evaluation of sealed sources of radium, began essentially simultaneously in the period of 1912 to 1913 at the world's three major National Standards Laboratories - the National Physical Laboratory in England, the Physikalischtechnische Reichsanstalt in Germany, and the National Bureau of Standards in the USA. The trigger for these starts was probably the Brussels Conference on Radiation Research held in 1910. As a result of this, an international standard of radium was deposited at the Bureau International des Poids et Mesures. Prior to this, "accurate" measurements of radium had been made in Vienna and comparisons between there and the BIPM provided the first traceable tie between the two.

In al1 of the national laboratories, the initial and primary purposes of the radiation measurements had been to evaluate the amounts of radioactivity in sealed radium preparations either in terms of milligrams of radium element or millicuries of activity. This was primarily a service to commerce, since the radium was then so expensive and so easily susceptible to deceptive practices. Of secondary interest, was medical dosimetry which for many years, beginning around 1915, was described in terms of milligrams or millicuries of radioactivity with a given filtration, a given distance, and a given time.

The first radium measurements at the National Bureau of Standards were carried out by $N$. E. Dorsey who continued with the work until the early 1920's, by which time he was suffering noticeable injury to his hands from handling the preparations. About 1925, a more extensive radioactivity program was started under Dr. L. F. Curtiss. This 
also consisted primarily in the calibration of sealed radium sources for commerce and medicine, but the work was on a very much stepped up scale because of the increased availability of radium. At that time, the radium program was a part of the Atomic Physics Section which in turn was in the Optics Division (after all, it was all radiation). Various researches with radioactivity were carried out at the same time.

$\mathrm{X}$-Ray programs started at all three of the National Laboratories during the war years, but these were limited mostly to the use of $x$ rays for inspecting objects and materials, rather than studies of the $x$ rays themselves. Probably the first program of $x$-ray dosimetry was undertaken at the PTR in the early $1920^{\prime} \mathrm{s}$. The NPL and the NBS began their programs in 1927. All three of these programs were directed toward development of standards and procedures for measuring $x-$ rays in connection with their therapeutic applications. Preceding this there had been a considerable amount of research on the measurement of $x$ rays carried out for the most part in universities.

The first proposal for a physical unit of measurement of $x$ rays was made by Villard in 1908, and his proposal was essentially the same as the definition of the roentgen adopted twenty years later. Friedrich and Kroenig proposed a physical unit of measurement in 1918, which was also essentially the same as Villard's proposal. In 1914 and 1922, Duane, in this country, using the same description for a unit, built a parallel plate ionization chamber which was proposed as a standard for $x$ rays. In 1921, Solomon in France described a unit known as the "Solomon $\mathrm{R}^{\text {" which was }}$ essentially a description of an ionization chamber system calibrated with a given radium preparation.

Behnken, in 1924, at the PTR, built a cylindrical pressure ionization chamber designed to measure in terms of the unit proposed by Villard and later by Friedrich. This chamber was abandoned because of measurement difficulties and replaced by a much largex cylindrical ionization chamber operating at atmospheric pressure which continued indefinitely in use. Glasser, in this country, in 1925, built a simple parallel plate ionization chamber and also developed a thimble chamber system, the outgrowth of which is still the primary medical $x$-ray measuring device in use in the United States. That was the general state of affairs preceding the $x$-ray measurement program started at the NBS in late 1927.

\section{Measurements for X-Ray Standards of Dose}

It has already been noted that by about 1925 there were at least three systems of $\mathrm{x}$-ray standards which were beginning to become recognized. There was the Friedrich Standard, a free air ionization chamber of cylindrical design; the Duane Standard, a free air chamber of parallel plate design; and the PTR chamber which operated under considerable air pressure and was also cylindrical in design. The first attempt to inter-compare standards was made by Behnken in 1927, at which time he brought to this country a thimble chamber and an electroscope which had been calibrated in Berlin against his pressure chamber. As a result of his measurements an agreement of 0.7 percent was given for the comparisons with Duane. A "large" discrepancy apparently existed between Behnken and Glasser. The NBS Standard was just being constructed at the time, and no comparisons were made.

By 1928, the NBS had completed its first standard free air ionization chamber of a parallel plate type. This was substantially different from the one proposed earlier by Duane, from which, on its face, one would expect substantial errors. Comparisons made between the NBS and Duane chambers in Boston in 1928 showed that indeed there were large differences of the order of 25 percent, but the comparison system was so indefinite that attempts were not made to track down the sources of difficulty. During the same year comparisons between the NBS and the Glasser standard showed close agreement. Both of these comparisons were just part of the day's work and have never been published.

In 1931, a more sophisticated comparison of standards was made by Taylor between the NBS, NPL, PTR, and Solomon. These employed for the first time the very much smaller parallel plate free air ionization chamber using a guarded electric field so as to reduce its size and weight. The initial difference between the NBS and NPL standards was some six percent. Most of the discrepancy was introduced because of an error in the NPL diaphragm. After correcting for various established differences, the agreement was of the order of 0.4 percent. 
At the Physikalisch-technische Reichanstalt, Behnken had his new cylindrical open air ionization chamber and agreement between that and the NBS chamber was .35 percent. These agreements look deceptively good, because such accuracy at that time was only barely within the limits of our technological competence.

It is interesting to note here that in about 1927 or '28, Behnken had discovered that their pressure ionization chamber was in substantial error because of ion recombination at high pressures. They quietly changed from the pressure chamber to a free air chamber without any official announcement. This probably accounted for the original large difference between the PTR and the Glasser Standards, and the agreement with the Duane Standard.

Comparisons with Solomon could not establish levels of agreement but were in essence comparing the magnitude of his measuring unit with that of the other laboratories. It turned out that the ratio varied from 2.1 to 2.3 over the energy range studied. Following these comparisons, Solomon adopted the same chamber that was used by the NBS.

The next major intercomparisons were carried out by H. O. Wyckoff in 1953 between the NBS and NPL. Considerable sophistication of the instrumentation and overall technology had taken place during the intervening years, but basically it was still a comparison between free air ionization chambers. Whereas in the earlier comparisons and measurements there had been talk about agreements within fractions of a percent, no one really believed that the measurements were that reliable. However, by the $1950^{\prime} \mathrm{s}$, the situation was different and when agreements were established, there was much higher confidence in their significance. Also, it might be pointed out that between 1931 and 1953 it had been found that the energy range of the NBS chamber at the earlier time was not sufficiently extensive and later measurements showed degrees of energy dependence which could not have been predicted in 1931 but which were clearly established by 1953.

\section{Measurements for Radiation Therapy}

The main drive for the development of radiation measurement standards came about through the recognized need for accurate dosimetry in the applications of radiation to patients. The problem became more acute with the advent of the Coolidge hot-cathode $\mathrm{x}$-ray tube, which by the early 1920 's promised to permit therapy up to $200 \mathrm{kV}$. Therapy at lower energies had been practiced for some time and the clinical attempts at dose control involved a variety of measurement means. For example, bariumplatino-cyanide pastiles were used for many years; these change color in accordance with the amount of radiation received. Another technique involved the use of photosensitive film or paper. Both of these techniques involved matching of color or darkening. Another system employed selenium cells which were photosensitive but had peculiar operating characteristics, at best.

Along with these older methods was a wide variety of ionization chambers which, while suitable for control purposes within a given clinic, defied any kind of real comparison because of the lack of a central comparison point, let alone a recognized standard. It is surprising, in looking back over this period extending up until about the middle of the $1920^{\prime} \mathrm{s}$, to see how relatively closely all of these various measurement systems were to each other in spite of the wide variations that existed relative to their fundamental operation.

Attention was directed to all of these problems at the First International Congress of Radiology held in London in 1925, and it is largely for that reason that activity in the area of radiation measurement sprang up quickly all over the world during the following period.

The big push in this country started in 1925 through action of the Radiological Society of North America that organized an $\mathrm{x}$-ray standardization committee. After reviewing the current situation, one of its first recommendations was to urge the start of a major program in the field of $x$-ray standards and measurements at the National Bureau of Standards. This was promoted through congressional pressures and by considerable correspondence with $\mathrm{Mr}$. Hoover who was then secretary of Commerce. The fruits of their efforts were shown by a lineitem appropriation in the budget for the National Bureau of Standards which was to become effective in July 1927.

The next major step took place at the Second International Congress of Radiology in 1928 at which time a definition of the roentgen was adopted. In the meantime, standards programs were well underway in the three major National Standards Laboratories 
as we1l as at numerous other points. Although several minor modifications in the wording were made later, particularly to insure its adoption to higher energy radiation, the definition of the roentgen remained essentially the same for the next 30 years.

With these steps having been taken, and accompanied by the development of reliable portable instruments, mostly using thimble chambers, it was then possible to rely on a central standard system for the uniformity of procedures in radiation therapy. The practical accomplishment of this has depended to a major degree upon the traceability of the measurements from the standards laboratory to the individual clinic, back to the standards laboratory, and then out to other clinics.

As far as clinical dosimetry is concerned, the overall system has been fairly good for many years - better in some areas than others. However, the system has not been good enough because in therapy, more than all other applications, high accuracy and traceability is essential to the best welfare of the patients involved.

As of today, while the problems remain somewhat the same in principle, they are often technically more difficult and complicated. To begin with we are dealing with higher energy photon as well as particle radiation. To be of ultimate clinical value there has to be a capability of comparability between the clinics and between the measurements with various other kinds of radiation.

In addition to the different radiations themselves there are now many different measurement techniques that may be used, such as those involving photoluminescence and thermoluminescence in addition to ionization chambers having special energy responses deliberately built into them.

To be used most effectively in the clinic, all of the radiations and all of the measurement systems will have the same accuracy requirements as the best we have achieved under the simpler systems in the past. This means that their errors must be no greater than five percent at the surface of the patient and probably should be better than that. The achievement of this level of accuracy requires a high degree of measurement assurance or traceability.

\section{Measurements for Radiation Protection}

The first tolerance dose for $x$-ray workers - a dose then considered to be without harm - was proposed by Mutscheller in 1925. It was described as one tenth of a skin erythema dose to a worker in a year. In turn, the erythema dose was described as the amount of radiation required to produce a defined reddening of the skin when delivered in one treatment. The traatment was described in terms of tube current and voltage, distance, time, and size of irradiated field. This was all based on Mutscheller's observation that a few $x-r a y$ workers who had been exposed for several years to radiation calculated to be about $1 / 100$ of an erythema dose over a one month period, showed no effects. He then estimated that since it would take some 8 years to accumulate an erythema dose, a tenth of an erythema dose in a year could be tolerated with a comfortable margin of safety.

In 1927 a survey established that a skin erythema dose was equivalent to $550 \pm 150$ roentgens ( 1927 PTR roentgens); thus $1 / 10 \mathrm{SED} / \mathrm{yr}$ amounted to $55 \mathrm{r}$ in a year. In 1934 the NCRP rounded this figure down to $0.1 \mathrm{r} /$ day or about $25 \mathrm{r}$ in a year. Later, using essentially the same data, the ICRP rounded the value down to $0.2 \mathrm{r} /$ day. No deleterious effect has ever been found for any such exposures. In 1949 the NCRP again lowered the maximum permissible dose for radiation workers (formerly tolerance dose) to $0.3 \mathrm{r}$ in a week ( $15 \mathrm{r}$ in a year). At the same time it recommended a level of $1 / 10$ of that for the general population. In 1956 the ICRP and NCRP further lowered the maximum permissible dose for radiation workers to 5.0 rem in a year and $1 / 10$ of that or 500 millirems for the general population.

Note two things: (1) no effect on man has ever been observed for exposures at any of the permissible levels used since 1925, and (2) there can be no ascribable accuracy to a permissible dose level.

Today there are discussions leading to still further reductions of the dose limits for the population; dose limits in the range of 5 - 25 millirems in a year are being considered. The question: what would be reasonable requirements for the accuracy of measurements made at these very low doses? Another question: to have some meaning, would not everyone need to be monitored continuously? 
Bear in mind that people who live in Bethesda - altitude 400 feet - receive at least 5 mrem a year more than do people living in downtown Washington. With such large normal variations in environmental radiation, is it necessary that measurements of the dose to the population be measured with an accuracy of say $+10 \%$ ? Or perhaps $\pm 200 \%$ ? Let us not completely lose our sense of humor.

A different set of accuracy criteria may well apply in a civil defense situation. Action decisions will almost certainly depend to a major extent upon radiation levels where people are and where they might move to. Yet the biomedical consequences of any of the exposures are not known and for all practical purposes must be assumed. Once assumed, they must be treated as real and measurement accuracy assigned accordingly; when the expected doses are relatively small - say a few $r$ in a day some laxity in accuracy may be tolerated if necessary. But at high doses where triage must be practiced to save the maximum number of people within the resources available, maximum accuracy is called for. Yes, even though you do not know the lethal dose limits.

\section{Measurements for Regulatory Purposes}

Measurements and measurement accuracy for regulatory purposes seem to fall outside of the normal scheme of laboratory

practices. Actually, it would seem that regulatory practices should be for one or both of two situations: (1) a strict control over the design intentions for a facility that may have the potential for causing injury to persons, and (2) a control program to assure that injuries are not being caused to persons.

The first is a rational approach to the problem provided it is carried out reasonably. Unfortunately (1) and (2) are rarely separated in spite of the fact that in the field of ionizing radiation the control levels that have been used for the past 40 years have been so low that no deleterious effects have ever been found for persons exposed within those limits.

I sometimes think that the real regulatory purposes may, consciously or unconsciously, be to assure compliance for compliance sake; surely not just to protect people.
The traceability and accuracy requirements for regulatory compliance programs must engender the strictest of all measurement programs. Regulations carry the force of law and associated penalties. But the law has no sense of humor; a radiation worker exposed to 4.995 rems in a year is deemed to be "safe" whereas another exposed to 5.005 rems in a year is presumed to have been injured. Now this can, of course, be regulated. All that is needed is instrumentation that can measure the workers' dose with an accuracy of $\pm 0.1 \%$. The situation becomes a bit more sticky when compliance necessitates a similar accuracy for measurement of, say, 5 or even 50 millirems in a year.

I have obviously exaggerated the situation to make a point. On the other hand, I have indeed encountered "inspectors" in the past who would see no difficulty with the problem I have posed. If they had a meter that they thought they could read to 5.005 rem they would accept the reading without question. "That's the Law" and I repeat, the law rarely, if ever, has a sense of humor.

Let us consider what may be reasonable and rational requirements for accuracy and traceability. The following may be suitable for discussion purposes but I do not pretend to necessarily recommend them; they are based mainly on the significance of the exposures involved:

(1) For occupational exposures near the upper limit of 5 rem in a year --- \pm 10 to $20 \%$.

(2) For occupational exposures of less than $1 / 2$ the upper limit ---- \pm 25 to $50 \%$.

(3) For non-occupational exposures of less than 25 mrem in a year ---factor of 10 .

(4) For non-occupational exposures of the order of 100 mrem in a year ---- factor of 3 .

(5) For non-occupational exposures of the order of 500 mrem in a year - $+50 \%$. 
My message:

1) Assure the existence of basic standards of the necessary accuracy.

2) Assure the existence of transfer standards of the necessary accuracy.

3) Develop adequate and reliable field instruments.
4) Develop adequate means for calibrating field instruments.

5) Assure that at a11 times any instrument reading anywhere can be traced to its basic calibration source.

6) In measurements anywhere and at any time, don't lose your sense of humor. 

In connection with a broad NBS study of the National

Measurement System, the measurement system for ionizing radiation has been investigated. Eight classes of radiation users were considered: medical, nuclear power, industrial radiation processing, defense, environmental, science, chemical analysis, and miscellaneous radiation applications. System structure including regulators, standards laboratories, manufacturers, as well as final users of radiation was studied.

Several common threads were observed in these studies for the various classes of users: (1) While there is often a need for new NBS standards where no standards exist, where NBS standards do exist their accuracy is generally sufficient for present needs. (2) There is a great need for measurement assurance, especially where regulatory requirements are involved. (3) A need exists to help users with training, convenient laboratory standards, and handbooks for guidance. In other cases, new or augmented institutional structures within the system (e.g. secondary standards laboratories) are needed.

(Ionizing radiation; measurements; standards, uses of radiation)

This paper will be primarily concerned with the measurement system for ionizing radiations. In the paper by Dr. Leiss, "National Measurement Standards for Ionizing Radiation", the questions of standards laboratory standards, dissemination of standards, and measurement assurance will be considered at greater length. The present contribution is based on a study carried out as one of a number of studies of various aspects of the measurement system by groups in many parts of the National Bureau of Standards. In this study of the measurement system for ionizing radiations, I should like to acknowledge the great help given by many members of the staff of the Center for Radiation Research, to whom I am much indebted.

\section{Purpose of the Study}

The objectives of the study were: (1) to understand how the radiation measurement system works in the U.S.; (2) to perform a kind of market research, contacting the customers of NBS services, learning their needs, and evaluating how well NBS or other organizations are filling those needs;

(3) to identify the needs of the measurers of radiation, be they calibration services, training, measurement assurance round robins--whatever; and (4) to develop National Bureau of Standards program responses to those needs.

Ionizing radiation is a convenient (but not precisely accurate) term used to describe a class of energetic radiations such as $x$ and $y$ rays, electrons, radioactivity, neutrons, and numerous other nucleons, nuclei, and mesons. In the measurement of ionizing radiation we are concerned with a rather large number of radiation quantities, some of which are listed in Table 1 with the traditional unit and the customary SI unit. However, the study was done, not on the basis of radiation quantities and units, but rather on the basis of radiation user communities, some of which are listed in Table 2. In each one of these main categories of radiation users, different groups of concerned organizations are grouped into the activity classes shown for the purposes of the study. The largest user categories are the medical community and the nuclear power community. Using the medical community as an example, we can list four direct users (or uses) of radiation: (1) radiation therapy, 


\begin{tabular}{|c|c|c|c|}
\hline Radiation & Quantities & $\begin{array}{l}\text { Uradional } \\
\text { Unit }\end{array}$ & $\begin{array}{l}\text { lustomary } \\
\text { SI Units }\end{array}$ \\
\hline \multirow[t]{4}{*}{$X$ and gamma rays } & Exposure & roentgen & coulomb kg ${ }^{-1}$ \\
\hline & Absorbed dose & rad & joule $\mathrm{kg}^{-1} \mathrm{a}$ \\
\hline & Energy spectrum ${ }^{\mathrm{C}}$ & $\mathrm{cm}^{-2} \mathrm{MeV}^{-1}$ & $\mathrm{~cm}^{-2}$ joule $\mathrm{e}^{-1}$ \\
\hline & $\begin{array}{l}\text { Energy flux density } \\
\text { (beam power density) }\end{array}$ & $\mathrm{MeV} \mathrm{cm} \mathrm{cm}^{-1}$ & watt $\mathrm{cm}^{-2}$ \\
\hline \multirow[t]{3}{*}{ Electron beams } & Absorbed dose & rad & joule $\mathrm{kg}^{-1 \mathrm{a}}$ \\
\hline & Energy spectrum ${ }^{c}$ & $\mathrm{~cm}^{-2} \mathrm{MeV}^{-1}$ & $\mathrm{~cm}^{-2}$ joule $\mathrm{e}^{-1}$ \\
\hline & Beam current and power & ampere, watt & ampere, watt \\
\hline \multirow[t]{3}{*}{ Radioactivity } & $\begin{array}{l}\text { Activity (nuclear trans- } \\
\text { formation rate) }\end{array}$ & curie & $s^{-1} b$ \\
\hline & $\begin{array}{l}\text { Emission rate (particle } \\
\text { or photon) }\end{array}$ & $s^{-1}$ & $s^{-1}$ \\
\hline & Power & watt & watt \\
\hline \multirow[t]{7}{*}{ Neutrons } & Emission rate & $s^{-1}$ & $s^{-1}$ \\
\hline & Fluence & $\mathrm{cm}^{-2}$ & $\mathrm{~cm}^{-2}$ \\
\hline & $\begin{array}{l}\text { Flux density (fluence } \\
\text { rate) }\end{array}$ & $\mathrm{cm}^{-2} \mathrm{~s}^{-1}$ & $\mathrm{~cm}^{-2} \mathrm{~s}^{-1}$ \\
\hline & Energy spectrum ${ }^{\mathrm{c}}$ & $\mathrm{cm}^{-2} \mathrm{MeV}^{-1}$ & $\mathrm{~cm}^{-2}$ joule $\mathrm{e}^{-1}$ \\
\hline & Absorbed dose & rad & joule $\mathrm{kg}^{-1} \mathrm{a}$ \\
\hline & Kerma & rad & joule $\mathrm{kg}^{-1} \mathrm{a}$ \\
\hline & Cross section & barn & $\mathrm{cm}^{2}$ \\
\hline \multicolumn{4}{|c|}{$\begin{array}{l}\text { a Unit gray (Gy) adopted for } 1 \text { joule/ } \mathrm{kg} \text {. One rad equals one } \mathrm{cJ} / \mathrm{kg} \text {. } \\
\text { b Unit becquerel }(\mathrm{Bq}) \text { adopted for } 1 \mathrm{~s}^{-1} \text {. }\end{array}$} \\
\hline
\end{tabular}

(2) diagnostic $x$-rays, (3) dental $x$-rays, and (4) nuclear medicine.

Similarly, manufacturers can be categorized into instrument makers, radiation source equipment manufacturers, and radiopharmaceutical manufacturers. The major regulators involved in this area are listed in Table 3. A very major part of the regulatory job falls on the state and local radiation control offices since, unless particular radiation responsibility is assigned to some Federal agency, the responsibility falls on the state or local government

Some of the important standards and calibration laboratories are listed in Table 4, the NBS being one of them. 0ther national standards laboratories such as the National Physical Laboratory in England, the Physikalisch- Technische Bundesanstalt in West Germany, the Laboratory for the Measurement of Ionizing Radiation (LMRI) in France, and the Central Bureau for Nuclear Measurements (BCMN) of Euratom located in Belgium are important laboratories with which we compare standards on a bilateral basis. The International Bureau of Weights and Measures (BIPM) is a central laboratory and secretariat for tying all of the national standards laboratories together into an internationa 7 measurement system. 
Table 2 ORGANIZATION OF THE IONIZING RADIATION MEASUREMENT SYSTEM STUDY

Main Categories of Users

1. Medical

2. Nuclear Power

3. Industrial Radiation Processing

4. Defense

5. Chemical Analysis

6. Science

7. Environmental Radioactivity

8. Miscellaneous Radiation Applications

Activity Classes

1. Direct Users of Radiation

2. Manufacturers

3. Regulators

4. Standards and Calibration Laboratories

5. Other Interested Groups

\section{Table 3 MAJOR REGULATORS OF RADIATION}

Nuclear Regulatory Commission Energy Research and Development Administration

Environmental Protection Agency

Bureau of Radiological Health, Food and Drug Administration

Occupational Safety and Health Administration, Department of Labor

Department of Transportation

State and Local Radiation Control Offices
Table 4 STANDARDS AND CALIBRATION LABORATORIES

National Bureau of Standards Bureau International des Poids et Mesures (BIPM), Sevres, France (International Bureau of Weights and Measures)

Other National Standards Laboratories

National Physical Laboratory, England, UK

Physikalisch-Technische Bundesanstalt, West Germany

National Research Council, Canada

Bureau National de Metrologie, France (radiation laboratory known as LMRI)

Central Bureau for Nuclear Measurements, Euratom (in Belgium), etc.

Regional Calibration Laboratories (recognized by the American Association of Physicists in Medicine)

Other Calibration Laboratories and Consultants

State and local laboratories Certified radiological physicists

\section{Table 5 IMPORTANCE OF RADIATION THERAPY}

One person in four will get cancer 50-60\% of cancer cases treated with radiation

Try for cure in 20-60\% of cases depending upon patient population received by hospital

Equipment 280 high energy electron accelerators (1973)

574 Co-60 (1970)

$30 \mathrm{Cs}-137$ (1970)

$5800 x$-ray sets (1972)

Accuracy required: $5 \%$ in dose to tumor

$3 \%$ in beam calibration 
Other standards laboratories are located in various government agencies in state and local governments and in regional calibration laboratories such as those sponsored by the American Association of Physicists in Medicine. Many calibrations are also provided by radiological physicists certified by the American College of Radiology.

Other interested groups include professional societies, such as the American Nuclear Society and the Health Physics Society; standards-setting organizations such as the National Council on Radiation Protection and Measurements (NCRP), the International Commission on Radiation Units and Measurements (ICRU), the International Organization for Standards (ISO), the International Electrotechnical Commission (IEC), and the American National Standards Institute (ANSI). Educational institutions and many government agencies are also very interested in the measurement system for ionizing radiation.

We now propose to take a more detailed look at the measurement system by locking at some of the user communities. The first community we shall consider is the Medical Community.

The first user group is that of radiation therapy. In Table 5 are shown some of the important characteristics of this field. The use of high energy electron accelerators is increasing very rapidly whereas the use of the $x$-ray sets is tending to decrease. What makes the measurement problem particularly difficult here is the need for measurements of significantly higher accuracy than the overall 5\% dose to the tumor generally accepted as needed. A modern electron linear accelerator used for radiation therapy is shown in Figure 1. Figure 2 is an example of an "interaction diagram" for the medical radiation measurement system for $x$ - and gamma rays. Note that the standards and calibration laboratories are shown at the top (they probably should be at the bottom in support of the system), the manufacturers are on the left, the regulators on the right, and the users shown at the bottom. The diagram gives us an indication of where more activity is needed. For example, the "fair" link is shown between the AAPM Regional Calibration Laboratories and the radiation therapy community because there are not enough such laboratories to service the whole community.

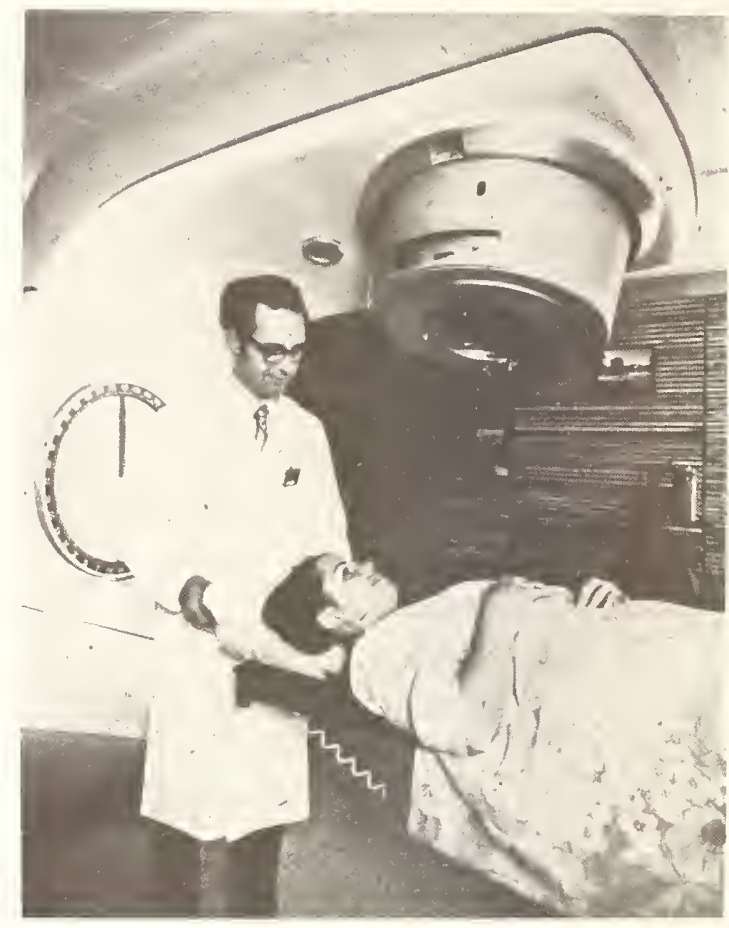

Figure 1. Modern electron linear accelerator (1inac) used for radiotherapy.

STANDARDS AND CALIBRATION LABS

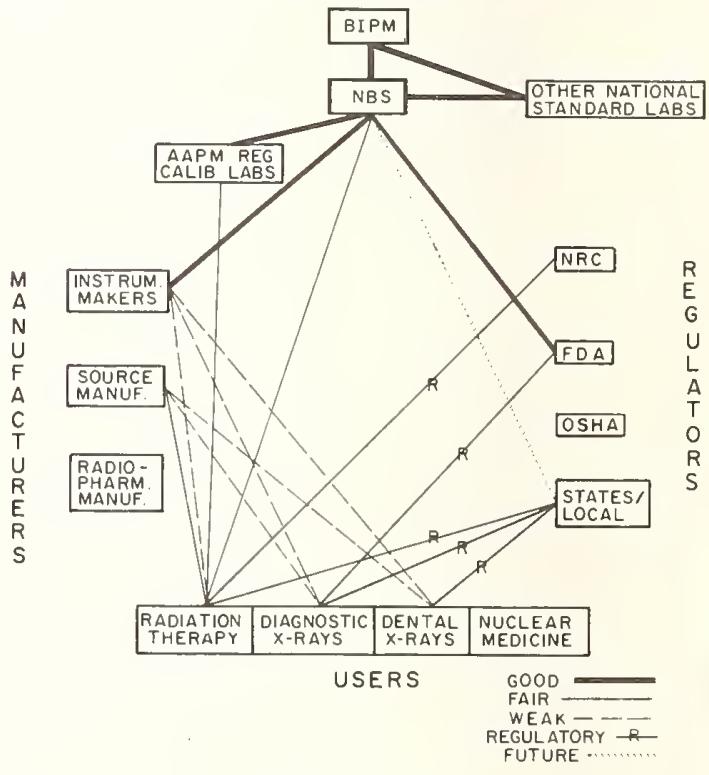

Figure 2. Medical radiation measurement system for $x$ and gamma rays, not including nuclear medicine. 
The measurement link between NBS and the state and local governments is shown as "future," but in fact, NBS now has a program in cooperation with the Conference of Radiation Control Program Directors aimed at establishing measurement links with this regulatory community. Figure 3 gives an indication of the need for accuracy in radiation therapy. The optimum dose will depend on the seriousness of the side effect. If the side effect itself is lethal, the optimum dose wi11, of course, be lower than if the side effect is tolerable, permitting the radiotherapist to opt for a higher chance of tumor cure. Figure 4 shows a study by the Radiological Physics Center located at M.D. Anderson Hospital in Houston, of the $x$-ray calibrations of 70 of 200 hospitals which are performing clinical trails for the National Cancer Institute. Note that $80 \%$ are within the $5 \%$ limit in the $x$-ray calibration. However, in dose delivered to the tumor (Figure 5) on 7 y $65 \%$ were within $\pm 5 \%$.

$X$-rays are used both in medicine and dentistry for diagnosis. The widespread use of $x$-radiation is indicated in Table 6 . Diagnostic $x$ rays are also important because over $90 \%$ of the radiation dose to the population is from diagnostic $x$ rays. However, new successes with techniques such as computerized tomography are making $x$-rays an even more powerful diagnostic tool. Unfortunately, however, the radiation dose given by these new diagnostic tools tends to be rather high. The needs of the medical radiation measurement system for diagnosis and therapy are listed in

Table 7 .

Nuclear medicine using radiopharmaceuticals is a very rapidly growing field as summarized in Table 8. An interaction diagram for the measurement system for nuclear medicine is shown in Figure 6 . Since the diagram was made, the interactions between NBS and the manufacturers, including measurement assurance programs, have been greatly increased. A summary of the needs of the nuclear medicine community are: (1) quality assurance system, (2) dose calibrator improvement and check kit, and (3) new radioactivity standards.

Nuclear Power Plants

Many nuclear power plants are now being constructed such as the one shown in Figure 7 at Three Mile Island (belonging to the Metropolitan Edison Company in Pennsylvania).

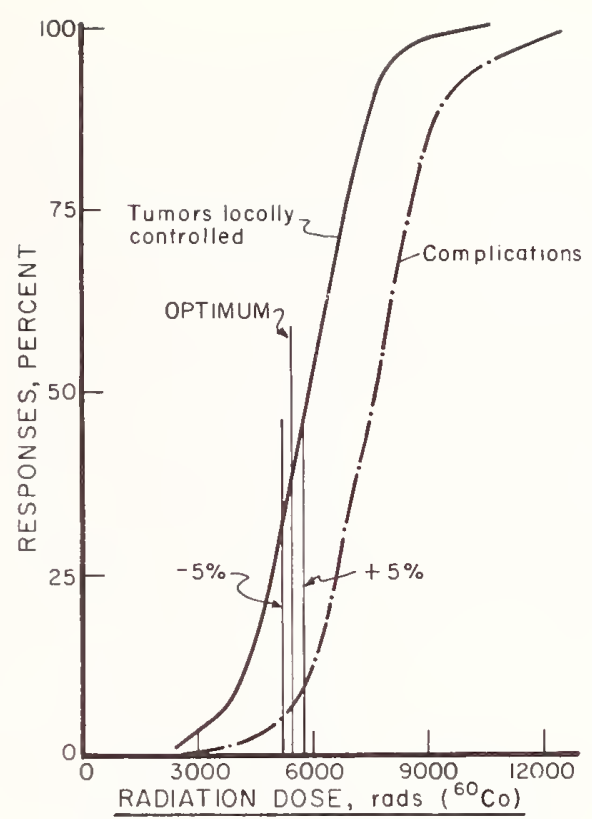

Need for accuracy in RADIATION THERAPY Based on HEAD and NECK TUMORS

Figure 3. Illustration of need for accuracy in radiation therapy. Determination of optimum dose is determined by the radiotherapist's judgment of the seriousness of complications. Too small a dose will produce fewer tumor cures than optimum. Too large a dose will produce a high probability of serious complication.

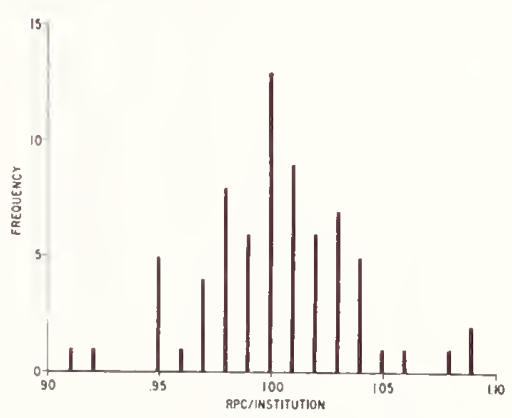

Figure 4. Comparison of radiation therapy machine calibrations by the Radiological Physics Center with those in use at institutions. Frequency refers to number of institutions; an average comparison is given if an institution had more than one radiation machine. 


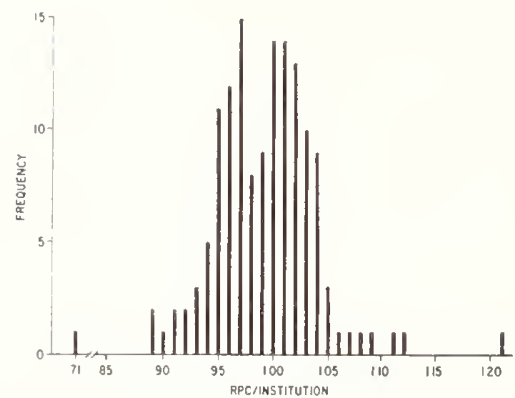

Figure 5. Comparison of tumor dose delivered (RPC) with that prescribed (institution). Radiation machines and types of treatment are counted separately in determining frequency.

Table 6 USE OF X-RAYS IN DIAGNOSIS

Diagnostic Medical

129 Million Persons, 210 Million Examinations (1970)

Examination Growth Rate $2 \% /$ Year

133,000 X-Ray Sets (FY 1974)

Dental

225 Mi11ion X-Rays (1970)

143,000 X-Ray Sets (FY 1974)

Table 7 NEEDS OF THE RADIATION DIAGNOSIS AND THERAPY MEASUREMENT SYSTEM

Calibration network using regional calibration laboratories for $x$ and gamma rays $\leq 1.25 \mathrm{MeV}$

Calibration transfer instruments for high energy radiation

Primary standards for absorbed dose

High-eriergy $x$-rays (1 inacs, betatrons, ....)

Fast neutrons

Pions (future)

Standards for imaging
Table 8 THE FIELD OF NUCLEAR MEDICINE

10 million applications of radiopharmaceuticals/year (1974)

98\% diagnostic

Growth rate $25 \%$ /year

Number of physicians doubled in five years

5000 U.S. Hospitals, use

2500 Private $\mathrm{Clinics}$ radionuclides

Radiopharmaceutical sales

$70 \mathrm{M} \$$ /year (1974)

Medical nuclear equipment $140 \mathrm{M} \$ /$ Year (1974)

Accuracy required: $\pm 10 \%$ (USP-FDA)

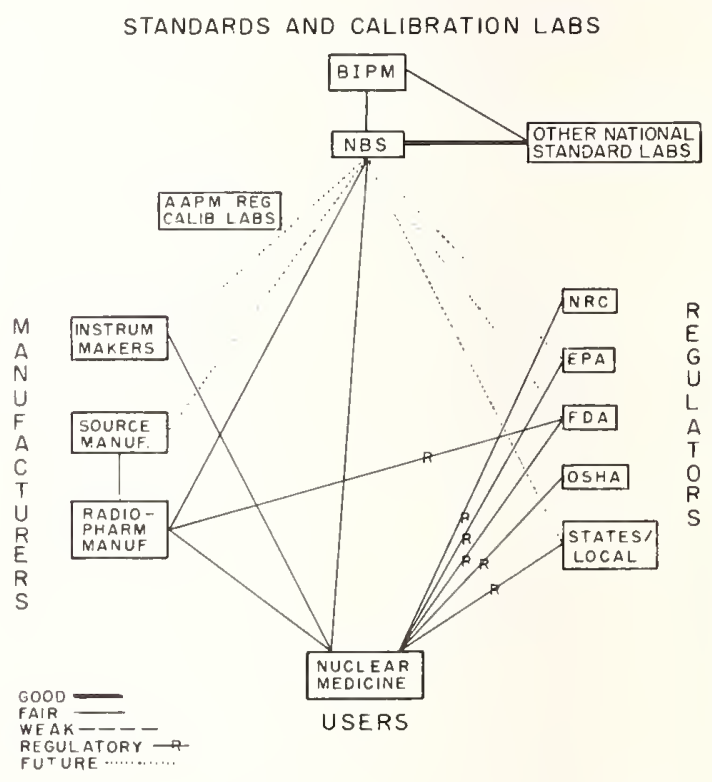

Figure 6. Interaction diagram for the measurement system for radioactivity standards and radiopharmaceuticals used in nuclear medicine. 
About 60 nuclear power plants are licensed to operate and currently provide about $8 \%$ of the electrical power in the U.S. In Figure 8 a distribution of the operational and planned nuclear power plants in the U.S. is shown. Note the concentration of nuclear power stations in the East and specifically in the Middle Atlantic area. The public is certainly clear on the question that nuclear power, if it is to exist, must be used safely. Some of the needs of the measurement system for nuclear power are summarized in Table 9. The NBS Neutron Standards Program was greatly augmented in 1971 essential1y as a response to the needs 1 isted in Table 9. Another area of extreme concern related to radiation safety has to do with the nuclear fuel cycle shown in Figure 9. A study of the nuclear fuel cycle and its measurement needs was carried out in 1974 by John Bartlett, a Presidential Intern at NBS. A summary of the results of this study is given in Table 10. It is interesting to note that the study was initiated by a concern for questions of measurement standards for "equity-in-trade" in dealing in nuclear fuel, but it turned out that the high accuracy needed was for safeguarding nuclear materials against diversion for unlawful purposes.

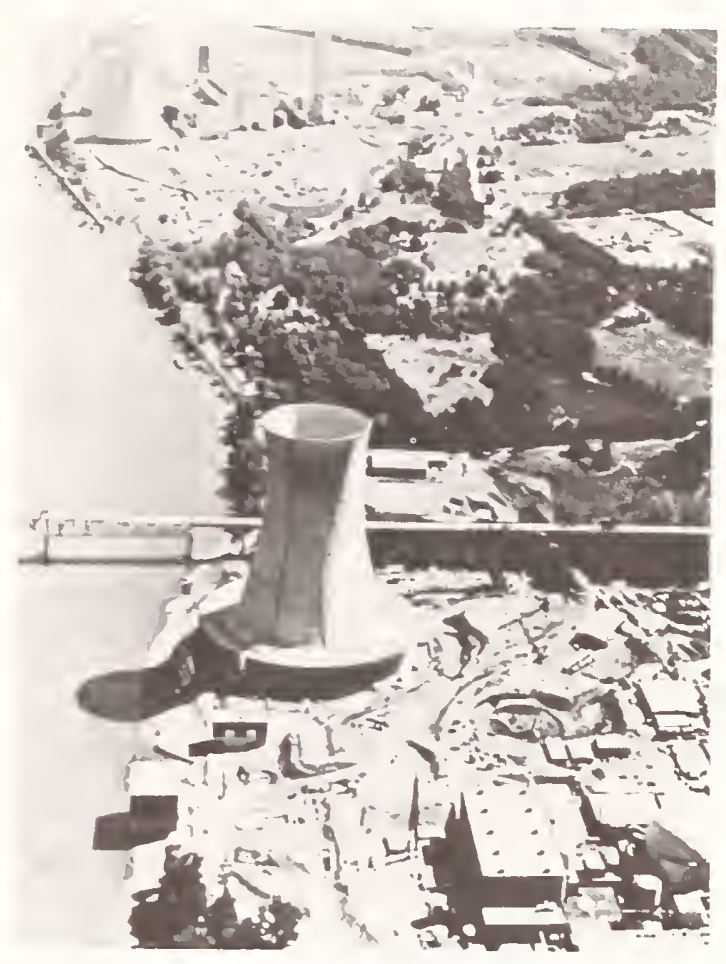

Figure 7. Modern nuclear power plant, Three Mile Island, No. 1, in Pennsylvania

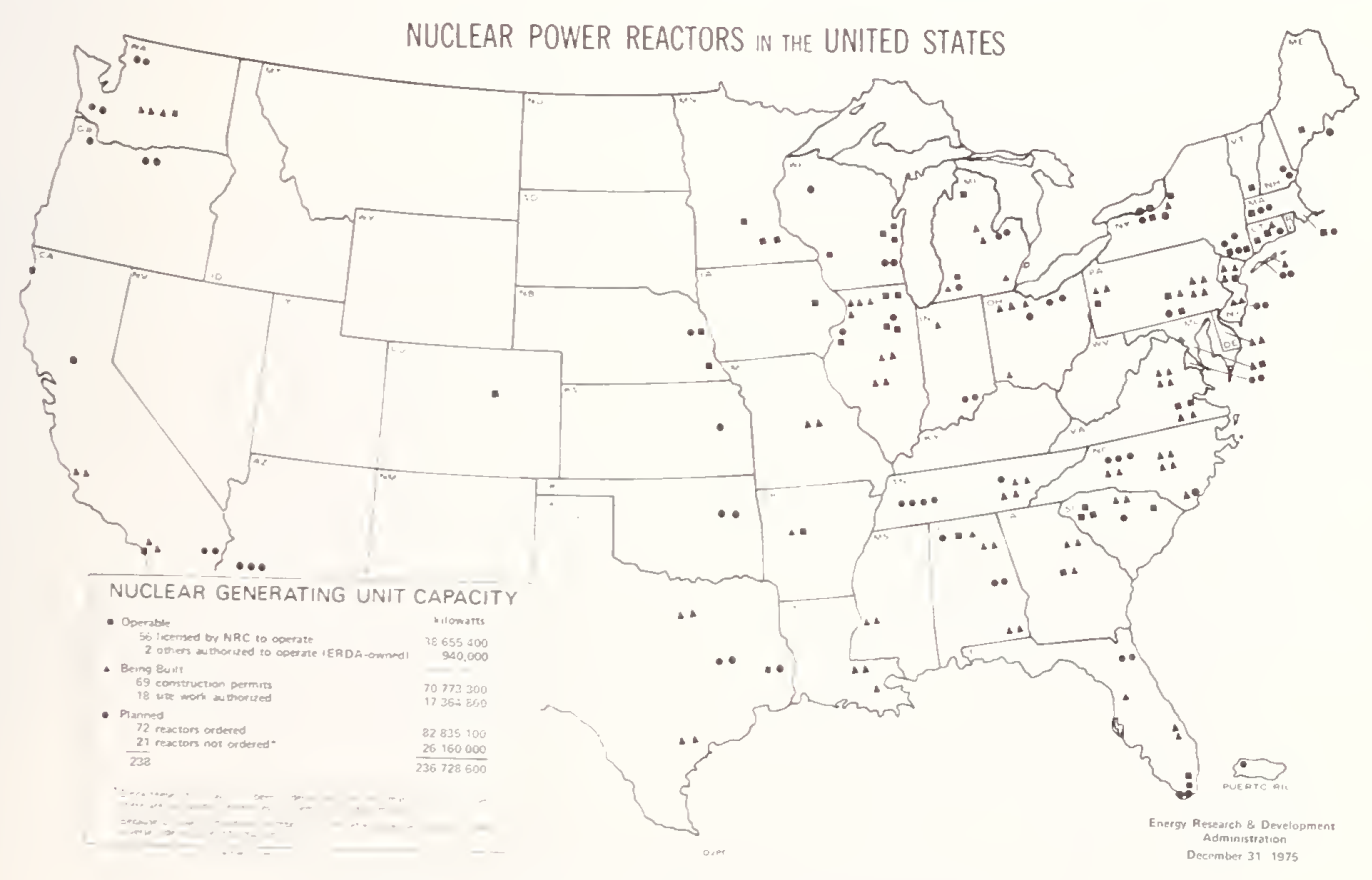

Figure 8. Nuclear power reactors in the United States according to Energy Research and Development Administration, December 1975. 
Table 9 NEEDS OF THE MEASUREMENT SYSTEM FOR NUCLEAR POWER (DESIGN DATA AND REACTOR OPERATIONS)

Neutron cross section measurement standards for:

Liquid-Metal Fast Neutron Breeder Reactor (LMFBR)

Light-Water Reactors (LWR)

Fusion Power (controlled thermonuclear and laser fusion)

In reactor measurement standards Performance vs. calculations

Fuel performance

Reactor 1 ifetimes

Reactor control

Safety

Table 10 RESULTS OF THE NUCLEAR FUELS MEASUREMENT SYSTEM STUDY (BARTLETT)

High accuracy needed for safeguards (not equity in trade)

Major Need:

National1y-based measurement assurance system for fuel material

Measurements (for demonstrably viable measurement capability)

Other Needs:

Regulations, guides, consensus standards

State-of-art performance in field with sophisticated measurement technology (approaches NBS capability)

Real-Time SNM control (replacing periodic inventory)

Table 11 SOME INFORMATION ABOUT THE INDUSTRIAL RADIATION PROCESSING FIELD

0.5 G\$ per year

Growth rate $30 \$ /$ year (recent yrs.)

Highly proprietary

Strong foreign competition

(Japan, France)

Successful areas:

Wire and cable insulation curing

Sterilization of medical disposables

Plastic automotive part curing

Electron beam welding (Activation)

Flame-resistant fabrics

Polyethylene foam

Heat shrinkable plastic film

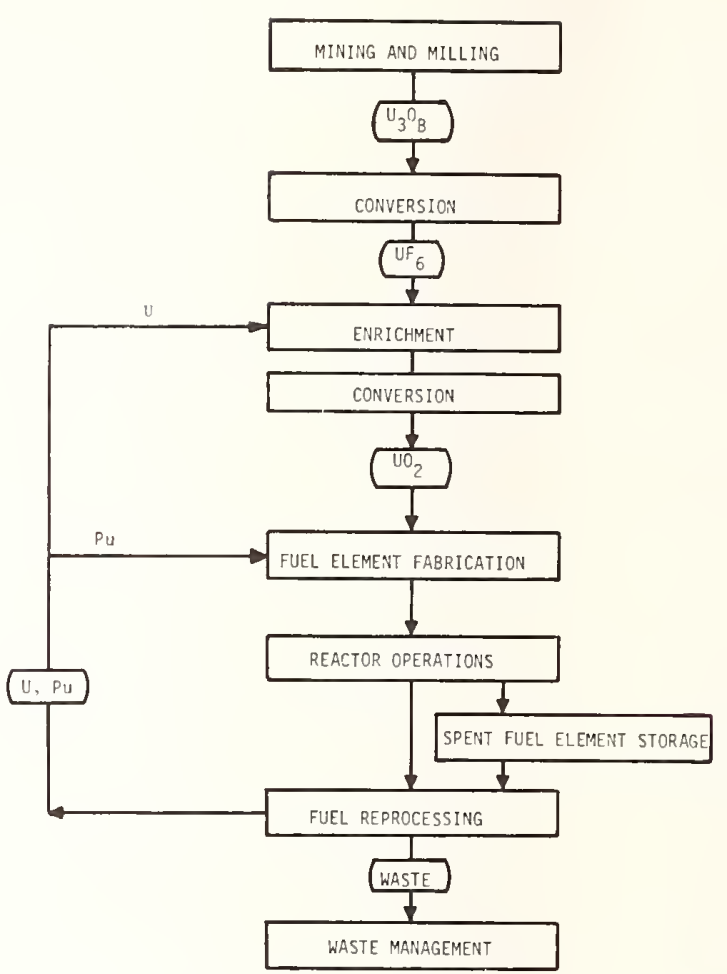

Figure 9. Nuclear fuel cycle.

Table 12 INDUSTRIAL RADIATION PROCESSING NEEDS AND RELATED NBS PROGRAM

Needs (some companies):

Calibrations of kilorad-megarad dosimetry systems

Dosimeters designed for specific tasks

Improved read-out methods

NBS Program:

Specific measurement services for processing industry

Preparing high radiation dosimeter calibration service $\left(\mathrm{e}^{-}+{ }^{60} \mathrm{Co}\right)$

NBS sources available to users

NBS program limited 


\section{Industrial Radiation Processing}

Some of the characteristics of the industrial radiation processing field are summarized in Table 11. Some of the needs of this industrial field as well as a modest size NBS program response are summarized in Table 12.

\section{Defense}

A summary of the needs of the Defense Department agencies in the area of electron, $x$ - and gamma ray measurement is given in Table 13. NBS response has been to provide monoenergetic radiation sources for calibration as well as absolute and relative dosimetry instrument calibration fields.

Chemical Analysis

Some of the characteristics of chemical analysis by activation and detection of the subsequent radiations are listed in Table 14. Although much activation analysis is done with neutrons, usually from a nuclear reactor, protons and charged particles are often advantageous for precise analysis of certain elements. In this field the need is for standard reference materials (SRM's) and no new specific radiation program need was identified.

\section{Radiation Measurement System for Science}

Many needs of scientists working with radiation are not radiation measurement as indicated in Table 15. Those needs that are radiation measurement are generally for source characterization, detector characterization, standard reference data, and assistance in theoretical interpretation of measurements--almost independent of whether the scientific field is geology, chemistry, biology and so on. NBS programs do impinge on these scientific needs. One need was identified for a data center for provision of charged particle range, stopping power, straggling, and delta ray production data especially below $10 \mathrm{MeV}$ relative to biology and such fields as ion implantation.

\section{Environmental Radioactivity}

The structure of the environmental radioactivity measurement system is indicated in Figure 10, while the needs and NBS response are summarized in Table 16. $A$ very vigorous measurement assurance
Table 13 DEFENSE RADIATION MEASUREMENT SYSTEM FOR X AND GAMMA RAYS

Interested agencies:

Defense Nuclear Agency

Defense Civil Preparedness Agency

Advanced Research Projects Agency

Air Force Tactical Command National Security Agency Army Materiel Command (Natick Laboratory)

Needs (electrons, $x$ and $y$ rays): High-flux, low energy pulsed x-ray calibrators (for hardness, survivability, simulators, plasma)

High-flux, D.C. fluence and dose measurements

NBS response:

Pulsed monoenergetic $x$-ray source $(.1-10 \mathrm{keV}, 1.5 \mathrm{keV}$ now)

D.C. monoenergetic sources $(.1-70 \mathrm{keV})$

Twin microcalorimeter + dye films

Standard electron irradiation field, 4 MeV Van de Graaff

Table 14 CHARACTERISTICS OF CHEMICAL ANALYSIS

Ultimate sensitivity excellent Non-destructive analysis often possible

Several elements can be determined in single sample

Avoids contaminated reagent problems

Carrier techniques can be used post-irradiation

Can distinguish different isotopes of same element

Neutrons, charged particles, photons all used 
program is now being carried out with the Nuclear Regulatory Commission, the Environmental Protection Agency and concerned state agencies to insure the reliability of environmental radioactivity measurements.

\section{Miscellaneous Radiation Applications}

Some of the characteristics of this field are summarized in Table 17. The key problem, as indicated, is in safe use of the equipment and an appropriate response seems to be to vigorously support the voluntary standards system.

\section{Personnel Monitoring}

Need for personnel monitoring is common to all users of radiation. As indicated in Table 18, the importance of personnel monitoring is not because it is a large business (it is quite modest in size) but because personnel monitoring is a chief factor making possible the safe use of radiation for its many desirable benefits. The structure of the radiation measurement system for personnel monitoring is indicated in Figure 11. Note the rather weak links indicated between NBS and the National Sanitation Foundation (NSF) and the suppliers of the commercial services, as well as the absence of a link between NBS and NSF, a chief tester of commercial services. This problem is being intensively studied by a number of government agencies. The needs of personnel monitoring are indicated in Table 19.

Summary

Some of the common threads found throughout this study, rather independent of the radiation user group being studied are: (1) a need for measurement quality assurance (especially in areas where radiation is regulated); (2) a need to reach out to measurers in the field with assistance, training, and regional calibrations of some kind; and (3) some new measurements standards are badly needed where such standards do not exist. A much more complete discussion, with references, of the measurement system for ionizing radiation should be available this year as an NBS internal report, NBSIR 75-946.
Table 15 SOME INFORMATION ON THE RADIATION MEASUREMENT SYSTEM FOR SCIENCE

Many needs from NBS are not "radiation" measurement: Atomic masses

Frequency

Length

Proton magnetic moment volt, etc.

Radiation Measurement Needs: Source characterization Detector Characterization Standard reference data Theoretical interpretation of measurements

NBS Program:

$>70$ calibrated radioactive sources

Data--atomic interactions of photons

Data--nuclear interactions of photons

Benchmark measurements

${ }^{\sigma}$ tot ${ }^{12}$ C; proton form factor)

Instrument development and characterization (Faraday cup, absorbed dose calorimeter, large NaI spectrometer)

Reference radiation fields Radiation transport

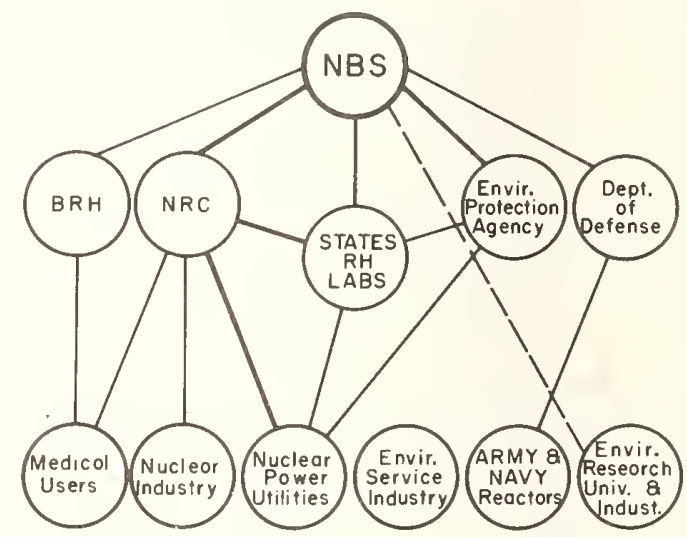

Figure 10. Structure of the environmental radioactivity measurement system. 
Table 16 ENVIRONMENTAL RADIOACTIVITY MEASUREMENT

Regulatory problems are important, NRC, ERDA, EPA are the interested Federal. agencies

Needs:

NBS standards

Traceability to NBS (measurement assurance)

Training and consultation

NBS Response:

Standards (mixed radionuclide, natural matrix, gaseous, low-level $\alpha$ and $\beta$ )

Lead 1 aboratory measurement assurance (NRC, EPA)

Round-robins to nuclear power industry

Low-level counting capability

International intercomparisons

Vational radiation measurement calibration system (States)
Tabie 17 MISCELLANEOUS RADIATION APPLICATIONS

Applications:

Radiographic equipment

Gauges

Irradiators

0i1 well logging apparatus

Self-luminous products

Smoke detectors

Static eliminators

Heat sources

$>70$ MS annually

Needs:

Adequate safety measures in use of this equipment

NBS Response:

Increase support for voluntary standards activities (NCRP, ANSI N43)

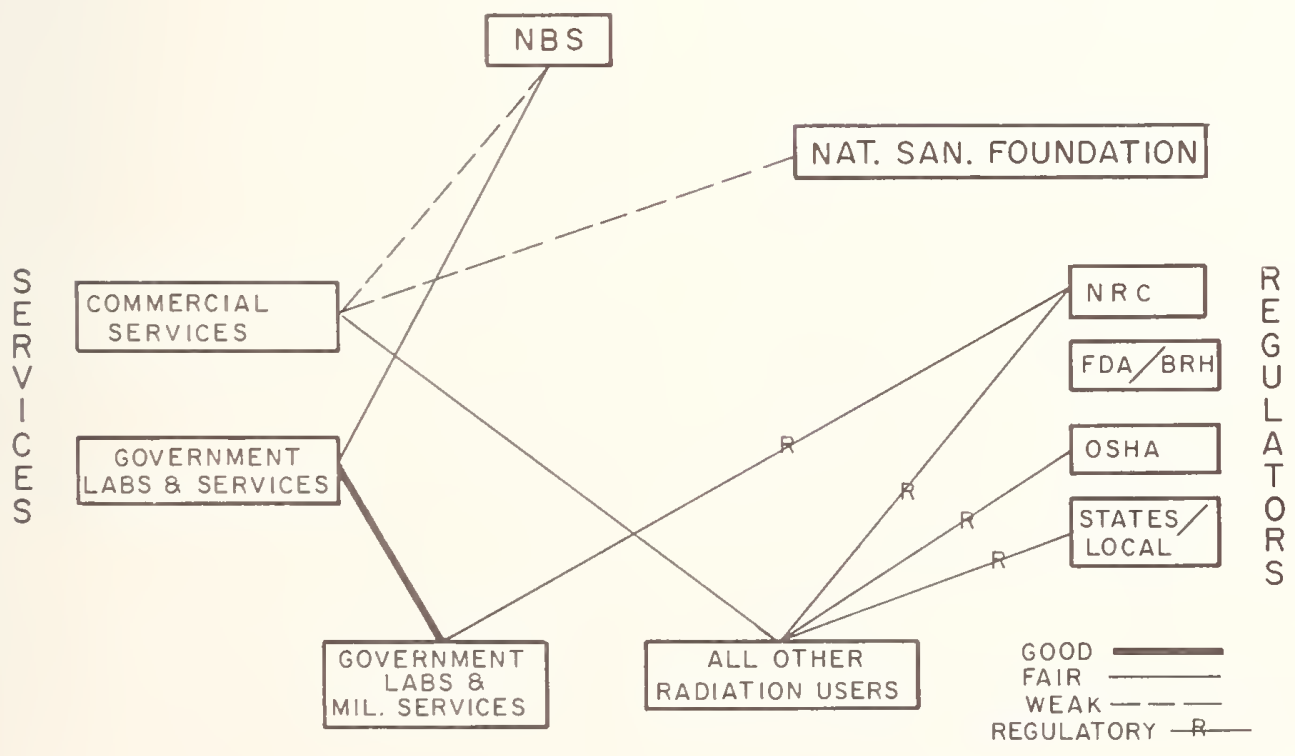

\section{PERSONNEL MONITORING MEASUREMENT SYSTEM}

Figure 11. Structure of the measurement

system for personnel monitoring. 
TabTe 18 MEASUREMENT SYSTEM FOR PERSONNEL MONITORING

14 commercial companies

$\$ 10 \mathrm{M} /$ year

Growth Rate 10\%/year

800,000 individuals monitored (commercial and government labs and military services)

Film badges and TLD are chief methods used

Makes possible benefits of ionizing radiation
Table 19 NEEDS OF THE PERSONNEL MONITORING MEASUREMENT SYSTEM

Satisfactory quality assurance Improved personnel monitors (especially for neutrons) Monoenergetic neutron calibration facility

Beta-ray source calibration facility 
THE FEDERAL REGULATORY AGENCY AND THE RADIATION MEASUREMENT SYSTEM R. H. Schneider

Food and Drug Administration 5600 Fishers Lane

Rockville, Maryland 20852

\begin{abstract}
The official actions of a federal regulatory agency can have unique health and economic impacts. It is often not feasible for other parties to review the processes leading to such actions in detail and appeal is tedious and expensive. As a matter of equity it is incumbent on the agency to assure that the errors associated with facts on which actions are based are sufficiently small as not to affect the course of such actions. When radiation measurements are used as part of the factual basis for regulatory action this means that these measurements must be an integral part of the national radiation measurements system. The manner in which this has been achieved within the Food and Drug Administration as part of the implementation of the Radiation Control for Health and Safety Act of 1968, (P.L. 90-602) is described.
\end{abstract}

In the abstract of this paper I discussed the philosophical underpinnings of the connection between the Federal Regulatory Agency with responsibilities in the radiation area and the radiation measurements system, which is indeed a fundamental and necessary connection. I stated that "as a matter of equity it is incumbent on the Agency to assure that the errors associated with facts on which actions are based are sufficiently small as not to affect the course of such actions." That is indeed the basis of the connection. In this paper I will discuss, in general, some of the problems that have arisen and the solutions to them which we have devised in the course of developing the regulatory machinery within the Food and Drug Administration for implementation of the Radiation Control for Health and Safety Act of 1968. The details of this process in the four areas of acoustic energy, microwave radiation, optical radiation and $x$-radiation will be discussed by members of my staff at later sessions of this symposium. I will then go beyond the bounds established in my abstract and discuss some of the other implications which radiation measurements and therefore the radiation measurements system can have for a regulatory agency.

The most surprising thing in my experience, in the eight years since the Radiation Control Act was passed, has been the realization that the national radiation measurements system was not ready for the Radiation Control for Health and Safety Act. There were, I believe, two reasons for that, the first a technical reason, is simply that the measurement technology which had been developed for many of the types of energy encompassed by the Act such as acoustical energy, radiofrequency and microwave energy, and optical energy, while very well developed, had been developed for use under circumstances quite different from those which apply when radiation measurements are needed for health protection purposes. I will illustrate this by one simple and obvious example. In a case where it is desired to evaluate the hazard potential of a product incorporating a radiofrequency or microwave generator, such as a microwave oven or a diathermy machine, it is quite often necessary to make measurements in the near-field or a radiator. The microwave instrumentation existing in 1968 consisted primarily of field strength meters which were used by antenna engineers to measure antenna patterns and gains in the far-field, because their only interest was in the antenna's ability to concentrate energy at great distances. Partly as a result of the Radiation Control Act a whole new science and technology of near-field measurements has blossomed within the past eight years. This has been stimulated further by the passage of the Occupational Safety and Health Act. There are many similar examples of new scientific and technical requirements being imposed on the radiation measurements system by the creation and implementation of regulatory authority.

Because of the state of unpreparedness of the radiation measurement system a considerable burden of measurement development was placed squarely on our shoulders and I believe that we have carried it well. The Division of Electronic Products of the Bureau of Radiological Health has, in the past eight years, made many substantial contributions to the radiation measurements system. It is appropriate to acknowledge here that many substantial contributions 
have also been made by various groups in the Institute for Basic Standards of NBS to assist us in developing the radiation measurements system to the point where it can meet the requirements of radiation measurements for health protection.

A second reason for the lack of preparedness of the radiation measurements system was essentially non-technical but rather administrative and logistical. I am not an expert on radiation law but I would like to offer the following suggestion for your consideration. Though previous acts of Congress had established various regulatory bodies having cognizance over radiation, the Radiation Control Act of 1968 was the first such act under which radiation measurements per se became the sole facts upon which regulatory actions were predicated. That means that an agent of the Food and Drug Administration in performing a radiation measurement can develop a datum which will determine whether or not a formal regulatory action will ensue. Some such actions in the recent past have had economic impacts tolled in the millions of dollars in situations where the risks to the nations health could have been substantial. Hark back to the matter of equity, which I raised at the beginning of this presentation. In such a situation, as a matter of equity, the nations health deserves that measurement to be as accurate as technically and economically feasible so that regulatory action is indeed pursued when a limit established in a regulation is in fact exceeded. It is also a matter of equity that the potential target of the regulatory action deserves that the measurement be as accurate as technically and economically feasible so that he does not suffer economic loss from a regulatory action which is pursued when in fact his product was in compliance with the $1 \mathrm{imit}$ established in the regulation.

The practical impact of this matter is profound. We are maintaining an instrument population now numbering in the hundreds scattered across the entire United States in active, if not always continuous, use with each and every one of these instruments having a non-zero probability on any given working day of turning out data which will have to be defended in a legal action. I submit that the radiation measurements system was not ready for this. We have, therefore, in the last eight years devoted substantial energy and resources to the development of equipment and systems for the logistical support, including calibration, of large numbers of radiation measuring instruments in the field. One of our later papers in the symposium discusses one of these systems.
We are confident that we have successfully integrated our regulatory programs with the national radiation measurements system, that we adequately treat the matter of equity and that we are prepared to make measurements of whatever, wherever, health protection demands.

Now I would like to talk a little bit about some of the other applications of radiation measurements in the programs of a Federal regulatory agency.

Radiation measurements offer the federal radiation control agency a means by which to assess the effectiveness of its own programs both regulatory and non-regulatory. At times the measurements which are most useful in assessing program effectiveness are not the same sort of measurements as are made for determining compliance with regulations, because, after al1, the intent of regulations is not to reduce the radiation insult to radiation measuring instruments but, rather to control, or reduce the radiation insult to health. In the design of regulations and the testing conditions which are used in tests of compliance with them we often, in the interest of efficiency, design tests and measurements which are not direct measurements of health insult. Therefore, when we want to assess the effectiveness of one of our programs we often face another measurements problem.

I now would like to describe to you a system which we have developed to assist us in meeting such needs in the area of medical radiation exposure. You will hear no more about this system at this conference so I will go into some detail. Publications describing it and its use are in preparation. This is a system for estimating absorbed doses in human organs by calculation rather than by measurement. There are situations where estimating by calculation is more costeffective than estimating by measurement and such calculated estimates are properly a part of the radiation measurement system.

A number of years ago we began a collaboration with members of the Health Physics Division of the Oak Ridge National Laboratory to apply a system developed by them for estimation of organ doses from internal radionuclides to the problem of the estimation of organ doses from external radiation sources such as medical $x$-ray machines. 1

The system consists of a detailed mathematical description of the geometry of a phantom which rather closely approximates 
reference man. The set of equations which constitutes the phantom includes descriptions of the phantoms' external surface and descriptions of many internal organs including a skeleton, lurigs, thyroid, both male and female gonads, a uterus, and many others. Mammaries are presently under development. The second component of the system is a Monte Carlo radiation transport code. The equations of the phantom define the geometrical regions in which the radiation transport code is active. The conversion of the system for estimation of doses from external sources was easily accomplished. 2,3 The development of phantoms representing children of different ages is well underway.

Using this mathematical model as a tool, we have developed yet another dose estimation system for more routine applications. It was immediately obvious that routine use of the transport code to generate dose estimates for a large number of specific clinical situations would be redundant and expensive. We therefore set out to generate a data base of reference organ dose data from which specific clinical situations could be simulated. This data base is organized according to the following scheme: The projections of the external surface of the phantom in each of the three views, anterior-posterior, lateral, and posterioranterior are segregated into $4 \mathrm{~cm} \times 4 \mathrm{~cm}$ square regions by a grid system and these resulting square regions are ordered and numbered. The photon energies of interest were considered to span the region between approximately $20 \mathrm{keV}$ and $150 \mathrm{keV}$. Seven photon energies, more or less uniformly spaced across this region were chosen. Transport calculations were then done for monoenergetic photon beams of each of the chosen energies into each of the surface regions of the phantom. Each such calculation produces a tabulation of dose factors in units of rad per incident roentgen for every organ in the phantom. The dose to any organ under given conditions of $x$-ray exposure can then be simulated by a weighted sum of selected cells of this data base. The weighting factors across the energy dimension are chosen to represent the photon spectrum used in the particular situation of interest and organ dose factors are selected from the tabulations for those surface regions included in the radiation field of the examination. The organ dose estimates are then obtained by normalizing with the exposure used in the examination under study.
The entire data base is maintained on a digital computer and routines have been written to generate the sums and weighting factors automatically from simple descriptions of the geometry and technique factors of the examination. These routines include provisions for assigning fractional weighting factors to those surface regions which are not entirely within the examination field.

The existence of this second system obviates the need for complete radiation transport calculation for the evaluation of organ doses from every clinical situation of interest. Good estimates of organ doses are now avajlable quickly and at minimal cost in computer or user time. We have already made extensive use of this system. As can be readily imagined, the system makes it very easy to investigate effects on organ doses of variations in technique factors or size and location of radiation fields. It permits the user to quickly and inexpensively perform a large number of dosimetry experiments by computer simulation rather than by many painstaking hours of laboratory work. We have, for example, found the system to be an invaluable tool in producing estimates of program efficacy for environmental assessment reports. The system was used to evaluate the impacts of the performance standards for medical $x$-ray equipment and our recent policy statement on gonadal shielding.

To date we have been able to perform several comparisons of estimates from this system with dose estimates in the literature. ${ }^{4}$ Such comparisons are of course always difficult because pairs of dosimetry experiments performed under identical situations are difficult to find. The estimates of doses to directly irradiated soft tissue produced by our system in general vary less than $15 \%$ from those in the literature which were obtained under similar irradiation conditions. Our estimates of doses to tissue not directly irradiated vary as much as 30\% from values in the literature. These comparisons have convinced us of the adequacy of the system for evaluation of the effects of variations in technique factors and irradiation geometry on absorbed doses in internal organs.

The activities of the federal regulatory agency in the radiation area need not a11 be regulatory, in fact, the Congress does not confer regulatory authority for the sake of regulations, but rather for the sake of some goal related to the nation's welfare. 
We continue to support, as we have over the years, non-regulatory programs in the field of radiological health because we have determined that certain problems are more effectively attacked by education, consultation, and persuasion, than by regulation. Non-regulatory programs also can benefit by integration into the national radiation measurements system, because wherever measurements are used credibility enhances their effectiveness. Two such programs in which the Bureau participates with the States are known by the acronyms DENT and NEXT.

DENT - Dental Exposure Normalization

Technique - is a program for measurement of the radiation exposures used for radiography by individual dental practitioners within a state and reduction of the exposures delivered by those individual practitioners whose values lie toward the upper end of the population distribution. ${ }^{5}$ Post cards containing Tithium fluoride thermoluminescent. dosimeters are mailed to each practitioner within a State with instructions for exposure under the same conditions the practitioner uses for bitewing radiographs. These are returned by mail and read and the population distribution generated from the resultant values. A point on the distribution is then selected which divides the population of practitioners into those who will not receive a follow-up visit and those who will. Those practitioners whose values are above this point are then visited by members of the State Health Department. Their radiographic techniques are reviewed and consultation and guidance for improvement is given. The subsequent reduction in radiation is demonstrated to the practitioner. The results of this program have been quite dramatic. In those states where follow-up studies have been done it has been demonstrated that lasting significant reductions in both population mean and extreme have been achieved. At the present time, Bureau support for the DENT program consists of maintenance and calibration of the dosimeters and readers that are used, processing of the data collected and consultation with State radiological health programs on operation of the individual projects.

NEXT - The National Evaluation of X-ray Trends - is a program about which you will hear a paper later in this conference, therefore I will mention it here only to state that the Bureau's participation on the topic of measurements has been to evaluate, by measurements of energy dependence, and calibrate the instruments which are used by the States in this program.

In closing I would like to mention briefly a new project within the Bureau of Radiological Health which at present is in the pilot stage. Because of the current interest in mammography we decided that it would be useful to have a program similar to the DENT program to evaluate the exposures being used in mammographic examinations at individual facilities, in an attempt to identify excessively high exposures resulting from poor technique, such as have been reported. We then were faced with the problem of developing a simple, mailable, inexpensive system capable of producing adequate estimates of exposure from the low energy $x$-ray spectra used in mammography. We began by investigating the photon energy dependence of the exposure response of lithium fluoride chips to low energy $x$-rays with both nearly monoenergetic fluorescent sources and broad spectrum low energy $x$-ray sources. We determined that when calibrated with an appropriate low energy $x$-ray spectrum the exposure response of the lithium fluoride chips did not vary more than plus or minus $20 \%$ over the range of beam qualities which we expect to encounter in a survey of mammography facilities. ${ }^{6}$ A pilot study of this system produced distributions of values for the various mammographic techniques which compare extremely well with reported values for the same techniques. We are continuing to develop the logistical support for this system and a second pilot study is underway.

In this presentation I have tried to give you some appreciation of both the scope and depth of the relationship between one Federal regulatory agency and the radiation measurements system by discussing the philosophy underlying that relationship and describing a variety of examples of the spectrum of activities of which the relationship is composed.

\section{References}

1. Snyder, W.S., M.R. Ford, G.G. Warner and H.L. Fisher, Jr., Journal of Nuclear Medicine, Supplement Number 3, Pamphlet 5, Society of Nuclear Medicine (August 1969). and

Snyder, W.S., M.R. Ford, G.G. Warner and S.B. Watson, ORNL-5000, Oak Ridge National Laboratory (November 1974). 
2. Poston, J.W., G.G. Warner, M. Rosenstein and R.H. Schneider, FDA-73-8029, Government Printing Office (March 1973).

3. Jones, T.D., J.A. Auxier, W.S. Snyder and G.G. Warner, Health Physics, 24, 241 (1973).

4. Rosenstein, M., Organ Doses in Diagnostic Radiology (in press).
5. DENT: A Quality Assurance Program in Dental Radiography, Gross, R., R. Bunge and C.L. Crabtree, Health Physics Mid-Winter Symposium, Denver, Colorado 2/9/-2/12, 1976.

6. Energy Response Curves for Various Dosimeters, T. Ohlhaber (to be published). 



\author{
STATE RESPONSIBILITIES AND THE RADIATION MEASUREMENT SYSTEM \\ M. W. Parrott \\ State Health Division \\ Radiation Control Section \\ PO Box 231 Portland, Oregon 97207
}

\begin{abstract}
The states' radiation control programs have attempted to respond to public health need through a radiation measurement system that requires much more than instrument

calibration. Few federal agencies can cover their responsibilities without state assistance. This paper explains these areas from one Radiation Control Director's point of view which may be shared by several others. Recommendations for some improvements are made.
\end{abstract}

(Radiation; regulations; ineffectiveness; measurements; compromise; states; abstract)

Traditionally, the states have had public health and safety responsibilities for their citizens which are not shared with the Federal government. Although 16 federal agencies attempt to regulate various health and safety aspects of radiation, they have been unable to adequately regulate these activities due, in part, to the magnitude of the enforcement problem. Therefore, a number of federal laws passed to "protect the public health" fall upon the states for enforcement. The states accept their traditional duties by assumption of responsibility (i.e., Nuclear Regulatory Commission/Agreement State and Occupational Safety and Health Act), by default by the federal agencies (i.e., U.S. Department of Transportation), or by the simple statement in a regulation that "certification" by state agencies is required of certain types of installations (i.e., Federal Aviation Administration baggage inspection by $x$-rays). Unfortunate$1 y$ and all too frequently, this "inspection and certification" may be accomplished with anything from a "wet finger" to equipment which represents the state of art in radiation measurement.

Unlike the ideal conditions present in a national standards laboratory, the radiation measurement system at the state level is not confined to the calibration of instruments alone. To make radiation measurements, a state inspector must carry any number of pieces of equipment many thousands of miles, under severe climatic conditions to any number of installations, a)1 of different character. The conditions of transport may vary from bus and foot travel in a large metropolitan area, or by car or airplane in some of the larger, less populated states. The inspections require different kinds of measurements of diagnostic, therapeutic and industrial byproduct, naturally occurring and cyclotron-produced radioactivity, diagnostic, therapeutic and industrial $x$-rays, lasers and microwaves. Usually, each measurement must be carried out in a short time with such accuracy as can be achieved under these somewhat hazardous conditions--hazardous in that each measurement may have to be defensible in court.

For clarification, perhaps it is appropriate to show what developed from the Workshop on "Criteria for an Adequate State Radiological Health Program for Radiation Sources not Covered under the Atomic Energy Act" at the 7 th Annual Conference of

Radiation Control Program Directors. ${ }^{1}$ This program is outlined in Table 1.

To adequately protect the public health by attaining a well-balanced radiation measurement system, one state agency, and probably a single federal agency should have the authority, responsibility, capability and demonstrated performance over al1 forms of radiation. Instrument capability may be present in any number of agencies as well as emphasis on a particular form of radiation. However, performance may be a totally different matter in the hands of people who are not technical1y competent to know the limitations of themselves or their instruments.

Unfortunately, the range of authority governing radiation measurements and regulations varies widely among the states from voluntary compliance to complete 
TABLE I. ELEMENTS FOR A MINIMUM STATE RADIOLOGICAL HEALTH PROGRAM FOR NON-ATOMIC ENERGY ACT SOURCES

I. X-Ray Sources
A. Machine Registration/Licensing
B. Standards
C. Compliance - Machine Inspection
D. Enforcement
- Facility Inspection
E. Constant Alertness to New Sources

II. Non-Ionizing Sources
A. Registration/Licensing
B. Standards
C. Compliance - Machine Inspection
D. Enforcement
E. Constant Alertness to New Sources

III. Natural Sources (Manufacture, Byproducts, Tailings, Phosphates, Radium, etc.)
A. Registration/Licensing of Radioactive Material
B. Radioactive Material and Environmental Standard
C. Compliance - Environmenta T Monitoring

- Radioactive Waste Control (Effluents, etc.)

- Surveillance over Product Manufacturing and Quality Control

D. Enforcement

- Facility and User of Radioactive Material Inspections

E. Constant Alertness to New Sources

IV. Cyclotron-Produced Radioactive Materials
A. Licensing Users and Equipment
B. Standards
C. Compliance for Production and Users
D. Enforcement

V. Other
A. Public Information and Technical Training
B. Program Effectiveness Evaluation
C. Emergency Response (Fixed Facility and Transportation)
Planning
Training
Response to Incidents
D. Legislation - Writing and Testifying
E. Budget
F. Personne1
G. Update and Publish Regulations 
legislative authority over all forms of radiation, ionizing and non-ionizing. Other variables among the states include personnel, available equipment, inspection procedures, travel and climate as well as the frequency, type and method of calibration. There is a total lack of uniformity in the radiation measurement system. This was pointed out very well in the beginnings of the States' participation in the Nationwide Evaluation of X-ray Trends (NEXT).

NEXT will be presented in more detail later in this symposium. The data that was sent in by the various states in that program was scattered throughout the spectrum of results. For several years, the procedures in this measurement system were followed rigorously while the instrumentation varied grossly. This has been somewhat rectified since the Food and Drug Administration Division of Electronic Products will not allow data to be incorporated into the overall system unless the survey instruments have been calibrated at FDA's Division of Electronic Products at no cost to the states. Automatically this introduces some other errors into that data bank. Some smaller states may not be able to send in their "on $1 y$ " survey instrument. Those states may also have the "worst" conditions in their state which will not show up in the overall evaluation of NEXT. Regardless of the bias that may be introduced into the NEXT data, the idea of having the procedures standardized and the instruments calibrated by competent physicists in the FDA is certainly a motion in the right direction.

What is the present world in the State programs? When it comes to environmental surveillance, the radiochemical analysis can be carried out to near perfection. The sample collection procedures have been standardized, the equipment has been developed to provide exceptional accuracy through sophisticated computer programs allowing simultaneous analysis of large numbers of radionuclides and the persons utilizing the equipment and doing the procedures are frequently highly skilled and motivated. They can obtain an ever growing list of radionuclides that have been prepared with meticulous accuracy at the National Bureau of Standards or indirectly by NBS through the two National Environmental Research Centers. This differs markedly from the support, personnel, equipment and procedures in all other parts or radiation control programs except possibly that part related to the Agreement Materials Program. All of this development is related directly to the nationwide interest in nuclear power while all other elements of a State Radiation Measurement Program suffer miserably. It should be noted that it may not be possible to maintain this high degree of accuracy in environmental surveillance. The Environmental Protection Agency Las Vegas facility has been furnishing a cross check program among a large number of laboratories in the U.S. to provide quality assurance to these laboratories. Many of the state environmental surveillance programs are doing the only work in this field around nuclear facilities. Now it appears that, as usual, as soon as federal funds are curtailed, the first programs that are eliminated are those that assist the states. This is going to happen to both the Northeastern and Las Vegas Laboratories. EPA has decided to cut back these programs, Consequently, one of the few ongoing Quality Assurance programs for the states is doomed.

This realistic world is such that the States' radiation measurement system is a compromise. A state inspector must do what is reasonable and possible. He must be reasonable. Interpretations of regulations, if there are any regulations, must be reasonable. This requires judgment. The judgment of an intelligent, we11-trained, experienced individual who is always aware of the fact that it is the protection of the public heaith that is of prime importance. The usual inspection of a hospital x-ray department is a pressure situation. How much time can a given $x$-ray room be out of business while the inspector is making measurements? The survey meter, more than likely, was calibrated with radium or cobalt which in no way can be accurate for $70 \mathrm{kVp}$ $x$-rays when related to $\mathrm{mr} / \mathrm{hr}$. These items severely curtail the accuracy with which the measurements can be made. The inspector must make measurements as to beam size and alignment, energy, scatter, adequacy of shielding and timer. In no way can the accuracy of any of these measurements be compared to, or attempt to emulate, those made under laboratory conditions. An x-ray inspector must inspect 400 to 500 machines over a large area of several thousand miles in a single year, go through temperature extremes, altitude changes and many other difficulties not encountered in a standards laboratory. So, how sophisticated must equipment be? In order to purchase any piece of equipment, he must go through a complete scrutiny by a budget analyst to prove the old equipment is too archaic or unusable to continue to be in service. Then he must, 
out of necessity, buy the lightest (remember he has to carry this under his arm along with any number of other items with which to make measurements), the strongest (remember the rough transport problem), most reliable (who can find a 67 volt battery in a town of 300) and above a 11 , cheapest available instrument. There has yet to be any mention of accuracy. With all of this, any inspector would be delighted with $+5 \%$ and why not? If shielding design requires a maximum of $2 \mathrm{mr} / \mathrm{hr}$ in an uncontrolled area, is this inspector going to complain to a facility if he gets $2.1 \mathrm{mr} / \mathrm{hr}$ ?

Most states show real concern for the necessity of a complete and adequate radiation measurement system. This can be demonstrated by a comparison of data published for 1970 by the Bureau of Census reported by

DeVore ${ }^{2}$ and data collected during the same period in the State of Oregon. Medical $x$-ray examinations were performed on 38 percent of the population in the United States during that year, or 77 million persons. In that same period 65 percent of the population of Oregon, or 7.35 million people, received medical $x$-ray examinations. Dental $x$-ray exams in the U.S. population totaled 59 million, or 29 percent, while they made up 62 percent of the examinations in Oregon for a total of 1.3 million persons. This, simply stated, shows the population of Oregon will have dental or medical x-rays an average of 1.27 times per year while the overal1 U.S. population would average .67 times per year. While the regulations enacted for Public Law 90-602 have provided for new equipment to be installed to assure adequate protection against radiation leakage from the overall system and positive beam limitation (automatic collimation), our experience during inspections has been that more than 99 percent of the units with positive beam limitation devices are operated in the manual mode. The positive beam limitation is cut out because the only facilities that can afford this kind of equipment have been using manual collimation for years and want to split film for economy and comparison, and many want to see cone cutting which is not permitted in the automatic mode.

Filtration, of course, is another area of concern. Although this, too, is provided for in the law, measurements must be made on each unit to determine if the filtration is adequate to eliminate the soft $x$-rays which are absorbed by the tissue rather than contributing to the radiograph.
It has been our experience that two-thirds of the installers fail to make the actual measurements to provide for adequate filtration due to lack of instrumentation. Consequently, they must go by a chart and add or subtract filters based on numbers. It should be mentioned that some suppliers have provided their installers with adequate instrumentation at considerable cost. Our studies indicate the lack of adequate filtration contributes on the average 500 milliroentgens of unnecessary radiation per film per x-ray machine. Of all patients receiving medical $x$-rays in the State in 1970, approximately 10 percent received unnecessary radiation due to inadequate filtration. About 11 percent received unnecessary radiation due to inadequate collimation. If each overexposure due to inadequate filtration alone is $500 \mathrm{milli-}$ roentgens and since there were about 135,000 patients overexposed in this manner in 1970, then there were 67,500 man roentgens of unnecessary radiation administered. ${ }^{3}$ It is much more difficult to quantify patient overexposures due to other noncompliances, such as inadequate collimation although an attempt is being made with the NEXT study. X-ray retakes due to poor techniques should also be considered when estimating the total amount of unnecessary radiation administered. The amount of unnecessary radiation described here is quite high when one considers that the maximum permissible exposure to any member of the general population is $500 \mathrm{mr}$ per year. It is our opinion that it is absolutely necessary to inspect and make the appropriate measurements on a 71 of these $x$-ray machines on a regular basis because of the great impact these have on the overa 11 radiation exposure burden.

$X$-ray inspection frequencies are usually set on the basis of kind of use and possibility of malfunction almost directly related to health vs. risk. The desired frequency of inspection is: dental every three years, general practitioners every two years, radiologists and hospitals every year. There are virtually no states able to maintain this kind of inspection frequency due almost entirely to the lack of public, practitioner and legislative awareness that these devices present a public health hazard several million fold greater than a nuclear power plant. Consequently, sufficient funds are rarely allowed to be directed toward this rather imposing public health problem. In Oregon, (the only state with which I am completely familiar) there are 3,588 registered $x$-ray machines. Nearly one-half of 
these are dental units. The rest are medical and a few industrial. In order to maintain the aforementioned inspection frequency, we would be required to inspect approximately 1,794 units per year. This would take between 4 and 4.5 inspectors. Oregon has authorized 4 inspectors but funded only 2 ; consequently, less than one-half of the required number of inspections will be made, mostly on recently installed units or at hospitals and other facilities requiring Medicare or hospital accreditation. It should be mentioned that there are some other critical areas of an inspection which take time but are very significant in reducing public exposure to unnecessary radiation. Fluoroscope output and $f i 1 \mathrm{~m}$ processing techniques must be thoroughly explained to those persons who are not adequately trained in the use of $x$-rays, which we find is usually the case.

Radioactive materials inspections differ significantly from other types of radiation inspections in the diversity of use of materials especially in industry and research, as well as the very rapidly growing medical uses of radioactive material. Safety practices are generally better than with other forms of radiation. One saving factor is that the degree of training required for the user far exceeds the training received by other users of radiation except perhaps radiologists.

This can also be attributed to the fact that applications for a radioactive materials license are completely and thoroughly reviewed in the licensing process. Source strength ranges from kilocurie quantities in medical therapy and industry through research uses to microcurie quantities for in vitro medical diagnostic tests. The inspectors themselves have received extensive training, both on the job and by special courses offered to Agreement States by the U.S. Nuclear Regulatory Commission. The range of instrumentation necessary includes neutron, alpha, soft beta and beta-gamma survey meters plus instruments for air flow measurements on hoods used for non-sealed sources. Our program for radioactive material has 184 licenses. This requires more than 200 amendments, 80 inspections and 30 investigations each year. This requires two men and the assistance of several others in the program during investigations.

Lasers and microwaves are almost totally foreign to state radiation inspectors. Although considerable discussion has been had on the subject and several state programs have legislative authority, they lack adequate background to adjudge something safe or unsafe. A number of guidelines have been set, but they differ considerably from one federal agency to another. One of the few positive approaches was made by the FDA Bureau of Radiological Health when they made microwave survey instrument comparisons and came up with two that were acceptable. Fortunately, these differed widely in price. We have yet to see any such work on lasers and can only guess at the appropriate instrumentation or maximum permissible exposure. Perhaps these non-ionizing sources are better off left to any other kind of regulatory entity than state radiation control programs.

The individual states' radiation control programs are the only method the United States has for making the measurements necessary to assure radiation protection.

What should the states have to make the radiation measurement system operate properly?

1. An independent evaluation of available instrumentation to determine relative merits for different uses and designs for instruments if none of these meet a11 the needs.

2. National calibration facilities for all types of sources and instruments and provision of transfer standards and Quality Assurance.

3. Assistance of equipping an inexpensive in-house "quick check" facility to determine reliability of in-use equipment.

4. Standardized procedures for all types of radiation measurements.

5. An annual peer review on all facets of the State's radiation program like those currently done by the NRC to help upgrade each program.

6. Totally funded continuing education like those provided by NRC which aid the new employee and sharpen the experienced employee.

7. A single federal agency responsible for radiation protection to provide the assistance cited in 1 through 6 above. 
These recommendations are currently the only visible means of assuring uniformity of radiation measurements from state to state.

This data from the real world of the states could be utilized to reach national decisions on radiation protection. Isn't that what the radiation measurement system is al1 about?

\section{References}

1. Conference of Radiation Control Program Directors 7 th Annual Meeting, Hyannis, Massachusetts, Apri1 28 - May 2, 1975.

2. DeVore, R.T. "Diagnostic X-Rays: How Safe Are They?" FDA Consumer, June 1973.

3. Boothe, G.F., K.L. Johnston, and E.A. Ranieri, unpublished data. 
J. S. Pruitt and R. Loevinger

National Bureau of Standards Washington, D.C. 20234

A cavity ionization chamber has been constructed for in-phantom calibration in terms of absorbed dose. Its principle characteristics are the following: a smooth external shape, allowing insertion in the phantom without unnecessary air gaps; a large collecting electrode and a small internal air gap ( $1.3 \mathrm{~mm})$, to meet Bragg-Gray cavity requirements; a uniform collecting potential gradient, allowing high potential gradients and low recombination loss; a guard electrode design which results in a stabilization time of a few seconds; construction of all three electrodes from pure graphite; and a day-to-day precision of about $0.1 \%$. The chambers have been used to make the high-precision depth-dose measurements in water and graphite needed to transfer calorimetric absorbed-dose calibration in graphite to absorbed-dose calibration in water.

(Absorbed dose; ionization chamber; graphite chamber; cavity chamber; calibration)

\section{Introduction}

This paper concerns cavity ionization chambers designed specifically for in-phantom measurements in high-energy beams. They have been successfully used as transfer instruments, to facilitate the transfer of calorimetric absorbed-dose measurements from a graphite phantom to a water phantom.

The two types of chambers described are 3-terminal graphite chambers, differing principally in the shape of the air cavity. The first has a thimble-shaped cavity and constitutes the chief justification for this report. It is a stable, high-precision instrument, capable of measurements with a longterm precision of $0.1 \%$. The second type has a simpler cavity, a cylindrical annulus. Its performance has been equally satisfactory in some respects, less satisfactory in others.

\section{The Graphite Thimble Chamber}

Figure 1 is a schematic cross-section of the thimble-type chamber. The polarizing, collecting, and guard electrodes are a 11 made of graphite with a density of $1.79 \mathrm{~g} / \mathrm{cm}^{3}$. The high-potential and guard insulators are made of acrylic plastic, with a $0.08 \mathrm{~mm}$ Mylar washer separating the guard and collecting electrodes in the air cavity. This small separation minimizes distortion of the electric field around the edge of the collecting volume, and minimizes accumulation of surface charge during irradiation. The components are assembled with epoxy resin, which fills extra-cameral volumes except for air bubbles.
The resin forms an intimate bond between electrodes and insulators, removing the need to coat insulator surfaces with a conducting material.

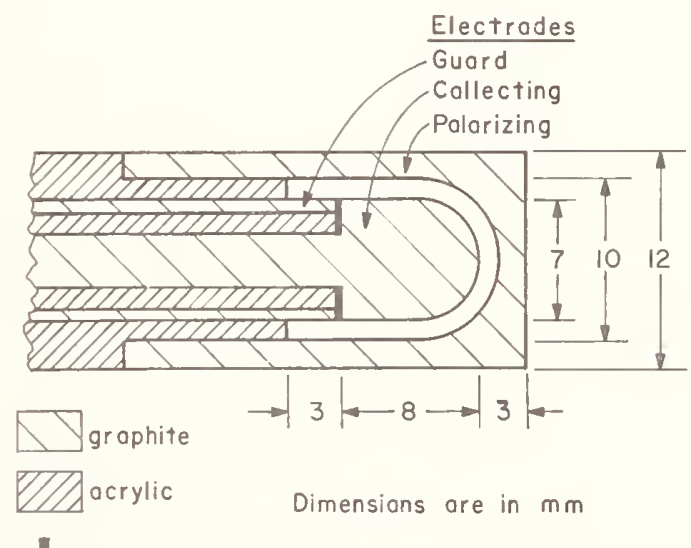

Mylar washer

Fig. 1. Schematic cross-section of a PL1 thimble ionization chamber.

When inserted in a cylindrical hole in the phantom, the hole is filled with a graphite rod of the same diameter as the chamber. The chamber walls are not quite thick enough to provide electron equilibrium for cobalt-60 gamma rays. About $2 \%$ of the chamber current comes from electrons generated outside the cylindrical surface of the 
chamber, while about $\frac{3}{4} \%$ comes from electrons generated in the matching graphite rod.

The guard electrode in the thimble chamber serves two purposes. It prevents current leakage between the polarizing and collecting electrodes, and it prevents the electrostatic field in the collecting volume from being influenced by changes in the charge distribution on the high-potential insulator. This feature reduces the chamber stabilization time to a few seconds and makes alternate measurements at both polarities more convenient. The difference between currents measured with positive and negative polarities depends to a small extent on the experimental situation, but is usually between 0.1 and $0.5 \%$ of the average. It can be made to disappear if a 11 parts of the chamber except the active volume are shielded from radiation, but this is hardly feasible in a phantom. In our practice all measurements with these chambers are averages of positive and negative currents.

The collecting volume is $0.3 \mathrm{~cm}^{3}$ and the air gap is $1.3 \mathrm{~mm}$ throughout, making it a good approximation to a Bragg-Gray cavity. The collecting electrode has a relatively large diameter, permitting the use of large polarizing potentials with a resulting high ion-collection efficiency. The ratio of the potential gradient to the polarizing potential varies from $10.7 \mathrm{~cm}^{-1}$ to $5.8 \mathrm{~cm}^{-1}$ at the inner and outer surfaces, respectively, of the hemispherical part of the cavity. When the polarizing potential $\phi=60 \mathrm{~V}$, the minimum gradient is $350 \mathrm{~V} / \mathrm{cm}$, and when $\phi=500 \mathrm{~V}$, the maximum is $5400 \mathrm{~V} / \mathrm{cm}$. Within this range of polarizing potentials, the potential gradients are large enough so that corrections for general recombination are negligible up to at least $10^{3} \mathrm{rad} / \mathrm{s}$ in a steady beam. These gradients are not large enough to cause sparking, which usually occurs at $\phi=1000 \mathrm{~V}$. Sparking does not seem to damage the chambers, probably because the position of maximum potential gradient is far from the insulators.

The three thimble chambers in operation at present a 11 have input impedances larger than $10^{14} \Omega$ and leakage currents of the order of $10^{-15} \mathrm{~A}$, independent of polarity. Their calibration factors in terms of absorbed dose to water in a cobalt-60 beam are al1 close to $10 \mathrm{rad} / \mathrm{nC}=0.10 \mathrm{~Gy} / \mathrm{nC}$ at STP. Their recombination characteristics are identical and can be summarized by the equation:

$$
\frac{I_{\phi}}{I_{60}}=K\left(1-\frac{A}{\phi}+B \phi\right)\left(1-\frac{C I_{60}}{\phi^{2}}\right)
$$

where $I_{\phi}$ and $I_{60}$ are the chamber currents or the measured dose rates for polarizing potentials of $\phi$ and $60 \mathrm{~V}$, respectively. The constants have the values

$$
\begin{aligned}
A & =0.069 \mathrm{~V} \\
B & =1.3 \times 10^{-5} \mathrm{~V}^{-1} \\
C & =0.20 \mathrm{~V}^{2} / \mathrm{nA} \\
& \simeq 0.020 \mathrm{~V}^{2} /(\mathrm{rad} / \mathrm{s})=2.0 \mathrm{~V}^{2} /(\text { Gy } / \mathrm{s})
\end{aligned}
$$

$K$ is a normalizing factor which does not differ significantly from unity for dose rates up to $100 \mathrm{rad} / \mathrm{s}=1 \mathrm{~Gy} / \mathrm{s}$. Recombination measurements with one of the thimble chambers are compared with Eq. (1) in Fig. 2

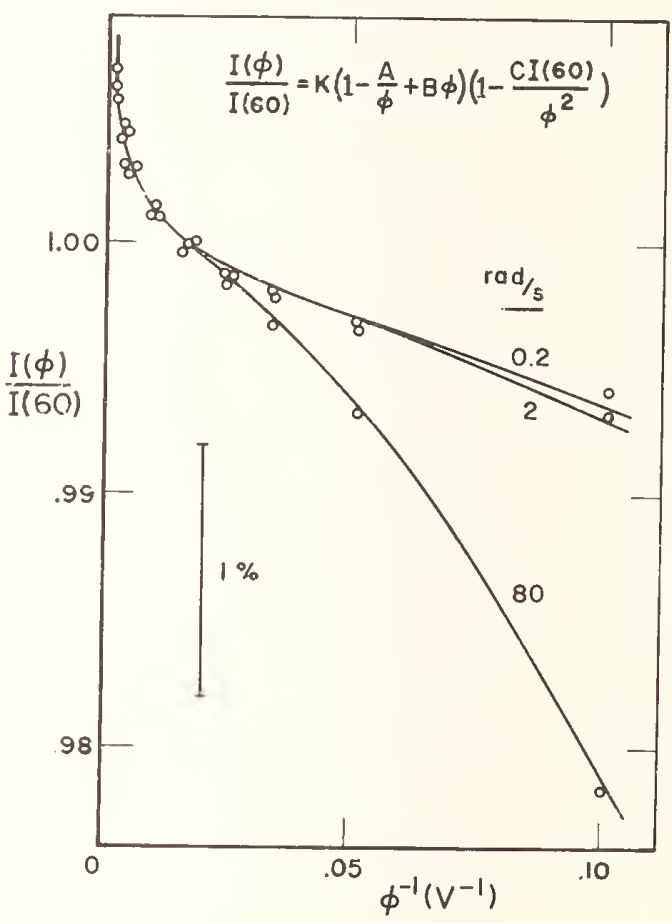

Fig. 2. Recombination measurements for thimble chamber PL1-10. The solid lines are predictions of the depicted equation.

The form of Eq. (1) is reminiscent of equations used to calculate recombination corrections to exposure determinations ${ }^{1}$, in which the first bracketed term, which is independent of exposure rate, is identified with initial recombination, and the second 
bracketed term with general recombination. The new feature in Eo. (1) is the 1 inear term in 0 in the first bracket, which describes the rise at the left side of Fig. 2 . It is only significant for $\phi>100 \mathrm{~V}$, and is smaller than $1 \%$ for $\phi$ as large as $500 \mathrm{~V}$.

This linear term could represent the beginning of ion multiplication in the chamber, enough to increase the current above the expected value, but not enough to sustain a chain reaction leading to a spark. The increase in current appears with both polarities, but is more pronounced for a negative polarizing electrode, as would be expected from the higher mobility of electrons, which probably constitute a significant fraction of the negative charge-carrying particles.

For this chamber, Eq. (1) cannot be used to describe recombination corrections in terms of $I_{\infty}$, the current at infinite potential, as is usually done with exposure measurements ${ }^{1}$, unless the potential is so low that $B$ c can be neglected. However, the first bracket of Eq. (1) is not relevant to a calibrated chamber if it is used at the calibration potential. The correction for different dose rates is given by the second bracket in Eq. (1).

The thimble chambers have been used to make extensive depth-dose measurements in graphite and water phantoms which will be reported elsewhere.

\section{The Cylindrical Chamber}

Three chambers have been constructed as shown in Fig. 3, with cylindrical symmetry and two guard electrodes. The purpose of this change was to simplify the shape of the air cavity and make it more amenable to mathematical analysis. The results to date are somewhat equivocal, probably because the assembly of a cylindrical chamber is considerably more complicated than for a thimble chamber. The input resistances are lower $\left(10^{10}-10^{12} \Omega\right)$ and the leakage currents are larger $\left(10^{-14}-10^{-12} \mathrm{~A}\right)$. Nevertheless, the leakage currents are independent of polarity, and the averaging of positive and negative polarity measurements appears to correct accurately for leakage. The depth-dose measurements made with one of these cylindrical chambers, which had a leakage current of $3 \times 10^{-13} \mathrm{~A}$, are indistinguishable from those made with the better-behaved thimble chambers. The recombination characteristics also appear to be similar, at least at low dose rates.

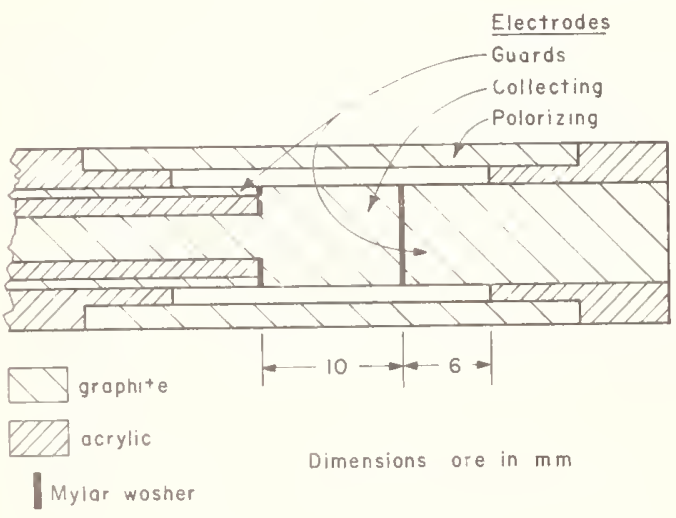

Fig. 3. Schematic cross-section of a PL2 cylindrical ionization chamber. Dimensions not shown are the same as for a PLl thimble chamber.

\section{$\underline{\text { References }}$}

1. See, for instance, T.P. Loftus and J. T. Weaver, "Standardization of ${ }^{60} \mathrm{Co}$ and ${ }^{137} \mathrm{Cs}$ Gamma-Ray Beams in Terms of Exposure," $\mathrm{J}$. Res. Nat. Bur. Stand. 78A, 465-476 (1974).

\section{Acknow redgement}

This work was supported in part by the National Cancer Institute. 

THE DEVELOPMENT AND APPLICATION OF MONOENERGETIC X-RAY SOURCES

J. H. Sparrow and C. E. Dick

National Bureau of Standards

Washington, D. C. 20234

High flux standard $x$-ray beams have been developed in the energy range from $\mathrm{i}$ - to $100 \mathrm{-keV}$. These beams which are produced by direct excitation of characteristic $x$ rays in elemental targets are useful for the investigation of phenomena associated with radiological imaging and the calibration of radiation detectors. The design and performance of the sources and some applications to radiation measurement systems will be discussed.

(Detector calibrations; fluorescence $\mathrm{x}$-ray sources; $\mathrm{K}$ x-ray sources; pulsed $x$ rays; radiographic imaging; steady-state $x$ rays)

With the current emphasis on product evaluation and safety, nondestructive examination, and the need to develop new energy sources, the use of $x$ rays is increasing rapidiy. They are being employed not only in the conventional industrial and medical radiographic applications but in new techniques such as $x$-ray fluorescence analysis and as diagnostic tools in plasma fusion research. blith all of these applications, however, a common need arises for a source of variable energy monoenergetic beams of $x$ rays for the examination of the energy response of systems and for calibrations of x-ray detector responses.

Historically, this need for monoenergetic $x$-ray sources has been filled by the use of radioactive isotopies or filtered bremsstrahlung spectra. Radioactive sources, however, are severely limited in their usefulness because of the limited number of lines available (particularly, in the energy range from $1 \mathrm{keV}$ to $100 \mathrm{keV}$ ) and because of the $1 \mathrm{imited}$ intensity range in small sources due to selfabsorption effects. Filtered bremsstrahlung spectra do not suffer from the drawback of limited intensity, but do not in many instances provide the degree of monochromaticity necessary for the characterization of energy response required.

Monoenergetic $x$-ray beams from $1-100 \mathrm{keV}$ can be produced by the excitation of characteristic x-ray lines. These lines can be excited by the interaction of either photons or charged particles in elemental targets. The photon excitation or fluorescence method has been widely utilized and is shown schematically in Fig. 1. Electrons preduced by a heated tungsten filament are focussed on a cooled anode assembly. $x$ rays and bremsstrahlung qenerated in the anode are then allowed to strike a secondarv fluorescent radiator. Because of the nature of the photoelectric process, the $x$ rays fluoresced in the secondary target are very nearly monoenergetic

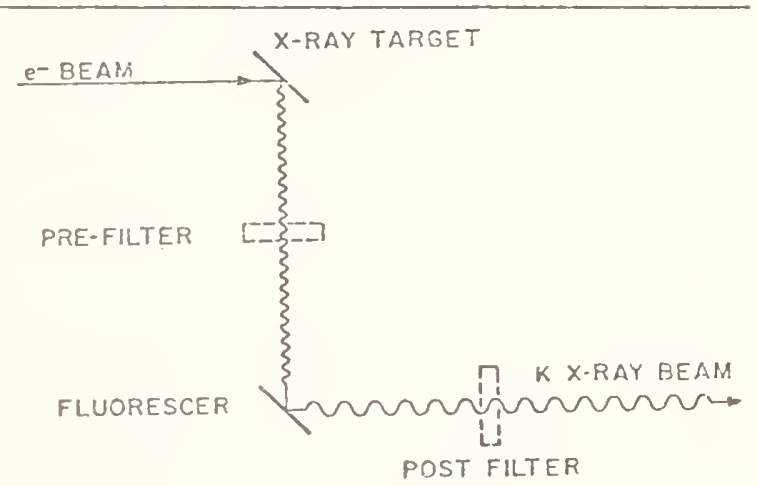

Fig. 1. Fluorescence method

(typically, for these sources, greater than $90 \%$ of the intensity is in the characteristic line of interest). 1 The principal dravback of this source lies in the two-step process involved. To achieve high output intensities, the primary anode requires cooling which complicates changing of the targets and altering of the x-ray energy. Because of these limitations, the intensities of fluorescent sources are limited to values below about $10^{10} \mathrm{~K}$ $x$ rays per steradian per second.

In order to obtain greater flux densities and facilitate changes in the $x$-ray energy, we have developed a source which utilizes only the first step of the fluorescence procedure, direct electron excitation of characteristic 7 ines in elemental targets. 2 Figure 2 illustrates the characteristic K line and bremsstrahluna angular distribution for a thin silver target for two different electron energies. Note that the bremsstrahlung is emitted preferentially in the direction of the incident electron momentum vector, while the $K \times$ rays are emitted isotropically. This suggests that with a proper choice of observation angles (near $180^{\circ}$ with respect to the electron direction) the ratio of $\mathrm{K} x$ ray to 


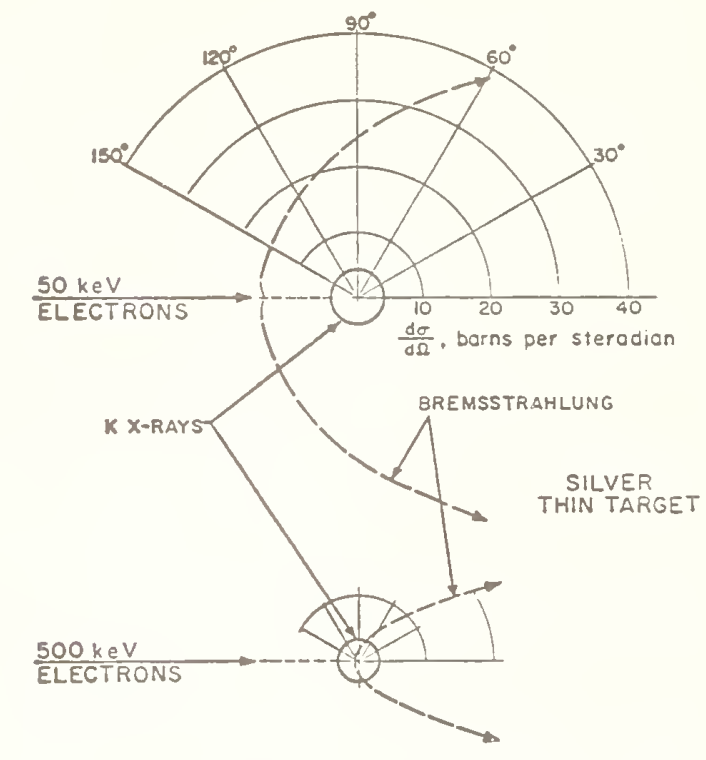

Fig. 2. Comparison of the angular distribution of $K \times$ rays (solid lines) and bremsstrahlung (dashed lines) for 50 and $500 \mathrm{keV} \mathrm{elec-}$ trons incident on thin silver targets.

bremsstrahlung output can be maximized. To investigate whether this ratio can be made sufficiently large for thick targets to provide adequate beam purities, an extensive experimental study of this process was undertaken. In these experiments, $x$-ray targets ranging in atomic number from beryllium to gold were bombarded with electrons having energies between $10 \mathrm{keV}$ and $3 \mathrm{MeV}$ from two low energy NBS electron accelerators. The $x$-ray spectra generated at $180^{\circ}$ were recorded as a function of the $x$-ray target thickness with various photon spectrometers. The results of this study have been published ${ }^{3}$ and can be summarized as follows:

a. The ratio of the $K \times$ ray to bremmsstrahlung intensities can be made large enough to provide beam purities in excess of $60 \%$ for the entire range of energies from 0.1 to 70 $\mathrm{keV}$.

b. This ratio is essentially independent of target thickness so that rugged easily handled targets are feasible.

c. The total output is determined by the current loading capacity of the target and even uncooled targets can provide flux densities of greater than $10^{10} \mathrm{~K} \times$ rays per steradian per second. d. The x-ray beams can be optimized for either maximum output or maximum purity by a simple adjustment of the experimental parameters.

e. The output can be extremely well characterized by a measurement of the electron beam current and energy.

Figure 3 illustrates the $K x$-ray output as a function of the incident electron energy for a variety of targets whose $K$ x rays range from 0.1 to $70 \mathrm{keV}$. These yield curves are used to determine the flux density for a particular combination of electron energy and target material. Fiqure 4 gives a schemat

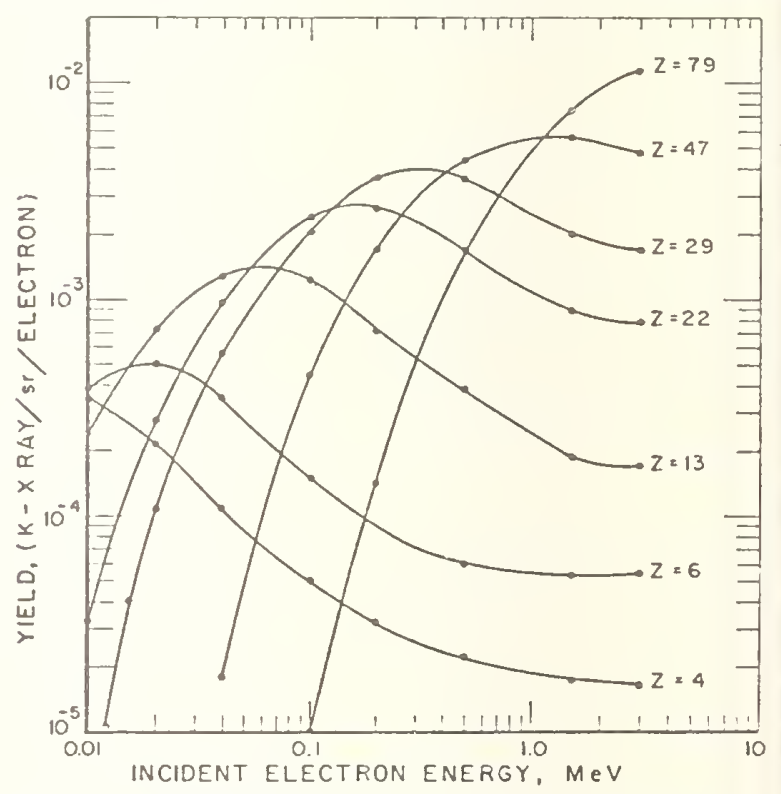

Fig. 3. Dependence of $k x$-ray yields for thick targets of $Z$ from 4 to 79 on incident electron energy.

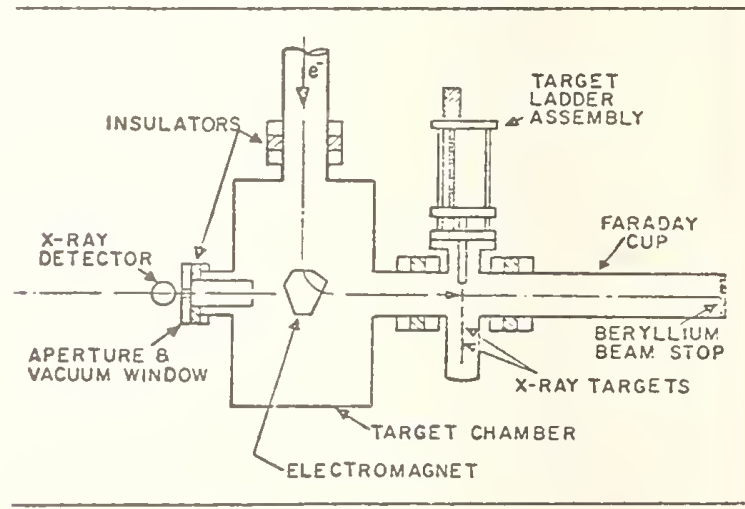

Fig. 4. Diagram of $K x$-ray source at $180^{\circ}$ geometry. 


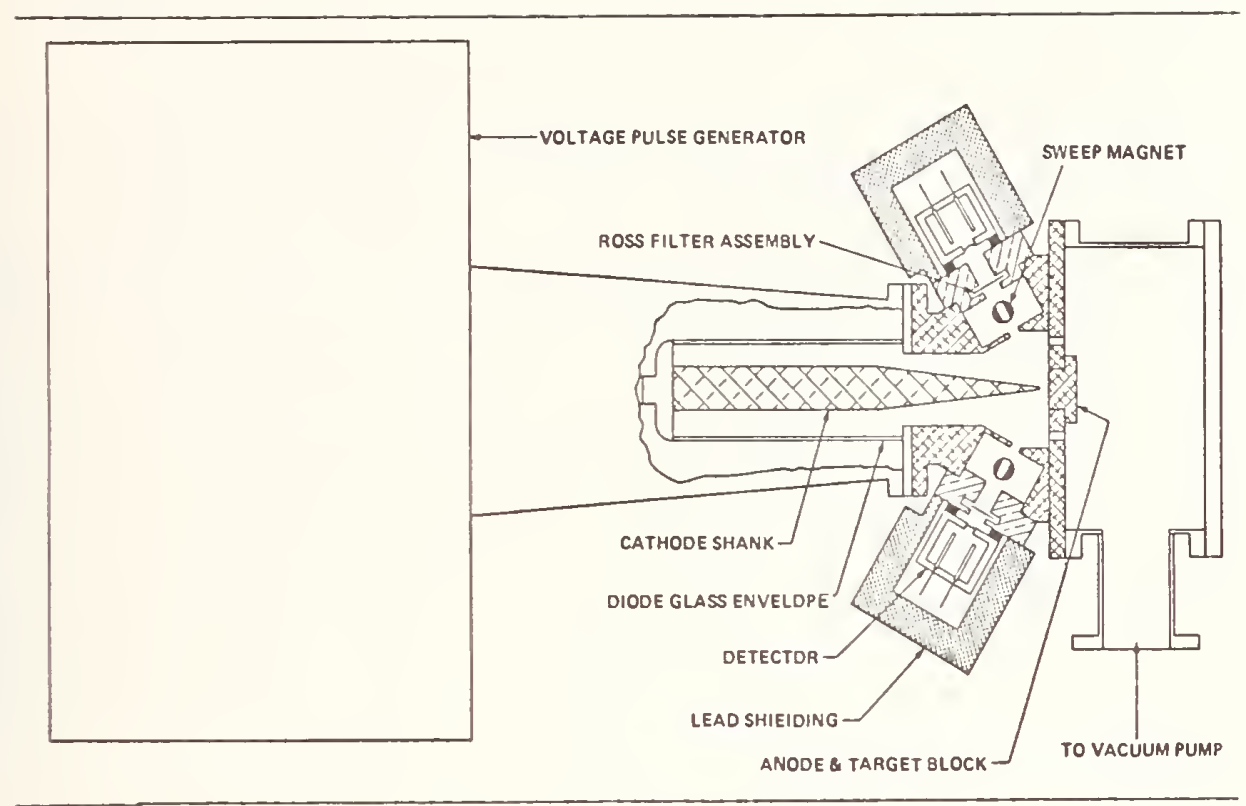

Fig. 5. Pulsed $K$ x-ray source

epresentation of the steady-state setup used for the measurements and as an x-ray source. Applications of this source will be described below.

In order to provide higher flux densities (up to $10^{18} \mathrm{~K} \times$ rays $/ \mathrm{sr} / \mathrm{s}$ ), a pulsed source has also been developed utilizing the concepts described above. Figure 5 depicts this source in which an intense electron beam generated by a high voltage pulser impinges on an elemental x-ray target. Typically, kiloamperes of beam: current are delivered to the target in a few nanoseconds thereby circumventing the target heat loading problems associated with high current steady state sources. Four identical ports in an $120^{\circ}$ geometry are equaliy spaced radially around the diode axis. This setup has been designed primarily as a calibration source for the calibration of radiation detectors utilized in high flux rate environments. In oprration, a parallel plate ionization chamber developed at NBS to measure the pulsed $x$-ray flux density is mounted on one of the four ports to measure the absolute output for each output pulse. This detector has been calibrated on the steady-state source described previously and its 7 inearity with flux density has also been measured. ${ }^{4}$ Detectors to be calibrated are placed on the other ports and compared to the standard detector. At present, this setup provides pulsed $x$-ray beams from 1.5 to $8 \mathrm{keV}$.

With the avajlability of monoenergetic $x$-ray sources with energies from 0.7 to $70 \mathrm{keV}$ and flux densities up to $10^{18} \mathrm{~K} \times \mathrm{rays} / \mathrm{sr} / \mathrm{s}$, a number of experiments utilizing these beams are underway at NBS. These experiments give some indication of the utility of such sources in a number of areas. The pulsed source has been used to calibrate a number of detectors. The calibration of a PIN solid state detector is shown in Fig. 6. These diodes are

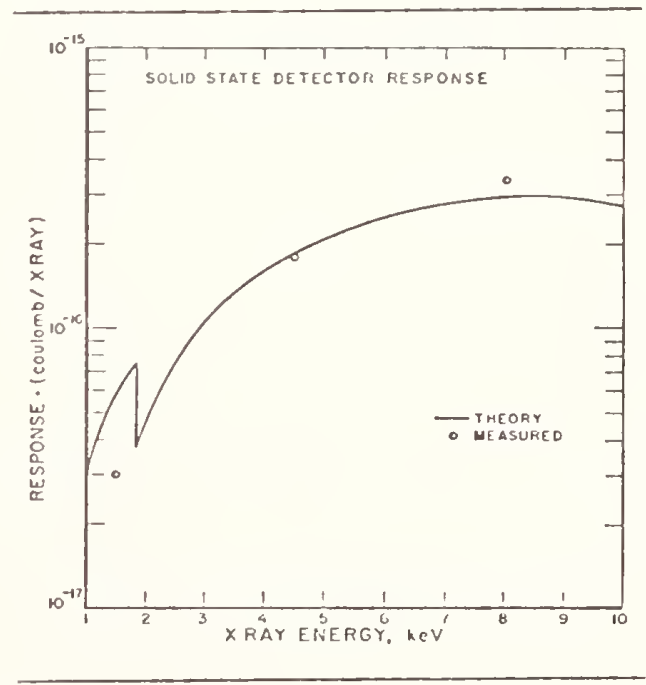

Fig. 6. The energy dependence of a PIN silicon diode as compared to calculated responses from energy absorbed in silicon.

utilized in the measurement of soft $x$ rays below $10 \mathrm{keV}$ in the diagnostics of plasma fusion devices. The shape of the $x$-ray spectrum can be related to the electron temperature in the plasma, a parameter of prime 


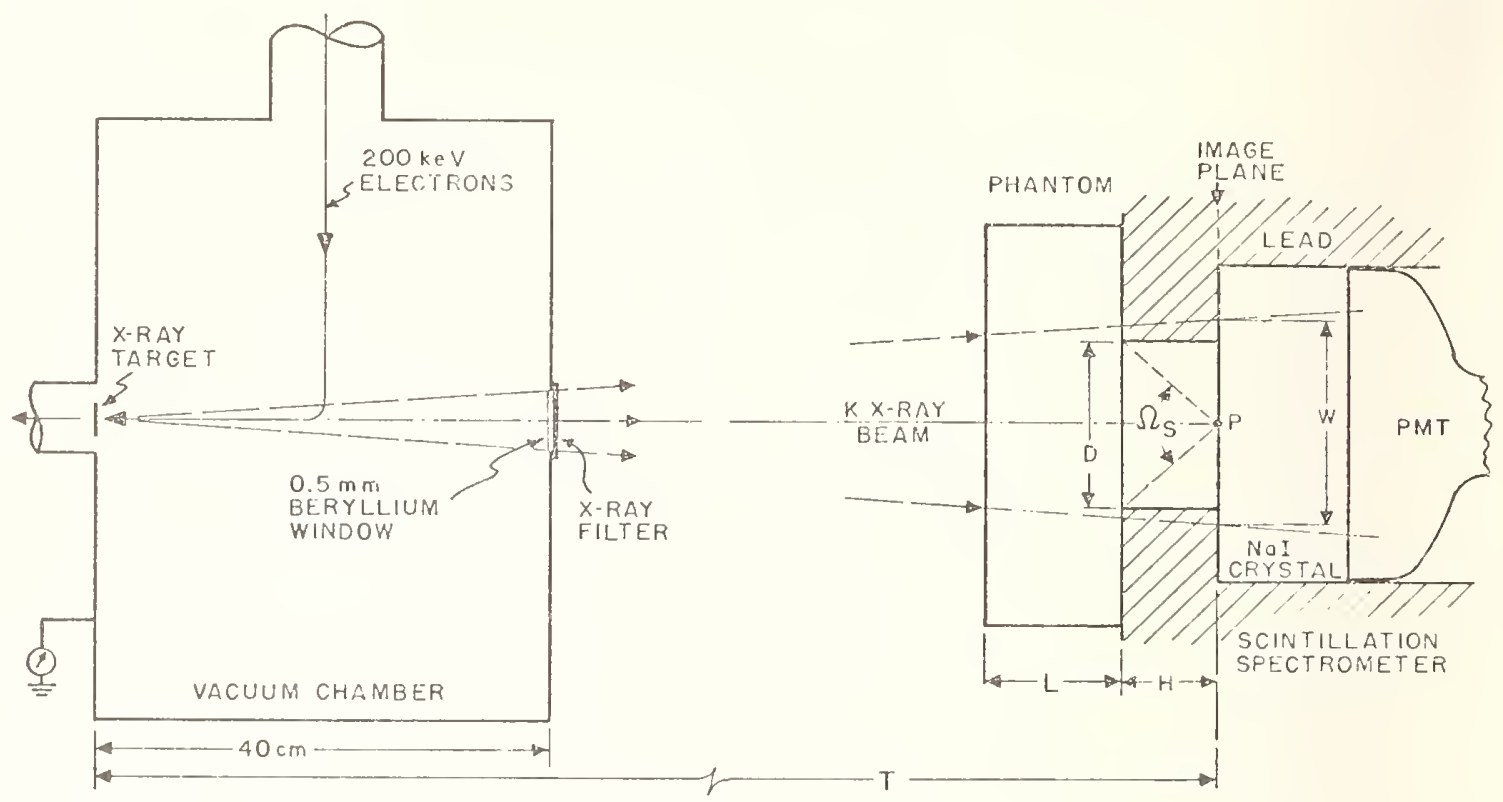

Fig. 7. Experimental arrangement for scatter measurements

importance in these experiments. Figure 6 illustrates the behavior of the diode as measured (open circles) and calculated (solid line) in this energy range. The calculated values are based on the mass attenuation coefficients and are corrected for deadlayer effects. Notice the variation in the response of this device from the calculated curve especially at low energies. This can lead to a significant error in the shape of the $x$-ray spectrum. This diode is designed for operation in high intensity environments and has a large steady-state leakage current which makes calibration in steady-state $x$-ray beams an extremely difficult procedure.

The steady-state $x$-ray sources are particularly well suited for measurements in radiographic imaging. Typical of these experiments is the measurement of the amount of scatter introduced into radiographic image signals as the $x$ rays pass through the object to be radiographed. This scatter is the principal source of loss of contrast and unsharpness in radiographs. ${ }^{5}$ Figure 7 shows the setup utilized to measure this quantity. A beam of monoenergetic $x$ rays impinges on a scatterer of polystyrene. The x-ray spectrum which is transmitted through the phantom is recorded by a scintillation spectrometer. The amount of scatter present in the image signal can be determined from measurements of the spectra recorded as a function of the solid angle, $\Omega_{s}$. Figure 8 shows the buildup of the scatter contribution to the image signal for a $10 \mathrm{~cm}$ thick and for a $21 \mathrm{~cm}$ thick phantom for $x$-ray energies of 32 and $69 \mathrm{keV}$. These data have been reported fully elsewhere. ${ }^{6}$

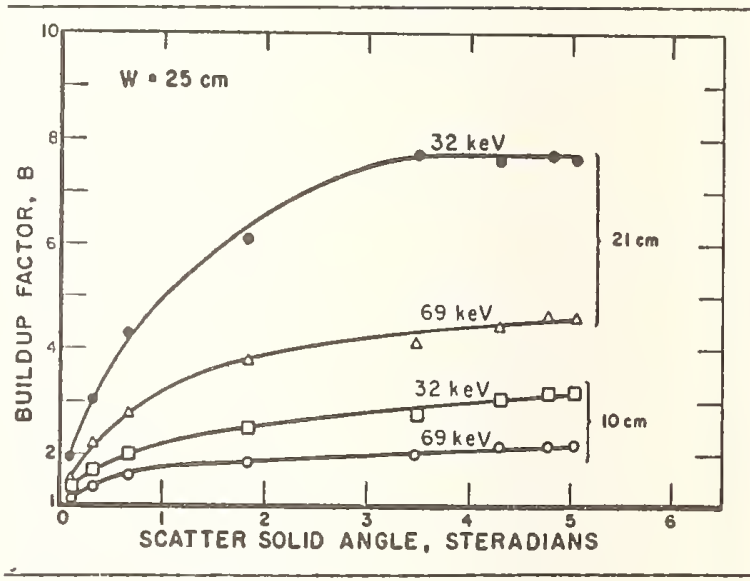

Fig. 8. Dependence of the build up factor, $B$, on scatter solid angle, $\Omega_{\text {}}$, for a 21 - and $10-\mathrm{cm}$. phantoms and for incident $x$-ray energies of 32 and $69 \mathrm{keV}$.

In conclusion, we have developed highly monoenergetic $x$-ray sources in the energy range from 0.1 to $70 \mathrm{keV}$ and with flux densities up to $10^{18} \times$ rays $/ \mathrm{sr} / \mathrm{s}$. These $\mathrm{x}$ rays are extremely useful in the calibration of detectors as a function of energy and flux rate and for any system whose energy dependence in this energy range must be ascertained. 


\section{Reference}

1. J.L. Gaines, P.J. Ebert, and G.R. Leipelt, 4. Lawrence Livermore Laboratory Memorandum UOPAD 67-107 (1967).

2. J.W. Motz, C.E. Dick, A.C. Lucas, R.C. Placious, and J.H. Sparrow, J. Appl. Phys. 42 2131-2133 (1971).

3. C.E. Dick, A.C. Lucas, J.W. Motz, R.C. Placious, and J.H. Sparrow, J. Appl. Phys. 44, 815-826 (Feb 1973).

J.H. Sparrow and C.E. Dick, Pulsed High Intensity Monoenergetic Low Energy X-Ray Source and X-ray Monitor, to be published.

5. Examples of such studies, which include references to other pertinent investigations, are given by G. Hettinger, Acta Radiol. 54129 (1960) and E.D. Trout and J.P. Kelly, Radiology 104, 161 (1972).

6. J.W. Motz and C.E. Dick, Medical Phys. 2, No. 5, 259-267 (1975). 

THE DESIGN OF A MOBILE RADIATION CALIBRATION LABORATORY

R. C. Placious and J. C. Humphreys

National Bureau of Standards

Washington, D.C. 20234

A mobile $x$ and $\gamma$-iay standards laboratory with facilities

for calibration and testing of radiation instruments has been proposed as an effective method for propagating the national standards for exposure directly to state agencies responsible for local radiation control. NBS studies indicate that this can be accomplished by equipping a trailer truck with various sources of radiation, reference class instrumentation, and power generating equipment. The trailer described here incorporates radiation shielding which wiil be optimized in relation to beam collimation, direction, scattering, and maximum weight. The design provides all proper safeguards prescribed by federal or state agencies for transport over the nation's highways.

(Mobile laboratory; radiation calibrations; radiation control; radiation measurement assurance; radiation standards)

\section{Introduction}

A mobile ionizing-radiation calibration facility has been proposed for calibrating instruments for state and local agencies at their laboratories. This mobile laboratory, designed by and constructed under National Bureau of Standards (NBS) supervision, would further the national goal of radiation measurement uniformity throughout the United States. According to recent NBS surveys few states at present maintain a calibration range for field or laboratory type radiation measuring instruments. An instrument to be checked or calibrated is sent to NBS, BRH (Bureau of Radiological Health), or private calibration laboratories. Because of costs and time delays, this generally means that only instruments classed as reference or laboratory standards are calibrated. The "turn around time" for these calibrations can be quite long.

As presently viewed by NBS, based on needs expressed by a number of states, the more immediate goals of the mobile laboratory are:

1. To calibrate the principal radiation measuring instruments of the various states.

2. To provide a tutorial or training service on calibration and measurement procedures, and the establishment of suitable calibration ranges.

3. To assist the states in solving unusual or unique radiation measurement problems that may arise in the course of their work.
A long range goal of the laboratory is to test the states' abilities to calibrate their own radiation instruments as part of a voluntary measurement assurance program.

All of these goals can be most effectively accomplished with the aid of the mo bile laboratory.

Design Considerations for a Mobile Calibration Facility

General Requirements

At this stage the criteria established for the mobile laboratory are:

a. The laboratory must meet all federal and state safety requirements for transportation and operation of the facility.

b. The laboratory must be capable of functioning without tying into the electrical service and other facilities of the local or state agency.

c. Insofar as practical, the laboratory should be designed to use commercially available equipment in its construction. The laboratory itself should be engineered and built by private contractors according to NBS specifications.

\section{The Radiation Sources}

of primary importance is the decision as to which radiation sources the laboratory will require for calibrating radiation instruments. Based on available survey information and personal contacts, it appears that an $x$-ray system capable of operating in the 25 to $300 \mathrm{kVcp}$ range, and a higher energy, longlived gamma ray source will be needed. 
The energy range of the $x$-ray system places it in the same region as is used for most medical diagnostic and radiographic procedures. This selection is not merely coincidental since monitoring the radiation levels of medical and dental x-ray units is one of the most important functions of the state radiation control agencies.

The $x$-ray source will have continuously variable, wel1-regulated tube potential and current, and would, if necessary, use both end-grounded and center-grounded tube heads. The tube current range should be from about 0.5 to $10 \mathrm{~mA}$ at $300 \mathrm{kV}$ and $20 \mathrm{~mA}$ at $150 \mathrm{kV}$. These specifications are within the 1 imits of presently available systems. Water cooling for the tube heads would be necessary. Based on measurements at NBS, such an $x$-ray source will provide an exposure-rate range from about $25 \mathrm{mR} / \mathrm{h}$ to $1000 \mathrm{R} / \mathrm{h}$, using the same filtration presently used at the NBS $x$-ray standards laboratory and the distance variation permitted by the length of the calibration range.

of the commonly used sources for higher energy instrument calibrations, generally ${ }^{137} \mathrm{Cs}$ and ${ }^{60} \mathrm{Co}$, the former was selected because of its longer half 1 ife and reduced shielding requirement. In addition, the energy dependence of instruments does not chànge significantly in the region between 0.6 and $1.2 \mathrm{MeV}$. The specific exposure rate for ${ }^{137} \mathrm{Cs}$ is about $0.32 \mathrm{R} / \mathrm{h}$ per curie at 1 meter. A source with activity of about 3.1 $\mathrm{C} i$ is required to produce an exposure rate of $1 \mathrm{R} / \mathrm{h}$ at one meter and about $60 \mathrm{mR} / \mathrm{h}$ at 4 meters.

The $x$-ray tube head (or heads) and the shielding cask holding the ${ }^{1{ }^{7}} \mathrm{Cs}$ source would be mounted "piggy-back" style on the same vertical axis and they would be alternately raised or lowered into position for a calibration. (See Fig. 1.) A single track

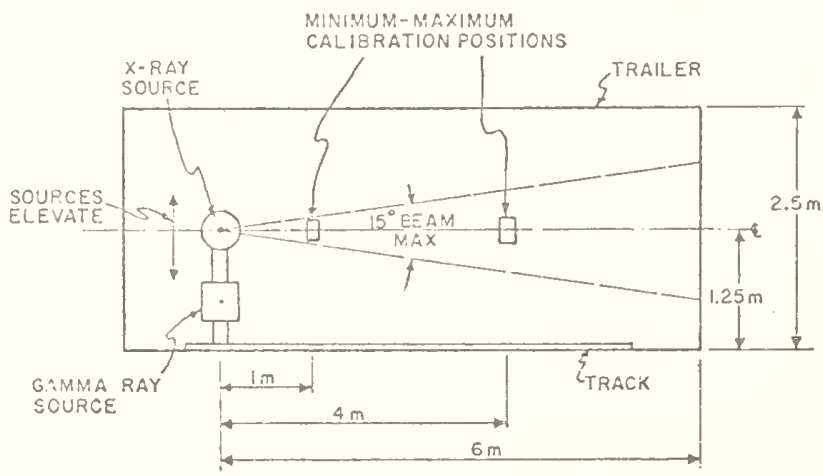

Fig. 1. Source and detector arrangement in the mobile laboratory. would extend down the center of the trailer for the length of the measurement room. The track will be designed so that the distance between a source and the reference point on an instrument being calibrated can be easily determined with high accuracy.

Shielding Requirements and Beam Collimation

The geometry described above and shown in Fig. 1 is designed to minimize scattered radiation in the calibration area. The useful beam for each source must be collimated so that no part of the primary beam strikes the side walls, floor or ceiling. The rear wal1 of the laboratory on which the direct beam impinges is expected to be 6 meters from the sources. The transverse crosssectional area of the laboratory is expected to be about $2.5 \times 2.5$ meters. Therefore, walls parallel to the beam axis will be about 1.25 meters from the center of the track. The maximum beam diameter at 6 meters from the source with good collimation should not exceed about 1.5 meters. This requirement indicates a beam cone of about $15^{\circ}$ total angle is required. Since most detectors used in radiation measuring instruments do not exceed $10 \mathrm{~cm}$ diameters, the maximum solid angle intercepted by the instrument being calibrated is at most 6 degrees at 1 meter and 1.5 degrees at 4 meters from the source. Beam uniformity must be such that over the useful beam size of $15^{\circ}$ the variation in flux must not exceed $1 \%$.

Where shielding is required lead has been chosen since it has the best shielding to volume ratio. Lead is also desirable since the radiation albedo decreases with increasing atomic number (see Fig. 2). In addition to the radiation shielding it may be necessary to provide magnetic and radio frequency shielding in the "skin" of the trailer itself. Sound and thermal insulation will also be needed. Fluorescence from the shielding material will be investigated and if significant, the proper material to reduce it without increasing albedo can be applied as an internal lamination to the lead wall.

The shielding thickness is graduated, with the heaviest shielding on the back wall opposite the source and on the wall separating the control area from the radiation area. The details of the radiation shielding are shown in Fig. 3. The wall between the control area and measurement area requires a minimum of 3 millimeters of lead to reduce the radiation to the same level $(2.5 \mathrm{mR} / \mathrm{h})$ as the exterior surfaces. 


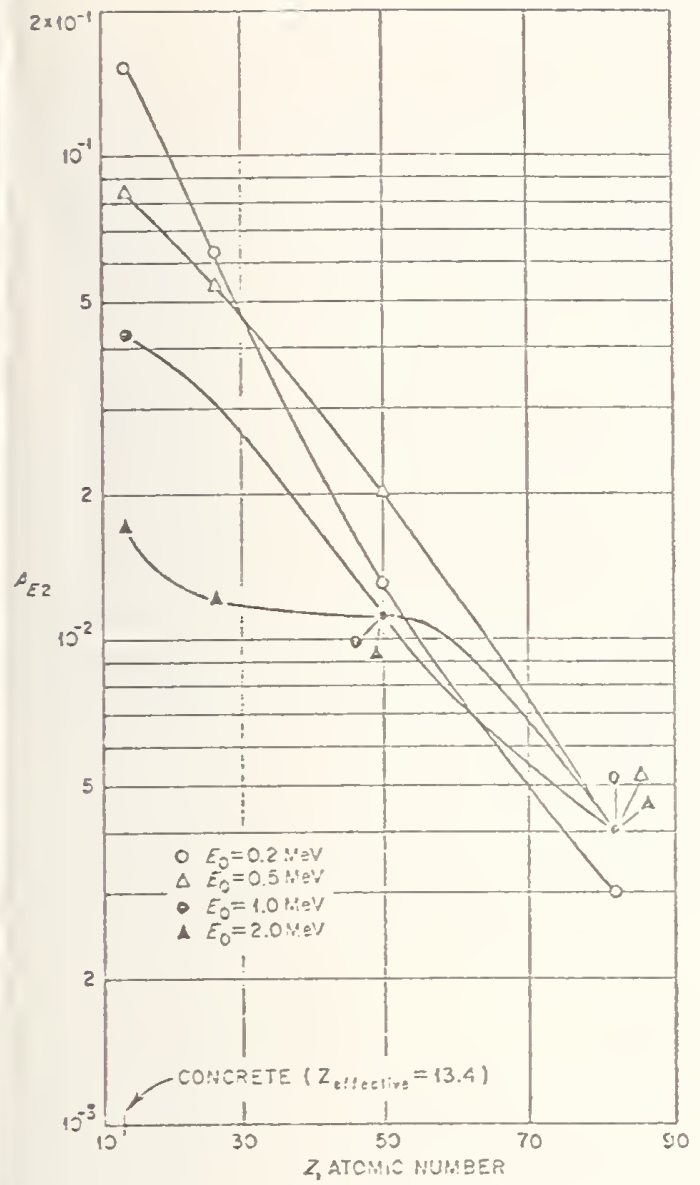

Fig. 2. Energy albedo of monochromatic photons with initial energies from 0.2 to 2.0 MeV normally incident on semi infinite media of different atomic number (from Berger and Raso) ${ }^{1}$

\section{Laboratory Facilities and Services}

Electrical service will be required in the $7 a b$ to operate the $x$-ray set, general lighting, instrumentation, and air conditioning. The power required should be provided by a self-contained motor-generator system with the alternative provision that power may be obtained by cable where adequate local ac service is available. The air-conditioning system would provide both cooling and heating capabilities as well as control of the relative humidity. The entire laboratory should be thermally well-insulated to minimize the power required for air conditioning. The system should be capable of maintaining the entire lab at a given temperature within $\pm 7^{\circ} \mathrm{C}$ and the relative humidity to $\pm 5 \%$. The $x$-ray unit will require a closed water cooling system for the tubehead with provision for heat exchange using the laboratory air conditioning unit or its own water cooler.

The following table indicates the estimated electrical power needs.

Table 1. Estimated Electrical Service Needed

\begin{tabular}{|c|c|}
\hline Item & AC Power Reguired \\
\hline ray U & $240 \mathrm{~V} \mathrm{ac}, 7 \mathrm{~kW}$ \\
\hline Temperature Control & $240 \mathrm{~V}$ ac, 15 \\
\hline
\end{tabular}

(Heating \& Cooling)

Motorized Tube Head-Source $240 \mathrm{~V}$ ac, $1 \mathrm{kl}$ ! Elevator \& Shutter Mechanism

Lighting, Ejectronics, etc. $120 \mathrm{~V}$ ac, $5 \mathrm{~kW}$

Item 2 includes the $3 \mathrm{~kW}$ of power needed for cooling the x-ray tube head. Total power needs are, therefore, about $28 \mathrm{~kW}$. This can

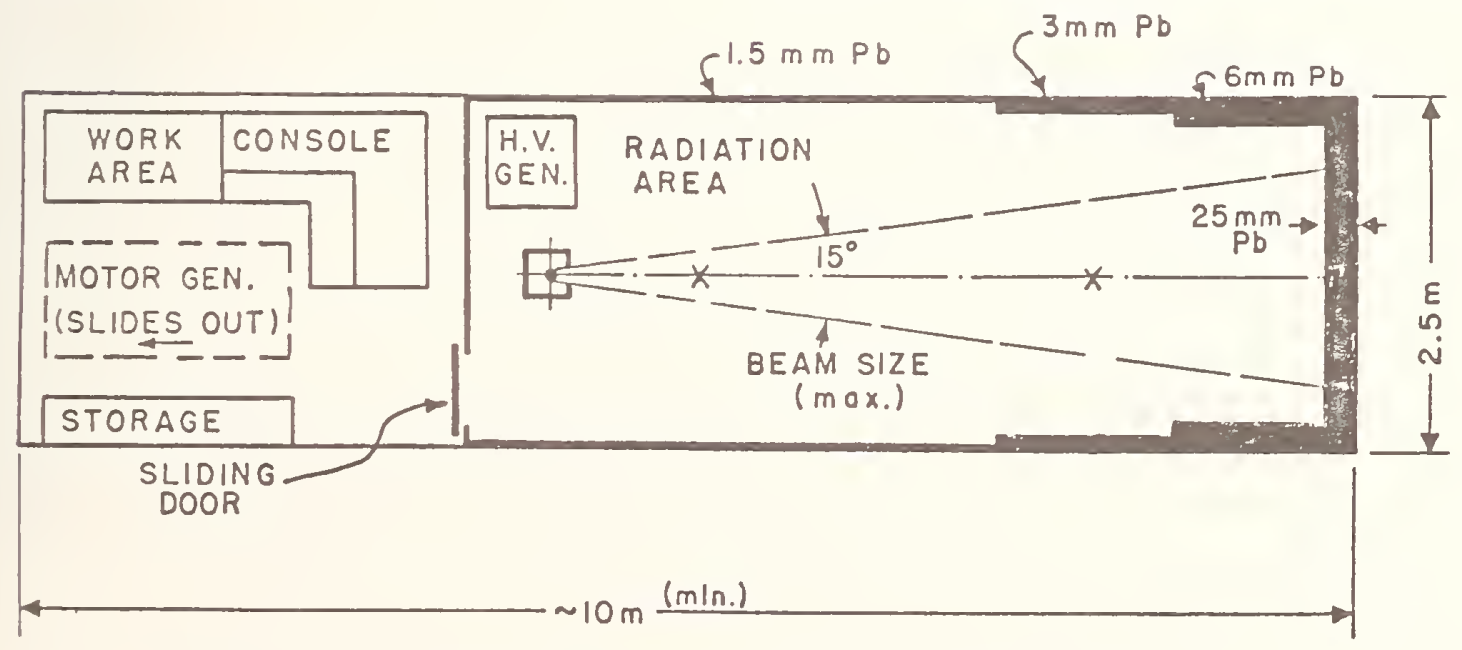

Fig. 3. Plan view of the mobile laboratory showing the shielding arrangement surrounding the radiation area. 
best be handled (perhaps) by two motor generators rather than one large one. It is assumed that most states will not be able to supply this much power from their laboratory 1 ines so the motor generator system should have a high usage factor.

Overall Layout and Weight Estimates of

\section{Laboratory}

The overall layout is shown in Fig. 3 . The radiation room occupies an area of about 7 by 2.5 meters wide $\times 2.5$ meters high and is expected to house the $x$-ray generators, the water cooler and circulator, the $x$-ray tube head and the ${ }^{137} \mathrm{Cs}$ source with its container. Access to this area is through a shielded sliding door properly interlocked and moritored for safety. The control area of about
$3 \times 2.5$ meters wide $\times 2.5$ meters high will contain all controls for operating the $x$-ray unit or the shutter for the source as well as a data acquisition and processing system. A cable rack properly shielded to prevent radiation leakage into the control room area will provide the means for sending and receiving information between control room and radiation room.

Some of the estimated weights and sizes of the equipment to be installed in the mobile facility are given in table 2. Noise and vibration from the air conditioning units, the water cooler or the motor generator set must be reduced to a very low level because of the sensitive electronic equipment used in this facility. This may require, for example, that these items be so designed that during operation they can be acoustically and mechanically insulated from the trailer (e.g., by outboarding on tracks).

Table 2. Size and Weight of Laboratory Equipment and Shielding

\section{Item}

1. X-Ray Units
(a) Generators (2)
(b) Control Unit
(c) Tube Heads
(d) Water Cooler

2. Isotope Source

3. Lead Shielding

Forward Wal1 (2.5 cm) Side Walls (2)

Back Wal1

4. Electrical Generator

5. Air Conditioning System

6. Control Console w/Instrumentation \& Work Bench
Estimated

Floor space Needed

$(\text { Meters })^{2}$

2

$1 / 2$

1

$1 / 4$

$1 / 2$

See

Figure 3

4100

1

700

1

2

300

600

$\mathrm{kg}$

560

40

90

180
Estimated Weight

Tota]

$6650 \mathrm{~kg}$ 
Choice of Vehicle for Laboratory

Consideration was given to two types of vehicles for the mobile laboratory; the recreational van such as many states and federal agercies use for environmental monitoring and other functions or a tractortrailer type truck. The recreational type van comes close to meeting the length requirements but the largest has a load-carrying capacity that is a factor of three too low. It would appear that a truck is the only practical solution. A representative of one of the major tractor manufacturers suggested that the tractor-trailer combiration would be the best arrangement for the load range $(20,000$ 1b.) the lab would require. One standard size trailer in this range is $12 \mathrm{~m}$ ( 40 feet) long, $2.5 \mathrm{~m}$ ( $\varepsilon$ feet) high, and $2.5 \mathrm{~m}$ ( 8 feet) wide. This trailer has a mass of $5000 \mathrm{~kg}$ $(11,0001 b$.$) , so a suitable tractor would need$ a "gross vehicle weight rating" of at least $14,000 \mathrm{~kg}(31,0001 \mathrm{~b}$.$) . This is no problem$ for existing tractors. Because of the delicate nature of the equipment in the trailer an air suspension shock prevention system will be prescribed.

\section{Calibration Standard}

The mobile laboratory calibration standard will be a transfer quality ionization chamber instrument, of high precision, calibrated against the national standard maintained at NBS. ${ }^{2}$ As indicated in reference 2, this instrument would probably be of guard ring design with low leakage and high consistency in exposure rate measurements. In all likelihood, two transfer quality instruments would be provided to cover both the high and low energy range. The redundancy necessary in a mobile laboratory dictates that each transfer quality instrument be provided with a back-up, which may or may not be of the same type. A11 standards maintained in the mobile laboratory will need to have a careful check on their energy dependence over the range from $25 \mathrm{keV}$ to $1.2 \mathrm{MeV}$.

\section{Operational Considerations}

It is expected that two people will be required to man the mobile laboratory in the field - one a physicist-supervisor-instructor, the other a driver-technician-laboratory operator. They would spend an average of three days out of the workweek at one site calibrating and the other two days in travel, set-up and routine checks and maintenance. Considering servicing, vacations, illnesses, etc., it seems reasonable to assume 40 places visited in one working year. This means all states could be covered about once every two years. Most likely Hawai i and Alaska would be required to send or bring their instruments to the nearest calibration facility on the American continent--perhaps California or the state of Washington.

\section{Conclusion}

A properly designed mobile radiation calibration laboratory, useful in the national radiation measurement assurance program, is most effectively realized in the tractor-trailer truck design described here.

While the present operational scheme has this facility traveling from state to state on a regularly scheduled basis, it could also serve as the means for tying "regional" calibration facilities to the national standards should such facilities be created. Also, if and when the states establish their own calibration ranges, the mobile laboratory could not only serve as an adequate design model but could be used to check the effectiveness and accuracy of the state calibration ranges.

On the basis of these potential benefits to the nation, the utility of the mobile facility seems to be proven.

\section{References}

1. M.J. Berger and D.J. Raso, Radiation Research 12, 20 (1960) Also, NBS

Report $59 \overline{82}$ (Feb 8, 1960)

2. NBS private communication with T.P. Loftus 



\section{AUTOMATED CALIBRATION AND RECORDKEEPING SYSTEM FOR X-RAY SURVEY} INSTRUMENTS

Thomas R. Ohlhaber, Frederick J. Prevo, Ph.D., and Thomas L. Miller Bureau of Radiological Health

Division of Electronic Products Rockville, Maryland 20852

A system for monitoring and controlling the calibration process for $x$-ray survey meters has been designed. A minicomputer with a realtime operating system and on-line disk storage provides the acquisition, reduction, and control parameters for the process and also maintains the recordkeeping for instruments dispersed over the country. The system is designed to rapidly provide information to multiple terminals about the calibration and availability status of survey instruments in order to facilitate the use of large numbers of instruments.

(Calibration; Recordkeeping; X-ray; Computer; Instruments; Automation)

\section{Introduction}

The administration and enforcement of Public Law 90-602, the Radiation Control for Health and Safety Act of 1968 , is a primary concern of the Bureau of Radiological Health. The implementation of performance standards for electronic products which produce $x$-radiation has been a result of Bureau activities under the Act in addition to general requirements for equipment which is not specifically covered by a performance standard.

The Bureau has also initiated cooperative proqrams which strive to reduce unnecessary exposure to manmade radiation (Nationwide Evaluation of $X$-ray Trends, Dental Exposure Normalization Technique). These programs monitor the ponulation exposure and also aim at reduction of exposure through assistance offered to the users of $x$-ray equipment.

The support of the Act and the cooperative proqrams require large numbers of $x$-ray measurement instruments consisting mainly of battery operated portable survey meters. Since these meters are used in the enforcement of a Federal law, the measurements performed with these meters must be defensible in leaal actions and possibly court actions. Therefore, the meters must be calibrated against recognized standards and all documentation of this process must be available. The care exercised in the Bureau is probably excessive for many of the meters calibrated; however, all instruments must possess well documented calibration records if they are to be used in possible legal actions.
Typically, in a six month period, the Bureau is responsible for calibrations on over 100 Victoreen Mode1 666's, 30 Victoreen Model 555's, 30 Victoreen Model 440 RFC's, $20 \mathrm{MDH}$ Model 1015's, 30 Victoreen Condensor R-meter sets, assorted Low Energy Pocket Chambers, a large number of thermoluminescent dosimeters, and assorted other ionization chamber type instruments. Where applicable, each instrument is given an electrical check of the readout electrometer, gross radiation check, leakage check of integration capacitor, response time check, and finally is calibrated against a radiation standard. It is obvious that a large number of instruments are involved and many parameters must be recorded. In order to continue providing calibration services for increasing numbers of instruments and to maintain records and inventory for these, it became necessary to computerize as much as possible of the routine work.

\section{Automated Calibration}

\section{The Calibration Process}

A11 x-ray survey meters exhibit readings which are affected in a non-linear fashion by the energy of the incident $x$ rays. In addition, most meters are also affected by temperature and pressure variations since they are open air ionization chambers. For these reasons, the calibration process must not only include information which correlates the reading of the meter with true Roentgens but must also correlate the response with specific energy, temperature, and pressure conditions.

A typical x-ray beam contains photons distributed over all energies up to the maximum applied $x$-ray tube potential 
(kilovoltage). In our facility, the combination of kilovoltage, first half-value layer and second half-value layer is being used for the $x$-ray beam quality (photon energy distribution) specification.

A meter must be compared to a known standard to determine the calibration correction. The standard used in this laboratory is a Victoreen Instrument Co., Model 4.15A Tranfer Chamber with a readout composed of a Keithley Instruments Inc., 216 Electrometer and a General Radio Co., type 1403 Standard Air Capacitor. Since this system is designed to measure integrated charge from the transfer chamber, exposure time information must also be obtained. To secure this information, an air operated shutter was designed to turn the beam on and off to provide accurate time information.

Since the comparison of the standard and the meter may be performed in sequence, the $x$-ray tube output must be stable. The best indicators of stability of the tube (without perturbing the radiation beam with another instrument) are the high voltage $(k V)$ and the $x$-ray tube current (mA). Table 1 lists the parameters entering into a calibration.

The first five items are essentially constants. Kilovoltage and tube current are monitored and controlled. Temperature and pressure readings are used to correct the instrument readings. The three remaining items are the primary variables used to compute the correction factor.

The simplest calibration which can be performed is a single point calibration. This entails the comparison of the meter to the standard with a single $x$-ray beam. In practice, most meters must be calibrated against a variety of $x$-ray beams since they are used at more than one energy.

By changing the kilovoltage and the filters in the x-ray beam, the entire process can be repeated for different sets of beam parameters.

\section{Choice of Control System}

It becomes evident that a large number of parameters are involved in the calibration of a meter. If all of these quantities are recorded and monitored by hand, the possibility of error increases while throughput decreases. Because of this, it was decided to automate as much of the process as feasible.
Taule 1. Parameters of Importance for a Calibration

First HVL at calibration position Second HVL at calibration position Capacitance of standard capacitor Correction factor of transfer chamber Correction factor of electrometer Kilovoltage of $x$-ray tube ( $k V)$ Stability of tube current (mA) Temperature

Atmospheric pressure

Time of exposure

Electrometer reading

Survey meter reading

Calibration control systems in the past have usually been run by talented and methodical individuals who monitored and controlled the complete process. Our intent was not to replace these people but rather to provide them with help in their more mundane tasks as well as to provide more salient information than was available previously. A competent operator can observe many types of errors in a system but is usually incapable of recognizing slow changes in the quality of his calibrations. For these reasons we decided to provide a system capable of monitoring the entire calibration process. After a review of available systems, it was decided that a system operating on a minicomputer was appropriate. Table 2 lists the equipment in this system. Since the system was intended to provide meaningful information for the operator and to speed up the collection and reduction of data, it was designed to operate in real time. The power of the computer is used to monitor the process and advise the operator of trends or errors.

\section{Equipment Changes Needed for Automatic Control}

Since the choice was made to use a minicomputer for control of the process, all equipment in the facility had to be made compatible with the computer. A decision was also made to utilize equipment which could be used manually if for any reason the automated system would fail. Thus, the power supply was fitted with digital $\mathrm{kV}$ and $\mathrm{mA}$ meters. The shutter controller was built with digital timers and comparators and with the ability for computer control of the exposure time. The electrometer is a diaital device with a remote readout at the control panel. Temperature, pressure, and humidity are also displayed digitally at the panel. The meter under test is usually an analog type instrument that cannot be interfaced directly to the computer. However, television 
Table 2. Digital Equipment Corporation PDP 11/40 Computer System

PDP $11 / 40$ processor with floating point and extended instruction set

$48 \mathrm{~K}$ core memory

Cartridge disk drives, 4.8 million words total storage

Line printer, 300 lines per minute

Assorted interfaces for data

acquisition and control

Printing terminals, one at each

calibration facility, one at the computer console

CRT terminals, located at convenient

locations for inventory update

RSX-17M real time operating system

monitors have been installed to read the instrument and a controller was designed to turn the function knobs of the instrument remotely. A terminal which has printing capability is also provided for manual entry of the meter reading.

We are presently modifying an instrument incorporating a digital electrometer (MDH model 1015) to provide a digital output that can be connected directly to the computer. If this survey meter is adopted for routine use, the necessity of manually entering the reading will be eliminated, therefore, providing additional time savings.

Concept of the Calibration Process under Computer Control

The calibration of a meter can now be performed without manual intervention. For each beam used the kilovoltage, first halfvalue layer, and second half-value layer are stored in the computer. The capacitance of the standard capacitor and the correction factors of the electrometer and transfer chamber are also stored in the computer. The room temperature and pressure can now be checked at each exposure, and more importantly, the stability of the x-ray tube can be continuously monitored. The computer can also check the sequential steps of a calibration and therefore standardize the process. The operator is free to observe the function of the meter under test and needs only to read the instrument after the exposure terminates. This savings of time results from the fact that no numbers are entered into the system except the actual meter reading.
Implementation of the Computer Controlled System

The flexibility of the computer based system allows many modes of operation. However, since the process of calibration must be accurate and reliable, caution must be exercised to insure that the increased complexity of equipment does not degrade the performance of the system. Therefore, the automation of this facility is being performed in stages with extensive tests performed at each stage to verify the performance of the system.

The first stage is to use the computer as a manual entry system assuring that each of the systems are performing accurately under manual control. At this stage, the computer merely contains a program which calculates the correction factors and types a calibration report. Since calibrations were previously performed in this manner, this provides a valid check on the individual items of the system. At present, our old manual facility also uses this program.

The second stage is to interface the computer so that it can read all the parameters of the process. The operator still manually initiates all functions, chooses beam parameters, and basically controls the process. The computer is the data accumulator, but minimal additional tests are performed on the data. This stage tests both the hardware and software of data accumulation.

The third stage is to allow the computer to perform an analysis of the process as related to its input and also as related to previous work. This quality control function is probably the most important function of the system. The operator now has information which informs him on the quality of the work today as compared to all previous work.

The final stage is to program the computer to control the actual process of calibration. Caution must be exercised here since some equipment (such as high voltage supplies) must be very carefully controlled because of the potential for harm to the equipment or the operator. At this point, the largest time saving is realized because all the routine button pushing is performed by the computer while the operator need only enter the survey meter reading and monitor the quality of the work. The goal of this system will be realized when the computer can read the 
meter as mentioned previously.

It is planned to provide for multiple simultaneous calibrations. This has been designed into the system along with automatic positioning of instruments in the beam. Tests must be performed on beam uniformity and cross-scatter before the first system could be used. In any case, the system will allow rapid change from one meter to the next with reduced calibration time resulting.

\section{Inventory Control}

The use of large numbers of survey meters in the field necessitates a system of recordkeeping that provides accurate information on the location and status of any meter. It was decided to incorporate this information on the same computer system, since the calibration of the meter and its status are pertinent to the use of the meter.

\section{Design of System}

Several factors should be accessible from a recordkeeping system if it is to be used. Information about a single meter should be retrievable by entering the model number and serial number and information on the status of a class of instruments should be available. Searches of this data base should be possible for any parameter stored. The updating of the information should be a simple, interactive process. Terminals should be located in convenient places where the information is generated, otherwise updates will tend not to be entered.

Implementation of the Inventory system

Since this system is designed for different types of survey meters, the form of the records may vary by type of instrument. In general, meters are either selfcontained or are composed of a readout (electrometer) and one or more sensors (chambers). Usually, the chambers are interchangeable and thus must have their own records. Table 3 shows a typical record format for a survey meter, and Table 4 shows the individual chamber records.

\section{Conclusion}

The system has been partially implemented at present. Al1 equipment is functional and the interfacing of sensors to the computer is in process. The programming of the computer to handle acquisition and reduction of calibration
Table 3. Electrometer Records

Model number of instrument Serial number of instrument Purchase date

System entry date

Date of last repair

Number of repairs on this instrument Name of user issued to

Address of user

Status Code

1. Returned for repair (to manufacturer)

2. Undergoing electrical check

3. Undergoing calibration

4. Ready for issue

5. Uncalibrated storage

6. Inhouse repair

7. Inhouse use

8. Issued

Use Code Number

1. BRH Inhouse

2. Compliance EDRO

3. Compliance state contract

4. NEXT program

5. State

6. Other

\section{Owner Code}

1. BRH

2. Proaram indicated in use code

Number of chambers mated with this electrometer ( $0=\mathrm{Self}$-contained)

Model and serial number of each mated chamber

Table 4. Chamber Records

Model number of chamber

Serial number of chamber

Purchase date

System entry date

Date of last repair

Number of repairs on this chamber

Last calibration date

Model and serial number of electrometer mated with

\section{Status Code}

1. Mated with electrometer

2. Returned for repair with electrometer

3. Returned for repair without electrometer

4. Storage (unmated) 
data is complete. The inventory control phase of programming has been initiated. Substantial time savings have already been noted. As rapidly as possible, the remainder of the system will be completed.
The system makes feasible the maintenance of a population of instruments numbering in the hundreds, in active continuous use, geographically distributed across the nation. It would be impractical to attack this task with conventional techniques. 

STANDARDIZATION OF A NATIONWIDE GAMM RADIATION CALIBRATION SYSTEM E.F. Williams, Ir., P.V. Barrans and G.H. Brodie

RADEF Instrumentation Test Facility Washington, D.C. 20374

A standardized y radiation calibration system has been developed and is presently being used to provide uniform $\gamma$ radiation fields for the calibration of $\sim 500,000$ y measuring, civil defense survey instruments. The instrumentation, procedures, data handing techniques and secondary standards developed to make the system directIy traceable to National Bureau of Standards are described and discussed. A simple test ard evaluation of the total system is described. The test data obtained appears to show that the true roentgen is probably being provided to 48 maintenance locations with an accuracy of better than $\doteqdot 6 \%$ and that the instruments can be calibrated to within $\div 14 \%$ of the true exposure rate at one point on each detection range.

( $\gamma$ radiation, roentgen, exposure rate, instrument, $\gamma$ response, ion chamber, standard, geometry, calibration)

\section{Introduction}

The Defense Civil Preparedness Agency ( $(C P A)$ of the Department of Defense has in readiness a nationvide radiological monito ing system designed to measure significant levels of radioactive fallout from a strategic nuclear attack on the United States. The primary radiation measuring instrument developed for this purpose is the CDV-715 survey meter. This instrument uses a hermetically sealed, non-pressurized ion chamber for a detector and has four ranges of $\gamma$ exposure rate sensitivity; 0-0.50, 0-5.0, $0-50$ and $0-500 \mathrm{R} / \mathrm{h}$. The current from the ion chamber develops a voltage across one of four high megohm grid resistors, one for each range. This voltage is applied to the grid of an electrometer tube. A $50 \mu \mathrm{A}$ indicating meter measures the current through the electrometer tube and is calibrated against the known $\gamma$ exposure rate with a separate variable shunt resistor for each range on the instrument. There are $v$ 500,000 of these instruments distributed throughout the United States in monitoring stations, fallout shelters and other emergency locations under the direct control of the local and State Civil Defense Authorities. The individual states are responsible for the maintenance and calibration of these instruments within their boundaries. This program is implemented by 48 separate State maintenance shops located within the 50 States and Puerto Rico. The device used to calibrate these instruments is the CDV-794 $\gamma$ Radiation Calibrator with one on more located in each shop. The calibrator contains $2130 \mathrm{Ci}$ of $137 \mathrm{Cs}-137 \mathrm{Ba}$ in a sealed source. The $y$ radiation from this source is collimated into a lead lined box containing the instrument in a fixed geometry. By the use of three tungsten attenuators, four discrete $\gamma$ exposure rates of $\sim 0.40,4.0,40$ and $400 \mathrm{R} / \mathrm{h}$ are produced at the instrument ion chamber. The shunt resistors on the instruments are adjusted by remote, mechanical means to produce the proper indication on the 50jA meter of the CDV-715 instrument on each range. The methods and instrumentation used in this system to calibrate the four $\gamma$ exposure rates produced in each CDV$794 \gamma$ Radiation Calibrator against the true roentgen as determined by the National Bureau of Standards (NBS) is subject of this report.

\section{Methods and Instrumentation}

The primary secondary standard $\gamma$ radiation field against which this system is standardized is produced by a $490 \mathrm{Ci} 137 \mathrm{Cs}$ $137 \mathrm{Ba}$ sealed source. This source is collimated by a shield which produces a y beam in free air 10 meters in useful length and 3.0 meters in diameter at its widest useful portion. This $\gamma$ radiation field is calibrated by feeding the ionization current collected from a Shonka high energy ionization chamber 1 into the input of a vibrating reed electrometer and a standard air capacitor with a low capacitance cable. As the capacitor charges, the electrometer meter current closes a sensitive relay starting a counter connected to a $1 \mathrm{kc}$ square wave frequency standard. The voltage on the capacitor is nulled with a precision voltage source until the total voltage reaches a predetermined value and the relay turns off the counter. The voltage collected is 210 times full scale to further reduce timing errors due to variations in the relay closing current. The total count gives the 


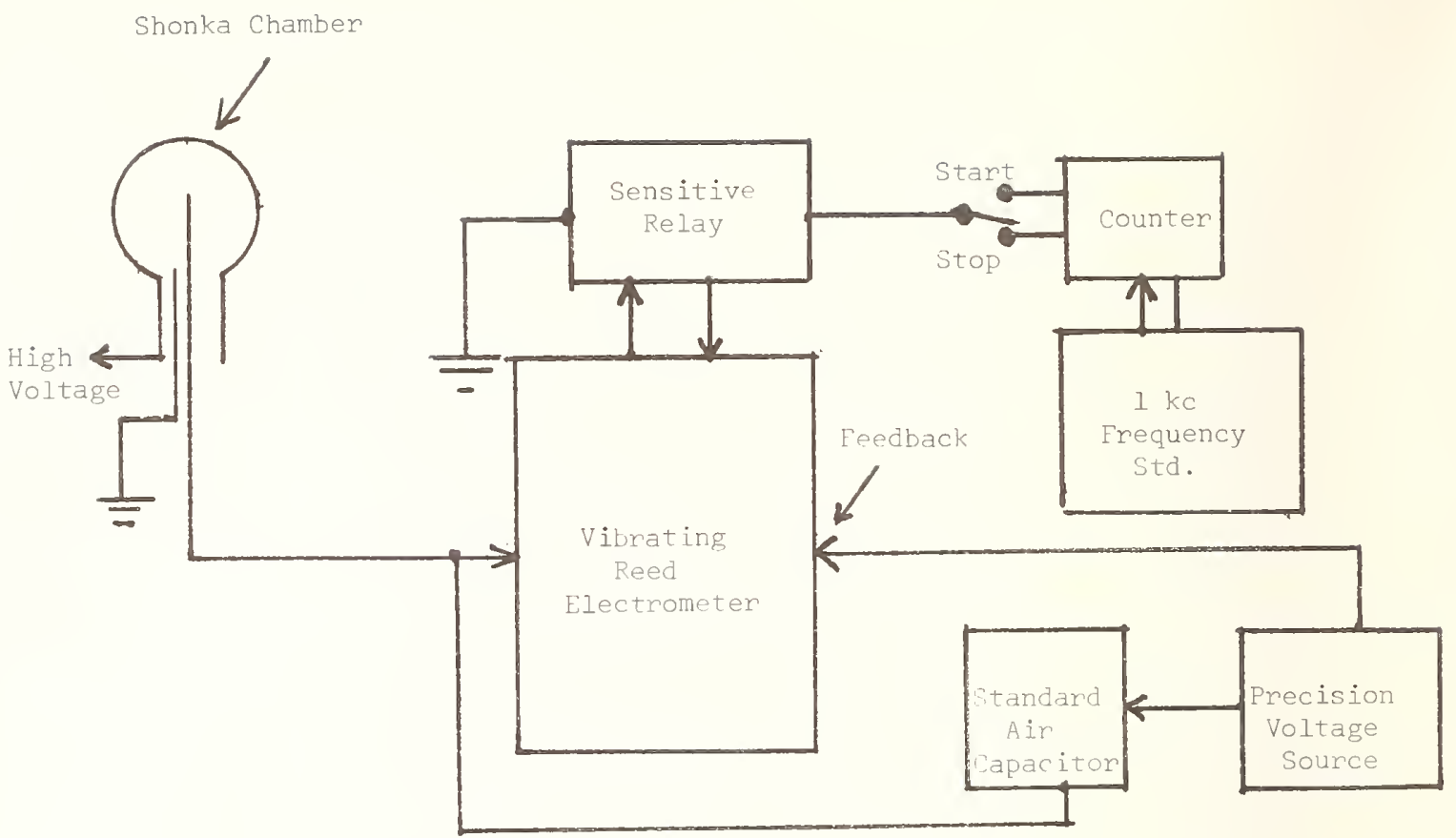

FIGURE NO. 1: BLOCK DIAGRAM OF THE SHONKA CHAMBER SYSTEM

exposure time in seconds and milliseconds. A block diagram of the basic system is given in Figure No. 1 above.

NBS has calibrated ${ }^{2}$ the shonka chamber, the voltage source, and the capacitors used in this system. Point by point calibration is accomplished by positioning the Shonka chamber in the collimated $\gamma$ radiation field and collecting ionization current at a number of points along the range. Radiation intensity is calculated by the equation:

$$
I=\frac{C V}{t} \cdot C F \cdot C F
$$

$$
\begin{aligned}
& \text { where: I = radiation intensity } \\
& C V=\text { capacity } \mathrm{X} \text { voltage } \\
& t=\text { time in seconds } \\
& \mathrm{CF}_{\text {NBS }}=\text { NBS Calibration Factor } \\
& \text { CFSTP = Standard temperature } \\
& \text { and pressure correct- } \\
& \text { ion factors }
\end{aligned}
$$

To eliminate misinterpreting data from graphs, the radiation intensities are converted to program cards for a programable calculator. The date and desired exposure rate are entered into the calculator and the distance is calculated. The calculator used is a rather basic one and in order to make the program usatle, some simplifying assump- tions must be made. The first is the as:sumption that the $y$ range follows inverse square law. After correcting for air attenuation and finding the effective source to detector distance for the source detector geometry, inverse square law is good over a limited range. From $50 \mathrm{~cm}$ to $\approx 6$ meters this program gives distances to better than l\% agreement with measured exposure rates. At a distance of $\approx 6$ meters scatter becomes significant and the program distances are not used for this application. Overall precision and accuracy for this total system which serves as the primary secondary standard for all DCPA r calibrations is within $\pm 4 \%$ of the NBS values for the roentgen. To calibrate the exposure rates from the CDV-794 calibrator against the $490 \mathrm{Ci}$ $137 \mathrm{Cs}$ - $137 \overline{\mathrm{Ba}}$ standard $\gamma$ field the CDV -765 Transfer standard ${ }^{3}$ was developed for DCPA. This device consists of an hermetically sealed, unpressurized, ionization chamber detector, electrometer integrator, and a digital readout, plus a logic and switching control system to permit automatic or semi-automatic operation. The ion chamber detector has an identical geometry to the standard CDV-715 ion chamber and is mounted in a standard CDV-715 instrument case along with a constant current tube which is used as an operational check for the instrument system. The ion chamber current is fed to 
tire input of a vibreting capacitor electroneter connected as a teedback integraton. the chamber is connected bj neans oI a 10 Æoot low capacity triaxial catie. The eleczrometer outcut voltage ¿̀s digitally displayed at tre end of the cperational cycle. the logic and switching circuits control the llectrometer input reed switches, select the irtegration perioj and range capacitor, poerate the readout and provide an automatic repeating cycle. Eigure :io. 2 below, is a block diagnam of the CDV-765 system showing the interrelationsip of the electrometer, digital readout, and control system. It should be noted that the operation oi the standand consists of selecting the iesired sensitivizy range and actuating the stant button. The rest of the operational arcle is fully automatic and cannot be itzered except jy using the reset Jutton to interrugt ar integrator cycle in pro-ress. The time delay circuits must operate sequentially before a new integraton stent is possible, and any disruption of their normal cycle only increases the total delay time. This was programmed into the control system to insure that the polystrene integraton capacitor is completely discharged before each new cycle is started to obtain consistent readings.

The CDV-765 instrument may be operated either by a single cycle with manual pushbutton restart, or may be operated with a completely automatic repeating cycle with a continuously updated reading.

If the master control switch is set on the low range and the exposure rate is 40 . $\mathrm{R} / \mathrm{h}$ on greater, an over-voltage detector circuit acts as soon as the electrometer output voltage exceeds full scale and shorts out the electrometer input circuit, dumping the charge on the integrating capacitor and protecting the circuits from jamming. The control circuit then disables itself and locks up, with an over range trip light (OR:) to show what has occurred. Until the reset button is pressed, no restart is possible. The CDV-765 is equipped with an aluminum clad, styrofoam lined, plywood shipping case which holds the console, detector cables and instruction manual and protects it from rough handling during shipment.

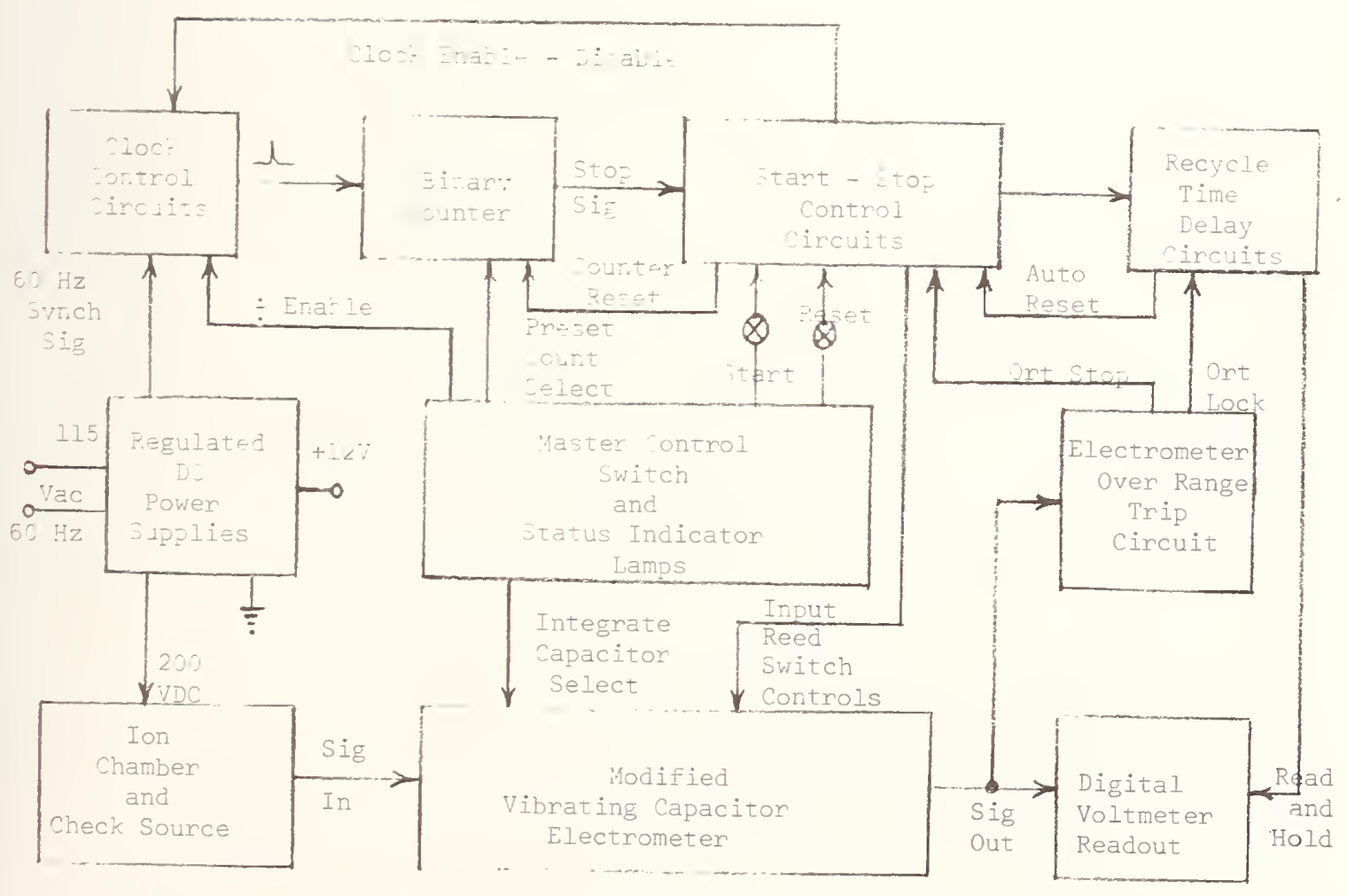

EIGURE NO. 2: BLOCK DIAGRAM OF THE CDV-765 TRANSFER STANDARD 
TABLE I: VARIATION IN CALIBRATION BETWEEN TWO TRANSFER STANDARDS

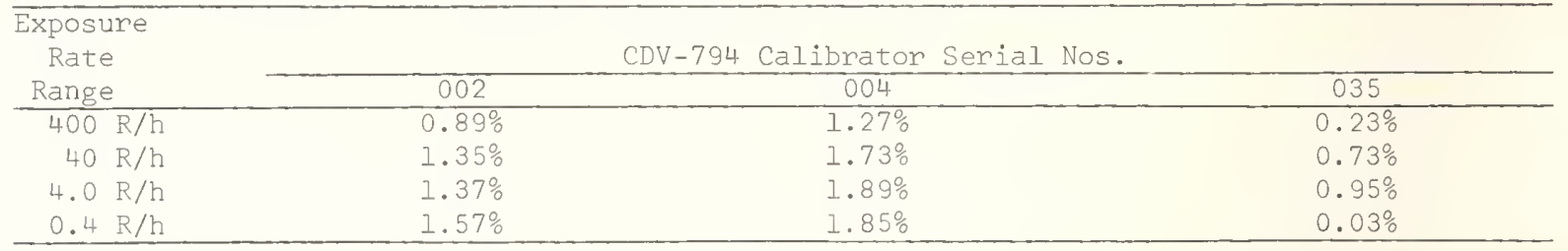

The CDV-765 is calibrated in the standard $137 \mathrm{Cs}$ - $137 \mathrm{Ba}$ y field at exposure rates of $0.40,4.0,40$ and $400 \mathrm{R} / \mathrm{h}$ and the output voltage recorded as a calibration factor at each rate in the instruction manual. This instrument is then shipped to a State maintenance shop where it is placed in the CDV-794 $\gamma$ Radiation Calibrator. The four discrete $\gamma$ exposure rates provided by the calibrator are measured with the transfer standard and are recorded. The exposure rate values determined by the transfer standard factors are then used by the state shop to set the response of the CDV-715 ion chamber instruments using the calibration potentiometers.

\section{Evaluation of the System}

In onder to determine the maximum possible precision for transferring the NBS roentgen to these CDV-715 instruments, oun Facility conducted a test and evaluation of each step in the system. Two CDV-765 Transfer Standards were calibrated in the $490 \mathrm{Ci}$ $137 \mathrm{Cs}$ - $137 \mathrm{Ba} \gamma$ field using the procedure described ahove. These two transfer standards were then used to calibrate the four discrete $\gamma$ exposure rates from three CDV-794 calibrators. The variation between the exposure rate for each transfer standand was then compared. The results of this comparison in terms of percentage is given for each discrete exposure rate for each calibrator in Table I above. The maximum variation response between the two transfer standards was $1.89 \%$ and the smallest difference was $0.03 \%$ for the 12 exposure rates evaluated. The mean variation between the determinations of the two standards was $1.16 \%$.
Twenty CDV-715 instruments were ther calibrated against the exposure rates determined by one of the transfer standards in CDV-794 y Radiation Calibrator, Serial No. 035. These 20 instruments were then placed in the other two CDV-794 calibrators and their response was compared against the exposure rate determined by the same transfer standard for the particular calibrator used. The variation between the CDV-715 instruments $\gamma$ response and the exposure rate in the calibrator was determined. The mean $\gamma$ response of the 20 instruments and the maximum and minimum response for the 20 instruments for each discrete exposure rate for both calibrators is given in Table II. As given in the data the largest variation for the 20 instruments over any of the eight discrete exposure rates was $7.1 \%$. The langest single variation observed for one instrument was $+3.8 \%$.

The 20 instruments were then placed in the standand ${ }^{137} \mathrm{Cs}$ - $137 \mathrm{Ba}$ y radiation field at the exposure rates obtained in CDV-794 Serial No. 035 and thein $\gamma$ response compared with the primary exposure rate value. The percentage of variation from the primary exposure rate value is given in Table III.

The maximum variation in response for the 20 instruments over any of the four discrete exposure rates is $8.0 \%$ with the largest single variation for one instrument being $-7.5 \%$. It should also be noted that in general the response of the CDV-715 instruments is less than the primary exposure rate to which they were exposed; particularly at the $0.40 \mathrm{R} / \mathrm{h}$ and the $4.0 \mathrm{R} / \mathrm{h}$ rates.

TABLE II: VARIATION IN RESPONSE OF 20 CDV-715 INSTRUMENTS IN CDV-794

\begin{tabular}{|c|c|c|c|c|}
\hline Instrument & & -794 Calibrator & fial Nos. & \\
\hline Calibration & & & 0 & \\
\hline Exposure & Mean & Extremes of & Mean & Extremes of \\
\hline Rate & Response & Response & Response & Response \\
\hline $400 \mathrm{R} / \mathrm{h}$ & $+0.5 \%$ & $+3.3 \%$ to $-3.0 \%$ & $+1.0 \%$ & $+3.8 \%$ to $-3.3 \%$ \\
\hline $40 \mathrm{R} / \mathrm{h}$ & $+0.3 \%$ & $+2.6 \%$ to $-1.3 \%$ & $+0.7 \%$ & $+2.9 \%$ to $-1.9 \%$ \\
\hline $4.0 \mathrm{R} / \mathrm{h}$ & $+0.4 \%$ & $+2.6 \%$ to $-1.3 \%$ & $+0.7 \%$ & $+2.5 \%$ to $-2.5 \%$ \\
\hline $0.40 \mathrm{R} / \mathrm{h}$ & $+0.3 \%$ & $+2.7 \%$ to $-2.8 \%$ & $+0.1 \%$ & $+2.5 \%$ to $-3.7 \%$ \\
\hline
\end{tabular}


TABLE III: TARIAIION IN RESPONSE JE 20 CDV-715 INSTRUMENTS IN PRIMARY Y FIELD

\begin{tabular}{rcr}
\hline Primany Eield & Mean Response & Extremes of Response \\
Exposune Rate & $-0.9 \%$ & $+2.6 \%$ to $-3.8 \%$ \\
\hline $400 \mathrm{R} / \mathrm{h}$ & $-1.4 \%$ & $+2.6 \%$ to $-3.9 \%$ \\
$40 \mathrm{R} / \mathrm{h}$ & $-3.5 \%$ & $+0.8 \%$ to $-5.8 \%$ \\
$4.0 \mathrm{R} / \mathrm{h}$ & $-4.3 \%$ & $+0.5 \%$ to $-7.5 \%$ \\
$3.40 \mathrm{R} / \mathrm{h}$ & & +0 \\
\hline
\end{tabular}

This effect aspears to be the result of the CDV-765 Transfer Standard being somewhat less sensitive to low energy scatter radiation than the CDV-715 instruments. However, further investigation of this phenomenon is necessary to determine the cause of this anomaly.

The variations that are additive from this eraluation are the maximum variation between transfer standards ( $1.9 \%$ ). the maximum variation in CDV-715 y response in the primary $y$ field ( $7.5 \%$ ) and the accuracy and precision with which the primary $y$ beam compares with the NBS roentg॰n $( \pm 4 \%)$. This would give a maximum observable error overall of $13.4 \%$. The statistical validity of using 20 instruments to represent $\sim 500,000$ instruments and the use of three calibrators to represent 60 is somewhat open to zuestion. However, these 20 instruments were normal stock and typical of what is Found in the State maintenance shop. It can be reasonably assumed that an accuracy of $\pm 14 \%$ can be achieved at the calibration point on each range of sensitivity with these instruments. The two transfer standards represent a significant number of instruments since eight instruments are actually used in this program. Therefore, the variation of $2 \%$ between standards and the $\pm 4 \%$ for the precision and accuracy of the primary $\gamma$ field should permit the transfer of the NBS roentgen to the CDV-794 calibrator within $\pm 6 \%$.

\section{References}

1. F.H. Attix and W.C. Roesch, 1966 Radiation Dosimetry, Second Edition, Volume II PP $54-55$ (New York. London: Academic Press)

2. T.P. Loftus, Aug. 1973, Report of Calibration, Shonka Ionization Chamber, DS $1725 / 73$, National Bureau of Standards

3. Victoreen Instrument Company, Final Report for Contract No. OCD-PS-65-182, Defense Civil Preparedness Agency Pub. 



\title{
A RELIABLE CALIBRATION PROGRAM \\ FOR IONIZING RADIATION MEASUREMENT
}

\author{
Ahren Jacobson \\ University of Louisville Radiation Center \\ Louisville, Kentucky 40201
}

\begin{abstract}
The recent availability in this country of the N.P.L. \#2560 Secondary standard instrument has afforded a new dimension to instrument calibration procedures. A description is presented of the accuracy, stability, and utility of the N.P.L. \#2560 for calibration of various instruments to measure a wide range of ionizing radiation energies.
\end{abstract}

(Dosimetry; calibration; ionization chamber; secondary standardization)

A principal reason for convening a symposium on "Measurements for the Safe Use of Radiation" arises from the acknowledged variability of such measurements being made throughout the country. Although these measurements are especially critical in clinical applications of ionizing radiations, there is also need for more reliable dosimetry in other areas, such as radiation safety and environmental monitoring procedures.

The foundation of any calibration methodology must employ a reliable primary standardization whose data can be utilized for secondary and tertiary instrument calibration. Primary standardization on a local basis can be demanding, both cost and ability-wise. To obtain this precise work at national, regional, or commercial standardization labo- ratories involves a host of problems. Turnaround time; fragility and bulk management by suitable transportation methods; and high cost are some of the more difficult problems frequently encountered.

It appeared prudent to choose the recent approach used in the United Kingdom for assurance of uniformity of radiation therapy dose measurement in all of their Regional Therapy Centers. Their method involved use of an ionization chamber specifically designed as a secondary standard by the National Physical Laboratory in England. (1) This instrument has now become available commercially* and we were fortunate to have acquired one using grant funds of the Regional Medical Program**.

* NPL Secondary Standard Therapy Level X-Ray Exposure Meter (\#2560) fabricated by Nuclear Enterprises Ltd., Reading, England.

** Ohio Valley Regional Medical Program project \#42C: "Regional Dosimetry Standards Development." 


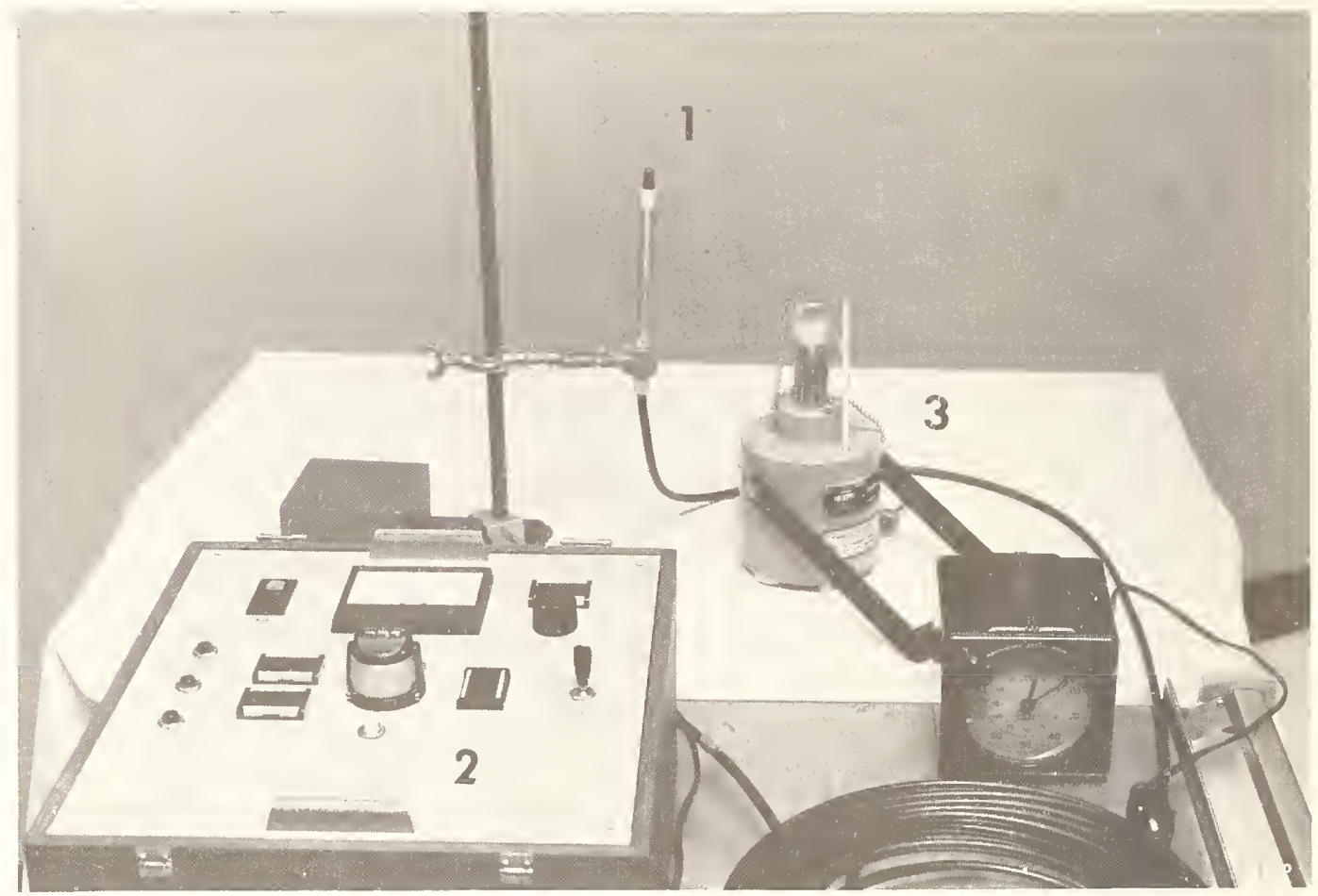

The 2560 dosimetry system consists of three basic components shown in Figure 1: (1) the ionization chamber, (2) measuring circuit, (3) and stability check source. Long term stability and excellent reproducibility and energy dependence characteristics are claimed for the precisely fabricated ionization chamber. It has a wall thickness of only $0.5 \mathrm{~mm}$ extremely pure extruded graphite that is remarkably durable. No conducting coating on the inner collecting surface is necessary, thereby eliminating the troublesome flaking problem found in conventional ion chambers. The chamber volume is $0.325 \mathrm{~cm}^{3}$ and it contains an ultrapure hollow Al collecting electrode. Having a hollow, low atomic number central electrode minimizes "shadow" effect at lower X-ray energies. Another outstanding feature of the ion chamber assembly is the incorporation of an amber insulator in the stem. Besides its exceptionally high electrical resistance quality, amber is essentially air-equivalent. Ionization current is integrated by a highly stable capacitor in the feedback path of an electrometer operational amplifier, which is part of a Townsend balance voltage and is measured potentiometrically by a 10 turn heliopot. The compact Sr-90 stability check source includes a thermometer for temperature determination. A reproducibility of \pm 0.03 percent is claimed for readings on this check source. Such data is possible because of the uniform radiation field obtained from two concentric circular foils of Sr-90 and a precision chamber positioning mechanism that assures reproducible irradiation geometry. 
Nuclear Enterprises Ltd. (the fabricator of the 2560) provided a primary standardization with the instrument. In addition to this, arrangements were made at NBS (2) to have an independent primary standardization performed there before the instrument was put into use. This was accomplished for two X-ray energies and Co-60. Also included in the NBS standardization were serial measurements on the Sr-90 stability check source supplied with the 2560. Although the Nuclear Enterprises primary standardization dealt with a variety of $\mathrm{X}$-ray energy points and no co-60 measurement, a comparison can be made of two sets of data for similar X-ray energy points from both laboratories. This comparison is shown in Table 1:

TABLE 1

$$
\text { I. E., LTD. }
$$

$760 \mathrm{~mm} \mathrm{Hg} 20^{\circ} \mathrm{C}$

$2.0 \mathrm{~mm}$ I I H.V.I. a $100 \mathrm{kVp}$

1.035

$3.25 \mathrm{~mm} \mathrm{Cu} \mathrm{H.V.L.} \mathrm{e} 250 \mathrm{kVp}$

1.040

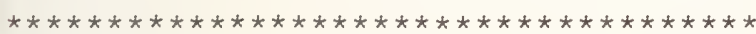

$!, B, S$,

$760 \mathrm{~mm} \mathrm{Hg} 22^{\circ} \mathrm{C}$

$1.86 \mathrm{~mm} \mathrm{AI} \mathrm{H.V.L.} \mathrm{e} 75 \mathrm{kVp}$

1.04

$3.25 \mathrm{~mm} \mathrm{Cu} \mathrm{H.V.L.} \mathrm{@} 250 \mathrm{kVp}$

1.05
Excellent agreement between these standardizations, separated by four months and the Atlantic Ocean, bear out the manufacturer's claim of reproducibility and long-term stability. Between primary standardizations, intermediate verification is conveniently accomplished by use of the Sr-90 stability check source.

Although we have only had the 2560 at our institution since November 1975, it has been instrumental in clearing up several dosimetry and calibration problems. A low-energy ion chamber, recently obtained from a well-known manufacturer, was found to have a substantial error in its primary standardization by intercomparison with our 2560. The particular error resulted from the use of an erroneous correction factor in the supplier's laboratory. This error was present in forty other instruments standardized for 30 other customers in this country and overseas. During the four month period that this error was propagated, none of the other 30 customers was aware of its presence until subsequent notification by the supplier. Undoubtedly, we would have accepted the low-energy standardization provided to us as well, were it not for the availability of the newly acquired 2560 system with its recent standardizations. Prior to acquiring the 2560 our reliance for constancy of ion chamber response was on the use of a radium constancy source, which of course, offers no absolute calibration. Coupled with this important deficiency, is the fact that the bulky radium constancy pot is not sent along with the ion chambers for primary standardization. It has been essential to virtually "hand-carry" the ion chambers to and from the standardizing laboratory to minimize chances of damage to the chambers.

The rugged and reasonably portable NPL 2560 system is expected to serve as an effective link between the primary standardization laboratory and our institution. Intercomparisons can be made with reasonable facility. Our recent visit by the Radiological Physics center team found the Co-60 intercomparison bet- 
ween our $100 \mathrm{R}$ high energy ion chamber and their instrument to be within $0.3 \%$ for several Co-60 exposures. The calibration factor for our chamber had been determined by simultaneous exposure of it and the 2560 ion chamber with build-up cap to large field Co-60 gamma rays. Minimal stem effect was determined for both chambers.

Figure 2 shows a co- 60 beam intercomparison of a Victoreen mediumenergy ion chamber with $4.5 \mathrm{~mm}$ lucite build-up cap and the 2560 ion chamber with its build-up cap. We use medium energy ion chambers in snug fitting cavities of polystyrene calibration phantoms for routine calibration of high energy machines. Therefore, it is imperative that a Co-60 calibration of the medium energy chambers be performed since we use $\mathrm{C}_{\lambda}$ factors on ion chamber data to determine absorbed dose in rads. Another useful application of this dosimetry system has been in the calibration of our Cs-137 and Ra-226 sources used for survey instrument calibrations.

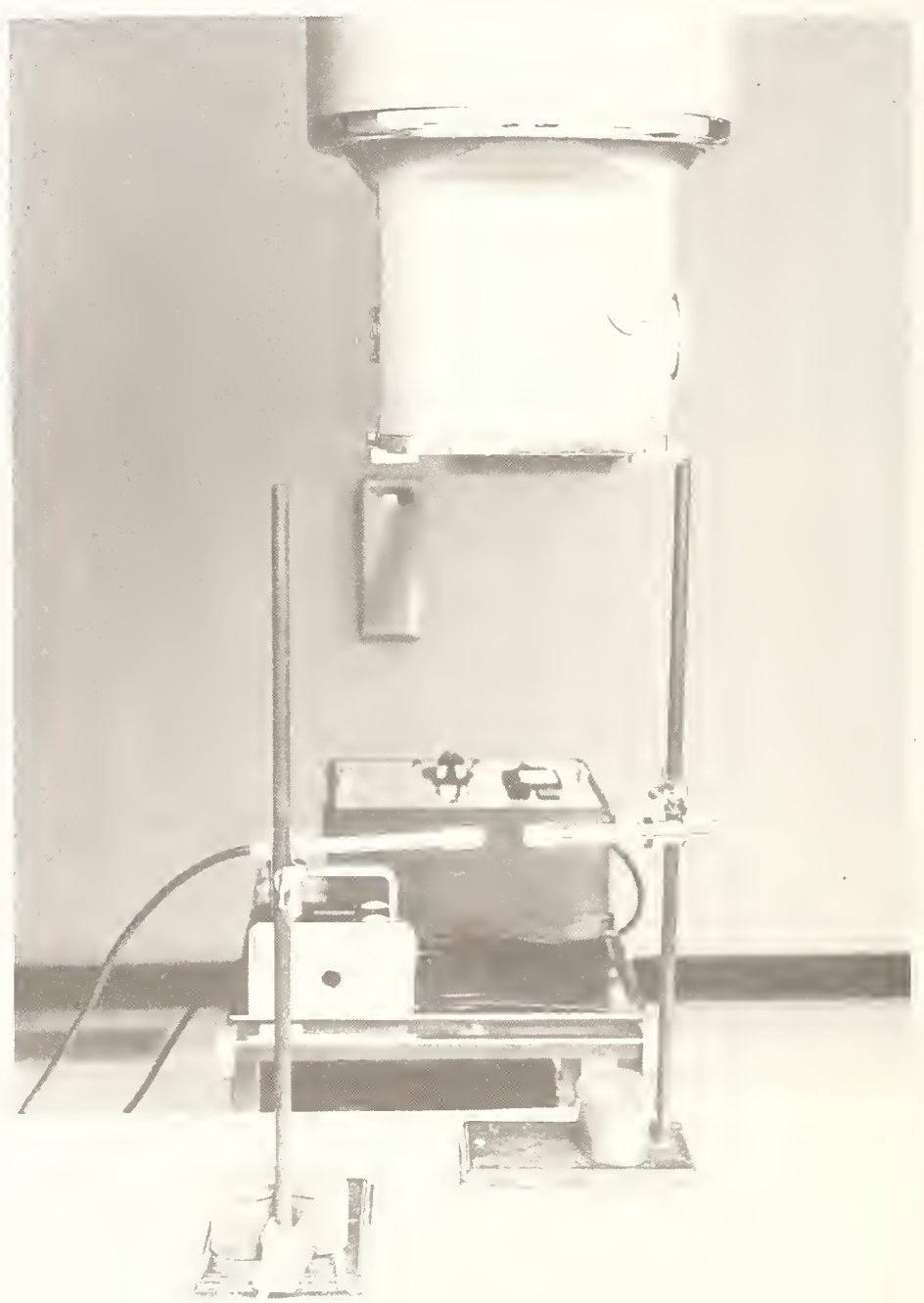




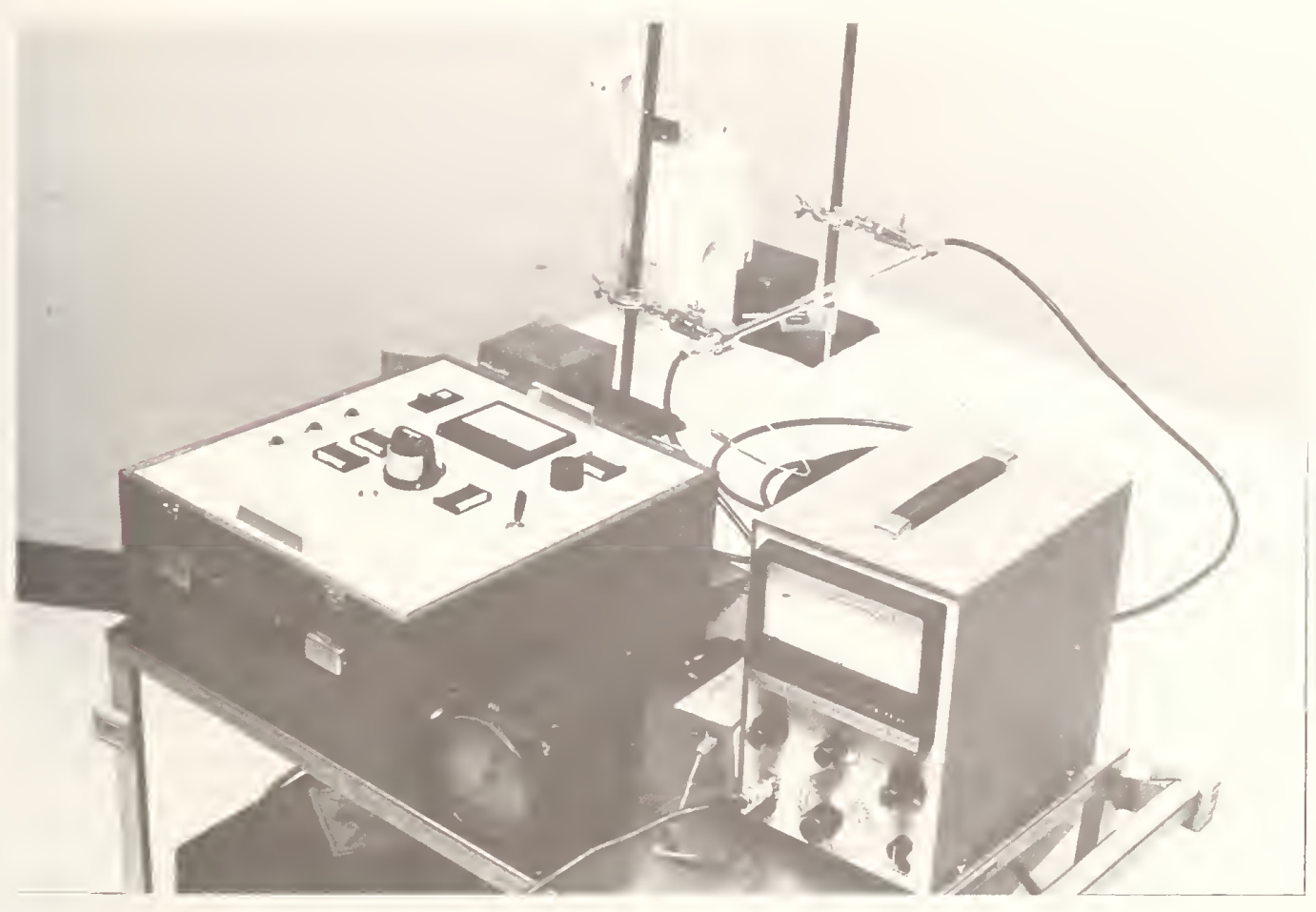

Figure 3 shows this use for a low intensity beam irradiator that is employed for some survey meter calibrations. In this instance the 2560 ion chamber and PrW chamber are both measuring the $\mathrm{Cs}-137$ beam for precise calibration of the irradiator at a $20 \mathrm{~cm}$ S.S.D.

These examples of the versatile application of the N.P.L. Secondary standard demonstrate its reliability in bridging ionizing radiation dosimetry between regional user and the primary standardization laboratory. The promising initial results of the finely designed and fabricated 2560 dosimetry system offer great hope for accomplishing uniform instrument calibrations locally. Intercomparisons on the local level are conveniently accomplished, thereby ameliorating the time consuming primary standardization problems regional users have encountered in the past.

(1) L.A.W. Kemp, Brit. J. Radiol., 45, 775 (1972).

(2) Dr. Robert Loevinger and T. R. Loftus were very cooperative in arranging for primary standardization of this new instrument. 



\section{AIF-NBS RADIOACT IVITY MEASUREMENTS ASSURANCE PROGRAM FOR THE RADIOPHARMACEUTICAL INDUSTRY}

$$
\text { R. Collé }
$$

Atomic Industrial Forum, Inc. Washington, D. C. 20014 and

National Bureau of Standards Washington, D. C. 20234

The National Bureau of Standards supervises and administers on behalf of the Atomic Industrial Forum (AIF) a radioactivity measurements technology quality assurance program for the radiopharmaceutical industry. The program provides for a monthly distribution of both millicurie- and microcurie-level Standard Reference Materials to each of eight participating commercial radiopharmaceutical manufacturers. A number of the standards are distributed as "blind" samples to establish traceability to the national radioactivity measurements system. In addition to identifying measurement discrepancies, assistance is provided to the participants in eliminating the sources of difficulties, and in correcting errors in their measurement techniques. In this report, results for the "blind"-sample traceability exercises are yiven, and a discussion of identified sources of measurement errors and recommendations are also presented.

(Assurance; measurement; radioactivity; radiopharmaceutical; standards; traceability)

In August of 1973 at a symposium quite similar to the present one, Seidel and Brantley ${ }^{1}$ reviewed a number of serious questions and problems which faced commercial radionuclide manufacturers as related to the needs for radioactivity standards. Some of the basic difficulties they cited were:

1) the lack of direct standards for approximately $75 \%$ of the more than 100 radionuclides produced by the industry at that time; 2) the sometimes inconvenient physical form or activity levels of the available standards; and 3) the failure to have industrywide adopted decay-scheme data upon which to base derived standard instrument calibrations in the absence of direct calibrations based on national standards. Although the American National Standards Institute (ANSI) Quality Assurance Program which was described at that time $^{1}$ (the current status of which will be the subject of the next paper ${ }^{2}$ ) addresses a number of these difficulties, it is concerned, however, only with microcurie-level measurements and its participants include hospitals, nuclear-power plants and industry. The Atomic Industrial Forum (AIF) and the National Bureau of Standards (NBS) therefore entered into an agreement on a research associate program whereby NBS will supervise and administer on behalf of AIF a measurements technology quality assurance program which caters more specifically to the needs of the radiopharmaceutical industry. Eight radiopharmaceutical suppliers are participating in this program. Concurrently, on the authority of an interagency agreement, NBS is providing the Food and Drug Administration (FDA) with services similar to those being given the AIF participants. These participants are listed in Table 1. The purpose of the program is to insure the continuous availability of national radioactivity standards at appropriate levels of activity for use by the radiopharmaceutical industry, and thus to establish a degree of uniformity in the measurements throughout the industry.

The major effort of the program consists of a monthly distribution of both millicurie- and microcurie-level Standard Reference Materials (SRM) to each of the participants. With the exception of a future ${ }^{133}$ Xe gaseous standard, all of the SRMs are solution standards contained in "5-m1" flame-sealed borosilicate glass ampoules. Those which have been already issued under the program are listed in Table 2. The choice of radionuclides, their activity levels as well as an order of priority for them are selected by a steering committee representing each of the AIF participants. Obviously these selections must be made in cooperation with NBS, taking into consideration the compatibility 
Table I

Participants ${ }^{\mathrm{a}}$

Standards Program for the Radiopharmaceutical Industry

Food and Drug Administration

Bureau of Drugs

Division of Drug Chemistry

Washington, D.C.

Atomic Industrial Forum Participants

Atomic Energy of Canada, Ltd.

Ottawa, Canada

General Electric Co.

Pleasanton, California

Mallinckrodt Nuclear

St. Louis, Missouri

Medi-Physics, Inc.

Emeryville, California

New England Nuclear

North Billerica, Massachusetts

E.R. Squibb \& Sons, Inc.

New Brunswick, New Jersey

Union Carbide Corp.

Tuxedo, New York

Amersham/Searle Corp.

Arlington Heights, Illinois

\footnotetext{
athe order in which participants are listed in this table does not correspond to the order in which the results are listed in Table 3 .

barticipation terminated November, 1975.
}

with their scheduling capabilities and existing standard developments. This does however provide a viable mechanism in which the radiopharmaceutical industry can direct requests to NBS to establish standards for those radionuclides which are of prime interest and major concern to them. As seen in Table 2, the trend is certainly toward shorter-lived radionuclides, reflecting the needs of the nuclear-medicine community which is served by the radiopharmaceutical industry. Although the trend is "healthy," particularly for patients, it does in itself present difficulties in the preparation and distribution of the standards. The millicurie-level standards made available by this program, compared with the typically microcurie-level standards normally handled at NBS, are of greater convenience and direct value for instrument calibrations at activity levels more closely matching those found in the manufacturing process.

A second major aspect of the program attempts to satisfy the increasing demands of both consumers and regulatory agencies to demonstrate "traceability" to the national radioactivity measurements system, embodied in the NBS. Satisfaction of this requirement also simultaneously fulfills the manufacturers' goal of achieving an industry-wide uniformity in measurements. Although the monthly distribution of standards serves to insure a regular supply of radioactivity standards to calibrate measuring equipment, the mere use of them does not constitute traceability. Despite the term's increased usage lately, a clear 
Table 2

Standard Reference Materials ${ }^{\text {a }}$

Issued Under the Standards Program for the Radiopharmaceutical Industry

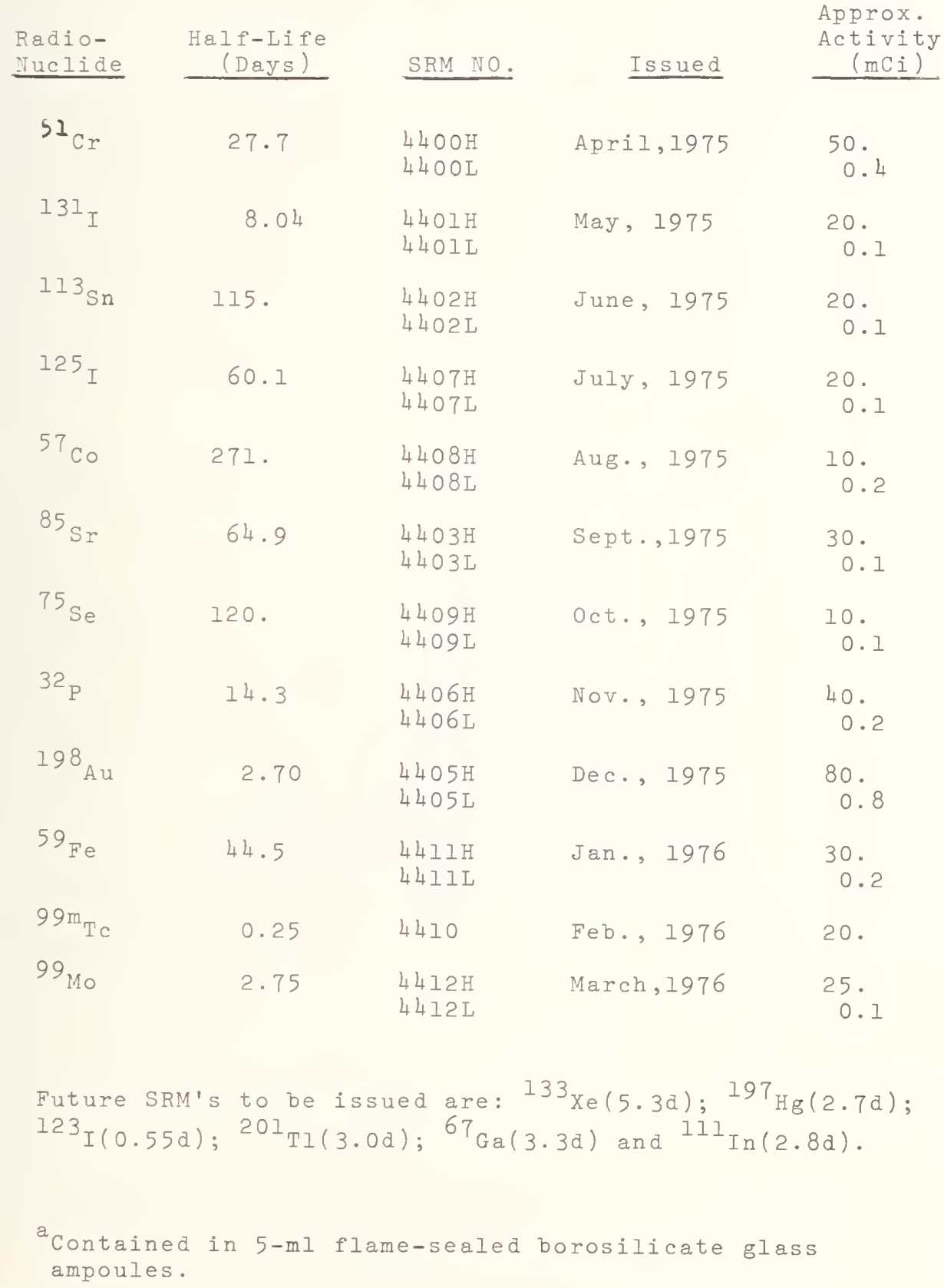


understanding of exactly what constitutes such traceability does not appear to be universal. A working definition has been given as "competence that can be periodically demonstrated."1 This can be accomplished by participation in a round robin distribution of calibrated, but "blind" samples of unknown (to the participant) quantity for assay by the participant. At the present time, approximately one-third of the monthly distributed SRMs for this program are issued as "blinds." These are selected by the steering committee with the understanding that participants may take substitutes and exempt themselves from the traceability exercises involving those radionuclides which they do not market. Upon receipt of their result for the "blind, "the participant receives a Certificate for the SRM. A detailed reporting form and questionnaire issued with the "blind" aids NBS in helping to identify errors or measurement discrepancies. To satisfy a request by the participants of this program for proof of demonstrated traceability, each participant in the "blind" round robin will receive a Certificate of Traceability to the National Measurements System. Participation in the "blind" round robins, although voluntary for the most part, is really of great advantage to the manufacturers in not only continually monitoring their measurements performance (i.e. assurance) but also documenting this performance (i.e. traceability). The NBS encourages a greater participation in this aspect of the program and would like to see the program gradually proceed from a distribution of standards to a distribution of "blinds."

The results for the "blind" round robin distributions for 1975 are summarized in

Table 3. They include measurements on both the H-level (multi-milicurie) and $\frac{L}{3}-1$ evel (multi-miçrocurie) standards for ${ }^{113} \mathrm{Sn},{ }^{125} \mathrm{I}$, ${ }^{85} \mathrm{Sr}$ and ${ }^{32} \mathrm{P}$ as well as a few additional

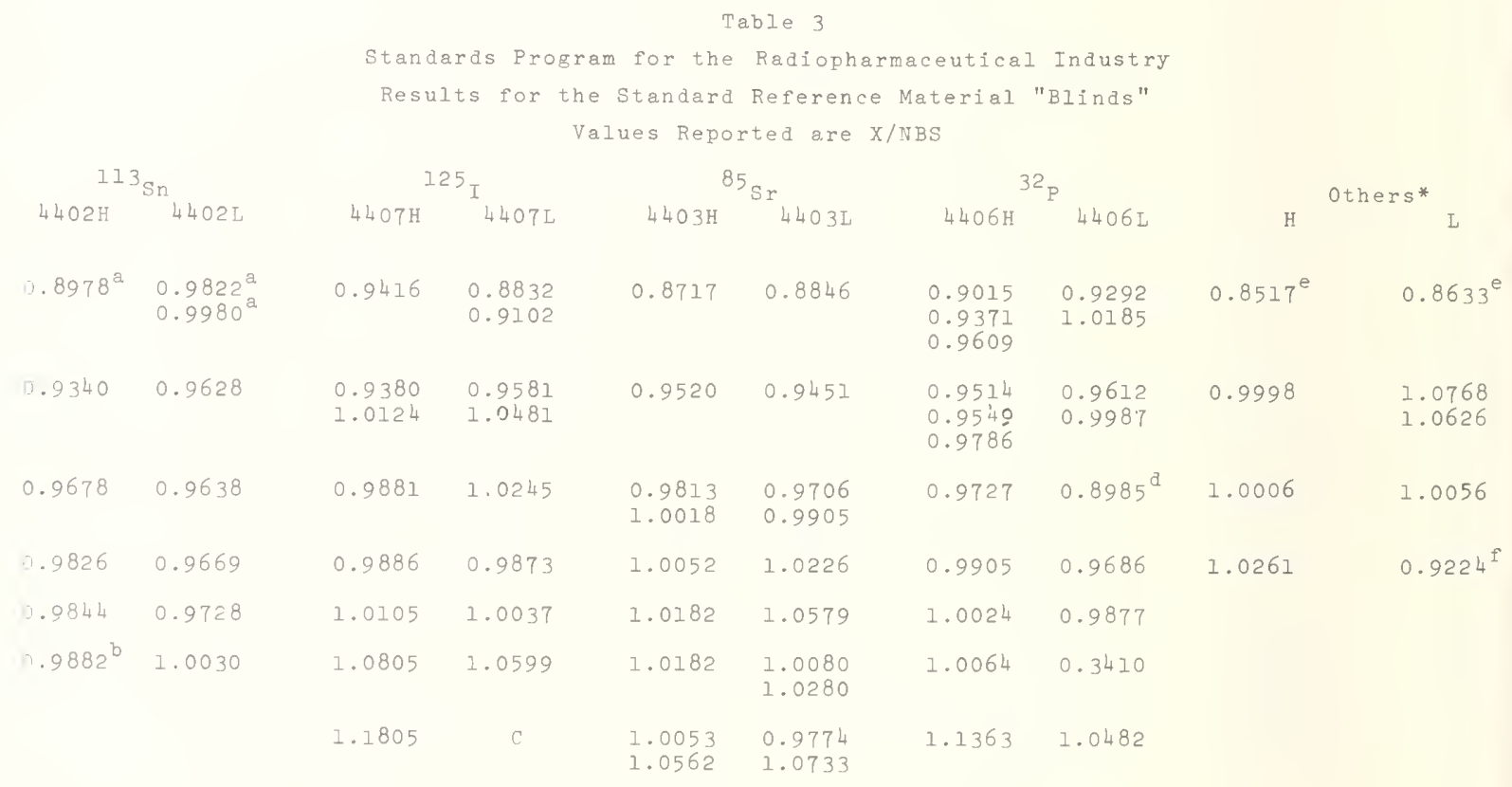

*one each for ${ }^{57} \mathrm{Co}(4408 \mathrm{H}$ and $4408 \mathrm{~L})$ and ${ }^{75} \mathrm{Se}(4409 \mathrm{H}$ and $4409 \mathrm{~L})$ and two each for $198 \mathrm{Au}(4405 \mathrm{H}$ and $4405 \mathrm{~L})$.

\footnotetext{
${ }^{2}$ Revisea values after increasing by $54 . \%$

${ }^{b}$ Revised value after increasing by $0.8 \%$.

Not measured.

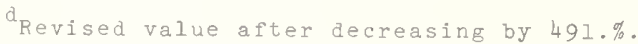

'on retest with $M-1$ evel activity $S R M, X / N B S=1.0282$

fRevised value after decreasing by $279 . \%$.
}

The results in each column of this table are listed in order of increasing values of x/NBS (for the $\mathrm{H}-$ level SRM) and does not correspond to the order in which the participants are listed in Table l. 


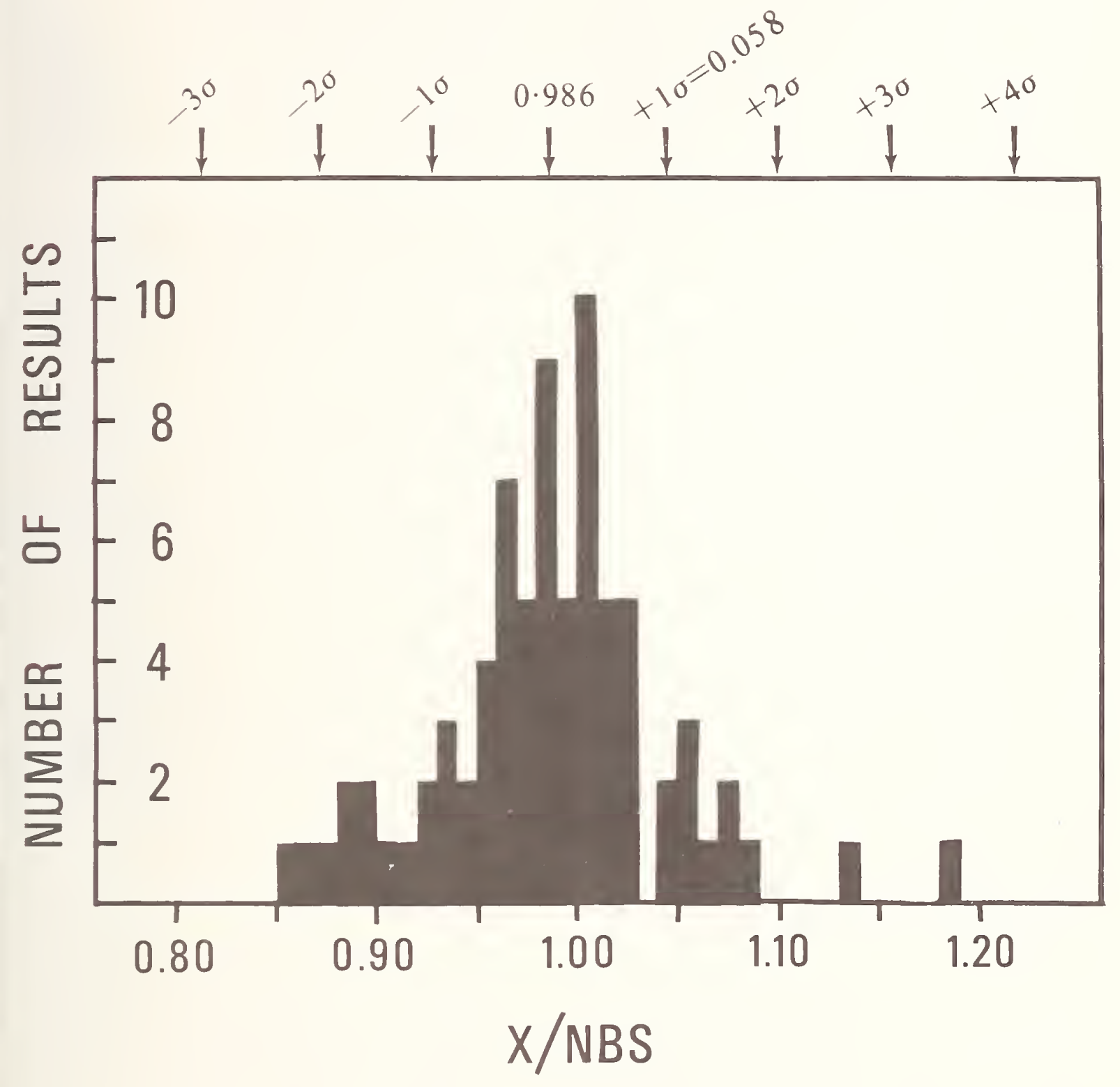

EIGURE 1

Distribution of reported results (data of Table 3 using revised values when applicable) for the 1975 SRM "blinds."

One outlier not plotted: ${ }^{32} \mathrm{P}, 4406 \mathrm{~L}(\mathrm{X} / \mathrm{NBS}=0.3410)$. 
results on ${ }^{57} \mathrm{Co},{ }^{75} \mathrm{Se}$ and ${ }^{198} \mathrm{Au}$. In most cases, the reported values were activity per unit weight, and after correcting for decay were divided by the NBS values to obtain $\mathrm{X} / \mathrm{NBS}$. There is a total of 78 reported values on 61 blind samples. Several participants reported the results of measurements by different methods on the same sample. Close inspection of Table 3 indicates that there are no major systematic trends. With the possible exception of ${ }^{113} \mathrm{Sn}$, where the reported values tend to be slightly low, all the radionuclides appear to have been measured about equally we11. Similarly, there are no major differences between the measurements of the $\mathrm{H}-1$ evel and L-level samples. Although not provided in the table, there was also no apparent evidence that one assay method or instrument was superior to another. In general, the results indicate a fairly satisfactory agreement with NBS values.

There are, however, a number of mistakes in data handling which should be distinguished from systematic or random errors in the measurements. These mistakes included a $54 \%$ error because of the incorrect use of a branching ratio (ganma-ray abundance), a $279 \%$ error due to a miscalculated decay correction, and a $491 \%$ reporting error resulting from the failure to distinguish between total activity and activity per unit weight. Oftentimes these can be detected directly from the questionnaire responses, when sufficient information is provided, and can be revised (as has been done in Table 3) to reflect the true measurement capability. Unfortunately these cases are not that rare, occurring nearly $10 \%$ of the time. A distribution of the results (data of Table 3 using the revised values when applicable) is provided in Figure 1. As can be seen, the reported values are distributed about the mean value of $\mathrm{X} / \mathrm{NBS}=0.986$ with a standard deviation of 0.058 . The values on the wings of the histogram, which are of the order of $10-20 \%$ deviation from the NBS values, are usually attributable to either unaccountable systematic errors such as in dilution or dispensing, or systematic errors such as in decay corrections, detection efficiency and instrument calibration factors which can be adjusted after receiving the NBS value and Certificate. A number of participants have been aided in identifying sources of discrepancies in their measurements and have been provided with suggestions for eliminating them and/or with information for improving their procedures. A good example of this is shown in the first values listed in the last column of Table 3 where measurements on both the H-level and L-level samples showed an approximately $15 \%$ discrepancy. On pursuing it with the participant, a long-existing systematic error in an instrument calibration factor (arising from an incorrect decay correction in a reference source) was discovered. On adjusting the calibration after receiving the NBS value, the participant was "retested" with an intermediate-level activity (M-1evel) sample of the same radionuclide. The reported value on this retest was within $3 \%$ of the NBS value.

I have tried to give an overview of the entire AIF-NBS Radioactivity Measurements Assurance Program for the Radiopharmaceutical Industry and hope you now have a better understanding of its objectives and progress. After spending considerable time discussing errors and discrepancies, I would, in conclusion, like to emphasize that the radioactivity measurements made by the radiopharmaceutical industry are now, after eliminating data-handling mistakes, generally within $\pm 20 \%$ of the NBS values. The program has been successful in meeting many of the needs of the radiophamaceutical industry and I believe we all look forward to its continued success.

\section{Acknowledgement}

It is a pleasure to acknowledge the extensive efforts of Dr. W. B. Mann, Miss L. M. Cavallo, Mr. R. W. Medlock and the many other personnel of the Radioactivity Section at NBS who have contributed to the success of this program.

\section{References}

1. C. W. Seidel and J. C. Brantley, "Activities of Commercial Radionuclide Producers" in Activities and Needs Related to Radioactivity Standards for Environmenta1 Measurements, Environmental Protection Agency Rpt. No. EPA-670/4-75-006, 39 (U.S. Govt. Print ing Office, Washington, D.C., 1975).

2. C. W. Seidel and J. M. R. Hutchinson, "ANSI Quality Assurance Program in Radioactivity Measurements" in proceedings of this symposium (1976).

3. L. M. Cava11o, B. M. Coursey, S. B. Garfinke1, J. M. R. Hutchinson and W. B. Mann, "Needs for Radioactivity Standards and Measurements in Different Fields, "Nucl. Instrum. Methods, 112, 5 (1973). 
AMERICAN NATIONAL STANDARDS INSTITUTE

QUALITY AS SURANCE PROGRAM IN RADIOACTIVITY MEASUREMENTS

C. W. Seidel

New England Nuclear Corporation

Boston, Massachusetts 02118

and

J. M. R. Hutchinson

National Bureau of Standards

Washington, D. C. 20234

The ongoing ANSI N42.2 program of writing procedural standards for radioactivity measurements with $\mathrm{NaI}(\mathrm{T} 1)$ and $\mathrm{Ge}(\mathrm{Li})$ detectors, liquid scintillation counters and ionization chambers is described.

(Detectors; germanium; ion chamber ; liquid scintillation; sodium iodide; standard)

In 1972 the Atomic Industrial Forum (AIF) appointed a subcomittee of Manufacturers of Radioactive Reference Standards with the objective of obtaining a high degree of consistency and reliability in commercially available radioactive reference standards and their accompanying Certificates of Calibration. Representatives of all major commercial manufacturers of radioactivity standards and the National Bureau of Standards (NBS) served on this comittee. Other companies and interested persons were 1 isted as observers and attended our meetings or were informed by mail of our discussions and decisions. This committee has now become a subcommittee, N42.2, of the American National Standards Institute (ANSI).

Several things were accomplished by the original committee that I feel are worth mentioning. As a result of meetings held in 1972 and 1973, the manufacturers of radioactive reference standards agreed to follow ICRU Report 12 (1968) in terms of the format for the Certificate of Calibration and, in reporting accuracies, to quote the random error at the $99 \%$ confidence level. In addition, they agreed to refer to this format in their product literature. Now purchasers of radioactive standards are able to make an intelligent choice of products by comparing specifications listed in a common format. The committee also began a blind round-robin measurements program to establish traceability to NBS.

The AIF comittee also sought to help the users of radioactive reference standards by promulgating guidelines or methods for the calibration of nuclear-radiation detectors that are in common use.

We believed that, with the increase in the use of radioactive materials in diverse scientific fields by people with non-nuclear backgrounds, there was a serious need for proper guidelines or written standards that would allow a knowledgeable person to set up the proper quality assurance program for his equipment. Many of us have heard of people who never calibrate their equipment, who try to measure weak beta-particle emitters through thick glass or I-125 through lead, who know little of decay schemes and abundances of particular radiations, and those who count samples too hot for their detector without knowing it. A survey in New England a few years ago uncovered a number of dose calibrators (ion chambers) that were out of calibration by a significant amount and on which a daily calibration check was rarely performed. Some of the participants in past NBS round robins completely misidentified the radionuclides, sometimes listing one whose gamma-ray spectrum bore little similarity to the actual radionuclide.

The Subcommittee believed that the much needed guidelines or methods of calibration would be more readily accepted by the multidisciplined users of radioactivity if they were formulated under the auspices of a national standards organization. It was also felt that the AIF as an industry oriented group was not the appropriate organization to sponsor such an effort. Therefore, we sought a home in the ANSI program.

In 1973 we tegan to work under the ANSI N43 and N44 committees, but since 1975 have functioned within the $\mathbf{N} 42$ committee, which is chaired by Lou Costrell of NBS. We are presently called the "N42.2 Subcommittee on Standard Methods to Calibrate Nuclear Detection Equipment" and are working 
on procedural standards to calibrate the dose-calibrator type of ionization chamber, $\mathrm{NaI}(\mathrm{T} 1)$ detector, $\mathrm{Ge}(\mathrm{Li})$ detector systems and liquid-scintillation counters. Table I 1 ists the task groups and their chairmen.

A number of task groups have been created to draft the written standards, and we have attempted to choose the members of these so as to be representative of industry, government and university areas, and any other groups that we find have an interest in our objectives. While we have 1 imited these task groups to 6-8 people in each, we do allow observers to participate in the discussions or to supply us with written suggestions. Currently, our subcommittee consists of 31 members and 71 observers.

Many observers represent other standards-writing organizations involved in similar areas such as the American Nuclear Society, Society of Nuclear Medicine, College of American Pathologists, American Society for Testing of Materials, National Committee for Clinical Laboratory Standards and others. Regulatory agencies such as the Nuclear Regulatory Commission (NRC) and Food and Drug Administration (FDA) are represented in either our active or observer membership. The observers also include organizations outside the United States such as the International Atomic Energy Agency and the National Laboratories in Canada, England and France.

For whom are we writing these standards? You might say that most of our interested clientele are included in the groups 1 isted in Table II.

We want to provide laboratory technicians and their supervisor with a guide on how to calibrate and to check the continued performance of their nuclear radiation détectors. We want to work with the interested regulatory agencies to provide meaningful written standards to which they can refer in their regulatory guide, or require their licensees to incorporate in their quality assurance procedures. And we want to aid the instrument manufacturers in the writing of their instrument manuals so that they can provide acceptable procedures for the setting up, the calibration and the checking of the operation of their instruments.

Our objective is to write a concise, clear guide that will serve as a standard from which an individual or laboratory may develop a program for the acceptable operation of its particular measurement systems.
Our standards will cover six basic items as 1 isted in Table III. The standards we are writing recommend both the method and frequency of calibrating the instruments, the types of standards that should be used, the minimum daily (or other interval) checks on instrument performance which should be made, the correct procedure for making the measurements and assessing errors, and a check list of potential problems. Such standards, if useful, are likely to be adopted or recommended by regulatory agencies such as the NRC or FDA.

One section of our standard is desig$\mathrm{n}$ ated as a Precaution section. Here we list common problems and state how to avoid them or cite references that report on how they may be properly handled. Examples of some precautions concern recombination effects in ion chambers at high activity levels, or the problems of counting high energy betaemitting radionuclides in ion chambers, coincidence losses in $\mathrm{NaI}$ (T1) detectors, and quenching or luminescence in 1iquidscintillation counting. We feel this is one of the most valuable sections of our standards.

An outline of our ion-chamber standard (N713) is shown in Table IV.

The standards for the ion-chamber detector and the germanium detectors recent $1 y$ went out for ballots, and we are now in the process of resolving the comments we received. We hope the other two standards will be ready for their first ballot later this year.

Our comittee looks forward to a rapid completion of our effort and the adoption of the written standards by the multidisciplined users of radioactive material.

We would also like to see greater participation in the blind round-robin program begun by the AIF comittee and now organized, promoted and directed by NBS and ANSI N42.2.

Under the blind round-robin program 4 to 6 radionuclide test samples are issued each year. The program was implemented to accomplish NBS traceability for the participants. For a nominal fee $(\$ 100$ to $\$ 150$ per standard) any interested party can obtain one of these "unknown" standards and a questionnaire. Each participant in the round robin is expected to complete his questionnaire within a few weeks stating (1) his measurement of the activity, (2) any detectable radioactive impurities, (3) method of measurements and (4) the determination 
of the accuracy of their measurements. Upon receip of the questionnaire. Lucy Cavallo, Coordinator of the program at NBS, will send the participant a Certificate of Calibration and collate the results. Summaries of the results of the program for 1973, 1974 and 1975 are shown in Tables V, VI and VII. Several participants in these round robins have been aided by the staff at NBS in correcting their measurement systems if their response has been in error.

The number of participants in this program is not large. Most of the major manufacturers of radioactive material, some National Laboratories, a hospital, and power reactor groups participate because of a need to be traceable to NBS. A low number of participants may also be noted in the College of American Pathologists testing program (which began before ours) although they have had some recent increases in the number of participants.

Another similar round-robin program oriented to specific requirements of the Radiopharmaceutical manufacturers and the FDA is now supported by the AIF to provide these manufacturers with NBS traceability. This program grew out of our initial roundrobin program and has been described by the previous speaker.

Increased participation in these roundrobin programs by various users such as hospitals and clinical laboratories is desirable and beneficial to the user. We look forward to your support and participation in our program.

\section{TABLE I}

\section{ANSI N42.2 Task Groups and Chairmen}

\section{Writing Groups}

N42.2.1 Germanium Detector Standard Gerald Martin, Jr., General Electric

N42.2.2 Ion Chamber Detector Standard Frank Masse, M.I.T.

N42.2.3 Liquid Scintillatioñ Counter Standard - Roger Ferris, Amersham/Searle

N42.2.4 Sodium Iodide Detector Standard Ron Coley, Commonwealth Edison

\section{Round-Robin Program}

L. M. Cavallo, National Bureau of Standards
TABLE II

\section{For Whom Are We Writing}

I. The technical user

II. The regulatory agencies (i.e., NRC, FDA)

III. The instrument manufacturer

\section{TABLE III}

What Are We Writing?

Standards that will provide a guide to:

I. Setting up instrument

II. Calibration

III. Performance checks

IV. Measurement procedures

V. Estimate of errors

VI. Potential problems (precautions)

\section{TABLE IV}

Ion-Chamber Detector Standard (N713)

I. Introduction

II. Scope and Purpose

II. Definitions

IV. Text of Standard

a. Operation

b. Calibration

c. Use

d. Standard Sources

e. Performance Monitoring

f. Accuracy and Reproducibility

V. Sources of Error

VI. Precautions

a. Non-linearity Effect

b. "Simulated" sources

c. Low-Energy Photon Emitters

d. Beta-Particle Enitters

e. Gaseous Radionuclides

f. Plate out of Radionuclides

g. Assay of a Radionuclide for which no standard is available 
1973 ROUND ROBIN

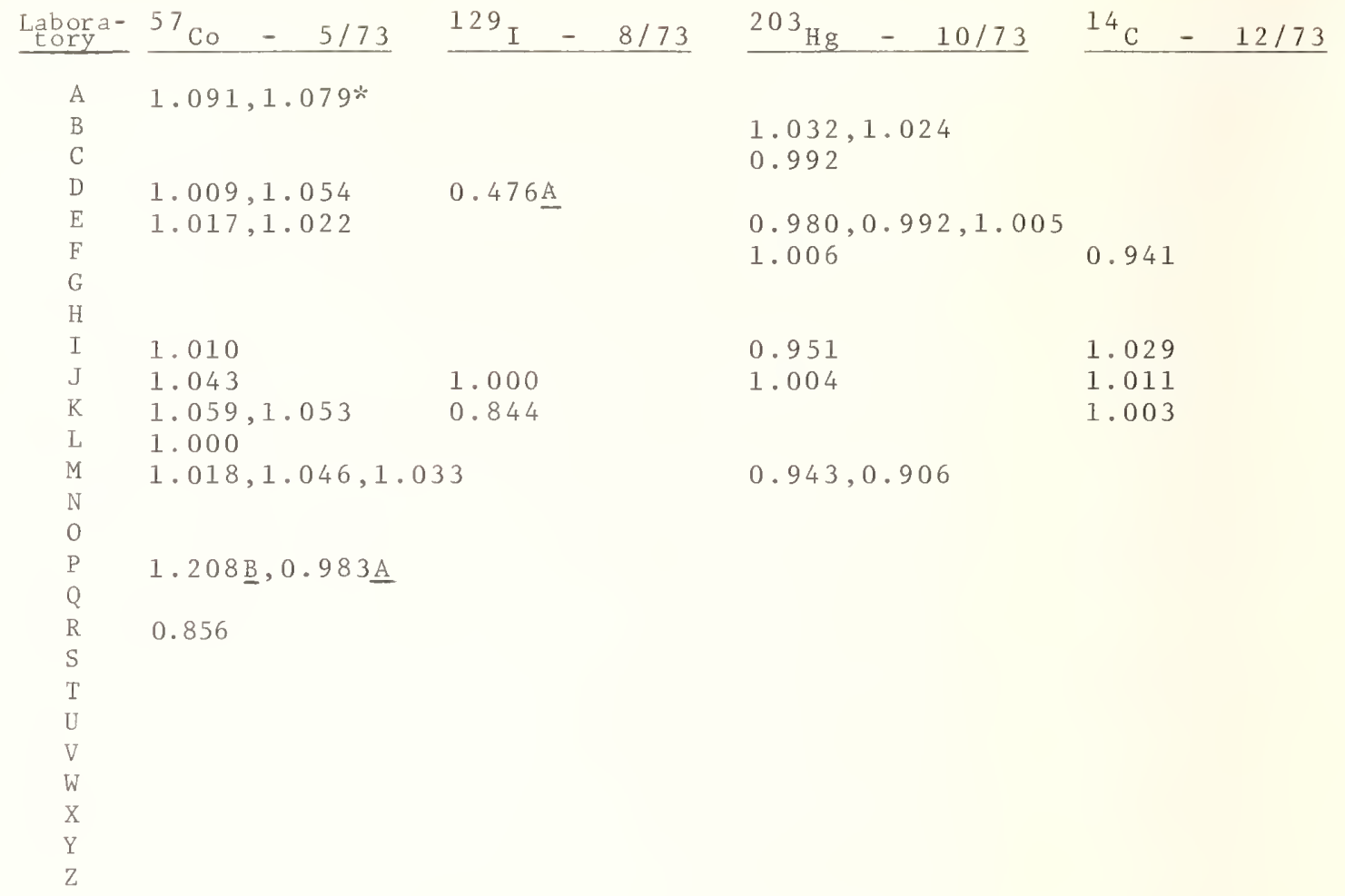

* Where more than one number is given, the laboratory reported more than one measurement.

$\underline{B}$ refers to the originally reported measurement; A refers to the value after correcting for computational or other data handling error. 

the laboratory reported more than one measurement. 


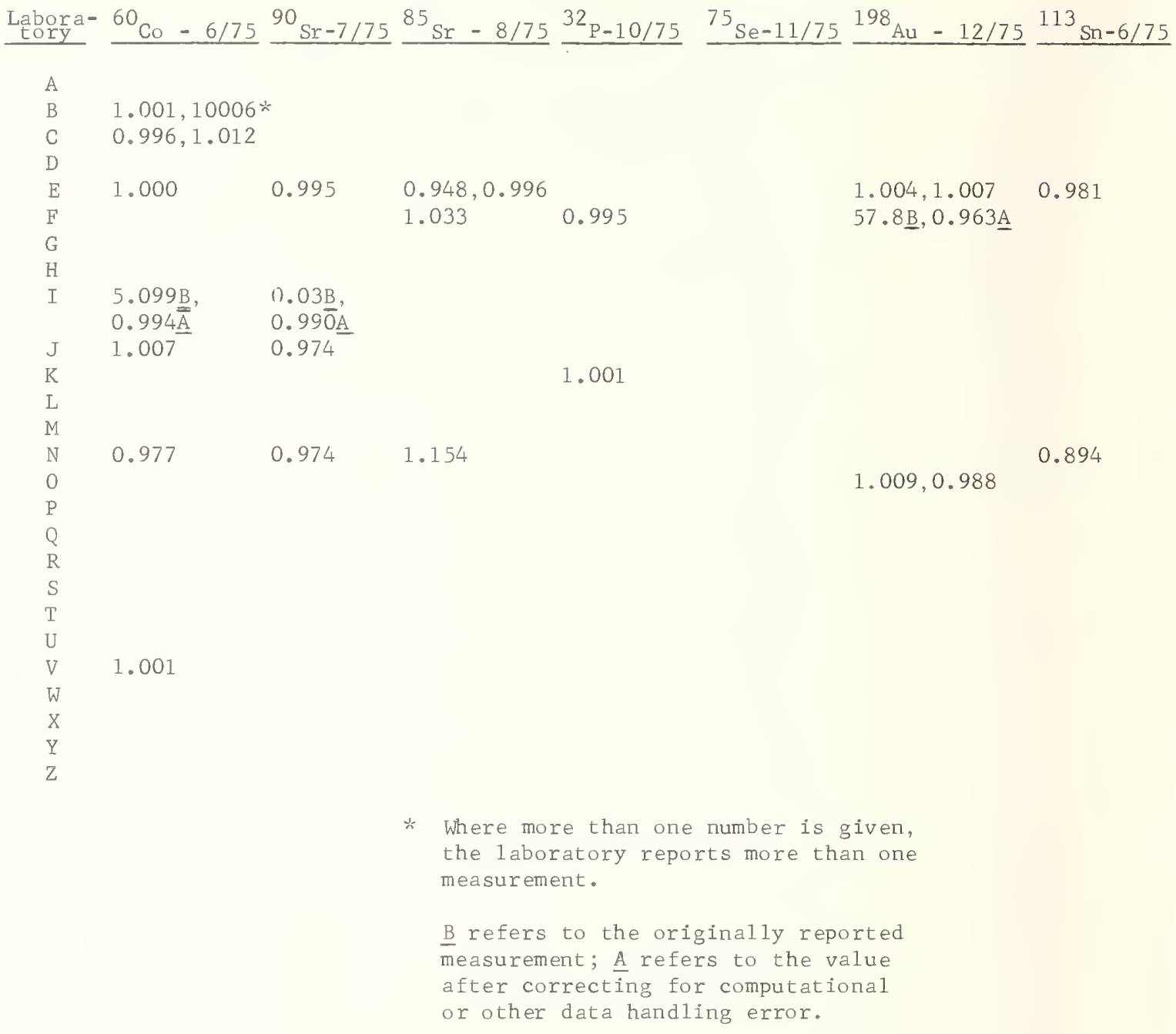


FILTERED BEAMS AT THE NBS REACTOR*

R. B. Schwartz and I. G. Schroder

National Bureau of Standards

Washington, D. C. 20234

and

E. D. McGarry

Harry Diamond Laboratories

Adelphi, MD 20783

Using a combination of resonant scatterers and filters, we are producing high intensity, very pure beams of $2 \mathrm{keV}, 25 \mathrm{keV}$, and $144 \mathrm{keV}$ neutrons at the NBS reactor. These beams have low background, are well calibrated and very stable, and are intended for use as calibration standards.

(Monoenergetic neutrons; neutrons; neutron beams; neutron filters; reactors; resonant scatterer.)

\section{Introduction}

One of the missions of the NBS Center for Radiation Research is the development and maintenance of standard neutron fields. These fields are to be used for the calibration of radiation instruments and of personnel dosimeters, as well as for other CRR programs. In this paper we shall be primarily concerned with those fields developed by means of filter techniques at the NBS reactor.

Our particular concern is with the energy range below $30 \mathrm{kev}$ (sometimes referred to as the "neglected energy range." ${ }^{1}$ Our concern arises from the fact that a very large fraction of the neutron dose-typically $30 \%-$ $40 \%$-in the working environment around a nuclear reactor is due to neutrons in this energy range. ${ }^{1}$ Despite the importance of these "intermediate" energy neutrons, very few instruments are ever directly calibrated anywhere in this range, and for a very good reason: it is very difficult to produce useful monoenergetic neutron fluxes of these energies.

At the NBS reactor, we have used filter techniques to produce high intensity neutron beams with energies of $2 \mathrm{keV}$ and $25 \mathrm{keV}$, and are soon to install a $144 \mathrm{keV}$ beam. Neutron filtering occurs at energies where there are very deep minima in the neutron total cross section. (These occasional minima are caused by interference between resonance and potential scattering.) An appropriate thickness of a material whose cross section shows such behavior will transmit essentially all neutrons whose energies correspond to the minimum in the cross section; but will attenuate neutrons of all other energies. The production of high intensity filtered beams was pioneered at the Materials Testing Reactor; ${ }^{2}$ unfortunately the MTR was shut down before the development of the beams could be completed. The filters themselves were subsequently transferred to NBS.

\section{$2 \mathrm{keV}$ Beam}

The $2 \mathrm{keV}$ beam is produced by a scandium filter: the minimum (or "window") at $2 \mathrm{keV}$ results from overlap of the interference minima from the resonances at 3.24 and $4.27 \mathrm{keV}$, producing a cross section with a minimum value of only $50 \mathrm{mb} \cdot{ }^{3}$ (See Figure 1.) Unfortunately, there are also several other windows in scandium at higher energies. These windows are responsible for a relatively high transmitted neutron flux in the energy region between $8 \mathrm{keV}$ and $800 \mathrm{keV}$. These higher energy neutrons form an unwanted background to the desired $2 \mathrm{keV}$ beam. In the work of Simpson et al. at the MTR, ${ }^{2}$ this background flux was equal to $2 / 3$ of the $2 \mathrm{keV}$ flux. This tyne of background can be tolerated in certain measurements, but would be

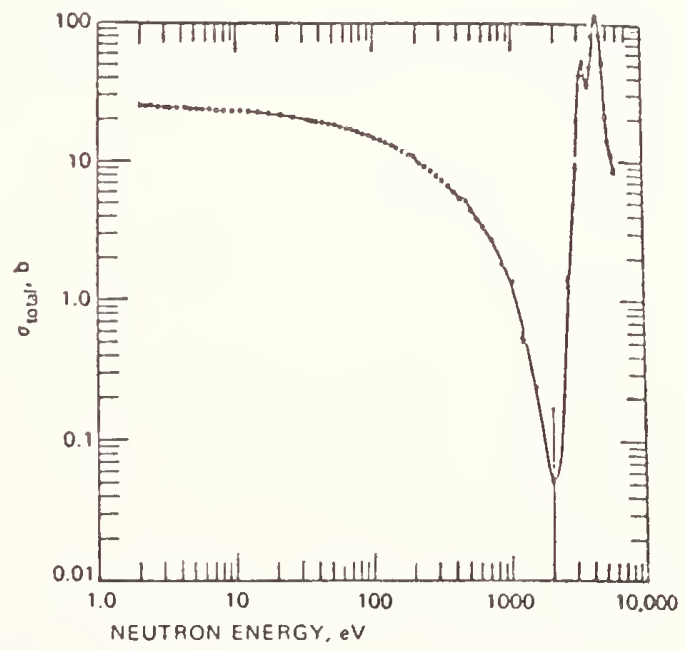

Fig. 1 Total neutron cross section of scandium from $1 \mathrm{eV}$ to $8 \mathrm{keV}$. The deep minimum at $2 \mathrm{keV}$ is responsible for scandium's functioning as a filter. Other minima at higher energies are not shown. 
disastrous for the calibration of dosimeters. Unfortunately, the problem is inevitable in any facility in which a scandium filter looks at a reactor core.

This background is essentially eliminated in the NBS installation by the use of a through tube in conjunction with a resonant scatterer. The through tube passes $10 \mathrm{~cm}$ outside of the edge of the reactor core, and the collimating system containing the filter only sees a scatterer at the center of the tube. (See Fig. 2) This design eliminates unwanted core neutrons and gammas. We use manganese to scatter the neutrons, making use of the large scattering resonance at $2.375 \mathrm{keV}$. Although the resonant energy does not exactly match the energy of the $2 \mathrm{keV}$ window, the resonance is sufficiently broad $(\Gamma=400 \mathrm{eV})$ that at $2 \mathrm{keV}$ the scattering cross section is still > 100 barns. ${ }^{4}$ Physically, the scatterer is a $3 \mathrm{~mm}$ thick $\mathrm{Mn}-\mathrm{Al}$ alloy, containing 57 atomic percent manganese.

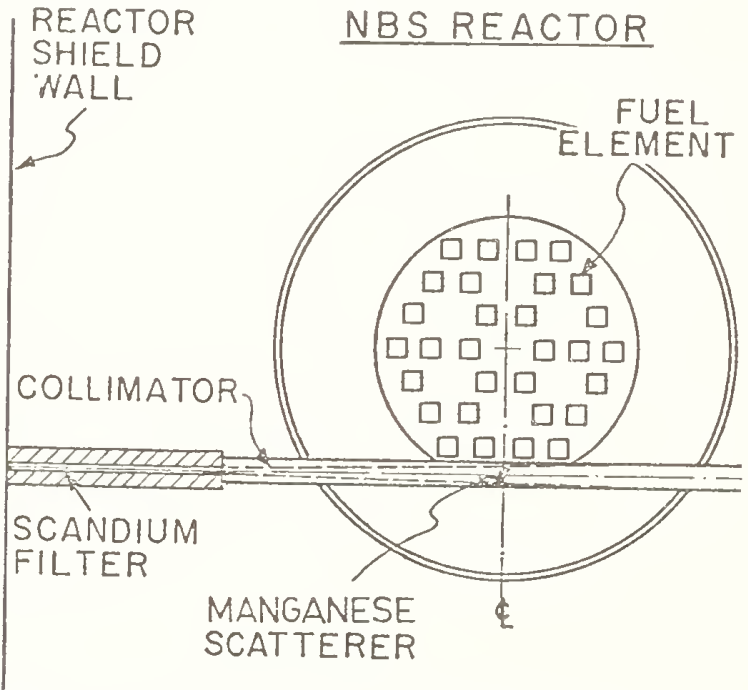

Fig. 2 Schematic representation of the $\mathrm{NBS}$ Reactor showing the filter, collinator, and scatterer in the through tube (not to scale.)

The advantage of this design may be seen in the spectra shown in Fig. 3 (These data were taken with a 1 atm. hydrogen proton-recoil counter). The solid line shows the spectrum obtained with a $110 \mathrm{~cm}$. long Sc filter; the dashed curve shows the effect of adding one $\mathrm{cm}$ of $\mathrm{Ti}$ to the $\mathrm{Sc}$. It can be seen that even without the $\mathrm{Ti}$, the main secondary peak at $29 \mathrm{keV}$ has an area of only $3 \%$ of the $2 \mathrm{keV}$ peak. The addition of the Ti reduces this peak (as well as the ones at 7 , 15 , and $40 \mathrm{keV}$ ) by a factor of about $2-1 / 2$, at the cost of only $17 \%$ of the $2 \mathrm{keV}$ peak.
In terms of total flux (i.e., the area under the peaks), the higher energy contaminants amount to approximately $6 \%$ of the $2 \mathrm{keV}$ flux without the titanium; the addition of the titanium; the addition of the titanium reduces their contribution of $\sim 3 \%$ of the $2 \mathrm{keV}$ flux.

The neutron intensity, as measured with a calibrated $\mathrm{BF}_{3}$ counter is $6 \times 10^{5}$ neutrons/ sec. The beam diameter at the reactor face is $1.7 \mathrm{~cm}$, diverging to $6 \mathrm{~cm}$ at $3 \mathrm{~m}$ from the reactor. The gamma ray background is $\sim 1 \mathrm{Mr} /$ hr.

\section{$25 \mathrm{keV}$ Beam}

The $25 \mathrm{keV}$ beam is produced by an ironaluminum filter, in another through tube in the reactor. For this case, the higher energy background problems are not so severe and it suffices to use a "flat" scatterer (i.e., graphite). The higher energy flux amounts to $1 \%$ of the $25 \mathrm{keV}$ flux. The $25 \mathrm{keV}$ flux is $2 \times 10^{5} \mathrm{n} / \mathrm{sec}$, with a gamma background of $\sim 1 \mathrm{mR} / \mathrm{hr}$.

\section{$144 \mathrm{keV}$ Beam}

A $144 \mathrm{keV}$ beam, using a silicon filter, will be installed later this month. The flux should be at least equal to that in the $2 \mathrm{keV}$ beam, with essentially no neutrons other than those at $144 \mathrm{keV}$.

\section{Summary}

The NBS filtered beams provide a very important calibration facility. All three will have low background, be very stable, and will be well calibrated. The three beams can be run simultaneously with no interference or cross-talk, and will serve as calibration standards. It is our intention that the "neglected energy range" be much less neglected in the future. 


\section{References}

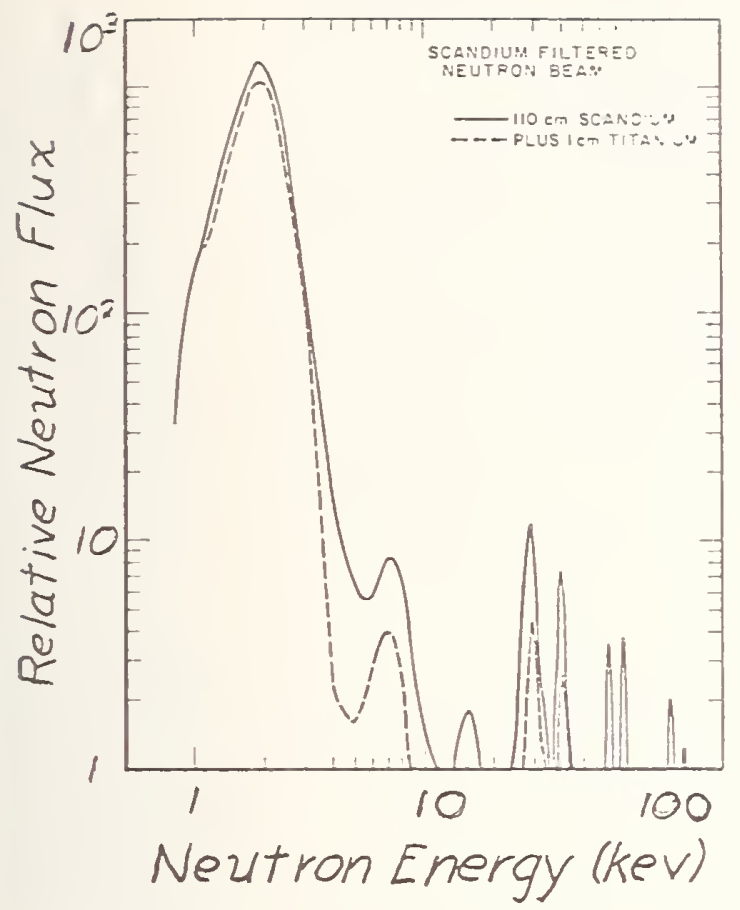

Fig. 3 Neutron spectrum through scandium filter. The solid curve represents the spectrum with a $110 \mathrm{~cm}$ scandium filter alone, and the dashed curve the spectrum observed with the addition of $1 \mathrm{~cm}$ of titanium.
*Work supported in part by the Energy Research and Development Administration

1) J. R. Harvey and S. Beynon, Proc. First Symposium on Neutron Dosimetry in Biology and Medicine, Neuherberg/Munchen (Germany), 1972 p. 955.

2) O.D. Simpson, J. R. Smith and J.W. Rogers, Proc. Symp. Neutron Standards and Flux Normalization; p. 362, CONF-701002, U.S. Atomic Energy Commission (1971); also, Proc. Third Conf. Neutron Cross Sections and Technology, p. 598, CONF-71031(1971).

3) W. L. Wilson, "Neutron Cross Section Measurements and Gamma Ray Studies of ${ }^{45}$ Sc"; M.S. Thesis, University of Idaho Graduate School, Dec. 1966 (unpublished).

4) M. D. Goldberg, S. F. Mughabghab, B. A. Magurno, and V. M. May; "Neutron Cross Sections, Volume IIA, Z=21 to 40 ;" BNL 325, Second Edition, Supplement No. 2 (Physics-TID-4500) February 1966. 

NEUTRON SOURCE CALIBRATIONS AT NBS FOR CALIBRATION CHECKS OF NEUTRON RADIATION INSTRUMENTS

V. Splegel

National Bureau of Standards Washington, D. C. 20234

The manganous sulfate bath method of neutron source calibrations at the NBS is described together with the corrections applied for neutron capture in the source itself, capture by competing nuclei of elther fast or thermal neutrons, and thermal neutron leakage. The advantage of commerclally avallable Am-Be $(\alpha, n)$ neutron sources as a calibration check for radiation instruments in the $\mathrm{MeV}$ range is stressed.

(Neutron source; callbration; manganous sulfate; radiation instruments)

\section{Introduction}

This paper describes the manganous sulfate bath method used at the NBS to calibrate the emission rate of neutron sources. For a more thorough review of and references to the measurement of neutron emission rates and neutron spectra see reference (I) by Caswell.

Neutron sources which are used for the calibration of neutron radiation instmments are selected on the basis of their size, stability, predictable and long half life, energy spectrum, and emission rate. The emission rate is used to calculate the strength of the neutron field at the position of the neutron radiation instrument. Neutrons emitted from these sources are not of uniform energy and therefore require fluencedose conversion factors to give a true calibration of a neutron dosimeter. It is not the purpose of this paper to describe such a complicated procedure for any given instrument, but to recommend that, once such a relationship is established, the operation is most easily checked over long periods of time by an $\operatorname{Am}-\operatorname{Be}(\alpha, n)$ neutron source.

Calibration of Neutron Emission Rates

At the NBS the manganous sulfate bath method ${ }^{2}$ is used to compare the neutron emission rate of an unknown source to the standard radium-beryllium, photo-neutron source, NBS-I. The principle of the method is shown in Figure 1. The source is placed in a thin-walled, alr-filled, teflon beaker at the center of a $125 \mathrm{~cm}$ dia- meter sphere of manganous sulfate solution. Due to the large sige of the bath very few neutrons escape. The remainder are captured by any of the following processes: (1) fast capture in the fluorine of the beaker, (2) fast capture in ${ }^{16}$ o or ${ }^{32} \mathrm{~S}$, (3) slow neutron capture in $55_{\mathrm{Mn}}$ or hydrogen, or (4) slow neutron capture in the beaker or the source itself. The slow neutron capture in ${ }^{55} \mathrm{Mn}$ produces ${ }^{56} \mathrm{Mn}$, which decays to ${ }^{56} \mathrm{Fe}$ with a half life of 154 minutes. It is the gamma activity of the ${ }^{56} \mathrm{Fe}$ which is measured at the sodium lodide crystal. The induced activity of the bath is measured for the unknown source and for the standard source, NBS-I. The ratio of the source emission rates is determined from the ratio of the two count rates after corrections have been applied for escape and capture in other nuclei.

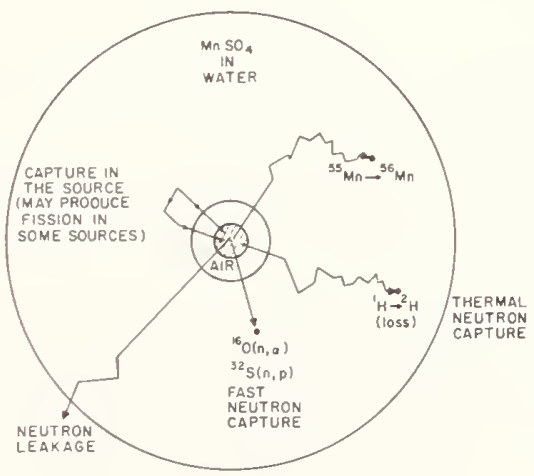

Fig. I Manganous Sulfate Bath Source Calibration (Schematic) 
A circulating manganous-sulfate bath system, as shown in Figure 2 , is used at the NBS. The circulating pump thoroughly mixes the bath solution by maintaining a flow rate of about 70 liters per minute through a dispersive nozzle. About 6 liters of solution per minute flow to a remotely located gamma-ray counting system, where counting can be continued and statistics accumulated as long as desired. The Sodium-Iodide crystal, located at the center of the Marinelil beaker, is used for neutron sources with emission rates between $10^{5}$ to $10^{8}$ neutrons per second. The second crystal, located above the Marinelli beaker, views a small fraction of the activated solution

through the lead shield and is used for neutron sources with an emission rate between $10^{8}$ and $10^{10}$ per second. The second crystal is needed for more intense neutron sources, because the main crystal counting system would be paralyzed by the resulting manganese activity. The relative efficiency of the two counting systems is measured by using an intermediate neutron source with an emission rate of about $10^{8}$ per second, which can be counted simultaneously by both systems.

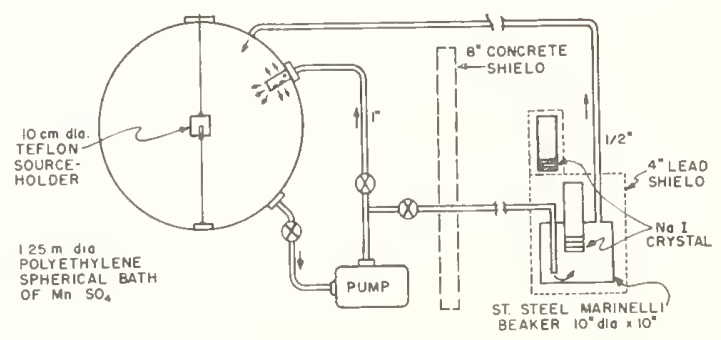

Fig. 2 Circulating Manganous Sulfate Bath and Shielded Gamma-Ray Detector

The uncertainties in a calibration are listed in Table I. The most recent absolute calibration of NBS-I by Noyce, et al. 3 was quoted with an error of $\pm 1 \%$. Escape from the 1.25 meter diameter bath is quite small even for the higher energy $(\alpha, n)$ neutron sources and essentially nonexistent for low energy $(\gamma, n)$ neutron sources. The error in the correction depends upon the size of the correction. A correction is applied for capture in the source itself. In the
Table I

Uncertainties in the Calibration of Neutron Sources

Systematic Errors

$(\alpha, n)$

$(\gamma, n)$

Emission Rate of NBS-I

Escape

$1 \%$

$0.2 \%$

$1 \%$

Absorption in Source 0.1-0.3\%

Fast Neutron Loss to

$1 \%$

$0.1 \%$

Reactions

\section{Random Error}

Counting of Activated $\quad 0.2 \% \quad 0.2 \%$ Bath

Total Error $\quad 1.6-1.7 \% \quad 1.3 \%$

case of sources containing fissionable material, such as plutonium, neutron multiplication by thermal capture in the fissionable material may partially cancel or be larger than losses due to capture in the source encapsulation. This correction is minimized by locating the source in an air cavity with thin teflon walls to reduce the thermal neutron flux. The correction is calculated ${ }^{4}$ for each individual source and its accuracy again depends on the size of the correction. The least well understood correction, which is very important for $(\alpha, n)$ sources because they have many neutrons with energies above 3 or $4 \mathrm{MeV}$, is the loss of fast neutrons due to reactions such as ${ }^{16} \mathrm{O}(\mathrm{n}, \alpha)$ and ${ }^{32} \mathrm{~S}(\mathrm{n}, \mathrm{p})$. Calculations of this correction by Murphey ${ }^{5}$ are used for our calibrations, but, because these disagree with some experimental measurements ${ }^{1}$, there still appears to be an uncertainty of about $1 \%$ in the correction for $(\alpha, n)$ sources.

The total errors Ilsted in Table I result from the linear addition of the quadratic sum of random errors and quadratic sum of systematic errors.

\section{${ }^{226} \mathrm{Ra}-\mathrm{Be}(\alpha, \mathrm{n})$ sources increase} in neutron source strength due to the growth of $210_{\text {Po. }} 210_{\text {Po decays with }}$ a half life of 138.38 days and is formed by the alpha decay of $210_{\mathrm{Pb}}$ in the radium decay chain with a 
half life of 22.3 years. The relative source strength as a function of time in years from the date of separation of $226_{\mathrm{Ra}}$ is given by the following relationship 6 ,

$$
\begin{aligned}
F(t)=1 & +.143\left(1.01437-1.03166 e^{-t / 31.138}\right. \\
& \left.+.01728 e^{-t / .5466}\right) .
\end{aligned}
$$

${ }^{239} \mathrm{Pu}-\mathrm{Be}(\alpha, \mathrm{n})$ sources increase in neutron source strength due to the growth of ${ }^{24 l_{\mathrm{Am}}}$. ${ }^{24 l_{\mathrm{Am}}}$ is formed by the beta decay of ${ }^{24} I_{\text {Pu with a half }}$ life of 14.89 years. If the atom percent of ${ }^{241}$ Pu in relation to the other neutron emitting Plutonium isotopes are known at the time of manufacture the growth of a $\mathrm{Pu}-\mathrm{Be}$ $(\alpha, n)$ source can be calculated by a rather complicated formula, which will not be included here, but may be found in an article by Caswell ${ }^{7}$.

Instrument Calibration Checks with Commercially Avallable Sources

The sources commonly used for

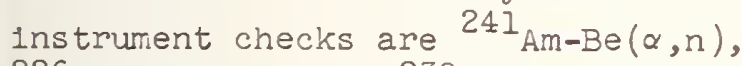

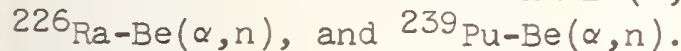
Their average neutron energies are approximately $3.9 \mathrm{MeV}, 2.8 \mathrm{MeV}$, and 3.4 MeV respectively. Only the Amerlcium sources have a single well known half life (433 yr), which is predictable over long periods of time. If great care is taken in determining isotopic composition of the source materlal before manufacture, an accurate effective half Iife can be determined for the other two sources. However, this information is not always available and is - ften lost or forgotten.

Another popularly used neutron source with emission rates up to $10^{10}$ per second is ${ }^{252} \mathrm{Cf}$ spontaneous fission. Its spectrum has been well studied $^{8}$ and it can be manufactured to approximate, very closely, a point source. However, it does have an inconveniently short half life of about 2.64 years and contains ${ }^{250} \mathrm{Cf}$ and ${ }^{254} \mathrm{Cf}$, which further complicates the determination of the effective half $11 \mathrm{fe}$.

\section{References}

1. R.S. Caswell. "Review of Measurements of Absolute Neutron Emission Rates and Spectra from Neutron Sources." Neutron Sources and Applications, Proc. ANS Nat'l Topical Meeting, April 19-2l, 1971, Augusta, Ga., CONF-710402, Vol. I, P. I-53 (1971).

2. K.W. Geiger. "Recent Improvements in the Absolute Calibration of Neutron Sources." Metrologia 4 , 8 (1968).

3. R.H. Noyce, E.R. Mosburg, Jr., S. Garfinkel, and R.S. Caswell. "Absolute Calibration of the National Bureau of Standards Photoneutron Source III. Absorption in a Heavy Water Solution of Manganous Sulphate." J. Nucl. Energy A/B 17, 313 (1963).

4. V. Spiegel, Jr. and W.M. Murphey. "Calculation of Thermal Neutron Absorption in Cylindrical and Spherical Neutron Sources." Metrologia 1, 34 (1971).

5. W.M. Murphey. "The Correction Factor for Fast Neutron Reactions on Sulfur and oxygen in the Manganous-Sulfate-Bath Cal1bration of Neutron Sources." Nucl. Instr. \& Methods 37, 13 (1965).

6. W.M. Murphey, National Bureau of Standards, Washington, D. C. 20234. "The Growth of $210_{\text {Po }}$ in $\mathrm{Ra}-\mathrm{Be}(\alpha, \mathrm{n})$ Neutron Sources." Internal Report, January 1967.

7. R.S. Caswell, R.H. Noyce, S.B. Garfinkel and E.R. Mosburg, Jr. "Recent Developments in Neutron Source Standardization." Neutron Dosimetry II, 547, IAEA, Vienna (1963).

8. J.A. Grundl and C.M. Elsenhauer. "Fission Spectrum Neutrons for Cross Section Validation and Neutron Flux Transfer." Conf. on Nucl. Cross Sections and Technology, washington, D. C. 1, 250 (March 1975). 



\section{Introduction}

In the past few years ultrasound techniques have become well known and are receiving widespread acceptance in clinical practice. For example, diagnostic ultrasound is rapidly finding use as a compliment to other techniques and often is the modality of choice for visualization of structures within the body supplanting procelures involving the use of ionizing radiation. Some of the main areas of application at the present time are visualization of abdominal organs, pregnant uterus, and the heart. It is estimated that approximately 35\% of the pregnant women in the United States currently under go ultrasonic insonations and that by 1980 this number will increase to about $90 \%$. Along with the increasing use of this modality come the responsibility for the assessment of the risk that may be involved with its use. This requires the measurement and characterization of ultrasonic fields by researchers engaged in the evaluation of biological effects associated with ultrasonic exposures. There is also the need to determine parameters of the ultrasonic field from fields produced by comercial ultrasonic devices in order to evaluate the potential risk their use may present on the basis of biological effects data. These measurements are especially necessary in view of the wide proliferation of such devices. In addition the establishment of any necessary performance criteria requires the quantification of the ultrasonic fields involved and calibration of measurement devices used.

Ultrasonic Quantities and Measurement Techniques

The ultrasonic field parameters which generally are of the greatest concern include total output power, intensity, and field distribution. In the case of ultrasonic intensity it is important that peak temporal and spatial values as well as average spatial and temporal values be measured. The need for peak intensity measurements is evident when one considers that peak intensities from ultrasonic diagnostic pulse echo equipment are on the order of $10^{4}$ times the spatial and temporal average intensities $(1,2)$. Other ultrasonic parameters which are of interest include acoustic pressure, particle velocity, speed of particle displacement, frequency spectrum and attenuation coefficients. Table 1 shows most of the quantities used along with appropriate SI units and quantity symbols.

Relationships exist among the quantities in Table 1. For traveling plane waves the Langevin radiation force (F) is related to the power by the relationship $\mathrm{F}=\mathrm{Pd} / \mathrm{C}$ where $\mathrm{P}$ is the acoustic power, $\mathrm{C}$ is the propagation velocity of the wave, and $\mathrm{d}$ is a factor determined by the type of interface an object presents to the ultrasonic field, values for $d$ are shown in Table 2. For a more detailed discussion of the topic the reader is referred to the following references (3, 4). The ultrasonic intensity (I) for progressive plane waves is given by $I=p v$ where $p$ is the rms value of the particle pressure and $\mathrm{v}$ is the rms value of the particle velocity. The relationship among acoustic pressure, particle velocity and specific acoustic impedance is given by the following formula $p=$ pocv. Unfortunately in the near field of ultrasonic transducers (applicable to most medical applications), the structure of the ultrasonic field is complex and measurement and calibration methods must properly take this into account Several methods for measuring acoustic field parameters exist. These may be grouped into six general techniques: (1) radiation force, (2) thermal as by calorimeters, thermal couples and thermisters, (3) electrical,i.e. equivalent circuits, (4) electroacoustic conversion as by piezoelectric, magneto strictive transducers and capacitance transducers, (5) optical, including both optical diffraction and interferometry, (6) reflection as by reciprocity calibration. The parameters measured by the different techniques are shown in Table 3.

Measurements using one or more of these techniques are being carried out in some university laboratories (usually associated with medical schools or hospitals), by certain manufacturers, and by the Bureau of Radiological Health and the National Bureau of Standards. The Bureau of Radiological Health maintains an inhouse capability to measure the acoustic field parameters from medical ultrasonic equipment in order to conduct biological effects investigations and evaluate ultrasonic products. This work is being carried out as part of the Bureau of Radiological Health's responsi- 
bility under the requirements of the Radiation Control for Health and Safety Act of 1968, P.L. 90-602. The law specifically directs the Bureau of Radiological Health to conduct a wide range of studies into possible conditions of exposure to electronic product radiation; to develop practical measurement techniques for the evaluation of radiation emission, and to investigate possible biological effects associated with such radiation.

Investigations by the Bureau of Radiological Health and reports from others have shown inadequacies in the calibration of ultrasonic medical devices (5). There has also been an inadequacy in the reporting of parameters used in biological effects investigation (6). This makes it difficult or impossible to correlate the results arrong investigators. These deficiencies have been due to not only to the lack of recognition of the importance of characterizing ultrasonic fields but also the unavailability of commercial equipment with which adequate and reliable measurements can be made. The lack of available instrumentation is currently a major limitation in the evaluation of ultrasonic devices. Currently there is no portable commercially available equipment which is capable of measuring ultrasonic quantities from pulse echo diagnostic equipment. Only one U.S. manufacturer is marketing a measurement device to determine total power from ultrasonic therapy transducers.

The remainder of this paper will discuss some of the techniques that can be used to measure the output from ultrasonic medical devices. A comparison of measurements made by the Acoustics Branch, Bureau of Radiological Health and the National Bureau of standards are shown.

Radiation Force Techniques

The radiation force technique is currently the most widely used method for measuring ultrasonic power. It measures the force produced by an ultrasonic field impinging on a target. The force produced is independent of frequency and is proportional to the total time averaged ultrasonic power in the field. For a progressive plane wave impinging on a perfectly absorbing target the radiation force is equal to the acoustic power divided by the speed of sound. One advantage of this technique is that it is an absolute method that can easily be calibrated by the use of weights. For example, an acoustic power of 1 milliwatt exerts a force of 0.068 milligrams weight on a totally absorbing target. The system used by the
Bureau of Radiological Health to measure the output from diagnostic ultrasonic devices uses a sensitive micro-balance from which a target suspended in water is attached. This system and other similar systems have been reported in the literature $(7,8)$. Table 4 presents some measurements made of the output from some pulse-echo diagnostic ultrasonic transducers. This data as well as that reported by Carson indicates that the temporal and spatial average power levels from pulse echo diagnostic equipment is usually less than 10 milliwatts per sq/cm (2). These low ultrasonic levels also illustrate that very sensitive systems must be used to measure the output from such equipment. For a totally absorbing target $10 \mathrm{~mW}$ corresponds to a force of only 0.68 mg weight. Although such measurements can be made in a laboratory environment using an analytical micro-balance system, reliable commercial radiation force systems have not yet been developed and marketed for the measurement of these diagnostic levels. Out put levels from ultrasonic therapy equipment is usually in the range of 1 to 20 watts (5). The forces produced by these levels as a result of radiation pressure, are about 3 orders of magnitude larger, than those produced by diagnostic pulse-echo devices and are readily measured. Unfortunately the availability of commercial instruments to measure the output of ultrasonic therapy equipment is very limited. There is a definite need for the development of a portable measurement system that can be used to check the calibration of such equipment.

A technique which has been used for the measurement of radiation pressure involves the measurement of the deflection of a target suspended in a sound field in a pendulum arrangement. This system has been reported in the literature and is used in our laboratories for calibration purposes $(7,9)$.

\section{Thermal Detectors}

Thermal methods of measuring ultrasonic field quantities take advantage of temperatures that rise due to the absorption of acoustic energy in a suitable material. The most widely used type of calorimeter is called a substitution calorimeter. The ultrasound is directed into an absorbing medium and the subsequent temperature rise measured. The system is calibrated by dissipating a known amount of energy in the absorbing medium by means of a resistance heating coil and measuring the resulting temperature rise. If the thermal loses of the calorimeter are the same in both cases then a direct determination of absorbed 
ultrasonic energy can be obtained. This may also be considered an absolute measurement technique. Calorimeters provide a unique means for the measurement of ultrasonic energy since they are least effected by the ultrasonic field shape and pulse duration and are not dependent on plane wave assumptions. Such a system developed by the Bureau of Radiological Health has a dynamic range from about 10 milliwatts to 50 watts (7). This calorimetric system was used in making comparative measurements by the radiation force float and acousto-optic methods using National Bureau of Standards sources. The results of this intercomparison are shown in Table 5 and 6 .

\section{Electrical or Equivalent Circuit Method}

A method employed by the National Bureau of Standards for the determination of ultrasonic power is the evaluation of the parameters of the equivalent circuit model of resonant transducers under loading conditions (11). This method involves calculation of the resonant radiation conductance of quartz transducers from admittance measurements made with a twin-tee circuit of NBS design. (12). Measurements of susceptance and conductance were made at the terminals of the transducer under three conditions; unloaded (transducer in air), loaded (transducer in water), and effectively clamped (at approximately twice the resonant frequency). The data is treated to yield values of conductances G' (unloaded), and G (loaded), and G" (determined from measurements at $2 W$ ), and from these, the diameters, $D^{\prime}=G^{\prime}-G^{\prime \prime}$ and $D=G-G "$, of the circles of motional admittance of the transducer can be calculated. Accurate values of the resonant frequencies (unloaded and loaded) are also obtained. From the circle diameters the resonant radiation conductance is calculated, $G_{Y}=D\left(1-D / D^{\prime}\right)$. Related methods for determining efficiency have been discussed by others $(13,14,15)$.

The National Bureau of Standards has recently announced the availability of these sources for calibration of measurement equipment. Continuous wave output levels in the range of microwatts to 1 watt are available. An overall accuracy of $5 \%$ or better is claimed at frequencies of $1,2,3$ and 5 megahertz. The maximum beam diameter is 2.5 centimeters. These sources are transportable and may be taken to customers' facilities for calibration of their measurement equipment. The availability of these sources in addition to an ultrasonic calorimeter measurement system to measure ultrasonic power directly is a significant advance in the capability to calibrate and measure the output from medical ultrasonic equipment.

Piezoelectric Method

A parameter of importance other than total acoustic power is the distribution of the energy in the ultrasonic field, both spatially and temporally. To be able to follow variations of the energy in both time and space a method is needed with a fast response time and small spatial dimensions. An appropriate technique involves detecting an electrical output from a small piezoelectric crystal place in the ultrasonic field. The voltage output from such a crystal (i.e., hydrophone) is proportional to the acoustic pressure and may be squared to obtain a signal proportional to the acoustic intensity. An absolute rather than a relative measurement of intensity can be obtained by calibrating the hydrophone using one of the other absolute methods. By scanning a hydrophone across the beam the intensity distribution across the field can be found and plotted.

\section{OPTICAL METHODS}

Acousto-Optical Diffraction Techniques

The literature in the area of acoustooptical diffraction techniques is well developed, consisting of two general categor ies (16). Schlieren visualization and pressure measurement. The study of ultrasonic fields by means of optical methods has the advantage of providing a means of measurement while avoiding the disturbance of the ultrasonic field. One of the measurement systems used by the Bureau of Radiological Health employs the Raman-Nath theory of light diffraction in which it is assumed that the ultrasonic field presents a phase grating to a normally incident beam of monochomatic light $(17,18)$. The irradiance of each of the diffraction orders of the resulting Fraunhofer pattern can be used to calculate the acoustic pressure within the ultrasonic beam. One of the disadvantages of this system is that it requires a knowledge of the ultrasonic field size and shape and thus is more applicable for work with sources of known configuration. The results of comparative measurements made with the radiation force and calorimetric methods using National Bureau of Standards sources is shown in Tables 4 and 5. An extension of this method for measurement of pulsed ultrasonic fields is currently being investigated by the Bureau of Radiological Health and offers possibilities as a portable measurement system. Results using this method were izported at the recent 
meeting of the Acoustical Society of America (19).

\section{Interferometric Optical Method}

A newly developed optical method that detects the movement of a thin membrane in a sound field but that does not require prior information with regard to ultrasonic field pattern or size has recently been developed (20). This is a scanning optical interferometer and as used in our laboratory can provide either qualitative and quantitative information about the spatial distribution of particle displacement amplitude of fields emitted by diagnostic ultrasonic transducers. It promises to provide a convenient and rapid method for the evaluation of ultrasonic transducers. A discussion of this system and its application to the evaluation of medical ultrasonic transducers is given in another paper in this symposium (21) .

Results of Intercomparison Measurements

Comparative measurements taken with the radiation force, calorimetric and acousto-optic methods used by the Bureau of Radiological Health were made with two National Bureau of Standards ultrasonic transducers. The ultrasonic output power from each transducer was determined by NBS using measurements of the impedance of the generating ultrasonic transducers and the applied voltage. These predicted outputs were compared with values measured by the Bureau of Radiological Health.

Tables 4 and 5 present the data collected for measurements on two air-backed quartz crystals, each with a resonant frequency of $2 \mathrm{MHz}$. The quartz crystals were circular disks with active surface diameters of $1.905 \mathrm{~cm}(3 / 4 \mathrm{inch})$ and $1.270 \mathrm{~cm}$ ( $1 / 2 \mathrm{inch})$ The variations in the radiation force readings in Table 4 at an applied voltage of 203.0 volts have not been explained. Previous intercomparisons between the radiation force and acousto-optic techniques have shown no statistical difference between the two measurement methods at the 95\% confidence level (22). For the measurements reported in Table 5 using the acoustooptic system, the highest possible power generated by the $1 / 2^{\prime \prime}$ crystal was too low to allow the conditions necessary to precisely align the sampling light beam in the acoustic field. The result is that the optical data in Table 5 is not as reliable as is possible with the present system. Alignment was not a problem with the $3 / 4$ " crystal. The limits of error in the rms voltage applied to the ultrasonic transducer are believed to be $+1 \%$ of the voltage. The maximum intercomparison differences were within $+20 \%$ of the mean, and for most measurements the difference was much within $\pm 5 \%$

\section{CONCLUSIONS}

Methods exist that can provide reliable means of measurement of ultrasonic field parameters. However, at the present time there is almost no commercially available ultrasonic measurement instrumentation. In addition there is a lack of availability of a low cost transfer standard which can be used by non-technical personnel to calibrate such equipment. The problems in both of these areas should be corrected in the future. Some commercial companies are beginning the development of instrumentation to measure ultrasonic field parameters, and the National Bureau of Standards is working toward the development of transfer standard sources.

Some measurement areas not discussed in this paper needing work include, invivo dosimetry, calibration in relation to frequency content of ultrasonic pulses, finite amplitude wave effects and methods for the characterization of vibrators, such as used in dentistry or the surgical removal of cataracts.

\section{References}

1. Carson, P.I.; Acoustic Exposure Determination in Diagnostic Ultrasound Responsibilities of Researchers Studying Biological Effects of Ultrasound Proceedings of the Society of PhotoOptical Instrumentation Engineers, Vol. 47, Application of Optical Implication in Medicine III: 16-117, Aug. 1-2, 1974.

2. Carson, P.L.; Exposure Measurements on Diagnostic Ultrasound Instrumentation Presentation to the Ultrasound SubCommittee of the Obstetrical Gynecological Device Classification Panel (FDA) Jan. 22, 1976.

3. Rooney, J.A., and Nyborg, W.L.; Acoustic Radiation Pressure in a Traveling Plane Wave, AJP, Vol. 40, 1825-1830, Dec. 1972

4. Hueter, T.F., B.H.R.H. Sonics Sec. 2.12 Radiation Pressure, Wiley, N.Y. 1955, 43-53. 
5. Stewart, H.F. et al; Survey of Use and Performance of Ultrasonic Therapy Equipment in Pinnelas County, Florida, DHEW Publication (FDA) 73-8039, May 1973.

6. Woodward, B. and Allen, A.J.; The Importance of Calibration in Medical Ultrasonics, British Journal of Radiology, 47, 707-711, October 1974.

7. Stewart, H.F.; Ultrasonic Measuring Techniques, Seventh Rochester International Conference on Environmental Toxicity Fundamentals and Applied Aspects of Non-ionizing Radiation, June 5-7, 1974.

8. Rooney, J.A.; Determination of Acoustic Power Outputs in the Microwatt-Milliwatt Range, Ultrasound in Medicine and Biology, Vol. 1, 1-4, 1973.

9. Rooney, J.A.; Acoustic Radiation Pressure and its Use in Power and Intensity Determining .

10. Hasegawa T., Yosioka K.; Acoustic Radiation Force on a Solid Elastic Sphere, J. Acoustical Soc. America 46, 17391143 (1969).

11. Zapf, T.L.; Calibration of quartz Transducers as Ultrasonic Power Standards by an Electrical Method 1974 Ultrasonics Symposium Proceedings, IEEE Cat. No. 74, CHO896-isu Nov. 11-14, 1974.

12. Huntley, L.E.; A Self-Calibrating Instrument for Measuring Conductance at Radio Frequencies, J. Res. National Bureau of Standards, 696, No. 2 . pp. 115-126 (1965).

13. Bobber, R.J.; Underwater ElectroAcoustic Measurements, Naval Research Laboratory, washington, D.C. July 1970 pp. 90-99.

14. Hueter, T.F. and Bolt, R.H.; Sonics, John Wiley and Sons, N.Y. pp. 99-122, 1955.

15. Kinsler, L.E. and Frey, A.R.; Fundamentals of Acoustics, Second Addition, John Wiley and Sons, N.Y. pp. 360-364, 1962
16. Kline, W.R. and Cook, B.D.; Unified Approach to Ultrasonic Light Diffraction, IEEE Transactions Sonics and Ultrasonics, SU-14, 123-134 (1967).

17. Raman, C.D. and N.S.N. Nath; The Diffraction of Light by High Frequency Sound Waves, Parc Indian Academy of Science 3, p. 406 (1935); 2, p. 413 (1935); 3, P. 75 (1926); 3, p. 119 (1926); 3, p. 459 (1936).

18. Kline, W.R., Cook, B.D. and Mayer, W.G, Light Diffraction by Ultrasonic Gradings, Acoustica, 15, p. 67 (1965)

19. Cook, B.D.; Investigation in Measurement of Pulse Ultrasonic Waves by Optical Techniques, University of Houston Contract No. FDA 74-39.

20. Mezrich, R., E.T. Zold, K.F., Vilkomerson, D.; Ultrasonovision; Interferometric Means for Measurement in Vizuilization of Ultrasonic Wave Front Interactions with Biological Tissue, Proceedings of the Society of PhotoOptical Instrumentation Engineers, Vol. 47, 69-74, Aug. 1-2, 1974.

21. Haran, M.E. and Stewart, H.F.; Optical Interferometeric Measurements of Ultrasonic Radiation and its Application to Medicine. Presented at the National Bureau of Standards 75 th Anniversary Symposiums - Measurements for the Safe Use of Radiation, March $1-4,1976$.

22. Haran, M.E., Cook, B.D. and Stewart, H.F.; Comparison of an Acousto-optic and a Radiation Force Method of Measuring Ultrasonic Power, Journal of The Acoustical Society of America, 1975. 
Table 1. Ultrasonic Quantities, Symbols and SI Units

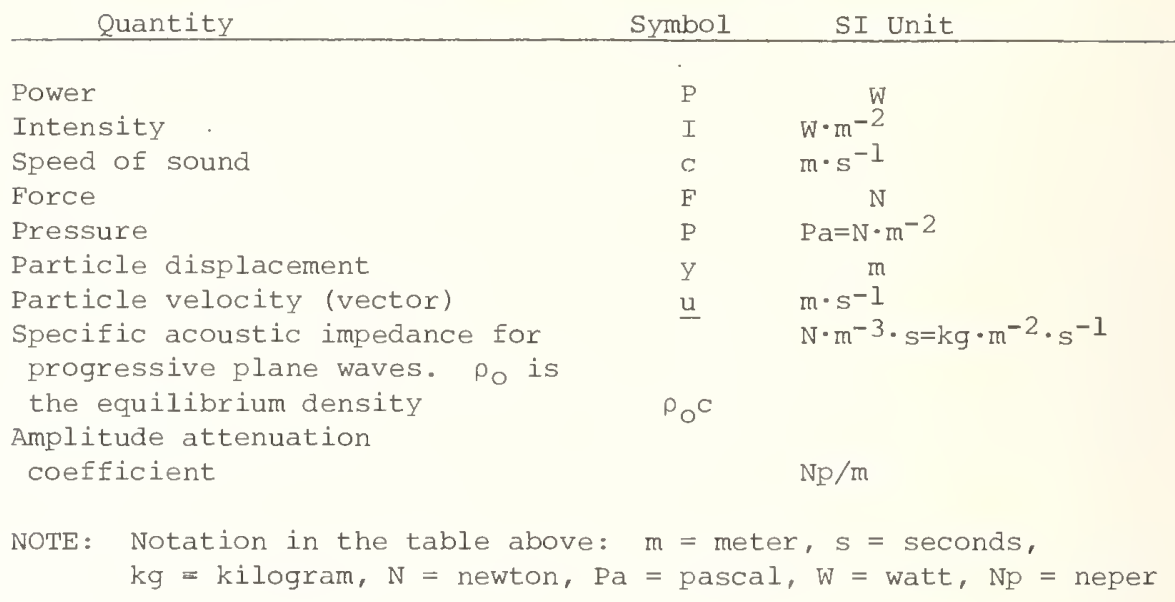

Table 2

Value of Constant D for Various Physical Situations

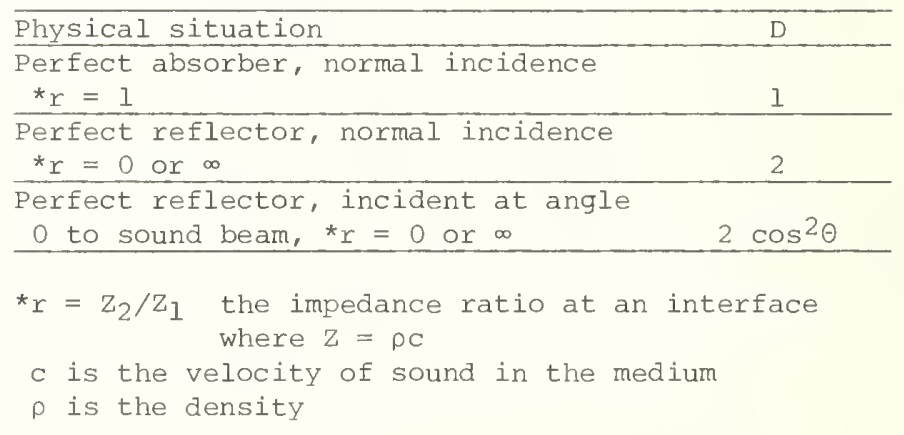

Table 3. Ultrasonic Quantities Measured by Different Techniques

Quantities Detected

$\begin{array}{lcr}\text { Techniques } & \text { Particle } & \text { Particle } \\ \text { Power Intensity Pressure Displacement Velocity }\end{array}$

\section{Calorimetry}

Thermal Probe

Optical

Diffraction

Radiation Force

(Total field)

Interferometric

Piezoelectric

Radiation Force

(Sphere deflection)

Equivalent Circuit

Reciprocity

$\mathrm{X}(1)$

NOTE: Notation in the above table: $\mathrm{x}=$ basic quantity measured 1 = time average only, 2 = temporal peak, 3 = peak pulse power 
Table 4

Measurements of Ultrasonic Output Use Radiation Eorce Method

\begin{tabular}{cccc}
$\begin{array}{c}\text { Generator } \\
\text { Manufacturer }\end{array}$ & $\begin{array}{c}\text { \# of Transducers } \\
\text { Measured }\end{array}$ & Range (mW) & Mean \\
\hline Picker & 57 & $<1$ to $13.6 *$ & 5.3 \\
Unirad & 2 & $<1$ & $<1$ \\
Smith Kline & 3 & $<1$ to 5.7 & 3.1
\end{tabular}

* Only two of the 57 measurements were above $10 \mathrm{mw}$. These two values were 10.5 and $13.6 \mathrm{mw}$.

Table 5. Comparative Measurements of Acoustic Power from 1/2" Quartz Transducer

Power (milliwatts) (See note b below)

\begin{tabular}{|c|c|c|c|c|}
\hline $\begin{array}{l}\text { Voltage } \\
\text { (volts) }\end{array}$ & $\begin{array}{c}\text { Electrical } \\
\text { Method } \\
\text { (NBS) } \\
\end{array}$ & $\begin{array}{c}\text { Rad. Force } \\
\text { Method } \\
\text { (BRH) }\end{array}$ & $\begin{array}{c}\text { Acousto- } \\
\text { Optics Method } \\
\text { (BRH) }\end{array}$ & $\begin{array}{c}\text { Calorimetric } \\
\text { Method } \\
\text { (BRH) }\end{array}$ \\
\hline $\begin{array}{l}203.0 \\
100.5 \\
30.26\end{array}$ & $\begin{array}{c}204(+0.4) \\
50.3(-1.9) \\
4.53(-4)\end{array}$ & $200(-1.6)$ & $\begin{array}{c}219(+7.7) \\
52.6(+2.5) \\
4.9(+4)\end{array}$ & $\begin{array}{r}190(-6.5) \\
51(-0.6)\end{array}$ \\
\hline \multicolumn{5}{|c|}{$\begin{array}{l}\text { a--rms voltage measured across the transducer. } \\
\text { b--numbers in ( ) indicate percent deviation from the mean } \\
\text { (measured-mean/mean). Where the mean is determined by averaging } \\
\text { the values obtained for all techniques at one voltage setting. }\end{array}$} \\
\hline
\end{tabular}

Table 6. Comparative Measurements of Acoustic Power from 3/4" Quartz Transducer

Power (milliwatts) (see note b below)

\begin{tabular}{lcccc} 
Vlectrical & Rad.Force & Acousto- & Calorimetric \\
Voltage & Method & Method & Optics Method & Method \\
(volts) & (NBS) & (BRH) & (BRH) & (BRH) \\
\hline
\end{tabular}

$\begin{array}{cclrl}232.6 & 603(-4.4) & 658(+4.4) & & \\ 203.0 & 459(-1.0) & 377(-18.7) & 479(+3.3) & 469(+1.1) \\ & & 535(+15.4) & & \\ 100.8 & 113(-0.5) & 111(-2.3) & 116.7(+2.8) \\ 50.54 & 28.5(-3.9) & & 30.8(+3.9) \\ 30.26 & 10.2(-3.8) & & 11.0(+3.8) & \end{array}$

a--rms voltage measured across the transducer

b--numbers in ( ) indicate percent deviation from the mean

(measured-mean/mean). Where the mean is determined by averaging

the values obtained for all techniques at one voltage setting. 

M. L. Swicord, H. I. Bassen and W. A. Herman

Electromagnetics Branch, Division of Electronic Products Bureau of Radiological Health, Food and Drug Administration 12721 Twinbrook Parkway

Rockville, Maryland 20852

A facility has been established for the evaluation and calibration of microwave power density instrumentation. It includes an anechoic chamber with a precisely positionable cart for measuring gain and chamber standing waves. The operable frequency range is from $900 \mathrm{MHz}$ to $10 \mathrm{GHz}$. A laser provides a means of aligning antennas. The absolute calibration of distance and microwave power are performed by NBS. The radiated electric field strength is then established by BRH as a primary standard using precise antenna gain measurement methods. An instrument evaluation facility includes an absorber-Iined temperature chamber, a probe/slotsource positioning system, and devices for measuring the polarization response, modulation response, and the linearity of the microwave survey instruments.

(Microwave Power Density; Microwave Radiation Measurement)

\section{Introduction}

Since the passage of the Radiation Control for Health and Safety Act of 1968 and the subsequent promulgation of the Microwave Oven Standard under that Act, the Bureau of Radiological Health has found it necessary to establish means of thoroughly evaluating and precisely calibrating microwave radiation instrumentation. These instruments are used in a wide range of programs including the enforcement of the Microwave Oven Standard, radiation hazard assessments, and radiation measurements performed on other electronic products such as microwave diathermy. This paper describes the facilities and methods established and used for these purposes.

\section{Plane Wave Calibration Facility, Physical Characteristics}

The main element of the calibration facility is an anechoic chamber. The chamber has approximate external dimensions of $3.6 \times 3.6 \times 9$ meters. This provides a usable interior longitudinal axis of 7 meters. A fiberglass cart, which is also partially covered with anechoic material, can be moved along the longitudinal axis of the chamber and remotely positioned to millimeter accuracy. The fiberglass cart rides on two stainless steel rails located below the anechoic floor material of the chamber. The rails are located off center so that no specular reflections can occur.
A chain drive parallels each rail and is connected to a motor outside the chamber.

A microwave oven survey probe to be calibrated is placed in a dielectric holder which, in turn, is mounted on the cart (Fig. 1). The probe is extended in front of the cart as far as possible on a dielectric rod. The readout device for the probe is then placed on the cart, behind anechoic material. The probe is irradiated by a truncated horn antenna. Standard gain horns are not used due to increased problems and uncertainties which occur when horn gains are experimentally determined in the Fresnel region or even the "pre-Fresnel" region of an antenna. A high power, rigid craxial transmission line is used as a forward power monitor with integral direction coupler between generator and antenna. While the probe is exposed to a precisely known plane wave, the meter readout indication is observed with a closed circuit TV camera utilizing a telephoto lens. The rear wall is covered with a high performance pyramidal absorbing material. Ports in the rear wall are provided for a camera, a spotlight and a laser. The split beam laser is used for the alignment of transmitting and receiving antennas with the central longitudinal axis of the chamber, and with the cart's rail system during the antenna gain measurement procedure. Transmitting antennas can be centered and aligned by placing a precisely machined, teflon-coated, insert containing a first-surface mirror in the waveguide throat of the transmitting horn as shown in figure 2. The reference laser beam is reflected 


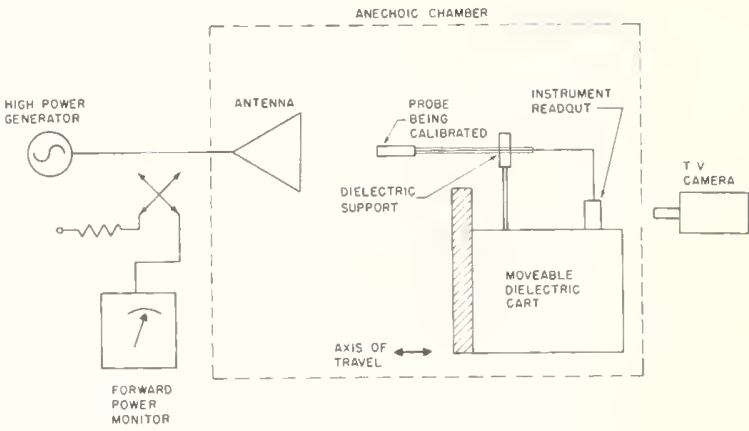

Figure 1: Probe Calibration in "Plane Wave" Facility

Figure 2: Horn Antenna with Alignment Mirror Inserted 
from this mirror and serves as a means for providing antenna alignment to better than one degree. This beam is also used for alignment of the receiving antenna on the cart. Cart wobble is minimized by alignment of the rails to eliminate lateral and vertical cart position variations during its trave1.

In the front, or transmitting end of the chamber, a connection is made from the microwave generator to the rigid coaxial feed system through high power flexible transmission line. Relative forward power is read on the coupler's side arm digital power meter. A single meter reading determines the absolute radiated power through precise "power equation" techniques developed by Dr. Glenn Engen of the National Bureau of Standards ${ }^{l}$. A description of this specific application has been published by the Bureau of Radiological Health ${ }^{2}$. An equipment console provides control signals to the cart and displays accurate position data. A shaft encoder is used to provide a digital cart position readout accurate to \pm 2 millimeters (calibrated against an NBScalibrated steel tape).

\section{Method of Generating a Precisely Known Plane Wave Power Density}

The procedure for establishing a calibrated plane wave field is as follows. The standing waves in the chamber are measured by mounting a probe, of the type to be calibrated, on the cart. The cart is then driven the entire length of the chamber and the output of the receiving probe vs. distance is recorded, while a constant power level is transmitted by a horn antenna. From this procedure, a relative "quiet zone" can be determined near the center of the chamber, for a particular frequency. Next, the horn gain is measured in this quiet zone via continuous path loss measurements between pairs of nominally identical horn antennas. The Power Equation methods are most appropriate for determining net power transfer (path 1oss) between transmitting and receiving antennas, since mismatches between these antennas and their respective source or load are precisely accounted for.

Antenna gain is then computed as a function of path loss between a pair of nominally identical antennas by the formula:

$$
G_{T} G_{R}=\frac{P_{R}}{P_{T}} \quad \frac{(4 \pi R)^{2}}{\lambda}
$$

where: $\mathrm{G}_{\mathrm{T}}=$ transmitting horn gain, $\mathrm{G}_{\mathrm{R}}=$ receiving antenna gain, $R$ = antenna separation distance at the aperture planes, $\lambda=$ wavelength of transmitted signals, and $\mathrm{P}_{\mathrm{R}} / \mathrm{P}_{\mathrm{T}}=$ received power/transmitted power ration (path loss).

Equation (1) assumes that no multipath reflections are received from chamber sidewalls or the cart and its supports and that no mutual reflections may exist between antennas. These conditions are met by providing a high performance anechoic chamber, proper antenna separation, and sufficient antenna directivity or gain. The gains $G_{T}$ and $G_{R}$ are a function of separation distance and approach a constant "far field" value at large separations.

Errors in path loss measurements due to antenna skin currents (side lobes), and leaking cable connections must be experimentally detected and minimized. Skin currents flowing on the outside of the horn antenna and on microwave feed cables can give rise to radiation side lobes. Since the method of antenna gain measurement used assumes that reciprocity exists, the gain of an individual antenna must be unaffected by its mounting structure and surroundings. These side lobes may be reflected to the opposite antenna by the materials surrounding the antenna (i.e. absorber behind the transmitting antenna, the cart on which the receiving antenna is supported, or the cables feeding each horn). Their effects are detected and minimized. No significant changes $(<0.1 \mathrm{~dB})$ in path loss due to sidelobes occur for any of the measurements in the final BRH calibration system.

The use of three pairs of antennas (with a total of three antennas utilized) allows the determination of the gain of a single antenna. This gain is position dependent, since the measurements may not be made in the true far field, especially at $915 \mathrm{MHz}$. The antenna separation is sufficient however, so that little spatial variation (less than 5 percent) occurs, over the aperture of the receiving antenna under worst case conditions. This implies that a small probe will observe the same power density as the receiving, antenna with its much larger effective aperture.

The calibration of absolute transmitted power from the horn antenna is accomplished via a special high-power coupler/bolometer (calibrated by NBS). This coupler/bolometer has a waveguide input flange of the same dimensions as the waveguide horn antennas 
(Fig. 3). To calibrate the "forward power monitor" which consists of a directional coupler, permanently mounted on the rigid coaxial feed system, the transmitting antenna is removed and the calibrated coupler/bolometer is substituted as a termination. The readings of the digital power meter on the forward power monitor are simultaneously compared with the substituted D.C. power supplied to the bolometer as measured by a precision bridge. The net transmitted power is absolutely calibrated using specialized techniques and the equipment illustrated in figure 4. The forward power monitor reading is related to transmitted power by leveling power $\mathrm{P}_{4}$, during all measurements. Waveguide offset shorts are used as references to provide accurate matching factor measurements.

Absolute power density is then computed at a point in the previously determined "quiet zone" of the chamber where both quasi-far field conditions and minimal standing waves (as seen by a small isotropic probe) exist. Power density is computed as:

$$
S=\frac{P_{T} G_{T}(R)}{4 \pi R^{2}}
$$

where: $\mathrm{S}=$ power density, $\mathrm{P}_{\mathrm{T}}=$ absolute transmitted power, $G_{T}(R)=$ transmitting horn gain (a function of distance) and, $\mathrm{R}=$ distance from the horn to the point where power density is computed. The standing waves (due to chamber wall reflections) which are seen by an isotropic probe under calibration must be accounted for to remove residual probe calibration errors. Then the process of calibrating the plane-wave

facility is complete. A worst-case absolute power density uncertainty of less than \pm 0.6 $\mathrm{dB}$ at $2450 \mathrm{MHz}$ and $\pm 0.8 \mathrm{~dB}$ at $915 \mathrm{MHz}$ are readily achieved using a very conservative error analysis.

\section{Probe Calibration Procedures in the Plane Wave Facility}

When calibrating a probe in the known plane-wave field, precautions must be taken to avoid additional errors due to

reflections from the dielectric cart, the probe support structure, and the instrument's cable and readout meter. Reflections from the cart are measured and reduced to acceptable levels $( \pm 0.2 \mathrm{~dB}$ at $2450 \mathrm{MHz}$ and $\pm 0.5 \mathrm{~dB}$ at $915 \mathrm{MHz}$ ). A large amount of additional scattering from the cable connecting the probe to the readout meter was detected. Shielding of the cable with absorber was necessary over portions where it was oriented vertically (aligned with the electric field). Even with this absorber, significant residual scattering occurs, particularly at the lower

frequencies. To eliminate the residual scattering errors a "multi-position averaging technique" was devised (Fig. 5). These standing waves had a known period of one-half wavelength. Therefore, several calibrations were performed on each probe, with the probe mounted ahead of the cart at various spacings. The spacing of the probe's sensor tip to the cart (D2) was varied in quarter wave increments for each calibration, while the distance from the probe's sensor to the transmitting horn (D1) was maintained at a constant value. An average of four such readings was computed. Cart and support reflection/errors are thus reduced to a value below $0.05 \mathrm{~dB}$ ( 1 percent) by this multi-position averaging technique.

Whenever the separation between two antennas is not great, compared to the parameter $\left(a^{2} / \lambda\right)$, where "a" is the maximum dimension of the transmitting horn, some amplitude and phase variations exist in the plane of the receiving antenna aperture used during gain measurement procedures. Spatial averaging of these fields occurs since the receiving antenna aperture is large. In contrast, a small antenna such as a radiation hazard meter, will see only the maximum field intensity if it is on the boresight of the transmitting antenna. At $2450 \mathrm{MHz}$ separations used exceeded $20 \mathrm{a}^{2} / \lambda$. At $915 \mathrm{MHz}$, since separations of $6.5 \mathrm{a}^{2} / \lambda$ were used, the spatial averaging error was experimentally evaluated by continuously scanning planes normal to the axis of propagation, with a small probe. Numerical integration was performed over a rectangular area with dimensions identical to the receiving horn aperture. The integrated power density over the aperture space differed from the central point value of power density by less than 5 percent. Because the effective aperture of the receiving antenna is much smaller than its physical aperture, this is a worst-case test of non-uniformity of the "plane wave" used to calibrate probes.

\section{Instrument Evaluation}

After calibrating an instrument in a known plane wave field, the response of that instrument to various field parameters existing during the measurement of a leaking microwave oven or other near-field source, must be evaluated. The ambient temperature, 


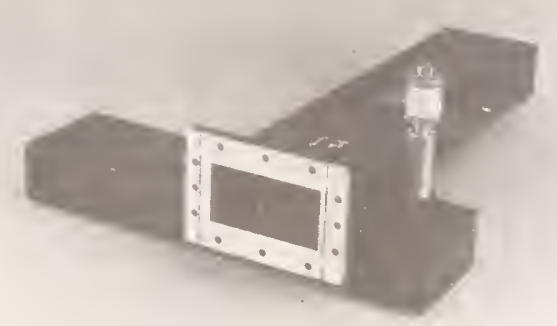

Figure 3: NBS-Calibrated Coupler-Bolometer

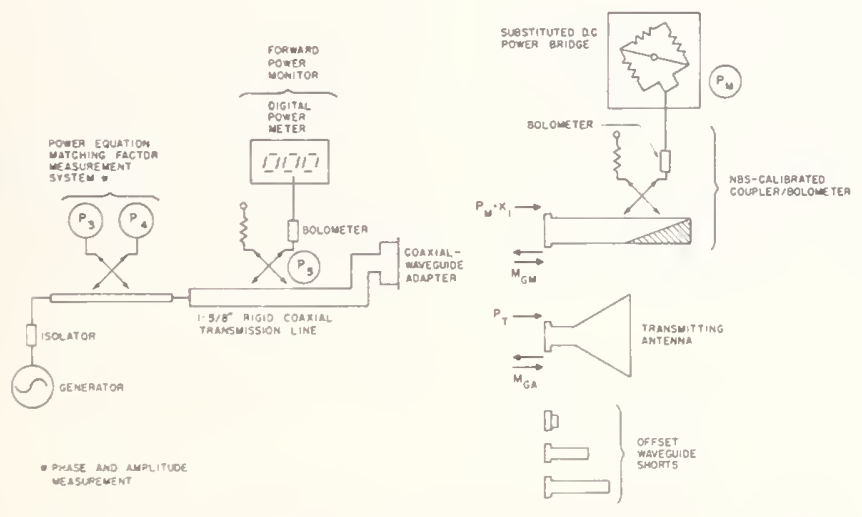

Figure 4: Technique for Forward Power Monitor Calibration

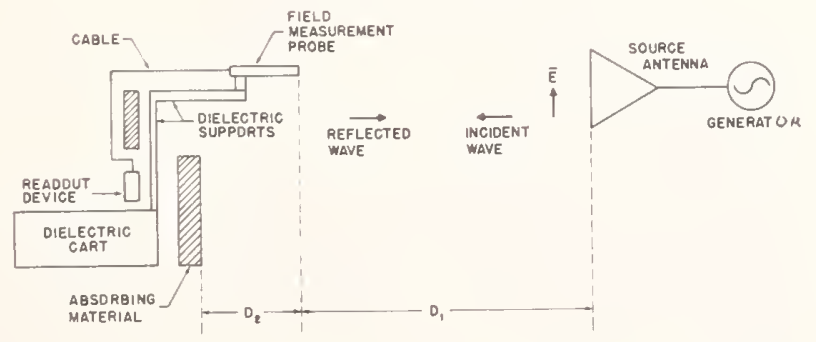

Figure 5: Mutti-Position Averaging Technique 
the polarization of the electric field, its amplitude modulation, and other parameters must be simulated and the instrument's response noted. The test apparatus shown in figure 6 provides a means for some of this evaluation. More absorber is used in actual practice than is shown. A probe, supported by a dielectric holder, is positioned in front of a slot source. The holder is mounted on rails as shown. The variation of probe response, as a function of distance from the slot source, can be observed and standing waves occurring in the system may be minimized by adding microwave absorber. The "near" vs. "far field" responses of various probes may be compared by moving them toward the slot source from the rear of the apparatus. A probe can be rotated to obtain the angular response (antenna pattern). During this rotation, the center of the receiving sensors of the probe is placed on the axis of rotation. A stable 20 watt source is used to provide field strengths of appropriate magnitude. Power to the system is leveled and a modulator, driven by a "function" or a pulse generator, allows complete control of the amplitude modulation impressed upon the radiated fields. This allows the evaluation of instrument linearity vs. field modulation while constant average power is maintained. The average power level may also be adjusted to determine the instrument linearity for $\mathrm{CW}$ radiation of varying amplitude.

Another system is used for determining the polarization response of a probe, i.e., the response to a linearly polarized E field as the probe is rotated about its handle axis (Fig. 7). Leveled power is fed to an absorber-lined box from an open-ended waveguide, while the probe body is supported by a plastic holder. The sensor is maintained at a constant distance from the waveguide. Rotation of the probe handle allows one to observe the polarization response or "polarization ellipticity" of the instrument.

A specially designed temperature response evaluation system has been developed. It consists of an absorber-lined environmental chamber ( $60 \mathrm{~cm}$ cubicle) capable of temperature variations from $-20^{\circ}$ $\mathrm{C}$ to $+50^{\circ} \mathrm{C}$ using liquid nitrogen cooling and electrical heating. The instrument under test is placed in a plastic holder at a fixed distance from a slot source (Fig. 8). The fields near the slot have been separately tested for temperature independence. The variation of relative probe response may be observed while constant net power is fed to the slot source at different ambient temperatures. The entire instrument, including readout, can placed in the chamber, with the instrument readout viewed through a small plastic window in the side of the chamber.

A final evaluation procedure must be performed to determine the radiofrequency interference (RFI) susceptibility of the probe body, cable, and readout meter to strong fields at frequencies which the microwave oven or other sources to be surveyed, operate. The sensor tip of the probe is totally enclosed in metal foil. The probe body, readout meter and cable (which is aligned with the electric field) are irradiated in the plane wave calibration facility. The response of the readout meter is noted and compared to the reading obtained with the sensor tip unshielded. Many instruments fail this test, due to faulty RFI gaskets in readout units, or insufficient filtering in the readout or probe body.

\section{Acknowledgement}

Much of the electronic and mechanical design for the various systems was performed by Mr. J. Duff and Mr. J. Bing. Many of the measurements involved in the various evaliation procedures were performed by Mssrs. D. Witters and J. Duff. Theoretjcal analysis of field curvature and other antenna gain considerations were performed by Mr. S. Neuder.

\section{References}

1. Engen, G. "Power Equations: A New Concept in the Description and Evaluation of Microwave Systems." IEEE Transactions on Instrumentation and Measurement Vol IM 20, No. 1, Feb. 1971.

2. Herman, W. and H. Bassen. "Precise Microwave Power Density Calibration Method Using the Power Equation Techniques." Dept. of Health, Education, and Welfare publication FDA 75-8028, March 1975. 


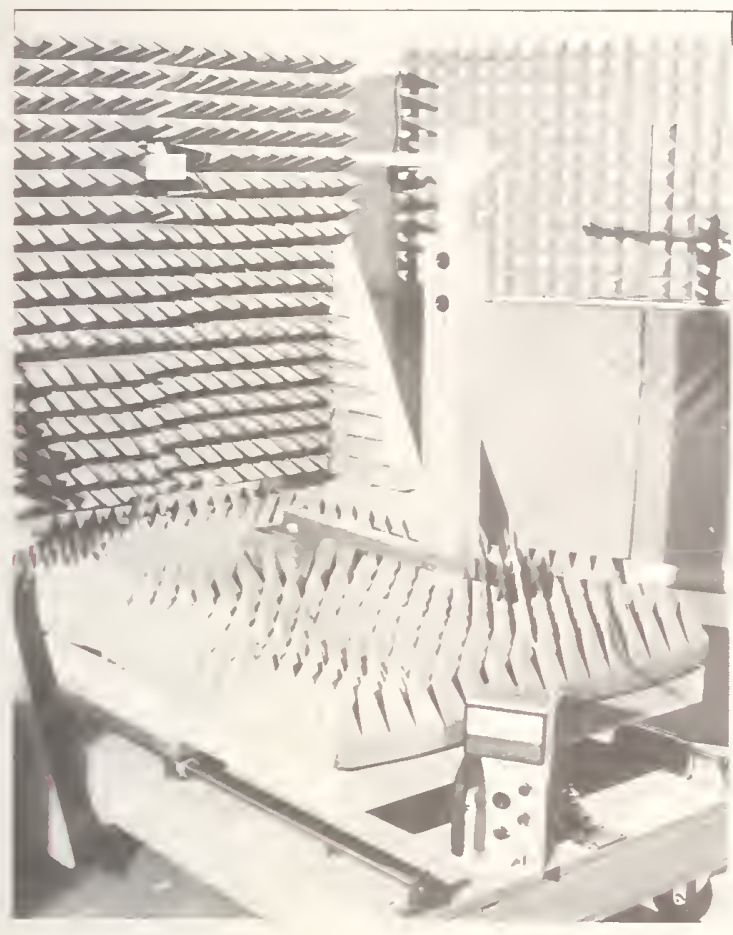

Figure 6: Probe Evaluation Test Apparatus

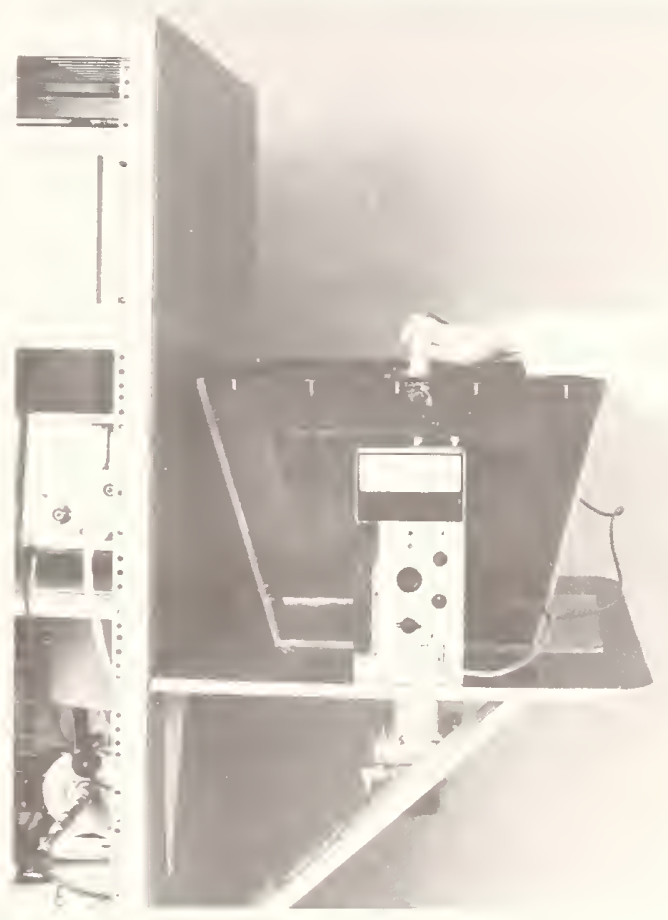

Figure 7: Probe Polarization Test System

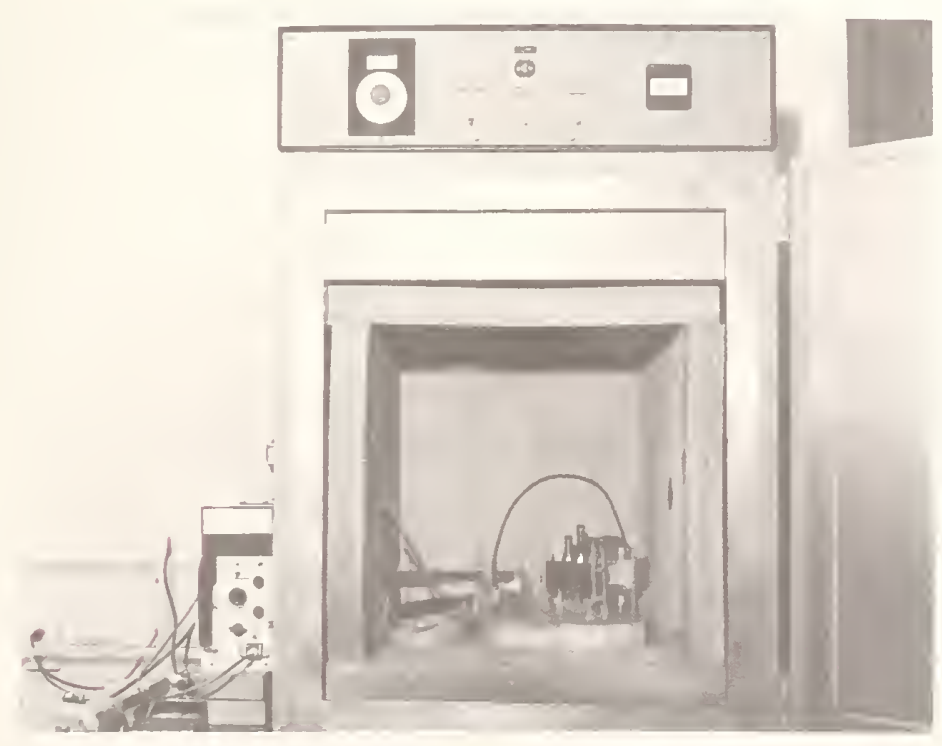

Figure 8: Probe Temperature Response

Evaluation System 



\section{NBS ULTRAVIOLET RADIOMETRIC STANDARDS}

$$
\text { W. R. Ott }
$$

National Bureau of Standards Washington, D.C. 20234

A brief review of the standard sources and detectors available as services from NBS will be given. Emphasis will be on the use of such standards to calibrate the radiant power of unknown sources or the response of radiation detectors and spectral radiometers at wavelengths less than $400 \mathrm{~nm}$. The following standards, listed in order of decreasing wavelength, are or will soon be available: the tungsten filament quartz-halogen lamp (above $250 \mathrm{~nm}$ ); the tungsten strip lamp (above $225 \mathrm{~nm}$ ); the low pressure mercury vapor lamp $(253.7 \mathrm{~nm})$; the deuterium arc lamp (165 to $350 \mathrm{~nm}$ ); the argon "mini$\operatorname{arc}^{\prime \prime}$ (115 to $400 \mathrm{~nm}$ ); photodiode detectors (20 to $254 \mathrm{~nm}$ ); and the synchrotron radiation source, SURF-II (5 to $400 \mathrm{~nm}$ ). The relative strengths and limitations of these radiometric standards with respect to accuracy, reliability, convenience, and intensity and wavelength range will be discussed.

(IIltraviolet, Radiometry, Standards, NBS, Sources, Detectors, Survey)

\section{Introduction}

In the wavelength region between 5-400 $\mathrm{nm}$, NBS offers a variety of standard sources and detectors which may be used to calibrate the radiant power of unknown sources or the response of radiation detectors and spectral radiometers. A brief review of the available services will be given and in addition several current projects which are meant to improve the accuracy, performance and variety of ultraviolet radiometric standards will be mentioned.

The ultraviolet wavelength region can be considered to extend from $400 \mathrm{~nm}$, the near ultraviolet, to about $4 \mathrm{~nm}$ where the soft $\mathrm{x}$-ray region begins. For perspective some key wavelengths in this region are illustrated in figure 1 . The critical atmospheric ozone absorption occurs between 200-280 $\mathrm{nm}$, the so-called UVC region. The UVA and UVB regions define the regions 315-400 nm and 280-315 nm respectively. Molecular oxygen absorption characterizes the region below $200 \mathrm{~nm}$ and thus the terminology "vacuum ultraviolet" to indicate the necessity for the absence of air in a measurement system designed to detect or utilize such radiation. Atomic hydrogen absorption sets in at $100 \mathrm{~nm}$ and sets a limit to the range of interstellar astrophysical measurements. However, the real importance of the ultraviolet wavelength region is contained in the energy scale corresponding to the wavelength scale in figure 1. The ultraviolet energy range spans from about $3 \mathrm{eV}$ to $300 \mathrm{eV}$, which is quite large as compared to the visible, for example, which extends only from $1.5 \mathrm{eV}$

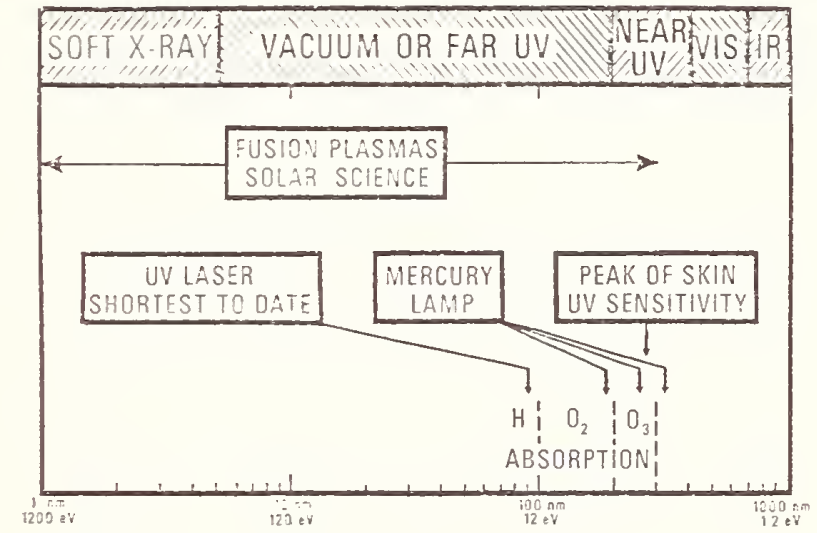

Figure 1. The ultraviolet region of the electromagnetic spectrum defined in terms of wavelength ( $\mathrm{nm})$, energy $(\mathrm{eV})$ and some key processes.

to $3 \mathrm{eV}$. This energetic radiation can be used to drive and control all sorts of chemical and biological reactions. For example extreme doses can cause skin cancer; controlled doses can heal. Continuous exposure to ultraviolet radiation causes the fading of paints and dyes; controlled exposures in specific processes are used to produce protective coatings on materials. Some documented examples of the various applications of ultraviolet radiation as both a natural and artificial element in our home and work environment are shown in Table 1 . 


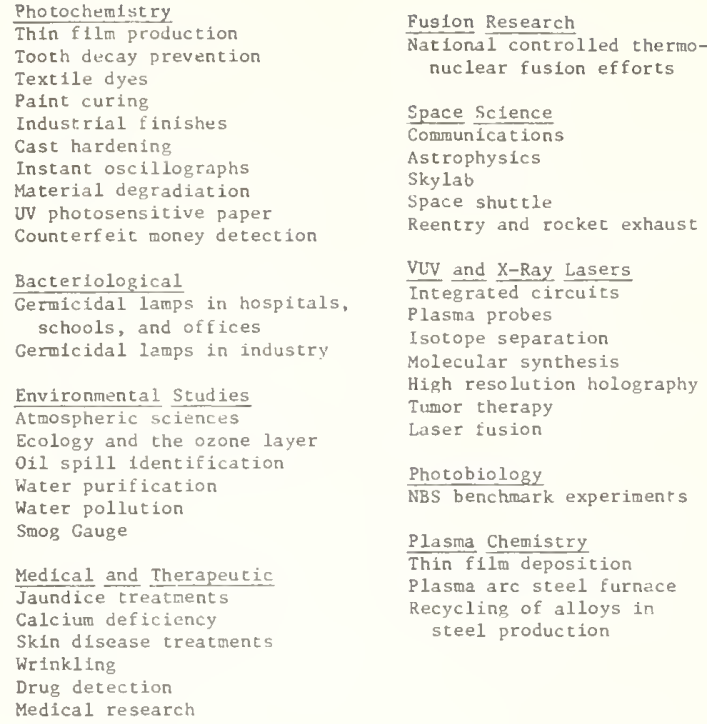

Table 1. List of applications requiring ultraviolet radiation measurements.

Why are accurate radiant power measurements needed in these areas and how does NBS interact with the measurement system? Basically, in three ways: (a) on the research leve1--new techniques require accurate documentation for the process or procedure to gain widespread commercial acceptance; (b) on the sales level--commercially available products need standards in order to meet specifications and to compete on a national and international market; and (c) on the applications level--the user needs standards in order to check specifications, to maintain quality control, and to monitor the health and safety of workers. As an example of these impacts, consider an industrial assembly line process in which certain manufactured items like paper products, glass, metal parts, microcircuits, or textiles are being coated by radiation curing. (a) For the uv radiation curing technique to be accepted in the first place, it would have to be well understood so that performance could be guaranteed. (b) The equipment which would enable the assembly Iine to operate at the highest speed is the most desirable (maximum ultraviolet flux in the most effective bandpass). (c) Periodic calibrations in the field are necessary to accurately monitor the intensity of bands of uv radiation hazardous to employees and to measure possible deterioration of the equipment due to radiation damage of the optical components.

\section{Radiometric Quantities}

In general, there are two ways to determine the radiant power of an unknown light source: (a) through the use of standard sources and (b) through the use of standard detectors. Standard sources are most useful when it is desired to know the emission characteristics of an unknown source. Standard detectors are most useful, on the other hand, when it is desired to know the radiant power at the location of a detector. For example, if the quantity of interest is spectral radiance [watts $\mathrm{cm}^{-2} \mathrm{~nm}^{-1} \mathrm{sr}^{-1}$ ], that is, if one is concerned with the power zadiated by a specified emitting surface $\left(\mathrm{cm}^{2}\right)$ in a certain wavelength band ( $\mathrm{nm}$ ) at a given solid angle (sr), then the light source to be investigated as well as the standard source may be set up in such a manner that the radiation from both passes through the same optical-spectrometric arrangement, thereby eliminating all geometric and other specific factors of the instrumentation. In short, the calibration is effected by a simple substitution of sources in the experimental arrangement. On the other hand, if the quantity of interest is spectral irradiance [watts $\left.\mathrm{cm}^{-2} \mathrm{~nm}^{-1}\right]$, that is if one is concerned with the radiant power incident on a specified surface area $\left(\mathrm{cm}^{2}\right)$ in a certain wavelength band ( $\mathrm{nm})$, then ideally a standard detector and filter arrangement is placed at the location of the irradiated surface to measure the power. Alternatively, if such a standard radiometer is not available, a standard source of spectral irradiance may be used in conjunction with a suitable diffusing element (to account for variations in the geometries of the standard and the unknown sources) to determine the response function of the user's diffuser-radiometer system.

At NBS, standard sources of both ultraviolet spectral radiance and spectral irinadiance are available. In the case of NBS standard detectors for the ultraviolet, the calibrated quantity is the absolute quantum efficiency [photoelectrons per incident photon] as a function of wavelength.

\section{Standard Sources}

The three primary source standards being used at NBS are the gold point blackbody cavity for which the spectral radiance is given by Planck's law, the wall-stabilized hydrogen arc for which the spectral radiance is given by accurately 
known quantum mechanical absorption coefIicients for atomic hydrogen, and the electron storage ring facility for which the spectral radiance is given by the theory of electron synchrotron radiation. The selection of one of these standards for a calibration application is influenced mostly by the specified wavelength region.

The primary standards are most often used to calibrate secondary or transfer standards of spectral radiance which can then be issued to customers. The secondary radiance standards are also used to generate spectral irradiance standards. The following ultraviolet standard sources, listed in order of decreasing wavelength, are available from NBS: the tungsten filament quartz-halogen lamp (above $250 \mathrm{~nm}$ ); the tungsten strip lamp (above $225 \mathrm{~nm}$ ); the low pressure mercury vapor lamp (253.7 nm); the deuterium arc lamp (165 to $350 \mathrm{~nm}$ ); the argon "mini-arc" (115 to $400 \mathrm{~nm}$ ); and the synchrotron radiation source, SURF-II (5 to $400 \mathrm{~nm})$. The relative strengths and 1imitations of these radiometric standards with respect to accuracy, reliability, convenience, and intensity and wavelength range will now be discussed.

\section{(1) Tungsten Lamps}

Tungsten strip lamps are used as transfer standards of spectral radiance, and tungsten quartz-halogen lamps are used as transfer standards of spectral irradiance. The tungsten resistive element is heated to incandescence by a specified dc electric current. Power requirements are about $8 \mathrm{~A}$ (a) $110 \mathrm{~V}$ for the irradiance standard and about 50 A @ $15 \mathrm{~V}$ for the radiance standard. Between 400 and $250 \mathrm{~nm}$, the spectral distribution of the quartz-halogen lamp and the tungsten strip lamp is approximately that of a $3000 \mathrm{~K}$ and $2700 \mathrm{~K}$ blackbody respective$1 y$.

Strengths: Techniques are well understood and calibrations based upon the conventional blackbody cavity are possible with uncertainties on the order of 1-3 percent.

Limitations: Relatively weak sources in the ultraviolet, especially below $250 \mathrm{~nm}$ where the application of these sources is limited by both low signal levels and significant scattered visible light. For example, the spectral radiance of a tungsten strip lamp at $230 \mathrm{~nm}$ is about 1000 times lower than it is at $400 \mathrm{~nm}$.

\section{(2) Low Pressure Mercury Vapor Lamps}

The irradiance of the $253.7 \mathrm{~nm}$ resonance line of mercury can be measured for various types of low pressure lamps supplied by the customer. The approximate uncertainty is $5 \%$ for irradiance levels on the order of 0.5 to $30 \mu \mathrm{W} \mathrm{cm} \mathrm{cm}^{-2}$.

Strengths: Calibration technique utilizes quartz-halogen spectral irradiance standard and can be extended to other line sources in the region above $250 \mathrm{~nm}$.

Limitations: The calibration of the customer's lamp is only as good as its stability and reproducibility.

\section{(3) Deuterium Arc Lamps}

These are available as transfer standards of spectral radiance (165-350 nm) and irradiance (200-350 nm) with calibration uncertainties of about $10 \%$. A short Lshaped arc discharge is formed between two electrodes set at $90^{\circ}$ in the sealed lamp and excites a molecular $D_{2}$ continum. The low pressure deuterium lamp spectrum peaks at about $200 \mathrm{~nm}$ where the spectral irradiance of a typical $30 \mathrm{~W}$ lamp is about 100 times greater than that of a $1000 \mathrm{~W}$ quartzhalogen lamp. Calibrations are based upon the hydrogen arc primary standard of spectral radiance. Irradiance calibrations have just recently become available down to $200 \mathrm{~nm}$. Plans are to extend this service down to $165 \mathrm{~nm}$.

Strengths: Very strong ultraviolet output, portable, low power requirements. Limitation: Alignment is difficult, and there are uncertain long-term aging effects.

\section{(4) Argon Mini-Arc}

The mini-arc is a one atm pressure wa11-stabilized arc discharge operated at power levels up to $1.5 \mathrm{~kW}$ depending upon the intensity desired. The spectrum is line-free between 194 and $350 \mathrm{~nm}$ and interrupted by only a few very narrow air impurity lines between 114 and $194 \mathrm{~nm}$. The spectral radiance of the argon continuum is determined in the region $114-350 \mathrm{~nm}$ by comparison with the hydrogen arc primary standard. The mini-arc has just recently become available. Future plans are to determine its suitability as a far ultraviolet spectral irradiance standard.

Strengths: Continuum is intense (a factor 1000 stronger than the tungsten strip lamp at $250 \mathrm{~nm}$ ), lamp aging is negligible, the uv wavelength coverage is the most extensive of all transfer lamps, 
and the alignment procedure is more reliable than for the deuterium lamp.

Limitations: The lamp system and ignition procedure are slightly more complicated than those previously described; not commercially available.

(5) Synchrotron Ultraviolet Radiation Facility (SURF-II).

The theory of synchrotron radiation from accelerated electrons is on excellent theoretical grounds, and the continuum enission can be quantitatively predicted when the energy of the electrons, the radius of the orbiting electrons, and the electron current are known. The radiation beam from the NBS $240 \mathrm{MeV}$ storage ring is highly collimated, polarized, and intense, on the order of $10^{11}$ photons $\mathrm{s}^{-1} \mathrm{~nm} \mathbf{m r a d}^{-1}$ in the wavelength range $10-400 \mathrm{~nm}$. Uncertainties in the flux determination are expected to be in the 5 to $10 \%$ range. There are no plans at present to calibrate transfer sources with the synchrotron radiation, but rather guests are invited to use the facility for their own purposes. Efforts are currently being made to organize and streamine the procedures so that such a collaboration can be carried out most efficient $1 y$.

Strengths: An intense source of continuum radiation which covers the entire visible and ultraviolet region. Limitation: Major facility, high vacuum requirements, guest worker arrangements must be made.

\section{Standard Detectors}

The primary standard for detector calibrations at NBS is a double-ionization chamber, a gas-filled detector in which each photon absorbed produces one electronion pair which is collected by a simple arrangement of parallel plates used to set up the collecting field. The transfer to the actual photodiodes which are available to customers is accomplished through the use of a uniformly grey (percent of radiation absorbed is independent of wavelength) thermopile whose efficiency has been calibrated with the ionization chamber at short wavelengths and checked with a spectral irradiance standard source at $253.7 \mathrm{~nm}$. Windowed photodiodes calibrated in this manner have been used to evaluate the response of other detectors such as photomultipliers, and the response of radiometers such as "hazard meters" which are designed to have a spectral response equivalent to a specified erythemal curve.

\section{(1) Window Photodiodes}

This transfer standard is basically a vacuum photodiode in which ideally the only parameter to be determined by reference to an absolute standard is the absolute photoelectric yield of the photocathode as a function of wavelength. The detectors are evacuated and sealed by an ultraviolet transmitting window to avoid atmospheric contamination of the surfaces. Calibration uncertainties are estimated to be about $6 \%$. The uniformity of the quantum efficiency of the cathode can be excellent, on the order of 1 percent. Aging effects are likewise negligible.

\section{(2) Windowless Photodiodes}

Basically this is the same type of detector as described above, but the lack of a window and the consequent occasional exposure of the photocathode to laboratory conditions affects the overall reproducibility. Nevertheless, there is evidence that natural aging effects cause not more than a 20 percent change in quantum efficiency over a three year test period. Strengths of (Z) and (2): Wide wavelength region of applicability (20 nm to $300 \mathrm{~nm}$ ); relatively high accuracy and stability.

Limitations of (2) and (2): These are low current devices (no amplification). If they are used to calibrate more sensitive photomultipliers, linearity and scaling problems with the latter introduce additional uncertainties.

\section{Summary}

Ultraviolet standard sources and detectors are available from NBS with uncertainties in the range of $3-10 \%$ depending upon the type of calibration required and the wavelength region of interest. In certain cases, arrangements can be made to use NBS radiometric facilities; collaborative efforts are also possible. Requests for services should either be directed to the appropriate sections $(232.03,232.04,232.07$, or 232.14) or, in general. to the NBS office of Measurement Services.

Part of the research described here was supported jy the National Aeronautics and Space Adninistration. 
QUALITY ASSURANCE FOR MEASUREMENTS IN THERAPYA

R. J. Shalek, P. Kennedy, M. Stoval1, J. H. Cundiff,

W. F. Gagnon, W. Grant, II I and W. F. Hanson

Physics Department, University of Texas System Cancer Center

M. D. Anderson Hospital and Tumor Institute, Houston, Texas 77030

Recommendations regarding quality control in physics relating to radiation therapy are derived from experience with the Radiological

Physics Center which is a national review program, and the Texas

Regional Medical Physicists, which is a regional consultation activity.

(Quality control; radiation measurement; tumor dose; radiation therapy; medical physicist).

During the last half a dozen years there has been a growing appreciation for the need for quality control in physics relating to radiation therapy. A much cited reference ${ }^{1}$ demonstrates a steep relationship between tumor control probability and radiation dose for T2-T3 lesions of the supraglottic larynx from analys is of clinical results. A dose change of 5 ; results in a change of tumor control probability from 38 to $72 \%$. This rather sharp dependence on the absorbed dose may be less critical but still true to some extent for radiation treatment at other sites. Findings which will be reported here include the evaluation of radiation measurement and calculation at 174 institutions visited by the Radiological Physics Center (RPC).b The RPC reviews the measurement and calculation systems employed by institutions participating in interinstitutional clinical trials involving radiation therapy; it also reviews the validity of tumor dose statements for individual patients entered into the trials. ${ }^{2,3}$ In addition some of the information reported here comes from experience with the Texas Regional Medical Physicists (TRMP)C. TRMP has acted as a consultant to physicists for about seven years; it does not serve the medical community directly. Its activities have become self-sustaining from fees collected. Services include: mailed thermoluminescent dosimeters for therapy machine output checks, dosimetry review visits, computer calculation of dose distributions for individual patients, analysis of films for verification of beam uniformity and light localizer coincidence, computer calculation of families of isodose curves from decrement line data from individual therapy machines, and rental of expensive but seldom used equipment.

A finding of these various experiences is that in most institutions the medical physicist is reponsible for the correctness of fulfillment of dose prescription, usually with no further check or review of his activities or conclusions. Three recommen- dations on quality control, which the data and findings reported in this paper support, are listed here to give emphasis to them:

1. One person should review and take responsibility for all steps from radiation measurement to fulfillment of dose prescription. The person best able to do this is usually the medical physicist.

2. Routine review of the accumulating radiation absorbed dose to patients under treatment can reduce random errors.

3. Review of dosimetry systems by outside individuals is important. These reviews may be informal.

Findings of the Radiological Physics Center and Texas Reqional Medical Physicists

A summary of the activities of the Radiological Physics Center appears in Table I. As of February, 1976, the RPC staff had visited 174 institutions for the purpose of reviewing dosimetry relating to interinstitutional clinical trials. A number of the institutions had been visited more than once. The criteria used by the RPC for acceptable dosimetry are agreement to $\pm 3 \%$ for machine calibration, and agreement to $+5 \%$ on fulfillment of tumor dose prescription. It is

TABLE I. Summary of activities, February 1976.

\begin{tabular}{cc}
\hline \hline Number of institutions visited & 174 \\
Number of machines reviewed & 352 \\
$\%$ calibration within $\pm 3 \%$ & $78 \%$ \\
Number of machines x protocols & $(275 / 352)$ \\
reviewed & 768 \\
$\%$ tumor dose within $\pm 5 \%$ & $88 \%$ \\
& $(678 / 768)$ \\
\hline
\end{tabular}

seen from Table 1 that at the time of the RPC review $78 \%$ of the measurement and dosimetry systems were within the $\pm 3 \%$ criteria for calibration and that $88 \%$ were within the $+5 \%$ criteria for the fulfillment of tumor dose as defined in the protocols. When the RPC 
finds a discrepancy exceeding the criteria it works with the institution to understand and resolve the discrepancy. Small discrepancies, often compensating, were found at all but one or two institutions; these were indicated in the reports to the institutions but there was no follow-up procedure when the overall discrepancy was less than $5 \%$.

Insight for quality control can be derived from a review of the information from the dosimetry reviews. In Figure 1 is shown a bar graph in which frequency of occurrence is plotted $v s$. the ratio of machine calibration determined by the RPC to the calibration in clinical use by the institution. It is seen that for the 352 machines measured, the mean of this ratio is very close to 1.00 which suggests that the calibration methods employed by the RPC are consistent with those employed generally. The extreme range of the ratio was .84 to 1.14 .

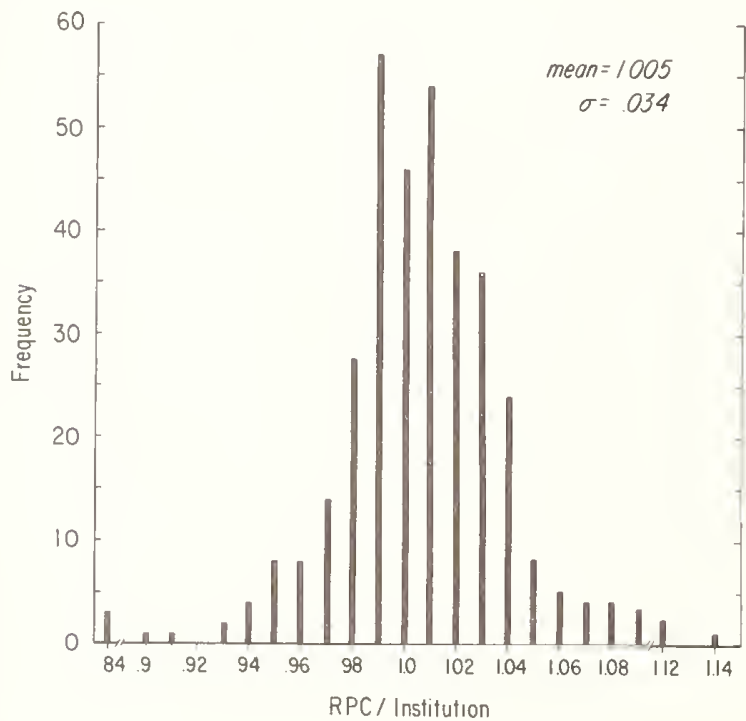

Figure 1. Frequency of occurrence versus the ratio of $\mathrm{RPC}$ calibration to that in clinical use on 352 radiation therapy machines reviewed at 174 institutions.

In Table I I is a listing of the types of measurement errors encountered, together with the number of instances and the maximum discrepancy for each type. The first entry, the chamber correction factor, is shown in greater detail by the bar graph in Figure 2. Of 167 intercomparisons between the RPC chamber and the chambers in use at institutions, the mean ratio of the chamber correction factor derived by the RPC for the institution's chamber to that
TABLE II. Measurement errors.

\begin{tabular}{lrc}
\hline \hline Error & $\#$ & Max. error \\
& & \\
Chamber correction fac.tor & 47 & $12 \%$ \\
Timer error & 38 & $9 \%$ \\
Field size dependence & 34 & $7 \%$ \\
Source movement mechanism & 4 & $16 \%$ \\
Anomalous source decay & 3 & $6 \%$ \\
Distance indicator & 4 & $5 \%$ \\
Light and radiation field & 58 & $15 \mathrm{~mm}$ \\
coincidence & 33 & $20 \%$ \\
Symmetry of beam & & \\
\hline \hline
\end{tabular}

in use by the institution was 1.005 with a standard deviation of $2.7 \%$. The RPC measuring system is a Farmer chamber and a Keithley 602 electrometer. The constancy of the RPC chamber and electrometer is measured before and after visits by utilizing a Strontium 90 constancy check source and a coba7t-60 irradiator. Over the same period of time, the standard deviation of these constancy measurements was $+0.4 \% .^{4}$ The range of the derived chamber factor expressed as a ratio of RPC to institution was 0.88 to 1.09 . In Table II I the chamber calibration results are expressed in another way according to the origin of the calibration factor. Chambers calibrated at the National Bureau of Standards (NBS), the

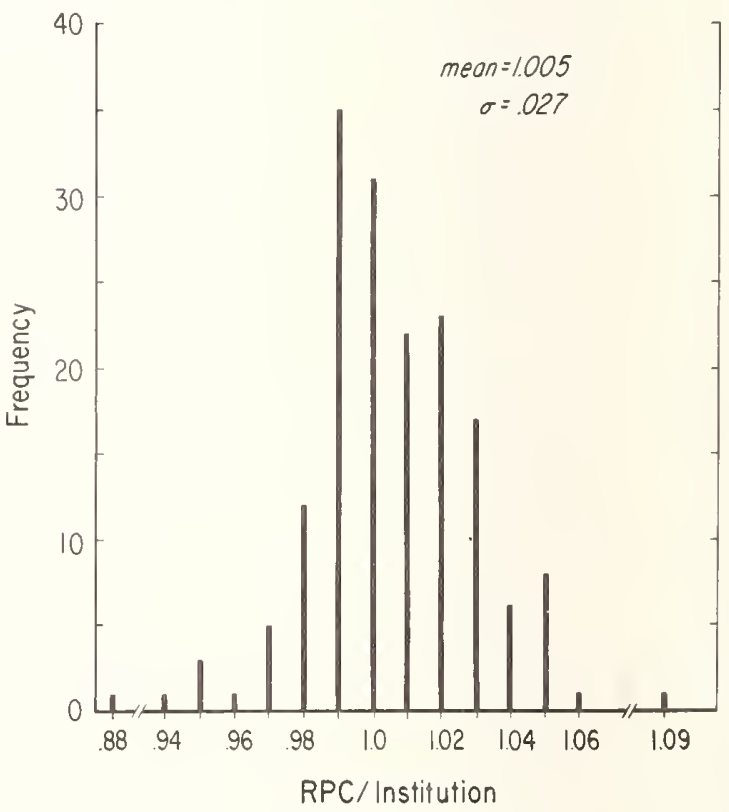

Figure 2. Frequency of occurrence versus the ratio of the chamber correction factor derived by the RPC to that in use at an institution for the instrument used to calibrate radiation therapy machines. There were 167 chamber intercomparisons. 
TABLE III. Chamber Intercomparisons.

\begin{tabular}{|c|c|c|c|c|}
\hline $\begin{array}{l}\text { Calibrating } \\
\text { Agency }\end{array}$ & $=$ & $\begin{array}{l}\text { mean } \\
\text { ratio }\end{array}$ & $\tau$ & $=3$ \\
\hline $\begin{array}{l}\text { :BS \& } \text { IIPL } \\
\text { RCL } \\
\text { ONE INTERMEDIATE } \\
\text { VICTOREEN } \\
\text { OTHER }\end{array}$ & $\begin{array}{l}31 \\
20 \\
23 \\
42 \\
51\end{array}$ & $\begin{array}{l}1.002 \\
1.002 \\
1.004 \\
1.012 \\
1.003\end{array}$ & $\begin{array}{l}.017 \\
.015 \\
.015 \\
.028 \\
.032\end{array}$ & $\begin{array}{r}1 \\
0 \\
1 \\
11 \\
9\end{array}$ \\
\hline
\end{tabular}

National Physical Laboratory (NPL), or Regional Calibration Laboratories (RCL) showed ratios very close to 1.00 with a standard deviation of about $1.5 \%$. The next category which is labeled "one intermediate" is those instruments which have a chamber correction factor derived by comparison to an instrument assigned a factor by NBS or NPL. In this category the intermediate instrument is not at an RCL or Victoreen Instrument Company. These also appear to have about the same mean and standard deviation as those from IIBS, NPL or an RCL. Those calibrated at Victoreen Instrument Company had a ratio 1.01 with a standard deviation of 2.8\%. Previous problems at that facility have been acknowledged ${ }^{\ddagger}$ and are thought to be corrected. Those instruments listed in the "other" category are usually the result of informal or undocumented intercomparisons. Here, though the mean was approximately 1.00 the standard deviation was over $3 \%$. It is important to indicate that it is possible for any calibrating laboratory to assign an inappropriate calibration factor or for a field instrument to change sensitivity after calibration. A check of the calibration may be done by informal intercomparisons and constancy checks of instrument performance. It is advisable to establish a constancy checking procedure with a radioactive source. This procedure should be used regularly but especially before and after chamber calibrations.

A strong case can be made for monitoring of the calibration of radiation therapy machines by mailed thermoluminescent dosimeters (TLD). This activity has been offered by the Texas Regional Medical Physicists since about 1970. The possibility of reviewing and rectifying discrepancies is an important aspect of the mailed TLD system. A number of calibration problems have been uncovered which were then resolved by telephone conversations or visits to the physicists at the institution concerned. In Figure 3 is shown the plot of the ratio of dose by TLD to the dose stated by an institution for a 6 MV X-ray machine and a cobalt-60 irradiator from

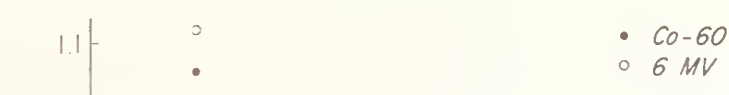

- Co-60

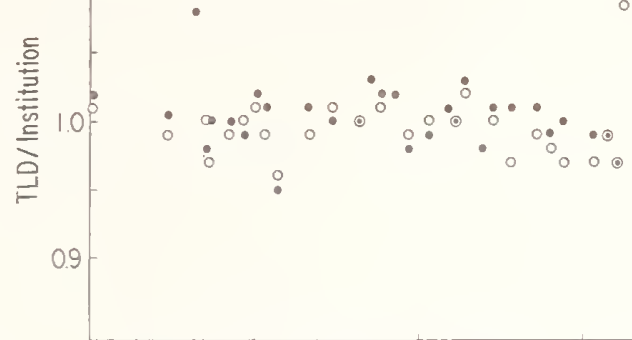

1973

1975

1976

Date

Figure 3. Ratio of radiation measured by alied TLD to that predicted by an institution from their machine calibration versus the date of irradiation. The open circles are for 6 :N $X$-rays and the solid circles are for cobalt-60 gamma rays.

the same institution. Prior to 1973 the $X$-ray points had considerable variability although on average would have been close to 1 (agreement). It is not entirely clear what caused the early observed variability. In our hands $+3 \%$ from an expected value is taken to be within the experimental error. When a discrepancy of $5 \%$ or greater is indicated, TRMP requests the initiating physicist to repeat the TLD irradiation.

In the AAPI protocol for high energy $x$-rays and gamma rays ${ }^{6}$ the recommended method of calibration is at $5 \mathrm{~cm}$ or more depth in water depending upon the energy. An alternative method in that document is calibration in air for cobalt-60 gamma rays and 2 MV X-rays. The RPC has collected data on many cobalt-60 machines indicating that these two methods of calibration appear to yield different calibration values. ${ }^{7}$ In Table IV the results of this study are listed. The uncertainty indicated is the standard deviation of the mean. The numbers in parentheses indicate the number of machines in each category. Thus, machine calibrations for cobalt-60 machines at $80 \mathrm{~cm}$ from the source may be 1 to $2 \%$ higher if the calibration is done at $5 \mathrm{~cm}$ depth in water than if calibration is in air. This comparison assumes the use of standard tables for backscatter factors and depth dose ${ }^{8}$. The reason for discrepancy is not entirely clear. It could be in the use of standard tables which do not precisely apply to a particular machine or there may be a more fundamental problem involving the use of cylindrical ionization chambers in air and in phantom. 
TABLE IV. Calibration in water/calibration in air, $10 \times 10 \mathrm{~cm}^{2}$ field, $80 \mathrm{~cm}$ SSD.

$5 \mathrm{~cm}$ Depth:

A11 Machines

Picker

AECL

$1.014 \pm .009$
$1.015 \pm .008$
$1.014 \pm .009$

$(42)^{*}$

$10 \mathrm{~cm}$ Depth:

A11 Machines

Picker

$1.028+.009$

$1.027 \mp .007$

AECL

$1.028 \mp .010$

$15 \mathrm{~cm}$ Depth:

A11 Machines

Picker

$A E C L$

$$
\begin{aligned}
& 1.027 \pm .010 \\
& 1.025 \pm .008 \\
& 1.028 \pm .011
\end{aligned}
$$

* Numbers in parentheses indicate number of machines in each category.

The other measurement discrepancies in Table II are more or less self explanatory. The entry, timer error, means the possible difference between the time or monitor units set and the effective irradiation span by the machine. The field size dependence. means the difference in measured exposure rate or absorbed dose rate with different field sizes set on the machine. At some institutions the same exposure rate or absorbed dose rate was utilized for all field sizes. Source movement mechanism refers to a few cobalt-60 machines in which the source was not centering over the aperture and to one machine with a problem with a mercury shutter. These machines had been calibrated with the source or shutter in proper position, but due to mechanical fault the source at the time of the RPC visit was not centering or the shutter not opening completely with a consequent reduction in dose rate and the appearance of asymmetry in the beam. These problems indicate the danger of doing only an initial machine calibration with subsequent calculation of clinical dose rate from source decay. At one institution more than two years separated the calibration and the discovery of the problem. The entry, anomalous source decay, refers to the few instances in which a cobalt-60 source was apparently decaying with an abnormal half-life. It is speculated that this result occurred in sources which were composed of cobalt-60 pellets of different specific activity. The pellets of greater specific activity were placed initially at the front of the source but as the source was moved the pellets became mixed, resulting in a changed gamma-ray attenuation and a decrease in exposure rate. The entry, distance indicator, applies principally to optical distance indicators which may be susceptible to alteration by jarring. It is worth mentioning that physicists should be careful that their method of measuring distance during calibration is the same as that used clinically. The entry, light and radiation field coincidence, refers to the geometric alignment of the localizing and treatment beam. The last entry, symmetry of beam, applies particularly to $x$-ray machines in which the compensating filters, the target, or the direction of the accelerated electrons is not on the axis of the collimator.

The errors involved in the determination of tumor dose includes the discrepancies which may have occurred in calibration and, in addition, the use of inappropriate factors in calculating tumor dose. The definition of tumor dose by the institution also enters. The relationship between the frequency of occurrence and the ratio of tumor dose determined by the RPC to that stated by the institution is shown in Figure 4 for simple radiation treatments.

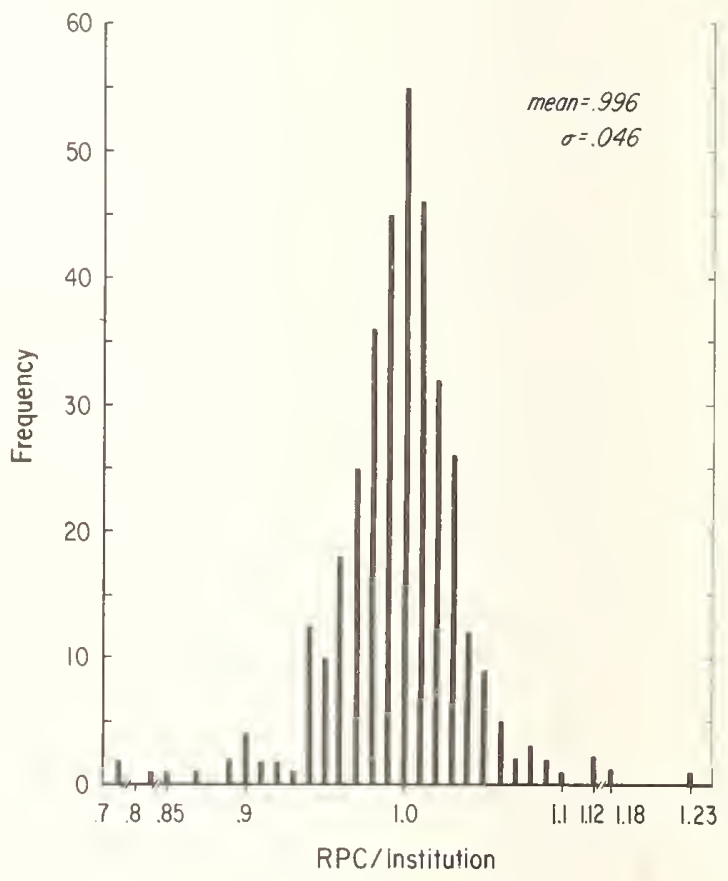

Figure 4. For uncomplicated treatments the frequency of occurrence versus the ratio of the fulfillment of tumor dose estimated by the RPC to that stated by the institution. Irregular fields (mantle, etc.) and tangential breast treatments are not included.

Each of the 352 treatment machines reviewed are counted once. 
Not included are data on irregular fields (mantle, etc.) and tangential breast treatments. This graph is indicative of the reliability of the fulfillment of tumor dose prescription for simple treatment techniques and would apply to protocol and non-protocol patients. Again the findings cluster around 1.00 with an extreme range of 0.71 to 1.23 .

In Figure 5 the frequency of occurrence is plotted vs. the ratio of RPC to institution for tumor dose as defined in the various protocols. If several points of calculation were called for in the protocol, the point showing the worst discrepancy

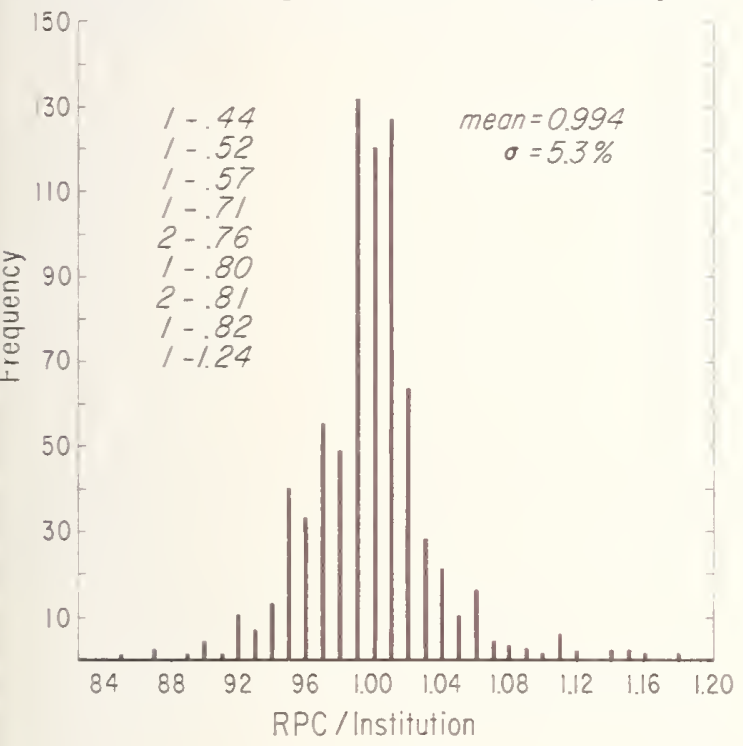

Figure 5. For all protocols the irequency 2 occurrence versus the ratio of the fulSiliment of tumor dose estimated by the RPC to that stated by the institution. Each treatment machine is multiplied by the number of protocols treated upon that machine to obtain the number of events. There was a total of 768 events. Where more than one calculation point is specified in the protocol, the point with the worst agreement is plotted.

is plotted here. These results are for 352 radiation machines and usually include multiple protocols per machine. The mean is close to 1.00 with a standard deviation of $5.3 \%$. The extreme range of 0.44 to 1.24 renders the distribution non-gaussian. It is interesting that the $+5 \%$ criteria for acceptable fulfillment of tumor dose is only slightly less than one standard deviation of the values found in practice.

The types of calculative errors encountered are shown in Table $\mathrm{V}$. A
TABLE V. Calculative errors.

\begin{tabular}{lcc}
\hline \hline Error & $=$ & Max. error \\
& & \\
Attenuation factor & 94 & $1.5 \%$ \\
f-factor or $\mathrm{C}_{\lambda}$ & 43 & $6 \%$ \\
Tray factor & 39 & $14 \%$ \\
Off-axis correction, & 37 & $13 \%$ \\
$\quad$ irregular fields & 49 & $20 \%$ \\
Wrong is depth dose & 63 & $7 \%$ \\
Backscatter factor & 134 & $1 \%$ \\
Rad (muscle)/rad $\left(\mathrm{H}_{2} \mathrm{O}\right)$ & \\
\hline \hline
\end{tabular}

frequent calculative error was the neglect of the attenuation factor of 0.985 required for cobalt-60 measurements made in air. The second entry, f-factor or $\mathrm{C}_{\lambda}$, is the neglect of or the improper transition from exposure to absorbed dose with a maximum error of $6 \%$. The larger errors in this category arose because of confusion of roentgen and rad units on the part of some institutions. Occasionally, the same numerical value was calculated in roentgen and reported to the clinical study in rad. The tray factor (attenuation in the tray for holding secondary blocking) is sometimes neglected, with errors of up to $14 \%$ The next entry, off-axis correction for irreqular fields, applies to those instances where the dose at selected points is determined by calculation and not derived from isodose curves ${ }^{9}$. This correction is not required in rectangular fields where isodose curves are available since in these instances the off-axis correction is included in the isodose curves. The entry, wrong percent depth dose, was usually a systematic mistake in the application of published data or in a few instances was the result of experimental depth dose measurements which were incorrect. The entry, backscatter factor, means lack of, or improper use of, backscatter factor. The last entry, $\mathrm{rad}(\operatorname{muscle}) / \mathrm{rad}\left(\mathrm{H}_{2} \mathrm{O}\right)$ refers to the question of whether absorbed dose should be calculated to muscle or water. The ratio is 0.99 at cobalt-60 and higher energies. No opinion is expressed here concerning which is more correct.

Information on the usefulness of an outside review procedure is shown in Table VI. The RPC made 69 revisits. Table VI is a listing of the number of institutions in which the review concluded that the fulfillment of tumor dose was improved, the same as, or worse than the earlier visit. The numbers are not strictly valid since the complexity of the protocols reviewed on different visits may not have been the same. 
TABLE VI. Impact of RPC visits.

Revisits

Number of revisits

Tumor dose ratio, RPC/Institution: Improved

Same (within $\pm 1 \%$ )

Worse

$11(16 \%)$

Correction of Major Problems

Number of problems exceeding $\pm 5 \%$ :

$\begin{array}{ll}\text { Found } & 62 \\ \text { Resolved } & 51 \\ \text { Not Resolved } & 11\end{array}$

Nonetheless, the numbers indicate that the visits were probably useful to institutions in tightening control over their techniques. The fact that $16 \%$ were in worse agreement at the time of the second visit was discouraging but may indicate that a continuing review effort is necessary. In the RPC reports which are submitted to the institutions, apparent discrepancies are discussed and recommendations to review the problems in question are made. When the total discrepancy at an institution exceeds $5 \%$, special efforts are made to resolve the problems. Of 62 such instances 51 have been resolved to date. Of the 11 unresolved problems, some are in negotiation, but a few are problems of long standing.

\section{Random Errors - Experience of RPC and Others}

The measurement and calculation problems discussed have been systematic in nature. The RPC has not kept a record of the many random arithmetic errors that have been discovered in the review of patient's dosimetry calculations, which it does for each patient entered into most of the studies. There have been literaily hundreds of these. The RPC findings are consistent with a retrospective review of patient charts by Sutherland ${ }^{10}$. He discovered numerous arithmetic errors, or errors in the choice of parameters for calculation. The regular review of the accumulating patient dose during treatment is an expensive but worthwhile procedure. Physicists at M. D. Anderson Hospital have been doing this weekly for more than 20 years.

Another level of quality control is the reproduceability of treatments from day to day upon patients. A study by Kartha and his associates ${ }^{1}$ involved the passive monitoring of four machine parameters on over 5,000 radiation exposures. The criteria used for declaring an accidental error were rather generous but even so it turned out that accidental errors occurred on the order of $3 \%$ of the time as shown in Table VII. If these were on a random basis, it is likely that the contributions of such errors to the uncertainty in total dose was not 1arge. Of course this institution may be above average in the care taken by personnel.

\section{TABLE VII ${ }^{11}$. Accidental errors of various types and frequency of each in percentage of the total.}

\begin{tabular}{lcccc}
\hline & $\begin{array}{l}\text { Minimum } \\
\text { criteria } \\
\text { used }\end{array}$ & $\begin{array}{l}\text { \# of } \\
\text { setups }\end{array}$ & $\begin{array}{l}\text { Acci- } \\
\text { dental } \\
\text { errors }\end{array}$ & $\%$ \\
Parameter & $1 \mathrm{~cm}$ & 2364 & 78 & 3.3 \\
Field size & $15^{\circ}$ & 1064 & 23 & 2.2 \\
$\begin{array}{l}\text { Gantry } \\
\text { Collimator }\end{array}$ & 100 & 1136 & 26 & 2.3 \\
$\begin{array}{l}\text { rotation } \\
\text { Treatment }\end{array}$ & $10 \mathrm{~min}$ & 1011 & 36 & 3.6 \\
time & $0.5 \mathrm{~min}$ & 5575 & 163 & 2.9
\end{tabular}

(Data are from Kartha, Chung-Bin, Wachtor and Henarickson ${ }^{11}$ with permission of the American Institute of Physics)

$\frac{\text { Uncertainties In The Delivery of }}{\text { Absorbed Dose }}$

When one set of measurements and calculations are compared with another it is necessary to consider the uncertainties in each. Is it possible to determine the tumor dose with substantially less uncertainty than the $+5 \%$ which is utilized by the RPC for acceptable fulfillment of tumor dose prescription? Loevinger and Loftus ${ }^{12}$ have considered this question by estimating the systematic and random uncertainties in the measurement chain from national radiation standards to the delivery of absorbed dose to a single point in a tissue phantom by coba 1t-60 gamma rays. The steps are: 1) choice of physical constants for the national radiation standard, 2) determination of exposure in the standard beam, 3) calibration of the secondary standard instrument for a regional calibration laboratory, 4) calibration of a field instrument by a regional calibration laboratory, 5) calibration of the treatment beam with the field instrument, 6) calculation of absorbed dose to a single point in a tissue phantom. In order to include in the model the variations in the quality of measurement 
procedures and instrumentation, starting with step 4, two models are defined. The minimal model represents a minimum standard of practice and instrumentation, while the optimal model represents the best current standard of practice and instrumentation. There is no limit on the magnitude of mistakes that can be made. Thus the large systematic errors plotted in Figure 5 would be outside both the optimal and minimal models. Large arithmetic errors that can be made would also be outside both models.

The institution and the RPC usually have instruments traceable to the same national standards. Thus it is only in steps 3 to 6 that differences in measurement and calculation may arise. Loevinger and Loftus concluded that the $95^{\circ}$ confidence limit for the combination of these steps ( 3 to 6 ) is +2 ? for the optimal model and $+5 \%$ for the minimal model. The 1 imits for steps 3 to 5 (i.e. to calibration of the treatment beam) were about the same. From these calculations of uncertainty, the choice of $+5 \%$ for the criteria for fulfillment of tumor dose is reasonable for simple treatments. For complex treatments such as with irregularly shaped fields the $+5 \%$ criteria may be too small. The $+3 \%$ critêria for machine calibration is reasōnable.

\section{Recommendations for Quality Control}

The implications for quality control from the above information suggests the following points:

1. One person should review and take responsibility for all steps from radiation measurement to fulfillment of dose prescription. The person best able to do this is usually the medical physicist.

2. Routine review of the accumulating radiation absorbed dose to patients under treatment can reduce random errors.

3. Review of dosimetry systems by outside individuals is important. These reviews may be informal.

4. Chamber calibration factors should be directly traceable to the National Bureau of Standards. That is, there should be not more than one intercomparison instrument between the national radiation standard and the field instrument used for therapy machine calibration.

5. Mailed thermoluminescent dosimeters are very useful for checking the calibration of radiation therapy machines. Provision for resolving discrepancies by telephone conver- sations or visits is important.

6. Monitoring of machine parameters in individual radiation treatment is probably useful. Time or monitor units set is likely the most important parameter.

7. The most common measurement and calculative errors are listed in Tables II and $V$.

a. Supported in part by NCI Grant CA 10953.

b. In 1968 the Radiological Physics Center (RPC) was established by the American Association of Physicists in Medicine (AAPM) upon suggestion of the then existing Committee for Radiation Therapy Studies (CRTS) which has been succeeded by the Committee for Radiation Oncology Studies (CROS). The Radiation Therapy Committee of the AAPM serves as the steering committee for the RPC. The present membership of that committee is as follows: Peter Wootton (Chairman), Farideh Bagne, Frederick L. Faw, James R. Goodgame, Mukund K. Kartha, John H. LeVan, Larry Simpson, Alfred R. Smith, Nagalingam Suntharalingam, Kenneth $A$. Wright, Robert $J$. Shalek (RPC), Max Boone (CROS), Jack S. Krohmer (ACR), Richard C. Granke (ANSI), and Robert M. Quil1 in (ANSI). The operating staff of the RPC include physicists:

Lawrence W. Berkley, Jack H. Cundiff, William F. Gagnon, William F. Hanson (Associate Director), and Robert J. Shalek (Director); technicians: Jamie Boling and Paula Kennedy; and secretary: Brenda Truitt. The interinstitutional clinical studies (with clinical chairmen shown parenthetically) being served by the RPC include: Acute Leukemia Cooperative Group B (James F. Holland, M.D.), Brain Tumor Study Group (Michael Walker, M.D.), Central Oncology Group (Theodore Grage, M.D.), Children's Cancer Study Group A (Denman Hammond, M.D.), Cooperative Hodgkin's Disease Clinical Trial (James Nickson, M.D.), Eastern Cancer Oncology Group (Paul Carbone, M.D.), Gynecologic Oncology Group (George C. Lewis, Jr., M.D.), National Bladder Cancer Cooperative Group A (George Prout, M.D.), National Surgical Adjuvant Breast Project (Bernard Fisher, M.D.), National Wilms' Tumor Study (Guilio D'Angio, M.D.), Radiation Therapy Oncology Group (Simon Kramer, M.D.), Southeastern Cancer Study Group (John R. Durant, M.D.), Southwest Oncology Group (Barth Hoogstraten, M.D.), Veteran's Administration (S. Stefani, M.D., E. Humphrey, M.D., R. Byhardt, M.D.), Western Cancer Study Group (Joseph Bateman, M.D.), Intergroup Ewing's Sarcoma Study (Mark Nesbitt, M.D.), Inter- 
group Rhabdomyosarcoma Study (Harold Maurer, M.D.). Studies which are now closed include: Cooperative Study on Radiotherapy for Carcinoma of the Prostate. (Juan del Regato, M.D.), Renal Cel1 Carcinoma Adjuvant Study (Clair Cox, M.D.), The Controlled Study to Determine the Value of Combined Irradiation and Chemotherapy for Squamous Cell Carcinoma of the Oral Cavity, Pharynx, and Larynx (Simon Kramer, M.D.).

c. TRMP counci]: Louis Deiterman, Jr. (Chairman), Lloyd Johnston (Vice-Chairman), Donald Arnold, Louis Levy, Robert Perry, and Ann Wright; operating personnel include: Cathy Kasper, Joye Roll, Robert J. Shalek (Director), and Marilyn Stoval1 (Associate Director).

\section{References}

1. Shukovsky, L.J. (1970) "Dose, time, volume relationships in squamous cell carcinoma of the supraglottic larynx" Am. J. Roentgeno1., Radium Therapy and Nucl. Med. $108,27$.

2. Golden, R., Cundiff, J.H., Grant, W.H., and Shalek, R.J., (1972) "A review of the activities of the AAPM Radiological Physics Center in interinstitutional trials involving radiation therapy" Cancer 29, 1468 .

3. Grant, W. (1974) "AAPM Radiological Physics Center: A status report" Medical Physics $1,41$.

4. Grant III, W.H., Cundiff, J.H., Hanson, W.F., Gagnon, W.F., and Shalek, R.J. (1976) "Calibration Instrumentation Used by the AAPM Radiological Physics Center" Med. Phys. in press.

5. McCarthy, W.A. (1975) "Importance of the calibration of all the parameters affecting radiation exposure measurement" Med. Phys. 2, 83.
6. AAPM (1971) American Association of Physicists in Medicine, "Protocol for the dosimetry of $X_{\text {- }}$ and gamma ray beams with maximum energies between 0.6 and $50 \mathrm{MeV}^{\prime \prime}$

Phys. in Med, and Biol. 16, 379.

7. Grant III, W.H., Cundiff, J.H., Gagnon, W.F., Hanson, W.F., and Shalek, R.J. (1976) "Calibration in water versus calibration in air for cobalt-60 gamma rays" Med. Phys. in press.

8. Cohen, M., Jones, D.E.A. and Greene, D., Eds. (1972) Central Axis Depth Dose Data for Use in Radiotherapy, a survey prepared under the auspices of the Hospital Physicists Association, published as Supplement No. 11, Brit. J. Radiol.

9. Cundiff, J.H., Cunningham, J.R., Golden, R., Lanz1, L.H., Meurk, M.L., Ovadia, J., Last, V. Page, Pope, R.A., Sampiere, V.A., Saylor, W.L., Shalek, R.J., and Suntharalingam, N. (1973) "A method for the calculation of dose in the radiation treatment of Hodgkin's disease" Am. J. Roentgenol., Radium Therapy and Nucl. Med. $117,30$.

10. Sutherland, W., (Oct. 1973), Oral presentation at Hospital Physicists Association - National Physical Laboratory meeting, Teddington, England.

11. Kartha, P.K.I., Chung-Bin, A., Wachtor, T., and Hendrickson, F.R. (1975) "Accuracy in patient setup and its consequence in dosimetry" Med. Phys. 2, 331. 12. Loevinger, R., and Loftus, T.P. (1976). "Uncertainty in the delivery of absorbed dose." Proceedings of the International Course on Ionizing Radiation Metrology, Varenna, Italy, September - October, 1974, in press. 
QUALITY ASSURANCE MEASUREMENTS IN DIAGNOSTIC RADIOLOGY

M. P. Siedband

Medical Physics Section

Department of Radiology

University of Wisconsin

Madison, Wisconsin 53706

The concatenation of elements in an $\mathrm{x}$-ray system necessitates a two level approach to system evaluation. The first level tests determine the weakest links of the chain while the second level tests provide more precise measures of elemental performance. Several types of first level pass-fail tests are described in terms of their utility in a routine quality assurance program for use in hospitals and in their relation to the second level tests. The requirements for accuracy and precision of the measurement apparatus are discussed.

\section{The Need for Quality Assurance Measurements}

The variations of performance of machines and their operators in obtaining diagnostic $\mathrm{x}$-rays have always been known, but only in recent years have the magnitudes of these variations been studied. The National Institute of Occupational Safety and Health (NIOSH) examined about 75,000 chest $x$-rays as part of a nationwide program to detect pneumoconiosis in coal miners. ' These films were about equally divided into good quality, fair quality and poor quality, and about three or four percent of the films were considered useless. A number of surveys of the performance of $\mathrm{x}$-ray apparatus have shown variations of over 3 to 1 in measured output for similar settings. ${ }^{2}$ The Nationwide Evaluation of X-ray Trends (NEXT) task force, formed in 1971 of seven state radiological health program representatives and a representative of the Bureau of Radiological Health, has developed a method for conducting surveys of diagnostic $x$-ray procedures, and has collected patient exposure data for a number of diagnostic examinations. The extremes of measured exposure varied by a factor of 10 for most examinations and over 100 in the worst instances. ${ }^{3}$ These surveys clearly show the need for the routine use of quality assurance measurements in diagnostic radiology.

In order to decide what types of measurements are appropriate, it is necessary to identify the areas where problems arise. There are a number of factors which can cause degradation of radiographs; we can tabulate the most common ones: 1) The operator may select the wrong $\mathrm{x}$-ray exposure factors, $\mathrm{kVp}$ and mAs, or may fail to use the collimator properly and expose too great or too small an area, or may have the patient positioned incorrectly requiring additional exposure. 2) The apparatus may slowly drift from its correct setting - as the tube filament ages the anode current will shift from its calibrated values even though the filament current remains stable. 3) The automatic replenishment system of film processors will add chemicals at an incorrect rate if a succession of films larger or smaller than a particular average size are processed. This is especially true when long lengths of fluoro spot film, $70 \mathrm{~mm}$ to $105 \mathrm{~mm}$, are processed. Improper operation of automatic film processors can result in underdevelopment of films requiring up to 4 times the normal $x$-ray exposure to obtain equivalent film density. 4) Component failure, the inadvertent absence of critical parts such as filters, or mechanical misalignment between the $x$-ray source assembly and the grid preceding the film cassette will obviously cause variations in equipment performance. For these causes of variation of film quality, the most common procedure is for the technologist to close his own servo loop by adjusting the $\mathrm{x}$-ray exposure factors as a result of his observations of the average film density.

Besides the causes of degradation of radiographic quality listed above, there are several other factors which must be considered in a quality assurance program. There are significant differences in the energy distributions and efficiencies of single phase, three phase, invertor type, capacitor discharge and constant potential generators. Generators other than constant potential and filtered polyphase machines tend to favor production of lower energy $x$-rays, which are almost entirely 
absorbed by the patient. Incorrect filtration will have little effect on the appearance of the finished films but a great effect on patient exposure. The film-screen combination must be chosen to give the best compromise between contrast, detail and noise in the final radiograph, and patient exposures for the examination being performed. Normal manufacturing tolerances in the thickness of the glass wall of the $x$-ray tube, variations in electron guns (beam concentration), anode heel effect, phototimers which must complete an integral number of half cycles of operation, and manufacturing variations in films and screens are also causes of variations in diagnostic radiographs.

One problem of quality assurance measurements in diagnostic radiology is the subtle nature of image degradation. Due to the internal impedence of the $x$-ray generator, a shift of tube anode current caused by normal drift or parts failure will cause a change in the opposite direction of the tube voltage and may result in no perceptible change in the density of the final radiograph. However, the contrast scale, that is, the film density change vs. object thickness change, is a function of the voltage applied to the $x$-ray tube, as is the absorption of certain contrast media, i.e., iodine based contrast agents. Since most diagnostic $x$-rays are negative, the absence of the image of an anomaly may be interpreted to mean the absence of the anomaly rather than the failure of the apparatus to image it.

In simplest terms, to use the radiographs themselves as quality control devices is just plain wrong. Since the radiograph incorporates the results of the individual elements, the quality of the power lines, the characteristics of the $x$-ray generator, filter, collimator, patient, grid, film-screen combination, film processor, $x$-ray illuminator and the human viewer, even if the image does not look quite right, it may still not be possible to determine the cause and take the correct remedial action. Tests using standard phantoms may be of value in assessing overall system performance. However, objective tests of each element of the system over the normal range of operation will permit the determination of the operating characteristics of each element, and show whether it is operating within normal bounds. Such objective tests would also permit the intercomparison of different $x$-ray systems and their optimization to produce the best radiographs and the least patient exposure. $\frac{\text { The Need for Three Levels of Quality }}{\text { Assurance Measurements }}$

The operator or $\mathrm{x}$-ray technologist taking the radiograph is the individual who observes the operation of the machine most closely. The radiation physicist, if one is used, will only survey the equipment intermittently. Medical physicists at hospitals often limit their efforts in diagnostic radiology to radiation protection or to the teaching of physics to residents so that they can pass their board examinations. Only rarely are they engaged in the day to day problems of quality assurance. Moreover the consulting costs of outside physicists are such that it is probable that they will not be called each time the operator suspects that the apparatus is not performing optimally. Thus it is important that the operator or $\mathrm{x}$-ray technologists have test tools and a series of procedures that he may use in a first level quality assurance program. Since it is possible to have films of proper density and reasonable appearance even though the apparatus is not functioning correctly, these procedures must be performed on a regular schedule. This first level quality assurance program must include a number of pass-fail tests, involve test exposures, include quality control tests of the processor, and be safe for the operator and the equipment. The equipment must not be dismantled or operated in an unusual way. Furthermore, the first level tests should be capable of being understood by the radiologist and by the technologist. For example, a series of test patterns may be radiographed to estimate the focal spot size of the $x$-ray tube rather than using a pinhole test for the same purpose. The test pattern may be readily related to anatomical parts so that the effect of improper focal spot size may be seen in terms of obvious effects rather than abstract numerical parameters.

During the past several years a program has been underway at the University of Wisconsin to teach first level quality control to $x$-ray technologists and physicists. ${ }^{4}$ A number of test tools were developed for this purpose. Many of these tools are modifications or improvements of earlier tools used at other institutions or in industry. The tools, which are described in more detail 
in the appendix, include an $x$-ray test cassette for estimating actual $\mathrm{kV}$ values, a focal spot test tool which incorporates a lead pattern comprising eleven groups of bars, a timing and mAs step wedge device which contains a motor driven spinning top and a six step copper wedge, a copper mesh pattern containing eight pieces of mesh ranging from 16 to 60 mesh holes per inch, an aluminum penetrameter, and a collection of standard devices such as a photographic sensitometer and densitometer and other devices. This collection of test tools permits the operator or technologist to assess the performance of his equipment on a scheduled basis or whenever malfunctioning is suspected. The sensitometer and densitometer would be used for a daily evaluation of performance of automatic film processors.

A second level of quality assurance testing would be that performed by a qualified physicist or engineer familiar with the inner workings of the $x$-ray apparatus and with the use of measurement devices capable of providing more accurate numerical results. The engineer developing new apparatus will find it necessary to determine in some detail whether his new system is better than the old one. The physicist performing acceptance tests of a new installation will want to know whether all of the specifications listed in the purchase order have been met and whether each element of the system has been installed and aligned properly. Detailed measurements of this type dictate the use of more sophisticated measurement apparatus and are far more painstaking and time consuming than pass-fail quality control tests. Tests at the second level would include those described in industry standards, in international standards, in the reports of professional societies, and those prescribed by national and international organizations concerned with radiation protection and measurements: At the second level of testing, the physicist responsible for recommending the type of radiation shielding to be used in an installation would evaluate the completed room with its installed apparatus to be certain that his recommendations have been followed.

A third level of testing ${ }^{5}$ is concerned with verifying that the installation meets state and federal standards of performance. Many of these tests are the responsibility of the manufacturer to certify that his apparatus performs in a constant and safe way. Compliance with state and federal requirements, however, does not necessarily mean that the $\mathrm{x}$-ray apparatus will meet the user's requirements regarding selection of the tube, generator, and associated apparatus to solve the particular diagnostic problem.

\section{What Goes Wrong}

A good quality assurance program should attempt to solve real problems. To understand the nature of the real problems, operator and equipment performance must be surveyed and the limitations of equipment design must be understood.

We will look first at the $x$-ray tube and generator system. The emission of the $x$-ray tube filament is proportional to the 8 th power of filament current

(Richardson-Dushman and Langmuir equations), approximately. Thus a $1 \%$ change of filament current results in an $8 \%$ change of emission. The high voltage transformer is made small to save costs and has a fairly high impedence to protect the system in the case of a tube or cable short circuit. The regulation is of the order of $4 \mathrm{kVp} / 100 \mathrm{~mA}$ (big systems) to $12 \mathrm{kVp} / 100$ $\mathrm{mA}$ (smaller systems). X-ray production is roughly proportional to $(\mathrm{kVp})^{2}$ and transmission through thick parts is proportional to $(\mathrm{kVp})^{3}$ so that film darkening is roughly proportional to $\left(\mathrm{kVP}_{\mathrm{p}}\right)^{5}$. Thus a $10 \%$ change of $\mathrm{kVp}$ will cause a change of up to $50 \%$ in film exposure.

Consider then, a generator which is supposed to operate at $80 \mathrm{kVp} / 400 \mathrm{~mA}$ but where the filament current drops by $4 \%$ due to temperature and aging effects. The emission will fall about $30 \%$, the $\mathrm{kVp}$ will rise about $10 \mathrm{kVP}$, and the film density may actually increase slightly. Most important, the contrast (especially of iodine compounds) will decrease; clearly, film density is no guide to generator calibration.

We consider next the $x$-ray tube collimator. The tube collimator serves four purposes: 1) to define the limits of the x-ray field, 2) to predict the position of the field with a localizing light and/or dial scale, 3) to add necessary beam filtration and 4) to reduce off-focus radiation. The most common field problem of collimators is that the light and radiation fields may not be congruent - the bulb may shift, the mirror may shift, or the entire assembly may 
shift out of position. The filter may be incorrect. Rotating anode $x$-ray tubes are imperfectly designed, with on $1 y$ $75 \%$ or so of the primary electron beam impinging on the focal spot. The remaining electrons are broadcast throughout the interior of the tube. ${ }^{6}$ A good collimator will use lead elements to define a minimum solid angle to exclude the off-focus radiation. If these lead elements are out of position (or absent) off-focus radiation may exit the tube.

The next stage in the diagnostic $x$-ray system is the imaging element. We will consider first image intensifiers. Most image intensifiers have a built-in vacuum pump which will use either an external power supply or will derive power from focus electrodes. In any case, when the tube is gassy, the point of best focus will be different from that obtained when the tube is "hard." It is normal for a new tube to be a bit gassy. When the tube has not been used for a while it may take several hours to pump down. The resistive voltage divider for the focus electrodes may drift as the parts age. Thus, the electrical focus may have to be adjusted from time to time. Limiting resolution of a high contrast copper mesh or lead grill pattern may not be sufficient to describe the performance of an imaging system. Other tests, for low contrast performance, are also required. A $2 \%$ penetrameter test or a measurement of the veiling glare (a particular type of contrast ratio test) will be helpful in measuring the imaging tube's ability to visualize the soft shadows of low contrast tumors.

Where films and screens are used for imaging, the film processor must be considered. The film processor is the most critical apparatus in an $x$-ray department. Variations of temperature and replenishment rates, contamination, light leaks (or unsafe safe lights), or improper set-up and operation are responsible for changes in base fog level, mean density (sensitivity), and gamma (contrast). 7,8 Splashing (fixer to developer) and/or contamination (residues of cleaning agents, rust of steel wool fibers) will cause random fluctuations of performance. Mechanical slippage will alter the time-temperature relationship required to obtain good films. The slow changes of performance which may be caused by shifts of temperature or replenishment rates must be plotted to be able to take corrective action. X-ray test exposures, e.g., exposure of a test wedge, are not valid tests of film processors because the normal variation of generator output ( $2 \%$ line, $2 \%$ display of $\mathrm{kVp}, 1 \%$ of filament and $8 \%$ of $\mathrm{mA}$ ) will total about $20 \%$ over long times, and will not yield values of film exposures, density, gamma, etc. which are useful for quality control purposes. A sensitometer and densitometer must be used.

\section{A First Level Quality Assurance Program}

The elements necessary for the implementation of a successful first level program may be listed as follows:

1) Realization of the necessity and benefits of the program by the department head and a11 subordinates, 2) regular scheduling of tests with provision for additional tests when problems are suspected, and following repairs, 3) daily film processor testing, 4) availability of simple tests which give valid test data when compared with more elegant methods, and 5) scheduled monitoring by a qualified medical physicist.

A series of first level tests which incorporates tests known in industry and in hospitals for many years has been incorporated into a quality control program developed at the University of Wisconsin. Similar programs have been developed by other universities and a protocol for first level testing is near completion by a Working Panel of the American Association of Physicists in Medicine.

The series of tests can be combined with visual inspection of the rooms and equipment. Cables should be examined for insulation damage. Mechanical devices and locks should be checked for proper appearance and functioning. The room inventory should be listed. Test data should be recorded and filed for each room and compared with current data to note changes. Test tools may be purchased, or tools similar to commercially available ones, may be built, but they should always be available for use. For most rooms, tests should be done every month; special procedure rooms may be tested more frequently. A test form should be developed for recording test data. Test limits should be provided by a first survey accomplished by a medical physicist or, instead of limits, actual values can be recorded and monthly variations noted. 
Some of the test tools and their applications are described in the following sections. The test tools permit testing without dismantling the system. Using the clamp-ammeter, adjusting electrical focus, estimating veiling glare and a few other tests may require some additional skill beyond that of some technologists but all are within the capabilities of field service personnel.

The Wisconsin $\mathrm{X}$-ray Test Cassette provides a means for estimating the tube potential and filtration of diagnostic $\mathrm{x}$-ray equipment. The technique used is a modification of methods described by Ardran and Crooks.

The Test Cassette is a modified $8^{\prime \prime} \times 10^{\prime \prime}$ x-ray cassette using a single intensifying screen covered by an optical attenuator in five selected areas.

Attached to the front of the cassette is a lead mask containing five pairs of columns of ten holes each. The mask is positioned so that the right hand member of each pair of columns is directly above one of the gray bars of the optical attenuator. Over the left hand column of holes are positioned copper step wedges, one step per hole. A sheet of copper is positioned above the first four pairs of columns for $\mathrm{kVp}$ measurements; the fifth pair is used to estimate the HVL. The front of the cassette is enclosed by a plastic cover with the various test areas identified.

The $1 / 16^{\prime \prime}$ copper sheet above the first four pairs of columns, which are used to measure tube potential, serves to "harden" the incoming primary $x$-ray beam by removing most of the low energy photons. No beam hardening absorber is used over the fifth pair of columns because the halfvalue layer depends on the energy distribution of the beam.

The result is a narrower $\mathrm{x}$-ray spectrum with an energy range over which the attenuation coefficient of copper is almost a linear function of energy. There is a particular thickness of copper absorber which, when placed over the unattenuated intensifying screen results in an optical density in the test film equal to that obtained with no absorber and with the optical attenuator. For a fixed optical attenuator the copper thickness required to accomplish this match increases with increasing $\mathrm{kVp}$. This "match" thickness is used to estimate $\mathrm{kVp}$ and HVL. For the $\mathrm{kVp}$ measurements, the "match" thicknesses are determined for the hardened beam; for the HVL measurement the non-hardened beam is used.

The four copper wedges used for $\mathrm{kVp}$ determination have different thickness ranges to allow $\mathrm{kVp}$ measurement over a wide range $(50-140 \mathrm{kVp})$ without sacrificing accuracy. The wedges are designed so that, from left to right, the "match" thickness for $60 \mathrm{kVp}, 80 \mathrm{kVp}, 100 \mathrm{kVp}$ and $120 \mathrm{kVp}$ are close to the center steps and the $\mathrm{kVp}$ per step is small.

In use, a total of five exposures is usually made. The cassette is loaded with any standard $\mathrm{x}$-ray film. The long (10") sides of the cassette should be parallel to the anode-cathode axis of the $x$-ray tube. The collimator is adjusted so that the entire length and width of a region are covered. Exposures are made one region at a time with the other regions of the cassette covered with lead sheets. The exposure is not critical. Typical mAs values at 1 meter are given in Table 1 for single phase $x$-ray units. However, variations in film type and processing may make it desirable to adjust these values. This will not affect the $k V_{p}$ accuracy of the test cassette. The mAs requirements for three phase units are about half of those for single phase units.

When exposing the half-value region, the collimator is opened to include the rectangular region marked "identification" where lead characters or other identification markers are placed to identify the pertinent data, such as machine and date.

After the film is developed the resultant image consists of 10 columns of dots in 5 pairs. Of each pair of columns, the right hand one will consist of dots of nearly uniform density, while the other column will show a density gradient. The net density of the uniform reference column should be between 0.5 to 1.5 . If not, the exposure should be repeated with an appropriate alteration in the mAs value in Table 1. The location in the columns where adjacent dots are of equal density is a measure of the $\mathrm{kVp}$. The use of a densitometer is preferable, but a good approximation $(+3 \mathrm{kV})$ may be made by eye. If an exact density match is not present, interpolation is used.

Each cassette must be calibrated with a known kVp x-ray unit. The calibration 
curve for three phase units lies above the curve for single phase units. The HVL region is calibrated at $60 \mathrm{kV}$ against known HVLs.

The cassette is capable of reading to an overall accuracy of within $3 \mathrm{kVp}$. It is suggested that machines within a department be calibrated with the $\mathrm{x}$-ray test cassette and monitored from time to time to be certain that $\mathrm{kVp}$ variations of less than $5 \mathrm{kVp}$ for 60 and $80 \mathrm{kVp}$ measurements and less than $8 \mathrm{kVp}$ for $120 \mathrm{kVp}$ measurements are maintained. Deviation from these values may indicate defective rectifiers or loss of one phase for polyphase machines. A high HVL may indicate that the $x$-ray tube is near the end of life or that the added aluminum filtration required for most systems has been removed, subjecting the patients to unnecessary exposure.

Similar test cassettes have been developed having only a single pair of columns centered over the $80 \mathrm{kVp}$ match point. These are useful for quick tests at high mA settings to verify both $\mathrm{kVp}$ and $\mathrm{mA}$ since an incorrect $\mathrm{mA}$ value will cause a shift of $\mathrm{kVp}$, the most common type of generator fault.

The Wisconsin Timing and mAs Test Tool is a plastic box containing a six step copper step wedge and a 1 rps motor rotating a brass plate which has four slits spaced $90^{\circ}$ apart. The timing test tool is used with an $8^{\prime \prime} \times 10^{\prime \prime}$ standard cassette and two lead blockers so that four exposures may be made on one film.

The timing device functions in a manner similar to the classical spinning top technique. In the spinning top test, which is suitable only for single phase $x$-ray generators, the individual pulsations of the $x$-ray generator were registered on the film and could be counted to determine exposure time. This may still be done using the timing test tool. However, because the timing test tool is driven by a synchronous motor, the continuous output of 3-phase and capacitor discharge machines may also be measured with respect to $x$-ray exposure time. Four exposures are made varying both exposure time and $x$-ray tube current but maintaining constant mAs. Exposure times may be determined by measurement of the length of the darkened arcs caused by the moving slits in the brass plate by using either a standard protractor or the special protractor provided with the test tool. If the exposure time is correct and if the images of the wedge pattern in the four exposures are close to one another in general appearance and optical density, it may be assumed that the $\mathrm{mA}$ calibration of the generator is also consistent (constant mAs).

In use, the timing tool is positioned on top of an $8^{\prime \prime} \times 10^{\prime \prime}$ cassette on the table top with a $40^{\prime \prime} \mathrm{FFD}$ and the collimator adjusted so that the beam just covers the active area of the timing test tool. The unused area of the cassette is covered with pieces of lead sheet. Four exposures are made on four different areas of the cassette at $80 \mathrm{kVp}, 1 \emptyset$ or $70 \mathrm{kVp} 3 \emptyset$. Typical values might be: Exposure 1: 1/5 of a second at $50 \mathrm{~mA}$; Exposure 2: 1/10 of a second at $100 \mathrm{~mA}$; Exposure 3: 1/20 of a second at $200 \mathrm{~mA}$; Exposure 4: 1/30 of a second at $300 \mathrm{~mA}$. The exposure times can be measured with a standard protractor. In the case of single-phase full wave rectified equipment, one may also count the number of impulses. An extra impulse at a low brightness level is permitted at the beginning of each arc on the film.

All four sets of the wedge pattern images should appear to be of approximately the same darkening (density). If the darkening varies by more than one "step", the machine should be adjusted by a qualified serviceman. The density uniformity of the timing arcs should also be examined; non-uniformity may indicate mA drift during the exposure or component weakness. (Exception: capacitor discharge systems, which should fall off in output during the on-time).

Suggested acceptance limits of exposure time are:

\begin{tabular}{llc}
$\begin{array}{l}\text { Time } \\
(\text { sec })\end{array}$ & $\begin{array}{c}\text { (3 Phase) } \\
\text { Angle }\end{array}$ & $\begin{array}{c}\text { (Single Phase) } \\
\text { pulses }\end{array}$ \\
\hline $1 / 5$ & $68^{\circ}-76^{\circ}$ & $24 \pm 1$ \\
$1 / 10$ & $34^{\circ}-38^{\circ}$ & $12 \pm 1$ \\
$1 / 20$ & $15^{\circ}-20^{\circ}$ & 6 \\
$1 / 30$ & $10^{\circ}-14^{\circ}$ & 4
\end{tabular}

A Pen Dosimeter and Clamp-Ammeter can be used to check system $\mathrm{mA}$ calibration. By checking a generator at 50 to $100 \mathrm{~mA}$ at long exposures with the dosimeter-ammeter method, the timing/mAs tool may be calibrated and then used to check higher mA values.

In the case of an ideal single phase generator, the turns ratio of the transformer will determine the ratio of 
secondary to primary current. Thus, the turns ratio can be determined by using the Wisconsin X-Ray Test Cassette to check the output $\mathrm{kVp}$ while reading the high-voltage transformer primary with the voltmeter section of the clamp-ammeter. The voltage drop within the transformer will range from about $4 \mathrm{kVp} / 100 \mathrm{~mA}$ for large units to $12 \mathrm{kVp} / 100 \mathrm{~mA}$ for smal1 units. When testing at $50 \mathrm{~mA}$, add 2 to $6 \mathrm{kVp}$ to the secondary reading - then

Ratio $=\frac{\{\mathrm{kVp} \text { (measured) }+2 \text { to } 6\} 1000}{(1.414)(\text { Primary Voltage) }}$

In the case of a three phase generator. assume that it consists of three single phase systems operating in parallel; determine the turns ratio of one of these "single phase generators" by measuring from any phase to neutral and comparing that voltage to the output kVp.

Because the clamp-ammeter is graduated in units of RiS amperes (0.707 I $)$ while the current sought is related to maxerage amperes ( $\left.0.636 \mathrm{I}_{\mathrm{max}}\right)$, the clamp-ammeter reading must be marrected by the ratio $0.636 / 0.707=0.90$. Ignoring exciting current losses, the secondary current into the $x$-ray tube is:

i sec. avg. $=\frac{(i \text { pri. RMS })(0.90)}{\text { Turns Ratio }}$

The result is multiplied by 3 for 3 phase units. Except for exciting current losses, which are usually low for operation at $80 \mathrm{kVp}$, transformer losses can be assumed to be entirely in the form of a secondary voltage drop. Waveform distortions will also cause some error giving readings up to $10 \%$ high, but usually can be ignored.

A pen dosimeter and a set of aluminum plates (EC-1100 alloy, $10 \mathrm{~cm} \times 10 \mathrm{~cm} \times 1 \mathrm{~mm}$, 10 total) may be used to measure the output at $80 \mathrm{kVp}$ at 1 meter and plot the attenuation to determine HVL. The output of 3 phase machines should average $6.0 \pm 2.0 \mathrm{mR} / \mathrm{mAs}$, 1 phase machines about $4.5+1.5 \mathrm{mR} / \mathrm{mAs}$ (assuming $2.5 \mathrm{~mm} \mathrm{Al}$ added). The HVL must exceed $2.5 \mathrm{~mm}$ Al. Of greater importance will be recording the output of each machine each month to detect gradual changes.

The Nine-Cent Collimator Test is a simple test to check coincidence of the light and $x$-ray fields using pennies and an $8^{\prime \prime} \times 10^{\prime \prime}$ cassette. The coins are placed in pairs at each edge, one coin at the edge, the second coin touching the first and aimed toward the center. The last coin is placed in the upper right quadrant. The light field is set to illuminate only the central coins and an exposure is made (100 cm, $60 \mathrm{kVp}, 2$ to $4 \mathrm{mAs}$ ) at 1 meter. Ideally, only the central coins are fully displayed i.e. the 1 ight and $x$-rays are coincident ( $\pm 1 / 2$ coin width is reasonable).

The Wisconsin X-ray Collimator Test

Tool consists of a base approximately

$\overline{20 \times} 25 \mathrm{~cm}$ in which are embedded two rectangular frames made of copper welding rod, and a brass ring. Mounted to this base is a Plexiglas plate in the center of which is a brass screw. The plate is supported by $15 \mathrm{~cm}$ aluminum rods.

The system is used only for testing overhead collimators. The test tool is placed on top of an $8^{\prime \prime} \times 10^{\prime \prime}$ or larger cassette on the table top. The focal spot of the $x$-ray tube is positioned 1 meter from the cassette. The light beam device of the collimator is set so that the edges of the light beam fall in the middle of the space defined by the inner and outer rectangles of copper rods. This should also set the lead shutters such that the $\mathrm{x}$-ray beam is confined to the same area if the system is aligned. An exposure is made at approximately $60 \mathrm{kVp}$ and 2 to $4 \mathrm{mAs}$. The small marker rectangle of the test pattern is placed so that it corresponds to the position of the patient's right shoulder so that the direction of collimator errors may be later determined. Lead numbers or other patient identification may be placed on the test tool for convenience in film identification. The film image should show only the innermost wire frame. Further, the $x$-ray image of the brass screw should be confined within the outer border of the image of the brass ring. The dimensions of these test objects have been chosen. so that a maximum $\mathrm{x}$-ray beam positional error of $+1.2 \%$ of the focus film distance and an angular displacement of $3^{\circ}$ will be allowed by the test tool.

\section{The Wisconsin Mesh Pattern is} designed to check the focus of fluoroscopic systems. The test tool is an $18 \mathrm{~cm}$ square plastic plate containing 8 wedge shaped pieces of copper mesh of $16,20,24,30,25,40,50$, and 60 mesh holes per inch. Lead numbers permit the identification of the mesh.

To avoid the effect of focal spot size on detector system resolution, the copper mesh should be placed close to the face of the image intensifier. Operate the 
system in the fluoroscopic mode at the lowest possible $\mathrm{kV}$ p setting and at approximately $1 \mathrm{~mA}$.

Because of the limitations of scanning, there will be a restriction on television resolution. However, all television systems should operate up to the limit of this scanning constraint. A standard television system will have 480 scanning lines in the visible field. Because of the random relationship between the scanning lines and the mesh position, this corresponds to 200 mesh holes per raster height in the television frame. Thus, a 6 " image intensifier system observed via television must be capable of resolving 30 mesh in the center field and a $9^{\prime \prime}$ system must display at least 20 mesh. It has become common practice to adjust the television to flatten the top and bottom of the round image so that most $9^{\prime \prime}$ systems should resolve 24 mesh. If the $6^{\text {" }}$ systems are similarly adjusted so that a $5^{\prime \prime}$ field is displayed, then they should resolve 35 to 40 mesh via TV. When 9" systems are in the magnified mode, they must resolve 30 mesh and may resolve 40 mesh via TV. Television systems must be adjusted for both optical and electrical focus.

Using the mesh tool, the systems should perform as follows (minimum values):

$\begin{array}{lllllll}\text { I.A. } & & & & & \\ \text { (telescope) } & 40 & \text { to } 60 & 30 & 35 & \text { to } 50 & 24 \\ \begin{array}{l}\text { I.A. } \\ \text { (viewer) }\end{array} & 40 & & 30 & 35 & & 24 \\ \text { TV } & 30 \text { to } 35 & 30 & 20 \text { to } 24 & 20 \\ \begin{array}{l}16 \mathrm{~mm} \\ \text { cine }\end{array} & 35 & & 30 & 30 & & 24 \\ \begin{array}{l}35 \mathrm{~mm} \\ \text { (and larger) }\end{array} & 40 \text { to } 60 & 30 & 35 \text { to } 50 & 24\end{array}$

The Wisconsin $\mathrm{X}$-ray Focal Spot Test Tool employs a heavy metal pattern which contains eleven pairs of bar pattern groups. Each pair of groups consists of three slots in a group in one direction and three slots at right angles to the first group. The spacing of the slots in the eleven grouppairs varies from 0.6 line pairs per $\mathrm{mm}$ to 3.35 line pairs per $\mathrm{mm}$. The test pattern is mounted in the center of a $10 \times 10 \mathrm{~cm}$ Plexiglas plate which contains a fluoroscent screen and a lead shield. This plate is mounted $15 \mathrm{~cm}$ over another thin Plexiglas plate. The test tool is designed to be used with any non-screened film.
In use, the focal spot of the $x$-ray tube is placed $45 \mathrm{~cm}$ from the test pattern. Film is placed $15 \mathrm{~cm}$ below the pattern. If the image of one bar and one space between bars is exactly the same as the dimension of the focal spot, then the $x$-ray image is incapable of producing a bar image. False resolution may be sometimes obtained, that is, instead of seeing three bars on each axis in the image at the film two or four bars may be seen. This will not occur with both sets of a group because of the asymmetry of the focal spot. Only when the dimension of the focal spot is less than the dimension of the bar and space in the plane of the focal spot can the image be resolved. X-ray focal spots are not symmetrical and the standards for performance of $x$-ray tubes permit one axis to be 1.4 times larger than the other axis. In addition, the standards also permit the actual dimensions of the smaller axis to be from 30 to $50 \%$ larger than the labeled value! Table 2 lists the number of groups that can be resolved for a given nominal focal spot size and the corresponding maximum actual focal spot dimensions. The bar patterns are listed in terms of line pairs per $\mathrm{mm}$.

A group of bars is said to be resolved when both sets of three bars can be seen clearly by eye in the $x$-ray image on the film. If two or four bars are seen or if the image is just a blur, the image is said to be unresolved. Obviously, larger focal spot tubes will resolve only the larger groups. Reference to the table indicates what one should expect of focal spots of given nominal sizes. For example, a $2 \mathrm{~mm}$ rated focal spot must resolve the first four groups, a $1.5 \mathrm{~mm}$ focal spot must resolve the first six groups, a $1.0 \mathrm{~mm}$ focal spot must resolve eight groups, and a $0.5 \mathrm{~mm}$ focal spot must resolve all groups.

Smallest

Groups

Clearly Pairs/mn

Nominal

Maximum Size Visible Size (mm) Focal Spot

\begin{tabular}{lllll}
\hline 1 & 0.6 & 2.8 & & \\
2 & 0.7 & 2.5 & & \\
3 & 0.85 & 2.3 & $2.60 \times 3.64$ \\
4 & 1.0 & 2.0 & $2.34 \times 3.28$ \\
5 & 1.15 & 1.8 & $1.95 \times 2.73$ \\
6 & 1.4 & 1.5 & $1.50 \times 2.18$ \\
7 & 1.7 & 1.3 & $1.4 \times 1.96$ \\
8 & 2.0 & 1.0 & $1.12 \times 1.57$ \\
9 & 2.5 & 0.8 & $0.90 \times 1.26$ \\
10 & 2.8 & 0.6 & $0.75 \times 1.05$ \\
11 & 3.35 & 0.5 & &
\end{tabular}


The Wisconsin Phantom and Penetrameter is used to estimate the low contrast performance of image intensifier systems and to check the performance of automatic brightness stabilizers and phototiming systems. The phantom and penetrameter consist of two phantom plates of aluminum $7^{\prime \prime} \times 7^{\prime \prime} \times 3 / 4^{\prime \prime}$, a plastic coated lead sheet $7^{\prime \prime} \times 7^{\prime \prime} \times 1 / 16^{\prime \prime}$ and a 1/32" thick aluminum penetrameter plate with holes of $1 / 16^{\prime \prime}$. $1 / 8^{\prime \prime} 3 / 16^{\prime \prime}$ and $1 / 4^{\prime \prime}$.

At $100 \mathrm{kVp} 2 \mathrm{~mA}$ all systems should display the $1 / 4^{\prime \prime}$ and $3 / 16^{\prime \prime}$ holes clearly and the $1 / 8$ " holes should be just visible. Better grade systems show the $1 / 16^{\prime \prime}$ holes clearly.

Automatic brightness stabilizers operating on fixed $\mathrm{mA}$, variable $\mathrm{kV}$, should stabilize at 85 to $115 \mathrm{kVp}$ for full phantom, 70 to $95 \mathrm{kVp}$ for $1 / 2$ phantom and go to the lowest kVp, below $60 \mathrm{kVp}$ for no phantom.

\section{The Wisconsin Veiling Glare Test Set} consists of a photometer incorporating an objective lens, aperture plate and a variable gain photomultiplier tube and output meter. Two lead strips are used as $x$-ray test objects, one is $1.5 \mathrm{~cm}$ and the other is $2.2 \mathrm{~cm}$ wide.

Image focus or the limiting resolution of high contrast objects are not sufficient criteria for describing the performance of image intensifier tubes. Systems may display the $4 \mathrm{lp} / \mathrm{mm}$ section of the lead grill pattern and yet yield poor patient images. A test such as that using the Wisconsin Phantom and Penetrameter is of value only as a pass-fail test. This veiling glare test yields a numerical value for contrast.

The main factor influencing loss of contrast in image intensifier systems is light scatter, in the input phosphor assembly, the output screen-window, and the collimating lens, and is independent of the radiation level. By using a lead strip as the test object and measuring the light level behind the image of the lead strip and in the area adjacent to the lead strip, a standard can be made for evaluating the light scatter, i.e., limiting contrast or veiling glare of image intensifier systems.

A lead strip (15 mm wide $30 \mathrm{~cm}$ long for $170 \mathrm{~mm}$ or smaller input tubes, $22 \mathrm{~mm}$ wide for larger tubes) is taped to the center of the face of the image intensifier assembly. The TV camera is removed to expose the collimating lens. A lead apron is used to drape the input of the system and protect the tester. The system is operated with the shutters open to just touch the edges of the input screen, using operating factors of $60 \mathrm{kVp}$ and $1 \mathrm{~mA}$. The photometer is positioned to monitor the light adjacent to the image of the lead strip (tilted slightly) and the gain set so that the meter reads $100 \mathrm{~mA}$ in the highlight. The photometer is then positioned to read in the area of the strip. Veiling glare is defined as:

$$
V G=\frac{100-\text { strip light level }}{100+\text { strip light level }} \times 100 \%
$$

The veiling glare will yield values of about $50 \%$ worse than conventional disc tests; e.g., a disc contrast ratio of 11:1 will have a veiling glare of about $80 \%$. Reasonable values of veiling glare for new CsI tubes should be:

80+ Cineradiographic, Special Procedures

72+ Two-Port Use, Fluorospot filming catheter positioning

$65+$ Single port TV or viewers, GI examinations

Below 65 reject for low contrast

The Wisconsin Tomograpinic Test Tool consists of the following:

5 plastic discs $9 \mathrm{~cm}$ in diameter; three of the plastic discs, 1, 2 and $4 \mathrm{~cm}$ thick, serve as spacers; one plastic disc $1.5 \mathrm{~cm}$ thick contains lead numbers ( 1 to 12 ) in a helix, with number $11 \mathrm{~mm}$, number $1212 \mathrm{~mm}$, etc., above the bottom of the disc;

1 plastic disc $1.3 \mathrm{~cm}$ thick contains 4 tilted copper mesh pieces of $1.2,1.6,1.8$, and 2.0 holes/mm (Fig. 3): and a lead aperture plate covered with white plastic. The lead is $1.5 \mathrm{~mm}$ thick with a central hole $2 \mathrm{~mm}$ in diameter.

The tomo test tool is used to: 1) determine the location of the fulcrum (plane of the cut), 2) determine the thickness of the cut, 3) test the overall resolution at the location of the cut and 4) test $x$-ray exposure uniformity over the trajectory of the moving source. 


\section{Quality Assurance for the Measurements}

As can be seen, the first level tests are not costly or complicated. For each first level test there is a more precise second level test: test cassette (4\%) voltage divider (1\%), focal spot tool (20\%)pinhole camera (5\%) - pen dosimeter (10 to $20 \%$ - electrometer (3-10\%) - coarse tests of collimation - precision fixtures, etc.

While certain tests at the first level are either obvious or self calibrating, e.g. the mesh pattern, the veiling glare tool, the tomo tool, other tests require a reference or standard base. The Wisconsin Test Cassette must have a test traceable to a standard. The "simple" pen dosimeter is not so simple and must be checked against an instrument of known (standard) performance. At least one manufacturer's product fails to meet published claims in most units. The dosimeters of other manufacturers have been shown to have a rather broad spread of sensitivities. Even the aluminum alloy used as an $x$-ray absorber has occasionally been improperly labeled so that copper bearing alloys yielded incorrect values of beam attenuation.

Second and third level tests, meaning those tests performed by physicists using more precise measurement devices and by state and federal government surveyors, can be considered as a form of quality assurance for the first level tests. The complete quality assurance program in a department of diagnostic radiology must include the first level tests on a frequent schedule with the detailed tests performed, say, on an annual basis.

The evidence of the surveys shows that the problem is not one of precision but of gross error solvable by means of simple tests and simple understanding of some rather basic principles. Emphasis should be placed on frequent testing and training at the user level to solve these problems.

\section{$\underline{\text { References }}$}

1. Trout, E.D., et al. Analysis of the Rejection Rate of Chest Radiographs Obtained During the Coal Mine "Black Lung" Program, Radiol. 109, 25-27, Oct. 1973.

2. Holloway, A.F., Campbe11, E.M., Essengerg, A., Exposure Characteristics of Diagnostic X-Ray Machines, Brit. J. Radiol. 45, 48-52, Jan., 1972.

3. Cameron, J.R., Wochos, J.F., Patient Exposure from Diagnostic X-rays: An Analysis of Two Years of NEXT Data. (To be published by Bureau of Radiological Health, 1976).

4. Cameron, J.R., Siedband, M.P., Quality Assurance in the Hospital, AAPM Summer School Syllabus, Houston, July, 1975.

5. NEXT Task Force, Suggested Optimum Survey Procedures for Diagnostic X-Ray Equipment, Bureau of Radiological Health, Washington, Ju1y, 1975.

6. Rao, G.U.V., Influence of Focus and Off-Focus Radiation on Radiographic Detail. Report to the Bureau of Radiological Health, DHEW 1966, (Also, Private Communication - 1971).

7. Lawrence, D.J., A Simple Method of Processor Control. Medical Radiography and Photography, Vol. 49 No. 1, Kodak, Rochester, 1973.

8. Gray, J., Photographic Quality Assurance in Diagnostic Radiology (In preparation for Bureau of Radiological Health, J. Vucich, Project Officer) Washington, 1976.

9. Jacobson, A.F., Cameron, J.R., Siedband, M.P., Wagner, J., Test Cassette for Measuring Peak Tube Potential of Diagnostic X-ray Machines. Medical Physics, Vol. 3, No. 1, AAPM, New York, 1976. 


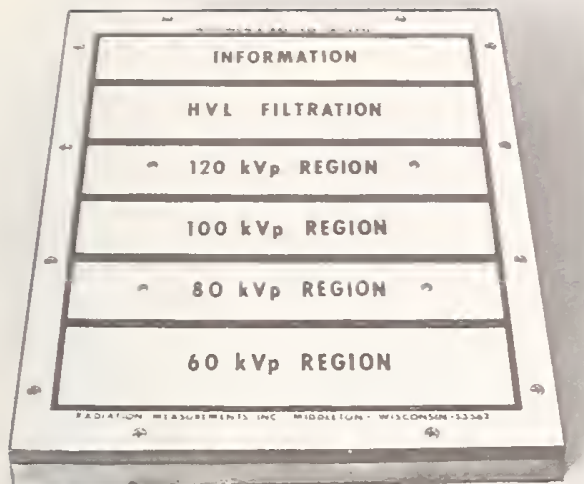

Fig. 1 X-Ray Test Cassette

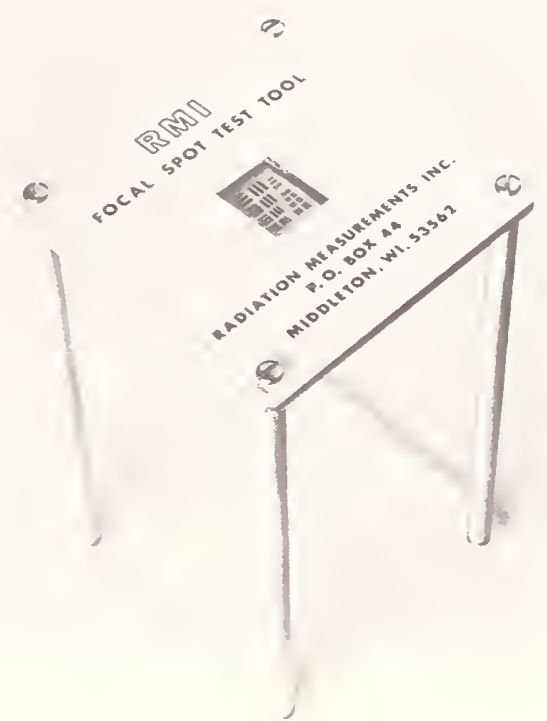

Fig. 3 Focal Spot Test Tool
Fig. 4 Test Images of $1 \mathrm{~mm}, 2 \mathrm{~mm}$ Spots

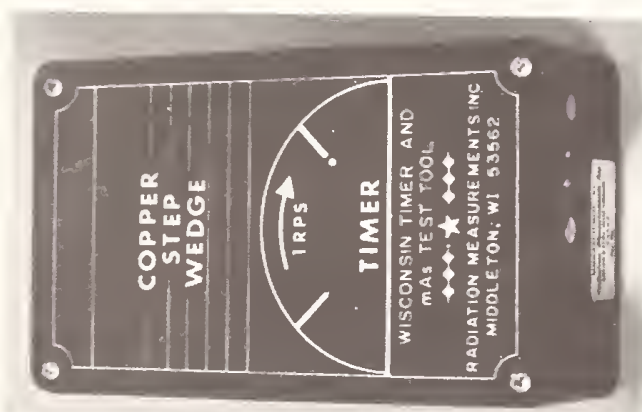

Fig. 5 Radiograph of Mesh Pattern 


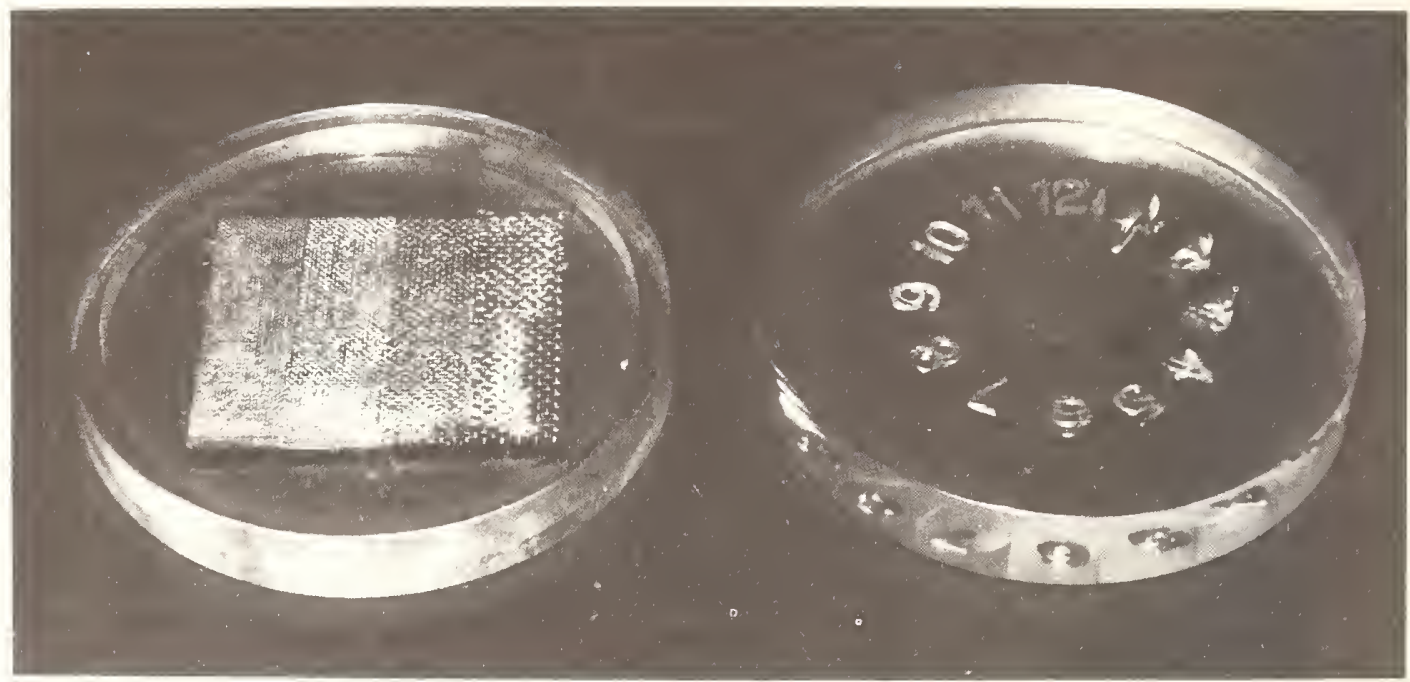

Fig. 6 Tomography Test Tool

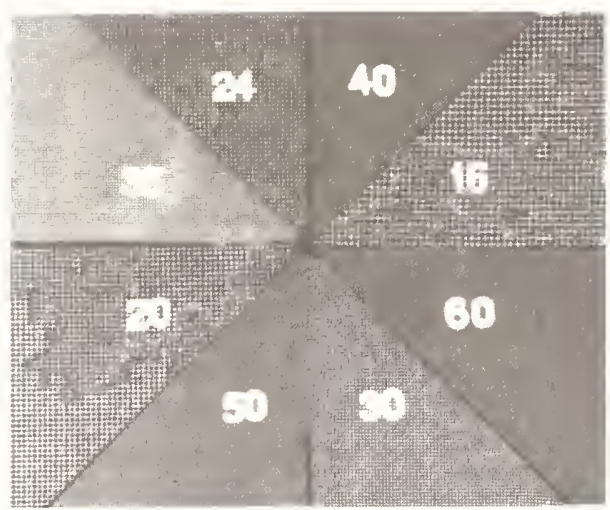

Fig. 7 Radiograph of a Mesh Pattern
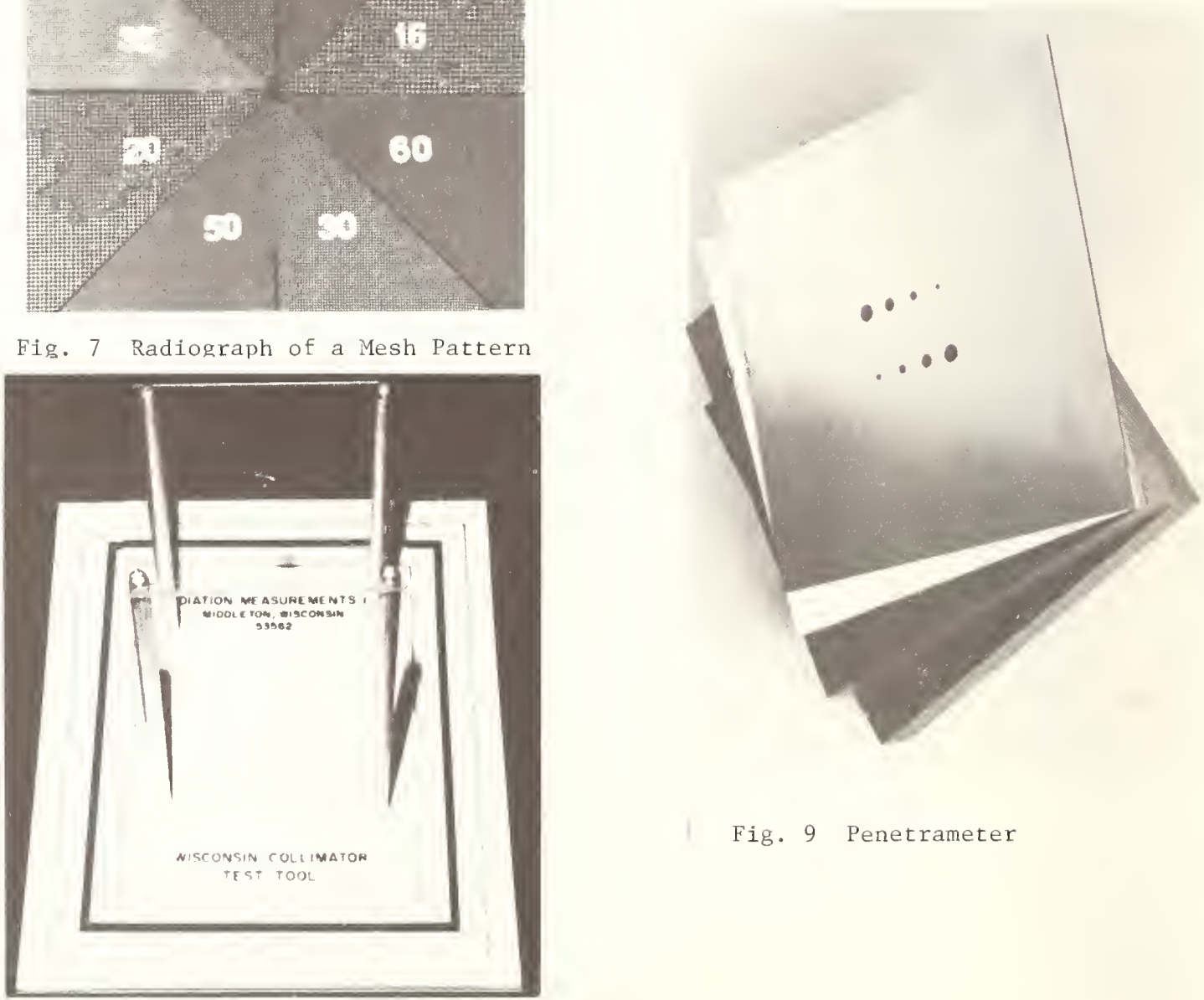

Fig. 8 Collimator Test Tool

Fig. 9 Penetrameter 
QUALITY ASSURANCE FOR MEASUREMENTS IN NUCLEAR MEDICINE

B. A. Rhodes, W. B. Hladik, J. H. Gallagher

Departments. of Diasnostic Radiology and

Pharmacy Practice

The University of Kansas Medical Center

Kansas City, Kansas 66103

This report reviews the measuring systems currently in use to assure safe and effective medical use of radioisotopes. One group of control tests measure the accuracy and precision of in vitro radiometric assays. Another group of measurements is applied to in vivo studies. This group includes measurements of quantity and quality of administered isotopes, performance of nuclear imaging devices and even the performance of physicians who interpret the images. Measures of overall system performance are also employed. The use of feedback loops to control and improve the quality of nuclear studies is an area of current exploration.

(Quality assurance; nuclear medicine; radiopharmaceuticals; 99 Technetium; gamma camera)

\section{Introduction}

The taking of measurements has been basic to nuclear medicine since its inception. Unlike most areas of medicine in which quantitative techniques are a relatively modern addition to the methodology, nuclear medicine began with the use of tracers to measure physiological functions not quantifiable by other means. A nuclear medicine measurement is, in fact, merely counting. Scintigraphy, the imaging process used in nuclear medicine, is produced by temporally integrating and mapping these counts. Specialists in nuclear medicine are technologically oriented and accustomed to the utilization of quantized raw data. Thus, it is natural for those in nuclear medicine to approach quality assurance via the use of quantitative techniques.

The purpose of this report is to summarize the current status of quality assurance measurement programs in nuclear medicine. These programs have been divided into those dealing with in vitro measurements and those dealing with in vivo measurements. They are further divided into internal or in-house programs and external or multi-institutional programs. Finally, the use of data feedback loops are discussed in terms of those mechanisms in current use and in terms of those additional mechanisms where automatic feedback looping might provide added quality assurance for diagnostic measurements in nuclear medicine.

\section{In Vitro Nuclear Studies}

One of the most rapidly increasing uses of radioisotopes is in the quantitative analysis of trace substances in plasma or serum by a technique usually referred to as radioimmuno assay. Utilizing substoichiometric amounts of specific molecular reagents such as antibodies to triiodothyronine and the radiolabeled form of the substance being analyzed, radioiodinated triiodothyronine in this example, it is possible to measure the level of the hormone in a small sample of blood. Such assays have been simplified by the use of disposible reagents and containers. Automatic techniques have been employed whereever possible to facilitate inexpensive, high volume laboratory service.

Several types of measurement errors have been identified in radioimmuno analysis. 1 These errors range from nonspecificity and loss of potency of the substoichiometric reagent, to radiochemical impurities, and to presence of unknown interferring substances in the original blood sample. These errors are in addition to the usual analytical errors of volume variations, and the statistical noise of nuclear counting procedures.

Two major types of control measurements are employed to assist in quality assurance. First are the in-house controls. Frequently this is a high and a low level 
pooled serum sample which is run along with each set of standards and unknowns. The results are plotted versus time to provide a day by day evaluation of the performance of an assay. Second are the interlaboratory controls. These are also aliquoted and sent to various participating laboratories as "unknowns". The analytical results are returned to a central administration where an interlaboratory comparison of data is made and reported back to the participants. One such program, used by over 6000 laboratories, is offered by the College of American Pathologists and is supported by subscriptions paid by the participants. ${ }^{2}$ The largest Federal Government program originates at the National Center for Disease Control (CDC) in Atlanta. The Clinical Laboratories Improvement Act passed by the U.S. Congress in 1967 gave CDC the authority to impliment proficiency testing programs for such tests as Radioimmuno Assays. 3

Individual results of interlaboratory comparison studies are reported in units of standard deviation intervals (S.D.I.). The S.D.I. is a multiple of the standard deviation which describes the frequency distribution of the particular intercomparison. It gives a numerical evaluation of a particular laboratory's response to the mean of peers. A positive or negative S.D.I. indicates a participants answer which is above or below the mean respectively. 2

\section{In Vivo Nuclear Studies}

Amount of administered radioisotope

Quality assurance measurements of radioisotopes administered internally are designed to control the safety of the test in addition to its accuracy and precision. The initial basic control test establishes the amount of radioactivity administered. Both underdosage and overdosage lead to unnecessary radiation exposure. If too small a dose is given, the patient is irradiated unnecessarily because insufficient radioactivity prevents the acquisition of the required data. If too large a dose is given, the patient exposure is proportionally higher and in some cases the detection instrument may be overwhelmed by the excess radioactivity, so that the desired information is either degraded or lost all together.

The usual procedure is to calculate the volume required to provide the necessary amount of the radioactive tracer. This is accomplished using data on the label which is provided by the radiopharmaceutical supplier. The dose is drawn into a syringe and the radioactivity checked using a dose calibrator. This is usually a large volume ion chamber, calibrated to give digital readout of $u C i^{\prime} s$ or $\mathrm{mCi}$ 's. Direct readings are available for the radioisotopes routinely used in clinical nuclear medicine.

Radioactivity standards used by industry to provide the assay reported on radiopharmaceutical labels can be traced back to standards prepared and certified by the National Bureau of Standards (NBS).$^{4}$ The accuracy of dose calibrators can also be regularly checked using standards tracable to NBS standards. Thus, the two in-house quality assurance checks are:

1) conparison of the calculated dose to the measured dose and

2) comparison of the dose calibrator reading to the value on the label of a calibration source.

An interlaboratory qua?ity assurance program is provided by the College of American Pathologists in cooperation with NBS. Radioactive samples, "unknowns", are distributed to subscribers by NBS. The radioassay results are collected from the participants and are compared to the NBS assay. Reports of these studies are published. 3 Initial surveys revealed significant inaccuracies in the assay of certain radionuclides. Subsequent surveys ${ }^{5}$ indicate improvement. This improvement probably is directly related to the fact that the surveys were made and the results publicized.

Problems occuring due to the administration of the radiopharmaceutical are less controlled than the amount of administered radioactivity. These can be classified as follows:

1) dose infiltration

2) partial loss of dose due to residual radioactivity left in the syringe

3) accidental overdosing

4) purposeful overdosing

A survey of 228 consecutive brain, lung and liver doses revealed three minor administration problems: the nature and frequencies of the problems are 1 isted in Table i. 
Table 1. Radiopharmaceutical Administration Problems

\begin{tabular}{|l|c|c|}
\hline Type & $\begin{array}{l}\text { Rate Per } \\
1000 \text { Administrations }\end{array}$ & $\begin{array}{l}\text { Procedure } \\
\text { (Scan) }\end{array}$ \\
\hline $\begin{array}{l}\text { Repeat Injection Required } \\
\begin{array}{l}\text { Syringe Flushed (Causes bolus } \\
\text { to have tail) } \\
\text { Dose Infiltrated }\end{array}\end{array}$ & 50 & Lung \\
\hline
\end{tabular}

A repeat injection is occasionally required because the initial dose is too low to carry out a study. They occurred in the dispensing of $99 \mathrm{~m} \mathrm{Tc}-\mathrm{microspheres}$ for lung scanning. About $5 \%$ of the time such a large amount of the dose remained in the syringe after injection that a second dose would have to be given. This delays the procedure and causes the patient the discomfort of a second venipuncture. The routine procedure for the injection of bolus doses of $99 \mathrm{mTc}$-DTPA for dynamic studies of brain flow preliminary to brain scanning is to make a single rapid injection without flushing the syringe. With almost every other procedure the syringe is routinely flushed to increase injection efficiency. However, for the brain studies flushing is avoided because it causes spreading and tailing of the bolus which degrades the data. This was the most frequent error. Infiltration or extravasation of enough of the dose to require reinjection was the least frequent problem and occurredin less than $2 \%$ of the administrations. Some infiltration is relatively common, but rarely does an injection have to be repeated.

The accuracy and precision of actual radiopharmaceutical administrations for lung scanning with ${ }^{99 \mathrm{~m}} \mathrm{Tc}-\mathrm{microspheres,}$ brain scanning with ${ }^{99 m} \mathrm{Tc}$-DTPA and bone scanning with $99 \mathrm{~m}_{\mathrm{T}}$-pyrophosphate are listed in Table 2.
The data was obtained by routinely measuring syringe residuals, correcting the residual for radioactive decay back to the time of administration, and then subtracting to get the actual dose delivered to the patient. The underdosage resulted primarily from occasional large syringe residuals. Overdosage was not a significant problem.

Purposeful overdosage is most likely to occur in situations where the radiopharmacy is located at a distance from the clinic. Thus, the radiopharmaceutical must be ordered in advance. The ordering or prescribing usually occurs the afternoon before the study. The order includes the time of study in addition to other pertinent data. On the morning of the study doses are drawn into the syringes in amounts to give the required number of $\mathrm{mCi}^{\prime} \mathrm{s}$ at the specified time. This means that before this time the dose is an overdose and later it becomes an underdose. The amount depends directly on the half-life of the isotope. Values for 1 and 3 hours differences of representative radioisotopes are given in Table 3. Since approximately $85 \%$ of the doses used clinically are $99 \mathrm{mT}$ c-labeled radiopharmaceuticals, doses of this isotope are the primary concern. For example, in an attempt to avoid underdoses, the prescription may specify a time later than the patient is scheduled. If however, the patient arrives earlier or on time and is

Table 2. Radiopharmaceutical Dosage Errors

\begin{tabular}{|l|c|c|c|c|c|c|}
\hline \multirow{2}{*}{ Agent } & \multirow{2}{*}{$\begin{array}{c}\text { Number of } \\
\text { Observations }\end{array}$} & $\begin{array}{l}\text { Intended } \\
\text { Dose }\end{array}$ & $\begin{array}{l}\text { Actual } \\
\text { Dose }\end{array}$ & Range & \multicolumn{2}{c|}{ Maximum Percentage } \\
\cline { 3 - 6 } Microspheres & 60 & 5.0 & 4.1 & $1.19-5.79$ & 76.2 & 15.8 \\
DTPA & 84 & 20.0 & 19.5 & $15.52-21.35$ & 22.4 & 6.8 \\
Pyrophosphate & 84 & 15.0 & 14.7 & $12.59-15.89$ & 16.1 & 5.9 \\
\hline
\end{tabular}


Table 3. Over and underdosage errors in radiopharmaceuticals

\begin{tabular}{|l|c|c|c|c|c|}
\hline \multirow{2}{*}{ Isotope } & \multicolumn{4}{|c|}{$\begin{array}{l}\text { Percentage error in dosage as time of administration } \\
\text { differs from time of calibration. Time is given in hours. }\end{array}$} \\
\cline { 2 - 6 } & -3 & -1 & 0 & +1 & +3 \\
\hline $113 \mathrm{~m}_{\mathrm{In}}$ & +248.1 & +51.5 & 0 & -34.0 & -71.3 \\
$99 \mathrm{~m}_{\mathrm{Tc}}$ & +41.4 & +12.2 & 0 & -10.9 & -29.3 \\
$67 \mathrm{Ga}$ & +2.7 & +0.9 & 0 & -0.9 & -2.6 \\
\hline
\end{tabular}

given the full dose, the patient is overdosed. The most extreme case is probably a $30 \mathrm{mc}$ i brain scan injected 3 hours early resulting in $12.4 \mathrm{mCi}$ excess dosage. Data defining the magnitude of this potential problem is not available.

Quality of the administered radiopharmaceutical

Measurement of the quality of radiopharmaceuticals includes several parameters in addition to those for non-radioactive drugs. The presence of the radioactivity within the molecule provides unique problems and some unique advantages compared to other drugs. In particular there are the problems of radionuclidic and radiochemical purities both of which are subject to change with time. There is the advantage that the radioactivity itself makes it possible to carry out biodistribution studies directly. The most common measurements are 1 isted in Table 4.

Table 4. Quality Control Measurements of Radiopharmaceuticals

1. Radionuclidic purity

2. Radiochemical purity

3. Appearance

4. Sterility

5. Apyrogenicity

6. Biodistribution

Radiopharmaceutical quality control measurements have been extensively reviewed in the published proceedings of national and international conferences such as the Proceedings of a Panel on Analytical Control of Radiopharmaceuticals organized by the International Atomic Energy Agency and held in Vienna, 7-11 July, $1969^{7}$; The First International Symposium on Radiopharmaceuticals, held in Atlanta, 12-15 February, $1974^{8}$ and Standardization Performance and Quality Control in Nuclear
Medicine, held at the NBS, 12-14, June $1975^{\circ}$. The stringent demands for more rapid and more sensitive quality control tests for radiopharmaceuticals have led to new test systems that are now finding applications in the testing of non-radioactive drugs. Two examples of this are the use of the limulus lysate gelation test for bacterial endotoxin 10 and the radiometric test for sterility.ll The limulus test has allowed the detection of pyrogen levels in cisternographic agents that are high enough to cause fever and aseptic meningitis in patients, but too low to be detected by the standard rabbit method recommended by the II.S. Pharmacopoeia.12,13 The data in Table 5 shows the reduction in numbers of adverse reactions to cisternographic agents that have occurred since the introduction of this new test.

Table 5. Adverse Reactions to Cisternography

\begin{tabular}{cc} 
Year & $\begin{array}{c}\text { Number of Reported } \\
\text { Reactions }\end{array}$ \\
\hline & 30 \\
1971 & 23 \\
1972 & 9 \\
1974 & 4 \\
1975 & 0
\end{tabular}

Data from the Adverse Reaction Subcommittee of the Society of Nuclear Medicine, H.L. Adkins, Chairman.

The data in Table 5 is an example of the effectiveness of radiopharmaceutical control testing at the present time. The number of patient administrations continues to increase while the total incidence of adverse reactions is decreasing. 
Performance of nuclear instrumentation

Performance testing of imaging devices is a standard of practice. The tests are usually classified according to the frequency of their use: routine- those done before each study, daily, or weekly or those done at intervals longer than a week. These are 1 isted in Tables $6,7,8$.

Table 6. Routine Tests of Nuclear Instrument Performance

1. Film check

2. Procedure parameters check

3. Calibration check

4. Collimator check

Table 7. Daily Tests of Nuclear Instrumentation Performance

1. Field uniformity response

2. Energy Calibration

3. Spatial resolution

4. Sensitivity
Table 8. Weekly Tests of Nuclear Instrumentation Performance

1. Linearity, size and distortion

2. Ancillary equipment function

These procedures for the performance of these tests are available in handbooks such as the Manual of Nuclear Medicine Procedures.14 Quality Assurance of Scintillation Camera (Workshop Manual) 15 and Quality Control in Nuclear Medicine 9 . The area of quality assurance testing is still evolving. Data on the frequency, significance and costs of nuclear instrumentation dys function has been reported. However, studies such as the one reported by Ashare and Podikal 16 review some of the kinds of problems which may be encountered. This study revealed that in certain instances the computer correction of gamma camera field non-uniformity introduced artifacts which degraded rather than improved image quality.

The College of American Pathologists also operates an interlaboratory nuclear instrumentation performance test program in which imaging phantoms are distributed to participants. 17

Training in the techniques of measuring the performance of detection and image instrumentation is a current major activity in nuclear medicine. The Bureau of Radiologic Health (BRH) of the FDA has taken a leading role in this area and is receiving the support of the Society of Nuclear Medicine in their efforts. The two day BRH Training program has been sponsored by local groups across the country.

\section{Observer Performance}

The performance of nuclear physicians in detecting abnormalities on radioisotope scans is measurable under certain specified conditions. 18 The methodology for taking this measurement is derived from signal detection theory. An observer is given a set of images with various signal to noise ratios and asked to report whether or not he observes a lesion. His answers are scored as true or false positives and true or false negatives. A plot of true positives versus false positives provides a point on a receiver operator characteristic (ROC) curve. By repeating the test under varying discrimination criteria data is obtained from which the ROC curve can be constructed. This curve is a quantitative measure of how well the observer is performing under the specific test conditions. The observer does not have to be a human, it may instead, be a computer programmed with a pattern recognition algorithym. These techniques are being used only experimentally at present. However, we can anticipate that their impact on nuclear medicine will have a positive effect on the improvement of physician performance.

\section{Overall System Performance}

Both information theory and operations research are providing techniques for system analysis. ${ }^{9}, 19$ Thus, a set of tools are being developed whereby the overall performance of imaging systems and their impact on patient care can be measured. Two measurements that have been used to evaluate nuclear medicine imagins systems are figures of merit and modulation transfer functions (MTF). A figure-of-merit permits a quantitative ranking of different information detecting or transmitting systems. The MTF permits a quantitative measure of the degradation of information as it is transferred through a system. We have yet to apply these techniques in routine quality assurance programs.

Methods for measuring the diagnostic usefulness of information provided for nuclear medicine studies are available.20 One procedure is to obtain from the refer- 
ring physician his probable diagnosis, and his certainty about each, both before and after he is provided with the results of the study. Change in his diagnostic certainty is measured by an index known as a $\log$ likelihood ratio. ${ }^{19}$ Another procedure is to measure outcomes of two procedural paths, one of which includes a nuclear test and the other a control which does not include the test. Patients with similar symptoms on admission are randomly divided between the two paths. Measurements are made of duration and costs of hospitalization, one-year survivals etc. which allow for quantitative comparisons of the outcomes of the two alternatives. As more reports are generated illustrating the value of these kinds of analysis we can expect that they will become more widely used.

\section{Feedback of Quality Control Data}

Quality assurance is achieved by the making of appropriate performance or quality measurements followed by a prompt response to correct the situation when indicated. When used, the feedback of quality control data is customarily prompt in nuclear medicine. The physician will look at the images before the patient is dismissed from the clinic. Radiopharmaceutical or nuclear instrumentation problems are often immediately apparent from the pictures. Corrections are ordered before the patient is released and before the next study is begun. Unlike regular drugs where altered biodistribution or metabolism is not apparent except by the use of carefully designed experiments, defective radiopharmaceuticals give visual biodistributions within minutes after each patient administration. Hence, a poor quality tracer is used only once before it is recognized, discarded and replaced with a better formulation or different agent. It also is common practice for the physician to have before him a picture of the daily flood field source as he reads gamma camera scintigrams. This allows for a comparison between the image of the patient and the quality control image.

Currently we are exploring the use of the computer to assist in the collection and evaluation of control data in hopes of further improving the quality of nuclear medicine studies. Two computer assisted feedback loops are under investigation: the radiopharmaceutical purity control of $99 \mathrm{~m}_{\mathrm{Tc}}-1$ abeled radiopharmaceuticals and the performance of the gamma camera. The hypothesis under test is that a prestudy verification of the current quality of these two system components will both improve patient care and reduce operating costs. The approach is to image a field flood, a phantom and a column chromatorgram of the radiopharmaceuticals before each days work. The images of these control objects will be compared to data stored in the computer and evaluated as to whether radiopharmaceutical purity and gamma camera performance are within prescribed tolerances. If not, the computer will be programmed to issue instructions as to which component is outside tolerance and to provide data characterizing the problem.

\section{Radiochemical Purity}

Persson 21 has shown that column chromatographic separation of $99 \mathrm{mTc}$-compounds can be achieved without elution of radioactivity from the columns. After development of the columns with saline or buffer, the fractionation of the ${ }^{99 \mathrm{~m}_{\mathrm{T}} \mathrm{T}-1 \mathrm{abel} e d}$ components are

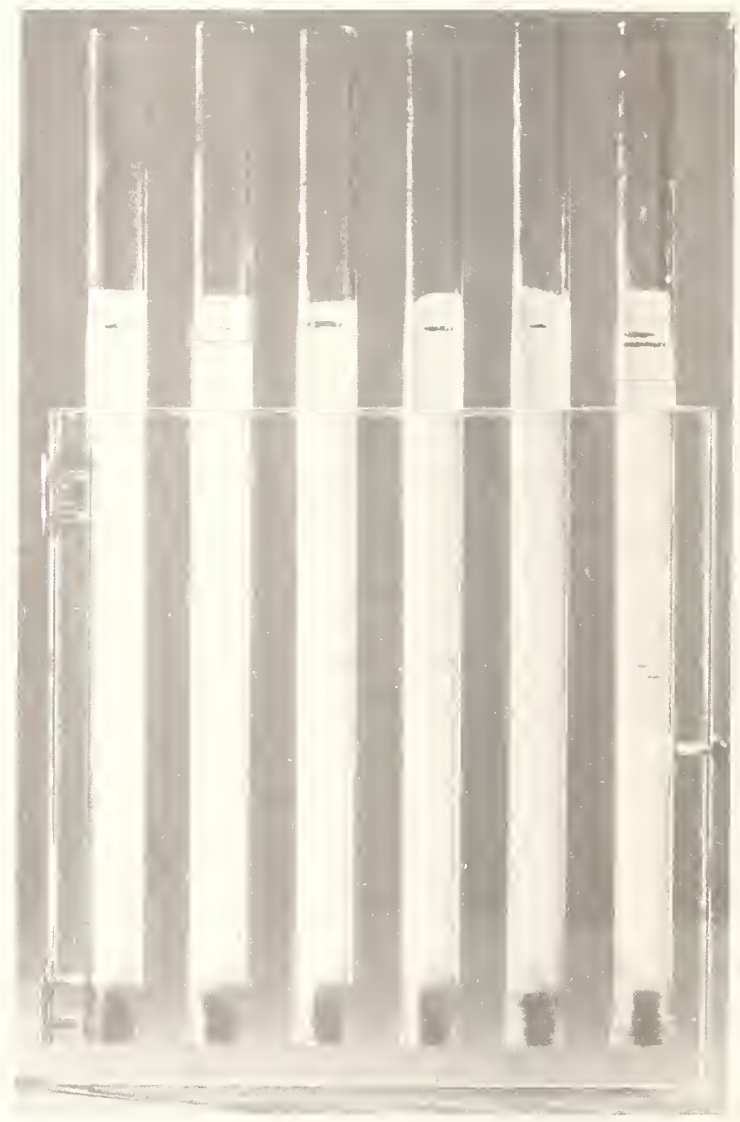

Figure 1: A set of gel chromatography columns in a special holder for use in the radiochemical purity testing of $99 \mathrm{~m}^{\mathrm{T}} \mathrm{c}-$ labeled radiopharmaceuticals. 


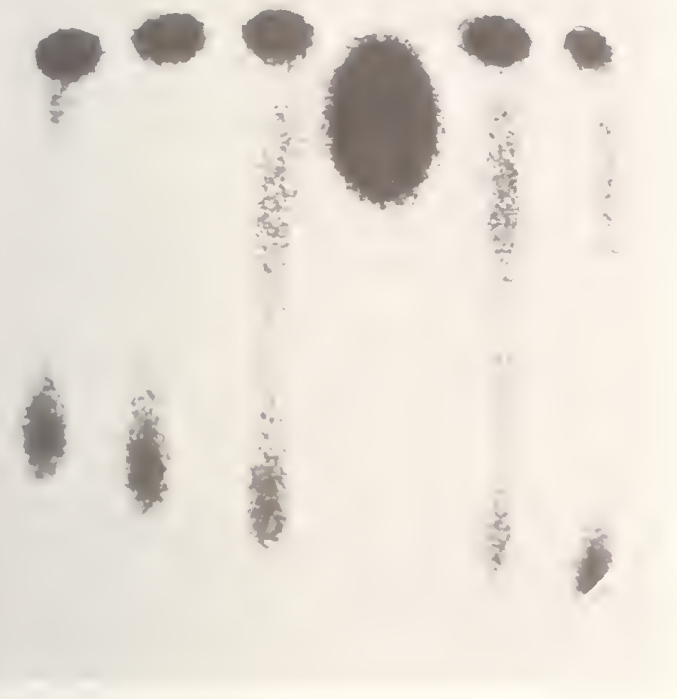

Figure 2: A gamma camera image of the columns shown in figure 1. Variations in radiochemical purity of the various $99 \mathrm{~m} T \mathrm{c}-\mathrm{s}$ colloid preparations are visualized. The differences can be quantitatively evaluated by computer analysis of the image.

visualized by scanning or gamma camera imaging, Figure 1 and 2. The data contained in the image can be transferred into a digital computer for quantitative analysis of radiochemical purity. Included in the computer programs are the routines for comparing the radiochemical purity to the expected values and for reporting the differences.

\section{Gamma Camera Performance}

In computerized gamma camera applications it is customary to image a field of uniform radioactivity called a field flood. The data obtained usually is not uniform however, because of regional variations in the sensitivity of response of the camera. Thus, a matrix provides the means of obtaining images equivalent to that which would have been obtained if the response of the camera was truly uniform. The correction matrix contains data which measures the uniformity of response of the camera. A recorded daily evaluation of this data documents the aspect of gamma camera performance. The collective record is therefore a reference measure of the quality of performance of the gamma camera. When a current days data is compared to the data of record it becomes possible to make a decision as to whether or not the performance has deteriorated to unacceptable levels. By doing this, a tighter quality feedback control loop is established.

Phantoms can be used to evaluate resolution, field size, and linear distortion. Again, day-to-day recording of these control values can create a record against which the current days performance can be measured. The comparison of the current operation to the record of past operations then provides additional feedback loops. The quality control feedback loops can be programmed so that at the end of data acquisition and analysis, a 'go' or 'no go' is displayed.

\section{Summary}

Measurements of the quality of nuclear measurement is becoming standard operating procedure in the clinical practice of nuclear medicine. Methods for quality assurance and performance testing of system components have been developed. Their use is providing evidence that quality assurance programs are effective in nuclear medicine. Further evidence of the impact of quality assurance programs is the results of the analys is of intra- and interlaboratory control samples. Investigation of quality assurance techniques continues with emphasis on measuring system performance, on determining appropriateness and cost-effectiveness of clinical nuclear medical measurements, and on establishing more effective feedback mechanisms.

\section{References}

1. Thorell, J. Quality Parameters of In Vitro Assays in Quality Control in Nuclear Medicine, B.A. Rhodes ed., C.V. Mosby Inc., St. Louis (In Press).

2. Hansell, J.R. Proficiency Testing of In Vitro Assays in Quality Control in Nuclear Medicine, B.A. Rhodes ed., C.V. Mosby inc., St. Louis (In Press).

3. Spierto, F.W., Knight, M.S. and Shaw, W. External and Internal Quality Control Programs for Clinical Radioassay Procedures in Quality Control in Nuclear Medicine, B. A. 
Rhodes ed., C.V. Mosby Inc., St. Louis (In Press).

4. Mann, W.B., Cali, J.P. Traceability of Radioactivity Measurements to National Standards in Quality Control in Nuclear Medicine, B.A: Rhodes ed., C.V. Mosby Inc. St. Louis (In Press).

5. Hauser, W. Radioactivity Measurements and The ir Quality Control in Quality Control in Nuclear Medicine, B.A. Rhodes ed., C.V. Mosby Inc., St. Louis (In Press).

6. Cavllo, L.M., Coursey, B.M., Garfinkel, S.B., Hutchinson, J.M.R. and Mann, W.B. Needs for Radioactivity Standards and Measurements in Different Fields. Nuclear Instruments and Methods 112, 5-18 (1973).

7. Analytical Control of Radiopharmaceuticals, International Atomic, Vienna, STI/ POB /253 (1970).

8. Subramanian, G., Rhodes, B.A., Cooper, J.F. and Sodd, V.J. Radiopharmaceuticals, The Society of Nuclear Medicine, Inc., New York (1975).

9. Rhodes, B.A., Quality Control in Nuclear Medicine, C.V. Mosby Inc., St. Louis (In Press).

10. Cooper, J.F., Hochstein, H.D. and Seligmann, E.B.: The Limulus Test for Endotoxin (pyrogen) in Radiopharmaceuticals and Biologicals. Bull. Parenteral Drug Assn. 26:153 (1972).

11. Chen, M., Rhodes, B.A., Larson, S.M. and Wagner, H.N.,Jr.: Sterility Testing of Radiopharmaceuticals. J. Nucl Med. 15: 1142 (1974).

12. Rhodes, B.A., Kamanetz, G.S. and Wagner, H.N.,Jr. The Use of Limulus Testing to Reduce the Incidence of Adverse Reactions to Cisternographic Agents. Neurology 24: $810(1974)$.

13. Cooper, J.F. and Harbert, J.C. Endotoxin as a Cause of Aseptic Menigitis after Radionuclide Cisternography. J. Nucl. Med. 16:809 (1975).

14. Keyes, J.W.,Jr., Carey, J.E., Moses, D.C. and Beierwaltes, Manual of Nuclear Medicine Procedures, 2nd Edition, The Chemical Rubber Co.

15. Quality Assurance of Scintillation Camera (Workshop Manual), Bureau of Radiological Health, DHEl. (In Press).
16. Ashare, A.B. and Padikal, Computer Correction of Gamma Camera Field Non-Uniformity: Possible pittfalls in Proceedings of a Symposium on Sharing of Computer Programs and Technology in Nuclear Medicine held in Atlanta, Ga., 26 January 1976, B. Howard Ed. (In Press).

17. 1974 Survey Manual, College of American Pathologists.

18. Goodenough, D.J. and Knowles, L.G. Measurements of Observer Performance in Quality Control in Nuclear Medicine, $\bar{B}$. A. Rhodes ed., C.V. Mosby Inc., St. Louis (In Press).

19. Potchen, E.J. Current Concepts in Radiology, Vol. 2, C.V. Mosby Co., St. Louis (1975).

20. Mclleil, B.J., Keeler, E. and Adelstein, S.J. Primer on Certain Elements of Medical Decision Making. N. Engl. J. Med. 293:211 (1975).

21. Persson, B.R.R. Gel Chromatography Column Scanning: A Method for Identification and Quality Control of $99 \mathrm{~m}^{\mathrm{T}} \mathrm{c}$ Radiopharmaceuticals in Radiopharmaceuticals, G. Subramanian, $\overline{B . A}$. Rhodes, J.F. Cooper and V.J. Sodd eds. The Society of Nuclear Medicine, New York (1975). 
STAIE AID FEDERAL REGLLATORY MEASURENENT RESPONSIBILITIES AROUND MEDICAL FACILITIES Lawrence H. Lanzl, Ph.D.

Department of Radiolcgy and Franklin McLear Memorial Research Institute (operated by the University of Chicago for the U.S. Energy Research:

and Development Administration under Contract E(1I-1)-69) and

Chairmar, Radiation Protection Advisory Council, Department of Fublic Health, State of Illinois

Raciation exposure to man is due chiefly to diagnostic x-ray procedures, in which radiation is intentionally directed toward a patient. Radiation therapy presents a lesser problem because a smaller percentage of the population is subjected to such treatment. Recently, some innovative steps were taken in the State of IIlinois to reduce patient exposure in four diagnostic procedures without reducing the benefits derived therefrom. However, if these procedures are to be carried out properly, it is necessary to increase the precision and accuracy of radiation exposure measurements to the onder of $\pm 2 \%$. The usual accuracy and precision of radiation protection measurements are of the order of $\pm 20 \%$. Thus, should the Illinois radiation protection rules become widely adopted, our national dosimetry network will need to upgrade exposure measurement techniques.

(Medical facilities, diagnosis, therapy, regulatory, measurement accuracy, traceability)

\section{Introduction}

The main theme of this Symposium concems the techrical communication system which insures that radiation measurements are correctly made, sufficiently accurate, and traceable to the national radiation standards. The measurements necessary for the safe use of radiation may be applied to two patient groups. One group is related to the safe use of radiation administered for the primary purpose of diagnosis to determine the state of an individlal's health. Radiation damage to human beings in this group is a secondary and negative byproduct of the use of the radiation.

In the second group, the primary purpose of administering radiation to the patient is to destroy human tissue, i.e., a malignant tumor. This use is designated as therapeutic.

In general, the regulatory agencies of federal, state, county, and city government have been very much concermed with the first, but not with the second group.

Below, two examples of types of radiation measurements that need to be made in medical facilities, one for each of the two patient groups, will be given. These examples will demonstrate the importance of precision and accuracy, and of traceability not only to the U.S. national radiation standards, but also to the world-wide dosimetry network. Some radiation measure- ments also need to be placed in a thind group. This group involves the measurements recessary for radiation and non-radiation workers in a medical facility as well as visitors to the facility.

\section{Regulatory Agencies}

All of the regulatory agencies set limits involving radiation measurements, and/or determinations which require some measurement. In some cases, the actual measurement is carried out by the agency and in others, the medical facility is obligated to carry out the measurements, or at least to demonstrate that it has the staff and equipment capable of doing so.

Some of the major regulatory and other agencies at the federal level that have an impact on medical facilities in the area of radiation measurements and their responsible areas of concem are:

\section{Department of Transportation}

Shipment of radioactive sources and pharmaceuticals to the medical facility and shipment of radioactive waste from the facility to the waste disposal site.

2. Food and Drug Administration, Bureau of Drugs-21 CFR 361.1, July, 1975

Limits the radiation dose from radioactive material that can be administered to an adult research subject in a study. The annual and total dose commitment to the whole body, active blood-forming ongans, 
lens of the eyes, and gonads shall not exceed 5 rems, etc.

3. Food and Drug Administration, Bureau of Radiological Health--Radiation Control

for Health and Safety Act. P.L. 90-602, 1968

The purpose of the law is to regulate and control the emission of electronic product radiation through the promulgation of performance standards for diagnostic $\mathrm{x}$-ray systems and their major components.

\section{Nuclear Regulatory Conmission}

Provides for the licensing of byproduct, source, or special nuclear materials in quantities in excess of specified exempt concentrations in gaseous concentrations and liquid and solid concentrations. Capability of making radiation measurements is obligatory under a license.

\section{Envinonmental Protection Agency}

Provides for federal radiation guidance in many areas including, for example, guides which govern the prescription of diagnostic $x$-rays and which assure the use of consistent techniques in the taking of $\mathrm{x}$-rays in federal agencies.

6. Department of Iabor--Occupational Fealth and Safety Act

Provides for limits of radiation exposure to workers.

Most, but not all state govermments have public health laws coupled with rules and regulations which require radiation measurements. If a state has a good control program, the cities usually delegate radiation regulatory matters to the state. Some cities, however, may have programs which are strong in themselves. In such cases, the city has more regulatory impact than the state.

Radiation Measurement, Accuracy, and Iraceability to the National Standara's

Those persons who write regulations and set numerical limits of any sont seldom, if ever, include in the regulation any statement about the uncertainty permitted in the limit due to the uncertainty of the measurement required by that limit. For example, we never see a level of uncertainty reganding a regulation for automobile traffic speed limits. It is usual, however, that the operational traffic officer decides on an allowable uncertainty before he issues a speeding ticket.
Likewise, in the area of radiation regulation, the various writers do not include information on required accuracy of the measurement of a limit.

There is an adage in physics that one should make a physical measurement with the accuracy and precision required to answer the question at hand, but not beyond what is needed. This certainly applies to measurements for the safe use of radiation. Generally, an accuracy of $\pm 20 \%$ is considered sufficient for operational health physics purposes. This degree of uncertainty reflects our lack of definitive information and knowledge on the radiation risk to human beings at the generally accepted maximum permissible dose-equivalent levels (see discussion on radiation protection philosophy in reference 1). However, situations arise where $\pm 20 \%$ accuracy is not sufficient. I will describe an example from the State of Illinois Rules and Regulations for the Protection against Radiation, which applies to the diagnostic group referred to above.

\section{Diagnosis}

\section{Dental}

Several years ago the Illinois Departmert of Public Health, Division of Padiological Health, began accumulating specific radiation-patient exposure data during regulatory inspections of various radiation facilities throughout the state. In an initial study, measurements were obtained and collected from all marufactimes of intra-oral dental $x$-ray machines. The prime objective of this study, as reported by M. E. Neuweg and P. N. Brunner ${ }^{2}$ centered around the "technique"

involved in achieving a diagnostic radiograph. "These were the use of slow speed dental film and improper film development technique. ... The total measured exposures ranged from less than $100 \mathrm{mR}$ per radiograph to greater than $7000 \mathrm{mR}$ per radiograph. All exposures were measured at the distance from the tip of the dental cone used by each facility in order to simulate entrance exposure to a patient. ... One hundred percent of all exposures identified above $500 \mathrm{mR}$ were conducted with slow speed dental film. 55\% of all incident exposures above $1000 \mathrm{mR}$ resulted from inadequate film development technique. .... Analysis of the exposure measurements was presented to our governor-appointed Padiation Protection Advisory Council, which consists of seven professionals, including health physicists, 
medical and dental licensed practitioners and industrial executives. As a result of this presentation, the following rule was developed by the State Flealth Department staff with the advice and approval of the Council: 'For routine intra-oral radiography, the incident exposure to an average patient shall rot exceed $1000 \mathrm{mR}$ per radiograph and shourd not exceed $500 \mathrm{mR}$ per radiograph.' This rule became effective on January 1, 1973."

"After a year of feedback information, we have received strong support from nimerous dentists, professional societies and other state radiation control programs. Wany licensed dentists in Illinois affected by the rule have not only complied with the exposume reduction to the patient but have also indicated to us the production of higher quality radiographs which are diagnostically beneficial to the practitioner. Use of higher sensitivity film and/or proper film development techniques assures compliance, but more importantly, true radiation hygiene is practiced by exposing each patient to only the minimum amount of radiation.

As of July 1973, the radiological health computer data base consisted of 3020 bitewing exposures. Eighty percent of exposures were within the 1000-mR-exposure limit. (See Figure 1.) From July 1973 through March 1974, operators of 221 dental $x$-ray machines had reduced the exposure to within the required exposure linit. Assuming an average patient exposure of 1870 $\mathrm{mR}$ before reduction, an average of $700 \mathrm{mR}$ per exposure after inspections, and an average workload of 50 exposures per week, 672,000 man-rems of unnecessary radiation exposure were eliminated the first year as a result of these radiation exposure regulations."

This work by Neuweg and Brunner and by the staff of the Illinois Department of Public Health represents a substantial step in eliminating radiation exposure without a negative impingement upon the practice of dentistry. It is clear that this work, leading to a safer use of radiation, would not have been possible without a measurement responsibility on behalf of a regulatory body.

\section{Medical}

\section{In a later work, Neuweg and}

Brunner 2 carried out a study to determine the range of radiation exposure levels used in several common diagnostic examinations. Radiographic techrique factors such as kilovoltage, milliamperage, time, and target-to-film distance were obtained for an "average-sizec" adult undergoing these examinations. Subsequently, using the same factors, they measured the exposure directly above the radiographic film with a direct-reading low-energy dosimeter, with the patient removed from the beam. Figure 2 is a plot of the number of exposures measured as a function of exposure in milliroentgens for 866 lateral lumbar spine exposures. It should be nuted that the exposures varied from 200 to 2,600 $m R$ per radiograph. This large range should be uncerstood in terms of the variety of techniques employed by diagnostic radiologists in a lange number of medical

facilities. In spite of this large range, the examining radiologists apparently are satisfied with the quality of the radiographic films which they obtain by use of their various methods. The data show that $75 \%$ of the lateral lumbar spine exposures were below $1,400 \mathrm{mR}$ each. Of the $866 \mathrm{ex}-$ posures, 216 were above $1,400 \mathrm{~m}$.

It was reasoned that, if $75 \%$ of the existing facilities could obtain a clinically acceptable radiograph by exposing the patient to a maximum dose of $1.400 \mathrm{mR}$ per radiograph, then all other facilities should be able to alter their techniques to reduce unnecessarily high radiation exposures. On this basis, a new rule was developed by the State Health Department staff and subsequently approved by the governor-appointed Illinois Radiation Protection Advisory Council. This rule states, "After January 1, 1975, the exposure measured at the tabletop of the technique used for an average adult patient for routine medical radiography will be the following: 'Lumbar spine (lateral) incident exposure shall not exceed 1400 milliroentgens per radiograph and should not exceed 1000 milliroentgens per radiograph. '"

This new approach to the reduction of patient exposure does not interfere with medical practice, except for requiring changes for those practitioners whose procedures result in higher patient exposures than those used by a substantial majority.

We have estimated the impact, in terms of reduced exposure, to Illinois patients due to the implementation of this rule together with companion rules for abdominal, cervical spine, and skull examinations. The estimates were made on the 
basis of U.S. census date for 1970 for the total Illinois population, and of the number of radiographs per year per person for a sample of Illinois for the four examinations in question. The data on the number of radiographs were taken from a report issued by the state of Illinois. 3 Using the exposure distribution of Figure 2 for lumbar spine and Figures 3, 4, and 5 for abdominal, cervical spine, and skull exposures, we estimate that the total exposure received by Illinois residents is as follows:

Lumbar spine 465,000 man-roentgens/year

$\begin{array}{lrl}\text { Abdomen } & 22,800 & " \\ \text { Cervical spine } & 38,600 & " \\ \text { Skull } & 20,800 & "\end{array}$

With the assumption that all techniques were modified to reduce the exposure to the Medical Radiographic Incident Exposure limits, the following figures show the amount by which the exposure to the Illinois patient population has been reduced starting on January 1 , 1975:

$\begin{array}{lrl}\text { Lumbar spine } & 60,000 \pm 20,000 & \text { man-R/year } \\ \text { Abdomen } & 3,600 \pm 1,800 & " \prime \\ \text { Cervical spine } & 1,500 \pm 450 " ~ \\ \text { Skull } & 2,500 \pm 1,500 " ~\end{array}$

The uncertainty in these figures is due to the statistical variation of the number of radiographs in the studies performed.

If the incident exposure had been reduced to less than the limit, a still greater overall reduction would have been achieved. We have also calculated exposure reductions for changes in technique so that those exposures above the incident exposure limits were reduced to the formen average values. These reductions are as follows:

Lumbar spine 100,000 man-roentgens/year

$\begin{array}{lll}\text { Abdomen } & 5,300 & " \\ \text { Cervical spine } & 1,700 & " \\ \text { Skull } & 5,600 & " 1\end{array}$

Further reduction in patient exposure may centainly be possible for other diagnostic procedures as well.
In Implementing these new medical limits, one cannot think in terms of the usual accuracy uncertainties of $\pm 20 \%$ that are in common use in health physics. Since a given medical facility could be shut down if the "she?I limit" were to be exceeded just because of a large measurement uncertainty, it would be unfair to the facility and, in turn, could lead to costly court adjudications. Thus, our system of accuracy in certain radiation protection problems like those described above and oun traceability to national radiation standards should be strengthened.

\section{Therapy}

It has only been in the last ten years that definitive studies were reported in the literature on tumor control probability as a function of total dose delivered to the tumor. Figure 6 shows the tumon control probability for two types of malignancies, squamous cell carcinoma of the supraglottis and Hodgkin's disease. The data are from a recent paper by Goitein 4 in which he discusses the work of Shukovsky ${ }^{5}$ and Kaplan 6 . In radiation therapy, the aim is a high probability of tumor control with as low a level of harmful side effects as possible. In the case of the supraglottis lesion, we see that an increase of only $10 \%$ in the dose delivered increases the tumor control probability from $20 \%$ to $75 \%$. Thus, the treatment dose needs to be selected with great care and treatment needs to be carried out with a high degree of precision and accuracy. There are very few data for other types of tumors that have been analyzed in detail in terms of tumor control probability versus dose, as in the examples above.

Complications from such high doses rave also been studied. In 1975 , Svensson et al. 7 from Sweden published a paper on radiation-induced lesions of the brachial plexus comelated to the dosetime-fraction schedule of a series of patients. Such complications may arise when a cancerocidal dose is delivered to regional lymph nodes in axillary ard supraclavicular field irradiations. The complications are neurologic in nature. The symptoms vary from slight numbness of the firgers to total paresthesia of the arm. The injury to the connective tissue cells in the plexus region is thought to be the cause of the lesions. 
Therefore, the Ellis formula is used as the one parameter in the analysis. This formula gives the Cumulative Raciation Effect (CRE), and takes into account the caily dose in rad, the repetition parameter, the total time for the treatment ir. days, and the total absorbed dose to the plexus for the whole coumse in rad. The results of Svensson et al. are plotted in Figure 6. This figure shows that the frequency of lesions as a function of $\mathrm{CRE}$ rises quite steeply.

The uncertainty of the shape of the frequency-CRE curve is deperdent on dosimetric, anatomic, and statistic factors.

The filled squares in the plot in Figure 6 are from the data of Stoll and Andrews 8 of Australia, for the same complication. The shift in the CRE scale represents a dose shift of $7 \%$. It is righly likely that a large portion of this shift is due to the accumulation of errors in the traceability of radiation standands from the hospital to the national radiation standards of one country, then to the Bureau International des Poids et Mesures, and ther through a second country's national radiation standands to the hospital in that country. As we develop more such data around the world, like those presented by Svensson et al., it becomes imperative that our dosimetry accuracy improve and remain at a high level, and that the uncertainties involved in traceability of actual clinical dose from one center to another become smaller.

The importance of accuracy and traceability cannot be stressed enough.

\section{References}

1. Review of the Current State of Radiation Protection Philosophy in National Council on Radiation Protection and Measurements. NCRP Report No. 43, January 15, 1975.

2. Neuweg, M. E., and Brunner, P. N. Radiation Exposure in the Healing Arts. Applied Radiology, Vol. 3, No. 6, p. 35, November/December 1974 .

3. Comprehensive Radiation Assessment Study, Illinois Department of Health and United States Environmental Protection Agency, Springfield, Illinois, 1975
4. Goitein, M., and Busse, J., Inmobilization Error: Some Theoretical Considerations. Radiology Vol. 117, lio. 2, p. 407. November 1975.

5. Shukovsky, L. J., Dose, Time, Volume Relationships in Souamous Cell Carcinoma of the Supraglottic Larynx. Am. J. Roentgenol. Vol. 108, P. 27, January 1970.

6. Kaplan, H. S., Evidence for a Tumoricidal Dose Level in the Radiotherapy of Hodgkin's Disease. Cancer Res. Vol. 26, p. 1221, June, 1966.

7. Svensson, H., restling, P., and Larsson, L.-G., Radiation-Induced Lesions of the Brachial Plexus Correlated to the Dose-Time-Fraction Schedule. Acta Radiologica, Therapy, Physics, Biology, Vol. 14, No. 3, p. 228, June 1975.

8. Stoll, B. A., and Andrews, J. T., British Med. J., Vol. I, p. 834, 1966. 
DENTAL-BITEWING

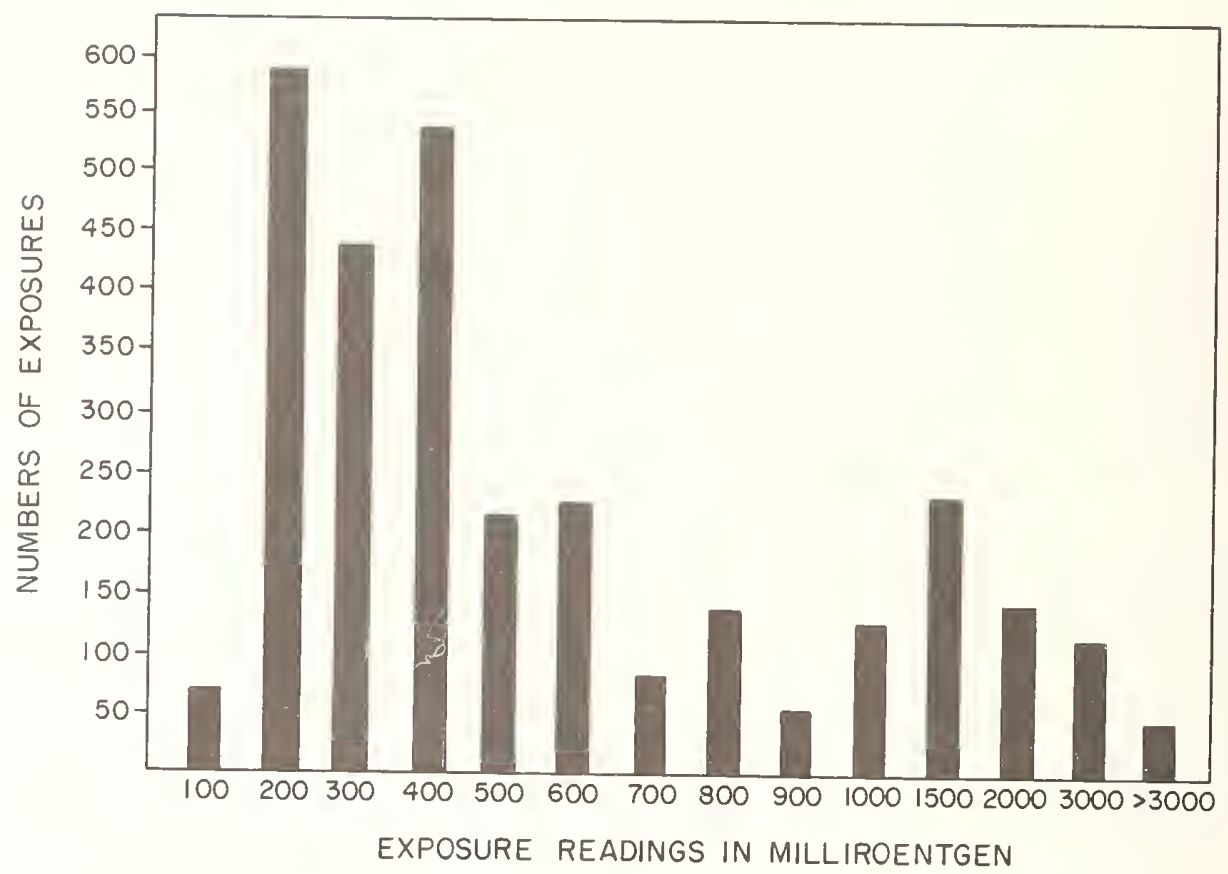

Figure 1

(Ref. 2)

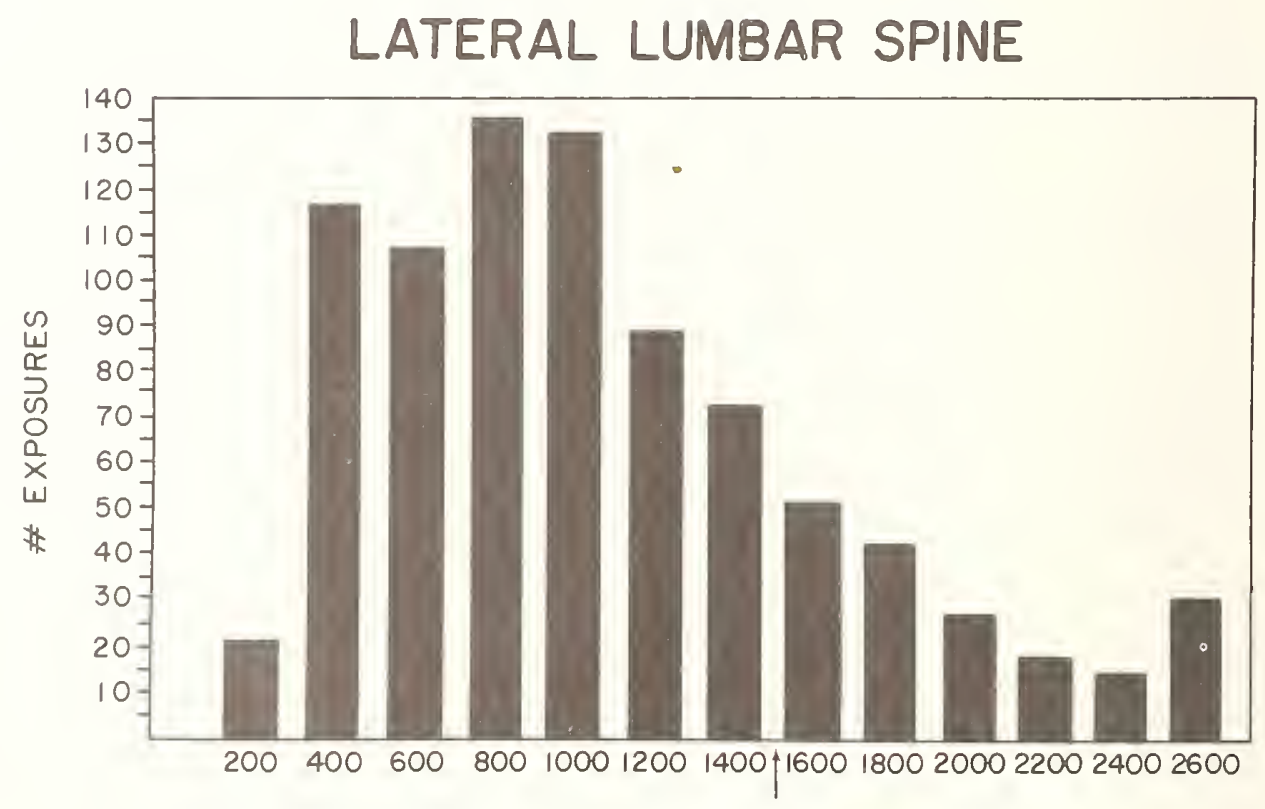

DOSE (MI LLIROENTGENS) MANDATORY LIMIT

Figure 2

(Ref. 2) 


\section{ABDOMEN}

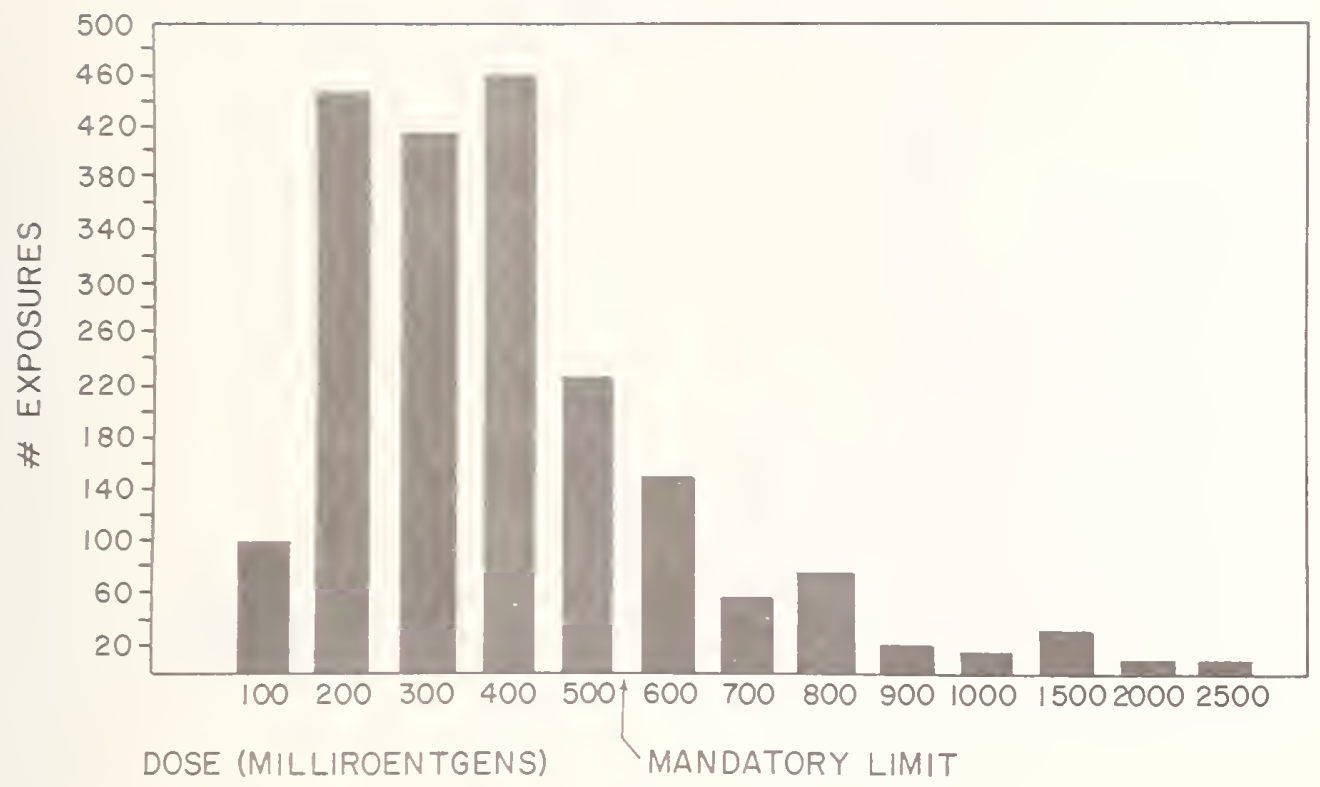

Eigure 3

(Ref. 2)

A.P. CERVICAL SPINE

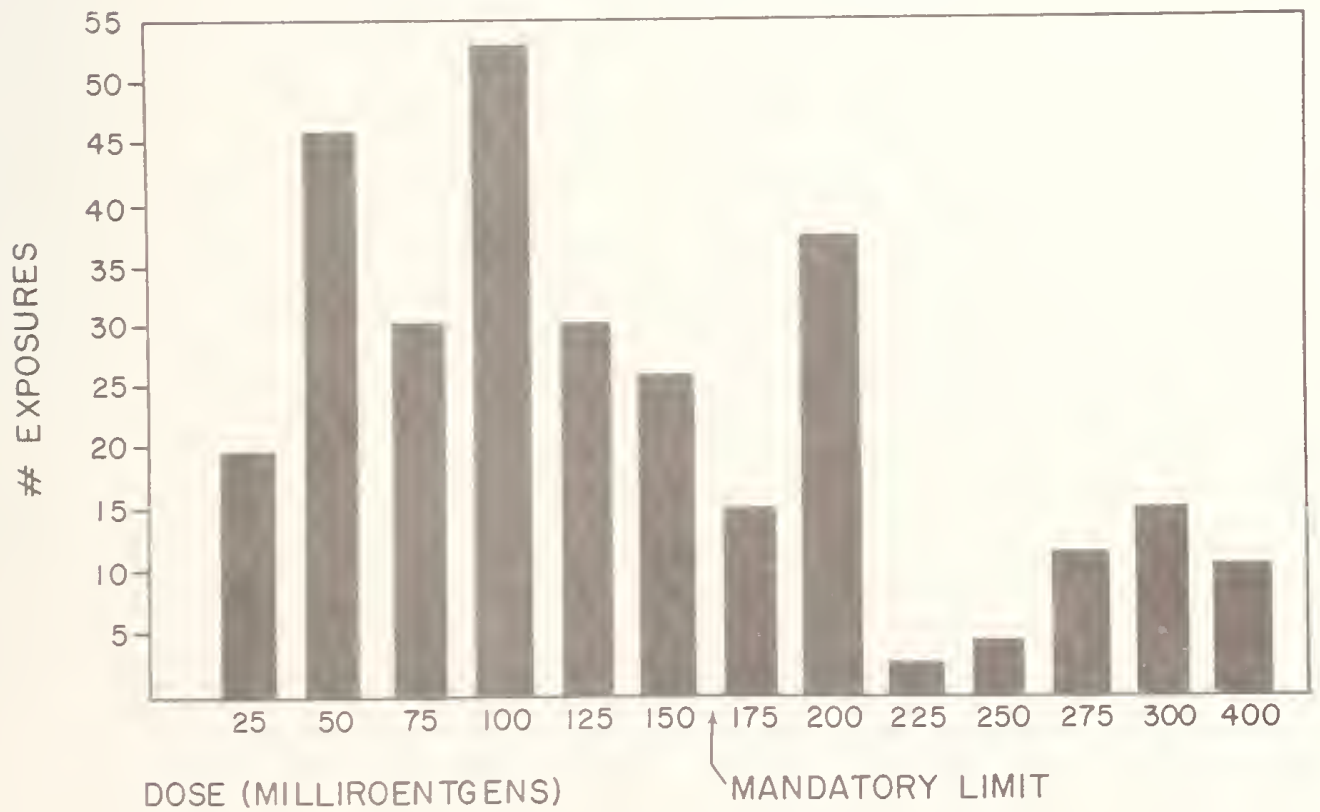

Figure 4

(Ref. 2) 
A.P. SKULL

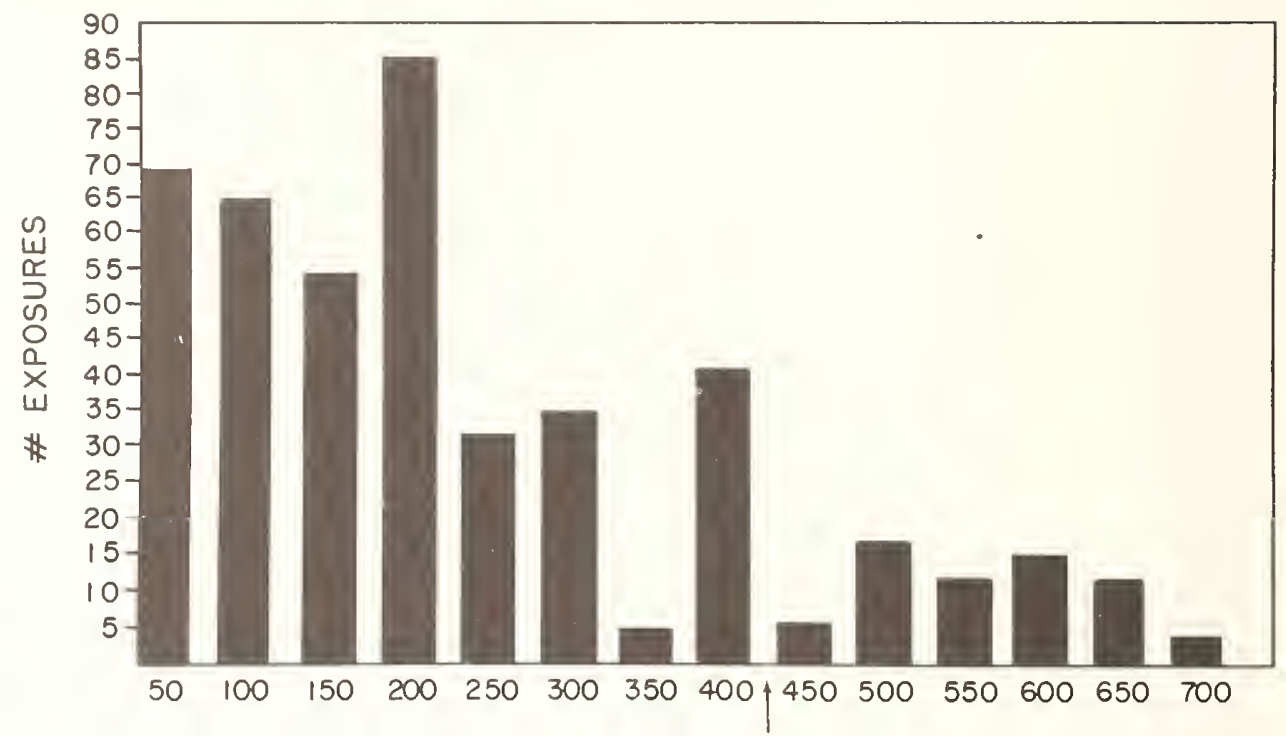
DOSE (MILLIROENTGENS) MANDATORY LIMIT

Figure 5

(Ref. 2)

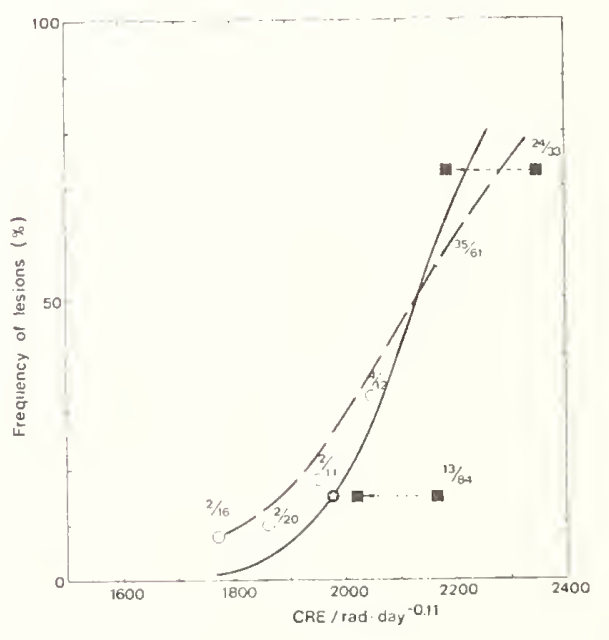

Figure 6

(Pef. 7) 
NATIONWIDE EVALUATION OF X-RAY TRENDS (NEXT)

PROGRAM - AND RADIATION MEASUREMENTS IN THE STATE John W. Shaver

University of California-San Francisco

Radiological Health Sciences Education Project

San Francisco, California 94117

A Task Force of state and Bureau of Radiological Health representatives has applied the "standard man" concept as a method of evaluating the impact of government control efforts on medical x-ray exposure. Exposure data are collected using standardized procedures and equipment, and Organ Dose Index values are obtained for selected critical organs. It is expected that these data can be correlated with governmental agency radiological health activities.

(Diagnostic, nationwide evaluation, physician, physicist, technologist, $x$-ray departments, $x$-ray trends)

An often quoted statistic is that medical and dental x-rays account for approximately $90 \%$ of the man-made radiation exposure to the U.S. population. ${ }^{1}$ This statistic, with which I have no quarrel, is sometimes used to justify or defend public health expenditures in $x$-radiation control activities. Although not generally stated, the implication is that if we in radiation control are effective in our programs, this percentage will be reduced. However, the conclusion can be reached that not only is this ratio reasonable but if we were more effective in all our radiation control efforts, it would probably increase and not decrease. Perhaps this needs explanation.

Medical and dental radiation is virtually the only radiation exposure to the population that is intentional; the rest is an unwanted by-product of the workplace, or from products or operations which utitize ionizing radiations. For example, the exposure to the population from the radioactive effluents and wastes of nuclear reactor operations is unwanted. The exposure of operators to radiation from industrial x-ray defraction units is unwanted. The exposure to operators of industrial radiography units is unwanted and the population exposure from color television sets is certainly unwanted.

ACTUAL
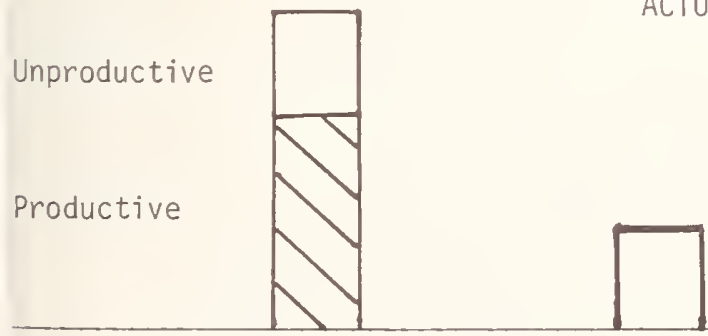

Other Source
However, patient exposure is intentional and essential if diagnostic radiology is to be used. Thus, it stands to reason that the intentional population exposure should far exceed the unwanted exposure. If we could ideally control all manmade exposure, medical and dental exposure should account for $100 \%$ of the man-made radiation exposure and not just $90 \%$. Figure 1 depicts this concept in graphic form. I mention this only because I do not find the use of this particular statistic presented as a "stand alone" statement a logical argument for defense of diagnostic $x$-radiation control budgets or expenditures. Of course, this is not saying that there is no room for improvement in the application of diagnostic radiation, but it is saying that problems must be clearly stated.

The problem as regards population exposure from diagnostic radiology is not that it accounts for $90 \%$ of the total manmade exposure, but rather that a portion of the diagnostic exposure is unproductive and can be reduced. Some have estimated the unproductive component to be on the order of $30 \% .2$ If this is the case, then the argument can be made that diagnostic radiology offers the greatest potential for reducing total population exposure. Figure 1 also shows this concept.

IDEAL 
Identifying problems, determiningtrends, and evaluating progress towards problem reduction were al1 considered in developing the system that I am about to describe. Increasing demands have been placed on government programs to precisely identify problems and then develop and efficiently manage specific programs to deal with the problems. The Conference of Radiation Control Program Directors recognized this coming trend several years ago and in conjunction with the Food and Drug Administration's Bureau of Radiological Health authorized the development of a system which would assist in meeting the demands for better data management. 3 It was in this light that the "NEXT" system was developed. Specifically the Conference called for the formation of a Task Force, in May 1971, to design a uniform program for surveys of x-ray facilities. The Task Force, co-sponsored by the Bureau of Radiological Health, was appointed in July 1971 and consisted of representatives from both state and federal radiological health agencies.

The Task Force adopted the project name of "NEXT", an acronym for Nationwide Evaluation of X-ray Trends. In reviewing its charge the Task Force identified four specific objectives.

1. Identify the optimum components of a radiation survey.

2. Provide a system for uniform data collection.

3. Provide a system to assign program priorities.

4. Provide a system to measure the effectiveness of the program.

To meet these objectives, the Task Force developed two major items:

1. An Optimum Survey Procedures Manual designed to meet, in part, Objectives 1 and 2 , and

2. An Organ Dose Index System (ODIS) designed to specifically meet objectives 3 and 4 .

\section{OPTIMUM SURVEY PROCEDURES MANUAL}

The Procedures Manual, entitled "Suggested Optimum Survey Procedures for Diagnostic X-Ray Equipment" was completed by a subcommittee of the Task Force and distributed in May, 1974. Development of the manual, which took over two years, was complicated in that the details of the Federal Diagnostic X-Ray Performance Standard were not finalized, which in turn complicated the revision of Part F, "Use of $X$-Rays in the Healing Arts," of the Council of State Governments Suggested State Regulations for Radiation Control. Since the procedures contained in the manual were to be sequenced in parallel with the requirements of Part $F$, for the convenience of state personnel, the Subcommittee had to lockstep its work with that of several other groups. As regards the term "optimum," it is applied to indicate that the procedures are not necessarily the most accurate but are a blend of the parameters of accuracy, required equipment, time needed to conduct the test, ease of performing the test in the field and overall costs. The tests were specifically developed with the state field inspector in mind. In many cases alternate test procedures are provided to give each state program as many options as possible to accomplish its work.

The Task Force does not view the Manual as an end point but rather as a starting point. As improved test equipment and procedures are developed, they will be evaluated and incorporated into the manual. As new diagnostic equipment is developed, new test procedures for its evaluation will be added. Furthermore, as the Manual is used, inevitable flaws will be found which will require revision. Nevertheless, the Task Force does modestly submit that the Manual may form the basis for a generally agreed upon "Standard Methods" for the evaluation of diagnostic $x$-ray equipment. The manual is proving helpful as a resource to train new personnel and as a source to refresh one's memory on tests that are infrequently performed. And finally, the Task Force feels that the use of this Manual does assist in meeting Objectives 1 and 2 .

\section{ORGAN DOSE INDEX SYSTEM}

As put forward in Objective 4, states felt an urgency to have available a specific parameter to measure program effectiveness. The days of counting how many facilities have had filtration added to their machines, or how many facilities have had proper collimation installed are over. Budget people are asking what impact your program has on the public's health, and asking that this impact be expressed in meaningful terms. In an effort to be responsive to this request, the Task Force selected radiation organ dose as a parameter which most closely satisfies these requirements. In particular, it was felt that if patient 
skin exposure and gonad dose (both ovarian and testicular), bone marrow and thyroid doses were obtained from statistically representative facilities throughout a state and tracked with time that the effectiveness of the overall radiological health effort could be monitored. This is what NEXT/ODIS is trying to accomplish.

The methodology required to calculate the radiation dose to a specific organ from diagnostic $x$-ray examinations is known. ${ }^{4-7}$ The methodology used in the $X$-ray Exposure Studies of the U.S. in 1964 and 1970, with certain modifications, was adopted by the Task Force for the Organ Dose Index System. As previously mentioned, besides skin exposure, the Task Force requested radiation doses be calculated for the testicles, ovaries, thyroid and bone marrow. Currently, only gonad doses are being calculated; however, the means to compute the other organ doses will be available this year.
To simplify the overall system, only 12 common diagnostic radiographic examinations were selected by the Task Force for inclusion into the system. However, the system is flexible and open-ended and if a need is felt to expand this list, it can be done. For example, as interest in mammagraphy continues to grow, it could be added to the system. The projections included are shown in Table 1. To simplify the dose calculation, a patient having standard anthropometrical characteristics was preselected (Table 1). Using this system the organ dose can be calculated from the following data:

1. Beam quality as measured by the half value layer and kilovoltage

2. Beam size, shape and location, and

\section{TABLE 1 - NEXT ODIS - Diagnostic X-ray Projection and Anthropometric Guidelines}

\begin{tabular}{|c|c|c|}
\hline Projection & $\begin{array}{l}\text { Body Part of } \\
\text { Interest }\end{array}$ & $\begin{array}{c}\text { Thickness } \\
\text { (Centimeters) }\end{array}$ \\
\hline Chest $(P / A)$ & Thorax & 23 \\
\hline Sku11 (Latera1) & Head & 15 \\
\hline $\begin{array}{l}\text { Abdomen (KUB) } \\
\text { Scout Film }(A / P)\end{array}$ & Abdomen & 23 \\
\hline $\begin{array}{l}\text { Retrograde Pyelogram, Scout Film } \\
\text { (Cysto Units) (A/P) }\end{array}$ & Abdomen & 23 \\
\hline Thoracic Spine (A/P) & Thorax & 23 \\
\hline Cervical (A/P) & Neck & 13 \\
\hline Lumbo-Sacral Spine (A/P) & Abdomen & 23 \\
\hline Ful1 Spine $(A / P)$ & $\begin{array}{l}\text { Chest \& } \\
\text { Abdomen }\end{array}$ & 23 \\
\hline $\begin{array}{l}\text { Feet (Weight Bearing) (D/P) } \\
\text { (Podiatrists Only) }\end{array}$ & Foot & 8 \\
\hline Dentai Bitewing (Posterior) & $\begin{array}{l}\text { Left Bicuspids } \\
\& \text { Molars }\end{array}$ & - \\
\hline Dental Periapical & $\begin{array}{l}\text { Central Incisor } \\
\text { (Maxillary) }\end{array}$ & - \\
\hline Dental Cephalometric (Lateral) & Head & 15 \\
\hline
\end{tabular}


3. Machine radiation output using the facility's technique for the selected examination. (Technique including kilovoltage, milliamperage, exposure time and target-to-film distance).

\section{HOW DO YOU IMPLEMENT THE SYSTEM?}

A statistically representative sample of the healing arts $x$-ray facilities in a particular jurisdiction is drawn by the radiological health program. During the inspection of a selected $x$-ray facility, an inspector determines if any of the 12 examinations are performed in the facility of the machine being inspected. If so, he asks the operator to set the technique (milliamperage, kilovoltage, exposure time, targetto-film distance and collimation) that would be used for a patient possessing the standard anthropometrical characteristics discussed above. For example, if a chest P/A is the most frequent examination performed with the machine in question, the operator is asked to set the technique that would be used for a patient with a $23 \mathrm{~cm}$ chest.

The inspector measures and records the needed parameters in accordance with standard procedures that have been developed using a test stand which standardizes field measurement techniques. Since the test stand is the critical component of the survey which fixes geometry and provides for reproductibility and uniformity of test measurements, a brief history of its develop-ment may be of interest. The idea of a test stand was presented to the Task Force in prototype form as the then called "North Carolina Chimney." Since we did not have a shop in North Carolina to construct anyining elaborate, we simply used two different diameter rainfall collection containers, stacked them one on top of the other and surrounded the entire contraption with scrap lead. A slot was cut out of the bottom to permit entry of the sma 11 probe of the Victoreen 666 instrument which was mounted on a special jig to provide for consistent positioning (See Figure 2).

To insure proper credit, the North Carolina Chimney was designed to simulate as best we could the filtration measurement geometry described by Dr. Dale Trout. ${ }^{8}$ We attempted to make his laboratory arrangement suitable for field use. From there, a more refined collapsible stand was constructed by the Bureau of Radiological Health's Northeastern Radiological Health Laboratory in Winchester, Massachusetts which was designed by Alvis Jordans. Later, a rigid stand was developed by the Bureau of Radiological Health's Division of Electronic Products (See Figure 3). I might add that the test stand concept is incorporated in both the Optimum Survey Procedures Manual and in the Bureau of Radiological Health's Routine Compliance Field Tests and that the same test stand can be used for both NEXT and field compliance surveys.

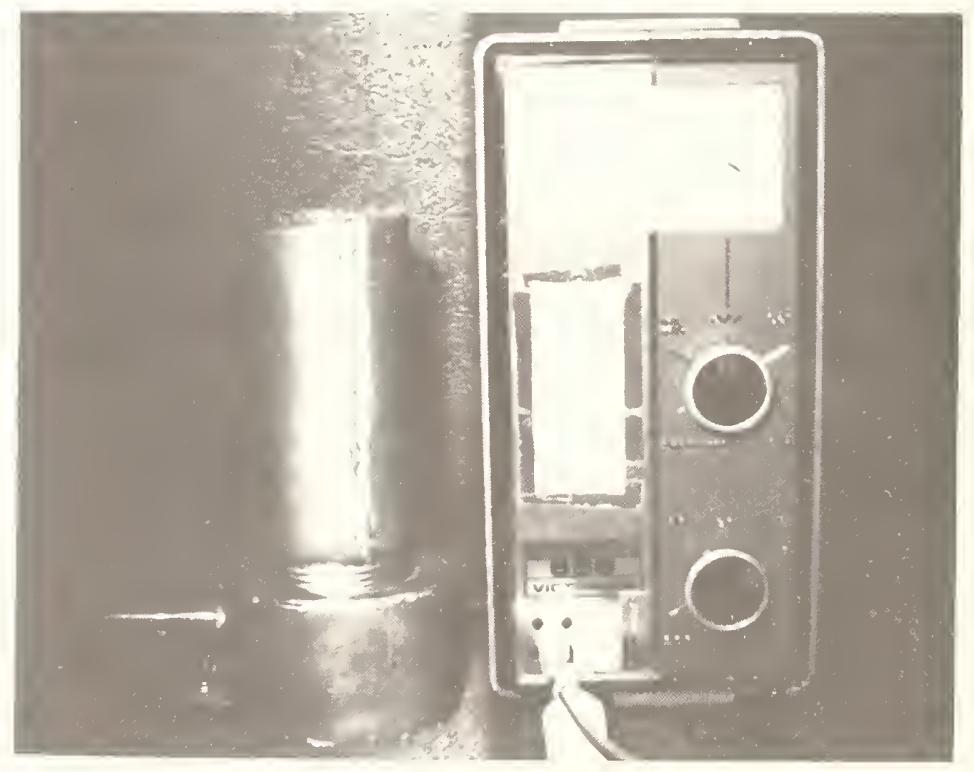

Figure 2: North Carolina Chimney 


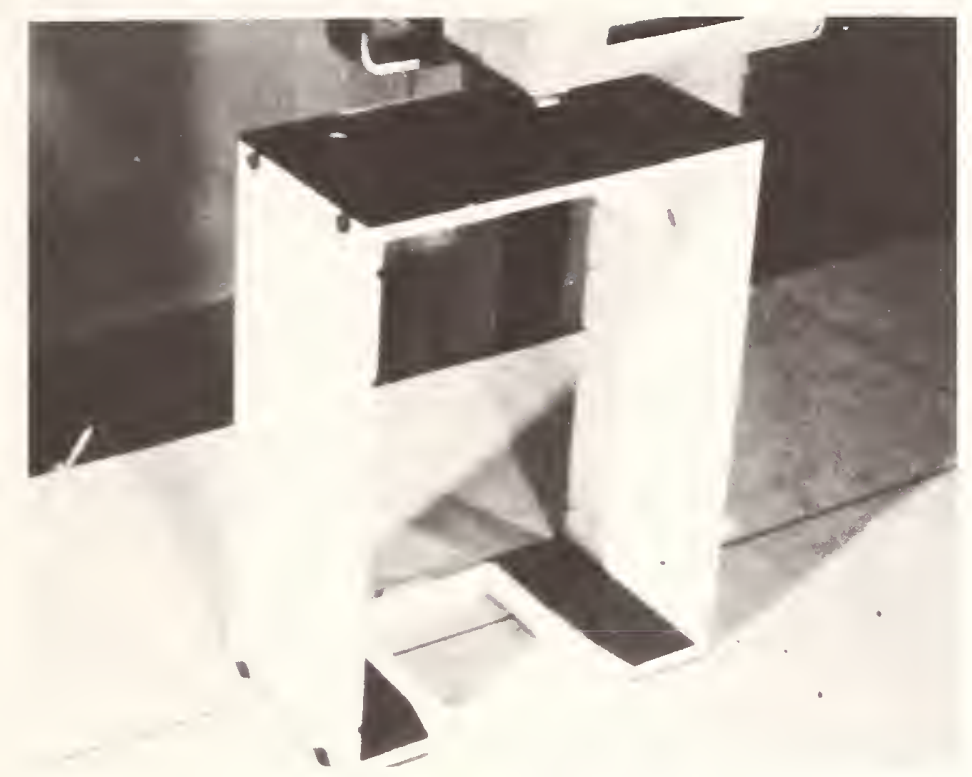

Figure 3: $B R H$ Test Stand

In the case of NEXT, these data (collected by using the test stand) are used to calculate patient exposure at skin entrance and organ dose indices. The term index is used because the dose is calculated for a standard patient possessing fixed characteristics. I emphasize that NEXT/ODIS does not provide estimates of population dose nor does it provide per capita dose information but it does provide information that can be used to effect changes in them.

\section{USE OF THE NEXT SYSTEM RESULTS OF THE DATA}

The most striking results from the NEXT system are the extreme variability of exposures calculated for the same standard patient undergoing the same radiological examination. For example, representative sample data collected in fiscal year 1974-1975 in facilities conducting lumbo-sacral spine examinations shows the following:

1. The Ovarian Dose Index varied from 5 to over 400 millirad.

2. The Testicular Dose Index varied from less that 0.5 to greater than 1000 millirad.

3. The Exposure at Skin Entrance varied from 70 to greater than $3800 \mathrm{mil1i-}$ roentgens with a median value of 560 mil1iroentgens.
4. The source-to-film distance varied from 30 to 75 inches.

5. The milliamperage-seconds varied from 30 to 300 .

6 . The x-ray beam size varied from a rectangular beam $5 \times 9$ inches to a circular beam 36 inches in diameter.

The mean exposure for this examination was recorded as 640 milliroentgens. A1though the lumbo-sacral examination is not performed as often as the chest P/A exam, if a program's objective is to optimize gonad dose, this particular examination stands out among the 12 as an opportunity for improvement. In this regard, a program may wish to concentrate it activities in those facilities conducting the high dose examinations. An initial sample can identify the types of facilities conducting these examinations and follow-up samples can plot the overall program effectiveness. It is hoped that the use of this system can assist program managers in setting more precise priorities and provide the needed data to more efficiently accomplish them. However, I should emphasize the NEXT is simply a planning and evaluation tool. Unless the problems identified by NEXT data are translated into action programs, changes will not occur. 


\section{SUMMARY}

By monitoring the Organ Dose Indices over time, trends in patient exposure can be detected. Comparisons between organ dose indices of participating jurisdictions and the nationwide data can also be made. This system appears to be a usefur people-oriented data item for radiological health agencies to assess program effectiveness and to do program planning. NEXT is certainly not a panacea for evaluating how well we are doing but it does provide a mechanism that can relate our efforts to our overall goal of reducing unproductive radiation exposure.

Thus, from the State program viewpoint, the efforts of this symposium are welcomed. The results will be beneficial if they suggest a means of increasing the accuracy and reliability under the constraints of cost and manpower available to State radiation programs. However, these suggestions should address not only the measurement of radiation exposure, for the measurement of many other parameters is involved in evaluating medical $x$-ray facilities, and all of these must be addressed to arrive at satisfactory measurements for the safe use of radiation.

\section{REFERENCES}

1. U.S. Environmental Protection Agency, Office of Radiation Programs. Estimates of Ionizing Radiation Doses in the United States, 19602000. ORP/CSD 72-1, Page 168.

2. National Academy of Sciences, National Research Counci1. The Effects on Populations of Exposure to Low Levels of Ionizing Radiation, Page 13, November 1972. Report of the Advisory Committee on the Biological Effects of Ionizing Radiations; Division of Medical Sciences; EPA; and BRH. Superintendent of Documents, U.S. Government Printing Office, Washington, D.C. 20402. Stock Number 55505-00005.
3. Third Annual National Conference on Radiation Control, DHEW Publication (FDA) 72-8021, BRH/ORO 72-2 (1971), Pages 324325, National Technical Information Service, Springfield, VA. 22151.

4. Population Exposure Studies Section, Bureau of Radiological Health. Population Dose from X-rays U.S. 1964. Washington, D.C.: Public Health Service Publication No. 2001, 1969.

5. A Panel Presentation by J.N. Gitlin, R.R. Fuchsberg, and R.F. Brown at the 49th Annual Meeting of the American College of Radiology, Miami, Florida April 3-8, 1972.

6. Proceedings. Third Annual National Conference on Radiation Control. Rockvi? 1e: DHEW Publication (FDA) 72-8021 BRH/ORO 722, 1971.

7. Gitlin, J.N., and P.S. Lawrence. Population Exposure to X-rays U.S. 1964. Washington, D.C.: Public Health Service Publication No. 1519.

8. Trout, E. Dale; J. Kelley, and A. Lucas. Determination of half-value layer. American Journal of Roentgenology 84: $729,1960$. 
LOW LEVEL ENVIRONMENTAL RADIATION MEASUREMENTS WITH A PLASTIC SCINTILLATION DETECTOR SYSTEM

A. P. Hull and G. S. Levine Brookhaven National Laboratory

Upton, New York 11973

A plastic scintillation detector-photomultiplier combination, operated in the current mode, has been developed at Brookhaven National Laboratory for the measurement of low level environmental radiation. Its output is digitized and processed by semiconductor circuits, which also provide for hourly compensation of variation in gain utilizing a built-in calibration source.

The instrument has been tested in the field for the past two years to establish its response capability to diurnal and longer term variation in background radiation, as well as small increments produced by a distant $\gamma$-field irradiation source. (Supported by U.S. ERDA.)

(Low level environmental radioactivity measurements scintillation detector)

\section{Introduction}

Iife has evolved and exists in a constant low level of external background radiation. Although this has been known since the discovery of natural radioactivity, the accurate determination of its levels did not become a matter of widespread interest until the development of nuclear reactors, and the consequent possibility of measurable dose increments to large populations from manmade radioactivity. The adoption by the Nuclear Regulatory Commission ${ }^{1}$ of $5 \mathrm{mrem} / \mathrm{yr}$ dose limit as an "as low as practicable" design objective for light water-cooled power reactor effluents has stimulated an interest in the accurate measurement of very small increments to background levels.

Until recently, ion chamber-electrometer combinations were the most suitable instruments for making low level measurements of environmental radiation. A simple 6 liter unpressurized bakelite wall ion chamber with a dynamic condenser electrometer (DCE) was developed at Brookhaven National Laboratory in the late $1940^{\prime} \mathrm{s}$. A number of these instruments were used for two decades in the field for the continuous measurements of the dose from the $41_{\text {Ar }}$ in the plume from the air cooled Brookhaven Graphite Research Reactor. 2 They were capable of detecting changes in radiation levels of about $1 \mu \mathrm{R} / \mathrm{hr}$, and of establishing yearly dose increments (above background) of about $5 \mathrm{mR}$. A somewhat more sensitive pressurized version, with a stainless stee1 wall, has been developed by the Health and
Safety Laboratory. 3 A commercial version is currently in wide use, particularly to estab1ish the sma11 environmental dose increments occasioned by the short-lived noble gases in the air effluents from commercial power reactors. Various devices, Geiger-Müller (GM) counters, film and thermoluminescent dosimeters (TLD) have also been employed. Unless specially shielded, the GM tube's variable energy response complicates the dose interpretation of its signal. Film is not a satisfactory environmental detector, due to its limited sensitivity $(\sim 20 \mathrm{mR})$ and vulnerability to temperature and humidity. Although several currently used TLD's are sensitive to doses as low as $1 \mathrm{mR}$, as passive devices they do not lend themselves readily to the measurement of plant effluent related doses of a few mR/yr in the presence of naturally occurring levels in the order of $100 \mathrm{mR} / \mathrm{yr}$.

At Brookhaven, a plastic scintiljator photomultiplier combination has been developed as a replacement for the ion chamber DCE. ${ }^{4}$ It provides for a high sensitivity with a small detector volume, $\beta+\gamma$ or $\gamma$ only response, an air equivalent energy response, and a high signal to inherent noise ratio. The D.C. output of the PM is digitized and processed by semiconductor circuits, hence it is a digital environmental monitor (DEM). It contains a built-in calibrator, which is utilized on an hourly basis to compensate for the temperature related changes in the gain characteristic of the photomultiplier. 
During the past two years the DEM has been operated side by side with the BNL ion chamber DCE (Figure 1) and TLD devices, measuring perimeter doses, and in other applications requiring great sensitivity. Results from this experience are described in the present report.

\section{Instrument}

The DEM is a self-contained, semiportable, line-powered low-level radiation detection system, with automatic standardization (Figure 2). The detector portion consists of a $2^{\prime \prime} \times 2^{\prime \prime}$ diameter tin loaded plastic scintillator (NE-106) coupled to a low noise photomultiplier (EMI $9635 \mathrm{~A}$ or $9750 \mathrm{~B}$ ) operated in the D.C. mode (Figure 3). The signal current is converted to pulses which are counted and recorded, repetitiously, by a digital printer for selected intervals between 1 and $16 \mathrm{hr}$. Internal standardization with an alpha source $\left({ }^{238} \mathrm{Pu}\right)$ is performed hourly to compensate for temperature effects on the gain characteristics of the photomultiplier. The detector section of the instrument is separate from the remainder of the instrument. In the DEM's which have been field tested to date, the detectors have been housed in an aluminum container whose wall thickness varies from $0.86-2.57 \mathrm{~g} / \mathrm{cm}^{2}$, thus making them essentially beta opaque.

\section{Calibration}

The instruments are periodically calibrated with a ${ }^{60}$ Co source mounted in a holder precisely located with respect to the detector so that the dose rate at the crystal is $5.42 \mu \mathrm{R} / \mathrm{min}(5 / 74)$, significantly greater than background, but well within the range capabilities of the instrument. Table 1 summarizes a number of calibrations for various individual instruments.

\section{Field Measurements}

Figure 4 illustrates the hourly dose recorded by a DEM located in a perimeter monitoring station adjacent to the BNL Ecology Forest ( $815 \mathrm{~m}$ distant). The local biosphere is exposed to the "skyshine" radiation of a $6350 \mathrm{Ci}{ }^{137} \mathrm{Cs}$ source for 20 hours each day. The source is lowered into its shield for 4 hours alternate mornings and afternoons. This near unique situation demonstrates several of the instrument's capabilities.

The diurnal pattern and the day to day variations while the source is exposed results from the temperature dependent density of the air scattering medium between the source and the monitoring station. The average background level, as established from the trough of the period when the source is down, was $6.3 \mu \mathrm{R} / \mathrm{hr}$. It is essentially constant except for two days, $12 / 25$ and $12 / 30$ during which precipitation occurred. The average source radiation increment, as determined from the difference between the adjacent "source up" and the "background" periods was $9.1 \mathrm{kR} / \mathrm{hr}$.

Comparisons with the other low level measurement devices operated side by side over time with the DEM at this location have revealed some systematic differences which imply that the DEM underestimates the true environmental dose by about $20 \%$. Attenuation and absorption in the enclosure and under response to the cosmic ray contribution by the plastic detector are possible explanations.

A DEM was recently used to evaluate the impact of the installation of a more intense $137 \mathrm{Cs}$ source in our calibration laboratory. A collimated $3 \mathrm{Ci}$ source, used to calibrate personnel dosimetry film, was deemed insufficiently intensive for high dose exposures. Measurements were made with the DEM to delineate the anticipated increase in facility background expected from a more intense source. The DEM, being reasonably portable, was set up, took baseline data with the source removed over the weekend, and because of its great sensitivity, provided the necessary information in several hours. The incremental addition, due to the present source, was found to be 31.1 at the point where personnel operate the free air sources during instrument calibration, and at worst $0.5 \mu \mathrm{R} / \mathrm{hr}$ in the repair area where the occupancy factor is highest.

\section{Conclusions}

The field testing has demonstrated that an instrument utilizing a scintillator, phototube detection scheme is a useful instrument for the measurement of background environmental radioactivity and small increments thereto. 


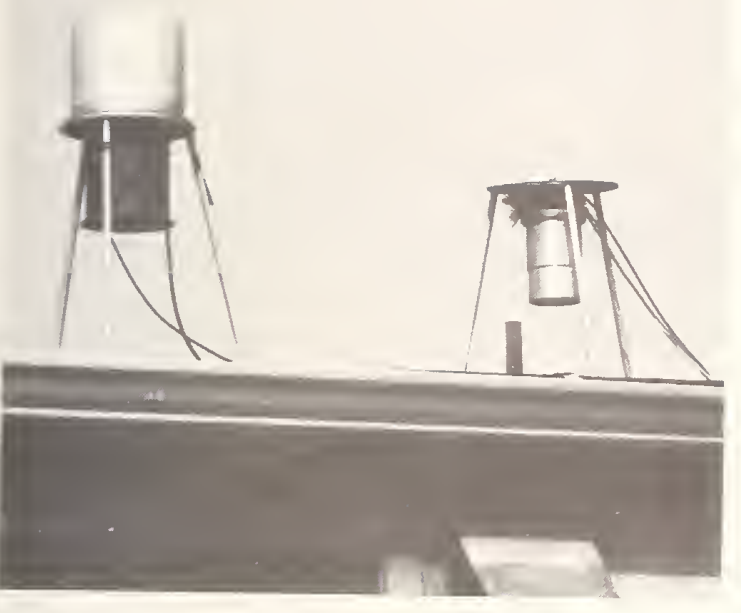

Figure 1 DCE \& DEM Perimeter Monitors

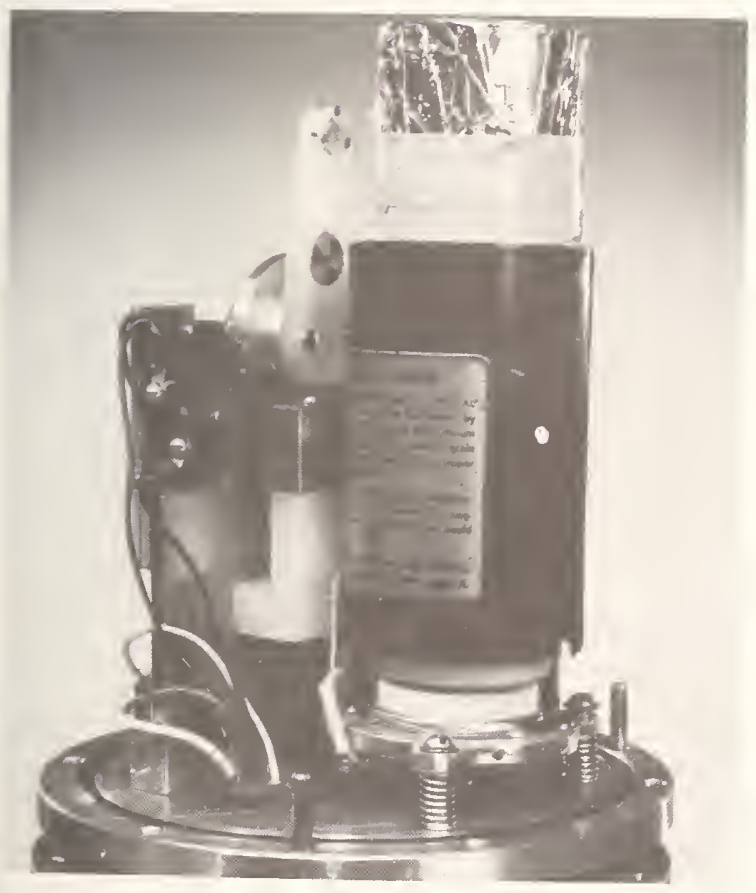

Figure 3 DEM Scintillator, Photomultiplier and Standardizing Source

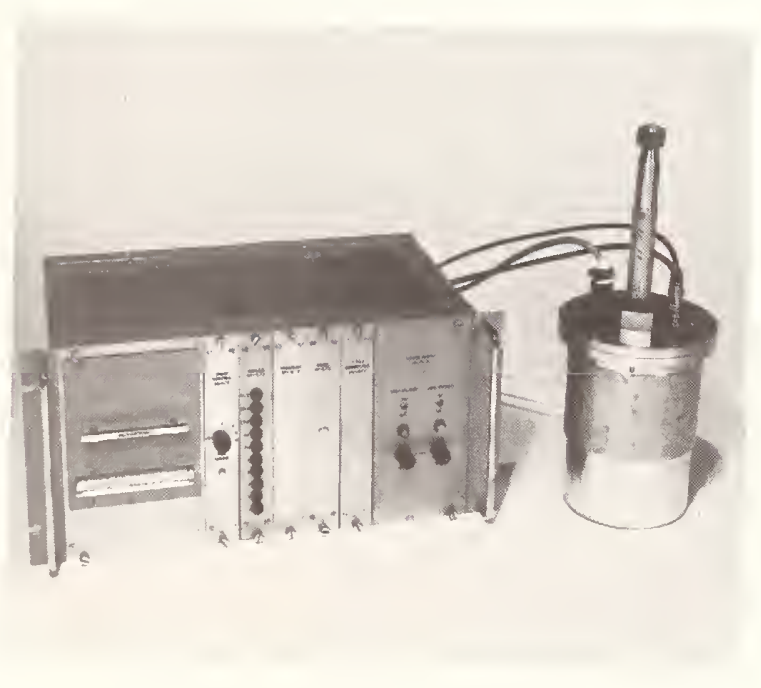

Figure 2 DEM Electronics \& Detector 
HOURLY INCREMENTAL DOSE

AT P-9 MONITORING STATION DEC.1974

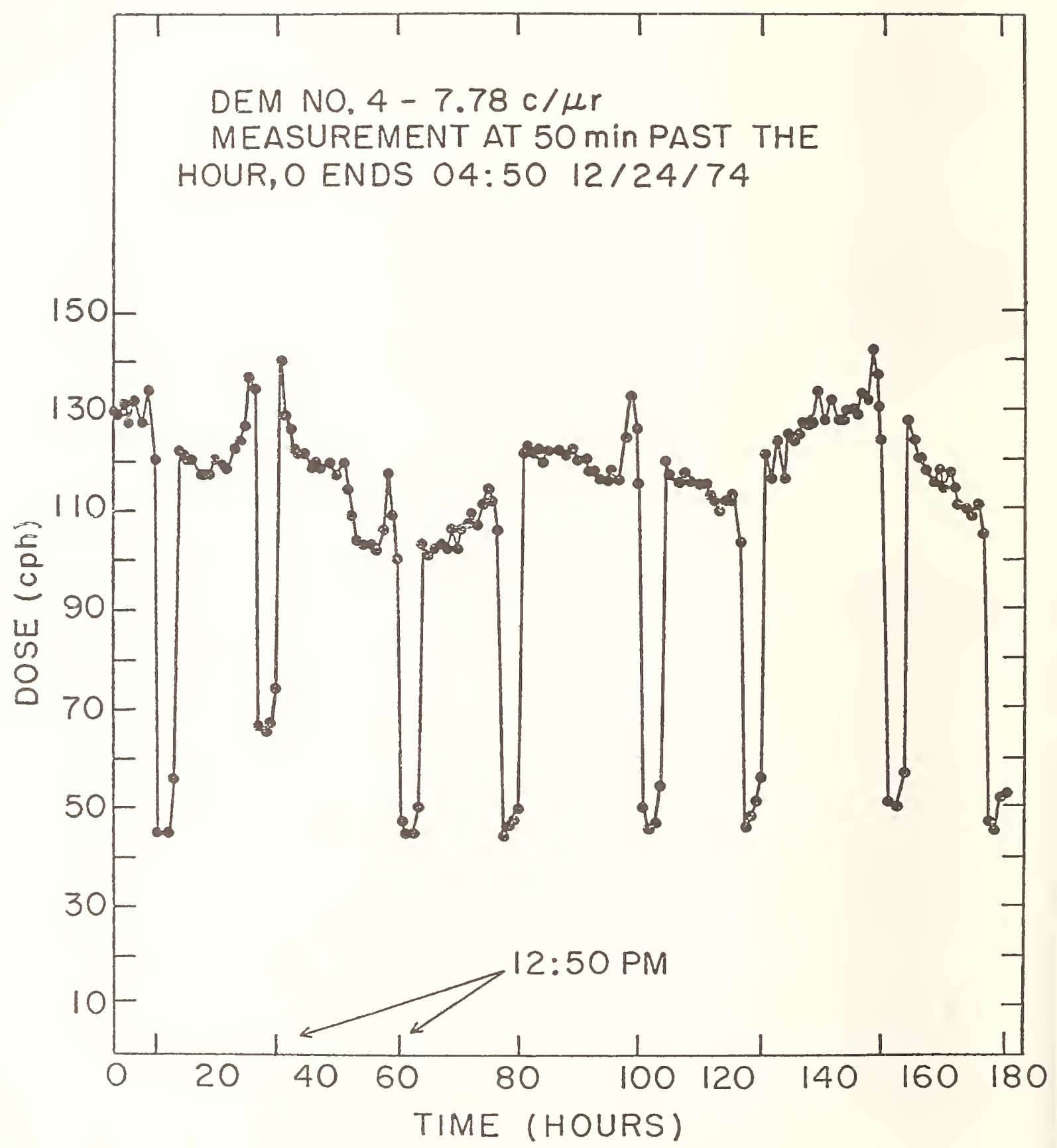

Figure 4 
1. U.S. Nuclear Regulatory Commission, "Opinion of the Commission", Docket RM 50-2 (4/30/75).

2. M.M. Weiss, "Area Survey Manual", Brookhaven National Laboratory Report BNL-344 (1955).

3. H.L. Beck, et a1., "Final Report on Continuous Monitoring with High Pressure Ionization Chamber", Health and Safety Laboratory Report HASL-290 (1975).

4. J.D. Chester, R.L. Chase, S. Woods, "A Digital Monitor", Brookhaven National Laboratory Report BNL-16922 (1972).

Table 1

DEM Calibrations

\begin{tabular}{|c|c|c|c|c|c|}
\hline Unit & Date & Source & Distance $(\mathrm{cm})$ & $c / \omega r$ & Notes \\
\hline 1 & $8 / 5 / 75$ & $137-\mathrm{Cs}$ & 55 & 9.9 & \\
\hline 1 & $8 / 5 / 75$ & $137-\mathrm{Cs}$ & 55 & 9.5 & $\begin{array}{l}\text { Additional collar } \\
\text { around scint. }\end{array}$ \\
\hline 2 & $12 / 17 / 74$ & $60-\mathrm{Co}$ & 55 & 7.15 & \\
\hline 2 & $1 / 24 / 75$ & $60-\mathrm{Co}$ & 55 & 7.36 & \\
\hline 2 & $4 / 5 / 75$ & $60-\mathrm{Co}$ & 55 & 6.35 & \\
\hline 4 & $5 / 6 / 74$ & $60-\mathrm{Co}$ & 55 & 9.39 & \\
\hline 4 & $5 / 7 / 74$ & $60-\mathrm{Co}$ & 55 & 9.50 & \\
\hline 4 & $7 / 2 / 74$ & $60-\mathrm{Co}$ & 200 & 8.95 & \\
\hline 4 & $9 / 11 / 74$ & $60-\mathrm{Co}$ & 55 & 8.58 & \\
\hline 4 & $9 / 16 / 74$ & $60-\mathrm{Co}$ & 55 & 8.60 & \\
\hline 4 & $1 / 24 / 75$ & $60-\mathrm{Co}$ & 55 & 7.78 & \\
\hline 4 & $4 / 29 / 75$ & $60-\mathrm{Co}$ & 55 & 8.43 & \\
\hline 4 & $11 / 5 / 75$ & $60-\mathrm{Co}$ & 55 & 8.71 & \\
\hline 4 & $11 / 18 / 75$ & $60-\mathrm{Co}$ & 55 & 8.37 & \\
\hline 4 & $11 / 25 / 75$ & $60-\mathrm{Co}$ & 55 & 8.10 & \\
\hline 4 & $11 / 25 / 75$ & $137-\mathrm{Cs}$ & 55 & 9.63 & \\
\hline 4 & $12 / 30 / 75$ & $60-$ Co & 55 & 8.03 & \\
\hline 4 & $12 / 30 / 75$ & $137-\mathrm{Cs}$ & 55 & 9.38 & \\
\hline 4 & $2 / 4 / 76$ & $60-\mathrm{Co}$ & 55 & 8.37 & \\
\hline 4 & $2 / 19 / 76$ & $60-\mathrm{Co}_{0}$ & 55 & 8.69 & \\
\hline 4 & $2 / 19$ & $137-\mathrm{Cs}$ & 55 & 9.70 & \\
\hline 5 & $2 / 7 / 75$ & $60-\mathrm{Co}$ & 55 & 11.85 & Unit has double scint. \\
\hline 5 & $11 / 3 / 75$ & $60-\mathrm{Co}$ & 55 & 14.62 & \\
\hline
\end{tabular}





\section{Introduction}

The analysis of environmental samples for radionuclides is fast becoming a large segment of the gamma-ray analyses performed. Many research laboratories and routine testing stations encounter a large number of samples. Some of these are relatively complex. In this paper we are concerned with the physical parameters of the measuring system; that is the characterization of the detector, the sample-detector relationship and the multichannel analyzer (analog to digital converter) and how these characteristics affect the accuracy of the measurement.

The counting sensitivity of a system can be used as a figure of merit for that system and can be defined as the minimum detectable limit or minimum acceptable limit. There are five factors affecting the sensitivity of a system. These are: the background, the system energy resolution, the detector efficiency and sample shape, the time allotted for the measurement, and the accuracy required for the result. We will discuss these parameters and their measurement and how they affect sensitivity for a series of standard germanium detectors.

Definition of Sensitivity

The definition of the sensitivity limits of a spectrometer have been previously described ${ }^{2}{ }^{3}$ in some detail and hence are only outlined here. In brief, two definitions are used. The minimum detectable activity is reached when the peak of interest is just observable above the interfering counts. This point has been previously determined ${ }^{3}$ when the peak standard deviation is one third that of the peak area. However, in order to give clarity to this study, and to present conservative data, this point was defined when the standard deviation of the peak area was one fifth that of the peak area. The minimum acceptable activity is reached when the experimentalist is just able to achieve the statistical precision required for a specific experiment.
It has been shown that either of the above sensitivity definitions is described 23 as:

$$
A e=\frac{1}{\varepsilon C T}\left(2 B+\frac{1}{2 C^{2}}+\frac{1}{2 C}\left(8 B+\frac{1}{C^{2}}\right)^{\frac{1}{2}}\right) \frac{1}{2}
$$

Where $A e=$ minimum detectable or acceptable activity (in gamma per min. emitted from the sample).

$\varepsilon=$ absolute full energy peak efficiency for that sample (count rate of peak of energy $\varepsilon$, divided by gamma rays of energy $\varepsilon$ emitted per unit time from the sample).

$C=$ Statistical precision of the peak area (standard deviation of peak area divided by the peak area).

$B=$ Total interfering counts under peak (these counts include both environment and sample generated scatter counts). Energy resolution is also incorporated in this parameter.

$T=$ Count time.

$T$ and $C$ are set by the experimentor whereas $\varepsilon, B$ and the energy resolution are determined by the construction of the spectrometer. Ae is determined for a spectrometer by substituting values for the counting parameters into the above equation.

\section{Background}

There are two groups of applications whose background contributions are sufficiently different to require separate consideration. These groups are separated by the origin of the background counts under the peak. In the first groups, the interfering background is due to the environment and in the second the interfering continuum has significant sample generated contributions.

The natural background can be reduced by placing the detector in a shield. 
Figures 1 and 2 show the background inside and outside the shield for 60 and $90 \mathrm{cc}$ standard detector in a $10 \mathrm{~cm}$ lead shield. The background is characterized in Tables 1 and 2.

The data in Tables 1 and 2 along with the energy resolution of the detector, $c=0.2$ and $T=40,000$ seconds (11.1 hours) yield the sensitivity for the detector both inside and outside the shield. This is shown in columns 1 and 2 of Table 3 . Column 9 shows the increase in sensitivity due to the shield.

Columns 4 and 5 show the change in sensitivity for lower energies when the sampie contains an intense high energy peak. As expected, the change is most pronounced at the lower energies (factor of 1.81 reduction in sensitivity), but is also seen at the higher energies (1.17).

Table 2 shows a list of peaks commonly found in the background spectrum. If the peak of interest coincides with one of the background peaks, then the background peak area must be accounted for in calculating the sensitivity. As a general rule, the sensitivity is reduced by a factor of two, if one of the interfering peaks is encountered.

\section{Energy Resolution}

The energy resolution of a system consisting of a detector, amplifier and multichannel analyzer is affected by every component in the system, how the resolution is calculated and how the data are collected. The energy resolution affects the analysis of the data in two ways: 1) the ability to separate two adjacent peaks is dependent on the width of the peaks and 2) the minimum detectable limit is dependent on the background under the peak, which is dependent on the peak base width.

In order to observe the influence of system energy resolution on the spectral statistical analysis, the experimental configuration in Figure 3 was used. This configuration allows the resolution to be broadened independent of the other counting parameters. The detector is a germanium detector with a resolution of $1.65 \mathrm{keV}$ FWHM at $1.3325 \mathrm{MeV}$. This resolution was measured with more than 20 channels in the peak and the FWHM was calculated from a peak with more than 10,000 counts in the peak channel. The following method was used for all the FWHM calculations: the contents of the maximum channei is divided by two to yield the half maximum. The full width points are found by linear interpolation between the contents of the channels surrounding the half maximum point.

The process of digitization in the analog-to-digital converter yields a set of numbers that represent the total number of pulses that lie between the lower and upper bounds of each channel. The numbers do not represent the average amplitude within each channel. As a consequence of this, the measured full-width-half-maximum is a function of the position of the true peak centroid relative to the channel boundaries. Figure 4 shows the variation of measured FWHM as a function of peak centroid position. As is expected, the curve is symmetrical about the midpoint between the channel boundaries and the resolution or FWHM broadens at the midpoint.

In addition, the number of channels or data points in the peak have an effect on the FWHM measurement. Figure 5 shows the broadening of the FWHM's as a function of energy/channel. The curves are steepest for the lowest resolution detector, that is, the smaller the FWHM the greater the broadening with increasing energy per channel. The energy scale corresponds to $750 \mathrm{keV}$ full scale in a 4096 channel analyzer at the low end to $4 \mathrm{MeV}$ full scale at the high end. As can be seen, the $1.65 \mathrm{keV}$ detector gives a resolution of 1.95 at $1 \mathrm{keV}$ per channel, while a 2.07 keV detector degrades to 2.32 $\mathrm{keV}$ and $2.99 \mathrm{keV}$ detector degrades to 3.02 keV. This indicates for many systems the detector resolution is not the limiting factor in determining the sensitivity of the system. The effect of energy resolution on the peak area statistical precision is shown in Figure 6. Figure 7 shows a sample spectrum used for this determination.

The spectrum in Figure 7 was analyzed when the energy resolution of the system was $1.7 \mathrm{keV}$ FWHM at $1.33 \mathrm{MeV}$. At $185 \mathrm{keV}$, the statistical accuracy to one $\sigma$ of the peak area was $\pm 1.67 \%$ and at $1.33 \mathrm{MeV}$ was $1.76 \%$. The energy resolution was successively degraded in steps using the techniques shown in Figure 3. At an energy resolution of $2.81 \mathrm{keV}$ at 1. $33 \mathrm{MeV}$, the statistical accuracy had been degraded to $1.80 \%$ for the $185 \mathrm{keV}$ peak and $1.96 \%$ for the $1.33 \mathrm{MeV}$ peak. These degradations are small compared with the energy resolution broadening. Similar degradations were observed for other peaks in the spectrum, some of which involved near doublet situations. 
The sample shape or the sample-detector geometry contributes significantly to the detectable limit. Three geometries were used to show this effect. These geometries were chosen because they maximize detection capability and are shown in Figure 8 . Geometry a) shows a disc of approximately $3 \mathrm{~cm} \mathrm{diam-}$ eter having a volume of $5 \mathrm{ml}$. Geometry b) shows the measurement made for the disc paralleling two detectors. Geometry c) shows the use of a $500 \mathrm{ml}$ Marinelli beaker placed over the end cap. Both disc and Marinelli beaker geometries of other dimensions are available, but the data in this paper can be extrapolated to those geometries.

Efficiencies were determined for the different geometries by filling them with a uranium ore standard which simulated the efficiencies for a typical soil sample. Measurement of liquid samples in the same geometries would show improved count rates due to the lower density and mean atomic number of the liquid. As a check upon the calibration of the ore, the disc sample was cross checked with known Cs 137 and Co60 sources. The ore samples provide an energy calibration from $185 \mathrm{keV}$ to $2614 \mathrm{keV}$. Figure 9 shows a typical spectrum measured for the Marinelli beaker with a $90 \mathrm{cc}$ detector.

Table 4 shows the efficiencies measured for $60 \mathrm{cc}$ and $90 \mathrm{cc}$ for these geometries at several energies. As seen in the Table, there is a large difference in efficiency among the geometries.

To compare the effectiveness of the $60 \mathrm{cc}$ and $90 \mathrm{cc}$ detectors, their efficiency ratios for the same sample geometry were determined as a function of energy and are shown in Table 5. At $185 \mathrm{keV}$, the ratio of the $60 \mathrm{cc}$ and $90 \mathrm{cc}$ for the disc source is approximately 1.1 . Since at this energy $75 \%$ of the incident photon beam interacts primarily within the first $11 \mathrm{~mm}$ of the detector, this ratio represents the difference in solid angle between the two detectors. At $2204 \mathrm{keV}$ this ratio is 1.34 and represents the combination of solid angle and detector dimension. It is important to note that these ratios represent solid angle differences and not detector quality differences.

It is interesting to note that the Marinelli efficiency ratios are greater than disc efficiency ratios, representing greater utilization of the sensitive germanium available. The ratio, however, reaches only 1.5 (the sensitive volume ratio of the two detectors) at energies in excess of $2 \mathrm{MeV}$, indicating that the volume of a large detector is only fully utilized for one sample geometry and energy range. In view of this fact, the optimization of a detector system requires that attention be placed upon solid angle considerations as well as detector size.

Referring back to Table 3, we see that the minimum detectable limit (in gammas/min) for the Marinelli beaker is higher than for the disc sample. Column 10 shows the ratio of $\mathrm{Ae}$ for disc sample and the Marinelli sample. Table 6 shows the minimum detectable limit expressed as gammas $/ \mathrm{min} / \mathrm{ml}$ of source for the disc, Marinelli and for the Marinelli volume reduced to a $5 \mathrm{ml}$ disc (approximate 100:1 volume reduction). These data indicate that the most sensitive geometry is the reduced sample and it is approximately 2.5 times as sensitive as the Marinelli beaker. However, the Marinelli geometry is approximately 40 times as sensitive as the unreduced disc sample.

Some samples cannot easily be reduced (soils, ores, ashes), and in some cases the number of samples prohibits reduction so the Marinelli beaker geometry represents an easy method for increasing sensitivity.

\section{Conclusion}

The general conclusion from this work is that standard detectors, shields, electronics and sample geometries provide sensitivities adequate for a large majority of the current environmental uses. In order to improve the sensitivity for a system, large expenditures are necessary in order to make small advances in $A$ e. For example, in the energy range near $200 \mathrm{keV}$ a $60 \mathrm{cc}$ detector degrades in uncertainty as the FWHM broadens. The uncertainty changes from $1.67 \%$ to $1.71 \%$ as the resolution changes from $1.7 \mathrm{keV}$ to $2.0 \mathrm{keV}$. There is a significant cost difference between a $1.7 \mathrm{keV}$ and $2.0 \mathrm{keV}$ resolution detector.

1) Wal ford, G.V., Al i aga-Kelly, D.T.W., and Gilboy, W.B., IEEE Transactions on Nuclear Science, NS-19, No. 1, Feb. 1972, p.127.

2) J.A. Cooper, NIM 82 (1970) 273.

3) G.V. Walford, and W.B. Gilboy, The Natural Radiation Environment 11, Houston, TX (1972) ERDA Conf. 72085-P1 135. 
TABLE 1

TYPICAL BACKGROUND CONTINUA OBTAINED FOR THE

60CC AND 90CC DETECTORS

\begin{tabular}{|c|c|c|c|c|c|c|}
\hline $\begin{array}{c}\text { Nominal } \\
\text { Energy }\end{array}$ & $\begin{array}{l}\text { 60cc No } \\
\text { Shield } \\
\text { cpm/keV }\end{array}$ & $\begin{array}{l}\text { 60cc With } \\
\text { Shield } \\
\text { cpm/keV }\end{array}$ & $\begin{array}{l}\text { Background } \\
\text { Reduction } \\
\text { Factor }\end{array}$ & $\begin{array}{l}\text { 90cc No } \\
\text { Shield } \\
\text { cpm/keV }\end{array}$ & $\begin{array}{l}\text { 90cc With } \\
\text { Shield } \\
\text { cpm/keV }\end{array}$ & $\begin{array}{l}\text { Background } \\
\text { Reduction } \\
\text { Factor }\end{array}$ \\
185 & 4.5 & 0.053 & 85 & 6.1 & 0.063 & 97 \\
609 & 0.34 & 0.013 & 26 & 0.53 & 0.017 & 33 \\
1238 & 0.12 & 0.0055 & 21 & 0.16 & 0.0059 & 29 \\
1764 & 0.014 & 0.0022 & 7 & 0.022 & 0.0030 & 7 \\
2204 & 0.012 & 0.0017 & 6 & 0.022 & 0.0023 & 9 \\
\hline
\end{tabular}

TABLE 2

TYPICAL BACKGROUND PEAKS OBTAINED FOR THE

$60 \mathrm{CC}$ AND $90 \mathrm{CC}$ DETECTORS

\begin{tabular}{|c|c|c|c|c|c|}
\hline Energy & Isotope & $\begin{array}{l}\text { Peak Count } \\
60 c c \text { No Shield } \\
\text { cpm }\end{array}$ & $\begin{array}{l}\text { Peak Count } \\
60 \mathrm{cc} \text { Shield } \\
\text { cpm }\end{array}$ & $\begin{array}{l}\text { Peak Count } \\
90 \mathrm{cc} \text { No Shield } \\
\text { cpm }\end{array}$ & $\begin{array}{l}\text { Peak Count } \\
90 \mathrm{cc} \text { Shield } \\
\mathrm{cpm}\end{array}$ \\
\hline 185 & ${ }^{220} \mathrm{Ra}$ & 2.7 & 0.13 & 3.1 & 0.22 \\
\hline 295 & $214 \mathrm{~Pb}$ & 5.4 & 0.08 & 7.9 & 0.11 \\
\hline 352 & $211_{\mathrm{Pb}}$ & 9.8 & 0.09 & 13.4 & 0.21 \\
\hline 511 & - & 3.7 & 0.60 & 5.8 & 1.14 \\
\hline 583 & ${ }^{208} \mathrm{Tl}$ & 3.0 & 0.237 & 4.2 & 0.36 \\
\hline 609 & ${ }^{214} \mathrm{Bi}$ & 8.2 & 0.081 & 11.2 & 0.15 \\
\hline 1378 & ${ }^{214} \mathrm{Bi}$ & 0.43 & $\begin{array}{l}\text { Not } \\
\text { Observable }\end{array}$ & 0.74 & $\begin{array}{l}\text { Not } \\
\text { Observable }\end{array}$ \\
\hline 1460 & $40 \mathrm{~K}$ & 11.9 & 0.06 & 21.9 & 0.087 \\
\hline 1764 & ${ }^{214} \mathrm{Bi}$ & 1.63 & 0.018 & 2.5 & Observable \\
\hline 2204 & ${ }^{214} \mathrm{Bi}$ & 0.42 & Observable & 0.74 & observable \\
\hline 2614 & ${ }^{208} \mathrm{Tl}$ & 1.77 & 0.084 & 3.1 & 0.15 \\
\hline
\end{tabular}

1) Not observable indicate $c>0.33$ for that peak. 
TABLE 3

MINIMUM DETECTABLE ACTIVITY (in $\mathrm{y} / \mathrm{min}$ ) FOR SEVERAL SAMPLE-DETECTOR CONFIGURATIONS

AND BACKGROUND RADIATION CONDITIONS AS A FUNCTION OF ENERGY

$\underline{60 c c}$

\begin{tabular}{c|c|c|c|c|c|c|c|c|c|c|}
\hline Energy $(\mathrm{keV})$ & $\mathrm{Ae}$ & $\mathrm{Ae}^{2}$ & $\mathrm{Ae}^{3}$ & $\mathrm{Ae}^{4}$ & $\mathrm{Ae}^{5}$ & $\mathrm{Ae}^{6}$ & $\mathrm{Ae}^{7}$ & $\mathrm{Ae}^{8}$ & $\mathrm{Ae}^{1} / \mathrm{Ae}^{2}$ & $\mathrm{Ae}^{2} / \mathrm{Ae}^{3}$ \\
\hline 185 & 11 & 1.6 & 4.9 & 2.9 & 4.9 & 16 & 1.0 & 3.4 & 6.875 & .32 \\
609 & 12 & 3.2 & 9.6 & 3.5 & 6.3 & 19.3 & 2.0 & 4.3 & 3.75 & .33 \\
1238 & 16 & 5.3 & 15 & 7.5 & 9.0 & 25.7 & 3.4 & 6.0 & 3.02 & .35 \\
1764 & 11 & 6.2 & 17.4 & 7.8 & 8.5 & 24 & 3.8 & 5.5 & 1.77 & .35 \\
2204 & 13 & 7.5 & 21 & 8.8 & 8.9 & 25 & 4.4 & 5.5 & 1.73 & .36 \\
\hline
\end{tabular}

$\underline{90 \mathrm{cc}}$

\begin{tabular}{|c|l|l|l|l|l|l|l|l|l|l|}
\hline Energy (keV) & $\mathrm{Ae}^{1}$ & $\mathrm{Ae}^{2}$ & $\mathrm{Ae}^{3}$ & $\mathrm{~A} \mathrm{e}^{4}$ & $\mathrm{Ae}$ & $\mathrm{Ae}$ & $\mathrm{Ae}$ & $\mathrm{Ae}$ & $\mathrm{Ae} / \mathrm{Ae}$ & $\mathrm{Ae}^{2} / \mathrm{Ae}^{3}$ \\
\hline 185 & 12.2 & 1.5 & 4 & 2.8 & 4.3 & 12 & 1.0 & 3.1 & 8.13 & .37 \\
609 & 13 & 3.0 & 7.8 & 6.7 & 5.4 & 14 & 1.9 & 3.5 & 4.33 & .38 \\
1238 & 17 & 4.4 & 11 & 6.6 & 7.2 & 18 & 2.7 & 4.8 & 3.86 & .4 \\
1764 & 10.5 & 4.2 & 10.5 & 6.6 & 6.8 & 17 & 3.2 & 4.4 & 2.5 & .4 \\
2204 & 12.6 & 6.0 & 15 & 7.1 & 7.0 & 17.4 & 3.6 & 4.3 & 2.1 & .4 \\
\hline
\end{tabular}

${ }^{1}$ No Lead shield, disk sample natural bg dominates interfering continuum.

$210 \mathrm{~cm}$ shield, disk sample, natural bg dominates.

$310 \mathrm{~cm}$ shield, Marinelli Beaker, natural bg dominates.

${ }^{4} 10 \mathrm{~cm}$ shield, disk sample with $\approx 1 \mathrm{nCi}$ of ${ }^{60} \mathrm{Co}$ and ${ }^{137} \mathrm{Cs}$ in disk.

${ }^{5} 10 \mathrm{~cm}$ shield, disk sample with $\approx 2 \mathrm{nCi}$ activity of $U$ and $\mathrm{Th}$ in disk.

${ }^{6} 10 \mathrm{~cm}$ shield, Marinelli Beaker with $\sim 5 \mathrm{nCi}$ activity of $U$ and Th in beaker.

$710 \mathrm{~cm}$ shield, two detectors in parallel with disk sample, natural bgrd dominates.

${ }^{8} 10 \mathrm{~cm}$ shield, two detectors in parallel with disk sample with $\approx 2 \mathrm{nCi}$ of $U$ and Th in disk.

$T=40,000 \mathrm{sec}, \quad C=0.2$

Ae in $\gamma /$ min emitted from sample. 
TABLE 4

ABSOLUTE FULL ENERGY PEAK EFFICIENCY $(\varepsilon)$ FOR SEVERAL SAMPLE - DETECTOR CONFIGURATIONS

\begin{tabular}{|l|c|c|c|c|c|} 
& $\varepsilon$ & $\varepsilon$ & $\varepsilon$ & $\varepsilon$ & $\varepsilon$ \\
Detector Configuration & At $185 \mathrm{keV}$ & At $609 \mathrm{keV}$ & At $1238 \mathrm{keV}$ & At $1764 \mathrm{keV}$ & At $2204 \mathrm{keV}$ \\
\hline $60 \mathrm{cc}$ Geometry a) & .082 & .025 & .012 & .0084 & .0067 \\
\hline $90 \mathrm{cc}$ Geometry a) & .091 & .030 & .015 & .011 & .009 \\
\hline $60 \mathrm{cc}$ Geometry b) & .16 & .050 & .024 & .017 \\
\hline $90 \mathrm{cc}$ Geometry b) & .18 & .059 & .030 & .022 \\
\hline $60 \mathrm{cc}$ Geometry c) & .025 & .0081 & .0042 & .0030 \\
\hline $90 \mathrm{cc}$ Geometry c) & .034 & .011 & .0060 & .0044 \\
\hline
\end{tabular}

TABLE 5

COMPARISON OF 60CC 90CC ABSOLUTE FULL ENERGY PEAK EFFICIENCIES

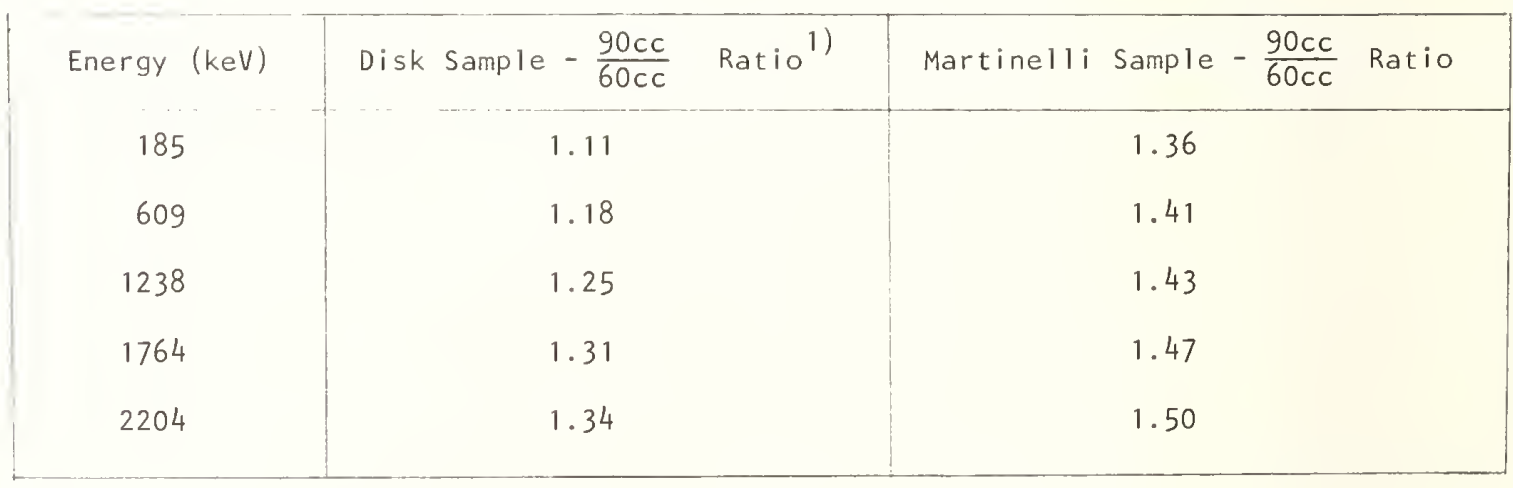

1) Ratio of absolute full energy peak efficiency for $60 \mathrm{cc}$ and $90 \mathrm{cc}$ 
TABLE 6

MINIMUM DETECTABLE ACTIVITY IN Y/MIN/ML OF SAMPLE MATERIAL FOR THE MARINELLI BEAKER AND FOR THE DISK SAMPLE

ONE 60CC DETECTOR

\begin{tabular}{|c|c|c|c|}
\hline Energy (keV) & $\begin{array}{c}\text { Ae (For Disk Sample) } \\
\gamma / \mathrm{min} / \mathrm{ml}\end{array}$ & $\begin{array}{c}\text { Ae (For Marinelli Beaker) } \\
\gamma / \mathrm{min} / \mathrm{ml}\end{array}$ & $\begin{array}{c}\text { Ae Marinelli Vol. Reduced } \\
\text { by Evaporation to 5ml Disk }\end{array}$ \\
\hline 185 & 0.3 & $8.9 \times 10^{-3}$ & $2.7 \times 10^{-3}$ \\
609 & 0.64 & $1.7 \times 10^{-2}$ & $5.6 \times 10^{-3}$ \\
1238 & 1.1 & $2.7 \times 10^{-2}$ & $9.6 \times 10^{-3}$ \\
1764 & 1.2 & $3.1 \times 10^{-2}$ & $1.1 \times 10^{-2}$ \\
2204 & 1.5 & $3.8 \times 10^{-2}$ & $1.36 \times 10^{-2}$ \\
\hline
\end{tabular}

ONE 90CC DETECTOR

\begin{tabular}{|c|c|c|c|}
\hline Energy (keV) & $\begin{array}{c}\text { Ae (For Disk Sample) } \\
\gamma / \mathrm{min} / \mathrm{ml}\end{array}$ & $\begin{array}{c}\text { Ae (For Marinelli Beaker) } \\
\gamma / \mathrm{min} / \mathrm{ml}\end{array}$ & $\begin{array}{c}\text { Ae Marinelli Vol. Reduced } \\
\text { by Evaporation to 5ml Disk }\end{array}$ \\
\hline 185 & 0.3 & $7.2 \times 10^{-3}$ & $2.7 \times 10^{-3}$ \\
609 & 0.6 & $1.4 \times 10^{-2}$ & $5.4 \times 10^{-3}$ \\
1238 & 0.9 & $2.0 \times 10^{-2}$ & $7.6 \times 10^{-3}$ \\
1764 & 0.8 & $1.9 \times 10^{-2}$ & $7.6 \times 10^{-3}$ \\
2204 & 1.2 & $2.7 \times 10^{-2}$ & $1.1 \times 10^{-2}$ \\
\hline
\end{tabular}

1Expressed as the activity in $\mathrm{Y} / \mathrm{min} / \mathrm{ml}$ of original sample volume.

$T=40,000$ secs.

$c=0.2$

Natural background dominates the total bg contribution. 


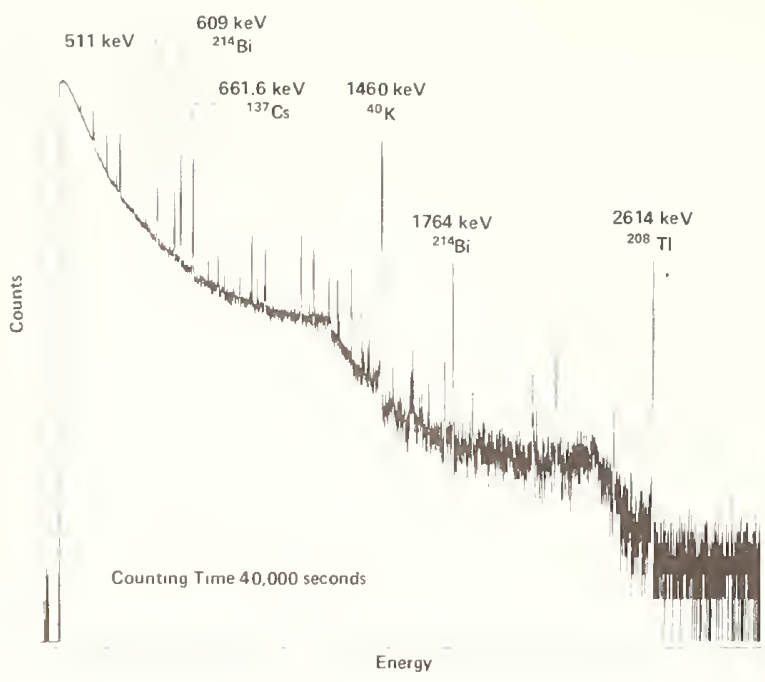

Fig. 1. Log Plot of Background Count Without Shielding for a 90 cc Detector.

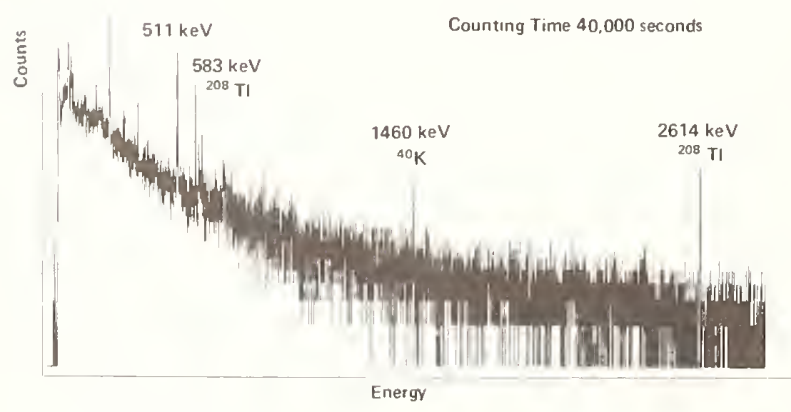

Fig. 2. Log Plot of Background Count with $10 \mathrm{~cm} \mathrm{Lead}$ for a $90 \mathrm{cc}$ Detector.

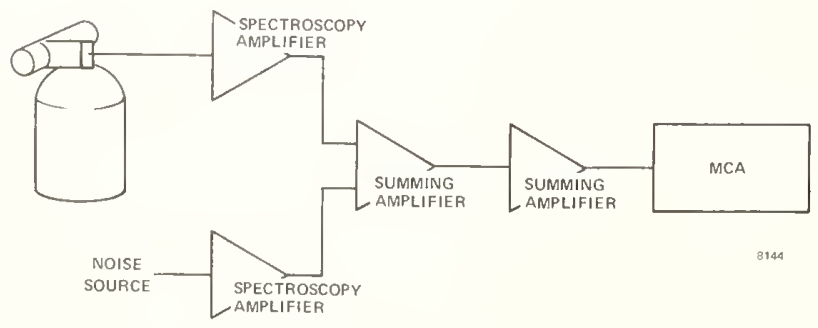

Fig. 3. Block Diagram of Electronic System Used in FWHM Studies.

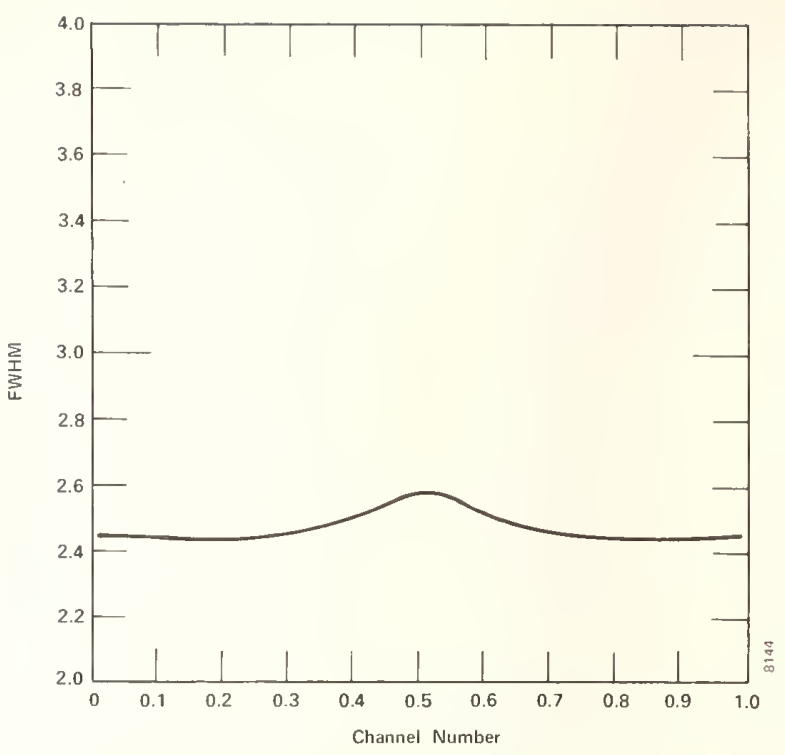

Fig. 4. Measured FWHM as a Function of Peak Centroid.

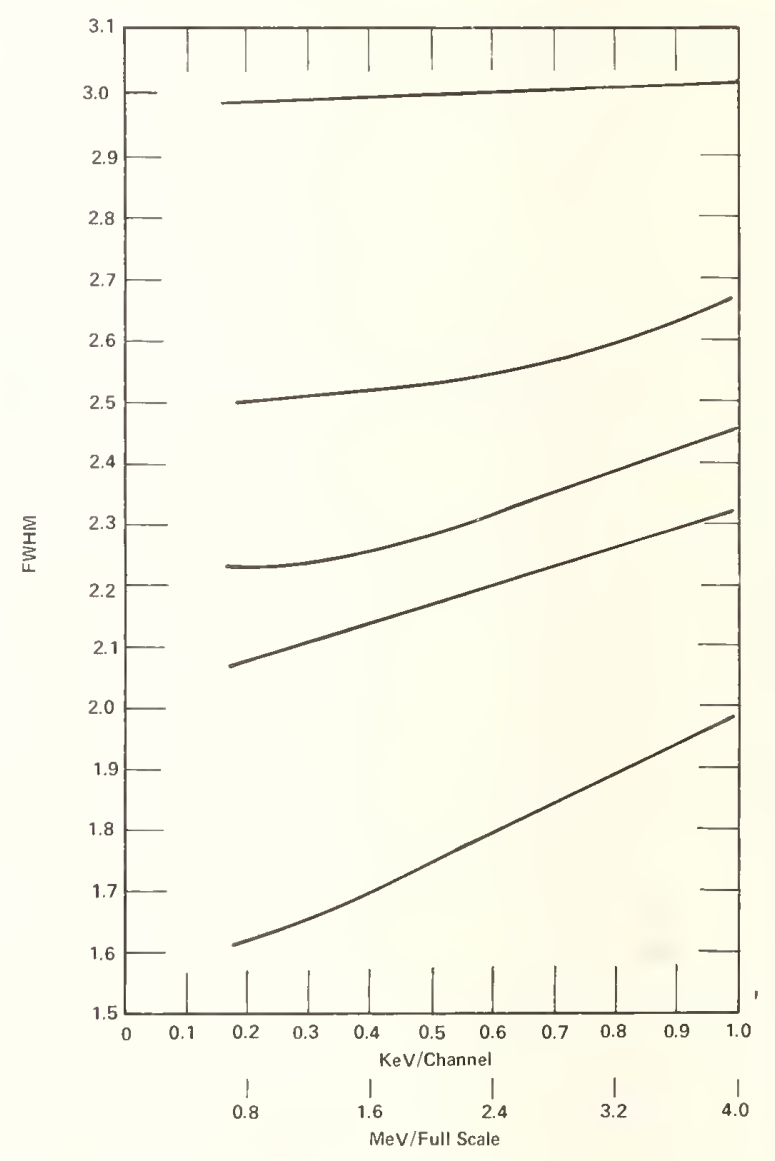

Fig. 5. Measured FWHM as a Function of Digitization Width. 


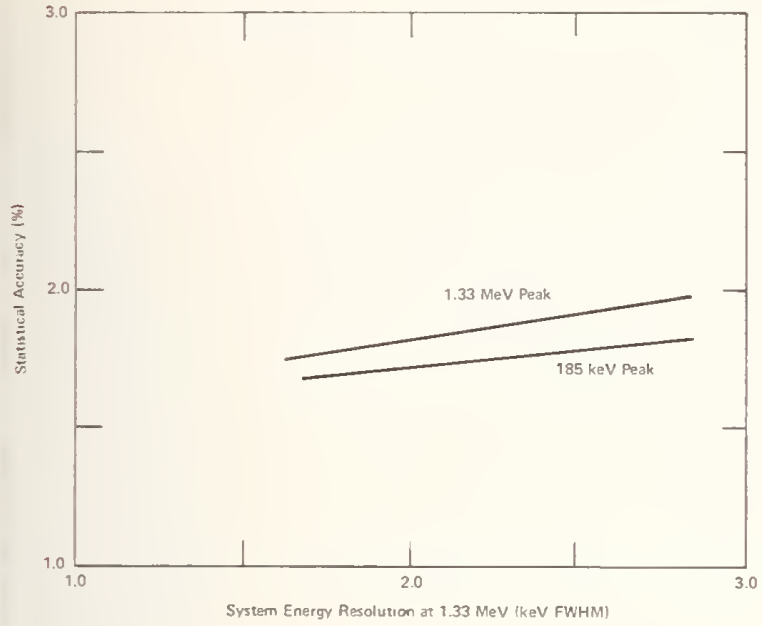

Fig. 6. Variation of Peak Statistical Accuracy (C) as a Function of Energy Resolution.

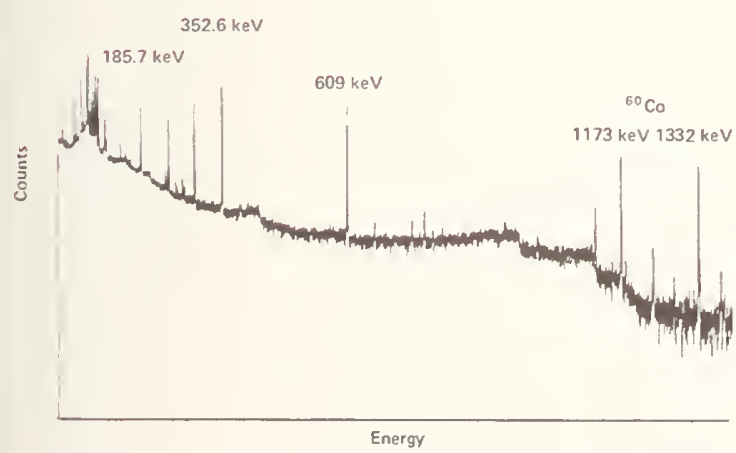

Fig. 7. Sample Spectrum from $1.7 \mathrm{keV}$ Detector with Uranium Ore and ${ }^{60} \mathrm{Co}$. (a) Disk Sample with One Detector

Detector Position

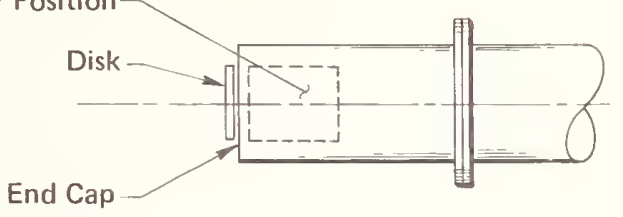

(b) Disk Sample with Two Detectors

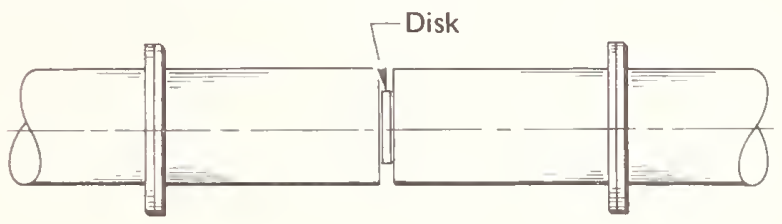

(c) Marinelli Beaker Placed upon Detector

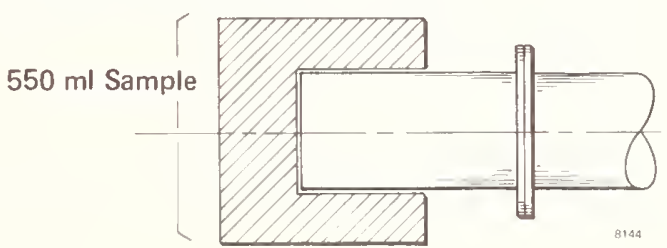

Fig. 8. Sample-Detector Geometries Used for Measurement of Weak Activities.

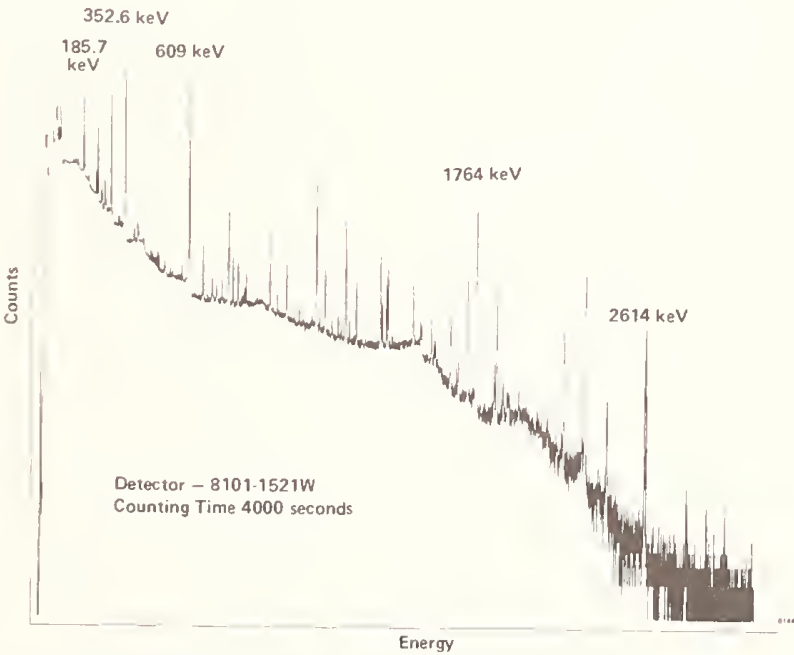

Fig. 9. Spectrum of a Uranium Ore Sample in a Marinelli Beaker Used for $90 \mathrm{cc} \mathrm{Ge}$ (Li) Detector Calibration. 



\author{
THE MINTMU-DETECTABLE-ACTIYITY CONCEPT \\ Joseph C. Lochamy \\ nuke Power Company \\ Charlotte, N. C. 28242
}

\begin{abstract}
Tarious definitions of "minimum detectable activity (MTA)" are discussed and statistically valid representatives are presented. The methods of Currie are given primary emphasis and include development of the Critical Leve1, Detection Limit, Less-Than Level, and Determination Limit in terms of count-rate.
\end{abstract}

(Background; Critica1 Level: Detection Limit: Netermination Limit: Less-Than Level: minimum detectable activity: statistical)

\section{Introduction}

A casual review of the literature references to "minimum detectable activity (MD)", "sensitivity", and "limits of detection" often gives the reader the feeling that everyone is going in different directions, without any rea11y-unified effort to define exactly what is being pursued. Out of all the different mathematical expressions for IDA used in low-level radiation counting, there are at least four different types of MDA implied. Nuite often a single mathematical representation is inappropriately called-upon to satisfy the requirements of several of these MDA concepts. These four concepts will be defined in terms of count-rate and, for convenience, will be called

$$
\begin{aligned}
& \text { the Critical Level }\left(\mathrm{L}_{\mathrm{C}}\right) \\
& \text { the Detection Limit }\left(\mathrm{L}_{\mathrm{d}}\right) \\
& \text { the Less-Than Level }\left(\mathrm{L}_{\mathrm{t}}\right) \\
& \text { and } \\
& \text { the Determination Limit }\left(\mathrm{L}_{\mathrm{q}}\right) \text {. } \\
& \qquad \text { Critical Level }
\end{aligned}
$$

Usually, some test is applied to lowlevel counting data to determine if the sample count-rate is statistically different from the background. This test is often expressed as a net count-rate equal to some multiple of the background standard deviation $\left(\sigma_{b}\right)$. This test is probably the most common conception of the MDA, and we will call it the Critical Level ( $\left.\mathrm{I}_{\mathrm{C}}\right)$. In words, the Critical Level $\left(\mathrm{L}_{\mathrm{C}}\right)$ is defined as that net count-rate which must be exceeded before the sample is said to contain any measurable radioactive material above background. Rather than choosing an arbitrary mathematical expression, let us define a statistically meaningful Critical Level $\left(\mathrm{L}_{\mathrm{C}}\right)$.

Figure 1 shows the normal distribution which would be expected for a mean net count-rate $\left(R_{S}\right)$ of zero in the presence of some background count-rate $\left(R_{b}\right)$. The value for $L_{C}$ is expressed as

$$
\mathrm{L}_{\mathrm{C}}=\mathrm{koO}_{0}
$$

where $k$ is the one-sided confidence factor and $\sigma_{0}$ is the standard deviation of a zero net count-rate. If $k$ is chosen so that $95 \%$ of the measurements of a true zero mean net count-rate are less than $\mathrm{L}_{C}$, that is $k=1.65$, then there is only a $5 \%$ chance that a true mean count-rate of zero will be falsely recorded as a positive value.

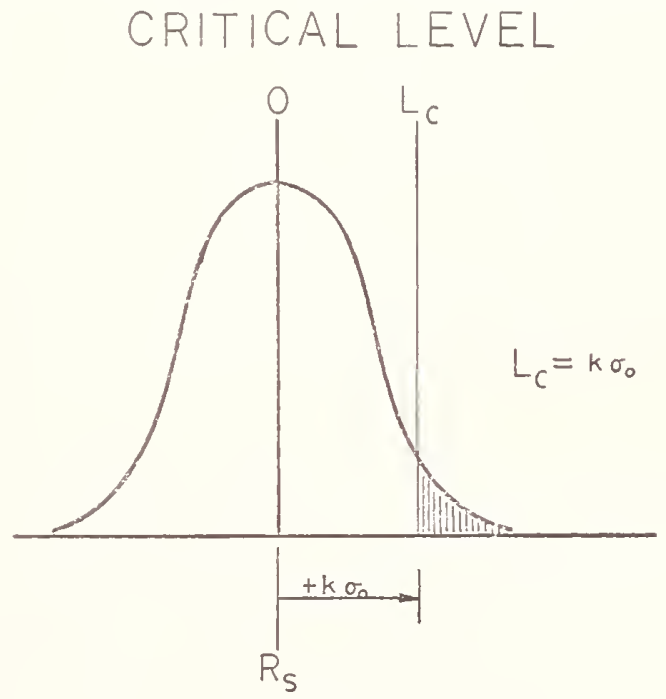

Figure 1

If the net count-rate exceeds $\mathrm{L}_{C}$, it is reported as positive with its two-sided confidence interval. If the net count-rate is less than $L_{C}$, it can only be said that the total count-rate $\left(R_{t}\right)$ is not statistically different from the background at the 0.05 level (or whatever $k$-factor level is chosen).

In the following block, the Critical Leve1 $\left(\mathrm{L}_{\mathrm{C}}\right)$ is derived in terms of the background standard deviation $\left(\sigma_{\mathrm{b}}\right)$. 
CRITICAL LEVEL $\left(\mathrm{L}_{\mathrm{C}}\right)$

From Figure 1: $\quad \mathrm{L}_{\mathrm{C}}=\mathrm{k} \sigma_{0}$

Since $R_{S}=R_{0}=R_{t}-R_{b}=0$

$$
\begin{aligned}
& \sigma_{0}^{2}=\sigma_{t}^{2}+\sigma_{b}^{2} \\
& \sigma_{t}{ }^{2}=\frac{R_{t}}{T_{t}}=\frac{R_{b}}{T_{t}} \\
& \sigma_{b}{ }^{2}=\frac{R_{b}}{T_{b}}
\end{aligned}
$$

Thus,

$$
\sigma_{0}{ }^{2}=\frac{R_{b}}{T_{t}}+\frac{R_{b}}{T_{b}}=\frac{R_{b}\left(T_{t}+T_{b}\right)}{T_{b} T_{t}}
$$

And, $\sigma_{0}=\sigma_{b}\left(1+\frac{T_{b}}{T_{t}}\right)^{1 / 2}$

Therefore,

$$
L_{c}=k \sigma_{b}\left(1+\frac{T_{b}}{T_{t}}\right)^{1 / 2}
$$

where $k$ refers to the one-sided confidence level.

In terms of count-rate,

$$
L_{c}=k\left[\frac{R_{b}}{T_{b}}\left(1+\frac{T_{b}}{T_{t}}\right)\right]^{1 / 2}
$$

If the background and sample counting-times are equal $\left(\mathrm{T}_{\mathrm{b}}=\mathrm{T}_{\mathrm{t}}=\mathrm{T}\right)$, then

$$
L_{c}=k\left(2 \frac{R_{b}}{T}\right)^{I / 2}
$$

The Critical Level is used only to determine if a measurement is statistically different from background. It should not be used as a practical MDA reporting-level and should not be reported as a "less-than" value.

\section{Detection Limit}

Often, when you are asked to specify your MDA, the question really being asked is "If I send you a sample, what does the count-rate have to be in order for you to be sure of detecting it?" In other words, what level can be detected on a routine basis? If you specify $\mathrm{L}_{\mathrm{C}}$, you may as well flip a coin to determine if the activity will be detected; that is, a true mean net count-rate of $\mathrm{L}_{C}$ has one chance in two of falling below $\mathrm{L}_{\mathrm{C}}$ for any single measurement.
The Netection Limit $\left(\mathrm{L}_{\mathrm{d}}\right)$ is the value that should he specified. The Detection Limit $\left(\mathrm{L}_{\mathrm{d}}\right)$ is defined as the smallest quantity of radioactive material which can be detected with some specified degree of confidence. If you are satisfied with a onechance-in-two degree of confidence, then

$$
\mathrm{L}_{\mathrm{d}}=\mathrm{L}_{\mathrm{C}}
$$

However, if you do not want the integrity of your instruments (or your own integrity) doubted $5 n \%$ of the time, you should specify a Detection Limit such that you will be sure to see the activity at least, say, $95 \%$ of the time.

\section{DETECTION LIMIT}

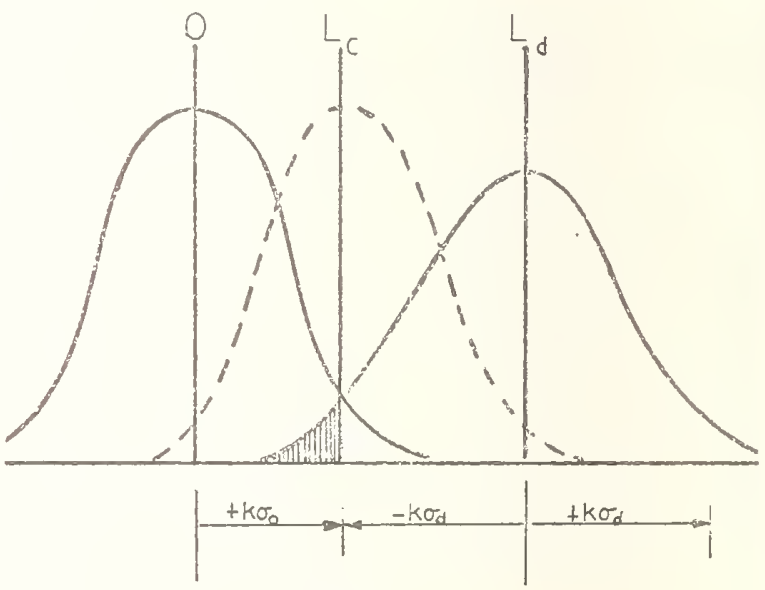

Figure 2

Figure 2 graphically represents the Detection Limit $\left(\mathrm{L}_{\mathrm{d}}\right)$, and shows its relationship to $\mathrm{L}_{\mathrm{C}}$. The value for $\mathrm{L}_{\mathrm{d}}$ is expressed as

$$
\mathrm{L}_{\mathrm{d}}=\mathrm{L}_{\mathrm{c}}+\mathrm{k} \sigma_{\mathrm{d}}
$$

where $k$ is, again, the one-sided confidence factor and $\sigma_{d}$ is the standard deviation of a net count-rate equal to $L_{d}$. If $k$ is chosen so that $95 \%$ of the measurements of a true mean net count-rate $\mathrm{L}_{\mathrm{d}}$ (that is, $\mathrm{k}=1.65$ ) are detected, then $\mathrm{L}_{\mathrm{d}}$ is a practical detection limit which you are $95 \%$ confident of detecting.

In the block on the following page, the Detection Limit is derived in terms of the hackground standard deviation $\left(\sigma_{\mathrm{b}}\right)$. Notice that for $\sigma_{b}=0$ (i.e. $R_{b}=0$ ), the Determination Limit still has a non-zero value. 


\section{DETECTION LIMIT $\left(\mathrm{Ld}_{\mathrm{d}}\right)$}

From Figure 2: $\mathrm{L}_{\mathrm{d}}=\mathrm{L}_{\mathrm{c}}+\mathrm{k} \sigma_{\mathrm{d}}$

Since $L_{d}=R_{d}=R_{t}-R_{b}$

$$
\begin{aligned}
& \sigma_{d}^{2}=\sigma_{t}^{2}+\sigma_{b}^{2} \\
& \sigma_{d}^{2}=\frac{R_{t}}{T_{t}}+\frac{R_{b}}{T_{b}} \equiv \frac{R_{d}+R_{b}}{T_{t}}+\frac{R_{b}}{T_{b}}
\end{aligned}
$$

But $\quad R_{d}=L_{d}$

Thus $\sigma_{d}^{2}=\frac{L_{d}}{T_{t}}+\frac{R_{b}\left(T_{t}+T_{b}\right)}{T_{b} T_{t}}$

and

$$
L_{d}=L_{c}+k\left[\frac{L_{d}}{T_{t}}+\sigma_{b}^{2}\left(1+\frac{T_{t}}{T_{t}}\right)\right]^{1 / 2}
$$

Since $L_{c}^{2}=k^{2} \sigma_{b}^{2}\left(1+\frac{T_{b}}{T_{t}}\right)$

$$
\mathrm{L}_{\mathrm{d}}=\mathrm{L}_{\mathrm{c}}+\left(\mathrm{k}^{2} \frac{\mathrm{L}_{\mathrm{d}}}{\mathrm{T}_{\mathrm{t}}}+\mathrm{L}_{\mathrm{c}}{ }^{2}\right)^{1 / 2}
$$

Rearranging and solving for $\mathrm{L}_{\mathrm{d}}$,

$$
L_{\mathrm{d}}=\frac{\mathrm{k}^{2}}{\mathrm{~T}_{\mathrm{t}}}+2 \mathrm{~L}_{\mathrm{c}}
$$

In terms of $\sigma_{b}$,

$$
\mathrm{L}_{\mathrm{d}}=\frac{\mathrm{k}^{2}}{\mathrm{~T}_{\mathrm{t}}}+2 \mathrm{k} \sigma_{\mathrm{b}}\left(1+\frac{\mathrm{T}_{\mathrm{b}}}{\mathrm{T}_{\mathrm{t}}}\right)^{1 / 2}
$$

Again, in terms of count-rate,

$$
L_{d}=\frac{k^{2}}{T_{t}}+2 k\left[\frac{R_{b}}{T_{b}}\left(1+\frac{T_{b}}{T_{t}}\right)\right]^{1 / 2}
$$

and for $T_{b}=T_{t}=T$, the practical

form is

$$
\begin{array}{r}
L_{\mathrm{d}}=\frac{\mathrm{k}^{2}}{\mathrm{~T}}+2 \mathrm{k}\left(2 \frac{\mathrm{R}_{\mathrm{b}}}{\mathrm{T}}\right)^{1 / 2} \\
\text { Less-Than Leve1 }
\end{array}
$$

Thus far, we have specified a Critical Level ( $\mathrm{L}_{\mathrm{c}}$ ) for determining if a measured count-rate is statistically greater than background, and a Detection Limit $\left(\mathrm{L}_{\mathrm{d}}\right)$ which the true net count-rate of a sample must exceed if we wish to be sure that a single determination will detect the activity. However, nothing has been said about the quantity of radioactive material which could be present even though it is not detected; that 1s, the measured count-rate is less than $\mathrm{L}_{\mathrm{C}}$. In general, neither $\mathrm{L}_{\mathrm{C}}$ nor $\mathrm{L}_{\mathrm{d}}$ satisfles the requirement for the Less-Than Leve1 $\left(\mathrm{L}_{\mathrm{t}}\right)$.
The Less-Than Leve1 $\left(L_{t}\right)$ is defined as the maximum true count-rate which a sample could have, based on the measured count-rate $R_{S}$, where $R_{S}$ is less than $L_{C}$. The mathematical expression for $L_{t}$ is given hy

$$
I_{\mathrm{t}}=\mathrm{r}_{\mathrm{s}}+\mathrm{k} \sigma_{\mathrm{s}}
$$

where $k$ is the one-sided confidence factor. If $95 \%$ confidence is desired $(k=1.65)$, this expression says that there is only a $5 \%$ chance that the activity actually present exceeds $I_{t}$.

In the special case where the sample net count-rate is exactly zero, then

$$
\mathrm{I}_{\mathrm{t}}=k \sigma_{0}=\mathrm{L}_{\mathrm{c}}
$$

But, in general, $\mathrm{L}_{t}$ is somewhere hetween $\mathrm{L}_{\mathrm{c}}$ and $\mathrm{L}_{\mathrm{d}}$. Of course, it is possible to have $R_{S}<n$, due to the statistical nature of low-level counting. In this case, it seems appropriate to let $\mathrm{I}_{t}=\mathrm{L}_{\mathrm{c}}$ for all $\mathrm{R}_{\mathrm{S}}<n$.

In terms of count-rate

$$
L_{t}=R_{s}+k\left(\frac{R_{t}}{T_{t}}+\frac{R_{b}}{T_{b}}\right)^{1 / 2}
$$

and if $T_{t}=T_{b}=T$, then the practical form is

$$
\begin{aligned}
& \mathrm{L}_{\mathrm{t}}=\mathrm{R}_{\mathrm{S}}+k\left(\frac{\mathrm{R}_{t}+\mathrm{R}_{\mathrm{h}}}{\mathrm{T}}\right)^{1 / 2} \\
& \text { DETERMINATION } \underline{\text { LIMIT }}\left(\mathrm{L}_{\mathrm{q}}\right)
\end{aligned}
$$

One last MDA concept is defined in the literature as the lowest net count-rate which can be detected with a specified relative standard deviation. This definition describes the Determination Limit $\left(\mathrm{L}_{\mathrm{q}}\right)$, and is expressed as

$$
\mathrm{L}_{\mathrm{q}}=f_{\mathrm{q}} \sigma_{\mathrm{q}}
$$

where $f_{a}$ is the reciprocal relative standard deviation desired.

In the hlock below, the Determination Limit $\left(\mathrm{L}_{\mathrm{q}}\right)$ is derived in terms of $\sigma_{\mathrm{b}}$.

$$
\begin{aligned}
& \text { DETERMINATION LIMIT }\left(\mathrm{L}_{\mathrm{q}}\right) \\
& \mathrm{L}_{\mathrm{q}}=f_{\mathrm{q}} \sigma_{\mathrm{q}}=f_{\mathrm{q}}\left(\frac{\mathrm{R}_{\mathrm{a}}+\mathrm{R}_{\mathrm{b}}}{\mathrm{T}_{\mathrm{t}}}+\frac{\mathrm{R}_{\mathrm{b}}}{\mathrm{T}_{\mathrm{b}}}\right)^{1 / 2} \\
& \mathrm{~L}_{\mathrm{q}}=f_{\mathrm{q}}\left(\frac{\mathrm{L}_{\mathrm{a}}}{\mathrm{T}_{\mathrm{t}}}+\frac{\mathrm{R}_{\mathrm{b}}\left(\mathrm{T}_{t}+\mathrm{T}_{\mathrm{b}}\right)}{\mathrm{T}_{\mathrm{t}^{\mathrm{T}} \mathrm{b}}}\right)^{1 / 2} \\
& \mathrm{~L}_{\mathrm{q}}=f_{\mathrm{q}}\left(\frac{\mathrm{L}_{\mathrm{q}}}{\mathrm{T}_{\mathrm{t}}}+\sigma_{\mathrm{b}}{ }^{2}\left(1+\frac{\mathrm{T}_{\mathrm{b}}}{\mathrm{T}_{\mathrm{t}}}\right)\right)^{1 / 2}
\end{aligned}
$$

\section{(Continued on next page)}


DETERMINATION LIMIT $\left(\mathrm{L}_{\mathrm{q}}\right)$ (Continued)

Solving for $\mathrm{L}_{\mathrm{q}}$

$$
\mathrm{L}_{\mathrm{q}}=\frac{f_{\mathrm{q}}^{2}}{2 \mathrm{~T}_{\mathrm{t}}}\left[1+\left(1+\frac{4 \mathrm{~T}_{t} \sigma_{\mathrm{h}}^{2}\left(\mathrm{~T}_{t}+\mathrm{T}_{\mathrm{b}}\right)}{f_{\mathrm{q}}{ }^{2}}\right)^{1 / 2}\right]
$$

In terms of count-rate,

$$
\mathrm{L}_{\mathrm{q}}=\frac{f_{\mathrm{q}}{ }^{2}}{2 \mathrm{~T}_{\mathrm{t}}}\left[1+\left(1+\frac{4 \mathrm{~T}_{t} \mathrm{R}_{\mathrm{b}}\left(\mathrm{T}_{\mathrm{t}}+\mathrm{T}_{\mathrm{b}}\right)}{f_{\mathrm{q}}{ }^{2} \mathrm{~T}_{\mathrm{b}}}\right)^{1 / 2}\right]
$$

The practical case where $T_{t}=T_{b}=T$ gives

$$
\mathrm{Lq}=\frac{f_{\mathrm{q}}^{2}}{2 \mathrm{~T}}\left[1+\left(1+\frac{8 \mathrm{Rb}^{\mathrm{T}}}{f_{\mathrm{q}}{ }^{2}}\right)^{1 / 2}\right]
$$

\section{REPORTING PRACTICES}

The Detection Limit $\left(\mathrm{L}_{\mathrm{d}}\right)$ and the netermination Limit $\left(\mathrm{L}_{\mathrm{q}}\right)$ are not used for routine counting and reporting. In those cases where you are required to specify your minimum detectable activity (e.g. to a regulatory agency), it is recommended that the Detection Limit be given then as a practical reporting limit. The Determination Limit $\left(\mathrm{L}_{\mathrm{q}}\right)$ is useful when a "sensitivity" with a specified relative standard deviation is required.

For routine low-level counting, only the Critical Level $\left(\mathrm{L}_{\mathrm{C}}\right)$ and the Less-Than Leve1 $\left(L_{t}\right)$ are of interest. Their use is as follows: a. If $R_{S}>L_{C}$, the result is reported as positive, with the two-sided confidence interval desired, $\mathrm{R}_{\mathrm{S}} \pm k \sigma_{\mathrm{S}}$, where $\mathrm{k}=1.96$ at the 0.05 level.

b. If $R_{S} \leq L_{C}, L_{t}$ is calculated using the one-sided confidence interval and reported as less than $R_{S}+k \sigma_{S}$, where $k=1.65$ at the 0.05 level.

\section{ACKNOWLEDGMENT}

This paper is designed to be primarily instructional rather than creative. The author has freely used material from the paper by J. K. Hartwell listed below.

\section{SOURCES}

J. K. Hartwe11, "Detection Limits for Radioisotopic Counting Techniques". ARH-2537. June 2.2, 1972.

L. A. Currie, "Limits for Oualitative Netection and Ouantitative Determination". Analytical Chemistm, Volume 40 , No. 3, 586-693. 1968.

B. Altshuler and B. Pasternak, "Statistical Measurements of the Lower Limit of netection of a Radioactivity Counter". Heatth Physics, Volume 9, 293-298. 1963. 
USE OF NBS MIXED-RADIONUCLIDE GAMMA-RAY STANDARDS FOR CALIBRATION OF Ge(Li) DETECTORS USED IN THE ASSAY OF ENVIRONMENTAL RADIOACTIVITY

\section{B. M. Coursey}

National Bureau of Standards Washington, D. C. 20234

The NBS mixed-radionuclide gamma-ray emission-rate standards were first distributed in 1972 for use in calibrating Ge(Li) detectors used for measuring environmental radioactivity (1). These sources allow the user to prepare full-energy-peak efficiency vs $y$-ray energy curves for the detector from measurements made with a single standard. This paper will discuss the development of these standards and examine their current use in the assay of radioactivity in liquid, gaseous, and soil samples.

(Radioactivity, standards, enviroment, gamma-ray measurements, Ge(Li) detectors)

\section{Design of Mixed Gamma-Ray Standards}

The five mixed- $\gamma$-ray Standard Reference Materials (SRM's) currently available are listed in Table 1A. A total of about 300 such sources are prepared annully from a single master solution containing the nine components shown in Table $1 \mathrm{~B}$.

\section{Spectroscopic Properties}

The $\mathrm{y}$-ray spectrum of a typical source, obtained with a Ge(Li) detector-multichannel analyser system, is shown in Figure 1. A typica1 "efficiency curve," full-energy-peak efficiency vs $y$-ray energy, for a Ge(Li) detector is shown in Figure 2. The eleven principal $y$-ray 1 ines in the present mixture were chosen to allow one to determine the entire efficiency curve over the energy region from $88 \mathrm{keV}$ to $1836 \mathrm{keV}$.

Others have suggested the use of single radionuclides which emit $\gamma$ rays of many energies for detector calibration $(2,3)$. Standards of a single radionuclide, such as $152 \mathrm{Eu}$, are clearly easier to use than mixed standards because only one half-life correction need be made. There are two advantages, however, in using mixed standard sources. First, one can choose the spacing of the $\gamma$-ray energies and, second, uncertainties in $\gamma$-ray emission rates are generally less for those radionuclides with simple decay schemes. Some precautions which should be taken in the design and use of a mixed standard are given here.

The first consideration in preparation of the standard is that accurate $\gamma$-rayemission rate standards of the individual components be available. The nominal uncertainties in the emission rates for the eleven principal $Y$ rays in the present standards are given in Table 1B. Research is continuing at NBS with $4 \pi \beta-y$ and $x-y$ coincidence counting techniques to improve the accuracy of these standards. Evaluations of nuclear-decay scheme parameters, such as half lives and $\gamma$ rays per decay, are continually reviewed to insure that the most recent data are provided on the certificates. In most instances, the decayscheme parameters are those suggested by the Oak Ridge Nuclear Data Project (4).

Decay schemes for components used in a mixed standard should be relatively simple to minimize interference from secondary events in the measurement of full-energy peaks. Single- and double-escape peaks from high-energy $\gamma$ rays, Compton edges, and weak $y$-ray transitions may present problems in the region of the spectrum where other calibration peaks are present. Three potential problems of this nature are associated with the present mixed standards when used with detectors of poor resolution. The single-escape peak from the $1836 \mathrm{keV}$ transition occurs at $1325 \mathrm{kev}$, and may only be partially resolved from the ${ }^{60} \mathrm{Co} 1333-\mathrm{keV}$ peak (see Figure 1). The $511-\mathrm{keV}$ annihilation peak from pair production may interfere with the measurement of the ${ }^{85} \mathrm{Sr} 514-\mathrm{keV}$ peak. And finally, the $\mathrm{Tl} x$ rays from the decay of $203 \mathrm{Hg}$ may not be resolved from the ${ }^{109} \mathrm{Cd} 88-\mathrm{keV}$ peak. The extent of these problems will depend on the resolution of the detector.

Whether a single radionuclide standard or a mixture is used for detector calibration, the problem of summing must be considered when the radionuclide measured emits coincident radiations (5). Summing can obviously occur in measurements of both the 
standard and the unknown and can only be reduced by using absorbers or counting at greater distances. Suming problems may be encountered in the present mixed standards, for example in the full-energy-peak measurements for ${ }^{60} \mathrm{Co},{ }^{139} \mathrm{Ce}$, and ${ }^{88} \mathrm{Y}$. Even when summing is not severe for the components of the standard it may be a problem for radionuclides in the sample such as ${ }^{13} 1_{I}$ and $154 \mathrm{Eu}$. Corrections require a knowledge of the decay scheme as well as full-energypeak efficiencies and total efficiencies for monoenergetic $y$ rays.

\section{Physical Properties}

The point sources, SRM 4215 and SRM 4216, are prepared by depositing an accurately-weighed aliquot, usually between 20 and 50 milligrams, of the calibrated radionuclide mixture onto polyester tape, drying the source, and covering it with another layer of the same tape. The tape, which is $0.006-\mathrm{cm}$ thick, is supported by a $5.2-\mathrm{cm}$ diameter aluminum annulus. The source is exposed to hydrogen sulfide during the drying period to precipitate the mercuric sulfide and prevent escape of ${ }^{203} \mathrm{Hg}$ by volatilization.

The solutions are distributed in breakseal glass ampoules or in "standard geometry" borosilicate-glass bottles fabricated at the NBS glass shop. Dimensions of the standard bottles are given in Table 2. It was found necessary to introduce standard bottles because the non-uniformity of commercially available bottles led to serious errors in the measurement process (1). Empty containers like the ones used for these standards are available from NBS.

A solution source, such as SRM 4242 , is prepared by depositing a weighed aliquot of the master solution into the bottle containing a weighed quantity of approximately $4 \mathrm{~N}$ hydrochloric acid (which has a density of $1.06 \pm 0.01 \mathrm{~g} / \mathrm{cm}^{3}$ ). The carrier content of the master solution is adjusted so that the ratio of stable carrier atoms to radioactive atoms is about $10^{4}$ for each of the parent radionuclides. Under these conditions we have found no evidence of deterioration of the solution, such as precipitation or "plate-out," over long periods of time.

The most recent of the mixed standard sources, SRM 4254, contains approximately $5 \mathrm{ml}$ of a $4 \mathrm{~N}$ hydrochloric acid solution in a break-seal glass ampoule. The solution also contains approximately 20 ppm cation carrier for each of the parent radionuclides in the mixture. It is intended to be opened by the user to prepare secondary standards as described in the following section.

Use of Mixed Gamma-Ray Standards in Environmental Laboratories

Table 3 lists the number of mixed $\gamma$-ray SRM's distributed between February 1972 and February 1976 and gives some indication of the kinds of users of these materials. Illustrations of how the sources are being used in the assay of liquid, gaseous, and soil samples are given in the following sections.

\section{Detector Calibration for Liquid Samples}

SRM 4242 (450 ml) and SRM 4243 (50 ml) are specifically intended to be used as sealed counting standards. As mentioned previously empty standard bottles are available from NBS which can be used to count the environmental liquid sample. Although few laboratories have adopted these standard geometry containers for routine use, the bottles do play an important role in intercomparative measurements between licensees, environmental consultants, and regulatory agencies.

It is often necessary to calibrate a detector for a small container, such as a 20-m1 liquid-scintillation vial, or a large volume container such as a 1-liter bottle. SRM 4254 has a higher radioactive concentration than the other two solution sources and may be quantitatively transfered or diluted to prepare such secondary calibration sources. Dilutions should be made with carrier solutions which are greater than $1 \mathrm{~N}$ hydrochloric acid and contain about 20 ppm of stable carrier for each of the parent radionuclides.

\section{Detector Calibration for Gaseous and Airborne Particulate Samples}

Mixtures of radioactive noble gases, such as effluents from nuclear power stations, are often measured in spherical glass ampoules. Radioactivity SRM's of $85_{\mathrm{Kr}}, 127,13 \mathrm{~lm}, 133 \mathrm{Xe}$, have been distrib-
${ }^{2}{ }^{2}$, uted by NBS in similar standard ampoules (see dimensions in Table 2). Recent measurements at NBS have shown (6) that efficiency curves applicable to mixtures of gases in such glass spheres may be obtained from measurements made with a mixed $y$-ray point source, SRM 4215, at the center of the sphere. The uncertainty 
in this method is generally less than $2 \%$ for $\mathrm{Ge}(\mathrm{Li})$ detectors with volumes less than $60 \mathrm{~cm}^{3}$ at source-to-detector distances greater than $10 \mathrm{~cm}$. The corrections necessary at closer distances are discussed in the reference.

Gamma-ray measurements are often used to assay radioactivity entrained on particulate air filters. Palms et al (7) have prepared secondary standards for use in detector calibration for such measurements. They prepared disk-shaped sources of differing thickness and diameter by introducing aliquots of an NBS solution standard into a "homogeneous polyester glass resin." The use of such sources appears justified providing consideration is given any difference in absorption or activity distribution between the filter and standard.

\section{Detector Calibration for Soil and Sediment Samples}

The current solution SRM's are not intended for use in soil measurements. The activity incorporated in such standards must be homogeneously distributed and the chemical composition and density of the standards should be similar to those expected for the unknown samples. Laichter et al (8), for example, have prepared solid y-ray emissionrate standards from evaporated sea water and tabulated attenuation coefficients to be used with different types of materials. In more recent work, Noyce et al (9) have described the preparation of the NBS river sediment standard, SRM 4350, which is primarily intended for use in radiochemical studies. SRM 4350 may be used for Ge(Li) detector calibration, but the low activities of the certified $\gamma$-ray-emitting radionuclides limit its use for this purpose. Soil y-ray emission-rate SRM's similar to the liquid standards described in this paper are under consideration at NBS.

\section{Acknowledgement}

A proper acknowledgement for a paper on the mixed $y$-ray standards should include a list of al1 17 members of the radioactivity section, as a group effort is involved in the preparation of these standards. Special acknowledgement must be given, however, to the three individuals most responsible for the design and development of these standards: Miss L. M. Cavallo, and Drs. J. M. R. Hutchinson and W. B. Mann.

\section{References}

1. Caval1o, L. M., Coursey, B. M., Hutchinson, J. M. R., Garfinkel, S. B., and Mann, W. B., Nucl. Inst. and Methods, 112, 5 (1973).

2. Seyfarth, H., Hassan, A. M., Hrastnik, B., Göttel, P., Delang, W., Nucl. Inst. and Methods, 105, 301 (1972).

3. Debertin, K., Schötzig, U., Weiss, H.M., PTB-Mitteilungen, 포, 187 (1975).

4. Current nuclear-decay scheme parameters are obtained from Dr. Murray Martin, Oak Ridge Nuclear Data Project, Oak Ridge National Laboratory.

5. McCallum, G. J., Coate, G. E., Nucl. Inst. and Methods, 130, 189 (1975).

6. Coursey, B. M., Hutchinson, J. M. R., Unterweger, M. P., submitted for publication.

7. Palms, J. M., Tanner, B. K., Wood, R. E., Boone, F. W., IEEE Trans. Nucl. Sci., NS-21, 585 (1974).

8. Laichter, Y., Notea, A., Shafrir, N. H., Nucl. Inst. and Methods, 113, 61 (1973).

9. Noyce, J. R., Hutchinson, J. M. R., Mann, W. B., Mullen, P. A., IEEE Trans. Nucl. Sci. (1976). 


\section{TABLE IA - NBS Mixed Radionuclide Gamma-Ray}

Emission-Rate Standards

\section{Description}

SRM 4215 Point Source

SRM 4216 Point Source

SRM 4242

SRM 4243

SRM 4254 450-ml Liquid Source

50-ml Liquid Source

5-ml Liquid Source
Approximate Total $\gamma$-RayEmission Rate on Date of Issue $(\gamma / s)$

$8 \times 10^{4}$

$4 \times 10^{4}$

$1 \times 10^{5}$

$2 \times 10^{5}$

$1 \times 10^{5}$

TABLE IB -- Calibration Lines for the 9 Components in the Mixed $\gamma$-Ray SRM's and Nominal Uncertainties in the $\gamma$-Ray Emission-Rate Values Reported

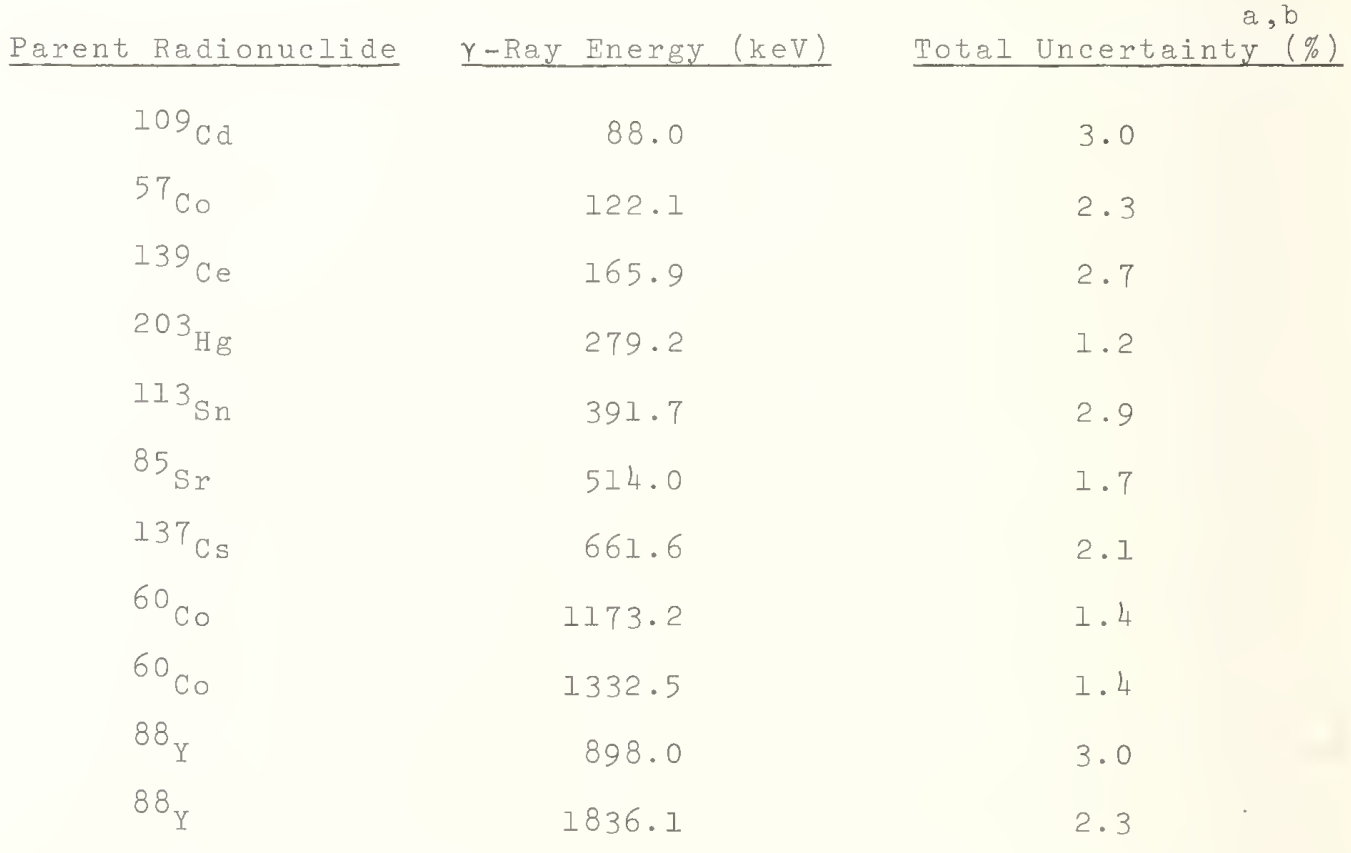

a Includes uncertainty quoted by compilers of decay-scheme data where appropriate

b Values shown here may differ slightly from one year's sources to the next 


\section{TABIE 2 - Standard Geometry counting Containers for TrBS Environmental Radioactivity Standards. Dimensions are given in centimeters.}

\begin{tabular}{|l|l|l|l|}
\hline & CYIITDER & CYIINDER & SPHERE \\
\hline Volume & $450 \mathrm{mI}$ & $50 \mathrm{ml}$ & $34 \mathrm{~cm}^{3}$ \\
\hline Intended Use For & Waste Water & Primary COOlant & IToble Gases \\
\hline SRY IO & 4242 & 4243 & $\begin{array}{l}4307^{\mathrm{a}} \\
4308\end{array}$ \\
\hline O. D. & $7.50 \pm 0.04$ & $3.80 \pm 0.02$ & $4.22 \pm 0.05$ \\
\hline Bottom & 0.5 & 0.3 & \\
\hline Wall & 0.275 & 0.2 & 0.1 \\
\hline \begin{tabular}{l} 
Height (Cyl.) \\
\hline $\begin{array}{l}\text { Internal Radius } \\
\text { (Sphere) }\end{array}$
\end{tabular} & 13.0 & 6.5 & 2.0 \\
\hline
\end{tabular}

a SRM 4307 and 4308 are gaseous $\gamma$-ray emission-rate standards (see text and reference 6).

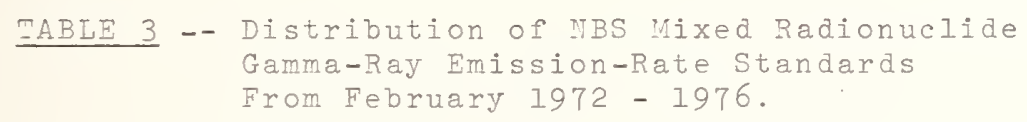

\begin{tabular}{|l|cccc|c|}
\hline USER CATEGORY & 1972 & 1973 & 1974 & 1975 & $1972-1976$ \\
\hline \multirow{2}{*}{ Tiuclear Power Stations } & 29 & 43 & 51 & 67 & 190 \\
National Laboratories & 26 & 43 & 54 & 60 & 183 \\
Other & 63 & 108 & 100 & 105 & 376 \\
\hline \multirow{2}{*}{ Total } & 118 & 194 & 205 & 232 & 749 \\
\hline
\end{tabular}




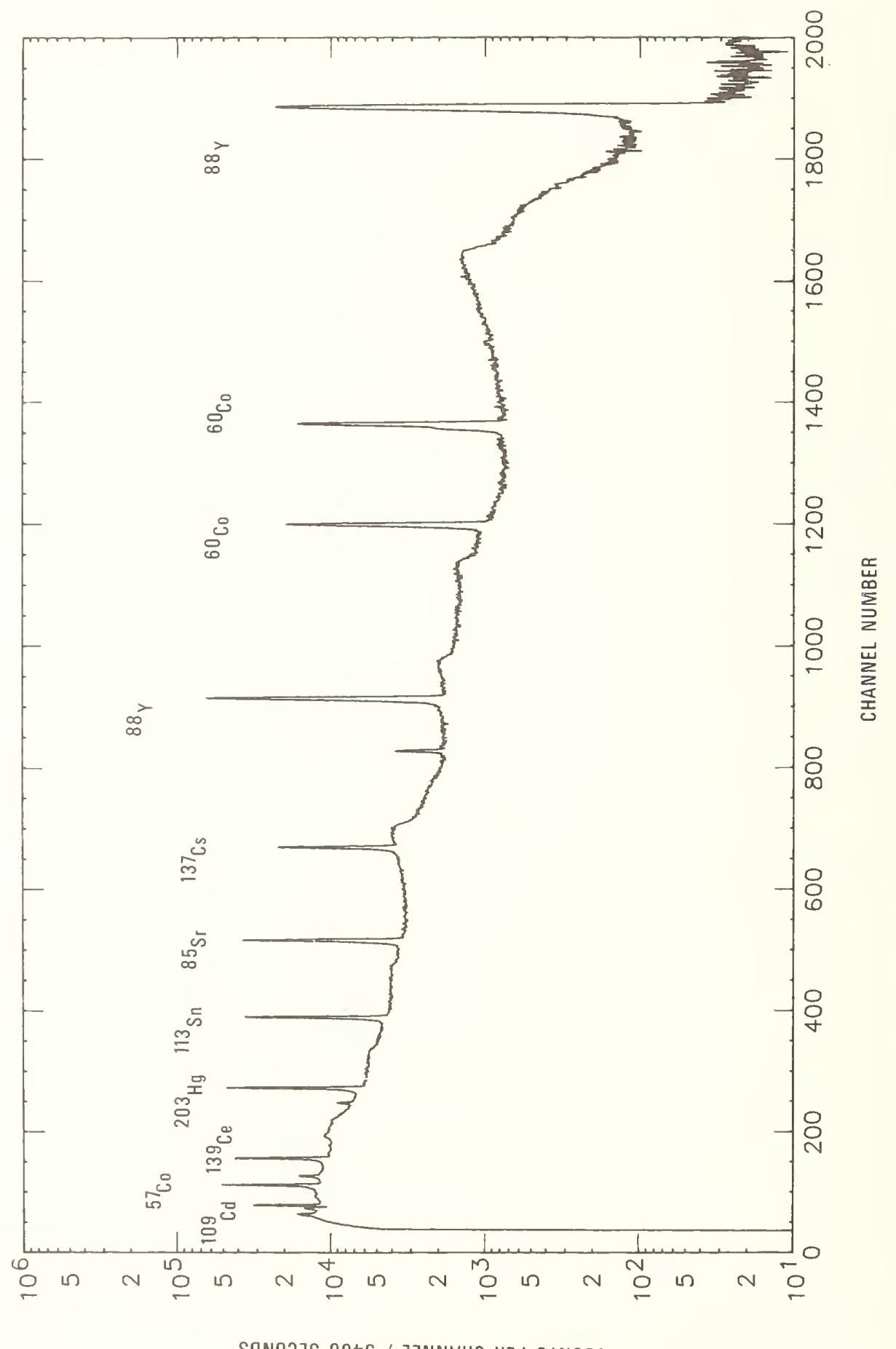

ฮ

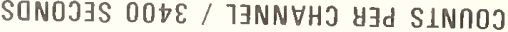



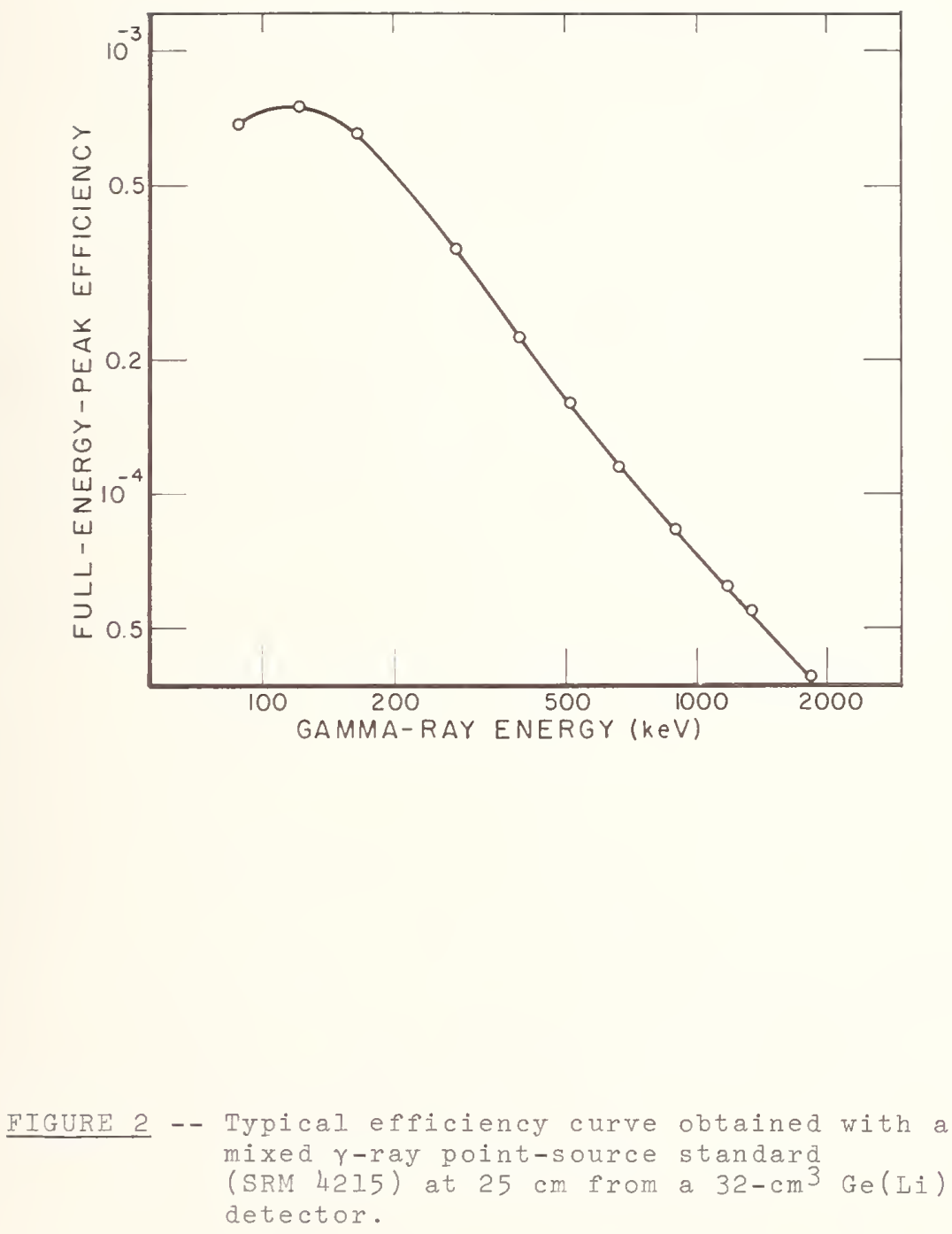

THE SAFETY AND RELIABILITY OF DECENTRALIZED TLD

Car1-0 Widel1

$\mathrm{AB}$ Atomenergi

Studsvik, Sweden

Decentralized reading of personnel TLD is in Sweden performed at the different nuclear power stations. The TLD results are automatically processed in the central dose register at Studsvik. In order to check and correct the readers, reference dosed dosimeters are used in each batch of TLD to be read. The reference dosed results are processed by the computer and will show the status of the different TLD readers. The reference dose irradiation is done with a Sr-90 source, which is placed above a rotating table in a brass shielding. The dosimeters are placed on this rotating table and will get a dose equivalent to 300 mrem gamma. In addition to this procedure a serie of calibration dosimeters is irradiated at a central calibration facility each month and read in the local TLD-reader.

\section{Introduction}

All nuclear energy installations in Sweden are performing their own personnel and environmental $\beta-\gamma$ dosimetry themselves. This is done by an automatic TLD-reader. The reader is coupled by the punched tape to a central dose register at Studsvik, figure 1. The terminals are connected to the computer via telephone lines.

In order to check the calibration of the TLD-system irradiated dosimeters are always used and evaluated together with the other dosimeters in the reader stack. In that manner the computer will get an information about the status of the TLD-reader and will automatically correct for deviations in the calibration.

\section{TLD-system}

The TLD-system consists of a dosimeter, figure 2, with four pellets on a slide. This slide is introduced into the reader and the pellets lifted up into the reading

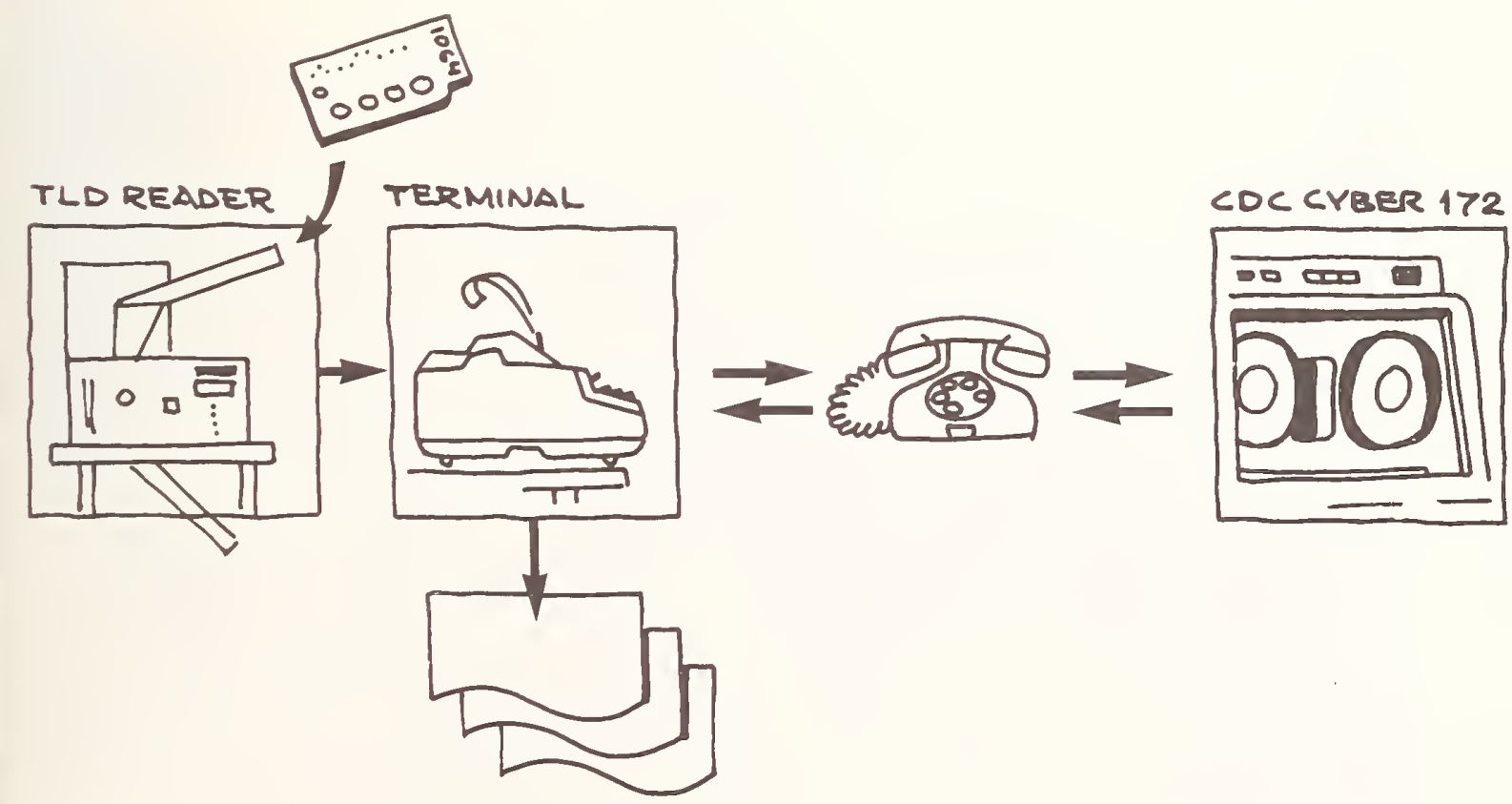

DOSE RECORDS

Figure 1. The Swedish Dose Register System 
chamber. Hot nitrogen is flushed on to the pellets and the light read by a peltier cooled photomultiplier. The TLD-reader is shown in figure 3 .

The dosimeter slide is identified by a hole coded number. This number is unique in this dosimetry system in Sweden. The dosimeter number is in the computer linked to the social security number of the wearer.

\section{Identification of persons}

In order to put the doses on the right person all doses are stored on the persons' social security numbers. These numbers consists in Sweden of ten digits, a six digits birth date, a three digits birth number and a check figure.

$$
\begin{aligned}
& \begin{array}{llll}
35 & 07 & 28 & -3517
\end{array} \\
& \text { Year Month Day Number Check } \\
& \text { Swedish social security number }
\end{aligned}
$$

Swedes are by this system very well identified.

Foreigners are handled in another way. For them the computer is given their birth date and the letter $U$. The computer is then listing all foreigners in the system with this birth date.
If the foreigner in question is not found on that list the computer proceeds and gives the man a three digit number and a check letter.

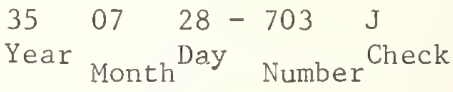

Foreigners number

The personal identification numbers have always a check function which allows the computer to check the correctness of the number.

\section{The calibration control of the TLD-system}

The results from the TLD appear on a punched tape. This punched tape is given to the computer via a local terminal at the nuclear power plant. The computer evaluates the doses from the TLD results. It is of course necessary to feed the computer with up-to-date calibration constants.

This is done by using special calibration or reference dosimeters which are always used when personnel dosimeters are read.

The calibration dosimeters are irradiated at the calibration $1 \mathrm{ab}$ at Studsvik. They are given doses from 20 to 10000 mrem.

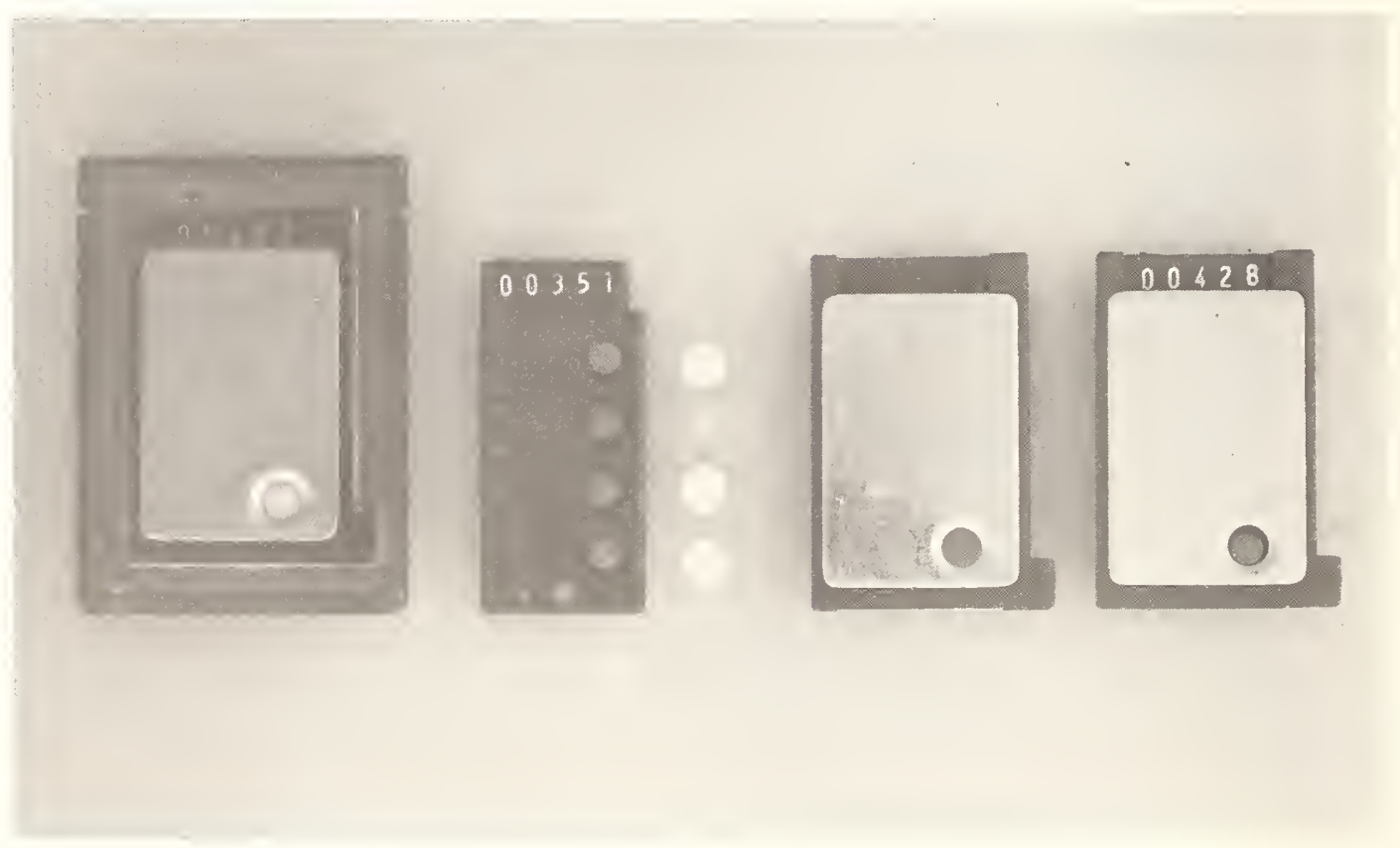

Figure 2. TL Dosimeter 
Dosimeter

number

Dose

mrem

m Gy

\begin{tabular}{|c|c|c|}
\hline 0 & & \\
\hline 1 & & 2 \\
\hline 2 & & 5 \\
\hline 3 & & 10 \\
\hline 4 & & 20 \\
\hline 5 & & 50 \\
\hline 6 & 1 & 00 \\
\hline 7 & 2 & 00 \\
\hline 8 & 5 & 00 \\
\hline 9 & 10 & 00 \\
\hline
\end{tabular}

This serie is used at each installation once a month.

Together with reading of other batches of dosimeters reference dosimeters are used. These dosimeters are irradiated in a reference dose irradiator with a Sr-90 source, figure 4 .

$\begin{array}{clc}\begin{array}{c}\text { Dosimeter } \\ \text { number }\end{array} & \begin{array}{l}\text { Dose } \\ \text { mrem }\end{array} & \text { m Gy } \\ 10 & \text { annealed dosimeter } \\ 11 & 300 & 3\end{array}$

In the reference dose irradiator the dosimeter slide with the pellets is placed on a rotating table. The table is rotated with a constant speed by a synchronous motor and the TLD pellets moved in under a $\mathrm{Sr}-90$ source with a large surface.

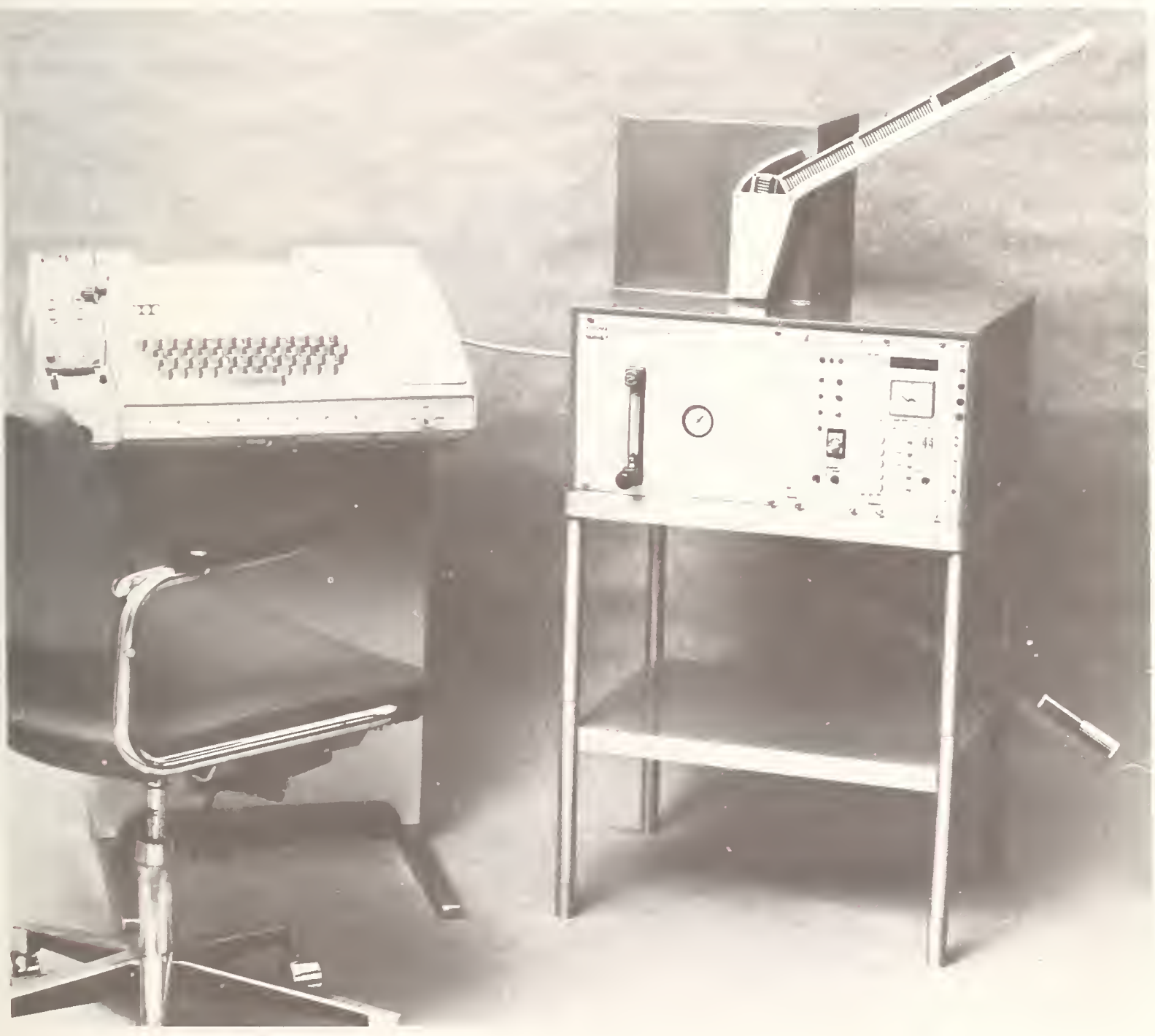

Figure 3. The Studsvik TLD-reader 
The TLD result is compared with the nominal value of the calibration or reference dosimeter which gives a calibration factor. This calibration factor is compared with that in the computer stored preceding calibration factor. The mean of the two calibration factors is used and stored back for further use in the computer.

If the new calibration factor deviates more than $\pm 5 \%$ from the old one a signal is given on the terminal. This signal has to be cleared before the evaluation can proceed.

In order to follow up the history of the calibration and reference dosimeters and the history of the calibration factors a record of the different monthly values is produced by the computer.

\section{Conclusion}

In this way a very good control of the standard of the whole country wide personnel dosimetry system can be obtained.

It is very convenient to use the reference dose irradiator for the day to day routine handing of the dosimeters. This reference dose irradiator can be used on the same table where the dosimeters are handled.

The use of social security numbers is of great advantage as the system can be used over the whole country without any identification problems.

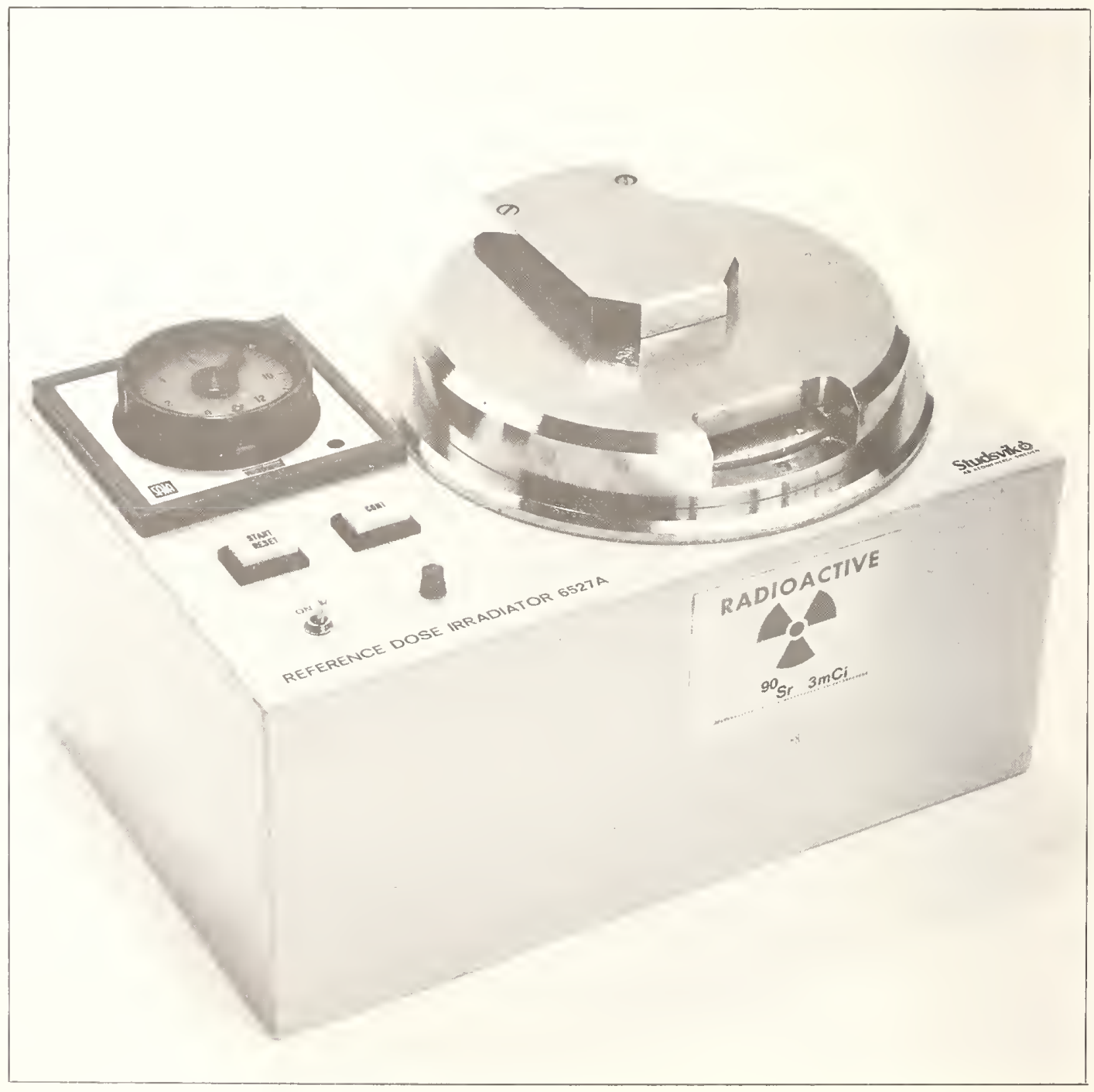

Figure 4. Reference Dose Irradiator 
Due to this central dose register and decentralized TLD-readings personnel can from the dose point of view move around Sweden without any restrictions or paper controls.
It has thus shown to be a very good tool for the safe use of radiation. We would like to see an international system of the same kind thus making it easier to handle dose records between countries. 



\title{
ERROR ANALYSIS OF ENVIRONMENTAL RADIATION MEASUREMENTS MADE WITH INTEGRATING DETECTORS
}

\author{
Gail de Planque Burke \\ Health and Safety Laboratory \\ U. S. Energy Research and Development Administration \\ New York, New York 10014 \\ Thomas F. GeselI \\ The University of Texas School of Public Health
}

Houston, Texas 77025

\begin{abstract}
Proper use of integrating detectors (e.g., TLDs) for environmental monitoring, particularly around nuclear facilities, requires a high degree of measurement accuracy and a meticulous assessment of measurement errors.

Mathematical expressions for the dose in air at a site are formulated in terms of factors affecting the measurement including detector efficiency and calibration techniques as well as characteristics inherent to the detector such as energy dependence and contributions to the total during storage and transport of the detector. The assessment of errors for individual terms in the equations is discussed and errors are combined to determine the accuracy of the estimated dose.
\end{abstract}

\section{(Error; Environmental Radiation Measurements; Integrating Detectors; TLD)}

\section{Introduction}

Integrating solid state detectors, for example, thermoluminescence dosimeters, are now used extensively for environmental radiation monitoring, particularly in the vicinity of nuclear facilities. Measurements made with a network of integrating detectors are often used either alone or in conjunction with other measurements to assure compliance with regulations such as Appendix I of $10 \mathrm{CFR} 50^{1}$ or the proposed Environmental Protection Agency standard concerning environmental radiation protection for nuclear power operations. 2 This use of integrating detectors requires not only a high degree of measurement precision and accuracy but also an assessment of measurement errors.

A recent intercomparison ${ }^{3}$ of measurements made with integrating detectors, primarily TLDs, demonstrated that reasonably accurate environmental radiation measurements are feasible. However, it is apparent that the errors quoted by many of the participants frequently underestimated the actual errors. The study involved the intercomparison of a set of dosimeters given a known dose in the laboratory and another set exposed for three months in the environment. In the latter case, the reported results differed from the mean of all the results by more than the participants' stated errors in 37 cases out of 54 . Somewhat better results were obtained for the laboratory-exposed dosimeters, where 22 of 54 cases differed from the mean by more than the stated error. These results indicate the need for a method of error assessment.

An ANSI Standard $d^{4}$ is available which designates performance, testing and procedural specifications for thermoluminescence dosimetry used for environmental measurements. While the standard specifies acceptable error limits and provides tests for determining individual errors, it does not recommend a method for the assessment of the total error.

This paper contains mathematical expressions for environmental radiation measure ments and a method for assessing the total errors. Our simple treatment provides a practical solution in a situation where quite often the practice is to report either no error or an error reflecting only measurement precision. The treatment presented uses thermoluminescence dosimetry as a particular 
case, however, the methods when suitably adjusted should be appropriate for most integrating detectors.

\section{Field Measurements}

To obtain a measurement of radiation dose in air at a particular location in the environment for a designated period, the ideal integrating detector would be "turned on", marking the beginning of the measurement interval, only after arrival at the specified location and "turned off", marking the end of the measurement interval, before removal from the location. In this ideal case

$$
\mathrm{x}_{\mathrm{FD}}=\left(\dot{\mathrm{X}}_{\mathrm{I}}+\alpha \dot{\mathrm{D}}_{\mathrm{F}}\right) \mathrm{t}_{\mathrm{F}} \mathrm{f}_{\mathrm{F}}
$$

where

$\mathrm{X}_{\mathrm{FD}}=$ the $\mathrm{TL}$ response of the field detector in units of exposure,

$\dot{\mathrm{X}}_{\mathrm{I}}=$ the exposure rate resulting from self-irradiation,

$\dot{\mathrm{D}}_{\mathrm{F}}=$ the average dose rate in air at the field location during the time the detector was operating at that location,

$t_{F}=$ the length of time the detector was operating at the field location,

$f_{F}=$ the fraction of the true exposure producing TL response which may differ from unity due to timetemperature-dependence (commonly occurring in the form of "fading"), and

$I / \alpha=$ the factor required to convert exposure to dose in air.

Quantities expressed in units of exposure, i.e., $x$, or $\dot{X} t$, indicate that the TL response of the detector obtained when measuring these quantities is equivalent to the TI response to a given exposure during calibration. Thus the conversion from TL response, expressed as exposure, to absorbed dose in air requires that the field energy spectrum and angle of incidence relative to the calibration energy spectrum and angle of incidence as well as the conversion factor from roentgens to rads, assuming electron equilibrium, be taken into account. In this case

$$
\alpha=\mathrm{ed} / 0.869
$$

where

I $R=0.869$ rads, assuming electron equilibrium, 5

e $=$ the fraction of the true dose producing $\mathrm{TL}$ response which may differ from unity due to the energy dependence of the detector response, and $\mathrm{d}=$ the fraction of the true dose producing TL response which may differ from unity due to the directional dependence of the detector response.

The time-temperature-dependence term results from the instability of many TL phosphors. Most often the detector undergoes a loss of stored signal with time, the rate of loss being dependent on temperature. Although it does not seem to completely describe the phenomenon, ${ }^{6}$ the RandallWilkins model ${ }^{7}$ can be used to determine corrections for time-temperature-dependence. Corrections are applied in two situations. When the exposure rate is constant during the measurement interval, the fraction of the actual or true exposure that results in TL response is

$$
f=\left(1-e^{-\lambda t}\right) / \lambda t
$$

where

$$
\begin{aligned}
\lambda= & B \exp (-\mathrm{E} / \mathrm{kT}), \\
\beta= & \text { the frequency factor (related to the } \\
& \text { presence of lattice vibrations in the } \\
& \text { phosphor), } \\
\mathrm{E}= & \text { the trap depth, } \\
\mathrm{k}= & \text { the Boltzmann constant, and } \\
\mathrm{t}= & \text { the length of the measurement interval. }
\end{aligned}
$$

When exposure has occurred before the beginning of the measurement interval and no further exposure occurs during the interval, the fraction of the true exposure resulting in $\mathrm{TL}$ response is

$$
f^{\prime}=e^{-\lambda t} \text {. }
$$

Other methods may, of course, be used in determining $f$ and $f^{\prime}$, but these factors will still affect the measured response of the detector in a similar fashion.

These terms represent the primary factors known to affect TLDs. If a different type of integrating detector is used, some factors may be eliminated, e.g., selfirradiation, while others may have to be added. However, the equation should be of an analogous form.

Equation (1) can be solved for $\dot{D}_{\mathrm{F}}$ or $\dot{\mathrm{D}}_{\mathrm{F}} \mathrm{t}_{\mathrm{F}}$ to determine the average dose rate or the total dose at the field location during the time the detector was operating at that location. Unfortunately, this ideal case is impossible practically for TLD unless the detector can be prepared (annealed) and analyzed (read out) right at the field location. In practice, the detector is prepared in a laboratory, perhaps stored briefly, transported to the field location, 
Ieft there for some time, transported back to the laboratory, and perhaps stored again before readout. Thus the entire measurement cycle may be composed of several intervals, of which the field interval is only one, and the total exposure obtained with the field detector includes exposures other than the field dose. Control detectors are commonly used to estimate the exposures received by the field detector during storage or transit or both. We now consider a few of the more common situations involving both field and control detectors for isolating the field dose.

The simplest practical case is that where the field detector is prepared as clase to the field location as possible with essentially no storage or transit prior to exposure in the field. At the conclusion of the field interval, a control detector is prepared and transported with the field detector to the laboratory and both are immediately read out. In this case the exposure for the field detector is given by

$$
\begin{aligned}
X_{F D}= & \left(\dot{X}_{I}+\alpha \dot{D}_{F}\right) t_{F} f_{F} f^{\prime} \\
& +\left(\dot{X}_{I}+\alpha \dot{D}_{T}\right) t_{T 2} f_{T 2}^{\prime}
\end{aligned}
$$

where

$\dot{D}_{T_{2}}=$ the average dose rate during retum transit (the subscript T2 indicating post-field transit),

$t_{\text {T2 }}=$ the length of time of the postfield transit interval,

$\mathrm{fr}_{\mathrm{T}}=$ the fraction of the true transit exposure producing TI response which may differ from unity due to time - temperature-dependence,

$f^{\prime} \mathrm{T} z=$ the fraction of the exposure received up to the beginning of the post-field transit interval producing TL response which may differ from unity due to time- temperaturedependence.

The exposure for the control detector is given by

$$
X_{C D}=\left(\dot{X}_{I}+\alpha \dot{D}_{T 2}\right) t_{T 2} f_{T 2}
$$

Subtracting equation (4) from equation (3) yields

$$
X_{F D}-X_{C D}=\left(\dot{X}_{I}+\alpha \dot{D}_{F}\right) t_{F} f_{F} f^{\prime}{ }_{T 2}
$$

assuming that self-irradiation is identical for both the field and the control detectors (if it is not, equation (5) will be more complex) and assuming that the $\alpha$ appropriate for the transit situation is the same as the $\alpha$ used for the field interval. The error involved in assuming the latter will be discussed below. Equation (5) can be solved for $\dot{D}_{F}$ or $\dot{D}_{F} t_{F}$ to determine the dose or average dose rate at the field location during the field interval. These quantities can thus be determined from two measurements and estimates of $\dot{X}_{I}, t_{F}, f_{F}$ and $f^{\prime}{ }_{T}$, the latter being the fraction of the true field dose producing a TI response which may differ from unity due to fading during transit.

If the retum transit is followed by a storage interval prior to readout, the field detector then yields

$$
\begin{aligned}
\mathrm{X}_{F D}= & \left(\dot{\mathrm{X}}_{\mathrm{I}}+\alpha \dot{\mathrm{D}}_{\mathrm{F}}\right) t_{\mathrm{F}} \mathrm{f}_{\mathrm{F}} \mathrm{f}^{\prime}{ }_{\mathrm{T} 2} f^{\prime} \mathrm{S}_{2} \\
& +\left(\dot{\mathrm{X}}_{\mathrm{I}}+\alpha \dot{\mathrm{D}}_{\mathrm{T} 2}\right) \mathrm{t}_{\mathrm{T} 2} \mathrm{f}_{\mathrm{T} 2} \mathrm{f}^{\prime} \mathrm{S} 2 \\
& +\left(\dot{\mathrm{X}}_{\mathrm{I}}+\dot{\mathrm{X}}_{\mathrm{S} 2}\right) \mathrm{t}_{\mathrm{S} 2} \mathrm{f}_{\mathrm{S} 2}
\end{aligned}
$$

where the subscript S2 indicates storage after the field interval and $\dot{\mathrm{X}}_{\mathrm{S}_{2}}$ is the exposure rate in the storage area determined with the type of detector used in the field and as controls. (IF $\dot{\mathrm{X}}_{S}$ is determined by other means, a factor analogous to $\alpha$ may be required so that this quantity is expressed in the proper units). In this case, the exposures received during the field and transit intervals are subject to possible fading during storage as indicated by $\mathrm{f}^{\prime} \mathrm{T}$ a in the first and second terms. The control detector yields

$$
\begin{aligned}
x_{C D}= & \left(\dot{X}_{I}+\alpha \dot{D}_{T 2}\right) t_{T 2} f_{T 2} f^{\prime}{ }_{S 2} \\
& +\left(\dot{X}_{I}+\dot{x}_{S 2}\right) t_{S 2} f_{S 2} .
\end{aligned}
$$

The difference, given by

$$
X_{F D}-X_{C D}=\left(\dot{X}_{I}+\alpha \dot{D}_{F}\right) t_{F} f_{F} f^{\prime}{ }_{T z} f_{S z}^{\prime}
$$

is identical to equation (5) except for $\mathrm{f}^{\prime} \mathrm{S} \mathbf{z}$.

A more complicated situation exists when the field and control detectors undergo storage and transit prior to the field inter val as well as after the field interval. In this case

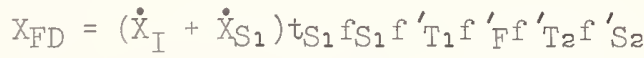

$$
\begin{aligned}
& +\left(\dot{X}_{\mathrm{I}}+\alpha \dot{\mathrm{D}}_{\mathrm{T} 1}\right) \mathrm{t}_{\mathrm{T} 1} \mathrm{f}_{\mathrm{T} 1} \mathrm{f}_{\mathrm{F}}^{\prime} \mathrm{f}_{\mathrm{T} 2}^{\prime} \mathrm{f}^{\prime} \mathrm{S} 2 \\
& +\left(\dot{\mathrm{X}}_{\mathrm{I}}+\alpha \dot{\mathrm{D}}_{\mathrm{F}}\right) \mathrm{t}_{\mathrm{F}} \mathrm{f}_{\mathrm{F}} f^{\prime} \mathrm{Tz} \mathrm{f}^{\prime} \mathrm{Sz} \\
& +\left(\dot{X}_{\mathrm{I}}+\alpha \dot{\mathrm{D}}_{\mathrm{T} 2}\right) \mathrm{t}_{\mathrm{T} 2} f_{\mathrm{Tz}} f^{\prime} \mathrm{Sz} \\
& +\left(\dot{X}_{I}+\dot{X}_{S 2}\right) t_{S 2} f_{S 2}
\end{aligned}
$$

where the subscripts SI and TI apply to prefield storage and transit, respectively. The 
control detector, prepared with the field detector and accompanying the latter at all times except during the field interval yields

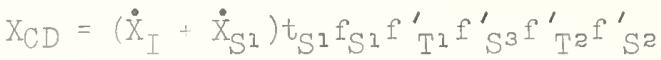

$$
\begin{aligned}
& +\left(\dot{X}_{\mathrm{I}}+\alpha \dot{D}_{\mathrm{T} 1}\right) t_{\mathrm{T} 2} \mathrm{f}_{\mathrm{T} 1} \mathrm{f}^{\prime} \mathrm{S}_{3} f^{\prime}{ }_{\mathrm{T} 2} \mathrm{f}^{\prime} \mathrm{S} 2 \\
& +\left(\dot{X}_{I}+\dot{X}_{S 3}\right) t_{S_{3}} f_{S_{3}} f^{\prime} T_{2} f^{\prime} S_{2} \\
& +\left(\dot{X}_{\mathrm{I}}+\alpha \dot{D}_{\mathrm{T} 2}\right) t_{\mathrm{T} 2} f_{\mathrm{T} 2} f^{\prime} \mathrm{Sz}_{2} \\
& \left(\dot{\mathrm{X}}_{\mathrm{I}}+\dot{\mathrm{X}}_{\mathrm{S} 2}\right) \mathrm{t}_{\mathrm{S} 2} \mathrm{f}_{\mathrm{S} 2}
\end{aligned}
$$

where the subscript S3 indicates storage during the field interval. This storage should be sufficiently near the field location so that transit time between the storage area and the field location is negligible and $t_{S_{3}}=t_{F}$. Subtracting equation (10) from equation (9) and simplifying yields

$$
\begin{aligned}
& \mathrm{X}_{\mathrm{FD}}-\mathrm{X}_{\mathrm{CD}}=
\end{aligned}
$$

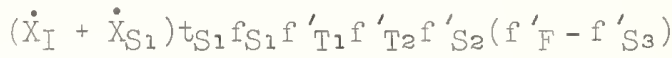

$$
\begin{aligned}
& +\left(\dot{X}_{I}+\alpha \dot{D}_{T 1}\right) t_{T 1} f_{T 1} f^{\prime}{ }_{T 2} f^{\prime} S_{2}\left(f^{\prime} F-f^{\prime} S_{3}\right) \\
& +\left(\dot{X}_{I}+\alpha \dot{D}_{F}\right) t_{F} f_{F} f^{\prime}{ }_{T 2} f^{\prime} S_{2} \\
& -\left(\dot{\mathrm{X}}_{\mathrm{T}}+\dot{\mathrm{X}}_{\mathrm{S} 3}\right) \mathrm{t}_{\mathrm{F}} \mathrm{f}_{\mathrm{S}_{3}} \mathrm{f}^{\prime} \mathrm{T}_{2} \mathrm{f}^{\prime} \mathrm{S}_{2}
\end{aligned}
$$

As in the earlier cases the terms pertaining to storage and transit after the field interval cancel. However, the terms pertaining to storage and transit prior to the field interval remain since the field detector and the control detector are located at different places during the field interval. Hence the fading undergone by the two detectors on the exposures received prior to and during the field interval may be different. The exposure received by the field detector is subject to fading in the field while that received by the control detector is subject to fading in the field storage area. In all other respects, the control accurately represents the extraneous exposures received by the field detector.

Other combinations of field and control detectors are possible and can be described by analogous equations. In general, the exposure received by a detector is given by

$$
\begin{aligned}
X= & \sum_{i=1}^{n}\left[\left(\dot{X}_{I}+\alpha_{i} \dot{D}_{i}\right) t_{i} \cdot\right. \\
& \left.\cdot f_{i}\left(f^{\prime}{ }_{i+1} \cdot f^{\prime}{ }_{i+2} \cdot \ldots \cdot f_{n+1}^{\prime}\right)\right]
\end{aligned}
$$

$$
\begin{aligned}
\text { where } & \\
f_{n+1}^{\prime}= & 1 \\
X & \text { the TI response of the detector in } \\
& \text { units of exposure, and } \\
= & \text { a single interval during the entire } \\
& \text { measurement cycle, e.g., the time } \\
& \text { spent in storage prior to field } \\
& \text { exposure. }
\end{aligned}
$$

When field and control measurements are combined, all terms will cancel except those having factors resulting from the detectors being in different locations during corresponding intervals.

\section{Error Assessment}

The field dose can be estimated from equations (1), (5), (8), (11) or similar equations depending on the manner in which the field and control detectors are used during the entire measurement cycle. For example, in the case represented by equation (5) the field dose rate is given by

$$
\dot{D}_{F}=\frac{X_{F D}-X_{C D}}{f_{F}^{f^{\prime}}{ }_{T} \alpha t_{F}}-\frac{\dot{X}_{I}}{\alpha}
$$

where $X_{F D}$ and $X_{C D}$ are TLD measurements and $\dot{\mathrm{X}}_{\mathrm{I}}, \alpha, \mathrm{t}_{\mathrm{F}}, \mathrm{f}_{\mathrm{F}}$ and $\mathrm{f}^{\prime}{ }_{\mathrm{T} \text { 2 }}$ are measured or estimated separately. The equation is of the form $V=f\left(x_{1}, x_{2}, \ldots x_{n}\right)$. If each factor in this equation is independent so that the possibility of error compensation exists, the error for $V$ is ${ }^{8}$

$$
\begin{aligned}
S_{V}= & {\left[\left(\frac{\partial V}{\partial x_{1}}\right)^{2}\left(S_{x_{1}}\right)^{2}+\left(\frac{\partial V}{\partial x_{2}}\right)^{2}\left(S_{x_{2}}\right)^{2}+\right.} \\
& \left.+\ldots+\left(\frac{\partial V}{\partial x_{n}}\right)^{2}\left(S_{x_{n}}\right)^{2}\right]^{\frac{1}{2}}
\end{aligned}
$$

where $\mathrm{S}_{\mathrm{X}_{i}}$ = the standard deviation for $\mathrm{x}_{i}$.

Assuming that each factor in equation (13) is independent, the error for the field dose rate in this case is given by

$$
\begin{aligned}
S_{\dot{D}_{F}}= & {\left[\left(\frac{\partial \dot{D}_{F}}{\partial X_{F D}}\right)^{2} S_{\mathrm{X}_{F D}^{2}}^{2}+\left(\frac{\partial \dot{D}_{F}}{\partial \bar{X}_{\mathrm{CD}}}\right)^{2} S_{\mathrm{X}_{\mathrm{CD}}^{2}}^{2}+\right.} \\
& +\left(\frac{\partial \dot{D}_{F}}{\partial \dot{X}_{I}}\right)^{2} S_{\dot{X}_{I}^{2}}^{2}+\left(\frac{\partial \dot{D}_{F}}{\partial f_{F}}\right)^{2} S_{f_{F}^{2}}^{2} \\
& +\left(\frac{\partial \dot{D}_{F}}{\partial f_{T}^{\prime}}\right)^{2} S_{f^{\prime}}^{2}+\left(\frac{\partial \dot{D}_{F}}{\partial t_{F}}\right)^{2} S_{t_{F}^{2}}^{2}+ \\
& \left.+\left(\frac{\partial \dot{D}_{F}}{\partial \alpha}\right)^{2} S_{\alpha}^{2}\right]^{\frac{1}{2}} .
\end{aligned}
$$


Analogous equations for detemining the total error can be derived for equations (1), 8), and (11). To compute the value of $\mathrm{S}_{\dot{D}_{F}}$, the errors for each individual factor must be obtained. A thorough treatment of the methods available for determining each factor and its respective error is beyond the scope of this paper. However, some basic concepts should be considered when assessing the individual emrors.

\section{Error issociated with $\alpha$}

"hile $\alpha$ functions as an independent term ir equation (13) its components e and $d$ given in equation (2) may not be strictly independent. The energy dependence term, e, arises because the response of the detector to environmental radiation including cosmic rays) representirg a broad energy spectrum is usually not identical to the response to the calibration source, generally ${ }^{226} \mathrm{Ra}$, ${ }^{137} \mathrm{CS}$, or ${ }^{60} \mathrm{Co} .{ }^{3}$ Although the detector response as a function of energy can be detamined by experiment or theory or both, a correction factor should take into account the detector response weighted by the particular environmental energy spectrum. Hence the error for this factor must reflect the accuracy with which both the detector response and the environmental spectrum are lmown.

The directional dependence term, $\mathrm{d}$, is required if the detector response is not spherically symmetric. In this case, the response of the detector will be different when the geometry of the incident flux in the environment differs from that encountered during calibration. While directional dependence can be estimated, the application of a correction for directional dependence requires that some assumption be made about the direction of the incident flux. Again, the error in d must reflect the accuracy with which both the directional dependence and the direction of the incident flux in the environment are known.

Since d is actually a function of energy, $e$ and $d$ are not strictly independent. However, in the interest of simplicity, they will be assumed to be independent in order to avoid calculating covariance terms for assessing the total error. The effect on the total error of assuming the independence of $e$ and d should be insignificant relative to the problems involved in accurately assessing the individual errors for both e and $d$.
Error Associated with $f$ and $f^{\prime}$

As indicated earlier, corrections for time-temperature-dependence may be necessary. Appropriate corrections can be based on theory, for example, the Randall-Wilkins model, or determined empirically by means of tests simulating actual storage, transit and field conditions. Even if the amount of fading or the fading rate as a function of temperature is known, corrections can be made only if certain assumptions are made about the actual exposure conditions. When fading occurs during interval i following the irradiation interval, the proportion of the signal retained at the end of interval i is given by $f^{\prime} i$, which should be detemined for the real temperature profile (ideally the temperature of the phosphor) or some approximation thereof. When fading and irradiation occur simultaneously, the proportional signal retained is given by $f$, which should also be determined for the real temperature profile. In the latter case, some assumption must be made about the irradiation rate; usually it is assumed to be constant.

Methods for minimizing the effects of time-temperature-dependence of TLD's and for determining these effects directly are discussed in the ANSI Standard. ${ }^{4}$ The errors for $f$ and $f^{\prime}$ are difficult to assess exactly and must include not only the errors for the amount of fading or the fading rate as a function of temperature, but the errors involved in the assumptions made regarding the actual exposure conditions.

As can be seen from equation (11), the fading factors prevent the cancellation of otherwise similar terms for the field and control detectors when both detectors are exposed prior to the field interval. If the errors for these factors are large, it is probably best to avoid this particular use of field and control detectors for isolating the field dose.

Error Associated with t

The error associated with $t$, which in equation (13) is the length of time of the field interval, results from the difficulty in precisely defining the beginning and end of the field interval. In most practical situations there is some small but finite amount of time during which the field detector and the control detector are separated, but during which either the field detector is not in the field or the control detector is not in storage (S3). During this time the field and control detectors 
may not be affected identically. Since determining this difference is often impractical, one way of approximating the possible error involved is by regarding the error as one of timing. However, when assigning this error, it is really the error in assuming that the two detectors were affected in an identical manner during this brief interval of separation that must be considered.

Error Associated with $\dot{\mathrm{X}}_{\mathrm{I}}$

Self-irradiation occurs in TI detectors when the phosphor itself or its packaging is radioactive. For example, self-irradiation occurs with most glass-encapsulated phosphors because of ${ }^{4} \mathrm{~K}$ in the glass. Usually $\dot{\mathrm{X}}_{\mathrm{I}}$ is determined empirically.

In the equations presented, it was assumed that $\dot{X}_{I}$ is identical for the field and control detectors. This assumption permits the cancellation of terms and hence considerably simplifies the equations defining $D_{F}$. This assumption can be made as long as the two values for $\dot{X}_{I}$ are reasonably similar, if this is true, the error for $\dot{X}_{I}$ should reflect the spread in the values of $\dot{\mathrm{X}}$ for the detectors in question. If the values differ substantially, then the assumption becomes unreasonable, the equations must be expressed in the appropriate more complex form and the errors for $\mathrm{X}_{\mathrm{I}}$ in each case should reflect the accuracy of the estimated exposure rate due to self-imadiation.

Error Associated with $\dot{\mathrm{X}}_{\mathrm{S}}$

When storage is necessary prior to or during the field interval, one or more terms of the form $\dot{X}_{S}$ enter the equation(s) for the field dose, for example, equation (11). In this case, even if $f^{\prime} F$ is identical to $f^{\prime} S_{3}$ and the term including $\dot{\mathrm{X}}_{S I}$ becomes zero, the errors involved must still be included in the total error. Hence $\dot{X}_{S_{1}}$ and $i t s$ error must be determined. $\dot{\mathrm{X}}_{S} \mathrm{~s}$ cannot be eliminated from equation (11) and must also be determined.

If $\dot{X}_{S}$ itself is measured with the detectors used in the field and as controls, then the error for this term is simply the measurement error. If it is determined in another manner, then corrections may be necessary to insure that the value truly represents the TL response in units of exposure of the field and control detectors measuring $\dot{X}_{S}$. For instance, if a different detector is used to determine $\dot{X}_{S}$, it is essential to consider the possible difference in the response of the two types of detectors to the radiation field in the storage area.

\section{Error Associated with $\dot{D}_{T}$}

When transit takes place prior to the field interval, the term $\dot{D}_{T}$ appears in the equations for the field dose. Again using equation (11) as an example, the tem containing $\dot{D}_{\mathrm{Tl}}$ will become zero if $\mathrm{f}_{\cdot \mathrm{F}}^{\prime}=$ $\mathrm{f}^{\prime} \mathrm{S}_{3}$. However, as was the case for $\dot{\mathrm{X}}_{\mathrm{S}}$, the errors must still be included in the total error.

Unfortunately, $\dot{D}_{\mathrm{T} 1}$ is usually unknown. If it were known, then there would be little need for using the control detector for determining this portion of the extraneous exposure. Not only is it unknown, but it is extremely difficult to estimate with any accuracy when the transit conditions are uncertain as would be the case if the detectors are transported by airplane. The results of the intercomparison ${ }^{3}$ mentioned earlier revealed that variations in the magnitude of the transit doses could not be explained by differences in transit distance. Further, attempts to estimate these transit doses taking into account typical airplane speeds and altitudes, appropriate cosmic radiation data, and reasonable estimates of exposure during ground level transit yielded poor results.

Thus when transit prior to the field interval is unavoidable and the transit conditions are highly uncertain, large errors may be introduced and this technique for isolating field dose may be unsatisfactory.

Empors Associated with $\mathrm{X}_{\mathrm{FD}}$ and $\mathrm{X}_{\mathrm{CD}}$

$\mathrm{X}_{\mathrm{FD}}$ and $\mathrm{X}_{\mathrm{CD}}$ are the quantities determined from the field and control detectors, respectively. The errors for $\mathrm{X}_{\mathrm{FD}}$ and $\mathrm{X}_{\mathrm{CD}}$ are measurement errors and may be derived differenty depending on the calibration method. Calibration techniques are discussed in the ANSI Standard ${ }^{4}$ and fall into two general categories, batch calibration and individual calibration. Whichever method is used, the conversion from TL response to exposure is described by

$$
X=\left(\frac{N}{N_{C A L}}\right) X_{C A I}
$$

where $\mathrm{N}$ is the TI response of field or control detectors and $\mathrm{N}_{\text {CAI }}$ is the TL response of either the same detectors (individual calibration) or another detector(s) from the same batch (batch calibration) given a known exposure $\mathrm{X}_{\mathrm{CAI}}$ from the calibration source. 
f the detectors are calibrated individually, the reasurerent error represents the precision with which the detector produces identical TL responses given identical exposures. Since the calibration exposures can never be reproduced exactly due to emors in position and timing, tests to determine the reproducibility of a single detector will include these emrors.

If batch calibration is employed, the measurement error represents the precision with which the detectors in the batch produce identical TL responses given identical exposures. Again, since the calibration exposures can never be identical, due to errors in position or timing or both, the unifomity of response for a batch of detectors will include these errors.

(Although when determining the measurement error for the batch calibration method, timing errors can be eliminated if all of the detectors to be tested are exposed simultaneously, it is probably more convenient to include this source of error in the measurement error for reasons which will be explained later.)

Tests for detemining the reasurement errors should be designed to incorporate the errors encountered in actual measurement situations. These include variations in detector preparation (cleaning, annealing, etc.) and readout (reproducibility of the reader heating cycle, stability of the reader electronics, etc.) as well as human errors (differences in carrying out all of the procedures involved). Variations due to physical differences among detectors or statistical variations in the physical processes (trapping efficiency, light emission, etc.) either among detectors or within a single detector as a function of time will be included automatically during testing. If both timing and position errors are included in the measurement error, then the error in $\mathrm{X}_{\mathrm{CAI}}$ represents only the error in the detemination of the exposure rate. from the calibration source at the calibration position (s).

Restricting the errors for $X_{F D}$ and $X_{C D}$ to measurement errors permits the error for $\mathrm{X}_{\mathrm{CAL}}$ to be considered in isolation. Since the error of $X_{C A I}$ affects not only XFD and $\mathrm{X}_{\mathrm{CD}}$ but usual1y $\mathrm{X}_{\mathrm{S}}$ and $\dot{\mathrm{X}}_{\mathrm{I}}$ as well, it will affect all of these tems systematically and should, therefore, enter into the field dose equation only once. If done this way, the term is then independent of all other terms and the error may be combined with all other errors in the manner described by equation (14).
Disregarding Certain Errors

Depending on the usage of the field dose estimates, it may be possible to ignore certain errors when assessing the total error.

For example, if simultaneous measurements at several locations within a small geographic area are made with a single batch of TLDs and the primary quantities of interest are their relative values, it would be reasonable to eliminate from the total error the error for $\mathrm{X}_{\mathrm{CAI}}$ since $\mathrm{X}_{\mathrm{CAI}}$ affects all of the measurements systematically. It might also be reasonable to ignore the errors for $\alpha$, $f$ and $f^{\prime}$ if there is some assurance that the effects of these terms are also systematic. Also, if measurements made at a single field location at various times are to be examined for time variations, it is again possible to ignore the error for $\mathrm{X}_{\mathrm{CAL}}$ and perhaps reasonable to ignore the error for $\alpha$. For both cases, the errors for the dose estimates are logically smaller than they would be if all sources of error were considered. Several other situations may permit the elimination of one or more error terms, but must be evaluated on a case-bycase basis.

If field doses determined from TLD measurements are to be compared with estimates made with a different detector calibrated against a different source, then all errors, including that for $\mathrm{X}_{\mathrm{CAL}}$ must be combined to determine the total error.

\section{Effect of Various Parameters on the Total Error for the Field Dose}

To determine the effect of individual parameters on the total error, typical values were chosen and then varied for the parameters in equations (5) and (11), and the total errors calculated. Equation (5) describes the relatively simple case involving no pre-field transit or storage and only post-field transit, whereas equation (11) describes the more complex case involving both pre- and post-field transit and storage.

Tables I and II contain the values chosen for nominal cases for equations (5) and (11), respectively. A percent error, representing one standard deviation, is assigned for each parameter. Both equations were solved for $D_{F}$ and the total error for $\dot{D}_{F}$ determined in the manner indicated by equation (14). All errors were considered in assessing the total error except that for XCAL which was omitted in order to focus on the errors inherent to the entire TLD 
measurement process. An error for $X_{C A I}$ can easily be combined with the total error using equation (14). For the simple case (Table I) and the more complex case (Table I) the errors for $\dot{D}_{F}$, excluding the errors for XCAL, are 6.6 and 7.0 percent, respectively.

Table III illustrates the effects of changes in individual parameters and their errors on the total error for $\dot{D}_{F}$. For each variation considered in Table III, aII values except $X_{F D}, X_{C D}$ and those 1 isted are the same as the values given in Tables $I$ and II. $X_{F D}$ and $X_{C D}$ were adjusted appropriateIy for each variation so that the estimated field dose rate, $\dot{D}_{F}$, would be about 7.82 $\mathrm{urad} / \mathrm{h}$ as in the nominal case.

The first three variations demonstrate the effects of changes in either the field fading or the error for field fading. Unless the fading is large, as in variation 2, the results are similar for the simple and complex cases. As fading itself increases, the error is greater in the complex case than in the simple one. This occurs mainly because the fading of the pre-field storage and transit exposures for the field detector is different from that for the control detector.

Variation 4 illustrates the effects of self-irradiation, assuming self-irradiation is identical for the field and control detectors. Variation 5 shows the effect of an increase in the uncertainty of the energy response. Since $e$ and $d$ appear together in the equations, the effects of any changes in d are the same as the effects of identical changes in $e$.

The effects of an increase in the transit dose rate and an increase in the error of the transit dose rate are illustrated in variations 6 and 7 . Although transit dose rate does not appear in equation (5), the total error still increases in the simple case because $\mathrm{X}_{F D}$ and $\mathrm{X}_{C D}$ are necessarily larger, resulting in larger measurement errors than in the nominal case. On the other hand, because transit dose rate is absent in equation (5), changes in the error for this term affect the total error only in the complex case.

Variations 8 and 9 indicate the effects produced when some of the previous variations are combined. Variations 10 and 11 are analogous to 6 and 7 in that the transit dose itself is increased and then the error for the transit dose rate is increased. The results show that increases in the error for the transit dose rate produce greater increases in the total error when the fading in the field is large. For a factor of five increase in the error for the transit dose rate, the total error increases by a factor of two when field fading, $f_{F}$, is 20 percent, whereas the increase in the total error is only a few percent when fading is only 3 percent.

Only a few of the possible situations are represented by the variations in Table III. However, any variation approximating a particular situation may be analyzed in the same manner.

\section{Conclusions}

A review of the results of an intercomparison of environmental radiation detectors indicated the need for developing a method of error assessment for environmental radiation measurements. Using thermoluminescence dosimetry as an example, we have formulated mathematical expressions for the dose in air for several cases where field and control detectors are used for isolating the dose at a f'ield site. Using standard statistical techniques, these equations can be used to determine the total error of an estimate of the field dose.

Typical cases were examined to reveal the effects of changes in the various parameters and their respective errors on the total error. The results indicate that the relative advantages of different applications of control and field detectors depend on measurement conditions.

The method presented here can readily be employed to estimate the total error for situations other than those considered. Also, when suitably adjusted, the techniques used should be appropriate for most integrating detectors.

In certain instances the method presented requires that some approximations be made, for example, certain parameters are assumed to be independent when this is not strictly true. Thus, from a statistical point of view, a more sophisticated treatment may be desirable and should be further investigated. The simple treatment presented is designed merely to provide an easily applied method for estimating total errors in a situation where frequently the practice is to report either no error or an error reflecting only measurement precision. 
The authors are grateful to K. J'Brien

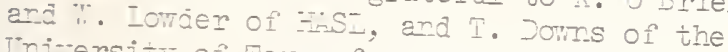
Uni-rersity of leyas for many helpful

aiscussiors. "ie also thenk R. Lare of the Uriversity of Texas for thein programing assistance.

\section{Dererences}

Tuclear Pegulatom Cormission (1975) "Radioactive liaterial ir Iight-iater-Cooled Vuclear Power Reactor Effluents (10 CFR 50, Aperdix -)," Federal Register, L0, lio. 87 ,
PD. 19439-19443.

${ }^{2}$ Environrental Protection LEency 1975)

"Enrironmental Radiation Protection fon Wuclear Zower Operations: Proposed Stand ards," Federal Register, LU, IIo. 104, 20. $23420-23425$.

${ }^{3} \mathrm{C} \in \mathrm{sel} I$, I. F., Eurke, G. de P. and Becker, K. (1976) "An -ntermatioral Intercomparison of Envimonental Dosimeters Under Field and laboratory conditions," Health Physics, 30 , po. $125-133$.
Americar liational Standard 1975) "Performance, Testing, and Procedural Specifications for Thermoluminescence Dosimetry (Environmental Applications)," ANSI N545 - 1975.

${ }^{5}$ Radiological Health Handbook (1970) U. S. Department of Health Education and Welfare, Rockville, Maryland.

"Burke, G. de P. (1974) "Thermoluminescence of CaF :iln Exposed at Elevated Temperatures to a Constant Rate of Iow-Level Radiation," pp. 315-345, in Proc. Fourth Int'l. Conf. on Iuminescence Dosimetry, Kraków, Poland.

${ }^{7}$ RandeII, J. T. and Wilkins, M. E. F. (1945) "Phosphorescence and Electron Traps," Proc. of the Royal Society, I84A, pp. 366-389.

8Eeers, Y. 1953) Introduction to the Theory of Error, Addison-Wesley, Cambridge, 


\section{TABLE I}

TEST PARAMETERS FOR EQUATION (5) NOMTNAI CASE

\begin{tabular}{llll}
\hline Parameter & \multicolumn{1}{c}{ Value } & Error* & Error (\%) \\
\hline$\dot{\mathrm{X}}_{\mathrm{I}}$ & $0.00 \mu \mathrm{R} / \mathrm{h}$ & $0.20 \mu \mathrm{R} / \mathrm{h}$ & - \\
$\mathrm{e}$ & 1.00 & 0.02 & 2.0 \\
$\mathrm{~d}$ & 1.00 & 0.02 & 2.0 \\
$\mathrm{t}_{\mathrm{F}}$ & $676 \mathrm{~h}$ & $2 \mathrm{~h}$ & 0.3 \\
$f_{\mathrm{F}}$ & 0.97 & 0.048 & 5.0 \\
$f^{\prime}{ }_{\mathrm{T} Z}$ & 1.00 & 0.005 & 0.5 \\
$\mathrm{X}_{\mathrm{FD}}$ & $7.00 \mathrm{mR}$ & $0.140 \mathrm{mR}$ & 2.0 \\
$\mathrm{X}_{\mathrm{CD}}$ & $1.10 \mathrm{mR}$ & $0.022 \mathrm{mR}$ & 2.0 \\
$\dot{D}_{\mathrm{F}}$ & $7.82 \mu \mathrm{rad} / \mathrm{h}$ & $0.69 \mathrm{\mu rad} / \mathrm{h}$ & 6.6 total \\
& & & $\begin{array}{l}\text { error } \\
\text { for } \mathrm{D}_{\mathrm{F}}\end{array}$ \\
\hline
\end{tabular}

*One standard deviation. 
TEST PARAIETERS FOR EQUATION (II) NOMINAI CASE

\begin{tabular}{|c|c|c|c|c|}
\hline Parameter & Value & Error* & Error & (8) \\
\hline$\dot{\mathrm{x}}_{I}$ & $0.00 u R / h$ & $0.20 \mu \mathrm{R} / \mathrm{h}$ & - & \\
\hline$\dot{\mathrm{X}}_{\mathrm{S} 1}$ & $2.00 u R / h$ & $0.20 u \mathrm{R} / \mathrm{h}$ & 10.0 & \\
\hline$\dot{\mathrm{X}}_{\mathrm{S}_{3}}$ & $2.00 \mu \mathrm{R} / \mathrm{h}$ & $0.20 \mu \mathrm{R} / \mathrm{h}$ & 10.0 & \\
\hline$a \dot{D}_{1}$ & $20.0 \mathrm{R} / \mathrm{h}$ & $20.0 \mu \mathrm{R} / \mathrm{h}$ & 100.0 & \\
\hline e & 1.00 & 0.02 & 2.0 & \\
\hline d & 1.00 & 0.02 & 2.0 & \\
\hline$t_{S 1}$ & $72 \mathrm{~h}$ & 0 & 0.0 & \\
\hline$t_{T 1}$ & $50 \mathrm{~h}$ & 0 & 0.0 & \\
\hline$t_{F}$ & 676 & 2 & 0.3 & \\
\hline$f_{S 1}$ & 1.000 & 2.005 & 0.5 & \\
\hline$f_{T 1}$ & 1.000 & 0.005 & 0.5 & \\
\hline$I^{\prime} T I$ & 1.000 & 0.005 & 0.5 & \\
\hline $\mathrm{f}_{\mathrm{F}}$ & 0.970 & 0.049 & 5.0 & \\
\hline$f^{\prime} F$ & 0.950 & 0.048 & 5.0 & \\
\hline $\mathrm{f}_{\mathrm{S} 3}$ & 1.000 & 0.005 & 0.5 & \\
\hline$f^{\prime} S_{3}$ & 1.000 & 0.005 & 0.5 & \\
\hline$f^{\prime} T^{2}$ & 1.000 & 0.005 & 0.5 & \\
\hline$f^{\prime} \mathrm{Sz}$ & 1.000 & 0.005 & 0.5 & \\
\hline $\mathrm{X}_{\mathrm{FD}}$ & $8.10 \mathrm{mR}$ & $0.16 \mathrm{mR}$ & 2.0 & \\
\hline $\mathrm{X}_{\mathrm{CD}}$ & $3.60 \mathrm{mR}$ & $0.07 \mathrm{mR}$ & 2.0 & \\
\hline$\dot{\mathrm{D}}_{\mathrm{F}}$ & $7.82 \mu \mathrm{rad} / \mathrm{h}$ & $0.73 \mu \mathrm{rad} / \mathrm{h}$ & 7.0 & $\begin{array}{l}\text { total } \\
\text { error } \\
\text { for } \dot{D}_{F}\end{array}$ \\
\hline
\end{tabular}

* One standard deviation. 
EFFECT OF CHANGES IN VARIOUS PARAMETERS AND THEIR ASSOC IATED ERRORS ON THE TOTAL ERROR

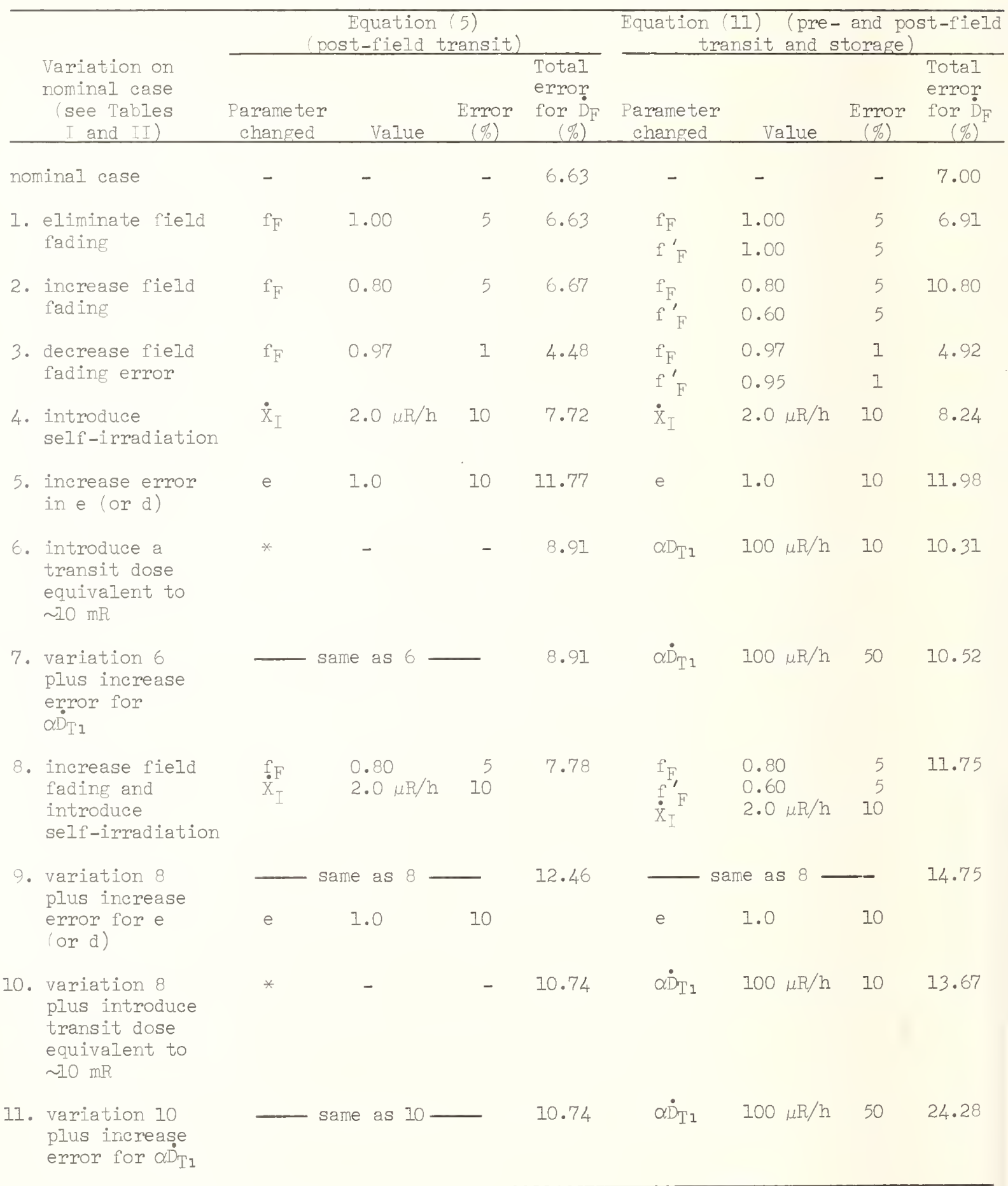

*Change occurs only in $\mathrm{X}_{\mathrm{FD}}$ and $\mathrm{X}_{\mathrm{CD}}$. 
RADIO-FREQUENCY ELECTROMAGNETIC RADIATION FROM PORTABLE AND MOBILE TELECOMMUNICATION TRANSMITTERS

$$
\text { J. F. Thiel }
$$

Texas Department of Health Resources Austin, Texas 78756

The increasing number of sources of electromagnetic radiation in the envircnment has placed more emphasis on the evaluation of such sources. Several of the more common sources of electromagnetic radiation to which individuals and equipment are exposed have been surveyed in an attempt to assess potential personnel hazards from the radiated fields. Measurements and examples of several typical types of antenna and transmitter combinations are presented.

\section{Introduction}

During the past several years the levels of radio-frequency electromagnetic radiation to which individuals have been exposed has grown dramatically. For example trersceivers used in the Citizens Radio Service license applications during the past eight (8) months have more than quadrupled over that received for the same period a year ago: forty thousarc epplications in September 1974; 160,000 in the month of September 1975; and currently 415,000 in December 1975. During this same period of time the number of licensed Citizens Band Stations has almost doubled from one million to two million with over 2,658,000 licensed as of January 1976. It is estimated that approximately 12,000,000 to 15,000,000 Citizen Band transmitters are presently in use in the United States. ${ }^{1}$ Reported cases of electromagnetic interference to other electronic devices, such as home entertainment equipment and antiskid braking systems on trucks has grown from approximately 45,000 during FY74-75 to an estimated 75,000 this year. During FY74-75, 69\% of the complaints received were from operations involving the Citizens Radio Service, a large majority of which were directly related to over-powered operation. Data which we have gathered indicates that approximately $0.5 \%$ of all transmitters used in the Citizens Radio Service are using power amplifiers to boost power output to between 75 and 100 watts radiated.

In 1959, the Department of the Navy initiated an effort to establish a national safety standard on the exposure of personnel to potentially hazardous electromagnetic radiation. The results of that $\epsilon$ ffort is the present American National Standards Institute Committee C95 on Radio Frequency Radiation Hazards. The current co-secretariates for the committee are the Institute of Electrical and Electronic Engineers and the Department of the Navy-Naval Electroni.c Systems Command.
The scope of the 095 committee project is as follows: "Hazards to mankind, volatile materials and explosive devices which are caused by man-made scurces of electromagnetic radiation. The frequency range of interest extends presently from $10 \mathrm{kHz}$ to $100 \mathrm{GHz}$. It is not intended to include inrrared, $\mathrm{X}$-rays or other ionizing radiations."

Presently existing standards published by the American National Standards Institute include ANSI C 95.1-1974, "Safety Level of Electromagnetic Radiation with Respect to Personnel." The applicability of this safety guide is as follows:

1. Normal environmental conditions - higher temperature or higher relative humidity requires that personnel exposure be lowered.

2. Dres; rot apply to the exposure of petients by a physician.

3. Frequency band of $10 \mathrm{MHz}$ thru $100 \mathrm{GHz}$.

4. All possible sources of electromagnetic radiation in the above range.

5. Continuous or intermittent exposure (time weighted average).

6. Whole--kody and partial-body exposure situation.

7. "Healthy people". For example, people without circulatory ailments.

As good as this standard is, it raises serious questions as to its applicability in specific circumstances.

Electromagnetic interference to electronic devices poses two distinct effects on the health and well-being of mankind. The first and most direct effect is that with electro-explosive devices and safety 
systems. The second and less direct effect is on home entertainment devices and medical prostetic devices such as the cardiac pacemaker.

In an attempt to review the magnitude of exposure of personnel, measurements have been made in the near field of antennas on mobile and portable telecommunications equipment.

Specifications of Typical Telecommunications Equipment

Antenna

Common antenna found to be used with mobile and portable equipment consists of the following:

1. Base loaded whip antennas whose electrical length is increased by the addition of inductive reactance at the base of the antenna. Base loaded antennas have been generally mounted on trunk decks or the areas surrounding the trunk lid. They are also found mounted on the top center of roofs and rarely on the front fenders.

2. Top loaded antennas whose electrical length is increased by the addition of inductive reactance at the top of the aritenna, have been generally found to be located on the rear deck or trunk of rehicles and sometimes mounted on tile mirrors of trucks.

3. Center loaded antennas whose electrical length is increased by the addition of inductive reactance at the center of the antenna, have been generally found to be located clipped to the rain gutters and mounted on mirrors of vehicles.

4. Quarter wave whip antennas have been generally found to be mounted on the rear fenders and bumpers of vehicles. They have also been found to be mounted on tool boxes in pick-up trucks directly behind the passenger cab.

5. Disguised antennas are generally mounted in locations similar to that used for normal car radio reception, with the addition of inductive load under the fender. These antennas are used for legal undercover work and more commonly by individuals who wish to disguise the fact that they have a radio in their vehicle to minimize theft of the radio.

All of the above antennas exhibit from unity to $5 \mathrm{dBi}$ gain in the far field.

Power Output of Equipment

Mobile aric portable equipment measured had power outputs ranging from 4 watts to 150 watts $\mathrm{CW}$ and to 200 Watts PEP. Portable equipment had nominally 2 to 4 watts power output in the VHF-Hi band with mobile units using external power packs having up to 25 watts output.

UHF equipment in the $400 \mathrm{MHz}$ thru 500 $\mathrm{MHz}$ band had nominal power outputs of 50 thru 110 watts using antennas with $5 \mathrm{dBi}$ gain. VHF-Hi equipment had power outputs of nominally 100 watts using antennas with $3 \mathrm{dBi}$ gain. VHF-Lo equipment had power outputs of nominally 100 watts with antennas of unity gain. Units used in the $27 \mathrm{MHz}$ band of the Citizens Radio Service were normally in the power output range of 50 thru 100 watts using unity gain antennas. It should be noted that the Citizens Radio Service is limited to 4 watts by Class D licenses issued by the Federal Communications Commission under $47 \mathrm{CFR} 95$ in the $27 \mathrm{MHz}$ ISM band.

Linear power amplifiers are manufactured by numerous concerns as broadbanded amplifiers designed for operation in the 1.6 thru 30 $\mathrm{MHz}$ range. Due to the high efficiency of the "broadband" design, it is an excellent amplifier for the 80 thru 10 meter amateur radio bands. However, many of these amplifiers are actually being used in violation of Federal Communications Commission rules and regulations in the 11 meter Citizens Band.

Several typical examples of these power amplifiers are given below in the table.

Because of the number of units in the field and training and background of the operators of the equipment, units of the Citizen Radio Service were chosen for measurement purposes. Part of any program for the purpose of radiation exposure control must analyze: who is being exposed, knowledge of the individuals being exposed of the potential exposure hazard, and potential number of individuals being exposed. 


$\begin{array}{lll}\text { A. } & 3.5 \mathrm{~W} & 100 \mathrm{~W} \\ \text { B. } & 2 \mathrm{~W} & 125 \mathrm{~W} \\ \text { C. } & 3 \mathrm{~W} & 80-110 \mathrm{~W} \\ \text { D. } & 4 \mathrm{~W} & 100 \mathrm{~W} \\ \text { E. } & 3 \mathrm{~W} & 300 \mathrm{~W} \\ \text { F. } & 1 \mathrm{~W} & 100 \mathrm{~W} \\ \text { G. } & 3 \mathrm{~W} & 100 \mathrm{~W} \\ \text { H. } & -1.5 \mathrm{~W} & 100 \mathrm{~W}\end{array}$

Measurements Made

Measurements of the electric field strength in the proximity of mobile and portable telecomunications transmitters were made using a General Microwave RAHAM Model 2 Power Density Meter. The Model 2 meter uses two diode detectors attached to orthogonal dipoles to measure the voltage sield strength to power density assuming a free space impedance of 377 ohms.

Experimental results indicate that when one measures within field strengths in the near field of an antenna that reproducible results can only be obtained if the measuring process does not significantly distort the radiated field.

At distances of 50 centimeters or less from antennas whose input power is 100 watts power densities (electric field strength equivalent of free space) in excess of one milliwatt per square centimeter can be found. As one approaches the loading coils on antennas shorter than one quarter wave length, power densities in excess of several hundred milliwatts per square centimeter are found.

Direct personal contact with an antenna whose input power is in excess of 35 watts may result in a visible radio-frequency burn.
Measurements were made on a two watt walkie-talkie operating in the VHF-Hi band using a bcrrowed Narda magnetic field probe. At approximately five centimeters from the base of the antenna a magnetic field intensity equivalent to a free space power density of 2.5 milliwatts per square centimeter was found. The maximum electric field strength equivalent to a free space power density of five milliwatts per square centimeter was found near the end of the antenna.

\section{Calculation Technique for} Radiation Hazard Evaluation

A technique for radiation hazard evaluation was described by Mumford ${ }^{3}$. The power density to which individuals are exposed from an electromagnetic radiation source depends on its radiated power in the direction of the individual being exposed. A conservative estimate of this power density in the near field of an antenna may be calculated assuming an isotropic point source at the center of radiation and the power density $W$ calculated from the equation:

$$
w=\frac{P}{4 \pi R^{2}}
$$

Where

$$
\begin{aligned}
\mathrm{W}= & \text { power density in watts } \\
& \text { per square meter } \\
\mathrm{P}= & \text { effective isotropic } \\
& \text { radiated power in watts } \\
\mathrm{R}= & \text { distance in meters } \\
\pi= & 3.1416 \ldots
\end{aligned}
$$

In order to have a conservative estimate of the power density in the near field one must assume 100\% ground reflection, which doubles the electric field strength

and quadruples the power density. 3 The equation below provides an estimate of the distance at which the power density exceeds the radiation protection guide given in American National Standard C 95.1-1974:

$$
d(m)=\sqrt{p} / 17.72
$$

If calculations based upon the above two equations indicates that there may be a problem then the actual antenna system and duty cycles of the transmitters will have to be considered.

Since "duty cycle" is a term used throughout the land mobile indussry, the appropriate multiplier for the type of equipment being evaluated should be used to 
take into account the fact that the radiated energy from mobile transmitters is not present under normal conditions for the entire averaging period used in the ANSI radiation protection standard. It is suggested that any portable or mobile installations that permi pperation by full duplex basis be considered to have a $100 \%$ duty cycle. Mobile units that are not capable of duplex operations but require the transmitter to be turned off before it is possible to hear the reply from the other party should be designated as using the EIA design, intermittent, duty cycle of $20 \%$ transmit and $80 \%$ stand-by or receive time, i.e. one minute transmitter on and four minutes transmitter off. A similar situation exists for portable transmitters. However, because of limitations of the power supply a more reasonable duty-cycle to assume would be $10 \%$ transmitter on and $90 \%$ off.

If after taking into account the duty cycle of the units being evaluated, one finds that individuals are still bifing exposed above the radiation protection guide as indicated by calculation, one should make an actual field measurement to determine the magnitude of potential personnel exposure. Such an evaluation should include measurements at locations normally occupied by individuals and measurements at some specific reference distance from the antenna say, one meter, to evaluate the situation.

\section{Conclusions}

Measurements made on a commonly available mobile and portable telecommunications system indicate that individuals may be exposed to levels of electromagnetic radiation comparable to the current radiation protection guide if they are at distances of less than one meter from the antenna. With the apparent increase and authorization of the land mobile radio spectrum which includes the 406-512 MHz and 806-960 $\mathrm{MHz}$ frequency band as well as numerous bands below $300 \mathrm{MHz}$. Consideration should be given to the applicability and interpretation of the current radiation protection guide. There exists a need for measurement standards in order to appropriately evaluate personnel exposure, both occupational and the general public. Effects of electromagnetic radiation on safety systems and flammable and volatile materials needs to be evaluated.

\section{References}

1. Private Communication, Richard Smith, Federal Communications Commission, February 1976.

2. Status Report on the Activities of the American National Standards C95 Committee on Radiofrequency Radiation Hazards, received from Professor Saul W. Rosenthal, chairman C95, December 8, 1975.

3. W. W. Mumford, "Some Technical Aspects of Microwave Radiation Hazards", Proc. IRE Vol. 49, pp. 427-447, February 1961.

4. Federal Communications Docket No. 19555, Implementation of the National Environmental Policy Act of 1969.

5. Federal Communications Commission, Report No. 7104, VHF-UHF Radiation Hazards and Safety Guidelines, J. Damelin, July $\overline{1971 .}$

6. Draft: Texas Regulations for the Control of RadiQ-Frequency Electromagnetic Radiation, Nov. 1975.

7. Private Communications, Homer I. Huddleston, Motorola, Inc., December 1975. 


\title{
AN AUTOMATED MEASUREMENT SYSTEM FOR DETERMINING ENVIRONMENTAI RADIOFREQUENCY FIELD INTENSITIES II
}

R. A. Tell, N. N. Hankin, J. C. Nelson, T. W. Athey, and D. E. Janes, Jr. U. S. Environmental Protection Agency

9100 Brookville Road

Silver Spring, Maryland 20910

\begin{abstract}
A measurement system is described which is used to measure environmental $\mathrm{RF}$ exposure levels. The system uses a spectrum analyzer interfaced to a minicomputer for data acquisition and is contained within a van for nationwide use. Special antenna systems allow isotropic field intensity measurements in selected bands between $0.5 \mathrm{MHz}$ and $10 \mathrm{GHz}$.
\end{abstract}

(Radiofrequency; spectrum analyzer; antenna; microwave; RF exposure; minicomputer)

\section{Purpose of System}

There are several reasons why the U. S. Environmental Protection Agency feels that data on environmental levels of electromagnetic radiation is needed: the considerable, current controversy over the existence of low-level or nonthermal effects of electromagnetic radiation: ${ }^{1,2}$ the demonstrated susceptibility of electronic devices to the existing environment; ${ }^{3,4}$ the gross uncertainties in estimates of population exposure; ${ }^{l}$ the rapid growth in the number and power of radiation producing devices; ${ }^{5}$ and the need to verify or improve predictive exposure models. 6,7 In order to obtain the required data and to determine the need for standards, EPA is conducting an environmental nonionizing radiation measurements program. The first phase of this effort was concentrated on field and analytical studies of selected high power sources. These sources have included aircraft, ${ }^{8}$ air traffic control, 9 and military radar; 10 satellite communications systems; 11 and UHF broadcast systems. 6 Genera11y, the survey instrumentation used in the field studies were broad band, high level, power density detectors. The purpose of this paper is to describe the instrumentation developed for the second phase of the program, a system for measuring and analyzing the multisource general electromagnetic environment.

\section{Basic Requirements of the System}

On the basis of the desired aspects of data interpretation for these measurements, a set of basic requirements was developed. The frequency range of the equipment should encompass all bands of interest from an exposure point of view. This means a fundamental detection capability from VLF to approximately $10 \mathrm{GHz}$ or $\mathrm{X}$-Band. Table 1 shows the bands of preliminary interest in our study. These particular bands were

Table 1

\section{INITIAL MONITORING BANDS OF INTEREST}

$$
\begin{gathered}
\frac{\text { FREQUENCY }}{0-2 \mathrm{MHz}} \\
54-88 \mathrm{MHz} \\
88-108 \mathrm{MHz} \\
150-162 \mathrm{MHz} \\
174-216 \mathrm{MHz} \\
450-470 \mathrm{MHz} \\
470-806 \mathrm{MHz} \\
1-10 \mathrm{GHz}
\end{gathered}
$$

\section{PRINCIPLE USE}

VLF Communications and AM Standard Broadcast

Low VHF Television Broadcast

FM Broadcast

VHF Land Mobile

High VHF Television Broadcast

UHF Land Mobile

UHF Television Broadcast

Radar 
selected primarily on the basis of highest potential environmental field intensities. The sensitivity of the system should be adequate for measuring relatively weak signals in order to ascertain the relative contribution to the total exposure picture from all signals. Frequency resolution was not a particularly critical factor; however, adequate selectivity should be used to allow differential evaluation of signal components in a given band. Stability commensurate with typical monitoring times was deemed to be sufficient.

Since previous work revealed the difficulty of making accurate field amplitude measurements 12,13 we have concentrated on the ability to accurately measure the signal's field strength or power density, i.e., to determine the actual exposure produced by signals in various bands. The desired accuracy is obtained with carefully calibrated antennas, using specially devised antennas where necessary. Consideration was given to the polarizations of different signals. Figure 1 illustrates conceptually the problem of determining the effective field strength of a signal where the resultant electric field vector is the vector sum of three mutually orthogonal components. Thus in certain cases, the use of an antenna sensitive to only one polarization is not desirable.
Another important requirement for the system was that of data analysis. The system was to incorporate a method for acquiring spectral amplitude data, correcting this data for system response functions, and analyzing the data for the pertinent information desired, providing if possible, an on-the-spot indication of results after some appropriate processing. This, in the field processing feature, would also minimize the possibility of undetected hardware or operator error at the field site.

The last major system requirement was that of mobility so that environmental evaluations could be performed at many locations throughout the country.

A generalized block diagram of the final measurement system is shown in figure 2. In essence the system is composed of a scanning spectrum analyzer with several different types of antenna systems, depending on the particular band of interest, interfaced to a minicomputer data acquisition system. Similar arrangements have been proposed $^{14}$ and are being used in surveillance applications from a spectrum management viewpoint.15,16 Analog signal amplitude information from the spectrum analyzer is digitized and input to the minicomputer where it is subsequently operated on by data correction and analysis routines. Trigger and timing signals are generated and
Figure 1. Relationship between power density 10 and electric field components

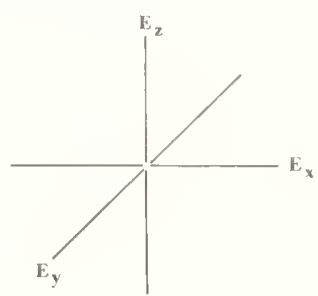

$\tau P D=\frac{E_{x}^{2}}{z_{0}}+\frac{E_{y}^{2}}{z_{0}}+\frac{E_{z}^{2}}{Z_{0}}$ $=\frac{\mathbf{E}_{\mathbf{R}}^{2}}{Z_{0}}$ where

$E_{R}=\sqrt{E_{x}^{2}+E_{y}^{2}+E_{z}^{2}}$

Figure 2. Block diagram of measurement system

\section{HORIZONTAL POLARIZATION VERTICAL POLARIZATION TURNST ILE}

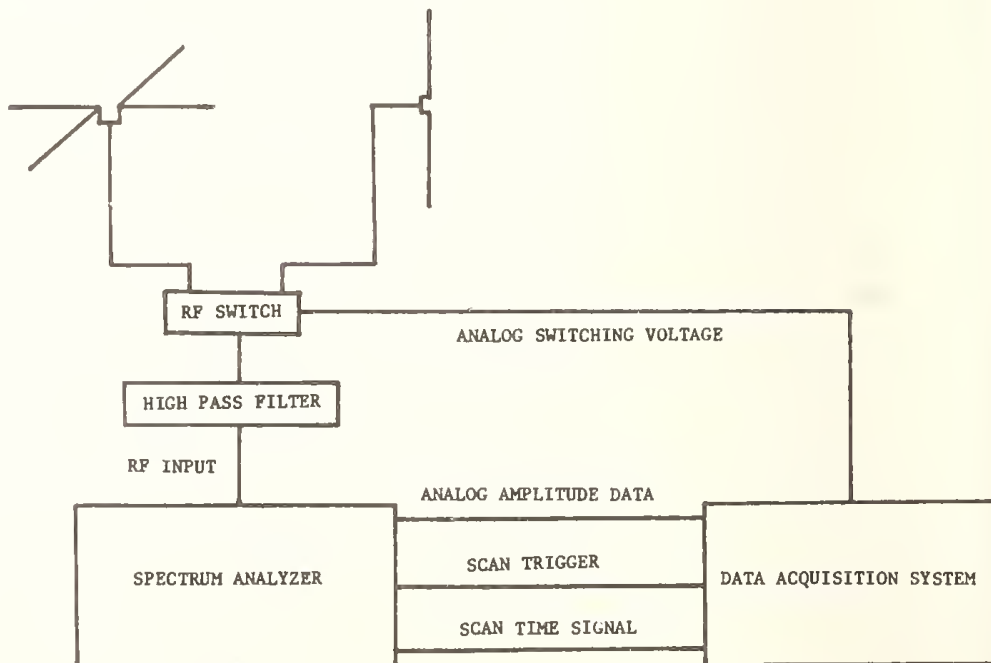


sensed by the computer to provide effective control of the spectrum analyzer. Other signals under program control are also provided for other events such as antenna switching.

\section{Antennas}

Table 2 lists the different antennas used with the system according to the different monitoring bands. In each case the antenna of selection provides, within reasonable limits, an accurate measure of the total, resultant field amplitude at any measurement point. In order to make maximum use of the spectrum analyzer as a scanning receiver, the antennas used exhibit an omnidirectional characteristic for those bands routinely containing many signals. In this fashion the analyzer may be repetitively swept over the given band and used to measure the amplitude of all signals in the band, regardless of the particular direction from which the signal approaches. Also, the antennas have been selected for being responsive to the significant polarization components in the bands of interest.

For frequencies in the $10 \mathrm{kHz}$ to approximately $60 \mathrm{MHz}$ range, a vertical monopole antenna with an active impedance matching circuit at the base is used. This antenna is referenced to a small square groundplane and is used to monitor signals mainly in the AM standard broadcast band. Because of minimal wave tilt in AM broadcast signals, the vertically polarized antenna provides in a single measurement, the true field amplitude. As the frequency increases above approximately $2 \mathrm{MHz}$ into the HF band, significant signal depolarization occurs, and a single measurement of the vertical field component is no longer sufficient for an accurate measurement.
Orthogonal dipole arrays are used for measurements in the VHF television and FM band since FM stations now use circular polarization with vertical field strengths in some instances $5 \mathrm{~dB}$ greater than the horizontal values. 17 In actual practice, a complete scan of the band is performed with one dipole connected, before electronically switching to the next dipole. Thus measurements of $E_{x}, E_{y}$, and $E_{z}$ are repetitively made in a sequential fashion where upon the resultant electric field strength or power density is determined. These antenna systems consist of thick dipolar elements, cut for resonance at mid-band and fed with $50 \Omega$ coax. By using large diameter elements a fairly uniform frequency response is obtained throughout each band for which the array is designed. Two sets of these antennas are used depending on whether the low VHF TV band or the high VHF TV band is being measured. In the case of television signals only two horizontal orthogonal dipoles are necessary to accomplish the required task, since depolarization of the horizontally transmitted TV signal is not significant at relatively short distances from the station. Using a system of three orthogonal dipoles, for measurements within the FM broadcast band, with an average gain figure, 18 an isotropic response with approximately $2 \mathrm{~dB}$ uniformity has been obtained.

Two different vertical coaxial dipole antennas are used for the land mobile bands. Gains above a $\lambda / 4$ radiator of $0 \mathrm{~dB}$ and $3 \mathrm{~dB}$ are used in the VHF and UHF bands respectively.

A commercial type of television receiving antenna is used for measurements in the UHF TV spectrum. It consists of a horizontally polarized, log periodic array which

Table 2

\section{MEASUREMENT SYSTEM ANTENNAS}

\section{APPLICATION}

VLF; AM Standard Broadcast

VHF-TV and FM

Land Mobile

UHF-TV

Radar

\section{DESCRIPTION}

Active Vertical Monopole

Orthogonal Dipoles

Vertical Coaxial Dipoles

Directional Log Periodic (Horizontally Polarized)

Vertical and Horizontal Saw-tooth, Planar Log Periodics 
exhibits a directional gain of about $5 \mathrm{dBi}$ across the UHF band ( $\mathrm{dB}$ above an isotrope). This antenna was selected over an alternative of crossed dipoles in order to provide an adequate gain across the band. It was found that when the dipole length was shortened enough to allow reasonable operation across most of the band, the effective area became so small as to adversely affect overall system sensitivity, except for exceptionally intense signals. Signal peaking is accomplished by use of a rotator on top of a telescoping antenna mast.

A pair of small, saw-tooth, planar, log periodics are used in the frequency range of 1 to $10 \mathrm{GHz}$. Two signal cables provide output from both the horizontally and vertically mounted antennas which are also rotatable from inside the van. A nominal $8 \mathrm{dBi}$ gain is apparent over their frequency range.

\section{System Calibrations}

Each antenna employed for routine monitoring was calibrated across its intended frequency band by referencing it, indirectly to a set of National Bureau of Standards (NBS) calibrated, standard dipoles. This was accomplished by first using the NBS antennas to calibrate a set of Singer tuneable dipoles which cover the frequency range of 30 to $1,000 \mathrm{MHz}$, and then using the Singer dipoles at appropriate frequencies to calibrate, by the comparison technique, the system monitoring antennas. This procedure required that an area electromagnetically quiet be found to properly employ the NBS standard dipoles since they rely on broadband, diode detection of the calibrating field. The presence of fields, other than the intended calibration field interfers with the overall calibration accuracy by adding in the undesired detection voltage of the extraneous fields. A calibration trip was arranged to the location of the National Radio Astronomy Observatory in Greenbank, West Virginia for this calibration purpose. Through special permission, intentional calibration fields were established for conducting the tests.

A least squares method was used to fit a curve to the Singer dipoles which resulted in a maximum deviance at measured points of $1.4 \mathrm{~dB}$ across the frequency range of 50$1,000 \mathrm{MHz}$. With the Singer dipole antenna factor now known functionally at any frequency, a comparison approach was used to calibrate the various systems of antennas. These calibrations were carried out with the antennas in their normally used position on the van. The calibrating field was established with a dipole antenna at a distance of 600 feet. Antenna calibration data was recorded at appropriate frequency intervals for each system for maximum signal pickup, i.e., main lobe alignment. In the case of orthogonally constructed dipoles, measurements were individually performed for each dipole in the array. These data were then subsequently fit using least squares techniques so that a functional expression of the given antenna's frequency response was developed. Now, except for the fact that the antenna data were for main lobe application, the antenna factors were ready for computer implementation. One final determination remained.

The monitoring van was driven to the Department of Commerce's Institute for Telecommunications Science's antenna range facility near Boulder, Colorado. Here, the van was placed on a 30 foot diameter turntable where radiation patterns for each of the antenna systems were measured. This was accomplished by radiating a stable field 2,225 feet from the van and, as the vanantenna system was rotated, using the monitoring equipment itself to record and analyze the pattern data. The minicomputer provided a convenient means for normalizing and plotting the received signal and storing the measured data on magnetic tape. Using this data, average antenna factor variations were computed and applied to the previously measured main lobe data to arrive at a final antenna factor, which when employed by the measuring system, would account for the total uncertainty in the measurement of resultant field strength or power density. The results of all of this antenna calibration work are contained in the application programs which are used by the system. Table 3 provides a summary of the total uncertainties measured for the overall system. The presence of the van had been presumed to be more significant in the pattern data than what was actually observed. The total system uncertainty can be characterized by a number of errors: inherent error in basic calibration accuracy of the NBS standard dipoles, the error due to using the Singer dipoles at frequencies between calibration points, the error associated with the fitting of a functional form to the measured calibration curves for the monitoring antennas, an error due to the angular dependence of the antenna system being used, and a system conversion error which is a measure of the spectrum analyzer-computer system's ability to accurately detect, plot, and record RF signal levels. Each of these factors are 
Table 3

SUMMARY OF OVERALL SYSTEM ERRORS (dB)

$\begin{array}{lcccccr}\text { Band } & \begin{array}{c}\text { NBS } \\ \text { Dipole }\end{array} & \begin{array}{c}\text { Singer } \\ \text { Dipole }\end{array} & \begin{array}{c}\text { Curve } \\ \text { Fit }\end{array} & \begin{array}{c}\text { Angular } \\ \text { Dependence }\end{array} & \begin{array}{c}\text { System } \\ \text { Conversion }\end{array} & \begin{array}{r}\text { Total Resulta } \\ \text { RMS Error }\end{array} \\ \text { AY } & 1.5 \mathrm{~dB} * & \text { N/A } & \pm 0.4 & \pm 0.5 & \pm 0.5 & \pm 1.6 \\ \text { Low VHF } & 1.0 & \pm 0.6 & \pm 0.8 & \pm 2.2 & \pm 0.5 & \pm 2.5 \\ \text { FM } & 1.0 & \pm 0.6 & \pm 0.4 & \pm 1.8 & \pm 0.5 & \pm 2.1 \\ \text { Low LM } & 1.0 & \pm 0.7 & \pm 0.7 & \pm 0.8 & \pm 0.5 & \pm 1.6 \\ \text { High VHF } & 1.0 & \pm 0.8 & \pm 0.5 & \pm 2.0 & \pm 0.5 & \pm 2.3 \\ \text { High LM } & 1.0 & \pm 0.5 & \pm 1.5 & \pm 1.1 & \pm 0.5 & \pm 2.0 \\ \text { UHF } & 1.0 & \pm 1.1 & \pm 1.2 & \pm 1.0 & \pm 0.5 & \pm 2.0\end{array}$

*Referenced directly to NBS field strength meter (Rhode and Swartz type HFH). The system as now configured is limited to discerning changes in RF level no smaller than $0.25 \mathrm{~dB}$ which is a limitation imposed by the resolution of the ADC.

provided in Table 3 and show that the system has a total uncertainty no greater than $2.5 \mathrm{~dB}$, a feat which is the result of extensive field work. The angular dependence error also includes a factor for a slight variation of the angular dependence upon frequency.

\section{Detection Hardware}

The principle detection equipment consists of a Hewlett Packard spectrum analyzer mainframe with variable persistence display and a number of plug-in RF sections to provide a total detection range from $20 \mathrm{~Hz}$ to $18 \mathrm{GHz}$. Normally the narrow band signal peak height is taken as the received signal's power except in the case of broadband radar spectra where determination of the impulse bandwidth, PRF, and pulse width are necessary to accurately obtain the peak and average exposure levels.

An additional device used in high intensity radar measurements is the high speed Biomation transient recorder. The recorder actually consists of a very high speed (100 MHz) analog-to-digital converter (ADC) and its own 2,048 word core memory and timing circuitry. By using a broadband diode detector connected to an antenna, a dc voltage replica of the radar's signal is obtained which can be digitized and stored in the memory. By using a dual time base mode, the pulse width and PRF may be deter- mined directly from a single recorder sweep. Figure 3 gives an illustrative example of this type of display where the pulse width is seen on the left and the PRF is apparent on the right. The time scale on the left half of the screen in $4 \mu \mathrm{sec} /$ division and on the right half is $1 \mathrm{msec} / \mathrm{div}$ showing a 10 usec wide pulse at a rep rate of $1 \mathrm{kHz}$. By judicious choice of trigger levels, the maximum amplitude of rotating radar beams

Figure 3. Detected RF pulse with transient recorder $4 \mathrm{\mu sec} / \mathrm{div}$ on left; $1 \mathrm{msec} / \mathrm{div}$ on right, $10 \mu \mathrm{sec}$ wide pulse at $1 \mathrm{kHz}$ PRF.

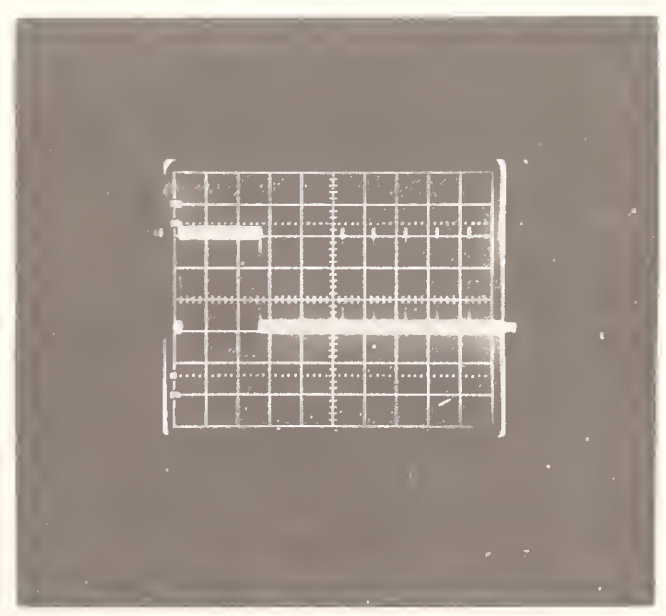


can be measured. Also, pulse widths from more than one radar may be determined simultaneously.

\section{Data Acquisition Equipment}

One of the main selection criteria for the data acquisition system was the development time necessary for preparing application software by personnel not previously familiar with real time programming tasks. For this reason a system oriented around an interactive interpreter with extensive hardware control under an extended version of BASIC (EBASIC) was chosen. It was deemed more important to get a number of conceptual acquisition and analysis processes actually operating on the computer in a fairly short period of programing time with the relatively long processing time associated with an interpreter as opposed to spending a long time preparing few programs which execute very rapidly.

Figure 4 is a block diagram of the data acquisition system. The system is configured around a Varian Data Machines central processing unit (CPU) with a $750 \mathrm{nsec}$ cycle time and $32 \mathrm{~K}$ of 16 bit word core memory. A $123 \mathrm{~K}$ word, fixed head, disc provides high speed mass storage for the system operating software, programs, and data. Additional slow access mass storage is provided by a dual transport digital magnetic cassette unit. A large screen CRT is used to display alphanumeric and graphic data while a standard ASR-33 teletype is the main communication link between the operator and the system. To facilitate interfacing to other instruments, a console is provided for access to various hardware features of the system which include a 13 bit resolution ADC multiplexed to 16 channels, two digitalto-analog converters (one 14 bit and the other 10 bit resolution) for outputing voltages, single bit inputs for sensing the status of various events, and electronically switched, control outputs. Additionally two channels of digital output and one channel of digital parallel 16 bit input are available. An interface connects the transient recorder for computer controlled high speed pulse acquisition and digital data transfer to main memory. Figure 5 is a photograph of the system as it appears in the measurement van. The RF detection equipment is in the rack to the left and the data acquisition system is beyond the operator. A hardcopy unit provides finished hardcopy output from the CRT display.

Connection to the spectrum analyzer is accomplished via the interface console. A trigger signal is developed by one of the digital-to-analog converters, and conversely the analog amplitude signal from the analyzer is read in via one of the ADC's multiplexed channels. Since the Hewlett

Figure 4. Block diagram of data acquisition system

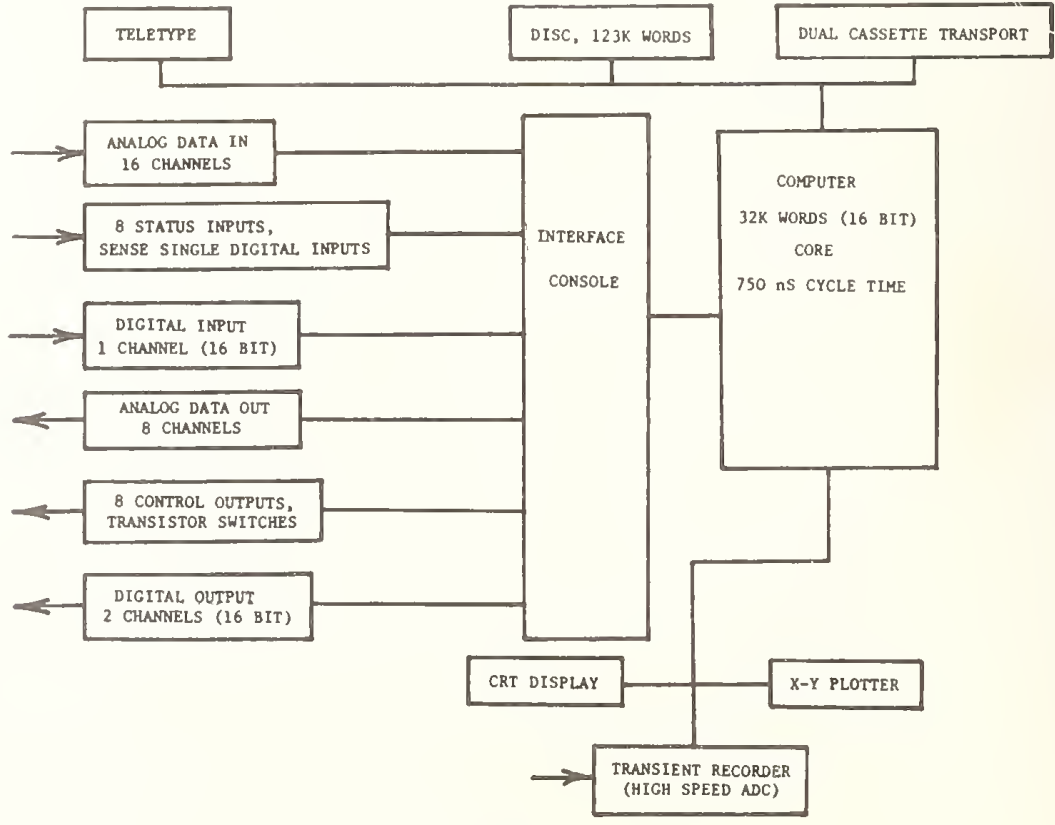




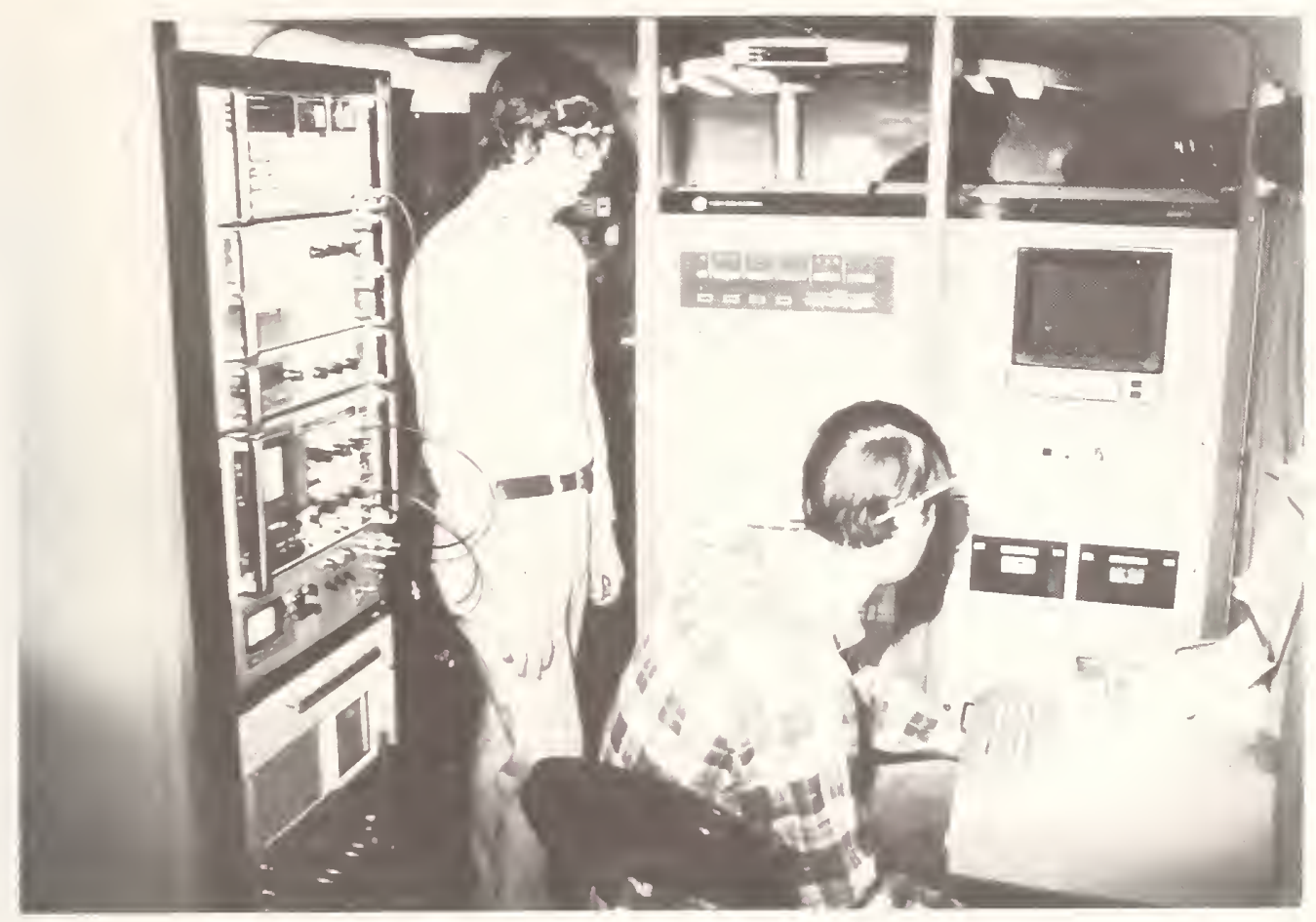

Figure 5. Radiofrequency measurement system in van

Packard analyzer conveniently allows for timing purposes, and has an output proportional to beam deflection on the analyzer's display, a very straightforward interface is possible.

The key to simplicity in the overall system, however, is the power of the Varian extended EBASIC software. Besides the normal programming instructions in EBASIC, extentions have been included to operate all peripheral hardware via calls to assembly language subroutines. This allows rapid program development through interactive communication with the system and yet very fast input-output (I/O) operation. A brief summary of the more useful software features includes:

- Versatile I/O commands for acquiring and outputting analog and digital data;

- Program and data file handling on both the disc and cassette; and

- Graphic display routines.

Analysis Techniques and Representative Data

Table 4 lists the main features of the data acquisition and analysis procedures we have developed. These features include: an absolute power calibration of the spectrum analyzer so that received antenna power is correctly indicated; signal averaging via software whereby spectral powers represented on a logarithmic scale in $\mathrm{dBm}$ are converted to absolute power, summed, averaged, and then converted back to $\mathrm{dBm}$ format for display purposes; peak power retention programs to retain the peak, or maximum, signal power ever observed over some specified monitoring time; measurement of elapsed monitoring time via reference to the CPU's real time clock; spectral power integration where signal powers may be summed across a given band yielding a measure of the total exposure power density; programs for measuring band occupancy to determine the percentage of time that signals exist during a given observation period; antenna calibrations to arrive at proper correction factors to apply to received power for determining field strength or power density; statistical treatment of the data to determine the length of monitoring time required at any given location to yield the required confidence in measurements of mean spectral power; CRT data display routines for plotting processed data and indicating pertinent control settings; and routines for storage 


\author{
Absolute Power Calibration \\ Signal Averaging - Determination of Mean Power Spectra \\ Peak Power Retention \\ Elapsed Monitoring Time Measurement \\ Spectral Power Integration \\ Per Cent Occupancy Measurement \\ Antenna Calibration \\ CRT Data Display \\ Digital Cassette Data Storage
}

of spectral monitoring data on digital cassettes.

Several of the software developments include the use of assembly language subroutines which provide for machine speed execution of various repetitive operations that would normally be substantially slower due to the overhead of the EBASIC processor. Notable in this area are routines for peak signal retention, signal averaging, and band occupancy.
Examples of system output for several bands are shown in figures 6-9. These represent actual environmental data collected during our first few field trips. Figure 6 shows the FM band for a site in Washington, DC. All system calibration factors are included so that the absolute field strength of any signal is obtained. The total power density from all signals in the band is calculated and printed near the top of the graph. The dynamic range capabilities of the system are also illustrated in

Figure 6. FM average field strength - Washington, DC

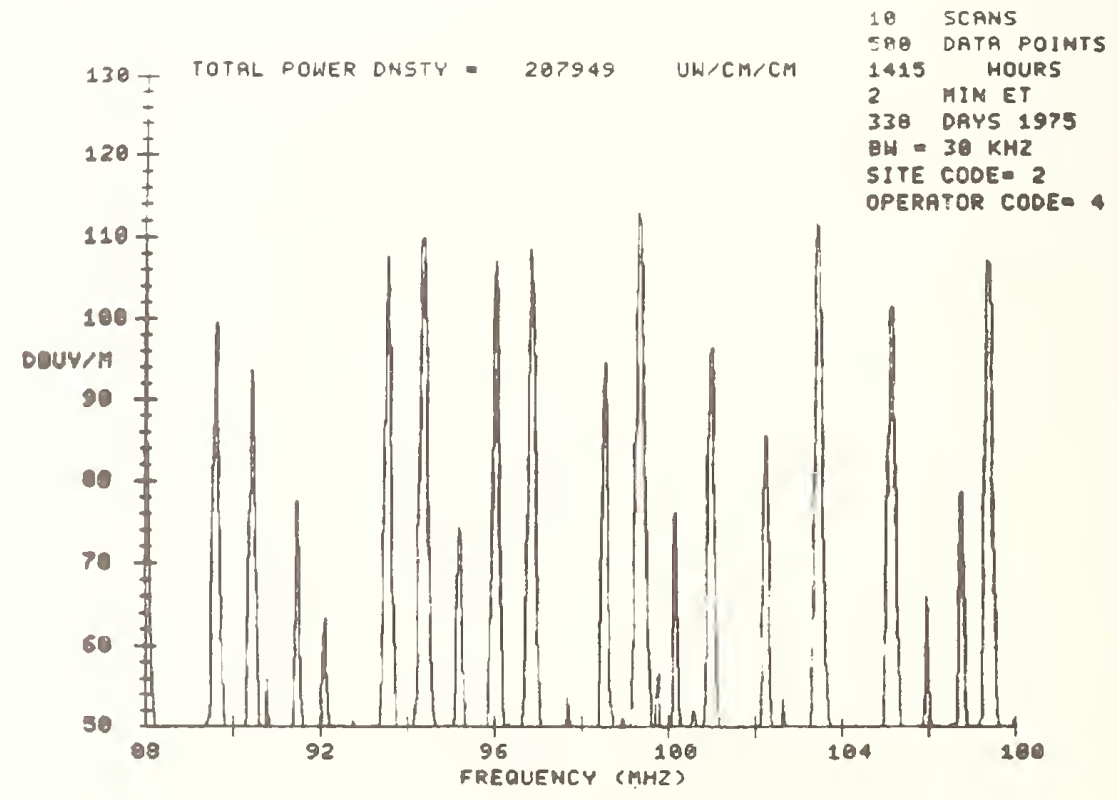


this figure, the highest levels being a factor of $10^{6}$ greater than the lowest.

Other pertinent parameters of the measurement are also printed on the graph.

Figure 7 is an example of data collected in the lower half of the VHF-TV band (channels 2-6). Only channels 4 (66-72 MHz) and 5 (76-82 $\mathrm{MHz})$ are on-the-air at this location. Part of the FM band is shown from 88-96 $\mathrm{MHz}$, but these signals are not included in the power density total for the band. Figure 8 shows the upper half of the VHF-TV band at another location. The AM standard broadcast band is shown in Figure 9.

Figure 7. Low VHF TV average field strength - Washington, DC

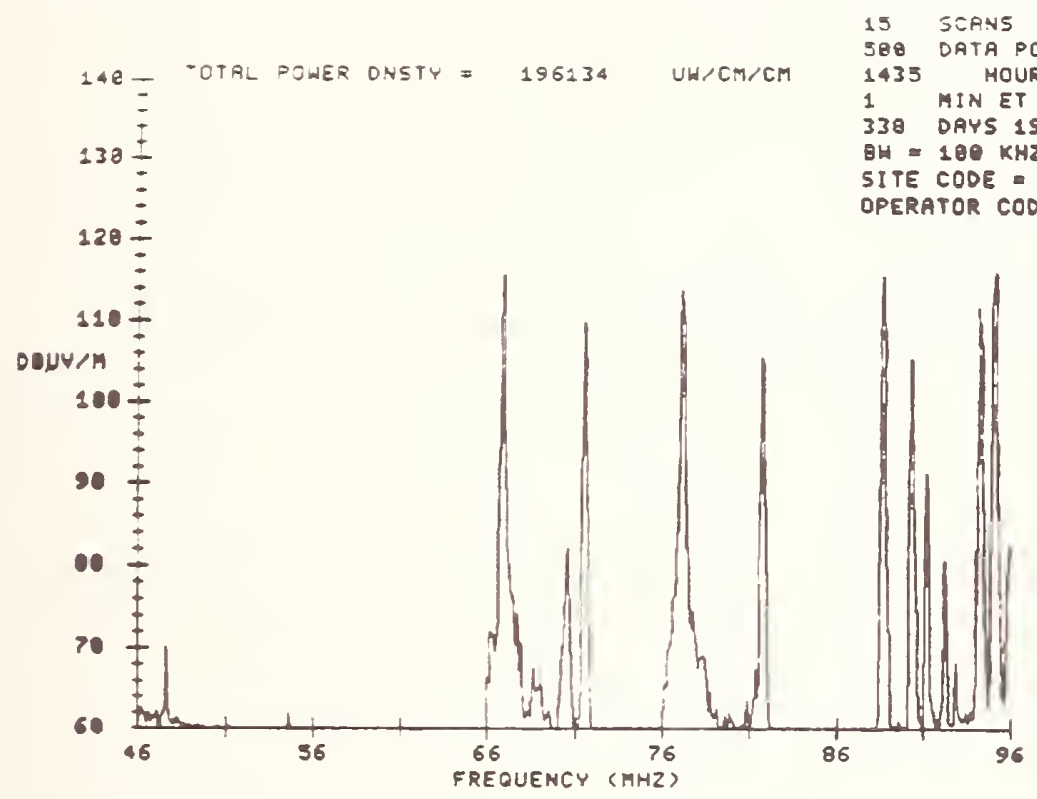

Figure 8. High VHF TV average field strength - Atlanta

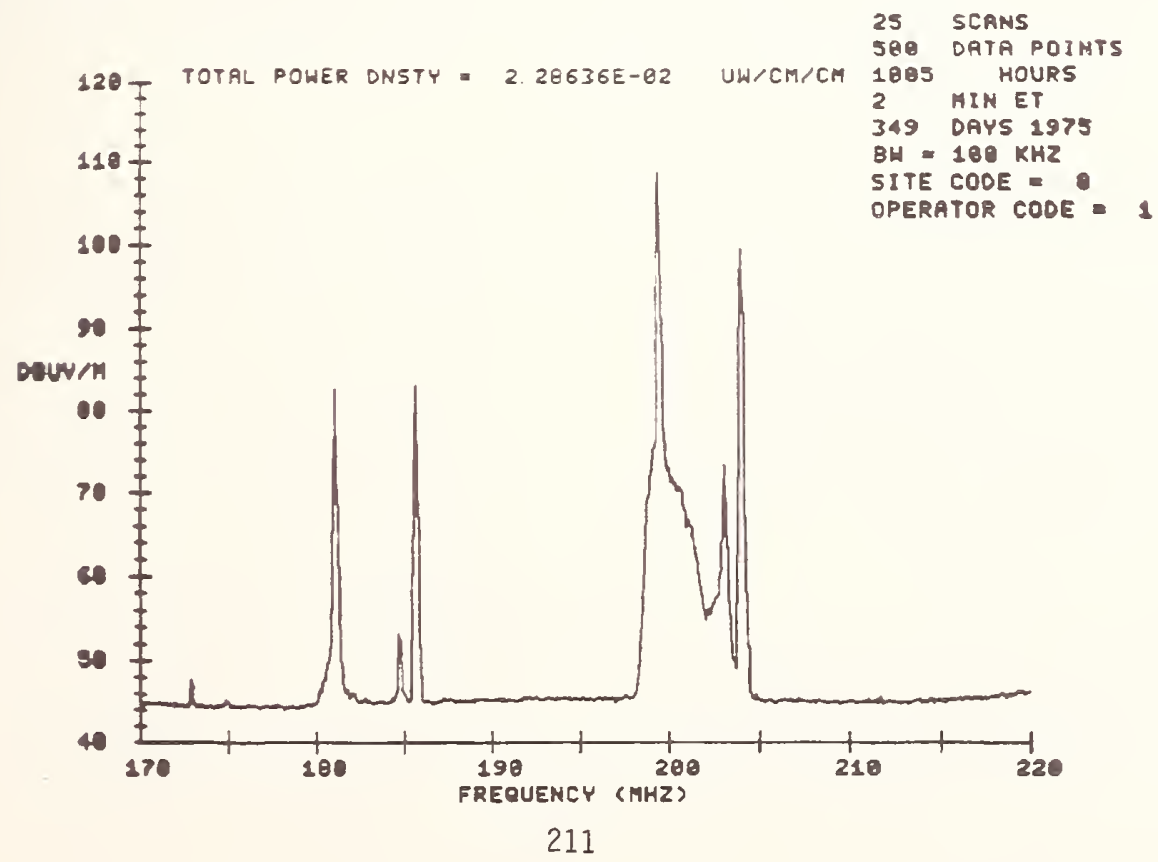


Figure 9. AM average field strength - Atlanta

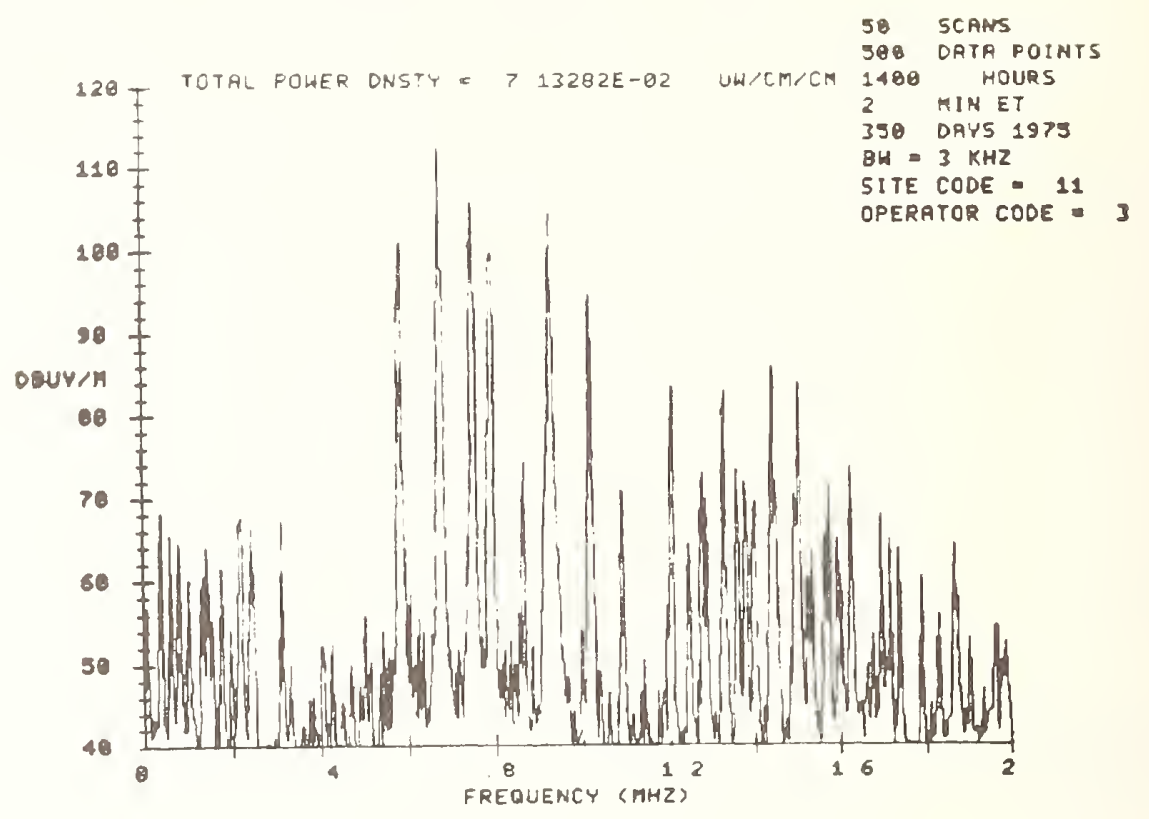

\section{Measurement Program Implementation}

The measurement system described is installed in a 27 foot Travco van for portable operation and has been used in various metropolitan area studies since October of 1975. A pneumatically, air-operated 26 foot telescoping mast attached to the rear of the van is used for elevating the antennas for monitoring. Two separate $6 \mathrm{~kW}$ electric generators are used to provide on-board power; one unit powers all utilities such as air conditioning, heating, lights, etc., the other unit is dedicated to the instrumentation.

Presently the van is being used to collect environmental data in cities on the East coast. As data becomes available more refined methods are being developed for selecting the total number and location of monitoring sites within a given metropolitan area. Initial monitoring locations are chosen on the basis of RF source distributions and population densities. As the monitoring program progresses, measurement in high density West coast cities are planned.

\section{References}

1. Electromagnetic Radiation Management Advisory Council (ERMAC), "Program for control of electromagnetic pollution of the environment: the assessment of biological hazards of nonionizing electromagnetic radiation," Office of Telecommunications Policy, Washington, DC, 1971.

2. Michaelson, S.M. and C.H. Dodge, "Soviet views on the biological effects of microwaves - an analysis," Health Physics, 21: 108-111 (1971).

3. Mitche11, J.C. et a1, "Empirical studies of cardiac pacemaker interference," Aerospace Medicine, 45: 189-195 (1974). [This paper gives references to earlier work.]

4. Vreeland, R.W., M.D. Sheperd, and J.C. Hutchinson, "The effects of FM and TV broadcast stations upon cardiac pacemakers," In Proceedings of IEEE Electromagnetic Compatibility Symposium, publication no. IEEE 74CH0803-7 EMC.

5. Lynn, J.F., "Sources of electromagnetic pollution or look what we are doing to ourselves," Workshop on Electromagnetic Pollution, Society of Automotive Engineers, Rockville, MD (1973).

6. Te11, R.A. and J.C. Nelson, "Calculated field intensities near a high power UHF broadcast installation," Radiation Data and Reports, 15: 401-410 (1974).

7. Athey, T.W., R.A. Te11, and D.E. Janes, "The use of an automated population data 
base in population exposure calculations," Health Physics Society Eighth Midyear Topical Symposium (1974).

8. Te11, R.A. and J.C. NeIson, "Microwave hazard measurements near various airborne radars," Radiation Data and Reports 15: 161-179 (1974).

9. Te11, R.A. and J.C. Nelson, "RF pulse spectral measurements in the vicinity of several ATC radars," Environmental Protection Agency, (EPA-520/1-74-005) Washington, DC (1974).

10. Hankin, N.N., R.A. Tell, and D.E. Janes, "Assessing the potential for exposure to hazardous levels of microwave radiation from high power sources," Health Physics Society Annual Meeting, 1974.

11. Hankin, N.N., "An evaluation of sate1lite communication systems as sources of environmenta1 microwave radiation," Environmental Protection Agency, (EDA520/2-74-008) Washington, DC, 1974.

12. Smith, S.W. and D.G. Brown, "Nonionizing radiation levels in the Washington, DC area," IEEE Transactions on Electromagnetic Compatibility, Vol. EMC-15, No. 1, pp. 2-6, February 1973.

13. Enva11, K., R.W. Peterson, and H.F. Stewart, "Measurements of electromagnetic radiation levels from selected transmitters operating between 54 and $220 \mathrm{MHz}$ in the Las Vegas, Nevada, area," USDHEW, PHS, BRH, Rockville, MD 20852 publication number BRH/DEP 72-4.
14. Hagn, G.H., S.C. Fralick, H.N. Shaver, and G.E. Barker, "A spectrum measurement/monitoring capability for the Federal government," final report on contract OEP-SE-70-102 prepared for Office of Telecommunications Policy by Stanford Research Institute, SRI project 8410, May 1971.

15. McMahon, J.H., "Capability of the FCC mobile monitoring van," in the proceedings of the Vehicular Technology Conference Record, Cleveland, Ohio, December 3, 4, and 5, 1973.

16. Barghausen, A.F. and L.G. Hailey, "Radio spectrum measurement system - current applications, experience, results," in proceedings of IEEE Electromagnetic Compatibility Symposium, July 16, 17, and 18, 1974, San Francisco, California, IEEE pub1ication 74CH0803-7EMC, Pp . 140-145.

17. Tell, R.A., "Signal polariations in the VHF and UHF broadcast spectrum," IEEE Transactions on Broadcasting, December 1974 .

18. Halberstein, "Gain averages as criteria for antennas EMC-performance," in proceedings of IEEE International Electromagnetic Compatibility Symposium, July 1972, IEEE publication 72CH0638-7EMC, pp. 333-336.

19. Schultz, L.D., A.D. Spaulding, and A.F. Barghausen, "Radio spectrum occupancy signals and noise," Ibid, pp. 42-49. 



\section{THE MEASUREMEIT OF OPTICAL RADIATIOHS:}

\section{SELECTED PRACTICAL COISIDERATIONS}

R. W. Peterson, J. Marshall Coakley, Kshitij Mohan and Robert James

Electro-Optics Branch, Division of Electronic Products Bureau of Radiological Health

Food and Rrug Administration

Rockville, llaryland 20852

This paper describes the measurement capabilities for coherent and incoherent optical radiations at the Bureau of Radiological Health and discusses some problems that arise in developing a program of measurements required for enforcing compliance with Federal standards and for related regulatory responsibilities.

(lasers, calorimeters, detectors, calibrations, radiometry)

\section{Introduction}

The biological effects from certain regions of the optical spectrum are vell established.1,2,3,4 Many of these effects constitute a health hazard. For example, retinal lesions, photokeratitis, erythema, and skin cancer are some of the known or suspected effects of exposure to certain spectral resions of the optical spectrum.

The wide use of lasers, WV sources, and other intense optical radiation sources and the potentlal hazards associated with their. use has emphasized the need for characterizing them, thereby increasing the burden on credible optical metrology.

The Bureau of Radiological Health, by virtue of the Radiation Control for Health and Safety Act of 1968 ( $P L$ 90-602) has been delegated regulatory responsibilities in several areas, among them promulgation of performance standards for sources of optical radiation.

A Federal safety standard for the performance of all lasers and laser products has been published and is due to become effective on
August 2, 1975. In order to enforce the compliance of industry with this standard, a comprehensive program for laser power measurements has been established in our laboratory. This paper, in part, will describe this measurement capability.

The proliferation of UV sources such as sunlamps, UV medical or dental devices, high intensity discharge lamps, etc. has resulted in many alleged radiation injuries. In order to characterize the optical performance of these sources, capabilities in radiometric measurements of incoherent optical radiation have been developed. Selected technical problems encountered in this program will be described.

\section{The lleasurement of Coherent Radiation}

At the heart of our measurement program for coherent optical radiation are two calorimeters referred to as the $\mathrm{C}$-series calorimeters 5,6 developed by the National Bureau of Standards in Boulder, Colorado. NBS maintains the national scales for laser power and energy measurements using a set of three of these calorimeters as the reference standard. Under an interagency agreement with IIP, a set of two calorimeters 
was purchased and is maintained in our laboratory. A joint NBS/BRH Measurement Assurance Program is used to intercompare the measurement capabilities at NBS and BRH four times a year.

The BRH calorimeters are used in turn to calibrate a variety of laser power and energy measuring detection systems, some of which are used for compliance related measurements.

Figure 1 shows a sketch of the C-series calorimeter. Each calorimeter consists of a copper cylinder blackened inside by copper oxide, or a similar material, that absorbs the incident energy. Surrounding the absorber is a constant temperature housing that thermally isolates the absorber from its surroundings as well as provides a constant reference temperature for the thermocouple sensors. The volume inside the temperature controlled housing is evacuated to a typical pressure of $10^{-5} \mathrm{~mm} \mathrm{Hg}$ or less. A window is necessary to allow optical radiation to pass to the absorber while maintaining the vacuum. Calibration heaters are attached to the absorber to permit electrical calibration and direct reference of measurements to primary electrical standards.
The c-series calorimeters are useful for measuring laser energy from 0.01 J up to $30 \mathrm{~J}$ to an overall accuracy of $\pm 1.2 \%$. It has a maximum Cll power limitation of 1 watt, with an energy density limitation of $0.1 \mathrm{~J} / \mathrm{cm} 2$. Nepending upon the window material used, measurements can be made over a wavelength range extending from the ultraviolet up through the far infrared of $\mathrm{CO} 2$ lasers.

In practice, the two calorimeters are used first to measure the ratio of a beam splitter. One of the calorimeters is then replaced by an instrument to be calibrated. By measuring the incident energy with the remaining C-series calorimeter and using the beam splitter ratio, the energy/power input to the instrument can be determined and the calibration factor established.

To assure confidence in our measurements, a record of the system's performance with time is maintained. One of the more meaningful records that may be kept is a control chart. l!e maintain a control chart of the electrical calibration factor vs. time for the C-series calorimeters.

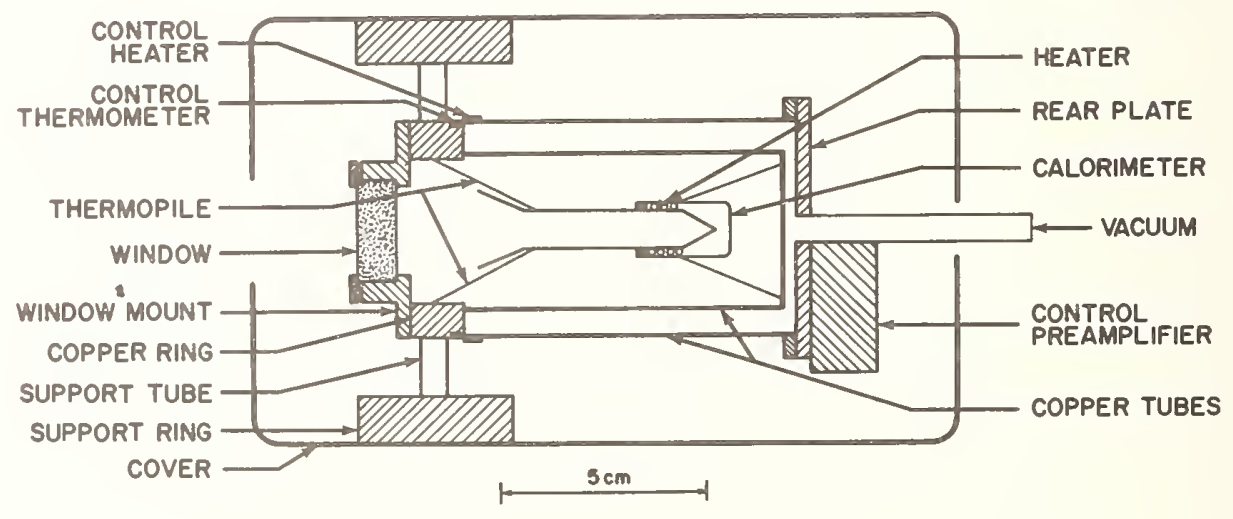

Figure I THE C-SERIES CALORIMETER 
These control charts indicate a standard deviation of less than $0.2 \%$ for 25 different runs spaced over several months.

However, for the actual optical measurements taken with the $\mathrm{C}$-series system, there are other sources of uncertainty also, such as the accuracy of the estimate of window transmission, absorption of the absorber surface, polarization of incoming beam, etc. The error inherent in the electrical calibrations is only one part of the overall error of the C-series system.

Figure 2 shows a control chart of a commercially available laser pover measurement device. The calibration factor plotted against the run number. Notice that the data are in general, randomly scattered about the mean. If the plot started indicating a trend, it would mean that the instrument's characteristics were changing and any measurement taken ,lith the instrument in this condition would have to be carefully evaluated. However, in this case, the points are scattered in a random fashion. You will notice that the one sigma levels for this instrument are considerably greater than they are for the $\mathrm{C}$-series calorimeter; $1.6 \%$ vs $0.2 \%$. Whenever the measurement system is a step removed from the reference system, the uncertainty is increased.

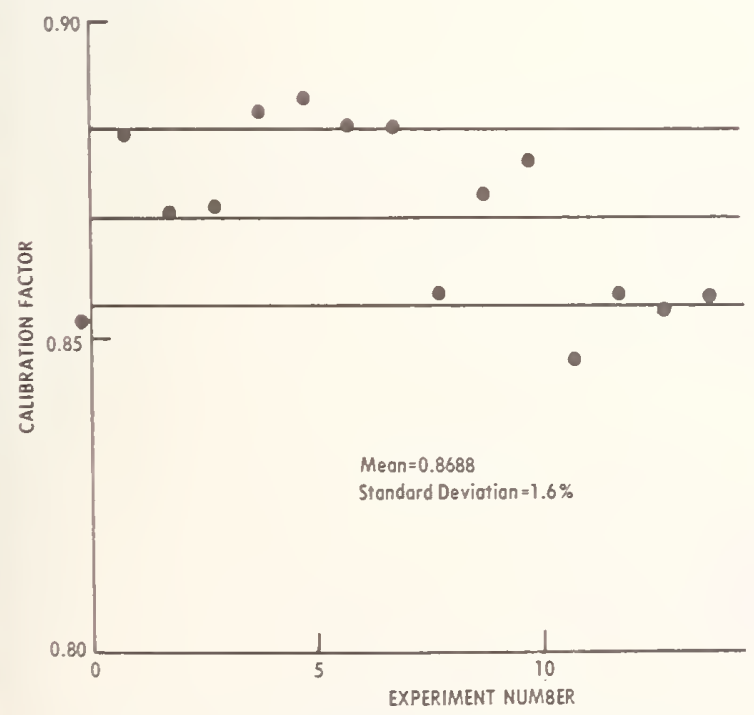

Figure 2 CONTROL CHART FOR A LASER POIER INSTRUMENT
Two other points should be made with respect to measurement of laser radiation. The first is that laser beams are extremely intense sources of $1 \mathrm{ight}$. Even the low powered lasers may be sufficiently intense to saturate, or even damage, certain detectors leading to erroneous results. Silicon detectors are especially susceptible to overloading.

The second point is that if a window or protective covering is used for a detector, it should be a wedge shape; i.e. have non-parallel front and rear surfaces. Since laser light is coherent, the use of optical flats would result in errors due to interference.

Finally, as with any metrology program, it is essential that control charts be maintained to monitor system performance.

Before instruments for the measurement of laser power or energy under noncontrolled conditions are calibrated, they must be evaluated to characterize their performance as a function of variations in the relevant parameters such as temperature, position of the incident beam on the detector, etc.

Te routinely make studies of the temperature and humidity dependence of detector response for "different detection instruments. The details of these measurements are contained in a separate publication 7 , and Figure 3 details the temperature dependence of response for a laser power meter which utilizes a silicon photodiode.

The spatial uniformity of the response of a detector surface is quite critical when making laser power or energy measurements. 1.4easurements of this characteristic are part of the routine evaluation of detectors in the Electro-optics Branch of the Bureau of Radiological liealth. These measurements are made by scanning a He-lle laser beam across the sensitive surface of a detector and displaying its response on an oscilloscope. Figure 4 is a schematic of this measurement facility. 


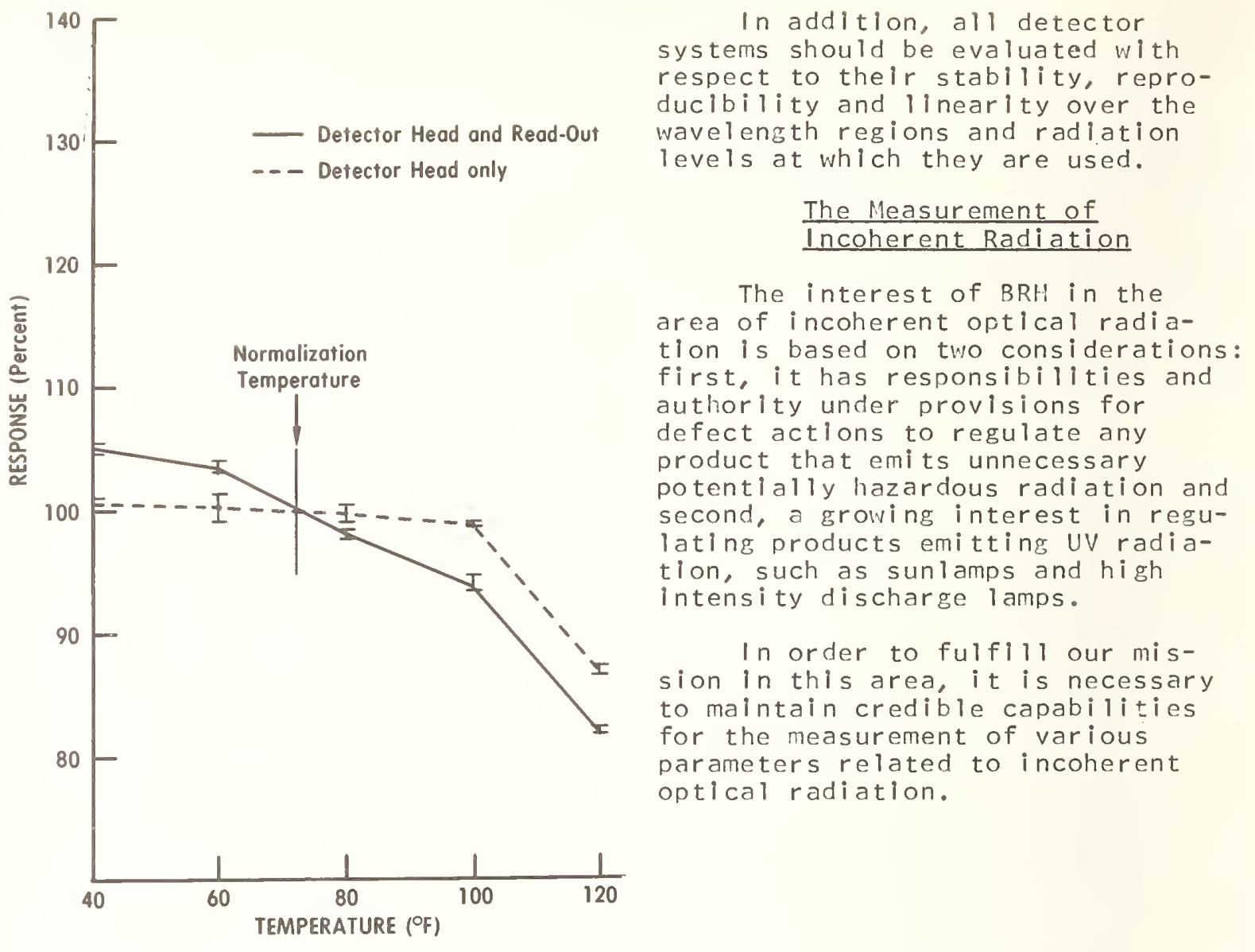

Figure 3 TEMPERATURE DEPENDENCE OF

RESPONSE - SILICON

POIVER METER

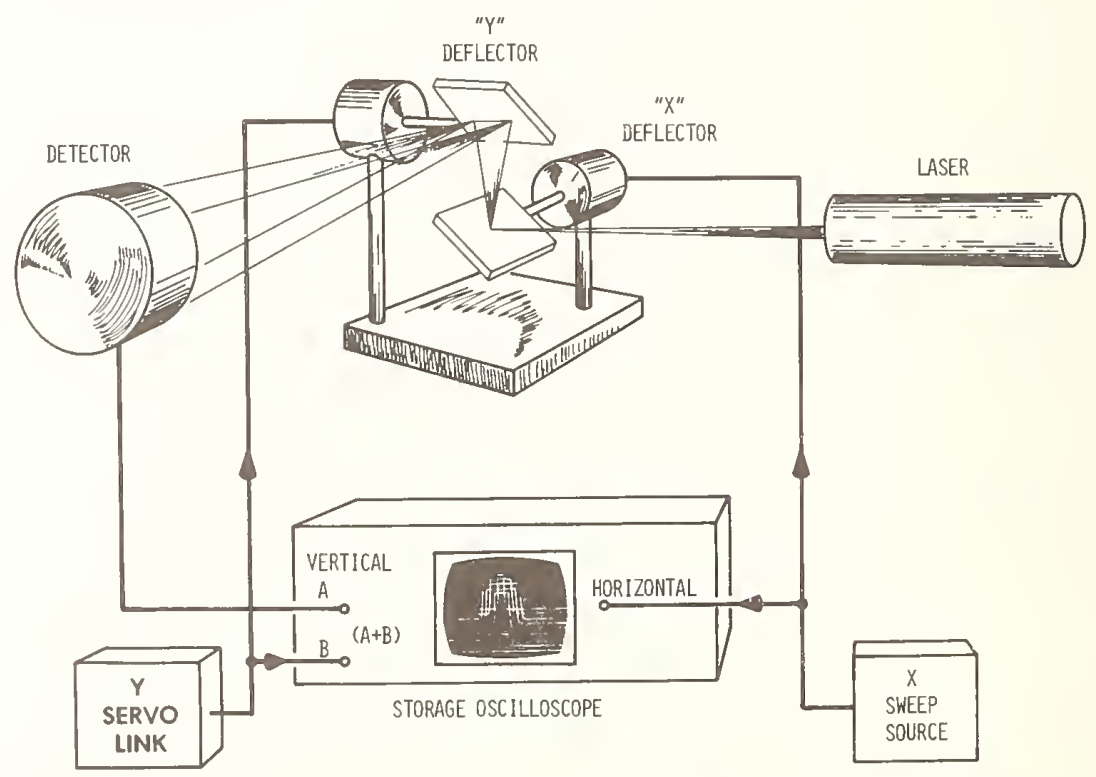

Figure 4 EXPERIMENTAL SYSTEM FOR MEASURING DETECTOR SURFACE UNIFORMITY 
The parameters of primary interest are spectral irradiance, spectral radiance and luminous flux. Our laboratory malntains a set of standards (obtained from the National Bureau of Standards) for each of these quantities. A set of working standards is callbrated against the standard from NBS and used in the routine measurements. Periodic intercomparisons with NBS and participation in Measurement Assurance Programs (MAP) ensures the continuing validity of all measurements that are made.

We will briefly describe now our spectroradiometric measurement facility and will give some representative results. Three different systems for the measurement of spectral irradiance have been established. The first is a rapid scanning spectroradiometer bullt around a silicon diode target vidicon tube and a minicomputer with which the spectrum can be measured in 400 nanometer sections from 300 to 1100 nanometers. This provides a capability for making nearly instantaneous measurements of spectral irradiance. It is used primarily for measuring radiation from sources that cannot be sustained for too long a period of time, such as welding arcs and photographic flash units. The second system is a set of portable spectroradiometers each consisting of a small single grating monochromator, a photomultiplier tube and an amplifier, high voltage supply and readout unit. These are used with a set of appropriate cut off $f i l t e r s$ to reduce the errors due to stray light. The third instrument, which is used for maintaining the scales of spectral irradiance at BRH, however, is more elaborate and is shown in figure 5. It consists of a double grating Czerny Turner monochromator which views a BaSO 4 plate at a 450 angle. The $\mathrm{BaSO}_{4}$ plate is in turn irradiated by a standard and the test source to provide the calibration of the spectral irradiance due to the test source. Measurements can be made from 250 to 800 nanometers using either a photomultiplier with an S-20 response or a solar blind photomultiplier tube which responds only to radiation shorter than $400 \mathrm{~nm}$. The wavelength is electro-mechanically scanned and data can be obtained almost continuously over the wavelength scale. The spectral bandpass of the instrument can be chosen betiveen approximately 0.5 and 2 nanometers.

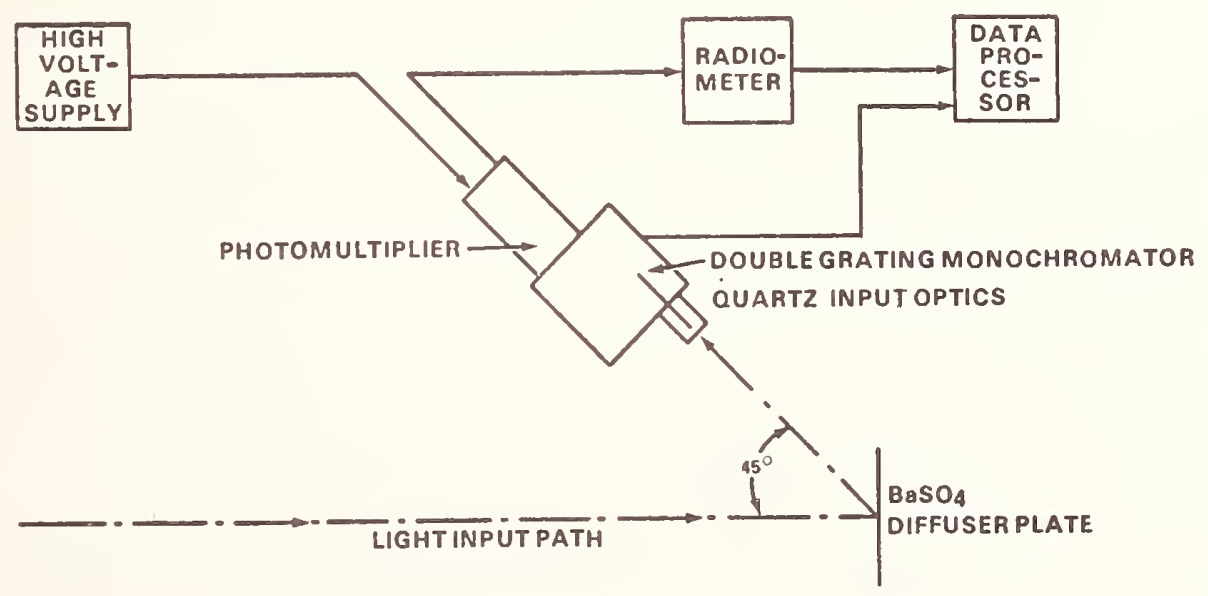

Figure 5 SPECTRORADIOHETRIC MEASUREMEHTS SYSTEM 
The primary measurement reference standards used are a 1000 watt FEL type quartz halogen lamp calibrated by IIBS for spectral irradiance between 250 and 1500 nanometers and a deuterium discharge lamp calibrated for spectral irradiance by a commercial laboratory between 180 and 400 nanometers. A deuterium lamp standard calibrated for spectral irradiance between 180 and 400 nanometers is now available from the National Bureau of Standards and our future measurements in that region will be based on this standard. The standard and the test 1 amps are operated at specified distances from the BaSO4 plate and a data acquisition system consisting of a Hewlett Packard llodel 2570 data coupler, a model 9810 calculator, an $x-\gamma$ recorder and a magnetic cassette data storage system is used for collecting, storing and reducing the data.

The uncertainties associated with the measurement of spectral irradiance depend greatly on the sources being measured. The uncertainties with respect to SI inherent in the calibration of our 1000 watt quartz halogen primary standard 1 amp are assigned by Migs and are $2.6 \%$ at $250 \mathrm{~nm}$ and $1.2 \%$ above $450 \mathrm{~nm}$.

Other sources of error that are taken into account are:

a. Uncertainties in the calibration of secondary standards

b. Error in the calibration of wavelength scale

c. Error because of temporal drifts in the system

d. Errors because of lack of 1 inearity in the instrument. (l/hen test sources emit at levels vastly different from the standard this may be a substantial error)

e. Errors because of stray radiation from wavelength regions other than that being measured f. Uncertainties because of lack of reproducibility, and

g. Uncertainties because of temperature dependence of instrument response

All these uncertainties are taken into account in the final estimation of the uncertainties associated with a measurement.

Figure 6 shows the spectral irradiance in :I cm-2 nm-1 at a distance of $30.5 \mathrm{~cm}$ from a device used for the polymerization of dental filling material by irradiation with UV radiation. Other sources that are measured in our laboratories range from units used in phototherapy for hyperbili rubinemia to sunlamps, fluorescent lamps, high intensity tischarge lamps, etc.

\section{References}

1. Mills, L. F., C. D. Lytle, F. A. Andersen, K. B. Heliman, and L. E. Bockstahler: A Review of Biological Effects and Potential Risks Associated With Ultraviolet Radiation as Used in Nentistry. U. S. NHEW Puhlication No. (FPA) 76-802I $(1976)$.

2. Freeman, R. G.: Data on the Action Spectrum for Ultraviolet Carcinogenesis. J. Nat. Can. Inst. 55A (1975).

3. Van Pelt, H. F., \%. R. Payne, and R. '\%. Peterson: A Review of Selected Bioeffects Thresholds for Various Spectral Ranges of Light. U. S. MHE H Publication (FDA) 74-8010, Rockville (1373).

4. Pitts, ח. G., and T. J. Tredici: The Effects of Ultraviolet on the Eye. Am. Ind. Hyg. Assoc. J. 32:235 (1971). 


\section{SPECTRAL IRRADIANCE OF A DENTAL DEVICE}

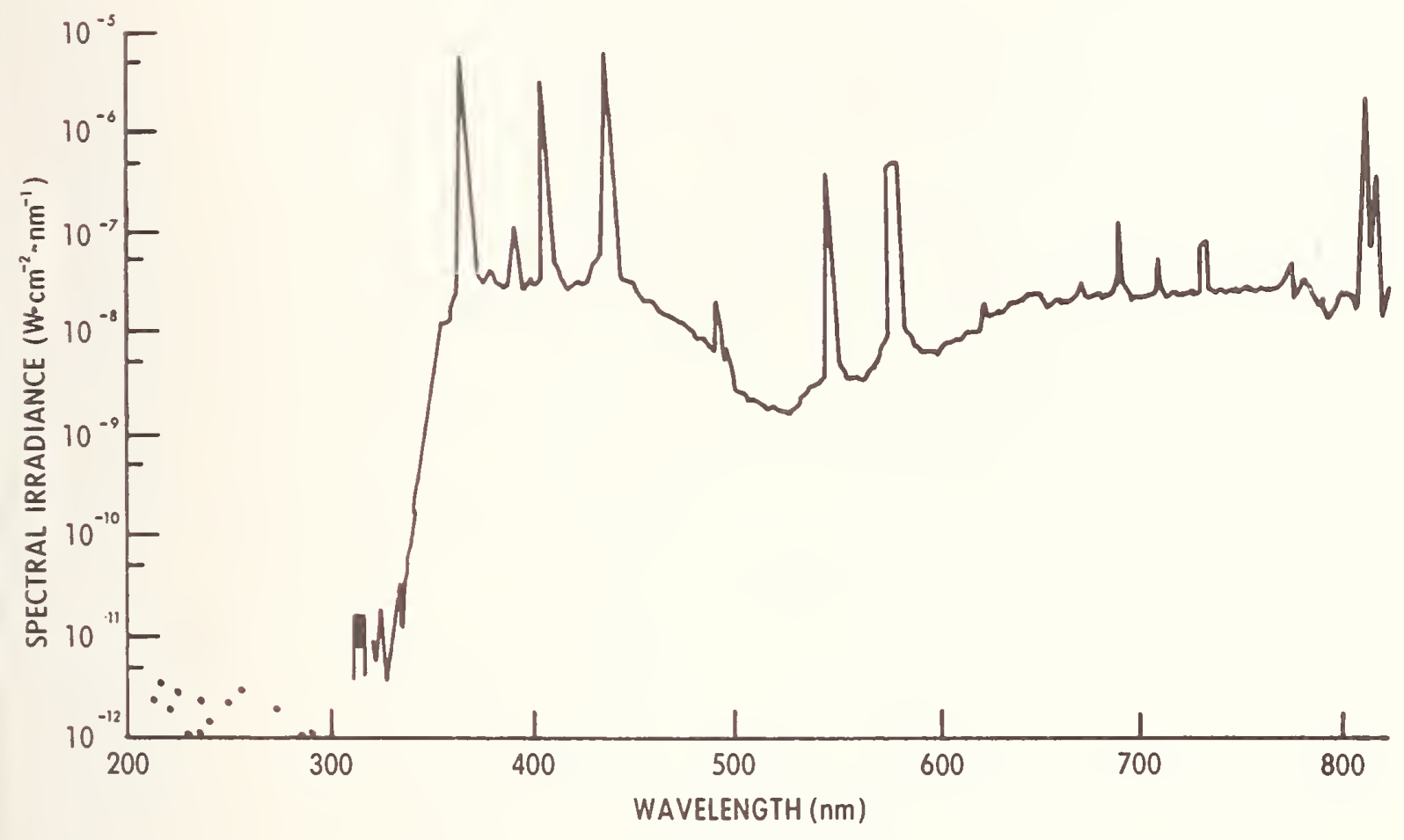

Figure 6 SPECTRAL IRRADI AIICE FROH 4 DENTAL JIV IRRADIATION DEVICE ( 30.5 CII FROIM THE APPLICATOR TIP)

5. Jennings, D. A., F.. D. Hest,

K. I. Eyenson, A. L. Rasmussen,

$1 !$. R. Simmons: Laser Power and

Energy Measurements. NBS

Technical llote 382. (oct.

$1969)$.

6. West, E. D.: Data Analysis

for Isoperibol Laser Calo-

rimetry. IIBS Technlcal lote

396. (Feb. 1971).

7. Landry, R. J. and R. U.

Peterson: Temperature dependent Response of Optical Radiation Measurement Instrumentation. To be published. 



\author{
D. F, Parsons \\ Roswe11 Park Memorial Institute \\ 666 Elm Street, Buffalo, N.Y. 14263 \\ V. A. Phillips and J. S. Lally \\ Members of the Radiation Safety Committee of the \\ Electron Microscopy Society of America*).
}

\begin{abstract}
A survey of the $x$-ray leakages from the electron microscopes used by members of the Electron Microscopy Soclety of America has been carried out. Few, if any, out of several thousand long term microscope users received an exposure detrimental to health. However, a sufficient number of design errors, and accidental over exposures have occured to make standardization of maximum allowed leakage desirable. The radiation hazards of high voltage electron microscopes are also discussed.
\end{abstract}

(Electron microscopes; x-ray leakage; health hazards; high voltage electron microscopes)

\section{Introduction}

A survey was completed in April 1972, by the above members of the Radiation Safety Committee of the Electron Microscopy Society of America. ${ }^{l}$ The basic findings have been published ${ }^{2}$ and a start made in standardization of $x$-ray leakage by publication of a Handbook for electron microscopists. ${ }^{3}$ This survey covered conventional fixed beam electron microscopes $(40-200 \mathrm{kV})$. Scanning microscopes and microprobe analyzers were not covered. Experience is gradually being acquired with high voltage microscopes (HVEM) installations of which there are now a sizable number (nine in USA, nine in U.K., four in France, one in the Netherlands, one in Germany, one in Russia, one in Sweden and seven in Japan). In the next few years, it is planned to reconstitute the Radiation Safety Comittee and to make a survey of HVEM x-ray hazards. A preliminary discussion is given in this report.

Since the publication of the survey, there has been considerable interest by State, County, City and Institution radiation safety officials in the results and recommendations. However, to-date, there has been no attempt to introduce standardization by a Federal Agency as recommended by the Committee. We hope that further discussions of our result at this meeting will be a first step towards standardization. Recently, there are signs that several manufacturers of electron microscopes have modified their instruments to conform to EURATOM standards of $x$-ray leakage (less than $0.1 \mathrm{mR} / \mathrm{hr}$ at a distance of $10 \mathrm{~cm}$, from all external surfaces of the equipment).

\section{$\underline{\text { Results }}$}

A survey of 589 electron microscopists gave the following main results:

1. Frequency of checking the microscope for leaks by electron microscopists and health inspectors. $8 \%$ never monitored, $44 \%$ monitored at one year or larger intervals, $10 \%$ monitored two or more times a year and $11 \%$ monitored continuously. Only $17 \%$ of those monitoring named the monitor and most monitors used showed wide variation of sensitivity with $\mathrm{x}$-ray energy.

2. Use of film badge. Only $17 \%$ used badges on their person.

3. Frequency of significant leaks. ( $>0.5$ $\mathrm{mR} / \mathrm{hr}$ ). Bearing in mind the general infrequency of adequate monitoring, nethertheless, $51 \%$ reported detecting $x$-ray leaks. However, on 1 y $18 \%$ recorded a figure. Of these, $41 \%$ found more than $0.5 \mathrm{mR} / \mathrm{hr}$. Hence, $\mathrm{x}-$ ray leakages of $>0.5 \mathrm{mR} / \mathrm{hr}$ must occur with considerable frequency. It was also found that a casual view of the magnitude of the leak exists since most regarded leaks of > $0.5 \mathrm{mR} / \mathrm{hr}$ as "probably not hazardous".

4. Site of Leaks. Gun 53\%, camera and viewing chamber $27 \%$, specimen chamber and column $16 \%$, high voltage tank and cable $3 \%$, luminous dial clock $2 \%$.

*The views expressed are those of the authors only. 
5. Variation of leaks with type of microscope. The fraction of each type showing leaks varied markedly with type indicating a need for design improvement in certain types. The worst hazard exists with some old early models which are still in use. A few of these may still not have lead glass windows. Manufacturing and installation errors have led to omission of some lead or lead-glass shielding and serious leaks. Poor vacuum has led to high gun current and high x-ray leakage. Manufacturers have had particular trouble in designing the specimen chamber, viewing chamber and camera to be leak free.

6. Accidents and deaths attributable to $x$-ray exposure. Failure to use lead glass windows caused facial burns and conjunctivitis. A claim was made against a manufacturer with respect to death from leukemia but the case was not proven. Two long term electron microscopists have recently reported traumatic type cataracts and the corresponding discovery of unrecognized high leak rates at the viewing chamber.

A survey of the death certificates of 37 electron microscopists was inconclusive because of the small sample available.

\section{Discussion}

We found only one well-organized institutional microscope monitoring program. City, County and State inspection appeared uniformly inadequate or inoperative. Sufficient incidents of over exposure have been found to justify some improvement in the $\mathrm{x}$-ray safety situation. The required steps are: (a) Agreement on maximum permissable leakage 1evel, (b) Agreement on monitoring instruments and procedure, (c) Improved education about $\mathrm{x}$-ray safety, (d) Legal implementation. The (Federa1) Regulations for the Radiation Control for Health and Safety Act of 1968 is in effect, but is not being applied to electron microscopes (only to television sets). This requires that exposure rates not exceed $0.5 \mathrm{mR} / \mathrm{hr}$ at $5 \mathrm{~cm}$. from any point on the external surface of the equipment. This is similar to the Euratom requirement which has already been met by some (but not a11) manufacturers. In actual effect are Radiation Protection Laws in some states which limit the permitted body dose. It appears simpler and safer to control $x$-ray leakage of the instrument than to limit the dose received, and the Federal Health and Safety Act of 1968 would be suitable for conventional microscopes. cult with the high voltage microscope and more careful shielding will be required to meet Health and Safety Act requirements. The much higher penetration of the $1 \mathrm{Mev}$ $x$-ray is offset at most points by the thicker shielding used on the column. The effects of using unusual beam conditions (misalignment or very high intensity) or of fitting different accessories with inadequate shielding are, of course, liable to be more drastic than the conventional microscopes.

HVEM's are "conditioned" once or twice a day by taking the acceleration voltage $20 \%$ higher than the voltage to be used for microscopy. Gas discharges occur in the accelerator tube during "conditioning" (once or twice a day) which can give rise to transient high levels of $\mathrm{X}$-ray radiation that require the microscope to be evacuated. The conditioning procedure is used to prevent similar discharges from occuring during normal operation and is therefore, a recommended safe procedure. Hence, in the design of a HVEM facility, attention has to be paid to the shielding of the walls around the microscope so that conditioning does not affect nearby personnel.

The EMSA Radiation Safety Committee will make recommendations about this following a study to be made in the near future.

\section{References}

${ }^{1}$ D. F. Parsons, V. A. Phillips and J. S. La11y. "Survey of X-ray Safety of Electron Microscopes". Report of the Radiation Safety Committee of the Electron Microscopy Society of America (April 10, 1972). Copies available from D. F. Parsons.

${ }^{2}$ D. F. Parsons, V. A. Phillips and J. S. Lally, Health Physics, 26, 439 (1974).

${ }^{3}$ Radiation Safety Committee of the E1ectron Microscopy Society of America. "Handbook of X-ray Safety for Electron Microscopists". Available (price \$1.00) from Dr. George G. Cocks, School of Chemical Engineering, olin Ha11, Cornel1 University, Ithaca, N.Y. 14850 . 
INTERCOMPARISON OF PERSONNEL DOSIMETERS*

H. W. Dickson, W. F. Fox, and F. F. Haywaod

Oak Ridge Natianal Loboratary

Oak Ridge, Tennessee 37830

The first intercomparisan of persannel manitaring dosimeters at Oak Ridge Nationol Labarotary's DOSAR Facility was canducted during the periad May 14-16, 1974. Ten independent laborataries ond companies porticipated in an intercomparison of neutron and gamma-ray dasimeters used for rautine persannel monitoring. The dasimeters, which were sent thraugh the mail, were expased at the Health Physics Research Reactar in three "standardized" radiation fields, which have been used far the past several years for intercamparing nucleor accident dosimeters. In addition, a 14-MeV neutron field was used as a fourth exposure candition. The results of the intercomparison show widely varying dose estimates; e.g., reported values af neutron dose equivolent have standard deviations ronging from 47-102\% af the mean. A secand intercamparisan was conducted in a similar manner an February 18-19, 1976; hawever, only a few preliminary results are available at this time.

(Dosimetry; intercomparison; neutron; gamma; reactor; persannel)

\section{Intraductian}

For the past ten years, the annuol dasimetry intercomporisons ${ }^{1-3}$ at the Oak Ridge $\mathrm{Na}$ tianal Laborotory's DOSAR Facility have pravided an opportunity for laboratories in the United States and foreign countries ta test dosimetry systems in simulated nucleor accident situations. These studies hove been successful in developing guidelines in instrumentation and procedures and in estoblishing "standardized" radiotion fields whose characteristics such os energy spectrum, intensity, ond unifarmity have been measured and accepted. The Health Physics Reseorch Reactar (HPRR) hos been used as o pulsed rodiotion source. The unshielded reactor and the reactor used with either of twa shields, a 12-cm-thick Lucite shield or a 13-cm-thick steel shield, provides three different neutron and gamma-roy spectra.

Many experimenters over the yeors have expressed interest in using the some "standardized" radiotian fields for the comparison of the response of rautine personnel dosimeters used at law radiatian levels typically encauntered in persannel monitoring. Recently other groups, including the Nuclear Regulatory Commission (NRC), become interested in the same project. As a result, the first Personnel Dosimetry Intercomparison Study (PDIS) wos conducteo' during the period Moy 1416, 1974, with ten groups porticipoting. The participants included Brookhaven Notional Laborotory; Dow Chemical Compony, Rocky Flots; Gesellschofł für Kernforschung (GFK), Karlsruhe,
Germany; Lowrence Livermore Labarotary; Los Alamos Scientific Labaratary; Naval Ordnance Laboratary; R. S. Landauer, Jr., and Company; Savonnah River Laboratory; and Unian Corbide Nuclear Divisian (Oak Ridge National Laboratory and $Y-12$ Plant).

\section{Radiation Sources}

The HPRR and a 14-MeV neutran generator were used to expose persannel dosimeters to mixed neutron ond gammo-roy fields. The reactar was operated in a steody-state made at a power level of one watt for a length of time necessary ta produce a radiation dose ronge commonly encountered in personnel monitoring. Since dase equivalents of 0 few hundred millirem ore cammonly encountered, this arder of magnitude was selected. In order to produce this range of radiatian levels, a free air tissue kerma af approximately 40 mrad wos selected for the neutron camponent; and the reactor operating time wos calculated based on this kermo. The neutron generotor was operated to produce a similar range af radiation levels. The resultont reactor runs were performed os shown in Toble 1.

Calculotions of the unshielded HPRR spectrum and the steel and Lucite degraded spectra have been mode using a discrete ardinates transport code. ${ }^{4}$ The results af these calculations, presented in Toble 2, represent the reactor spectro used for this intercomporison study.

\footnotetext{
${ }^{*}$ Research sponsared by the Energy Reseorch \& Development Adm. under contract with Union Corbide.
} 
Table 1. Summary of Reactor Operations for Intercomparison

\begin{tabular}{ccccc}
\hline \hline Run No. & Shield & Power & Time (min.) & Fissions \\
\hline 1 & Unshielded & 1 watt & 5.0 & $9.25 \times 10^{12}$ \\
3 & Steel & 1 watt & 13.9 & $2.57 \times 10^{13}$ \\
\hline
\end{tabular}

During the course of this intercomparison, the DOSAR Low-Energy Accelerator (DLEA) was unavailable for the production of neutrons; consequently, the 14-MeV neutron exposures were made using a Phillips, sealed-tube, neutron generator whose radiation field components were not as well known as those for the DLEA. In addition to the 14-MeV neutrons, there was a significant exposure due to low-energy $x$-rays $(E \leq 150 \mathrm{keV}$ ) emitted from the vicinity of the ion source.

\section{Experimental Details}

All badges were placed on water-filled trunk portions of Bomab phantoms. These were placed at three meters from the reactor in the case of reactor exposures and at one meter from the Phillips tube in the case of the 14-MeV exposure. When shields were used, they were placed at two meters. The placement of dosimeters on the phantoms is shown in Fig. I, while a typical experimental arrangement with reactor and shields in place is given in Fig. 2.

Generally, the dosimeters were mailed or shipped to the DOSAR a few days in advance of the intercomparison. The dosimeters were refurned in a similar manner the day after the intercomparison exposures were completed. Exceptions to this procedure were that dosimeters from local laboratories were hand-carried back and forth. Because dosimeters from the GFK laboratory, Karlsruhe, Germany, arrived late, it was necessary to make an additional exposure on an independent but "identical" basis. The types of dosimeters used by the participants are listed in Table 3. Information provided to the participants included the reactor operation data shown in Table 1, the position of their dosimeters as shown in Fig. I, and the calculated neutron spectra shown in Table 2.

\section{Reference Dosimetry}

Sulfur pellets exposed on the reactor during the intercomparison exposures were used to estimate kerma for the three-meter position. The estimated kerma values were 36,42 , and $35 \mathrm{mrad}$ for the unshielded, steel-shielded, and Luciteshielded runs, respectively. The count rates on the sulfur pellets were quite low, and a standard deviation of $\pm 20 \%$ was expected due to counting statistics and other sources of error. Based upon the neutron spectra that have been published ${ }^{4}$ for the three exposure configurations used, the dose and dose equivalent can be calculated. Using the dose conversion factors given in Radiation Dosimetry ${ }^{5}$ for that section of a phantom designated element 57, the dose conversion factors for the HPRR spectra were calculated and are shown in Table 4. Using the fission yield and the calculated leakage of the HPRR, the neutron fluence was calculated for each reactor run. By applying the previously determined dose conversion factors and average quality factors $(\overline{\mathrm{QF}}){ }^{6}$, the dose and dose equivalent were calculated and are given in Table 5 .

In the case of the 14-MeV neutron exposure, the dose equivalent was monitored by a tissueequivalent proportional counter used in an integrating mode and placed at the approximate position of the dosimeters. This monitor indicated a dose equivalent of 325 mrem for the operation. Due to variations in the angular intensity of the radiation around the neutron generator fube, it is expected that the actual dose equivalent varied from phantom to phantom. The high $x$-ray exposure levels that were encountered were not anticipated, and no provision was made to monitor them. A summary of the reference values of neutron dose and dose equivalent for the four exposures is presented in Table 6. 
Table 2. Calculation of HPRR Spectrum for NAD Intercomparison

\begin{tabular}{|c|c|c|c|c|c|c|c|c|c|c|}
\hline \multirow{3}{*}{$\frac{\text { Group }}{1}$} & \multirow{2}{*}{\multicolumn{2}{|c|}{ Upper Energy (ev) }} & \multirow{2}{*}{\multicolumn{2}{|c|}{ Mid Energy (ev) }} & \multirow{2}{*}{\multicolumn{2}{|c|}{ No Shield }} & \multicolumn{4}{|c|}{$N(E) \Delta E^{*}$} \\
\hline & & & & & & & \multirow{2}{*}{$\begin{array}{r}\text { Lucite } \\
3.31\end{array}$} & \multirow{2}{*}{$\frac{\text { Shield }}{\text { E7 }}$} & \multirow{2}{*}{$\frac{\text { Steel }}{1.35}$} & \multirow{2}{*}{$\frac{\text { Shield }}{\text { E7 }}$} \\
\hline & 1.49 & E7 & 1.22 & E7 & 9.53 & E7 & & & & \\
\hline 2 & 1.0 & E7 & 8.19 & E6 & 1.18 & E9 & 3.63 & E8 & 1.5 & E7 \\
\hline 3 & 6.7 & E6 & 5.77 & E6 & 3.43 & E9 & 4.29 & E8 & 3.8 & E8 \\
\hline 4 & 4.97 & E6 & 3.87 & E6 & 1.44 & E 10 & 2.58 & E9 & 1.57 & E9 \\
\hline 5 & 3.01 & E6 & 2.12 & E6 & 3.76 & E 10 & 5.56 & E9 & 7.94 & E9 \\
\hline 6 & 1.5 & E6 & 1.16 & E6 & 3.16 & E10 & 3.19 & E9 & 1.21 & E 10 \\
\hline 7 & 9.07 & E5 & 6.08 & E5 & 4.61 & E10 & 3.69 & E9 & 3.34 & E 10 \\
\hline 8 & 4.08 & E5 & 2.13 & E5 & 3.39 & E 10 & 3.08 & E9 & 5.02 & E 10 \\
\hline 9 & 1.11 & E5 & 9.80 & E4 & 2.60 & E9 & 4.18 & E8 & 2.13 & E9 \\
\hline 10 & 8.65 & E4 & 7.64 & E4 & 2.0 & E9 & 3.81 & E8 & 2.91 & E9 \\
\hline 11 & 6.74 & E4 & 5.95 & E4 & 1.5 & E9 & 3.49 & E8 & 1.41 & E9 \\
\hline 12 & 5.25 & E4 & 4.63 & E4 & 1.21 & E9 & 3.24 & E8 & 1.25 & E9 \\
\hline 13 & 4.09 & E4 & 3.61 & E4 & 9.71 & E8 & 3.05 & E8 & 5.61 & E8 \\
\hline 14 & 3.18 & E4 & 2.81 & E4 & 8.40 & E8 & 2.98 & E8 & 6.64 & E8 \\
\hline 15 & 2.48 & E4 & 2.19 & E4 & 7.35 & E8 & 2.76 & E8 & 2.5 & E8 \\
\hline 16 & 1.93 & E4 & 1.70 & E4 & 6.37 & E8 & 2.66 & E8 & 1.01 & E8 \\
\hline 17 & 1.50 & E4 & 1.03 & E4 & 1.58 & E9 & 7.60 & E8 & 1.14 & E8 \\
\hline 18 & 7.10 & E3 & 4.88 & E3 & 1.39 & E9 & 7.23 & E8 & 1.02 & E8 \\
\hline 19 & 3.35 & E3 & 2.03 & E3 & 1.62 & E9 & 9.48 & E8 & 1.16 & E9 \\
\hline 20 & 1.23 & E3 & 8.48 & E2 & 1.04 & E9 & 6.97 & E8 & 4.2 & E8 \\
\hline 21 & 5.83 & E2 & 3.54 & E2 & 1.24 & E9 & 9.21 & E8 & 4.47 & E8 \\
\hline 22 & 2.14 & E2 & 1.47 & E2 & 8.45 & E8 & 6.91 & E8 & 3.14 & E8 \\
\hline 23 & 1.01 & E2 & 6.96 & El & 7.76 & E8 & 6.90 & E8 & 2.88 & E8 \\
\hline 24 & 4.79 & El & 3.73 & El & 4.72 & E8 & 4.59 & E8 & 1.69 & E8 \\
\hline 25 & 2.90 & E 1 & 2.26 & E 1 & 4.54 & E8 & 4.60 & E8 & 1.67 & E8 \\
\hline 26 & 1.76 & El & 1.37 & El & 4.34 & E8 & 4.61 & E8 & 1.61 & E8 \\
\hline 27 & 1.07 & El & 7.34 & & 6.09 & E8 & 6.93 & E8 & 2.11 & E8 \\
\hline 28 & 5.04 & & 3.93 & & 3.82 & E8 & 4.58 & E8 & 1.28 & E8 \\
\hline 29 & 3.06 & & 2.18 & & 4.84 & E8 & 6.11 & E8 & 1.71 & E8 \\
\hline 30 & 1.56 & & 1.25 & & 3.04 & E8 & 3.79 & E8 & 1.21 & E8 \\
\hline 31 & 1.0 & & 8.06 & $E-1$ & 2.81 & E8 & 3.41 & E8 & 9.16 & E7 \\
\hline 32 & 0.65 & & 5.41 & $E-1$ & 2.42 & E8 & 2.86 & E8 & 7.83 & E7 \\
\hline 33 & 0.45 & & 2.12 & $E-1$ & 1.78 & E9 & 2.67 & E9 & 5.63 & E8 \\
\hline \multirow[t]{2}{*}{34} & 0.1 & & 2.24 & $\mathrm{E}-2$ & 3.36 & E9 & 1.95 & E10 & 1.09 & E9 \\
\hline & 5.0 & $E-3$ & & & & & & & & \\
\hline
\end{tabular}

This number is the area of the histogram for each energy interval. 

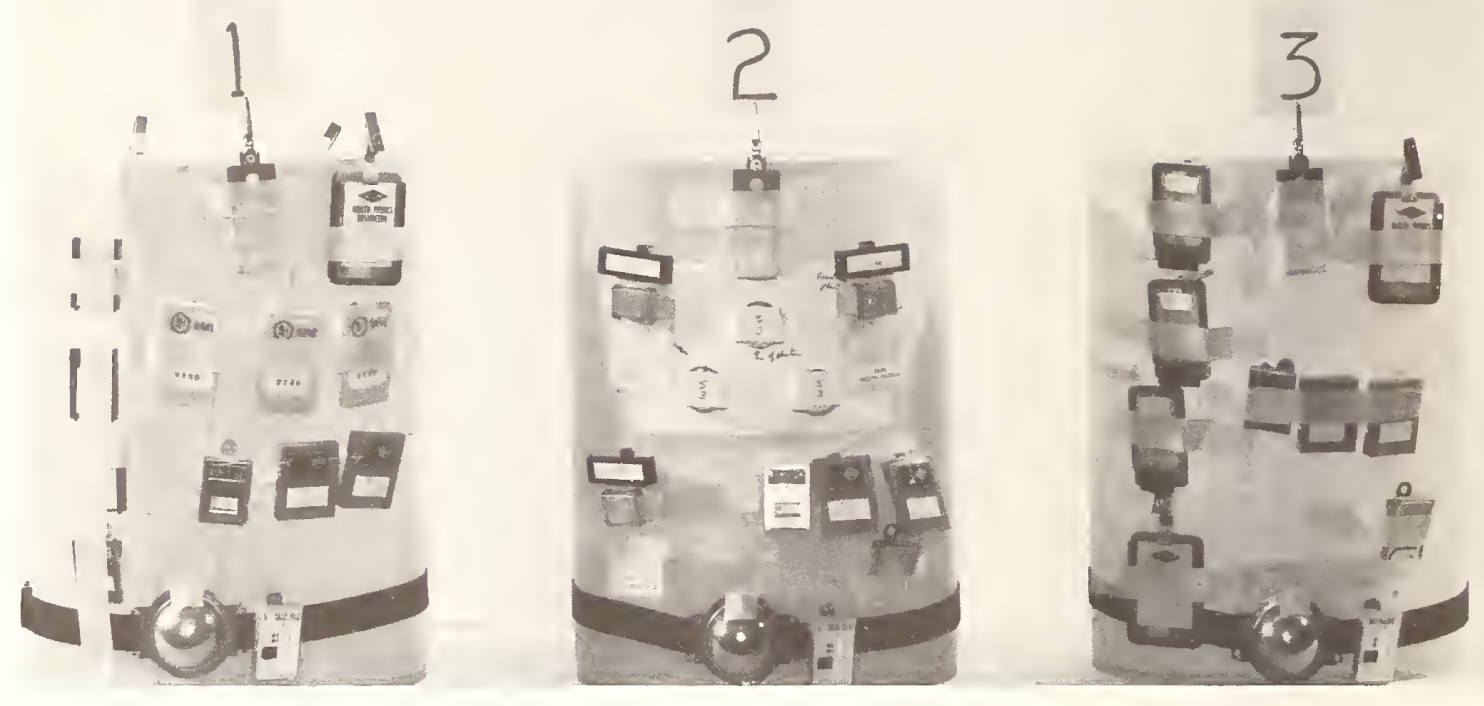

FRONT

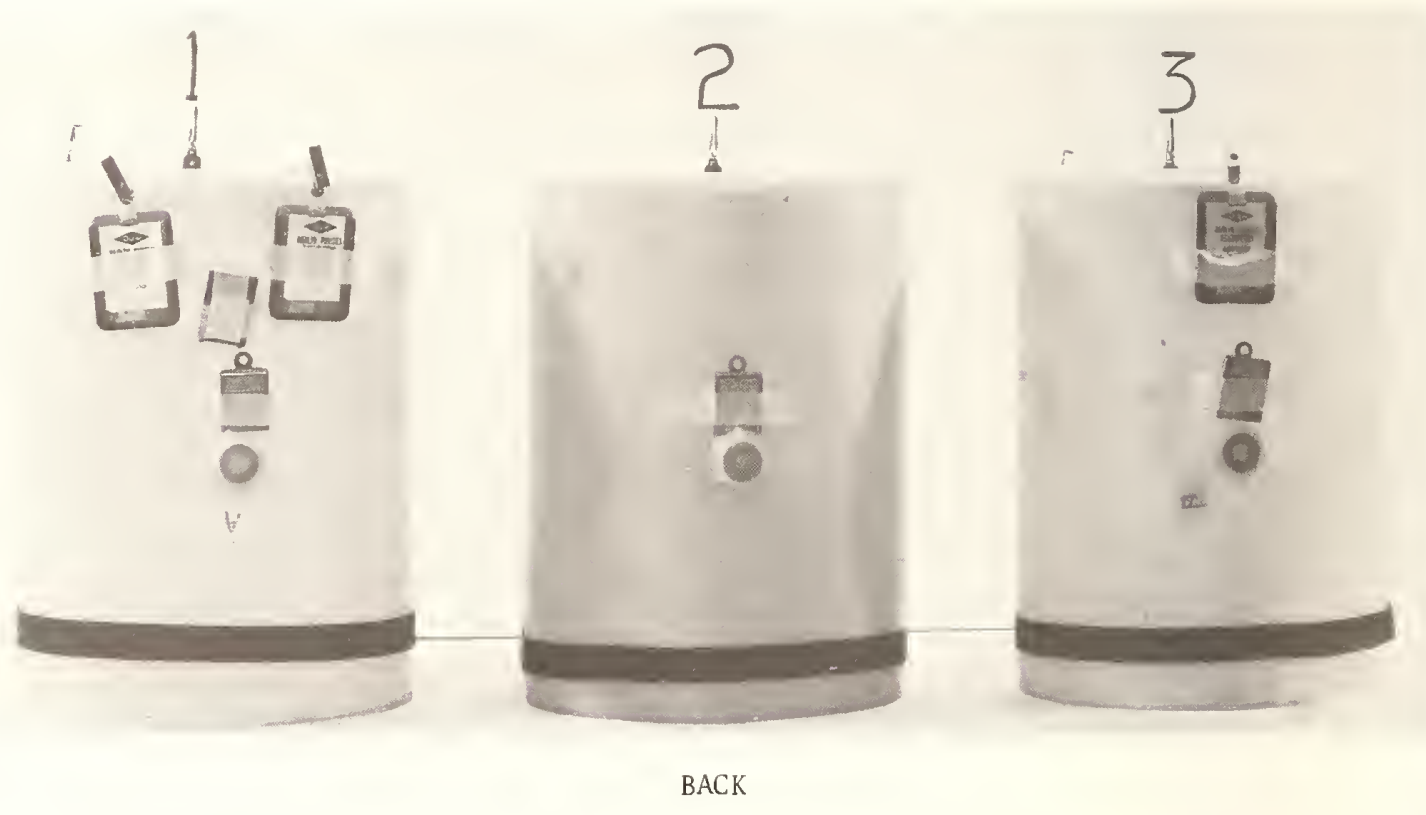

Fig. 1. Typical Placement of Dosimeters on Phantom Section 


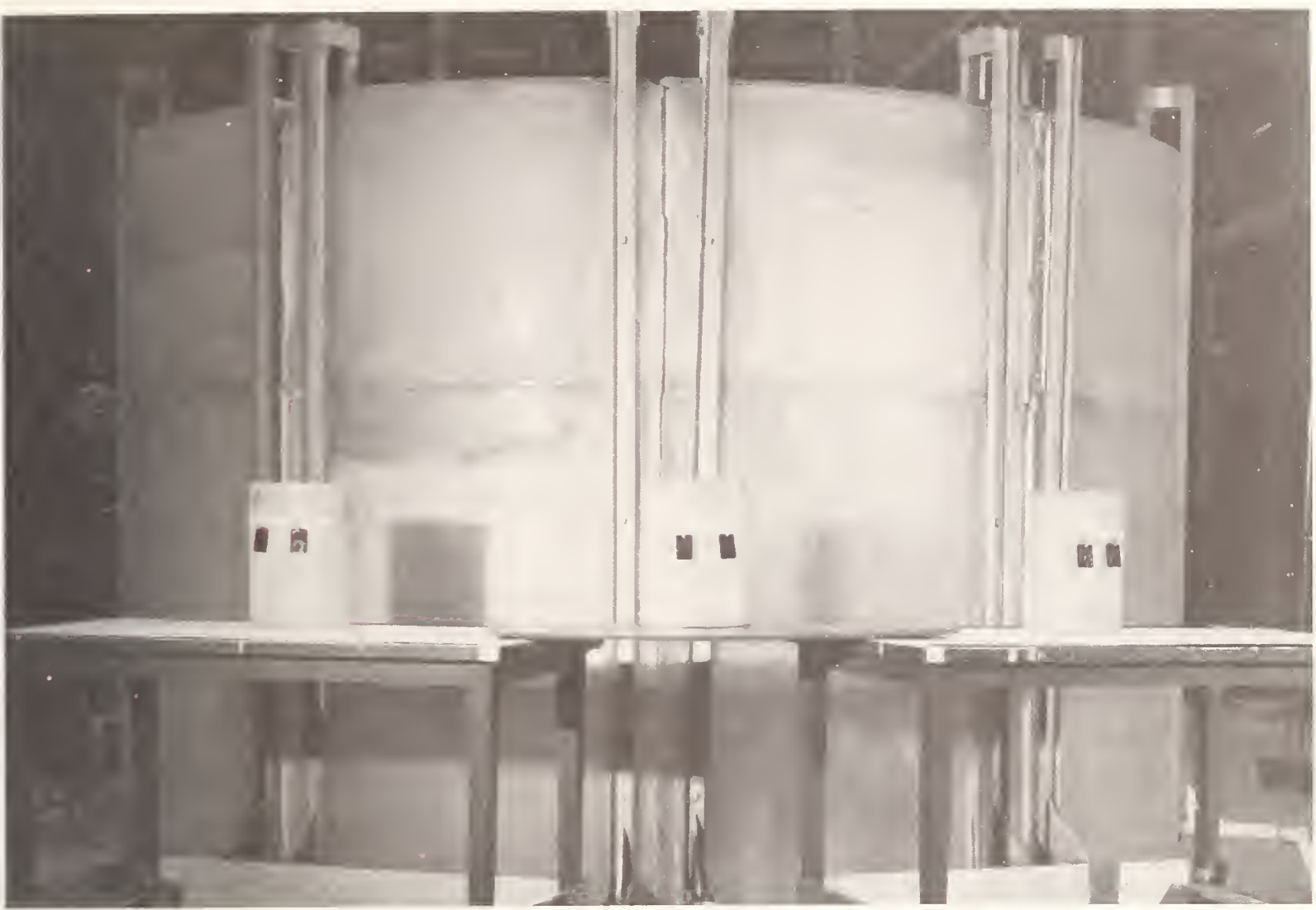

Fig. 2. Typical Experimental Arrangement for Reactor Exposures

Table 3. Dosimeters Used by Participants

\begin{tabular}{clc}
\hline & \multicolumn{2}{c}{ Dosimeter } \\
Group & \multicolumn{1}{c}{ Neutron } \\
\hline A-1 & TL pair & Gamma \\
A-2 & Thorium & TLD \\
A-3 & Standard interpretation & - \\
A-4 & NTA film & - \\
B & TLD albedo & Film \\
C-1 & TLD albedo & TLD \\
C-2 & NTA film & - \\
D & TLD albedo & Film \\
E & TLD albedo & TLD \\
F-1 & NTA film & TLD \\
F-2 & TLD albedo & Film \\
F-3 & TLD albedo & TLD \\
G & NTA film & TLD \\
$H$ & TLD albedo & Film \\
I & TLD albedo & TLD \\
$J$ & & TLD \\
\hline
\end{tabular}


Toble 4. Neutron Dose Conversion Factors and Averoge Quolity Factors for HPRR Spectra

\begin{tabular}{lcc}
\hline \multicolumn{1}{c}{ Shield } & $\begin{array}{c}\text { Dose Conversion Factor } \\
\left(\operatorname{mrod} \mathrm{cm}^{2} \times 10^{-7}\right)\end{array}$ & $\overline{\mathrm{QF}}$ \\
\hline Unshielded & 25.5 & 9.4 \\
Steel & 17.9 & 9.5 \\
Lucite & 14.6 & 8.9 \\
\hline
\end{tabular}

Gommo exposures voried; but, using previous intercomparison results, gamma doses of 5.6, 4.7, and $34.5 \mathrm{mrad}$ were calculoted based on the number of fissions that occurred on the three respective runs during the operotion of the reactor. In actuality, the dosimeters accumulated odditional gomma exposure from the residual activity in the reactor core. The exposure rote at three meters from the unshielded reactor was approximately 15 $\mathrm{mR} / \mathrm{hr}$. A good estimate of the gammo dose is not possible due to the vorying lengths of time the dosimeters remoined in proximity to the reoctor ond the varying attenuation through the shields. However, it is reosonoble to ossume that 15-20 mrod could be added to the dose delivered during the octuol reactor operation. This would suggest total gammo doses of 20-25 mrad for the unshielded and steel-shielded runs and $50-55 \mathrm{mrad}$ for the Lucite-shielded run.

\section{Results}

The results of all participants are given in Tables 7 and 8 for the four exposure configurations used during the intercomparison. The averages of the participants' estimotes were 453 $\pm 213 \mathrm{mrem}$ for the bare reactor, $554 \pm 346$ for the steel-shielded reactor, and $675 \pm 687$ for the Lucite-shielded reactor. Some of the participants gave several dose estimates either due to the use of multiple dosimeters or due to vorious meons of interpreting their results. The average includes all estimates that the porticipants claimed to be valid, even those taking into account actual knowledge of the spectro. The results of the GFK laboratory (I) are included but not averaged with the others. This is because the GFK dosimeters arrived late and had to be exposed at a different time but under "identical" conditions. The opera. tion of the reoctor should be reproducible to within a few percent, and the GFK results should be in good agreement with the others for the three reoctor runs. In the case of the 14-MeV exposure, the conditions for GFK were oltered by plocing o lead shield around the sealed source tube to attenuote the low-energy photons $(\leq 150$ $\mathrm{keV}$ ) from the accelerotor. This exploins the significantly smoller gommo dose reported by GFK.

Table 5. Absorbed Neutron Dose and Dose Equivalent Calculated from HPRR Fission Yields

\begin{tabular}{cccc}
\hline Reactor Run & $\begin{array}{c}\text { Fluence } \\
\left(\mathrm{cm}^{-2} \times 10^{7}\right)\end{array}$ & $\begin{array}{c}\text { Dose } \\
(\mathrm{mrad})\end{array}$ & $\begin{array}{c}\text { Dose Equivalent } \\
(\mathrm{mrem})\end{array}$ \\
\hline 1 & 1.82 & 46.4 & 436 \\
2 & 3.11 & 55.7 & 529 \\
3 & 2.60 & 38.0 & 338 \\
\hline
\end{tabular}

Table 6. Reference Values of Neutron Dose ond Dose Equivolent

\begin{tabular}{|c|c|c|c|c|c|}
\hline \multirow[b]{2}{*}{ Run } & \multirow[b]{2}{*}{ Spectrum } & \multicolumn{2}{|c|}{ Dose (mrod) } & \multicolumn{2}{|c|}{ Dose Equivalent (mrem) } \\
\hline & & Calculated & Measured & Calculoted & Meosured \\
\hline 1 & Unshielded HPRR & 46.4 & $36 \pm 7.2$ & 436 & - \\
\hline 2 & Steel-shielded HPRR & 55.7 & $42 \pm 8.4$ & 529 & 一 \\
\hline 3 & Lucite-shielded HPRR & 38.0 & $35 \pm 7.0$ & 338 & - \\
\hline 4 & $14 \mathrm{MeV}$ & 43.9 & - & - & 325 \\
\hline
\end{tabular}


ble 7. Results of Personnel Dosimeter Intercomparison Expressed in Dose Equivalent (mrem)

\begin{tabular}{|c|c|c|c|c|c|c|c|c|c|c|c|}
\hline \multirow{3}{*}{$\frac{\text { Phantom }}{D E_{n}}$} & \multicolumn{5}{|c|}{ Unshielded Reactor } & \multicolumn{6}{|c|}{ Steel-Shielded Reactor } \\
\hline & No. 1 & Phantom & No. 2 & Phanto & $\mathrm{m} \mathrm{No.} 3$ & Phantom & No. 1 & Phantc & No. 2 & Phantc & $\mathrm{m} N 0.3$ \\
\hline & $D E_{\gamma}$ & $D E_{n}$ & $\overline{D E_{\gamma}}$ & $\overline{D E_{n}}$ & $\mathrm{DE}_{\gamma}$ & $D E_{n}$ & $\overline{D E_{\gamma}}$ & $\overline{D E_{n}}$ & $\overline{D E_{\gamma}}$ & $\mathrm{DE}_{\mathrm{n}}$ & $\mathrm{DE}_{\gamma}$ \\
\hline 582 & 25 & - & - & - & - & 612 & 15 & - & - & - & - \\
\hline 520 & - & - & - & - & - & 484 & - & - & - & - & - \\
\hline 435 & - & - & - & - & - & 223 & - & - & - & - & - \\
\hline 350 & - & 350 & 30 & 350 & 30 & 330 & 20 & 330 & 20 & 330 & 20 \\
\hline 510 & 22 & - & - & 510 & 22 & 550 & 11 & - & - & 550 & 11 \\
\hline- & - & 307 & - & - & - & - & - & 378 & - & - & - \\
\hline- & - & 307 & $20^{a}$ & - & - & - & - & 302 & $12^{a}$ & - & - \\
\hline 380 & 23 & 350 & 23 & 370 & 23 & 410 & 16 & 410 & 17 & 420 & 17 \\
\hline- & - & 540 & 20 & - & - & - & - & 690 & 20 & - & - \\
\hline 140 & 31 & 140 & 23 & 160 & 23 & 50 & 19 & 20 & 19 & 40 & 19 \\
\hline 952 & 23 & 865 & 25 & 921 & 28 & 1120 & 24 & 1112 & 17 & 1259 & 24 \\
\hline 662 & 23 & 594 & 26 & 512 & 19 & 1050 & 22 & 1144 & 20 & 870 & 21 \\
\hline 220 & 30 & - & - & - & - & 400 & 20 & - & - & - & - \\
\hline 420 & 30 & 405 & 25 & 395 & 25 & 565 & 20 & 690 & 20 & 620 & 20 \\
\hline- & - & 383 & 35 & - & - & - & - & 402 & 14 & - & - \\
\hline- & - & - & - & - & $35 / 14^{b}$ & - & - & - & - & - & $28 / 45^{b}$ \\
\hline 470 & 25.9 & 429 & 24.0 & 460 & 24.3 & 527 & 18.6 & 564 & 18.1 & 584 & 19.4 \\
\hline
\end{tabular}

not mrem $\quad{ }^{b}$ First number given is based on film dosimeter; second number is based on a TLD.

able 8. Results of Personnel Dosimeter Intercomparison Expressed in Dose Equivalent (mrem)

\begin{tabular}{|c|c|c|c|c|c|c|c|c|c|c|c|}
\hline & $c i t \epsilon$ & Shie & ed $R e$ & actor & & & $14-M$ & $e V N$ & tron & Gene & tor \\
\hline hanto & No. & Phant & No. 2 & Phant & $\mathrm{m} N \mathrm{No} 3$ & Phanto & No. 1 & Phantc & No. 2 & Phanto & No. 3 \\
\hline$D E_{n}$ & $\mathrm{DE}_{\gamma}$ & $\overline{D E_{n}}$ & $\overline{D E_{\gamma}}$ & $\overline{D E_{n}}$ & $\mathrm{DE}_{\gamma}$ & $\overline{D E_{n}}$ & $\overline{D E_{\gamma}}$ & $\mathrm{DE}_{\mathrm{n}}$ & $\overline{D_{\gamma}}$ & $\overline{D E_{n}}$ & $\mathrm{DE}_{\gamma}$ \\
\hline 431 & - & - & - & - & - & - & - & - & - & - & - \\
\hline 451 & - & - & - & - & - & - & - & - & - & - & - \\
\hline 434 & - & - & - & - & - & 587 & 505 & 587 & 505 & 587 & 505 \\
\hline 400 & 90 & 400 & 90 & 400 & 90 & 220 & 490 & - & - & - & - \\
\hline 383 & 51 & - & - & 383 & 51 & - & 435 & - & - & - & 435 \\
\hline- & - & 443 & - & - & - & - & - & 308 & - & - & - \\
\hline- & - & 297 & $63^{a}$ & - & - & - & - & 283 & $307^{a}$ & - & - \\
\hline 420 & 76 & 400 & 70 & 440 & 69 & 1600 & 310 & 1400 & 160 & 1400 & 120 \\
\hline- & - & 380 & 60 & - & - & - & - & 390 & 600 & - & - \\
\hline 100 & 89 & 140 & 89 & 90 & 89 & - & - & - & - & - & - \\
\hline 2515 & 95 & 2437 & 84 & 2422 & 86 & - & - & - & - & - & - \\
\hline 870 & 89 & 1000 & 83 & 1272 & 97 & - & - & - & - & - & - \\
\hline 500 & 85 & - & - & - & - & 100 & 700 & - & - & - & - \\
\hline 405 & 65 & 405 & 65 & 425 & 65 & 315 & 320 & 225 & 265 & 225 & 270 \\
\hline- & - & 418 & 65 & - & - & - & - & 341 & $24^{c}$ & - & - \\
\hline- & - & - & - & - & $67 / 54^{b}$ & - & - & - & - & - & 375 \\
\hline 628 & 80 & 656 & 75.5 & 776 & 74.2 & 564 & 460 & 532 & 367 & 737 & 341 \\
\hline
\end{tabular}

not mrem birst number is based on film dosimeter; second number is based on a TLD.

ce shielded with lead

231 
A summary of the results is presented in Table 9. It is reasonable to expect a more favorable agreement between the several participating laboratories if the results of experimental devices and nonroutine dosimeters are ignored or if a selective data handling technique is used. For example, if the extreme data points for each of the phantoms in Tables 7 and 8 are excluded, the resultant average dose equivalent estimates are $431 \pm 112,539 \pm 238$, and $501 \pm 240 \mathrm{mrem}$, respectively. Also, if the upper and lower extremes are excluded from the 14-MeV results, the average neutron dose equivalent becomes $409 \pm$ 154 mrem.

This study was found to be valuable to the participants, and the wide range of results indicate some problems with the response and/or the interpretation of the dosimeters used. The second Personnel Dosimetry Intercomparison Study was held February 18-19, 1976. The reference dosimetry was upgraded to provide for more accurate low-level gamma-ray as well as neutron dosimetry. At this time, only preliminary results are available and it has not been determined as yet if the results are in better agreement than those of the first study. This addition to our dosimetry intercomparison program was judged to be worthwhile, and plans are under way to continue these studies in the future.
1. F. F. Haywood, 1970 Intercomparison of Nuclear Accident Dosimetry Systems at the Oak Ridge National Laboratory, ORNL-TM3551 (Feb. 1972).

2. J. W. Poston and F. F. Haywood, 1972 Intercomparison of Nuclear Accident Dosimetry Systems at the Oak Ridge National Laboratory, ORNL-TM-4387 (July 1972).

3. H. W. Dickson, F. F. Haywood and K. Becker Tenth Dosimetry Intercomparison Study, August 27-September 7, 1973, ORNL-TM-4566 (Mar. 1975).

4. J. W. Poston, J. R. Knight and G. E. Whitesides, "Calculation of the HPRR Neutron Spectrum for Simulated Nuclear Accident Conditions," Heal th Phys. 26, 217 (1974).

5. F. H. Attix and W. C. Roesch (eds.), Radiation Dosimetry (Academic Press, New York, 1968).

6. M.S. S. Murthy, R. C. Bhatt and S. S. Shinde, "Estimation of Quality Factor and RBE for Degraded Fission Neutron Spectra," Health Phys. 27, 9 (1974).

Table 9. Summary of Results

\begin{tabular}{lccc}
\hline \multicolumn{1}{c}{ Spectrum } & $\begin{array}{c}\text { Neutron Dose Equivalent } \\
\text { (mrem) }\end{array}$ & $\begin{array}{c}\text { Gamma } \\
\begin{array}{c}\text { Dose Equivalent } \\
\text { (mrem) }\end{array}\end{array}$ \\
\hline Bare HPRR & $453 \pm 213^{a}$ & $24.6 \pm 5.9$ \\
Steel-shielded HPRR & $554 \pm 346$ & $18.1 \pm 4.3$ \\
Lucite-shielded HPRR & $675 \pm 687$ & $75.1 \pm 14.2$ \\
14 MeV & $587 \pm 501$ & $384 \pm 151$ \\
\hline \multicolumn{2}{c}{$a_{\text {Error given for one standard deviation }}$} & &
\end{tabular}




\section{ENVIRONIVENTAI RADIATION MEASTREMENTS}

James E. McIaughiin

Health and Safety Laooratory

U. S. Emergy Research and Development Administration

INew Yorix, N.Y. 10014

Deterninations of raziation dose rates and radionuclide concentrations depend on systematic considerations of detector characteristics and instrument system calibrations, as iell as the composition, source distributions, and fluxenergy and angle distribut-ons of the environmental rajiation fiela. Routine in situ measurements around nuclear facilities are made on rariy to monitor changes in certain rajiation parameters. Even these measurements should be based on absolute deterninations in order to attribute changes correctly to the source. The systematic considerations required for monitoring environmental sources are discussed and realistic examples of gamma-ray measurements are presented along rith a suggestion for beta ray measurement.

(Environmental gamma-rays, sourve distributions, instruments, calibration, ìield testing)

\section{Introduction}

Environmental raxiation measurements are made for a variety of purposes, but primarily to aid in assessing $\operatorname{man}^{\prime}$ 's exposure to environmental sources. Measurements małe in connection with nuclear facilities monitoring provide data on measurable radiation and radionuclide levels. Then relationships between radionuclides in effluents and the consequent doses to individuals or population grouos can be established or - Terified and any radionuclide conzentration trends detected.

liany of the research and measurement efforts in this field have been described at numerous meetings, notably at the Iatural Radiation Environnent Symposia in 1964 and 19721,2. Whether external radiation measurements are male becajse of scientific curiosity or for practical purposes, e.g., geoscientific surveys or nuclear facilities monitoring, the problems are complex and fornidable, and should be addressed in a systematic fashion.

The employment of hand-held survey instmuments, ionization chambers, thermoluminescence dosimeters, and ganma-ray spectrometers for in situ measurements of the external radiation field is reviewed and the validity of such measurement is discussed. Some of this information has been developed for the National Council on Radiation Protection and Measurements.3

The basic problem is that there are no environmental external radiation calibration standards. One must take account of the instrument and radiation field characteristics that are considered integral parts of a measurement system. The system is then "calibrated" by relying on a complex and sometimes tenuous argument supported by theoretical considerations and independent measurements. This is often difficult when one wishes to quantify one of the smaller and often variable components of environmental radiation, e.g., that due to manmade radionuclides.

\section{Theoretical Considerations}

The two main contributors to the penetrating component of an environmental radiation field are cosmic rays, mostly muons, and gamma rays, mostly photons from terrestrial sources. The exact composition and intensity of the field, in terms of either the ionization rate in air 
near the ground or absorbed dose rate in free air, vary with time and with location. The detection and quantification of either these natural chaiges in environmental background or changes attributable to the addition of manmade sources depends on the degree to with one can determine the responses of raliation detectors to photons and muons.

In most applications, one can subtract the response to cosmic rays corresponding to the measurement altitude from the total detector response. If the latter is expressed as a free air dose rate, the values for the mid-geomagnetic latitudes corresponding to the continental United States show in Figure 1 can be subtracted.3,4 These values were inferred from measurements of cosmicray ionization by several investigators. The data neglect the effect of changes due to solar modulation during the solar cycle, solar flares, and atmospheric temperature and pressure.3 At or near sea level, i.e. in most inhabited locations, these

changes are usually less than 10 percent in magnitude and are negligible for many measurement programs.

The determination of the cosmicray response of a detector is not a straightforward process. The charged-particle equilibrium in the detector that exists for terrestrial photons may not exist for cosmicray muons and electrons. So one must either perform a complex detector evaluation based lacgely on theory or evaluate the response empirically as a function of altitude as indicated in the following section. 3, 4

The gamma-ray calibration of a radiation detector with a known point radionuclide source, such as 226Ra, or a known photon beam, is applied to convert the field measurement to a convenient parameter like dose rate. This procedure can be seriously in error if the detector response is strongly dependent on the (photon) energy or if the response is anisotropic. The major environmental sources are the $233 \mathrm{~J}$ and the $232 \mathrm{Th}$ series and $40 \mathrm{~K}$ in the ground. The latter two contributors tend to dominate the gamma-ray absorbed dose rate in free air about one meter above ground, the height usually employed for convenience.

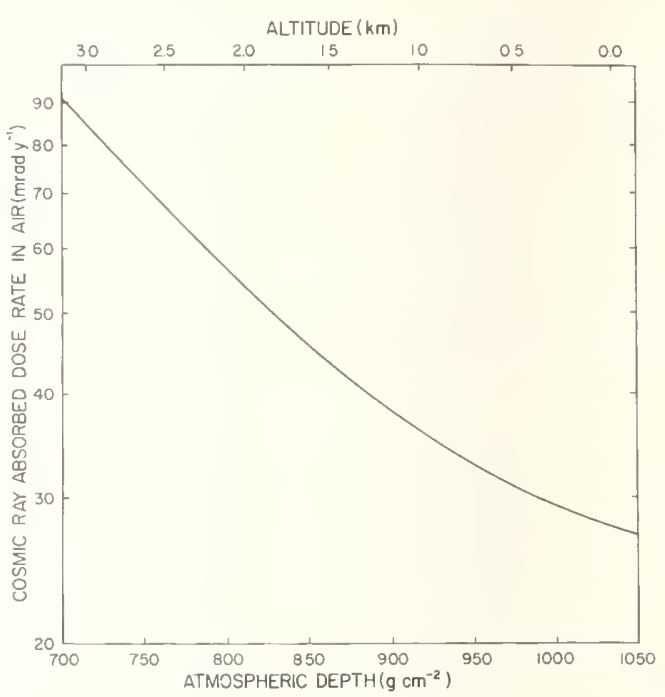

Figure 1. Absorbed dose rates in free air from cosmic-ray charged particles at geomagnetic latitude $50^{\circ} \mathrm{N}$ (Ref. 4).

The calibration problem can be illustrated with a comparison of the energy spectrum from a sealed source of 20Ra with that in a "typical" field situation. Typical contributions to dose rate were derived from many field measurements made in the eastern United States.5 The measurements were coupled with theoretical calculations of the radiation field above a half-space containing the sources shown in Table 1, under the assumption that the series are in radioactive equilibrium. Disequilibrium can exist for $238 \mathrm{~J}$, but not in any important way for $232 \mathrm{Th}$, because of the short helf-life of $220 \mathrm{Rn}$.

The energy spectrum for this typical situation is shown in Figure 2 as the "boxed" histogram: The spectrum from a 226Ra source, shown as the "shaded" histogram, is much harder, so the dependency of the detector response on energy cannot be neglected. 

Table 1. $\frac{\text { Contributions to Dose Rate }}{\text { One Meter Above Ground }}$

Absorbed Dose Rate

Ratiation Source in Free Air (urad/h)

$4 \mathrm{O}_{\mathrm{K}}$

$238_{T J}+$ daughters

$232_{\text {Th }}+$ daxghters

$2 \cdot 2$

$137 \mathrm{Cs}, 95 \mathrm{Zr}-95 \mathrm{No}$

222 Rn dairghters in air

TOTAL

a. The ${ }^{222} \mathrm{Rn}$ daughter contribution is variable according to the soil type and local atmospheric conditions. 6

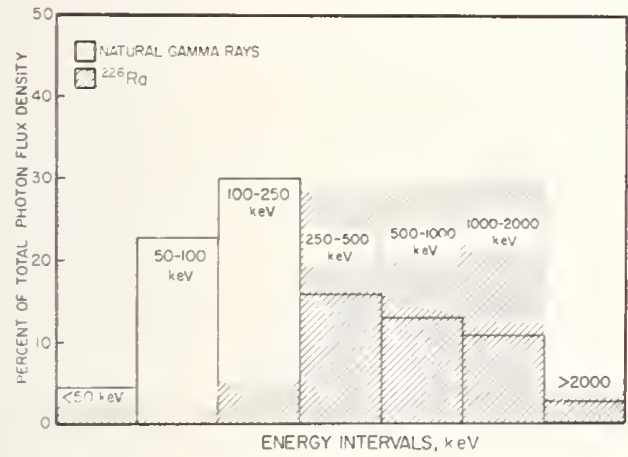

Figure 2. Comparison of ${ }^{226} \mathrm{Ra}$ calibration source energy spectrum and that from environmental natural ganma rays (Latter from Ref. 5)

The angular distribution of the photon flux from the ground, the predominant environmenta.l source, is also a significant consideration in field measurements. One can often characterize the half-space source as having a uniforn distribution of natural emitters with depth and an exponential distribution of deposited manmade nuclides or recently deposited natural emitters. The calculations show that a significant fraction of the photon flux incident on a detector is at large angles from the nornal. Figures 3 and 4 show the angular distributions from a $6 \mathrm{O}-\mathrm{KeV}$ and a $662-\mathrm{keV}$ source at $30 \mathrm{~cm}$. and $100 \mathrm{~cm}$. above the ground, respectively. The $\alpha$ values represent the vertical distributions in the ground and are in effect reciprocals of relaxation length. So, $\alpha=0.10 \mathrm{~cm}^{-1}$ represents a deeply distributed 60 $\mathrm{keV}$ source and $\alpha=1000 \mathrm{~cm}^{-1}$ epproximates a plane source.

The angular photon distributions illustrate the problem of improving detector response merely by increasing detector size. Only a small fraction of the flux originates from sources directly beneatin the detector; most of the flux is incident from angles closer to the horizontal and hence plane detectors may not be desirable. This observation, for example, affects an improvement of plane, photon detectors designed to measure actinides deposited on the ground based on an increase of the plane detector area. An ideal detector should be one with its sensitive surface oriented to intercept photons from angles of $60-90^{\circ} . ?$

\section{The $\alpha=0.33 \mathrm{~cm}^{-1}$ curve for a} high energy photon emitter like $137 \mathrm{Cs}$ represents a typical distribution from world wide fallout in a location that has not been disturbed. Here about one half of the flux is incident from angles above about $70^{\circ}$ and, consequently, a detector placed 1 meter above the ground in effect "samples" a large volume of ground, perhaps one that is tens of meters in diameter.

Calculations have also shown that virtually the entire dose rate at 1 meter above ground with a uniforn distribution of the natural emitters is attributable to about the top $25 \mathrm{~cm}$. This is significant in selecting a measurement location, because one only needs to test that the horizontal variation across the 10 20 meter area being measured is minimal with a survey instrument. This simple test and considerations of source distributions in the ground and flux distributions have indicated that in situ spectrometry can be employed in environmental and geological survey to quantify soil concentrations of ganma-ray emitting nu- 
clides. The circumstances for this application have been reported 8 and are reviened in a later section.

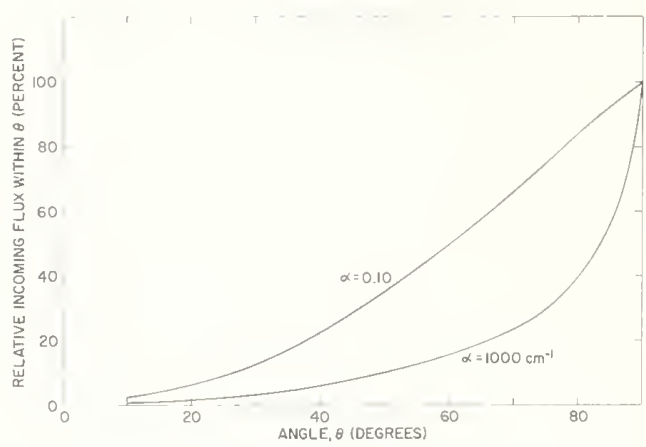

Figure 3. Angular distribution of photons at $30 \mathrm{~cm}$ above $60 \mathrm{keV}$ source in the ground for two vertical source distributions (Ref.?).

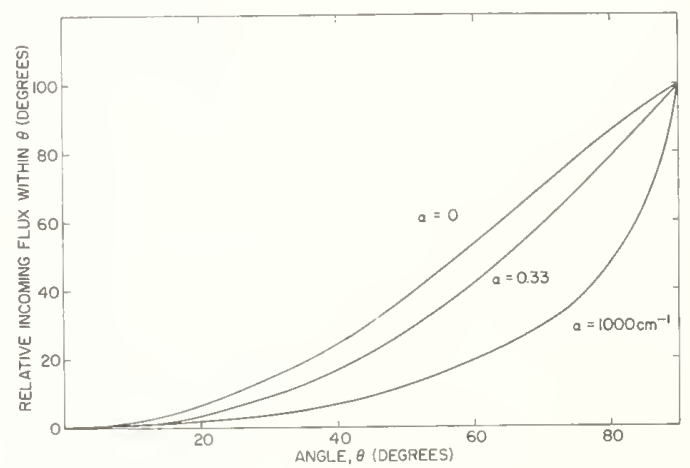

Figure 4. Angular distribution of photons at $100 \mathrm{~cm}$ above $662 \mathrm{keV}$ source in the ground for three vertical source distributions (Ref. 8).

The calculations we have employed to characterize tine typical gamma-ray field were based on a soil composition with 10 percent water by weight. The water content can vary widely, of course, but a comparison of mass attenuation coefficients for dry soil and that containing 25 percent water indicated the resulting exposure rates or dose rates would vary by approximately 5 percent, a value that is within the estimated uncertainty of the calculations and also the field measurements.
For certain applications, the effect of airborne radon daughters on detector response is not negligible. Variations of dose rate in the lower atmosphere can be substantial and should be evaluated in connection with measurements on aircraft and around nuclear facilities 3 Theoretical calculations have been made to identify the magnitude of these variations at heights above the ground between 1 meter and several hundred meters. Figure 5 depicts the relative contributions to the ionization rate in air for the principal environmental sources. The contributions from radon daighters for the extreme meteorological conditions are a small part of the total but they vary by about a factor of four, the maximum being well within $1 \mathrm{\mu rad} / \mathrm{h}$ at ground level. 6

Theoretical treatments of the type identified here have been reported (see for example Refs. 5 and 8). The tabulated energy and angle distributions can be folded into measured detector response functions of energy and angle to obtain correction factors for detectors with non-flat or anisotropic responses.

\section{Survey and Continuously-Monitoring Instruments}

Instruments employing scintillation or G.M. counters have been widely used for measuring radiation from environinental sources. However, one should not be misled by the apparent simplicity of survey instruments. Quantitative measurement requires that one account for contributions from all sources of penetrating radiations, interference by any beta-ray component, and departures from linear energy response. A practical instrument should:

a. be sufficiently sensitive to allow the measurement of environmental gamma rays down to about 1 $\mu \mathrm{R} / \mathrm{h}$,

b. have a sufficiently short time constant to permit the making of many measurements, but sufficiently long to minimize rapid fluctuations,

c. have a uniform and nearly isotropic response over the gamma-ray 
energy range of interest,

d. be insensitive to climatological shanges, e.g., temperature changes, and

e. be small and lightweight, if it is to be used for hand-held sirveys.

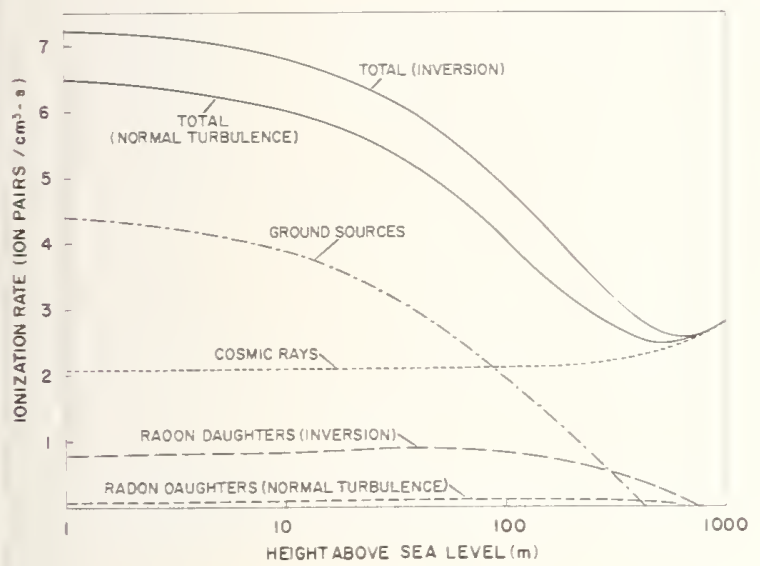

Figure 5. Calculated gamma- and cosmic-ray ionization rate in free air as a function of height above the ground (Ref. 6).

The responses of organic scintillators have been extended to lowenergy photons, as required for measrring many environmental gamma-ray fields (see previous section), by the addition of high-Z material. 9, 10 For example, coating anthracene with a thin layer of $\mathrm{ZnS}(A g)$ extends its "flat" response down to a bout $30 \mathrm{keV}$. Figure 6 illustrates the combined response of these materials in terms of exposire rate. The detector response below $1300 \mathrm{keV}$ is energy independent within about \pm 15 percent, when employed in al instrument having a D.C., as opposed to a count rate, output. A similar response and a detection limit of about $1 \mathrm{\mu R} / \mathrm{h}$ has been achieved with a tin-loaded plastic detector; the digital data readout of this instmment makes it sitable for axtomatic data recording and unattended monitoring.10

Inorganic scintillation detectors, e.g., INaI(Tl), show enhanced low energy responses because of their greater effective atomic numbers. Interpretation problems resilt from departures from uniform response of this type of detector and the greater sensitivity to low energy photons. The tacit assumption is often made that the total counting rate above some discriminator threshold level is proportional to exposure rate, but the reduced low-energy response should be assured by setting the discriminator sufficiently high so that any gain shift or zero shift has a negligible effect.11 This is importait because the environmental low energy photon flux density is relatively large a.1d is variable (see Figure 2).8 Hence, the determination of exposure rate based on calibrations with radioactive sources such as $137 \mathrm{Cs}$ or $225 \mathrm{Ra}$ can be seriously in error (see previous section).

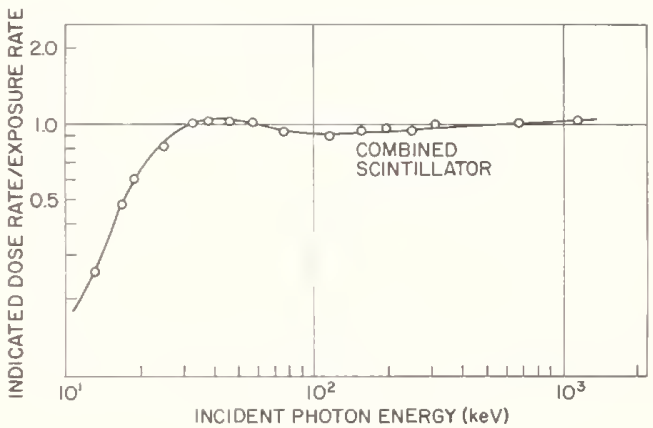

Figure 6. Ganma-ray response function of ZnS-plastic combination scintillator (Ref. 9).

Field measurements of radioactive areas in Colorado and on Bikini Atoll have illustrated the problem of directly applying point source laboratory calibration data to NaI(Tl) responses. The detector response to one of the usual highenergy point sources per unit exposure rate will probably be smaller tha: the corresponding value for the largely scattered and low-energy grama-ray field. In the cases mentioned this led to overestimates of the field exposure rates by factors of 1.5 to $2.12,13$

The NaI(Tl) detector sizes are often $2.5 \times 2.5 \mathrm{~cm}$ or only slightly 
larger and the high-energy response may, therefore, be energy dependent. The use of these detectors for special studies, e.g., the environmental measurement of $6-7 \mathrm{MeV}$ photons from $16 \mathrm{~N}$ produced in direct-cycle light-water power reactors, can lear to significant underestimates, if they are based on the incomplete. laboratory calibration described above.

An acceptable calibration is to determine the response of the survey instrument to know sources in the laboratory and compare this response with a well-calibrated ionization chamber. This procedure should account for the fact that photons from the point source, unlike those in the environment, are not generally isotropically incident. A convenient method is to base the calibration on simultaneous in situ measurements for different environmental radiation fields. In these procedures one needs to account for differences in response of each detector to cosmic radiation.

Ionization chambers are attractive because measurements with this instrument have been made for many years in connection with cosmic-ray research and the early environmental monitoring programs.14 - 17 These measurements were historically interpreted in terms of the ionization rate in free air and then converted to estimates of exposure rate or absorbed dose rate in free air, based on reasonable assumptions.

Problems of insufficient sensitivity and reliability, as well as the unwanted response to internal rarioactive contamination, limited the use of air-filled chambers and many low-pressure chambers. These problems are largely alleviated by the employment of high-pressure ionization chambers (HPIC) of the type employed by Millikan, Spiers and others.14,16 The HPIC exhibit suitable signal-to-noise ratio, discrimination against beta rays, greatly reduced response to radioactive contamination, and remarkable resistance to environmental stresses.

Several types of this instrument were developed and employed in a lengthy series of high-altitude, balloon-borne experiments.1? The small, solid-state electrometer was designed for minimal variation with temperature in the range encountered in most field workl8. The system finally evolved for monitoring environmental gamma rays has the characteristics listed in Table 2.

Table 2. Nominal Design Specifications of HPIC System.

Chamber wa.l1: $0.30 \mathrm{~cm} \cdot(2.36$ $\left.\mathrm{g} / \mathrm{cm}^{2}\right)$ stainless steel.

Chamber volume: 8 l $(25-\mathrm{cm}$ dia.) sphere.

Chamber filling: 20 - 25 atm. ultra-high-purity argon.

Collecting voltage: $300 \mathrm{~V}$.

Electrometer: HASL-designed solid-state electrometer (Reference 18).

Data recorder: ADC to scaler, voltmeter to strip-chart or cassette tape.

The pulse-scaler output is used for rapid in situ exposure rate measurements and the cassette tape output for continuous unattended monitoring. The detector employed with each system should be manufactured to the specifications given, and evaluated and calibrated to avoid departures from the desired characteristics.

The response of this or any chamber to the environmental gammaand cosmic-radiation field is represented by

$$
R=k_{c} I_{c}+k_{\gamma} I_{\gamma}+R_{\alpha},
$$

where $R$ is the system response in appropriate current, voltage, or pulse rate units, $I_{c}$ and $I_{\gamma}$ are the contributions from cosmlc and gamma radiation, respectively, $\mathrm{R}_{\alpha}$ is the response to any internal radioactive contamination, and the $k$ factors are the conversions to absorbed dose rate in free air or to exposure rate. A HPIC is considered unacceptable if $R_{\alpha}$ exceeds a value that is equivalent 
tc ajout 0.I , $\mathrm{R} / \mathrm{h}$ from an external gamma-ray sourze. Analyses of these and other uniantec effects have been described.19,20 The tro main calibration problems are described simoly in terms of the parameters in equation (I). In these non-airlike detectors, $x_{C}$ and $x_{y}$ are usually not equal and there is no knotr

"standard", pure, cosnic- radiation field for a direct detemination of

$k_{c}$. The factor, $k_{Y}$, depends on

energy so tie use of a talue derived Irom a laboratory calibration with point sources or beans may not be ralid for fielà measurements.

The deternination of $k_{c}$ derives from a complex combination of botin tinoretical calculations of the interaction of muons and electrons in the chamber, as well as the consistency of the result with many field measurements made at various altitudes and with data in the literature (see review in Reference 20). The theoretical calculation takes account of the slight attenration in the steel wall of the cosmic-ray electrons and the net buildup of electrons passing through the chamber because of pair production interactions. The net build-up or transition effect in our chanber was calculated to be about 20 percent.21 Since the electrons make up roughly one-half of the total chargedparticle flux in the lower atmosphere and the muons are practically unattenuated by the chamber rall, the net enhancement of the ionization current from the chamber wall is about 10 percent. The estimated value for the chamber in Table 2, $\sim 3 \times 10^{-14}$ amperes per $\mathrm{uR} / \mathrm{h}$, is consistent with the resilt derived from many measurements of nearly pure cosmic ray and mixed cosnic-and ganma-ray fields. In these measurements $R$ was detemined by subtracting $I_{\gamma}$ and $k_{\gamma}$ values obtaiñed from in sltu spectrometry and laboratory caliorations. BJ normalizing an experimental $k_{c} I_{c}$ curve as a function of altitude to $I_{c}=2.1$ ion pairs $/ \mathrm{cm}_{-}$ s-atm. at sea leveI for STP, an $I_{C V S}$ altitude profile, corresponding to the dose rate profile in Figure 7 , and values for $k_{C}$ were derived. $1 \overline{7}^{\prime}$ The uncertainty of the product $k_{c}$
$I_{c}$, estimated to be about \pm 5 percent (s.d.), is due to tine small time and space variations in cosnic-ray ion- 10 ization rate in the mid-latitudes 7,10 The uncertainty in $I_{C}$ is similar. although some of the literature indicates the error in $I_{c}$ may be somewhat larger.22,23 However, $I_{c}$ is generally a minor contribution to the total ionization rate so the larger error would increase the uncertainty only slightly for most applications. For the nominal. HPIC in Table $2, k_{c}$ equals $x_{y}$, within 2 percent, though one should not assume this equality pertains for other HPICs or other detectors.

The gamma-ray calibration of any detector is accomplished in a more direct fashion. First, the response to primary photons from a known point source is obtained in the laboratory. It is necessary to subtract the response to scattered photons in a particular location (obtained by shielding the detector from the primary photons) from the total response obtained without a shadow shield. Then, the resulting $k_{\gamma}$ should be corrected for differences in the calibration source energy spectrum and that expected in the field. The field spectrum is "softer" than the laboratory, so the laboratory $k_{\gamma}$ for the particular HPIC must be corrected to obtain a $k_{y}$ for the expected field spectrum. 20

The overall accuracy of this instrument for measurements of gamnaray or gamma plus cosmic radiation is about \pm 5 percent (s.d.). This estimate derives in part from the consistency of ion chamber and gamma-ray spectrometric measurements in many different radiation fields, that is discussed in the next section.

Useful comparisons with calculated responses of real chambers have been made that take account of the effect of wall attenuation and buildup, as well as energy absorption in the argon gas as a function of photon energy. The comparison is shown in Figure 7 where the response on the ordinate, $F$, is

$$
F(E, x, P V)=B_{\gamma} e^{-\mu x} \frac{\left(\mu_{e} / 0\right)}{\left(\mu_{e} / p\right) \operatorname{Ar}}
$$


and $\mu_{e} / 0$ is the mass energy absorption coefficient, $\mu$ the linear absorption coefficient, $x$ the mean thickness of the steel wall for isotropically incident photons, and $B_{y}$ the ratio of ionization in argon due to all photons to that produced by the primary photons from monoenergetic sources. The competing effects of the various factors in equation (2) yield a fairly flat energy response for the $2.36 \mathrm{~g} / \mathrm{cm}^{2}$ wall chamber, while the thin-wall ( $\left.0.40 \mathrm{~g} / \mathrm{cm}^{2}\right)$ device is obviously unsuitable.

For the thick-wall chamber in Figure 7, the $k_{\gamma}$ value for the typical natural gamm-radiation field in Figure 2 is $2.5 \times 10-14$ amperes per $\mu \mathrm{R} / \mathrm{h}$, a value that is only 1.03 times that obtained from the laboratory calibration with a 226Ra source. Because of the non-uniform response of the thin-wall chamber, the laboratory $k_{\gamma}$ must be multiplied by 1.23.

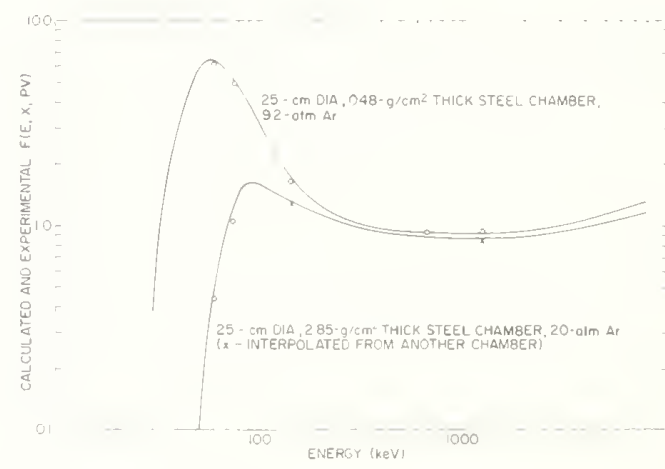

Figure 7. Ganma-ray response

function thin wall and thick wall high pressure argon ionization chambers (Ref. 20).

\section{Thernoluminescence Dosimeters}

The thermoluminescence phenomenon has been widely exploited for environmental as well as for geological measurements. This extensive technology has undergone intercomparison tests under environmental conditions and guidance on performance specifications and testing procedures was recently recommended in a national standard 24,25 . As for any field instrument, themoluminescence dosimiters (TID) should be little affected by environmental conditions. The TID should be protected against moisture and the response corrected for the effects of any trace radioactivity in the phosphor or its packaging and large temperature changes on the measured response. This is accomplished by relying on special control dosimeters and, in some cases, on temperature profile data for the usually lengthy exposure times. The national standard offers partial guidance on a test aimed at developing suitable correction factors for thermoluminescence fading. The empirical determination of fading and self-irradiation corrections is difficult and time-consuming, so one is often inclined to rely on approximate corrections.

An indication of the attainable overall accuracy is indicated by a comparison of 7 IIF TID and HPIC measurements obtained from the HASI monitoring site in Iloyd, New York, near Poughkeepsie. The results in Figure 8 cover about fifteen months and are reported as the average exposure rate for each four-week monitoring period. The measurements were male and analyzed according to the procedures described in Section $\mathrm{C}-01$ and C-O4 of the HASI Procedures Manual and they, of course, resemble the guidance in the national standard. 25,26

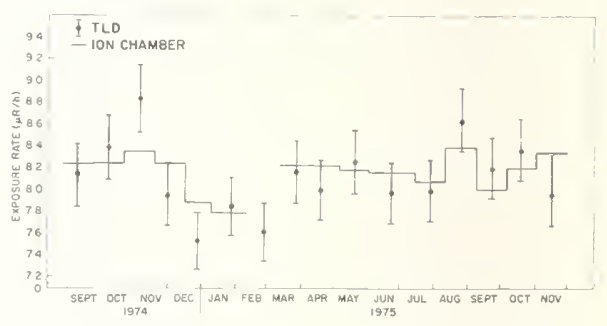

Figure 8. Average environmental exposure rates from TLD and HPIC measurements at Lloyd, New York.

Tine error bars in the figure (standard deviations) represent the accuracy of the field measurements, if one were to neglect the small laboratory calibration errors. The precision of the ionization chamber ineasurements is not indicated, but is about \pm 1 percent (s.d.). A measure of agreement between the two de- 
jectors was taken to be the difference between the average exposure rates divided by the ionization chanber result. The arerage agreement for the total period indicated in Figure 8 is within 0.5 percent and the mean difierence for 4 - week measurement periods is 2.7 percent. It is believed that the overall accuracy for these tyoe of terrestrial ganna ray olus cosmic ray measurements is about \pm 5 percent (s.d.). $\therefore$ part of the uncertainty is atiriouted to the relative responses of MTD, LiF in this case, to cosmic-ray muons and electrons.

The recent international intercomparison Las afforded additional insignt into the TTD capabilities as now employed.24 In this test fortyone organizations provided TID sets for exoosure to a gamma-ray calibration source and to the natural radiation environment for three months at a location near Houston, Texas. The agreenent indicated oy all of the dosimeters was $28 \pm 5 \mathrm{mR}$ (s.d.) for the laboratory exposure and $16 \pm 4$ $m R$ (s.d.) for the field. It is interesting to note that about onefourth of the participants corrected for fading by relying on either teinperature profile data or by controls, "inile the majority made no correction.

A general evaluation of error propagation has been developed for TID and other integrating dosimeters and is reported at this symposium.27

Thermally-stimulated exo-electron emission (TSEE) is a detector surface phenomenon related to themoluminescence that may be suitable for environmental use. Results from the intercomparison and more recent field tests indicate that TSEE is a promising development, but difficulties arise during exposure that are attributed to moisture on the detector surfaces. 28

\section{In Situ Gamma-Ray Spectrometers}

Field spectrometry does not yet fit the technical and economical requirements for routine environmental monitoring. However, spectrometry is important, and possibly necessary for special environmental and geological surveys. It aids in determining the radionuclide composition of environmental sources and their relative contributions to the radiation field. In some cases, one can quantify radionuclide concentrations in the ground and monitor the changing amount of environmental moisture and its asricultural importance. $8,29,30$

In situ spectrometers usually have minimum responses to cosmic-ray muons muons, so the gamma-ray responses can be employed to evaluate the responses of rajiation detectors or to provide independent verification of these responses (see previous section).

Moderately-large ( $10 \times 10-\mathrm{cm}$ ) $\operatorname{NaI}(\mathrm{Tl})$ and $1 \operatorname{arge}(60-130 \mathrm{~cm} 3)$ Ge(Ii) detector systems have been successfully employed by a number of organizations for radiation measurements in the general environment and around nuclear facilities. Measurements of natural enitters with uncollimated NaI(Th) detectors tare several tens of minutes, wille Ge(Ii) measurements can usually be accomplished in an hour or less. For example, the several pulse height spectra obtained at the same 1ocation, but at different times, near a boiling water power reactor were useful in assuring that the response of a HPIC monitor was attributable to the noble gas plume. 3,30

The areas of the measured photopeaks are proportional to the flux densities of uncollided photons from particular radionuclide emissions. If the source geometry is reasonably well known, these photopeak areas can be correlated to individual contributions to the exposure rate above ground or the absorbed dose rate in free air. The correlations depend on the radionuclide distribution in the ground and the energy and angle spectra above it. An important advantage of in situ spectrometry is that an uncollimated detector above the ground in effect samples a very large volume of soil and comparable counting statistics are obtained in a small fraction of the time required for the laboratory spectrometric analysis of a collected sample. 
A limitation of in situ spectrometry is that the accuracy of determinations of the exposure rate above the ground or radioniclide concentration in the ground depend on the source distribution and composition.8 Determinations of rationiclide concentrations depend strongly on the distribution with soil depth, while exposure rate determinations are much less dependent.

The detector response in terms of total counts in an absorption peak from the uncollided photon flux density is determined by calibrating the detector in the laboratory with small point sources. The analysis of an in situ spectrum and its relation to exposure rate or concentration depends on the following parameters:

$\left(N_{0} / \varphi\right)=$ the count rate in a particul-ar photopeak per unit flux density of photons of energy, E, incident along the detector axis of symmetry;

$\left(\mathbb{N}_{f} / \mathbb{N}_{0}=\right.$ the ratio of photopeak count rate in the field, where these photons are not necessarily incident along the detector axis of symmetry, to the count rate from an equal flux density along the detector axis. For a uniform angular response $\mathrm{N}_{f} / \mathrm{N}_{O}$ is unity. This ratio depends on the source energy, distribution and soil properties;

( $\varphi / C)$ the total uncollided flux density at the detector per unit soil concentration ( $\mathrm{pCi} / \mathrm{g})$ or $\mathrm{mCi} / \mathrm{km}$ ) of a particular nuclide as a function of energy, source distribution, and soil properties;

(ं) = the exposure rate in $\mu \mathrm{R} / \mathrm{h}$ at one meter above the ground from all photons from a particular nuclide including the secondary photons produced in the soil and air; and

$(\varphi / \dot{X})=$ the ratio of the flux density at the detector due to photons of energy, E, from the decay of a particular nuclide and any daighters to the corresponding total exposure rate for that nuclide and its daighters.

Then the photopeak count rate is related to the exposure rate in air above the ground by:

$$
\left(\mathbb{N}_{f} / \dot{X}\right)=\left(\mathbb{N}_{f} / \mathbb{N}_{0}\right)\left(\mathbb{N}_{0} / \varphi\right)(\varphi / \dot{X}) \text {, }
$$

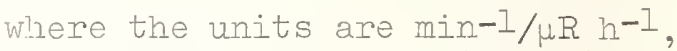
and, similarly, to radionuclide concentrations in the ground by

$$
\left(\mathbb{N}_{f} / C\right)=\left(\mathbb{N}_{f} / \mathbb{N}_{0}\right)\left(\mathbb{N}_{0} / \varphi\right)(\varphi / C),
$$

and the units are min-l/pCi $\mathrm{g}^{-1}$ or $\mathrm{m} \mathrm{Ci} / \mathrm{km}^{2}$.

The high resolution of Ge(Ii) detectors allows one to measure absorption peak areas with little interference from neighboring peaks as well as several peaks from the sane nuclide. However, the counting efficiency is somewhat lower and slightly longer in situ counting times are required to obtain statistical precision comparable to that of $\mathrm{NaI}(\mathrm{T} l)$.

The first two terms in Eqs. 3 and 4 represent the angular response correction and counting efficiency, respectively, of the particular detector. The ratios $\varphi / \mathrm{X}$ and $\varphi / C$ depend only on source composition and geometry and can be used for any detector. Some values for these parameters are given in Ref. 8 and other values can also be inferred if one employs decay scheme data.

The most important parameter for determining the exposure rate attributed to a particular. radionuclide or natural series is $\varphi / \dot{X}$. Model calcuIations have shown that while $\varphi$ and $X$ vary over a range of about ten for different ground source depth distributions, the ratio $\varphi / \dot{X}$ is nearly flat from a plane - to a deeply-distributed source. 5 Thus, even if one has only a poor knowledge of the actual depth distribution, the error in the $X$ inferred from field measurements is reasonably limited. 
This has been illustrated by the comparison of spectrometric measurements of natural emitters and independent HPIC measurements. The summed spectrometric photopeak contributions usually equal the HPIC results within about 5 percent. 8 Such data have been used to estimate the overall accuracies of exposure rates determined from spectrometric measurements of sources in the ground. 22 For Ge(Ii) spectrometry, the estimates range from six percent ( $40 \mathrm{~K}$, $232 \mathrm{Th}$ series) to twelve percent (239u series). The poorer accuracy of the uranium series is related to source geometry uncertainties caused by radon migration.

In situ determinations of radionuclide concentrations in the ground are more difficult and their accuracy depends strongly on the accuracy of $0 / C$ which in turn depends directly on the vertical source distribution. Test measurements with $\operatorname{NaI}(T l)$ and comparison with laboratory analyses of collected soil samples have indicated agreement within a factor of two for $137 \mathrm{Cs}$ when the vertical distribution is assumed and within twenty percent when it is determined for the sample collection site. 8 Field tests in the Livermore Valley were made with an unshielded $70 \mathrm{~cm}^{3}$ closed-end coaxial Ge(Li) detector. 30 Soil samples were collected in increnents to a depth of $25 \mathrm{~cm}$ to determine the vertical distribution and the total activity for the radionuclides inferred. The results from one location used for field calibration, shown in Figure 9, indicate as expected that natural radioactivity is distributed uniformly in undisturbed places, while $137 \mathrm{Cs}$ from world-wide fallout decreases exponentially with depth. The average concentrations for the thorium and uranium series and for $40 \mathrm{~K}$ and $137 \mathrm{Cs}$ from the in situ and laboratory analyses agreed within a few percent. In cases where the depth distribution was not measured, agreement was within 15 percent for the natural, uniformly distributed nuclides, and within a factor of 2 for 137 Cs.

A similar experiment was made at the HASI monitoring site in Lloyd, New York as part of a long-term regional monitoring effort.31 Soil samples were collected in increments to about $20 \mathrm{~cm}$ depth, stones were removed, the soil dried and the concentrations of $40 \mathrm{~K}$, the $238 \mathrm{U}$ and $232 \mathrm{Th}$ series and $137 \mathrm{Cs}$ determined with a large field $\mathrm{Ge}(\mathrm{Li})$ spectrometer mounted temporarily in the laboratory. The depth profiles were qualitatively similar to the profiles in Figure 9. Given the radionuclide concentrations and assuming the in situ (soil plus rocks) composition, the exposure rate above the in situ soil was calculated to be $8 . \overline{2} \pm \overline{0.4}$ $\mu \mathrm{R} / \mathrm{h}^{6}$, This value assumed 15 percent $222 \mathrm{Rn}$ migration from the soil, but accounted for the resulting radon daughters in the air. The exposure rate was determined from an independent HPIC measurement to be $8.3 \pm 0.4 \mu \mathrm{R} / \mathrm{h}$. The former error is based on laboratory counting statistics (s.d.) and latter is the estimated HPIC accuracy (s.d.).

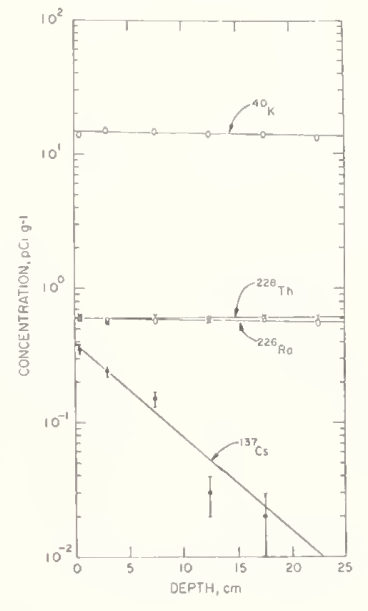

Figure 9. Measurement of radionuclide concentration with soil depth (Ref. 30).

\section{Field Tests of Instruments and Desirable Developments}

The determination of dose to man is the major one of the three principal purposes of measurements identified in the introduction. While doses are often calculated, assessments for nuclear facilities ought to depend in some way on measurements or on experimentally - verified computational methods. 
Table 3. Estimated Noble Gas Plume Exposure Near a BWR Obtained from HPIC Monitoring Record (Aug. 1973 - Sept. 1974) (Ref. 33).

\begin{tabular}{|c|c|c|c|}
\hline $\begin{array}{c}\text { Distance and Direction } \\
(\mathrm{km})\end{array}$ & $\begin{array}{l}\% \text { of Time in } \\
\text { Operationa }\end{array}$ & $\begin{array}{l}\text { Background } \\
\text { Expo sure }(m R)^{b}\end{array}$ & $\begin{array}{l}\text { Plume } \\
\text { Exposure }(\mathrm{mR})^{\mathrm{C}}\end{array}$ \\
\hline 1.3, NNE & 97 & 84 & 3.5 \\
\hline 2.6, ENE & 98 & 78 & 3.1 \\
\hline $4.6, \quad \mathrm{E}$ & 86 & 71 & 0.86 \\
\hline 4.6, NNE & 52 & 44 & 0.10 \\
\hline $5.2, N E$ & 95 & 84 & 2.1 \\
\hline 6.8, NNE & 36 & 30 & 0.1 \\
\hline 8.0, ENE & 65 & 58 & 0.7 \\
\hline
\end{tabular}

a. Refers to time of successful HPIC data acquisition.

b. Includes the cosmic-ray contribution equivalent to about $35 \mathrm{mR}$.

c. Plant effluent monitoring indicated the noble gas activity release rate during the period ranged from 4 to $140 \times 103 \mu \mathrm{Ci} / \mathrm{s}$.

A three-year long experiment to monitor radioactive noble gases around a boiling water power reactor (BWR) was concluded in 1974. The twofold purpose was to develop and field-test measurement methods suitable for documenting ambient environmental gamma-ray levels and quantifying any contributions attributable to plant operation, and to obtain data for verifying comnonly-used methods for computing the diffusion and dispersion of radionuclides in the air. Several reports describe the results, their significance and comparisons of measurement and calculation. $32-35$

In one part of the experiment, seven HPIC monitoring systems were deployed at locations between 1.3 and $8.0 \mathrm{~km}$ from the BWR. 18, 20 A variations analysis was applied to the measurements to separate the relatively rajidly - varying plume contribution from the slowly - varying background. A summary of the results from the period August 1973 through August 1974 is given in Table 3. The analysis produces an essentially unique identification and quantification of the noble gas contribution to the total environmental gamma-ray field.
As indicated in a previous section, the overall accuracy of this type of measurement for the total exposure rate (or absorbed dose rate in free air) is estimated to be within 5 percent (s.d.). If the HPIC produces data for a year, or a large fraction thereof, it was concluded from the tabulated and other data that the exposure attributable to a nearby nuclear facility can be determined within $1 \mathrm{mrad} / \mathrm{y}$.

Comparison of these measurements with several common computational methods indicated agreement, usually well within a factor of two. Identification of these models can be obtained from the references.

It should be noted that, given this type of verification, one can estimate population doses in terms of man-rad. It was estimated that an environmental "cost" of the one year operation of the BWR in question was 12 man-rad within $16 \mathrm{~km}$, and about $60 \mathrm{man}-r a d$ within $80 \mathrm{~km} .35$ of course, those of us who develop and perform measurements are not certain we understand the significance of these population dose estimates. 
In another oart of the experiment, TID measurements were made for nontily periods at about tiwo dozen locations, including the seven HPIC locations. A semi-emoirical calculation, correlating the soil moisture and exposure rate above ground ras made to estimate the expected background value.32 The difierence between the exoected value and the total exposure rate after correcting for the cosmic-ray contribution (equivalent to $3.7 \mathrm{k} / \mathrm{h}$ ) ras attributed to the noble gas plume. The agreement between the total exposures derived from measurements at four locations and calculations of the type used for the HPIC comparisons ras generally witinin a factor or two. The agreement at a location $14 \mathrm{~km}$ from the BWR was poor, because the error in the inferred plume contribution was abovt equal to the error estimated for the soil-noisture, exposure model.

Based on a typical background corresponiing to $10 \mu \mathrm{R} / \mathrm{h}$, or $88 \mathrm{mR} / \mathrm{y}$ including the cosmic-ray contribution to the annual environmental exposure from a nuclear facility would have an error of about $\pm I m R$ (s.d.). This is based on twelve successive, montin1.J TID measurement periods and acquisition of suitable climatological data for tine soil moisture - exposure model.32 Hence, if one vere to estimate the annual increment over this "tgpical" background with this method to be $10 \mathrm{mR}$, the associated error rould be acceptably small. It should be noted that the error would be somewhat reduced in areas where background is relatively low (see Table 3).

A parallel demonstration was undertaken on behalf of the IVuclear Regulatory Commission by the Health Services Laboratory of ERDA.36 Five IPIC detector units were modified to provide exposure rate vs. time data to a small computer located in a special trailer facility. Simultaneous recording, processing and transmitting of data from a nearby meteorological tower were performed and the results were retrieved either from the local facility or in HSL located at the National Reactor Testing Station in Idaho. Though "hardwire" transmission was employed in this experiment, and this tended to limit the area monitored, a brief test of FM radio telemetry was demonstrated to be feasible. A similar system has been successfully used at the $13,000 \mathrm{~km} 2$ Idaho site.

The demonstration was conceived as a possible prototype for a large area monitoring program. The multiple application of a complex system has recently undergone investigation. In this effort, remote NaI(Tl) detectors are expected to produce data on environmental levels and on effluents at selected release points on an automatic and continious basis.

An important problem for the development of instruments of this type is the lack of systematic instrument research and development in the Government and industry. The gamna-ray monitoring efforts described in this paper relied heavily on detectors and instruments developed during the last two decades. If the use of nuclear energy is to continue, continued development measurement methods is needed. One class of methods, pertaining to plutonium and other actinides is being investigated and has been reviewed.?

The development of an environmental beta-ray monitor is underway. Monitoring of $85 \mathrm{Kr}$ released from a reactor fuel reprocessing facility is also being attempted. Separation of any $85 \mathrm{Kr}$ contribution from the relatively substantial total beta-ray levels are based on the analysis method developed for the HPIC. Though insufficient data is available for dose estimation, several months of operation indicates that the G. M. count rate monitors are functioning reasonably well. $3 \overline{7}^{\prime}$

It seems apparent that combinations of continuous monitors that have been thoroughly field tested can be deployed around nuclear power plants, and possibly reactor fuel reprocessing plants, in fairly limited quantities.10,20, 33,37 These systems in conjunction with a larger number of monthly TLD measurements, in the experience of HASL are a practical program for monitoring external radiation. The measurements can be interpreted to form a record of any addi- 
tions to the natural environmental external dose rates.

Rapid surveys for obtaining information on sites for routine monitoring or on long-term deposits of radionuclides are best accomplished with suitably calibrated, possibly flat-response, survey instruments, and in situ spectrometers. The data on qualitative variations of gammaray levels and on dose rates attributable to specific natural radionuclides and pre-existing mannade nuclides can aid greatly in preparing a facilities monitoring program such as may be recommended or required. 38

The costs of equipment procurement for a monitoring program and of its operation and data processing are high, but they appear to be defensible and not necessarily extraordinary when one considers the cost and value of many current monitoring efforts.

\section{Acknowledgments}

The author gratefully acknowledges the many efforts of his associates in the Radiation Physics Division, especially H. I. Beck, G. de P. Burize, C. V. Gogolak, K. M. Miller and P. D. Raft, who performed the work of the Division's program described herein, as well as the contributions of W. M. Lowder, who in a sense was the originator of the first HASI investigations in environmental radiation in the 1950s. In addition, without the support of the Instrumentation Division, many of the measurements would not have been possible. Finally, acknowledgment is due P. G. Voilleque, formerly of the ERDA Health Services Laboratory for the use of unpublished information.

\section{References}

1. Adams, J. A.S. and Lowder, W.M., Eds., The Natural Radiation Environment, Univ. of Chicago Press, Chicago (1964).

2. Adams, J.A.S., Lowder, W.M. and Gesel1, T., Eds. (1972). The Natural Radiation Environment II, Report

CONF-720805 (U.S. Energy Research and Development Administration, Wash.).
3. Environmental Radiation Measurements, National Council on Radiation Protection and Measurements (to be published).

4. Iowder, W. M. and Beck, H. I., Cosmic ray ionization in the lower atmosphere, J. Geophys. Res. 71, 4661 $(1966)$.

5. Beck, H. I., The physics of environmental radiation fields, p. 101 in The Natural Radiation Environment II, USERDA Report CON-720805 (1972).

6. Beck, H. I., Gamma radiation from radon daughters in the atmosphere, J. Geophys. Res. 79, 2215 (1974).

7. Beck, H. I., McLaughlin, J. E., and Miller, K. M., In situ determinations of environmental plutonium and its related nuclides, IEEE Trans. Nuc. Sci. (to be published, 1976).

8. Beck, H. I., DeCampo, J. and Gogolak, C. V., In Situ Ge(Ii) and NaI (Tl) Gamma-Ray Spectrometry for the Measurement of Environmental Radiation, USERDA Report HASI-258 (1972)

9. Kolb, W. ond Lauterbach, U., A scintillation exposure rate meter for environmental radiation survey, p. 245 in The Natural Radiation Environment II, Report CONF-720805 (1972)

10. Chester, J. P., Chase, R. I. and Wood, S., A digital environmental monitor, Report BNL-16922 (1972).

11. Wollenberg, H. A. and Smith, A. R., Studies in terrestrial $\gamma$ radiation, p. 513 in The Natural Radiation Environment, Univ. of Chicago Press, Chicago (1964).

12. Gudiksen, Paul H. and Crites, Thomas R., A comparison of gamma-ray exposure rate measurements at Bikini Atoll, p. 727 in Operational Health Physics, Proceedings of Ninth Midyear Topical Symposium (Health Physics Society, 1976).

13. Lowder, W. M., Private Communication (1971). 
14. Millikan, R. A. and Neher, H. V., A preaision world survey of sea-level cosmi-ray intensities, Phys. Rev. 50,15 (1936).

15. Kuper, J.B.H. and Chase, R. I., Monitor for low intensity gamma rays, Rev. Sci. Instrum. 21, 356 (1950).

16. Spiers, F. W., McHugn, M. J. and Appleby, D. B., Environmental $\gamma$-ray dose rate to populations, surveys made with a portable meter, p. 385 in Tne Natural Radiation Environment, Univ. of Chicago Press, Chicago (1964).

17. Lowder, W. M., Raft, P. D. and Beck, H. I., Experimental determination of cosmic-ray charged particle intensity profiles in the atinosphere, p. 908 in Proc. of National Symp. on Natural and Manmade Radiation in Space (E. A. Waman, ed.). NASA rpt. NASA TII X-2440 (1972).

18. Negro, V. C., Watnick, S., Raft, P. D., A temperature-compensated electrometer for environmental measurements, IEEE trans. Nucl. Sci. NS-21, 805 (1974).

19. Shamos, M. H. and Liboff, A. R., New ionization chamber technique for the measurement of environmental radiation, Rev. Sci. Instrum. 39, 223 (1968).

20. DeCampo, J. A., Beck, H. I. and Raft, P. D., High Pressure Argon Ionization Chamber Systems for the Measurement of Environmental Exposure Rates. USAEC Report HASL-260 (1972).

21. Beck, H. I., Monte Carlo calculations of electromagnetic shower transition effects, Nucl. Instrum. Meth. 91, 525 (1971).

22. Iowder, W. M. et al, Experimental investigations of the environmental radiation field, p. 255 in the Natural Radiation Environment II, USERDA Report CONF-720805 (1972).

23. George, M. J., New data on the absolute cosmic ray ionization in the lower atmosphere, J. of Geophys. Res. 75,3693 (1970).

24. Gesell, Thomas F., Burke, Gail de P., and Becker, Klaus, An international comparison of environmental dosimeters under field and laboratory conditions, Health Physics 30 , 125 (1976).

25. Performance, testing, and procedural specifications for therinoluminescence dosimetry (environmenta applications), ANSI N 545 - 1975 , American National Standards Institute, New Yorix (1975).

26. Harley, J. H., Ed., HASI Procedures Manual, USERDA Report HASL-300 ( 1972 ).

27. Burke, Gail de P. and Gesell, Thomas F., Error analysis of environmental radiation measurements made with integrating dosimeters, proceedings of this symposium (1976).

28. Gamnage, R. B. and Haywood, F. F., Experiences in environmental monitoring with BeD ceramic dosimeters, p. 324 in Operational Health Physics, Proceedings of Ninth Midyear Topical Symposium (Health Physics Society, 1976).

29. Kogan, R. M., Nazarov, I. M. and Fridman, Sh. D., Gamma Spectrometry of Natural Environments and Formations (Atomizdat, Moscow) (English translation, Israel Program for Scientific Translations, Jerusalem, (1971).

30. Anspaugh, I. R., Phelps, P. I., Gudiksen, P. H., Lindeken, C. I. and Huckabay, G. W., The in situ measurement of radionuclides in the environment with a Ge(Li) spectrometer, p. 279 in The Natural Radiation Environment II, USERDA Report CONF-720805 (1972).

31. Miller, K. M., private communication (1976).

32. Burke, G. de P., Variations in Natural Environmental Gamma Radiation and Its Effect on the Interpretability of TID Measurements Made Near Nuclear Facilities, USERDA Report HASL-289 ( 1975 ).

33. Miller, K. M., Gogolak, C. V. and Raft, P. D., Final Report on Continuous Monitoring with High Pressure Argon Ionization Chambers Near the Millstone Point Boiling Water Power Reactor, USAEC Report 


\section{HASI-290 ( 1975 ).}

34. Gogolak, C. V., A Data Set for Noble Gas Plume Exposure Model Validation, USERDA Report HASL-295 (1975).

35. Gogolak, C. V. and Miller, K. M., Determination of gamma-ray exposure in the vicinity of a boilingwater power reactor, p. 207 in Population Exposure, Report CONF741018 (1974).
36. Voilleque, Paul G., private communication'(1975).

37. Beck, H. I. and Freeswick, D. C., A continuous monitoring system for $85 \mathrm{Kr}$ in the environment, Trans. Am. Nuc. Soc. 21, 92 (1975).

38. Environnental Radioactivity Surveillance Guide, USEPA Report ORP/SID 72-2 (1972). 


$$
\text { J.M. Matuszek }
$$

Radiological Sciences Laboratory

Environmental Health Center

Division of Laboratories and Research

New York State Department of Health Albany, New York 12201

During recent years, federal and state regulatory agencies have exhibited a propensity for issuing environmental radiation regulations regardless of the quality of the data base available for derivation of the standards. Particular examples of this trend are the AEC/NRC Regulatory Guide 4.3, EPA's Federal Safe Drinking Water Regulations, and some state and local edicts. Though the federal agencies appear to be improving in their efforts to obtain a sound environmental data base in certain areas, recent estimates of health effects attributable to ${ }^{14} \mathrm{C}$ effluents show that some interest continues for the "estimate and speculate" method of arriving at environmental radiation standards. Some state and local regulatory agencies, more closely attuned to political and media pressure, have rendered decisions or made statements which are not readily supported by the data obtained from environmental measurements. Environmental radiochemical laboratories, on the other hand, have found that quality assurance requirements ordinarily acceptable for routine surveillance programs are not always sufficient at low radioactivity levels for the regulatory needs developed. As even more stringent analytical requirements are imposed, the staffs of both the laboratories and the regulatory agencies must be fully cognizant of the statistical limitations of environmental data developed at very low radioactivity levels and respond accordingly in making regulatory decisions or setting environmental regulations.

(Regulations; radioactive effluents; environmental samples, laboratories; radioanalytical data; quality assurance)

\section{Introduction}

Regulatory actions based on environmental measurements involve a double responsibility: the laboratory must provide the most reliable data possible within analytical and statistical limitations, and the regulatory agencies must take care to apply the data only to the extent permitted by these limitations. All too often, one or both of these requirements are not met. The result is adverse public reaction, administrative problems, and/or added expense to resolve questions arising.

During the 1960 s environmental radioactivity was caused predominantly by fallout from nuclear weapons testing. Effluents from the few operating experimental nuclear reactors were rarely above ambient fallout levels, except for samples collected very close to the point of discharge. Radioanalytical requirements were relatively simple. Radioactivity levels from fallout in most environmental samples were well above the minimum detection limits of ordinary counting systems, and the counting requirements centered on obtaining satisfactory accuracy and precision for each measurement.

In the 1970s ambient fallout levels have decreased, and analytical sophistication has increased to the point where nuclear reactor effluents can be identified even at some distance from the source. These reactor-related radioactivity levels are one or more orders of magnitude below previous fallout levels, and the corresponding dose levels to the public are extremely small. Nevertheless, regulatory agencies have been developing environmental radiation regulations which tax radioanalytical capabilities technically and financially.

In this brief presentation I cannot hope to discuss all the radiological environmental regulations and decisions made during the past few years. Instead, I shall touch on some of the regulations made by the U.S. Nuclear Regulatory Commission (NRC) and 
the U.S. Environmental Protection Agency (EPA) which affect the greater body of environmental radiochemical laboratories and environmental regulatory agencies. Some decisions by state and local regulatory authorities, though not universal in impact, will be mentioned as examples of the environmental measurement problems which occur because of the more direct response by these agencies to public opinion. Finally, I shall offer some examples of the regulatory problems caused by inadequacies in data reported by state regulatory agencies or by radioanalytical laboratories.

For the sakie of time, the scope of this discussion is limited to topics associated with radioactivity levels in the environment, leaving discussion of ionizing environmental radiation to Dr. McLaughlin, the preceding speaker, and leaving unaddressed the less controversial topic of nonionizing environmental radiation.

Requlatory Imposition of New Analytical Tasks

Though the NRC has existed for only one year, much of the staff remains from its parent, the U.S. Atomic Energy Commission (AEC). Many regulatory decisions made by the AEC, which are still in effect as NRC Regulatory Guides or as technical specifications at various nuclear facilities, imposed new or more difficult tasks on radiochemical measurement laboratories.

The EPA's Office of Radiation Programs has existed somewhat longer. However, recent regulations by EPA have also imposed analytical tasks which are out of proportion to anticipated benefits. The situation in the radiochemical measurement field resembles that described succinctly by Hirschfeld for analytical developments in general:

"The Delaney amendment and other masterpieces of legal and bureaucratic regulation formally embody the principle of today's maximum analytical capability being tomorrow's minimal legal requirement. This automatic no-win situation for the developers of analytical techniques, besides its eventual effects on the publication of analytical research, will keep a substantial fraction of the analytical workload in perpetual proximity to the technological and eventually fundamental limits."

New radioanalytical methods are being developed to meet the sensitivity and precision requirements of the new regulations. All too often, considerable financial and technical effort is required of the radioanalytical laboratories to incorporate or develop these new techniques. Such methods -- in proximity to technological limits -are difficult to use for routine analysis and provide unreliable results when operated by technicians. Hiring better trained staff to meet the more demanding technical requirements leads to still another cost burden for the laboratory. These additional costs, which are eventually absorbed by the public, are for the most part not readily justified when one realistically considers the beneficial effects on health which are likely to accrue from many of the stringent new regulations.

Requlatory Imposition of Unreliable or Technologically Limited Analytical Tasks

Certain of the radiological environmental regulations have imposed analytical tasks which approach the technological limits of radioanalytical systems. Particularly important among these are NRC's Regulatory Guide $4.3^{2}$ (in association with requirements of 10CFR50, Appendix I, ${ }^{3}$ and of Regulatory Guide 1.424) and EPA's Interim Primary Drinking Water Regulations 5 (IPDWR). The IPDWR also prove difficult to justify with regard to health benefits which may be derived, but they impact the measurement laboratory most heavily through the regulated imposition of unreliable analytical tasks.

Appendix I and Regulatory Guide 4.3. At the beginning of this decade the AEC was increasingly pressured by various environmental groups to provide evidence of safe operation of nuclear power reactors. One result of this pressure was the development of 10CFR50, Appendix I. The requirements expressed in Appendix I have never achieved formal legal status but are being imposed on operators of new nuclear power reactors through the license approval process.

Based on dose levels and concentrations established in Appendix I, the AEC required laboratories (in Regulatory Guide 1.42) to obtain analytical precision of $\pm 25 \%$ at an unspecified confidence level for 131 I levels of $0.5 \mathrm{pCi} /$ liter at time of sample collection, or approximately $0.2 \mathrm{pCi} / \mathrm{liter}$ at time of counting. The reactor operators complained to the AEC that the analytical requirements were beyond the scope of existing measurement capabilities. A radiochemist on the Directorate staff was assigned the task of quickly "developing" a satisfactory method. 

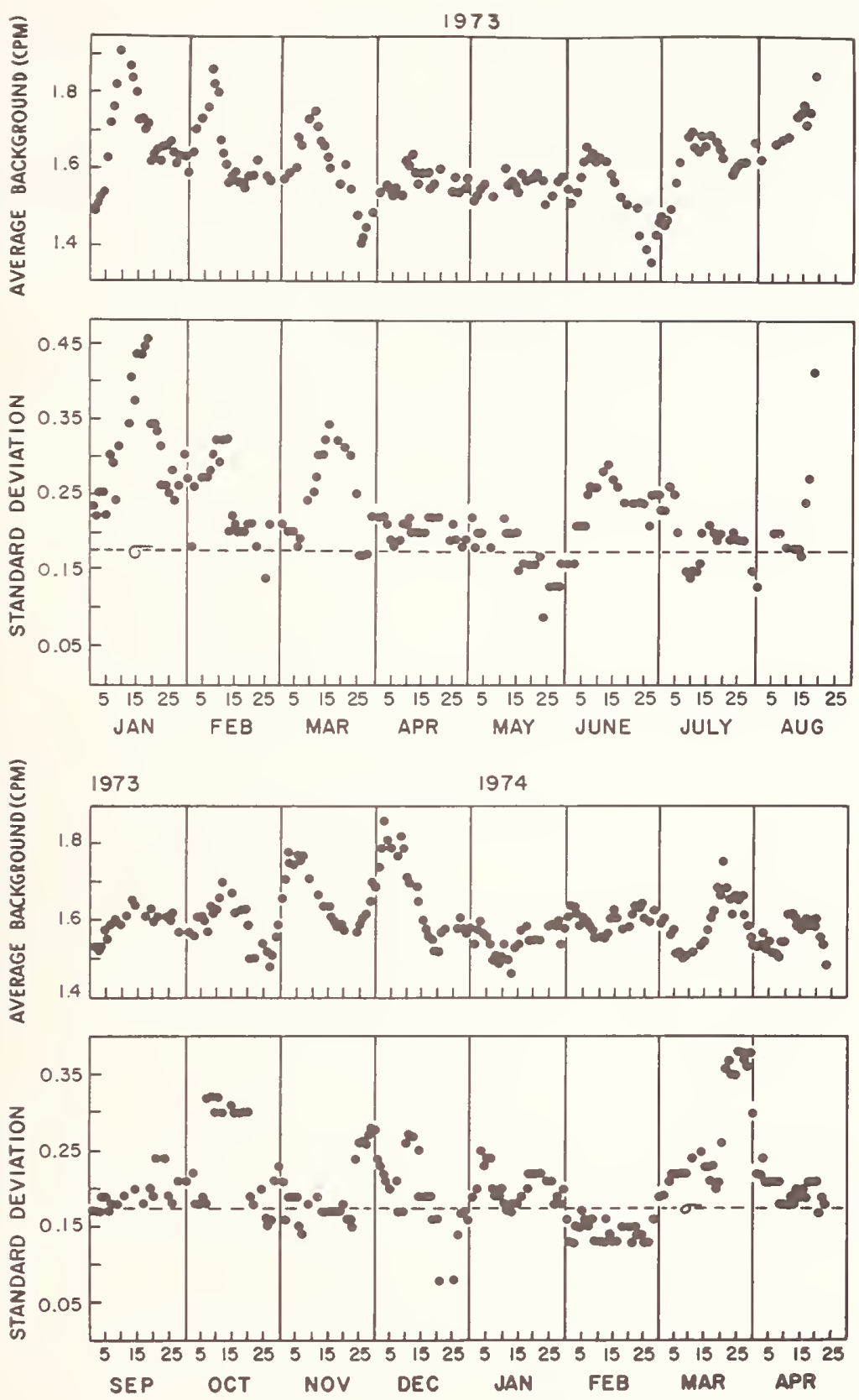

Fig. 1. Running 10-point average background and standard deviation values for a lowbackground beta-particle-counting system (from Ref. 8).

Combining analytical methods from several laboratories, including those of our Radiological Sciences Laboratory (RSL), 6 he derived at an untested analytical method which would presumably suffice. When preliminary tests of the method were conducted at an AEC contractor laboratory, that laboratory apparently ignored several important statistical parameters which would have precluded use of this method for the purpose intended. Despite its technical weaknesses, the method was promulgated 7 as Regulatory Guide 4.3 . 


$$
\begin{aligned}
N & =\text { Net } \mathrm{pCi} / \text { liter } \\
& =\frac{(\text { net counts) (factor for protein-bound fraction) }}{(\text { count time)(chem yield)(counter eff) (sample vol) (decay) (dpm/pCi) }} \\
& =\frac{(360 \pm 75)(1.05 \pm 0.05)}{(1000 \pm 0.1)(0.6 \pm 0.1)(0.28 \pm 0.02)(4 \pm 0.01)(0.5 \pm 0.005)(2.22)} \\
& =0.5 \\
\frac{\mathrm{SD}}{\mathrm{N}} & =\left[\left(\frac{75}{360}\right)^{2}+\left(\frac{0.05}{1.05}\right)^{2}+\left(\frac{0.1}{1000}\right)^{2}+\left(\frac{0.1}{0.6}\right)^{2}+\left(\frac{0.02}{0.28}\right)^{2}+\left(\frac{0.01}{4}\right)^{2}+\left(\frac{0.005}{0.5}\right)^{2}\right]^{\frac{1}{2}} \\
& =10^{-1}(4.34+0.23+0.00+2.04+0.51+0.00+0.01)^{\frac{1}{2}} \\
& =10^{-1}(7.13)^{\frac{1}{2}} \\
& =0.27
\end{aligned}
$$

We have shown in a previous publication 8 that the analytical method published in Regulatory Guide 4.3 cannot achieve the sensitivity and precision required by the NRC. First of all, the background counting rate of commercial $\beta$-counting systems can differ significantly from predicted statistical behavior (Fig. 1). The fluctuations in background rate occur even though the counting systems are powered through voltage stabilizers and line filters and even though the humidity and temperature of the countingroom air are closely controlled. The reason for periodic behavior of the background fluctuations is not clear. NaI(TI) systems used for $\gamma$-spectrometric analysis of $131 \mathrm{I}$ exhibited similar nonstatistical background fluctuations. Regardless of the source of background fluctuations, this nonstatistical behavior of the counters can result in falsepositive 131 I values.

The RSL also found large quantities of stable iodine in milk from dairy farms in New York State. Investigation by the New York State Department of Environmental Conservation (NYSDEC) attributed the high levels of stable iodide to a fungicide fed to barned cattle in wet, cold climates.9 Apparent chemical recovery factors could be quadrupled by the stable iodide in the milk, producing a fourfold underestimate of 131 I levels. Addition of carrier sufficient to overcome the natural iodide content would reduce the $\beta$ counting efficiency to unacceptably low values. Uneven distribution of carrier on the sample mount can also cause large counting errors.

A mathematical analysis of the several accountable sources of error (Table I) indicates that background contributions to the counting error and chemical recovery errors would be the predominant terms setting the analytical precision for 131 I in milk. The chemical recovery error could be reduced by measuring stable iodide in each milk sample -- at the cost of doubling the number of analyses.

Periodic nuclear weapons tests were found to increase ambient 131 I levels to three times the specified level of $0.5 \mathrm{pCi} /$ liter (Fig. 2). Incorrect attribution of elevated 13lI levels to local nuclear power reactors can be avoided only by analysis of several control samples collected remote from each reactor.

An interlaboratory comparison study 10 conducted by the AEC substantiated the inability of some 15 laboratories to achieve the precision and accuracy required now by the NRC.

Regardless of all these problems, reactor operators are still required by NRC regional inspectorsll to obtain 131 I data at the sensitivity and precision specified by Regulatory Guide 4.3.

Interim Primary Drinking Water Requlations. A heavier cost burden to most states in the way of increased analytical workload 
will be created by the IPDWR. In 1971 the EPA Office of Radiation Programs began developing standards for radioactivity in drinking water. The regulations were finally published in 1975.5 During that period the draft IPDWR were revised time and again as serious technical errors were discovered, yet the various versions showed little cognizance of many practical aspects of performing environmental measurements.

Many of the analytical methods, sensitivity requirements for several radionuclides, and cost estimates for certain analyses recommended in the IPDWR are incompat- ible with normal laboratory practices. For example, the sensitivity requirement for gross-alpha measurement was at one time less than the fundamental limit by a factor of 4 . In this case, recommendations from several laboratories did lead to correction of the technical impossibility being imposed.

Originally, specific $226 \mathrm{Ra}$ limits in IPDWR were set on the basis of population served. Such a requirement would have caused difficult enforcement problems for state water-supply agencies. That requirement was changed only after a concerted effort was organized by several states and

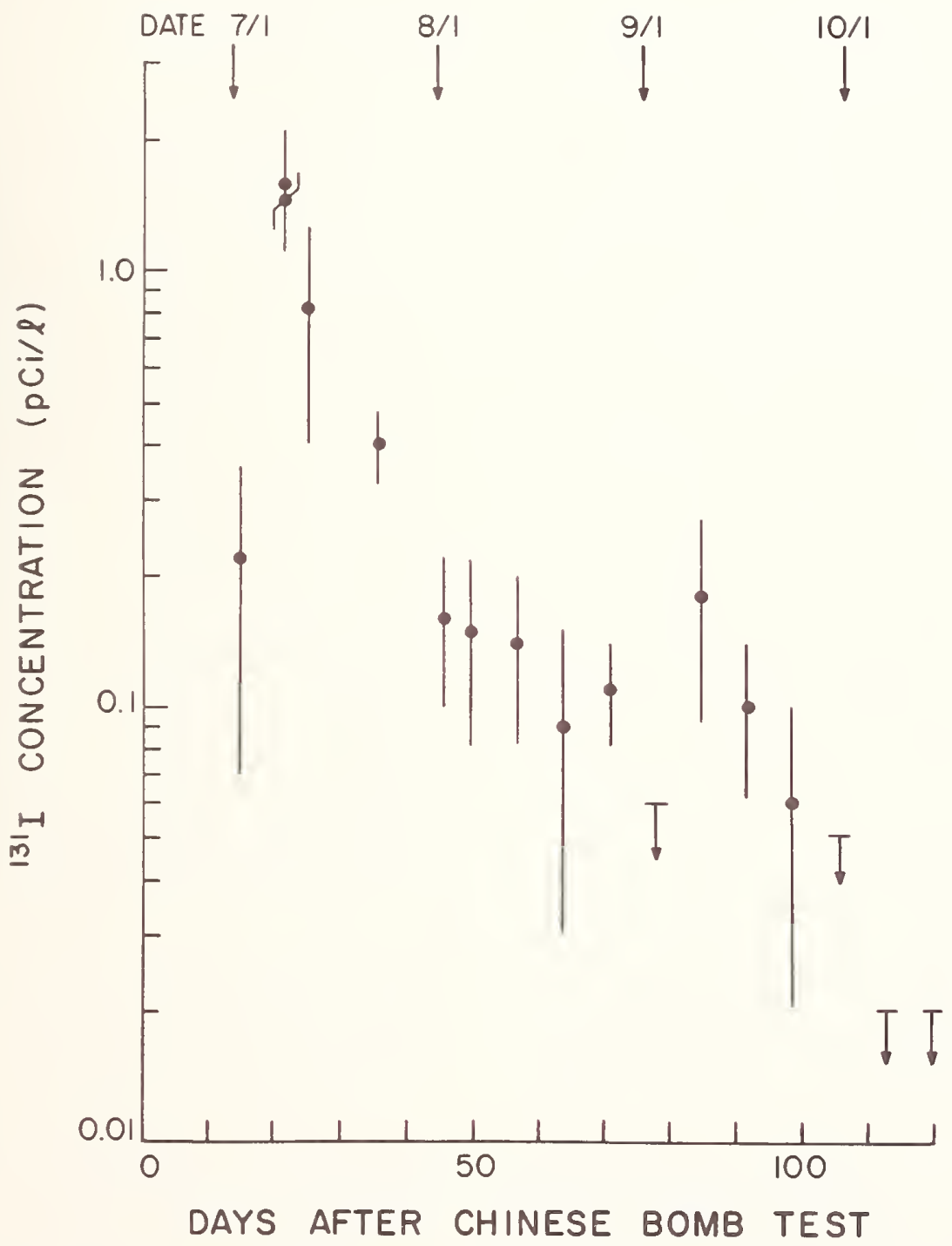

Fig. 2. Iodine-131 concentration in milk collected during 1974 in areas of New York State remote from operating nuclear facilities (from Ref. 8). 
the problem brought to the attention of EPA's Office of Water Supply.

A new requirement calling for measurement of both 226Ra and 228Ra found its way into the final version of the interim regulations. The EPA cost estimate for measuring ${ }^{226} \mathrm{Ra}$ and $228 \mathrm{Ra}$ is based on a single separation of $\mathrm{Ba}(\mathrm{Ra}) \mathrm{SO}_{4}$, though counting of $\mathrm{Ba}(\mathrm{Ra}) \mathrm{SO}_{4}$ for ${ }^{226} \mathrm{Ra}$ is technically inferior to the emanation method of analysis. Furthermore, the recommended separation procedure has been found unreliable as an analytical method for 228Ra analysis.

An important incongruity in the IPDWR permits concentrations of natural radioactivity which will result in dose levels of 60 mrem per year per individual, while only 4 mrem per year per individual is allowed from man-made radioactivity. Measurement requirements for man-made radioactivity are at or below the current technological limit for $13 \mathrm{I}$ and $90 \mathrm{Sr}$. Measurement of $13 \mathrm{II}$ at 1 $\mathrm{pCi} / 1$ iter will require $\beta / \gamma$-coincidence counting or excessively long counting periods on conventional $\beta$-counting systems unless special arrangements are made for rapid transport of the samples to the laboratory, where they must be analyzed immediately on receipt. A slower-paced analytical effort would require shipment of large sample volumes (up to 4 liters of water). $90 \mathrm{Sr}$ measurements at $0.5 \mathrm{pCi} /$ iter will also require large sample volumes, as will the ${ }^{134} \mathrm{Cs}$ and $137 \mathrm{Cs}$ measurements. Specially developed counting systems, field concentration of certain radionuclides, or transportation of up to 10 or more liters of water may be necessary to meet all of the IPDWR requirements. Such stringent sensitivity requirements for man-made radioactivity are incompatible with the 15-fold greater levels permitted for natural radioactivity.

The allowances in IPDWR for compositing samples prior to analysis of ratural radioactivity must be carefully examined by state laboratories and regulatory agencies. Precipitation and adherence of many radionuclides to sample containers may prove a significant problem if samples are stored over an extended period. Measurement laboratories may be required to develop new techniques to assure representative sample transfer when samples are composited.

The stringent sensitivity requirements for man-made radioactivity are also incompatible with the monitoring frequency allowed in the IPDWR. Measurements for environmental 131 I cannot be considered meaningful if obtained on the basis of a single quarterly grab sample. The IPDWR statement that if maximum contaminant levels are found, "monitoring shall be continued with monthly samples or the analyses of quarterly composites of three monthly samples," is inconsistent with the physical decay processes involved. Because of its 8-day half-life, the decay of 131 I from the time of collection to the time of measurement will be incalculable for samples composited over a period of three months. Even ${ }^{89} \mathrm{Sr}$ decay will not be readily calculable for composited samples.

Additional questions concerning the viability IPDWR may also be raised. For example:

- Calculation of health risks should not be based on individual consumption of 2 liters of drinking water, but rather about half that amount.

- The recommendation that laboratories use outdated radioanalytical methods, such as those of the American Public Health Association, will cause added cost burdens to the measurement laboratories while providing inferior results. Some of the radioanalytical methods recommended, including those of the American Society for Testing and Materials, cannot meet the sensitivity requirements of the IPDWR. Laboratories will find it necessary to develop or adapt methods at the limits of current technology for some radionuclides.

- Monitoring of "any community water system contaminated by effluents from nuclear facilities" presents an interpretive dilemma. Contamination by $129 \mathrm{I}$ and HTO in gaseous effluents from fuel-reprocessing facilities or ${ }^{131}$ I and other radionuclides in liquid effluents from hospitals may also require consideration.

- The permitted use of utility discharge data made concentration estimates at the water intake dependent on water dispersion models, which may result in serious overor underestimates of actual concentrations.

- Failure to require laboratories to exhibit proficiency through participation in a nationwide quality assurance program may jeopardize the reliability of results.

To summarize, the specific analytical requirements now imposed on the measurement laboratories by the IPDWR are out of line with the dose justification, the sampling and compositing allowances, the costs allowed for analyses, and the quality assurance requirements. 
Reculatorv Proarams Recuiring Eurther Iechnical Develooment

Certain regulatory programs, including some of long standing, require further technical development. Though Emergency Response Planning has only recently achieved notoriety, state representatives to the Confererce of Radiation Control Program Directors have attempted for the past few years to draw attention of responsible federa- agencies to deficiencies in the program. Iechnical problems have also developed in iRd's Independent Measurements Program.

Emeroency Resoonse Planning. As a condition for a license to operate a nuclear power reactor, the facility operator must develop with the State an emergency response plan, under the guidance of the NRC and EPA.

The IIRC, as lead agency for emergency planning, recently has come under considerable pressure from various groups concerned about technical and financial inadequacies in emergency planning for accidents at nuclear facilities. This problem was highlighted in February 1976 by extensive media coverage f alleged deficiencies at several reactor sites. EPA, the U.S. Energy Research and Development Administration (ERDA), the U.S. Defense Civil Preparedness Agency (DCPA), the Federal Disaster Assistance Administration (FDAA), state agencies, and facility operators also have extensive responsibilities in the emergency response planning program.

Current problems with environmental measurements for emergency planning involve plume transport and protective action guides. In-depth studies of plume transport are only now being started. To the credit of the NRC, basic research programs are being developed to icentify gaseous and particulate species which may be released in a reactor accident and to resolve the chemical behavior of these species as a plume moves.

On the other hand, insufficient technical and financial assistance has been provided to state and local agencies which must eventually administer any emergency-response action. WASH-1293 provides a checklist for cadre operations 12 which the states are expected to use as the basis for their own plans. However, there are important deficiencies in the WASH-1293 dose estimates and in EPA's protective action guides.13

Very little is known concerning radioiodine species which may be released in a reactor emergency, though 131 I dose to thyroid is postulated to be the predominant factor determining the need for evacuation.13 Reliable methods for sampling various chemical species of radioiodine must be developed. Laboratory measurements for the several iodine radionuclides released may prove too complex for $\mathrm{NaI}(\mathrm{Il}) \mathrm{r}$-spectrometry, but few enviromental radiochemical laboratories have even one Ge(Ii) system for high-resolution r-spectrometry. Prediction of radioiodine dose is further complicated by inadequate plume transport models for the terrain surrounding most reactor sites in the eastern U.S., particularly at distances beyond 5 or $6 \mathrm{~km}$.

Present evacuation plans are therefore scientifically deficient. Such plans for densely populated areas are dependent on predictive transport models, but the sourceterm radioiodine species are not yet adequately identified, and the transport mechanisms are still poorly defined. State and local authorities may be forced to base evacuation plans in part on the emergency response capability of environmental radiochemical laboratories. Few state laboratories, however, are prepared to produce definitive results within an hour or two following an accident. Even should the laboratories be prepared, the procedures for collection, shipping, and handling of emergency environmental samples differ markedly from those used for routine surveillance programs.

Research requirements for emergency planning include identification of radionuclide species which may be released in an accident at each nuclear facility and development of site-specific mathematical models for evacuation planning. Site-specific data can be obtained only by appropriate laboratory and field studies at each nuclear facility.

\section{Independent Measurements Program. The} Independent Measurements Program evolved approximately five years ago, when the AEC began considering environmental data as well as discharge data for regulatory purposes. This program appears to have been a response to criticism from environmentalists and other antinuclear groups, who charged that a conflict of interest existed in the AEC as it was then structured. The states were to be introduced into part of the monitoring sequence, which otherwise was performed by the individual operator or contractor laboratory. 
Problems immediately surfaced. Firstly, the individual contracts with the states were severely underfunded by the AEC. One goal of the AEC funding may have been to induce the states to provide supplemental funds, but this is always a difficult proposition. Secondly, most states did not have the technical or administrative resources to implement such a program. Thirdly, technical support by the AEC developed late in the program, and even that support was less than adequate.

The obstacles to technical and administrative support on the state level are not always realistically appreciated by federal agencies. State bureaucracies are often slow to move, whether the need is for added funds or for chrnges in job titles to attract trained personnel. Several states did obtain supplemental funds and did begin work. However, technical capabilities at many of the state laboratories were impaired because properly trained nuclear chemists and physicits could not be hired in existing job titles or at existing salary levels. Even the reactor operators experienced problems in obtaining trained laboratory staff because of the limited number of experienced environmental radiochemists immediately available.

Laboratory facilities in several states were not adequate to cope with the variety of samples collected. These limited facilities, not only in many state laboratories but also at the reactor sites, made difficult the processing of high-activity inplant samples in the same laboratory with low-activity environmental samples. In some instances counting equipment was set up in the same room or in close proximity to where chemical spearations were being performed.

As for technical support, the samples provided to the laboratories were often nonrepresentative. Many corrosion-product radionuclides were found to be fixed permanently on container walls when in-plant samples were shipped or stored for more than a few days. This problem was only discovered 2 or 3 years after the program was initiated. The shipping problem was solved by adding paper pulp to the large-volume samples, but difficulties in aliquoting radioactive material adsorbed to the pulp increased problems of sample cross-contamination.

Technical support for quality assurance has also been less than adequate. Results for intercomparison samples run at state, reactor, and AEC (now ERDA) laboratories are made available to the laboratories only after a year or more, so that recognition of any performance inadequacies is delayed.

Requlations Imposing Impractical Analytical Tasks

As in the case of the IPDWR, regulations or regulatory decisions have been issued by various agencies without full consideration of health benefits to be obtained. Analytical tasks in these cases do not necessarily approach or exceed technological limits, but the tasks are likely to set excessive workloads which cannot be readily justified by cost/benefit considerations.

Control of $85 \mathrm{Kr}$ Effluents in the Nuclear Power Cycle. EPA regulations 14 for control of $85 \mathrm{Kr}$ effluents appear to refiect the agency's philosophical approach to regulatory responsiblities. As a matter of polciy, 15 EPA's Office of Radiation Programs develops all regulations on the basis of a very restrictive interpretation of the BEIR Committee recommendations.16

At the 1973 Noble Gases Symposium, 17 the EPA made a well-orchestrated presentation designed to convince the technical community that $85 \mathrm{Kr}$ releases from the nuclear fuel cycle must soon be brought under control. Eight papers were presented extolling the need for separating $85 \mathrm{Kr}$ from gas effluents generated in the nuclear fuel cycle.

Recommendations for $85 \mathrm{Kr}$ control have appeared again in EPA's most recent proposed rulemaking action.14 Health effects savings realized by reducing environmental $85 \mathrm{Kr}$ effluents are partially offset by increased occupational health effects from storage of ${ }^{85} \mathrm{Kr}$. Yet it appears that radiochemical laboratories may soon be required to conduct routine measurements of environmental $85 \mathrm{Kr}$ levels.

\section{Control of $14 \mathrm{C}$ in the Nuclear Power} Cycle. EPA's most recent proposals for rulemaking action 14 also suggest that $14 \mathrm{C}$ is the single most important radionuclide in the nuclear fuel cycle in terms of "possible health effects." Little substantive evidence is provided in support of this contention. Calculated health effects 18 supporting the proposals are identical to those presented by Magno et al.19 at the 13th AEC Air Cleaning Conference. In that study a series of speculative calculations were derived from an undefined model to obtain estimates of health effects. No experimental data were submitted to verify the estimates of $14 \mathrm{C}$ production at nuclear power reactors, nor were any environmental measure- 
ments presented.

The RSI has performed a limited number of measurements 20,21 on in-plant samples. These values indicate that reactor releases O ${ }^{2} \mathrm{C}$ may be within the range estimated by Hagno. However, no data have as yet been obtained at the fuel reprocessing sites, where Magno predicted most $14 \mathrm{C}$ woulo be released to the environment. Lacking a specific model description of exchange processes anticioated for various chemical species of ${ }^{4} \mathrm{C}$ which may be released, it is difficult to accept the startlingly high estimates of health effects obtained by EPA.

Again, radiochemical laboratories may be reouired to develop methods for routine analysis of $14 \mathrm{C}$ released to the environment. Reactor laboratories may also be required to develop analytical methods for regulatory control.

Burial Site Effluents. State regulatory agencies may on occasion yield to intense public pressure by arbitrarily modifying environmental standards.

Rainwater infiltrating trenches at the low-level radioactive waste burial site in West Valley, N.Y. had raised the water level in some of the trenches to the point of overflow. Prior to overflow, the site operator had requested a permit to pump the water from the three affected trenches and release the water at a controlled rate to local streams. Concentrations of radionuclides in such a discharge would have been several orders of magnitude below corresponding concentrations in releases that had previously been permitted from the fuel reprocessing plant on the same site. After two years of attempting to arrive at a decision acceptable to all state agencies involved, overflow eventually forced the state agencies to permit pumping and discharge.

The NYSDEC environmental radiation code 22 includes discharge limits identical to those in other state codes and in federal code 1OCFR20.23 However, state and local environmental groups became actively involved in the situation, and some of the news releases were irresponsible. One Buffalo television station issued a news bulletin that State Police would be aligned along the entire 30mile course of Cattaraugus Creek to prevent access by the public during the period of discharge.

Finally, NYSDEC set the discharge limits at the levels in the State Health Department's drinking water code, even though the nearest public water supply intake is on Lake Erie, 35 miles and a 500-fold dilution downstream. Since levels permitted by the drinking water standards are approximately one-thirtieth of those for discharge, the RSL was required to perform more sensitive analyses than would otherwise be necessary. We were also required to analyze more samples than are normally necessary for control purposes in order to maintain an extensive public record. In addition, the site operator was required to perform a large number of analyses to maintain careful control of discharge rates.

The site operator expended approximately $\$ 100,000$ in waste treatment and discharge control. The analytical costs to the state exceeded $\$ 50,000$. Integral dose savings were extremely small, a potential saving of at most one death every $10^{5}$ years in a population of $2 \times 106$.

\section{Environmental Data and Public Relations}

Environmental measurement laboratories dealing directly with state or local regulatory agencies are likely to find stringent ouality assurance a paramount consideration, even for single samples. This is a consequence of the closer public review of data reported by the state or local agencies.

Reporting Format. The laboratory staffs normally report all results, including statistical errors. Actual detection limits for each sample are reported for radioactivity levels below the limit of detection. On the other hand, the regulatory agency usually.reports the environmental data received from the laboratory in the simplest format possible. Levels below the limit of detection are often reported as "nondetectable" (ND), a term the public tends to view as "zero."

Another common problem area develops when the regulatory agency attempts to make specific environmental evaluations from data for a limited number of samples or from results with inadequate controls. Generalizations which seem innocuous to the reporting agency may incite strong public reaction due to the public's sensitivity to radiological safety. This in turn may create difficult technical decisions and interpretations for the regulatory agency and more work for the radioanalytical laboratory.

The following examples illustrate the care necessary to ensure data reports which do not induce needless public alarm.

"Hot" Milk. Measurements of HTO concentration in milk are performed routinely by the 
RSL as part of the NYSDEC's statewide surveillance program. A problem developed in 1973 when a single sample was reported 24 to have a positive level of HTO, while all previous values for that site had been reported to the public as "ND," indicating results below the sensitivity of the analytical method.

For years $90^{\mathrm{Sr}}$ levels in milk from this dairy farm on Long Island had been among the highest measured in the state. Coincidentally, this farm was close to Brookhaven National Laboratory, which has long been a thorn to downstate environmental groups. In 1973 the annual average levels 25 of $90 \mathrm{Sr}$ in milk from this farm were the highest in the state, about double the statewide average. An NYSDEC review of farming practices indicated this farmer fed his cattle scrap potatoes from nearby farms and did not fertilize his fields as heavily as do other dairy farmers on Long Island. This contention was supported by the low 90Sr levels in milk from another nearby farm where different feeding and fertilizing methods were used. Also, analyses of whole and peeled potatoes (peels and meat analyzed separately) showed that $90 \mathrm{Sr}$ levels in the feed potatoes were similar to levels from other locations in the state.

Unfortunately, just at the time state and local officials were attempting to resolve the $90 \mathrm{Sr}$ dilemma, a group of milk samples were analyzed for HTO content on a liquid scintillation counter with poor sensitivity. (The counter normally used was inoperative.) One positive result was obtained, a value of $1,500 \pm 1,200 \mathrm{pCi} / 1$, and unluckily that value was for a milk sample from the farm with elevated 90Sr levels. All previous HTO results for milk from that farm had been publicly reported by NYSDEC as "ND," though the analytical results reported by the RSL. were "<500 pCi/1." Local officials had interpreted the "ND" values to mean "zero," so when the $1,500 \mathrm{pCi} / 1$ value was reported by NYSDEC without an associated analytical error, it appeard to show a huge increase in HTO activity. A local press release alleged that releases from Brookhaven had caused the increased activity.

The RSL and NYSDEC staffs spent much time and effort explaining the statistical behavior of radiochemical data to the press, local officials, the local citizenry, the operator of the milk cooperative receiving that milk, and the farmer. A lawsuit was threatened by the farmer until he started selling his milk again. The issue finally lost its high public profile, but the farmer removed his farm from the state surveillance network. This experience illustrates how important it is for the laboratory to maintain a consistent measurement capability. Equally important for the regulatory agency is the inclusion of analytical errors with reported data.

"Hot" Fish. In its 1973 Annual Report, 25 NYSDEC reported that two fish samples from the Peconic River collected below the discharge from Brookhaven had $90 \mathrm{Sr}$ and $137 \mathrm{Cs}$ levels higher than those from elsewhere in the state. NYSDEC attributed the elevated radioactivity levels to effluents from Brookhaven. Shortly after the report was issued, a fish kill occurred in the Peconic River. The RSL received a large number of rotten fish collected along the stream bank for assay because the local environmental groups had attributed the fish kill to radioactivity discharged by Brookhaven. This proved not to be true. Radioactivity levels in the dead fish were similar to those obtained statewide.

"Hot" Kelp and Mussels. A delayed reaction occurred following the report25 of elevated levels of $131 \mathrm{I}$ in kelp and ${ }^{134} \mathrm{Cs}$ in mussels of $F$ ishers Island, which lies in the mouth of the Connecticut River near the Connecticut coast. These elevated levels were attributed by NYSDEC to the "nuclear power plants that eventually discharged into Long Island Sound." Several months later this statement was relayed by a county health official to a Connecticut newspaper editor, who credited the elevated levels to discharges from the Millstone Point reactor in Connecticut. The press coverage drew an angry response by environmentalists on Long Island, calling for the congressman representing that district to investigate the issue. As a result, the RSL, the Connecticut Health Department Laboratory, and Millstone's contractor laboratory became involved in an analytical round robin, analyzing fish, mussels, lobsters, and kelp for various radionuclides to find the origin of the elevated radioactivity levels in specimens from Long Island Sound.

The Rochester Committee Samples. In 1968 members of the Rochester Committee for Scientific Information surreptitiously collected water samples within the exclusion area at the nuclear fuel reprocessing plant in New York. The samples were sent to an AEC laboratory for analysis with no indication as to the sampling location. The $90 \mathrm{Sr}$ levels in these samples were incorrectly overestimated because a technician inadvertently applied an aliquot factor twice, 
causing a 1,000-fold error in the reported result. The Rochester Committee seized on the high values to issue a "report" to the press alleging inordinately high releases by the reprocessing plant to offsite surface waters.26 The AEC laboratory later issued correct analytical values, but, the Rochester Committee did not issue a corresponding correction of its hazard estimates.

The Shippinqport Incident. In 1973 Dr. Ernest Sternglass claimed that the Shippingport reactor had seriously endangered the population in Aliquippa, $\mathrm{Pa}$. As part of his evidence he asserted that gross-beta values in stream samples downstream from the plant were significantly higher than those for upstream samples. His position was apparently substantiated when the Pennsylvania Radiological Laboratory reported high $90 \mathrm{Sr}$ levels in samples from the Shippingport environs. However, a subsequent intercalibration study between the state laboratory, an AEC laboratory, and the reactor operator proved that the state laboratory had incorrectly calibrated the counting system used for measurement of $90 \mathrm{Y}$, the $90 \mathrm{Sr}$ daughter. Where most laboratories obtain a counting efficiency of approximately $50 \%$ for $90 y$, the state laboratory had for some time been using a counting efficiency value of only $15 \%$.

Sternglass's claim of excess deaths due to the Shippingport reactor received extensive press coverage, including a series of special state hearings on nuclear reactor safety. Subsequent corrections by the AEC and the state laboratory, showing that downstream levels of $90 \mathrm{Sr}$ were indistinguishable from upstream levels, received little public attention. The absence of specific reactorrelated radionuclides indicated that the elevated gross-beta activity was probably due to natural radioactivity ( $40 \mathrm{~K}$ ) introduced into the river by sewage from the city of Pittsburgh, another fact which failed to receive follow-up coverage by the press.

Realistic Measurement/Requlation Relationship

Laboratory/regulator relationships are not always negative, as this survey of problem areas might suggest. The bulk of environmental data are reported with no adverse public reaction. Environmental results have been used to formulate regulations, though not as often as would seem desirable.

Control of Effluents from a Nuclear Fuel Reprocessing Plant. Responsibility for performing environmental research is a difficult issue to resolve, though independent state programs can be useful for the regula- tory process. Because of work performed by the State of New York, controls on nuclear fuel reprocessing effluents were imposed by the AEC which now appear realistic and adequately supported by environmental data.

Prior to 1971 radioactivity levels in stream samples containing liquid effluents from the fuel reprocessing plant in New York often were at the limits of 10CFR20.23 The state's surveillance program provided the AEC with data concerning concentrations of $90 \mathrm{Sr}, 137 \mathrm{Cs}$, and other fission products in offsite surface waters. On the basis of the data provided, the operator of the reprocessing plant was required by the AEC to install a liquid waste treatment facility which resulted in a 100- to 1,000-fold reduction of liquid effluent radioactivity levels.

\section{A similar situation arose with ${ }^{129} \mathrm{I}$ in} gaseous effluents from the same plant. Measurements by the RSL and the EPA produced evidence 9 that 129 I releases were greater than plant discharge records would indicate. Levels of 129 I in animal thyroids and milk samples collected offsite indicated that thyroid doses to individuals could approach unacceptable levels. Again, presentation of the evidence to the $A E C$ led to the imposition of new technical specifications for 129 I at this and other commercial fuelreprocessing plants under construction in the United States.

\section{New Environmental Research Programs.} The NRC has apparently increased its efforts to gather environmental data before issuing regulatory decisions related to environmental issues. The agency has conducted studies to identify the chemical species of radioiodines released from nuclear power reac$\operatorname{tors}^{27}$ and is cooperating with ERDA to develop new plume-transport models. Translation of the ideas into funded programs has been slow, but the delays are apparently those to be expected as a new agency begins implementing its research programs.

The EPA has funded a study to measure hospital-related radionuclide levels in sewage treatment plant effluents. With the U.S. Geological Survey, it is conducting extensive studies to develop standards for low-level waste-burial sites. Toward its responsibilities for regulating nonionizing radiation, EPA has also performed a number of field evaluations of environmental levels of microwave radiation and is preparing to evaluate radiation fields generated by ultra-high voltage transmission lines. 
Pressures from various sources often make it difficult to take sufficient time to develop substantive environmental data before preparing regulations. However, such research is an inescapable responsibility of the regulatory agencies and the measurement laboratories.

\section{Acknowledgement}

The suggestions offered by Dr. Alan Levensohn are very much appreciated.

\section{$\underline{\text { References }}$}

1. T. HIRSCHFELD, Analytical Ghemistry 48 , 16A-31 A (January 1976).

2. "Measurements of Radionuclides in the Environment: Analysis of I-13l in Milk," Regulatory Guide 4.3, U.S. Atomic Energy Commission, Directorate of Regulatory Standards (Sep. 1974).

3. "Numerical Guides for Design Objectives and Limiting Conditions for Operation to Meet the Criterion 'As Low As Practicable' for Radioactive Material in Light-Water-Cooled Nuclear Reactor Effluents," 10CFR50, Appendix I, Federal Register 36 FR 11113 (June 1971).

4. "Interim Licensing Policy on As Low As Practicable for Gaseous Radioiodine Releases from Light-Water-Cooled Nuclear Power Reactors," Regulatory Guide 1.42, U.S. Atomic Energy Commission, Directorate of Regulatory Standards (June 1973)

5. "Interim Primary Drinking Water Regulations - Proposed Minimum Containment Levels for Radioactivity," Federal Register 40 FR 34324 (August 14, 1975).

6. J.J. GABAY, C.J. PAPERIELLO, S. GOODYEAR, J.C. DALY, and J.M. MATUSZEK, "A Method for Determining Iodine-129 in Milk and Water," Health Phys., 26, 89 (1974).

7. B.E. VAUGHN, Pacific Northwest Laboratory Annual Report for 1973, Part 2, Ecological Sciences, BNWL-1850 PT 2, p. 126 (1974).

8. J.M. MATUSZEK and C.J. PAPERIELLO, Nuclear Technology 27, 514-522 (1975).

9. J.M. MATUSZEK, J.C. DALY, S. GOODYEAR, C.J. PAPERIELLO and J.J. GABAY, "Environmental Levels of 129I," Proceedings of the Symposium on Environmental Surveillance Around Nuclear Installations, Warsaw, Poland, Nov. 5-9, 1973. International Atomic Energy Agency, Vienna, 1974, Vol. II, pp. 3-20.

10. G. HAMADA and B. WEISS, "An Intercomparison of Various Analytical Laboratories in Determining Low Concentrations of Iodine-131 in Milk Samples," Conf. Bioassay, Environmental and Analytical Chemistry, October 1974, Cincinatti, Ohio, unpublished. Copies available from Nuclear Regulatory Commission.

11. U.S. Nuclear Regulatory Commission, Docket No. 50-220, Inspection No. 75-13, License No. DRR-63, (September 16, 1975).

12. "Guide and Checklist for the Development and Evaluation of State and Local Government Radiological Emergency Response Plans in Support of Fixed Nuclear Facilities," WASH-1293, U.S. Nuclear Regulatory Commission, December 1974.

13. "Manual of Protective Action Guides and Protective Actions for Nuclear Incidents," EPA-520/1-75-001, U.S. Environmental Protection Agency, September 1975.

14. "Environmental Radiation Protection for Nuclear Power Operators - Proposed Standards," Federal Register 40 FR 23420 (May 29, 1975).

15. "POLICY STATEMENT-Relationship Between Radiation Dose and Effect," Office of Radiation Programs, Office of Air and Waste Management, U.S. Environmental Protection Agency, Washington, D.C. (March 3, 1975).

16. "The Effects on Populations of Exposure to Low Levels of Ionizing Radiation," Report of the Advisory Committee on the Biological Effects of Ionizing Radiations, National Academy of Sciences/ National Research Council (November 1972).

17. R.E. STANLEY and A.A. MOGHISSI (editors), "Noble Gases," CONF-730915 (1975).

18. "Environmental Radiation Protection Requirements for Normal Operations of Activities in the Uranium Fuel Cycle," (Draft Environmental Statement), U.S. Environmental Protection Agency (May 1975).

19. P.J. MAGNO, C.B. NELSON and W.H. ELLETT, "A Consideration of the Significance of Carbon-14 Discharges from the Nuclear Power Industry," 13th AEC Air Cleaning Conference (San Francisco), U.S. Environmental Protection Agency (1974).

20. C.O. KUNZ, W.E. MAHONEY and T.W. MILLER, "l4C Gaseous Effluent from Pressurized Water Reactors," Proceedings of the Health Physics Society's Eighth Midyear Symposium on Population Exposures, Knoxville, Tenn., Oct. 21-24, 1974.

21. C.O. KUNZ; W.E. MAHONEY and T.W. MILLER, "14C Gaseous Effluents from Boiling Water Reactors," Transactions of the American Nuclear Society, Annual Meeting, New Orleans, Louisiana, June 8-13, 
1975, Irans. ANS, pp. 91-92.

22. New York State Code of

Rules and Regulations 6NYCRR Part 380,

"Rules and Regulations Relating to Prevention and Control of Environmental Pollution by Radioactive Materials," New York State Department of Environmental Conservation, Albany, N.Y. (October 1974).

23. Code of Federal Regulations 10 CFR Part 20, "Standards for Protection Against Radiation," U.S. Nuclear Regulatory Commission, Washington, D.C.

24. "Radioactivity in Air, Milk and Water for July-September, 1973," Environmental Radiation Bulletin Number 3, 1973, N.Y. State Department of Environmental
Conservation, Albany, N.Y. (1974).

25. "Annual Report of Environmental Radiation in New York State - 1973," New

York State Department of Environmental Conservation, Albany, N.Y. (1974).

26. "Strontium-90 in Buttermilk Creek," Industrial Radioactive Waste - Bulletin No. 2, Rochester Committee for Scientific Information (February 24, 1968).

27. B.H. WEISS, P.G. VOILIEQUE, J.H. KEILER, B. KAHN, H.L. KRIEGER, A. MARIIN and C.R. PHILLIPS, "Detailed Measurements of ${ }^{13}$ I in Air, Vegetation and Milk Around Three Operating Reactor Sites," NUREG-75/021, U.S. Nuclear Regulatory Commission (March 1975). 

A. N. Jarvis and D. G. Easterly

U.S. Environmental Protection Agency Las Vegas, Nevada 89114

Policy decisions concerning environmental quality must be based on valid and comparable data. The distribution of radioactive reference samples and conducting interlaboratory studies aids in the critical evaluation of such data and helps assure that measurements are correctly made, accurate, traceable to national radiation standards, and comparable. Standards distribution and intercomparison studies are discussed. Traceability studies between the National Bureau of Standards and the U.S. Environmental Protection Agency are reviewed. Identification and documentation of potential radioactive pollutants that could result from expanded energy programs are also discussed.

(Quality assurance, quality control, radioactivity, calibration, reference methods, intercomparison studies)

Policy decisions concerning environmental quality must be based on valid and comparable sample analysis. Interlaboratory studies and the distribution of radioactive reference samples aid in the critical evaluation of environmental data and help ensure that measurements are correctly made, accurate, and traceable to the national measurements system.

Since its inception in 1970, the U.S. Environmental Protection Agency (EPA) has conducted a radiation quality assurance program designed to encourage the development and implementation of quality control procedures. Various aspects of this program have been described in EPA publications. $1,2,3,4$ some of those in attendance at this symposium may be familiar with, and in some instances may be participants in, the Radiation Quality Assurance Program conducted by the Environmental Monitoring and Support Laboratory-Las Vegas (EMSL-LV). However, for the benefit of those neither involved in, nor familiar with this program, I will give a brief overview of our activities.

The radiation quality assurance program has three major aspects. These are:

- Standards distribution

- Methods evaluation

- Laboratory intercomparison studies

The standards distribution program involves the preparation and distribution of calibrated samples which are used by laboratories for instrument calibration and chemical yield determinations. Currently there are in excess of 30 different radionuclides available on request. About 100 samples are being shipped each month. During the second quarter of FY-76 (October-December 1975), 290 samples, comprising 23 different radionuclides, were distributed to laboratories throughout the country.

Methods evaluation involves the selection and collaborative testing of tentative reference methods. At present, collaborative tests of methods to determine plutonium239 in soil are in progress. In addition, as a result of the EPA's proposed Interim Drinking Water Regulation ${ }^{5}$ the tentative reference method for the measurement of gross alpha and gross beta radioactivities in environmental waters ${ }^{6}$ is being tested. Tentative reference methods for the analysis of tritium, strontium-89 and 90 , and radium in water are in preparation and will be collaboratively tested this year.

The laboratory intercomparison studies program includes the preparation and distribution of samples (milk, water, food, air filters, and gases) containing known amounts of one or more radionuclides. A major objective of this program is to assist laboratories involved in environmental radiation measurements to develop and maintain both intralaboratory and interlaboratory quality controls. Approximately 100 laboratories currently participate in one or more of the cross-check programs.

Periodically the data from the intercomparison studies are summarized and published as EPA reports. These summarizations enable us to get some indication of the quality of environmental radiation measurements. A summary of the milk data was recently published ${ }^{7}$ 
and summaries of the water and diet crosschecks are in preparation.

Having briefly described the EMSL-LV quality assurance program, I will outline current and future needs, and, in some instances, describe what is being done in an effort to meet these needs.

We see an urgent need for the preparation of additional reference materials, standards, and quality assurance guidelines, and for increased efforts in the areas of traceability and training.

It is imperative that we identify, produce, and make available additional reference materials. Both the EPA and the National Bureau of Standards (NBS) have attempted during the past several years to increase the number and kinds of standards and reference materials required for environmental monitoring. Unfortunately the production and testing of reference materials are laborious and time-consuming procedures. As a consequence, availability often lags well behind the need. This lag time has been increased as a result of the rapid expansion of the energy program, the debate on the role nuclear power will play in this expansion, the problems associated with phosphate mining and processing, and the promulgation of the Interim Drinking Water Regulations.

There is further need to identify, quantify, assign priorities to, and then prepare the reference materials required in the monitoring of those pollutants which may arise from the expanded energy program, as well as those required to monitor phosphate production activities and drinking water. The EPA, through its laboratory in Las Vegas, is taking action in these areas of interest and concern. An investigation of the potential pollutants which may arise as a result of the expanded energy program has been initiated and should be completed within a year.

In cooperation with the NBS we have made a mixed gamma standard available to laboratories. A "round-robin" study, involving the measurement of polonium-210 by 30 laboratories, is in progress. Mancos shale, a widespread and well-characterized geological type, is currently being analyzed (primarily for radium) and should soon be available as a reference material. An aqueous plutonium-239 sample should also become available within the year.
As a result of renewed and increasing interest in both the nuclear fuel cycle and naturally - ccurring radionuclides, three types of samples are being prepared which should be of interest to those involved in environmental monitoring activities. The samples include pitchblende, uranium mill tailings (including a 16-mill composite), and thorium ore. It is planned to use these materials both as reference materials and, diluted with soil, in the intercomparison studies program.

Negotiations with NBS for the production of aqueous solutions containing radium228 are currently in progress.

As they have indicated, the NBS cannot maintain direct traceability to every user of radioactive materials. Consequently, they have outlined a plan ${ }^{8}$ wherein the radioactivity laboratories of the EPA, the Energy Research and Development Administration, the National Radiation Council, the Food and Drug Administration, and others would be traceable to the national measurements system. These laboratories would in turn assure traceability to state laboratories, nuclear power facilities, and contract laboratories.

Beginning in 1974, the EMSL-LV instituted continuing intercomparison studies with the NBS--the goal being to establish the traceability of the EMSL-LV measurements to those of the NBS. A summary of the results obtained during 1974 and 1975 has been published. ${ }^{9}$

Another need is for the publication of guidelines describing radioanalytical methodology, calibration techniques, and quality control procedures. I was much encouraged by carl seidel's paper ${ }^{10}$ describing the calibration guidelines being prepared in the American National Standards Institute quality assurance program. Guidelines such as these have long been needed by user laboratories.

Agency priorities, in particular the energy program and the drinking water regulations, have required us to turn our attention to the methods and guidelines needed by laboratories which will be involved in monitoring water supplies and those monitoring in the environs of nuclear facilities. Therefore, as described above, much effort has been devoted to the preparation of reference methods for the analysis of water. However, recognizing the need for other guidelines designed for technicians, we 
began the preparation of guidelines on gamma spectroscopy and liquid scintillation counting which deal with standards preparation, calibration, and quality control procedures.

This overlapping of activities within the radiation measurements community indicates what I believe to be another of the major needs. There is a need for better communication, increased liaison, and more cooperation between Federal, state, and private agencies. Since we are all working on the improvement of our only product, radiation data, it seems only reasonable that we should assist each other as much as possible. I would, therefore, urge that during the remainder of this symposium we give thought to better ways to more quickly exchange information and interact in areas of common interest.

Still another need in the area of environmental radiation measurements- an area which has been too long neglected--is that of sampling and sample-handling procedures. For years many laboratories have been much concerned with, and devoted considerable effort to, decreasing the uncertainty of an analytical measurement by 0.5 percent or 0.25 percent, or even 0.005 percent. But there has been too little interest in and too few attempts to determine (much less report!) the uncertainties associated with the collection and handling of samples, and the chain of custody records essential in legal proceedings.

One of my personal concerns, and one which I consider to be a pressing current problem, is the lack of training received by the analysts and technicians responsible for radiation measurements. We are all aware that the quality of the data is dependent upon the competence and reliability of individual analysts and technicians. As so eloquently stated by L. Cavallo and her colleagues at the NBS, 11 "... the long-term credibility of our curie lies in a piece of apparatus and the skill of its custodian." Unfortunately the training of technicians and analysts has been much neglected. Federal agencies have abolished training programs and almost eliminated training grants. A few institutions and agencies offer short- or long-term training courses. However, high tuition fees and the cost of travel, coupled with travel restrictions, have prevented the attendance of those who are most in need of training. Consequently, I would suggest that the organizations sponsoring this symposium make a concerted effort to initiate the allocation of the resources required to institute training in radioanalytical techniques and quality control procedures. Moreover, I would urge that we all explore ways in which less expensive training can be provided and the means whereby we can assist one another.

\section{References}

1. The U.S. Environmental Protection Agency's Quality Assurance Program, M72-199, December 1972.

2. Radioactivity Standards Distribution Program 1975, EPA-680/4-75-002a, April 1975.

3. Environmental Radioactivity Laboratory Intercomparison Studies Program, EPA680/4-75-002b, May 1975.

4. Measurement Methods Standardization Strategy Document, USEPA, September 1973.

5. Proposed Maximum Contaminant Levels for Radioactivity, Federal Register, Thursday, August 14, 1975, Vol 40, No. 158, pp 34324-34328.

6. Tentative Reference Method for the Measurement of Gross Alpha and Gross Beta Radioactivities in Environmental Waters, EPA-680/4-75-005, June 1975.

7. Preliminary Milk Report, EPA-680/4-75007 , June 1975.

8. W. Mann, "Radionuclide Metrology and Quality Assurance," Activities, and Needs Related to Radioactivity Standards for Environmental Measurements, ed. by J. E. Eldridge and B. Kahn, pp 47-54, EPA-680/4-75-006, June 1975.

9. Radiation Quality Assurance Intercomparison Studies, 1974-1975, EPA-600/475-014, October 1975.

10. C. W. Seidel and J. M. R. Hutchinson, ANSI Quality Assurance in Radioactivity Measurements, CSI-8, Symposium on Measurements for the Safe Use of Radiation, March 1976.

11. L. M. Cavallo, et al., Needs for Radioactivity Standards and Measurements in Different Fields, pp 5-18, Nuclear Instruments and Methods, 112 (1973). 



\author{
PERSONNEL MONITORING MEASUREMENTS* \\ Richard V. Griffith \\ Lawrence Livermore Laboratory \\ Livermore, California 94550
}

A personnel monitoring program must include a well integrated combination of dose determination methods, and should not depend on a single dosimetry system. Many of the necessary techniques have become well developed and dependable, such as the personnel gamma dosimeters in use today. However, other monitoring methods are still not adequate. The two most important personnel monitoring problems remaining are development of personnel neutron dosimeter and in-vivo measurement of plutonium at sublung burden levels. Although there are a few techniques under development to attack these problems, satisfactory long-term solutions will require much more work. As the developments in nuclear power and medicine continue, the need for solutions to these problems will intensify.

(Dosimetry; betas; neutrons; gammas; $x$ rays; calibrations; plutonium)

\section{Introduction}

A successful personnel monitoring program requires a number of well integrated monitoring techniques. Dependence on the dosimeter or other single system for evaluation of personnel radiation exposure will paint an incomplete picture, and eventually will return to haunt those responsible for the radiation protection program. A thorough investigation of the working environment before and during occupation by the worker is essential to prevent surprises. An interview and resurvey following an indicated high exposure are also very important, although the scenario leading to an exposure is often difficult to recreate because of changes in the work area. An example of the problems created by dependence on the dosimeter is the number of high exposures indicated following nonoccupational medical treatment with radioisotopes. This particular problem even reaches those responsible for environmental surveillance in the form of elimination into the facility sewage system.

A more basic need for a total monitoring program is the inherent inadequacy of a single dosimeter to adequately characterize the radiation distribution experienced by a human wearer. The dosimeter tells us something about the intensity of the radiation at the point of measurement. It tells us little about the distribution through the body, and in fact, cannot even be used to determine the radiation direction. It does not know whether the wearer was leaning over a glove box or working outside a reactor shield. Only the individual and those concerned with his safety can tell us that. The dosimeter cannot provide data which can be used to determine individual organ doses, but it can provide information that must be used by a trained professional to do that.

It is also important to note that a complete personnel monitoring program includes the need for internal exposure information as well as that from external fields. Here again, reliance on a single assay method is generally inappropriate. Chemical bioassay, whole-body counts, nose swipes, and airsampling data must be combined to make a complete evaluation. There are certainly times when one technique is more feasible than others, but continuing total dependence on one method is poor practice and unjust to the employee.

One point that is stressed at LLL, incidentally, is that it is the responsibility of the health physicists and other safety professionals to provide a safe working environment, but that the employee is responsible for his own safety - that is, it is his responsibility to use the Laboratory facilities safely. That is as true for radiation safety as for any other aspect of safety. In that sense the individual is part of his own personnel monitoring program.

\section{External Dosimetry}

Having said all this, however, the personnel dosimeter must be considered the

\footnotetext{
"Work performed under the auspices of the U.S. Energy Research \& Development Administration under contract No. W-7405-Eng-48.
} 
single most important element in a personnel monitoring program. Its primary value is that it can be used to identify those individuals that have experienced a radiation environment in excess of the lower limit of detection of the system. The dosimeter's secondary value is that it can be used to provide an estimate of the relative severity of the radiation experience at the location of the dosimeter. The degree of confidence in the accuracy of that estimate is a function of the optimism of the dosimetrist.

The performance of the dosimetry system depends on three factors:

- Inherent characteristics of the detecting element.

- Quality of the calibration.

- Precision of the readout system.

Confining the discussion to the first two of these factors, it is helpful to begin by reviewing the preliminary results of a recent survey on personnel monitoring, including 83 personnel monitoring services in 33 countries. 1 The survey questions deal with a number of aspects of the dosimetry system, but I sha11 only touch on the types of detectors used and their relative merits.

The survey covered monitoring of personnel for $x$ rays and gammas, betas, and neutrons. Of course, the bulk of the users are monitored for $x$ rays and gammas on $1 y$, with only $15 \%$ of al1 people monitored being issued a neutron dosimeter. In the case of gammas, $x$ rays and betas, it is no surprise that TLD and film are the dosimeters of choice, with on $1 y$ $3 \%$ of the services using radiophotoluminescent glass. The move is clearly to TLD. In the U.S., $72 \%$ of the personnel monitored by the services responding to the survey are now or will soon be monitored by TLD exclusively, with LiF used by over $75 \%$ of the services. Internationally, the trend is the same, although lagging somewhat. The principle advantage cited by TLD proponents is an easily automated readout, with the energy response second. Those preferring film cite the availability of a permanent record as the main advantage, with secondary interest in energy evaluation and the high information content of the film "image." With regard to sensitivity, estimates of "lowest measurable dose" by TLD service range from 5 to 100 mrem, with an average of 22 mrem. For film, the range was 5 to 150 mrem with a 40-mrem average.
The survey clearly identifies development of fast neutron dosimetry as the major problem in personnel monitoring. Over twothirds of the responses cited a fast neutron capability as the improvement most needed in their present systems. Forty-two services reported doing research and development. Of these, the largest number fifteen - are working on neutron dosimetry. Others reported work on system improvements and new or improved TLD phosphors. It is of interest that only three are working on beta dosimeters and two are doing $\mathrm{R}$ and $\mathrm{D}$ to improve calibration and standards techniques.

\section{Dosimetry Development}

What is really going on in dosimetry development? For $\mathrm{x}$ rays and gammas we are in the position of fine-tuning reasonably dependable systems. People are becoming quite comfortable with TLD, primarily LiF. A great deal of information has built up over recent years for both film and LiF, and some of the disadvantages cited for TLD by dosimetrists are not considered to be as severe as they once were. Few people now feel the need for the dosimeter itself as part of the permanent record. The energy determination question is being partially resolved by use of multiple crystal badges with built-in absorbers to provide penetrating-nonpenetrating dose information. Another approach is to use additional TLD phosphors with different energy response, such as $\mathrm{CaF}_{2}$. The image aspect of film may still be an advantage in some cases, however. 2

Minor problems with LiF cause a continuing but low-1evel search for other phosphors, but with greater emphasis on use for environmental monitoring than personnel dosimetry. As experience with long-term use and recycling of LiF grows, the dosimeters are found to be durable. However, changes in sensitivity with repeated high-

temperature annealing have been reported. ${ }^{3}$ Our own experience is a loss of sensitivity by as much as $30 \%$ after seven years. In practice, of course, the calibration program compensates for that effect. Another disadvantage is that the glow peak is somewhat lower $\left(\sim 200^{\circ} \mathrm{C}\right)$ than for other phosphors, resulting in some fading at elevated environmental temperatures (up to $8 \%$ per month). ${ }^{4}$ The annealing of LiF is complicated and time-consuming. ${ }^{5}$ It is not as sensitive as other phosphors such as $\mathrm{CaF}_{2}$ 
and $\mathrm{Mg}_{2} \mathrm{SiO}_{4}(\mathrm{~Tb})$, but sensitivity is not a limitation for personnel monitoring and the other problems do not seem to outweigh the advantages of LiF, including stability and flat energy response. 6,7

Research continues on other detection mechanisms, primarily those involving thermally or optically activated chargetransport effects such as radiation induced thermally activated currents (RITAC), radiophotoluminescence (RPL), and thermally stimulated exo-electron emission (TSEE). 8-13 of these, RPL is the oldest, having been studied for over 25 years. Very little current research is being done on it for personnel dosimetry. RPL has suffered rejection because of inadequate sensitivity, but recent $1 y$ a detection level of a few mrem has been reported. ${ }^{14}$ The 1 argest U.S. use of RPL was in the DT-60 dosimeters issued by the Navy. The most attractive feature is a dosimeter capable of maintaining a permanent integrated exposure.

TSEE involves emission of trapped electrons from the surface of an insulator by heating. The emission of electrons with energies of a few $\mathrm{eV}$ has a temperature dependence similar to a TL glow curve. Both GM and proportional counters have been used for detection. The attraction of TSEE has been its potential sensitivity (a few $\mu R$ ) and the fact that the electrons are detected only from a very thin layer on the surface (a few $\mu \mathrm{m}$ ). The thin layer emission is thought to give it a Linear Energy Transfer (LET) dependent response. The major obstacle to the use of TSEE has been the care required in handing and the protection necessary to prevent nonradiationinduced signals from surface damage. TSEE dosimeters are also sensitive to light and humidity. 15

RITAC has been investigated only relatively recently. RITAC really includes a family of thermally activated current phenomena: thermally stimulated conductance (RITSC), thermally activated polarization (RITAP) and thermally activated depolarization (RITAD). RITAC involves measurement of thermally induced current with a potential ( $1-5 \times 10^{2} \mathrm{~V} / \mathrm{mm}$ ) applied across a letector such as $\mathrm{CaF}_{2}$ or $\mathrm{Al}_{2} \mathrm{O}_{3}$ (sapphire). The current through the detector is measured vith a picoammeter as it is heated, and cecorded as a function of temperature to rield glow peaks similar to TL or TSEE. The tt tractive features of RITAC are that it has l sensitivity comparable with TL but uses a simpler readout system, and needs no annealing before reuse.

There are, of course, a number of other methods of gamma detection, but they suffer from poor sensitivity, high cost, or poor stability. The likelihood of seeing a gamma dosimeter to replace TLD, film or RPL in the near future is very small. Any new unit will not only have to be superior to existing dosimeters, but must overcome the wealth of experience dosimetrists have with their systems. A lot of time and money is invested in a personnel dosimetry system, so the justification for changing it must be very solid.

Challengers in the neutron dosimetry field face a much brighter future. There is simply not a satisfactory neutron dosimeter available. There are more methods for detection of neutrons than ganmas; however, the personnel neutron dosimetry problem is more difficult than that for gamma dosimetry:

- The neutron energy range is wider ( 9 decades as opposed to 3 or 4 for gammas and $x$ rays).

- The quality factor for fast neutrons is ten times that for gammas, requiring ten times the sensitivity for the same dose-equivalent level.

- The gamma sensitivity of some neutron detection processes impairs discrimination between the two radiations.

Three neutron dosimeter types are in use today - NTA film, TL albedo and fission track etch dosimeters. None of these can be considered a long-term solution to the general problem of personnel neutron dosimetry. NTA film has been used most extensively, but has been severely criticized because of its fading properties, need for laborious manual counting and gamma sensitivity. ${ }^{4,16}$ Much of the concern about fading can be overcome by adequate protection, but the counting is still tedious and does not lend itself to automation. 17,18 In addition, it is not well suited for use in areas where there are a large number of low-energy neutrons. 19

TL albedo dosimeters have become popular in the last few years, primarily because they are sensitive and use a TLD readout. 20,21 The dosimeter depends on body 


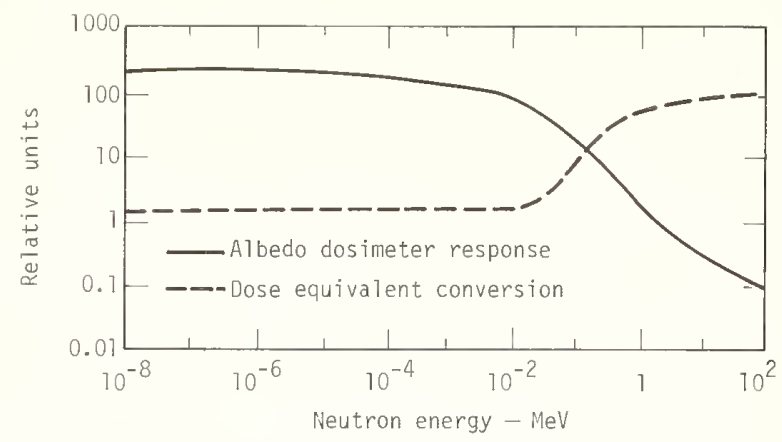

Fig. 1. Comparison of relative response of TLD albedo dosimeter with neutron dose equivalent flux to dose equivalent conversion.

thermalization and reflection of fast neutrons for its response. The major limitation is that they are severely energydependent. Figure 1 is a schematic comparison of the calculated response of an albedo system compared with the NCRP 38 dose-equivalent response curve. 22,23 Below about $5 \times 10^{-3} \mathrm{MeV}$ the responses are roughly parallel, but for higher energies they invert. For fast neutrons where we need doseequivalent information the most, the sensitivity is the least. Use of these dosimeters requires that either the spectrum be well known or a thorough investigation of each positive reading be made to establish a spectral calibration factor. Efforts have been made to eliminate the spectral dependence of these dosimeters, but they have not been completely successful. 20,24

Fission track detectors have found limited use in the U.S., but have been more accepted internationally. 25-28 Although they are not as sensitive as albedo dosimeters, the major objection is the need for personnel to carry radioactive materials such as ${ }^{23} \mathrm{U},{ }^{238} \mathrm{U},{ }^{23}{ }^{7} \mathrm{~Np}$ or ${ }^{232} \mathrm{Th}$ in their badges. Many health physicists feel it inappropriate to give a dose of one radiation in order to measure the dose of another.

Perhaps as much in desperation as for any other reason, a number of potential neutron detection methods have been considered for personnel monitoring:

- ${ }^{6} \mathrm{Li}(\eta, \alpha){ }^{3} \mathrm{H}$ and ${ }^{10} \mathrm{~B}(\eta, \alpha)^{7} \mathrm{Li}$ with cellulose nitrate track registration. 29,30

- Be recoil radiators with cellulose nitrate track detectors for fast neutron measurement. 31

- Detection of fast-neutron-induced direct recoils in cellulose nitrate. 32,33

- Detection of fast-neutron-induced direct recoils in polycarbonate film by spark counting. 34

- Detection of fast-neutron-induced direct recoils in polycarbonate sheet by electrochemical etching. 35,36

- Formation of tracks from $(n, p)$ and $\left(\eta\right.$, alpha) reactions in $\mathrm{AgCl} .^{37}$

- Development of an hydrogeneous TLD. 38

- Detection of damage in silicon diodes. 39

- Detection of self-activation in TLD materials. 40

- Use of LET-dependent differential glow peak formation in TL materials. 41,42

- TSEE with hydrogenous radiators. 43

These methods depend either on detection of the tracks of individual charged particles resulting from neutron-induced nuclear reactions, or on some bulk effects such as activation, atom displacement or electron trap formation. Track detection is-attractive because it allows one to look at individual neutron interactions. A more important advantage is that the registration process requires a minimum LET for track formation, so the technique is generally gamma insensitive. ${ }^{44}$ The main disadvantage is that readout is laborious and not easily automated. Other solid-state dosimetry techniques are more easily automated, but the response decreases with increasing LET, making the relative gamma-to-neutron sensitivity much too high. 45

The emphasis in track registration is to develop simple readout techniques for detection of tracks, particularly from fast neutron interactions. The fast neutron interactions usually involve detection of direct recoil tracks in plastics such as polycarbonate or cellulose nitrate by 
chemical etching. Detection methods include track contrast enhancement by including dye in the film, semiautomatic detection by spark counting, or track enlargement by electrochemical etching. ${ }^{46-48}$ We have found that the efficiency of the red dye contrast enhancement method is very sensitive to film thickness and etching parameters. ${ }^{33}$ Use of the technique routinely would require very careful control of etching conditions, to a point that might not be practical in a large-scale field program. In addition, automated counting would require an expensive image analysis system. Spark-counting direct recoils in polycarbonate film seems to lack sufficient sensitivity for routine personnel work, and a recent study suggests poor reproducibility. 34,49

The use of high-frequency alternatingcurrent etching to enlarge damage tracks is one of the more promising track etch methods for fast neutron detection. An irradiated foil of polycarbonate is placed between two volumes of etchant at room temperature, and an ac field is applied across the sample to enlarge the tracks to dimensions of tens of microns in diameter (Fig. 2a). They are visible with the unaided eye, but need some magnification for counting. The plastic must be selected and handled carefully because tracks form on scratches and other artifacts, resulting in a significant background. However, with a few square centimeters of suitable material, it should be possible to detect fast neutron doses of 100 mrem. One interesting aspect of the enlarged tracks is that most of them are below the surface, with only a fine ( $0.5-\mu \mathrm{m})$ track connecting to the surface. We discovered this while trying to look at the tracks with a scanning electron microscope. After having a lot of trouble locating a track, we found a slight depression in the sample surface (Fig. 2b). As we increased the magnification the depression collapsed, revealing a subsurface cavity (Fig. 2c). We found a number of these pits on further investigation, which also collapsed under the increased dose from high-magnification electron scans.

Silver halide single crystals have been used to detect heavy ions and cosmic rays. ${ }^{50}$ The latent tracks are made visible by decoration techniques using UV illumination or UV illumination in combination with an electron beam. 37,50 Although neutron detection has been reported, the application
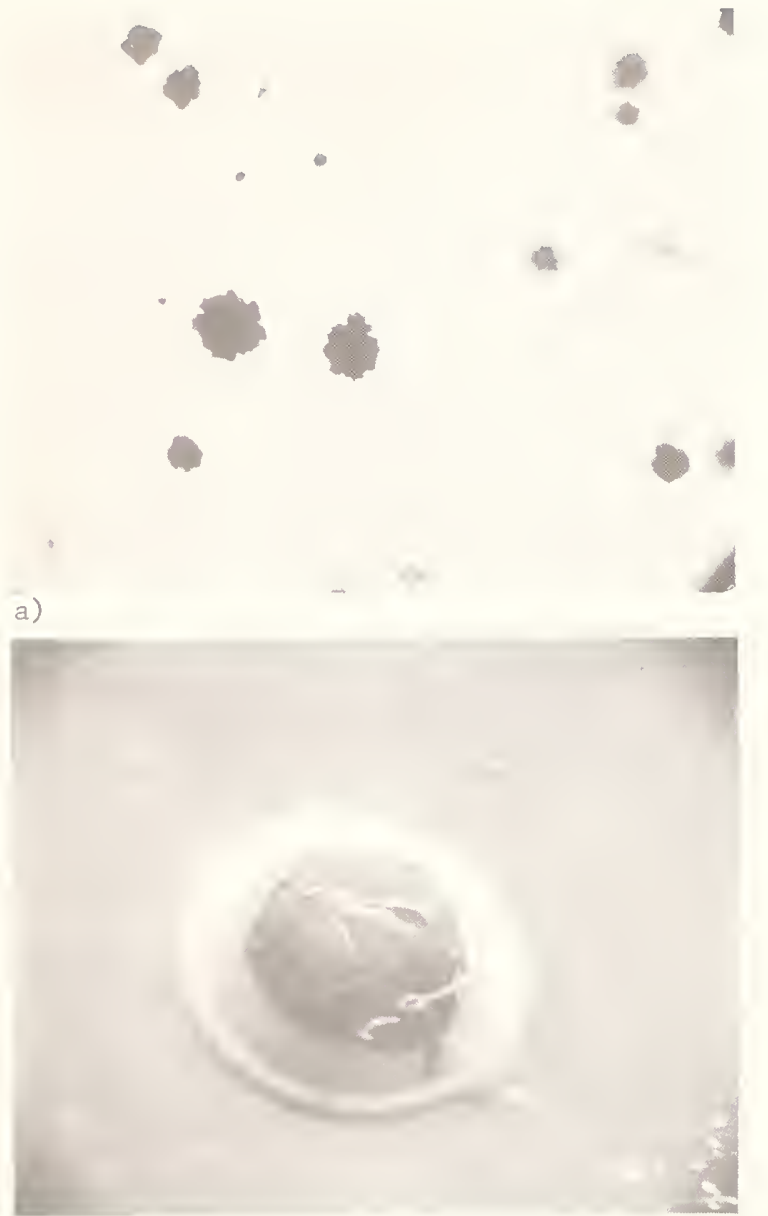

b)

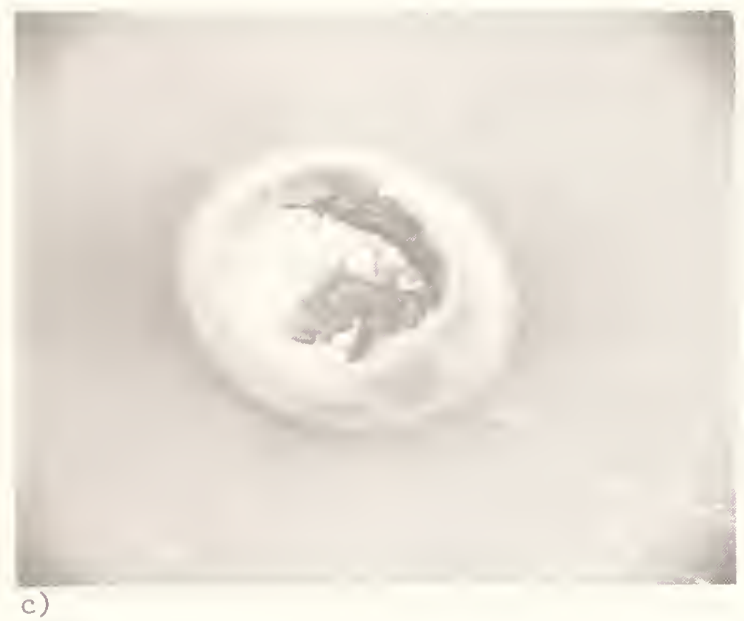

Fig. 2. (a) Optical microphotograph of electrochemically enlarged recoil tracks in polycarbonate (102.5x). (b), (c) Scanning electron microphotograph (S.E.M.) of electrochemically enlarged recoil track before and after collapse of polycarbonate surface (1344x). 
of these crystals to personnel dosimetry has not been pursued. Unlike NTA track films, gamma background does not develop in the crystal.

As we shift attention to other solid state detector techniques, we find that silicon diodes have been discussed for many years, but still suffer from lack of sensitivity and high cost. TLD autoactivation is the build-up of TL signal from the decay of neutron activation products after preliminary readout and annealing. The short half-life of activation products in TL materials precludes its use for routine personnel dosimetry. It might, however, have application in criticality dosimetry.

People have attempted to associate hydrogenous material with TLD by use of polyethylene radiators, or by inclusion of heat-resistant hydrogenous material in the TLD matrix. 38,51 Neither of these approaches has been particularly successful, with gamma sensitivity and difficulty in producing phosphors as the main problems. Others have looked at LET dependent flow peaks in materials such as $\mathrm{CaF}_{2}: \mathrm{Tm}$, however, the success has been limited by lack of sufficient neutron sensitivity and high gamma response. Finally, Becker ${ }^{43}$ has used TSEE with polyethylene radiators, but the gamma sensitivity and care required in handling have prevented use in routine personnel monitoring.

The beta monitoring problem seems to be of least interest to dosimetrists. Film or TLD are used routinely for beta dosimetry, and are satisfactory for most routine applications. However, certain applications such as measurement of weak betas from tritium do require more study. TSEE is a possible solution. It is sensitive and the thin sensitive layer is particularly well suited to measurement of low-energy electrons. In addition, the TL signal from a detector like $\mathrm{BeO}$ can be used to measure the gamma background. 52 Again, however, the necessary dosimeter protection and careful handling have prevented routine use of TSEE.

\section{Calibration}

Calibration and quality control are a vital part of a personnel monitoring program. Without careful attention to these considerations, the effort expended in the rest of the dosimetry program is wasted. The following are some points that need to be stressed in that regard:
- Calibrations must be made in terms of physical measurements. The method of conversion to dose equivalent is up to the dosimetrist, but the primary calibration must be capable of description in physical units. I often hear people ask for calibration data in rem because that is what the field health physicist is obliged to use, but a calibration that cannot be reproduced physically will only lead to problems when subsequent data comparisons are made.

- Radiation fields must be careful1y characterized. This is a particular problem in neutron dosimetry where dose equivalent is often determined with a remmeter. Measurement of primary fields should be made with more than one instrument, and preferably more than one instrument type. At LLL, for example, our neutron fields are determined by spectrum measurement, long-counter measurement, computer calculation, as well as by calibrated remmeters. In the near future, we will also be making LET and absorbed dose measurements.

- Every effort should be made to duplicate the radiation field quality found in working environments. It is really upsetting to talk about using a PuBe neutron source to calibrate dosimeters for use around reactors and other fields with low-energy neutrons. The growing availability of ${ }^{252} \mathrm{Cf}$ sources makes it quite practical to provide moderated fission spectra using spheres of polyethylene, metal such as aluminum, or metal shells which can be filled with water or $\mathrm{D}_{2} \mathrm{O}^{53}$ of course, spectral duplication implies detailed field surveys, but simple techniques to do this have been developed. 54

- Intercomparisons with other laboratories are very valuable. They can be done through formal intercomparison programs, or more easily in many cases informal dosimeter exchanges with friends at other laboratories. 55 Organized intercomparisons can provide a rigorous test of the dosimetry system, while informal exchanges have the advantage that the data need not be published and the results can be compared in a short time. 
To finish the question of calibrations, I want to acknowledge some development in radiation field production. Monoenergetic x-ray fields require careful development. The most popular technique is the use of secondary fluorescent radiators excited by either machine-produced $\mathrm{x}$ rays or isotopic sources. Isotopically excited sources in particular can produce high levels of Compton scatter which make a significant dose contribution. Even machine-generated $\mathrm{x}$-ray sources must be carefully filtered so that the dose comes from the fluorescent $x$ rays and not scatter. The filtration reduces $x$-ray intensity severely, and may require a rather large $x$-ray generator to make up for the loss in intensity.

Two alternative $\mathrm{x}$-ray production techniques are under study at LLL. In one case, special tubes, referred to as TRansmission Anode X-ray (TRAX) tubes, have been built so that the electrons strike the back of the characteristic target which is part of the

a)
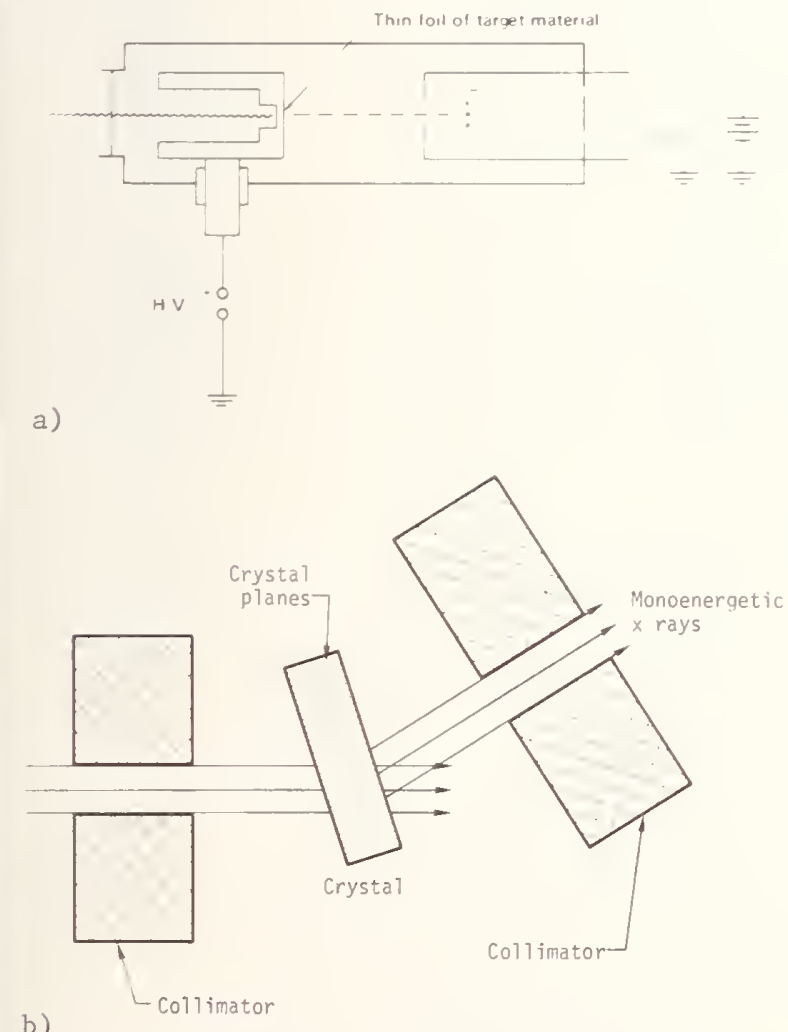

Fig. 3. (a) Production of transmission anode $x$-rays (TRAX). (b) Production of monoenergetic rays by anomalous transmission through crystals (Borrmann effect). tube. 56 The target filters the radiation, preferentially transmitting its own characteristic $x$ rays (Fig. 3a). Silver and copper tubes have been made which produce characteristic $\mathrm{x}$ rays at levels approaching $10 \mathrm{R} / \mathrm{min}$ at $10 \mathrm{~cm}$ from the tube face. The cost of these tubes is significantly lower than that required for a high-intensity $\mathrm{x}$-ray generator.

Another technique under study is production of $\mathrm{x}$ rays by anomalous Borrmann transmission through single crystals such as germanium. 57 The energy of $x$ rays scattered from crystal planes is a function of the angle between the beam and crystal planes (Fig. 3b). Rotation of crystal and second collimator is expected to provide us with a dial-an-energy, monoenergetic $x$-ray source. Although the $x$-ray yield is much less than from the previously discussed TRAX tubes, it may be a very useful system for instrument energy dependence measurements.

I would like to say a word in support of the NBS filtered beam monoenergetic neutron facility. 58 This facility should be a valuable addition for calibration of neutron dosimeters and detectors. The availability of 2-keV neutrons will give us valuable data, particularly for moderated detectors, that was not available before.

\section{In-vivo Dosimetry}

A complete discussion of personnel monitoring must include internal dosimetry. Estimates of dose from internally deposited radioactive material have the advantage that the material is available for measurement and sampling for a sufficient length of time to permit use of sophisticated detector systems and acquisition of adequate data. In fact, most radionuclides can now be assayed quite accurately. However, some isotopes have unfavorable decay schemes with little or no gamma emissions, and are very difficult to detect.

The most important example of an unfavorable decay scheme is ${ }^{239} \mathrm{Pu}$ which has no $\mathrm{K}$ $\mathrm{x}$ rays and on $1 \mathrm{y} 0.04717-\mathrm{keV} \mathrm{L} x$ rays per alpha disintegration. We obtain a count rate of only a couple of counts per minute from a lung burden of ${ }^{239} \mathrm{Pu}$ in an average male, using a $300 \mathrm{~cm}^{2}$ chest counter. It is possible to detect plutonium in vivo at that level, only with elaborate detection equipment using anticoincidence techniques. 59 Uncertainty in the geometric distribution 
in the lung makes accurate measurement even more difficult.

There is now a concerted effort to improve ${ }^{239} \mathrm{Pu}$ measurement capability on a nationwide basis, including work by the ERDA-established Intercalibration Committee for Low-Energy Photon Measurements. 60 The committee, made up of members from ERDA contractor laboratories, is primarily involved with a program of chest-counting facility intercomparisons. The intercomparison program includes circulation of a set of heavy-element sources of ${ }^{2}{ }^{8} \mathrm{Pu}$, ${ }^{239} \mathrm{Pu},{ }^{241} \mathrm{Am}$ and other transuranic isotopes. 60 In addition, three solid tissue-equivalent torso phantoms with removable major organs are under construction to be used in an ongoing ERDA sponsored multi-1aboratory heavy-element intercomparison program. 61,62 Finally, in the future, we hope to conduct an in-vivo calibration study similar to that performed by the U.K. involving inhalation of aerosols labelled with low levels of ${ }^{10} \mathrm{Pd}$ which has an $\mathrm{x}$-ray used to simulate plutonium. 63

There seems to be a physical limitation on the lower limit of detection possible with chest counters. However, techniques such as solid-state detector arrays or use of multiwire proportional counters may be very helpful for location of material in the lung and improved estimation of $x$-ray region human backgrounds. An esophageal probe has been used for truly internal counting resulting in a lower limit of detection, but the technique requires medical attendance and does not have widespread appea1. 64

\section{Summary}

A complete personnel monitoring program must include a well integrated set of dose assessment techniques. This is particularly true in view of a growing nuclear energy program and increasing use of radiation in medicine. I have discussed a number of techniques for both external and in vivo dosimetry. There are a number which could not be included, but must be considered in the overall dosimetry program. Use of biological dosimetry, for example, can be very useful in some applications, and development of analytical techniques such as chromosome aberration detection will certainly become increasingly more valuable.

The backbone of a monitoring program the personnel dosimeter - has distinct limitations, and it must be used with those limitations in mind. Personnel neutron dosimetry is currently the most difficult external monitoring problem. Although a number of techniques are under study, the most satisfactory solution may require use of composite techniques such as addition of fast neutron track detectors to more sensitive, but less accurate albedo dosimeters.

Careful calibration of personnel dosimeters is vital to the monitoring program. We must recognize the need for calibrations which can be reproduced in physical terms. We must characterize the calibration fields carefully and accurately, and use fields which simulate real life environments. It will be important to have more intercomparisons, particularly on an informal basis, with emphasis on improving our systems rather than being graded on performance.

The need for internal dosimetry is not as widespread as for external monitoring, but the quality of the dosimetry is just as important. The most difficult in-vivo measurement problem is reduction of the ${ }^{23}{ }^{9} \mathrm{Pu}$ limit of detection. There seems to be no single solution, but like neutron dosimetry, it will require a coordinated combination of techniques.

\section{Acknowledgments}

I wish to thank Ken Crase, George Campbe11, Dale Hankins, Tom Crites, Wade Patterson, and Dave Myers for their contributions to the preparation of this paper.

\section{References}

1. K. Becker, Oak Ridge National Laboratory, Rept. ORNL-TM-5102, (1975).

2. U. Madhvanath, P. H. Patel, R. V. Dhond, K. S. Shenoy, and A. B. Kadam, Health Phys. 30, 119 (1976).

3. F. M. Cox, A. C. Lucas, and B. M. Kapsar, Health Phys. 30, 135 (1976).

4. K. Becker, Oak Ridge National Laboratory, Rept. ORNL-TM-4297 (1973).

5. K. Becker, Solid State Dosimetry (CRC Press, 1973) p. 38.

6. W. Binder, S. Disterhoff, and J. R. Cameron, Proc. Second Intern. Conf. Luminescence Dosimetry, paper CONF-680920 (1968), p. 43. 
7. B. D. Bhasin, R. Sasidharan, and C. M. Sunta, Health Phys. 30, 139 (1976).

8. G. D. Fullerton and P. R. Moran, Medical Phys. 1, 161 (1974).

9. P. R. Moran, E. B. Podgorsak, G. E. Fuller, and G. D. Fullerton, Medical Phys. 1, 155 (1974).

10. E. R. Ballinger and P. S. Harris, Los Alamos Scientific Laboratory, Rept. LA-2998, Report 4526, (1959).

11. R. Maushart and E. Piesch, Luminescence Dosimetry, AEC Symp. Ser. 8, U.S. Atomic Energy Comm. (1967), p. 300.

12. J. Kramer, Proc. Second Intern. Conf. Luminescence Dosimetry, paper CONF-680920, (1968), p. 180.

13. K. Becker, R. B. Gammage, K. W. Crase, and J. S. Cheka, Proc. Third Intern. Symp. on Microdosimetry, Stresa, 1971, EUR-4810, Euratom, Luxembourg (1972), p. 675 .

14. F. Hillenkamp and D. F. Regulla, Proc. 3rd Intern. Conf. on Luminescence Dosimetry, Riso Report No. 249, Part II, Danish AEC Research Establishment, (1971), p. 718.

15. J. S. Nagpal and R. B. Gammage, Rad. Eff., 20, 214 (1973).

16. P. N. Kirshnmoorthy, G. Venkataraman, D. Singh, and D. Dayashankar, Proc. Symp. Neutron Monitoring for Radiation Protection Purposes, Vol. II, IAEA, Vienna (1973), p. 343.

17. J. Jasiak and T. Musialowicz, Proc. Symp. Neutron Monitoring for Radiation Protection Purposes, Vol. II, IAEA, Vienna (1973), p. 191.

18. A. Knight, Health Phys. 27, 606 (1974).

19. D. E. Hankins, Health Phys. 9, 31 (1963).

20. J. E. Hoy, Savannah River Laboratory Report DP-1277, E.I. duPont de Nemours \& Co., (1972).

21. D. E. Hankins, Los Alamos Scientific Laboratory, Rept. LA-5261 (1973).

22. R. G. Alsmiller, Jr. and J. Barish, Health Phys. 26, 13 (1974).
23. NCRP Report No. 38, Protection Against Neutron Radiation, National Council on Radiation Protection and Measurements, Washington, D.C. (1971).

24. T. R. Crites, Lawrence Livermore Laboratory, Rept. UCRL-77028 Rev. 1 (1975).

25. T. J. Yule, Fourth AEC Workshop on Personnel Neutron Dosimetry, Battelle Northwest Laboratory, Rept. BNWL-1777 (1973), p. 51.

26. C. H. Distenfeld, Fourth AEC Workshop on Personnel Neutron Dosimetry, Battelle Northwest Laboratory, Rept. BNWL-1777 (1973), p. 20.

27. W. G. Cross and H. Ing, Fourth AEC Workshop on Personnel Neutron Dosimetry, Battelle Northwest Laboratory, Rept. BNWL-1777 (1973), p. 15.

28. S. Pretre, Proc. Symp. Neutron Monitoring for Radiation Protection Purposes, Vol. II, Vienna (1973), p. 99.

29. B. J. Tymons, J. W. N. Tuyn, and J. Baarli, Proc. Symp. Neutron Monitoring for Radiation Protection Purposes, Vol. II, Vienna (1973), p. 63.

30. Dayashankar and G. Venkatarian, Proc. Symp. Radiation Protection Monitoring, IAEA, Vienna (1968), p. 47.

31. R. V. Griffith, D. R. Slaughter, and T. R. Crites, Fifth ERDA Workshop on Personnel Neutron Dosimetry, Pacific Northwest Laboratory, USERDA, Washington, D.C., BNWL-1934 (1975), p. 38.

32. K. Becker, Topics in Radiation Dosimetry, F.H. Attix, Ed., (Academic Press, New York, 1972), p. 122.

33. R. V. Griffith and J. C. Fisher, Hazards Control Progress Report No. 49, Lawrence Livermore Laboratory, Rept, UCRL-50007-74-2, (1975), p. 1 .

34. K. Becker and M. Abd-E1 Razek, Nucl. Instr. Meth. 124, 557 (1975).

35. M. Sohrabi, Health Phys. 27, 598 (1974).

36. R. V. Griffith and J. C. Fisher, Hazards Control Progress Rept. No. 51 , Lawrence Livermore Laboratory, Rept. UCRL-50007-75-2 (1976). 
37. G. Henig, E. Schopper, F. Granzer,

K. Dardat, J.A. Schott, A. Wensel,

H. Bucker, G. Horneck, G. Haase, and

F. Zorgiebel, Proc. 8th Intern. Conf. on Nucl. Photography and Solid State Track Detectors, Institute of Atomic Physics, Bucharest (1972), p. 384.

38. K. Becker, T. D. Tham, and F. F. Haywood, Proc. Third Intern. Congress of the Intern. Radiation Protection Assoc., CONF-730907-P1, USAEC, Washington, D.C., (1973), p. 584 .

39. H. Kruger, G. Tumbrägel, R. Metzner, and $\mathrm{H}$. Koch, Proc. Symp. Neutron Monitoring for Radiation Protection Purposes, Vol. II, IAEA, Vienna (1973), p. 401 .

40. D. Pearson, J. Wagner, P. R. Moran, and J.R. Cameron, University of Wisconsin, Rept. Co0-1105-175 (1971).

41. B. G. Oltman, J. Kastner, and C. Paden, Proc. Second Intern. Symp. on Luminescence Dosimetry, paper, CONF-680920 (1968), p. 623.

42. G. W. R. Endres, Fifth ERDA Workshop on Personnel Neutron Dosimetry, Pacific Northwest Laboratory, USERDA, Washington, D.C., BNWL-1934 (1975), p. 32.

43. K. Becker, Solid State Dosimetry, (CRC Press, 1973), p. 111.

44. R. L. Fleischer, P. B. Price, and R. M. Walker, Nuclear Tracks in Solids (University of California Press, 1975).

45. K. Becker, Proc. Fourth Symp. on Microdosimetry, Vol. 2, Verbina, Italy, (1974), p. 899.

46. J. Barbier, Trans. Am. Nucl. Soc. 13, 530 (1970).

47. W. G. Cross and L. Tommasino, Health Phys. 15, 196 (1968).

48. L. Tommasino, Comitato Nazionale Energia Nucleare, RT/PROT (71), 1, 1970.

49. J. Jasiak and E. Piesch, Nucl. Instr. Meth. 128, 447 (1975).

50. C. B. Childs and L. M. Slifkin, Rev. Sci. Instr. 34, 101 (1963).

51. C. M. Sunta, K. S. V. Nambi, and V. N. Bapat, Proc. Symp. Neutron Monitoring for Radiation Protection Purposes, Vol. II, Vienna (1973), p. 57.
52. R. B. Gammage and J. S. Cheka, Nucl. Instr. Meth. 127, 279 (1975).

53. R. V. Griffith, R. Kloepping, H. W. Patterson, and T. Crites, Hazards Contro1 Progress Report No. 48, Lawrence Livermore Laboratory, Rept. UCRL-50007-74-1 (1974), p. 8 .

54. D. E. Hankins, Lawrence Livermore Laboratory, Rept. UCRL-77198 (1975).

55. H. W. Dickson, W. F. Fox, and F. F. Haywood, in Proceedings, this Symposium.

56. W. L. Pickles and J. L. Cate, Jr., Proc. 22nd Annual Conf. on Applications of X-ray Analysis, Denver, (1973), p. 337.

57. C. T. Prevo, Lawrence Livermore Laboratory, personal communication, Feb. 1976.

58. R. B. Schwartz and I. G. Schroder, in Proceedings, this Symposium.

59. E. G. Shapiro and A. L. Anderson, IEEE Trans. Nuc1. Sci. NS-21, 201 (1974).

60. K. L. Swinth and P. N. Dean, Health Phys. 25, 599 (1973).

61. P. N. Dean, R. V. Griffith, and A. L. Anderson, IAEA Intern. Seminar on Diagnosis and Treatment of Incorporated Radionuclides, Vienna, Dec. 1975 (to be published).

62. R. V. Griffith, A. L. Anderson, and P. N. Dean, Hazards Control Progress Rept. 51, Lawrence Livermore Laboratory, Rept. UCRL-50007-75-2 (1976).

63. D. Newton, F. A. Fry, B. T. Taylor, M. L. Eagle, and R. C. Sharma, oral presentation at Workshop on The Measurement of Plutonium and Other Transuranic Elements in Vivo by External Counting, Twentieth Annual Meeting of the Health Physics Society, Buffalo, N.Y., July 1975.

64. K. L. Swinth, J. F. Park, G. L. Voelz, and J.H. Ewinn, Hanford Biology Symp. on Radiation and the Lymphatic System, Richland, Wash., CONF-740930, 30 Sept.2 Oct. 1974. (In press.) 


\begin{abstract}
Efforts in the United States to provide some measure of uncertainty to the interpretation of routine personnel monitoring badge results have received the attention of various groups, individuals and governmental agencies for over 20 years. However, in general, health physicists and the users of these personnel monitoring devices still do not have great confidence in the results. This paper will review the history of the significant activities in trying to develop a system for testing the performance of personnel dosimetry services. This will also include a discussion of the current published standards and those in draft with respect to their different approaches and objectives. In particular, the paper will describe activities of the Nuclear Regulatory Commission in considering mandatory performance testing of personnel dosimetry and the work of a Health Physics Society Working Group which could be the basis for an NRC proposed rule.

(Personnel dosimeters; film badges, TLD, HPS Working Group)
\end{abstract}

In most instances, measuring exposures of individual workers to harmful conditions is a laborious procedure. In general, one must utilize bulky and complicated sampling equipment, perform time consuming chemical analyses or physical measurements; relate the measurement to a worker's breathing rate and work habits; and finally, making further assumptions, arrive at an annual or periodic exposure.

Health physicists have considerable advantage over other health professionals in making these evaluations. For ionizing radiation one has available various kinds of simple personal monitoring devices. The most common and universally used are the badge type dosimeter, i.e., either film or thermoluminescent dosimeters (TLD). Next to the radiation warning symbol, the public probably recognizes this symbol of the nuclear industry most easily. These devices 1) are small and lightweight, 2) require no power source, 3) are inexpensive to use, 4) have a sensitivity less than one percent of the regulatory limit, 5) can differentiate between the different types of ionizing radiation, and 6) have an accuracy sufficient to meet health protection requirements.

The technical literature provides sufficient evidence as to the accuracy of both film and TLDs. However, tests of personnel dosimetry suppliers under controlled conditions have shown that these devices do not always measure up to their capability. Consequently, the question "How accurate are the results obtained from personnel dosimeters processed on a routine basis by commercial, military, governmental or other private services?" has been of considerable import in this country for at least twenty years. This paper will consider efforts to establish a national personnel dosimetry testing laboratory and the criteria for evaluating personnel dosimetry performance. It will seek to explore why no effective and nationally recognized personnel dosimetry testing service exists after considerable effort by federal and state agencies, commercial processors, and other industry and private groups. Finally, this paper will attempt to draw some conclusions for future action from the past effort to avoid repeating past mistakes.

There have been several attempts to evaluate film dosimeter processor performance beginning in 1955 and continuing sporadically through 1975. In addition, much effort has been expended on establishing personnel dosimetry performance criteria. In spite of all the previous efforts, the problem of assuring adequate personnel dosimetry processor performance to users of these devices has remained unresolved.

Since 1955, several studies ${ }^{1-7}$ evaluated routine film and TLD dosimeter performance. In general, the conclusions that can be drawn from these studies indicate that some processors demonstrate a capability more than adequate to satisfy health protection needs. However, these studies also show that performance by a substantial fraction of participants is not satisfactory 
for use by health physicists and the results are misleading to the users. These studies have almost universally concluded that there is a need for an independent, objective method of periodically evaluating personnel dosimetry services. The latest study, conducted by Battelle, Pacific Northwest Laboratories in 1974 indicates that there has been little improvement in the performance of personnel dosimetry processors 7 .

The retracing of any recent history regarding the attempt to establish a personnel testing laboratory or performance criteria should begin with the most formal and significant event in the long history, i.e., the September 4, 1963 Federal Register publication by the U. S. Atomic Energy Commission - Notice Regarding Need for Establishment of Film Dosimetry Calibration Laboratory ${ }^{8}$. This notice did several things:

1. Announced that the AEC was considering the desirability of issuing a proposed regulation which would require $\mathrm{AEC}$ licensees to utilize film dosimetry services which met certain minimum performance criteria;

2. Proposed a minimum set of performance criteria to provide standards of accuracy for evaluating film dosimetry services utilized by licensees;

3. Indicated that the Commission was studying the possibility of utilizing a laboratory for Commission contract activities; and

4. Requested expressions of interest of organizations and other persons desiring to establish and operate such a laboratory.

A significant number of comments were received and a large majority of these favored the establishment of the laboratory. A number of groups expressed interest in operating such a laboratory although most indicated their interest in operating such a laboratory was contingent upon financial support from the Federal Government. The AEC was concerned about what official or quasi-official recognition could be granted to a non-governmental laboratory by a regulatory or other governmental agency. The Commission was not sure what criteria should be used by a laboratory in a testing program and what criteria could be used to evaluate the testing laboratory. The Commission wanted to involve industry in evaluating licensees; also, to have AEC contractors tested by a group over which they had direct control, e.g., another AEC contractor.

In trying to develop a program, the AEC then considered, among other proposed actions initiating a program in which the National Bureau of Standards would develop guides for the establishment and operation of a film dosimetry calibration laboratory. Because of the Bureau's prestige and general preeminence in the standards field, it was felt that they might play a key role in a testing or calibration program even though NBS was unwilling to be involved in the approval of specific film dosimetry services. However, in view of the impending NBS move to Gaithersburg in 1966 and because of previous commitments, NBS indicated that they would have to delay start of the work and completion of the task would take three years. Because of the priorities that the AEC placed on this program, the Battelle Pacific Northwest Laboratory was asked to conduct a similar program. "The primary purpose of the Battelle study will be to consider ways and means to appraise any evaluation laboratory that might be established. Performance criteria and procedures for use by evaluation laboratories also will be drawn up. These criteria would help to provide standards for measuring continuing accuracy of service."9

Independent of these AEC activities, the National Sanitation Foundation (NSF) was showing interest in the evaluation of $\mathrm{film}$ dosimetry services. The U. S. Public Health Service (PHS) began supporting a program at NSF in 1963 which aimed at the development of procedures and performance standards for evaluation of film dosimetry services. NSF felt that when procedures and standards were developed, the film dosimetry industry would voluntarily support an evaluation program leading to the award of the NSF Seal of Approval. 6 NSF believed that this approval would be recognized by the AEG and others. The objectives of the NSF program were consistent with the needs expressed by the AEC and the program received broad support from the film dosimetry industry.

The NSF Foundation Council of Public Health consultants unanimously approved Standard No. 16, "Standards of Performance for Film Badge Services" on May 4, 1966. The NSF advisory committee on film badge services represented members of government agencies, professional organizations, AEC contractors, and industry. The basis used in the establishment of control limits for film badge service accuracy was primarily developed from a study conducted by NSF involving approximately two thousand film badges from twenty five different organizations. 4,6 
The NSF standard considers types of radiation at different energy ranges. The type of radiation with which a badge is irradiated is not revealed to the participants. In order to successfully meet the performance standard the participants' interpretation must be within certain control limits. As stated above, these control limits were established on the basis of the performance of a group of film processors that participated in an initial study. These limits are specified in terms of the width of an error band within which all but one of the participants' results must fall.

After reviewing the objectives of the NSF program and anticipating its usefulness in defining performance criteria that reflect the capabilities of film dosimeter technology the Commission felt it was not in a position to support NSF financially or approve their standard, although AEC staff participated in program development meetings. The Commission considered it desirable to supplement the efforts of INSF by establishing an alternate parallel program to permit the Commission to determine the adequacy of any evaluation program that might be estab1ished.

The Battelle study begun in 1965 took a considerably different approach to the evaluation of performance. Battelle utilized two distinct parameters for use in establishing performance; the systematic bias and the random variations. These parameters were considered to some extent to be unique and that the analysis of performance should consider them separately. A participant's results would be corrected for a bias greater than $10 \%$ before the variance criteria would be applied. Performance criteria are specified in terms of the parameters of the equation $D_{i}=\alpha+\beta E_{i}+\varepsilon_{E_{i}}$ where: $D_{i}$ is the actual delivered dose; $E_{i}$ the reported dose; $\alpha$ and $\beta$ are systematic bias parameters; and $\varepsilon_{\mathrm{E} i}$ is a random variable for which the variance is computed.

The Battelie report 5 also concluded that for its evaluation of the performance of the services tested that excessive bias and variance existed among film dosimeter processors. It concluded that a film dosimeter evaluation program should be implemented but reached no conclusion whether it should be voluntary or implemented by regulatory requirements. The 1967 Battelle report has not had a significant effect on the testing of personnel dosimeters since an evaluation laboratory has not been estab1ished.
Following publication of the Battelle study, the Commission considered requiring all film badge services for AEC contractors to meet performance standards compatible with the standards recommended in the Battelle report. In addition, commercial film dosimeter vendors which were not providing a service to an AEC contractor but were desiring to bid on such services would demonstrate their performance through an evaluation laboratory. The Commission considered soliciting bids on a competitive basis, for a film dosimetry evaluation laboratory service to judge the performance of AEC contractor and commercial film vendor services to AEC contractors. This would have allowed the AEC contractor and other private laboratories who could meet the conditions of the call for bids to compete on a competitive bid basis. However, a unique situation played a role in delaying implementation of that plan. Up until 1967, all AEC facilities utilized film rather than TLDs. One reason for this was the Commission position that the permanence of film gave it an overriding advantage over TLDs. During 1967, it was decided that the permanent record of the film should not be a deciding factor and that TLDs could be considered for use. Consequently, during the late 1960 's many AEC contractors began changing over from film dosimetry to TLD. No data base for routine performance existed, In addition, there obviously were no authoritative TLD performance criteria. In that respect, the film badge criteria proposed by Battelle were not an authoritative standard and the Commission felt some reluctance to use such a standard without consensus approval.

In 1969, the American National Standards Institute (ANSI) requested the Health Physics Society to write a document that could be used as an ANSI standard. The Society accepted the task and as a result the ANSI standard (N13.7-Criteria for Film Badge Performance) was approved on July 11,197210 . This standard was based largely on the Battelle study and the approach to judging performance criteria acceptance are identical.

AEC Regulatory formally endorsed the ANSI criteria for film badge performance in 1972. This endorsement was by means of Regulatory Guide 8.3 which also pointed out the Commission's exceptions to certain parts of the standard.11 It should be remembered that regulatory guides are merely" recommendations and do not directly place requirements upon licensees. The publication of this regulatory guide has not had a 
profound effect on film dosimetry processors or licensees. To the best of our knowledge, no licensee or processor has ever performed the tests specified in the Standard and guide. In this same year, an ANSI committee was formed to consider standards for performance for TLDs. A draft of this document which has been circulated for letter ballot indicates that the approach which is being considered differs considerably from the film badge performance standard.12 This standard has not been approved by the American National Standards Institute, as of this report.

NSF continued to conduct its program for evaluating film badge performance on a voluntary basis. The first "Approval Listing for Film Badge Services" was issued by NSF on July 1, 1967 and listed 9 approved services ( 7 commercial services, 1 military and $1 \mathrm{AEC}$ contractor). In addition, there were two other participants in the program but not desiring to have an NSF approval. However, this small number of participants was not considered adequate to hire full time NSF personnel to run the program or upgrade the physical facilities and instrumentation available. As a result, NSF was subjected to some criticism with regard to the technical adequacy of their program and administrative errors in implementing the early program. Some of this may have been aggravated by the loss of services of the individual who played the major role in initiating the program. For whatever the reason, the NSF program has continued to operate but has not gained the amount of official or technical recognition to enable it to have attained the impact originally envisioned or gained the confidence of users or regulatory agencies. The NSF program continues to operate, but with fewer participants than when it began.

1973 saw the introduction of a new participant in the attempts to evaluate personnel dosimetry performance. The Conference of Radiation Control Program Directors holds an annual meeting sponsored by the Bureau of Radiological Health (FDA), the Environmental Protection Agency (EPA), and the Nuclear Regulatory Comnission.

During these meetings, workshops on various subjects of interest and concern to the State Radiation Program Control Directors are held. During the 1973 meeting, the conference held a workshop which was to consider how a personnel dosimetry certification program could be established to assure that dosimetry results were accurate.
The major recommendations of the workshop were that a federal agency be designated to direct a continuing performance testing program and that the executive committee of the conference establish a task force of federal and State representatives to consider this proposal during the next year. 13 Such a task force was established and included representatives of three states plus individuals from FDA, EPA, OSHA, AEC (Regulatory) and the NBS.

The workshop had endorsed the idea of NBS involvement to provide credibility to a testing program. As had been recognized several years earlier, the NBS stature and unique capabilities were felt to be factors that could provide any program developed with the necessary prestige and technical backup which could not be obtained from a regulatory agency. As a result of the initial meetings of this task force it was concluded that the only practical way of having personnel dosimetry suppliers submit to testing was through some regulatory device. From a practical standpoint, the only agencies which had the authority either directly or indirectly to compel suppliers to participate in a testing program were OSHA and AEC. OSSHA had limited interest in such a program so it was left up to AEC to determine whether it could either condition licenses to require use of tested dosimeters or change the regulations to require their use. Although AEC had no control over $X$-ray users, it was felt that essentially a11 dosimetry suppliers would have to submit to a testing program to remain competitive. In early 1974 , the Director of Regulatory Standards directed his staff to develop regulatory requirements to require all licensees to use personnel dosimetry services evaluated as adequate by a testing or intercalibration laboratory. In order to assist AEC in writing a rule and establishing criteria for the program, a work group composed of directly concerned federal agencies was formed which included representatives of NBS, FDA, EPA, ERDA and various NRC offices. This work group had periodic meetings which began to crystallize a testing laboratory concept and identified remaining problems. Conceptually, it was felt that the program probably would operate in the following manner: Part 20 of the NRC regulations ${ }^{14}$ requires the use of personnel dosimetry under certain circumstances, i.e., when there is a possibility of an individual exceeding $25 \%$ of the maximal permissible exposure or where an individual enters a high radiation area. This section could be amended so that all licensees must use dosimetry services whose performance is tested 
to meet certain minimum criteria. The testing could then be performed by a testing laboratory using standards set by the Commission. The accuracy of a testing laboratory's calibrations and the manner in which the testing laboratory could be technically evaluated by the NBS which in turn could be supported by NRC under an interagency agreement.

When the writing of the proposed rule began, it was felt that two things should be accomplished: 1) establishing a testing laboratory in the proper regulatory framework; and 2) establishing standards for the testing of personnel dosimetry suppliers. In order to get the program moving, it was felt that it would be advantageous to adopt the ANSI film badge standard rather than going through the process of promulgating new standards. It was believed that in that way there would be only one problem-establishment of a testing laboratory. However, as drafting began on a proposed rule and discussions were held with various individuals, it became evident that the existing ANSI criteria for film badge performance would not be satisfactory for the use envisioned. It was generally agreed that the ANSI criteria are a good set of sophisticated criteria which may be useful for judging state of the art for performance of film. However, it became apparent that what was needed for the regulatory process are simpler standards based primarily on health physics considerations and only secondarily on the dosimetry system limitations. A second problem as noted above, is that there are no ANSI criteria for TLDs. These may be forthcoming but will also raise similar problems as the film criteria. An alternative to the ANSI criteria which was considered was the National Sanitation Foundation No. 16. Although this standard had some advantages, its rather simplistic statistical criteria and emphasis of system performance over health physics consideration directed us toward consideration of new performance criteria. The NBS, under sponsorship of FDA, attempted to develop these new criteria. This initial attempt of developing criteria based on health physics needs served as a spur to establishing a committee to produce a standard which could eventually receive ANSI approval.

During the 1975 meeting of the Health Physics Society, the standards committee approved the formation of a working group to produce a standard test method and performance criteria to be used by a testing laboratory to determine the output of a sup- plier of personnel dosimetry services. This group has been designated Working Group 15 and is being chaired by Dr. Margarete Ehrlich of the National Bureau of Standards.

Included on the working group are two commercial personnel dosimetry suppliers, a military processor, an ERDA contractor, a reactor health physicist, representatives from two potential testing laboratories, NSF and Battelle and the author is the NRC representative. Eventually, it is anticipated that these efforts will form the technical basis of a regulatory action by the NRC.

The working group anticipates that a final draft will be submitted to the parent Health Physics Society Standards Committee in early May 1976. It would be improper for me to discuss the details of the efforts of the working group but a brief discussion of the philosophy and some of the basic ideas that are emerging would be of interest.

Nowhere in the proposed standard is it planned to make direct reference to the type of personnel monitoring device employed. The performance tests and test criteria will be the same for all devices presently employed and contemplated for future use. Performance criteria will be based, as much as feasible, on the recommendations of the ICRU ${ }^{15}$ and the ICRP16 for monitoring accuracies adequate in the field of radiation protection. Only where it is known that the recommended tolerance limits cannot be met, at the present state of the art, will wider limits be set. An early revision date for reconsideration of these limits will also be recommended.

The various categories for which a supplier could request testing is shown in figure 1. The first three categories refer to photons in three different dose equivalent ranges between 30 or $50 \mathrm{mrem}$ and $3 \mathrm{rem}$. Further tests for accident levels of 3 to 800 rads are specified in the first two categories. The next category is for beta particles with the testing laboratories prepared to use a sealed strontium 90 source or a uranium slab. For the fast neutron tests, either californium-252 whose spectrum is close to a moderated fission spectrum or a source of higher average energy, such as plutonium-beryllium will be available. Mixtures of high and low energy photons; photons and beta particles; and fast neutrons and photons constitute the last three categories. 


\section{RADIATION CATEGORIES AND TEST RANGES}

\begin{tabular}{|c|c|c|c|}
\hline & RADIATION CATEGORY & \multicolumn{2}{|c|}{ TEST RANGE } \\
\hline 1 & Photons, $200 \mathrm{keV} \leq \mathrm{E}_{\mathrm{eff}}<3 \mathrm{MeV}$ & $\begin{array}{l}\text { (a) Protection: } \\
\text { (b) Accident: }\end{array}$ & $\begin{array}{l}30 \mathrm{mrem} \text { to } 10 \mathrm{rem} \\
10 \mathrm{rad} \text { to } 800 \mathrm{rad}\end{array}$ \\
\hline 11 & Photons, $30 \mathrm{keV} \leq \mathrm{E}_{\mathrm{eff}}<200 \mathrm{keV}$ & $\begin{array}{l}\text { (a) Protection: } \\
\text { (b) Accident: }\end{array}$ & $\begin{array}{l}30 \mathrm{mrem} \text { to } 10 \mathrm{rem} \\
10 \mathrm{rad} \text { to } 800 \mathrm{rad}\end{array}$ \\
\hline III & Photons, $16 \mathrm{keV} \leq \mathrm{E}_{\mathrm{eff}}<30 \mathrm{keV}$ & \multicolumn{2}{|c|}{$50 \mathrm{mrem}$ to $10 \mathrm{rem}$} \\
\hline IV & Beta Particles & \multicolumn{2}{|c|}{$150 \mathrm{mrem}$ to $10 \mathrm{rem}$} \\
\hline V & Fast Neutrons & \multicolumn{2}{|c|}{$100 \mathrm{mrem}$ to $5 \mathrm{rem}$} \\
\hline VI & $\begin{array}{l}\text { Photon Mixtures } \\
\text { (Categories I, II, and III) }\end{array}$ & \multicolumn{2}{|c|}{$50 \mathrm{mrem}$ to $10 \mathrm{rem}$ (total) } \\
\hline VII & $\begin{array}{c}\text { Photons and Beta Particles } \\
\text { (Categories i, and iV) }\end{array}$ & \multicolumn{2}{|c|}{150 mrem to 10 rem (total) } \\
\hline VIII & $\begin{array}{l}\text { Photons and Fast Neutrons } \\
\text { (Categories I, and V) }\end{array}$ & \multicolumn{2}{|c|}{100 mrem to 10 rem (total) } \\
\hline
\end{tabular}

Each full category test will be conducted over a period of several months. It was considered rather important to test a supplier over an extended period of time in order to be able to more effectively judge a supplier's performance for the entire period under consideration. In general, a supplier will not know which radiation types and categories were used for the irradiations of particular badges. There will be an exception in the accident range where the testing laboratory will identify the badges involved just as the users do when an accident has occurred.

Pass-fail criteria will be based on the weighted sum of bias and standard deviation. These quantities will be computed from the results of the current test and the results of past tests of the particular supplier in the category. Bringing in past test results will enable one to draw conclusions regarding the long term consistency of a supplier's performance. This is important if inferences are to be made about his daily processes from the results obtained from a relatively smal1 test sample. The bias for the process will be determined from those irradiation levels where it is found that the performance is independent of the radiation level. A supplier will fail a test in a given category if the weighted sum of the bias and the standard deviation for the current test lies outside the specified tolerance, either for his interpretation of total dose equivalent or for his interpretation of the dose equivalent due to penetrating radiation alone. Depending on the category, the tolerance will be set at 30 to $50 \%$.

The pass-fail criterion will also take into account the length of a supplier's monitoring period. This was considered important because if a supplier offers a weekly service, for example, imprecision in his individual interpretations will cancel out more readily over a period of a year than if he offered a monthly or quarterly service. Therefore, the weight attached to the bias relative to that attached to the standard deviation will be greater the shorter the monitoring period for which a supplier's service is offered.

The standard will also stipulate that a11 badges will be calibrated in terms of 
dose equivalent. This could be accomplished by computing the dose equivalent from the measured quantities (e.g., exposure, absorbed dose, flux density) at the prescribed depth in a tissue equivalent phantom or the personnel dosimeters can be mounted on a phantom during irradiation.

NRC is delaying proposal of a rule requiring testing of personnel dosimetry until the performance standard has been approved and the technical requirements of the performance standard can be effectively incorporated. If an existing standard could have been adopted in a rulemaking action, publication for public comment would already have been made. However, we believe that the delay will have significant benefits.

Recognizing that implementation of a performance testing program is complex and will have a great impact on the dosimetry suppliers and licensees, the NRC has decided to hold a public meeting on this subject prior to publishing a proposed rule for comment.

One would hope that the past efforts in this endeavor would provide some guidance for this current attempt at developing a system for testing the accuracy of personnel dosimetry services. Considering the past obstacles which caused delay and defeat, three points can be identified, which should be considered in the development of any mandatory testing program:

1. It is impractical to assume that a highly competitive industry will take a unified action to police itself in such a manner as to satisfy regulatory authorities without adequate regulatory incentives.

2. A regulatory authority will not be in a position to approve or recognize the testing laboratory results unless that testing laboratory is either an authoritative body, an arm of the regulatory authority or a contractor to the regulatory authority. Providing continuing regulatory recognition to a testing or certification organization implies that the regulatory agency can oversee the operation of that organization and/or the testing organization has national recognition and peer acceptance.

3. The criterion for judging acceptability of a supplier's process should be based on the health physics needs of the users rather than the capability of individual systems developed from prior testing. This will provide incentive for improving dosimetry performance independent of the system used for evaluating that performance.

\section{REFERENCES}

1. Staff Reports: Intercomparison of Film Badge Interpretations. Isotopics (No.2) 5:8-33, April 1955.

2. W. A. Longmead, N. Adams, Investigations of the Accuracy Attained in Routine Film Badge Dosimetry, United Kingdom Atomic Energy Authority, AHSB(RP)R62, 1966.

3. R. O. Gorson, N. Suntharalingam and John W. Thomas, Results of Film-Badge Reliability Study, Radiology, 84, No.2, 333-346, February 1965.

4. D. E. Barber, Film Badge Service Performance, Radiological Health Data and Reports, Vol. 7, No. 11, 623-626, November 1966.

5. C. M. Unruh, H. V. Larson, T. M. Beetle, and A. R. Keene, The Establishment and Utilization of Film Dosimetry Performance Criteria, BNWL-52, Battelle Pacific Northwest Laboratory, September 1967 .

6. D. E. Barber, Standards of Performance For Film Badge Services, Public Health Service Publication No. 999-RH-20, September 1966.

7. L. L. Nichols, Performance Tests of Commercial Personnel Monitoring Services, Presented at Health Physics Society Meeting, Buffalo, N.Y., July 16, 1975 .

8. U. S. Atomic Energy Commission, Notice Regarding Need For Establishment of Film Dosimetry Calibration Laboratory 61F.R. 9411, September 1963.

9. U. S. Atomic Energy Commission, Press Release No. IN-616, September 9, 1965.

10. Criteria For Film Badge Performance, ANSI N13.7-1972, American National Standards Institute, July 11, 1972.

11. U. S. Atomic Energy Commission, Regulatory Guide 8.3, Film Badge Performance Criteria, February 1973.

12. Thermoluminescent Dosimetry Standards For Performance, Draft ANSI Standard, HPSSC/N B/42/WG-1.

13. Workshop No. 3, Personnel Dosimetry Evaluation and Control, 5th Annual National Conference on Radiation Control, DHEW Publication (FDA) 74$8008,342-343$, October 1973.

14. U. S. Nuclear Regulatory Commission, 
10 CFR Part 20, Standards for Protection Protection Against Radiation.

15. Radiation Protection Instrumentation and its Application, Section C, ICRU Report 20, Washington, D.C., 1971.
16. General Principles of Monitoring for Radiation Protection of Workers, ICRP Publication 12, 1969. 
OPTICAL INTERFEROMETRIC MEASUREMENTS OF ULTRASONIC RADIATION AND ITS APPLICATIONS TO MEDICINE

M. E. Haran and H. F. Stewart Bureau of Radiological Health Food and Drug Administration Rockville, Maryland 20852

A modified Michelson interferometer is currently being used to map particle displacement amplitude within a beam of pulses ultrasound. one leg of the interferometer is raster scanned across a 4 micrometer thick, opaque gold coated membrane upon which the sound is incident. The reference leg of the interferometer has been modified to provide stability. Sensitivity has been found to be at least 10 picometers which corresponds to 1.0 micro watts $/ \mathrm{cm}^{2}$ at $1.5 \mathrm{MHz}$. particle displacement amplitude is displayed in two modes: 1) displacement vs. position for each horizontal scan, 2) displacement vs. position for an entire frame by z-axis modulation of a CRO. Frame rates of $2 / \mathrm{sec}$. can be achieved. This system has been used to determine measured ultrasonic field patterns. Parameters of interest include peak intensity, intensity distribution, ratio of peak to average intensity and calculation of beam area. Methods for specifying beam areas will be discussed as well as the application of the system to evaluate medical diagnostic and therapy transducers. This system was developed by RCA Laboratories, Princeton, New Jersey.

\section{INTRODUCTION}

A modified Michelson interferometer is currently being used by the Bureau of Radiological Health (BRH) to map particle displacement within a beam of pulsed ultrasound. This system, developed by RCA Laboratories, Princeton, N.J., employs a $15 \mathrm{~mW}$ HeNe laser beam to raster scan a four (4) micrometer thick, opaque gold-coated membrane upon which sound is normally incident. Since the membrane is extremely thin compared to the acoustic wavelengths of interest, it is transparent to the sound while experiencing the same particle displacement as the surrounding transmission medium. At each point in the raster scan, the particle displacement amplitude is measured and displayed in two modes: 1) displacement vs. position for each horizontal scan, 2) displacement vs. position for an entire frame by $\mathrm{z}$-axis modulation of an oscilloscope. The first display has been calibrated to sensitivities corresponding to $10^{-6}$ watts $/ \mathrm{cm}^{2}$ of acoustic intensity.

Under the purview of the Radiation Control for Health and Safety Act, Public Law 90-602, the Bureau is currently investigating the output characteristics of medical ultrasonic devices. Some of these characteristics that can be measured by this interferometric system are peak intensity, intensity distribution, ratio of spatial peak to average intensity, and calculation of beam area. The last parameter is discussed in some detail since calculation of an area from experimental data requires a definition of beam area. This definition will influence the methods of data coliection as well as computational techniques. In this paper two methods for specifying beam area are discussed and compared. In addition, the interferometer is discussed in some detail and examples are given of its measurement capability.

\section{Description of system}

The optical interferometer is of the Michelson type and has been referred to as the "ultrasonovision." Figure 1 shows the basic optical arrangement. The beam from the NeHe laser is split into the two legs of the interferometer. In the reference leg is mirror $\mathrm{Ml}$. In the scanning leg are galvanometer mirrors M2 and M3 which provide the vertical and horizontal scanning, respectively. Lens L4 collimates the raster scanning beam to provide normal incidence to the thin, gold coated membrane (pellicle). The transducer is located behind the pellicle.

Characteristics of interferometers is the brightness curve (figure 2) relating the relative phase difference between the legs of the interferometer with the optical irradiance measured at the photodetector. The optimum operating condition for the ultrasonovision occurs when the relative phase difference is $\pi / 2$. At that point any phase change caused by motion of the pellicle will 
cause a maximum brightness change and the functional relationship between them will be nearly linear. To guarantee this operating condition an open loop technique is employed in which the reference mirror Ml is "wiggled" along the optic axis at a frequency of approximately $30 \mathrm{kHz}$. The amplitude of the wiggler is such that the relative phase difference is swept through at least I radians. At the photodetector the combined effect of the pellicle motion at the acoustic frequency and the wiggler motion is a signal varying in time as the acoustic pulse and contained in an envelope having the wiggler frequency. The peak of that envelope occurs when the relative phase difference is $\mathrm{i} / 2$. Therefore, peak detection of the photodetector signal results in the displacement amplitude at that scan location in the acoustic field. Another advantage of this technique is to eliminate noise due to thermal drift and vibration.

The operation of the interferometer is described by the following analysis.1. Consider an acoustic wave of angular frequency $\omega_{\mathrm{S}}$ and displacement amplitude $\Delta$ passing through the pellicle. When one point on the pellicle is interrogated by the laser, the photodiode generates a current $i_{S}$ having the form

$$
i_{S}=2 n \sqrt{I_{R} I_{p}} \cos \left[\Phi+\frac{4 \pi \Delta}{\lambda} \sin \left(\omega_{S} t\right)\right]
$$

where $\eta$ is the quantum efficiency of the photodiode, $I_{R}$ and $I_{p}$ are the irradiances in the reference and pellicle legs of the interferometer, respectively, $\Phi$ is the relative phase difference between legs, and $\lambda$ is the optical wavelength. The d.c. terms of this equation have been eliminated by the use of high pass filter. If $\Delta / \lambda<1$ and $\Phi=$ I/ $/ 2$ then equation (1) reduces to

$$
i_{S}=8 \pi n \frac{\Delta}{\lambda} \sqrt{I_{R} I_{p}} \sin \left(\omega_{S} t\right),
$$

where the signal amplitude is directly proportional to the displacement amplitude of the acoustic wave. This approximation is good for acoustic intensities up to several watts $/ \mathrm{cm}^{2}$.

The introduction of the wiggler for the reference mirror alters equation (2) such that

$$
\begin{aligned}
i_{S}= & 8 \pi n \frac{\Delta}{\lambda} \sqrt{I_{R} I_{P}} \sin \left(\omega_{S} t\right) \\
& \sin \left[\Phi+\frac{\pi}{2} \sin \left(\omega_{w} t\right)\right] .
\end{aligned}
$$

If the wiggler displacement is greater than $\lambda / 4$ and the ratio of the acoustic frequency to wiggler frequency is sufficiently high, then the relative phase difference will be $\mathbb{I} / 2$ at the same time $\sin \left(\omega_{\mathrm{S}} t\right)=1$. Therefore, peak detection of the wiggler envelope will yield the displacement amplitude at the point of interrogation.

The sensitivity of the system is determined by thermal noise and shot noise. At low light levels thermal noise predominates. For a pellicle leg irradiance of $0.1 \mathrm{mw}$ and unity signal to noise ratio, the particle displacement is on the order of $0.03 \AA$. From equation (3) it can be seen that lower displacement amplitudes can be detected for higher irradiances at a fixed photodetector signal. It has been estimated that the ultimate system sensitivity is $10^{-10} \mathrm{~W} / \mathrm{cm}^{2}$ (acoustic) at $1.5 \mathrm{MHz}$. The $\mathrm{BRH}$ system presently can detect displacements lower than $0.1 \AA$ corresponding to $10^{-6} \mathrm{~W} / \mathrm{cm}^{2}$ (acoustic) at $1.5 \mathrm{MHz}$.

The system was calibrated by illuminating the photodetector with a known intensity of light and measuring the resultant voltage after the photodetector preamplifier. An NBS C-series laser calorimeter was used to determine the irradiance. The total estimated uncertainty is on the order of $+4 \%$.

\section{Examples}

Several transducers were scanned at various distances along the propagation axis. These scans show the ability of the ultrasonovision to measure the radiating patterns from both NDT and medical transducers.

Figure 3 shows the development of the acoustic radiation from a $2.0 \mathrm{MHz}$, air-backed ceramic transducer. The pulse width was of the order of $9 \mu \mathrm{s}$. and the crystal diameter was $1.3 \mathrm{~cm}$. At each location in the field two displays are shown. The first is a brightness modulated picture of the beam cross-section. The grey scale was individually adjusted for each photograph to show maximum detail. Therefore, no comparion can be made between grey levels of any two photos. The second display is a single horizontal scan through the beam center. The horizontal axis corresponds to scan position and the verticle axis corresponds to particle displacement amplitude. The verticle and horizontal scales are the same 
within each scan series of a transducer for purposes of comparison.

An immersion NDT transducer is shown in figure 4. It is nominally rated at $1.0 \mathrm{MHz}$, 1.0 inch diameter, and $6.0 \mathrm{~cm}$ focal length. Using a Hewlett-Packard 4815A RF vector impedance meter the resonant frequency was found to be $1.16 \mathrm{MHz}$. This transducer was driven at its resonant frequency with a pulse width of 9.0us. According to theory, the diffraction pattern produced at the focus of a finite aperture lens is the product of the Fourier transforms of the aperture function and the field distribution across that aperture. For a plane distribution across a circular aperture, the focal pattern takes the form of a Besinc function ${ }^{2}$. This function is characterized by a large central peak (Airy disc) and several concentric rings (Bessel rings) of lower amplitude.

Referring to figure 4 , it can be seen that the distribution at the transducer face does not occupy the full aperture nor is it plane.

Measurement of the Airy disc shows it to correspond to an aperture diameter of 0.83 inches. This is confirmed in the scan of the transducer face.

Figures 5 through 8 are scans of medical diagnostic transducers. Each is nominally rated as having a $2.25 \mathrm{MHz}$ resonant frequency. As before, the vector impedance meter was used to determine the resonant frequency and each transducer was driven at the resonant frequency with a 9.0us. pulse width to produce each figure.

Figure 5 shows a nominal 19 mm diameter, $10 \mathrm{~cm}$. I.F. (internally focussed) transducer driven at its resonant frequency of $2.12 \mathrm{MHz}$. At the focus $(10 \mathrm{~cm})$ the Bessel rings are not well defined because of the lack of uniform amplitude distribution at the transducer face.

The transducer depicted in figure 6 has a nominal $13 \mathrm{~mm}$ diameter radiating area, no focussing, and a $2.05 \mathrm{MHz}$ resonant frequency. The uneven amplitude pattern at the transducer face is carried through in near field in the unusual structure of the field pattern.

In figures 7 and 8 are two biopsy transducers having resonant frequencies of 2.91 $\mathrm{MHz}$ and $2.52 \mathrm{MHz}$, respectively. The first shows a relatively uniform amplitude distribution at the transducer face and the field development is relatively even. The second transducer radiates primarily from the upper right quadrant of the face, and this is reflected in the development of the field. It is interesting to note that these last two transducers were production line rejects because they failed leakage current tests but not for their acoustic behavior.

Beam Area

During medical diagnostic imaging or flaw detection, the beam area (lateral dimensions for pulses) strongly determines the effective resolution of adjacent objects. The definition of beam area or radiating area at the crystal face must be sufficiently broad to include the various outputs demonstrated in the previous figures. In its specification for ultrasonic therapeutic equipment, the American National Standards Institute (ANSI) defines effective radiating area as "that [area] which is 1.11 times the area, measured at the applicator face and using circular baffles..., which transmit 90 percent of the effective acoustic power. " 3 The difficulty with this definition is its assumption of a radially symmetric distribution of acoustic intensity. Significant errors can occur when applying this definition to transducers of the type shown in figure 9. This is a commercial therapy transducer of multiple crystal construction, the output from which is shown in this schlieren photograph.

As an alternative, the radiating area (or beam area depending on location) can be defined as the area in a plane normal to the beam axis consisting of the locus of all points at which the intensity is greater than a given percentage of the maximum intensity in that plane. Using this definition several transducers were scanned with the ultrasonovision at a distance of $4 \mathrm{~mm}$ from the transducer face. Each scan was digitized and area versus percent of peak intensity calculated on a Hewlett-Packard 9820 programable calculator. The results are plotted in figures 10 through 12. Each transducer is a therapy applicator and was driven at its nominal resonant frequency (1.0, 1.0, and $0.925 \mathrm{MHz}$ respectively).

Figures 10 through 12 show the beam areas calculated from the peak intensity, calculated from the ANSI definition, and that area specified by the manufacturer. Figure 12 corresponds to the multiple crystal therapy applicator whose schlieren 
photograph was shown above. The large discrepancies between measured areas and the manufacturers specification is apparent.

\section{SUMMARY}

The optical interferometric system has been shown to be an effective method to determine ultrasonic field patterns. With a sensitivity below $0.1 \AA$, this system can detect acoustic displacement amplitudes corresponding to intensities below 1.0 microwatts $/ \mathrm{cm}^{2}$. It has been used on therapy, diagnostic and NDT type transducers to measure peak intensity, intensity distribution and beam area.

In the calculation of beam area, an alternative to the ANSI definition has been suggested using the peak intensity in the plane of measurement. This definition has been shown to be more general and applicable to transducers of multiple crystal design as well as those with asymmetric radial intensity distributions. It is felt that this alternative definition would better apply to the specification of transducer characteristics.

\section{References}

1. R.S. Mezrich, K.F. Etold, and D.H.R. Vilkomerson "System for Visualizing and Measuring Ultrasonic Wavefronts", RCA Review, Vol. 35, No. 4, Dec 1974, p. 483-519.

2. G.B. Parrent, Jr., and B.J. Thompson Physical Optics Notebook, Society of Photo-optical Instrumentation Engineers Redondo Beach, Calif. 1969, p.5.

3. American National Standard Specification for Ultrasonic Therapeutic Equipment z24.18-1956, para. 2.3.4. 


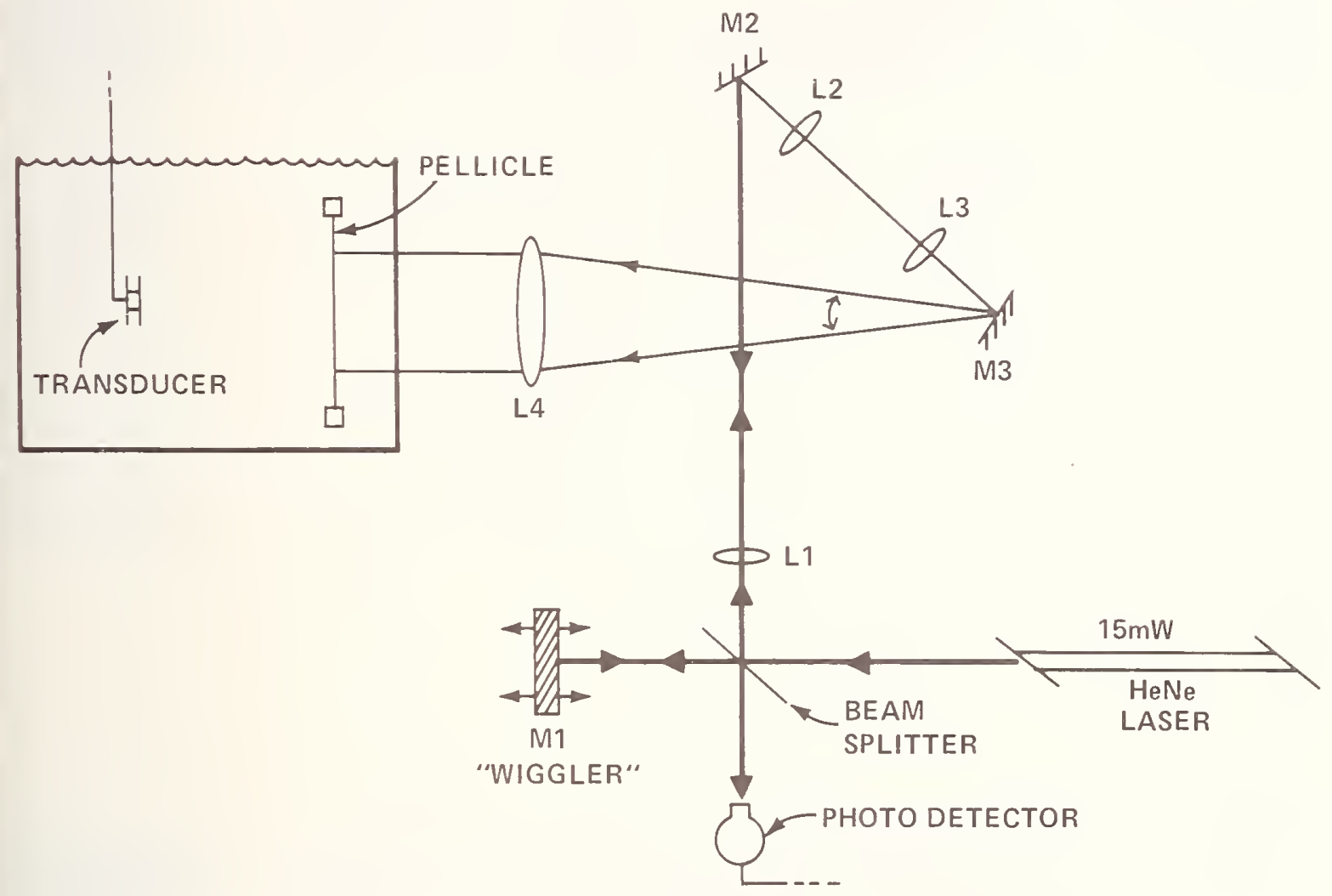

Figure 1. Diagram of Scanning Optical Interferometer 


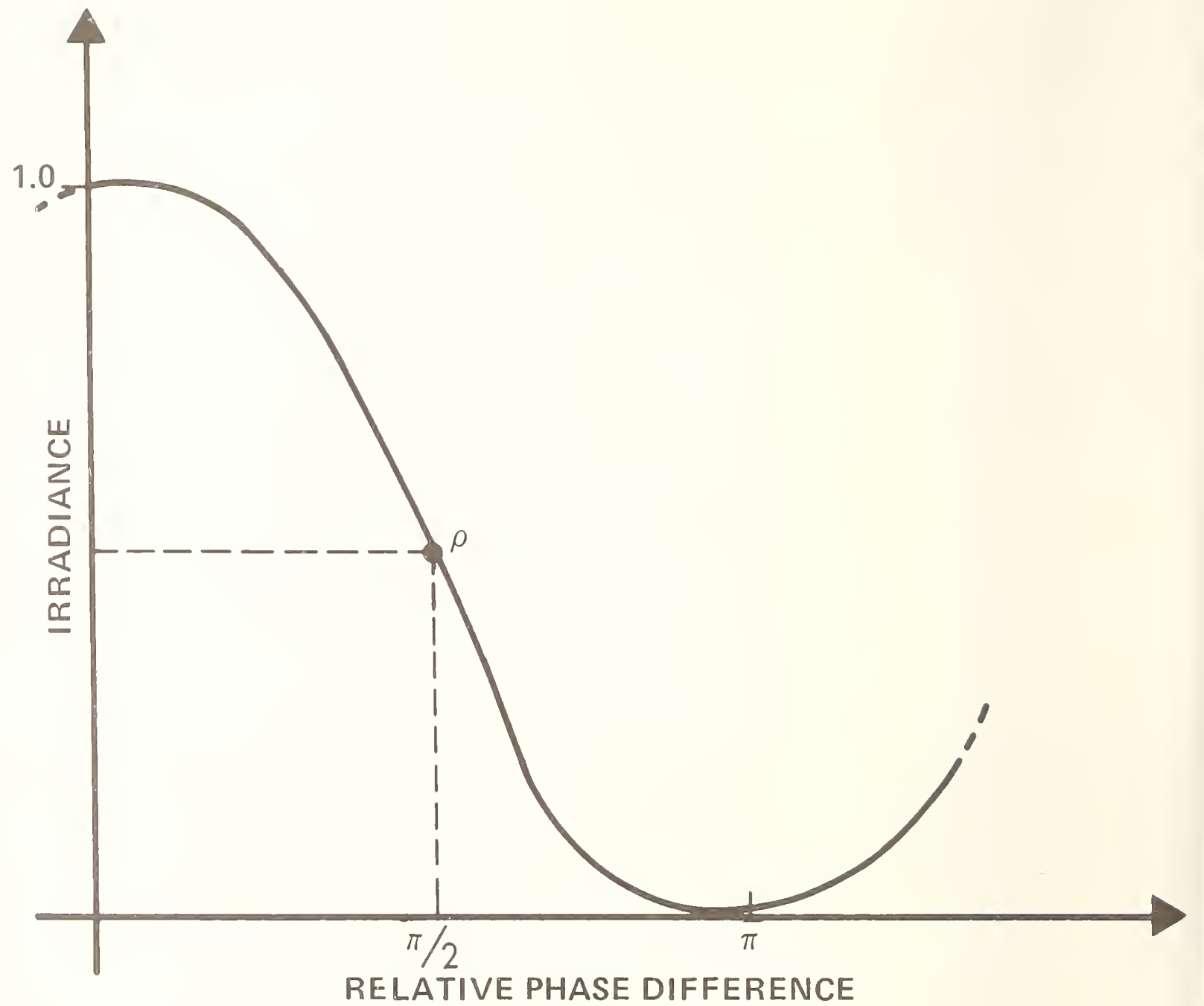

Figure 2. Brightness Curve Showing the Relationship of Relative Phase Difference Between Interferometer Legs And Optical Irradiance Upon Recombination 

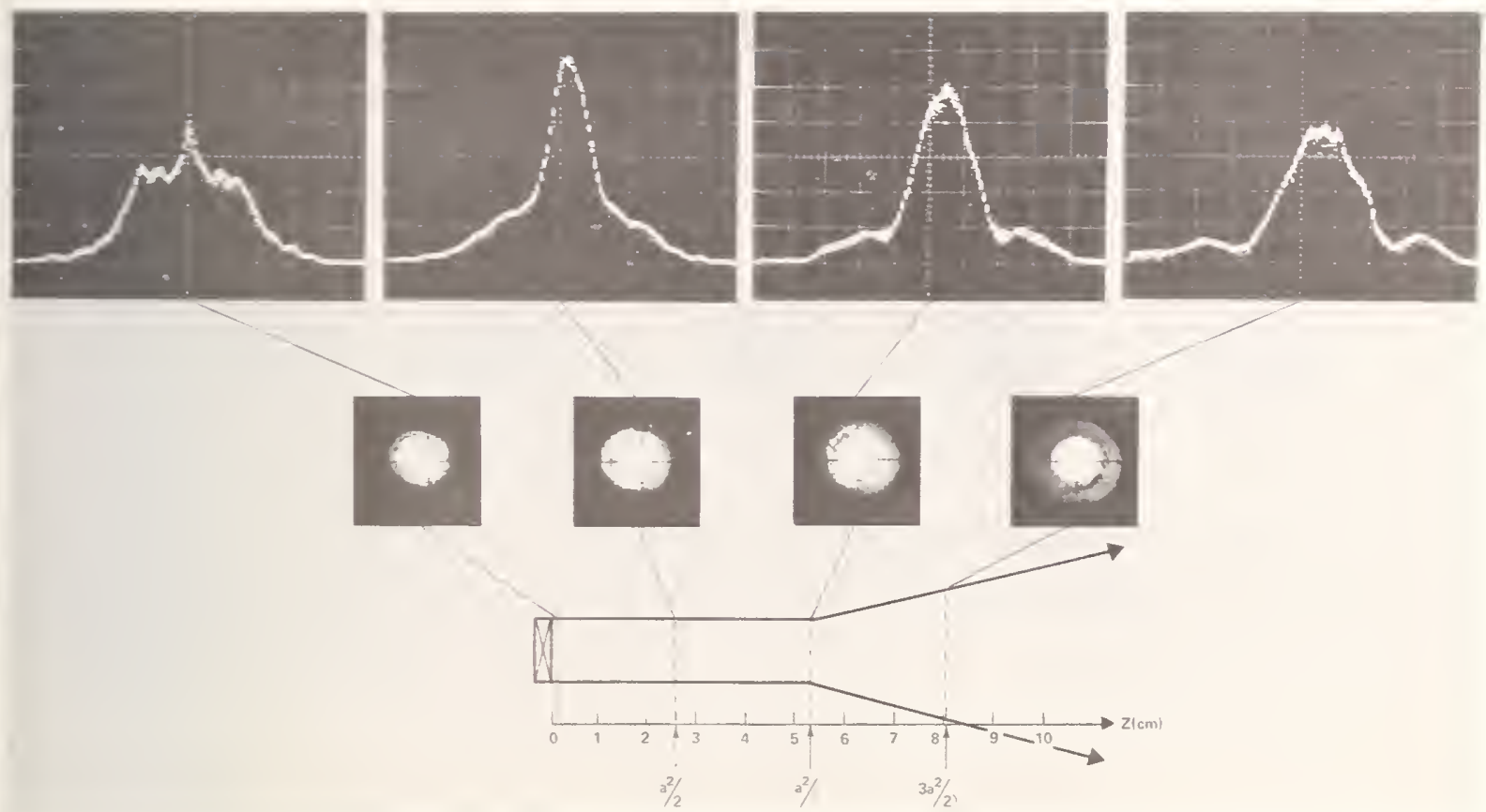

Figure 3. Multiple Scans of Air-Backed Ceramic Transducer ( $f=2.0 \mathrm{HMz}, \mathrm{d}=1.3 \mathrm{~cm}$ ). Scan Width of Singe Traces is $3.0 \mathrm{~cm}$.
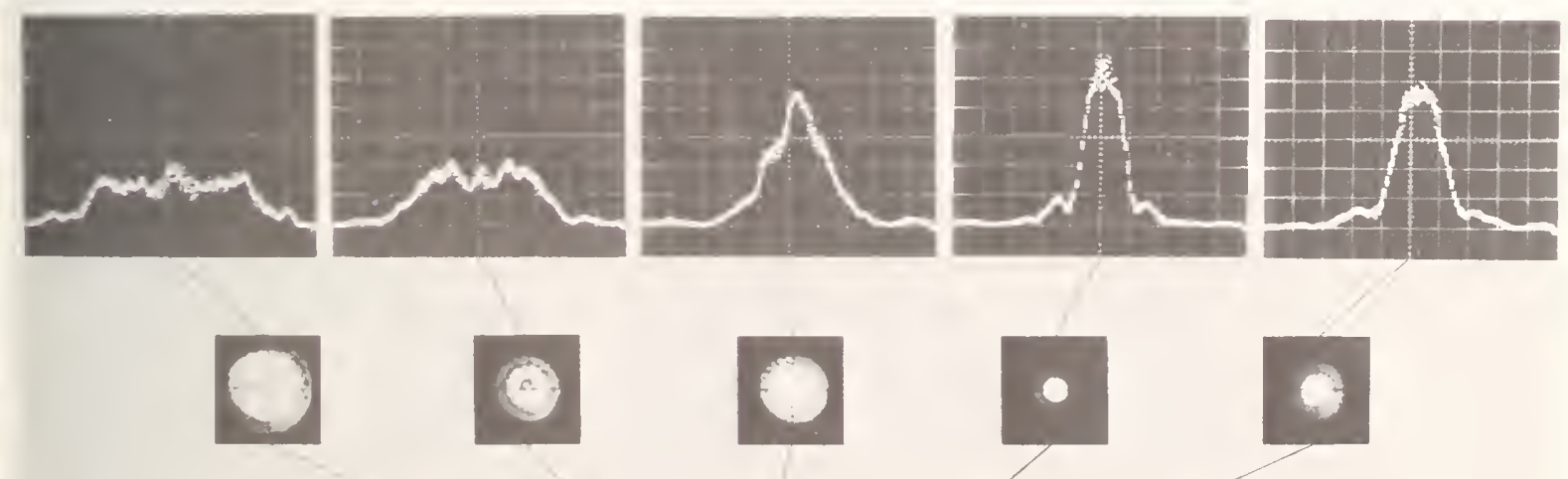

Figure 4. Multiple Scans of NDT Transducer ( $f=1.16 \mathrm{MHz}, d=2.5 \mathrm{~cm}$, focus $=6.0 \mathrm{~cm}$ ). Scan Width of Single Traces is $3.2 \mathrm{~cm}$. 

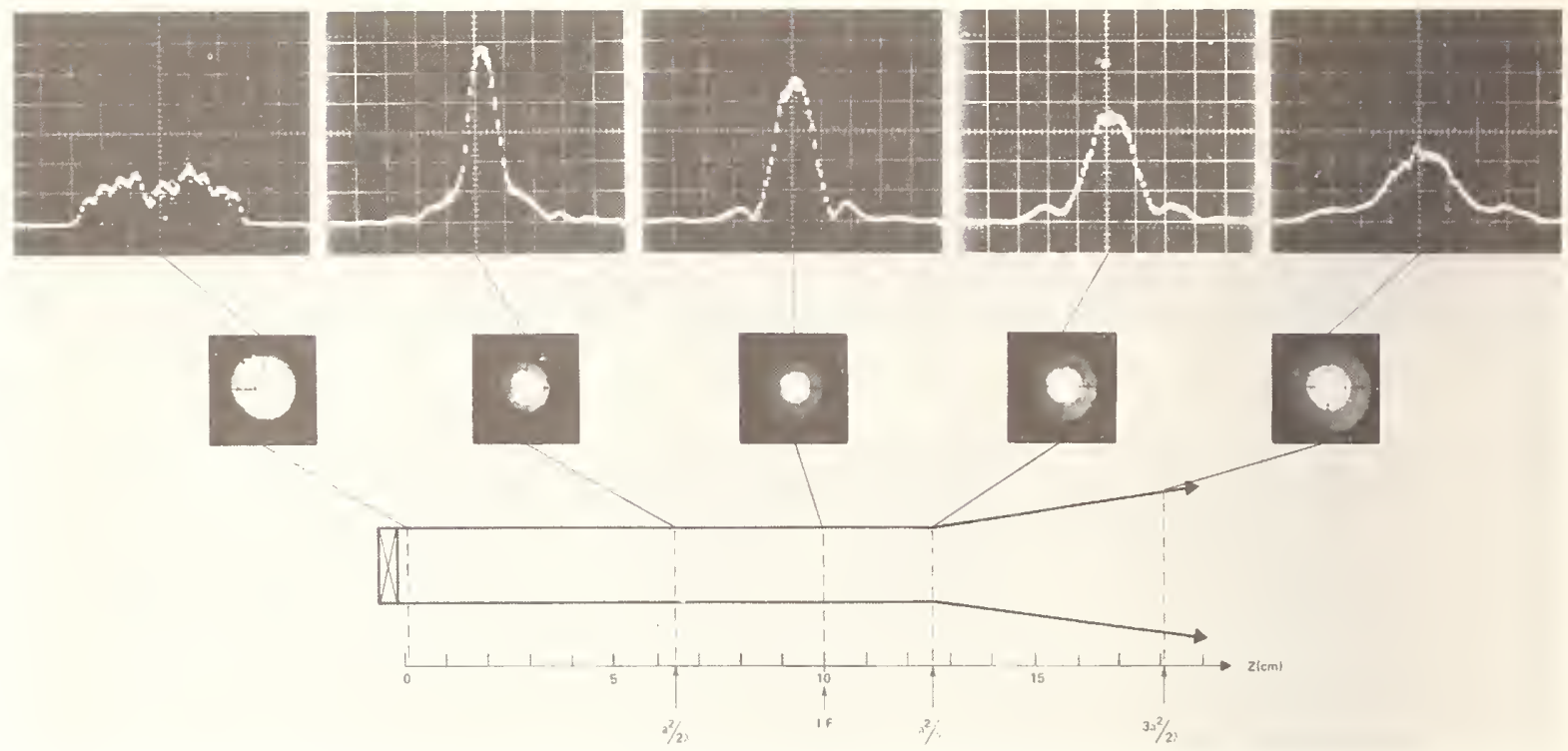

Figure 5. Multiple Scans of Diagnostic Transducer ( $f=2.12 \mathrm{MHz}, d=1.9 \mathrm{~cm}, I . F .+10 \mathrm{~cm}$ ) Scan Width of Single Traces is $3.5 \mathrm{~cm}$.
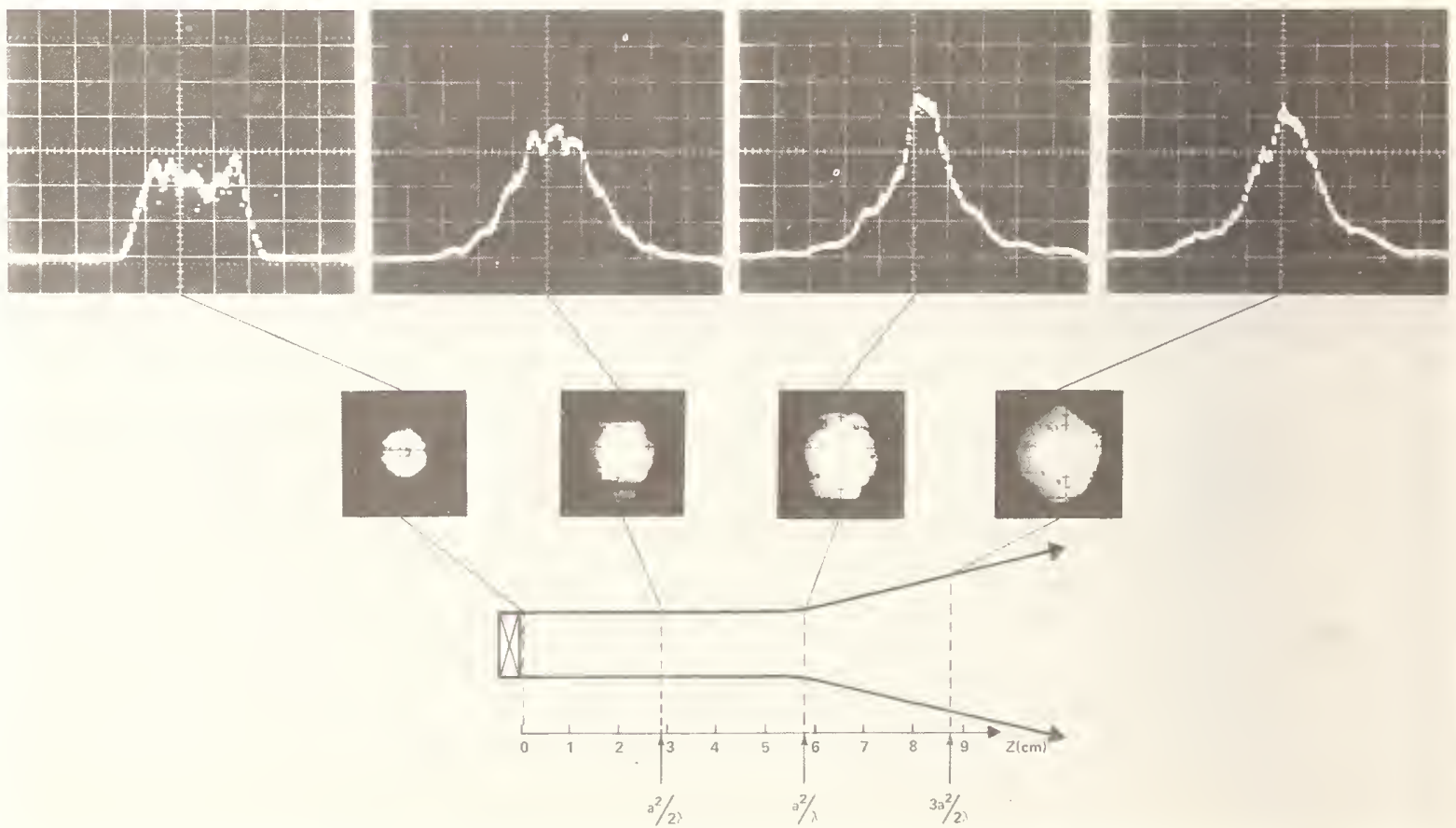

Figure 6. Multiple Scans of Diagnostic Transducer $(f=2.05 \mathrm{MHz}, d=1.3 \mathrm{~cm})$. Scan Width of single Traces is $3.5 \mathrm{~cm}$. 

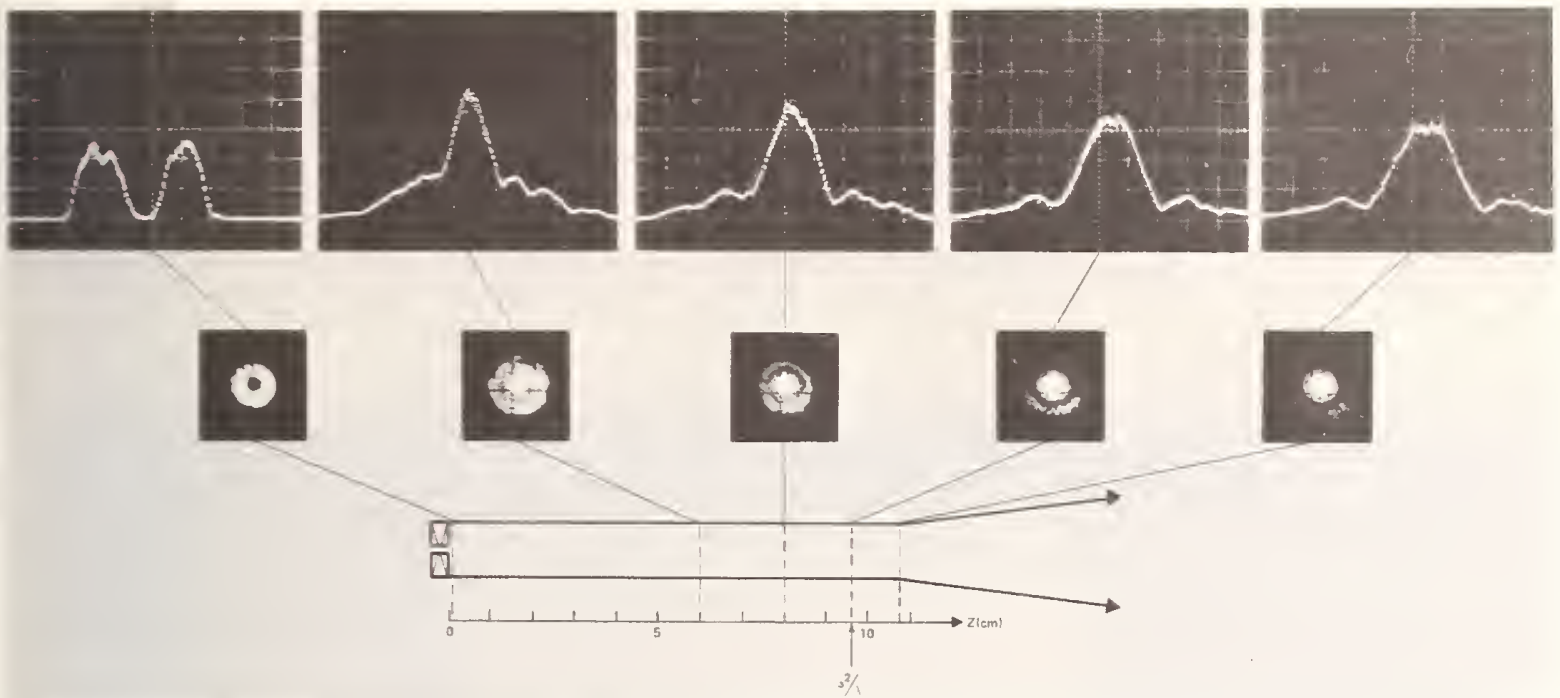

Figure 7. Multiple Scans of Biopsy Transducer ( $f=2.91 \mathrm{MHz}, d=1.3 \mathrm{~cm}$ ) . Scan width of single Traces is $3 \mathrm{~cm}$.
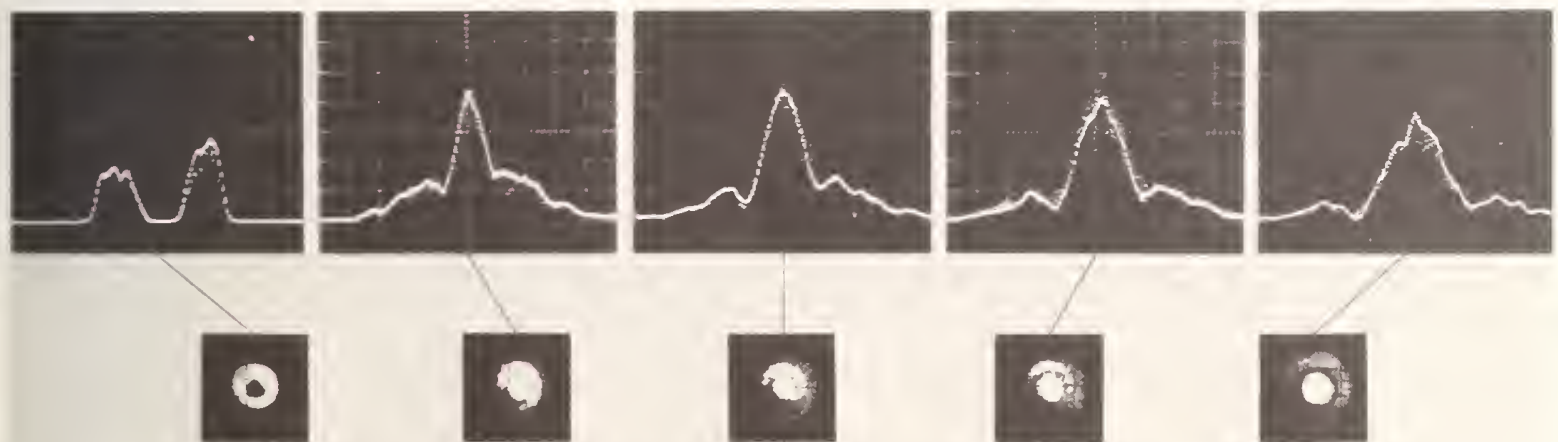

a.

Figure 8. Multiple Scans of Biopsy Transducer $(f=2.52 \mathrm{MHz}, d=1.3 \mathrm{~cm}$ ) . Scan width of Single Traces is $3 \mathrm{~cm}$. 


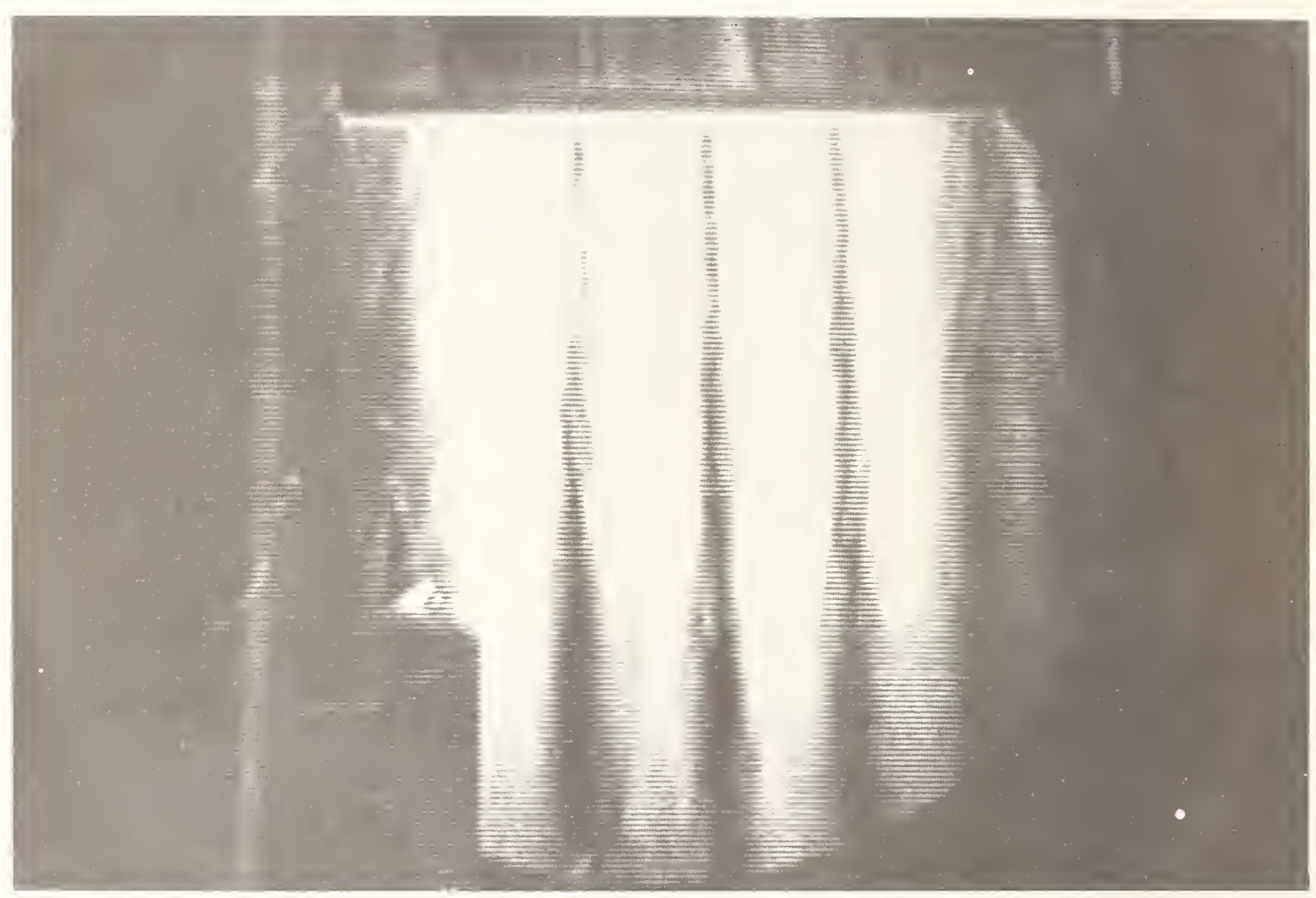

Figure 9. Schlieren Photograph Showing Output From A Commercial Multiple Crystal Therapy Applicator. 


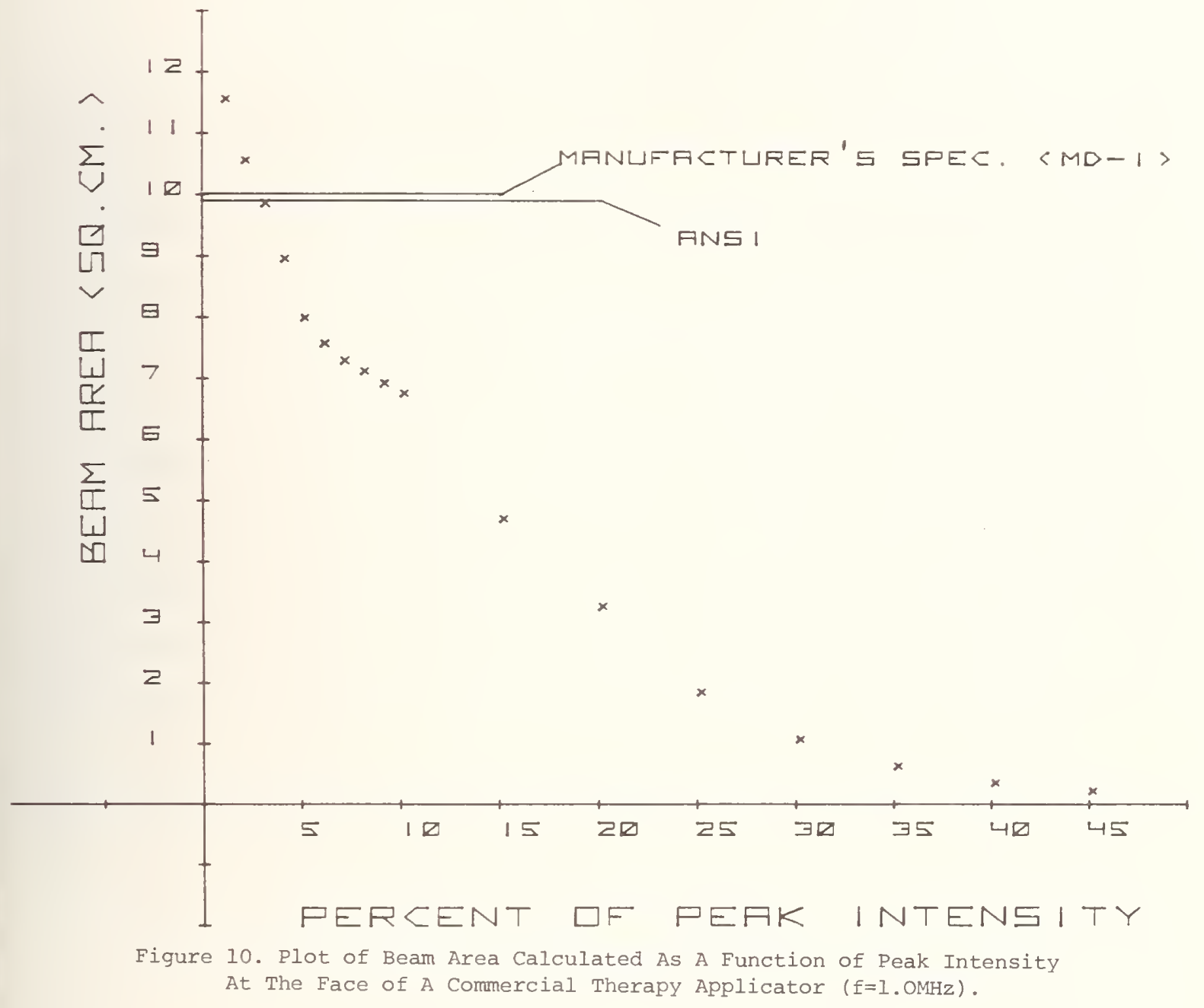




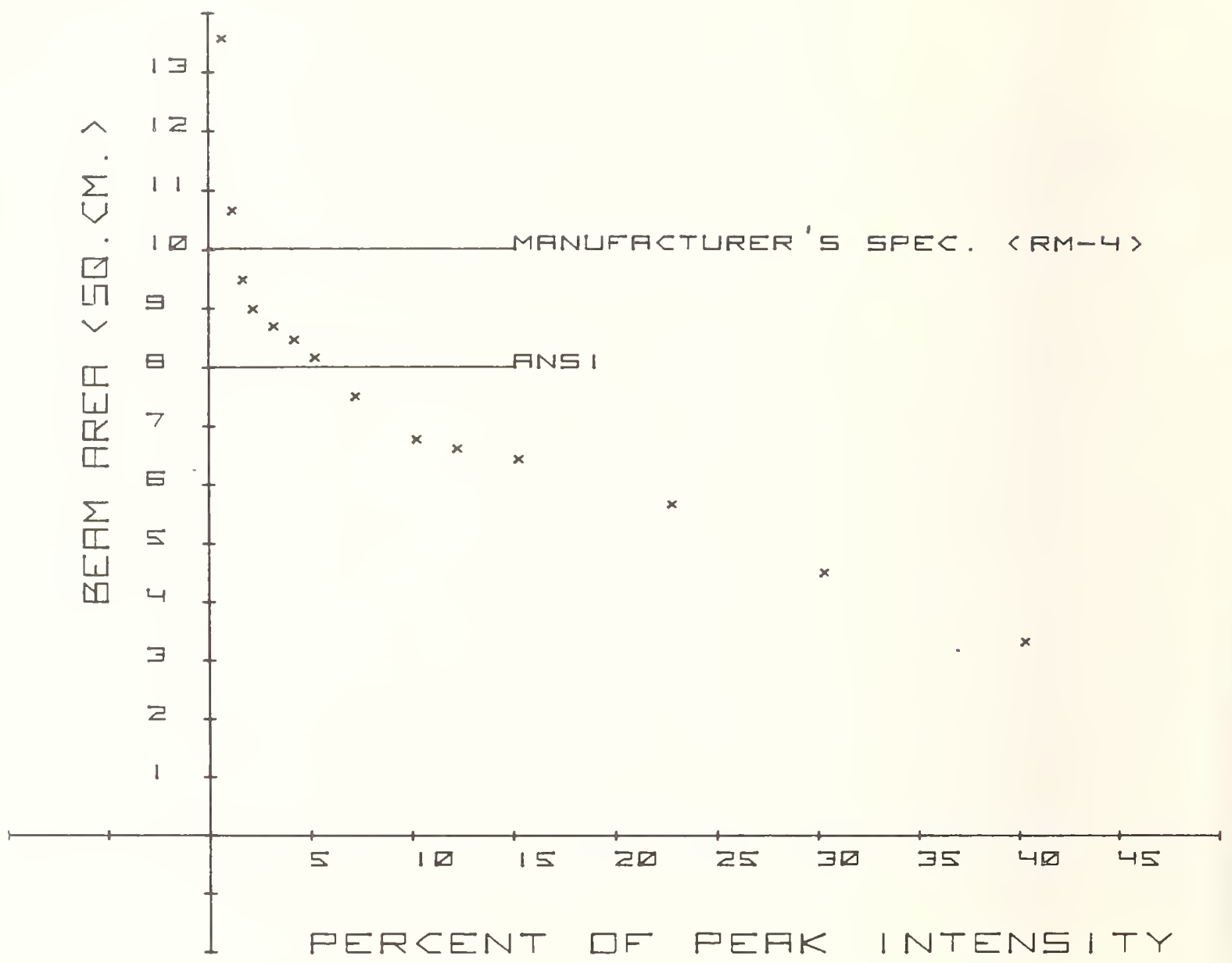

Figure 11. Plot of Beam Area Calculated As A Function of Peak Intensity At The Face of A Commercial Therapy Applicator ( $\mathrm{f}=1$. OHMz). 


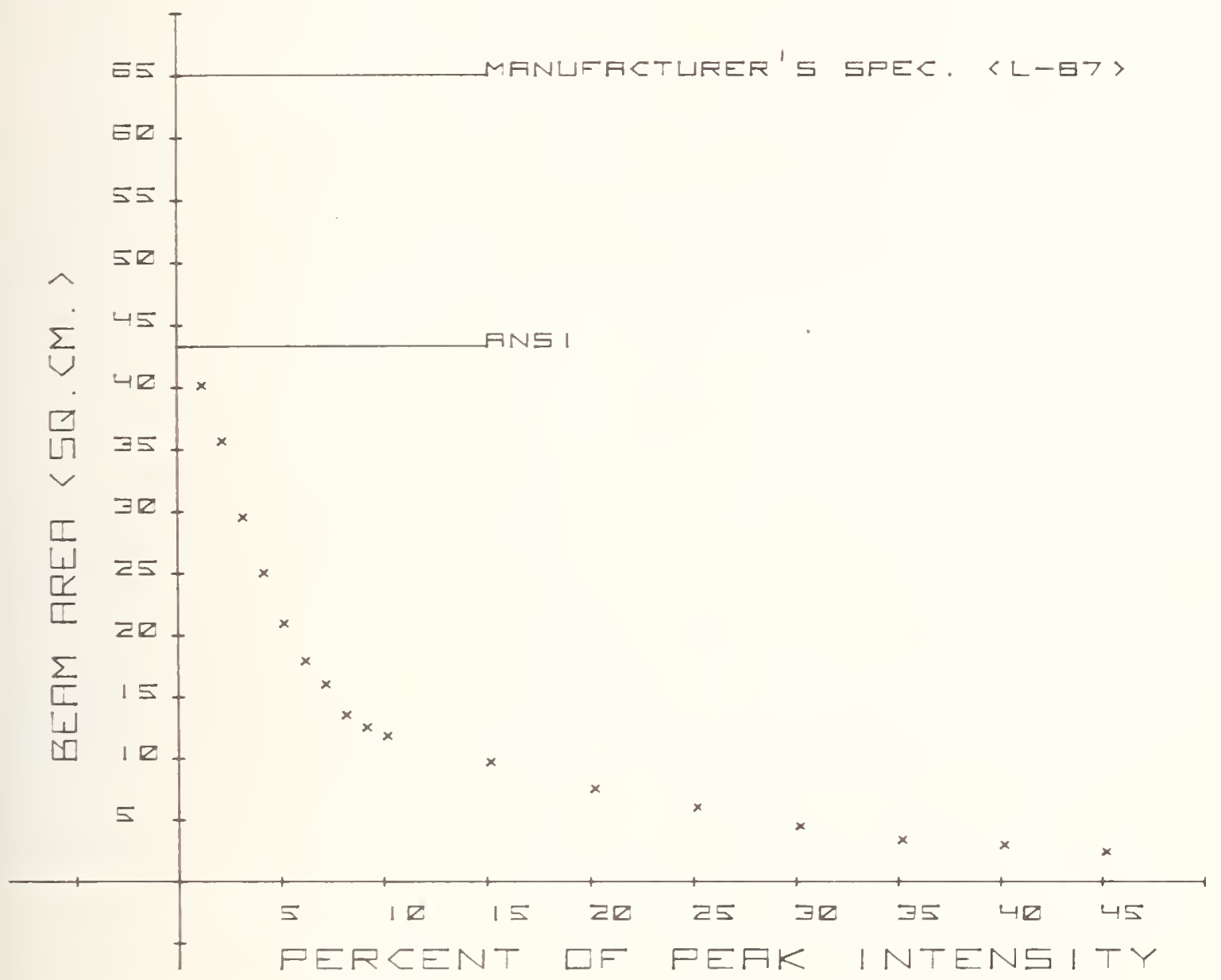

Figure 12. Plot of Beam Area Calculated As A Function of Peak

Intensity At The Face of The Commercial

Therapy Applicator of Figure 9. ( $\mathrm{f}=925 \mathrm{kHz})$. 

PHOTON MASS ENERGY-ABSORPTION COEFFICIENT AIR/MEDIUM RATIOS FOR IONOMETRIC DOSIMETRY $0.1 \mathrm{keV}$ TO $20 \mathrm{MeV*}$

\section{J. H. Hubbel1}

National Bureau of Standards

The determination of photon absorbed dose in a medium by means of an air-ionization chamber depends in part on the ratio of the mass energyabsorption coefficient $\mu_{\text {en }} / \rho$ for air to that for the chamber wall material, to account for the differing atomic compositions. In this paper, mass attenuation coefficients $\mu / \rho$ developed at the National Bureau of Standards by the X-Ray and Ionizing Radiation Data Center from the latest theoretical cross section data are compared with measurements for selected low-Z elements of dosimetric interest. From this cross section data base, mass energy-absorption coefficients $\mu$ en $/ \rho$ are derived for air and selected media, along with air/medium ratios, over the photon energy range $0.1 \mathrm{keV}$ to $20 \mathrm{MeV}$ including values at the cesium-137 and cobalt-60 energies. Comparisons are made with earlier published $\mu_{\mathrm{en}} / \rho$ values and air/medium ratios.

(Attenuation coefficient; cross section; dosimetry; energy-absorption coefficient; gama-ray; photon; x-ray)

\section{Introduction}

The mass energy-absorption coefficient $H_{\text {en }} / \rho$ enters into medical and industrial irradiation $\mathrm{x}$-ray metrology in various essential ways. For example, as has recently been discussed by Loevinger ${ }^{1}$ and by Niatel, Loftus and Oetzmann, ${ }^{2}$ present reference standards for ganma-ray beam calibration in the cesium-137 $(0.66 \mathrm{MeV})$ and cobalt-60 (1.17, $1.33 \mathrm{MeV})$ energy region rely on air cavity ionization detectors by which the exposure rate $\dot{x}$ at a point in the radiation field is determined as

$$
\dot{\mathrm{X}}=\frac{I}{\mathrm{~m}} \cdot \frac{1}{\overline{\mathrm{f}}} \cdot \frac{\left(\mu_{\text {en }} / \rho\right)}{\left(\mu_{\text {en }} / \rho\right)_{\text {wall }}} \cdot \pi \mathrm{K}_{j}
$$

where $\mathrm{I} / \mathrm{m}$ is the measured ionization current per unit mass of air in the cavity, $\bar{f}$ is the ratio of the average mass collision stopping powers for air and the cavity wall material and $\Pi \mathrm{K}_{\mathrm{j}}$ is the product of additional correction factors.

As defined in ICRU Report $19^{3}$ and schematized in Fig. 1, the mass energy-absorption coefficient $\mu_{\text {en }} / \rho$ may be written as

*Work supported by the NBS Office of Standard Reference Data.

$$
\begin{aligned}
\mu_{\text {en }} / \rho & =\frac{\mu_{\text {inc. }}}{\rho} \cdot \mathrm{f}_{\text {inc. }} . \\
& +\frac{\mu_{\tau}}{\rho} \cdot \mathrm{f}_{\tau}+\frac{\mu_{K}}{\rho} \cdot \mathrm{f}_{K}
\end{aligned}
$$

in which $\mu_{i n c} / \rho, \mu_{\tau} / \rho$ and $\mu_{K} / \rho$ are the contritributions to the mass attenuation coefficient $\mu / \rho$ from incoherent (bound-electron Compton) scattering, photoeffect and pair-production (including triplet) respectively. These

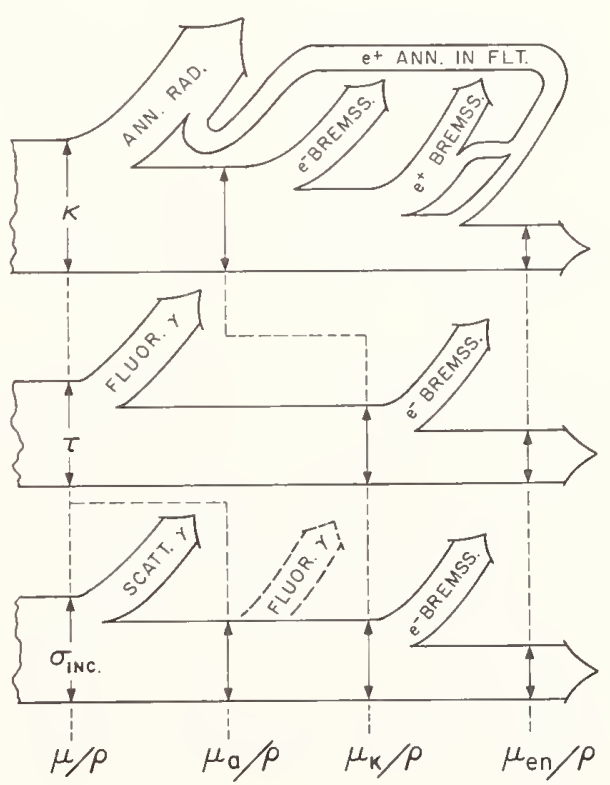

Fig. 1. 
partial coefficients $\mu_{i} / \rho$ are related to the respective atomic cross sections $\sigma_{i}$ as

$$
\mu_{i} / \rho=\sigma_{i} \cdot\left(N_{A} / M\right)
$$

where $\mathrm{N}_{\mathrm{A}}$ is Avogadro's number and $\mathrm{M}$ is the atomic weight. In Eq. (2) each factor $\mathrm{f}_{i}$ weights the respective partial cross section $\sigma_{i}$ by the average fraction of the initial photon energy transferred to kinetic energy of charged particles. The remaining fraction $\left(1-f_{i}\right)$ of the initial photon energy includes secondary photons from the primary interaction, such as Compton-scattered and fluorescence photons, and from subsequent radiative processes of the charged particles such as bremsstrahlung and positron annihilation.

Previous Compilations of $\mu_{\text {en }} / \rho$

The first major reviews and tabulations of the mass energy-absorption coefficient were the independent efforts of R. T. Berger ${ }^{4}$ and J.W.Allison. ${ }^{5}$ The Berger tabulation (under the name "energy transfer coefficient") included 15 elements plus air and water over the range $3 \mathrm{keV}$ to $10 \mathrm{MeV}$ and took into account positron annihilation in flight as well as Compton scattered photons (but neglecting electron binding effects), fluorescence, and bremsstrahlung by Compton and pair electrons. A procedure for including photoelectron bremsstrahlung was described but not used by Berger. Allison's tabulation (under the name "absorption coefficient") for seven elements plus air and water for energies $10 \mathrm{keV}$ to $100 \mathrm{MeV}$ was similar to Berger's except for the inclusion of photoelectron bremsstrahlung and omission of positron annihilation in flight.

As part of the continuing X-Ray and Ionizing Radiation Data Center program at NBS the Berger results were updated in NSRDSNBS $29^{6}$ for 18 elements plus air and water over the range $10 \mathrm{keV}$ to $10 \mathrm{MeV}$ using revised basic cross section data and $f_{i}$ values interpolated from Berger. ${ }^{4}$ Earlier versions of this revised set of $\mu_{\text {en }} / \rho$ values were also published in Radiation Dosimetry, ${ }^{7}$ the IAEA Engineering Compendium on Radiation Shielding and The Physics of Radiology. 9 Storm and Israel $1^{10}$ used similar procedures to derive $\mu_{\text {en }} / \rho$ values for a 11 elements $Z=1$ to 100 over the range $1 \mathrm{keV}$ to $100 \mathrm{MeV}$. They included electron binding effects in their incoherent (Compton) scattering cross sections, combined with free-electron energyabsorption fractions. The Storm and Israel energy-absorption fractions in the range 10 to $100 \mathrm{MeV}$ were taken from Al.1ison ${ }^{5}$ and hence do not include positron annihilation in flight.

Additional tabulations of $\mu_{\text {en }} / \rho$ include those given in ICRU Report 10b (NBS Handbook $85)^{11}$ relying primarily on the Berger ${ }^{4}$ data, for 12 elements and 8 mixtures over the range $10 \mathrm{keV}$ to $10 \mathrm{MeV}$. This tabulation was revise and extended down to $1 \mathrm{keV}$ in ICRU Report $17^{1}$ for 12 elements and 9 mixtures over the energy range 1 to $150 \mathrm{keV}$. In deriving the latter tabulation, the basic interaction cross sections used were combined and smoothed from (1) NSRDS-NBS 29,6 (2) NcMaster et a1, 13 and (3) an earlier version of Storm and Israel. 14 Present $\mu_{\text {en }} / \rho$ Compilation

The mass energy-absorption coefficients presented in table 1 for the five low $-Z$ elements $\mathrm{H}, \mathrm{C}, \mathrm{N}, \mathrm{O}$ and $\mathrm{Ar}$, and seven mixtures of these elements, extend previous $\mu_{\text {en }} / \rho$ compilations down to $0.1 \mathrm{keV}$ and incorporate new theoretical and experimental cross-section data evaluated by the NBS X-Ray and Ionizing Radiation Data Center from the published and report literature. The air composition was taken as $.755 \mathrm{~N}, .2320$ and .013 Ar by weight. The treatment of the incoherent scattering, photoeffect and pair production cross sections indicated in Eq. (2) is described below.

Incoherent (Bound-Electron Compton) Scatterin

The combined quantity $\left(\mu_{\text {inc. }} / \rho\right) \cdot \mathrm{f}_{\text {inc. }}$ was computed by numerical integration, over all outgoing photon angles, of the product of (1) the differential Klein-Nishina formula (2) the incoherent scattering function $\mathrm{S}(\mathrm{q}, \mathrm{Z})$ as recently tabulated by Hubbell et a1, 15 (3) the ratio $\mathrm{T} / \mathrm{k}$ of the Compton electron kinetic energy $\mathrm{T}$ to the incident photon energy $k$ and (4) the bremsstrahlung loss correction $[1-G(T, Z)]$ where $G(T, Z)$ is the "radiation yield" tabulated by Berger and Seltzer. ${ }^{16}$ The combined radiative and double-Compton correction as calculated by 
等

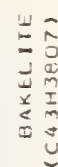

I $z$

㞶

늠

$\pm z$

uै

势$$
\text { (1) }
$$

总

$\frac{x}{4}$$$
z
$$

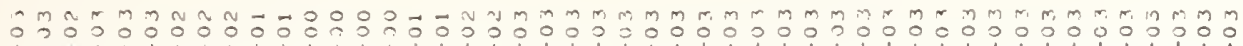

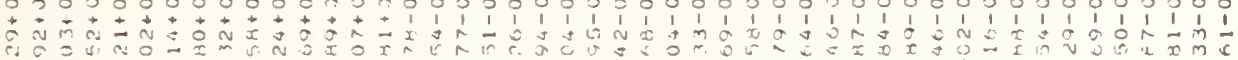

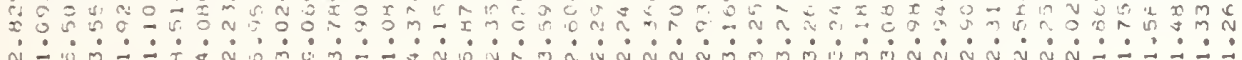

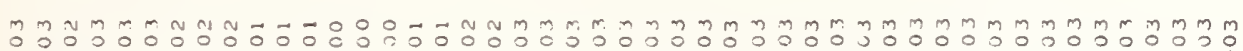

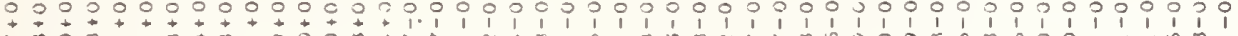

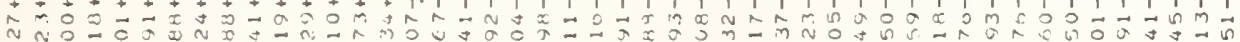

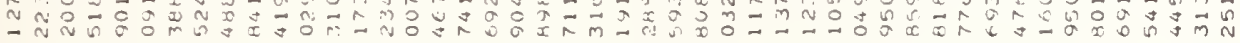

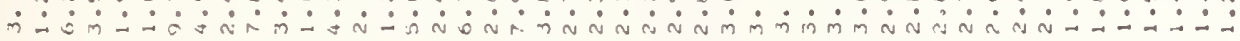

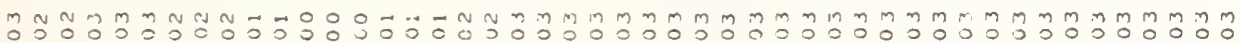

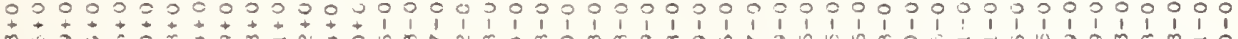
a

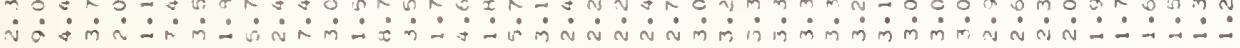

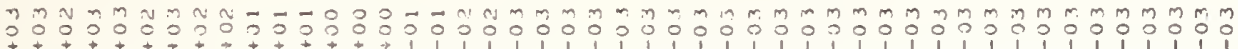

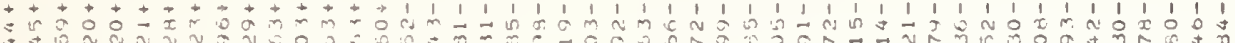

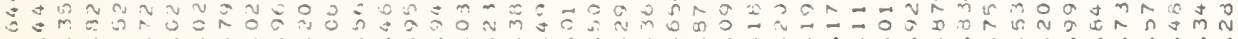

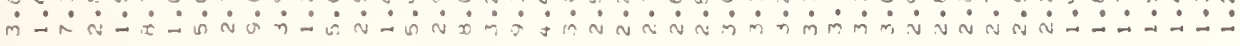

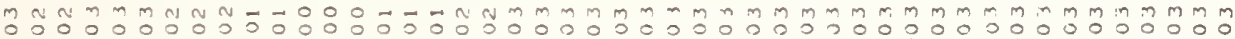

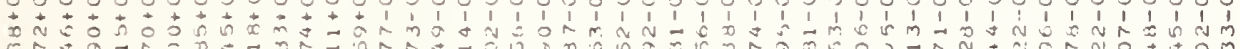

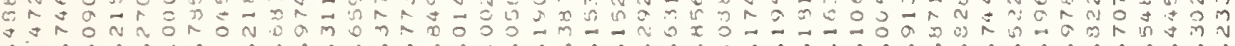

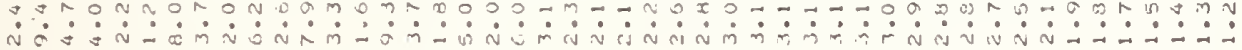

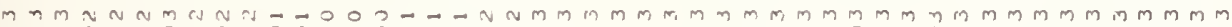

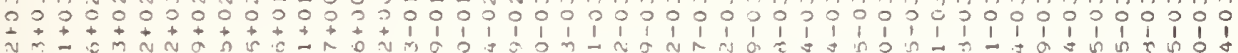

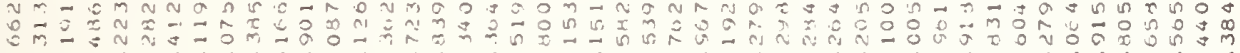

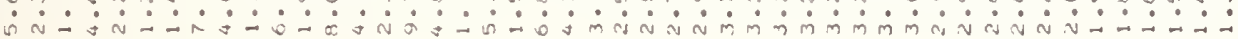

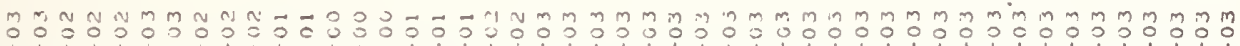

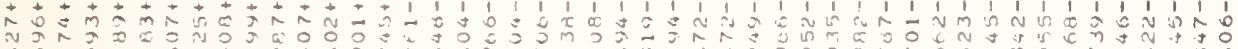
x $x^{x} \div$

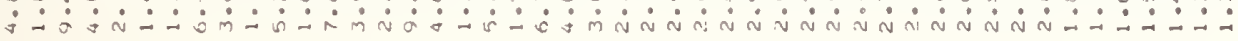

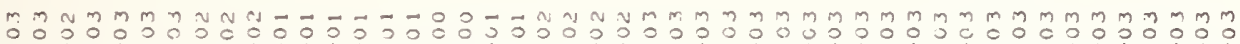

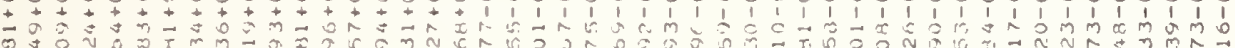

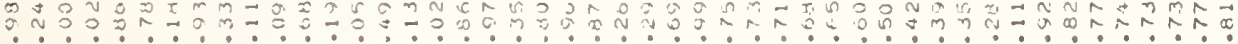

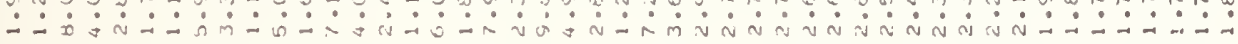

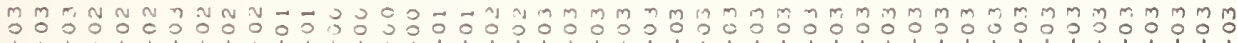

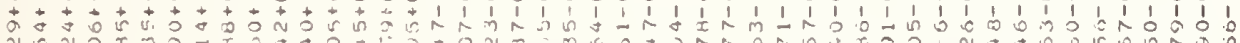

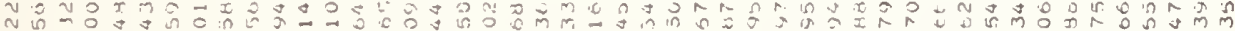

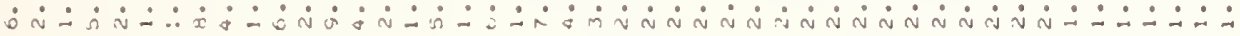

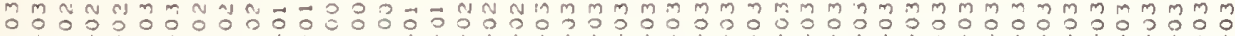
t.

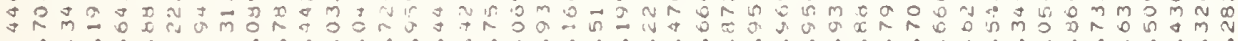

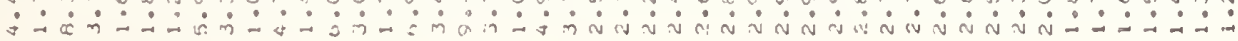

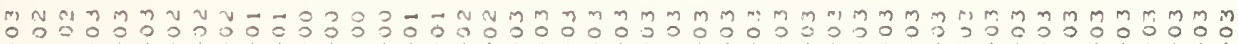

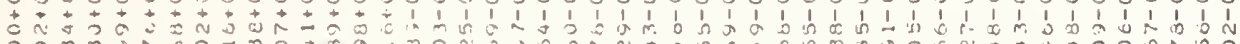

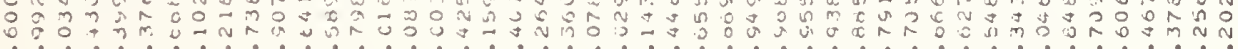

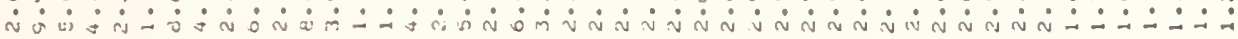

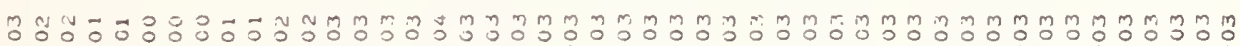

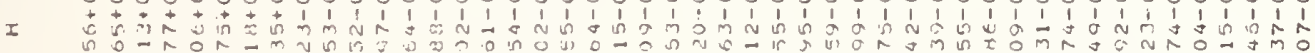

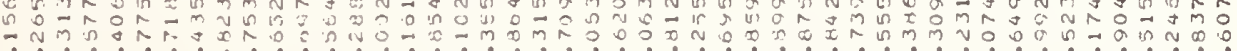

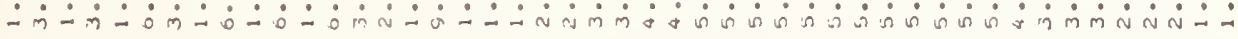

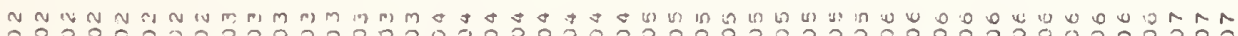

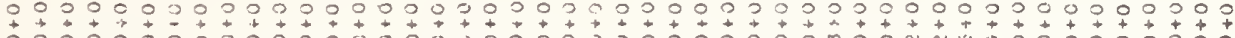

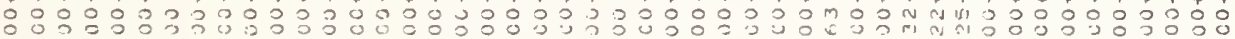

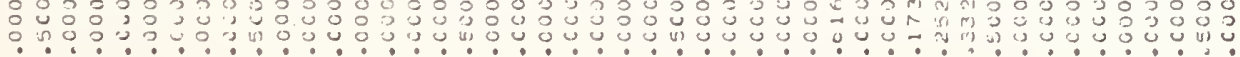

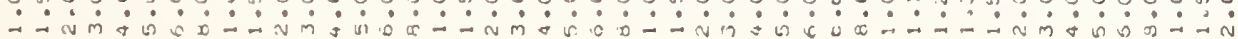


Mork $^{17}$ was included as a multiplicative correction outside the integral sign. Fluorescence resulting from Compton-created vacancies was neglected.

\section{Photoeffect}

Photoeffect energy-absorption fractions $\mathrm{f}_{\tau_{\mathrm{K}}}, \mathrm{f}_{\tau_{\mathrm{I}}}, \ldots$ were calculated for the $\mathrm{K}$, L, ... subshell groups using the procedure described by Berger ${ }^{4}$ and used by Allison ${ }^{5}$ to take into account photoelectron bremsstrahlung as well as primary fluorescence, modified as suggested by Carlsson ${ }^{18}$ to include additional fluorescence cascade effects. Fluorescence and absorption-edge energies were taken from Bearden, ${ }^{19}$ the bremstrahlung data were again taken from Berger and Seltzer, ${ }^{16}$ fluorescence yield data from Bambynek et $\mathrm{al}^{20}$ and from Freund ${ }^{21}$ and data on $\mathrm{L}-$ shell vacancies resulting from primary-event $\mathrm{K}-$ shell vacancies were taken from Robinson and Fink. 22

The total photoeffect energy-absorption cross section $\tau$. $f_{\tau}$, where $\tau$ is related to $\mu_{\tau} / \rho$ according to Eq. (3), was then calculated as

$$
\tau \cdot \mathrm{f}_{\tau}=\tau_{\mathrm{K}} \cdot \mathrm{f}_{\tau_{\mathrm{K}}}+\tau_{\mathrm{L}} \cdot \mathrm{f}_{\tau_{\mathrm{L}}}+\ldots
$$

where theoretical photoeffect subshell-group cross sections $\tau_{K}, \tau_{L}, \ldots$ were taken from Veigele et $a 1^{23,24}$ in the region 0.1 to $1 \mathrm{keV}$ and from Scofield ${ }^{25}$ from $1 \mathrm{keV}$ to 1.5 MeV with some adjustments to fit measured total attenuations coefficients as shown for example in Fig. 2 for nitrogen. In Fig. 2

all cross sections are multiplied by $\mathrm{E}^{2}$ to better display differences between the selected experimental, 26-31 theoretical ${ }^{23-25}$ and compiled ICRU Report $17^{12}$ values. The Scofield values were extrapolated above $1.5 \mathrm{MeV}$ by an empirical formula (Eq. 2. - 1) given in NSRDSNBS 29. 6

\section{Pair and Triplet Production}

The pair production energy-absorption fractions $\mathrm{f}_{K}$ in Eq. (2) were not recalculated for the present tables, but were interpolated

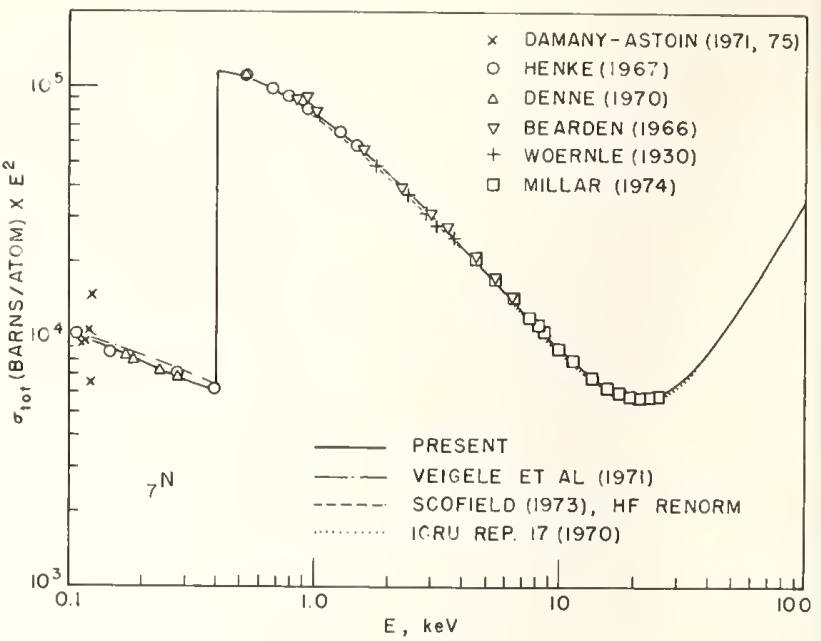

Fig. 2 .

and extrapolated from the 1.5 to $10 \mathrm{MeV}$ values given by $R$. T. Berger ${ }^{4}$ (Table $\mathrm{X}$ ) which take into account positron annihilation in flight. The graphical extrapolation to $20 \mathrm{MeV}$ results in uncertainties in $\mathrm{f}_{K}$ at $20 \mathrm{MeV}$ of the order of $+5 \%$ for the low $Z$ materials in this report to $\bar{\imath} \pm 15 \%$ for $\mathrm{Pb}$.

However, the pair production $k_{n}$ (nuclear field) and triplet $k_{e}$ (electron field) cross sections were recalculated since new mass attenuation coefficient measurements by Henry and Kennett, ${ }^{32}$ Ahrens et $a 1,{ }^{33}$ Bar-Noy and Moreh $^{34}$ and others indicate the need of revision of earlier NBS values ${ }^{6,10}$ in the threshold ( $1.022 \mathrm{MeV}$ for $K_{n}, 2.044 \mathrm{MeV}$ for $k_{e}$ ) to $20 \mathrm{MeV}$ region.

The pair production cross section $k_{n}$ used in the present work was combined from (a) the near-threshold exact Coulomb-field relativistic results of $\emptyset$ verb $\phi$, Mork and 0lsen, 35 (b) the near-threshold HFS (Hartree-FockSlater) screened results by Tseng and Pratt ${ }^{36}$ and (c) the Mork-01sen ${ }^{37}$ radiative correction factor. These results join smoothly (differing by $0.2 \%$ or less at $30 \mathrm{MeV}$ ) with new highenergy screened results calculated using

Eq. (2. - 31) in NSRDS-NBS $29^{6}$ in which the empirical correction factor is replaced by an updated correction factor fitted by Ahrens 38 to recent mass attenuation coefficient measure ments. 
The triplet cross section $k_{e}$ for energies less than $15 \mathrm{MeV}$, where screening is assumed negligible, was taken directly from the new free-electron results by $\mathrm{Haug}^{39}$ who extends the 2.044 - $10 \mathrm{MeV} \mathrm{Mork}{ }^{40}$ results up to 2.55 $\mathrm{GeV}$ by an improved numerical integration procedure. These results join smoothly (differing by $1.5 \%$ or less at $15 \mathrm{MeV}$ ) with higher-energy screened results calculated using a procedure first suggested by Bethe, 41 according to Mork, ${ }^{40}$ in which the wheelerLamb ${ }^{42}$ TF (Thomas-Fermi) screened triplet result is corrected for electron-proton differences neglected by wheeler and Lamb. In the present calculation this correction is obtained by subtracting the bare-proton Naximon ${ }^{43}$ results from the Haug ${ }^{39}$ free-electron results.

The pair-production energy-absorption cross section was then calculated as

$$
k \cdot f_{K}=\left(k_{n}+k_{e}\right) \cdot f_{K}
$$

TAELE 2. MASS ENERGY ABSORPTICN COEFFICIENT RATIOS. AIR/MEDIUM.

\begin{tabular}{|c|c|c|c|c|c|c|c|}
\hline $\begin{array}{c}\text { EAERGY } \\
\text { EV }\end{array}$ & $\begin{array}{l}\text { AIR' } \\
\text { CAREON }\end{array}$ & $\begin{array}{r}\text { AIR/ } \\
\text { HATER }\end{array}$ & $\begin{array}{c}\text { AIR/ } \\
\text { PULYSTYR. }\end{array}$ & $\begin{array}{l}\text { AIR/ } \\
\text { LUCITE }\end{array}$ & $\begin{array}{c}\text { AIR, } \\
\text { POLYETH. }\end{array}$ & $\begin{array}{l}\text { AIR/ } \\
\text { SAKELITE }\end{array}$ & $\begin{array}{r}\text { ALR' } \\
\text { AMRER }\end{array}$ \\
\hline 1. $\mathrm{CCCO}+0$ ? & 1.8505 & .0527 & 1.4303 & 1.3248 & 2.0176 & 1.5438 & $1.70=6$ \\
\hline i. E ECUO+02 & 1.8575 & .0170 & 2.0022 & 1.3124 & 2.1012 & $1.550 \mathrm{~L}$ & 1.7300 \\
\hline $2 . c c 00+02$ & $1 \cdot \sin 21$ & .7555 & $1.5 \theta t 3$ & 1.2475 & 2.1057 & 1.5282 & 1.7217 \\
\hline 3. $\mathrm{cccc}+02$ & .0924 & $.5: 24$ & .1001 & .1451 & .1078 & .1164 & .1152 \\
\hline $4 . \mathrm{CCCO}+02$ & - $c S 12$ & - St年3 & .0583 & .1440 & .1064 & .1151 & .1140 \\
\hline E. $\mathrm{CCCO}+02$ & 1.0774 & $11+5035$ & $\therefore 1673$ & 1.7004 & 1.2572 & 1.3597 & 1.3460 \\
\hline $6 . \mathrm{CcCO}+02$ & 1.5073 & $.92 \equiv 1$ & $1 \cdot \dot{0} 331$ & 1.2704 & 1.7590 & 1.3118 & 1.5346 \\
\hline E. ccco +02 & i. 5064 & .9025 & $\therefore .6574$ & 1.2791 & 1.8282 & 1.4201 & 1.5748 \\
\hline 1. $c \mathrm{ccc}+03$ & 1.0275 & .8854 & 1.7041 & 1.2903 & 1.9002 & 1.4502 & 1.6163 \\
\hline $1.5 c c 0+03$ & 1.7791 & - E6s? & 1.9270 & 1.3273 & 2.0767 & 1.5259 & 1.72 .30 \\
\hline $2 . \mathrm{CcO}^{2}+23$ & 1. स1त3 & . $\varepsilon \supset 74$ & 1.9705 & 1.3330 & 2.1227 & i. 5464 & $1.74 d 1$ \\
\hline $3 . c c 00+03$ & 1.3559 & $.8<55$ & 2.0150 & 1.3305 & 2.1713 & 1.5619 & 1.7722 \\
\hline $4 . \operatorname{cccu}+03$ & 2.1134 & . $\$ \leqslant 01$ & 2.2957 & 1.5014 & 2.4731 & 1.7639 & 2.0003 \\
\hline E. $\therefore C=0+03$ & $2 .: 096$ & .9455 & 2.3513 & $1.521 \pi$ & 2.5329 & 1.7950 & 2.0454 \\
\hline $6 . c c c 0+03$ & 2.2038 & .9504 & 2.3537 & $1.537 \mathrm{~s}$ & 2.5786 & 1.81839 & 2.0755 \\
\hline 8. $20 \mathrm{co}+0 \mathrm{z}$ & 2. $-\varepsilon \varepsilon 50$ & .9524 & $2.4 \leq 44$ & $1 \cdot 5 \leqslant 33$ & 2.6438 & 1.84495 & 2.1154 \\
\hline $1.0000+04$ & 2.3200 & $.96,06$ & 2.5143 & 1.5742 & 2.7078 & 1.8340 & 2.1580 \\
\hline $2.5 C C C+04$ & 2.4030 & .5731 & 2.6002 & 1.6134 & 2.7968 & 1.9340 & 2.2106 \\
\hline $2 . \operatorname{ccc} 0+04$ & 2.4394 & .5018 & 2.6302 & 1.6300 & 2.8191 & 1.9564 & 2.2404 \\
\hline 3 . icuoto 4 & 2.3479 & .9903 & 2.4042 & 1.6029 & 2.6143 & 1.9032 & 2.1412 \\
\hline$\because . \mathrm{CCOO}+\mathrm{OA}_{4}$ & 2.0547 & $.38<3$ & 2.1020 & 1.4409 & 2.1443 & 1.7203 & 1.8658 \\
\hline $5.1 ; 0 \mathrm{CO}+04$ & 1.7112 & .9722 & 1.6919 & 1.3374 & 1.6756 & 1.4891 & 1.5505 \\
\hline $6 \cdot c c 0 C+04$ & 1.4477 &.$\$ 545$ & 1.3570 & 1.2016 & 1.3563 & 1.2987 & 1.3106 \\
\hline $8 \cdot \operatorname{ccc} 0+04$ & 1.1798 & .5272 & $1 .: 123$ & $1.04 / 45$ & 1.0504 & 1.0925 & 1.0678 \\
\hline 1. $c \subset \leq 0+05$ & 1.0821 & . $\$ 135$ & 1.0120 & .9816 & .9587 & 1.0138 & .5754 \\
\hline $1.5 c 00+05$ & 1.0187 & $.40=0$ & .9479 & .438 .9 & .8916 & .9618 & .9222 \\
\hline 2. $c(c c+0 s$ & $1.00 E 4$ & .9007 & .9355 & .9304 & .9822 & .9515 & .9111 \\
\hline $3 . \mathrm{cccO}+05$ & 1.0010 & .8990 & .9301 & . 5267 & .6769 & .9470 & .9062 \\
\hline$\therefore$. CCCu+05 & - Sऽऽ 8 & .0954 & .9289 & .9259 & .5757 & .9460 & .9051 \\
\hline $5.0 c c 0+05$ & $.5 \$ 94$ & - 2.993 & .9284 & .9255 & .8752 & .9456 & .9047 \\
\hline $8 . c c c 0+05$ & .9992 & .8992 & . 9282 & .925 .3 & .8749 & .9454 & .5044 \\
\hline $6 \cdot \varepsilon: \leqslant 3+05$ & .5991 & .8991 & - 5281 & .9253 & .8748 & .9453 & .9043 \\
\hline e.cccotos & .5589 & • வேり 1 & .9279 & .9251 & .8746 & .9452 & .5041 \\
\hline $1 \cdot \operatorname{ccov} 006$ & • $ธ \dashv € 7$ & .8990 & $.5 \angle 75$ & .9250 & .8743 & .9450 & .9039 \\
\hline $1 \cdot i 7 \equiv 2+0 \epsilon$ & .9986 & . 8490 & .9274 & .9248 & .8741 & .9448 & .9037 \\
\hline 1. $\leqslant 522+0 \epsilon$ & .9985 & .8989 & .9274 & .5248 & .8740 & .9448 & .9037 \\
\hline $1.332 E+06$ & .5985 & - 8989 & .5273 & .9248 & .8740 & .9448 & .5036 \\
\hline $1.5 c 00+0 \epsilon$ & .5986 & . 8905 & .9274 & .9246 & .8741 & .9449 & .9037 \\
\hline 2. $\mathrm{ccc} C+\mathrm{c}^{\circ}$ & .5996 & . 3995 & .9286 & .9258 & .8757 & .9459 & .9051 \\
\hline $3 . c 000+06$ & 1.0043 & .9017 & . 9354 & .5306 & - 4835 & .9510 & .9110 \\
\hline A. CCOO+OE & 1.0106 & .9048 & .9444 & .9370 & . 8941 & .9580 & .9206 \\
\hline S.cccoto6 & 1.0175 & . 9082 & .9542 & .9441 & .9059 & . 5655 & .9304 \\
\hline $0 . \mathrm{CCCO}^{\circ}$ & 1.0243 & .5116 & .9640 & .9511 & .9177 & .9731 & .9402 \\
\hline $8 \cdot C C 00+05$ & 1.0371 & .9179 & .9828 & .9643 & .9406 & .9873 & .5589 \\
\hline 1. $c 000+07$ & 1.0488 & .9236 & 1.0000 & .9704 & .9617 & 1.0003 & .9761 \\
\hline $1.5(\mathrm{CO}+07$ & 1.0710 & .9355 & 1.0342 & 1.0003 & 1.0046 & 1.0258 & 1.0106 \\
\hline $2 . \mathrm{ccc}<+07$ & $1.08 \leqslant 5$ & .5437 & 1.0589 & 1.0173 & 1.0354 & 1.0439 & 1.0357 \\
\hline
\end{tabular}

Table 2 lists the ratio $\left(\mu_{\text {en }} / \rho\right)$ air $\left(\mu_{\text {en }} / \rho\right)_{\text {medium }}$ as called for in the exposure-

in which $K$ is related to $\mu_{K} / \rho$ according to Eq. (3).

\section{Air/Medium Ratios}


the remaining mixtures in Table 1 , also vs carbon. The unusually large and small ratios in the region 0.3 to $0.5 \mathrm{keV}$ result from displacements of the $\mathrm{K}$ absorption edge in $\mathrm{C}$, $\mathrm{N}$ and $\mathrm{O}$.
Comparison of Present Results with Previous Compilations

In tables 3 and 4 the present $\mu_{\text {en }} / \rho$ values for air and carbon are compared with

Table 3. Air Mass Energy-Absorption Coefficient $\mu_{\text {en }} / \rho, \mathrm{m}^{2} / \mathrm{kg}$

\begin{tabular}{|c|c|c|c|c|c|c|c|}
\hline & Berger ${ }^{4}$ & Allison ${ }^{5}$ & ICRU $10 b^{11}$ & $\begin{array}{l}\text { NSRDS } \\
\text { NBS } 29^{6}\end{array}$ & $\begin{array}{l}\text { Storm } \\
\text { Israel }\end{array}$ & ICRU $17^{12}$ & Present \\
\hline $\mathrm{E}(\mathrm{MeV})$ & 1961 & 1961 & 1962 & 1969 & 1970 & 1970 & 1976 \\
\hline
\end{tabular}

Table 4. Carbon Mass Energy-Absorption Coefficients $\mu_{\text {en }} / \rho, \mathrm{m}^{2} / \mathrm{kg}$

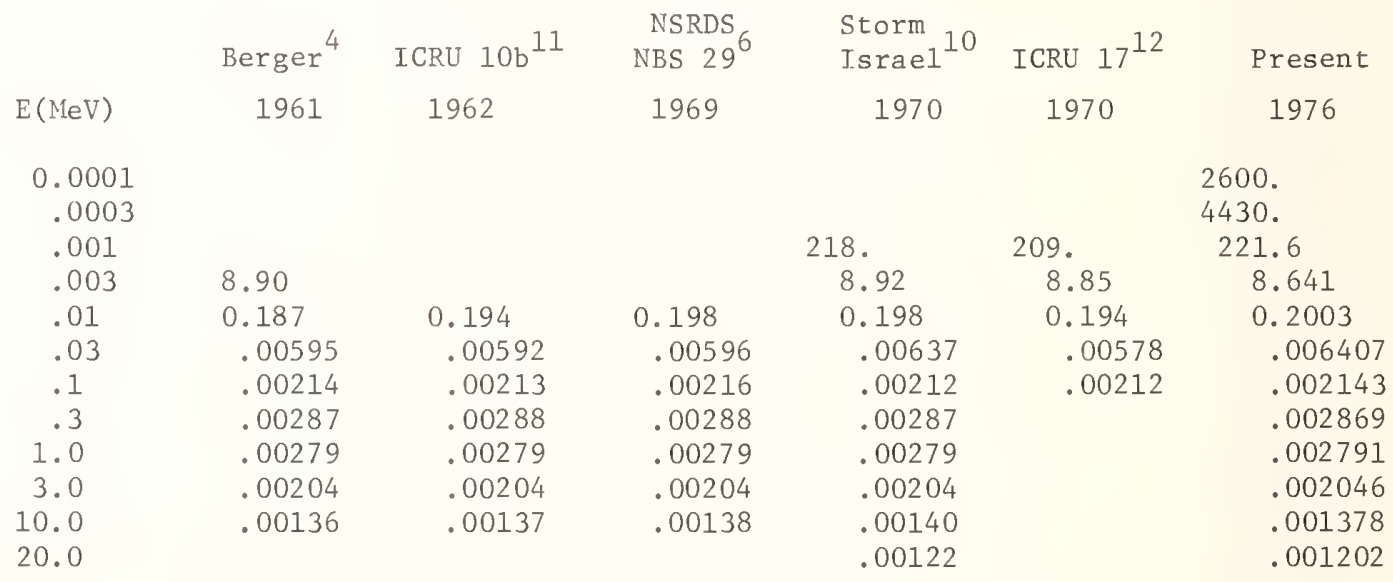

values from the previous compilations $4-6,10-12$ surveyed in this report. Of particular interest are the ICRU Report $17^{12} \mu_{\text {en }} / \rho$ values at $30 \mathrm{keV}$ for both air and carbon which are seen to be $5 \%$ lower than the average of the other compilations including the present results. The ICRU Report $17 \mu_{\text {en }} / \rho$ values appear to be systematically $5 \%$ to $10 \%$ low for $\mathrm{C}, \mathrm{N}$ and $\mathrm{O}$ over the range 20 to $50 \mathrm{keV}$.
In table 5 the present air/carbon ratio given in table 2 is compared with this ratio derived from air and carbon $\mu_{\text {en }} / \rho$ data from the previous compilations as listed in tables 3 and 4 . Where Compton scattering predominates (100 keV to $10 \mathrm{MeV}$ for the low-Z materials in this report) the different compilations yield air/carbon ratios differing by less than $0.5 \%$. Below $100 \mathrm{keV}$, where the photoeffect predominates, the differences are seen to range up to $5 \%$. 
Table 5. Air/Carbon Ratios $\left(\mu_{\text {en }} / \rho\right)$ air $/\left(\mu_{\text {en }} / \rho\right)$ carbon Compared with Previous Compilations

\begin{tabular}{|c|c|c|c|c|c|c|}
\hline & Berger ${ }^{4}$ & ICRU $10 b^{11}$ & $\begin{array}{c}\text { NSRDS } \\
\text { NBS } 29\end{array}$ & $\begin{array}{l}\text { Storm } \\
\text { Israel }\end{array}$ & ICRU $17^{12}$ & Present \\
\hline $\mathrm{E}(\mathrm{MeV})$ & 1961 & 1962 & 1969 & 1970 & 1970 & 1976 \\
\hline 0.0001 & & & & & & 1.857 \\
\hline .0003 & & & & & & .092 \\
\hline .001 & & & & 1.64 & & 1.628 \\
\hline .003 & & & & 1.80 & & 1.860 \\
\hline .01 & 2.43 & 2.40 & 2.33 & 2.31 & 2.37 & 2.321 \\
\hline .03 & 2.44 & 2.48 & 2.48 & 2.34 & 2.42 & 2.348 \\
\hline .1 & 1.079 & 1.085 & 1.083 & 1.080 & 1.080 & 1.082 \\
\hline .3 & 1.000 & 1.000 & 1.000 & 1.000 & & 1.001 \\
\hline 1.0 & 1.000 & 1.004 & .996 & 1.000 & & .999 \\
\hline 3.0 & 1.005 & 1.005 & 1.005 & 1.005 & & 1.0043 \\
\hline 10.0 & 1.051 & 1.051 & 1.051 & 1.050 & & 1.047 \\
\hline 20.0 & & & & 1.090 & & 1.087 \\
\hline
\end{tabular}

Acknowledgement

The author thanks M. J. Berger and R. Loevinger for suggesting this work and for their helpful comments.

\section{References}

1. Loevinger, R., "Realization of the Unit of Exposure. II. Cavity Chambers," Proc. Int. Course on Ionizing Radiation Metrology, Varenna, Italy (1974).

2. Niate1, M. -T., Loftus, T. P., and Oetzmann, W., Metrologia 11, 17 (1975).

3. Int. Commission on Rad. Units and Meas., ICRU Rep. 19 (1971).

4. Berger, R. T., Radiat. Res. 15, 1 (1961).

5. Allison, J. W., Aust. J. Phys. 14, 443 (1961).

6. Hubbe11, J. H., Nat. Stand. Ref. Data Ser., Nat. Bur. Stand. 29 (1969).

7. Evans, R. D., Radiation Dosimetry, Vol. 1, p. 128, Attix, $\overline{\text { F. H., and Roesch, }}$ W. C., eds., (Academic Press, N. Y., 1968).

8. Hubbell, J. H., and Berger, M. J., Engineering Compendium on Radiation ShieldIng (IAEA), Vo1. I, p. 180, Jaeger, R. G., ed. in chf. (Springer-Verlag, Berlin, 1968).

9. Johns, H. E., and Cunningham, J. R., The Physics of Radiology, 3rd ed., p. 735 (Thomas, Springfield, I11., 1969).

10. Storm, E., and Israel, H. I., Nucl. Data Tables A, 7, 565 (1970).

11. Int. Commission on Rad. Units and Meas., ICRU Rep. 10b (NBS Handbook 85, 1962).

12. Int. Commission on Rad. Units and Meas., ICRU Rep. 17 (1970).

13. McMaster, W. H., Del Grande, N. Kerr, Mallett, J. H., and Hubbel1, J. H., Lawrence Livermore Rep. UCRL-50174, Sec. II, Rev. 1 (1969).
14. Storm, E., and Israel, H. I., Los Alamos Sci. Lab. Rep. LA-3753 (1967).

15. Hubbell, J. H., Veigele, Wm. J., Briggs, E. A., Brown, R. T., Cromer, D. T., and Howerton, R. J., J. Phys. Chem. Ref. Data 4, 471 (1975).

16. Berger, M. J., and Seltzer, S. M., NASA SP-3012 (1964).

17. Mork, K., Phys. Rev. A 4, 917 (1971).

18. Carlsson, G. A., Health Phys. 20, 653 (1971).

19. Bearden, J. A., Nat. Stand. Ref. Data Ser., Nat. Bur. Stand. 14 (1967).

20. Bambynek, W., Crasemann, B., Fink, R. W., Freund, H.-U., Mark, H., Swift, C. D., Price, R. E., and Rao, P. V., Rev. Mod. Phys. 44, 716 (1972).

21. Freund, H.-U., X-Ray Spectrom. 4, 90 (1975).

22. Robinson, B. L., and Fink, R. W., Rev. Mod. Phys. 32, 117 (1960).

23. Veigele, Wm. J., Briggs, E., Bates, L., Henry, E. M., and Bracewe11, B., Kaman Sciences Rep. KN-71-431 R, Vol. 1, Rev. 1 (1971).

24. Henry, E. M., Bates, C. L., and Velgele, Wm. J., Phys. Rev. A 6, 2131 (1972).

25. Scofield, J. H., Lawrence Livermore Lab. Rep. UCRL-51326 (1973).

26. Damany-Astoin, N., pers. comm. (Oct. 29, 1975). Also de Reilhac, L., and DamanyAstoin, N., J. Phys. (Paris) C4, 32 (1971).

27. Henke, B. L., Elgin, R. L., Lent, R. E., and Ledingham, R. B., Norelco Rep. 14, 112 (1967).

28. Denne, D. R., J. Phys. D 3, 1392 (1970).

29. Bearden, A. J., J. Appl. Phys. 37, 1681 (1966).

30. Woernle, B., Ann. Phys. (Leipzig) 5, 475 (1930).

31. Millar, R. H., and Greening, J. R., J. Phys. B 7, 2332 (1974). 
32. Henry, L. C., and Kennett, T. J., Can. J. Phys. 49, 1167 (1971); 50, 2756 (1972).

33. Ahrens, J., Borchert, H., Czock, K. H., Eppler, H. B., Gimm, H., Gundrum, H., Krönig, M., Riehn, P., Sita Ram, G., Zieger, A., and Ziegler, B., Nucl. Phys. A251, 479 (1975).

34. Bar-Noy, T., and Moreh, R., Nucl. Phys. A229, 417 (1974).

35. Øverb $\phi$, I., Mork, K. J., and Olsen, H. A., Phys. Rev. A 8, 668 (1973).

36. Tseng, H. K., and Pratt, R. H., Phys. Rev. A 6,2049 (1972).
37. Mork, K.; and 01sen, H., Phys. Rev. 140, B1661 (1965); 166, 1862 (1968).

38. Ahrens, J., pers. comm. (Sep. 6, 1974).

39. Haug, E., Z. Naturforsch. 30a, 1099 (1975)

40. Mork, K. J., Phys. Rev. 160, 1065 (1967).

41. Bethe, H. A., and Ashkin, J., Experimental Nuclear Physics, Vol. 1, p. 334, Segré, E., ed., (Wiley, New York, 1953).

42. Wheeler, J.A., and Lamb, W. E., Phys. Rev. 55, 858 (1939); 101, 1836 (1956).

43. Maximon, L. C., J. Res. Nat. Bur. Stand., Sect. B 72, 79 (1968). 


\author{
M. J. Berger and S. M. Seltzer \\ Radiation Theory Section \\ S. R. Domen and P. J. Lamperti \\ Dosimetry Section \\ National Bureau of Standards \\ Washington, D. C. 20234
}

\begin{abstract}
This paper describes work at NBS dealing with the conversion of ionization measurements to absorbed-dose values in high-energy electron dosimetry. This work was done by two approaches: experimentally, through the comparison of calorimeter and ionization-chamber measurements in a graphite phantom, for incident-beam energies from 15 to $50 \mathrm{MeV}$; theoretically, via transport calculations of electron flux spectra and application of BraggGray cavity theory, for beam energies from 1 to $60 \mathrm{MeV}$ and for various media including water, tissue, graphite and plastics. The pertinent dose/ionization conversion factor has been determined with an estimated accuracy of $1 \%$ as a function of the beam energy and of the depth in the phantom. Experimental evidence has also been obtained on the influence of lead scattering foils on the value of the conversion factor.
\end{abstract}

(Electrons; dosimetry; ionization chamber; calorimeter; dose/ionization conversion; stopping-power ratios)

This talk is concerned with one aspect of electron dosimetry, namely, the translation of measured ionization data into absorbed-dose values. Let $D$ (med) be the absorbed dose, and J(air) the ionization measured with an airfilled ionization chamber. In order to be able to interpret the ionization measurement, one must know the dose/ionization conversion factor $C($ med/air $)=D($ med $) / J($ air $)$.

According to the Bragg-Gray theory of cavity ionization, the conversion factor is proportional to the medium/air stopping-power ratio $\mathrm{s}_{\text {air }}^{\text {med }}$ Thus

$$
C(\text { med/air })=(\bar{W} / e) P S_{\text {air }}^{\text {med }},
$$

where $\bar{W}$ is the mean energy needed for the production of an ion pair in air, $e$ is the charge of the electron, and $p$ is a correction factor ${ }^{1}$ (usually close to unity) that takes into account the perturbation of the electron flux by the presence of the air cavity. We have adopted the value $(\bar{W} / e)=33.7$ joules $/$ coulomb.

$$
\mathrm{S}_{\text {air }}^{\text {med }} \text { is the ratio of the average electron }
$$
stopping power in the medium to that in air, the average in both cases being taken over the electron spectrum at the point of measurement. In the case of irradiation by high-energy

\footnotetext{
"This work was supported in part by the National Cancer Institute.
}

electron beams, the average stopping-power ratio $S_{\text {air }}^{\text {med }}$ is not constant but varies as much as thirty percent depending on the incident beam energy $T_{0}$ and the depth $z$ in the phantom. This is due to the combination of the energydependent polarization effect (which reduces the stopping power in water or tissue relative to that in a gas) and the change of the electron spectrum with depth.

The dependence of $\mathrm{S}_{\text {air }}^{\mathrm{med}}$, and therefore $C$ (med/air), on $\mathrm{T}_{0}$ and $z$ is especially important for the calibration of ionization chambers. It cannot be disregarded even when only the relative shape of the depth-dose curve is of interest. This is illustrated by means of the example in Fig. 1, which shows curves of $\mathrm{D}$ (med) vs $z$ and of $J$ (air) vs $z$, for a beam of 20-MeV electrons incident onto a water phantom. Both curves are normalized to unit peak height. It can be seen that close to the phantom surface J(air) is $5 \%$ higher than $\mathrm{D}$ (med), whereas at depths beyond the peak it becomes from 5 to $10 \%$ smaller. The peak of the curve of ionization vs depth, in this example, occurs at $z=3.34 \mathrm{~cm}$, whereas the peak of the depthdose curve lies somewhat deeper, at $z=3.80 \mathrm{~cm}$.

The variability of the dose/ionization conversion factor is inconvenient. However, air-filled ionization chambers have so many practical advantages that they continue to 


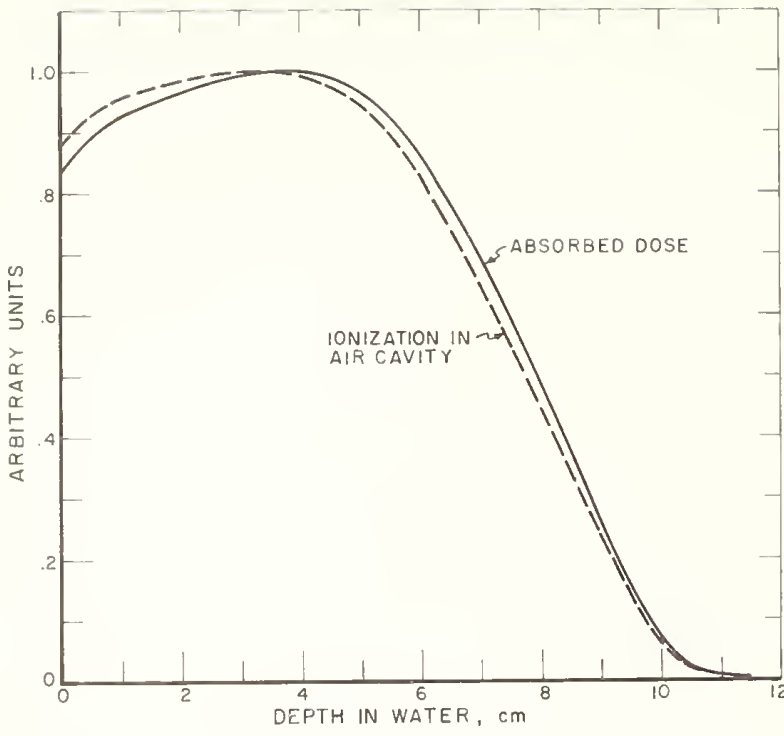

Fig. 1. Curves of absorbed dose vs depth and of ionization $v s$ depth, calculated for a $20-\mathrm{MeV}$ beam incident onto a water phantom. The beam is assumed to be nondivergent and to have a circular cross section with an area of $16.5 \mathrm{~cm}^{2}$. were obtained; and a portable calorimeter which can serve as a field instrument. 14 Tests with $20-\mathrm{MeV}$ and $50-\mathrm{MeV}$ electrons have shown that the two calorimeters give results that agree to within $0.1 \% .^{15}$

The experimental arrangement is illustrated by Fig. 2 which shows the electron beam from the NBS Linac passing through the accelerator window and a lead scattering foil into a graphite phantom (density $1.7 \mathrm{~g} / \mathrm{cm}^{3}$ ).

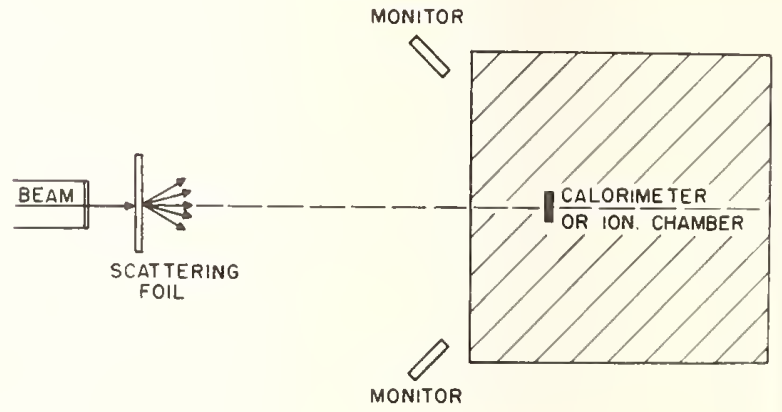

Fig. 2. Layout of the NBS experiment.

be used widely for electron dosimetry. Considerable effort on the part of radiological physicists has therefore gone into mapping out the conversion factor as a function of beam energy and depth. Most of the systematic determinations have been made in water, through the intercomparison of ferroussulfate dosimeter and ionization chamber measurements. ${ }^{2-6}$ A more limited number of experimental determinations have been made with calorimeter and ionization chamber measurements in graphite. ${ }^{7-9}$ An excellent review of the literature up to 1972 can be found in a report of the ICRU. ${ }^{10}$

As part of an absorbed-dose standards program, coordinated experimental and theoretical work has also been done at the National Bureau of Standards to determine dose/ionization factors for electron dosimetry. 11,12 The purpose of this talk is to describe briefly some of the results obtained.

In the experimental work at NBS, a specially designed heat-loss-compensated calorimeter has been used as the primary standard of absorbed dose. ${ }^{13}$ In fact two instruments have been built: a large calorimeter permanently installed at the NBS Linac facility, with which the results in this paper
Radiation measurements at the same depth were carried out, alternately, with the graphite calorimeter, and with a graphite-walled parallel-plate air-filled ionization chamber. The collecting volume of the ionization chamber was placed at the same effective depth, and had the same size and shape, as the calorimeter core. The calorimeter/ ionization chamber measurements were done for six beam energies from 15 to $50 \mathrm{MeV}$, at depths in graphite from 0.9 to $51 \mathrm{~g} / \mathrm{cm}^{2}$, and with different lead scattering foils with thicknesses from 0.144 to $1.584 \mathrm{~g} / \mathrm{cm}^{2}$. The overall uncertainty of the experimental values of $\mathrm{C}$ (graphite/air) is estimated to be not greater than $1 \%$.

The theoretical evaluation of the dose/ was used to calculate electron flux spectra at various depths in homogeneous phantoms irradiated with broad, parallel monoenergetic electron beams. Then, the required average medium/air stopping power ratios were evaluated from the electron spectra, within the framework of the Spencer-Attix ${ }^{18}$ version of Bragg-Gray cavity ionization theory. These calculations were comparable to earlier work by Kessaris, 19 but included energy-loss ionfzation factor was carried out in two stages. First, the Monte Carlo method 16,17 
straggling instead of the continuous-slowingdown approximation and avoided various other approximations made by Kessaris. Theoretical stopping-power ratios were obtained for incident beam energies from 1 to $60 \mathrm{MeV}$, in various media of low atomic number (water, muscle tissue, graphite, polystyrene and acrylic plastic). The overall uncerținty of the theoretical results is estimated to be $1 \%$.

In order to treat the case of a strictly monoenergetic incident beam, and to allow comparison with the calculations, the experimental data were extrapolated to zero thickness of the lead scattering foil. It was also assumed that the perturbation factor $p$ in the NBS experiment is unity. Figure 3 compares the variation of the experimental and theoretical values of $\mathrm{C}$ (graphite/air) as a function of incident beam energy $T_{0}$, at

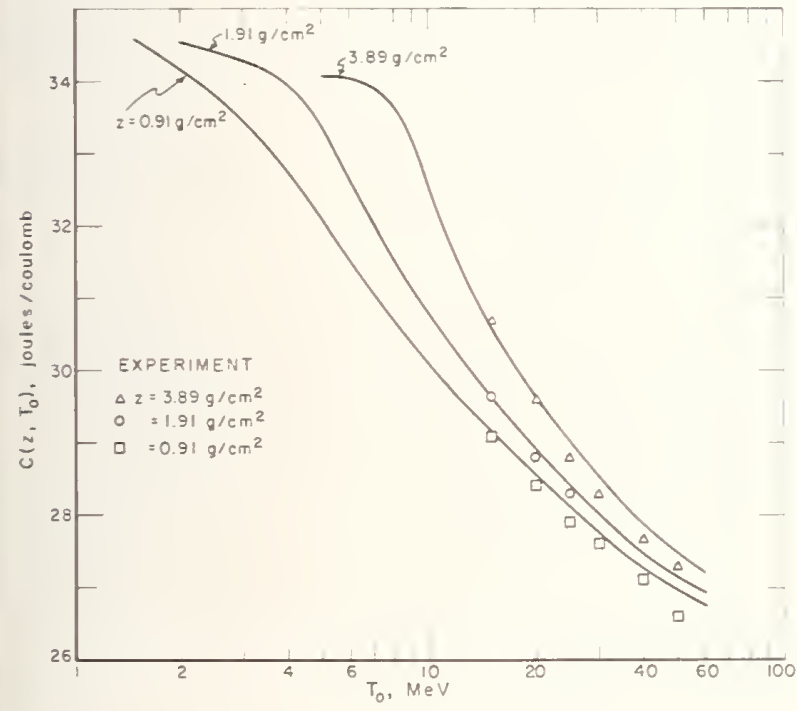

Fig. 3. Conversion factor C(graphite/air) as a function of beam energy. The curves are calculated and the points are experimental.

three depths. The experimental results lie slightly below the calculated results, the mean deviation for the data shown amounting to $0.5 \%$ and the root-mean-square deviation to $0.7 \%$. Figure 4 shows the dependence of C(graphite/air) on the depth in a graphite phantom, for an incident beam energy of 30 $\mathrm{MeV}$. The experimental results lie somewhat below the calculated results for shallow depths, and are in close agreement for intermediate and great depths. The conversion factor increases (almost linearly) up to a

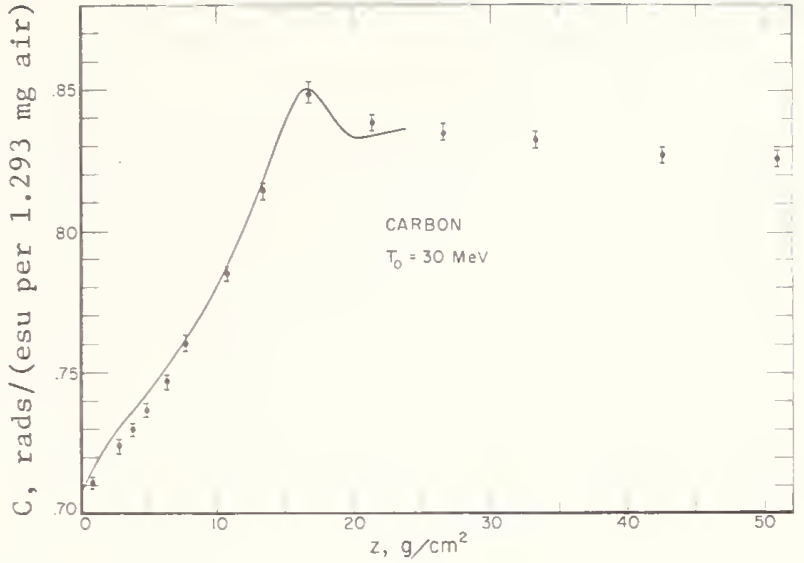

Fig. 4. Conversion factor C(graphite/air) as a function of depth in a graphite phantom. The curve is calculated and the points are experimental. To change to units of joules/coulomb, multiply the ordinate by $38.8=33.7 / 0.869$.

depth of $15 \mathrm{~g} / \mathrm{cm}^{2}$ where it reaches a peak value. This depth corresponds to the practical range of $30-\mathrm{MeV}$ electrons in graphite, i.e., the greatest depth to which the incident electrons can effectively penetrate. The value of the conversion factor at depths greater than the practical range is determined by the spectrum of Compton and pair electrons that have been set in motion by penetrating secondary bremsstrahlung.

Comparisons between experimental and calculated conversion coefficients in graphite have been made for sixty-three combinations of beam energies and depths. The experimental values tend to be slightly lower than the calculated values at shallow depths. Overall there is good agreement, usually within 1\% which is well with the combined limits of experimental and theoretical uncertainty.

In view of this satisfactory agreement we think it possible to rely on the theoretical approach to obtain conversion factors for conditions not readily accessible with the NBS experimental setup. Illustrative calculated results are shown in the next two figures. Figures $5 \mathrm{a}$ and $5 \mathrm{~b}$ give curves of the stopping-power ratio $\mathrm{s}_{\mathrm{air}}^{\text {water }}$ vs depth, systematically displayed for eighteen beam energies from 1 to $60 \mathrm{MeV}$. These results can be used to evaluate $\mathrm{C}$ (water/air) according to Eq. (1). Just as in the example in Fig. 4, the curves reach a peak value at or near a depth corresponding to the practical range 


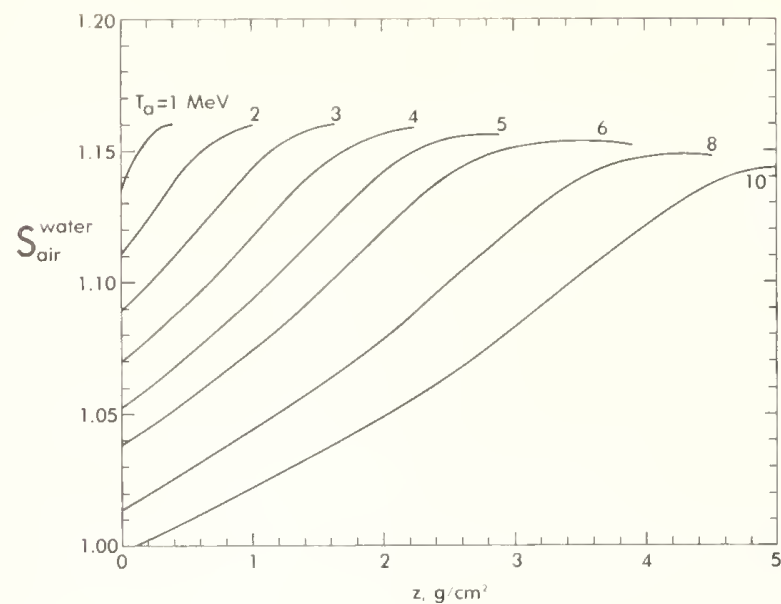

Fig. 5a. Calculated water/air stopping power ratios, for beam energies from 1 to $10 \mathrm{MeV}$.

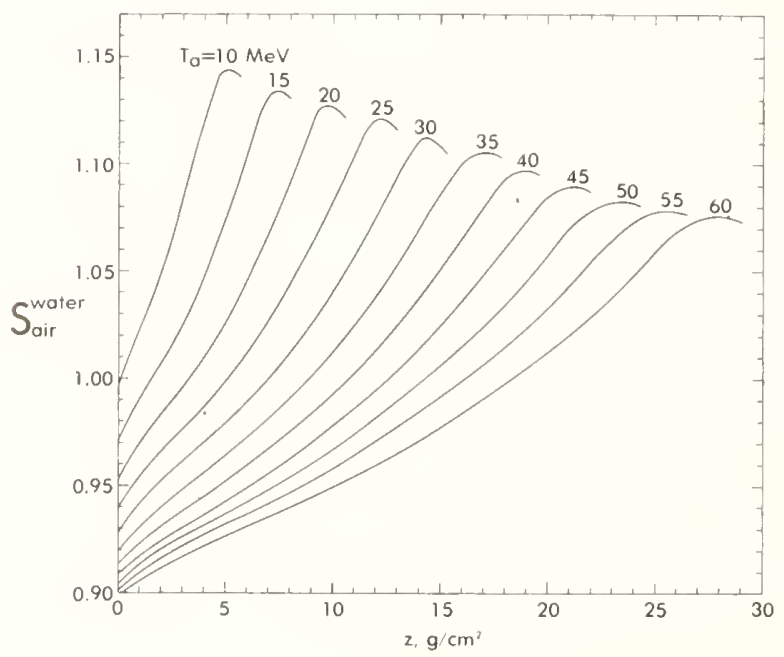

Fig. 5b. Calculated water/air stopping power ratios, for beam energies from 10 to $60 \mathrm{MeV}$.

of the incident electrons. Figure 6 compares dose/ionization conversion factors in water with those in muscle tissue, polystyrene, and graphite. The results are given as functions of the beam energy, at a depth of $2 \mathrm{~g} / \mathrm{cm}^{2}$ which may be of interest for calibrations. Stopping-power ratios in tabular form have been given in reference 11 for water, and will be given for other media in a separate report.

Various medical physics organizations have carefully analyzed the experimental and theoretical literature on dose/ionization conversion factors and have recommended the adoption of certain values of C(water/air) for instrument calibration. Provided the

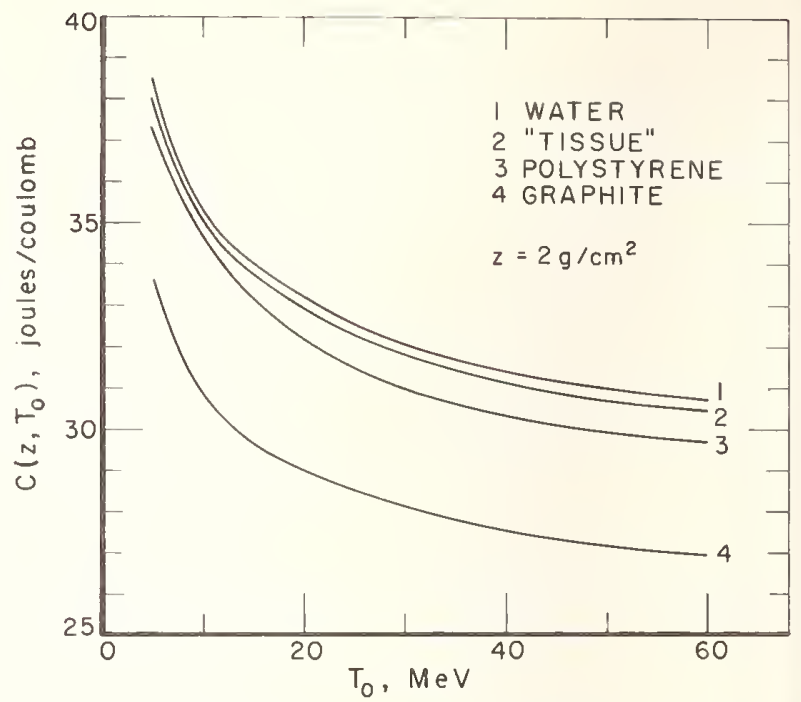

Fig. 6. Calculated dose/ionization conversion factors in various media at a depth of $2 \mathrm{~g} / \mathrm{cm}^{2}$. Assumed composition of tissue is: $10.20 \% \mathrm{H}, 12.30 \% \mathrm{C}$, $3.50 \% \mathrm{~N}, 72.90 \% \mathrm{O}, 0.08 \% \mathrm{Na}, 0.02 \% \mathrm{Mg}$, $0.20 \% \mathrm{P}, 0.50 \% \mathrm{~S}$, and $0.30 \% \mathrm{~K}$ by weight.

same assumptions are made about the values of $(\bar{W} / e)$ and the perturbation factor $p$, the percentage deviations of these recommended values from the values calculated by us are as follows:

Recommended by: Percent Deviation

$\operatorname{ICRU}^{10}$
$\mathrm{HPA}^{20}$
$\mathrm{NACP}^{21}$
Mean R.M.S. $-1.0$ 1.1 1.3 $\mathrm{NACP}^{21}$ $-0.0_{3}$ 0.4

It appears from this comparison, and from other more detailed comparisons in reference 11 , that the new NBS results are quite consistent with the older results in the literature. There are only minor changes in the value of the dose/ionization conversion coefficients. However, the accuracy has been significantly improved to the 1-percent level (necessary for standards work), and conversion factors have been made available for a wider range of beam energies, depths and materials than heretofore.

The discussion so far has been confined to the idealized case of irradiation with monoenergetic beams. In practical applications (e.g., in radiation therapy) the electron beam must pass through various kinds of material (scattering foil, collimator, 
nonitor) before reaching the phantom. Even a beam which is initially quite monoenergetic (which is not the case for all accelerators) will usually arrive at the phantom with a rather complex energy spectrum.

Scattering foils which are used to broaden the beam are perhaps the most important factor influencing the beam spectrum. The passage of the beam through the foil has the following effects: (1) the electrons lose a certain amount of energy and their spectrum is broadened; (2) their angular distribution is diffused, which in turn modifies their penetration into the phantom; (3) a significant amount of bremstrahlung is produced in the foil which penetrates into the phantom and gives rise to a spectrum of Compton and pair electrons and their secondaries.

The influence of scattering foils is reduced when a magnetic field is present which removes a large fraction of the scattered lowenergy electrons from the beam. This was the case in a series of experiments made by Svensson ${ }^{3,6}$ and coworkers at various medical betatrons. Svensson estimated the most probable energy $E_{p}$ of the electrons reaching the phantom. It has been shown ${ }^{11}$ that his experimental values for C(water/air), for beam energies from 10 to $30 \mathrm{MeV}$, are in good agreement with theoretical values calculated for monoenergetic beams of energy $E_{p}$.

The NBS experiment provided a good deal of information about the effect of lead scattering foils, in the absence of a magnetic field. As an example, Fig. 7 shows the percentage change of $\mathrm{C}$ (graphite/air) that occurs when a lead scattering foil is inserted into

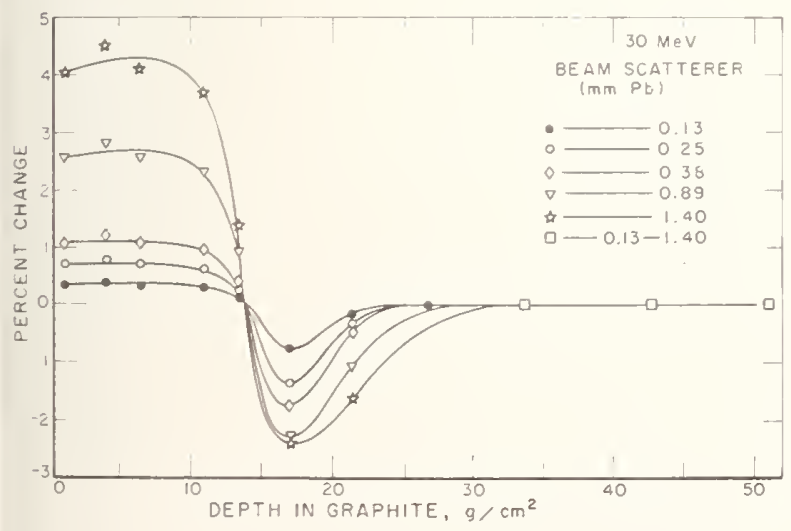

Fig. 7. Change of $C$ (graphite/air) due to the introduction of a lead scattering foil. Results have been determined experimentally for a $30 \mathrm{meV}$ beam of electrons. a 30-MeV beam. Up to depths equal to the practical range of the incident electrons, there is an increase which can be as great as several percent for the thickest foils. This increase is approximately proportional to the foil thickness, and is presumably due to the lowering of the mean energy of the electrons in their passage through the foil. At depths beyond the practical range there is a reversal of the effect, C(graphite/air) being reduced slightly. This may be due to hard bremsstrahlung produced in the lead foil, but no thorough analysis has as yet been made. Finally, the values of $C$ (graphite/air) at very great depths appear to be unaffected by the presence of a foil.

\section{References}

1. Harder, D., Biophysik 5, 157 (1968); see also reference 10 .

2. Almond, P. R., J. Appl. Radiat. and Isotopes 21, 1 (1970).

3. Svensson,, H., Hettinger, G., Acta Radiol. Ther. Phys. Bio1. 10, 369 (1971).

4. Wambersie, A., Dutreix, A., Prignot, M., Studia Biophysika, 26, 167 (1971).

5. Svensson, H., Pettersson, C., Hettinger, G., Acta Radiol. Ter. Phys. Biol. 10, 504 (1971).

6. Svensson, H., Acta Radiol. Ther. Phys. Biol. 10, 631 (1971).

7. Bradshaw, A. A., Phys Med. Biol. 10, 355 (1965).

8. Pinkerton, A. P., Ann. New York Acad. Sci., 161, 63 (1969).

9. Sakata, S., Nippon Acta Radiol. 32, 619 (1972).

10. International Commission on Radiation Units and Measurements (ICRU), Report 21 , Radiation Dosimetry: Electrons with Initial Energies Between 1 and $50 \mathrm{MeV}$, Washington (1972).

11. Berger, M. J., Seltzer, S. M., Domen, S. R., and Lamperti, P. J., Biomedical Dosimetry (IAEA-SM-193/39), International Atomic Energy Agency, Vienna, 589-609 (1975).

12. Domen, S. R., and Lamperti, P. J., in press for publication in Medical Physics.

13. Domen, S. R., and Lamperti, P. J., J. Res. NBS, 78A, 595-610 (1974).

14. Kase, K. R., and Domen, S. R., Nuc1. Instr. Meth. 118, 469 (1974).

15. Domen, S. R., Mikado, T., and Lamperti, P. J., NBS-IR-76-1023 (1976).

(Available as PB 256191 from the National Technical Information Services, Springfield, Va. 22161.)

16. Berger, M. J., Ch. 5, Methods in Computatational Physics (Adler, B., Fernbach, S., Rotenberg, M., Eds.), Academic Press, New York (1963).

17. Berger, M. J., Seltzer, S. M., Ann. New York Acad. Sci. 161, 8 (1969).

18. Spencer, L. V., Attix, F. H., Radiat. Res. 3, 239 (1955). 
19. Kessaris, N. D., Radiat. Res. 43, 288 (1970); also private communication.

20. Hospital Physicists' Association (HPA), Report No. 4, A Practical Guide to Electron Dosimetry, London (1971).
21. Nordic Association of Clinical Physics (NACP), Acta Radiol. Ther. Phys. Biol. 11, 603 (1972). 


\title{
DOSE PROFILES IN TISSUE AND IN AIR FOR THERAPEUTIC ELECTRON BEAMS
}

\author{
G. Osman
}

Roswell Park Memorial Institute Buffalo, NY 14263

Electron dose profiles in body tissues and in air were obtained in this work theoretically and experimentally for 3 to $30 \mathrm{MeV}$ incident beam energies. A knowledge of dose profiles in air is necessary for any extended SSD treatments or air gap beyond the end of collimator. Theoretical dose profiles were predicted at various depths in tissue using the Osman's Model for therapeutic electron beams. Experiments were carried out with unfiltered beams in air and in tissue equivalent materials and results were compared with those predicted from our theoretical model. During the initial depth of penetration in muscle, fat, bone, lung and air, the electron dose profiles showed flat-top distributions within the geometrical configuration of the beam. At considerable depths in air or tissue (comparable to the range and field size), electron dose profiles have shown Gaussian distributions of various half-widths. Between the two extremes, electron dose distribution experiences a gradual transition from flat-top to the Gaussian. Slopes of these Gaussian curves varied inversely with the incident electron energy. The Gaussian dose distributions in tissues are generally not clinically acceptable. The flat distributions, however, can be obtained by proper design of scattering foils and electron cones. Tissue dose profiles as obtained from our mode1 could provide useful input data for programing treatment planning with high energy electron beams, using mini-computers. It is expected that the predicted dose distribution by our model could also account for cone design and body-inhomogeneity.

(Electron disometry, pencil beam, therapeutic beam, dose profiles)

\section{Introduction}

High energy electron beams produced by linear and circular accelerators are now in common use in radiation therapy. Electron beams with initial kinetic energies between 3 and $30 \mathrm{MeV}$ have been found to form useful therapeutic agents. Considerable physical and clinical experience has been gained in the last two decades.1-6 Electron beams offer absorption characteristics in tissue enabling treatments of any superficial volume and depth with good protection of underlying vital organs and surrounding tissues. Electron depth dose curves lose their sharp cut-off at high energies and the dose distribution is very sensitive to a change in mass density. It was also found that a linear energy-range relationship could be a reliable and convenient basis for determining the kinetic energy of theincidentelectron beams within the useful therapeutic energy range. It is important to be aware of a number of discrepancies ${ }^{7}$ in field uniformity and depth dose curves between different machines or even for identical units depending on the scattering foils used and the design of accelerator collimating system, field defining cones and the transmission monitoring devices. For this reason, depth dose on the major axis as a function of portal size and depth below the surface is a function of the machine generating the beam. For an acceptable therapeutic machine, the unit should deliver a uniform flat field of variable size, the initial depth dose curves should parallel the surface in a homogeneous medium and the $\mathrm{x}$-ray contamination should be at a minimum level. We have, therefore, assumed in our model for therapeutic electron beams, that the clinical beam is adequately uniform at the portal entry and central axis depth dose data for individual fields must be experimentally determined. When the electron isodose surfaces are not parallel to the phantom surface under normal incidence e.g. near the edge of the field, measures should be taken to correct the initially incident beam. ${ }^{8}$ In the present investigation, when a uniform therapeutic electron beam enters a tissue or air medium directly from the collimating device of the clinical machine, a flat-top dose distribution was observed at shallow depths changing gradually to gaussian as the depth in medium is increased. However, the tendency of electron isodose surfaces to remain parallel to the entrance surface can be extended all the way up to the end of the range by proper design of their scattering foils and electron cones placed directly on the skin except for very large fields or for total body superficial therapy. The interactions of a narrow beam of fast electrons with air, water or other media is we11 known. Theoretical and physical analyses for these 
interactions are reviewed in 1iterature?,10, 11 In these studies, the main interest was the interactions of electron pencil beams with matter. In an attempt to study the mode of interactions of a broad therapeutic electron beam with air and body tissues and to relate the dose distribution at a particular depth to the basic parameters of the medium (e.g. the average density and the effective atomic number), we present our theoretical electron model in which we consider the broad therapeutic electron beam to be an integral of electron pencil beam components 12,13 to which the basic theories of interactions apply. We have also studied the divergence of electron pencil beams since they are the input elements in our model. Seeking a workable mathematical expression that can lead to a simple computer program, this work was aimed to find a simple relation between the percent depth dosages at locations away from the central axis and those on the central axis at the same depth in air or in tissue and for any field configuration. The axial percent depth dose as a function of transverse displacement off the central axis of the therapeutic electron beam in planes perpendicular to the central axis are called: dose profiles.

\section{Experimental Methods}

Electron absorbed dose is measured ${ }^{14}$ by the fluence of electrons together with the stopping power of the medium. Electron dose in the air was measured with a calibrated parallel plate build-up chamber connected to a Keithley electrometer. Readings at each location refer to local peak dose values in a polystyrene phantom. Suitable build-up polystyrene plates were added for equilibrium dose measurements. Readings were then corrected for tissue equivalence and energy was calculated from absorption range measure ments carried out separately. This method can be considered fairly accurate since there was no beam diffuser, flattening filters, scattering foils or direct in-beam monitoring device. Dose distribution in electron pencil beams in air were measured at the equilibrium depth by a $0.2 \mathrm{~cm}^{3}$ Baldwin-Farmer ion chamber mounted in a water phantom-automatic remote scanner. The chamber moves in planes perpendicular to the beam for dose profile measurements. The chamber was calibrated for Cobalt 60 beams. Two separate methods for tissue dose measurements were used. The first method of measurement was made with an automatic remote scanning water phantom of $30 \times 30 \times 30 \mathrm{~cm}$ dimension. The second procedure utilized a solid "SCRAD" phantom made of polystyrene blocks and slabs of $25 \times 25 \mathrm{~cm}$ and of sufficient depth for each particular energy. Translite Kodak films were pressed evenly between two flat polystyrene slabs: A11 phantoms were exposed at right angles to the central axis of the therapeutic beam. Electron beam dose profiles were then obtained by measuring the optical densities on an automatic microdensitometer. Films were processed under controlled and reproducible conditions and readings were normalized to ionization measurements. Density readings were all taken in the linear range of the characteristic curve for Translite films. Optical film density measurements were checked and found to be in good agreement with those obtained from small probe ion chamber measurements in polystyrene phantom.

Fig. (1)

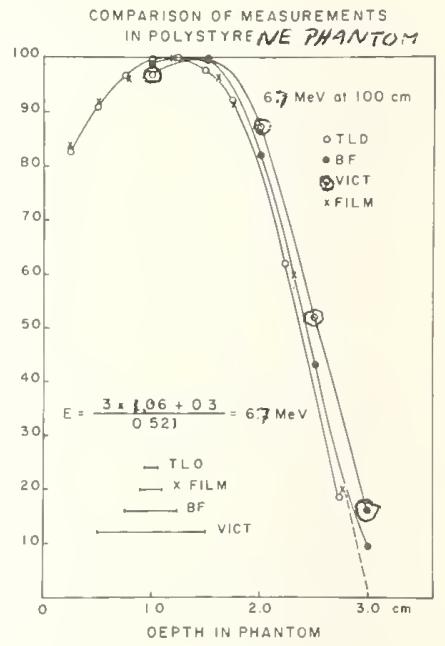

Figure (1) shows a comparison of dosimetric techniques where different dosimeters were used to obtain depth dose curves. The insert in this figure shows the relative probe dimensions while the scanning spot of the microdensitometer used in this experiment was of $2 \mathrm{~mm}$ diameter. X-ray contamination in the therapeutic beam was measured by the ionization current with the ion chamber placed beyond the range of the electrons. For narrow beam experiments, a well defined pencil beam of electrons was obtained by further collimation of the uniform electron therapy beam delivered from the Roswell Park Memorial Institute Clinic 6 and 35 linear accelerators. The pencil beam collimator consists of two parallel lead sheetabsorbers, each $2 \mathrm{~mm}$ thick, $5 \mathrm{~cm}$ apart with a circular pin-hole of $1 \mathrm{~mm}$ radius exactly in line with the central axis of the therapeutic beam. The lead sheet-absorbers were mounted at the end of the machine collimator 
perpendicular to the central axis of the therapeutic beam.

Fig. (2) Energy Distribution of Electron Beams in Planes Perpendicular to The Central axis.

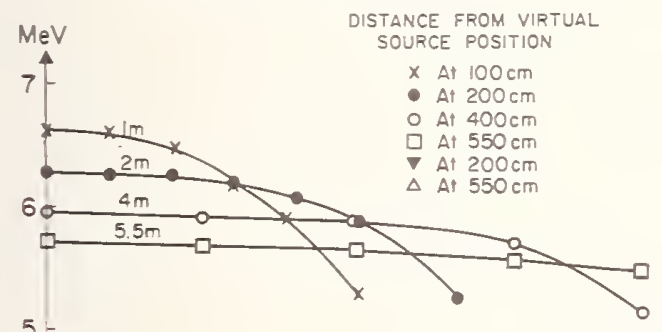

5

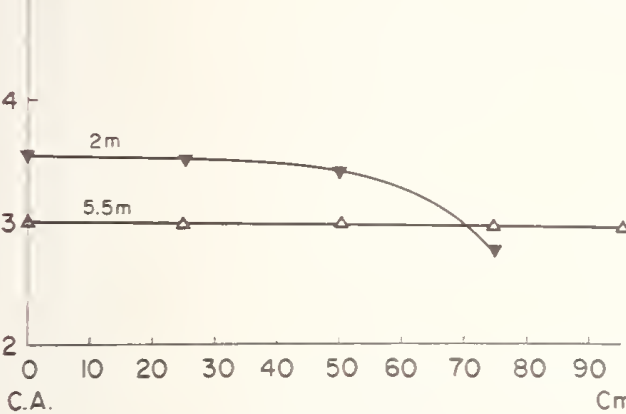

Distonce from C.A

A spatial distribution of the electron fluence in energy was made in air of the treatment room within the limits of the clinical beam in order to detect any energy losses upon penetrating into the air medium. Fig. (2) shows the results of measurements at 1,2 4 and 5.5 meters SSD for a clinical electron beam of initial beam energies 6.7 and 3.6 $\mathrm{MeV}$ at isocenter. Energy was calculated from Markus relation between the energy at the surface of normally incident electrons on the build-up chamber and the practical range. It is concluded from this experiment that energy fluence from the central axis remains fairly constant within the 50 percent decrement surface of the beam. Beyond this region a gradual drop in energy was observed as the transverse displacement is increased.

This is probably due to the greater path length and the wide range of energies result ing from electron back scattering at further locations. In addition to the energy loss function, the energy of electrons that have suffered extreme deviations differs from the energy at same depth on the central axis, resulting in a straggling distribution effect.

Consequently, the energy based on absorption range measurements beyond the 50 percent decrement surface has very little meaning. It is also concluded from this exper-
Fig. (3) Diagram Showing the Geometry of the Scattering Angles.

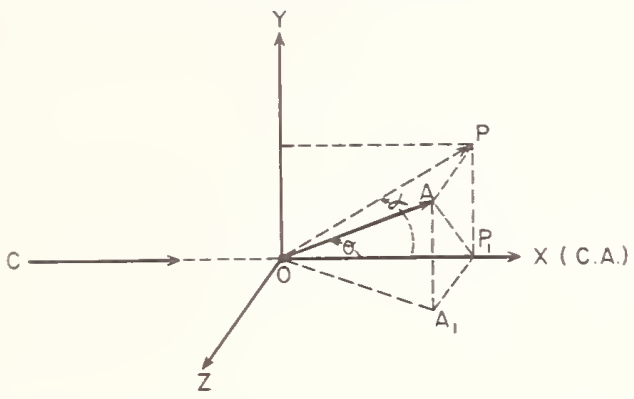

iment that spatial dose distribution of absorbed energy can be accurately determined within the 50 percent decrement curve.

\section{Theory of Electron Beams}

\section{(i) Pencil Beams:}

Electron dose distribution can be assumed to be proportional to the electron number distribution i.e. the fluence, provided the incident energy is normal to the surface and essentially constant across the dose profiles within the useful width of the beam. In fact the overall correction factor which converts number of electrons into dose is a function of the mean energy of the primary beam at the depth of measurement. The number distribution of electrons due to one single pencil beam is based on the central limit statistical theory when applied on individual scattering events. The distribution function is, therefore, a Gaussian with the mean value lying along the original direction of the pencil beam. Figure (3) shows the geometry for scattering angles where $\theta$ is the gross scattering angle of the electrons which is a function of depth $\alpha$ is the projected angle of scattering and for small angles such as those encountered in elastic scattering, $\left\langle\alpha^{2}\right\rangle$ is one-half of $\left\langle\theta^{2}\right\rangle$. Dose profiles in planes at right angles to the pencil beam as the electrons traverse a thickness $x$ in a medium is given by 18

$$
D(\theta)=D(0) \exp \left[-\left(\theta-\mu_{\theta}\right)^{2} / \sigma_{\theta}{ }^{2}\right]
$$

where $D$ represents the electron dose at a 
particular location on the profiles.

Fig. (4) IHEORETICAL $607 \%$ DECREMENT LINES OF A PENCIL BEAM OF ELECTRONS $\sigma$. RMS ANGLES OF DEVIATION at VARIOUS SS D. In $\boldsymbol{Q}$ ir \& NET ANGLES OF DEFLECTION AT TREATMENT PLANE $56 \mathrm{~m}$ S.S.D.

Since the Gaussian peaks along the original direction of the beam, the mean of the scattering angles $\mu_{\theta}=0$ In the projection plane the last equation will reduce to: $D(\alpha)=D$ (0) $\exp \left(-\alpha^{2} / 2 \sigma^{2}\right)$ (i) if $D(x)$ is normalized such that the area under the curve is unity, then $D(0)=1 /(2 \pi)^{\frac{1}{2}} \sigma$ where $\sigma$ is the standard deviation of the projected scattering angles, defined as the square root of the average of the squares of the angular deviations from the mean $i . e .1$

$$
\begin{aligned}
& J=\left\langle\left(\alpha-\mu_{\theta}\right)^{2}\right\rangle^{\frac{1}{2}}=\left\langle\alpha^{2}\right\rangle^{\frac{1}{2}}=R . M . S \\
& D(\alpha) / D(0)=60.7 \% \quad \text { (2) }
\end{aligned}
$$

The root-mean-square projected transverse displacement " $d$ " in the dose profiles can be calculated from the following equation ${ }^{19}$ :

Theoretical derivations of the divergence of electron pencil beams have been the subject of a large amount of literature based on multiple scattering. The theories most relevant to the pregsent work are those of Ashkin and Bethe ${ }_{1}$ and of Rossill from which $\sigma,\left\langle\theta^{2}\right\rangle^{\frac{1}{2}}$ and $\left\langle\alpha^{2}\right\rangle^{\frac{1}{2}}$ lon be cbtained.

$$
\begin{aligned}
& \sigma^{2}=\frac{\left\langle\theta^{2}\right\rangle}{2}=\left\langle\alpha^{2}\right\rangle=\frac{4 \pi N z(z+1) e^{4} x}{P_{x}^{2} V_{x}^{2}} \ln \left[2 \pi^{\frac{1}{2}} z^{\frac{2}{3}} N^{\frac{1}{2}} x^{\frac{1}{2}}\left(\frac{\hbar}{m V_{x}}\right)\right] \\
& \sigma^{2}=\frac{\left\langle\theta^{2}\right\rangle}{2}=\left\langle\alpha^{2}\right\rangle=\frac{4 \pi N z^{2} e^{4} \cdot x}{P_{x}^{2} V_{x}^{2}} \ln \left[\frac{137 P_{x}}{z^{\frac{1}{3}} \text { me }}\right]
\end{aligned}
$$$$
=9.108 \times 10^{-28} \mathrm{~g} \equiv 0.511 \mathrm{MeV}
$$$$
\mathrm{e}=\text { Electronic charge }
$$$$
=4.8 \times 10^{10} \mathrm{esw}
$$

$\hbar=$ Planck's Const. divided by $2 \pi$

$=1.05445 \times 10^{-27}$ erg.sec

Figure (4) shows the Aivergence of two elec tron pencil beams in air obtained theoretically. Because the decrement curves are symmetrical around the initial direction of the pencil beam, only one half. for each di-

$\mathrm{N}=$ Number of scattering centers $/ \mathrm{cm}^{3}$

$\mathrm{C}=$ Speed of light in Vacuum

$=2.4979 \times 10^{10} \mathrm{cin} / \mathrm{sic}$

$\mathrm{P}_{\mathrm{x}}=$ Average momentum of electrons at a depth $x$ $\mathrm{cm}$ in medium

$\mathrm{V}_{\mathrm{X}}=$ Average velocity of electrons at a depth $x$ $\mathrm{cm}$ in medium

$z=$ Effective atomic number ${ }^{20}$ of material transversed by the incident electrons.

$$
=2.94 \sqrt{a_{1} z_{1}^{2.94}+a_{2} z_{2}^{2.94}+a_{3} z_{3}^{2.94}+\cdots}
$$

where $a_{1}, a_{2}, a_{3}, \ldots$ are the fractional contents of electrons belonging to elements $z_{1}, z_{2}, z_{3}, .$. of the medium. $\mathrm{m}=$ Rest mass of the electron vergence cones is given. In this figure, the 60.7 percent decrement curves were obtained based on elastic multiple scattering theories of Bethe and Ashkin and Rossi"

for 3.6 and $6.7 \mathrm{MeV}$ initial beam energies. The upper half refers to the divergence of a $3.6 \mathrm{MeV}$ electron pencil beam while the lower half refers to the $6.7 \mathrm{MeV}$ pencil beam. The projected R.M.S. angular deviation $\left\langle\alpha^{2}\right\rangle^{1 / 2}=\sigma$ and the corresponding projected transverse displacement "d" were calculated for each energy at different depths $x$ from equations 3, 4 and 5. After defining the points of a particular transverse displacement " $\mathrm{d}$ " and the corresponding 
tangential directions, the decremental curves were drawn for each energy. The net deflection angle $\phi$ can also be obtained for any depth in tissue or any treatment plane as shown in this figure.

(ii) Therapeutic Beams

Figure (5) shcis the basis of our electron Model. The dose distribution in a therapeutic beam at a depth $\mathrm{x}$ in tissue or in air medium can be considered to be the resultant of a number of dose contributions of elec-1 trons coming from different locations within the collimated broad beam. When a clinical machine gives a uniform dose at the end of the collimator, it is reasonable to consider the therapeutic beam as being made up of an infinite number of identical and initially parallel pencil

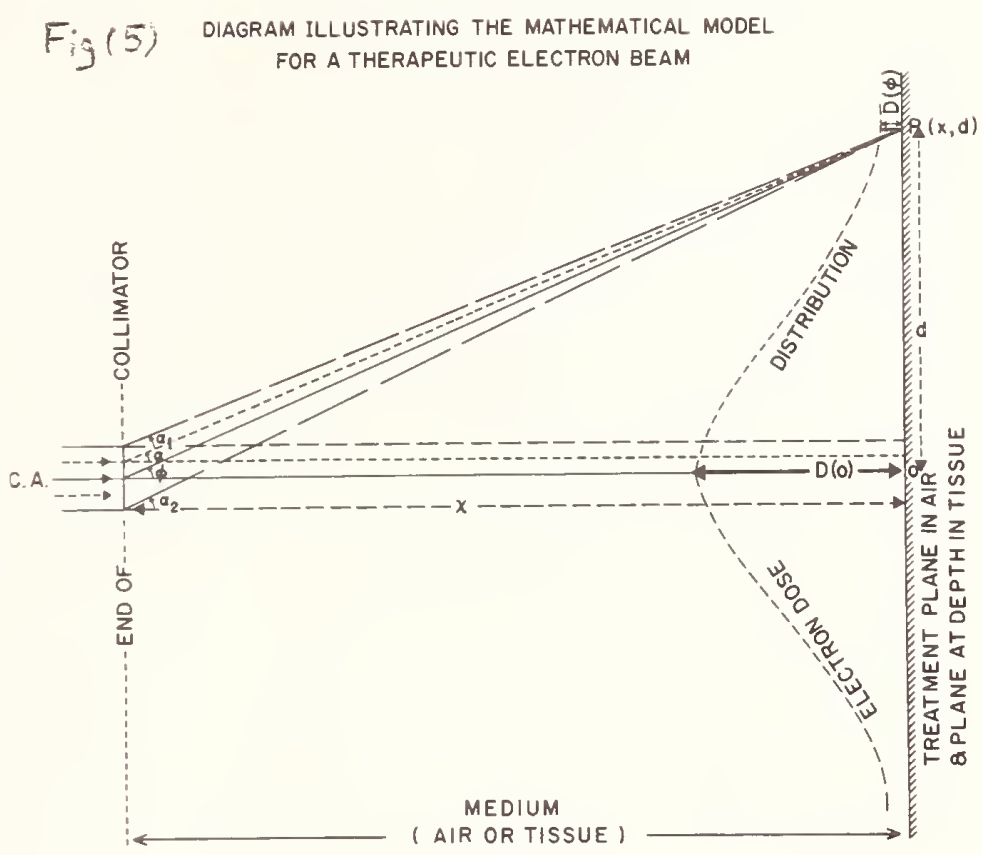

beam components each of minute width,

Each pencil beam, emerging at

different locations within the

width of the beam, will diverge into a cone on entering the uniform medium such as those shown in Fig. (4). Considering a point at a location $\mathrm{p}$ at a depth $\mathrm{x}$ in medium and distance $d$ off the central axis of the therapeutic beam, or at a deviation $\phi$ from the beam central axis, the resultant dose $\mathrm{D}_{\mathrm{p}}(\phi)$ will be the summation of different dose components (the overlapping gaussians) contributed from each pencil beam. The resultant dose profiles in air or in tissue can, therefore, be predicted from the scattering characteristics of individual electron pencil beans. Therefore the resultant dose $D_{p}(\phi)$ can be expressed as an integral between $\alpha_{1}$ and $\alpha_{2}$ as the limits as shown in Fig. (5).

$$
\begin{aligned}
& D_{p}(\psi)=\frac{1}{(2 \pi)^{\frac{1}{2}}} \int_{\alpha_{1}}^{\alpha_{2}} \frac{1}{\sigma} \exp \left(-\alpha^{2} / 2 \sigma^{2}\right) d \alpha \\
& \alpha_{1}=\tan ^{-1}\left(\frac{d-l}{x^{2}}\right)=\tan ^{-1}\left(\tan \phi-\frac{l}{x}\right) \\
& \alpha_{2}=\tan ^{-1}\left(\frac{d+l}{x}\right)=\tan ^{-1}\left(\tan \phi+\frac{k}{x}\right)
\end{aligned}
$$

where $l$ is half the initial beam width at end of colifmator. The parameter $\sigma$ in this basic equation can be substituted from the we11 known expressions of R.M.S. angular deviation for electron pencil beams. The above mentioned integral is the basis for the resultant dose profiles which can be applied for air, tissue or any other medium. The body inhomogeneity can be easily accounted for, from the basic parf neters such as

average density and atomic number. The projected R.M.S. angular deviation " $\alpha$ " is expressed in terms of electron energy (e.g. momentum, velocity), depth of penetration $\mathrm{x}$ and the average density of the medium. The integral in equation (6) cannot be integrated in terms of a closed form but can be numerically integrated by applying "Simpson's rule"2l.

\section{(iii) Method of Calculation}

Our main objective is to obtain theoretically derived values of the electron dose distribution across the therapeutic beam in tissues which can predict accurate experimental results. The resultant dose profiles were calcula ed from eq (6) with the central axis dose normalized to 100 percent. The computer print out values are the theoretically predicted dose distribution at successive planes i.e. dose profiles. Theoretical dose profiles leading to electron dose distribution were obtained in air, subcutaneous fat, lung, muscle and bone for initial electron beam energies ranging from 3 to $30 \mathrm{MeV}$ taking into account the energy loss in the media concerned. The central axis dose, however, was taken for all media from experimental depth dose curves at the particular energy and SSD concerned. Dose profiles on planes perpendicular to the central axis were calculated at successive increments and for 
adequate lateral displacements. In muscle, dose profiles were obtained to a depth of $15 \mathrm{~cm}$ with successive increments of $1 \mathrm{~cm}$ depth and up to $8 \mathrm{~cm}$ lateral displacement on either side for a portal field of $10 \mathrm{x} 10 \mathrm{~cm}$ with increments of $2 \mathrm{~mm}$ between successive locations. In air, for a therapeutic electron beam with initial configuration of $20 \mathrm{~cm} x$ $20 \mathrm{~cm}$ at the end of the collimator of the linear accelerator, dose profiles for all points between central axis and $100 \mathrm{~cm}$ displacements on either side were calculated at successive increments of $2 \mathrm{~cm}$ between two locatio:s. For relatively large distances, i.e. from 3.5 to $5.6 \mathrm{~m}$ they were also calculated between the beam central axis and $300 \mathrm{~cm}$ lateral displacement at successive increments of $5 \mathrm{~cm}$ between each two locatiors.

\section{(iv) Formulae Used in the Calculations}

The spatial dose distribution of the absorbed energy of electrons was calculated from eq (6) on the basis of our theoretical Model. The parameter $\sigma$ in this basic equation was taken from the pencil beam expressions for electrons as the basic elements. The R.M.S. angular deviation $\left\langle\cdot \gamma^{2}\right\rangle^{\frac{1}{2}}=\sigma$ is expressed in terms of depth of penetration $\mathrm{x}$, average density 5 of the medium, effective atomic number $z$ of the medium, number of scattering centers/ $\mathrm{cm}^{3}$ " $\mathrm{N}$ " and the electron energy at a depth in the medium (average momentum $P_{X}$ and average velocity 22 $\mathrm{V}_{\mathrm{x}}$ ). For any tissue the following relation was applied in our calculations:

$\rho R_{\mathrm{p}}=0.530 \mathrm{E}-0.106$ $\rho \mathrm{R}_{\mathrm{p}}=\mathrm{in} \mathrm{g} / \mathrm{cm}^{2}$ and $\mathrm{E}$ is the kinetic energy of the incident electrons in $\mathrm{MeV}$.

In muscle we have used the following equation: ${ }^{2} \mathrm{R}_{\mathrm{p}}=0.521 \mathrm{E}-0.376$, knowing the initial energy and density of medium, the practical range $R_{p}$ could be calculated. The effective total energy $E_{x}$ of the electrons $z_{4}$ at depth $\mathrm{x}$ in the medium was calculated from

$$
E_{x}-0.511=E\left[1-x / R_{p}\right]
$$

$P_{x}$ and $V_{x}$ can be obtained from the effective electron energy $E_{x}$ at depth $x$ in the medium from the end of collimator or pinhole by the following relations ${ }^{19}$ :

$$
\begin{aligned}
& E_{x}=\left[c^{2} P_{x}^{2}+m^{2} c^{4}\right]^{\frac{1}{2}}=E_{c}-\left(\frac{d E_{i}}{d x}\right) x \\
& V_{x}=c^{2} P_{x} / E_{x}
\end{aligned}
$$

$E_{C}$ is the initial electron total energy at the end of collimator=Kinetic energy $E$ in

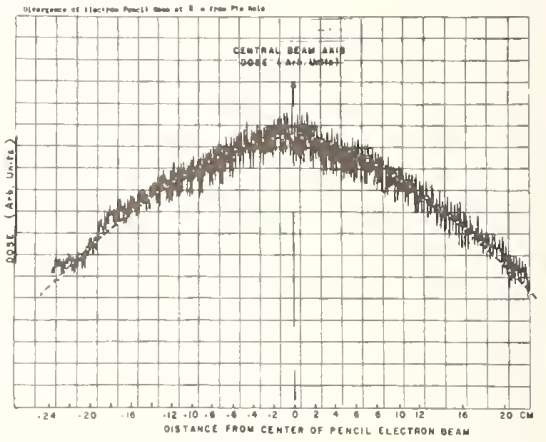

$\mathrm{MeV}+0.511$. The number of scattering centers " $\mathrm{N}$ " in different body tissues or in air can be calculated from simple relations. In our calculations we have used the following methods:

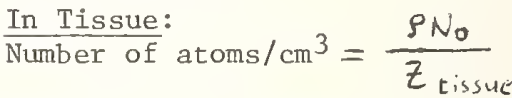

where No is the number of electrons/g. The following table 25 gives $\rho$, No and $z$ for different tissues which were used in our calculations :

$\begin{array}{lccc}\text { Tissue } & \begin{array}{c}\text { Density } \\ \rho \mathrm{g} / \mathrm{cm}^{3}\end{array} & z & \text { No } \\ \text { Muscle } & 1.00 & 7.42 & 3.36 \times 10^{23} \\ \text { Fat } & 0.91 & 5.92 & 3.48 \times 10^{23} \\ \text { Bone } & 1.85 & 13.8 & 3.00 \times 10^{23} \\ \text { Lung } & 0.51 & 7.53 & 3.8 \times 10^{23}\end{array}$

(1) Number of atoms $/ \mathrm{cm}^{3}$ of air "N" $=\rho \frac{6.02 \times 10}{2 Z_{\text {air }}}$ where $\rho=\rho_{0} F_{T, p}$ $\rho$ is the density of air (at prevailing temperature $T$ and pressure $P$ ) in $\mathrm{g} / \mathrm{cm}^{3}$.

$\rho_{0}$ is the density of air at "normal $T$ and $P$ " $=1.2929 \times 10^{-3} \mathrm{~g} / \mathrm{cm}^{3}$. 
$F_{T, P}=$ Temperature and pressure correction factor. Since the prevailing conditions in our treatment room are $\mathrm{P}=745 \mathrm{~mm} \mathrm{Hg}$ and $\mathrm{T}=$ $295 \circ \mathrm{K}$

$$
\begin{aligned}
\rho & =1.2929 \times 10^{-3}\left(\frac{745}{760}\right)\left(\frac{273.16}{295}\right) \\
& =1.115 \times 10^{-3} \mathrm{~g} / \mathrm{cm}^{3} \text { of cir }
\end{aligned}
$$

IV Results:Experimental and Theoretical

(i) Pencil Beams and Gaussian Fitting: Equation (1) can be written: $D(j)=D(c) \operatorname{cxp}\left(-0.693 \mathrm{~d}^{2} / \mathrm{d}_{\frac{1}{2}}^{2}\right)$ where $d$ is the hall width of the gaussian distribution i.e. when $d=d_{\frac{1}{2}}, D(d)=\frac{1}{2} D(0)$
and has the value:

$$
d_{\frac{1}{2}}^{2}=2 x^{2} \sigma^{2} \ln 2
$$

Fig. (6) shows the experimental results for an electron pencil beam diverging in air. Dose distributions at 1,2 and 3 meters from pin-hole were recorded at the equilibrium position in water phantom. Experimental curves were fitted with theoretical gaussian distributions as obtained from eq (7). We can compute $100 \mathrm{D}(\alpha) / \mathrm{D}(0)$ for different $\left(d / d \frac{1}{2}\right)$ with $d \&$ and $D(o)$ taken from experimental data. 'In all these experiments, the chart recorded lines represent experimental data while the dotted curves represent eq (7) with $d_{\frac{1}{2}}=8,24$ and $38 \mathrm{~cm}$ respectively. It is, therefore, concluded that applying the multiple scattering elastic theories is justifiable and the divergence of the electron beam should be accurately known from the basic parameters of the medium such as $\rho \& z$. From these equations, it is also possible to account for body inhomogeneity as $\rho$ and $z$ varies with depth.

Fig. (7). Experimental Electron Dose Profiles for $3.6 \mathrm{MeV}$ Therapeutic Beam in Air at $100 \mathrm{~cm} \mathrm{SSD}$.

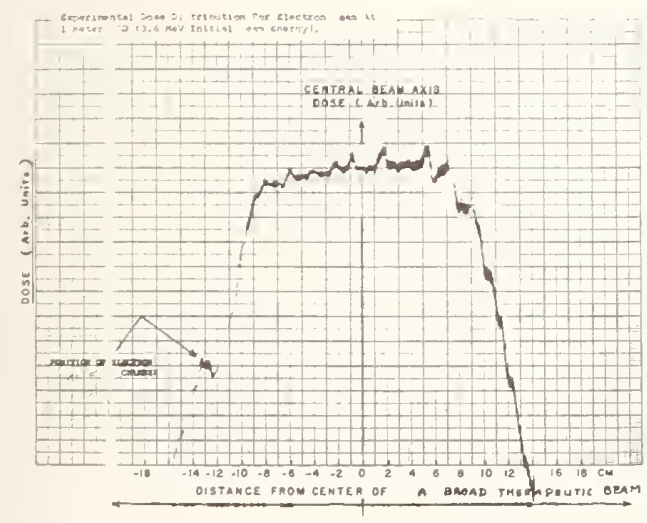

Fig. (8)

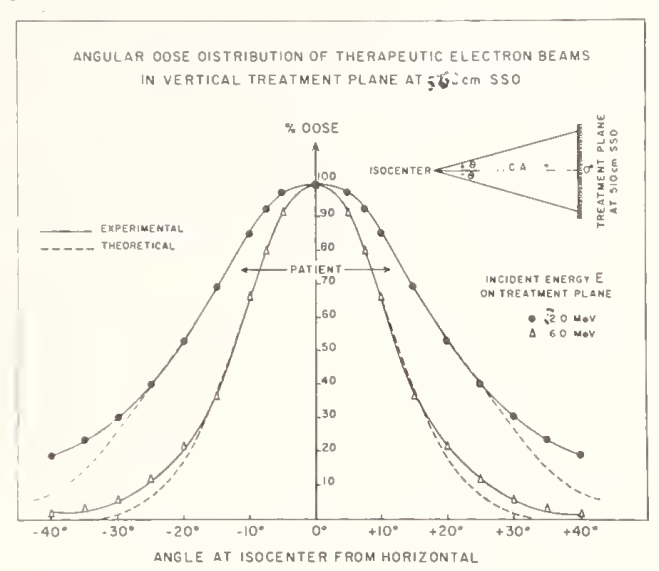

(ii) Therapeutic Beams:

The present results refer to a broad therapeutic electron beam as used in radiation therapy. No electron cone or beam diffuser were used in the following experiments in air or in tissue.

(a) Dose Profiles in Air

The Roswell Park Memorial Institute linear accelerator delivered the electron therapeutic beams across the length of the treatment room with a horizontal central axis as used in total body superficial treatments. The main interactions of the travelling electrons is with air molecules and an analysis of dose distribution was reported ${ }^{12,17}$. The transmission electron ion chamber was removed and placed outside the accelerator head, intercepting the edge of the beam. This ion chamber has no effect on the beam and served as the monitor unit indicator of the linear accelerator. Figure (7) shows the experimental dose distribution for a 21 x $21 \mathrm{~cm}$ electron beam of $3.6 \mathrm{MeV}$ initial energy at $100 \mathrm{~cm}$ SSD or at about $40 \mathrm{~cm}$ from the end of the machine collimator. The position of the transmission electron ion chamber can be observed in the chart recorded diagram of the dose profile Fig. (7). Figure (8) shows the angular dose distribution for 3.0 and $6.0 \mathrm{MeV}$ incident electron beam energy at $560 \mathrm{~cm}$ SSD for same field as obtained from experimental results together with their gaussian fits. From Figs. 7 and 8 it is clear that electron dose distributions vary from an essentially uniform field to gaussian distributions. The agreement with gaussian curves indicates that electrons are mainly suffering multiple elastic scattering. Figs. 9 and 10 show the dose profiles in air for 
3.6 and $6.7 \mathrm{MeV}$ incident electron energy as obtained from our theoretical Model.

\section{Fig. $(9)$ \% DOSE PROFILESIN AIR FOR THERAPEUTIC ELECTRON BEAM \\ AT SHORT SSD}
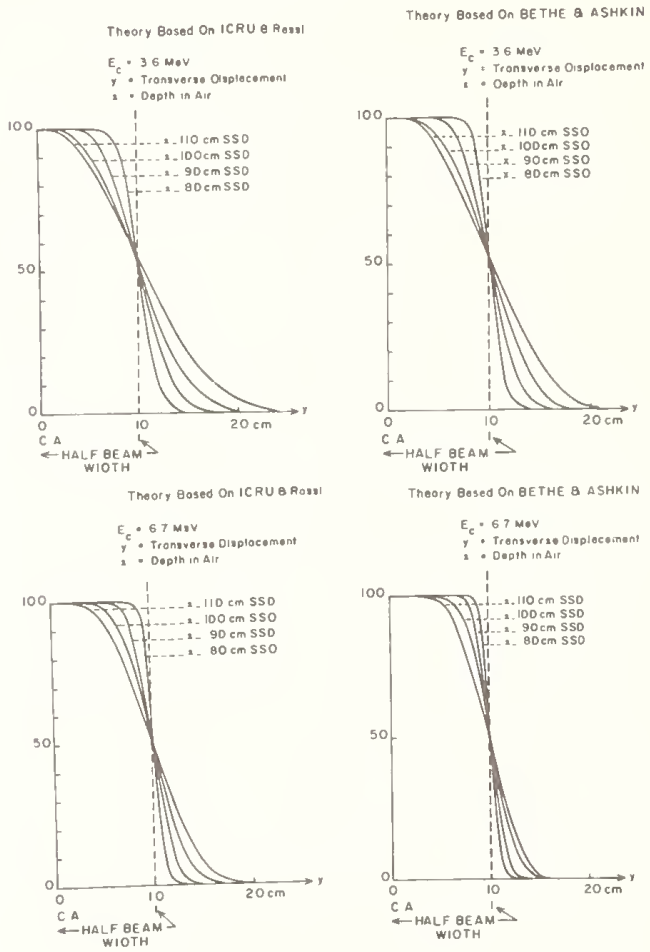

Fig. (11) Off-Axis Electron Dose Distribution of a Therapeutic Beam at Different Treatment Distances.

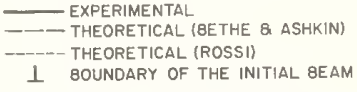

PERCENTAGE OF CENTRAL AXIS "CA" DOSE VS. TRANSVERSE DISTANCE " $d$ "

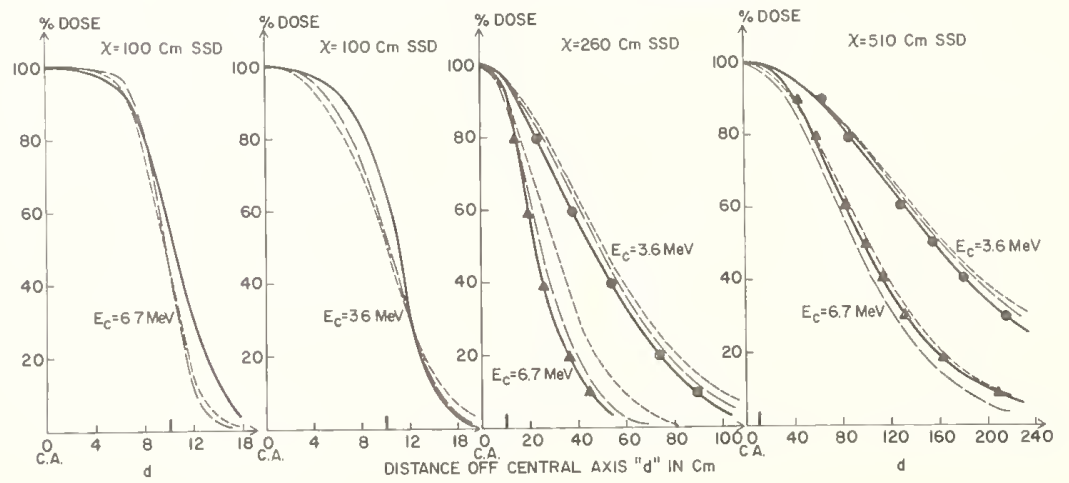

$\%$ DOSE PROFILES IN AIR

FOR THERAPEUTIC ELECTRON BEAM

AT EXTENDED SSD
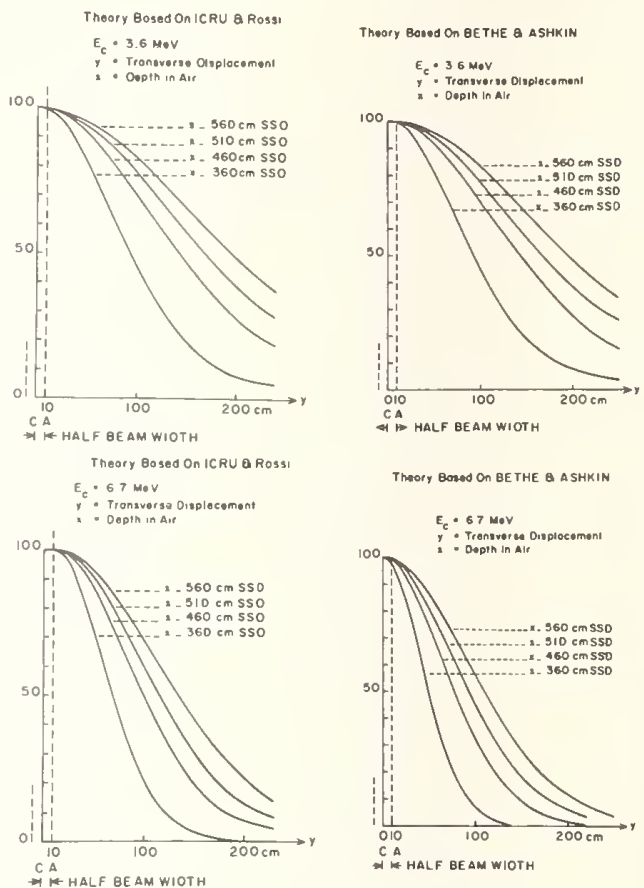

THAory BOrod ON BETHE Q ASHKIN

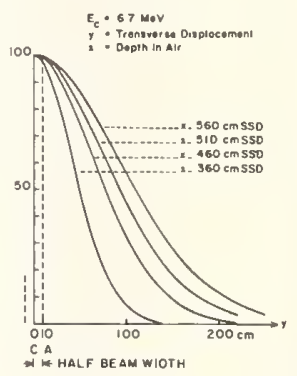

Fig. (11) shows a comparison of electron dose profiles in air of a therapeutic beam at different treatment distances for 3.6 and $6.7 \mathrm{MeV}$ initial beam energies. Two pencil beam theories were applied as the basic element in our Model for obtaining the electron dose distributions in the profiles, at short, intermediate and long SSD as shown in this figure. 
(b) Dose Profiles in Tissue: Fig. (12) shows an example of the chart recorded electron dose profiles across $30 \mathrm{MeV}$ beam at various depths in water phantom for a $10 \times 10 \mathrm{~cm}$. The gradual transition from flat-top uniform distribution to gaussian is observed as the depth in phantom is increased. Dose profiles in muscle were predicted from our theoretical model using the physical properties of the tissue concerned as explained in Section III. Figure 13 shows these profiles at various depths and for $6.7,10,20$ and 30 $\mathrm{MeV}$ normally incident electrons of the therapeutic beam and for a $10 \times 10 \mathrm{~cm}$ port field. Fig. 14 gives a comparison of dose profiles for $30 \mathrm{MeV}$ incident electron beam as obtained from experimental results and from our theoretical model for electron beams.
Fig. (12) Experimental Dose Profiles across $30 \mathrm{MeV}$ Electron Beam at Various Depth In Phantom for a $10 \times 10 \mathrm{~cm}$ Field Without Electron Cone or Beam Diffuser.

$$
x=\text { Depthin Phantion }
$$

$$
y=\text { Transverse Displacement }
$$
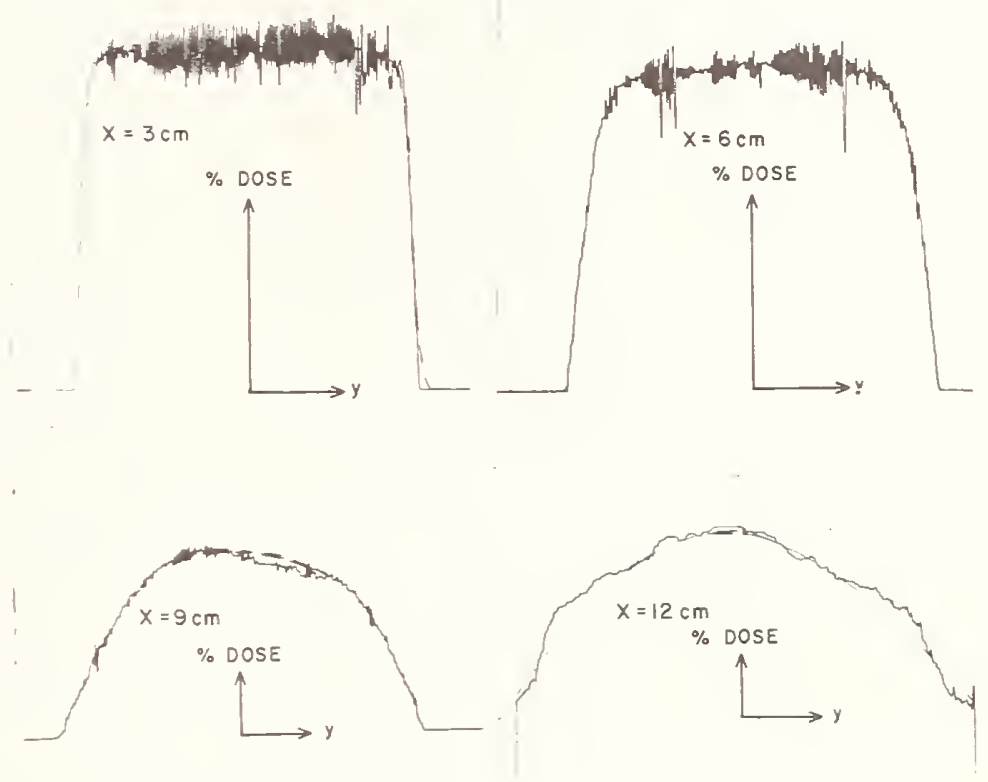

FIGI3 \% DOSE PROFILESIN MUSCLE FOR THERAPEUTIC ELECTRON BEAM
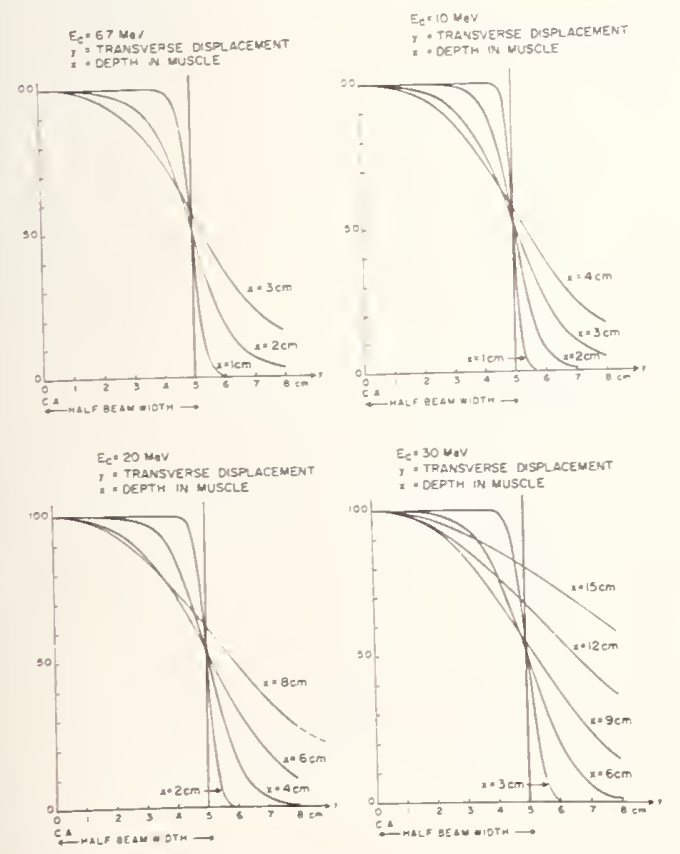

FIg( 14 ) COMPARISON OF THEORETICAL \& EXPERIMENTAL DOSE PROFILES FOR $30 \mathrm{MeV}$ ELECTRON 8 EAM FOR $10 \times 10 \mathrm{~cm}$ FIELD
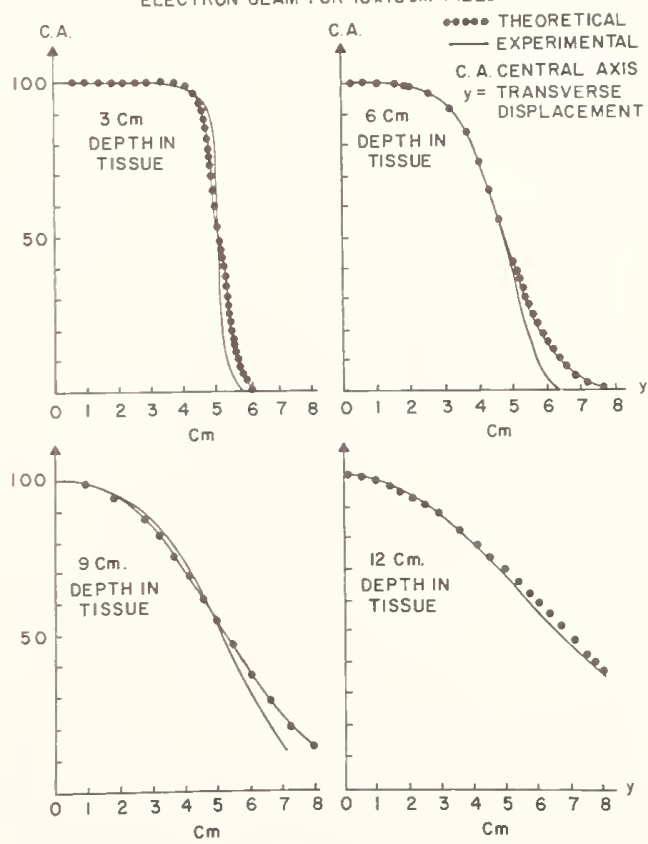
FIG. $15 \%$ DOSE PROFILES IN LUNG FOR THERAPEUTIC ELECTRON BEAM
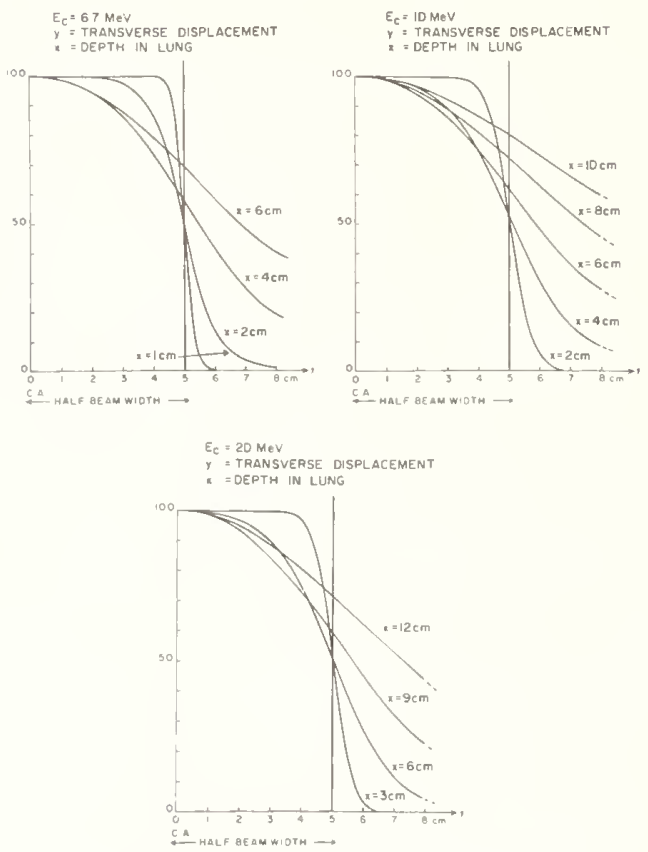

FIG. $16 \%$ DOSE PROFILESIN BONE FOR THERAPEUTIC ELECTRON BEAM
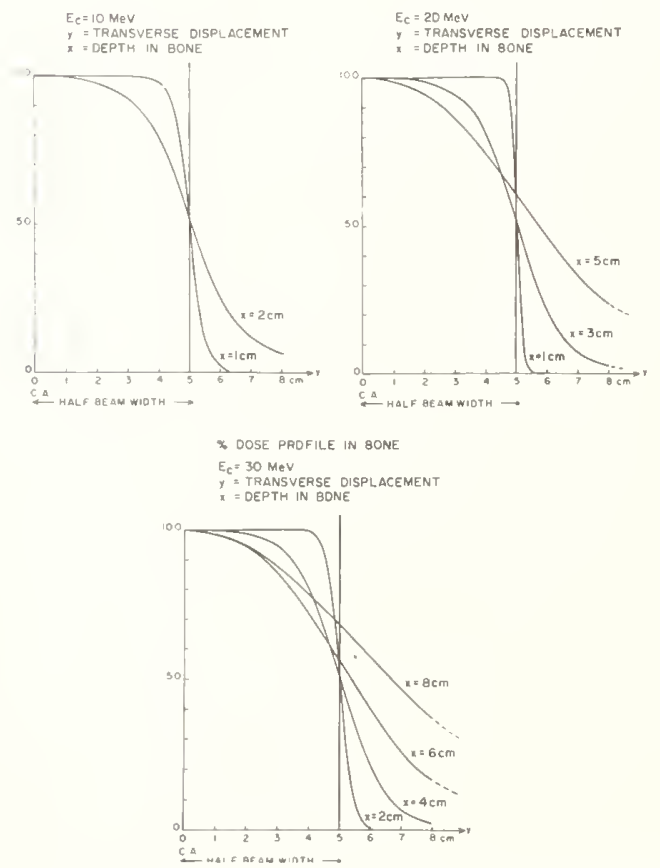

Figurs $(15,16$, and 17) are another examples of electron dose profiles in different tissues e.g. 1ung, bone and subcutaneous fat as predicted from our theoretical model. In both experimental and theoretical results, the electron depth dose in tissue
FIG $17 \%$ DOSE PROFILES IN FAT FOR THERAPEUTIC ELECTRON BEAM
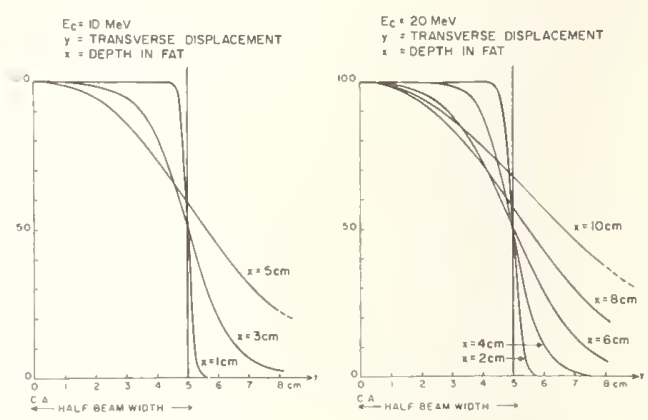

$$
\begin{aligned}
& E_{\mathrm{c}}=30 \text { MeV } \\
& y=\text { TRANSVERSE DISPLACEMENT } \\
& i=\text { DEPTH IN FAT }
\end{aligned}
$$

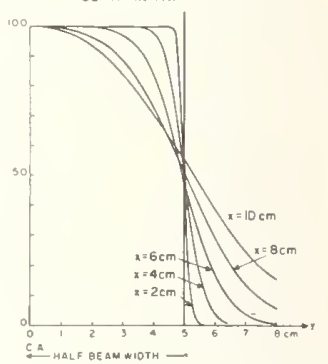

changes from uniform flat-top distribution to gaussian as the depth in tissue is increased. Tissue iose profiles as obtained from our electron model are in very good agreement with experimental data. Based on this evidence, a computer program has been derived as explained in Section III in application of our model and on the knowledge of the physical parameters of the concerned tissue.

\section{(c) X-Ray Contamination In Tissue}

$\mathrm{X}$-ray contamination was measured in polystyrene phantom for port fields of $10 \mathrm{x}$ $10 \mathrm{~cm}$ and for normally incident electron beams of $4,10,15,20,25$ and $30 \mathrm{MeV}$ initial kinetic energies. Readings were then corrected for tissue equivalence. In fact this experiment was part of electron depth dose curves after the beams have reached their practical range as shown in Fig. (18).

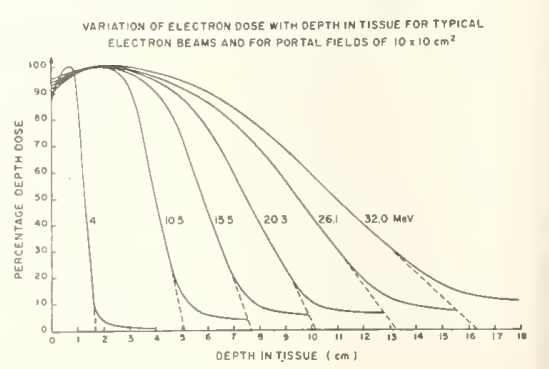


Beyond the electron range each curve levels off reaching a fairly constant value. The $\mathrm{X}$-ray contamination dose was found to increase with increase of the energy of the incident beam, rising from about /, percent at $4 \mathrm{MeV}$ to about 10 percent for the 30 $\mathrm{MeV}$ beam. In Fig. (18) the kinetic energy of each beam as calculated from the practical range were $4,10.5,15.5,20.3,26.1$ and $32 \mathrm{MeV}$ respectively.

(d) Electron Cones and Scattering Foils:

Flatness of electron beams used in radiation treatments has been improved by introducing one or more scattering foils inserted in the beam 26-30. These foils may be flat or shaped or combination ${ }^{31}$. Obviously this will increase the $\mathrm{X}$-ray contamination and therefore they should be avoided for whole body superficial electron treatments 32 . When using scattering foils they should be as thin as possible so that the contamination is minimized. However, in some clinical accelerators, the uniformity of the electron beam and the shape of isodose curves are not adequate for treatments except when using heavy scattering foils. Electron cones have also been designed so that lead blocks of adequate thickness intercept the edges of the electron beams. These electron cones are usually made of lucite and meant to make immediate contact with the skin. The lead blocks are built towards the end of the electron cone and acts as a diaphragm. The diaphragm size is usually smaller than the field size and an experimental relation between field size versus diaphragm size could be easily established. The scattered electrons from the wall material contributes to the skin dose and may also vary the shape of depth dose and isodose curves. Both electron cones and scattering foils are made to extend the electron beam flatness over the full range of electron energies and field sizes.

Fig. (19). Isodose Distribution of $14 \times 14 \mathrm{~cm}$ Field for $10 \mathrm{MeV}$.

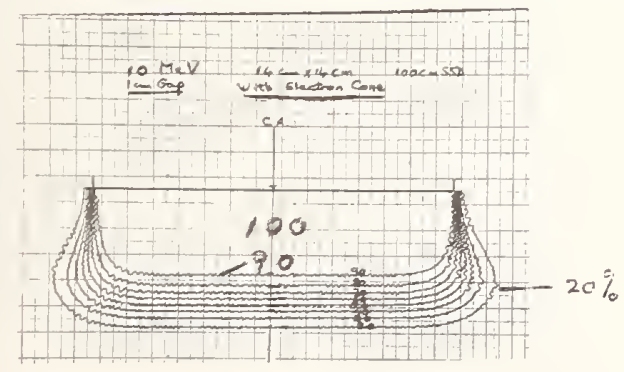

Figure (19) shows a typical electron isodose distribution for a $10 \mathrm{MeV}$ initial beam energy and for a port field $14 \mathrm{x} 14 \mathrm{~cm}$ using electron cone and a thin scatterer of 0.02 " $\mathrm{Pb}+.012$ " Al. These isodose curves were obtained using the $0.2 \mathrm{~cm}^{3}$ Farmer probe mounted on an automatic scanner in water phantom.

\section{(e) Air-Gaps:}

In some clinical situations it is necessary to treat a patient at distances greater than the length of the electron cone leaving an air gap between the end of the electron cone or collimator and the skin.

These air gaps could be small when electron cone fails to make good contact with the skin, or intermediate e.g. in the perineum or in the head and neck region, or could be large in the treatments of extensive or whole body superficial skin lesions. The air-gap has also strong influence on the electron dose profiles particularly at shallow depths.

Figs. (20 and 21).

Typical electron Dose Profiles with 1 and $2 \mathrm{~cm}$ AirGaps.
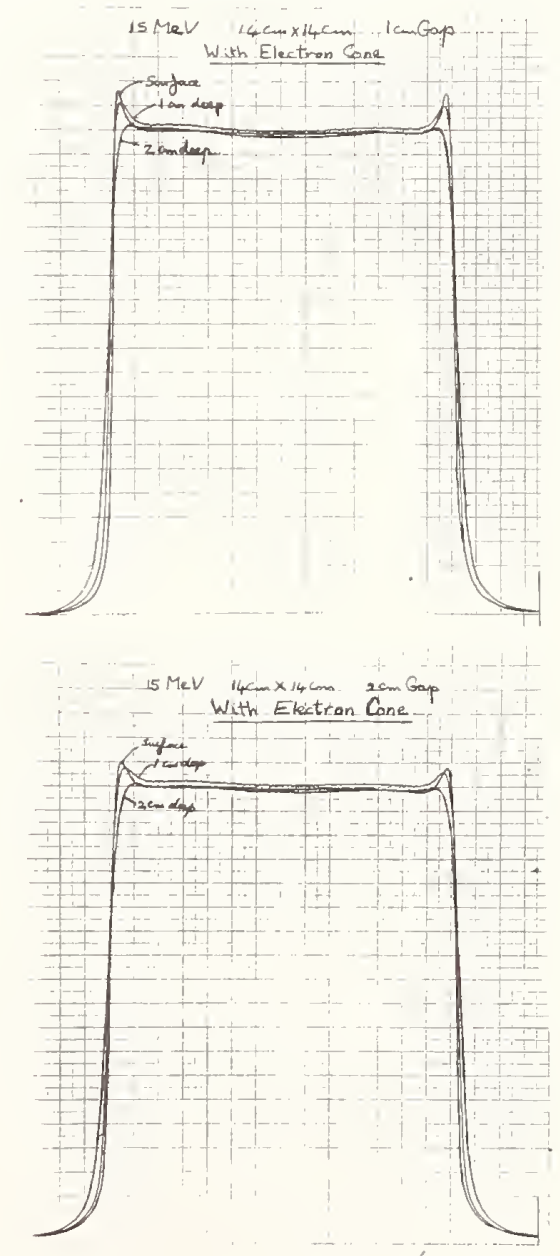
Fig. (22) .

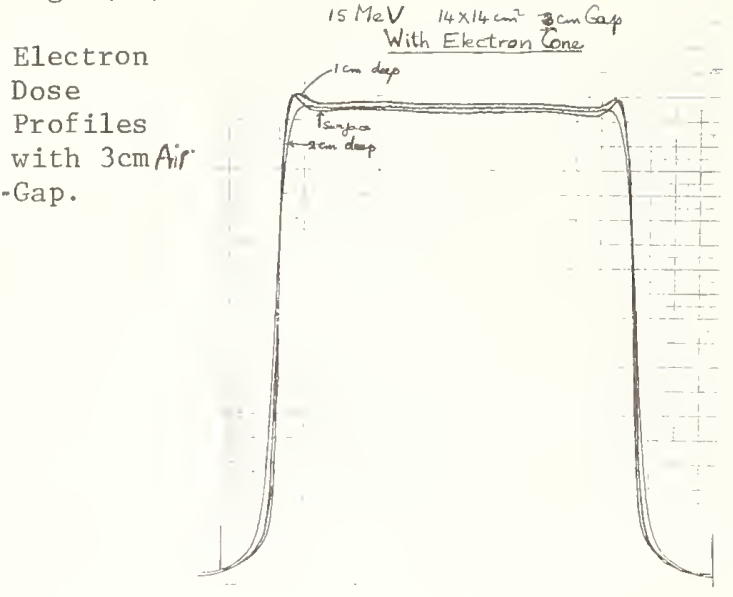

(i) Small Gaps: Experimentation at Roswell with 1 to $5 \mathrm{~cm}$ air gaps showed an arise or inflections in each profiles range near the edges of the beam. However, these inflections are eliminated at about $2 \mathrm{~cm}$ depth from the skin surface for 10 and $15 \mathrm{MeV}$ electron beam energies of the depth dose curves. Figures $(20,21$ and 22) show examples of these results for $15 \mathrm{MeV}$ electron beams and for air gaps of 1,2 and $3 \mathrm{~cm}$. Electron dose uniformity at the surface of phantom and at $1 \mathrm{~cm}$ depth is improved as the air-gap increases from 1 to $5 \mathrm{~cm}$. However, dose profiles have optimal uniformity at $2 \mathrm{~cm}$ depth for the range of energy under investigation.

(ii) Intermediate Gaps: In this case consideration should be given to the change in beam flatness. As mentioned in Section II, a gradual transition from flat-top distribution to gaussian depending on distance. With such intermediate gaps of about $20 \mathrm{~cm}$ probably the gaussian distributions will not yet be formed, but there is a definite increase in the beam half width while the sharp and rapid fall-off in the electron dose profiles are gradually diminished. In this case the profile shape will be somewhere between the uniform or flat-top distribution and the gaussian depending on distance and energy.

(iii) Large Gaps: Significantly large air gaps about several times the initial width of the beam, the distribution curves of electron dose profiles are essentially gaussians, such as those obtained in total skin irradiations for the treatments of extensive skin lymphomas. In fact the relative dimensions of field size to SSD is very small, consequently the therapy beam in this case could be considered as a pencil beam where electron cones or scattering foils are avoided. The uniform dose distribution can best be obtained by a dual electron beam. In this case the angles of the central axis of the beam above and below the horizontal are a function of beam energ高2.

\section{Discussion and Conclusion:}

A study has been made on electron dose distribution in air and in tissue for therapeutic beams of 3 to $30 \mathrm{MeV}$ incident beam energies. Electron dose profiles were experimentally determined and were compared to those predicted from theoretical considerations. An electron model for clinical beams has been presented and is based on considering the therapeutic beam to be made up of an infinite number of pencil beam components. The divergence of electron pencil beams has been studied in the present work and dose profiles were found to be in excellent agreement with theoretical gaussian curves over a wide range of transverse displacement except for the tail ends of the distributions where the experimental curves indicate broader dose profiles than the gaussians. It was also found in the present work that the root-mean-square angular deviations of an electron pencil beam is in fact, the 60.7 percent decrement curve. Therefore the average divergence of an electron beam could be easily visualized. It is also concluded that the scattering is mainly elastic obeying a mutiple small angle scattering formula. The non-gaussian parts of the curves may be explained by a major change in the mode of electron interactions. This is probably due to the transition from small angle multiple scattering to large angle single scattering acts. Instead of interacting with the nuclear coulomb field of the atomic nuclei without loss of energy, there will be electron-electron collisions with the atoms of the stopping medium accompanied by energy loss. This, in fact, is a minor process of about 14 percent after $\mathrm{X}$-ray contamination was removed, as indicated by the experimental curves when compared with the gaussian fits. Therefore, the spatial dose distributions are dominated by a multiple scattering process. Scattering of the incident electrons with the electrons of the absorbing medium results in large angle single scattering of $\phi>1$ mill; radian accompanied by energy loss due to collision. Elastic scattering of the incident electrons by the atomic nuclei is a 
more probable interaction with $\phi<<1$ milli radian. It is the large number of such scattering acts at successive collisions which produces the multiple scattering events. Moreover, in all tissues or air media,slopes of the gaussian distributions varied inversely with the initial normally incident electron energy. As the energy of the beam is increased, narrower gaussian distributions are expected particularly at considerable depths in the medium. Tissue dose profiles as obtained from our electron model are in very good agreement with experimental data. Electrons scattered by the cone and diaphragm as well as air gaps between patient and collimator may contribute substantially to the surface absorbed dose. Changes in variables, standard conditions or formulae used could be easily accounted for in the presented program. It was not our aim to investigate the validity of the existing theories on electron pencil beams. This aim can probably be achieved by using a more realistic theoretical model taking into account the quantum mechanical manybody effect (i.e. the various interactions among electrons in the therapeutic beam), the scattering within collimator and symmetry monitor etc. However, our simple model has sufficiently served the purpose and our theoretical predictions were consistent with our experimental results. The validity of our mathematical model is based on the accuracy of the available theories of multiple scattering expressions for narrow electron beams. With the proper choice of the pencil-beam theory which is used as the basic element in our model, an agreement with the experimental results of about 5 percent is achieved. Our theoretically predicted results can be used for routine programming of treatment planning with high energy electron beams using mini-computers. Further, it is also possible to account for body inhomogeneity, not from the stopping power formula, but from the basic parameters of the media concerned.

\section{ACKNOWLEDGEMENT}

The authors acknowledge considerable assistance from Dr. H. Yu in particular in the computer and theoretical aspects of this investigation. The authors also are grateful to Dr. T. Dougherty for his comments on the text and to $\mathrm{Mr}$. H. Kowal for helping in all measurements on the Clinac 35 .

\section{REFERENCES}

1. H.A. Bethe, Handbuch der Physik, Julius Springer, Berlin, 24 (1933).

2. H.W. Lewis, Phys. Rev. 78, 526-9 (1930).

3. J.S. Laughlin and J.S. Beatty, Phys. Rev. 83, 692 (1951).

4. L.V. Spencer and U. Fano, Phys. Rev. 93,6 (1954).

5. I. Berger and Seltzer, NASA-SP-3012 Suppl. to circular No. 577 (1958).

6. J. Ovadia et al, Radiology 72, 99 (1959).

7. I.A.E.A. Atlas VoI. 1, Electron Beams, p. 131-155 (1965).

8. R. Loevinger, C.J. Karzmark and M. Weissbluth, Radiology 77, 906-927 (1961).

9. J. Ashkin and H. A. Bethe, Experimental Nuclear Physics Vol. 1 Part II, John Wiley and Sons, Inc., 166 (1953).

10. B.B. Rossi, High Energy Particles, Englewood Cliffs, N.Y. (1964).

11. N.D. Kessoris, Phys. Rev. 145, 164-173 (1966).

12. G. Osman, Proceedings of AAPM, Phys. in Med. and Biol. 18, No. 4 (1973).

13. S.C. Lillicap, W. Patricia and J.W. Boag, Phys, in Med. and Biol. 20,30 (1975).

14. I.C.R.U. Report 21 (1972)

15. J. Dutreix and A. Dutreix, Annals of the N.Y. Academy of Sciences 161 , 33-43 (1969).

16. AAPM Protocol "SCRAD", Phys. in Med. and Biol. 11, 4 505-520 (1966).

17. G. Osman, Proceedings of AAPM, Med. Phys. Vol. 1, No. 2, p. 97 (1974). Also Med. Phys. Vol. 2, No. 3, p. 155 (1975). 
18. A. Hald, Statistical Theory with Engineering Applications, John Wiley and Sons Inc. 119-188 (1952).

19. J.D. Jackson, Classical Electrodynamics, John Wiley and Sons Inc. 456-463 (1967).

20. W.V. Mayneord, Acta of the International Union Against Cancer, $2: 271$ (1937).

21. A.D. Booth, Numerical Methods, Academic Press Inc., 31-34 (1957).

22. L. Katz and A.S. Penfold, Rev. Mod. Phys. 24, 28 (1952).

23. B. Markus, Strahlentherapie 116, 280 (1961).

24. D. Harder, Symposium on High Energy Electrons, Montreux 1964, Springer Verlag, Berlin and Heidelberg. Also: W. Pohlit, Annals of the N.Y. Academy of Sciences 161, 195 (1969).
25. H.E. Johns and J.R. Cunningham, The Physics of Radiology, Thomas Charles (1974).

26. M. Sempert and R. Wideroe, Elektronenstrahlen, Springer Verlag, Berlin, 182 (1958).

27. A. Dahler, Proceedings of the International Symposium on High Energy Electrons, Montreux, p. 98, Springer Verlag, Berlin (1964).

28. C. Lindskoug, and K.A. Johansson, Acta. Radiol. Ther. Phys. Biol. 10, 21 (1971).

29. H. Svensson, and G. Hettinger, Acta. Radiol. Ther. Phys. Biol. 6, 404 (1967).

30. B. Lindskoug, and A. Dahler, Collimating System for Electron Beams, Acta. Radiologica, New Series Vol. 10 454-463 (1971).

31. J.A. Rawlinson, The Scientific Program of R.S.N.A., 186 (1975).

32. G. Osman, Proceedings of the Third International Conference on Medical Physics, Sweden (1972). 


\title{
CALORIMETRIC DOSE MEASUREMENTS IN FAST NEUTRON AND COBALT-60 GAMMA-RAY FIELDS*
}

\author{
J.C. McDonald and J.S. Laughlin \\ Memorial Sloan-Kettering Cancer Center \\ New York, N.Y., 10021 \\ L.J. Goodman \\ Radiological Research Accelerator Facility \\ Brookhaven National Laboratory \\ Upton, N.Y., 11973
}

\begin{abstract}
A portable calorimeter, constructed of A-150 tissue equivalent (T.E.) plastic, was employed to measure dose in a cyclotronproduced fast neutron field and in a ${ }^{60}$ Co gamma-ray field. The precision of these measurements ranged from $\pm 7-2 \%$ depending upon dose rate and signal to noise ratio. The absolute accuracy of measurements in the neutron field is estimated to be $\pm 5 \%$ and those in the ${ }^{60}$ Co field to be $\pm 2 \%$. A method for determining the magnitude of endothermic radiochemical effects which occur in T.E. plastic will be described along with other sources of uncertainty in calorimetric dosimetry.
\end{abstract}

(Tissue equivalent; calorimeter; fast neutron; dosimetry)

\section{Introduction}

Cyclotron-produced fast neutron fields are presently employed for radiobiological research and $\mathrm{clinical}$ investigations of their potential advantages for radiotherapy. In order to properly evaluate the results of these studies, it is necessary to develop a high degree of precision and accuracy in dosimetric measurements. At our laboratory calorimeters have been designed, constructed and employed as primary dosimetric standards for measurements in electron and photon beams ${ }^{1-7}$. This work has recently been expanded to include measurements in fast neutron fields ${ }^{8}$. The advantages of calorimetric dosimetry are that dose is determined directly by measurement of the temperature rise in an isolated test volume, calibration is carried out by electrical heating of the test volume thus obviating a radiation calibrating source and calorimeter response does not require a knowledge of stopping power ratios or $W$ values. There are also some disadvantages, such as relatively poor sensitivity and the physical size of some calorimeter systems. The instrument described in this report is compact and has been designed to be portable, in addition, measurements have been carried out at dose rates as low as 0.5 $\mathrm{mGy} \cdot \mathrm{s}^{-1}\left(3 \mathrm{rad} \cdot \mathrm{min}^{-1}\right)$, and a precision of $\pm 2 \%$ has been achieved for dose rates of 1.9 $\mathrm{mGy} \cdot \mathrm{s}^{-1}\left(11 \mathrm{rad} \cdot \mathrm{min}^{-1}\right)$.

The material selected for use in construction of this calorimetric dosimeter is A-150 T.E. plastic ${ }^{9}, 10$ since it is almost universally employed for ionization chambers and proportional counters now used in fast neutron and pion dosimetry. Although the composition of the T.E. plastic is not identical to the formulation adopted by the ICRU to characterize tissue ${ }^{11}$, the kerma correction which is employed to convert dose in T.E. plastic to dose in tissue is smal1 ( $\leq 5 \%)$ for most photon and neutron energy spectra of current interest in radiobiology and radiotherapy. Unfortunately, nearly all irradiated plastics are characterized by a thermal defect $2,12-15$ which is the quantity of absorbed energy that does not result in the production of heat.

When fast neutrons and pions interact with tissue and tissue equivalent plastic, an array of secondary charged particles is produced which includes protons, alpha

*This research is supported in part by ERDA Contracts $E(11-1)-3522, E(11-1)-3243$ and by NCI Grants CA-08748, CA-12536. 
particles and nuclei of carbon and oxygen. The numbers and energy spectra of these secondary charged particles are determined by the initial energy spectrum of the primary particles. The thermal defect in T.E. plastic has been measured using $1.7 \mathrm{MeV}$ protons ${ }^{10}$, however, there have been no such measurements to date for higher energy protons, alpha particles or heavy ions for which the thermal defect may be quite different. A method for extending the thermal defect measurements of Fleming and Glass 12 witT be described in this report, since this method wi11 help to reduce the uncertainty associated with the determination of dose in fast neutron fields using a T.E. plastic calorimeter.

\section{Calorimeter Elements}

Core

The central absorbing element, or core, is cylindrical in shape; $2 \mathrm{~cm}$ in diameter and $0.2 \mathrm{~cm}$ thick. This design was chosen to insure reasonably good spatial resolution in fields whose intensity decreases rapidiy with depth. The core is thermaliy isolated from its surroundings by hollow nylon suspension points which provide a minimal thermal conduction path. The opposing faces of the core have been coated with a thin Tayer of conducting silver dag providing electrical contacts for calibration heating of the core, and also lowering radiative heat loss from the core to its surroundings by producing a lower emissivity surface compared to uncoated T.E. plastic. A thermistor has been bonded into the core with an electrically insulating epoxy which has relatively good thermal conductivity. The cross-sectional view of the calorimeter, shown in Figure 1, indicates some of the details of construction.

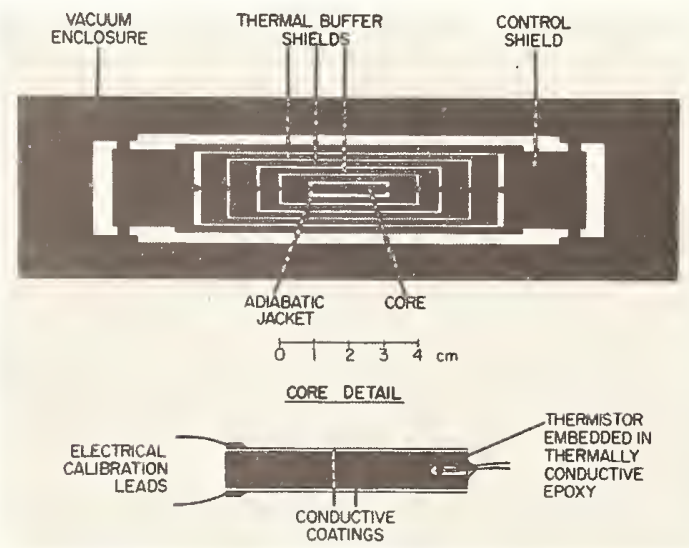

Figure 7 . Cross-section of the calorimeter.
Since it is necessary to determine the quantity of energy deposited per unit mass in a defined medium, a precise amount of energy must be introduced into the core. The electricaliy conductive faces are used to pass current through the T.E. plastic which results in a heating pattern similar to that produced by the radiation field. The resistivity of A-150 T.E. plastic has been measured as a function of temperature and was found to be $48.21 \mathrm{ohm}-\mathrm{cm}$ at $30^{\circ} \mathrm{C}$. The electrical calibration circuit and the method used to measure the current flowing through the core will be described below.

The composition of the core assembiy is shown in the following table. The percentage of material other than T.E. plastic has been kept to a minimum in order to maintain a nearly homogeneous medium in which dose is measured.

\begin{tabular}{l|c|c}
\hline Component & Weight & Percentage \\
\hline Tissue equivalent plastic & .7365 & 98.29 \\
Conducting paint & .0055 & .73 \\
Conducting epoxy & .0027 & .36 \\
Thermal epoxy for thermistor & .0022 & .29 \\
Thermistor head & .0020 & .27 \\
Electrical leads on core & .0004 & .05 \\
& $.7493 \mathrm{gm}$ & $\frac{99.99 \%}{}$ \\
\hline
\end{tabular}

TABLE I, Calorimeter Core Composition. Jacket

Surrounding the core is a "pil1-box" shaped adiabatic jacket whose inside dimensions are approximately $0.05 \mathrm{~cm}$ larger than the core in all directions. The gaps between all calorimeter elements, which are necessary for thermal isolation, have been kept to a minimum practical size in order to maintain homogeneity throughout the instrument. The jacket is electrically heated in the same manner as the core, by means of conducting silver dag contacts. During electrical calibration the jacket and core heating rates are adjusted so that the initial temperature rise in both elements is nearly equal. This quasi-adiabatic operation, initially described by Laughtin ${ }^{1}$, provides a method of reducing heat loss from the core to its surroundings, and evaluating the magnitude of this heat loss during irradiation and cali- 
bration.

Thermal Buffer and Control Shields

The three bodies surrounding the core and jacket serve as static thermal buffer shields whose thermal capacity tends to damp out temperature oscillations before they reach the core and jacket. These elements also rise in temperature during irradiation which helps to reduce the heat loss of the core and jacket. Radiative losses between elements have been minimized by evaporating gold coatings on the faces of these buffer shields to reduce their emissivity. The control shield, indicated in Figure 1, serves as a constant temperature enclosure which is regulated to within $\pm 0.001{ }^{\circ} \mathrm{C}$ of a set point a few degrees above room temperature. The inner components are housed within a vacuum enclosure also constructed of T.E. plastic. The pressure within this enclosure is estimated to be $<.013 \mathrm{~Pa}\left(10^{-4}\right.$ torr) which is necessary to reduce heat losses from convection and air conduction.

\section{Signal Detection and Calibration Systems}

A $d-c$ Wheatstone bridge is employed to measure the resistance of the core thermistor. This circuitry must be quite precise and capable of resolving small resistance changes since the bridge imbalance that occurs during calibration or irradiation may be due to a change in resistance of only a few ohms in approximately 30,000 ohms. The precision of the bridge system is estimated to be $\pm 0.05 \%$. The specific heat of A-150 T.E. plastic is approximately $1.8 \mathrm{~J} \cdot \mathrm{g}^{-1} \mathrm{OC}^{-1}$ so that the temperature rise which occurs upon absorption of $0.17 \mathrm{mG}$

( $1 \mathrm{rad}$ ) is approximately $7.5 \times 10^{-6}$ o C in a one gram sample. The temperature increase and resulting relative resistance change, $\Delta R / R$, when a known amount of energy is deposited in the core by electrical heating serves as the calibration. The instruments used for measurement and calibration are shown in Figure 2.

The calorimeter in its styrofoam thermal enclosure appears at the far right, and the Wheatstone bridge is just to the left of the calorimeter. The bridge enclosure is also insulated with styrofoam in order to minimize the effect of room temperature changes on resistance values. The bridge potential of 12.15 volts is supplied by three mercury batteries, and the variable resistance arm is remote con- trolled by three stepping motors so that re-balancing can be achieved without entering the irradiation area. The temperature regulator for the calorimeter control shield and a microvoltmeter, which serves as a null detector, are in place above the bridge. The remote controller for the bridge and the calibration circuit appear at the far left and the strip chart recorder, which provides a graphic record of the core temperature versus time by indicating the bridge imbalance potential, is in the left foreground.

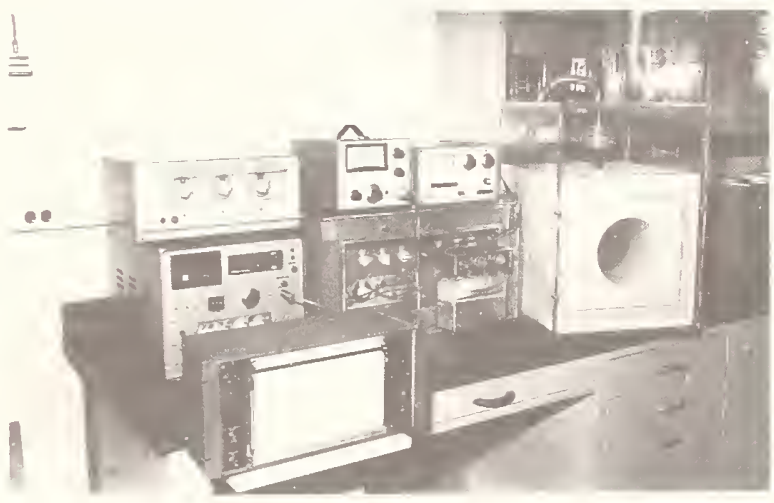

Figure 2. Tissue equivalent plastic calorimeter, along with the measurement, calibration and control systems.

A Leeds and Northrup N.B.S. type standard resistor of 1000 ohms is connected in series with the calorimeter core (3.41 ohms) and the potential across both resistive elements is measured with a digital voltmeter calibrated using an Eppley standard cell. The electrical power dissipated in the core during calibration is known to an accuracy estimated to be $\pm 0.25 \%$, and the precision of electrical calibration measurements ranges from $\pm 0.5-1 \%$ depending on the heating time and the magnitude of the relative resistance change.

Dose Measurements in Radiation Fields

\section{Calorimeter}

A mixed fast neutron and gamma-ray field was generated at the Sloan-Kettering Institute cyclotron by using a beam of deuterons to bombard a thick Be target. The neutron spectrum produced by the ${ }^{9} \mathrm{Be}(d, n)^{10_{B}}$ interaction has a mean energy of approximately $3.5 \mathrm{MeV}^{16}$ (for $\simeq 7.5 \mathrm{MeV}$ deuterons). The percentage of gamma-ray dose present at the position of the calorimeter core has been computed to be approxi- 
mately $6 \%$ from paired ionization chamber measurements in a water phantom ${ }^{17}$. The cyclotron area is an electrically noisy environment which is also subject to somewhat larger temperature variations than other laboratory areas. In spite of these difficulties, a series of measurements was carried out at a dose rate of $1.9 \mathrm{mGy} \cdot \mathrm{s}^{-1}$ (11 $\mathrm{rad} \cdot \mathrm{min}^{-1}$ ) with a precision of $\pm 2 \%$ (standard deviation about the mean). An example of one such irradiation run is shown below in Figure 3.

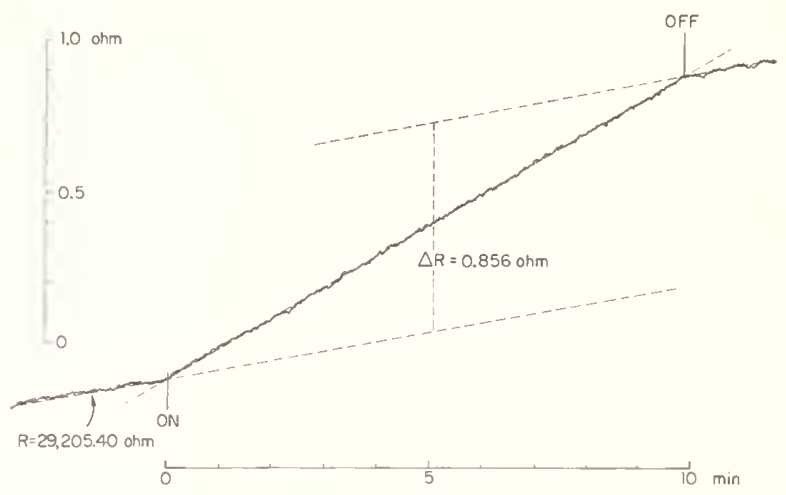

Figure 3. Core temperature vs. time recording for neutron irradiation.

A series of measurements was also carried out in a ${ }^{60}$ Co gamma-ray field at Memorial Hospital. The calorimeter was set up in the evening and allowed to equilibrate overnight. The thermal drift rate on the following day was approximately $6 \times 10^{-6}$ ${ }^{0} \mathrm{C} \cdot \mathrm{min}^{-1}$ for the duration of exposures which took place over a period of two hours. The dose rate at the calorimeter core was $10.22 \mathrm{mGy} \cdot \mathrm{s}^{-1}\left(60.09 \mathrm{rad} \cdot \mathrm{min}^{-1}\right)$ and the precision of this series of measurements was $\pm 1.2 \%$. The absolute accuracy of the calorimetric measurements is estimated to be approximately $\pm 2 \%$ in ${ }^{60}$ Co field and $\pm 5 \%$ in the cyclotron-produced fast neutron field. The uncertainty in the thermal defect is a contributing factor affecting the accuracy of these measurements, and a method of reducing this uncertainty will be described below.

\section{Ionization Chamber}

An ionization chamber which is cylindrical in shape, has been constructed of T.E. plastic. The collecting volume is guarded and has a diameter of $2 \mathrm{~cm}$ and a thickness of $0.2 \mathrm{~cm}$. T.E. gas flows through the chamber at a rate of approximately $0.08 \mathrm{~cm}^{3} \cdot \mathrm{s}^{-1}\left(5 \mathrm{cc} \cdot \mathrm{min}^{-1}\right)$ at a pressure of $2 \mathrm{kPa}$ ( 15 torr) above atmospheric pressure. Collection efficiency at $400 \mathrm{~V}$ in ${ }^{60}$ Co gamma-ray and fast neutron fields at dose rates of about $10 \mathrm{mGy} \cdot \mathrm{s}^{-1}$

$\left(60 \mathrm{rad} \cdot \mathrm{min}^{-1}\right.$ ) has been measured to be $99.85 \%$ and $99.3 \%$ respectively (assuming columnar recombination only). The charge measured when using positive and negative collecting potentials agrees to within $\pm 0.6 \%$.

An irradiation phantom which places both the calorimeter core and the collecting volume of the ionization chamber at the same depth in T.E. plastic has been constructed, and is shown below in Figure 4. The dimensions of the phantom approximate those of the calorimeter and inserts have been machined to accept other dosimeters.

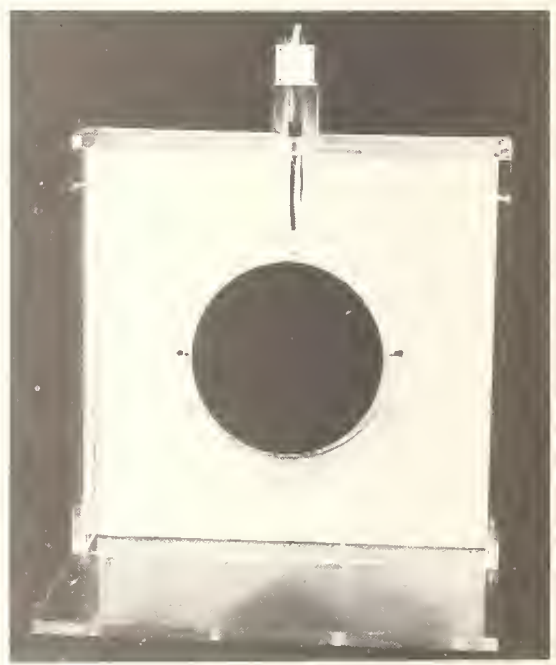

Figure 4. T.E. ionization chamber in place within a T.E. plastic irradiation phantom.

$\frac{\text { Measurement of the Thermal Defect }}{\text { In A-150 T.E. Plastic }}$

One of the sources of uncertainty in calorimetric dosimetry which employs T.E. plastic is the thermal defect. The amount of energy which is consumed by endothermic radiochemical reactions when A-150 T.E: plastic is irradiated with 7 MV $x$-rays has been determined by Bewley 13 to be $(4 \pm 2) \%$. Fleming and Glass ${ }^{12}$ employed a differential calorimeter in which absorbers of T.E. plastic and aluminum were irradiated with 1.7 MeV protons. This method was quite 
precise and yielded a value for the thermal defect extrapolated to zero dose of $(4.2 \pm 0.2) \%$. As accumulated dose increased radiation damage eventually caused lowering in this value to $3.67 \%$ for doses above $10^{6} \mathrm{~Gy}\left(10^{8} \mathrm{rad}\right)$. Over the useful range of a calorimetric dosimeter, the thermal defect would be relatively constant, however, this accumulated dose dependence is a source of uncertainty which will be investigated further.

The thermal defect value measured by Fleming and Glass for T.E. plastic could be employed for neutrons of relatively low energy since the dose is predominately deposited by recoil protons. However, for higher energy cyclotron-produced fast neutron beams an increasingly larger fraction of dose is deposited by alpha particles and heavy recoil nuclei. Radiochemical yields for these densely ionizing charged particles are usually quite different than those obtained with sparsely ionizing radiation. In order to properly evaluate the thermal defect for fast neutron irradiated A-150 T.E. plastic, the technique of Fleming and Glass will be extended by using different energy protons and other charged particles.

This project will be a collaborative effort of the Sloan-Kettering Biophysics Laboratory and The Radiological Research Accelerator Facility (RARAF). Thermal defect measurements will be performed on a number of T.E. plastic samples from three laboratories in the United States and one in Europe. An attempt will be made to evaluate samples molded at the extremes of temperature and pressure which will demonstrate whether the thermal defect is dependent on sample processing technique. Hydrogen, helium, carbon and possibly oxygen ions of various energies will be accelerated at the RARAF 4 MV Van de Graaff. The calorimeter constructed by Fleming and Glass will be employed, however, certain critical internal components have been rebuilt or replaced. A block diagram of the system is shown in Figure 5.

This calorimeter system has been described previous $1 y^{12}$ and a brief summary of its operation will now be given. The charged particle beam from the accelerator is swept across the absorber face by electrostatic deflection plates, in the manner of a video raster, in order to produce a uniform irradiation pattern. The calorimeter is adiabatically controlled by a thermopile which detects the temperature difference between the absorber and the surrounding adiabatic jacket. The thermopile signal is amplified and used to control a thermal regulator which provides current for a heater surrounding the adiabatic jacket.

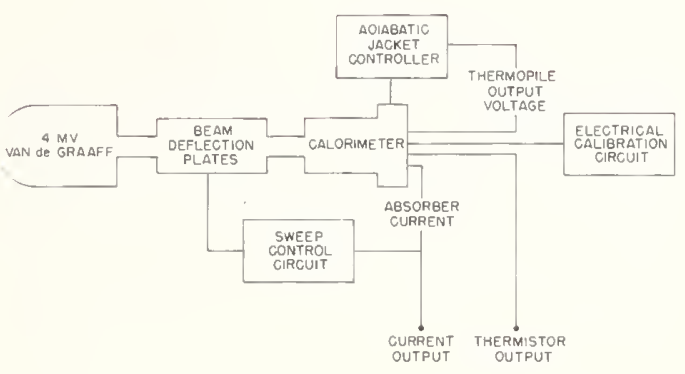

Figure 5. Block diagram of the experimental system.

Since the absorber and adiabatic jacket are constrained to rise in temperature simultaneously, the measuring thermistor is placed in the jacket in order to minimize power dissipation in the absorber. Electrical calibration is achieved through the use of a resistance element between the T.E. plastic and aluminum faces.

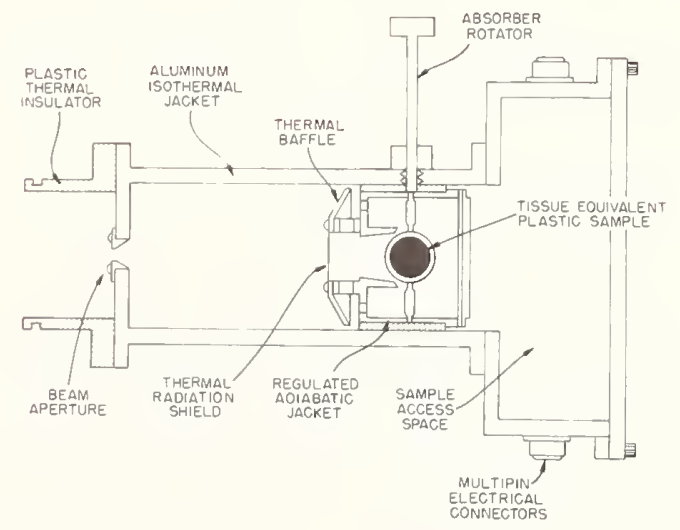

Figure 6. Cross-sectional view of thermal defect calorimeter.

The cross-sectional diagram in Figure 6 shows the various elements of the calorimeter. The aluminum isothermal jacket is maintained at a set temperature by a thermal regulator, and its interior is at vacuum. Within this element is the adiabatic jacket, which is thermally and electrically insulated from the outer aluminum 
wall. The adiabatic jacket is maintained at a negative potential in order to suppress the emission of secondary electrons from the absorber and assure a reliable measurement of the beam current. The measuring thermistor and thermopile are mounted with a high thermal conductivity epoxy and are electrically guarded from the negative high potential to prevent leakage currents.

The absorber can be rotated so that either face will completely stop the charged particle beam. One face is aluminum $0.018 \mathrm{~cm}$ thick and $1.4 \mathrm{~cm}$ in diameter and the other is $0.025 \mathrm{~cm}$ thick T.E. plastic of the same diameter. The ratio of power measured using both sides of the absorber yields the thermal defect. [etails of the absorber assembly are shown below in Figure 7.
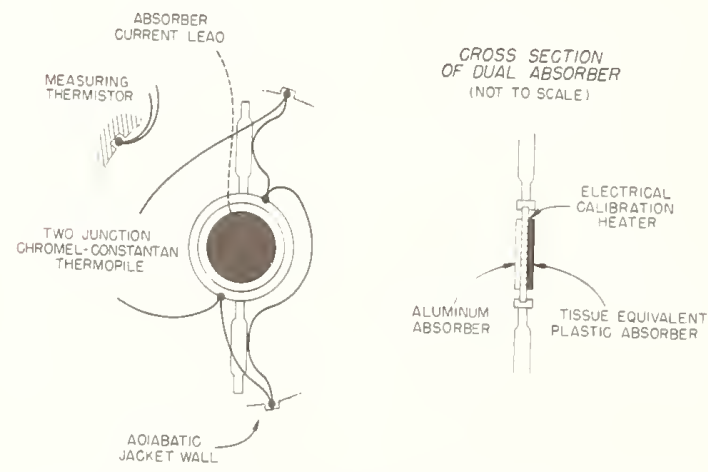

Figure 7. Details of the thermal defect calorimeter absorber assembly.

\section{Summary}

A series of measurements has been carried out in ${ }^{60}$ Co and fast neutron fields using a portable tissue equivalent calorimetric dosimeter. These measurements have a precision of from \pm 1 to $2 \%$ depending somewhat on dose rate and signal to noise ratio. The absolute accuracy at present is estimated to be approximately $\pm 5 \%$. Much of this uncertainty is associated with the thermal defect, and measurements are in progress which will help reduce this uncertainty. Comparisons will then be completed for calorimetric dose and ionometric specific charge in cyclotron-produced fast neutron fields.

\section{References}

1. J.S. Laughlin, AEC Progress Report for Contract AT(30-1)-1451 (1954)

2. P. Milvy, S. Genna, N. Barr and J.S. Laughlin, Proc. 2nd Int. Conf. Peaceful Uses of Atomic Energy, Geneva (1958), p. 142.

3. J. Geisselsoder, K. Koepke and J.S. Laughlin, Rad. Res. 20, 423 (1963).

4. J.S. Laughlin, Proc. XI Int. Cong. of Radiol., Rome (1965), p. 1772.

5. J.S. Laughlin and S. Genna, Radiation Dosimetry, ed. F.H. Attix, W.C. Roesch and E. Tochilin (Academic Press, New York, 1966), Vol. 2, pp. 389-439.

6. A.P. Pinkerton, Ann. N.Y. Acad. of Science 161, 63 (1969).

7. J.C. McDonald, A.P. Pinkerton, H. Weiss and E.R. Epp, Rad.Res. 49, 495 (1972).

8. J.C. McDonald, J.S. Laughl in and R.E. Freeman, Med. Phys. 3 No. 2 (in press 1976).

9. J.J. Spokas, Presented at Rad. Res. Soc. Meeting Miami Beach, May 11-15, 1975 .

10. J.B. Smathers, V.A. Otte, A.R. Smith, P.R. Almond, F.H. Attix, J.J. Spokas, W.M. Quam and L.J. Goodman, Presented at R.S.N.A. - A.A.P.M. meeting Chicago, Nov. 30-Dec. 5, 1975.

11. I.C.R.U. Report 10B (1964),p.4.

12. D.M. Fleming and W.A. Glass, Rad.Res. 37, 316 (1969).

13. D.K. Bewley, E.C. McCullough, B.C.Page and S. Sakata, Phys. Med. Biol. 17, 95 (1972).

14. M. Sabel, Th. Schmidt and H. Pauly, Health Physics 23, 744 (1972).

15. G. von Weimer, Atomkernergie, 20, 327 1972 
16. F.T. Kuchnir, L.S. Skaggs, A.J. Elwyn, F.P. Mooring and N.A. Frigerio, in Cyclotrons 1972, Proc. of the 6th Int. Cyc7. Conf. (American Institute of Physics, New York, 1972).
17. T.R. Canada, J.C. MCDonald, B.R. Freed, T.Y.T. Kuo and J.S. Laughlin, Rad. Res. 60 , 205 (1974). 

SCATTERED RADIATION FROM A NEUTRON COLLIMATOR*

F.H. Attix, L.S. August, and P. Shapiro

Naval Research Laboratory

Washington, D.C. 20375

Fast-neutron beams are being employed in radiotherapy trials and associated radiobiology studies at numerous centers in the U.S., Europe, and Japan. Since collimated beams of various sizes and shapes are employed, it is desirable to know the composition of the scattered radiation component contributed by the collimator. A simple method is shown for deducing the field composition in terms of a 3-component model, from measurements made with three ionization chambers (tissueequivalent, graphite, and magnesium). The dose contributed by the scattered radiation in the present example was found to be predominantly due to fast neutrons indistinguishable from those in the primary

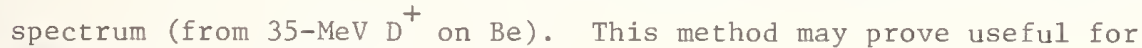
measurements in phantoms as well.

(Neutrons; scattering; collimation; albedo; dosimetry; ion chambers; $\mathrm{n}-\gamma$ dosimetry)

\section{Introduction}

It is well known that the presence of a neutron-beam collimator near a source tends to increase the absorbed dose (tissue) ${ }^{1}$ in the beam, compared to what it would be at the same point with the collimator removed. This is, of course, due to the contribution of scattered and secondary radiation by the collimator. It is the purpose of this note to describe quantitatively a specific example of this effect as a function of collimator size, and to show that about $90 \%$ of the observed dose increase can be ascribed to fast neutrons which have undergone little energy loss in being scattered by the collimator.

\section{Collimators}

Figure 1 is a schematic diagram (either plan view or elevation) of the collimator design originally employed at the NRL Cyclotron to provide a neutron beam for the

MANTA $^{2}$ program. Each collimator is made of Benelex $401^{4}$ having a thickness of $66 \mathrm{~cm}$, with aperture walls sloped so that their extension toward the source is tangential to the circular beryllium target $2.5 \mathrm{~cm}$ in diameter. The extension of the collimator walls away from the source intersects the 125-cm SSD plane to give the nominal beam size for that collimator. The $35-\mathrm{MeV}$ deuteron beam which generates the neutrons in the Be target is focused in a central spot less than $1 \mathrm{~cm}$ in diameter, which

* This work was partially supported by the National Cancer Institute under Interagency Agreement No. 1Y01C040001-01.

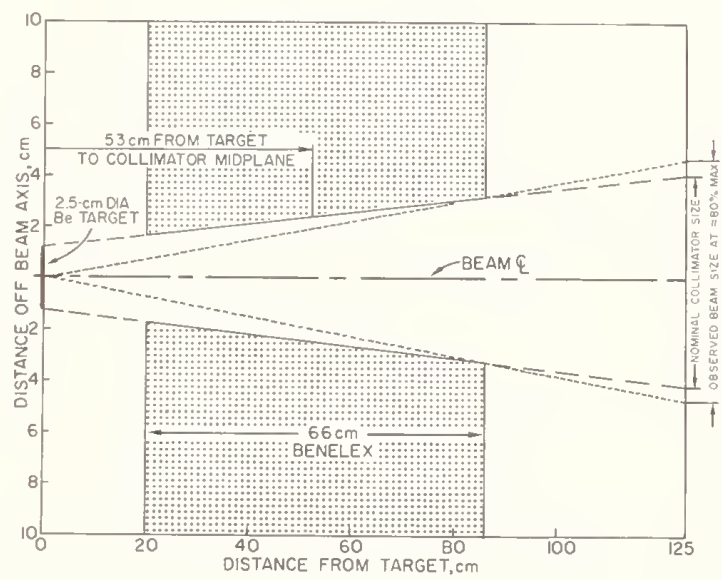

Figure 1. Schematic diagram of the Benelexcollimator geometry. The 35-MeV deuteron beam is incident on the thick beryllium target from the left. Ion chamber measurements were made on the beam axis in free space $125 \mathrm{~cm}$ from the target. A monitor chamber (not shown) was located $10 \mathrm{~cm}$ from the target. Note the exaggerated scale perpendicular to the beam axis.

yields a beam size at $125 \mathrm{~cm}$ SSD somewhat larger (at $\approx 80 \%$ of max.) than the nominal designation, as approximated by the dotted rays shown emanating from the target.center in Fig. 1. Typical isodose curves have been reported in an earlier paper. ${ }^{5}$ The present study was made with three such collimators having square apertures which pass neutron beams of $5 \times 5,10 \times 10$, and $15 \times 15 \mathrm{~cm}$ in nominal size. A flat polystyrene-walled 
monitor chamber, centered on the beam axis $\approx 10 \mathrm{~cm}$ from the target, provided exposure control for the present measurements. The effect of neutrons backscattered from the collimators into the monitor chamber was found to be negligible, inasmuch as the ratio of monitor reading to deuteron charge on the Be target was independent of collimator size.

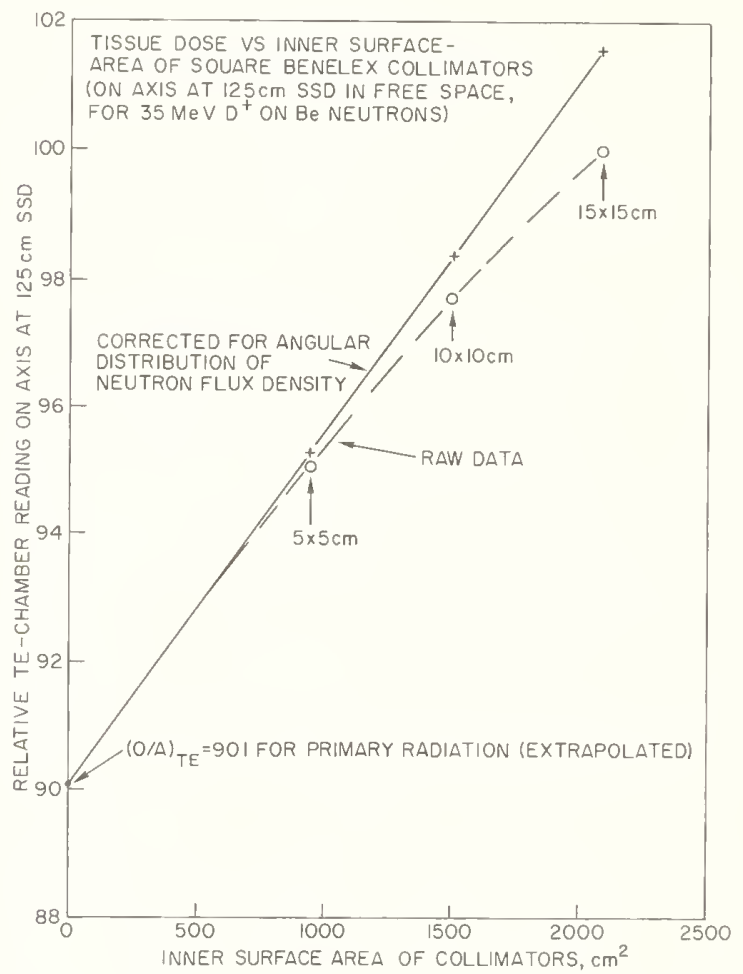

Figure 2. Graph of the TE-plastic chamber reading (for a constant charge collected in the monitor chamber) vs the inner surface area of the collimators. The lower points (circles) are raw data; the upper points (crosses) have been corrected for the angular distribution of the neutron flux density according to Serber stripping theory, and therefore should apply to an isotropic neutron source.
Measurements

The lower points in Fig. 2 represent the ionization readings obtained with a $\mathrm{TE}-\mathrm{plastic}$ chamber $^{6}$ located on the beam axis at $125 \mathrm{~cm}$ from the target, for a constant monitorchamber reading. The abscissa of the graph was arbitrarily chosen to be the inner surface area of the collimators, since that surface probably contributes most of the scattered neutrons reaching the chamber. Although there was no a priori reason for believing that the chamber reading due to the scattered radiation component would be proportional to such a simple parameter, the raw data points in Fig. 2 are seen to be approximately collinear, but with a slight downward curvature.

\section{Correction for Angular Dependence of Neutron Flux Density}

August et $a 1 .^{7}$ have shown that the present neutron beam is generated primarily by the deuteron stripping reaction described by Serber, ${ }^{8}$ and as a result the neutron flux density is strongly peaked at $0^{\circ}$ (the direction of the deuteron beam axis) and decreases rapidly with increasing angle. This forward peaking persists even for thick targets. 5,7 Because of the angular dependence, relatively fewer neutrons can strike the collimator wal1 and be scattered than would occur for an equivalent isotropic source, and the disparity increases with collimator aperture size.

A correction to the data in Fig. 2 was made for this angular dependence, to raise the points to where they would be if the source were isotropic. Initially we obtained a first approximation of the TE-chamber response due to primary radiation only, by extrapolating the raw data points to the zero-area axis in Fig. 2. This quantity was then subtracted from each of the raw data points to obtain the approximate scattered-

Table I. Correction for Angular Dependence of Flux Density in the Neutron Beam from $35-\mathrm{MeV} \mathrm{D}{ }^{+}$on Be

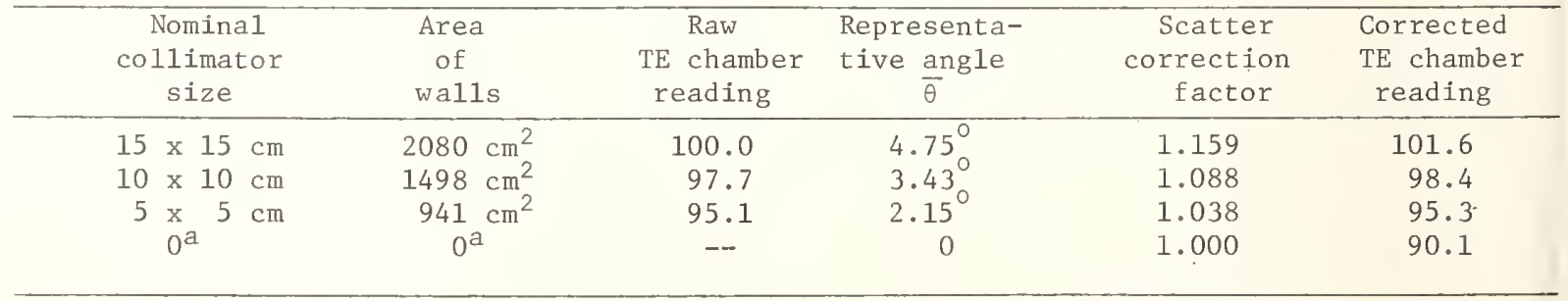


radiation contribution to the TE-chamber reading for each collimator. The appropriate angular corrections were then applied to these scatter components, which were then recombined with the primary radiation component and then replotted. Iteration of this procedure rapidly converged on the upper points and solid curve shown in Fig. 2, which are strictly linear and extrapolate with a slope

of $5.5 \times 10^{-3} \mathrm{~cm}^{-2}$ to a relative TE chamber reading of 90.1 for the primary radiation component.

Table I contains the numerical data for this correction. The angle $\bar{\theta}$ taken to be representative of each collimator was assumed to be that lying between the beam axis and a ray drawn from the target center to a point $P$ in the perpendicular midplane of the collimator $53 \mathrm{~cm}$ away (see Fig. 1). Imagining the square aperture cross-section which lies in that plane, point $\mathrm{P}$ was taken to be located on the collimator wall, one-fourth of the way from one corner of that square to the next adjacent corner. Letting the length of one side of that square be $\mathrm{d} \mathrm{cm}, \bar{\theta}$ is given by

$$
\bar{J}=\arctan \frac{\mathrm{d} / 2}{53 \cos (\operatorname{arc} \tan 0.5)}
$$

The fact that a straight line results from these considerations tends to support the idea that the tissue dose component on axis due to scattered radiation is closely proportional to the collimator wall area for an isotropic neutron field, at least within the range of square Benelex collimators studied here. This could be further verified with $14-\mathrm{MeV}$ neutrons from the $\mathrm{D}-\mathrm{T}$ reaction, for which the neutron field is nearly isotropic. It seems reasonable, moreover, that the increasing scatter trend with collimator size must eventually reverse itself for very large collimator apertures, in order for the dose to return smoothly to its uncollimated level again when the aperture has been enlarged to the point of eliminating the collimator entirely. That interesting transition would be a suitable subject for further experimentation as well as Monte Carlo calculations.

The last column in Table I shows that the $T E$ chamber reading contribution due to scattered radiation from these collimators ranges from $\approx 6$ to $13 \%$ of that delivered by primary radiation alone. It is important, both for radiobiological and radiotherapeutic applications, to know the composition of such a sizeable scattered radiation component. A satisfactory method has been devised for obtaining this information in terms of a simple three-component model.

\section{Three-Chamber, Three-Component Method}

Let us assume the total radiation field at the point of interest to be made up of only three components:

a. Fast neutrons having a spectrum practically the same as the primary neutron spectrum (see Refs. 3 and 5)

b. A slower monoenergetic neutron component, $E \approx 0.1 \mathrm{MeV}$, low enough in energy to provide a negligible response in nonhydrogenous chambers, but high enough to give normal response per tissue rad in a $\mathrm{TE}$ plastic chamber

\section{c. $y$ rays from whatever source.}

We make measurements of this mixed field for each of the collimators with three ionization chambers:

\section{The TE-plastic chamber with TE-gas flow,}

2. A graphite chamber (EG\&G Model IC-17G) containing static air, and

3. A magnesium chamber (EG\&G Model IC-17M) with argon gas flow.

For equal arbitrary monitor readings the relative ionization charge readings $Q$ divided by the $\gamma$-ray sensitivity A (coulombs/tissuerad) are listed in Table II for each collimator.

Also shown in parentheses are the ratios for each chamber of $(Q / A)$ relative to that for the $5 \times 5 \mathrm{~cm}$ collimator. It is evident that the three chambers show quite similar variations of Q/A vs collimator size. This can only occur if the tissue dose component due to scattered radiation consists mostly of fast neutrons, rather than the slower neutron component of $\gamma$ radiation in this 3 -component model, as can be seen from the following argument.

Suppose the scatter contribution to the tissue dose, as measured approximately by the $\mathrm{TE}$ chamber, were due solely to the slower neutron component ( $b$ above). Since the response of both the G-air and $\mathrm{Mg}-\mathrm{Ar}$ chambers is negligible for such neutrons, as recently

verified by Kuchnir et a1., ${ }^{9}$ those chambers would show little or no change in output vs collimator aperture size, which is contrary to the observed findings in Table II. Thus the slower-neutron component must be relatively small. 
Table II. Relative Response (Q/A) for Equal Monitor-Chamber Readings

\begin{tabular}{rrrr}
\hline $\begin{array}{c}\text { Nominal } \\
\text { colimator } \\
\text { size }\end{array}$ & TE-TE & G-air & Mg-Ar \\
\hline $15 \times 15 \mathrm{~cm}$ & $100.0(1.052)$ & $32.76(1.053)$ & $16.80(1.059)$ \\
$10 \times 10 \mathrm{~cm}$ & $97.7(1.027)$ & $31.92(1.026)$ & $16.29(1.027)$ \\
$5 \times 5 \mathrm{~cm}$ & $95.1(1.000)$ & $31.10(1.000)$ & $15.86(1.000)$ \\
\hline
\end{tabular}

Suppose, on the other hand, that the scatter component were due entirely to $\gamma$ rays. The TE chamber, for which the ratio of neutron-to- $\gamma$ sensitivity per tissue rad $(\mathrm{B} / \mathrm{A})_{\mathrm{TE}}$ is $\approx 1$ independent of neutron energy, sees a dose increase of $\approx 5 \%$ in going from the smallest to the largest collimator, on top of a primary beam due almost entirely to fast

neutrons. 10 The response (Q/A in Table II) of the G-air chamber to these neutrons is evidently only about one-third as great, so that the $\gamma$-ray contribution to this chamber's response would appear relatively three times as large, or $\approx 15 \%$. Likewise the relative response of the $\mathrm{Mg}$-Ar chamber to the same $\gamma$ ray contribution would appear to be $\approx 6 \times 5 \%=30 \%$, since this chamber is only about $1 / 6$ th as responsive as the TE chamber to the fast neutrons. The data in Table II give no indication of marked differences in variation among the three chambers; therefore no substantial part of the scatter dose component can be due to $\gamma$ rays.

By process of elimination we are therefore drawn to the conclusion that the scattered radiation from the collimators must consist mostly of fast neutrons (a above) whose energies have been altered very little by a glancing elastic collision with the collimator wall.

The data in Table II can be incorporated into three simultaneous linear equations to obtain a quantitative solution for the three dose components due to $\mathrm{a}, \mathrm{b}$, and $\mathrm{c}$ in the simple model proposed above. Using the notation established earlier 5,11 we can write for each collimator:

$$
\begin{aligned}
& \mathrm{TE}-\mathrm{TE}\left(\frac{\mathrm{Q}}{\mathrm{A}}\right)_{\mathrm{TE}}=\mathrm{D}_{\gamma}+\left(\frac{\mathrm{B}}{\mathrm{A}}\right)_{\mathrm{TE}} \mathrm{D}_{\mathrm{fn}}+\left(\frac{\mathrm{B}}{\mathrm{A}}\right)_{\mathrm{TE}} \mathrm{D}_{\mathrm{sn}} \\
& \mathrm{G}-\operatorname{air}\left(\frac{\mathrm{Q}}{\mathrm{A}}\right)_{\mathrm{G}}=\mathrm{D}_{\gamma}+\left(\frac{\mathrm{B}}{\mathrm{A}}\right)_{\mathrm{G}} \mathrm{D}_{\mathrm{fn}}+(0) \mathrm{D}_{\mathrm{sn}} \\
& \operatorname{Mg}-\mathrm{Ar}\left(\frac{\mathrm{Q}}{\mathrm{A}}\right)_{\mathrm{Mg}}=\mathrm{D}_{\gamma}+\left(\frac{\mathrm{B}}{\mathrm{A}}\right)_{\mathrm{Mg}} \mathrm{D}_{\mathrm{fn}}+(0) \mathrm{D}_{\mathrm{sn}}
\end{aligned}
$$

where $\mathrm{D}_{\gamma}$ is the $\gamma$ ray dose (tissue) $\mathrm{D}_{\text {fn }}$ is the fast-neutron dose (tissue) from
component a above

$D_{s n}$ is the slower-neutron dose (tissue) from component $\mathrm{b}$ above

and $(B / A)_{G} \approx(B / A)_{M g} \approx 0$ for slower neutron component $\underline{b}$.

We assume the values $(B / A)_{T E}=0.991$ and $(\mathrm{B} / \mathrm{A})_{\mathrm{G}}=0.319$ for our primary neutron spectrum, in agreement with earlier studies of this neutron beam.12 This leaves us with three equations in four unknowns, since we have not yet established a reliable value for (B/A) $\mathrm{Mg}$ with argon gas flow for the NRL primary neutron spectrum. 13 Instead, we will treat (B/A) $\mathrm{Mg}$ as a fourth unknown in addition to $D_{\gamma} D_{f n}$, and $D_{s n}$. It is reasonable to suppose that $D_{\text {sn }}$ increases approximately in proportion to the scatter component of the TE chamber readings (raw data in Fig. 2) so that, referring to Table I,

$$
\begin{aligned}
& \left(\mathrm{D}_{\mathrm{sn}}\right)_{5 \times 5 \mathrm{~cm}}=\mathrm{n}(95.1-90.1)=5.0 \mathrm{n} \\
& \left(\mathrm{D}_{\mathrm{sn}}\right)_{10 \times 10 \mathrm{~cm}}=\mathrm{n}(97.7-90.1)=7.6 \mathrm{n} \\
& \left(\mathrm{D}_{\mathrm{sn}}\right)_{15 \times 15 \mathrm{~cm}}=\mathrm{n}(100-90.1)=9.9 \mathrm{n}
\end{aligned}
$$

where $\mathrm{n}$ is a proportionality constant which is a single unknown to be solved for in place of the three different values of $D_{\text {sn }}$ for the three collimators. In this way we can solve the data in Table II for each pair of collimators, for which there will be 6 equations in 6 unknowns. This allows three different solutions for the three pairs of collimators employed.

The values obtained for $(\mathrm{B} / \mathrm{A}) \mathrm{Mg}$ in the three solutions were as follows: 
Table III. Components of Dose (Tissue) in Benelex-Collimated

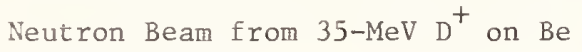

\begin{tabular}{|c|c|c|c|c|}
\hline $\begin{array}{l}\text { Nominal } \\
\text { collimator } \\
\text { size }\end{array}$ & $\mathrm{D}_{\text {fn }}$ & $\mathrm{D}_{\mathrm{sn}}$ & $\mathrm{D}_{\gamma}$ & $\mathrm{D}_{\mathrm{T}}$ \\
\hline $15 \times 15 \mathrm{~cm}$ & 109.4 & 0.44 & 1.13 & 111.0 \\
\hline $10 \times 10 \mathrm{~cm}$ & 107.1 & 0.37 & 0.95 & 108.4 \\
\hline $5 \times 5 \mathrm{~cm}$ & 104.4 & 0.28 & 0.92 & 105.6 \\
\hline $0^{a}$ & $99.2^{\mathrm{a}}$ & $0^{a}$ & $0.8^{a}$ & $100 \cdot 0^{\mathrm{a}, \mathrm{b}}$ \\
\hline
\end{tabular}

Extrapolated

bormalized to 100 rads, which equal 1 gray (Gy).

$$
\begin{array}{r}
\frac{\text { Collimator Pair }}{5 \times 5 \mathrm{~cm}, 10 \times 10 \mathrm{~cm}}-\frac{(\mathrm{B} / \mathrm{A}) \mathrm{Mg}}{0.1583} \\
5 \times 5 \mathrm{~cm}, 15 \times 15 \mathrm{~cm}-\frac{.1584}{.1587} \\
10 \times 10 \mathrm{~cm}, 15 \times 15 \mathrm{~cm}-\frac{.1585}{\mathrm{Avg} .}
\end{array}
$$

The spread of these values is only about $\pm 0.1 \%$, indicating good internal consistency among the data in Table II. However the $2.2 \%$ discrepancy from the earlier value for $(B / A)$ Mg (see Ref. 13) must await later resolution. In the present context 0.1585 is clearly the more appropriate value.

Table III contains a résumé of the average values obtained in these calculations for $D_{f n}, D_{s n}, D_{\gamma}$ and their sum $D_{T}$. Also shown are the corresponding data extrapolated to the case of zero aperture area, using the same iterative technique already described in relation to Fig. 2. All the figures were then normalized to a value of $100 \mathrm{rad}$ for $\mathrm{D}_{\mathrm{T}}$ with zero aperture scatter. The value of $0.8 \mathrm{rad}$ for $\mathrm{D}_{Y}$ in that case was obtained as the difference between the extrapolated values of $D_{T}$ and $D_{f n}$, and it agrees with the figure for "aperture $\gamma$-rays" given in Ref. 10 for a 2-cm diameter collimator. These $\gamma$-rays evidently originate in the target, and the collimators in the present study are seen to add only about $0.1 \mathrm{rad}$ more $\gamma$-ray dose per collimator size, so that the $\gamma$-ray dose percentage only varies between 0.9 and $1.0 \%$ of $\mathrm{D}_{\mathrm{T}}$ over the range of collimator sizes emptoyed here.

\section{Although $D_{\text {sn }}$ was constrained by} assumption to vary in proportion to the TE chamber reading, the $\mathrm{D}_{\mathrm{sn}}$ contribution is so small $(0.3-0.4 \%)$ that the substitution of any other reasonable assumption about its dependence upon collimator size would not change the data in Table III significantly.

\section{Conclusions}

The most important finding in Table III is that, of the 5.6 to $11 \%$ dose increase caused by the collimators, over 9/10ths is due to the fast neutron component $\mathrm{D}_{\mathrm{fn}}$. Thus these collimators act to intensify the neutron beam in the desired direction while degrading the quality and purity of the beam only to a negligible degree.

This has the practical consequence that one should not expect detectable differences in the RBE or OER of any biological system exposed in free space to the collimated vs uncollimated neutron field, assuming independence of dose rate. Likewise in the use of various-sized Benelex collimators in neutron radiotherapy, the quality and purity of the incident beam may be assumed to remain unchanged, although the characteristics of the mixed field in the body would be expected to depend upon beam dimensions. other types of collimators may behave similarly to those studied here, but that remains to be demonstrated elsewhere.

In this connection a recent time-offlight study by Hertel ${ }^{14}$ at Texas A\&M with a 
49- $\mathrm{MeV} \mathrm{D}{ }^{+}$on Be neutron beam showed no significant degradation in the energy spectrum (within the range $7-53 \mathrm{MeV}$ ) on axis for a $7 \times 7-\mathrm{cm}$ square pyramidal collimator vs a straight-sided circular one $3.8 \mathrm{~cm}$ in diameter. Both were made of iron filings and water-extended polyester.

It should be pointed out that, having once established a consistent set of values for $(B / A)_{T E},(B / A)_{G}$, and $(B / A)_{M g}$ for the primary neutron spectrum, it should then be possible to solve for $D_{f n}, D_{s n}$, and $D_{\gamma}$

at any measurement location, including points within a phantom. The conventional twodetector, two-component $(n, \gamma)$ method fails when applied within a phantom unless one knows the neutron spectrum at each point and the neutron energy dependence of the non-

hydrogenous detectors. 1516 In other words, one must know the effective (B/A) of the non-hydrogenous detector for the neutrons present at the point of interest. In the present model we do this by allowing the parameter $D_{\text {sn }}$ to grow and take on the appropriate value as a weighting factor so that the effective B/A for the graphite or magnesium chamber becomes

$$
(B / A)_{\text {eff }}=\frac{(B / A) \cdot D_{f n}+(0) \cdot D_{s n}}{D_{f n}+D_{\text {sn }}}
$$

Although we do not use (B/A) explicitly in the method, this viewpoint helps clarify the role of the $\mathrm{D}_{\text {sn }}$ component.

Finally, one can employ the present results to obtain an estimate of the effect of the collimator on the neutron output measurements given by August et a1.17 They reported that the neutron tissue dose rate ${ }^{1}$ on the central axis at $125 \mathrm{~cm}$ from the $\mathrm{D}^{+}$ on Be neutron source, with a 5 x $5 \mathrm{~cm}$ beam defined by the same collimator as one of those used in the present experiment, was given by

$$
Y=a\left(E_{D}\right)^{b} \operatorname{rad} \mu A^{-1} \min ^{-1}
$$

$$
\text { where } \underline{a}=1.24 \times 10^{-4}
$$$$
\underline{\mathrm{b}}=2.99
$$

and $E_{D}=$ the deuteron beam energy (MeV).
This a value takes into account a $3 \%$ downward correction for $\gamma$ radiation in the beam on the basis of the best estimate then available. In the present Table III it will be seen that $0.9 \%$ should be used in place of $3 \%$, and the value of a is increased thereby to $1.27 \times 10^{-4}$ for the $5 \times 5 \mathrm{~cm}$ collimator. Since the value of a should be proportional to $\left(D_{T}-D_{\gamma}\right)$ in Table III for the other collimators, one obtains $\underline{\mathrm{a}}=1.33 \times 10^{-4}$ for the $15 \times 15 \mathrm{~cm}$ collimator, $1.30 \times 10^{-4}$ for the $10 \times 10 \mathrm{~cm}$, and $1.20 \times 10^{-4}$ for the uncollimated source.

A very slight collimator effect on the value of $\underline{b}$ in the above equation, due to the energy dependence of the neutron-flux angular distribution, can be neglected in comparison with the change in a. Moreover August et a1. ${ }^{7}$ have shown from Serber stripping theory considerations that b should approximate 3 .

If the corresponding neutron dose rates are desired for the bare source unencumbered by the neutron attenuation of $3.5 \mathrm{~mm} \mathrm{Al}$ backing the target and $9.5 \mathrm{~mm}$ polystyrene in the monitoring ion chamber ${ }^{17}$, the foregoing values of a should all be increased by the factor $1.1 \overline{4} 5$.

Shapiro et al. ${ }^{18}$ made time-of-flight studies of this beam with no collimator, and with the monitor chamber removed but the Al target-backing plate in place. For this case the value of $\mathrm{a}=1.20 \times 10^{-4}$ should be increased by the factor 1.105 to remove the attenuating effect of the $9.5 \mathrm{~mm}$ of polystyrene, so that the above output equation gives $54.9 \mathrm{rad} / \mathrm{min}$ at $125 \mathrm{~cm}$ for $10 \mu \mathrm{A}$ of deuteron current on the target. The time-offlight results indicated that the corresponding neutron flux density at the same place was $8.4 \times 10^{9} \mathrm{n} / \mathrm{cm}^{2}-$ min, from which one can obtain a value $6.5 \times 10^{-9} \mathrm{rad} \mathrm{n}^{-1} \mathrm{~cm}^{2}$ for the average energy-transfer coefficient of this neutron beam in muscle tissue. This agrees fortuitously well with the value given by Bach and Caswel1 19 for $E_{n}=14 \mathrm{MeV}$, which is approximately the average as well as modal energy of the neutron spectrum obtained in the time-of-flight measurements (see Ref. 3 or 5). 


\section{References}

1. Throughout this paper we will employ the terms dose and absorbed dose (tissue) in place of kerma (tissue) by invoking the equality which results from the approximation of charged particle equilibrium at the point of interest.

2. The Middle Atlantic Neutron Therapy Association (MANTA), is a consortium of medical centers engaged in a clinical trial of the effectiveness of fast neutrons in cancer radiotherapy under the sponsorship of the National Cancer Institute (see Ref. 3). The Principal Investigator is Charles C. Rogers, M.D.

3. R.B. Theus, R.O. Bondelid, F.H. Attix, L.S. August, P. Shapiro, R.E. Surratt, and C.C. Rogers, Cancer 34, 17 (1974).

4. Benelex 401 is a composition product of density $\rho \approx 1.42 \mathrm{~g} / \mathrm{cm}^{3}$ made of pressed wood chips and lignin by the Masonite Corporation, 29 North Wacker Drive, Chicago, Illinois.

5. F.H. Attix, L.S. August, P. Shapiro, and R.B. Theus, The physics and dosimetry of fast neutrons for radiotherapy. NRL Memorandum Report 3123, 1975.

6. Model IC-17, manufactured by EG\&G, Inc., 130 Robin Hill Road, Goleta, California. This is a spherical chamber of nominal value $1 \mathrm{~cm}^{3}$, having walls of type A-150 tissueequivalent plastic $5 \mathrm{~mm}$ in thickness. TE gas was flowed through the chamber continuously.

7. L.S. August, F.H. Attix, G.H. Herling, P. Shapiro, and R.B. Theus, 1976, Stripping theory analysis of thick-target neutron production for $D+B e$. In press.

8. R. Serber, Phys. Rev. 72, 1008 (1947).

9. F.T. Kuchnir, C.J. Vyborny, and L.S. Skaggs, A precise method for measuring the neutron response of a "neutron-insensitive" dosimeter. IAEA-SM-193/51, 1975.

10. F.H. Attix, R.B. Theus, and C.C. Rogers, Measurement of dose components in an $n-\gamma$ field, Proceedings of the 2nd Symposium on Neutron Dosimetry in Biology and Medicine, Neuherberg. Edited by G. Burger and H.G. Ebert, EUR 5273 d-e-f, p. 329, 1975. Commission of the European Communities, Luxembourg.
11. F.H. Attix, R.B. Theus, P. Shapiro, R.E. Surratt, A.E. Nash, and S.G. Gorbics, Phys. Med. Biol. 18, 497 (1973).

12. Note that $(B / A)_{G}=0.318$ for an airfilled graphite chamber, as given in Ref. 10, has been remeasured recently at 0.319 by the same authors using an improved technique to be reported later.

13. A preliminary determination of $(B / A)_{M g}=0.162$ was obtained, but this is evidently too large since it gives some slightly negative $D_{S n}$ and $D_{Y}$ values in solving the above equations using the data in Table II.

14. N.E. Hertel, Fast Neutron Spectra Produced by a 49-MeV Deuteron-Beryllium Reaction and its Modification by Selected Absorbers. Master's Thesis, Texas A\&M University, (1975).

15. G.H. Herling, Monte Carlo Calculations of the Neutron and Photon Spectra for the INDI Project, NRL Report No. 7886 (1975).

16. F.H. Attix, R.B. Theus, S.G. Gorbics, and C.C. Rogers, Report on NRL Measurements as a Participant in the INDI, NRL Memorandum Report No. 3051 (1975).

17. L.S. August, R.B. Theus, F.H. Attix, R.O. Bondelid, P. Shapiro, and R.E. Surratt, Phys. Med. Biol. 18, 641 (1973).

18. P. Shapiro, R.B. Theus, R.E. Surratt, L.S. August, F.H. Attix, R. O. Bondelid, and C.C. Rogers, (Abstract), Transactions of the American Nuclear Society, 19, 52 (1974). Also unpublished data of Shapiro.

19. R.L. Bach and R.S. Caswell, Radiation Research 35, 1 (1968) and 60, 363 (1974). 

K.R. Kase, Ph.D., B.E. Bjarngard, Ph.D., B.J. Maddox, M.S., G.K. Svensson, Ph.D.

Joint Center for Radiation Therapy and Department of

Radiation Therapy, Harvard Medical School

Boston, Massachusetts

Accurate and precise dose delivery is necessary in radiation therapy because of the steep tissue response-dose curve. This paper discusses the precision of dose measuring devices, therapy machines, patient dose delivery at the Joint Center for Radiation Therapy. Ion Chambers used for therapy machine calibrations show a precision of 0.7 to $1.3 \%$ while a thermoluminescent dosimetry (TLD) system shows a precision of $1.5 \%$. Therapy machines are calibrated on a weekly schedule and the variation in dose delivery between calibrations varies from 1 to $2 \%$. Daily constancy checks on the linear accelerators using TLD show a variation of about $2 \%$ from day to day. Patient dose measurements using TLD at orthovoltage energies appear to have a precision of about $5 \%$ except for very small treatment fields where it is about $12 \%$. Patient dosimetry at megavoltage $\mathrm{X}$-ray energies shows a precision of $3.5 \%$ while at megavoltage electron energies it is about $5 \%$.

(Therapy, Dosimetry, Precision, Ion Chamber, TLD, Patient)

\section{Need for Dose Control}

The need for accurate and precise dose delivery in radiation therapy is primarily a consequence of the steep tissue responsedose curve, which implies that a small change of dose can make a large difference in tumor control probabilityl, 2 or in severity of complications. It must be possible to deliver repeatedly and reproducibly high doses to the treatment volume and at the same time spare adjacent normal tissue or critical organs. To plan a treatment for optimum ratio of tumor dose to healthy tissue dose a large amount of empirical data are required. These include the relative distribution of dose for various field sizes and depths, the therapy machine output expressed in rad and a quantitative description of the influence of different beam modifying devices such as wedges and compensators on the dose distributions. Measurements for this data base are made under good experimental conditions and should ideally be versatile enough to allow corrections for almost all variations of field configuration and patient geometry.

There are also a number of corrections one would like to apply to the machine output to correct for tissue heterogeneity and nonuniform field configuration. Because exact information on individual tissue composition is lacking many of these corrections can not be done with any great accuracy. Consequently one would expect that the dose delivered to a point in a patient is known with even less accuracy. However, the capability of precisely repeatable dose delivery is a necessity for patient treatment.
To be certain that therapy machines deliver a precisely repeatable dose to the treatment volume when operated in accordance with the treatment plan, periodic measurements of the radiation fields must be made. Absolute accuracy of these measurements is assured by proper calibration of dose measuring devices and nationwide intercomparisons. For accelerators and X-ray machines, whose characteristics depend on the reliability of electrical systems, a program of frequent constancy control is required which can supplement the more time-consuming calibrations. However, we must also be convinced of the precision of these dose meters and therapy machines, i.e. for a given programmed dose the variability in dose delivered and the variability in dose measured must be carefully assessed and determined to be within the limits that can be tolerated.

This paper discusses the dose measurement systems routinely in used at the Joint Center for Radiation Therapy (JCRT) and studies undertaken to determine the precision of these measurement systems and patient dose delivery. The measure of precision used is the coefficient of variation $\left(\frac{\sigma}{\bar{X}} \times 100\right)$ of a series of measurements.

Dose Measurement Systems

\section{Ionization Chambers}

At the present time the standards for dose measurements at the JCRT are Victoreen R-meters model 621 for megavoltage machines and model 131 for orthovoltage machines. 
Every two years one set of these chambers is calibrated at the NBS, and will be referred to as the local standard irstruments. Another set was calibrated at the JCRT against the local standard. This set is used in routine calibrations and will be referred to as field instruments.

Periodic constancy checks are made on these four chambers to insure that they hold their calibration. These checks are done by exposing the chambers in a fixed geometry to a $137 \mathrm{Cs}$ source for a fixed time. Results of these checks corrected for $137 \mathrm{Cs}$ decay are plotted in Fig. 1 . These constancy checks show a coefficient of variation of $0.7-0.8 \%$ for the local standard instruments and $1.3 \%$ for the field instruments over a 22 month period. During this period the reader for the field instruments became defective and was replaced. This together with the harsher environment experienced by the field instruments accounts for their greater variability relative to the local standard instruments.

\section{Thermoluminescent Dosimeters}

The other dose measurement system in routine use at the JCRT is a TLD system using $\mathrm{Li}_{2} \mathrm{~B}_{4} \mathrm{O}_{7}: \mathrm{Mn}$ Teflon disks. These dose meters were calibrated against the local standard instruments in the beam of a 4 MV Linac and at orthovoltage energies used in therapy. TLD disks have been used for routine daily checks on therapy machine calibration, for depth dose measurements in X-ray and electron beams, and for some patient dosimetry.

The method we use for dose interpretation involves a double readout cycle which determines the sensitivity of each dosimeter each time it is used. This improves precision by eliminating the effects of dosimeter history and day-to-day variations in operator technique and reader performance. Following exposure to the unknown dose the dosimeter is annealed at $80^{\circ} \mathrm{C}$ for $5 \mathrm{~min}$ and then its light output $\left(\mathrm{L}_{1}\right)$ is measured. The dosimeter is then irradiated to a standard dose in a $137 \mathrm{Cs}$ irradiator, annealed at $80^{\circ} \mathrm{C}$ for 5 min and its light output $\left(\mathrm{L}_{2}\right)$ determined. The unknown dose is then calculated from:

$$
\mathrm{D}=\left(\mathrm{L}_{1} / \mathrm{L}_{2}\right) \mathrm{C}
$$

where $\mathrm{C}$ is the calibration constant for the radiation producing $\mathrm{Ll}$.
Precision of the TLD system was tested by exposing 21 dosimeters to ${ }^{137} \mathrm{Cs}$ in a series of 8 identical exposures over a period of 3 weeks. The coefficient of variation in this series was calculated for each dosimeter and plotted in Fig. 2. The average coefficient of variation for the 21 dosimeters in this study was $1.5 \%$.

\section{Other Measurement Devices}

In addition to R-meters and TLD, film has been used for field size and uniformity and relative depth dose measurements. Film also plays an important role in routine monitoring of the relative distribution of exit dose in mantle field treatments. The information obtained is subsequently used in the calculation of the patient midline dose. Fricke dosimeters have been used for absolute dose measurements in X-ray and electron beams but no detailed study has been done on the precision of our Fricke system. Several other ionization chambers have been used from time to time, but only for relative dose measurements.

$$
\begin{gathered}
\text { X-Ray Treatments on } 4 \text { and } 8 \mathrm{MV} \\
\text { Linear Accelerators } \\
\hline
\end{gathered}
$$

\section{Weekly Calibrations}

Therapy machines are calibrated at approximately weekly intervals using the field instrument. The R-meter is exposed in a polystyrene phantom under standardized conditions (Fig. 3). If this measurement indicates that the dose delivered to $\mathrm{D}_{\max }$ is more than $1 \%$ different from the dose indicated by the monitor chamber on the machine, appropriate adjustments are made on the machine. Machine output is then remeasured and if 3 consecutive measurements show dose delivery within $1 \%$ of the programmed dose, the machine is considered calibrated.

In general the therapy machines hold their calibration very well. A study of calibration measurements over a period of 12 months indicated that the mean difference between $\mathrm{R}$-meter and machine monitor readings prior to machine adjustment was very close to zero on all machines (Fig. 4). The coefficient of variation for four machines ranged from $1.2 \%$ to $2.1 \%$. The coefficient of variation of an R-meter measurement is $1.3 \%$, thus the maximum coefficient of variation in the dose delivered by a therapy machine is about $1.7 \%$, since some imprecision will arise in the calibration setup. 
Each morning prior to starting treatment the calibration of each therapy LINAC is checked by exposing 2 TLD at $D_{\max }$ to a predetermined fixed dose ( 300 rad on the machine monitor) in a standardized geometry (Fig. 3). The TLD measurement must fall within $+5 \%$ of the programmed dose. If it does not, a second measurement is taken and corrective action would be initiated if the second measurement is also more than $5 \%$ different from the programmed dose.

A six month period in 1975 was broken into intervals corresponding with the intervals between machine calibration adjustments. The percent difference between the morning check measurement and the machine monitor dose was averaged over this interval to get an indication of the precision of our dose control base on these daily TLD checks. The results are shown in Fig. 5 and indicate that the relationship between the TLD measurement and the machine monitor followed the same pattern on all machines. The coefficient of variation ranged from 2.1 to $2.4 \%$ for the four machines. Since the maximum coefficient of variation of the dose delivered by the machine is about $1.7 \%$ from above, the minimum coefficient of variation of a TLD measurement is in the range 1.2 to $1.7 \%$ which compares well with that found in the laboratory study reported above.

An interesting observation is that the periods in which differences between the TLD and machine monitor exceed $4 \%$ are generally associated with the replacement of a machine component such as an accelerator wave guide, magnetron or pulse transformer. Because our morning check measurements are made at a depth of $1 \mathrm{~cm}$ on the $4 \mathrm{MV}$ and $2 \mathrm{~cm}$ on the $8 \mathrm{MV}$ LINAC while the machine calibration measurement is made at $5 \mathrm{~cm}$ depth, a large difference between the dose measured by the TLD and the machine monitor would indicate a shift in the depth dose characteristics of the beam. This might indicate that a temporary but significant energy shift occurs as a result of these component replacements.

\section{Patient Dosimetry}

Routine dose measurements are done on patients treated with a mantle field. TLD measurements are made during the early portion of the treatment on the surface of the patient in polystyrene build-up blocks. Measurements made at various points in the field (Fig. 6) are used to verify that the prescribed dose is being delivered and to measure dose uniformity throughout the treatment field. Undertreated areas as determined by the TLD are boosted.

Doses delivered to 52 patients treated with mantle fields since Jan. 1975 have been measured. The distribution of dose measurements is shown in Fig. 7 . Results of these measurements show that 47 of these patients received midline doses within $+5 \%$ of the prescribed dose. The coefficient of variation in the measurement of the ratio of Measured Dose/Prescribed Dose for the 52 patients is $3.5 \%$, which is an indication of the precision of dose delivery for mantle fields.

\section{X-Ray Treatments at Orthovoltage Energies}

Orthovoltage $\mathrm{X}$-ray treatments are done at the JCRT using one of two beam qualities: $140 \mathrm{kVp}, 3.5 \mathrm{~mm}$ Al HVL or $300 \mathrm{kVp}, 1.5 \mathrm{~mm}$ $\mathrm{Cu}$ HVL. Tissue areas treated vary from $1.5 \mathrm{~cm}$ equivalent square to $14.0 \mathrm{~cm}$ equivalent square fields. Patient doses are verified by exposing a pair of TLD dosimeters in the center of the treatment field on the skin surface during treatment. Treatment fields were defined using a lead mask at the surface of the patient and overlapping the mask with the machine collimators.

Over a period of time covering 116 treatment measurements it was observed that although there is a large amount of scatter in the data, measurements of dose delivered were generally slightly lower than the dose expected at the skin surface based on the prescribed dose (Table 1). Also the measurement precision appeared to be worse for the very small field sizes.

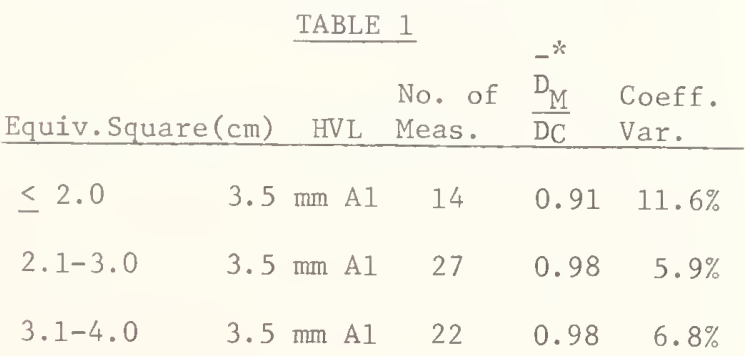

$* \bar{D}_{M}$ is the mean of the ratio of measured to $\overline{\mathrm{D}_{\mathrm{C}}}$ calculated skin dose. 


$\begin{array}{lcccc}4.1-5.5 & 3.5 \mathrm{~mm} \mathrm{Al} & 12 & 0.98 & 5.3 \% \\ 4.1-5.0 & 1.5 \mathrm{~mm} \mathrm{Cu} & 12 & 0.99 & 3.1 \% \\ 5.1-6.0 & 1.5 \mathrm{~mm} \mathrm{Cu} & 6 & 0.97 & 4.9 \% \\ 6.1-7.0 & 1.5 \mathrm{~mm} \mathrm{Cu} & 5 & 0.97 & 7.1 \% \\ 7.1-8.0 & 1.5 \mathrm{~mm} \mathrm{Cu} & 5 & 0.95 & 3.0 \% \\ 8.1-10.0 & 1.5 \mathrm{~mm} \mathrm{Cu} & 8 & 0.94 & 8.3 \% \\ 10.1-12.0 & 1.5 \mathrm{~mm} \mathrm{Cu} & 5 & 0.93 & 4.6 \%\end{array}$

A series of experiments were undertaken to determine what factors other than TLD calibration might cause a difference between measured and calculated skin dose. A $30 \times 30 \mathrm{~cm}$ polystyrene box filled with water was used as a phantom. Pairs of TLD were placed at the water surface in the center of the phantom for the measurements. Field size was defined in 3 ways: (1) the field size was set with the machine collimator; (2) field size was defined with lead strips at the water surface and the machine collimators were set to overlap the lead by $1 \mathrm{~cm}$ on all sides; (3) field size was again defined with lead strips but the machine collimators were set at $10 \times 10 \mathrm{~cm}$. The majority of patient treatments are done under condition 3. Another measurement was done setting field size with the machine collimators and exposing pairs of TLD with no water in the phantom. This was done to study the effect of field size without the influence of backscatter.

Field flatness was measured under conditions 1 and 3 above using TLD microrods (1 $\mathrm{mm} \times 6 \mathrm{~mm}$ ) spaced at $0.5 \mathrm{~cm}$ intervals across the field.

Results of the first set of measurements are given in Table 2. The dose was first measured at the center of a $10 \mathrm{~cm}$ $x 10 \mathrm{~cm}$ field. The dose expected for smaller field sizes was then calculated using published backscatter factors ${ }^{3}$ in the following way:

$D_{F}=D_{10 \times 10}\left(\frac{B_{F}}{B_{10 \times 10}}\right)$ where

$D_{F}=$ dose expected for field size $F$.

$\mathrm{D}_{10 \times 10}=$ dose measured in $10 \mathrm{~cm} \times 10 \mathrm{~cm}$ field.

$\mathrm{B}_{\mathrm{F}}=$ backscatter factor for field size $\mathrm{F}$. $\mathrm{B}_{10 \times 10}=$ backscatter factor for $10 \mathrm{~cm} \times$ $10 \mathrm{~cm}$ field.
Results shown in Table 2 are the ratio of the measured dose ( $\left.D_{M}\right)$ to the expected dose $\left(D_{F}\right)$ for various field sizes under the three conditions.

It is apparent that dose delivered is within a few percent of the expected dose when field size is defined by a lead mask and the machine collimators are opened to $10 \times 10 \mathrm{~cm}$. However, if the field size is defined by the machine collimators, doses delivered

\section{TABLE 2}

$$
\begin{gathered}
\mathrm{D}_{\mathrm{M}} / \mathrm{DF}_{\mathrm{F}} \\
\text { Field Defined } \\
\text { by: }
\end{gathered}
$$

Field Collimators Lead Mask \& Mask

Size HVL Only Collimator Only

$\begin{array}{lllll}2 \times 2 & 3.5 \mathrm{~mm} & 0.80 & 0.89 & 0.99 \\ & \mathrm{~A} 1 & & & 1.03 \\ 2 \times 3 & & 0.89 & 0.94 & 1.00 \\ 3 \times 3 & & 0.92 & 0.93 & 1.01 \\ 4 \times 4 & & 0.98 & 0.98 & 1.01 \\ 5 \times 5 & & 0.99 & 1.06 & 0.97 \\ 2 \times 2 & 1.5 \mathrm{~mm} & 0.79 & 0.93 & 1.00 \\ & \mathrm{Cu} & & & 1.01 \\ 2 \times 3 & & 0.87 & 0.95 & 1.00 \\ 3 \times 3 & & 0.93 & 1.00 & 1.05 \\ 4 \times 4 & & 0.95 & 0.99 & 1.01 \\ 5 \times 5 & & 0.96 & & \end{array}$

to the small fields are significantly less than expected. The measurement in air (no water in phantom) showed that the dose measured decreased with field size and that this non-uniformity accounts for the discrepancy between dose measured and dose expected when the machine collimators are used to define the field size. The precision of the dosimetry in these experiments was quite good (less than $3 \%$ coefficient of variation).

The field flatness measurement confirmed that the dose is very non-uniform for small fields when the field is defined by the machine collimators. This occurs because there is no scattering into the beam from outside its edges. Field 
uniformity is much improved when a lead mask is used to define the field size. However the dose still falls off rapidly at the edges of the field. Since the dosimeters used are about $1 \mathrm{~cm}$ diameter, placement is critical in small fields which probably accounts for the large variations in dose measurement for patients treated with very small fields.

\section{Electron Treatments at 4, 7 and $11 \mathrm{MeV}$}

Measurements to characterize the radiation field when treating patients with intermediate energy electron beams, characterization of the electron depth dose for the treatment configuration is most important. At the JCRT the radiation field is defined at the patient using a lead mask cut to the desired field size and shape and placed in contact with the patient. An adjustable aluminum collimator defines the electron beam impinging on the lead mask. This collimator is set so that the electron beam is 1 to $2 \mathrm{~cm}$ larger than the cutout in the lead mask at the surface of the patient. The collimator jaws on the therapy machine are set to 30 x $30 \mathrm{~cm}$. In this configuration we have been able to achieve reasonably consistent depth dose curves and field uniformity 4.

Prior to beginning treatment on a patient, the lead mask for that patient is used to define the electron field for a depth dose measurement in a polystyrene phantom at the treatment energy.

Thermoluminescent dosimeters are used for this measurement which provides the information required to calculate the machine meter dose needed to deliver the prescribed treatment dose.

\section{Patient Dosimetry}

Pairs of TLD are placed in the center of the radiation field on the patient during each treatment. These dosimeters are used both to verify the dose delivered during a particular treatment and to measure the cumulative dose during the treatment period. Based on the delivered dose as measured by the TLD, the treatment plan may be modified to deliver higher or lower doses for the remainder of the treatment. To date 24 patients have been treated in this manner. Nineteen have received cumulative doses within $5 \%$ of the prescribed dose.
Day-to-Day variations in dose delivered could be caused by variations in the TLD, machine fluctuations or patient setup. TLD precision was discussed above where it is shown that the coefficient of variation in a series of exposures is about $1.5 \%$. Electron beam stability was checked in a series of measurements in which a Baldwin-Farmer ionization chamber and TLD were exposed together to electron beams of 4,7 and $11 \mathrm{MeV}$. These measurements showed that the coefficient of variation in the comparison between TLD, Baldwin-Farmer chamber and the monitor chamber on the machine was about $2 \%$. In a more extensive series of comparisons between the Baldwin-Farmer chamber and machine monitor chamber the coefficient of variation of the ratio, B-F dose/monitor dose, was $2.0 \%$ for $4 \mathrm{MeV}, 2.6 \%$ for $7 \mathrm{MeV}$ and $0.9 \%$ for $11 \mathrm{MeV}$ electrons.

The ratio of monitor dose (MD) to dose delivered during patient treatment as measured by TLD should remain constant throughout the treatment period for any given patient. Variations in this ratio from patient to patient are caused by adjustments in monitor chamber sensitivity, SSD, field size, etc., but are taken into account in the treatment plan. The coefficient of variations in the ratio MD/TLD over the treatment sessions for a single patient is a measure of the precision of dose delivery for electron treatments. Table 3 presents the mean of the ratio $M D / T L D$ and the coefficient of variation in the ratio for patients treated to date.

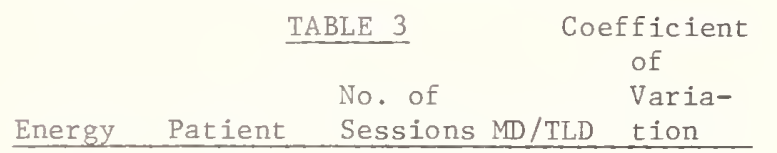

$\begin{array}{llrll}4 & \mathrm{a} & 9 & 0.968 & 7.4 \% \\ 4 & \mathrm{~b} & 13 & 0.384 & 3.8 \% \\ 4 & \mathrm{c} & 8 & 0.462 & 4.7 \% \\ 4 & \mathrm{~d} & 5 & 0.450 & 9.8 \% \\ 7 & \mathrm{e} & 7 & 1.258 & 5.8 \% \\ 7 & \mathrm{f} & 10 & 0.855 & 6.1 \% \\ 7 & \mathrm{~g} & 13 & 0.281 & 7.1 \% \\ 7 & \mathrm{~h} & 10 & 0.324 & 5.7 \% \\ 7 & \mathrm{i} & 5 & 0.283 & 4.2 \% \\ 7 & \mathrm{j} & 6 & 0.792 & 2.4 \% \\ 7 & \mathrm{k} & 8 & 0.870 & 1.9 \% \\ 7 & \mathrm{l} & 4 & 0.997 & 3.3 \% \\ 7 & \mathrm{~m} & 4 & 0.907 & 4.5 \% \\ 7 & \mathrm{n} & 9 & 1.048 & 8.7 \% \\ 7 & \mathrm{o} & 3 & 1.101 & 3.2 \%\end{array}$




$\begin{array}{llrll}11 & \mathrm{p} & 3 & 0.677 & 0.8 \% \\ 11 & \mathrm{q} & 9 & 0.681 & 6.6 \% \\ 11 & \mathrm{r} & 15 & 0.577 & 6.6 \% \\ 11 & \mathrm{~s} & 10 & 0.479 & 3.8 \% \\ 11 & \mathrm{t} & 5 & 0.566 & 4.7 \% \\ 11 & \mathrm{u} & 15 & 0.477 & 5.2 \% \\ 11 & \mathrm{~V} & 18 & 0.743 & 3.3 \% \\ 11 & \mathrm{~W} & 10 & 0.794 & 2.9 \% \\ 11 & \mathrm{x} & 4 & 0.823 & 4.4 \%\end{array}$

As seen in Table 3 the precision varies widely from one patient to another from less than $1 \%$ to almost $10 \%$, while the average value is about $5 \%$. An analysis of the 203 sessions involving 24 patients shows that in $85 \%$ of the sessions the dose measured was within $+10 \%$ of the prescribed dose and in $61 \%$ of the sessions the measured dose was within $\pm 5 \%$ of the prescribed dose.

Based on these data the uncertainty in dose delivery averages about $8.5 \%$ at the 95\% confidence level. However, because of as yet unexplained non-statistical fluctuations in dose delivered, we must continue to measure patient dose during each treatment session.

\section{Conclusions}

Dose control in radiation therapy depends on the precision of many instruments and techniques. Using the coefficient of variation as a measure of precision we have attempted to determine the individual precision of some of these factors and relate that to the precision of patient dose delivery. Table 4 summarizes our findings and gives the precision with which we can calibrate therapy machines, verify their calibration, measure patient dose and verify treatment plans.

\section{TABLE 4}

Instrument or Technique

Precision

R-meter and Therapy Machine Calibration

$1.3 \%$

TLD Controlled Experiment

$1.5 \%$

LINAC Dose Delivery

$1.7 \%$

Verification of IINAC

Calibration
Patient Dose Measurement and Treatment Verification

- Orthovoltage

$3 \%$ to $12 \%$

- Mantle Fields $3.5 \%$

- Electrons

$5.1 \%$

Based on these results our policy is to investigate any measurement which exceeds these values and if deemed necessary repeat the measurement, adjust the machine calibration, or revise the patient treatment plans.

As a result of this study we have decided that continued daily verification of therapy machine calibration is essential. However, we will soon change from using TLD at one point for this purpose to a system similar to that recently published by Jones and Schumacher 5 using diode detectors at 3 positions in the beam. This will allow an immediate check on dose output, beam symmetry and depth dose without waiting 2-3 hours for the TLD to be read out.

\section{References}

1. M.L. Mendelsohn, Front. Radiation Ther. Onc. 6, 512 (1972) (ed. J.M. Vaeth).

2. W.D. Bloomer \& S. Hellman, New Eng. J. Med. 293, 80 (1975).

3. British J. Radiol. Supplement No. 11 (1972).

4. B.E. Bjarngard, R.W. Piontek and G.K. Svensson, Med. Phys. 3, (1976) (in press).

5. D. Jones and D. Schumacher, Am. J. Roentgenol. 123, 198 (1975). 


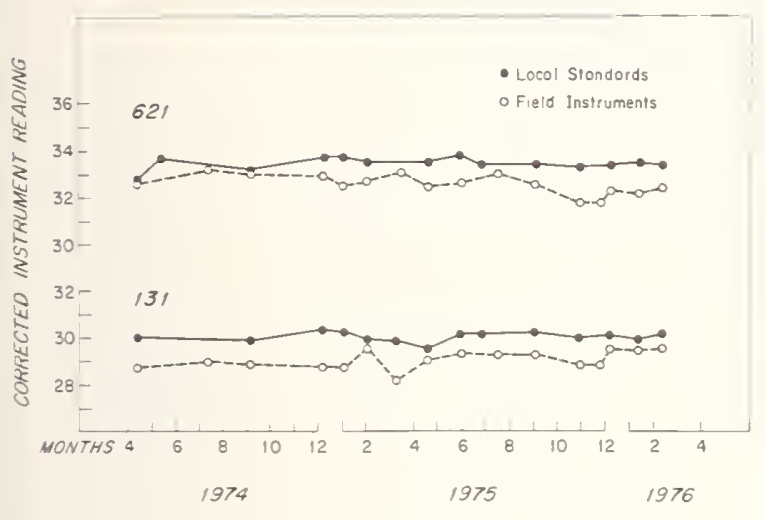

Fig. 1. Constancy measurements for Victoreen R-meters over 22 month period corrected for source decay.

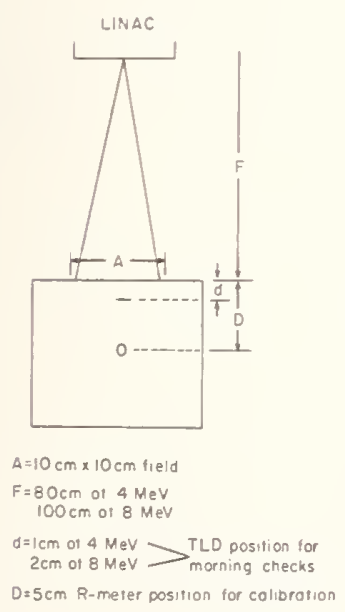

Fig. 3. Setup for LINAC calibrations and morning checks.

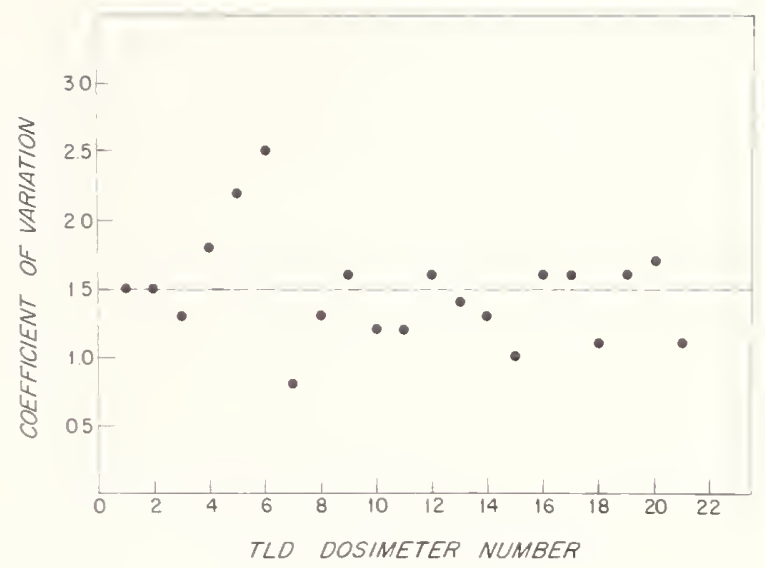

Fig. 2. Coefficient of variation for 8 identical exposures of 21 TLD over a 3 week period.

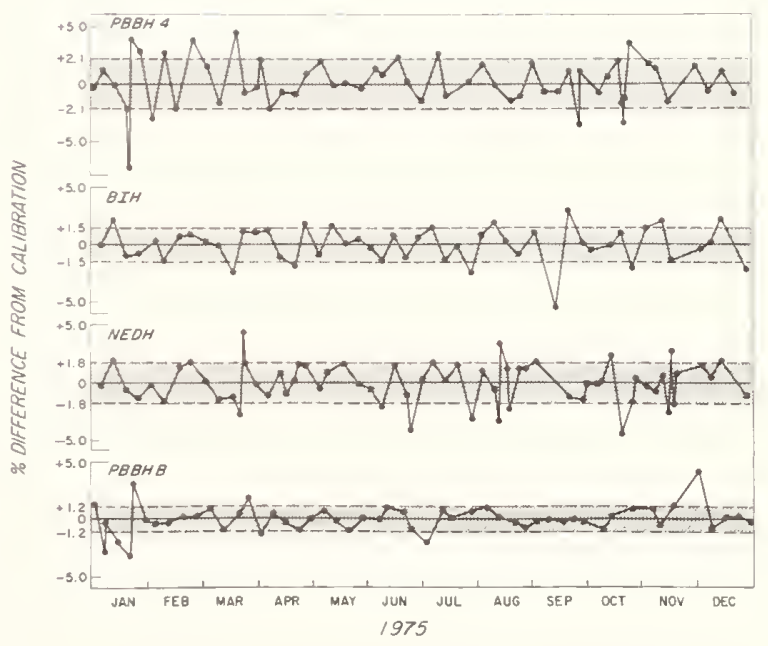

Fig. 4. \% Difference between machine monitor and $\mathrm{R}$-meter dose prior to machine adjustment for $4 \mathrm{MV}$ and 8 MV LINAC's. 


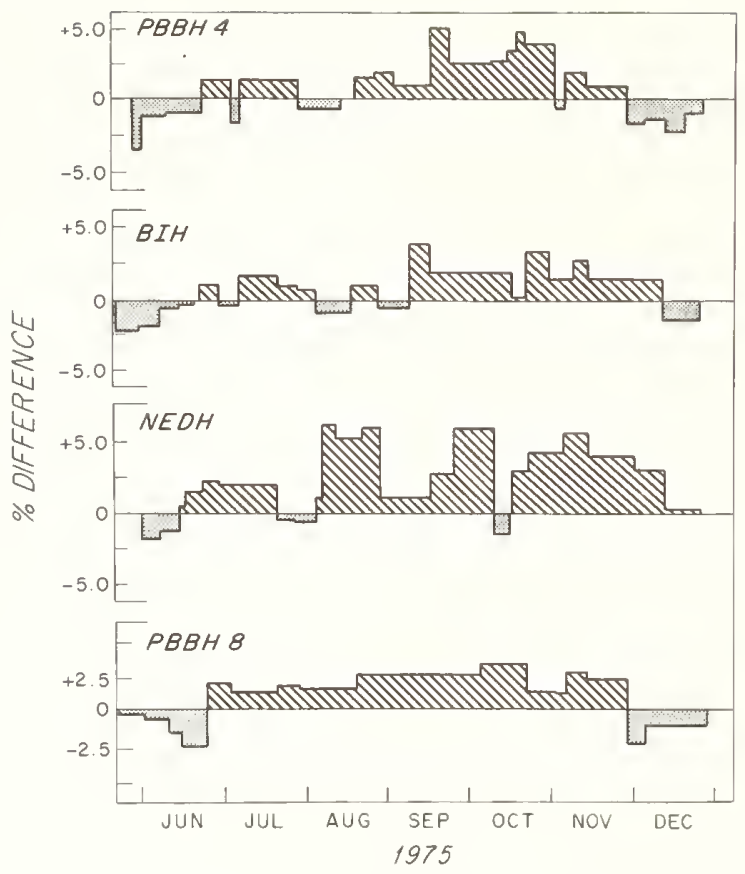

Fig. 5. \% Difference between morning dose check and machine monitor dose averaged over the period between calibration adjustments for $4 \mathrm{MV}$ and 8 MV LINAC's

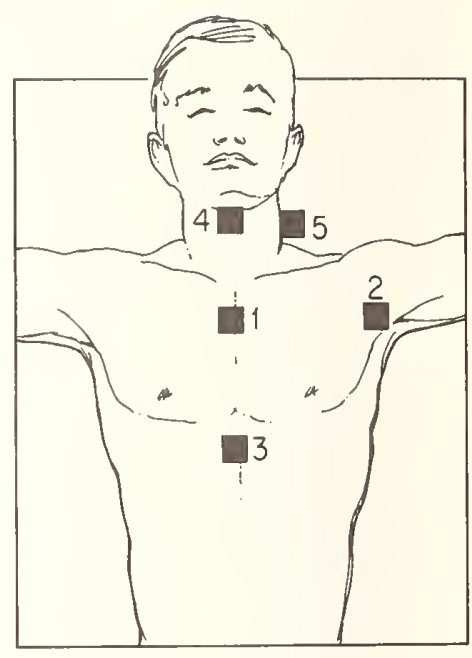

Fig. 6. Points of dose measurement for mantle fields.

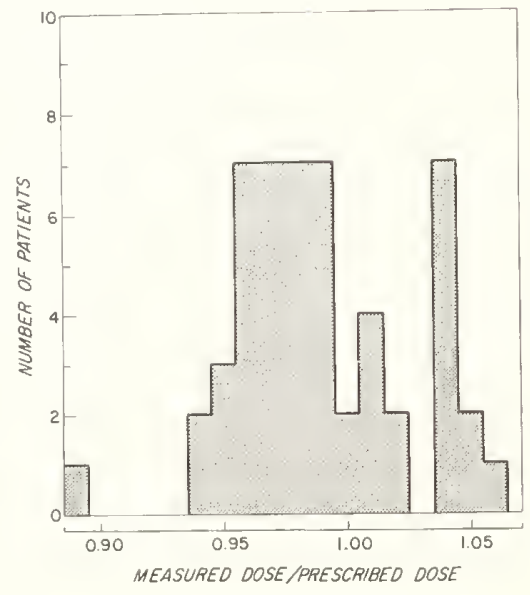

Fig. 7. Distribution of mantle patient dose measurements. 


\title{
DOSIMETRY PROCEDURES AT THE NORTHWEST MEDICAL PHYSICS CENTER \\ Douglas Jones, Douglas Schumacher \& John Washington Northwest Medical Physics Center \\ University of Washington, RC-08 Seattle, Washington 98195
}

\begin{abstract}
The Northwest Medical Physics Center provides physics support to 28 radiotherapy institutions in the Northwest from a central location. The methods employed in on-site physical measurements, organization of data and long-term quality assurance are described.
\end{abstract}

(Calibration; megavoltage radiotherapy; quality assurance)

\section{Introduction}

The Northwest Medical Physics Center developed from funds from the Washington/ Alaska Regional Medical Program in the years 1969 through 1971. The Center supplies radiotherapy physics support to 28 radiotherapy departments in Washington, Oregon and Alaska at which over 7,000 cancer patients are treated annualiy. The growth in participation in the Center is illustrated in Table I, and the radiation therapy equipment currently in use at the participating institutions is shown in Table II.

\section{TABLE I}

Participation in the Center

$\begin{array}{cccc}\text { Year } & \begin{array}{c}\text { Number of } \\ \text { Participants }\end{array} & \begin{array}{c}\text { Number of New } \\ \text { Cancer Patients }\end{array} \\ 1970 & 16 & 3,182 \\ 1971 & 22 & 4,103 \\ 1972 & 23 & 5,505 \\ 1973 & 27 & 6,500 \\ 1974 & 29 & 7,778 \\ 1975 & 28 & 7,400^{\star}\end{array}$

*Data for 1975 is estimated.

TABLE II

Radiation Therapy Equipment January, 1976

Machine Type Number

Superficial X-Ray

Orthovoltage $X$-Ray 9

Cs-137

Co-60

4 MV Linear Accelerator

8 MV Linear Accelerator

1

The Center provides a broad range of physics support services to radiotherapists which have been described ${ }^{1}$. The purpose of this report is to describe the methods employed to ensure accurate dose delivery at these institutions with an emphasis on megavoltage photon therapy which is the modality employed in over $94 \%$ of the treatments delivered in our region.

\section{Equipment Available \& Its Calibration}

Routine Field Instruments

The Farmer dosimeter, Mode1 2502/3, with the associated $0.6 \mathrm{cc}$ chamber is the basic field instrument. In our experience, the new graphite wall chamber has proved too fragile for routine field work, and the nylon wall chamber is preferred for this purpose. The precision of readings with this instrument is defined by a standard deviation of less than $0.2 \%$ of the mean value. The nonlinearity of our particular instrument over its range is less than $0.5 \%$ and a calibration curve has been constructed to account for this deviation. Other chambers which are used with this system in specialized applications include a $0.03 \mathrm{cc}$ parallel plate chamber with a $2.3 \mathrm{mg} \cdot \mathrm{cm}^{-2}$ window and a $30 \mathrm{cc}$ chamber for low level measurements. A Keithley Model 602 Electrometer has been modified to accommodate the Farmer chambers and is used when dose rate measurements are required. The local standard to which these field ionization chambers are referred is a $0.6 \mathrm{cc}$ Farmer graphite chamber which is calibrated annually at a Regional Calibration Laboratory. Depth dose and off axis dose measurements are routinely made using a $50 \times 50 \times$ $38 \mathrm{~cm}$ water phantom to which is attached a linear scanner having variable speed, remote operated drive. The position of the chamber is sensed electronically allowing automatic plotting of beam profiles. Prior to 1975, a silicon diode was used as the detector for off axis dose measurements but studies showed that this device overresponds at the periphery of large fields from 4 MV $x-r a y$ machines and its use was discontinued. Where indicated, film is employed for 
measurements, and Kodak RP/V is preferred for its slow speed. Our experience is that unit density is obtained from a dose around $50 \mathrm{rads}$. The dose/density relationship of the $\mathrm{film}$ is measured on each occasion.

Plastic and tissue-equivalent rubber phantoms are occasionally used for check measurements or depth dose checks at beam orientations other than vertically down. The reference material is water and when plastics are used, the depth is scaled on the basis of linear attenuation coefficients rather than simple density. For example, a scaling factor for lucite for Co-60 gamma rays of 1.15 is employed and good agreement with depth dose measurements in water has been obtained on this basis.

The routine armamentarium for field work is completed by a survey meter. The Eberline Rad-Owl has proved satisfactory for our purposes. The facility to integrate exposure is a particular advantage. This instrument is calibrated by a standardized Cs-137 source.

\section{Laboratory Apparatus}

Several film densitometers are available for quantifying the film images produced in the field. The one most commonly used is a Joyce-Loebl Scanning Microdensitometer. The automatic and high resolution features of this instrument are particularly advantageous. A Teledyne-Isotopes Model 7300B Thermoluminescent Dosimetry Reader is available for the evaluation of LiF-Teflon and $\mathrm{Li}_{2} \mathrm{~B}_{4} \mathrm{O}_{7}: \mathrm{Mn}$-Teflon dosimeters which are the thermoluminescent dosimeters usually employed at the Center. A chemical dosimetry system built around a Beckman DoubleBeam Spectrophotometer is available for Fricke dosimetry. A considerable assortment of test equipment and general laboratory apparatus together with a wel1-stocked workshop complete the set of tools required by a physicist supporting radiotherapy departments in the 1970 's.

The data processing capabilities of the Center should be mentioned in this general category. These range from pocket calculators through desk top calculators, typified by the Hewlett-Packard Model 9810, to a CDC 6400 Computer which are a11 used for data manipulation and reduction.

Measurements at the Institutions

Initial Acceptance Testing

In 1976, we anticipate responsibility for the acceptance testing of one $10 \mathrm{MV}$, two 6 MV and six 4 MV radiotherapy machines.
With a load of this magnitude, a systematic approach is necessary. Table III is a guide employed by us structured on the AAPM recommendations ${ }^{2}$.

\section{TABLE I I}

The Guide used by the Center in Acceptance Testing of Megavoltage Photon Radiotherapy Machines Safety Considerations

1. Leakage Radiation, Beam off

2. Leakage Radiation, Beam On

3. Exposure in the Environs

4. Door Interlock

5. Couch Interlock

6. Beam Direction Interlocks

7. Emergency off Switches

8. Signs and Labels

9. Radiation On Indicators

10. Backup Dosimetry

11. Operating Procedures

Mechanica 1 Aspects

1. Dimensions

2. Isocenter Location and Stability

3. Optical Distance Indicator

4. Collimator Symmetry in Rotation

5. Light Field Size

6. Radiation Field Size

7. Coordination of Light and Radiation Fields

8. Radiation Field Symmetry

9. Isocenter Indicators

\section{Dosimetry Data}

1. Shutter Time

2. Quoted Rmm

3. Dose Rate as a Function of Field Size

4. (TAR) o off Axis

5. Off Axis Dose in Water (Flatness)

6. TAR or TMR Measurement

7. Surface Dose

8. Interface Dose Between Couch

9. Output as a Function of Gantry Angle

10. Stability in Rotation

11. Output as a Function of Distance

12. Timer and Integrator Linearity

\section{External Inhomogeneities}

1. Wedges

2. Compensators

3. Bolus

4. Block Transmission

5. Couch

6. Trays

\section{Machine $\log$}

1. Beam Monitor Readings

2. Frequent Check Dosimeter Setup

3. Review Measurements and Checks to be made by Local Staff 
The guide also defines the form of the report, which will be discussed later. The tasks enumerated in Tabie III will be recognized by those experienced in the field. Our approach and methods are elaborated in the subsequent sections and where appropriate, examples are given to illustrate specific tasks.

\section{Safety Considerations}

The objects of this section of the review are to ensure that the machine complies with local regulations and that hazards to patients and operators are minimized. Head leakage measurements in the beam off condition are only relevant to isotopic machines when measurements are made in accordance with the methods described in NCRP Report No. $33^{3}$. There are two considerations in measurements of the head leakage in the beam on condition. The first is if the housing is designed to minimize total body exposure to the patient and the second is if high leakage areas will result in high exposures in the environs of the room. In the case of electrical machines, a search is made for high leakage areas by wrapping the head with film. If Kodak RP/V film is used for this purpose, the machine should be set to deliver a dose on the order of 7,000 rads at one meter. Following the demarcation of these areas, the magnitude of the leakage is determined using an ionization chamber. An interesting characteristic of the Varian Clinac 4 operating with the uranium flattening filter is that measurements with the jaws closed indicate leakage in excess of $0.1 \%$ of the primary but this zone of high leakage is obscured by the upper jaws so that for clinically useful beams, the leakage is within specification.

The checking and evaluation of interlocks has to be done with reference to the day-to-day operation of the machine. It is necessary that the local technical staff be on hand during these tests so that they fully comprehend the function of all the interlocks and that the purpose of ongoing checks is realized. Tests are conducted to ensure the efficient operation of backup dosimetry systems also.

\section{Mechanical Aspects}

There is a class of measurements to be made on a therapy machine which relate to its construction and accurate design. Generally, a test which does not require the use of an ionization chamber falls into this category. The dimensions of the machine should be noted with particular reference to points of measurement such as the isocenter and wedge location. The stability of the isocenter during gantry, collimator and couch rotation is assessed using slit beams on film. The accuracy of the optical distance indicator is assessed over its range. The rigidity of the collimator system is checked by film exposures at various gantry arigles. The size of the light and radiation fields and their coordination are checked over the range of field sizes available using film. We have found it convenient to use a marked polystyrene plate with radiopaque markers for this purpose. The field symmetry is assessed by dose measurements at symmetrical locations in the beam.

Dosimetry Data

Besides the items mentioned in Table III, measurements are made to provide the radiotherapist with clinical data for special treatment regimes such as moving strip therapy and whole body irradiation. But in general, the purpose of this section is to develop a comprehensive set of data to allow computer or hand calculations of dose distributions.

The output of the machine as a function of field size is assessed and a separation made of collimator and phantom scatter. The variation in energy across the field of low energy linacs is an important consideration in dose calculations. Recently Bruels ${ }^{4}$ described a routine to accommodate this effect in clarkson-type irregular field calculations which is currently under review at the Center. Initial measurements were made using a narrow column of water, but in order to expedite this measurement, a semi-empirical relationship was developed to convert attenuation measurements in lead to those in water. For beams of nominal energy of $4 \mathrm{MV}$ the equation has the following form: HVL Water $=7.11$ (HVLLead) $+4.91 \mathrm{~cm}$. Typical values for the change in energy are shown in Table IV. The dose off axis is assessed by scanning in a water tank. A range of field sizes are normally measured and the data tabulated in the form of off center ratios to enable computer calculation of patient dose distributions. For depth dose determination, the standard practice at the Center is to measure tissue air ratios for beams up to $4 \mathrm{MV}$ and tissue maximum ratios for higher energies. A computer program, Tarman, has been developed and is used to generate percentage depth 
dose tables and tables of the dose along the central axis in isocentric treatments. We are currently extending this program to smooth and extrapolate tissue air ratio data to produce scatter air ratio tables for large radii. Surface dose measurements are made under various conditions and serve to illustrate reduction in skin sparing under such conditions as increasing field size and inclusion of plastic block trays in the beam. Where the couch is fitted with a cutout section, an assessment is made of the loss of skin sparing introduced by the patient support structure. The operation of the machine in the rotation therapy mode is evaluated in order to ensure uniform output as a function of gantry angle. The output is correlated to inverse square law to establish the location of the virtual source which is used in depth dose computations. General1y, a 11 measurements are made with the machine running at the dose rate to be employed clinically. However, the deviation of the output due to nonlinearities in the integrator circuitry is determined over the range of operation of the unit.

\section{TABLE IV}

HVL, Water, cm

Machines

Distance Off Axis at $100 \mathrm{~cm}$

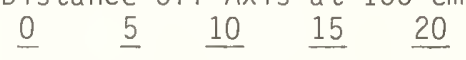

$\begin{array}{llllll}\text { Clinac } 4 & 12.9 & 12.5 & 12.0 & 11.5 & 11.1 \\ \text { Pb Filter } & & & & & \\ \text { Clinac } 4 & 13.5 & 13.4 & 13.1 & 12.7 & 12.4 \\ \text { UFilter } & & & & & \\ \begin{array}{l}\text { Therapi-4 } \\ \text { SS Filter }\end{array} & 12.4 & 12.3 & 12.1 & 11.5 & ---- \\ \begin{array}{l}\text { Therapi-400 } \\ \text { SS Filter }\end{array} & 12.1 & 12.1 & 12.1 & 11.9 & 11.3 \\ \text { LMR-4 } & 12.5 & 12.3 & 11.8 & 11.3 & 10.9 \\ \text { Pb Filter } & 12.9 & & & & \end{array}$

External Inhomogeneities

This term refers to measurements of the effect of the introduction of any absorbers in the beam. The standard algorithm employed at the Center for dose computations is that developed at Memorial Hospital ${ }^{5}$ with some local modifications ${ }^{6}$. The computation of the dose under wedges is based upon the assumption of exponential absorption on a ray through the wedge. This technique does not adequately account for phantom scatter and other effects, and thus it is necessary to fit wedge dimensions to experimental data. As a routine, wedged beams are scanned at several depths in a water phantom and the wedge dimensions are modified to yield a best fit to the empirical data.

The evaluation of compensation, bolus and blocking techniques employed would be the subject of a report in themselves. The general approach we make is to ensure that sufficient information is collected to enable the calculation of dose distributions. In this regard, mention should be made of recent work at the Center using the concept of negative fields ${ }^{7}$ to calculate the dose distribution under narrow blocks.

The attenuation of the support structures of the couch are determined and this information is used in dose calculations where the couch interposes the beam. The transmission factors of blocking trays are determined and if the variation with field size is less than $\pm 1 \%$, a single factor is quoted.

Machine Log

The foregoing has provided an outline of the initial measurements on a megavoltage radiotherapy machine. It must be stressed that while we do use this guide, it is construed as an outline and that individual variations in the installation wil1 require innovation on the part of the responsible physicist.

Following this extensive series of tasks, we see the essential task to be one of ensuring the fidelity of the original data and providing adequate documentation to this effect. For electrical machines, we consider the particularly suspect parameters to be the dose delivered per unit monitor setting and, to a lesser extent, beam energy and symmetry for bent beam or multimode machines.

We have developed a device called the beam monitor ${ }^{8}$ which consists of two diode detectors located at 5 and $17 \mathrm{~cm}$ depth in a phantom with electronic circuitry attached to the phantom. Our design considerations in developing this device were that it be simple and convenient to use and that long-term drift of the readings of the integrated current from the diodes be 1 ess than $3 \%$ per year. Seven instruments were produced which have been in use on the machines in our region over the last three years. A commercial version of this unit is now available 9 . A standard procedure is in use which consists of two exposures of the device under well defined conditions at the beginning and end of the treatment day. At the time of a calibration 
using ionization chambers, the physicist will establish the readings obtained by the beam monitor under these standard conditions. At the end of each month, a form developed by the Center is completed in which the average readings are shown as well as the deviations from the exact values. A copy of this is returned to the Center for review. When an isotopic source is available at an institution, this is used for a weekly check on the constancy of the beam monitor. This system has proved very useful in diagnosing problems with the machine and has enabled the analysis of the long-term stability of the machines in our

region ${ }^{10}$. A typical example of a completed analysis form is contained in an appendix to this paper.

Another parameter which requires regular review is the coordination of the light and radiation fields. For this purpose, we have developed a frequent check dosim-

eter $^{11}$ which consists of a piece of DuPont Radiation Imaging film contained in a specially marked cassette. The cassette has two rectangles scribed on its surface with corners defined by radiopaque markers.

These devices are exposed each month on the therapy machines in the region and returned to the Center where the coordination of the image of the radiation field produced on the film to the scribed rectangle is determined. The device also contains two precalibrated $\mathrm{Li}_{2} \mathrm{~B}_{4} \mathrm{O}_{7}: \mathrm{Mn}-\mathrm{Tef}$ lon dosimeters which are used to check the output of the machine.

Check lists have been designed for use by the local staff which define the parameters to be checked and their frequency. For example, a check list for an isocentric 4 MV machine requires a daily check on the accuracy of the optical distance indicator, a weekly check on the accuracy of sidelights and a monthly check on all safety interlocks. A copy of this form is returned to the center for review. The form employed is shown in the appendix.

\section{Information Flow \& Documentation}

Methods of documentation are extremely important in this field and the methods employed at the Center today are the result of considerable effort and time. While simple in concept, they are worthy of description here.

A central repository for all data pertaining to a therapy machine is maintained. This takes the form of a ring binder which is kept close to the therapy machine. An exact replica is maintained at the Center to ensure the application of uniform data in dose calculations. The format of these binders follows that described in Table III. We have not found it possible to formalize the information further than a narrative style report structured on the topics shown in the table. Except that as already discussed, the presentation of depth dose is formalized by the output format of the program Tarman and as in most repetitious tasks, a uniform pattern tends to be enforced in all sections. The Machine Log Section also contains forms for recording downtime on the unit and narrative reports by the maintenance engineer and the technicians on the machine operation. In summary, this approach produces a data base for use by the radiation oncologist, physicist, dosimetrist, technician and maintenance engineer and by this means, ensures uniform data and clear communication of information. The structure is logical and in our experience, it has proved easy to access information on the machine.

There exists a manual of operations describing all the services offered by the Center. With reference to the object of this paper, the manual contains a section dealing with the standards employed by the Center and this general statement makes it unnecessary to repeat in reports such items as the value of the displacement factor employed or the method used to determine shutter time.

The Center conducts annual workshops for participants to review the operation of the services provided by the Center and while these tend to emphasize the treatment planning services offered by the Center, there is discussion of other dosimetry procedures.

\section{Future Trends}

\section{Electron Beam Therapy}

There is currently only one center employing this modality but this is expected to expand in the coming years. The Center is developing the tools and techniques necessary to support this modality in the same rigorous fashion currently employed for megavoltage photon beam.

\section{Patient Information Systems}

While this topic is only obliquely related to the general subject of this paper, the Center is developing a system to automate the therapy record and thus obviate arithmetic errors that do occur in manual updates of treatment records. A development from this project will be a 
unified data base on radiation therapy in the Northwest.

\section{Conclusion}

The remote nature of the Center and its scale of operation have required us to formalize, in many aspects, the physics support of radiotherapy. It is a matter of semantics to decide whether a physicist is remote if he or she is in the next office, building, town or state from the therapy facility so that some of the techniques we have described should be universally applicable.

\section{Acknow ledgment}

We wish to express our appreciation to the Board of Directors of the Northwest Medical Physics Center for their constant and enthusiastic support of the activities of the Center.

\section{References}

1. Jones, D.: Five years activity of a regional physics program. Presented at the American College of Radiology Workshop on Current Trends in Radiotherapy, San Diego, December, 1974.

2. Task Group 10, P. Wootton, Chairman, AAPM: Code of practice for $x$-ray therapy linear accelerators. Medical Physics 2:110, 1975.

3. Medical x-ray and gamma ray protection for energies up to $10 \mathrm{MeV}$ : Equipment design and use. NCRP Report No. 33, 1968.

4. Bruels, M.C. and Hanson, W.F.: Off axis large field corrections for linacs. Medical Physics 2:150, 1975. (Abstract)

5. DeClemente, $A_{\text {. }}$, et al: The new Memorial Hospital external beam treatment planning program. Memorial Hosp., New York, New York, 1971.

6. Jones, D. and Washington, J.: The quantitative description of a radiation therapy plan. Radiology 115:451, 1975.

7. Jones, D., Gifford, J. and Washington, J.: On the use of fields of negative weight to simulate blocks in radiotherapy calculations. Int'l. J.Rad. Onc., Biol. \& Phys. In press.
8. Jones, D. and Schumacher, D.: An instrument for the rapid check of output, energy and field symmetry of supervoltage therapy equipment. Am. J. Roentgenol. 123:198, 1975.

9. Monitrex-100. Lawrence Soft Ray Corp. Mountain View, California.

10. Jones, D., Rubach, A. and Schumacher, D.: Variations in linear accelerator radiotherapy machine output. Medical Physics 2:160, 1975. (Abstract)

11. Jones, D.: A system for the frequent check by mail of the operation of radiotherapy machines. AAPM Quarterly Bulletin 7:100, 1973. (Abstract)

\section{Appendix}

The following forms are employed at the Center for the analysis of beam monitor readings and for checks made by the local staff. 
Machine I.D.:

Month and Year:

\section{DAILY CHECK LIST}

Optical Distance Indicator - Check accuracy at usual treatment distance and initial if within $\pm 3 \mathrm{~mm}$.

\begin{tabular}{|c|c|c|c|c|c|c|c|c|c|c|c|c|c|c|c|}
\hline Day & 1 & 2 & 3 & 14 & 15 & 6 & 7 & 8 & 9 & 10 & l 11 & 12 & 13 & 14 & 15 \\
\hline 16 & 17 & 18 & 19 & 20 & 21 & 22 & 23 & 24 & 25 & 26 & 27 & 28 & 29 & 30 & 31 \\
\hline
\end{tabular}

\section{WEEKLY CHECK LIST}

Sidelight Accuracy - Set up $4.5 \mathrm{~mm}$ diameter pinhead at isocenter. If sidelights impinge on sphere, initial and date.

Initial Initial Initial Initial

Da te Date

Date Date

\section{MONTHLY CHECK LIST}

Door Interlock - Check function and initial if o.k.

Emergency Off Switch - Check function and initial if o.k. $\frac{\text { Source On Indicator Lights - Check that all lights function }}{\text { and initial if } 0 . k \text {. }}$

Frequent Check Dosimeter - Expose NMPC frequent check dosimeter as per instructions on the first Wednesday of each month.

PLEASE MAKE COPIES OF THIS FORM FOR YOUR USE. RETURN ONE COMPLETED COPY TO THE NORTHWEST MEDICAL PHYSICS CENTER EACH MONTH AND RETAIN ORIGINAL IN BLUE BINDER. 
ANALYSIS OF BEAM MONITOR READINGS

Month of Jenuary

\title{
First Readings \\ Number of Readings \\ Average Reading
}

\begin{tabular}{|c|c|c|c|}
\hline \multicolumn{2}{|c|}{ A.M. } & \multicolumn{2}{c|}{ P.M. } \\
\hline Upper & Lower & Upper & Lower \\
\hline 18 & 18 & 18 & 18 \\
\hline 204 & 146 & 207 & 149 \\
\hline
\end{tabular}

\section{Second Readings}

Number of Readings

Average Reading

\begin{tabular}{|c|c|c|c|}
\hline \multicolumn{2}{|c|}{ A.M. } & \multicolumn{2}{c|}{ P.M. } \\
\hline Upper & Lower & Upper & Lower \\
\hline 18 & 18 & 18 & 18 \\
\hline 203 & 148 & 207 & 151 \\
\hline
\end{tabular}

DISTRIBUTION OF SECOND READINGS

Exact

$\pm 1 \%$ Bracket

$\pm 2 \%$ Bracket

$\pm 3 \%$ Bracket

$+4 \%$ Bracket

$\pm 5 \%$ Bracket

\begin{tabular}{|c|c|c|c|c|c|}
\hline Upper & \multicolumn{2}{|c|}{$\begin{array}{c}\text { Number of Readings } \\
\text { Inside Bracket }\end{array}$} & \multicolumn{2}{|c|}{ Lower } & \multicolumn{2}{|c|}{$\begin{array}{c}\text { uumber of Readings } \\
\text { Inside Bracket }\end{array}$} \\
\hline 209 & A.M. & P.M. & & A.M. & P.M. \\
\hline 211 To207 & 0 & 11 & 154 To 150 & 2 & 12 \\
\hline 213 To 205 & 8 & 14 & 155 To 149 & 4 & 5 \\
\hline 215 To 203 & 10 & 16 & 157 To 147 & 11 & 17 \\
\hline 217 To 201 & 13 & 18 & 158 To 146 & 15 & 18 \\
\hline 219 To 192 & 18 & 18 & 160 To 144 & 18 & 18 \\
\hline
\end{tabular}

ISOTOPIC SOURCE CHECK ON BEAM MONITOR

\author{
Upper \\ Lower \\ Average reading of the diode per rad \\ $1.95 \quad 2.03$
}

PLEASE MAKE COPIES OF THIS FORM FOR YOUR USE AND RETURN ONE

COMPLETED COPY TO THE NORTHWEST MEDICAL PHYSICS CENTER EACH MONTH 


\title{
INTERCOMPARISON OF PRESCRIPTION DOSE DELIVERY IN THE NEW ENGLAND REGION
}

J.S. Blinick* and J. Spira,

Boston University Medical Center

Boston, Massachusetts 02118

and

D. Cail and P. Plazeski, Boston City Hospital

Boston, Massachusetts 02118

\begin{abstract}
An intercomparison of radiation dosimetry among 16 institutions within the New England area was performed using a hand carried water phantom-ionization chamber system. Participants were asked to deliver 200 rads using their own beam data and computations to the chamber for each of eight field size and depth combinations. Average measured doses ranged from 200 to 204 rads. For all combinations, the standard deviation of the measured values was $3 \%$.
\end{abstract}

(Dosimetry; radiation; measurements; intercomparisons; supervoltage; therapy)

\section{Introduction}

Intercomparisons of radiation dosimetry are an important means of assuring accurate dose delivery to radiation therapy patients.

An informal intercomparison among several hospitals in Boston was conducted in 1973 using a handcarried water phantom-ionization chamber system. 1 The results of this pilot study indicated close agreement between the hospitals. However, some discrepancies were found that exceeded $5 \%$, and these were subsequently investigated and corrected by the institutions themselves.

On the basis of these results, it was felt that an expanded intercomparison would be of use to radiation physicists in the area. The New England Radiological Physics Organization (NERPO) agreed to sponsor the survey, and a total of 21 supervoltage units $\left(14{ }^{60}\right.$ Co units, 4 linear accelerators, and 3 betatrons) were measured at 16 different institutions in Maine, Massachusetts, New Hampshire, and Vermont.

\section{Procedure}

\section{Equipment}

Al1 measurements were made with a water phantom and ionization chamber system. The phantom measured $30 \times 30 \times 30 \mathrm{~cm}$. and, except for the front face, was made from lucite. The front face was made from polystyrene and was inscribed with lines outlining different field sizes. An adjustable lucite sleeve was used to hold a $0.6 \mathrm{cc}$ Farmer ionization chamber. The chamber and its electrometer were calibrated at ${ }^{60} \mathrm{Co}$ before, during, and after the study with a Shonka-type transfer standard chamber ${ }^{2}$ which in turn was calibrated by the National Bureau of Standards. Water for the phantom was obtained at each institution, and was adjusted whenever possible to the same temperature as the ambient air. The ambient pressure was measured with a portable aneroid barometer which was frequently compared to a standard mercury-in-glass barometer.

\section{Measurements}

The equipment was hand carried to each institution by one member of a three man measurement team. The phantom was filled with water and placed on the treatment table. It was then centered and adjusted to the usual source to surface distance (SSD) used by the institution by means of the devices normally employed by that institution. Once the setup was completed, and the 
water and chamber temperature stabilized, the participants were asked to deliver $200 \mathrm{rads}$ to the chamber for each of eight different field size and depth combinations. A minimum of two readings were taken for each combination. If these readings did not agree to within the reading error of the instrument, a sufficient number of additional readings were taken to obtain a meaningful average. The water temperature and ambient pressure were measured before and after the series of ionization measurements.

A novel feature of this study was the presence at each trial of an impartial observer chosen from among the members of the NERPO radiation therapy physics committee. ${ }^{3}$ These "inspectors" provided an extra check of the measurement setup, and helped prevent any bias on the part of the members of the measurement team from affecting the results. No committee member served as an inspector at his own institution.

Calculations

The ionization chamber readings were converted to absorbed dose by means of the standard equation ${ }^{4}$

$$
\mathrm{D}=\mathrm{RNC}_{\mathrm{tp}} \mathrm{C}_{\lambda}
$$

where $\mathrm{D}$ is the absorbed dose in rads, $R$ is the chamber reading, $N$ is the chamber calibration factor for ${ }^{60} \mathrm{Co}$, $\mathrm{C}_{\text {tp }}$ is the temperature-pressure correction factor, and $\mathrm{C}_{\lambda}$ is the over-

al1 correction factor.

Values for $\mathrm{C}_{\lambda}$ were obtained from a tabulation in Report 14 of the ICRU. ${ }^{5}$ A value of 0.87 , obtained by extrapolation of these tables, was used for the $42 \mathrm{MeV}$ and $45 \mathrm{MeV}$ betatrons.

Separate correction factors were calculated for the temperatures and pressures measured before and after the ionization measurements. The average of these factors was then used in Eq. (1).

\section{Reports}

Reports indicating the measured dose values for each field size and depth combination and the percentage difference of these values from the nominal prescribed dose of 200 rads were sent to the physicist affiliated with each institution shortly after the measurements at that institution were completed. In addition, at the conclusion of the survey, a summary of the results was also sent to each physicist to allow him to compare his results with the average values.

\section{Results}

Frequency distributions summarizing the results of the survey are shown in Fig. 1. It can be seen that for most of the field size and depth combinations, the majority of the measured dose values fall within the range of $196-204$ rads $( \pm 2 \%$ of the nominal prescribed dose). However, some values were obtained well outside this range. The lowest reading was $184 \mathrm{rads}$, and the highest $216 \mathrm{rads}$, both of which represent an $8 \%$ deviation from the prescribed dose. Table I shows the average measured dose value and standard deviation for each field size and depth combination. The average measured dose values should not be given too much significance. It may be noted that a reduction of $1 \%$ for the chamber calibration factor or $\mathrm{C}_{\lambda}$ would bring the average measured values even closer to the nominal prescribed dose.

A more relevant parameter for the purpose of intercomparison is the standard deviation. For all field size and depth combinations, the value for the standard deviation was about $3 \%$. These results are comparable to those obtained by the Radiological Physics Center in their study. ${ }^{6}$ The data indicate that the uniformity of basic dosimetry within the region is quite good considering the diversity of machines and institutions surveyed. 
FIGURE I. FREQUENCY OF MEASURED DOSE VALUES
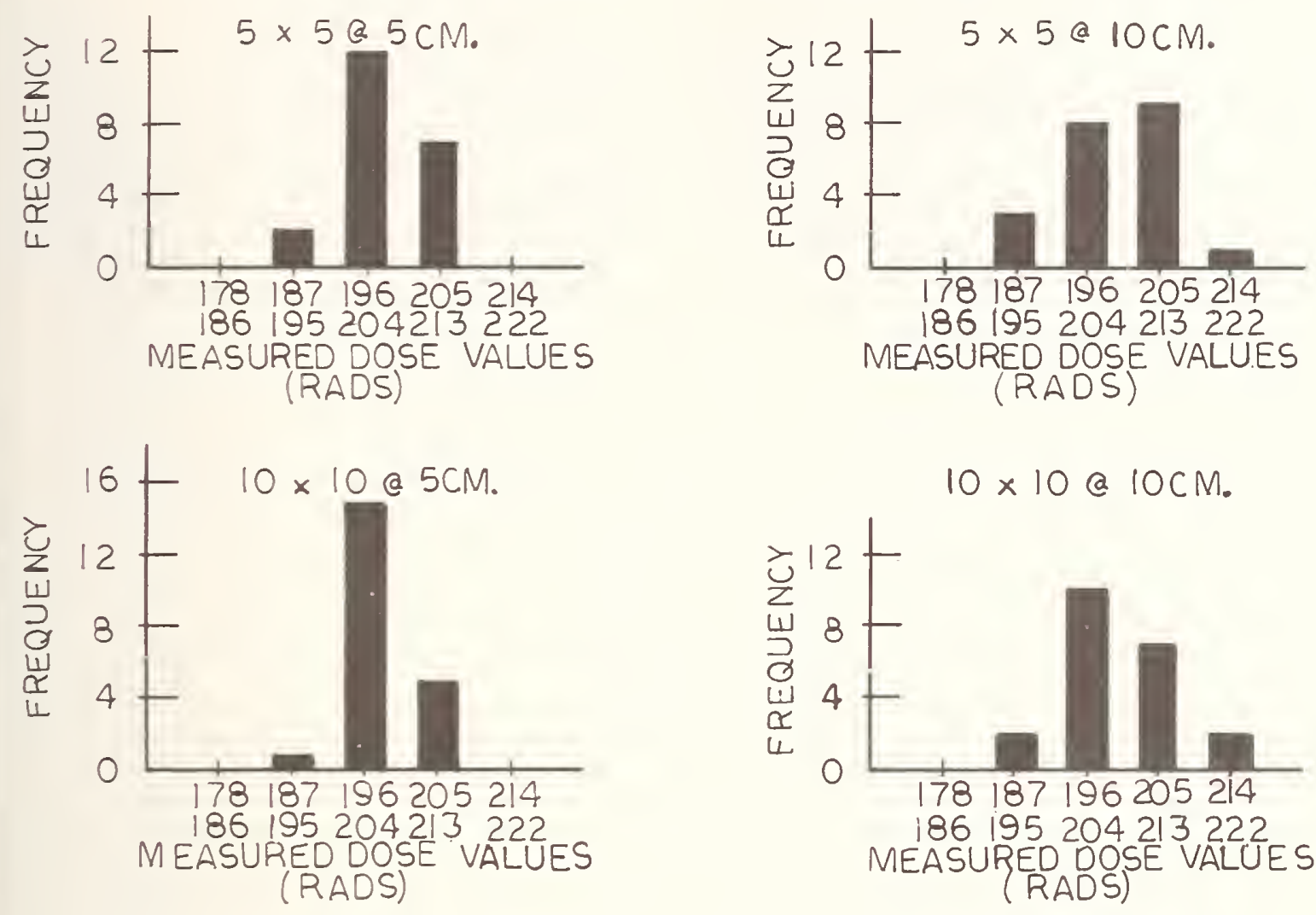

$10 \times 10 @ 10 \mathrm{~cm}$.
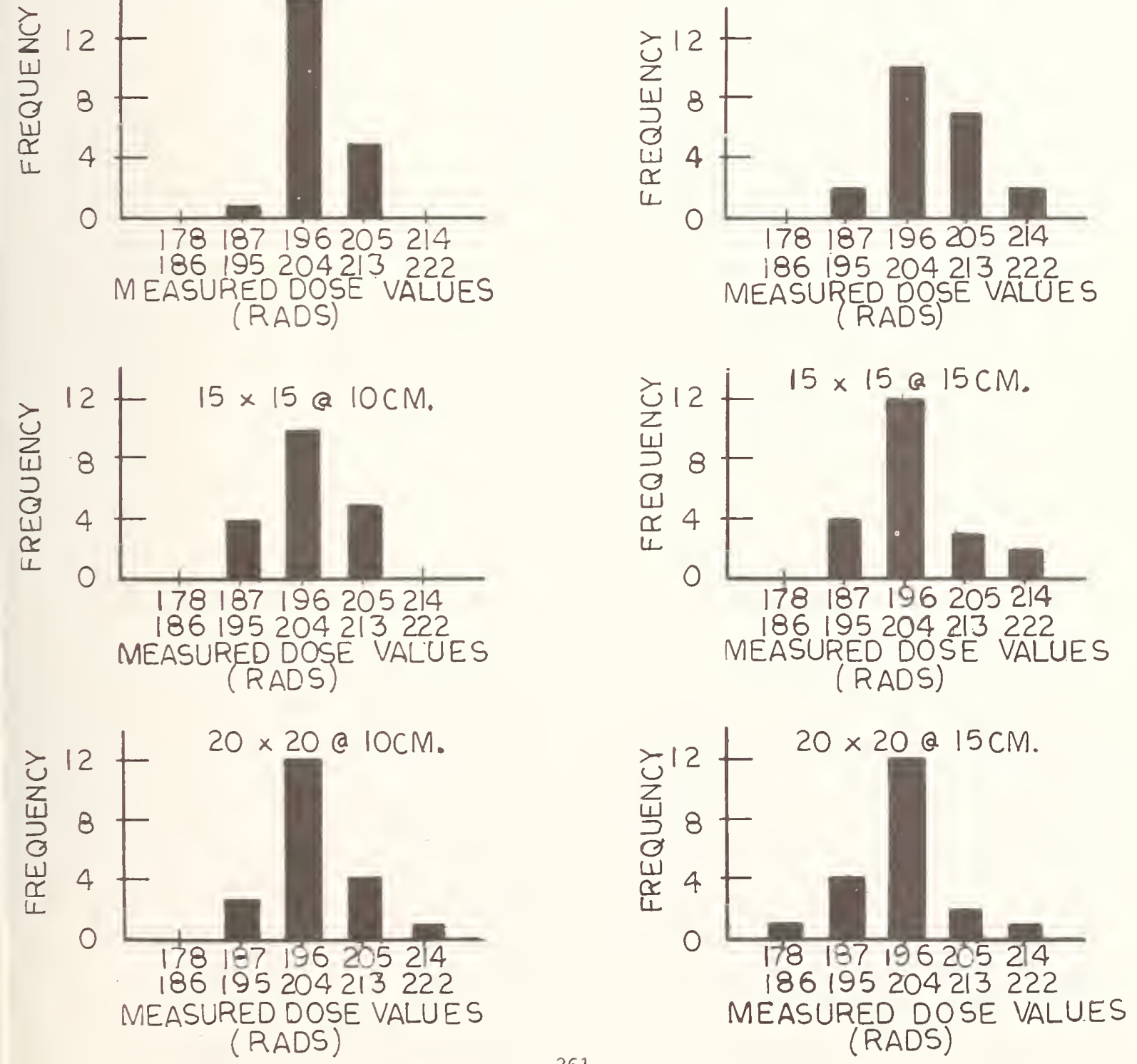
Table II shows a comparison of the results for ${ }^{60}$ Co units at large institutions (those having one or more full-time physicists) and small institutions (those having only parttime or consultant physics services). Only the data for a $10 \times 10 \mathrm{~cm}$. field at $10 \mathrm{~cm}$. depth is presented, but the results were essentially the same for all combinations. It can be seen in this study that 1 , the average measured dose is closer to the nominal value, and 2 , the standard deviation is less for the large institutions.

It should be emphasized that irradiating a water phantom is not the same as irradiating a patient. Factors such as technologist error, setup reproducibility, and proper chart maintenance have not been considered in this study. Nevertheless basic dosimetry, as evaluated in this study, is fundamental to the accurate treatment of radiation therapy patients.

Table I

Measured Doses - Average and Standard Deviation

$\begin{array}{rrrr}\begin{array}{c}\text { Field Size } \\ (\mathrm{cm} \times \mathrm{cm})\end{array} & \begin{array}{c}\text { Depth } \\ (\mathrm{cm})\end{array} & \begin{array}{c}\text { Average Value } \\ (\text { rads })\end{array} & \begin{array}{c}\text { Std. } \\ \text { Deviation } \\ (\%)\end{array} \\ 5 \times 5 \times 5 & 5 & 202 & 2.8 \\ 5 \times 10 \times 10 & 10 & 203 & 2.8 \\ 10 \times 10 & 5 & 202 & 2.5 \\ 15 \times 15 & 10 & 204 & 2.9 \\ 15 \times 15 & 10 & 202 & 3.0 \\ 20 \times 20 & 15 & 202 & 3.2 \\ 20 \times 20 & 10 & 202 & 3.0 \\ & 15 & 200 & 3.5\end{array}$

Table II

Measured Doses - Average and Standard Deviation ${ }^{60} \mathrm{Co} 10 \times 10 \mathrm{~cm}$. Field at $10 \mathrm{~cm}$. Depth

$$
\begin{gathered}
\text { Average Value } \\
\text { (rads) }
\end{gathered}
$$

Std. Deviation

204

210

$(\%)$

1.6

2.5

\section{Large Institutions (8) \\ Small Institutions (6) \\ Summary}

Measurements using a handcarried water phantom-ionization chamber system were made on 21 supervoltage units at 16 institutions.

Eight different field size and depth combinations were examined. For all combinations, the average measured dose was within $2 \%$ of the nominal prescribed dose. Standard deviations of the measured doses were about $3 \%$, indicating good uniformity of dose delivery at the institutions studied.

\section{Acknowledgments}

The authors would like to thank the members of the NERPO therapy physics committee who voluntarily provided their time and expertise to act as inspectors. The cooperation and hospitality of the participating institutions are also greatly appreciated. We would like to thank Dr. Edward S. Sternick for his assistance in the statistical evaluation of the data. 


\section{References}

*Present address: Maine Medical Center, Portland, Maine 04102

1. J. Spira and D. Cail, unpublished

2. Obtained from Physical Sciences Laboratory, Illinois Benedictine College, Lisle, Illinois 60532

3. Members of the committee at the time of this survey were
J. Spira, Chm.
Boston, MA
F. Bagne,
Hanover, N.H.
A. Boyer,
Boston, MA
A. Pinkerton,
Manchester, N.H.
D. Reinhard,
G. Svensson,
K. Wright,
Boston, MA
Boston, MA
Boston, MA

4. H. E. Johns and J.R. Cunningham, The Physics of Radiology, (3rd ed. Charles C. Thomas, Springfield Illinois, 1969), p. 288.

5. "Radiation Dosimetry: X-Rays and Gamma Rays with Maximum Photon Energies Between 0.6 and $50 \mathrm{MeV}^{\prime \prime}$ ICRU Report 14 (1969).

6. W. Grant, III, Medical Physics 1 , 41 (1974). 

M. Ehrlich and P. J. Lamperti

National Bureau of Standards

Washington, D.C. 20234

\begin{abstract}
A service for checking the dosimetry of electron beams in the energy range from 5 to $50 \mathrm{MeV}$ was established in 1967, mainly for use by radiation-therapy departments. Since then, 34 radiation-therapy departments and several other groups have participated, a relatively large number of them only intermittently or for a total of only a few tests. There has been 1 ittle improvement of overall performance over the years, less than one-half of the total number of irradiated dosimeters involved in any one test showing agreement to within 5 percent between the dose reported by the participants and the NBS dose interpretation, and up to one-quarter of the dosimeters showing disagreements of more than 10 percent. Only in a few exceptional cases was there an improvement in individual performance with continuing participation. It is concluded that means must be sought, in collaboration with the therapy community, to secure a more favorable impact of this service on electron-therapy dosimetry.
\end{abstract}

(Electrons; dosimetry; service; radiation therapy; performance; impact; improvement)

\section{Description of Service}

Since 1967, the National Bureau of Standards (NBS) has been providing a service for testing the status of dosimetry for electron beams $i r_{1}$ the energy range from about 5 to $50 \mathrm{MeV}$. The service, which is directed mainly toward radiation-therapy applications in the United States, was established upon the request of the American Association of Physicists in Medicine (AAPM), with the initial financial assistance of the Bureau of Radiological Health (BRH). Ferrous-sulfate (Fricke) dosimeters, prepared and checked at NBS by means of cobalt-60 pre-irradiation, are shipped to the participating institutions with the request to irradiate them in a polystyrene phantom, using the dosimeter depth and the phantom and field sizes specified by the AAPM. ${ }^{1}$

Figure 1 shows the dosimeter assembly, consisting of Fricke solution in a stoppered spectrophotometer cell, cradled in a polystyrene block. Each participant receives two control dosimeters and at least two dosimeters to each of which he is asked to deliver between 50 and 80 Gy (5000 and 8000 rad). After irradiation, the dosimeters are returned to NBS for evaluation of their response in terms of absorbed dose in water. Finally, the participants are informed of their own test results and those of all other participants, whose identity, however, is not divulged. They then are free to discuss their results and possible problems with NBS. Technical details on dosimeter preparation and on the pre-irradiation checks have been published. ${ }^{2}$
Figure 1. Dosimetry Assembly

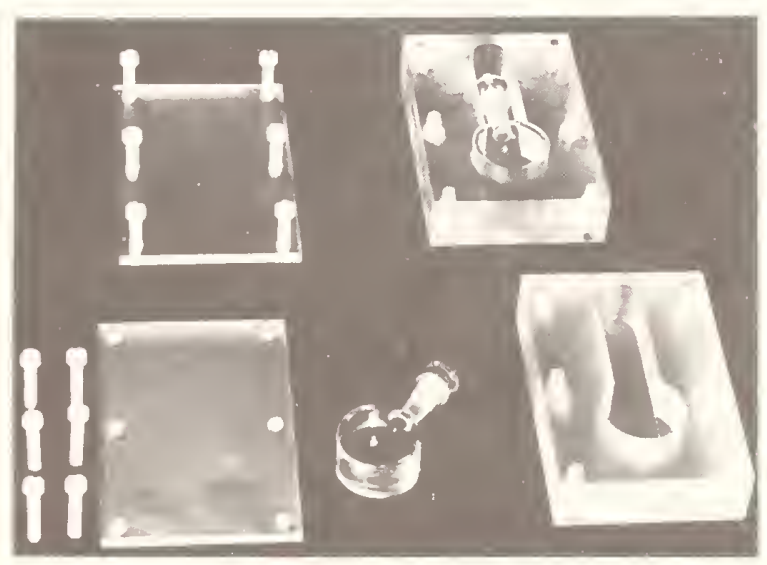

\section{Inherent Uncertainties}

Irradiation of each dosimeter batch prior to shipment permits (1) a statistical study of the random variations from dosimeter to dosimeter in the response to a given irradiation level, as measured spectrophotometrically by the change in optical density; and (2) the detection and elimination from further use of dosimeters with responses far removed from the mean for the particular batch. Furthermore, it permits the evaluation of the absorbed doses administered by the participants from higher (more precise) optical densities. Both before and after shipment, the relative standard deviation from the mean response, which is a measure 
of the random uncertainty of the method, is about 1.2 percent for the currently used dosimeters.

The overall uncertainty of the method was determined by irradiating batches of dosimeters at two dose levels, shipping them cross-country and back as if they had been irradiated by participants, and evaluating their response in terms of absorbed dose in the usual manner. The difference between actual dose delivered to the dosimeters and dose evaluation from dosimeter response, which is taken to be a measure of the total uncertainty of the method, was found to be about 4 percent at the 99-percent confidence level. Not considered are uncertainties (1) due to the assumption of a constant efficiency (a G value of $15.5 / 100 \mathrm{eV}$, as recommended by the AAPM ${ }^{1}$ ) of the Fricke-dosimetry system over the electron-energy range from 5 to $50 \mathrm{MeV}$, and (2) due to inhomogeneities in absorbed dose over the 1-cm depth of the dosimeter's sensitive volume. Thus, the service does not provide a beam calibration, but simply a check on the uniformity of electron dosimetry throughout the United States at a given electron energy. However, since the participants are informed of the details of the NBS evaluation procedure, they are in a position to derive information about their beam calibration from the supplied test results.

\section{The Participating Therapy Departments}

During its first year of operation, the service was subsidized by BRH and thus could be offered quarterly and at a fraction of actual cost. After this period, the frequency was reduced to twice yearly, and the charges were increased to cover the actual cost of the service. During the past nine years, a total of about 40 groups have participated. Of these, 34 are therapy users of electron beams, two use their beams for biological research and development, one is a food processor. On a few occasions, some non-medical groups desiring cobalt-60 gamma irradiations have been accepted. Some of the groups originally participating have dropped out while others joined later. However, ten of the original groups are still participating.

Table 1 gives a survey of the machines used by the current participants who employ their beams for therapy. There are 18 users of betatrons in the energy range from 5 to $45 \mathrm{MeV}$ and 9 users of 1 inear accelerators, in the energy range from 6 to $50 \mathrm{MeV}$.

Table 1. MACHINES USED BY CURRENT PARTICIPANTS

\begin{tabular}{|c|c|c|c|c|c|}
\hline $\begin{array}{l}\text { Type and } \\
\text { Maximum } \\
\text { Energy (MeV) }\end{array}$ & Manufacturer & Number & $\begin{array}{r}\text { Number } \\
<10 \mathrm{MeV}\end{array}$ & $\begin{array}{l}\text { sed in Energy } \\
10-30 \mathrm{MeV}\end{array}$ & $\begin{array}{l}\text { Range } \\
>30 \mathrm{MeV}\end{array}$ \\
\hline \multirow{6}{*}{ BETATRON, } & Applied Rad. (Siemens) & 1 & 1 & 1 & -- \\
\hline & Siemens & 2 & 2 & 2 & -- \\
\hline & ATC (Allis-Chalmers) & 1 & 1 & 1 & -- \\
\hline & ATC (Allis-Chalmers) & 11 & 8 & 11 & -- \\
\hline & Siemens & 1 & 1 & 1 & -- \\
\hline & Brown-Boveri & 2 & 2 & 2 & 2 \\
\hline \multirow[t]{6}{*}{ LINAC, } & Varian & 2 & 2 & -- & -- \\
\hline & Varian & 2 & 2 & -- & -- \\
\hline & Applied Rad. (Siemens) & 1 & 1 & 1 & -- \\
\hline & Varian & 2 & 2 & 2 & -- \\
\hline & Non-commercial & 1 & 1 & 1 & 1 \\
\hline & HV Engineering-Varian & 1 & - & 1 & -- \\
\hline
\end{tabular}




\section{Performance}

Figure 2 gives a review of the performance of a 11 therapy participants in each of the nine years of operation of the service, indicating the deviations between NBS dose interpretation and the participants' reported dose, as well as the energies of the electron beams employed. It is evident that some of the current users of $6-\mathrm{MeV}$ beams do not take into account that the average absorbed dose in the sensitive volume of the dosimeters is of the order of 10 percent smaller than the dose at the peak of the 6-"eV depth-dose curve. During the past year a statement of caution regarding this effect was mailed to the participants involved.

The results shown in Fig. 2 are reviewed numerically in Table 2, in which the number of participants also is broken down according to whether or not they had participated before. On the whole, the performance is seen to have improved only very little over the years, up to one-half of the total number of dosimeters having interpretations that differ from the reported dose by more than 5 percent, with differences of more than 10 percent occurring in up to onefourth of all dosimeters.

\section{Examples of Individual Performance}

Further insight is gained through a detailed study of the performance of individual groups. In Table 3 , the performance of Group I is seen to have remained essentially unchanged throughout the years, agreement to within 5 percent occurring about 70 percent of the time. Group II is better, with about 80 percent of the dose interpretations agreeing to within 5 percent. Group III is seen to have improved, showing no disagreement larger than 5 percent over the last five years. The performance of Group IV remains poor, less than one-half of its reported doses being within 5 percent of the NBS dose interpretation, and as many differing by more than 10 percent. Group V shows some improvement.

Groups VI and VII are included here because there is evidence that participation

\section{Table 2. PERFORMANCE OF ALL PARTICIPANTS}

\begin{tabular}{|c|c|c|c|c|c|c|c|c|}
\hline \multirow{3}{*}{$\begin{array}{l}\text { Calendar } \\
\text { Year }\end{array}$} & \multirow{2}{*}{\multicolumn{2}{|c|}{$\begin{array}{l}\text { Number of } \\
\text { Participants }\end{array}$}} & \multicolumn{6}{|c|}{ Number of Dosimeters with Difference $\Delta^{*}$ of } \\
\hline & & & $|\Delta|$ & $5 \%$ & $5 \%<1 \Delta$ & $\leq 10 \%$ & $|\Delta|>$ & $10 \%$ \\
\hline & $01 d$ & New & absolute & $\begin{array}{c}\text { relative } \\
(\%)\end{array}$ & absolute & $\begin{array}{c}\underset{(\%)}{\operatorname{relative}} \\
\end{array}$ & absolute & $\begin{array}{c}\text { relative } \\
(\%)\end{array}$ \\
\hline 1967 & 0 & 14 & 33 & 66 & 14 & 28 & 3 & 6 \\
\hline 1968 & 10 & 2 & 39 & 48 & 24 & 30 & 18 & 22 \\
\hline 1969 & 10 & 5 & 25 & 45 & 16 & 29 & 14 & 25 \\
\hline 1970 & 12 & 3 & 34 & 56 & 12 & 20 & 15 & 25 \\
\hline 1971 & 17 & 0 & 30 & 52 & 13 & 22 & 15 & 26 \\
\hline 1972 & 13 & 2 & 35 & 74 & 5 & 11 & 7 & 15 \\
\hline 1973 & 17 & 2 & 38 & 63 & 9 & 15 & 13 & 22 \\
\hline 1974 & 12 & 2 & 38 & 78 & 6 & 12 & 5 & 10 \\
\hline 1975 & 17 & 3 & 38 & 51 & 24 & 32 & 12 & 16 \\
\hline
\end{tabular}

*The symbol $\Delta$ stands for the difference between the absorbed dose reported and the NBS absorbed-dose assignment. 
Figure 2.

PERFORMANCE OF ALL PARTICIPANTS IN EACH OF 9 YEARS OF SERVICE

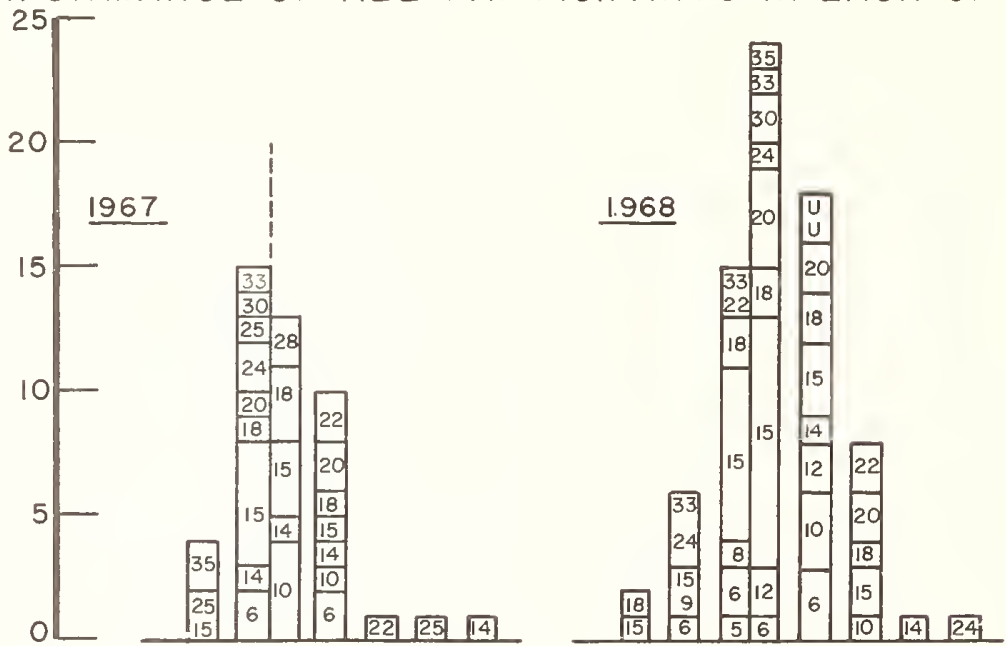

969
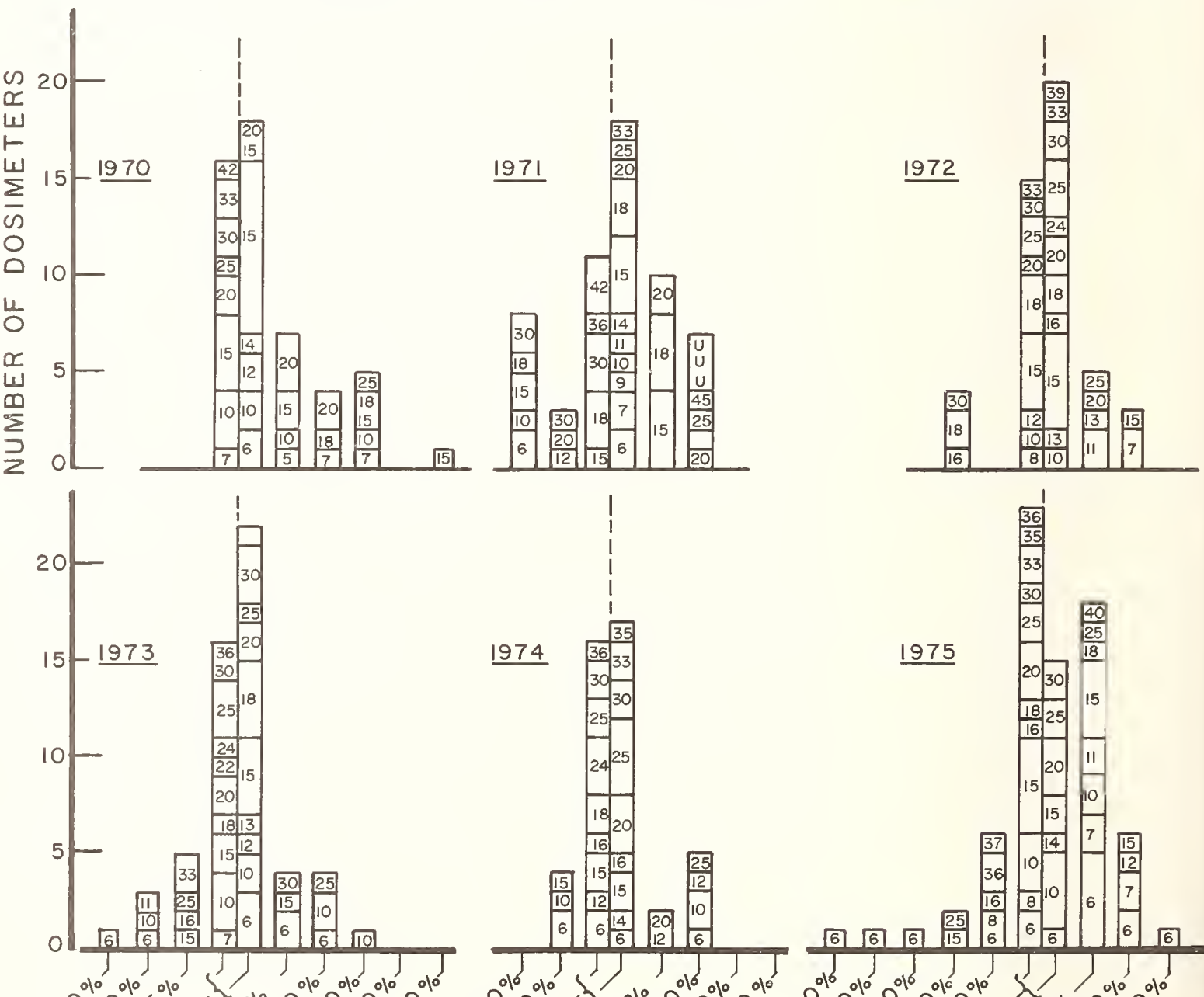

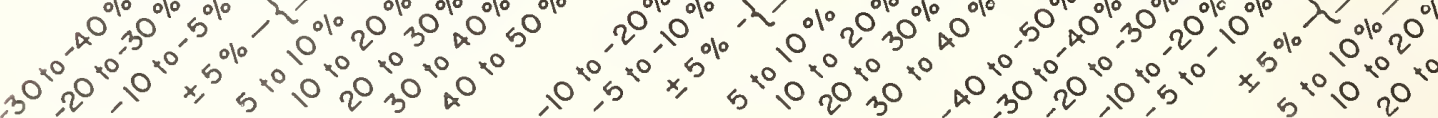


Table 3. FOLLOW-UP ON PERFORMANCE OF INDIVIDUAL GROUPS

\begin{tabular}{|c|c|c|c|c|c|c|c|c|c|c|c|}
\hline \multirow{2}{*}{$\begin{array}{l}\text { Group } \\
\text { No. }\end{array}$} & \multirow{2}{*}{$\Delta^{\star}$} & \multicolumn{9}{|c|}{ Number of Dosimeters in a Calendar Year } & \multirow{2}{*}{$\begin{array}{l}\text { Percentage of } \\
\text { Total Number } \\
\text { Per Group }\end{array}$} \\
\hline & & 1967 & 1968 & 1969 & 1970 & 1971 & 1972 & 1973 & 1974 & 1975 & \\
\hline \multirow{3}{*}{ I } & $|\Delta| \leq 5 \%$ & 2 & 4 & 1 & 3 & 2 & 3 & 3 & 4 & 3 & 66 \\
\hline & $5 \%<\mid \Delta ! \leq 10 \%$ & 0 & 1 & 0 & 0 & 1 & 0 & 1 & 0 & 1 & 11 \\
\hline & $|\Delta|>10 \%$ & 0 & 1 & 4 & 0 & 2 & 1 & 0 & 0 & 1 & 24 \\
\hline \multirow{3}{*}{ I I } & $|\Delta| \leq 5 \%$ & 2 & 5 & 2 & 4 & 4 & 4 & 1 & 4 & 4 & 81 \\
\hline & $5 \%<|\Delta| \leq 10 \%$ & 0 & 1 & 2 & 0 & 0 & 0 & 3 & 0 & 0 & .16 \\
\hline & $|\Delta|>10 \%$ & 0 & 0 & 0 & 1 & 0 & 0 & 0 & 0 & 0 & 3 \\
\hline \multirow{3}{*}{ I I I } & $|\Delta| \leq 5 \%$ & 3 & 1 & 2 & 2 & 2 & 2 & 3 & 4 & 2 & 78 \\
\hline & $5 \%<|\Delta| \leq 10 \%$ & 1 & 3 & 2 & 0 & 0 & 0 & 0 & 0 & 0 & 22 \\
\hline & $|\Delta|>10 \%$ & 0 & 0 & 0 & 0 & 0 & 0 & 0 & 0 & 0 & \\
\hline \multirow{3}{*}{ IV } & $|\Delta| \leq 5 \%$ & & 1 & 1 & 5 & 0 & 2 & 2 & 2 & 0 & 42 \\
\hline & $5 \%<|\Delta| \leq 10 \%$ & & 0 & 2 & 1 & 0 & 0 & 0 & 1 & 1 & 16 \\
\hline & $|\Delta|>10 \%$ & & 3 & 0 & 2 & 2 & 0 & 2 & 1 & 3 & 42 \\
\hline \multirow{3}{*}{ v } & $|\Delta| \leq 5 \%$ & 1 & 0 & 0 & 1 & 0 & 2 & 4 & 2 & 3 & 38 \\
\hline & $5 \%<|\Delta| \leq 10 \%$ & 1 & 2 & 1 & 2 & 3 & 2 & 0 & 2 & 0 & 38 \\
\hline & $|\Delta|>10 \%$ & 0 & 2 & 3 & 1 & 1 & 0 & 0 & 0 & 1. & 24 \\
\hline \multirow{3}{*}{ VI } & $|\Delta| \leq 5 \%$ & 2 & 0 & 0 & 1 & 2 & 2 & 1 & 1 & 2 & 50 \\
\hline & $5 \%<|\Delta| \leq 10 \%$ & 2 & 3 & 1 & 1 & 0 & 0 & 1 & 1 & 0 & 41 \\
\hline & $|\Delta|>10 \%$ & 0 & 1 & 1 & 0 & 0 & 0 & 0 & 0 & 0 & 9 \\
\hline \multirow{3}{*}{ VII } & $|\Delta| \leq 5 \%$ & 2 & & & 3 & 4 & & & & 1 & 63 \\
\hline & $5 \%<|\Delta| \leq 10 \%$ & 1 & & & 1 & 0 & & & & 2 & 25 \\
\hline & $|\Delta|>10 \%$ & 1 & & & 0 & 0 & & & & 1 & 13 \\
\hline \multirow{3}{*}{ VIII } & $|\Delta| \leq 5 \%$ & & & 4 & 0 & 0 & & & & & 29 \\
\hline & $5 \%<|\Delta| \leq 10 \%$ & & & 2 & 0 & 1 & & & & & 21 \\
\hline & $|\Delta|>10 \%$ & & & 3 & 3 & 1 & & & & & 50 \\
\hline
\end{tabular}

*The symbol $\triangle$ stands for the difference between the absorbed dose reported and the NBS absorbed-dose assignment.

assisted them in finding and removing certain difficulties in their operation of which they had not been aware prior to their participation in the service. For instance, Group VI discovered a faulty integrator, while the monitor chamber of Group VII was found to heat up and thus show an increase in sensitivity during irradiation. Unfortunately, Group VII seems to be unable to afford regular participation, and after a 3-year interruption now performs as poorly as it did in 1967. Group VIII is an example of a participant who felt that his system was not made to perform over the long exposure times required for the test irradiations. Therefore, after three years of poor performance, he dropped out, declaring that he would consider resuming participation when a more sensitive dosimetry system becomes available. 


\section{Conclusions}

After nine years of operation of the NBS electron-dosimetry uniformity service, it seems that the impact of the service on the therapy community does not match the effort expended. Participation is relatively poor and irregular, and improvement in performance is an exception instead of the rule. Therefore it was decided to initiate an active dialogue with the therapy community, in an effort to explore (1) means for a more personal interchange with the participants and for increasing participation; and (2) the priority to be given to a change-over to a more sensitive dosimetry system, and inclusion of high-energy bremsstrahlung beams. It is hoped that this presentation will assist in stimulating such a dialogue.

\section{References}

1- The Subcommittee on Radiation Dosimetry (SCRAD) of the AAPM, Phys. Med. Biol. 11, 505 (1966).

2- M. Ehrlich, P. J. Lamperti, Phys. Med. Biol. 14, 305 (1969). 
THYROID MONITORING AND MINIMIZING I-125 UPTAKE

M. M. Gabel, K. W. Price and G. R. Holeman

Health Physics Division

Yale University

1136 Kline Biology Tower

219 Prospect Street

New Haven, Connecticut 06520

A method is described for the quantitative determination of I-125 uptake by the thyroid gland of all users of I-125. Causes for uptake are discussed and the need for monitoring is illustrated.

(Thyroid monitoring; I-125 uptake; bioassay)

\section{Introduction}

The rapidly increasing use of $I-125$ labeled compounds in biomedical research has presented several radiation protection problems. In order to keep exposures to I-125 to "as low as practicable," Yale has included in its Cancer Center a radioisotope facility, intended as a safe, supervised, multi-use research laboratory. One of the first uses of the facility was for iodination experiments. The control program for I-125 usage has evolved with time and has become rather complex. The following information summarizes some of our experiences with I-125, its control and methods of dose assessment.

\section{Materials and Methods}

\section{I-125 in the Thyroid}

To measure the amount of radioactive material which has accumulated in the thyroid, a detector can be placed over the neck in the region of the thyroid. Equipment which has been used for thyroid monitoring is as follows: $31 / 32^{\prime \prime} \times 1 / 32^{\prime \prime}$ NaI crystal, high voltage power supply, preamplifier, amplifier, 100 channel analyzer and printer.

The count rate obtained from the detector is related to the amount of activity in the thyroid by calibration. The method of calibration includes the use of an anthropomorphic phantom filled with water, representing the thyroid of an average man. The thyroid cavity of the phantom will hold a calibrated I-125 source.

The standardized I-125 source in the range of five microcuries is counted for four minutes. A four-minute background count is also made by removing the source from the phantom. In order to maximize counting statistics, the optimum channel width must be obtained. This may be found by adding the counts in successively increasing channels on either side of the peak channel (see

Table I). The ratio of the counts in $\pm X$ channels inclusive of the peak channel, and the corresponding background counts may be plotted against the number of channels on either side of the peak (see Figure I). The number of channels on either side of the peak channel, yielding the highest value, determines the optimum channel width.

Once the channel width has been established, system efficiency may be measured. This is the ratio of the gamma rays which interact in the crystal to the total gamma rays emitted by the source or

$$
E=\frac{c p m \text { in optimum channel width }}{\text { actual activity in dpm }}
$$

A minimum detectable level of activity present in a four-minute count at a $95 \%$ confidence level may now be found using the following equation:

$$
Y=\frac{K}{2} \pm \frac{t_{G}}{2} \sqrt{\frac{K^{2}}{t_{G}^{2}}+\frac{4 t_{B}}{t_{G}}+\frac{4 K_{B}}{t_{G}}}
$$

where: $t_{G}=$ sample count time

$$
\begin{aligned}
t_{B}= & c p m, B k g \\
\sigma_{B}= & \text { standard deviation Bkg } \\
Y= & \bar{x}_{G} \text { gross counts needed } \\
K= & \text { number of standard deviations } \\
& \text { for } X \% \text { accuracy; } K=2 \text { for } 95 \% \\
Y= & \text { number of counts to be accumu- } \\
& \text { lated in time } t_{G}{ }^{\circ}
\end{aligned}
$$

The Minimum Detectable Activity may then be defined as 


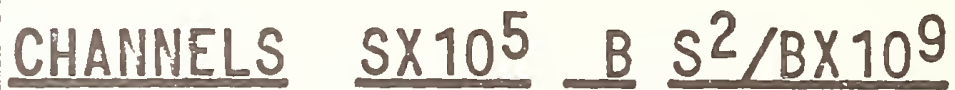$$
\begin{array}{llll} 
\pm 2 & 1.44 & 9 & 2.3
\end{array}
$$

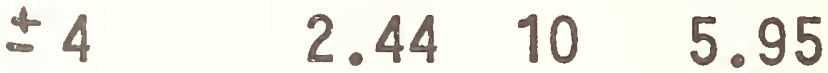

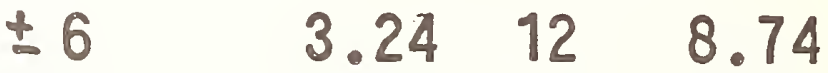$$
\begin{array}{llll} 
\pm 8 & 3.83 & 16 & 9.14
\end{array}
$$$$
\pm 10 \quad 4.22 \quad 20 \quad 8.9
$$$$
\pm 12 \quad 4.48 \quad 24 \quad 8.36
$$

$$
M D A=\left(\frac{\frac{Y^{2}}{4}-t_{B}}{E}\right)_{C}
$$

where: $M D A=$ minimum detectable activity in 4-minute count at a $95 \%$ confidence level

$$
\begin{aligned}
Y= & \text { number of counts to be accu- } \\
& \text { mulated in time } t_{G} \\
t_{G}= & \text { sample count time } \\
t_{B}= & \text { Bkg cpm } \\
E= & \text { system efficiency, } 1.2 \% \\
C= & \text { conversion constant, } \\
& 4.5 \times 10^{-7} \frac{\mathrm{dpm}}{\mu \mathrm{Ci}}
\end{aligned}
$$

The system is checked weekly with the calibrated I-125 source for accuracy and stability. To date, the system has remained consistent.

$$
\text { Sixty-eight individuals working with }
$$
I-125 were given thyroid counts utilizing the equipment described above. Iodine 125 concentration in the thyroid was measured by placing the probe in the region of the thyroid, between the laryngeal prominence and the manubrium. A standard value for background was obtained by counting the thyroids of 15 individuals not exposed to I-125. The results of these measurements yielded a consistent value for these control individuals. The background counts per minute are then subtracted from the gross counts per minute of the individual being counted.

Questions may arise as to the reproducibility of positioning the probe in successive counts. This was measured experimentally by altering the normal counting geometry. The source was placed at various distances about the detector probe. The count at each distance was taken as a percentage of the total counts at the normal counting geometry. The results are shown graphically in Figure II. It can be seen that the best results are obtained when the probe is flush against the object being counted. A concentric deviation of more than 1/2" from the center of the probe, in the flush position, will result in a greater 
Figure I

1-125 PEAK AT $~ 636 \mathrm{KEV/CHANNEL}$ FOR 4 MINUTE COUNT

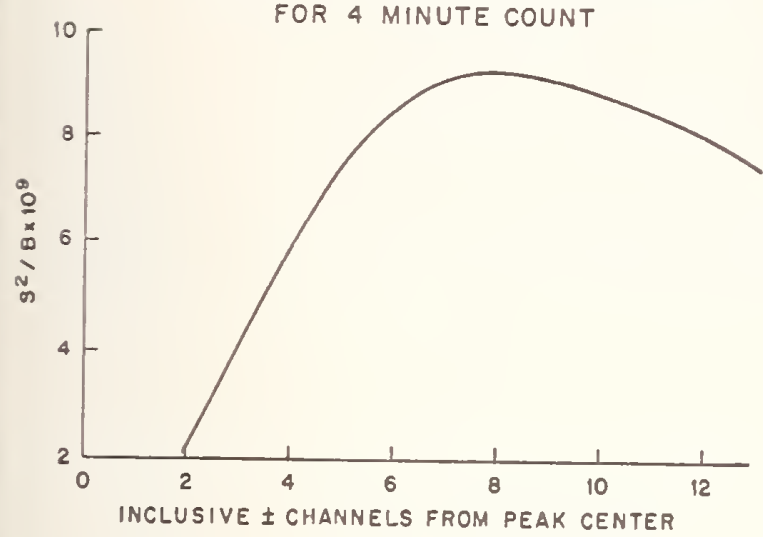

than $15 \%$ error.

I-125 in the Urine

In addition to the analysis of individual thyroids using the thyroid monitor, a urine sampling program was initiated for the determination of I-125 whole body content. The objectives of the urine sampling program were (a) to try to correlate urine sample activities with actual measurements of thyroid I-125 content, and (b) to try to determine if a urine sampling procedure is sufficient for routine screening for I-125 uptakes. Therefore, in instances where a thyroid count indicated activity in the organ, a urine sample was taken immediately afterwards. Urine samples were analyzed utilizing a liquid scintillation spectrometer and urine sample activity in $\mu$ Ci/liter was determined.

For an in-house program for urine anal$y s i s$, one wishes to reduce the time involved per sample and at the same time have an acceptable sensitivity for detecting the isotope in question. Instagel was chosen as a counting medium for all urine samples. The anount of urine added to the Instagel was determined by pipetting several different volumes of samples into $10 \mathrm{ml}$ of gel. This procedure was repeated for 20 different urine samples. The resultant cocktail mixture was inspected visually for gelling quality. If too little urine was present in the sample, a gelling did not occur. When an excess of urine was added to the gel, a precipitate formed. It was determined that the best mixture was $3 \mathrm{ml}$ of urine and $10 \mathrm{ml}$ of gel. This proportion always provided an acceptable sample that gelled and was not severely quenched. Although this mixture does not always yield an optimum quenching condition, the amount of time saved is
Figure II

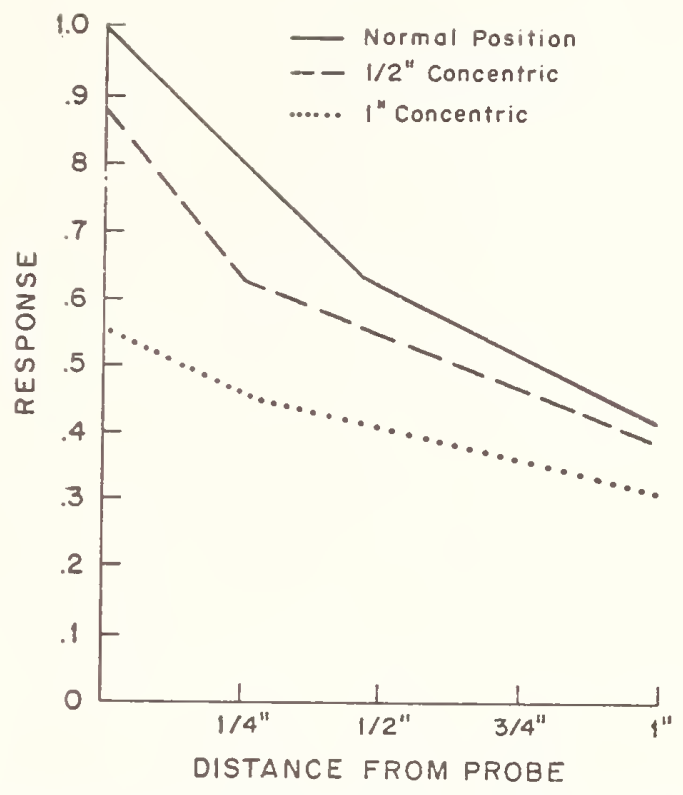

significant and the loss of counting efficiency is not unacceptable.

Urine samples were counted in a Tri-Carb Liquid Scintillation Spectrometer. Counting efficiencies were determined by adding a known amount of calibrated I-125 standard to various urine-gel samples. An efficiency curve was eventually detemined and is plotted in Figure III. In Figure III the counting efficiency is plotted versus the automatic external standard ratio (AES ratio). It may be seen that the counting efficiencies range from $28 \%$ to $39.4 \%$ depending on the degree of quench of the sample. In order that a quench curve, such as shown here, may be used, a system reliability check must be made with each batch of samples analyzed. As values of I- 125 counting efficiency were determined, a set of $\mathrm{six}$ sealed quenched standards and a blank system standard were counted also. As a result, AES ratios of the sealed standards and blank standard are related to system performance. With each batch of samples analyzed, the quench curve shown in Figure III is applied if the sealed standards efficiency curves do not deviate from the expected values.

When one begins to analyze urine samples of occupationally exposed individuals at very low counting rates, the question of what sample background to use becomes a significant factor in determining the net activity. We have chosen to use as a sample background counting rate that activity which is an average of non-occupationally exposed individuals. A total of 15 urine samples 
Figure III

COUNTING EFFICIENCY VS. AES RATIO FOR 1-125 IIV URINE, 3 ML URINE, $10 \mathrm{ML}$ GEL

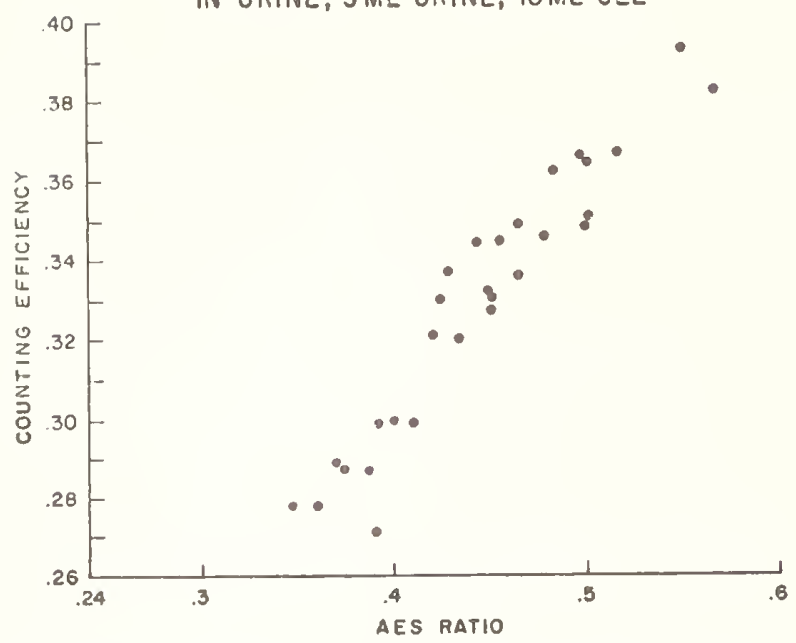

were collected from the Yale Health Services Center and analyzed. It was determined that these individuals were not involved in radioactive isotope work at the University. Each sample was counted for a 50-minute period, and all counts were averaged and a standard deviation computed. It was found that the background counting rate was $11.62 \pm 1.07 \mathrm{cpm}$ in the I-125 channel of the liquid scintillation spectrometer. This background was applied to all urine samples if no background was available for the individual in question. In determining the net sample activity, a gross count rate of at least three sigma $(11.62+3 \times 1.07)$ or 14.83 $\mathrm{cpm}$ was required. Any sample analyzed that yielded a count rate less than $14.83 \mathrm{cpm}$ was considered to be not different from the background level. This minimum detectable level corresponds to a net sample activity of $(1.72 \pm 0.37) \times 10^{-3} \mu \mathrm{Ci} / 1$ and $(1.22 \pm 0.46)$ $\times 10^{-3} \mu \mathrm{Ci} / \mathrm{l}$ of urine for the worst and best quenched samples, respectively. These minimum detectable levels are based on a 50minute counting time per sample.

Once the urine activity at a specific point in time is determined, an approximation to the original amount of I-125 uptake may be calculated. The fractional retention equation for stable iodine in the human body is given by (ICRP, 1967):

$$
\begin{aligned}
R(t) & =0.7 \exp \left(\frac{-.693 t}{T_{b 1}}\right) \\
& +0.3 \exp \left(\frac{-.693 t}{T_{b 2}}\right)
\end{aligned}
$$

where $\mathrm{T}_{\mathrm{bl}}$ and $\mathrm{T}_{\mathrm{b} 2}$ are the biological half lives of iodine in the whole body and thyroid, respectively. The excretion function is of interest when analyzing urine sample data, and may be obtained by taking the differential of $R(t) .1$ In addition, $d R(t) / d t$ is multiplied by $\exp \left(-\lambda_{r} t\right)$ to account for radioactive decay. The equation for the fraction excreted per day via all routes is given by:

$$
\begin{aligned}
Y(t)= & -\exp \left(-\lambda_{r} t\right) \frac{d R(t)}{d t} \\
& \text { or } \\
Y(t)= & -\exp \left(-\lambda_{r} t\right) \\
& \left\{\frac{-.7(.693)}{T_{b 1}} \exp \left(\frac{-.693 t}{T_{b 1}}\right)\right. \\
& \left.-\frac{(.3)(.693)}{T_{b 2}} \exp \left(\frac{-.693 t}{T_{b} 2}\right)\right\}
\end{aligned}
$$

where $\lambda_{r}$ is the radioactive decay constant for $\mathrm{I}-125$. The values of $\mathrm{T}_{\mathrm{bl}}$ and $\mathrm{T}_{\mathrm{b} 2}$ are assumed to be 0.353 days and 96.37 days, respectively. These values represent averages of the data.l The initial amount of I-125 uptake $U_{0}$ may now be computed by:

$$
X(t)=\frac{Y(t) U_{0} F_{u}}{G}
$$

where $X(t)=$ urine concentration in $\mu \mathrm{Ci} /$ liter at time $t$

$$
\begin{aligned}
& Y(t)=\text { fractional excretion equation, } \\
& \mathrm{F}_{u} \quad \text { fraction of activity lost that }
\end{aligned}
$$

The approximate value for $G$ may be calculated from the expressions ${ }^{2}$

$$
\begin{aligned}
& G=0.02 \mathrm{~W} \text { adult males, } \\
& G=0.017 \mathrm{~W} \text { adult females, }
\end{aligned}
$$

where $W$ is the mass of the individual in kilograms. The initial uptake $U_{0}$ is then finally given by: 
$U_{0}=\frac{G X(t)}{\left[\frac{.485}{T_{b l}}{ }^{\left.\frac{1}{T_{r}}+\frac{1}{T_{b 1}}\right) .693 t}+\frac{.208}{T_{b 2}} e^{-\left(\frac{1}{T_{r}}+\frac{1}{T_{b 2}}\right) .693 t}\right.} \mu C i$

Now that $U_{0}$ is determined, the amount of

I-125 in the thyroid at any time $T$ after an acute uptake may be computed by the use of the retention equation for the thyroid alone. This is

$U(T)=0.3 U_{0} e^{-\left(\frac{1}{T_{t}}+\frac{1}{T_{b} 2}\right) .693 T} \mu C i$

Therefore, by determining the urine sample activity at time $t$ days after an acute uptake of I-125, the amount in the thyroid at $T$ days after the uptake may be calculated. This value may then be compared with the measured quantity present at time T. To date a few comparisons of thyroid burdens as detemined from urine analysis data and thyroid count data were made. The results of these comparisons will be given later in the present paper.

Another objective of the urine analysis was to determine how effective urine sampling would be in routine monitoring procedures for I-125 uptake. In calculating the sensitivity of urine analysis, the minimum detectable activity was assumed in the sample under worst and best quenching conditions $(1.72 \mathrm{x}$ $10^{-3} \mu \mathrm{Ci} / 1$ and $1.22 \times 10^{-3} \mu \mathrm{Ci} / 1$, respectively). This activity was converted to I-125 uptake $\mathrm{U}_{0}$ by the use of equation (5). It was assumed that the minimum detectable activities were measured at various times $t$ after the uptake. In addition to the calculation of $U_{0}$ from urine data, the initial uptake was calculated from a thyroid count $T$ days after the exposure. The thyroid activity $U(T)$ may then be related to $U_{0}$ by the use of equation ( 6 ). The minimum detectable activity in the thyroid was used at various times $T$ for the variable $U(T)$. The minimum detectable thyroid activity is approximately $0.0002 \mu \mathrm{Ci} \pm 0.00007 \mu \mathrm{Ci}$ at the two-sigma level of confidence. The results of the calculations are plotted in Figure IV, which is a plot of the minimum uptake detectable of I-125 based on urine analysis and thyroid count data. It is very evident from the plot that the thyroid counting technique is much more sensitive than is urine sampling. However, the urine sample should not be ruled out completely. One must be certain to obtain a urine sample within the first few days of a suspected uptake to determine if an uptake was recent or occurred some time

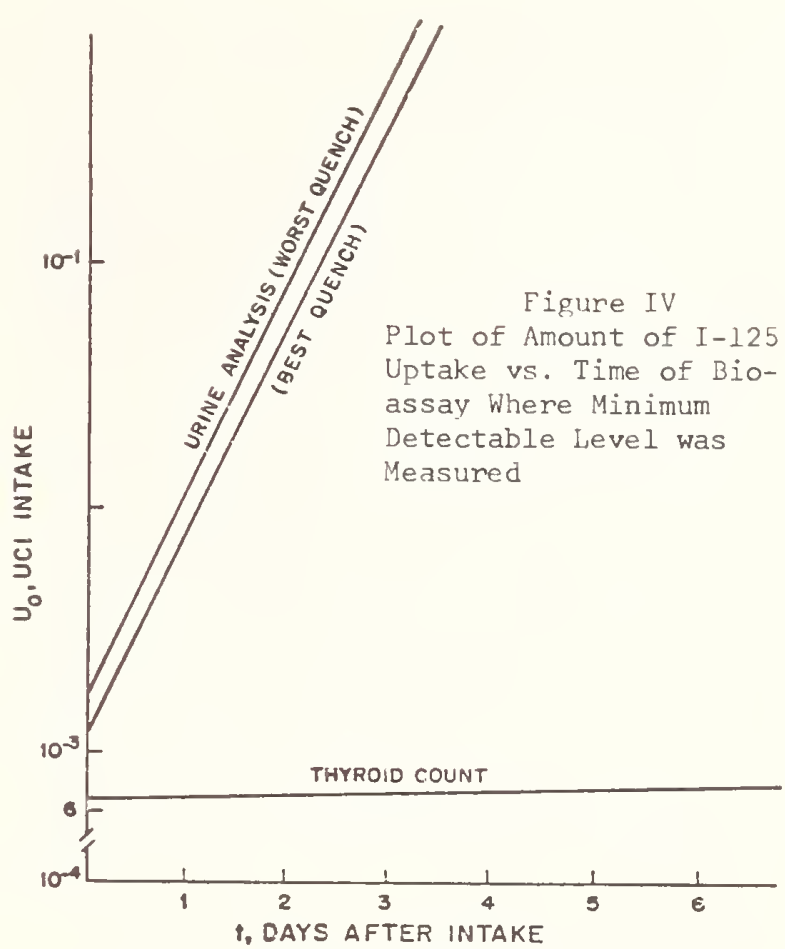

in the past. Our data to date, as summarized in Table II, have allowed us to determine which laboratory procedures are contributing to thyroid burdens. By using the thyroid count and the urine sample in conjunction, the time exposure may be determined with acceptable accuracy.

\section{Table II}

Summary of the Factors Affecting Uptake*:

No. of Users

Form $\left\{\begin{array}{l}\text { Free } \\ \text { Bound }\end{array}\right.$

\begin{tabular}{|c|c|c|c|c|}
\hline \multirow{2}{*}{\multicolumn{2}{|c|}{ U }} & N.D. & $\begin{array}{l}\text { N.D. }- \\
.005 \mu \mathrm{Ci} \\
\end{array}$ & $.005 \mu \mathrm{Ci}$ \\
\hline & & 26 & 19 & 23 \\
\hline Form & $\left\{\begin{array}{l}\text { Free } \\
\text { Bound }\end{array}\right.$ & $\begin{array}{l}16 \% \\
22 \%\end{array}$ & $\begin{array}{r}22 \% \\
6 \%\end{array}$ & $\begin{array}{r}31 \% \\
3 \%\end{array}$ \\
\hline Qty. & $\left\{\begin{array}{l}<500 \mu \mathrm{Ci} \\
>500 \mu \mathrm{Ci}\end{array}\right.$ & $\begin{array}{l}27 \% \\
11 \%\end{array}$ & $\begin{array}{r}9 \% \\
19 \%\end{array}$ & $\begin{array}{r}6 \% \\
28 \%\end{array}$ \\
\hline Exp. & $\left\{\begin{array}{l}<1 \text { year } \\
1-5 \text { years } \\
>5 \text { years }\end{array}\right.$ & $\begin{array}{r}23 \% \\
13 \% \\
1 \%\end{array}$ & $\begin{array}{r}19 \% \\
6 \% \\
3 \%\end{array}$ & $\begin{array}{r}19 \% \\
10 \% \\
4 \%\end{array}$ \\
\hline Septum & $\left\{\begin{array}{l}\text { On } \\
\text { off }\end{array}\right.$ & $\begin{array}{l}24 \% \\
15 \%\end{array}$ & $\begin{array}{l}15 \% \\
13 \%\end{array}$ & $\begin{array}{r}4 \% \\
29 \%\end{array}$ \\
\hline Hood & $\left\{\begin{array}{l}\text { Yes } \\
\text { No }\end{array}\right.$ & $\begin{array}{l}10 \% \\
28 \%\end{array}$ & $\begin{array}{r}22 \% \\
6 \%\end{array}$ & $\begin{array}{r}25 \% \\
9 \%\end{array}$ \\
\hline
\end{tabular}

*Expressed as $\%$ of total 
Summary of Results

I-125 in the Thyroid

Of the 68 users who were counted, $38 \%$, or 26 , had no detectable uptake, while $28 \%$ and $34 \%$ had uptakes which were greater than non-detectable but less than $0.005 \mu \mathrm{Ci}$ and $0.005 \mu \mathrm{Ci}$ or greater, respectively. Sixtynine percent of the individuals were using the free form of $\mathrm{I}-125$, and the remaining $31 \%$ a bound form of I-125. In terms of quantities used, $34 \%$ of those counted were utilizing 500 or less $\mu \mathrm{Ci}$ in their laboratory procedures and the remaining 59\% were using more than $500 \mu \mathrm{Ci}$. The vast majority of individuals, $61 \%$, had one year or less experience. Twenty-nine percent had from one to five years experience and onl.y eight percent had more than five years experience. Fifty-seven percent of those counted reported that they removed the vial septum prior to working with the material and $43 \%$ remarked that the vial septum had not been removed. Finally, the hood was utilized by $57 \%$ of the individuals counted.

\section{Characteristics of Investigators with Non-Detectable Uptake}

As mentioned, 26 of the 68 individuals counted had non-detectable I-125 uptake. of this group, $58 \%$, or 15 , were using the bound form of I-125 while 11 , or $42 \%$, employed the free form. This pattern departs from the pattern established for the group as a whole where $69 \%$ and $31 \%$ were using the free and bound forms, respectively, and points out that the widespread use of the less volatile bound form may in part have contributed to this group's non-detectable uptake.

Also, those with non-detectable uptakes utilized smaller quantities, generally associated with the bound form, than the total group. Seventy percent of those with nondetectable uptakes were experimenting with less than $500 \mu \mathrm{Ci}$ and $30 \%$ with 500 or more $\mu \mathrm{Ci}$. This compares with $42 \%$ using less than $500 \mu \mathrm{Ci}$ and $58 \%$ using 500 or more $\mu \mathrm{Ci}$ for the group as a whole. Obviously, quantity used should be positively related to uptake.

Prior experience should correlate highly with uptake, but in the present context, $62 \%$ of those with no detectable uptake had one year or less experience. Thirty-five percent had from one to five years prior experience and the remaining $3 \%$ had more than five years experience. Uptake may have been minimized in this largely inexperienced group due to the widespread use of the bound form of I-125 and the small quantity. In the absence of these offsetting factors, it is likely that uptake would increase with decreases in prior experience.

Sixty-two percent of those counted with non-detectable uptakes reported working with the vial septum on, while $32 \%$ reported it had been removed. This departs from the total sample where $57 \%$ of the 68 counted reported removing the septum.

The overall pattern seems to indicate that those with a non-detectable uptake used the less volatile bound form, less than 500 $\mu \mathrm{Ci}$ and did not remove the vial septum. There is no doubt that these factors are crucial in minimizing uptake, but there may also be a chronic uptake from repeated experimentation with these small quantities and it is unlikely that such a largely inexperienced group ( $94 \%$ of those working in this group had less than six months experience) could have yet become subject to this effect. Future counting of these individuals may reveal detectable uptakes as this influence takes effect.

Characteristics of Individuals with a Greater than Non-Detectable but Less Than $0.005 \mu \mathrm{Ci}$ Up \pm ake

Nineteen of the 68 individuals counted had uptakes which were greater than nondetectable but less than $0.005 \mu \mathrm{Ci}$. Of this group, 79\% were experimenting with the free form of I-125 and 21\% with the bound form. This differs from the group with a nondetectable uptake where $58 \%$ used the bound and $42 \%$ the free form and suggests that uptake is correlated with the form utilized.

Sixty-nine percent of the individuals with greater than non-detectable to 0.005 $\mu \mathrm{Ci}$ uptake utilized $500 \mu \mathrm{Ci}$ or more and $31 \%$ used less than $500 \mu \mathrm{Ci}$. Again, those with non-detectable uptakes exhibited a different pattern. In their case, $70 \%$ experimented with $500 \mu \mathrm{Ci}$ or less.

Those with a greater than non-detectable to $0.005 \mu \mathrm{Ci}$ uptake had less prior experience than those with non-detectable uptakes. In the former case, $68 \%, 21 \%$ and $11 \%$ had one year or less, one to five years and more than five years prior experience, respectively. In the latter case, $62 \%$ had one year or less prior experience. Limited prior experience coupled with the use of the more hazardous free form and 500 or more $\mu_{1} \mathrm{Ci}$ seems in the present context to have contributed to a higher uptake.

Use of the septum had inconclusive 
results. Fifty-three percent of those counted reported not removing the septum and $47 \%$ removed it. For the group as a whole, $57 \%$ removed the septum while $43 \%$ did not remove it, and for those with non-detectable uptakes $62 \%$ did not remove the septum and $38 \%$ did.

Seventy-nine percent of those with a greater than non-detectable to $0.005 \mu \mathrm{Ci}$ uptake used the hood while $21 \%$ did not use the hood. A far smaller group of those with nondetectable uptake used the hood where only $27 \%$ reported its use.

The combined evidence from this group and those with non-detectable uptakes indicates that primary factors in determining uptake are form, quantity used and prior experience. To this point, however, the influence of the septum and the hood on uptake is uncertain.

Characteristics of Individuals with a Greater than $0.005 \mu \mathrm{Ci}$ Uptake

Twenty-three of the 68 individuals counted had a greater than $0.005 \mu \mathrm{Ci}$ uptake. Far more individuals in this group used the free form of I-125 than did in any other group. Here $91 \%$ used the free form where only $79 \%$ did so in the greater than nondetectable to $0.005 \mu \mathrm{Ci}$ uptake group. Nine percent of the current group used the bound form.

Five hundred or more $\mu \mathrm{Ci}$ were used by $83 \%$ of the individuals with a greater than $0.005 \mu \mathrm{Ci}$ uptake and $17 \%$ used 500 or less $\mu \mathrm{Ci}$. Only the group with greater than nondetectable to $0.005 \mu \mathrm{Ci}$ uptake came close where $69 \%$ reported using 500 or more $\mu \mathrm{Ci}$.

Progressively, it can be seen that as uptake has increased, so had the percentage of individuals using the free form and the quantity utilized. Therefore, there appears to be a strong correlation between uptake and these variables.

Experience has not increased with the level of uptake. On the other hand, in this group uptake and experience appear to be negatively correlated. For those with greater than $0.005 \mu \mathrm{Ci}$ uptake, $57 \%$ reported one year or less experience. Thirty percent reported one to five years experience and $13 \%$ more than five years experience. Sixtytwo percent of those with no detectable uptake reported one or less years experience and $68 \%$ of those with a greater than nondetectable to $0.005 \mu \mathrm{Ci}$ uptake reported one year or less experience.
The septum was reported removed by $87 \%$ of the individuals with greater than 0.005 $\mu \mathrm{Ci}$ uptake and not removed by $13 \%$. Fortyseven percent of those with greater than non-detectable to $0.005 \mu \mathrm{Ci}$ uptake reported the septum off and $38 \%$ of those with nondetectable uptakes reported it off. The increasing percentage of those removing the septum seems to indicate that uptake is in part influenced by the absence of the septum.

Seventy-four percent of those with greater than $0.005 \mu \mathrm{Ci}$ uptake reported that the hood was used, while $25 \%$ reported that it was not used. The majority of the group with a greater than non-detectable to 0.005 $\mu \mathrm{Ci}$ uptake also reported that the hood was used. In this group, $79 \%$ reported that the hood was used. Widespread use of the hood in combination with a detectable uptake would indicate that inexperience coupled with unfamiliarity with experimental procedures has resulted in the ineffective utilization of the hood and the resultant pattern.

Current Yale University guidelines suggest that only users of one millicurie or more of I-125 and performing iodinations need be counted for thyroid uptake. To have adhered to this guideline would have meant that the number of people counted would have been reduced from 68 to 36 or by $32 \%$. More important, however, of the total 68 individuals counted, 40 or $59 \%$ had detectable uptakes. Of this group, 12 or $30 \%$ were experimenting with less than one millicurie and were not iodinating. Obviously, to have adhered in this case to the guideline would have meant that a sizeable group with detectable uptakes would have been overlooked.

I-125 in the Urine

Urine samples were obtained from twelve individuals immediately after a thyroid count indicated a thyroid uptake of more than $.001 \mu \mathrm{Ci}$. This cutoff level was based on the minimum detectable activity in the urine of $1.72 \times 10^{-3} \mu \mathrm{Ci} / 1$ and $1.22 \times 10^{-3}$ $\mu \mathrm{Ci} / 1$ for worst and best quenched samples, respectively. Table III gives, the results of both the thyroid counts and the urine analysis. Of the 12 urine samples analyzed, four showed a detectable I-125 concentration.

Utilizing the urine sample data along with equation ( 7 ), the initial uptake, $\mathrm{U}_{\mathrm{O}}$, was calculated. Once $U_{0}$ is determined, the retention function given by equation (3) is 
Table III

Urine Concentrations at Time $t$ Vs. Thyroid Burdens at Time $t$

\begin{tabular}{|c|c|c|c|}
\hline$t$ & $\mathrm{~T}$ & $\begin{array}{l}\text { Amount in } \\
\text { Thyroid } \mu \mathrm{Ci} \\
\end{array}$ & $\begin{array}{l}\text { Urine } \\
\text { Activity } \mu \mathrm{Ci} / \mathrm{I}\end{array}$ \\
\hline $1.125 d$ & Id & 0.141 & $3.52 \times 10^{-2}$ \\
\hline 2 wks. & 2 wks. & 0.0165 & N.D. \\
\hline $43 d$ & $43 d$ & 0.0066 & N.D. \\
\hline $.75 d$ & $.75 d$ & 0.0024 & $2.76 \times 10^{-3}$ \\
\hline $3 d$ & $3 d$ & 0.006 & N.D. \\
\hline $15 d$ & $15 d$ & 0.007 & N.D. \\
\hline $15 d$ & $15 d$ & 0.003 & N.D. \\
\hline $88 d$ & $88 d$ & 0.005 & N.D. \\
\hline $1.5 d$ & $1.5 d$ & 0.0038 & N.D. \\
\hline $8 d$ & $8 d$ & 0.0105 & N.D. \\
\hline $1.125 d$ & $1.125 d$ & 0.007 & $2.89 \times 10^{-3}$ \\
\hline Id & Id & 0.0096 & $2.23 \times 10^{-3}$ \\
\hline
\end{tabular}

applied in order to determine the thyroid activity at the time of the thyroid count. A comparison of calculated and measured thyroid burdens is given in Table IV. The thyroid count data was assumed to yield the actual amount present. The percent error given in the last column of Table IV reflects the ability of the urine sample data to approximate the measured thyroid burden. The authors feel the results are better than what one would expect, the worst error being $34.4 \%$ too low. One must remember that in calculating a thyroid burden from urine data, errors result from estimating total daily urinary excretion, assumptions in biological half lives of stable iodine in the body and thyroid, and the actual time the uptake occurred. It is extremely difficult to correlate urine sample activity and thyroid burden. This is easily seen by referring to Figure $V$. Plotted in Figure $V$ is the excretion function for I-125 given in terms of the fraction of the initial uptake excreted per day, including radiological decay. The amount excreted from the thyroid is insignificant as compared to the initial whole body excretion. The body eliminates the isotope rather quickly, and for typical thyroid burdens of 0.01 to
Table IV

Calculated and Measured Thyroid Burdens

$$
\text { A B }
$$

Measured Thyroid Activity

Thyroid Calculated

Activity
$\mu \mathrm{C} i$ Analysis, $\mu \mathrm{Ci} \frac{\mathrm{A}-\mathrm{B}}{\mathrm{A}} \times 100 \%$

No.

0.141

0.12

$14.9 \%$

2

0.007

0.0059

$15.7 \%$

3

0.0024

0.0031

$29.2 \%$

4

0.0096

0.0063

$34.4 \%$

$0.001 \mu \mathrm{Ci}$, no detectable activity is present in the urine. We have found that in order to make comparisons between urine activity and thyroid burden, a sample must be taken within three days of the uptake. After three days the urine activity is below the minimum detectable level of our system.

Figure $\mathrm{V}$

EXCRETION OF I-125 VS. TIME

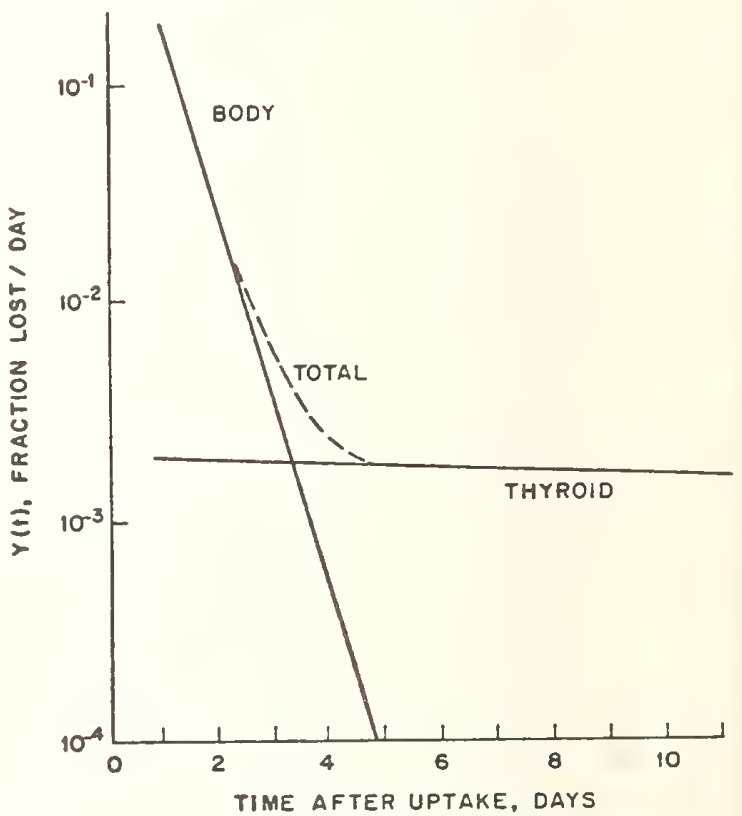


It should also be pointed out that a thyroid count always has a better sensitivity than does a urine sample. Plotted in Figure IV is the minimum uptake $U_{0}$ of I-125 that may be determined from urine sample data and thyroid count data. In constructing the plot, it was assumed that the minimum detectable level was measured in the urine or thyroid $t$ days after an uptake. The initial uptake was then calculated and is the minimum detectable. Urine sample sensitivity increases with time while thyroid count sensitivity remains approximately constant.

\section{References}

1. International Commission on Radiological Protection (ICRP), Publication 10, 1967 (Oxford: Pergamon Press).

2. International Commission on Radiological Protection (ICRP), Publication 23, 1975 (Oxford: Pergamon Press). 

ATTENUATION PHANTONS FOR PATIENT EXPOSURE MEASUREMENT DURING RADIOGRAPHIC EXAMINATIONS UTILIZING PHOTOTIMING TECHNIQUES Pei-Jan Paul Lin

Northwestern University Medical School

Chicago, I11inois 60611

Aluminum phantoms have been obtained for patient simulation in radiographic procedures utilizing phototiming techniques. Thickness of these phantoms have been determined by (a) monitoring radiographic parameters of phototimed examinations and (b) attenuation measurements of $x$-rays in aluminum. Four of the eight selected radiographic procedures, namely (1) A-P cervical spine, (2) Lateral lumbar spine, (3) A-P abdomen, and (4) A-P skull examinations, have been subject to patient exposure limitation in the State of Illinois since January 1 , 1975. Use of these aluminum phantoms in compliance testing and estimation of patient exposure are discussed.

(Phantoms; attenuation; exposure; phototiming; radiographic examination; $x$-ray tube potential)

Introduction

To measure patient exposure from medical radiographic examinations utilizing phototiming techniques, it is necessary to recreate the clinical situation with an appropriate phantom in place of a patient. The thickness of the phantom employed should correspond to the body part of interest to simulate $x$-ray beam attenuation. The $\mathrm{x}$-ray exposure is then terminated at a preset radiation level striking the film-cassette system.

The purpose of this study is to assess phantom thicknesses for. $x$-ray attenuation of various routine radiographic examinations. Patient exposure can then be estimated with the use of the phantoms when the phototiming technique is employed for the examinations. Eight radiographic examinations are chosen for this study. These eight examinations are (1) A-P cervical spine, (2) Lateral lumbar spine, (3) A-P abdomen, (4) A-P sku11, (5) A-P thoracic spine, (6) A-P lumbar spine, (7) high voltage P-A chest, and (8) P-A chest. The examinations (1) through (4) have been subject to patient exposure limitation in the State of Illinois since January 1, 1975 ${ }^{1}$. These eight radiographic examinations are most often performed, therefore are chosen for this investigation.

\section{Experimental Methods}

Monitoring Clinical Fáctors
Accurate assessment of the clinical situation is necessary to facilitate proper measurement from which the attenuation phantoms can be derived. Monitoring and recording several physical quantities during actual radiographic examinations ensures such an accurate assessment. These physical quantities are the radiographic technique factors employed for the examinations, including $x$-ray tube potential ( $\mathrm{kVp}$ ), tube current (mA), phototimer density selection, source-to-film distance, and the thickness of the body part to be examined. The exposure time or the value of milliampere-second (mAS) must be measured and recorded.

In this study, a Machlett Dyna1 izer $\mathrm{II}^{2}$ system was employed during actual radiographic examinations to monitor values of $\mathrm{kVp}, \mathrm{mA}$, mAS and exposure time. The experimental arrangement is illustrated in Figure 1 .

\section{Attenuation Phantoms}

With sufficient data obtained from the arrangement shown in Figure 1 , the patient is then replaced by an appropriate phantom. The thickness of the phantom is varied while al1 other factors are held constant. Thus the exposure time or the mAS value which provides adequate radiographic film density for a given examination is dependent upon the thickness of the phantom only. By placing phantoms of different thickness in place of the patient, the mAS values 


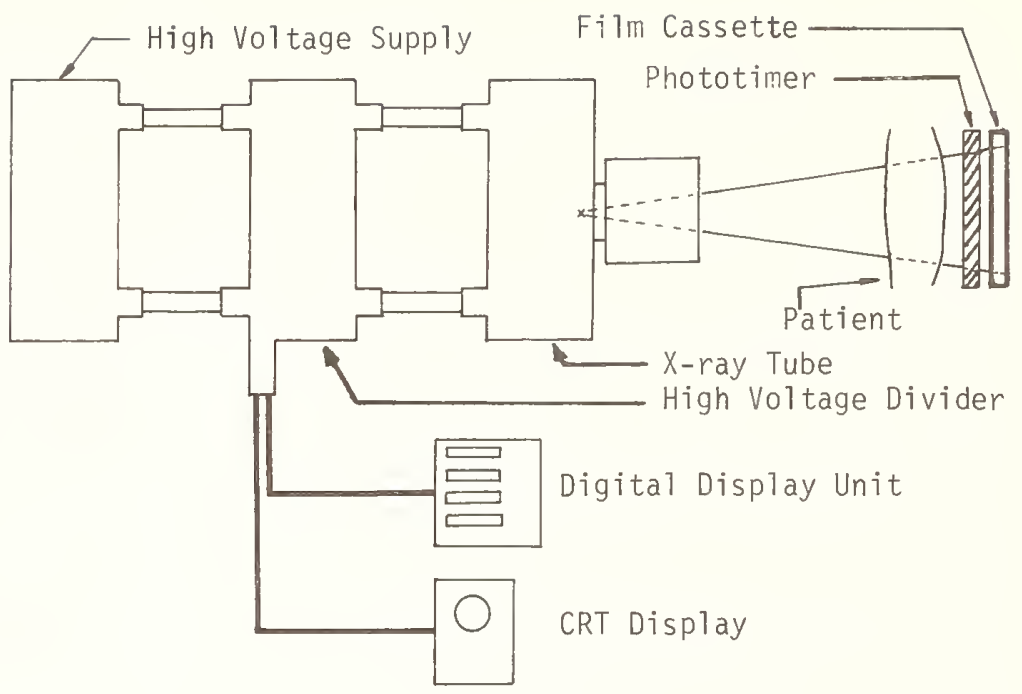

Figure 1. Schematic illustration for the Experimental Arrangement.

are measured. The phantom thickness which provides the same exposure time or the mAS value as the average patient is a realistic phantom for this examination. The phantoms, with their thickness determined for various examinations, can then be utilized for patient simulation in $x$ ray beam attenuation to activate the automatic termination of exposure. Primarily, because of convenience and reasons that will be discussed in a later section, aluminum is chosen for simulation of patient attenuation. The phantom consists of a number of aluminum (type 1100) sheets of dimensions 12 "x12"x1/16". The total thickness of the phantom can be adjusted in increments of $1 / 16^{\prime \prime}$.

Although it is designed for dosimetry verification in radiation therapy, the Rando Phantom ${ }^{3}$ is also utilized for the purpose of comparison with the aluminum phantom. With soft tissue equivalent material incorporating a human skelton, the Rando Phantom is a potential1y useful phantom for phototiming attenuation measurements.

\section{Results and Discussion}

\section{Clinical Factors}

The thickness of the body parts and the $x$-ray tube potentials employed for the radiographic examinations investigated are shown in Table I. The values in the second and the fifth columns under the headings "Technique Chart" are those obtained from the technique charts posted in the $\mathrm{x}$-ray rooms. The second column indicates the average thickness of the body parts and the fifth column identifies the $x$-ray tube potential dial settings. Notice that the thickness for each examination shown in columns of "Technique Chart", "Rando Phantom", and

"Patient" are very close to each other. The Rando Phantom is, therefore, indeed a "standard" man phantom with respect to its physical size.

Figures 2 through 8 depict the variation of mAS values as a function of the patient thickness for the examinations investigated. The $\mathrm{x}-\mathrm{ray}$ tube potentials are held constant at the values indicated in Table I. The mAS values measured with the Rando Phantom are also included in the figures. The Rando Phantom appears to be denser than an average patient for A-P skul1, A-P thoracic spine, and Lateral lumbar spine examinations. Consequently, for these examinations, the Rando Phantom requires higher mAS values to obtain proper radiographic film density. However, the Rando Phantom is, radiographically speaking, a "standard" man for the rest of the examinations included in this study. The mAS values which will provide adequate film density for an average patient may be obtained from Figures 2 through 8 . "These mAS values facilitate the determination of the phantom thickness as will be shown in the next section.

Attenuation Phantoms 
Table I. Radiographic examinations investigated.

\begin{tabular}{|c|c|c|c|c|c|c|c|}
\hline \multirow{2}{*}{\multicolumn{2}{|c|}{ Examination }} & \multicolumn{3}{|c|}{ Thickness of Body Part (cm) } & \multicolumn{3}{|c|}{$x$-ray Tube Potential (kVp) } \\
\hline & & Technique Chart & Rando Phantom & Patient $(4)^{1}$ & Technique Chart $^{2}$ & Manufacture ${ }^{3}$ & Measured 4 \\
\hline (1) & A-P Cervical Spine & $12-13$ & 13.0 & $12.8(32)$ & 65 & 60 & 75 \\
\hline (2) & A-P Lumbar Spine & $20-21$ & 20.5 & $20.1(41)$ & 75 & 70 & 85 \\
\hline (3) & Lat. Lumbar Spine & $25-27$ & 25.5 & $25.9(39)$ & 85 & 77 & 95 \\
\hline (4) & A-P Thoracic Spine & $21-22$ & 22.0 & $20.9(45)$ & 75 & 73 & 85 \\
\hline (5) & A-P Abdomen & $20-21$ & 20.5 & $21.2(43)$ & 75 & 80 & $80^{7}$ \\
\hline & A-P Skul1 & $19-20$ & 19.0 & $19.0 \quad(23)$ & 70 & 70 & 80 \\
\hline (7) & P-A Chest ${ }^{5}$ & $21-22$ & 21.5 & $21.1(50)$ & 115 & 120 & 120 \\
\hline (8) & $P-A$ Chest $\cong$ & --- & 21.5 & --- & $\ldots$ & 70 & 70 \\
\hline
\end{tabular}

The number in the parenthesis indicates the total number of patients.

The grid employed for these examinations has $12: 1$ grid ratio.

3 Manufacturer suggested $X$-ray tube potential for use with a grid having 8:1 grid ratio.

4 X-ray tube potential measured at the transfoner terminals in series with the $X$-ray tube.

5 High voltage chest radiography. Grid ratio is 12:1.

- Chest radiography. No grid is employed.

7 Large focal spot is employed for this examination.

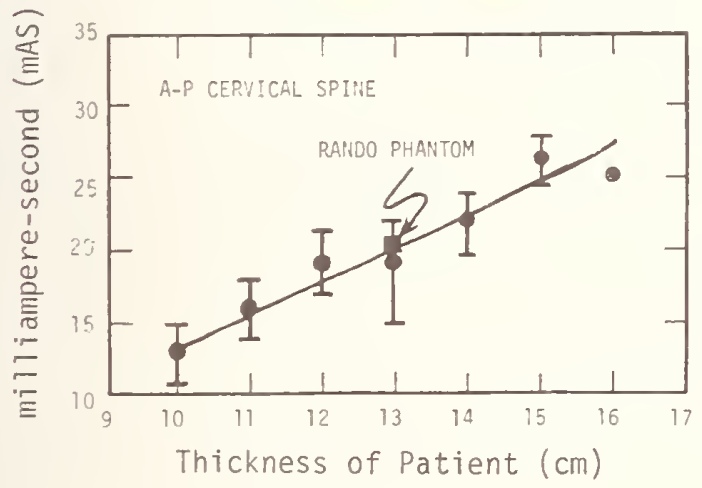

Figure 2. mAS vs patient thickness, for A-P cervical spine examination.

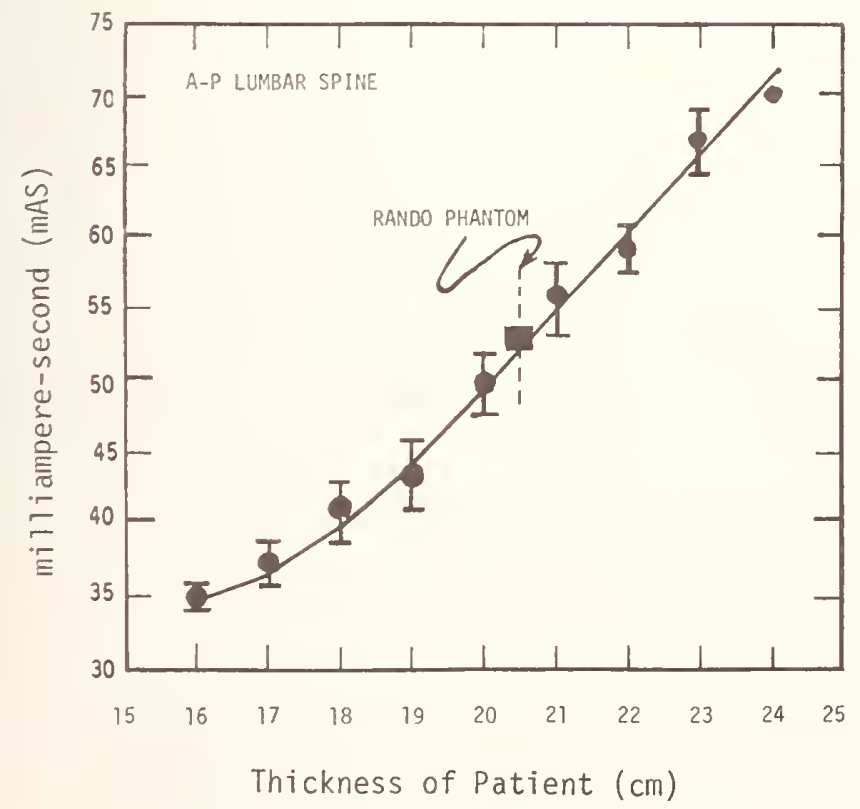

Figure 3. mAS vs patient thickness, for A-P lumbar spine examination. 

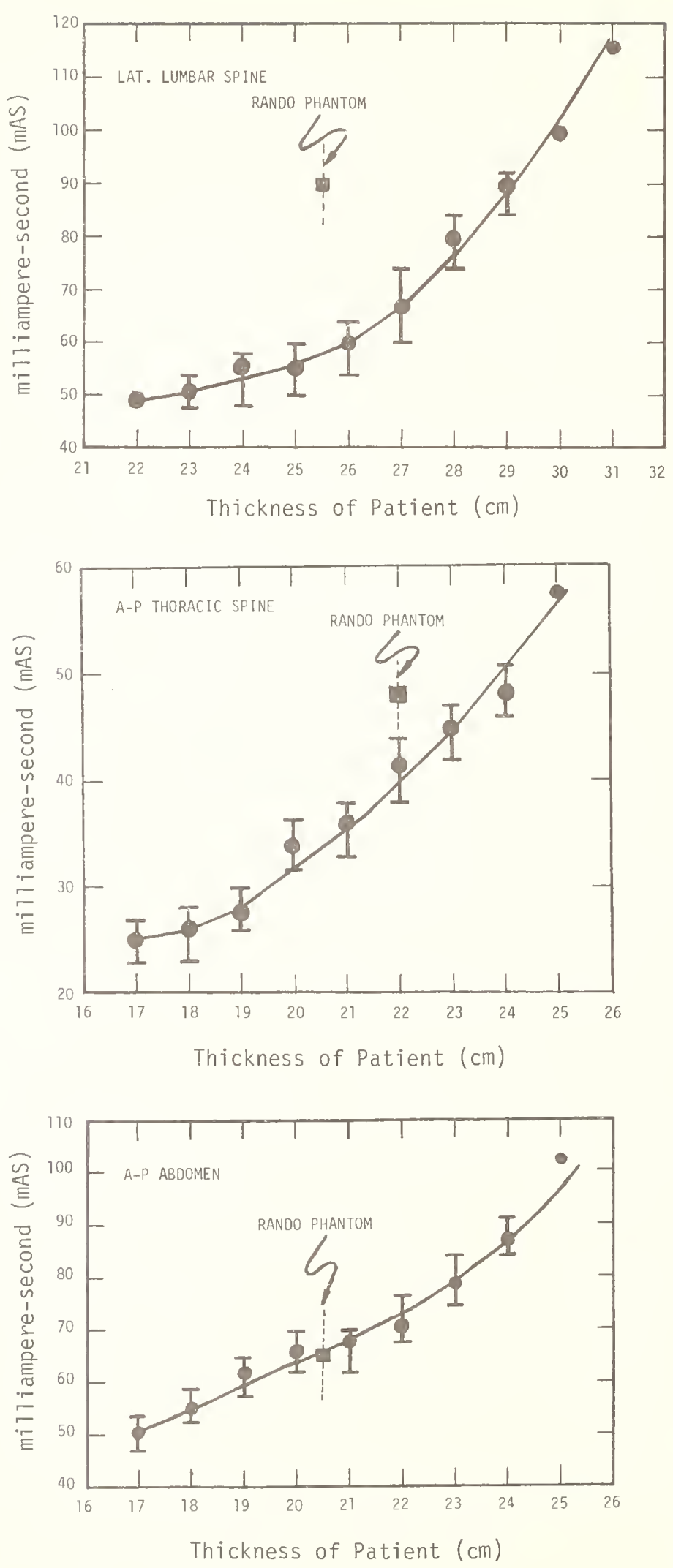

Figure 4. mAS vs patient thickness, for Lateral

lumbar spine examination.

Figure 5. mAS vs patient thickness, for A-P thoracic spine examination.

Figure 6. mAS vs patient thickness, for A-P abdomen examination. 


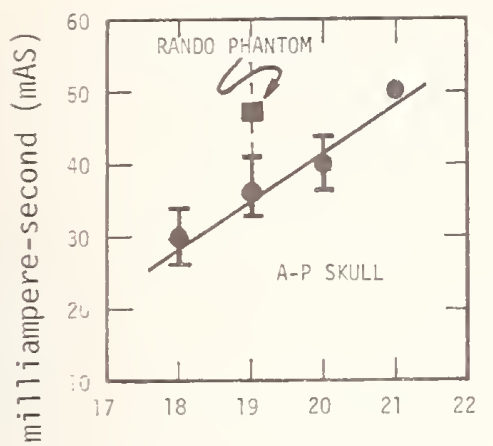

Thickness of Patient $(\mathrm{cm})$

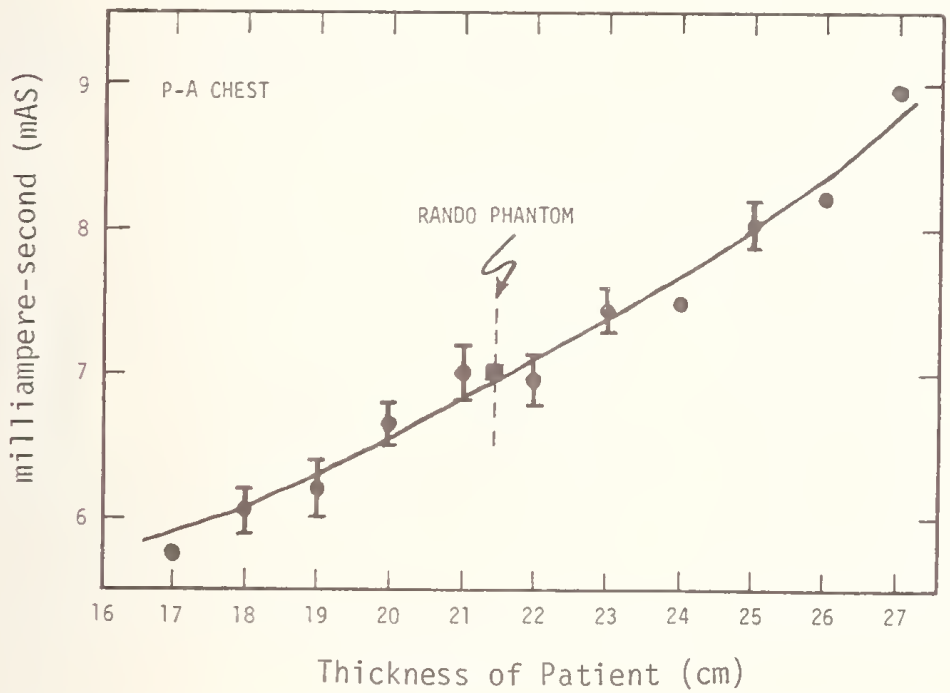

Figure 7. mAS vs patient thickness, for A-P sku11 examination.

Figure 8. mAS vs patient thickness, for high voltage P-A chest examination.

The attenuation measurements with the phantom, based on the results obtained in the previous section, are then performed to determine the thicknesses of aluminum which provide the same mAS values as the average patient. The thicknesses of aluminum determined by the attenuation measurements are to be utilized as attenuation phantoms for the examinations included in this study. The thickness of the phantoms are specified in Table II. These aluminum phantoms are only valid at the $\mathrm{x}$ ray tube potentials (measured) of respective examinations.

The attenuation properties of the aluminum phantoms and the Rando Phantom with respect to the variation of $x$-ray tube potential are depicted in Figures 9 through 16. The aluminum phantom tends to be "too thin" and "too thick" at $x$-ray tube potentials "higher" or "lower", respectively, than at the $x$-ray tube potential that is employed for the examinations. This can be explained by noting that the attenuation of $x-$ rays in the photon energy range of diagnostic radiology (average energy less than $75 \mathrm{keV}$ ) is approximately proportional to the cube of the atomic number and the thickness of the attenuation media ${ }^{4}, 5$ :

For the purpose of comparison, attenuation measurements with copper and water were also performed to determine the thickness required to simulate an A-P abdomen examination. The thickness obtained were $2.5 \mathrm{~mm}$ for copper and $22 \mathrm{~cm}$ for water. These two phantoms provide the same mAS value as the 2 " aluminum phantom or the average patient for A-P abdomen examination at $80 \mathrm{kVp}$. Figure 17 depicts the attenuation properties of the $2.5 \mathrm{~mm}$ copper phantom and the $22 \mathrm{~cm}$ water phantom with respect to thevariation of $x$-ray tube potential. Comparison of Figures 13, and 17 clearly indicates that (1) the water phantom and the Rando Phantom behave 

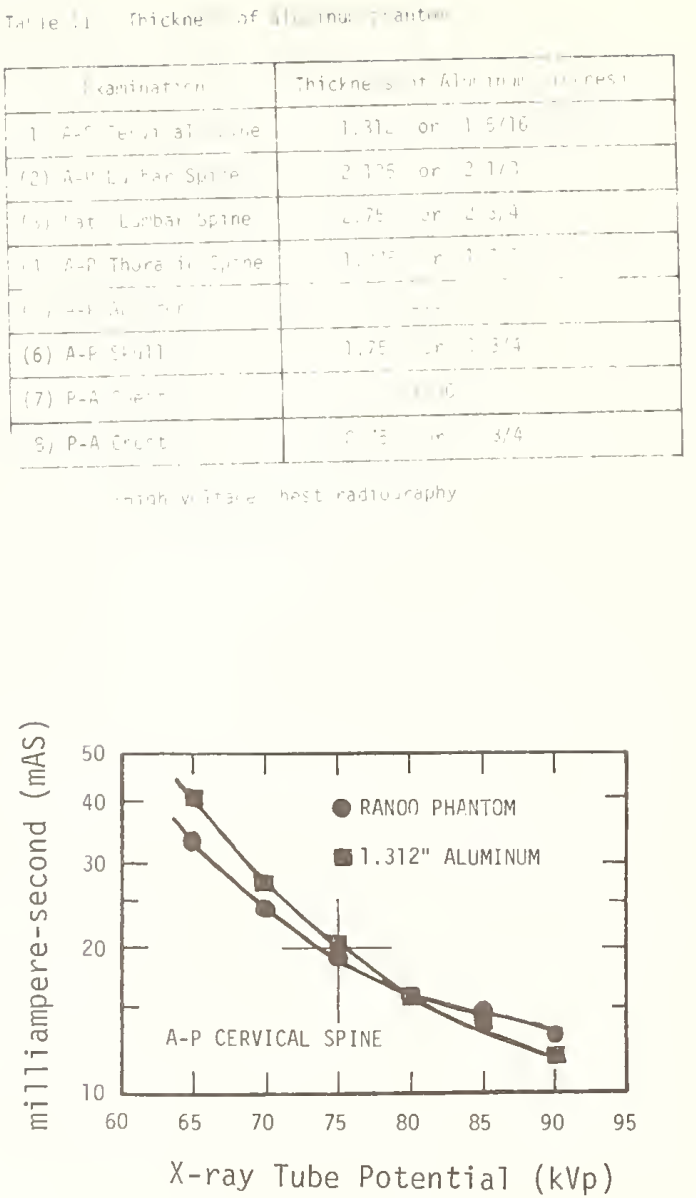

Figure 9. mAS vs kVp, for A-P cervical spine examination.

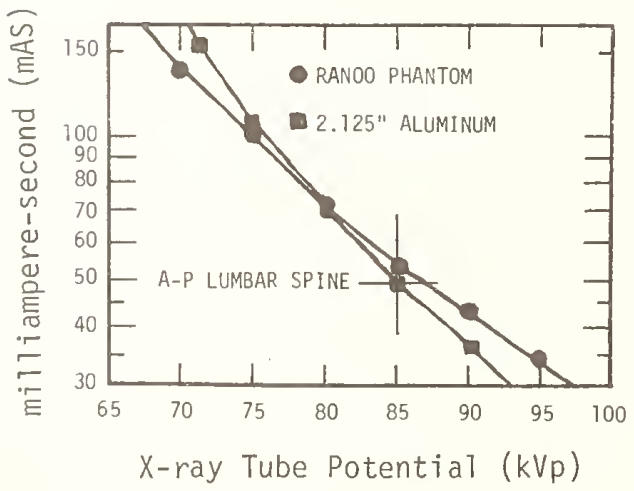

Figure 10. mAS vs kVp, for A-P lumbar spine examination.

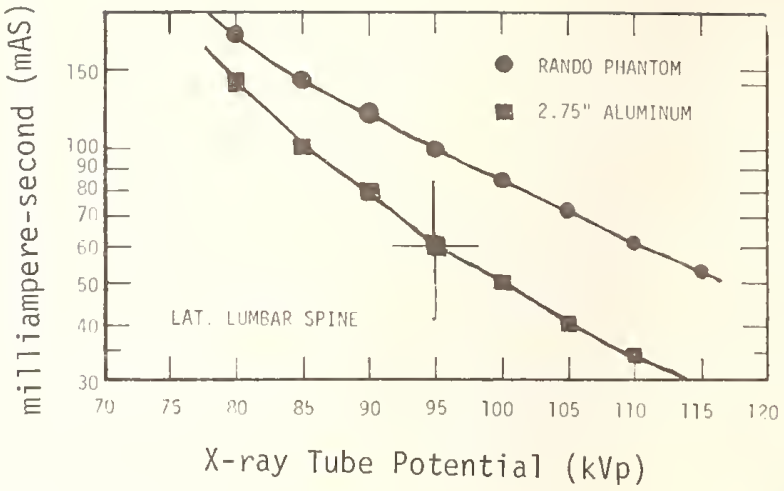

Figure 11. mAS vs kVp, for Lateral lumbar spine examination.

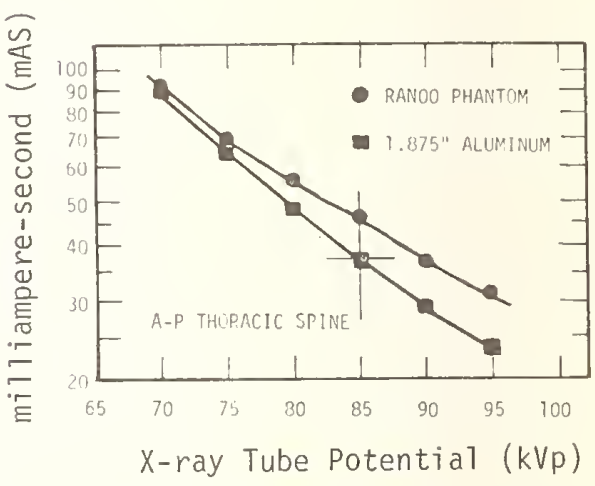

Figure 12. mAS vs kVp, for A-P thoracic spine examination.

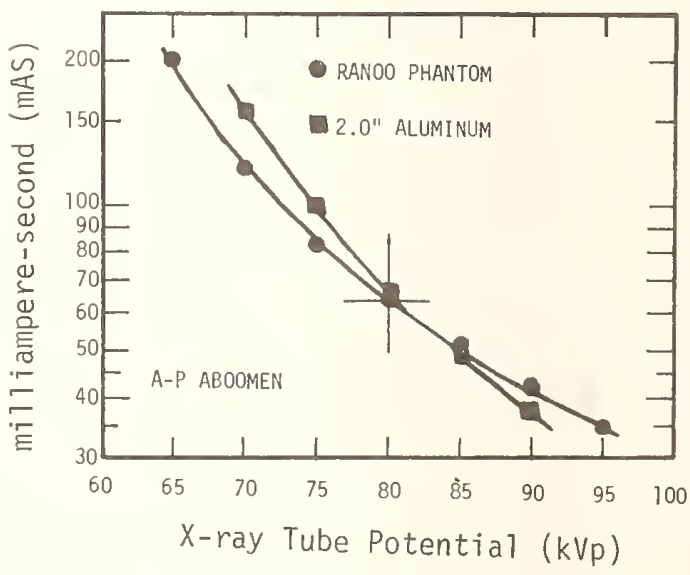

Figure 13. mAS vs kVp, for A-P abdomen examination. 


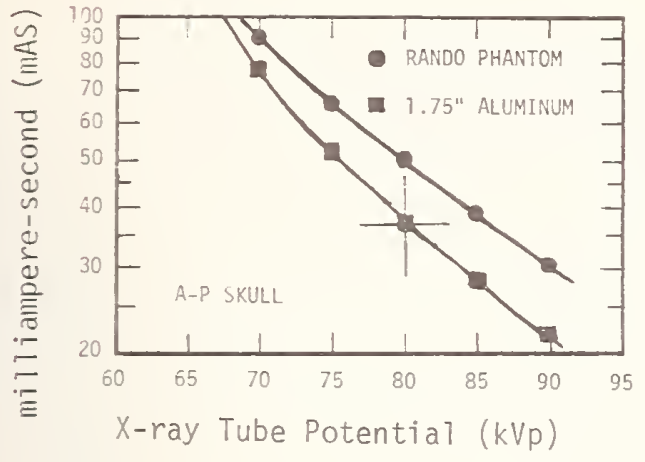

Figure 14. mAS vs $\mathrm{kVp}$, for $\mathrm{A}-\mathrm{P}$ skull examination.

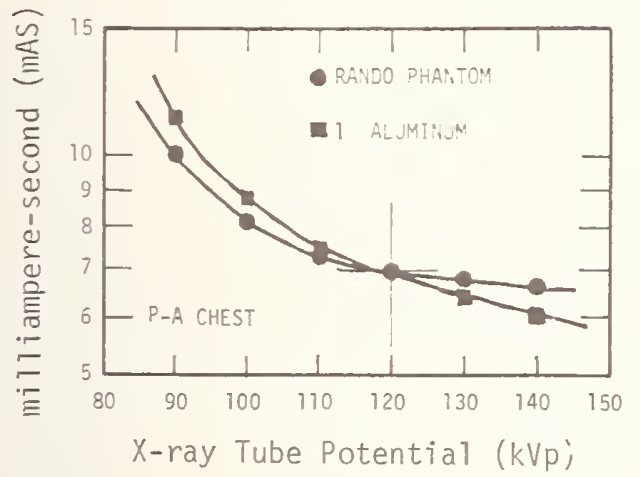

Figure 15. mAS vs kVp, for high voltage $\mathrm{P}-\mathrm{A}$ chest examination.

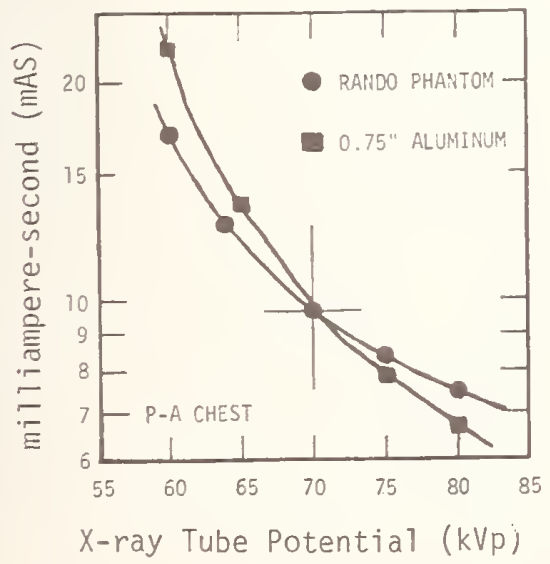

Figure 16. mAS vs kVp, for P-A chest examination. (Routine chest radiography)

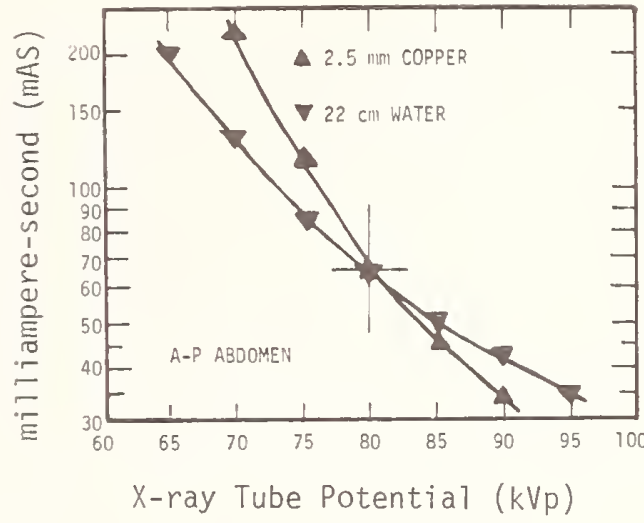

Figure 17. mAS vs $\mathrm{kVp}$, for A-P abdomen examination.

in a very similar manner, (2) the copper phantom has a steeper curvature, and (3) the aluminum phantom has an intermediate characteristic. The water phantom is probably the best choice for patient simulation, however, transportation and handling of this phantom in $x$-ray rooms is often inconvenient. Due to its relatively high atomic number $(z=29)$, the copper phantom does not attenuate $x$-rays in a manner similar to that of muscle (average $z=7.4$ ), or water. Therefore, copper may not be an appropriate choice for attenuation phantom when the variation of $x$-ray tube potential is anticipated. Aluminum $(z=13)$, on the other hand, has an attenuation property which is not significantly different from that of muscle or water. In addition, its reasonable thickness for handling is most suitable in field applications.

\section{Use of the Phantoms and Summary}

For patient exposure measurement or estimation of, for example, the abdomen examination, the $2^{\prime \prime}$ aluminum phantom is placed on the examination table. The radiation field is aligned with the phantom and the phototimer-film cassette system. The geometry and the parameters of radiographic techniques are arranged identically with those of the clinical situation for an average patient. An ionization chamber is positioned $21 \mathrm{~cm}$ (the average thickness) above the center of the phantom. The exposure obtained is an index of the estimated 
Table III. Thickness of aluminum phantom for various kVp values.

\begin{tabular}{|c|c|c|c|c|c|c|c|c|c|}
\hline $\begin{array}{l}\text { A-P Cervical } \\
\text { Spine }\end{array}$ & $\begin{array}{l}\text { kVp } \\
\text { inch }\end{array}$ & $\begin{array}{c}65-70 \\
11 / 4\end{array}$ & $\begin{array}{rr}71 & -79 \\
1 & 5 / 16\end{array}$ & $\left|\begin{array}{c}80-85 \\
13 / 8\end{array}\right|$ & A-P Abdomen & $\begin{array}{l}\text { kVp } \\
\text { inch }\end{array}$ & $\begin{array}{l}70-75 \\
115 / 16\end{array}$ & $\begin{array}{c}76-84 \\
2\end{array}$ & $\begin{array}{rr}85 & -90 \\
2 & 1 / 16\end{array} \mid$ \\
\hline $\begin{array}{c}\text { A-P Lumbar } \\
\text { Spine }\end{array}$ & $\begin{array}{l}k V p \\
\text { inch }\end{array}$ & $\begin{array}{r}75-80 \\
21 / 16\end{array}$ & $\mid \begin{array}{cc}81 & -89 \\
2 & 1 / 8\end{array}$ & $\left|\begin{array}{rr}90 & -95 \\
2 & 3 / 16\end{array}\right|$ & A-P Sku11 & $\begin{array}{l}\text { kVp } \\
\text { inch }\end{array}$ & $\begin{array}{l}70-75 \\
111 / 16\end{array}$ & $\begin{array}{cc}76 & -84 \\
1 & 3 / 4\end{array}$ & $\begin{array}{l}85-90 \\
113 / 16\end{array}$ \\
\hline $\begin{array}{l}\text { Lat. Lumbar } \\
\text { Spine }\end{array}$ & $\begin{array}{l}\text { kVp } \\
\text { inch }\end{array}$ & $\begin{array}{c}85-90 \\
25 / 8\end{array}$ & $\mid \begin{array}{c}91-99 \\
23 / 4\end{array}$ & $\left|\begin{array}{c}100-105 \\
27 / 8\end{array}\right|$ & P-A Chest & $\begin{array}{l}\text { kVp } \\
\text { inch }\end{array}$ & $\begin{array}{c}110-115 \\
7 / 8\end{array}$ & $\begin{array}{c}116-124 \\
1\end{array}$ & $\begin{array}{c}125-130 \\
11 / 8\end{array}$ \\
\hline $\begin{array}{l}\text { A-P Thoracic } \\
\text { Spine }\end{array}$ & $\begin{array}{l}\text { kVp } \\
\text { inch }\end{array}$ & $\begin{array}{l}75-80 \\
113 / 16\end{array}$ & $\mid \begin{array}{cl}81 & -89 \\
1 & 7 / 8\end{array}$ & $\begin{array}{rr}90 & -95 \\
1 & 15 / 16\end{array} \mid$ & P-A Chest & $\begin{array}{l}k \vee p \\
\text { inch }\end{array}$ & $\begin{array}{c}60-65 \\
11 / 16\end{array}$ & $\begin{array}{c}66-74 \\
3 / 4\end{array}$ & $\begin{array}{c}75-80 \\
13 / 16\end{array}$ \\
\hline
\end{tabular}

exposure that may be received by an average patient at the surface of the primary beam entrance without a backscatter correction.

The measurement protocol for patient exposure limitation implemented by the State of Illinois calls for positioning of the ionization chamber at the tabletop ${ }^{1}$. The exposure obtained above, therefore, should be corrected by the inversesquare law for compliance testing. There was no statement made regarding the $x$-ray tube potential by the State of Illinois when the abdomen phantom was specified $(2.1 \mathrm{~mm}$ of copper). However, it is clearly demonstrated in Figures 9 through 17 that an appropriate compensation for correcting the exposure values due to the variation of $x$-ray tube potential is necessary. The investigator finds a correction chart derived from attenuation measurements with various thickness of aluminum phantoms, shown in Table III, to be useful. This chart is currently employed at our institution for patient exposure estimation of radiographic examinations utilizing phototiming techniques.

\section{Acknowledgments}

The author would like to express his appreciation to L. Lanzl for his useful suggestions, to P. Johnson for her reviewing of the manuscript, and to the technologists involved, especially $M$. Klein, for their technical assistance in obtaining parts of the data for this study.

\section{References}

1. Rules and regulations for protection against radiation, adopted April 18, 1961, amended May 1, 1974. Illinois Department of Public Health.

2. Dynalizer II $x$-ray equipment calibration and verification system, available from the Machlett Laboratories, Inc., Stamford, Connecticut.

3. The Rando Phantom, available from Alderson Research Laboratories, Inc., Stamford, Connecticut,

4. William R. Hendee, Medical Radiation Physics (Y)ar Book Publishers, Inc., Chicago, Illinois)

5. Harold E. Johns and John R. Cunningham, The Physics of Radiology (Charles C. Thomas Publisher, 1969, Springfield, I1linois) 


\author{
EFFECTS OF MEASURING APPARATUS ON X-RAY ATTENUATION MEASUREMENTS \\ Tommie J. Morgan, Ph.D., Libby B. Smith, M.S., \\ and Zia R. Hashmi, Ph.D. \\ Medical Physics Branch \\ Division of Electronic Products \\ Bureau of Radiological Health \\ Food and Drug Administration \\ Rockville, Maryland 20852
}

\begin{abstract}
Measurements of $x$-ray exposure are affected by the photon energy dependence of instrument response, and measurement geometry.
\end{abstract}

The effects of these factors on measured $x$-ray attenuation curves and estimates of first half-value layer in aluminum under certain test conditions are described, and estimates of the magnitudes of the effects are given.

A method for correcting half-value layer estimates obtained under less than ideal, but well-controlled, conditions is discussed.

(Half-value layer; geometry effects; chamber energy response; photon attenuation)

\section{Introduction}

An investigation was conducted to determine the effects of measurement geometry and radiation detector energy dependence on $x$-ray beam half-value layer determinations within the diagnostic energy range. Two discrete $x-r a y$ beam qualities were selected for this investigation. The two $x$-ray beam qualities selected were approximately $1 \mathrm{~mm}$ Al half-value layer (HVL) and $4 \mathrm{~mm} \mathrm{Al} \mathrm{HVL,}$ with nominal $48 \mathrm{kVp}$ and $90 \mathrm{kVp}$ tube potentials, respectively. It was felt that these two beam qualities were representative of the lower and medium beam qualities encountered in diagnostic radiology. In addition, it was suspected that the greatest error in half-value measurements might occur at the lower beam quality.

This investigation encompassed three geometries commonly employed in $x$-ray attenuation measurements, and three instruments having significantly different energy response functions. The response of one instrument had a rather significant but not necessarily atypical photon energy dependence. An attempt was made to quantify the nagnitude of the effect of the individual parameters on the determination of the two half-value layers.

\section{Experimental Design and Equipment Description}

Two $x$-ray beam qualities were selected for this investigation. The peak tube potentials were $48 \mathrm{kVP}$ and $90 \mathrm{kV}$.
Test Geometries. Three geometries were selected for evaluation in this investigation. These geometries are commonly employed in attenuation measurements in the diagnostic energy range. Geometry A can be described as follows: the distance from the source to the center of the sensitive volume of the ionization chamber was approximately $75 \mathrm{~cm}$; the source to aperture distance was approximately $30 \mathrm{~cm}$; the field was defined by the beam-limiting device of the $x$-ray source assembly, i.e., the collimator of the $x$-ray system itself; the $\mathrm{x}$-ray field cross-sectional area at the plane of the center of the sensitive volume of the ionization chamber was approximately $220 \mathrm{~cm}^{2}(12 \mathrm{~cm} \times 18 \mathrm{~cm})$; the attenuators were perpendicular to the central ray of the beam placed approximately midway between the $x$-ray source and the center of the sensitive volume of the ionization chamber. It may be noted that this geometric configuration corresponds to that routinely employed in the Bureau of Radiological Health (BRH) routine compliance testing of radiographic x-ray systems, utilizing the BRH test stand and associated hardware. 


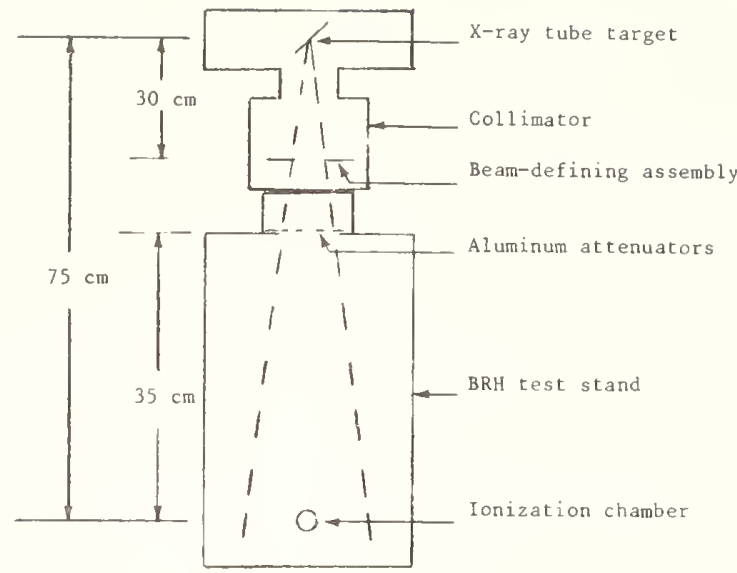

Geometry A

Geometry B can be described as follows: the distance from the $x$-ray source to the center of the sensitive volume of the ionization chamber was approximately $75 \mathrm{~cm}$; the source to aperture distance was approximately $38 \mathrm{~cm}$; the beam-defining assembly was a $15 \mathrm{~cm}$ x $15 \mathrm{~cm}$ square lead/Lucite sandwich with a $5 \mathrm{~cm} \times 5 \mathrm{~cm}$ centered aperture; the $x$-ray field cross-sectional area at the plane of the center of the sensitive volume of the ionization chamber was approximately $100 \mathrm{~cm}^{2}(10 \mathrm{~cm} \times 10 \mathrm{~cm})$. The configuration described as geometry $\mathrm{B}$ can be achieved by using the BRH test stand and associated hardware, and corresponds closely to the test configuration used in gathering HVL data for NEXT (Nationwide Evaluation of X-Ray Trends.)

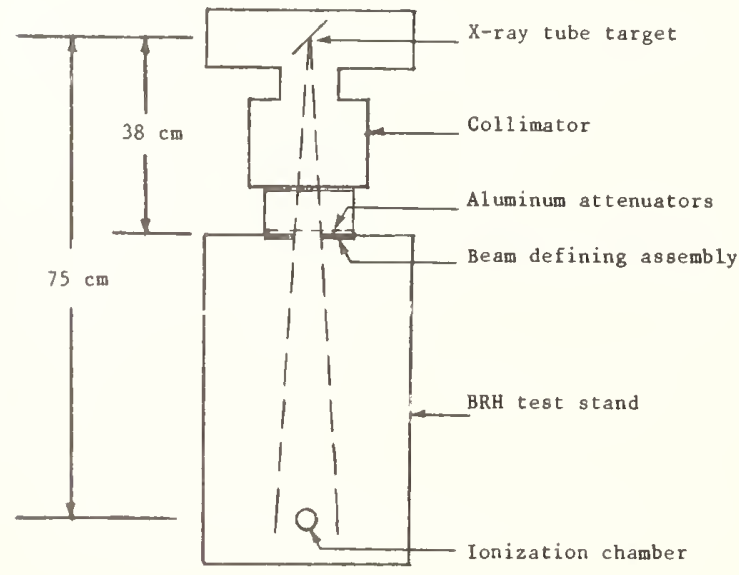

Geometry B
Narrow beam geometry (geometry C), was used to determine the reference half-value layers throughout this investigation. Narrow beam geometry is described as follows: the distance from the $x$-ray source to the center of the sensitive volume of the ionization chamber was approximately $100 \mathrm{~cm}$; the beam-defining assembly was midway between the $x$-ray source and the ionization chamber, perpendicular to and centered on the central ray of the $x$-ray beam; the $x-r a y$ field was large enough to expose uniformly only the entire sensitive volume of the ionization chamber; and the attenuators were placed on the source side of and in direct contact with the beam-defining as sembly.

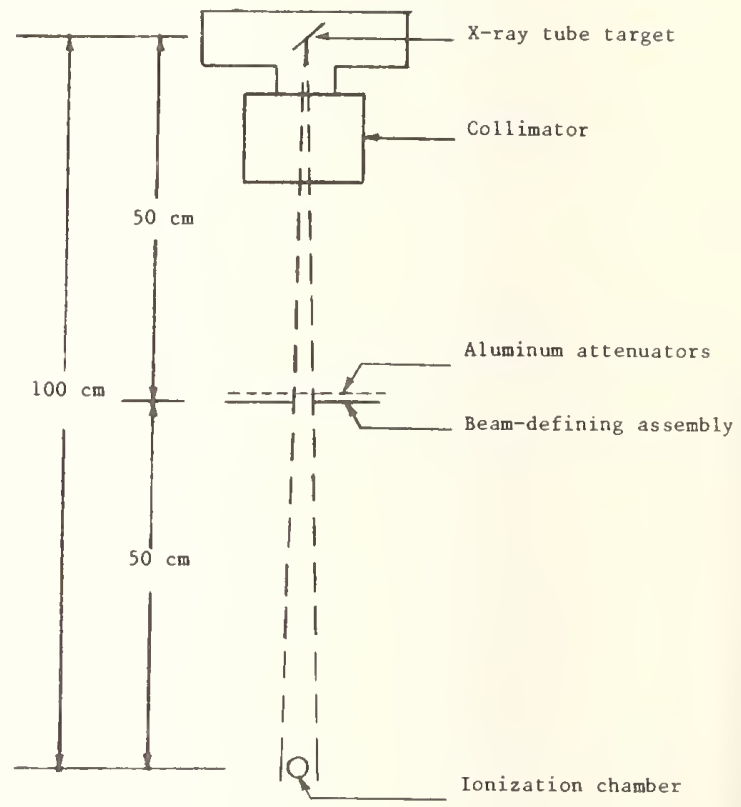

Geometry C

The BRH test stand used in geometries $A$ and $B$ is a rectangular Lucite frame which provides fixed geometry for placement of the ionization chamber, the beam-defining assembly, and the aluminum attenuators.

Instrumentation. Three ionization chambers and their respective readout systems were selected for this investigation. A Victoreen 555 Radocon II with a 555-1 DAS chamber was used in making the reference half-value layer determinations. The energy dependence of this ionization chamber is depicted in figure 1 . This chamber is a propionate cylinder with a metal wire as the central anode. 


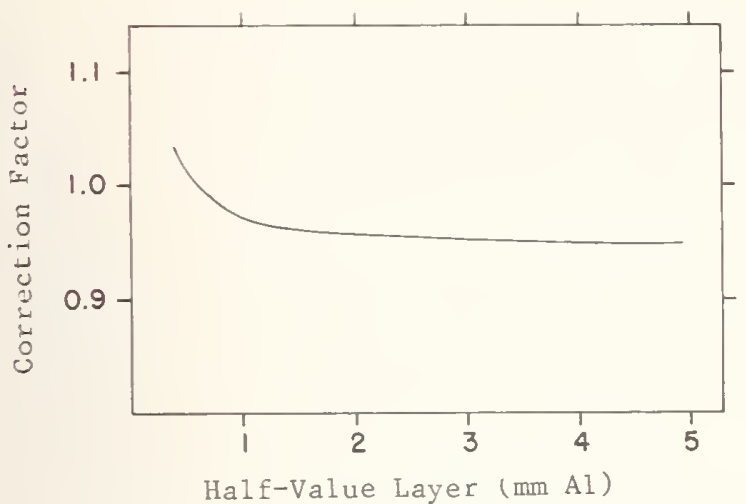

Figure 1. Energy Dependence of a Victoreen 555-1 DAS Chamber.

The second instrument selected for evaluation in this investigation, an MDH 1015A $\mathrm{X}$-Ray Monitor with a 10x5-6 chamber, involved the use of an ionization chamber composed of two concentric polystyrene cylinders. The energy response of this chamber is depicted in figure 2 .

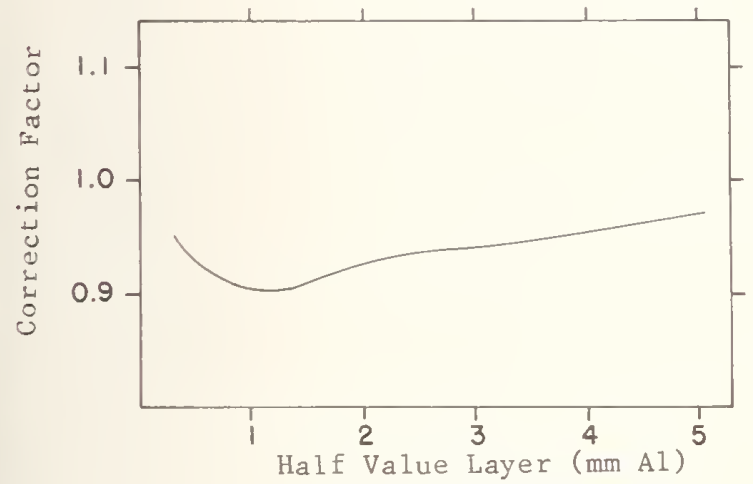

Figure 2. Energy Dependence of an $\mathrm{MDH} 10 \times 5-6$ Chamber.

The 666-10 ionization chamber selected for the use with the third instrument employed in this investigation, a Victoreen 666 Survey Meter, can be described as a right cylinder, constructed of phenolic, of parallel plate design with a round disk anode. The characteristic energy response of this chamber model is depicted in figure 3.

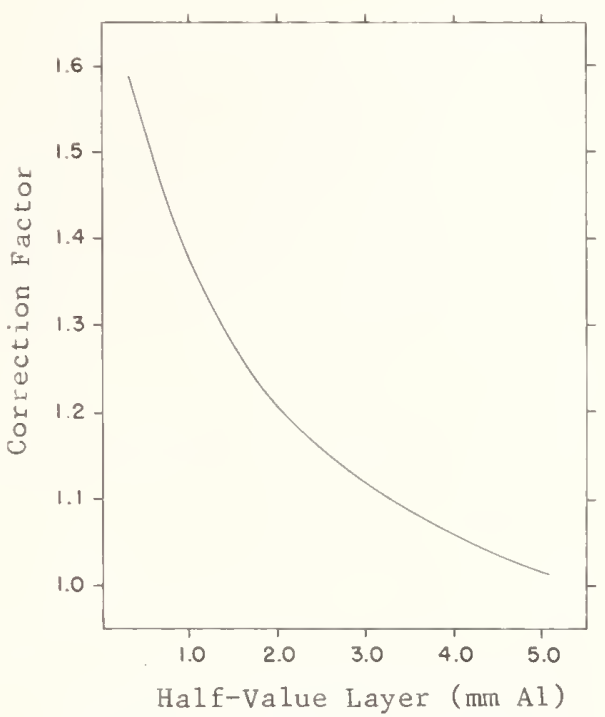

Figure 3. Energy Dependence of a Victoreen 666-10 Chamber.

Throughout the course of this investigation the peak tube potential, the product of the tube current and exposure time, and the line voltage regulation of the $x$-ray machine were monitored continually and found to be reproducibly stable. The peak tube potential was monitored with a Philips model 8122100 voltage divider equipped with a digital readout system. Calibration of this voltage divider was traceable to the National Bureau of Standards. The tube current/exposure time product measurements were made with a Hewlett-Packard model 4288 clip-on probe milliammeter, modified to provide the exposure time/tube current product. The line voltage regulation was observed with a specially designed BRH line voltage regulation monitoring system.

\section{Data and Results}

Extended attenuation curves were deve1oped with the use of the Victoreen 666 Survey Meter in all three geometries: $A, B$, and C. The MDH 1015 X-Ray Monitor was also used in the three geometries. The Victoreen 555 Radocon II was used only in geometry $\mathrm{C}$. The relationships among instruments and geometries are depicted in Table 1 .

From the extended attenuation curves the first half-value layers (observed, uncorrected) were determined. The results of these uncorrected data are presented in Tables 2 and 3 . It is obvious from 


\begin{tabular}{lccc}
\hline Instrument & & Geometry \\
\hline Victoreen 666 with $666-10$ chamber & $\mathrm{X}$ & $\mathrm{X}$ & $\mathrm{X}$ \\
\hline MDH $1015 \mathrm{~A}$ with $10 \times 5-6$ chamber & $\mathrm{X}$ & $\mathrm{X}$ & $\mathrm{X}$ \\
\hline Victoreen 555 with $555-1$ DAS chamber & $\mathrm{X}$ \\
\hline
\end{tabular}

Table 1. Instrument/Geometry Combinations Employed.

\begin{tabular}{|c|c|c|c|}
\hline \multirow{2}{*}{ Instrument } & \multicolumn{3}{|c|}{ Geometry } \\
\hline & A & B & $\mathrm{C}$ \\
\hline Victoreen 666 with $666-10$ chamber & 0.98 & 1.00 & 1.03 \\
\hline MDH $1015 \mathrm{~A}$ with $10 \times 5-6$ chamber & 0.87 & 0.84 & 0.87 \\
\hline Victoreen 555 with 555-1 DAS chamber & & & 0.88 \\
\hline
\end{tabular}

Table 2. Observed First Half-Value Layers for X-Ray Beam at $48 \mathrm{kVp}$.

\begin{tabular}{|c|c|c|c|}
\hline \multirow{2}{*}{ Instrument } & \multicolumn{3}{|c|}{ Geometry } \\
\hline & $\mathrm{A}$ & $\mathrm{B}$ & $\mathrm{C}$ \\
\hline Victoreen 666 with $666-10$ chamber & 5.02 & 4.62 & 4.70 \\
\hline MDH $1015 \mathrm{~A}$ with $10 \times 5-6$ chamber & 3.85 & 3.80 & 3.76 \\
\hline Victoreen 555 with 555-1 DAS chamber & & & 3.74 \\
\hline
\end{tabular}

Table 3. Observed Half-Value Layers for X-Ray Beam at $90 \mathrm{kV}$. 
Tables 2 and 3 that significant differences occur in the determination of the half-value layer with different geometries and instruments. The implication is that these variations can be attributed to the different chamber energy responses and geometries.

An attempt was made to correct the observed half-value layers determined in geometries $A$ and $B$ for the energy responses of the Victoreen 666-10 and MDH 10×5-6 chambers, such that the observed half-value layers would be equal to the reference half-value layer. The iterative technique of correcting half-value layers for the ionization chamber energy dependence, as suggested by Morrison ${ }^{1}$, was applied to the data. This technique attempts to regress the observed attenuation curve to the true attenuation curve, independent of the energy dependence of the ionization chamber employed. The number of iterations required to achieve this goal is a function of the severity of the energy dependence of the particular ionization chamber employed.

Figure 4 shows the uncorrected attenuation data (Geometry C) as well as the results of the first iteration applied to this data for a beam having a reference half-value layer of $3.75 \mathrm{~mm} \mathrm{Al}$. Inherent in the iterative technique is an implied knowledge of the energy response of the particular ionization chamber employed as well as the selection of the appropriate correction factor for the individual points on the attenuation curve. Since half-value layer measurements are relative measurements and not absolute exposure measurements, it is frequently concluded that it is unnecessary to use any calibration data for the ionization chamber employed in such measurements. It is obvious from figure 4 that this is not the case at all, and that it is necessary to apply the appropriate corrections for the particular beam quality being determined.

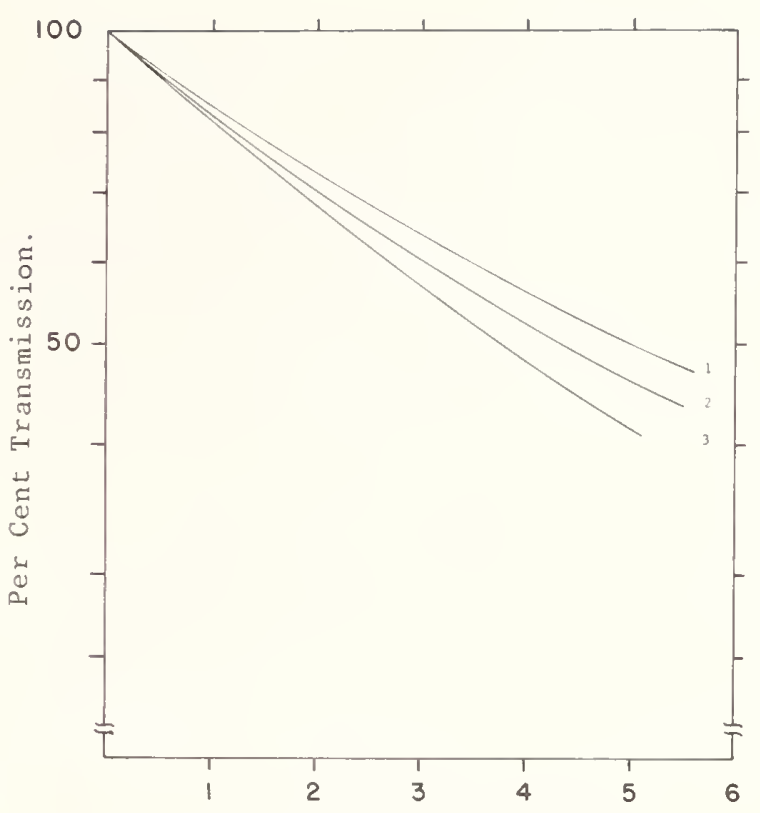

Absorber Added (mm Al)

Figure 4. Attenuation Curves for a Beam of $3.75 \mathrm{~mm}$ Al Reference Half-Value Layer. 1. Observed attenuation curve for Victoreen 666-10 chamber in geometry A. 2. Above attenuation curve, corrected by one iteration. 3. Reference attenuation curve for Victoreen 555-1 DAS chamber.

It is imperative to recognize that in order to use the iterative technique effectively on attenuation data, it is necessary to know the energy response of the ionization chamber employed over a range of energies significantly exceeding that corresponding to the particular observed half-value layer; e.g., for an observed half-value layer of $3.74 \mathrm{~mm} \mathrm{Al}$, it is necessary to know the energy response of the ionization chamber to approximately $8 \mathrm{~mm}$ Al half-value layer if the iterative technique is to be effectively applied to the attenuation data. It is also worth noting that the application of the iterative technique requires that the attenuation data be extended so that the initial radiation exposure rate is reduced to $25 \%$ or less, and, if possible, to $12 \%$.

Instrument response is not solely dependent on the energy dependence of the ionization chamber, and certain other parameters can significantly affect the instrument response in an attenuation measurement. Some of these parameters are instrument precision, sensitivity, and scale linearity for analog readout systems. In addition, all radiation measurements have an associ- 
ated inherent uncertainty dependent on the reproducibility of the detector readout system, the accuracy of the calibration of that particular system, the exposure rate, the number of observations, and the magnitude of the particular exposure being measured. It is appropriate to re-emphasize the importance of selecting the appropriate energy response data for the particular ionization chamber employed as well as selecting from the energy response data the appropriate correction factor for the particular attenuation data being corrected.

\section{Conclusions}

From Table 2, which gives the results for the lower beam quality as measured with the three geometries and three instruments, it can be seen that at this lower half-value 1 ayer $(0.88 \mathrm{~mm} \mathrm{Al}$ reference value) the magnitude of the percentage error is significant. The apparent geometry and instrument effects result in a measured half-value layer which can be as much as $15 \%$ greater than the reference value. The first application of the iterative technique gave a good approximation of the lower reference half-value layer. Application of the iterative technique seems to be valid, within reasonable limits, regardless of the severity of the energy response of the particular ionization chamber employed for measurement of the lower half-value layer.

Table 3 shows the results of the several instrument/geometry combinations employed in measuring the quality of the beam with a reference half-value layer of $3.75 \mathrm{~mm}$ A1. It can be seen that the magnitude of the effects of the several instrument/geometry combinations is more severe at higher energies than at lower energies. For the instrument/geometry combinations evaluated here, the worst-case condition reflected a half-value layer approximately $34 \%$ larger than the reference value. The first application of the iterative technique to the data in Table 3 for the Victoreen 666-10 chamber did not correct the observed halfvalue layer to the reference value. There is strong indication that it is not the iterative technique that is at fault, but the lack of knowledge of the energy response of the particular chambers employed for beam qualities greater than $5 \mathrm{~mm} \mathrm{Al} \mathrm{half-}$ value layer.

Another interesting point is the effect of geometry on the determination of the half-value layer for a particular beam. Within the framework of this investigation, the effects of geometry provided a source of variation not greater than $4 \%$. It is not reasonable to believe that the $4 \%$ variation can be attributed solely to geometry, since there are other variations that could contribute to this value. From this, the conclusion can be made that it is much more important to review the energy response of the ionization chamber being employed in haif-value layer measurements than it is to put severe restrictions on the geometry for making the measurement. If the data are to be used for comparative evaluations of the same particular piece of $x$-ray equipment, then a fixed reproducible geometry for making the attenuating measurement is very important; however, in the event that one is simply making a single estimate of the half-value layer, it is much much more important to have précise knowledge of the energy response of the ionization chamber employed. It is also worthwhile to note that the effects of the different source to chamber distances employed here and the effects of the x-ray field size were essentially insignificant in the case of the chamber with minor energy dependence (i.e., the MDH 10x5-6 chamber).

The implication is that a geometry which is simple to establish and reproduce is an acceptable configuration in making routine beam quality evaluations of diagnostic $x$-ray equipment. Geometry effects are minimal in such determinations of halfvalue layer when such measurements are made with an instrument having a minor energy dependence.

1 Margaret T. Morrison and G.W. Reed, Brit. J. Radiol., 25:270, 1952. 
kVP MEASUREMENT FOR DENTAL X-RAY UNITS

M. S. Moroz and J. A. Vukan

Pennwalt Corp., S. S. White Div.

Holmdel, New Jersey 07733

The kVp measurement of a sealed $x$-ray system using filtered radiation from a scatterer is discussed and compared to direct voltage measurement techniques.

(kVp; dental; x-ray)

\section{Introduction}

Federal legislation has made necessary the accurate measurement of all parameters associated with $\mathrm{x}$-ray production. Among these parameters is the peak potential impressed on the $\mathrm{x}-\mathrm{ray}$ tube by the high voltage generator.

An accurate measurement of this parameter is important for two (2) reasons:

1. It is an indication of the penetrating power of an $\mathrm{x}-\mathrm{ray}$ beam.

2. The radiation incident upon the patient is dependent on the $\mathrm{kVp}$.

The measurement of this parameter in a dental $x$-ray unit offers a particular challerge to the investigator. The diagnostic source assembly contains the $x$-ray tube housing, beam limiting device and the high voltage generator. These are immersed in oil or gas and sealed in a metal enclosure with expansion compensators. The high voltage area of the diagnostic source assembly is therefore inaccessible without breaking the seal on the metal enclosure.

Five (5) methods of measuring this parameter for dental $\mathrm{x}$-ray systems are discussed below:

1. Direct high voltage measurement in an open oil filled tank using a high resistance voltage divider and an oscilliscope.

2. Direct high voltage measurement in an open oil filled tank using a peak reading $\mathrm{kV}$ meter of the charging capacitor type.

3. Direct pulse height analysis using a 1024 channel multichannel analyzer, GeLi detector and heavy filtration of the $x$-ray beam.

4. Pulse height analysis of a scatter spectrum from an element whose " $\mathrm{K}$ " line is at or near the peak $x$-ray potential indicated, using a 1024 channel multichannel analyzer and a sodium iodide scintillation detector.

5. Method 非 4 modified by a filter with an atomic number one higher than that of the scatterer placed between the scatter source and the detector.

$$
\text { The open tank/high voltage divider }
$$

The apparatus used for this measurement is depicted in Fig. 1.

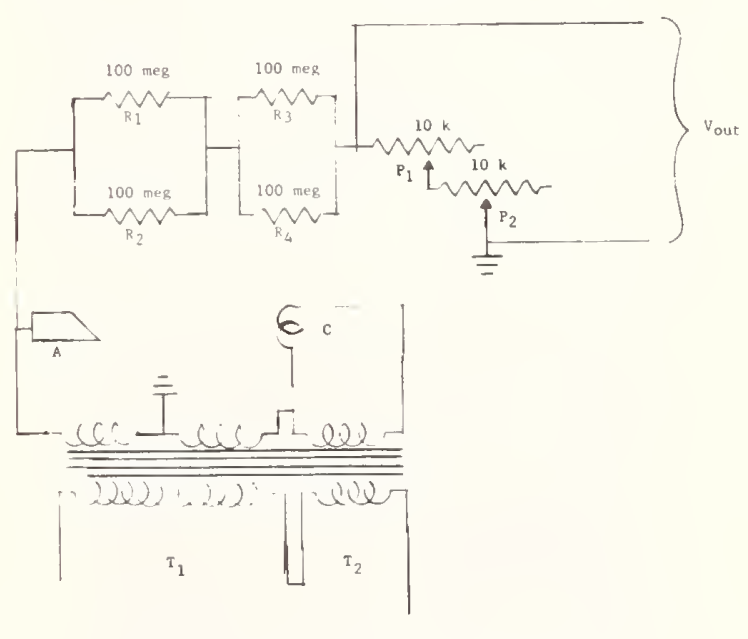

Fig. 1

Schematic of Open Tank/High Voltage Divider Apparatus

The entire assembly was put into an electrically insulated tank, evacuated to 200 microns and held there for 24 hours. Hot transformer oil $\left(140^{\circ} \mathrm{F}\right)$ was allowed to cover the assembly and the unit was held at 200 microns for another 24 hours. The transformer $\left(\mathrm{T}_{1}\right)$, the $\mathrm{x}$-ray tube $(\mathrm{C}, \mathrm{A})$ and transformer $\left(\mathrm{T}_{2}\right)$ constitute the electrical components of a dental diagnostic source as sembly. 
To calibrate this apparatus, a pure sine wave was impressed on the primary of $\mathrm{T}_{1}$; the primary of the filament transformer $\left(\mathrm{T}_{2}\right)$ was disconnected so that no distortion of the sine wave was introduced into the secondary. A Hipotronics AC/DC kilovoltmeter, Model 110, was put in parallel to the divider. The meter used has an N.B.S. traceable calibration. The oscilliscope was calibrated for a full scale reading of the highest peak potential to be measured by adjusting potentiometers $P_{1}$ and $P_{2}$. This produces the proper peak reading corresponding to the rms reading on the Hipotronics meter. A pure sine wave was needed at the output of transformer $\mathrm{T}_{1}$ to ensure that the conversion from rms $\mathrm{kV}$ to peak $\mathrm{kV}$ is without harmonic error.

The Hipotronics meter was then removed, the primary of transformer $T_{2}$ was re-con-

nected and the entire assembly was connected to the proper $\mathrm{x}$-ray control. Readings were then taken at the maximum rated peak $x$-ray potential of the $\mathrm{x}$-ray system and compared to its specifications. The $\mathrm{kVp}$ was varied when it was possible, or the mA was varied and the indicated value of $\mathrm{kVp}$ was compared to the actual.

Fig. 2 is a picture of the waveform seen on the oscilliscope face; note that due to the half wave loading of the high voltage transformer by the $\mathrm{x}$-ray tube, the waveform departs from that of a true sine wave.

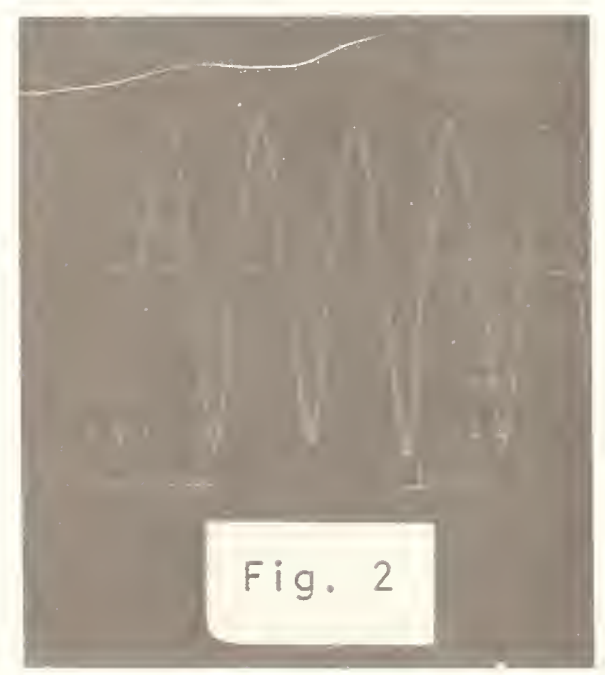

Waveform at $90 \mathrm{kV}$ indicated, $10 \mathrm{~mA}$ indicated obtained by the voltage divider method.
The advantages of the method are listed below:

1. The actual secondary waveform is seen and $c$ an be studied if the oscilliscope is a storage type oscilliscope.

2. The mA can be displayed with the secondary voltage waveform to determine the effects that changing the $\mathrm{mA}$ has on the $\mathrm{kVp}$.

3. The primary voltage can be studied along side the secondary voltage and the effects that waveform changes in the primary have on the secondary waveform can be determined.

4. The kVp reading is instantaneous.

These reasons are all good research tools and this method allows one to use them. However, what effects are ignored by this method or what new effects are introduced by this method?

A list of the major aberrations of this system are in order:

1. This system ignores the possible capacitance currents that a rapidly varying high voltage field generates when surrounded by a grounded metal enclosure.

2. The introduction of a resistive load unbalances the two (2) coils in the transformer.

3. The resistive load also tends to lower the kVp reading since the voltage regulation in dental diagnostic source assemblies is low.

4. The resistors used ( $100 \mathrm{meg}, \frac{1}{2} \%$ ) have a high voltage coefficient.

5. The width of the oscilliscope trace also lends itself to inaccuracy.

The error is estimated at a full-scale inaccuracy of $2-5 \%$ by this method, depending on the type oscilliscope used and the individual researcher. However, this is a valuable research tool in determining high voltage wave shapes and factors effecting them.

\section{Method 非2}

Direct high voltage measurement in an open oil tank using a charging capacitor type $\mathrm{kVp}$ meter

The meter used in this method was an ITT Jennings Model 12102 high voltage meter with a calibration traceable to N.B.S. 
This meter has an oscilliscope output and digital readout with an option of reading the positive half cycle, the negative half cycle or rms kilovoltage.

The diagnostic source assembly was evacuated in an open tank and filled with oil by a method similar to that described in Method 非.

N.B., in certain cases, when the $x$-ray tube and high voltage generator are sealed by bolting the outer tank together, the diagnostic source assembly may be put into a larger tank of oil and the bolts removed and the diagnostic source assembly opened in the oil to avoid the necessity of re-evacuation. This procedure is somewhat difficult and many dental diagnostic source assemblies are of a welded or soldered construction and this procedure is not applicable.

After the diagnostic source assembly was put into oil, the high voltage input lead from the metering circuit was connected to either the anode or cathode. The diagnostic source assembly was connected to the control designed for it. The control was then set for the maximum rated $\mathrm{kVp}$ and $\mathrm{mA}$. An exposure was made of at least five (5) seconds duration and a reading was taken. The exposure was of long duration because the rise time of the meter was quite long. Charge time is a very important factor in the use of peak reading devices of this nature.

If there is more than one technique factor available, the machine should be tested at every combination of preset techniques and in the case of continuously variable techniques it should be tested in a series of combinations at predetermined steps, i.e. variations of $10 \mathrm{kV}$ if the $\mathrm{kVp}$ is continuously variable at each of the preset $\mathrm{mA}^{\prime} \mathrm{s}$.

The positive aspects of this type of measurement are easily recognizable.

1. The machine has a digital readout and readability is good.

2. One has the option of viewing the waveform on an oscilliscope independent of the readings.

3. One has the option of reading the magnitude of the positive peak, negative peak or the rms $\mathrm{kV}$.

However, this type of measurement has a few subtleties not as readily apparent as the positive aspects:

1. The long rise time forces the researcher into making a long exposure. In dental diagnostic source assemblies, this is undesirable due to duty cycle considerations (most dental $\mathrm{x}$-ray control panels do not have a time setting greater than five (5) seconds).

2. Due to the long rise time of this unit, the instantaneous peak voltage cannot be measured. Some dental machines may produce a kVp that varies in magnitude on alternate conducting half cycles, ref. Fig. 2. Others may have a low $\mathrm{kVp}$ on the initial impulses of x-ray which then stabilizes to a higher value of $\mathrm{kVp}$. Finally, the $\mathrm{kVp}$ may be a high value which stabilizes to a lower value of $\mathrm{kVp}$. None of these cases can be detected with this type of instrumentation due to the long rise time of the metering circuit. The $\mathrm{kVp}$ value obtained by this method may at times be erroneous.

Fig. 3 is an example of an $x$-ray unit whose $\mathrm{kVp}$ does not stabilize in the first few $\mathrm{x}$-ray impulses. The $\mathrm{kVp}$ is very low initially and stabilizes at a higher value.

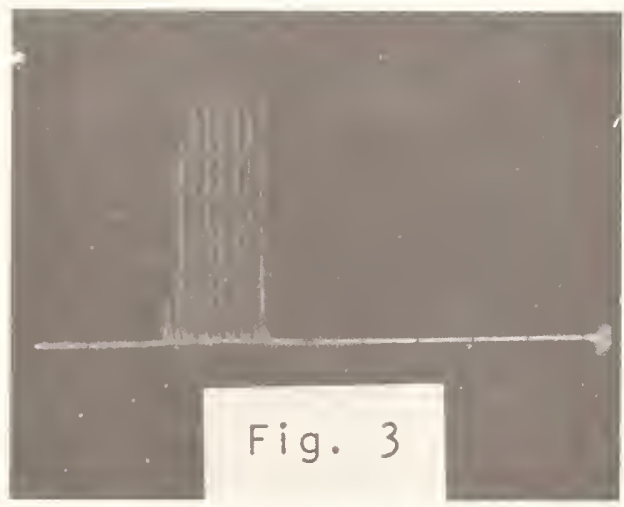

Positive half cycle of the $\mathrm{kVp}$ of a dental $x$-ray machine

3. The nature of the circuitry itself tends to allow the calibration to change over relatively short periods, 1-3 months, necessitating frequent re-calibration.

The biggest problem associated, both, with this method (非) and the previous method (非1) is that in order to test the diagnostic source assembly, the high voltage generator and the $x$-ray tube must be removed from their housing. The tube housing, expansion devices or both may be damaged by the process. A significant amount of the testing of dental machines involves machines that are already installed, 
Methods 非 1 and 非 2 have a serious drawback for this application.

The accuracy of Method 非 2 is dependent on the type of system being tested. For instance, a unit in which the $\mathrm{kVp}$ varies on alternate peaks, Method 非 2 yielded a value 4-6 kV lower than the true value in the range 80-100 kVp; another example is the unit whose $\mathrm{kVp}$ drops down to a point of stabilization, the initial high $\mathrm{kVp}$ is never seen by the observer only the long term stabilized kVp. The error here was measured to be $8-12 \mathrm{kV}$ in the 50-100 kVp range. However, if the unit being tested has the same peak $k V$ for the first pulse of $x$-ray as for the 300th pulse of $x$-ray and for all those in between or if the kVp stabilizes at a point higher than the kVp of the first $x$-ray pulse and remains stable, then measurement of the peak $\mathrm{kV}$ would be within the machine's accuracy of $\pm 1 \%$.

\section{Method 非3}

Pulse height analysis of a heavily filtered $x$-ray beam using a

GeLi detector

This method, depicted in Fig. 4, was tried briefly. This apparatus was an $x$-ray source, narrowly collimated by a heavy lead collimator. The $\mathrm{x}$-ray beam was then heavily

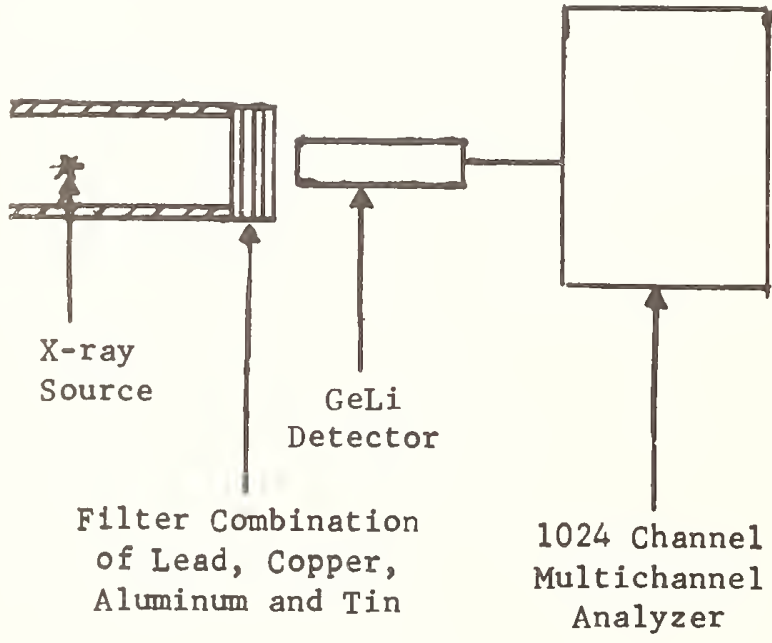

Fig. 4

Block diagram of the apparatus for direct pulse height analysis of a heavily filtered $x$-ray beam

filtered by a filter comprised of aluminum, copper, lead and tin. The GeLi detector was brought in as close to the source as physically possible. The system was calibrated prior to the test using two (2) radioactive isotopes which have gamma emission lines bracketing the indicated $\mathrm{kVp}$. This was repeated after the location of the spectrum end was found. The spectrum end was found by drawing a line along the noise level at the end of the spectrum and drawing a line on the downward slope of the last peak; where these two (2) lines intersected was considered to be the end of spectrum. The $\mathrm{kVP}$ was then calculated by the formula:

$k V p=P_{1} \pm\left|\frac{\left(P_{1}-P_{2}\right)\left(C_{P_{1}}-C_{e}\right)}{\left(C_{P_{1}}-C_{P_{2}}\right)}\right|$

Where:

$\mathrm{P}_{1}=$ The energy of the calibration peak nearest the end of the spectrum.

$P_{2}=$ The energy of the other calibration peak. $\mathrm{C}_{\mathrm{P}_{1}}=$ The channel number of calibration

$\mathrm{C}_{\mathrm{P}_{2}}=$ The channel number of calibration

$\mathrm{C}_{\mathrm{e}}=$ The channel number of the end of spectrum.

$+/-\quad$ is determined by:

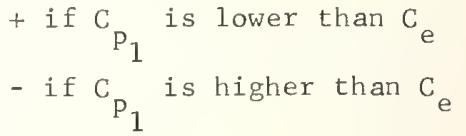

The calibration points, both at the start and the end of a test, were each used to calculate a separate $\mathrm{kV}$ p and the spread between the two (2) values was used as an indication of error. The $\mathrm{kVp}$ was found to be repeatable by this method to $\pm 3 \mathrm{kV}$ in the 50-100 kVp range. Also noted during the course of experimentation was that equally good results in this application could be obtained by using a sodium iodide detector. The sodium iodide detector had the added advantages of not needing cryogenic temperatures and being less bulky to handle (use of the GeLi detector was discontinued in favor of a sodium iodide detector).

The above method was reasonably repetitive but relies too much upon the judgement of the individual experimenter. It has the advantages of:

1. Not disturbing the electrical potentials 
being measured.

2. The diagnostic source assembly need not be disassembled to measure the kVp.

3. The highest kVp pulse is measured each time and not the steady state kVp.

4. Results are not dependent on the secondary wave shape.

There are a few problems with this system when applied to dental $x$-ray units. These are:

1. The inherent storage characteristics demand extremely long cumulative exposure times.

2. Long term drift of the system adds an additional I $1 \mathrm{kV}$ error to the absolute measurement.

3. Due to the pulsed half sine wave, the electron flow in the conducting half cycle see the peak potential only intermittently, not continuously as in the case of a fully filtered d.c. potential across the tube. In essence, what this means is that the true value of the $\mathrm{kVp}$ may be buried somewhere in the noise of this experiment and that even though the values are relatively repeatable they are in fact too low to be the actual value. This is indicated by the results obtained in Method $\equiv 5$. The values obtained in Method $t=3$ tend to be $2-4 \mathrm{kV}$ lower than those of Method $\$ 5$.

A typical spectrum generated by this method is shown in Fig. 5. It can be easily seen that the noise level at the end of the spectrum is barely differentiated from the signal, an inherent problem in this method.

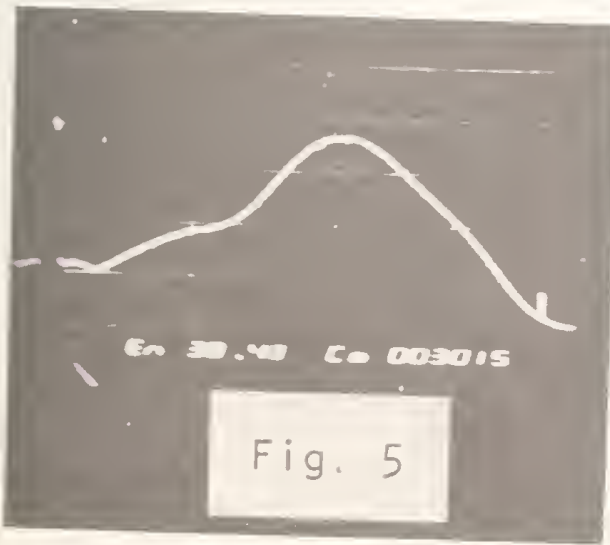

Typical spectrum from a dental diagnostic source assembly derived from the analysis of a highly filtered $x$-ray beam
Method $\equiv 4$

$\mathrm{kVp}$ measurement of an $\mathrm{x}-\mathrm{ray}$ beam by analysis of the scatter spectrum of a known pure element

The apparatus (seen in Fig. 6) is relatively simple; the $\mathrm{x}$-ray beam was highly collimated by a lead collimator, it then passed through a thick lead aperture which allows a beam to exit which is slightly Known Absorber Whose Atomic Number is one higher than the scatterer (used in Method (15)
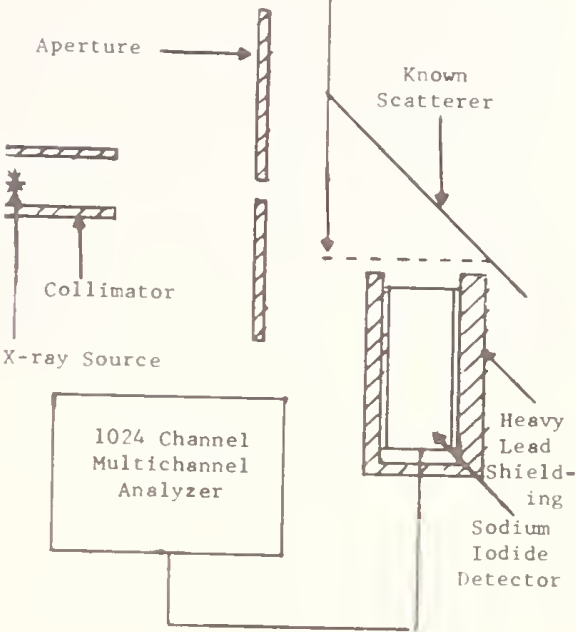

Fig. 6

Block diagram of the apparatus used in Methods 非 4 and 非5. The additional filter is not used in Method 非4.

smaller than the cross section of the scatterer that the beam sees. The scatter spectrum was. then detected by a sodium iodide detector which was connected to the 1024 channel multichannel analyzer. The scatterer was an ultra-pure foil and has a " $K$ " line near the indicated $k V p$ being meas-
ured.

For this experiment, the following scatterers were chosen:

1. $50 \mathrm{kVp}$ - Terbium has a $\mathrm{K}_{\beta}$ of $50.391 \mathrm{keV}$.

2. $70 \mathrm{kVp}$ - Platinum has a $\mathrm{K}_{\beta}$ of $75.736 \mathrm{keV}$.

3. $90 \mathrm{kVp}$ - Lead has a $\mathrm{K}_{\mathrm{ab}}$ of $88 \mathrm{keV}$.

Each line has a width so a reasonable variation from the nominal $\mathrm{kVp}$ can be deter- 
mined.

The apparatus was calibrated by radioactive isotopes before and after an analytical run was made to collect the scatter spectrum. The isotopes were chosen so that their gamma emission lines bracketed the nominal kVp.

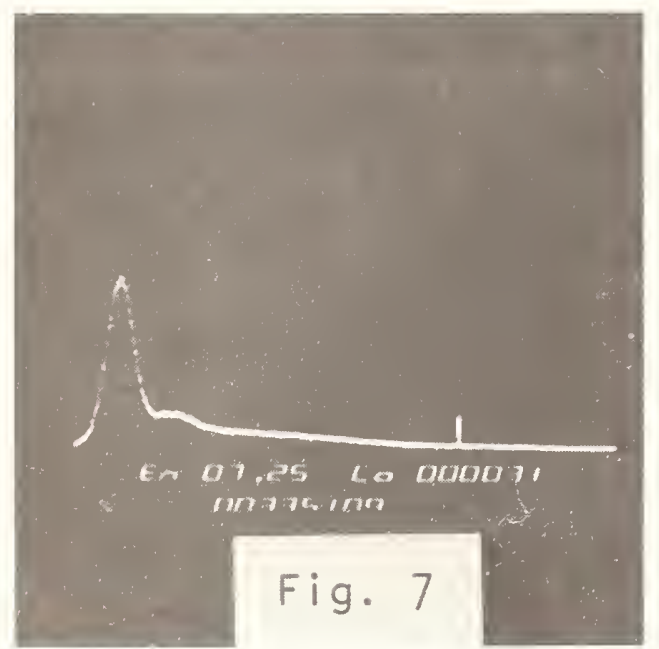

Typical scatter spectrum from platinum generated by a $68 \mathrm{kVp}$ $\mathrm{x}-\mathrm{ray}$ field

Due to the strength of the " $\mathrm{L}$ " and " $\mathrm{M}$ " lines of the scatterer, the higher energy "K" lines were obscured.

Fig. 7 is a picture of the spectrum given off by this scatterer (platinum in this case) in the region of $70 \mathrm{kVp}$. The unit was subsequently measured at $68 \pm 0.9 \mathrm{kVp}$, however, in the best judgement of the experimenter the $\mathrm{kVp}$ was determined to be $73 \pm .5 \mathrm{kVp}$ with Method 非4. The $68 \mathrm{kVp}$ figure was verified by both Method 非 5 and Method 非1.

A method was sought to enhance the " $\mathrm{K}$ " line radiation or reduce the " $L$ " line radiation. The solution became obvious and Method 非 5 was tried. Method 非 4 was discarded due to the fact that the information desired was buried in the noise level.

\section{Method 非 5}

$k V p$ determination of a dental diagnostic source assembly by analysis of the scatter spectrum of an ultra-pure foil which is filtered by another pure foil whose atomic number is one greater than that of the scatterer.

The apparatus for this method is depicted in Fig. 6. The $\mathrm{x}$-ray beam exited from the collimator and passed through the aperture which allowed a stream of photons through which had dimensions equal to the projected dimensions of the scattering foil. The scattering foil was angled at $45^{\circ}$ to the central ray of the system. The axis of the scintillation detector passed through the center of the scatterer and was orthogonal to the central ray. If desired, the axis of the detector may be orthogonal to the surface of the scatterer to gain in spectrum intensity, but the surface of the absorber should be perpendicular to the axis of the scintillation probe. The scatterer/absorber pairs chosen for this experiment were:

1. $50 \mathrm{kVp}$ - Terbium/Dysprosium.

2. $70 \mathrm{kVp}$ - Platinum/Gold.

3. $90 \mathrm{kVp}$ - Lead/Bismuth.

The $70 \mathrm{kVp}$ materials were chosen because these materials are commonly found in the pure form. The $90 \mathrm{kVp}$ materials were chosen because they were the closest non-radioactive substances that could be found.

Fig. 8 is a spectrum obtained by analyzing the filtered scatter spectrum from a diagnostic source assembly. This is the same diagnostic source assembly that produced the spectrum of Fig. 7. The difference lies in the fact that the spectrum of Fig. 7 was further filtered by a gold foil filter to obtain Fig. 8. It can be seen that less experimenter judgement is needed to determine the $\mathrm{kVp}$ than in the previous two (2) methods.

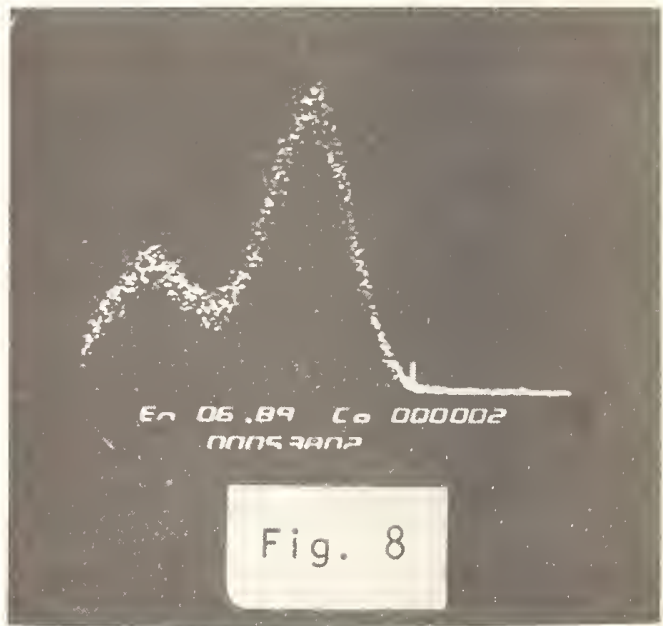

Same spectrum as Fig. 7 except the scatter spectrum was further filtered by a gold foil 
The positive aspects of this type of measurement are:

1. Less room for experimenter errors in judgement.

2. This method does not disturb the electrical system of the $x$-ray machine.

3. The diagnostic source assembly does not have to be disassembled to gain access to the high voltage areas.

4. The measurement of $\mathrm{kVp}$ is independent of secondary waveform.

The disadvantages of this method are:

1. The equipment is not portable for field use.

2. The length of time needed for a complete analytical run ranges from 1-6 hours depending upon the $x-r a y$ system's duty cycie. This method is impractical as a quality control method in a high volume production situation.

\section{Conclusions}

A comparison of the results obtained by the above methods are expressed in Table $\#_{1} 1$. It can be seen that Methods $\neq 1$ and $\# 5$ agree

\begin{tabular}{|c|c|c|c|}
\hline Method & 70 & $\mathrm{kVp}$ & $90 \mathrm{kVp}$ \\
\hline$* 1$ & 68 & $\mathrm{kVp}$ & $k V p$ \\
\hline$\neq=2$ & 69 & $k V p$ & $88.6 \mathrm{kVp}$ \\
\hline 非 & 67 & $k V p$ & $89.8 \mathrm{kVp}$ \\
\hline$\$ 4$ & 73.5 & $\mathrm{kVp}$ & indeterminate \\
\hline$\# 5$ & 68 & $k V p$ & $91.5 \mathrm{kVp}$ \\
\hline
\end{tabular}

Table 拈

A set of typical values for the inter-comparison of methods discussed in this paper rather closely with each other. The differences are mainly attributed to the loading effects of the high voltage divider and the line width of the oscilliscope trace. other discrepancies are attributed to the effects of a low signal to noise ratio and errors in operator judgement because of the high noise levels.

Research will continue to obtain a portable and/or fast response $\mathrm{kV}$ measurement system that can measure the kVp accurately in the field and on the production line.

\section{Bibliography}

1. Clark, G. L., ed., "The Encyclopedia of $\mathrm{X}$-rays and Gamma Rays," Reinhold Publishing Corp., New York (1963)

2. Green, M., Cosslett,V. E., "The Efficiency of Production of Characteristic X-Radiation in Thick Targets of a Pure Element," Proc. Phys. Soc. LXXVIII, 6 (1961)

3. Richards, A. G., et al, "Samarium Filters for Dental Radiography," Oral Surgery (May 1970)

4. Richards, A. G., private communication, (1974)

5. Rothenberg, L. N., Williams, D. J., "Compliance Test Methods for Diagnostic Medical X-ray Equipment," Vo1. I, Vol. II (June 1973)

6. U. S. Department of Health, Education and Welfare, "Draft of Proposed Test Methods for Determining Compliance with the diagnostic $\mathrm{X}$-ray Equipment Performance Standard (42CFR78.213-1, 78.213-2, 78.213-3)"

(March 1972) 



\author{
NATIONAL IONIZING RADIATION STANDARDS \\ James E. Leiss \\ National Bureau of Standards \\ Washington, D. C. 20234
}

Ionizing radiation in its many forms is used in an increasing number of ways to help solve important problems of our technological society. As these applications become more mature, the needs for reliable radiation measurement continue to increase, both to achieve the maximum benefit from the radiation use and also to assure that these applications are carried out safely. To meet these measurement needs, the Mational Bureau of Standards provides a wide variety of measurement standards and services. New standards are also being developed to meet a variety of new needs.

It is recognized that these radiation measurement standards by themselves are of limited usefulness unless they can be related to measurements made at the user level. Various mechanisms exist for this, including measurement calibrations, measurement traceability testing, and measurement assurance programs. The National Bureau of Standards carries out an increasing number of activities of this type. In some cases new or augmented institutional arrangements within the national measurement system for ionizing radiation appear necessary.

(ionizing radiation, standards, measurement assurance, radiation users)

\section{Introduction}

The history of x-rays and of ionizing radiation in general is a remarkable example of rapid technology transfer from the basic physics discovery to practical applications. Almost from the day of their discovery in 1896 the value of x-rays for visualizing the interior of the human body was recognized and applied in the medical profession. Within about five years of their discovery $x$-rays were used to assist doctors in the setting of broken bones, locate tumors, relieve the suffering of cancer, and "cure" a variety of human ailments. Many of these applications, of course, were not really successful and some of them are only in very recent years reaching the point of significant success.

The harmful effects of ionizing radiation were also recognized very early in the history of $x$-rays, mostly because of the severe skin burns received by some of the early radiation workers. Thus ionizing radiation is a classic example of new scientific discovery which has led to very important benefits for mankind, while at the same time carrying significant risk to man if not used carefully and intelligently. The problem of balancing these benefits and risks continues today in the national debate regarding nuclear power plants and in the debate over $x$-ray screening of women for breast cancer.

Our conference title, "Measurements for the Safe Use of Radiation," is thus very timely since it relates directly to one as- pect of this important benefit-risk problem. Reliable and accurate measurement relates directly to achieving benefits from the use of ionizing radiation, and also is essential if meaningful measurements are to be made to protect the public from undue harmful effects. As uses and the effects of ionizing radiation become better understood, this need for accurate and reliable radiation measurement will become increasingly important.

The use of ionizing radiation within our society is widespread and applications are growing. One categorization of these applications which we find useful in thinking of our programs at NBS is given in Table I where I have tried to identify radiation applications by their relationship to important national interests.

TABLE I. Ionizing Radiation Application Areas

- Health and Public Safety

- Energy and Environment

- Industrial Productivity

- National Defense

- Basic Measurement Science

Our conference this week is devoted primarily to the first of these areas, Health and Public Safety. NBS, howeyer, must attempt to provide appropriate measurement standards and measurement assistance to all of these applications. What I hope to do in this paper is to give you at least a partial look at some of our activities related to all 
of these radiation applications. I will attempt to do this by taking you on a brief tour of some of our facilities and some of the activities carried out at these facilities. Some of the other speakers from our laboratory have spoken on some of these same activities; however, my objective is to give you an overview, particularly of some of our ionizing radiation measurement standards and of measurement techniques which are being developed. I will also try to briefly relate these activities to why we are doing the work.

Dr. Caswell in his paper has discussed "The Stardards Laboratory and the Radiation Measurement System." This is primarily a look at the national radiation measurement system and how we at NBS appear to relate to this system. Without too much overlap with his presentation, I also will discuss some of the activities at NBS related to radiation measurement calibrations and measurement assistance to the radiation user community as well as some of the problems which we perceive with the present operation of this national measurement system.

\section{NBS Radiation Laboratories and Activities}

Although ionizing radiation is used extensively in many different activities within the National Bureau of Standards, the responsibility for ionizing radiation standards and measurement science is located within the Center for Radiation Research (CRR). Most of the activities of the Center are located in the Radiation Physics Building. An aerial view of this laboratory is shown in Fig. 1. The large stack is for exhaust air from our 1 inear accelerator complex, and the horizontal pipes going out from the building (up to 200 meters) are evacuated flight tubes for neutron time-offlight measurements. A number of different radiation sources are located within this building, and are required for the many different types of radiation standards and services which we provide. A list of some of these radiation sources is given in Table II. Some of our activities using these sources will be discussed below.

\section{TABLE II. CRR Radiation Facilities}

- 0.5-, 1.5-, 4-, and 150-MeV high intensity accelerators.

- 250-MeV electron storage ring (for synchrotron 1ight).

- 3-MeV Van de Graaff (used as a neutron source).

- 2-MeV and 600-keV flash x-ray machines.

- Five constant potential $x$-ray machines (50-250 kV).

- Various gamma, beta, and alpha radiation sources.

- Isotope separator.

NBS also has a 10 megawatt research reactor which is operated by the Reactor $\mathrm{Ra}-$ diation Division in the Institute for Materials Research. The Center for Radiation Research is an active user of this facility in part of our neutron measurement standards programs. A view of the NBS reactor is shown in Fig. 2.

One of our users of the reactor is in the area of reactor dosimetry to support the growing nuclear power industry. Fig. 3 is an artist's view of an intermediate energy standard neutron field (ISNF) which has been developed within the graphite thermal column of the reactor. A spherical cavity has been created within the thermal column with small fission plates on the surface. This creates a nearly pure fission neutron spectrum within the cavity. An inner spherical shell of B-10 moderates this neutron spectrum to a spectrum similar to that of a breeder reactor. Instruments or activation foils being calibrated can be located as shown within this inner region (inside the B-10 she11).

The instrument shown at the center of Fig. 3 is shown in more detail in Fig. 4. It is a double fission chamber developed at NBS for reactor dosimetry and reaction rate measurements. This instrument can operate in a wide variety of environments, including those within an operating reactor. Duplicates of this instrument are now used in many reactor research centers throughout the world.

A second use which we make of the reactor is for the production of low-energy monoenergetic filtered neutron beams. These beams and their use to calibrate neutron personnel monitors were discussed by $\mathrm{Dr}$. Schwartz in his paper, "Filtered Neutron Beams at the NBS Reactor." We are now working actively with a number of ERDA sponsored laboratories to develop improved personnel monitoring and calibration techniques using these beams. 
The largest of our radiation facilities within the Radiation Physics Building is the $150 \mathrm{MeV} 7$ inear electron accelerator shown in Fig. 5. This facility produces high intensity electron beams from 10-150 MeV which can be directed into a number of different experimental areas. Some of the magnet assemblies used to direct the beam to different areas are shown in Fig. 6. This was one of the first fully achromatic beam switching systems ever developed and has contributed greatly to the ease with which such accelerators can be applied to a diverse research program. I wish to discuss three of our applications of this accelerator, primary beam monitoring standards, calorimetric dosimetry standards for electron beam cancer therapy, and measurement of standard reference neutron cross sections.

Before the advent of modern linear accelerators most high energy electron accelerators were of relatively low current and used primarily for production of bremsstrahlung beams. Beam measurements were done mostly with ionization chambers. The NBS P-2 ionization chamber, shown in Fig. 7, was developed as a primary beam monitoring standard for such accelerators. This NBS P-2 chamber has been duplicated by many national standards laboratories throughout the world, and is still the primary standard in many countries. Faraday cup monitors are used with modern high intensity accelerators (external electron beams), to collect beam charge. Fig. 8 shows the IIBS primary electron beam standard. This is a Faraday cup collector which will operate reliably up to $100 \mathrm{~kW}$ of average beam power. We have also developed non-intercepting monitors for measurement of these high energy electron beams using ferrite transformers. Fig. 9 shows that we know the performance of our primary standard to about $500 \mathrm{ppm}$. Only the most exacting of physics research activities need such measurement accuracy and we now only maintain this standard.

High energy electron beams are used extensively in cancer therapy. As discussed by several speakers during this conference, reliable radiation dosimetry is essential for successful cancer radiation therapy. Fig. 10 shows an experimental arrangement at the NBS linac for development of calorimetric absorbed dose standards. The large wheel shown in the figure contains a variety of carbon absorbers used to obtain absorbed dose measurements at different depths in the carbon medium. This apparatus contains both a calorimeter which measures absorbed dose direct$1 y$ and an ionization chamber of nearly identical geometry. The objective in the use of this apparatus is to measure the relative response of the calorimeter and of the ionization chamber, and to compare this experimental ratio with theoretical calculations. Fig. 11 shows one such comparison for $25 \mathrm{MeV}$ electrons. Agreement between experiment and theory is within one percent. We have also developed a portable calorimeter which is suitable for field use. This instrument, shown in Fig. 12, has been successfully used in the field. Recently it was carried to the Saclay laboratory and compared with the French national standard using Co-60 radiation, with agreement to within a few tenths of a percent.

The safe and economical development of nuclear power plants, especially the breeder reactor, requires a vast amount of accurate nuclear data. Most measurements of such nuclear data are actually measurements of the ratio of an unknown cross section to some reference cross section. Thus the data files which are used in reactor design can only be as good as the reference cross sections. There is great need to improve and verify the accuracy of these reference cross sections. At the present time the major use of our linear accelerator is as a pulsed neutron source. Using time-of-flight (see Fig. 1) to measure neutron energy, we have an active effort underway to accurately measure a set of standard reference neutron cross sections, especially in the energy region of greatest interest to breeder reactors and fusion reactors. The energy dependence of some of these standard reference neutron cross sections is shown in Fig. 13. For neutron energies below one MeV the accuracies we have achieved at present are about 3 percent. Our target goal is an accuracy of 1 percent.

A primary standard of neutron source strength which has been maintained at NBS for a number of years is the $\operatorname{Ra}-B e(\gamma, n)$ neutron source shown in Fig. 14. Both this source and unknown'sources which are sent to NBS for calibration are measured in a manganous sulfate bath. The arrangement is shown schematically in Fig. 15. Most of the neutrons are captured by $M n-55$ to form $M n-56$. The radioactive decays from $M n-56$ are detected with scintillation counters. One special source at NBS which has been calibrated in this manner is a specially encapsulated Cf-252 source shown in Fig. 16. The postage stamp gives a size comparison and Fig. 17 shows an x-ray of the source. The small dark spot in the center is the Cf-252 material, very nearly a point neutron source. One example of the use of this CF-252 source is shown in Fig. 18, in which two of the NBS double fission chambers shown in Fig. 4 are placed symmetrically around the CF-252. Through the chain of measurements indicated 
here and in the previous discussion, we have established a direct measurement chain from reaction rates and dosimetry within nuclear power reactors to the primary neutron source standards.

Within the areas of national defense, industrial radiation processing, and con-. trolled thermonuclear research there is continuing need to make radiation measurements under conditions of extremely high radiation intensity, and often in low pulse repetition rate situations. Fig. 19 shows one of the radiation sources used in developing standards for such radiation applications. It is a $2 \mathrm{MeV}, 5000$ ampere pulsed accelerator with beam pulse length of a few nanoseconds. Either $x$-rays or the direct electron beam from the accelerator can be used. One example of how this accelerator is used is indicated on the left side of Fig. 20. When electrons strike a target material, the bremsstrahlung production goes primarily in the forward direction. However, the production of $K x$-rays is nearly isotropic. Thus by looking in the backward direction, beams of nearly monoenergetic $K x$-rays are available and can be used for instrument calibrations. Using such sources we have provided calibrations for weapons testing programs for several years, and are now providing such calibrations to laboratories in the controlled thermonuclear research program. We also use this radiation source to investigate the rate dependence of radiochromic dye dosimeters used in industrial processing. Fig. 21 shows the change in optical density of solutions of these dye dosimeters for different absorbed dose levels. The dye dosimeters developed at NBS are remarkably dose rate independent.

A last dosimetry technique which has been developed using these high current pulsed accelerators is indicated in Fig. 22. This figure shows the interference fringe pattern of laser light produced when one beam burst from the accelerator passes through a liquid cell in one arm of a two arm interferometer. The fringes are a result of the radiation heating of the liquid cel1. This technique has the great advantage that the absorbed dose measurement is absolute, knowing the heat capacity of the liquid and the change of the optical index of refraction with temperature. It has the further advantage that the entire depth dose distribution is obtained with one radiation burst, which is very important in some cases.

In more conventional radiation calibration activities, $x$-ray and Co-60 calibration ranges are utilized to calibrate ionization chamber instruments for medical radiation monitoring applications. Fig. 23 shows a view of an x-ray calibration range, which is now almost completely automated. Fig. 24 shows a Co-60 calibration range. For x-ray calibrations a free-air chamber is the primary reference standard. For Co-60 the reference standards are graphite cavity ionization chambers. A number of these graphite cavity chambers are shown in Fig. 25.

An active area of standards development within CRR is that of radioactivity standards. These standards are needed for nuclear medicine, scientific research, instrument manufacturers, and environmental monitoring. Most of the radioactivity standards which we produce are distributed by NBS through the Standard Reference Materials program. We also have substantial involvement in measurement assurance testing programs relating to radioactivity measurements. Some examples of these programs are discussed later in this report.

Fig. 26 shows a photograph of some of the radioactivity standards which we develop. Included are liquid and gaseous sources at different activity levels, point sources, and sources which have been developed for specific applications. An example of this latter case is a series of mixed radionuclide sources which simulate the type of radiation measurement problem encountered in monitoring the radioactive effluent from nuclear power plants. These sources are now in use by most operating nuclear power plants in the United States for calibration and testing of their instruments and spectrum analysis programs.

Some examples of the apparatus used in the development of these radioactivity standards is given in the next few figures. Fig. 27 is a view of the high resolution isotope separator operated by the Radioactivity Standards Section. Ore example of the use of this facility in standards development is indicated in Fig. 28. Krypton gas is passed through the isotope separator and imbedded in pure aluminum foil as shown in this figure. The $\mathrm{Kr}-84$ component of this is cut-out and irradiated in the NBS reactor. Through the neutron capture process in $\mathrm{Kr}-84, \mathrm{Kr}-85$ is formed, resulting in an isotopically pure source of $\mathrm{Kr}-85$. Such sources are valuable in environmental monitoring of the $\mathrm{Kr}-85$ release from nuclear power plants. A second piece of apparatus useful in development of environmental standards is shown in Fig. 29. This is a two-crystal high-efficiency, low background scintillation spectrometer. The arrangement of this spectrometer is shown in Fig. 30. One great advantage of this spectrometer is that its response is nearly in- 
dependent of sample size and shape. For complex spectra the energy resolution of this spectrometer is not adequate and high-resolution germanium spectrometers must be utilized. Fig. 31 illustrates such an example, the gamma ray spectrum from Columbia River sediment. This material has been prepared as Standard Reference Material and the activity of many of the nuclides present has been certified.

For the more conventional radioactivity standards more conventional apparatus is used. Fig. 32 shows some of the gas handling apparatus used in the preparation of gaseous radioactivity standards. Fig. 33 shows portions of a beta-gamma coincidence apparatus used in checking the activity of standard sources. This apparatus is highly automated so that many standard sources can be measured economically.

\section{Measurement Dissemination Activities}

In the above discussion I have tried to give an overview of many needs for NBS standards which result from the diverse applications of ionizing radiation in our society. I would like now to discuss the difficult problem of getting the output of our work to the radiation users. For many of our activities the need is to provide standards for a specialized type of measurement to a relatively small community of users. Getting our services to these groups is not too difficult. Where we experience our greatest problems is with large diverse user groups.

The various measurement dissemination mechanisms which we now use are listed in Table III.

TABLE III.

NBS Standards Dissemination Mechanisms

- Calibrations

- Standard Reference Materials

- Publications

- Measurement Traceability Testing

- Measurement Assurance Programs

The first of these, calibrations, is usually only applicable to a general situation where there is a common calibration need of a relatively large users group. There is also the problem that calibrations of an instrument for a user really provides little assurance that the user is making measurement adequate to his needs. The various ionizing radiation calibrations formally offered by NBS are listed in Table IV. In addition to these, many calibrations are provided on an individual basis.
TABLE IV.

NBS Calibration Services in Ionizing Radiation

- Neutrons

-- Neutron source strength

-- Neutron instrument (thermal neutrons)

-- Neutron irradiation of foils

- Radioactivity

-- Gamma-ray emitters

-- Alpha particle emitters

-- Beta-ray emitters

- Radiological equipment

-- $X$ and gamma ray equipment

-- Gamma ray sources

-- High energy electron beam dosimetry

-- Thin film dosimeters (high dose)

- Many special calibrations upon request

Standard Reference Materials offered by NBS in the ionizing radiation area are radioactivity standards, and essentially all of our radioactivity standards are available in this manner. These also have a difficulty in that purchase of SRM's provide no assurance that sufficiently accurate measurements are actually being made in the field.

The measurement dissemination mechanisms which appear most valuable and are increasingly being employed by NBS are those of Measurement Traceability Testing and Measurement Assurance. In essence these could be called Measurement Quality Control programs. Table $\checkmark$ lists a number of traceability and measurement assurance programs which have been conducted recently. I wish to discuss three of these as examples.

TABLE V. NBS Measurement Assurance and Traceability Testing Programs

- Radiopharmaceutical Users (CAP)

- Radiopharmaceutical Producers (AIF)

- Radioactivity Reference Standards (ANSI)

- Dose Calibrators (CAP)

- Environmental Radioactivity (Users)

- Measurement Traceability Testing (NRC, EPA, FDA)

- Nuclear Fuel and Reactor Dosimetry (ILRR)

- Radiation Hardness Testing (ASTM).

- Personnel Monitors (Pilot Test)

- Brachytherapy Source Dosimetry (Pilot Test)

- Electron Therapy Dosimetry 
The first example of measurement assurance testing is in the area of environmental radioactivity monitoring. In this program mixed-radionuclide standards are offered to users by NBS. These are initially distributed to purchasers without the calibration certificate. After the purchaser measures the content of this standard and reports his observations to NBS, the calibration certificate is sent to him. This type of test is extremely valuable. It allows a normal calibration standard to serve the dual purpose of standard and mechanism for measurement assurance testing. The results of one such test are indicated in Fig. 34.

A second example of a measurement assurance test is a program conducted with the College of American Pathologists (CAP). In this case, samples of I-125 were distributed by NBS as unknowns to the participants. The participants measured the sources with their dose calibrators, identified the dose calibrator model, and reported their findings to NBS. The results of this test are shown in Fig. 35. Two significant facts are apparent from this test. First, in no single case did a participant's measurement come within the accuracy limits recommended by the U.S. Pharmacopeia. Second, there is clearly a bias of almost a factor of two in the readings of dose calibrators from one particular manufacturer. The problem was found to be an error essentially in dial calibration, and was reported to the manufacturer.

The third example is in the area of measurement assurance programs for electron therapy dosimetry. Fig. 36 shows the results of measurement assurance testing by NBS for a 9-year period. Chemical dosimeters are distributed to participants by NBS. The participant irradiates the dosimeter in a phantom according to a prescribed protocol, sends the irradiated dosimeter back to NBS with his measure of the absorbed dose given to the dosimeter. NBS in turn sends back to the participants the actual dose indicated by our reading of the dosimeter, the distribution of performance by all participants, and suggestion that corrective action be taken if appropriate. The results of this measurement assurance program indicate that a substantial fraction of participants lie outside of the accuracy $1 \mathrm{im}-$ its normally considered desirable for radiation therapy, and furthermore the situation has not appreciably improved during the nine years that the program has been in operation. Clearly many of the participants do not use the information available to them from this measurement assurance program.
Problems of the National Measurement system for Ionizing Radiation

The examples given above of the results from NBS measurement assurance testing programs clearly indicate the great value of such programs in evaluating radiation measurement performance at the user level, and the value that such programs can have in identifying problems. These programs unfortunately clearly reveal that many users are not making measurements at an accuracy which is considered generally desirable. It is also clear, and best illustrated in Fig. 36, that users often do not take corrective measures even when a measurement assurance test clearly reveals the need for corrective action.

In attempting to understand this situation we have concluded that there are a number of specific problems which exist within the national measurement system for ionizing radiation. These are the following:

- Lack of user recognition of need.

- Need to document accuracy goals and procedures.

- Lack of adequate national measurement dissemination system.

- Lack of follow-up and corrective action.

- Some standards do not exist.

- Inadequate resources at all levels.

We believe that the challenge to the national measurement system is clear. It is to achieve adequate and assured radiation measurement accuracy at the user level. I believe that the evidence presented in this paper and in other papers of this conference clearly indicate that in some areas this goal is not being achieved at the present time. A number of corrective actions which we believe would help this situation are the following:

- Develop concensus documents of accuracy needs.

- Organize national measurement assurance programs with follow-up procedures, including substantially complete user participation.

- Establish educational programs where appropriate.

- Develop missing standards for the cases where they do not exist.

- Recognize adequate measurement accuracy as a legitimate business cost.

Above all else, it is necessary for us in NBS and the user community to recognize that we each have a role to play in making the national measurement system work effectively, and for each of us to effectively carry out our appropriate role. 


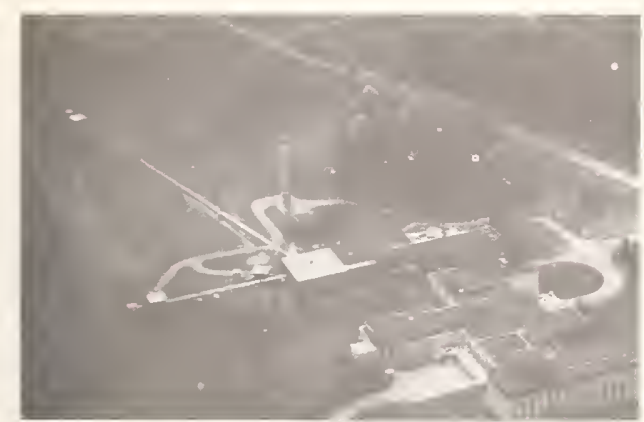

Fig. 1 Aerial view of NBS Radiation Physics building. The long horizontal pipes (up to 200 meters) are evacuated neutron flight paths for neutron standard cross section measurements.

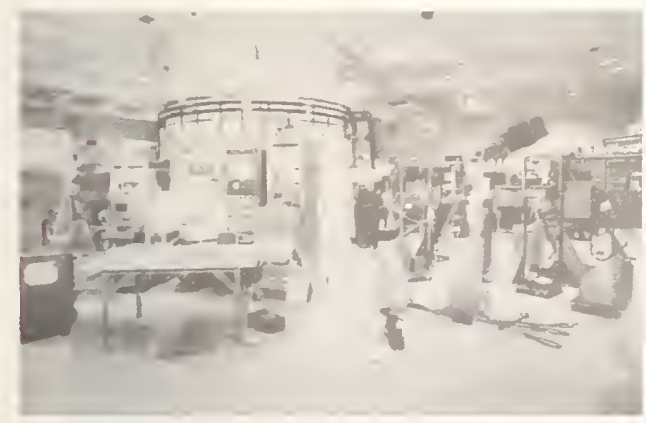

Fig. 2 View of NBS 10-megawatt reactor.

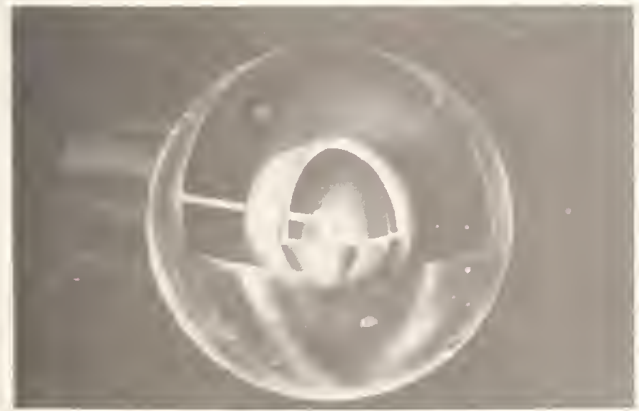

Fig. 3 Artist's view of Intermediate-Energy Standard Neutron Field (ISNF). This calculable reference field matches the typical spectrum within a breeder reactor.

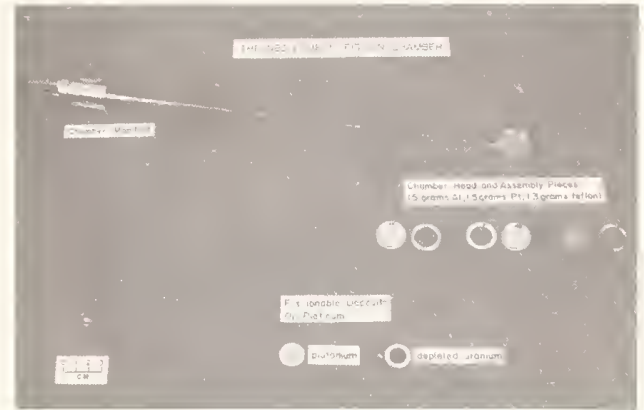

Fig. 4 NBS double fission chamber. This standard instrument is now in use in reactor research laboratories throughout the world.

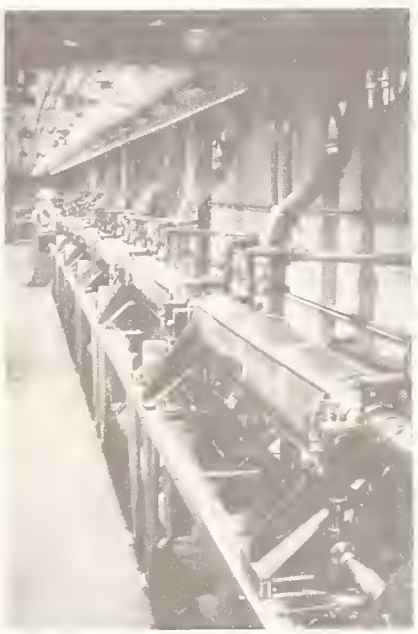

Fig. 5 View of NBS 150-MeV linear electron accelerator.

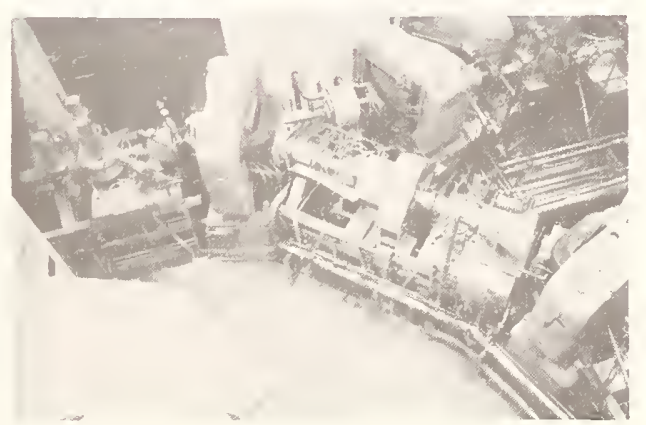

Fig. 6 Beam handling system which deflects the NBS linac beam into different experimental areas. 


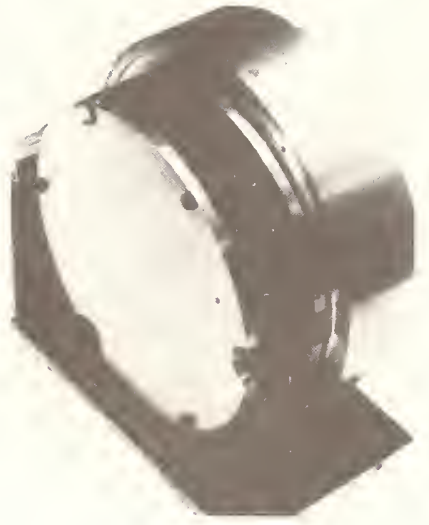

Fig. 7 NBS P-2 ionization chamber, a primary standard instrument for measurement of low intensity high-energy bremsstrahlung beams.

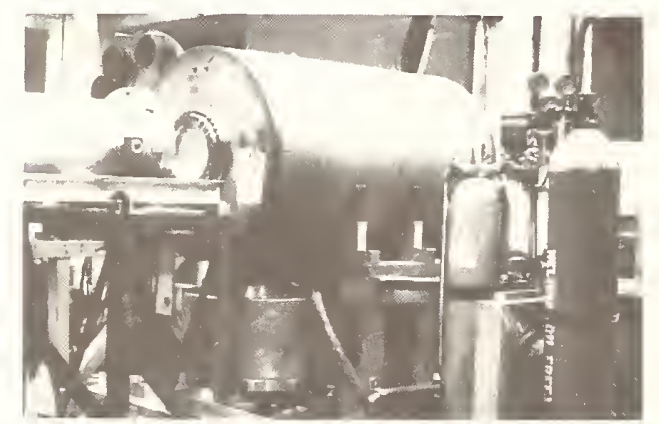

Fig. 8 NBS primary standard Faraday cup for measurement of high-energy electron beams.

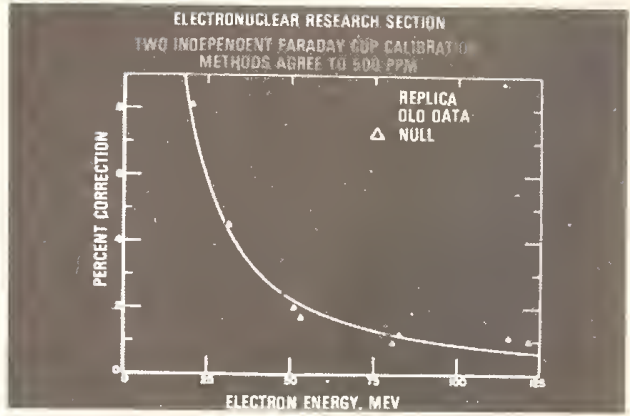

Fig. 9 Indication of accuracy of NBS Faraday cup. Independent calibration is provided by an absolute non-intercepting current transformer monitor. The correction indicated is largely due to backscattered electrons from the Faraday cup.

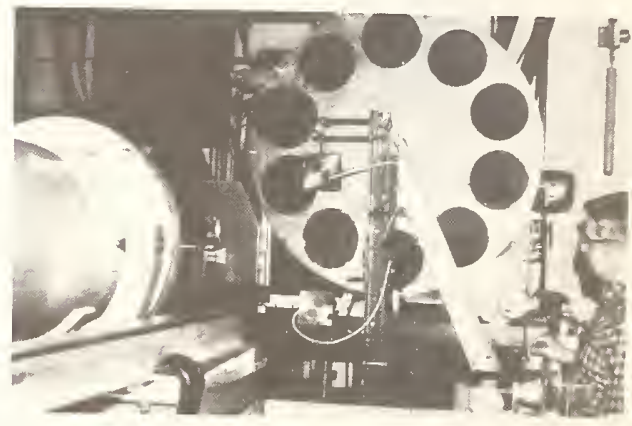

Fig. 10 Apparatus for development of absorbed dose standards for electron therapy. Apparatus is located in one of the electron 1 inac measurement rooms. 


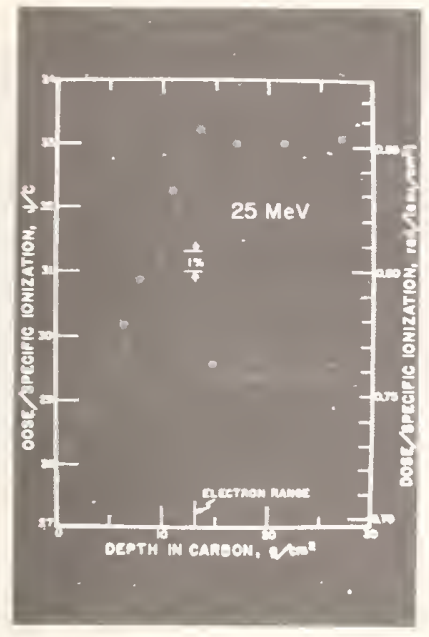

Fig. 11 Ratio of absorbed dose as calorimetrical1y measured to specific ionization observed in an ionization chamber. The theoretical curve was calculated by Berger and Se]tzer.

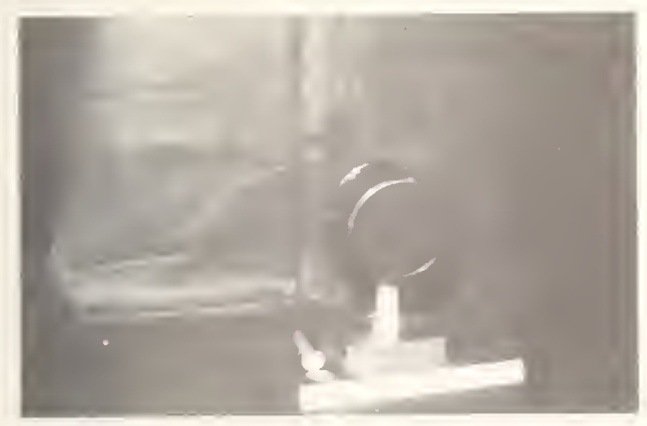

Fig. 12 Portable calorimeter for absorbed dose measurements in field applications.

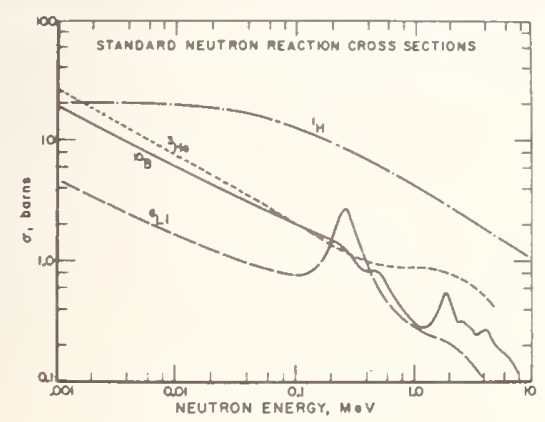

Fig. 13 Energy dependence of several standard reference neutron cross sections. Most neutron data used in nuclear power development is measured relative to such reference cross sections.

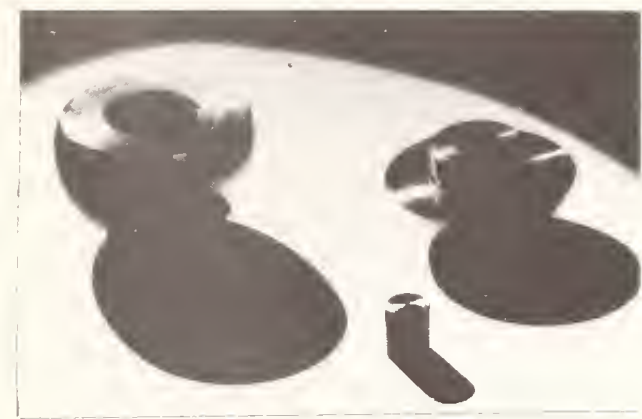

Fig. 14 NBS primary standard neutron source (NBS-1). This Ra-Be $(\gamma, n)$ source is the U.S. primary standard.

\section{MANGANOUS SULFATE BATH SOURCE CALIBRATION}

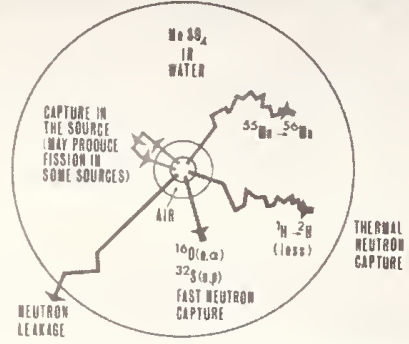

Feb. 15 Schematic illustration of the physical processes which must be considered in the manganous sulfate bath technique for neutron source calibration. Although capable of absolute source calibration, it is usually used to obtain the ratio of the strength of an unknown source to a standard source. 


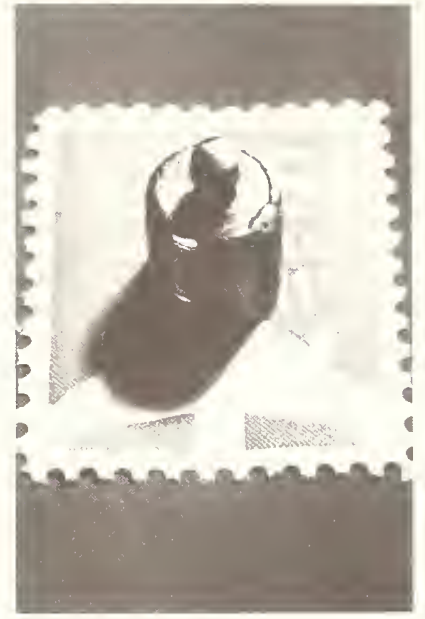

Fig. 16 View of NBS specially encapsulated cf-252 neutron source. The postage stamp gives an indication of the actual size of this source. The strength of this source has been measured relative to NBS-1 using the manganous sulfate bath technique.

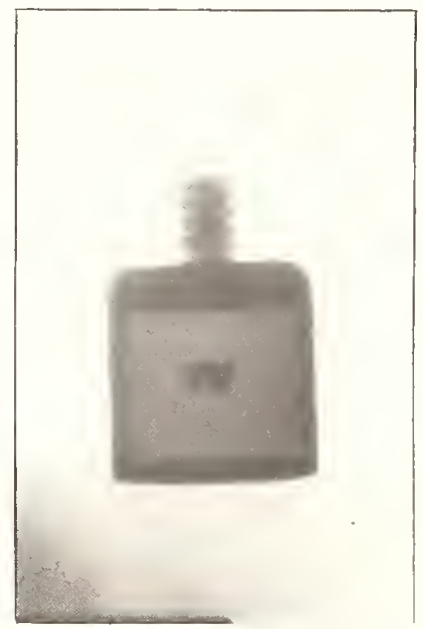

Fig. 17 X-ray of NBS Cf-252 neutron source. The dark spot in the center is the actual neutron emitting material, and closely approximates a point source.

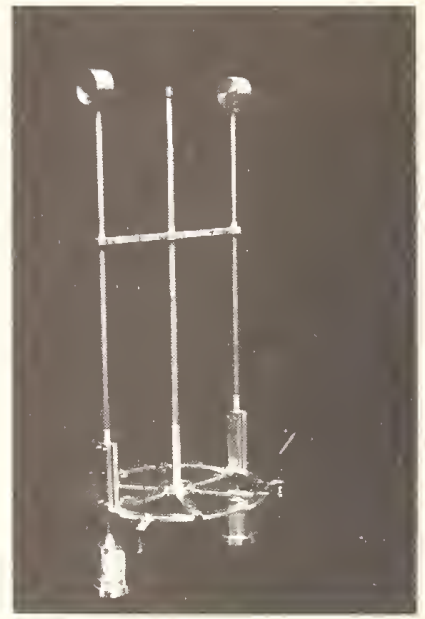

Fig. 18 Arrangement of NBS double fission chambers with Cf-252 neutron source for determination of absolute fission chamber response to $\mathrm{Cf}-252$ neutrons.

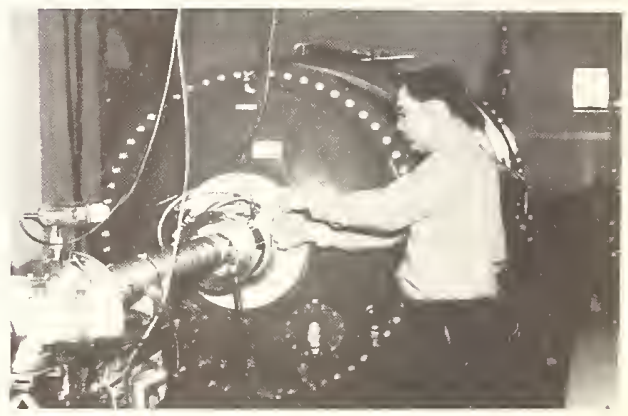

Fig. 19 View of 2-MeV flash x-ray machine. This accelerator is capable of 5000 ampere current with few nanosecond pulses. The apparatus on the left is used for calibration of detector response to low energy $K$ x-rays. 


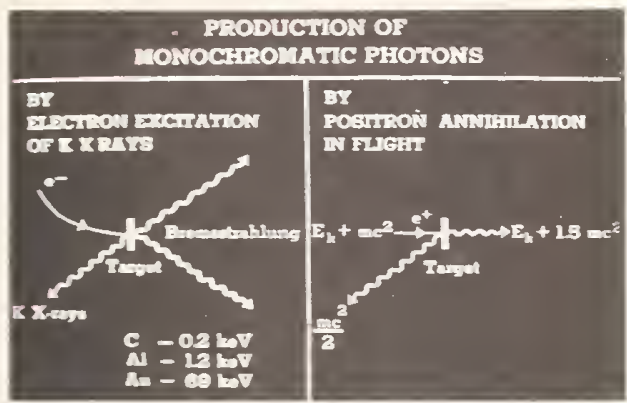

Fig. 20 Arrangements used by NBS to produce monochromatic photon bears. At the left, $K$ $x$-ray beams largely free of bremsstrahlung are produced by viewing targets at large angles. At the right, positrons produced in the NBS 7 inac are annihilated in flight to produce nearly monochromatic beams in the forward direction.

\section{IIIIII}

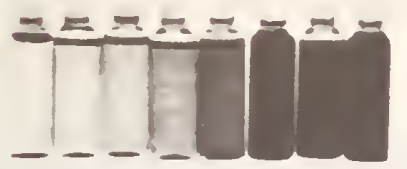

KILORADS

$\begin{array}{llllllll}0 & 5 & 10 & 15 & 25 & 50 \quad 75 & 100\end{array}$

Fig. 21 Response of radiochromic dye dosimeters to different radiation doses. These dosimeters have been shown to be nearly dose rate independent over a wide range of dose rates.

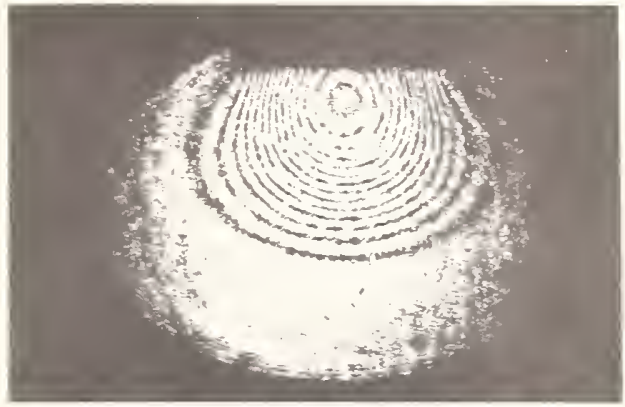

Fig. 22 Interference fringes produced in laser Tight by radiation dose heating of liquid in one arm of a double arm interferometer. The electron beam enters from the top. Knowing the change of optical path length with temperature and the heat capacity of the liquid, absolute dosimetry is achieved by counting interference fringes.

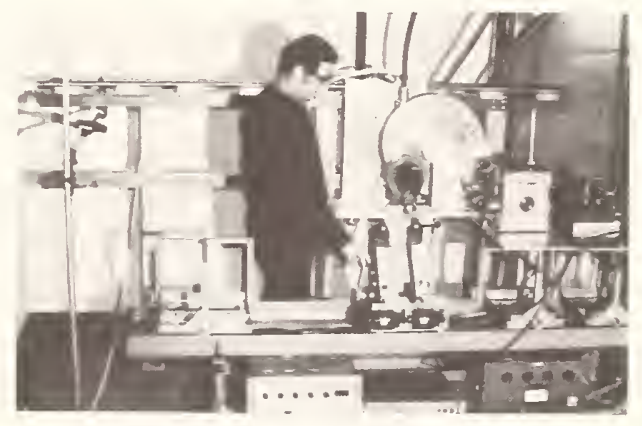

Fig. 23 View of $x$-ray exposure calibration range. Operation of these calibration activities is now largely automated. 


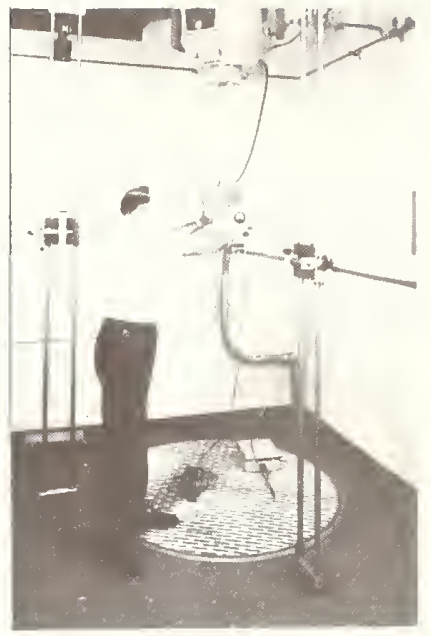

Fig. 24 View of Co-60 calibration range. A radiation survey instrument is being mounted for calibration.

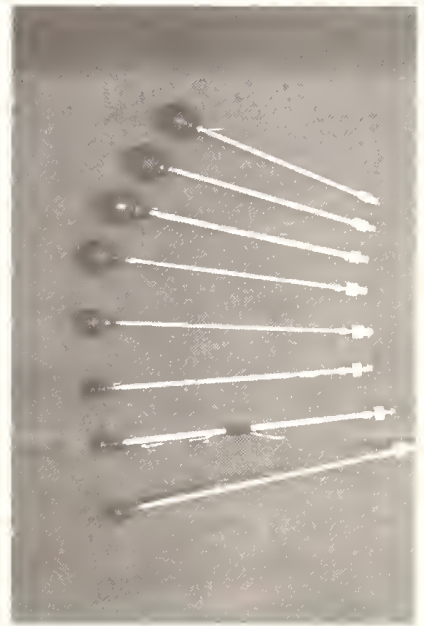

Fig. 25 Graphite cavity ionization chambers for Co-60 radiation. The national standard is the average response of several of these instruments.

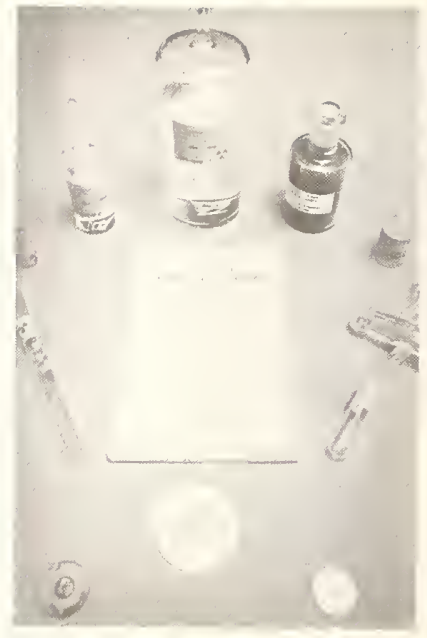

Fig. 26 Examples of several radioactivity standards offered by NBS as Standard Reference Materials.

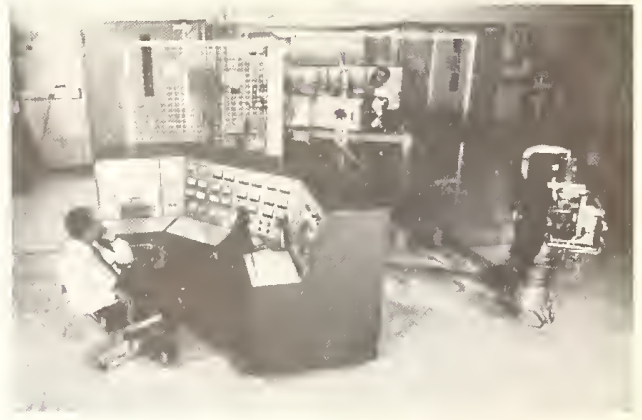

Fig. 27 View of NBS isotope separator used in preparation of some radioactivity standards.

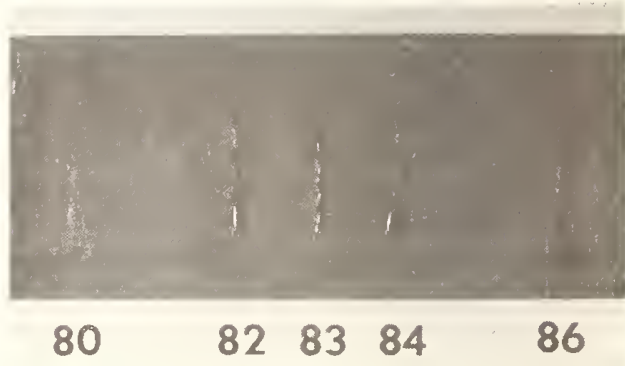

Fig. 28 Ion-implanted krypton gas in aluminum foi]. The $\mathrm{Kr}-84$ portion under neutron irradiation undergoes neutron capture and becomes $\mathrm{Kr}-85$, an important gaseous effluent from nuclear power plants. 


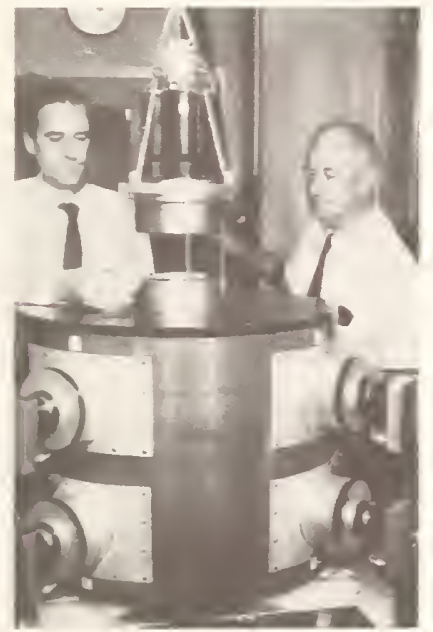

Fig. 29 View of two-crystal spectrometer for development of environmental radioactivity standards.

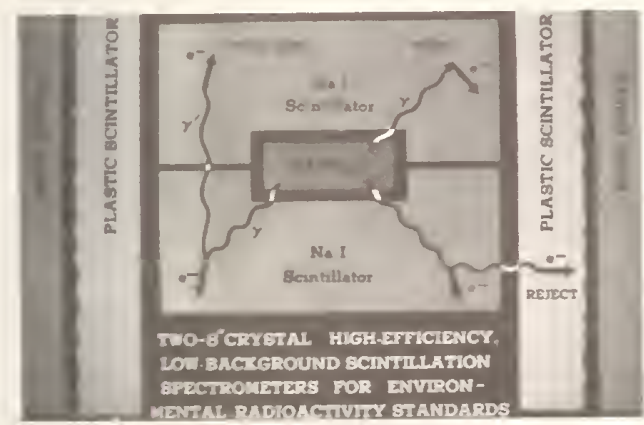

Fig. 30 Illustration of detector, sample and shield arrangement of two-crystal spectrometer.

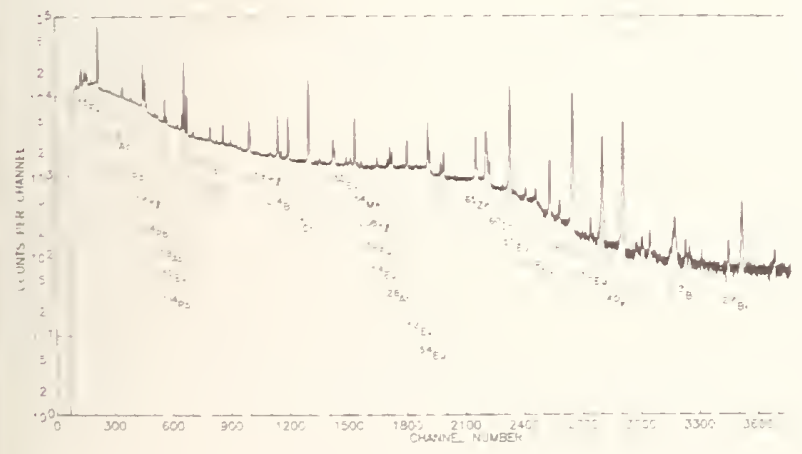

Fig. 31 Gamma ray spectrum of Columbia River sediment obtained with a high resolution gernamium detector. This material is now offered by NBS as a Standard Reference Material.

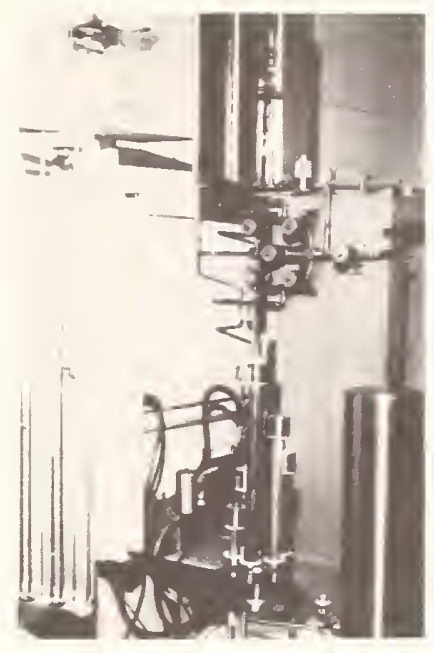

Fig. 32 Gas handling and counter system used in preparation of gaseous radioactivity standards. Included in this arrangement are counters of several different lengths to allow correction for counter end effects.

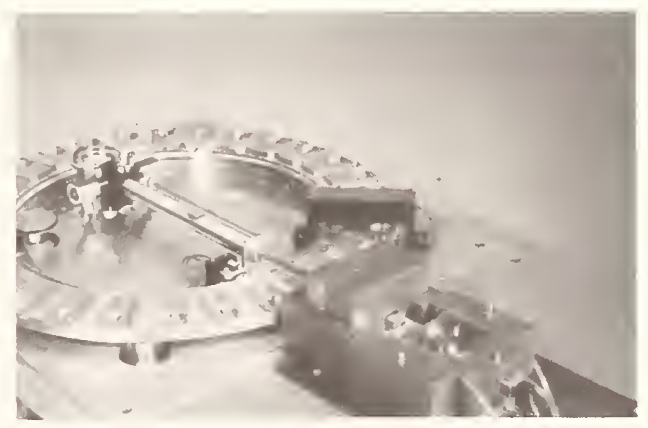

Fig. 33 Automatic sample changer and betagamma coincidence counting apparatus for measurement of "point-source" radioactivity standards. 


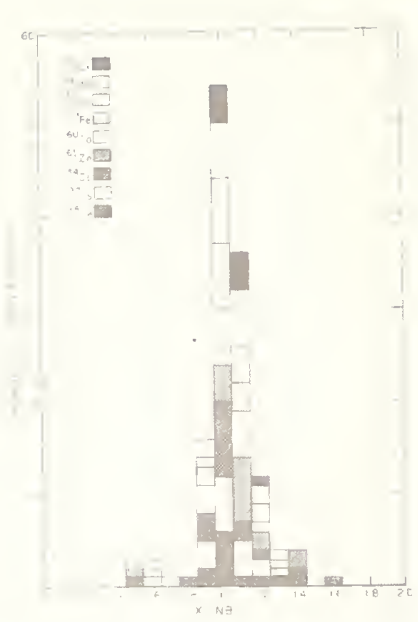

Fig. 34 Results of measurement assurance test of user ability to measure mixedradionuclide solutions distributed by NBS.

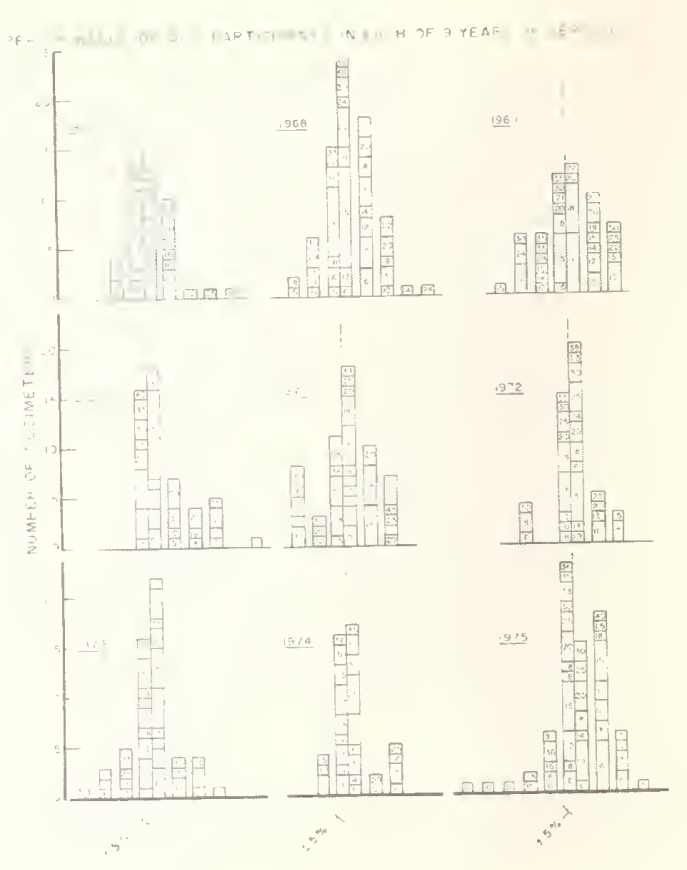

Fig. 36 Results of measurement assurance testing of dosimetry for electron beam therapy over a nine-year period. The lack of improvement at the user level during this period is striking.

IODINE-125, OCT., 1972. DOSE CALIBRATOR RESULTS

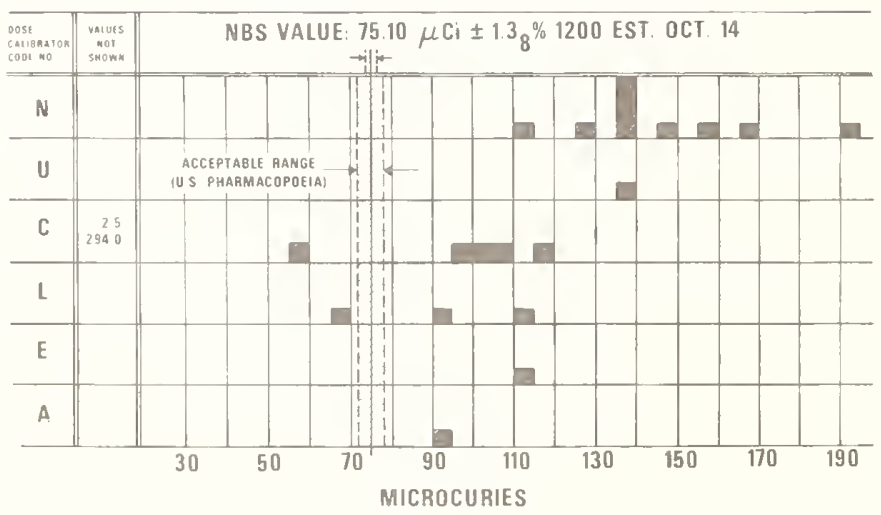

Fig. 35 Results of NBS-CAP measurement assurance test of dose-calibrators in field environment to measure I-125 samples distributed by NBS. Dose calibrators of type $\mathrm{N}$ exhibit a clear bias (later confirmed). 
R. C. Baird and A. A. Sanders

-iationa- Bureau of Standards Bouläer, Colorado 80302

Caionimeters have been deve-oved by IBS as accurate $\pm 1 \%$ ) standards For caitirating laser power anà energy measuring devices. A pilot measuremert assurarce program (WP) is underway, with 12 industrial orgarizations participating. The anticipatea accuracy is $\pm 5 \%$. A forma: $\because$ - service wiz de announcea aboú JuIy 1976. Accurate staräards for $r=$ aná microwave lields are not as well developed because of the inherent-j greater difficu-ty in measuring these fields causeả o: ififraction, interference, reactive rielas, modulation, etc. aSS has cevelopec accurate measurement ana calibration techniques which will ce ruly imp-emented about August 1976 when a new anechoic (ref-ection-iree) chamber will be completed. IBS is also developing stabje transfer standaras suitable for measurement assurance programs,

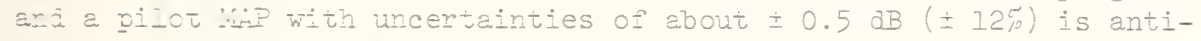

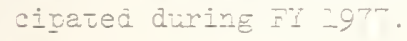

Wor-ionizirg E. rakiation hazards; laser calorimeters; RF and microwa:e measurements; cailbrajions; standarảs; measuremet assurance programs)

\section{Introziation}

Requirements to compare anà correlate measurements made by different Laboratories, to settIe disputes arising in the marret Elace, to determine bionogicai effects of radiation, ana to assure tine saiety o: workers anc the cublic exposeā to ror¿onizing radiation have producea an ircreasing demana by a number ồ iaboratories ano orgarizations for measurement traceabilizy to nationa: standaras. This caper reviews the present siatus of starbaras for laser, Eicrowave, and re spequencies, giving rarges of applicability and associated zeasurement uncertainties. A brief review of how one car establish iraceability to these national standaras trrough a measurement assurance progrem (IAP) is included. In the case of microwave and ri measurezents, some discussion of the aifficult measurement froblems that have impeded the developrent of adequate standards is also zresented.

\section{Zaser Standards}

\section{Lescrittion of TBS Stanciards}

The "HBS stanäards for power anà energy reasurements are the $C, Y$, and $Q$ series calorimeters. 1-3 Their essertial features include a constant temperature jacket surroundire a cavity which absorbs an incident Iaser beam. The temperature rise due to the absorbed optical radiation is measured and compared to the quantity of electrical energy causing the sane temperature rise. The calorimeter is thus a comparative device which compares the energy of the optical raiiation from a laser with an equivalent quantity $\hat{\imath}$ electrical energy.

The approximate ranges and accuracies of these calorimeters are shown in Figures 1, 2, and 3. Each figure shows the laser power (or energy) range of the calorimeter. For example, Figure 1 demonstrates that the C-series calorimeter is useful, for the nominal accuracy shown, for energies in the range of 0.1 joule to 22 joules. Experience has demonstrated that 300 seconds is the maximum laser (or electrical) power injection time compatible with reasonable precision. Thus, since 0.1 joule is the minimum amount of energy that can be accurately measurea, this sets a lower power limit of $0.3 \mathrm{milliwatts.} \mathrm{The} 300$ second injection time also determines the left slanted boundary. The response of the calorimeter becomes non-linear for energies above the upper energy boundary, and the boundary on the right is the maximum power that can be produced by the electrical heaters used in the calibration. Laser powers in excess of this boundary may also damage the calorimeter.

Figure 4 depicts the optical arrangement of a measurement system formed from these calorimeters (NBS maintains two or more of each type of calorimeter). A laser beam is incident upon the beam splitter which both reflects and transmits portions of the beam. The action of the beam splitter causes the incident beam to be dispersed into two or more beams; one of these dispersed beams is incident on each calorimeter. Since the power in the transmitted beam is usually larger than that in the reflected beam, we normally refer to them 


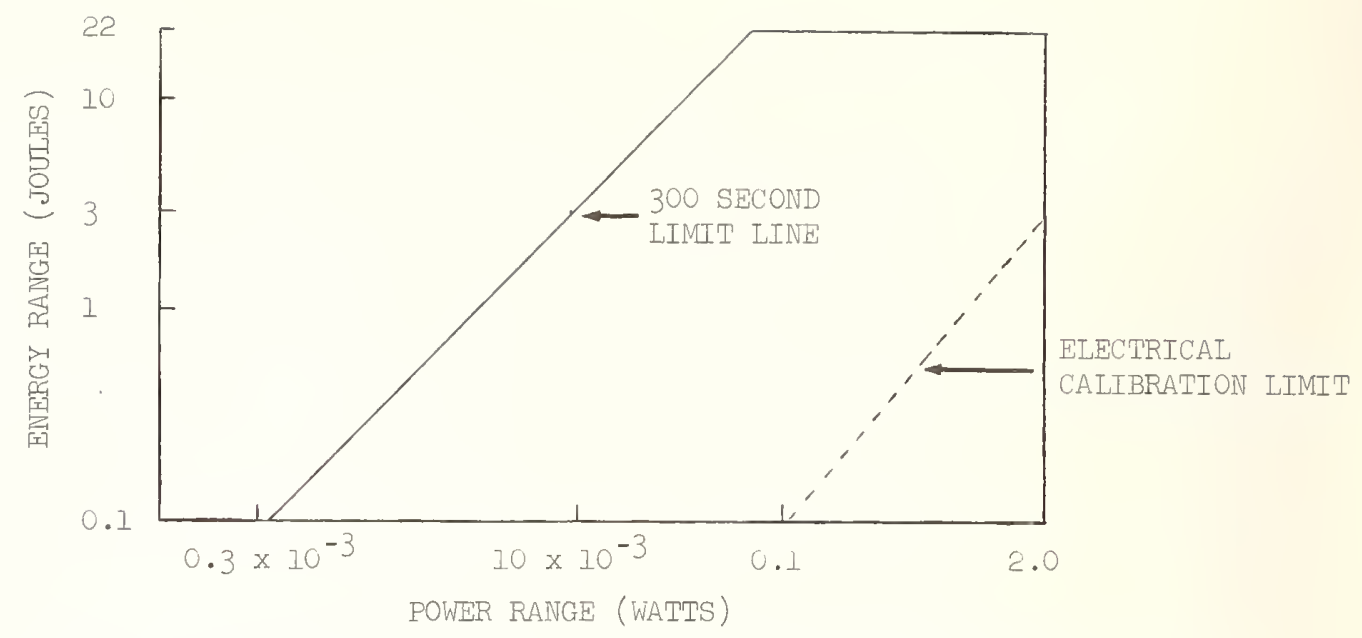

FIGURE 1. The enclosed region defines the energy-power range for the $C$ calorimeter. The nominal uncertainty is one percent.

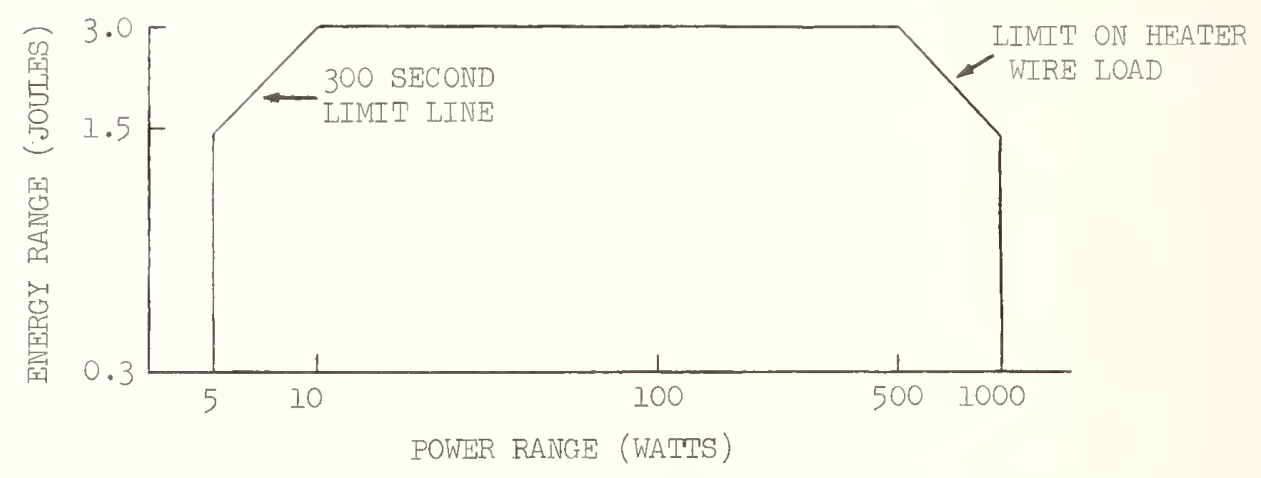

FIGURE 2. The enclosed region defines the energy-power range for the $K$ calorimeter. The nominal uncertainty is four percent.

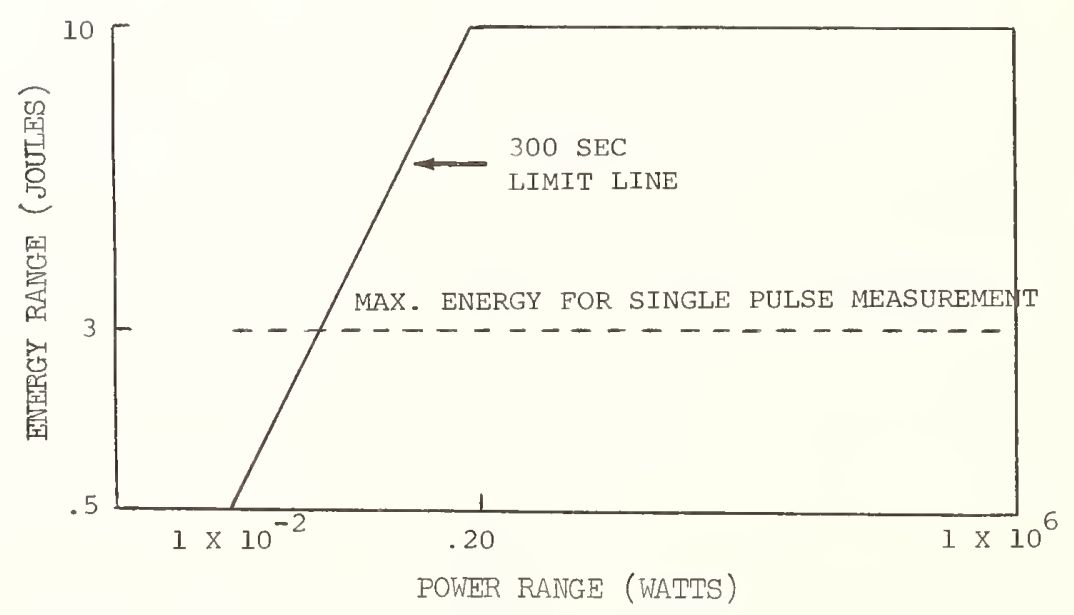

FIGURE 3. The enclosed region defines the energy-power range for the $Q$ calorimeter. The nominal uncertainty is two percent. 
NBS LASER MEASUREMENT SYSTEM

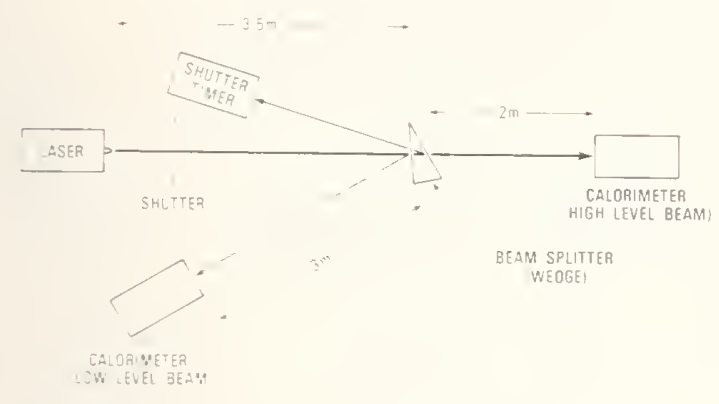

FIGURE 4. Diagram of IIBS calorimeter system used for calibrajing laser power and energy meters.

respectively as the high and low level beams. The actual ratio of power in the two beams is referred to as the beam splitter ratio and can be measured. Once this ratio is known, an unknown power or energy meter can be substituted for one of the calorimeters. The response of the remaining calorimeter and knowledge of the beam splitter ratio, permits one to determine the amount of energy incident on the unknown instrument and hence its calibration constant. This beam splitter measurement technique helps prevent imprecision in the measurement caused by instabilities of laser radiated power.

The calorimeters are strictly energy measurement devices and are therefore insensitive, within reasonable limits, to the temporal characteristics of the laser. However, they can be utilized in certain circumstances to calibrate power meters. Consider, for example, the case where the power in the laser radiation is nominally constant (CW). By means of a timed shutter, the actual time that the laser radiation is incident upon the calorimeter can be determined. A measurement of the total energy and knowledge of this time interval allows one to determine the average power incident on the calorimeter. Since the beam splitter ratio is known, the power incident on the unknown power meter is also known. Thus calibration of $\mathrm{CW}$ power meters can be accomplished. Other power measurements are possibie to the extent that the temporal characteristics of the laser are known, but the detailed measurement of laser power is still an important area of research.
The inaccuracy of these calorimeter measurement systems is comprised of systematic uncertainties and imprecision. Systematic errors are composed principally of the uncertainties in the electrical energy to which the optical radiation is compared, uncertainties in the percentage of radiation absorbed by the calorimeter, and any inequivalence in the response of the calorimeter to electrical versus optical energy. These uncertainties have been carefully studied and bounded. Errors due to imprecision are determined through a careful and thorough internal quality assurance program.

\section{Laser Measurement Dissemination Program}

Considerable effort is being expended to disseminate measurement services and traceability to the national standards to other laboratories. In some cases copies of the calorimeter have been built for other government laboratories, e.g., Newark Air Force Station, the Bureau of Radiological Health, and Fort Belvoir. By means of measurement assurance programs (MAPS) with NBS, these laboratories can establish essentially the same measurement capability as IVBS. The Air Force laser calibration laboratory at Newark Air Force Station is currently duplicating most of the laser power and energy measurement systems maintained by NBS. They will then be able to extend calibration and traceability to other DoD laboratories and DoD contractors.

However, duplicating and maintaining these calorimeter measurement systems is an expensive endeavor and not practical for most laboratories. For these laboratories we are offering some limited calibration services and are establishing measurement assurance programs. We feel that reliable measurement assurance programs have the greatest potential for providing measurement traceability in an efficient and cost effective manner.

\section{Laser Measurement Assurance Programs}

The measurement assurance program (MAP) is one of the primary vehicles used by NBS to provide measurement services to other laboratories. Within this concept, measurements are viewed as the product of a complete measurement system. Hence, the total measurement is evaluated including the measurement environment, operator, data reduction, laboratory, standards, and anything else that may influence the measurement. 
In practice a laser MAP might work as follows. A participant in the laser MAP will establish his own measurement system and required level of accuracy. He would then establish an internal program for documenting the precision of his system (NBS guidance and suggestions will be available for each of these tasks). This might, for example, consist of intercomparing two or three different power or energy meters and maintaining records and control charts. Instead of having devices calibrated, the participant would occasionally be sent a transfer standard by NBS. (A transfer standard is simply a stable optical detector or power meter that has been extensively evaluated with respect to its stability, accuracy, and suitability for use as a standard in intercomparisons.) The calibration constant of this transfer standard would not be known to the participant in advance. He would calibrate the transfer standard by his measurement system and forward the results and data to NBS. NBS would analyze the data and forward a formal report of the precision and accuracy to the participant. Any systematic error would have been determined and appropriate corrections could be made. Transfer standards would be forwarded on some periodic basis (or more often if required). The level of accuracy is determined primarily by the user. The results of the intercomparison and the participant's own documentation of his measurement system furnish detailed information on the system measurement accuracy and provide traceability to the national standards maintained by NBS. The user may now use his measurement system to extend calibration to other devices or establish internal MAP's of his own.

NBS now has underway a pilot laser MAP involving twelve laboratories. This pilot program is restricted to the measurement of approximately 1 milliwatt of Helve $(632.8 \mathrm{~nm}$ ) laser radiation. A formalized laser MAP for this frequency and power level will be available approximately July 1976. The cost of the service to a participant will be approximately $\$ 1500.00$ per year.

An extensive evaluation of potential transfer standards is being conducted by NBS for these MAP's. The total uncertainties of the transfer standards for the $1 \mathrm{~mW}$ MAP is about 3-5\%. By means of this MAP a participant should be able to document measurement accuracies of $5-10 \%$ at a nominal cost. Transfer standards for other wavelengths, energies, and powers are currently being evaluated and additional laser MAP's will be instituted as needs are established.
$\underline{R F}$ and Microwave Standards

Electromagnetic hazards ( $E M H)$ and electromagnetic interference (EMI) are problem areas of increasing importance and concern due to expanding use of electromagnetic (EM) radiation for communications and industrial purposes, and the growing use of electronic equipment that may be affected by FM radiation. These areas require accurate measurements of EM fields for adequate monitoring and control of undesired radiation, and for determining biological effects and the susceptibility of sensitive electronic devices to EM fields. It is worth pointing out that very real (though indirect) hazards can result from the effects of EM fields on certain electric and electronic devices--for example, the unplanned detonation of electro-explosive devices and the failure of automatic braking systems on vehicles. The development of adequate measurement instruments and techniques, standards, and calibration methods has been painfully slow in both the FMH and EMI areas due to several severe measurement problems. Although the following discussion of these problems is oriented toward EMH, it is also generally applicable to EMI. Measurement Problems at RF and Microwave
Frequencies

With lasers, one is usually dealing with a well defined beam of reasonably small cross section. The total energy or power in the beam can be measured by the techniques previously described, and the power density $\left(\mathrm{W} / \mathrm{m}^{2}\right)$ can be inferred from the size of the beam. In contrast, rf or microwave energy is usually distributed throughout large volumes of space so the best one can do is to measure the pertinent field parameters in a localized region of the field. Perhaps as a carryover from the practice in laser and ionizing radiation safety, workers in $r f$ and microwave radiation safety also began to characterize field levels in terms of power density. However, it is now recognized that power density is not always a good parameter to use because: (I) it is not measured directly, but is derived from measurements of electric and/or magnetic field strength; (2) it does not always provide a good indication of the hazard and (3) it cannot be accurately measured or calculated in many hazardous situations. Since hazards are usually most closely related to the electric field E (vectors are indicated by underbars), we feel that measurements of $|\underline{E}|^{2}$ or the electric energy density $U_{E}$ are most

appropriate. (There is some evidence that 
$|\underline{H}|^{2}$ or $U_{H}$ may be important at the lower

frequencies. ${ }^{4-6}$ ) Measurements of $E$ would be equally good, but it is much easier to measure $|E|^{2}$. Probes based on resistive heating, and composite probes which indicate the sum of the squares of three orthogonal components of $\underline{E}$ are now available for measuring $|\underline{E}|^{2}$ directly. The rationale for choosing $|\underline{E}|^{2}$ will become more apparent from the following discussion.

Under far-field plane-wave conditions any one of a number of parameters is a valid index of the field. These parameters include electric field strength $\mid \underline{E}\}$, magnetic field strength $|\underline{H}|$, time average power density $|\underline{S}|$, electric energy density $U_{E}$, magnetic energy density $\mathrm{U}_{\mathrm{H}}$, and the total energy density $U$. If any one of these parameters is known, all of the others can be obtained from relatively simple rigorous relationships. Under these conditions power density is a suitable parameter. Unfortunately, both EMH and EMI tend to occur in near-field situations where the EM field configurations may be very complicated, where the simple plane-wave relationships among the various field parameters no longer hold, and where conventional field intensity meters are totally inadequate for measurements. The situation is further complicated by the wide frequency range $\left(>10^{10}\right)$ involved. Wavelengths extending from thousands of kilometers to millimeters are common, and different measurement approaches are required for different portions of the spectrum. The principal complexities and problem areas that need to be coped with are the following. (For a more detailed discussion see reference 7. )

Diffraction and Interference. Since the wavelengths associated with $r f$ and microwave radiation are not, in general, short compared to the dimensions of the objects or subjects involved, diffraction and interference effects can cause significant spatial variations in field intensity. These variations show up as standing waves, hot spots, dead spots, etc., with the distance between maxima (minima) being the order of a wavelength. Interference occurs when multiple or extended sources are involved, and where there is scattering from the ground, walls, or other objects. This condition is the rule rather than the exception for most hazardous situations, and is a good example of a case where power density is not an appropriate measure of the field. If we consider two waves of equal amplitude and frequency, travelling in opposite directions, the power density is exactly zero. However, the fields (and therefore the hazard) can be large where the resulting standing wave is a maximum. The foregoing implies that, at least for wavelengths larger than the diameter of the human eye, the field intensity at the maxima of standing wave patterns should be used as the hazard indicator. Thus, for frequencies up to about $10 \mathrm{GHz}$, a good hazard meter should employ a field sensor that is smaller than the shortest wavelength of interest.

Polarization. In the near field and in the presence of multipath interference, the polarization can be arbitrary, is probably not known in advance, and may change radically from point to point. It is not practicable to determine power density in a general field of this type since one would need to measure the equivalent of the amplitudes and relative phases of all six $\underline{E}$ and $\underline{H}$ field components--a very difficult measurement task. Further, if directional probes or probes that respond to a single component of $\underline{E}$ or $\underline{H}$ are used, the measurement procedures for finding the maximum response are tedious and time consuming, and one is never really sure that the probe has been oriented for maximum response.

Reactive Near-field Components. Within distances of approximately one wavelength from some sources and antennas, strong reactive fields can exist which contribute to the hazard but not to the time-average energy flow (power density). Such fields are more common at the lower frequencies, but can be important up to about $1 \mathrm{GHz}$.

Interactions With the Field Being Measured. Several types of interactions occur which can cause serious errors in EM field measurements. For instance, a measuring instrument or object close to a source, particularly in a reactive zone, can interact with the source and alter the amount of energy emitted. Even at large distances where the source is not affected, a dielectric or conductive body can strongly affect the field in the immediate vicinity and perturb the measurement. Thus, the metallic transmission lines used with early model hazard meters have been observed to produce variations in field intensity of 10 to $20 \mathrm{~dB}$. A good probe should, therefore, be designed to produce very little field perturbation. The close proximity of an operator can also cause significant errors, even with the best of probes. 
Complicated Modulation of the Field.

Fields may vary drastically with time, depending on the type of modulation present, and this can affect the potential hazard. For example, with pulsed fields some effects may be functions of peak field strength instead of the average; some effects may even depend on the pulse shape and repetition rate. Meters with fast response times, capable of measuring both peak and average fields are needed but are difficult to design and produce.

\section{Summary. In the complicated fields} associated with EMH and EMI, measurements may vary with time, position, frequency, polarization, modulation, probe orientation, etc., and the simple plane-wave relationships between parameters are not valid. A good general purpose instrument capable of accurately measuring such fields should, therefore, possess most of the following characteristics which we believe to be essential or highly desirable. (1) The instrument should measure $|\underline{E}|^{2}$ or $U_{E}$, and possibly $|\underline{H}|^{2}$ or $U_{H}$, since measurements of $\underline{E}$ and $\underline{H}$ are often too difficult and time consuming. (2) The probe should be small enough to resolve significant spatial variations in the field. (3) For most purposes the probe response should be isotropic, i.e., independent of the orientation of the probe in the field. (4) An ideal instrument should also be capable of measuring both average and peak field levels. Useful probes that satisfy the first three conditions are now available, ${ }^{8}$ but there is still a need for probes with faster response times for peak measurements.

\section{Standards and Calibrations}

Existing calibration methods are based on the premise that a known field intensity can be established through measurement, calculation, or a combination of both. The device to be calibrated is placed in the "standard" field and the meter indication is compared with the known field value. There are three basic methods of producing the "standard" calibrating field: (1) the freespace standard field method, (2) guided wave methods and, (3) the standard probe or "transfer standard" method. The choice of technique will depend on such things as the type and size of probe, frequency range, available facilities and equipment, and the accuracy requirements. The basic concepts of each method are presented below; see reference 9 for more details.
Free-space Standard Field Method. The objective of this method is to establish a known plane-wave calibrating field in free space by means of the basic experimental arrangement shown in Figure 5. The power density $|\underline{S}|$ at a point on the transmitting axis at a distance d from the transmitting antenna is given by

$$
|\underline{E}|=\frac{P_{T} G}{4 \pi d^{2}}
$$

where $P_{T}$ is the net power delivered to the transmitting antenna, and $G$ is the effective gain of the transmitting antenna. The gain is normally determined in advance, and $P_{T}$ and d are measured as part of the regular calibration procedure. The rms electric field strength $|E|$ can be obtained from the relation $|\underline{E}|=\left.\varepsilon T E\right|^{2}$, where $\varepsilon$ is the permittivity of the medium.

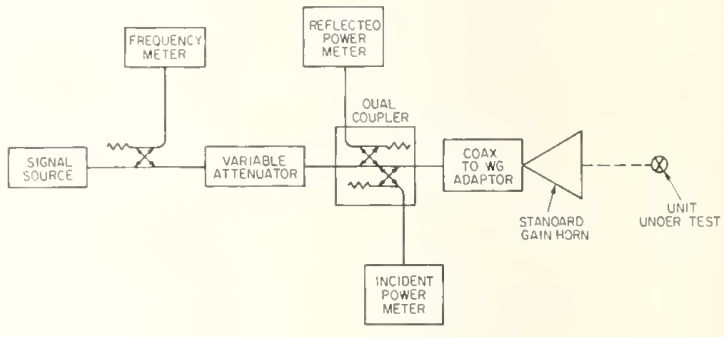

FIGURE 5. The basic experimental arrangement for the free-space standard field method of calibration.

The principal sources of error in this method are multipath interference and uncertainties in the gain determination. Multipath effects are often overlooked, but every calibrating facility will have some scattering associated with it which may cause the field in the calibrating region to be significantly different from that predicted by equation 1 . Even high quality anechoic (reflectionless) chambers are not perfect and should be carefully evaluated if the best accuracy is desired. Reference 10 contains a useful discussion of this problem. It is relatively easy to obtain accurate $( \pm 2 \%)$ gain values for large distances; ${ }^{11}$ in fact, the far-field gain of some standard horns can be calculated to about \pm 7 percent.12 However, large distances require greater transmitter power and the multipath situation is worse. On the other hand, there are some fundamental difficulties 
associated with accurate gain determinations at short distances. ${ }^{9}$ These problems are gradually being overcome and it seems possible to reduce the total maximum uncertainty of this technique to less than $\pm 0.5 \mathrm{~dB}(10 \%)$ for relatively close distances and moderate transmitter powers.

Guided-Wave Methods. Several types of guided-wave structures have been considered for generating known fields for calibration purposes. These include waveguides, parallel plane lines, parallel wire lines, and TEM cells. 13 Only waveguides and TEM cells will be considered here because they are fully shielded systems that do not radiate into the surrounding environment and are, therefore, much preferred. The main advantage of a guided-wave system is that considerably less power and space are required. One disadvantage is that the maximum transverse dimensions of the structure must be less than $\lambda / 2$ at the highest frequency in order to avoid higher order modes which result in more complicated field distributions. Hence, the method is only useful for frequencies below 1 or $2 \mathrm{GHz}$, since the device being calibrated must be small compared to the guide dimensions.

Figure 6 shows how a section of rectangular waveguide can be used for calibrations. A reflectionless load is connected to the output end to prevent standing waves which would cause serious errors in

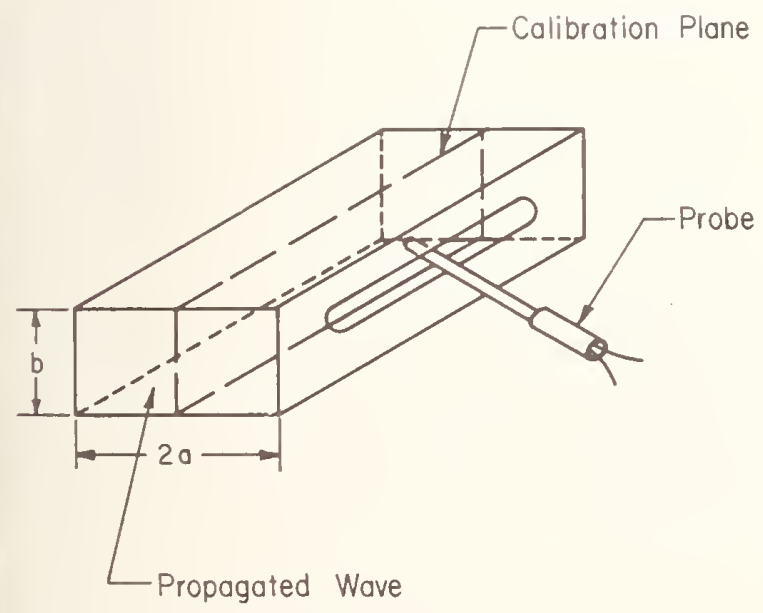

FIGURE 6. A section of rectangular waveguide modified for use as part of a probe calibration system. the calibration. The probe to be calibrated is inserted into the waveguide through a hole in either the top or side wall and positioned in the center of the guide where the field is most uniform. The access holes should be as small as possible. Equations for calculating the field distribution from $\mathrm{P}_{\mathrm{n}}$ (the net power delivered to the section) and the guide dimensions can be found in any book on waveguide theory. It is difficult to estimate the total uncertainty of this method because the field intensity will be modified by the size and nature of the probe being calibrated. Woods ${ }^{14}$ describes a system which operates from 400 to $600 \mathrm{MHz}$ with an estimated uncertainty in the field intensity of $\pm 12 \%$, and Aslan 15 slaims an uncertainty of approximately $\pm 5 \%$ in WR 430 guide $(1.7-2.6 \mathrm{GHz})$.

The basic TEM Cell is illustrated in Figure 7. It consists of a section of twoconductor transmission line operating in the transverse electromagnetic mode (TEM), hence the name. The main body of the cell consists of a rectangular outer conductor and a flat center conductor located midway between the top and bottom walls. The dimensions of

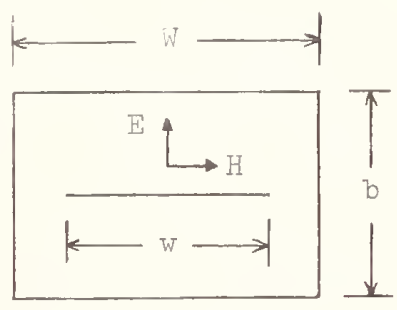

CROSS SECTION

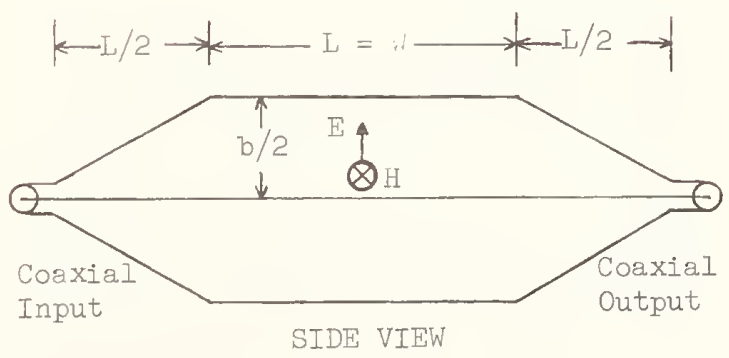

FIGURE 7. A type of TEM transmission cell used at the NBS for calibrating hazard meters and for measuring susceptibility of electronic equipment to EM fields. These cells can also be used as exposure chambers for studying biological effects. 
the main section and the tapered ends of the cell are usually chosen to provide a 50-ohm impedance along the entire length of the cell. Hence, when the cell is carefully made, terminated in a reflectionless load, and connected to a 50-ohm coaxial cable, the input VSWR is usually less than 1.05 and always less than 1.1 for all frequencies below the cutoff limit. The principle advantages of the cell (compared to a waveguide) are: (1) the field intensity in the center of the cell midway between the top wall and center conductor can be made uniform over a much larger region, and (2) the wave impedance of the TEM field is nominally 377 ohms, the same as a plane wave in free space. These features are the principal reasons for using TEM cells for calibrations. 13

The cells can be made in various sizes to suit particular needs and cover certain frequency ranges. However, since the width ( $W$ in Fig. 7) must be less than $\lambda / 2$ to avoid multimoding, the upper useful frequency of the devices is probably about $500 \mathrm{MHz}$. As in a waveguide, the introduction of a probe into the test region will change the field distribution in the vicinity of the probe, but if the probe is much smaller than the transverse cell dimensions, this error will be small. Fully evaluated cells should be capable of about the same accuracy as waveguides $(5 \%-10 \%)$, and are expected to play an important role in EMH and EMI measurements.

Transfer Standard Method. This method may be the simplest and best method of calibrating hazard meters for general fiield use. The idea is to have a stable and reliable probe that has been accurately calibrated (by one of the previous techniques) and use it as a "transfer standard." That is, the standard probe is used to measure the field intensity at a particular point in space (or in a guided system) produced by an arbitrary transmitter. The probe to be calibrated is then placed in the same location in the field and the meter reading compared with the known value of the field. The only requirements on the transmitter are that it generate a field which has the desired magnitude, is constant in time, and is sufficiently uniform over the calibrating region. Accuracies of about $\pm 0.5 \mathrm{~dB}(12 \%)$ can probably be achieved with this method. The biggest advantages of this approach are convenience, reliability, and simplicity. The ideal probe for use as a transfer standard should be stable, rugged, and not easily burned out; it should have a large dynamic range, cover a broad frequency range, and possess an isotropic response pattern. Suitable probes are becoming available. 8 Organizations that cannot justify the construction and maintenance of a calibration facility could have a transfer standard calibrated by NBS or some other reputable laboratory and make measurements that would be satisfactory for most purposes.

\section{Practical Measurement Accuracy. It is} important to understand that one cannot expect to achieve the full calibration accuracy when using meters for practical measurement applications. Some of the reasons are as follows. (a) Hazard meters are usually calibrated in nominally planewave fields. Such fields are seldom encountered in practice, and the sensor may not respond in the same way to non-planar fields. (b) In most calibration methods, only the sensor (probe) is exposed to the field, while in practice the complete system, including the indicating unit and connecting cable, is immersed in the field. (c) With hand-held meters, the presence of the operator can significantly affect the reading. The additional uncertainity caused by these factors is difficult to assess and will vary with the type of meter. However, if good measurement procedures are followed, accuracies of \pm 1 or $2 \mathrm{~dB}(10-50 \%)$ can probably be achieved under typical measurement conditions.

\section{Measurement Assurance Programs at NBS}

The Electromagnetics Division of NBS (Boulder) will be in a position to offer MAP's in rf and microwave measurements in about the fall of 1976 . During recent years NBS has concentrated on developing the required calibration systems and techniques and instruments for measuring EM fields since adequate instrumentations and measurement techniques did not exist. By the summer of 1976 we will have an anechoic (reflectionless) chamber, adequate for free space calibrations down to about $500 \mathrm{MHz}$. We plan to employ a series of TEM cells to extend the calibration capabilities down to very low frequencies, so we should be in a position to calibrate instruments and conduct MAP's in any frequency range of interest.

There is still a need for probes with better characteristics for use as transfer standaras, but progress in this direction is being made, both commercially and at NBS. The NBS effort is based on the 3-axis dipolediode probe described by Bowman. ${ }^{8}$ We believe it is possible to make such a probe 
with an improved temperature coefficient and with sufficient stability, sensitivity, dynamic range, and ruggedness to qualify as an excellent transfer standard. The units employ very small sensors and high resistance Iines that do not unduly perturb the fields being measured. In any event, existing probes could be used for interim IIAP's and the overall accuracy could be improved later on as better transier standards become available.

\section{References}

1. E.D. West and K.I. Churney, "Theory of isoperibol calorimetry for laser power and energy measurements," J. of Applied Physics, 41, 2705-2712, May 1970.

2. E.D. West, W.E. Case, A.L. Rasmussen, and L.B. Schmidt, "A reference calorimeter for laser energy measurements," $J$. of Research of the ITational Bureau of Standards-A. Physics and Chemistry, 76A, Io. 1, 13-26, January-February, 1972 .

3. D.I. Franzen, "An absolute reference calorimeter for measuring high power laser pulses," manuscript in preparation, National Bureau of Standards, Boulder, Colorado 80302.

4. C.C. Johnson, C.H. Durney, and H. Massoudi, "Long wavelength electromagnetic power absorption in prolate spheroidal models of man and animals," IEEE Trans. on Microwave Theory and Techniques, MTT-23, 739-747, September 1975.

5. O.P. Gandhi, "Frequency and orientation effects on whole animal absorption of electromagnetic waves," IEEE Trans. on Biomedical Engineering, BME-22, 536542, November 1975.

6. A.W. Guy, M.D. Webb, and C.C. Sorensen, "Determination of power absorption in man exposed to high frequency electromagnetic fields by thermographic measurements on scale models," submitted for publication in IEEE. Trans. on Biomedical Engineering, April 1975.
7. P.F. Wacker and R.R. Bowman, "Quantifying hazardous electromagnetic fields: Scientific basis and practical considerations," IEEE Trans. on Microwave Theory and Techniques, MTT-19, 178187, February 1971.

8. R.R. Bowman, "Some recent developments in the characterization and measurement of hazardous electromagnetic fields," Proceedings of International Symposium on Biologic Effects and Health Hazards of Microwave Radiation, 217-227, Warsaw, october 1973. (Copies are available from the author at the National Bureau of Standards, Boulder, Colorado 80302.)

9. R.C. Baird, "Methods of calibrating microwave hazard meters," Proceedings of International Symposium on Biologic Effects and Health Hazards of Microwave Radiation, 228-235, Warsaw, October 1973. (Copies are available from the author at the National Bureau of Standards, Boulder, Colorado 80302.)

10. R.R. Bowman, "Field strength above $1 \mathrm{GHz}$ : Measurement procedures for standard antennas," Proc. IEEE, 55, 981-990, June 1967.

1. A.C. Newell, R.C. Baird, and P.F. Wacker, "Accurate measurement of antenna gain and polarization at reduced distances by an extrapolation technique," IEEE Trans. on Antennas and Propagation, AP-21, 418-431, July 1973.

12. E.V. Jull, "Errors in the predicted gain of pyramidal horns," IEEE Trans. on Antennas and Propagation, AP-2l, 25-31, January 1973.

13. M.L. Crawford, "Generation of standard EM fields using TEM transmission cells," IEEE Trans. on Electromagnetic Compatability, EMC-16, 189-195, November 1974

14. D. Woods, "Standard intensity electromagnetic field installation for calibration of radiation hazards monitors from $400 \mathrm{MHz}$ to $40 \mathrm{GHz}$," Non-ionizing Radiation, 1, 9-17, June 1969.

15. E. Aslan, "Simplify leakage probe calibration," Microwaves, 14, 52-57, December 1975. 

E. Dale Trout, Oregon State University--Moderator

G. S. Parker, Massachusetts Department of Public Health

J. R. Cameron, University of Wisconsin

W. A. Mil1s, U.S. Environmental Protection Agency

R. S. Caswel1, National Bureau of Standards

Dr. Trout:

The Panel consists of the four Chairmen of the Invited Paper Sessions-each comes from a different area of interest. Each represents a consumer area for measurement. There is Gerald Parker who represents the State's Regulatory Program, John Cameron from Medical Physics, Bil1 Mi1ls from a Federal Regulatory Group and Randy Caswell from the source of the standards which we use. To be sure that we didn't waste a lot of time I, with Jim's assistance, put some questions to these fellows which we asked them to consider during the course of the Symposium.

1. Did the Symposium contribute to the stated objective?

That is, did it draw attention to the rapidly increasing need for high quality measurements of ionizing and non-ionizing radiation throughout the country?

2. In the session you chaired what were the major contributions to the objective of the Symposium?

3. Is the current measurement system working in your area of interest and if not, how can it be improved?

4. Should there be further symposia sponsored by NBS and other sponsoring organizations? If so, how often? What is your recommendation for a title and an objective for the next symposium?

We will first of all give each of the four Chairmen five minutes in which to speak to the four propositions we put in front of them; then ten minutes for them to kick these things back and forth among themselves. Now to take them in order of their sessions...Gerry, tell us about it.

Mr. Parker:

Thanks Dale. After seeing Jim Leiss' slides I'm not sure there's much that the panelists can add on what's occurred here. I will try to amplify on a few of these items and answer some of the questions that Dale has raised with us. As far as the high quality radioactivity standards that we've heard about, I think that Laurie Taylor perhaps summed it up when he said we' 11 have to have a little sense of humor about this. Why do we need $0.5 \%$ when the last speaker told us the things that he would use in the field would be about $+50 \%$ and we heard one of the state program directors saying the same thing. Perhaps, we are over-emphasizing the need for that degree of accuracy. Now in the first session we heard from the Federal agencies, and the Bureau of Radiological Health talked about P.L. 90-602. One of the items of compliance in the $x$-ray performance standard promulgated under P.L. 90-602 was positive beam limitation. Some investigators indicate that perhaps we could reduce exposures by $90 \%$ if we could limit the beam to the image receptor. 
We heard about therapy calibration centers and availability of precise standards. Yet we heard that $6 \%$ of the practitioners that were doing therapy were delivering doses more than $15 \%$ off from the calculated dose. Larry Lanzl showed some curves with very steep slopes of dose vs. treatment effectiveness. If the delivered dose is more than $15 \%$ off from the desired dose, then the clinical implications are rather frightening; i.e. the therapy has no effect whatsoever. So I wonder, why have we been spending hundreds of thousands of dollars on therapy calibration centers when perhaps what we should be doing is concentrating on the user--the people that are going to be using these therapy units. The standards are available but apparently they are not being used correctly.

I was rather shocked about laboratories. Fifty percent of the environmental radioactivity laboratories in the country can't even do a gross beta within three standard deviations. Twenty-three percent.can't even do a Cesium-137 within three standard deviations. The standards are there, where are the procedures? Where is the training? What are we going to do about these users?

As far as measuring systems are concerned, what are the things that we need? We could use some low energy dosimeters for use around mammographic units. Those of you who've tried to do some measurements of mammographic units find it very difficult to find a dosimeter that's down in that energy range with the exception of TLD chips. I think NBS should take a real close look at this problem. This is an important problem because of the possibility of radiation induced tumors. I believe the states need the mobile calibration lab that Randy spoke about. However, I think NBS should understand the states aren't going to have the money to pay for this kind of service, and NBS better be able to support it themselves if they want to put a mobile lab out there, and provide this service to the states.

I don't think most people realize that the state surveyors don't go around with three quarter ton trucks when we go out to survey an installation! We have to carry our instrumentation, and I think you ought to concentrate on getting instruments small enough so that they are really portable and can give us reproducible results. We don't need $0.5 \%$ accuracy. We'11 settle for $5,10,15$ and as the 1ast speaker said even 25\%. What do I propose? I propose the next time NBS wants to have a meeting they bring in more people living out in the real world. Find out what's going on out there in the states where the people have to visit al1 these installations, have to do al1 these measurements and have to make the decisions whether a medical office will stay open, whether a reactor will continue to operate. How can we make these decisions when the laboratories cannot produce accurate results and when the health and radiation physicists at therapy installations are not able to deliver the desired dose? Thank you.

Dr. Trout:

Dr. Cameron:
The next Chairman represents the area of medical physics and John, I want you to clean up your stories because remember, this is being taped and it has to come out rated $G$. John Cameron.

I'11 try one of my general stories that I have left over from an earlier meeting concerning the person who asked a Frenchman what the temperature was and the Frenchman answered that it was 38 degrees. The fellow says is that in Fahrenheit or Celsius? He said no, no, it's right here in Paris. 
Dr. Trout:

Dr. Cameron:
The answer to the first question: Did this Symposium contribute to the stated objective? My answer would be, in general, yes. The answer to the second question, what were the major contributions: I would like, in an effort to reduce time, to read a few pages I have written here for that.

Up to 5,000 words.

Bob Shalek reviewed the experiences of the Radiological Physics Center which he has directed. His measurements at 175 radiation therapy installations indicated that about $20 \%$ had errors 1 arger than $5 \%$ in delivering the prescribed tumor dose and $6 \%$ had errors exceeding $15 \%$. These measurements were made at centers which are large enough to participate in a national treatment trial. Essentially no data exists on the much larger number of smaller centers doing radiotherapy, many of whom do not have full time physicists to help them. The accuracy of calibration of ionization chambers by NBS and the two Regional Calibration Laboratories (RCL's) sponsored by the AAPM was good. However, one commercial calibration laboratory produced calibrations that sometimes exceeded errors of $5 \%$. It would appear that the country would benefit from more RCL's but subsidization will probably be necessary if they are to be started. In general, quality assurance in radiotherapy appears satisfactory, at least in the larger centers. Measurements should be made at the large number of small centers doing radiotherapy to determine how well they are doing.

Mel Siedband reviewed the need for more quality assurance in diagnostic radiology and described very simple test tools to aid in these measurements. Of the three major areas of radiology: therapy, nuclear medicine and diagnosis, it appears that the largest area, diagnostic radiology, is most in need of improvement in quality assurance. Relatively few medical physicists work in diagnostic radiology, and at NBS very few calibrations involve instruments for diagnostic radiology. The situation is improving but there is a long way to go. The advent of computerized tomography is bringing more physicists into diagnostic radiology and once they are there they will undoubtedly have a beneficial effect on the general level of quality assurance. Most states have an inspection program for diagnostic $x$-ray equipment. Unfortunately, many $x$-ray users confuse this inspection with quality assurance and feel that everyone is $0 . k$. because the state inspector checks their equipment every three years.

Buck Rhodes discussed the status of quality assurance in nuclear medicine. As the newest major field of radiology, this area is in pretty good shape, at least compared to diagnostic radiology. There is a need for specification of guidelines or standards in the biodistribution of radionuclides. There is also a need for more education of nuclear medicine technologists in the area of quality assurance.

The next speaker, Larry Lanzl, discussed state and federal radiation regulations related to medical facilities. He described in some detail the radiation control program in 111 inois that limits the exposure to typical patients from some standard $x$-ray projections. The limits were determined largely on the basis of data obtained from the N.E.X.T. Program. In the discussion period, some questions were raised about the advisability of such limits. However, it seems unlikely that the rules will be rigorously enforced and I view the rules as a step in the right direction. I think more states should follow the Illinois lead in this area. The regulation implies that measurements will be made, otherwise the rules will be ignored. In general, when you start to measure radiation the exposure(s) tend to decrease as the users become more aware of the overexposures. 
The last speaker in the session, John Shaver, discussed the N.E.X.T. Program. Nearly all states now participate in this program. I think this is the best program yet developed for obtaining realistic data on patient exposures from diagnostic x-rays. N.E.X.T. data show large variations of exposure and exposure area products from one facility to another. However, the actual situation is probably somewhat worse since it is likely that the $x$-ray operator is somewhat more careful during an inspection than at other times. We have done an analysis of the N.E.X.T. data at Wisconsin to see how the radiation to the standard patient depends on the type of facility and the training of the operator. This is a different analysis than BRH has done. The results will be available, hopefully, this year when BRH reproduces our report.

In summary, I think quality assurance in daily radiotherapy practice is in fair shape. Quality assurance in nuclear medicine appears o.k. but good field data is needed, and quality assurance in diagnostic radiology needs all the help it can get. In answer to the fourth question, are further symposia needed or should they be sponsored by NBS, I think meetings are needed in the specialized areas rather than the general areas. General meetings like this are useful but not too often, maybe every three or four years. Diagnostic radiology could use many more meetings. Thank you.

Dr. Trout:

Next we have a Chairman from a Federal regulatory group and if you don't believe that the Chairman of one of these sessions has a bad time you weren't here yesterday afternoon when John Matuszek was working Bi11 over.

Dr. Mills:

I thought I'd have a few slides to show to rebut everything John Matuszek said, but that didn't seem appropriate, so let me speak to the points that were raised. It seems to me that in looking at the program across the board, you've had a breadth of representation but perhaps not to the depth the states and the users, like Gerry Parker, would suggest. I would tend to agree with Gerry. I think you've been told how standards are designed and how they can be used. Now what happens after that, it seems to me, is really up to you. If you don't go back and use the information then the Symposium hasn't accomplished much. Certainly there's a need for these standards and certainly there's a need to push it to the greatest extent possible. As far as the session I chaired, I' 11 run through briefly the sumilary that I got from the speakers.

Jim McLaughiin, I thought, described well the ability to go out and make measurements around nuclear power plants at very low levels. For the most part, we're talking about gamma measurements and perhaps beta measurements. The levels of interest are at the least, a few millirem above background, and at the most, several times background. This is the area of environmental concern. It isn't with doses of 100 rads. It isn't doses of one rad even in a short period of time. It's of the order of background and I think Jim described some systems that can be used in this regard.

Matuszek impressed you with the ability of the laboratories to come down hard on regulatory agencies and show how absurd some of the regulations are. I'd get angry with that except for the fact that John's objective is the same as my own. That is, protection. I can argue with him about differences in many things but as long as the objective is protection of the public or protection of the worker, then I think these things can be worked out.

National Evaluation of X-Ray Trends 
Art Jarvis' job was to describe how the quality assurance program in EPA has worked. Art expressed some concern that it would go down the drain. Everything, I think, will be done to assure its survival because it seems to me that the quality assurance program is mandatory at environmental levels. Most of us who are concerned with the environment just can't make decisions without some reliability in the measurements.

Dick Griffith discussed some of the personnel dosimetry problems, emphasizing that he thought that the low L.E.T. radiations were in good shape. However, for the neutron dosimetry, high L.E.T., there is certainly a lack of confidence. I don't know how much this means from the standpoint of the numbers of people in the exposed population i.e. how many receive neutron doses of magnitude, but I can visualize that this is a problem.

Bernie Weiss gave encouragement to the fact that we're beginning to make some inroad into standards for the performance of personnel dosimeters. I'd only add to this that if he can do this for essentially an occupational situation, there is some hope that we can apply these same kinds of performance standards in the general environment.

So, in general, I got from this session that we can make measurements at low levels in the environment and we must be as precise as we can be in those we make. If you sit in the public arena like some of us do, it doesn't do much good to rely to a large extent on models. If you get a public inquiry and you respond that the model shows such and such it doesn't satisfy many people. But they are satisfied if you have gone out and made a measurement. They have more confidence in that. I think that does more to sell the need for measurement capability, for example, in nuclear power than anything else. If you can say you've made a measurement you can go a long way in satisfying many of the concerned. Unfortunately, while our physics, our instrumentation, our electronics, and our radiochemistry have gotten very precise, keep in mind that the biology that goes with it is not that precise. We can't do anything about that, we have to live with it. This is not to say, however, that we shouldn't keep our sights on the preciseness of the measurements.

As far as the future of the Symposium. I would emphasize from an environmental standpoint that we have two major problems at the present time that I see in the ionizing field. We have to be able to measure plutonium and we have to be able to do it in a precise way. There are many areas in which plutonium contamination is being discussed and action will have to be taken. We've also got the problem of emanations from tailing piles, from phosphate mining in which $\alpha$-daughters from radon is an environmental problem that requires improvement in our ability to measure. The last point is for the non-ionizing field. I would plead for more environmental measurements, hopefully by the time we can scope that problem the biology will be sufficient that we can say there is a non-problem. But at this stage in the game we just can't make that statement. So I would say that in three to five years, if we could come in with a program designed to look at the radiochemical procedures, in particular in a-measurements, this would make many of us in the environmental arena very happy.

Dr. Trout:

While Randy's collecting his thoughts I want to tell you what was one of my most enjoyable moments. I enjoyed thoroughly Harold Stewart's presentation when he got up here and developed some standards with nothing but some basic high school and college physics. He didn't have a transistor on the screen, and I didn't see a computer anywhere. 
Now from the NBS, that provides the product that goes on the shelf for the consumer to use, here's Randy Caswell of the Bureau.

Dr. Caswe11:

First, I'd like to say something concerning measurements which were mentioned in connection with safeguards. NBS is trying to establish a program of measurements and standards of special nuclear material which is not part of the environmental problem.

Now as we come back to the question of the meeting, one of the participants in the meeting remarked to me yesterday that this was a different meeting than he expected to find. I guess he was expecting more technical measurements and what he found was a meeting that included some of that but also included a lot of questions on the nature of measurement policy. That got me thinking about who are the people who came to the meeting. As I indicated in my talk the first day, one of the ways of looking at the system is to divide people up into whether they are direct users of radiation or manufacturers, or standards and calibration lab people, or regulators, or some of the other interested groups such as ANSI, NCRP, and standards setting organizations. So I took the list of participants and went through it and counted. I arbitrarily decided which you were, according to the institution you came from, and probably made some mistakes. If you were from a University or Hospital, I thought you were a direct user. If you were from BRH I thought you were a regulator, and so on. I came out with some percentages of the audience, and these are: $53 \%$ of the audience are direct users of radiation, 18\% represent manufacturers of instruments or of sources or radiopharmaceutical manufacturers, $10 \%$ are from standards 1abs, $16 \%$ are from regulatory agencies of state or federal government or 1oca1, and 3\% are from other categories. The person who made this original suggestion to me said, you looked at the wrong set of numbers--what you really should have looked up was the fraction of the papers that were in the various categories. So I said, I'll do that too, that's even easier because there are less papers. It turns out that $39 \%$ of the papers were from users, $7 \%$ from manufacturers, which is surprisingly low to me, $28 \%$ from standards 1 abs, $24 \%$ from regulators and $2 \%$ from other organizations. I think these numbers in a way are not too surprising in the sense that part of the goal of the meeting was a communication from standards people and regulators to users. It's not surprising to me anyway that these two groups are well represented in the program. I thought you might be interested in that.

The one other point I want to key on, which impressed me in the meeting, is the importance of feedback in various ways in the measurement system. I think, certainly one of the purposes of the meeting was to have constructive feedback from one group to another and I think this was largely achieved. I heard lots of feedback during the meeting. This also reminded me, looking at it from an NBS standpoint, that the way you get standards out to the user and help the user in getting demonstrable accuracy occurs in four steps. First, develop a standard. Second, issue a standard or calibration. Third is to carry out measurement assurance to see whether the measurements are really correct, or whether there is some problem. Fourth, the very important feedback or correction step. I think 15 or 20 years ago at NBS we did largely the first two. We developed the standards and we issued them or we made calibrations available. He who wanted to come and get a calibration could do so and he who chose not to--nobody bothered him. He didn't have to do so. The third step, which is very active now, is the one of measurement assurance and there were lots of results of measurement assurance tests and round robins reported at the meeting. But I think the final step, and the one where the problem is now, is in the feedback or correction step. I think, for example, Bob Shalek's paper 
showed beautifully what the feedback or correction steps could do to improve measurements in a system. Some mechanism is needed to find out what's wrong, to correct it and improve the system. I think the areas where we are in trouble is where we have not, for some reason or another, been able to carry out that fourth step. Therefore, I think that's what we should perhaps be focusing on.

I'11 just comment on one of the other questions--remarks on the measurement system, and on my area of interest--I sort of have two hats. As a member of the office of our Center, I'm interested in the broad system and I think here the pattern is clearly specified. Some areas seem to be in good shape and other areas seem to need much more work. My other hat is that of a specialist in neutron dosimetry. There we have sort of a strange situation in that the laboratories doing therapy in the United States are very closely tied to each other on an arbitrary scale, but there is no standard produced by the National Bureau of Standards, and there is no well-determined absolute scale. I think before we can feel that this area is in really satisfactory shape we do need absolute standards.

Dr. Trout:

Up to this point the Panel has been speaking to the audience. For the next ten minutes I'm going to ask them to speak to one another. Bill, say what you think to the other panelists, ask them questions and the rest of you hold your answer until I get to you. What would you like to say to the three other panelists?

Dr. Mills:

Dr. Cameron's discussion on the medical--let me be the first to endorse what he said about the Illinois situation. We have a great deal of interest in the dose ranges used in the medical area and it seems to me that that's an area in which there can be a very great reduction in the exposures, the unnecessary parts of the exposure, and a lot of man-rems (if you want to use man-rems) can be saved in this area. So I don't know as I really have a question addressed to that point, but I certainly go along with the fact that if we want to make a contribution in terms of reduction of exposure, and if it depends on good standards in the medical field, I certainly think this is a very viable area to go into. I guess I really don't have too much complaint with what the other panel members have said because Matuszek isn't on this program. I would like the chance to defend the drinking water standards, but he's not here so it would just be wasted effort, I think. I certainiy think state labs will have to share a large burden of the drinking water act, not just for radiation but for the other pollutants as well, and are going to have to have federal funds to do it. There's no question. They just can't do that job by themselves.

Dr. Trout:

It looks as though we have a universally approved message here to OMB. John, what can you say? Don't join the mutual admiration society with Bill there. Give us a little conflict.

Dr. Cameron:

I was just going to comment to Gerry's question about the need for a low energy chamber for measuring radiation in the mammography region. The Memorial Sloan Kettering group has developed a very nice chamber which, I think, they are now putting on the market. They have found somebody to produce it and Garrett Holt would be the person to contact about that. It has accuracy, flatness of energy response relative to air of about $1 \%$ in the energy region from a half value layer of $.3 \mathrm{~mm}$ to about $1.5 \mathrm{~mm}$ of aluminum. I think to a large extent it's not real1y necessary. I would like to argue that measuring mammography exposures to $1 \%$ or even $5 \%$ is a bit of overki11. I think one of the last speakers, Jim Leiss, made the point that there's no point in measuring something more accurately than you need it. We measured quite a few 
Mr. Parker:

Dr. Cameron:

Mr. Parker:

Dr. Cameron:

Dr. Caswel1 mammographic exposures in connection with our Midwest Center for Radiological Physics. We found one center that over a period of about a month had tripled this exposure. It had gone from about $1 R$ to $3 R$. This is the kind of thing we're looking for. We aren't looking for the $1 \%$ or $5 \%$ changes. TLD is quite adequate in this case, even if you get on 1 y 10 or $20 \%$ accuracy. I am often impressed by high accuracy, but I think in the field we don't really need it.

John, I couldn't agree with you more. We don't really need that precision. I think that was my point that I made in my talk and it's true. I spoke to Garrett about his dosimeters while he was here. We haven't seen how they will operate in the field. The proof will be if 50 states are able to get reproducible results from these dosimeters. When you're getting 47 rads per mammographic film you really don't need $5 \%, 10 \%$ or $20 \%$ accuracy. I'd $7 i k e$ to go back to two of the papers in your session John. Dr. Shalek's and Dr. Rhodes'. I'11 mention Buck's first because that was a minor point. He spoke about brain scanning using $30 \mathrm{mCi}$, and when questioned by the audience he dropped it to 20 and I'd like to know why you, as someone from a large physics organization, didn't say "hey, what the he11 are you using 20 for, why don't you get down to 10 ?"

Partly because I left the nuclear medicine field when they were still using mercury and giving the kidneys about 200 rads. I plead ignorance in this particular case.

With the Shalek thing I just don't see your optimism that this shows good results. Here we have sophisticated centers and we get that kind of data. Can you imagine what's going on in the smaller therapy installations?

One of the errors he pointed out was caught at wisconsin. He found out we had a ${ }^{60} \mathrm{Co}$ source that decayed with a half 1 ife of 3.5 years instead of 5.3. Nature got the numbers reversed! Apparently the source consisted of ${ }^{60} \mathrm{Co}$ of various specific activities, with the hotter ${ }^{60} \mathrm{Co}$ in front. The source was apparently not properly made and the colder ${ }^{60} \mathrm{Co}$ gradually moved forward giving more self absorption to the hotter ${ }^{60} \mathrm{Co}$. Even relatively good places can make mistakes!

I hate to be agreeable with a fellow member of the Panel but I do agree with the person who said that probably what is needed next are special technical meetings in certain areas and that some period like three years is probably an appropriate period for repetition of a general type of meeting. Secondly, one of our problems is how to make the regional calibration laboratory system work or be viable. I believe we have three now and I think they're a fine system because I think people in medical fields like to come to a medical-type institution for their calibration. But unless we can find some means of making these economically viable and create more successful ones, the approximately 10,000 institutions in the field are not going to be reached with calibration. That's a problem that I would encourage the audience to think about and try to help. We're interested in that too, and we should work together to try to solve it. Just one other thing I wanted to add since I have the microphone. This measurement system study that I talked about is one of a number and they will be coming out in about three months as NBS reports on such subjects as electromagnetics, medical ultrasonics, acoustics, radiometry and photometry, spectrophotometry, far ultraviolet radiometry, optics, lasers, ionizing radiation and so on. If you're in such a position where you would like to see what somebody who tried to study part of a system found out; in about three months it will be easy to get a hold of such information by writing to NBS. 
Roger Aamodt

National Institutes of Health

Building 10, Rm. B3B25

9000 Rockville Pike

Bethesda, Maryland 20014

Siro Abe

National Institute of Radiological

Sciences

9-1, Anagawa-4

Chíba-Shi JAPAN 280

Robert Adams

Case Western Reserve University

2065 Adelbert Road

Cleveland, Ohio 44106

John Alexander

Oak Ridge National Laboratory

Health Physics Division

Box X

Oak Ridge, Tennessee 37830

Michael Altebrando

Grumman Aerospace Corporation

Bethpage, New York 11714

Charles Armentrout

Department of Physical Sciences

and Engineering

University of Maine

96 Falmouth Street

Portland, Maine 04103

John Arras

SAF Department

Armed Forces Radiobiology

Research Institute

NNMC

Bethesda, Maryland 20014

Frank Attix

Naval Research Laboratory

Code 6603A

Washington, D.C. 20375
Migue1 Awschalom

Fermi National Accelerator Lab

P.O. Box 500

Batavia, Illinois 60510

Norman Baily

University of California, San Diego

School of Medicine

Department of Radiology M-010

La Jolla, California 92093

Ramon Baird

Electromagnetics Division

National Bureau of Standards

Boulder, Colorado 80302

Daniel Balkunow

E. R. Squibb and Sons, Inc.

Georges Road

New Brunswick, New Jersey 08903

Stephen Balter

Philips Medical Systems, Inc.

710 Bridgeport Avenue

Shelton, Connecticut 06484

Paul Barrans

RADEF Instrumentation Test Facility

U.S. Army Communications Command

Building 22, Paulding Street

Washington Navy Yard

Washington, D.C. 20374

Harry Bartlett

E. R. Squibb and Sons, Inc.

Georges Road

New Brunswick, New Jersey 08903

Arden E. Bicker

Reynolds Electrical and Engineering Co.

P. 0. Box 14400

Las Vegas, Nevada 89114

Ra1ph Bish

CAPINTEC Instruments Inc.

540 Alpha Drive

Pittsburgh, Pennsylvania 15238 
Nick Blaskovich

U.S. Department of HEW-NIOSH

944 Chestnut Ridge Road

Morgantown, West Virginia 26505

Joseph Blinick

Maine Medical Center

22 Bramhall Street

Portland, Maine 04102

Leroy Booth

Naval Research Lab

Washington, D.C. 20375

Arthur Boyer

Massachusetts General Hospital

Boston, Massachusetts 02114

Svresh Brahmavar

Medical Center of Western Massachusetts 759 Chestnut Street

Springfield, Massachusetts 01107

Robert Britain

Bureau of Radiological Health

Division of Compliance

Rockville, Maryland 20852

William Britt

The Machlett Laboratories, Inc.

1063 Hope Street

Stamford, Connecticut 06907

George Brodie

RADEF Instrumentation Test Facility, USACC Building 22, Paulding Street

Washington Navy Yard

Washington, D.C. 20374

David Brown

Bureau of Radiological Health

12720 Twinbrook Parkway, 非204

Rockville, Maryland 20851

Frank Brown

Eastman Kodak Company

Health, Safety and Human Factors

Laboratory

B-320, Kodak Park

Rochester, New York 14650
Joseph Brown

Nuclear Regulatory Commission

5903 Greentree Road

Bethesda, Maryland 20034

Henry Buchanan

Yankee Atomic Electric Company

20 Turnpike Road

Westborough, Massachusetts

01581

Gail de Planque Burke

U.S. ERDA, HASL

376 Hudson Street

New York, New York 10014

David Burton

Litton Bionetics

5516 Nicholson Lane

Kensington, Maryland 20795

Robert Butenhoff

Division of Biomedical and

Environmental Research

U.S. ERDA

Washington, D.C. 20545

John Cameron

University of Wisconsin

Sterling Hall

Madison, Wisconsin 53706

Victoria Castro

American Oncologic Hospital

Central and Shelmire Avenues

Philadelphia, Pennsylvania 19111

Randa11 Caswe11

Center for Radiation Research

National Bureau of Standards

Washington, D.C. 20234

Gu1 Chughtai

Radiation Therapy

Georgetown University Medical Center

Washington, D.C. 20007

Robert Clark

Searle Analytic Inc.

2000 Nuclear Drive

Des Plaines, Illinois 60018 
D. Collins

U.S. ERDA Savannah River

P.O. Box A

Aiken, South Carolina 29801

John Conne11y

NUS

4 Research Place

Rockville, Maryland 20850

Grace Conrad

ArIington County Virginia

Department of Inspection Services

Court House

Arlington, Virginia 22201

John Cooper

Nuclear Science Center

Auburn University

Auburn, Alabama 36830

Tommy Corbin

Lawrence Radiation Lab

End of Hearst Avenue

Berkeley, California 94720

Karin Corey

136 High Street

Leonia, New Jersey 07605

Bert Coursey

Center for Radiation Research

National Bureau of Standards

Washington, D.C. 20234

Sue Crain

Monsanto Research Corporation

Mound Laboratory

Miamisburg, Ohio 45342

Roland Davis

Los Alamos Scientific Laboratory

P.0. Box 1663

Los Alamos, New Mexico 87544

Neal De Gaston

Orange County Medical Center

University of California, Irvine

101 The City Drive

Orange, California 92668
Herbert Deich

$\mathrm{MDH}$ Industries, Inc.

426 Duarte Road

Monrovia, California 91016

Andrzej Demidecki

American Association of Physicists

in Medicine

Suite 307

6900 Wisconsin Avenue

Chevy Chase, Maryland 20015

James Deye

George Washington University

Medical Center

Radiology Department

901 23rd Street, N.W.

Washington, D.C. 20037

Charles Dick

National Bureau of Standards

Washington, D.C. 20234

Howard Dickson

ORNL

P.O. Box X

Oak Ridge, Tennessee 37830

J. William Dirksen

University of Nebraska Medical Center

42nd and Dewey Avenue

Omaha, Nebraska 68105

Ponce Dixon

U.S. Army Edgewood Arsenal, APG

2127 Round Hill Road

Fallston, Maryland 21047

Elwyn Dolecek

Rutgers University Radiation Safety

Department

Doolittle Building, Rm. 3572

New Brunswick, New Jersey 08903

Steve Domen

National Bureau of Standards

Washington, D.C. 20234 
Richard Donovan

RAB Department

Armed Forces Radiobiology Research Inst. NNMC

Bethesda, Maryland 20014

Keith Eckerman

Nuclear Regulatory Commission

Washington, D.C. 20555

Elmer Eisenhower

National Bureau of Standards

Washington, D.C. 20234

Jack E11iott

Lawrence Livermore Laboratory

P.O. Box 808

Livermore, California 94550

David Espejo-Villalobos

Hospital San Jose De Monterrey

Ave. Morones Prieto Pte N ${ }^{\circ} 3000$

Monterrey, N.L. Mexico

Frederick Faw

Arnot-Ogden Memorial Hospital

Grove and Roe Avenues

Elmira, New York 14901

Sherman Fivozinsky

National Bureau of Standards

Washington, D.C. 20234

Ralph Flint

Battelle Columbus Labs

505 King Avenue

Columbus, Ohio 43201

Gary P. Foster

Division of Labor and Industry

203 E. Baltimore Street

Baltimore, Maryland 21202

Judith Foulke

U.S. Nuclear Regulatory Commission

office of Nuclear Material Safety

and Safeguards

Washington, D.C. 20555
Douglas Fuhrman

Teledyne Isotopes

50 Van Buren Avenue

Westwood, New Jersey

07675

\section{Gary Fullerton}

University of Minnesota

Ther. Radiology Department

Box 494 Mayo Building

Minneapolis, Minnesota 55455

Marianne Gabel

Yale University

219 Prospect Street

New Haven, Connecticut 06520

David George

National Naval Medical Center

Bethesda, Maryland 20014

T. F. Gesel1

University of Texas School of

Public Health

P.O. Box 20186

Houston, Texas 77025

Wallace Giffen

Mis-Hospital Shared Engineering

Services, Inc.

Box 1441

Springfield, Ohio 45501

David Gilliam

National Bureau of Standards

Washington, D.C. 20234

Richard Gilmore

School of Medicine

Marshall University

Huntington, West Virginia 25701

Leon Goodman

Columbia University

Brookhaven National Lab

Building 902B

Upton, New York 11973 
Richard Granke

1311 Downs Drive

Silver Spring, Maryland 20904

Richard Griffith

Lawrence Livermore Laboratory

Trailer 21, Room 103, L-520

P.O. Box 808

Livermore, California 94550

Michael Grissom

EXP Department

Armed Forces Radiobiology Research Institute NNMC

Bethesda, Maryland 20014

James Grolton

Georgetown University Medical Center

Radiation Control office

3800 Reservoir Road, N.W.

Washington, D.C. 20007

Walter Gundaker

FDA-Bureau of Radiological Health

5600 Fishers Lane (HFX-470)

Rockville, Maryland 20852

Charles $\mathrm{Hall}$

E. I. duPont

Photo Products Department

Chestnut Run

Wilmington, Delaware 19898

Mahmoud Hammoudah

Howard University College of Medicine

Radiotherapy Department

Washington, D.C. 20060

James Hancock

Traps, Inc. and DePaul Hospital

1130 Hanover Avenue

Norfolk, Virginia 23508

Gerald Hanson

Pan American Health Organization

525 23rd Street, N.W.

Washington, D.C. 20037
Rupert Henry

General Electric Company

Building 273, Rm. 299

Schenectady, New York 12345

John Hickey

U.S. Nuclear Regulatory Commission

Nicholson Lane Building

Washington, D.C. 20555

Carolyn Holman

Sawyer and Joiner, M.D., P.A.

85 W. Sturtevant

Orlando, Florida 32806

J. Garrett Holt

Memorial Hospital

1275 York Avenue

New York, New York 10021

Yingchieh Hsu

Nuclear Medicine

Veterans Administration Hospital

University Drive C

Pittsburgh, Pennsylvania 15240

Jimmy Humphreys

National Bureau of Standards

Washington, D.C. 20234

Robin Hutchinson

National Bureau of Standards

Washington, D.C. 20234

Thomas Jackson

Dosimeter Corporation

6106 Interstate Circle

Cincinnati, Ohio 45242

Ahren Jacobson

University of Louisville

Radiation Center

500 South Floyd Street

Louisville, Kentucky 40201

Arthur Jarvis

Office of Quality Assurance-Radiation P.O. Box 15027

Las Vegas, Nevada 89114 
Ronald Johnson

National Bureau of Standards

Washington, D.C. 20234

Tommy Johnson

Naval Research Lab

Code 6070

Washington, D.C. 20375

Donald Jones

ERDA

1253 Norton

Idaho Falls, Idaho 83401

Robert Jucius

General Electric

Medical Systems Division

P.0. Box 414

Milwaukee, Wisconsin 53201

Kenneth Kase

Harvard University Medical School

50 Binney Street

Boston, Massachusetts 02115

Albert Kasenter

Henry Ford Hospital

Department of Radiology

2799 W. Grand Boulevard

Detroit, Michigan 48202

\section{David Katzman}

Teledyne Isotopes

50 Van Buren Avenue

Westwood, New Jersey 07675

Eric Kearsley

RAB Department

Armed Forces Radiobiology Research Inst. NNMC

Bethesda, Maryland 20014

Ronald Keyser

ORTEC

100 Midland Road

Oak Ridge, Tennessee 37830
Allan Kolber

NYU Institute of Environmental Medicine 138 Village Circle West

Paramus, New Jersey 07652

Dean Kunihiro

RAB Department

Armed Forces Radiobiology Research Inst. NNMC

Bethesda, Maryland 20014

Paul Lamperti

National Bureau of Standards

Washington, D.C. 20234

Robert S. Landauer

R. S. Landauer, Jr. and Company Science Road

Glenwood, Illinois 60425

Lawrence Lanzl

University of Chicago

950 East 59th Street

Chicago, Illinois 60637

Harold Larson

Battelle Northwest Laboratories

P.O. Box 999

Richland, Washington 99352

Jay Lee

U.S. Nuclear Regulatory Commission

ETSB/SSEA/NRR

Washington, D.C. 20555

Paul Leggett

Bureau of Radiological Health

Division of Compliance

Rockville, Maryland 20852

Vernon Leininger

Department of Radiology

University of Connecticut

Farmington, Connecticut 06032 
James Leiss

Center for Radiation Research

National Bureau of Standards

Washington, D.C. 20234

Irving Lerch

Radiation Oncology

New York University Medical Center

566 First Avenue

New York, New York 10016

Stuart Levin

Pennsylvania Department of

Environmental Resources

1204 Kossman Building

100 Forbes Avenue

Pittsburgh, Pennsylvania 15222

Gerald Levine

Brookhaven National Lab

Building 535

Upton, New York 11973

Pei-Jan Lin

Northwestern University Medical School

303 East Chicago Avenue

Chicago, Illinois 60611

Otha Linton

American College of Radiology

6900 Wisconsin Avenue

Chevy Chase, Maryland 20015

Joseph Lochamy

Duke Power Company

422 S. Church Street

P.O. Box 2178

Charlotte, North Carolina 28242

Robert Loevinger

National Bureau of Standards

Washington, D.C. 20234

John Lombardi

U.S. Army Ordnance Center and School

1315 Charleston Drive

Edgewood, Maryland 21040
Joseph McDonald

Sloan-Kettering Institute

1275 York Avenue

New York, New York 10021

Norman McElroy

George Washington University

Medical Center

2300 K Street, N.W.

Washington, D.C. 20037

Stephen A. McGuire

Nuclear Regulatory Commission

Washington, D.C. 20555

James McLaughlin

U.S. ERDA

Health and Safety Laboratory

376 Hudson Street

New York, New York 10014

Elaine Mackowiak

Temple University

School of Pharmacy

3307 N. Broad Street

Philadelphia, Pennsylvania 19140

Henry Magee

Radiation Health Division (Code 105.05)

Pearl Harbor, Hawaii 96610

Adam Malik

Union Carbide Corporation-Linde Division

175 E. Park Drive

P. 0. Box 44

Tonawanda, New York 14150

Marjorie Malmberg

NUS Corporation

Rockville, Maryland 20850

John Matuszek

New York State Department of Health

Radiological Sciences Laboratory

30 Russe11 Road

Albany, New York 12206 
Karl Mendenhall

RAB Department

Armed Forces Radiobiology Research Inst. NNMC

Bethesda, Maryland 20014

William Mills

Criteria and Standards Division (AW-460)

U.S. Environmental Protection Agency

401 M. Street, S.W.

Washington, D.C. 20460

Thomas Mitchell

Georgetown University Medical Center

Radiation Control Office

3800 Reservoir Road, N.W.

Washington, D.C. 20007

R. W. Modrel1

Goodyear Tire and Rubber Company

1144 East Market Street

Akron, Ohio 44316

Robert Mogle

Virginia Polytechnic Institute

and State University

Robeson Hall, Rm. 13

Blacksburg, Virginia 24061

Tommie Morgan

Bureau of Radiological Health

5600 Fishers Lane

Rockville Maryland 20852

Michael Moroz

Pennwalt Corporation

S. S. White Division

P.O. Box 100, South Street

Holmde1, New Jersey 07733

Robert Morton

Nuclear Medicine Branch

National Naval Medical Center

Bethesda, Maryland 20014

C. E. Moss

NIOSH

PAEB/DBBS -4th F1oor

R. A. Taft Lab

4676 Columbia Parkway

Cincinnati, Ohio 45226
William Moss

Los Alamos Scientific Laboratory MS 486

P.0. Box 1663

Los Alamos, New Mexico 87545

Joseph Motz

National Bureau of Standards

Washington, D.C. 20234

\section{Victor Múnoz}

Centro Regional de Calibracion

y Dosimetria S.S.A.

Dr. Balmis No. 148

Mexico D.F. Mexico

John Munro

Technical Operations, Inc.

40 South Avenue

Burlington, Massachusetts 01803

Ravi Nair

Ohio State University Hospital

410 W. 10 th Avenue

Columbus, Ohio 43221

Christopher Nelson

USEPA/Office of Radiation Programs (AW-461)

401 M Street, S.W.

Washington, D.C. 20460

W. Roger Ney

National Council on Radiation

Protection and Measurements

7910 Woodmont Avenue, Suite 1016

Washington, D.C. 20014

Jagannadha Rao Nibhanupudy Howard University Hospital Radiotherapy Department 2041 Georgia Avenue, N.W. Washington, D.C. 20060

Lowell Nichols

Battelle Northwest

P.O. Box 999

Richland, Washington 99352 
Edward Nickoloff

The. Johns Hopkins University

School of Hygiene and Public Health Rm. 1102

$615 \mathrm{~N}$. Wolfe Street

Baltimore, Maryland

21205

Dale Nix

Tennessee Valley Authority

204 Greenfield Road

Florence, Alabama 35660

James Noyce

National Bureau of Standards

Washington, D.C. 20234

Thomas Ohlhaber

FDA, Bureau of Radiological Health

12720 Twinbrook Parkway

Rockville, Maryland 20852

W. F. Ohnesorge

Oak Ridge National Laboratory

P.O. Box X

Oak Ridge, Tennessee 37830

G. Osman

Department of Radiation Medicine

Roswell Park Memorial Institute

Buffalo, New York 14263

William Ott

National Bureau of Standards

Washington, D.C. 20234

John Paciga

New Brunswick Electric Power Commission

527 King Street

Fredericton, New Brunswick Canada

Bhudatt Paliwal

University of Wisconsin-Madison

Radiology Department

475 N. Charter Street

Madison, Wisconsin 53706

Peter Paras

Bureau of Radiological Health

1901 Chapman Avenue

Rockville, Maryland 20852
Gerald Parker

Massachusetts Department of Public Health 80 Boylston Street

Rm. 835

Boston, Massachusetts 02116

Marshall Parrott

Radiation Control Service

State Health Division

P.0. Box 231

Portland, Oregon 97207

Sidney Parry

USNRC-Division of Organization and

Personnel

Training and Development Branch

Washington, D.C. 20555

Donald Parsons

Roswell Park Memorial Institute

666 Elm Street

Buffalo, New York 14263

Indu Pate 1

Howard University

College of Allied Health

Washington, D.C. 20060

Gerald Peters

Naval Surface Weapons Center

White Oak Laboratory, Code WR41

Building 2-013

Silver Spring, Maryland 20910

Paul Pfalzner

Ontario Cancer Treatment and

Research Foundation

1053 Carling Avenue

Ottawa, Canada K1Y 4 E9

Leigh Phillips

Brookhaven National Laboratory

Building 535

Upton, New York 11973

Robert Placious

National Bureau of Standards

Washington, D.C. 20234 
Robert Poe

ERDA

Division of Operational Safety

Washington, D.C. 20545

John Potter

Nuclear Regulatory Commission

Washington, D.C. 20555

\section{William Powers}

Naval Research Laboratory

Washington, D.C. 20375

\section{S. Guru Prasad}

Evans Hospital

2650 Ridge Avenue

Evanston, Illinois 60201

Mark Presser

Victoreen Instrument Company

10101 Woodland Avenue

Cleveland, Ohio 44104

Frederick Prevo

Bureau of Radiological Health

12720 Twinbrook Parkway

Rockville, Maryland 20852

John Pruitt

National Bureau of Standards

Washingt on, D.C. 20234

William Quam

EG\&G

130 Robin Hill Road

Goleta, California 93017

Mohammed Quddus

Mechanical and Aerospace Engineering Dept.

University of Delaware

Newark, Delaware 19711

Philip Quinn

FDA/WEAC

109 Holton Street

Winchester, Massachusetts 01890
Gustavo Quintanilla-E.

ITESM Physics Department

Sucursal De Correos "J"

Monterrey, N.L. Mexico

Buck Rhodes

University of Kansas Medical Center

Rainbow Boulevard at 39th Street

Kansas City, Kansas 66103

Gordon Riel

Naval Surface Weapons Center

White Oak

Silver Spring, Maryland 20910

Francis Roddy

Bechtel Power Corporation

15740 Shady Grove Road

Gaithersburg, Maryland 20760

Jerry Rosen

University of Pittsburgh

Graduate School of Public Health

Pittsburgh, Pennsylvania 15213

Paul Rosenbaum

Nuclear Radiation Consultants

P.O. Box 647

New Haven, Connecticut 06503

David Ross

SINB

7 Dunwoody Park-Suite 104

Atlanta, Georgia 30341

Harvey Rudolph

Bureau of Radiological Health

12720 Twinbrook Parkway

Rockville, Maryland 20852

Warren Rumble

National Institutes of Health 9000 Rockville Pike

Bethesda, Maryland 20014

Roger Schneider

Division of Electrontc Products Bureau of Radiological Health

5600 Fishers Lane

Rockville, Maryland 20852 
Robert Schwartz

National Bureau of Standards

Washington, D.C. 20234

Richard Sha

Radiation Management Corporation

Science Center Building 非, Suite 400

3508 Market Street

Philadelphia, Pennsylvania 19104

Robert Shalek

Physics Department

M.D. Anderson Hospital

Houston, Texas 77025

John Shaver

University of California Medical Center

350 Parnessus Avenue, Suite 307

San Francisco, California 94117

Bruce Shields

Union Carbide Corporation

Tarrytown Technical Center

Spine Building

Tarrytown, New York 10591

Melvin Siedband

Medical Physics-Department of Radiology

University of Wisconsin Medical School

Madison, Wisconsin 53706

Robert Simpson

Bureau of Radiological Health

5600 Fishers Lane

Rockville, Maryland 20852

Lester Slaback

SAF Department

Armed Forces Radiobiology Research Inst. NNMC

Bethesda, Maryland 20014

Robert Sleever

White Sands Missile Range

Nucleonics Calibration

P.O. Box 182

White Sands Missile Range, New Mexico

88002
Richard Smale

Los Alamos Scientific Laboratory

$\mathrm{H}-1$, MS403

P.0. Box 1663

Los Alamos, New Mexico

87545

Robert Smith

Westinghouse Hanford

Box 1970

Richland, Washington 99352

George Snyder

U.S. Navy

19066 Montgomery Village Avenue

Gaithersburg, Maryland 20760

Julian Sparrow

National Bureau of Standards

Washington, D.C. 20234

E. W. Spiers

Toronto General Hospital

College Street

Toronto, Ontario M5G1L7

Jacob Spira

Boston University School of Medicine

80 East Concord Street

Boston, Massachusetts 02118

Larry Stephenson

Texas Department of Health Resources 1100 W. $49 \mathrm{th}$

Austin, Texas 78756

Eugene Stickley

Medical College of Virginia

Richmond, Virginia 23298

Robert Strauss

Picker Corporation

595 Miner Road

Cleveland, Ohio 44143

Kar1 Stuermer

Victoreen Instrument Company

10101 Woodland Avenue

Cleveland, Ohio 44104 
Monte Sula

United Nuclear Industries, Inc. Box 490

Richland, Washington 99352

N. Suntharalingam

Department of Radiation Therapy

Thomas Jefferson University Hospital

Philadelphia, Pennsylvania 19107

Arata Suzuki

CAPINTEC Instruments Inc.

540 Alpha Drive

Pittsburgh, Pennsylvania 15238

Michael Svoren

LSU Schoo1 of Medicine

P.O. Box 3932

Shreveport, Louisiana 71130

John Taschner

Bureau of Radiological Health

18917 Alpenglow Lane

Brookeville, Maryland 20729

Lauriston Taylor

National Council on Radiation Protection and Measurements

7910 Woodmont Avenue, Suite 1016

Washington, D.C. 20014

Warren Taylor

Sandia Laboratories-Division 9531

P.O. Box 5800

Albuquerque, New Mexico 87115

José Telich

Hospita1 Españo1

Av. Ejercito Nacional 613

Mexico 5, D.F. Mexico

Jerry Tetenes

Albert Einstein College of Medicine

1825 East Eastchester Road

Bronx, New York 10461
Joseph Thiel

Texas Department of Health Resources 1100 West 49th Street

Austin, Texas 78756

Danie1 Thompson

Sandia Laboratories

Albuquerque, New Mexico

87115

Winston Thompson

Howard University

College of Allied Health

Washington, D.C. 20059

Hans Toveda 1

$\mathrm{AB}$ Atomenergi

Studsvik, Fack

S-61101 Nykoping, Sweden

E. Dale Trout

Oregon State University

X-Ray Science and Engineering Lab

Corval1is, Oregon 97331

George Tucker

Sandia Laboratories

Albuquerque, New Mexico

87115

Arnold VanArt

Litton Medical Systems

515 E. Touhy Avenue

Des Plaines, Illinois 60018

Wesley Van Pelt

Hoffmann-La Roche Inc.

340 Kingsland Street

Nutley, New Jersey 07110

David Verrel1i

Research Program Coordinating Office Armed Forces Radiobiology Research Inst. NNMC

Bethesda, Maryland 20014 
G. Walford

ORTEC Inc.

100 Midland Road

Oak Ridge, Tennessee 37830

William Walker

Malcolm Grow USAF Medical Center

Box 3525

Andrews Air Force Base

Washington, D.C. 20331

L. Walsh

Ontario Hydro

Pickering G.S.-Operations

Pickering, Ontario Canada L1V2R5

John Wang

Federal Communications Commission

2025 M Street, N.W., Rn. 7202

Washington, D.C. 20554

Joseph Wang

Naval Surface Weapons Center

White Oak Laboratory

Silver Spring, Maryland 20910

John Washington

Northwest Medical Physics Center

$\mathrm{RC}-08$, University Hospital

Seattle, Washington 98195

Goldie Watkins

New York State Department of Health

Empire State Plaza

Albany, New York 12237

Arthur Weis

CAPINTEC Inc.

136 Sumit Avenue

Montvale, New Jersey 07645

Bernard Weiss

Radiological and Environmental Protection

Branch

Office of Inspection and Enforcement

Nuclear Regulatory Commission

Washington, D.C. 20555
M. Weiss

Bell Laboratories Inc.

600 Mountain Avenue

Murray Hill, New Jersey 07974

Bryan Westerman

Department of Nuclear Medicine

Northwestern Memorial Hospital

Fairbanks and Superior

Chicago, Illinois 60611

Robert Wheeler

R. S. Landauer, Jr. and Company Science Road

Glenwood, Illinois 60425

Carl-O Widell

$\mathrm{AB}$ Atomenergi

Studsvik

S-61101 Nykoping, Sweden

John Widman

Veterans Administration Hospital

Nuclear Medicine Service

Allen Park, Michigan 48101

Richard Wilder

Toledo Ohio Board of Health

635 N. Erie Street

Toledo, Ohio 43624

G. Wilkening

Bel1 Laboratories

600 Mountain Avenue

Murray Hill, New Jersey 07974

Edward Williams

RADEF Instrumentation Test Facility

U.S. Army Communication Command

6626 Reynard Drive

Springfield, Virginia 22152

C. Wright

E. I. DuPont De Nemours and Company Savannah River Plant Aiken, South Carolina 29801 
James Wyckoff

National Bureau of Standards

Washington, D.C. 20234

George Zacharopoulos

Montefiore Hospital and Medical Center

111 East 210 Street

Bronx, New York 10467

Al Zirkes

ICN Dosimetry Service

26201 Miles Road

Cleveland, Ohio 44128 
Athey, T.W. $\quad 203$

Attix, F.H. $\quad 335$

August, L.S. $\quad 335$

Baird, R.C. $\quad 417$

Barrans, P.V. $\quad 59$

Bassen, H.I. 99

Berger, M.J. $\quad 307$

Blinick, J.S. $\quad 359$

Bjarngard, B.E. $\quad 343$

Brodie, G.H. $\quad 59$

Burke, G.D. $\quad 187$

Cail, D. 359

Cameron, J.R. $\quad 427$

Caswel1, R.S. 13, 427

Coakley, J.M. 215

Collé, R. 71

Cooper, J.A. $\quad 159$

Coursey, B.M. $\quad 173$

Cundiff, J.H. 111

Dick, C.E. 47

Dickson, H.W. 225

Domen, S.R. $\quad 307$

Easter7y, D.G. 263

Ehrlich, M. $\quad 365$

Fox, W.F. 225

Gabe1, M.M. $\quad 371$

Gagnon, W.F. $\quad 111$

Gallagher, J.H. 131

Gesel1, T.F. 187

Goldman, D.T. 3

Goodman, L.J. $\quad 327$

Grant, W., III $\quad 111$

Griffith, R.V. $\quad 267$

Hankin, N.N. 203

Hanson, W.F. 111

Haran, M.E. 91, 285

Hashmi, Z.R. 389

Haywood, F.F. 225

Herman, B.A. 91

Herman, W.A. 99

Hladik, W.B. 131

Holeman, G.R. $\quad 371$

Hubbell, J.H. 299

Hull, A.P. 153

Humphreys, J.C. 47

Hutchinson, J.M.R. $\quad 77$
Jacobson, A. 65

James, R. 215

Janes, D.E., Jr. 203

Jarvis, A.N. 263

Jones, D. 351

Kase, K.R. $\quad 343$

Kennedy, P. $\quad 111$

Keyser, R.M. 159

Lally, J.S. 223

Lamperti, P.J. $\quad 307,365$

Lanzl, L.H. $\quad 139$

Laughlin, J.S. $\quad 327$

Leiss, J.E. $\quad 403$

Levine, G.S. $\quad 153$

Lin, P.P. $\quad 381$

Lochamy, J.C. $\quad 169$

Loevinger, R. $\quad 37$

McDonald, J.C. $\quad 327$

McGarry, E.D. 83

McLaughiin, J. E. 233

Maddox, B.J. 343

Matuszek, J.M. 249

Miller, T.L. 53

Mills, W.A. $\quad 427$

Mohan, K. 215

Morgan, T.J. 389

Moroz, M.S. 395

Nelson, J.C. 203

Ohthaber, T.R. $\quad 53$

Osman, G. $\quad 313$

Ott, W.R. 107

Parker, G.S. $\quad 427$

Parrott, M.W. $\quad 31$

Parsons, D.F. 223

Peterson, R.W. $\quad 215$

Phillips, V.A. 223

Placious, R.C. $\quad 47$

Plazeski, P. $\quad 359$

Prevo, F.J. 53

Price, K.W. $\quad 371$

Pruitt, J.S. $\quad 37$

Rhodes, B. 131 
Sanders, A.A.

Schneider, R.H.

Schroder, I.G.

Schumacher, D.

Schwartz, R.B.

Seide 1, C.W.

Seltzer, S.M.

Shalek, R.J.

Shapiro, P.

Shaver, J.W.

Siedband, M.P.

Sorrows, H.E.

Smith, L.B.

Sparrow, J.H.

Spiege 1, V.

Spira, J.

Stewart, H.F.

Stova 11, M.

Svensson, G.K.

Swicord, M.L.

Taylor, L.S.

Te11, R.A.

Thiel, J.F.

Trout, E.D.

Vukan, J.A.

Walford, G.V.

Washington, $J$.

Weiss, B.H.

Widel1, C.

Williams, E.F. 
NBS-114A (REV. 7-73)

\begin{tabular}{|c|c|c|c|}
\hline $\begin{array}{l}\text { U.S. DEPT. OF COMM. } \\
\text { BIBLIOGRAPHIC DATA } \\
\text { SHEET }\end{array}$ & $\begin{array}{l}\text { 1. PUBLICATION OR REPORT NO. } \\
\text { NBS SP-456 }\end{array}$ & $\begin{array}{l}\text { 2. Gov't Accession } \\
\text { No. }\end{array}$ & 3. Recipient's Accession No. \\
\hline \multirow{2}{*}{\multicolumn{3}{|c|}{$\begin{array}{l}\text { 4. TITLE AND SUBTITLE } \\
\text { Measurements for the Safe Use of Radiation } \\
\text { Proceedings of an NBS } 75 \text { th ANNIVERSARY Symposium } \\
\text { held at the National Bureau of Standards } \\
\text { Gaithersburg, Maryland, March 1-4, } 1976\end{array}$}} & $\begin{array}{l}\text { 5. Publication Date } \\
\text { November } 1976\end{array}$ \\
\hline & & & 6. Performing Organization Code \\
\hline \multicolumn{3}{|c|}{ 7. AUTHOR(S) } & 8. Performing Organ. Report No. \\
\hline \multirow{2}{*}{\multicolumn{3}{|c|}{$\begin{array}{l}\text { 9. PERFORMING ORGANIZATION NAME AND ADDRESS } \\
\text { NATIONAL BUREAU OF STANDARDS } \\
\text { DEPARTMENT OF COMMERCE } \\
\text { WASHINGTON, D.C. } 20234\end{array}$}} & 10. Project/Task/Work Unit No. \\
\hline & & & 11. Contract/Grant No. \\
\hline \multirow{3}{*}{\multicolumn{3}{|c|}{$\begin{array}{l}\text { 12. Sponsoring Otganization Name and Complete Address (Street, City, State, ZIP) } \\
\text { National Bureau of Standards } \\
\text { American Association of Physicists in Medicine } \\
\text { Conference of Radiation Control Program Directors } \\
\text { Health Phy sics Society }\end{array}$}} & $\begin{array}{l}\text { 13. Type of Report \& Period } \\
\text { Covered }\end{array}$ \\
\hline & & & 14. Sponsoring Agency Code \\
\hline \multirow{2}{*}{\multicolumn{4}{|c|}{$\begin{array}{l}\text { 15. SUPPLEMEN TARY NOTES } \\
\qquad \text { Library of Congress Catalog Card Number: 76-600060 }\end{array}$}} \\
\hline & & & \\
\hline \multicolumn{4}{|c|}{$\begin{array}{l}\text { 16. ABSTRACT (A 200-word or less factual summary of most significant information. If document includes a significant } \\
\text { bibliography or literature survey, mention it here.) }\end{array}$} \\
\hline \multicolumn{4}{|c|}{$\begin{array}{l}\text { These proceedings are the compilation of papers presented at the } \\
\text { Symposium, "Measurements for the Safe Use of Radiation", held in } \\
\text { Gaithersburg, Maryland, March 1-4, 1976. The symposium addressed the } \\
\text { present status and future requirements for measurements of ionizing and } \\
\text { non-ionizing radiation. Emphasis was placed on the needs for } \\
\text { standardization and measurement assurance activities in medical, } \\
\text { environmental, and personnel protection applications. }\end{array}$} \\
\hline
\end{tabular}

17. KEY WORDS (six to twelve entries; alphabetical order; capitalize only the first letter of the first key word unless a proper name; separated by semicolons)

Calibrations; environment; measurements; medical; radiation; standards

18. AVAILABILITY $\bar{x}$ Unlimited

For Official Distribution. Do Not Release to NTIS

$X$ Order From Sup. of Doc., U.S. Government Printing Office

Washington, D.C. 20402, SD Cat. No. C13.10:456

$\square$ Order From National Technical lnformation Service (NTIS) Springfield, Virginia 22151

\begin{tabular}{|l|c|}
\hline $\begin{array}{c}\text { 19. SECURITY CLASS } \\
\text { (THIS REPORT) }\end{array}$ & 21. NO. OF PAGES \\
UNCL ASSIFIED & 437 \\
\hline $\begin{array}{c}\text { 20. SECURITY CLASS } \\
\text { (THIS PAGE) } \\
\text { UNCLASSIFIED }\end{array}$ & $\begin{array}{c}\text { 22. Price } \\
\$ 5.15\end{array}$ \\
\hline
\end{tabular}





\section{NBS TECHNICAL PUBLICATIONS}

\section{PERIODICALS}

JOURNAL OF RESEARCH reports National Bureau of Standards research and development in physics, mathematics, and chemistry. It is published in two sections, available separately:

- Physics and Chemistry (Section A)

Papers of interest primarily to scientists working in these fields. This section covers a broad range of physical and chemical research, with major emphasis on standards of physical measurement, fundamental constants, and properties of matter. Issued six times a year. Annual subscription: Domestic, $\$ 17.00$; Foreign, $\$ 21.25$.

- Mathematical Sciences (Section B)

Studies and compilations designed mainly for the mathematician and theoretical physicist. Topics in mathematical statistics, theory of experiment design, numerical analysis, theoretical physics and chemistry, logical design and programming of computers and computer systems. Short numerical tables. Issued quarterly. Annual subscription: Domestic, $\$ 9.00$; Foreign, $\$ 11.25$.

DIMENSIONS/NBS (formerly Technical News Bulletin)-This monthly magazine is published to inform scientists, engineers, businessmen, industry, teachers, students, and consumers of the latest advances in science and technology, with primary emphasis on the work at NBS. The magazine highlights and reviews such issues as energy research, fire protection, building technology, metric conversion, pollution abatement, health and safety, and consumer product performance. In addition, it reports the results of Bureau programs in measurement standards and techniques, properties of matter and materials, engineering standards and services, instrumentation, and automatic data processing.

Annual subscription: Domestic, $\$ 9.45$; Foreign, $\$ 11.85$.

\section{NONPERIODICALS}

Monographs-Major contributions to the technical literature on various subjects related to the Bureau's scientific and technical activities.

Handbooks-Recommended codes of engineering and industrial practice (including safety codes) developed in cooperation with interested industries, professional organizations, and regulatory bodies.

Special Publications-Include proceedings of conferences sponsored by NBS, NBS annual reports, and other special publications appropriate to this grouping such as wall charts, pocket cards, and bibliographies.

Applied Wathematics Series-Mathematical tables, manuals, and studies of special interest to physicists, engineers, chemists, biologists, mathematicians, computer programmers, and others engaged in scientific and technical work.

National Standard Reference Data Series-Provides quantitative data on the physical and chemical properties of materials, compiled from the world's literature and critically evaluated. Developed under a world-wide program coordinated by NBS. Program under authority of National Standard Data Act (Public Law 90-396).
NOTE: At present the principal publication outlet for these data is the Journal of Physical and Chemical Reference Data (JPCRD) published quarterly for NBS by the American Chemical Society (ACS) and the American Institute of Physics (AIP). Subscriptions, reprints, and supplements available from ACS, 1155 Sixteenth St. N.W., Wash. D. C. 20056.

Building Science Series-Disseminates technical information developed at the Bureau on building materials, components, systems, and whole structures. The series presents research results, test methods, and performance criteria related to the structural and environmental functions and the durability and safety characteristics of building elements and systems.

Technical Notes-Studies or reports which are complete in themselves but restrictive in their treatment of a subject. Analogous to monographs but not so comprehensive in scope or definitive in treatment of the subject area. Often serve as a vehicle for final reports of work performed at NBS under the sponsorship of other government agencies.

Voluntary Product Standards-Developed under procedures published by the Department of Commerce in Part 10, Title 15, of the Code of Federal Regulations. The purpose of the standards is to establish nationally recognized requirements for products, and to provide all concerned interests with a basis for common understanding of the characteristics of the products. NBS administers this program as a supplement to the activities of the private sector standardizing organizations.

Consumer Information Series-Practical information, based on NBS research and experience, covering areas of interest to the consumer. Easily understandable language and illustrations provide useful background knowledge for shopping in today's technological marketplace.

Order above NBS publications from: Superintendent of Documents, Government Printing Office, Washington, D.C. 20402 .

Order following NBS publications-NBSIR's and FIPS from the National Technical Information Services, Springfield, Va. 22161.

Federal Information Processing Standards Publications (FIPS PUBS)-Publications in this series collectively constitute the Federal Information Processing Standards Register. Register serves as the official source of information in the Federal Government regarding standards issued by NBS pursuant to the Federal Property and Administrative Services Act of 1949 as amended, Public Law 89-306 (79 Stat. 1127), and as implemented by Executive Order 11717 (38 FR 12315, dated May 11, 1973) and Part 6 of Title 15 CFR (Code of Federal Regulations).

NBS Interagency Reports (NBSIR)-A special series of interim or final reports on work performed by NBS for outside sponsors (both government and non-government). In general, initial distribution is handled by the sponsor; public distribution is by the National Technical Information Services (Springfield, Va. 22161) in paper copy or microfiche form.

\section{BIBLIOGRAPHIC SUBSCRIPTION SERVICES}

The following current-awareness and literature-survey bibliographies are issued periodically by the Bureau:

Cryogenic Data Center Current Awareness Service. A literature survey issued biweekly. Annual subscription: Domestic, $\$ 20.00$; Foreign, $\$ 25.00$.

Liquified Natural Gas. A literature survey issued quarterly. Annual subscription: $\$ 20.00$.
Superconducting Devices and Materials. A literature survey issued quarterly. Annual subscription: $\$ 20.00$. Send subscription orders and remittances for the preceding bibliographic services to National Bureau of Standards, Cryogenic Data Center (275.02) Boulder, Colorado 80302 . 
U.S. DEPARTMENT OF COMMERCE

National Bureau of Standards

Washington, D.C. 20234

POSTAGE ANO FEES PAID

OFFICIAL BUSINESS U.S. DEPARTMENT DF COMMERCE $C O M-295$

Penalty for Private Use. $\$ 300$ 


